KAPL, InC.

Knolls Atomic Power Laboratory

P.0. Box 1072

Schenectady, NY 12301-1072

Bechte/ Bettis, Inc.

Bettis Atomic Power Laboratory

P. O. Box 79

West Mifflin, PA 15122-0079

MDO-723-0044

January 31, 2006

Page 1

The Manager

Schenectady Naval Reactors Office

U.S. Department of Energy

Schenectady, New York

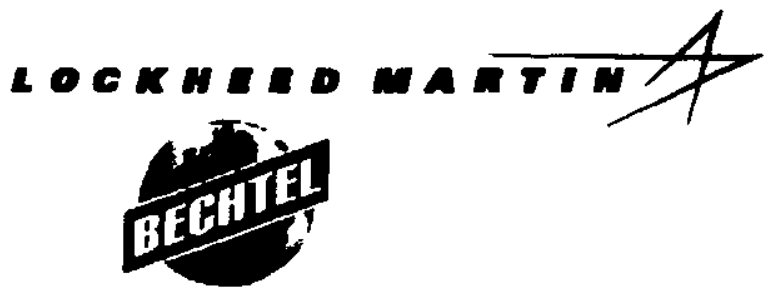

B-MT(SRME)-52

Subject: $\quad$ JOYO-1 Irradiation Test Campaign Technical Close-out, For Information

Enclosures: (1) JOYO-1 Structural Refractory Metal Materials Specimen Fabrication Efforts

(2) JOYO-1 Superalloy Specimen Fabrication Efforts

(3) JOYO-1 Refractory Metal (Liner Materials) Specimen Fabrication Efforts

(4) JOYO-1 Silicon Carbide Specimen Fabrication Efforts

(5) JOYO-1 Beryllium Oxide Specimen Fabrication Efforts

(6) JOYO-1 Temperature Monitors and Dosimetry Fabrication Efforts

(7) JOYO-1 Capsule Design Close-out Report

(8) Alternative Disposition of JOYO-1 Structural Materials Irradiation Rigs

(9) JOYO-1 Post-Irradiation Examination

Dear Sirs:

The JOYO-1 irradiation testing was designed to screen the irradiation performance of candidate cladding, structural and reflector materials in support of space reactor development. The JOYO- 1 designation refers to the first of four planned irradiation tests in the JOYO reactor. Limited irradiated material performance data for the candidate materials exists for the expected Prometheus-1 duration, fluences and temperatures. Materials of interest include fuel element cladding and core materials (refractory metal alloys and silicon carbide (SiC)), vessel and plant structural materials (refractory metal alloys and nickel-base superalloys), and control and reflector materials (BeO). Key issues to be evaluated were long term microstructure and material property stability. The JOYO-1 test campaign was initiated to irradiate a matrix of specimens at prototypical temperatures and fluences anticipated for the Prometheus-1 reactor [Reference (1)]. Enclosures 1 through 9 describe the specimen and temperature monitors/dosimetry fabrication efforts, capsule design, disposition of structural material irradiation rigs, and plans for post-irradiation examination. These enclosures provide a detailed overview of Naval Reactors Prime Contractor Team (NRPCT) progress in specific areas; however, efforts were in various states of completion at the termination of NRPCT involvement with and restructuring of Project Prometheus. 
The Manager, SNR

The Manager, PNR
MDO-723-0044

B-MT(SRME)-52

Page 2 of 16

\section{Background Review}

The Experimental Fast Reactor JOYO was selected as the test reactor for the initial materials screening test based on a review of candidate international fast spectrum test reactors such as BOR-60 (Russia), Phenix (France), and others [Reference (2)]. A fast-neutron-spectrum test reactor was needed to provide near-prototypical conditions, accelerate testing, and to minimize transmutations due to thermal neutrons. JOYO is located at the O-arai Engineering Center (OEC) in O-arai, Japan and is operated by Japan Atomic Energy Agency's (JAEA) Experimental Reactor Division within the Irradiation Center of OEC. JAEA was formed in a recent merger between Japan Nuclear Cycle Development Institute (JNC) and Japan Atomic Energy Research Institute (JAERI). JOYO's rated power is $140 \mathrm{MW}_{\mathrm{t}}$, with a peak fast flux of approximately $5.5 \mathrm{x}$ $10^{15} \mathrm{n} / \mathrm{cm}^{2}-\mathrm{s}$ (E>0.1 MeV). JOYO is a loop-type sodium-cooled liquid metal reactor (LMR) that utilizes a Mixed Oxide Fuel (MOX) as its fuel. Operating cycles of 60 effective full power days (EFPD) with approximately 20 days between cycles for refueling and experiment handling are typical. Nominal operating schedules include five cycles per year followed by approximately six to twelve months of down time for inspections and maintenance. Irradiation experiment planning, design and analysis are responsibilities of the Irradiation and Administration Section within the Irradiation Center.

NRPCT had limited experience with large international contracting efforts. Because of this and the aggressive schedule, Pacific Northwest National Laboratory (PNNL) was selected to contract directly with JAEA on behalf of the NRPCT. PNNL has international contracting experience and contacts with JAEA, as well as a business relationship with other Japanese firms. NRPCT was on target to deliver test specimens, capsules, and associated equipment for a June 2006 JOYO-1 test commencement. Specific challenges and status of tasks required to deliver specimens and complete the JOYO-1 irradiation test are described below. This letter constitutes the bulk of the close out documentation for the NRPCT JOYO-1 irradiation test campaign.

The materials testing planned for JOYO- 1 was designed to address uncertainties and data gaps in post-irradiation mechanical properties in temperature and fluence ranges envisioned for potential Prometheus-1 designs. Examples of irradiation effects on candidate material properties that were to be addressed by this test are shown in Table 1. The relative priority of each effect on each material type was indicated by the number of check marks. JOYO-1 testing was planned as part of a suite of key irradiation and out-of-pile tests. In addition to a more prototypical spectrum achievable in JOYO, extensive irradiation campaigns on fuel materials were planned for the Advanced Test Reactor (ATR) in Idaho and on structural materials in the High Flux Isotope Reactor (HFIR) at Oak Ridge National Laboratory (ORNL). HFIR testing was planned to provide complementary studies on refractory metal alloys and SiC materials. Silicon carbide materials can be tested in a thermal reactor because they are judged to have less concern with transmutation effects due to thermal neutron fluxes. Transmutation can adversely affect irradiated properties of susceptible materials. Collectively, these irradiation tests (including JOYO) were planned to aid in establishing an irradiated material property database for a reliable design. Individually, JOYO-1 results would also have been used to develop focused material matrices for subsequent test campaigns. 
Table 1 - Irradiation Effects of Interest for Alternative Core Structural Materials

\begin{tabular}{|c|c|c|c|c|c|}
\hline Material & $\begin{array}{c}\text { Microstructural } \\
\text { Stability }\end{array}$ & $\begin{array}{c}\text { Toughness } \\
\text { Degradation }\end{array}$ & $\begin{array}{c}\text { Irradiation- } \\
\text { enhanced } \\
\text { Creep Crack } \\
\text { Growth }\end{array}$ & $\begin{array}{c}\text { Irradiation-induced } \\
\text { or Enhanced } \\
\text { Creep/Swelling }\end{array}$ & $\begin{array}{c}\text { Degradation of } \\
\text { Thermal } \\
\text { Conductivity }\end{array}$ \\
\hline $\begin{array}{c}\text { Refractory } \\
\text { Metals }\end{array}$ & $\checkmark \checkmark \checkmark$ & $\checkmark \checkmark$ & $\checkmark$ & $\checkmark \checkmark \checkmark$ & $\checkmark$ \\
\hline $\begin{array}{c}\text { SiC/SiC } \\
\text { Composite }\end{array}$ & $\checkmark$ & $\checkmark \checkmark$ & $\checkmark$ & $\checkmark \checkmark$ & $\checkmark \checkmark$ \\
\hline $\begin{array}{c}\text { Ferrous } \\
\text { Alloys }\end{array}$ & $\checkmark$ & $\checkmark \checkmark$ & $\checkmark \checkmark \checkmark$ & $\checkmark$ \\
\hline $\begin{array}{c}\text { Ni -based } \\
\text { Superalloys }\end{array}$ & $\checkmark \checkmark \checkmark$ & $\checkmark \checkmark$ & $\checkmark$ & $\checkmark$ & $\checkmark$ \\
\hline
\end{tabular}

The reffactory and SiC materials are being considered primarily for cladding and core applications, while conventional alloys are under investigation as potential vessel and plant structural applications.

Principal effects to be evaluated in JOYO-1 included irradiation embrittlement, void and defect swelling, irradiation creep, microstructural (phase) stability, transmutation effects, and thermal and physical property stability. To understand irradiation effects on these properties, baseline unirradiated material properties were documented in Reference (3). A rationale for JOYO-1 material choices and test conditions is detailed in Reference (1) with further refinement of the materials test matrix established in Reference (4). This refinement was necessary to finalize specific issues identified in the test matrix: (1) specify target temperatures of all capsules, (2) balance the number of superalloy specimens, and (3) specify the mix of $\mathrm{SiC}$ forms (monolithic and composite).

Additional details of irradiation effects on nickel-base superalloys and beryllium oxide (BeO) properties were provided in References (5) and (6), respectively. To conclude the JOYO-1 test planning phase, a design review was conducted with an independent committee [Reference (7)]. The temperature ranges included in this test approximate the temperatures expected for reactor operation. Materials would have been tested in the range of $850 \mathrm{~K}$ to $1050 \mathrm{~K}$ (nominal) for the vessel and plant structural applications (e.g., piping) and $850 \mathrm{~K}$ to $1350 \mathrm{~K}$ (nominal) for the cladding and core structural applications. The actual temperature experienced by each specimen was to be controlled through manipulation of: axial location in the reactor, gas gap thickness, gas content, and gamma heating rates of the specimen holders.

\section{Test Specimen Summary}

This section details the work carried out through the beginning of fiscal year 2006 to fabricate specimens for irradiation in JOYO-1 and related out-of-pile testing. Table 2 lists the various materials and specimen types for the JOYO-1 irradiation test campaign. A total of 1180 material specimens were in the process of fabrication to support JOYO testing. Approximately 515 of these specimens were to be tested during the JOYO-1 test campaign. Figure 1 identifies the approximate specimen loading configuration and location of each capsule as positioned in the two Structural Materials Irradiation Rigs (SMIR) in the JOYO reactor. 
Specimen fabrication was in various states of progress at project restructuring. Novel testing designs, such as bend stress relaxation (BSR) and creep crack growth compact tension specimens were proposed to evaluate irradiation effects on creep (in SiC) and crack growth with in-situ loading (in Mo-47.5 Re and nickel-base superalloys). BSR specimen fabrication was completed; however, locating a vendor to pre-crack the in-situ loaded crack growth specimens was problematic. Proof-of-principle design and fabrication work had already been established for BSR specimens at ORNL. Aside from BSR and crack growth with in-situ loading, specimen types fabricated for JOYO-1 were conventional, but sub-sized, ASTM specimen types. All types are traceable or comparable to those established in various ASTM testing standards, as miniature specimen sizes are accommodated by these standards. Plans also included out-ofpile comparisons between uniaxial (ASTM) and biaxial creep testing. Biaxial creep specimens have been demonstrated to provide data comparable to these uniaxial specimens.

Table 2

\begin{tabular}{|l|c|c|c|c|c|c|r|r|}
\hline \multicolumn{7}{|c|}{ JOYO-1 Specimen Count -- Rigs A \& B } \\
\hline Material & $\begin{array}{c}\text { Biaxial } \\
\text { Croep }\end{array}$ & $\begin{array}{c}\text { Fracture } \\
\text { Toughness } \\
\text { Bars }\end{array}$ & $\begin{array}{c}\text { Compact } \\
\text { Tonsion } \\
\text { Discs }\end{array}$ & Tensile & $\begin{array}{c}\text { Density \& } \\
\text { Diffusivity } \\
\text { Discs }\end{array}$ & $\begin{array}{c}\text { Comprossion } \\
\text { Cylinders }\end{array}$ & BSR & $\begin{array}{c}\text { Material } \\
\text { Totals }\end{array}$ \\
\hline Astar-811C & 24 & & & 53 & 18 & & & 95 \\
\hline Mo-47.5Re & 22 & & & 67 & 17 & & & 106 \\
\hline Re & 22 & 48 & 16 & 39 & 32 & & & 157 \\
\hline W & & 8 & & 24 & 22 & & & 54 \\
\hline W-25Re & & 8 & & 23 & 21 & & & 52 \\
\hline Alloy 617 & 9 & 54 & 8 & 65 & 40 & & & 176 \\
\hline Haynes 230 & 9 & 54 & 8 & 54 & 41 & & & 166 \\
\hline Nimonic PE16 & 9 & 54 & 8 & 61 & 41 & & & 173 \\
\hline monolithic SiC & & & & 12 & 24 & & 16 & 52 \\
\hline Composite SiC & & & & 24 & 24 & & & 48 \\
\hline BeO & & & & & 32 & 16 & & 48 \\
\hline \multicolumn{1}{|c|}{ Specimen Totals } & 95 & 234 & 40 & 446 & 333 & 16 & 16 & 1180 \\
\hline \multicolumn{1}{|c|}{ TotaI } & & & & 1180 & & & & \\
\hline
\end{tabular}

Materials were included in the JOYO-1 specimen matrix based on the most promising properties for the applications listed below, as determined from available literature and databases.

- Fuel element cladding and core internals (refractory metals and SiC materials)

- Vessel and plant structural components (refractory metals and nickel-based superalloys)

- Control and reflector materials (BeO)

Specific choices for combinations of materials and specimen types took into account:

- Gaps or lack of fast spectrum test data for specific mechanical properties

- A potential for a particular material to represent an entire class of materials 
The Manager, SNR

MDO-723-0044

The Manager, PNR

B-MT(SRME)-52

Page 5 of 16

- A potential for a particular specimen type to provide the most pertinent and widest range of relevant property information (balancing breadth and depth)

- Constraints on specimen delivery due to material availability, fabrication and processing complexity, machining difficulty, and vendor experience/capability.

- Irradiation capsule volume constraints, thermal and fluence responses of candidate materials

- Post-irradiation testing capabilities and shipping and waste logistics.

During specimen fabrication, some machining and processing difficulties were realized, but the flexibility built-in to the capsule assembly process allowed for limited specimen delivery delays. The NRPCT was in a position to deliver assembled capsules in time to support reactor insertion need dates. Specimen fabrication and processing efforts have been documented [References (8), (9), and (10)] and proper inventory and storage of fabricated parts has been completed.

\section{Structural Refractory Metal Materials}

Three refractory metal alloys, representing different alloy classes, were candidates for cladding and core applications for Prometheus-1. These classes included tantalum-base (ASTAR$811 \mathrm{C}$ ), niobium-base (FS-85), and molybdenum-base (Mo-47.5Re) alloys. Only one alloy was chosen from each of these classes because of limited space available for test specimens in the JOYO rigs. Alloys were chosen based on their individual attributes, their ability to represent alloys of the base metal as a class, and their availability in the time frame and quantities needed for the test. NRPCT was interested in post-irradiation thermal creep, irradiation creep, irradiation embrittlement, microstructure stability and toughness performance of these alloys.

The fabrication of the refractory metal alloys was being undertaken by vendors familiar with the processing requirements of these unique and high-potential alloys. The tantalum and niobiumbase alloys are the easiest of the refractory alloys to process but also are most prone to contamination during annealing in poor environments or via contact with non-refractory metal alloys during other process steps. This concern is not limited to processing but also applies to usage. Molybdenum alloys are less prone to contamination during processing or usage but are also tougher to process and weld because they usually exhibit a Ductile-Brittle-TransitionTemperature (DBTT) that is below room temperature in the wrought condition but above room temperature in the welded condition. Molybdenum-rhenium alloys are believed to exhibit significantly improved weld behavior to both pure molybdenum and most commercial molybdenum alloys although this was still under active investigation at the time of project restructuring. Contamination and processing issues remain the major concerns for employment of refractory metal alloys. An additional concern existed with respect to evaluation of irradiated tantalum alloys because of the high residual activity remaining after exposure. The activity would not have allowed transmission electron microscopy exams and may have hindered handling of specimens in a timely manner.

Tensile specimens of all three alloys were in the process of being fabricated. Biaxial creep specimens required the most significant time investment because of the multi-step fabrication process. Biaxial creep specimen parts had been fabricated for ASTAR-811C and FS-85. Material delivery for Mo-47.5Re was behind schedule, and tubing was still being formed at Rhenium Alloys (Cleveland, $\mathrm{OH}$ ). Processing of these specimen parts and other specimens is detailed in References ( 8 and 9). A welding process for ASTAR-811C and FS-85 biaxial creep specimens was being developed at PNNL. Electron beam welding of end-caps to tubes was successfully developed for both materials. Laser-seal welding of the pressurization hole in the 
The Manager, SNR

MDO-723-0044

The Manager, PNR

B-MT(SRME)-52

Page 6 of 16

top end-cap was still being developed and would have required further work. Prior success with biaxial creep tube sample fabrication of these alloys indicated that this would not be a major issue with respect to schedule. Details of biaxial creep specimen fabrication efforts are provided in Reference (9).

Enclosure 1 and References $(8,9$, and 10) provide details of the proposed JOYO-1 refractory metals specimen matrix, along with specimen disposition, materials issues, and vendor fabrication details.

\section{Structural Nickel-based Superalloys}

Nickel-based superalloys were under consideration for structural plant and core applications for Prometheus-1. Three structural nickel-base superalloys were chosen for the first JOYO irradiation test: Alloy 617, Haynes 230, and Nimonic PE16. Similar to the refractory alloys, the main interest was with nickel-base superalloy post-irradiation thermal creep, irradiation creep, microstructural stability and toughness performance.

Procurement of stock nickel-base superalloys and subsequent machining into specimens was progressing successfully. These alloys were available commercially in sufficient stock supplies of several product forms to begin machining, although the sample microstructures of these samples may not have been commensurate with that in any final material specification. Hence, nickel-base superalloy specimen fabrication was on schedule. Over 500 nickel-base superalloy specimens were fabricated to support the JOYO-1 test matrix as well as out-of-pile testing to differentiate thermal effects from radiation effects. Based on a few stock limitations, plans to procure larger quantities of the materials and product forms chosen for eventual Prometheus-1 structures required longer lead purchase orders.

Biaxial creep specimen fabrication was progressing toward a full qualification procedure. This included welding end caps, pressurized seal welding, and hermeticity verification. Machining of nickel-base superalloy tensile, fracture toughness and density disc specimens was completed without significant difficulty. Development of precracking parameters for nickel-base superalloy subsized fracture toughness bars was initiated in-house. Drawings for $0.2 T$ compact tension disc specimens and the fixtures necessary to bolt-load them (for in-pile crack growth rate testing) were created. Development of a precracking and bolt-loading procedure was planned for these first-of-a-kind specimens.

Enclosure 2 provides details of the proposed JOYO-1 superalloy specimen matrix, along with specimen disposition, material issues, and vendor fabrication details.

\section{Refractory Metal Liner Materials}

Refractory metal liner materials were considered for use as a diffusion barrier (liner) between the fueled region and cladding. Additionally, a liner may act as a barrier to contain fission products and serve as an interface between potentially incompatible materials. Over 150 specimens were to be fabricated for the JOYO-1 irradiation test, as well as replicates for out-ofpile testing to evaluate thermal degradation with respect to radiation effects. Refractory metals and alloys of interest included tungsten, tungsten-25\% rhenium (W-25Re), and rhenium. As pure tungsten has poor fracture toughness, the NRPCT pursued potassium doping to improve high temperature properties. Doping tungsten to a level of 50-100 ppm potassium allows for 
The Manager, SNR

The Manager, PNR

MDO-723-0044

B-MT(SRME)-52

Page 7 of 16

improved creep strength with no significant activation or neutronic effects [Reference (11)]. Specimen types included tensile, fracture toughness, and density discs.

Refractory metal liner materials of interest are not routinely manufactured and typically are not kept in-stock at material suppliers. Initial plans were to fabricate specimens from material forms (thin plate materials) that would be prototypic of those anticipated for production liners. Several factors, however, resulted in a re-evaluation of liner production methods. These included:

- availability/cost

- ductility and associated fabrication methods

- concerns about joining/welding

Materials received were rhenium sheets, potassium-doped tungsten rod, W-25Re sheet and small diameter $(0.025 ")$ W-25Re rod (sinter-swaged). Contracts for fabrication of these materials were not placed prior to project restructuring. Similar to the case of the nickel-base superalloys, vendors had significant reservations about their ability to pre-crack the sub-sized fracture toughness specimens. Therefore, an in-house effort was initiated to develop a precracking process. Three fracture toughness bars of $\mathrm{W}-25 \mathrm{Re}$ and potassium-doped tungsten were electro-discharge machined in-house to perform an expedited pre-cracking evaluation. Testing to obtain pre-cracking results was not completed.

Enclosure 3 contains the details of the proposed JOYO-1 refractory metal liner specimen matrix, along with specimen disposition, material issues, and vendor fabrication details.

\section{Silicon Carbide (SiC)}

The NRPCT considered silicon carbide in various forms for Prometheus core and cladding structural materials. For a ceramic fuel cladding material system, significant challenges include assuring hermeticity with respect to fission products and avoidance of brittle failure. For Prometheus, a hermetic ceramic clad design was pursued that would have consisted of a combination of monolithic and composite SiC materials in a duplex and/or triplex configuration. These configurations, i.e., the laminate (duplex/triplex) structures, were under development but would not have been ready for a JOYO- 1 insertion. Therefore, specimens fabricated from the basic constituent materials, monolithic and composite $\mathrm{SiC}$, were prepared for the JOYO-1 irradiation testing. Future irradiation testing in HFIR and JOYO were planned for the more complex arrangements.

Fabrication of monolithic and composite SiC specimens for the JOYO-1 irradiation test campaign was completed. Chemical vapor deposited (CVD) monolithic SiC was chosen for JOYO- 1 as a baseline material. The $\mathrm{SiC}$ composite consisted of Hi-Nicalon Type S fibers in a Chemical Vapor Infiltration (CVI) SiC matrix. For these materials, miniature tensile specimens and thermal diffusivity discs were fabricated. Use of a miniature tensile specimen design permitted inclusion of sufficient replicates to produce statistically reliable results. This was important for brittle materials like monolithic $\mathrm{SiC}$, which typically have large strength variability.

For monolithic SiC only, Bend Stress Relaxation (BSR) specimens for (irradiation) creep estimation were procured for JOYO-1. This testing may have provided new data for irradiation induced stress relaxation, thus providing a potential design relaxation for stress build up in a SiC core/clad design. In this technique, the BSR fixture holds monolithic thin strip $(50,100$ and 200 $\mu \mathrm{m}$-thick) specimens at a defined radius during irradiation. Creep estimation is determined by comparing the specimen radius before and after testing. BSR techniques allow detection of 
The Manager, SNR

The Manager, PNR
MDO-723-0044

B-MT(SRME)-52

Page 8 of 16

small creep rates $\left(\sim 10^{-11} \mathrm{~s}^{-1}\right)$ that are observed in $\mathrm{SiC}$.

Initial thermal diffusivity and tensile testing was completed for both monolithic and composite $\mathrm{SiC}$ to characterize their baseline properties, however, testing of the monolithic tensile specimens was unsuccessful because they broke in the clamps rather than at the gage section. To alleviate this issue, additional testing would have been required to identify appropriate modifications to the clamping devices.

Enclosure 4 contains the details of the SiC specimen matrix, along with specimen disposition, material issues, and vendor fabrication details.

\section{Beryllium Oxide (BeO)}

Beryllium oxide and beryllium were being considered for neutron reflectors in the Prometheus-1 reactor. Beryllium oxide has very low neutron absorption and high number density, is excellent at moderating fast flux neutrons, and has high temperature capability $\left(T_{m}=2840 \mathrm{~K}\right)$ due to its ceramic nature. In addition to its moderating capability, previous nuclear design studies favored BeO due to its high thermal conductivity for such applications. Irradiation swelling was the main concern for reflector materials.

Upon program restructuring, it had not been concluded that $\mathrm{BeO}$ was more desirable than beryllium as a radial reflector material in low temperature applications from either a mass, cost, fabricability or simplicity standpoint. Beryllium oxide was, however, considered a leading material candidate for high temperature axial reflector applications. For JOYO-1 irradiation testing, a single grade of $\mathrm{BeO}$ was selected: $\mathrm{BW}-1000$ manufactured by Brush Ceramic Products. This grade was selected because of its fine grain size (10 $\mu \mathrm{m}$ nominal) and historical documentation implying improved itradiation performance of fine grain sized BeO [Reference (12)]. BeO discs were proposed for thermal diffusivity, density and swelling measurements while cylinders were proposed for compression, density and swelling measurements. Material property testing of unirradiated BeO was completed for baseline purposes.

Beryllium oxide as a particulate is a hazardous material that may cause lung diseases and skin irritations. Brush Ceramic Products, as well as other DOE facilities investigated (BWXT/Y-12 and Los Alamos National Laboratory) have taken extensive measures to control this hazard, including protective equipment, engineered ventilation controls at source points and throughout buildings including interlocked air showers, dressing rooms, training, and administrative controls. To avoid potential health hazards at NRPCT facilities, formal cleaning, handing and packaging requirements were established for all BeO specimens prior to shipment. Because loose BeO particulate was of greatest concern from a health and safety standpoint, all processes generating powder (e.g., machining, grinding etc.) were to be completed by Brush Ceramic Products at their facility. Upon project restructuring, Brush Ceramic Products stopped BeO test specimen fabrication. No BeO was sent to NRPCT facilities.

Enclosure 5 contains details of the BeO specimen matrix, along with material issues, vendor fabrication details and special requirements associated with BeO health and safety (i.e. cleaning, handling and shipping).

\section{Temperature Monitors and Dosimetry}

Passive temperature monitors and dosimeters were being fabricated for use in the JOYO-1 
The Manager, SNR

The Manager, PNR
MDO-723-0044

B-MT(SRME)-52

Page 9 of 16

irradiation test. Temperature monitors were machined from silicon carbide for temperatures below $1250 \mathrm{~K}$ and from zirconium carbide $(\mathrm{ZrC})$ for temperatures above $1250 \mathrm{~K}$. Machining of the $\mathrm{SiC}$ passive temperature monitors was completed, while machining of the $\mathrm{ZrC}$ monitors was incomplete. The machining vendor had difficulty fabricating the $\mathrm{ZrC}$ monitors because the base material ( $\mathrm{ZrC}$ rod stock) was breaking into many pieces during the machining operation. $A$ limited investigation did not identify reasons for the unexpected breaking; therefore a corrective action could not be identified. Machining was stopped, and the remaining $\mathrm{ZrC}$ was returned.

A limited neutron flux dosimetry set (also called flux wire assemblies) consisting of iron, nickel, and titanium wires was specified for use with selected JOYO capsules. These wires were a sub-set of a "full" flux dosimetry package sometimes used in JOYO by JAEA. The sub-set was selected because the full JOYO package required use of the space between capsules in the SMIR, thus reducing the space available for specimens. Since the limited dosimetry set required less space, it allowed the dosimetry to be located closer to the test specimens. This would produce results more representative of the specimen exposures. The iron, nickel, and titanium wires were judged to provide sufficient neutron spectral sensitivity to confirm (or adjust) calculated neutron fluence values. NRPCT planned to install a flux wire assembly into five of the ten JOYO-1 capsule types. Flux wire assemblies were ordered, delivered, inspected, and certified per the purchase order and sketch requirements.

Enclosure 6 contains the details of the temperature sensors and dosimetry, along with their disposition, material issues, and vendor fabrication details.

\section{Summary of International Contracting and Interactions with JAEA}

NRPCT contracted with PNNL to facilitate contracting for JOYO-1 irradiation testing with JAEA. NRPCT selected PNNL for several reasons. PNNL had recently conducted a review of the world's fast spectrum test reactors and was familiar with the NRPCT planned testing. PNNL has experience in international irradiation programs and placing contracts with Japanese firms, including an understanding and experience with appropriate export control laws. During the contract period, PNNL placed the contract with JAEA and served as a communication liaison while they reviewed JAEA quality program(s), export issues, and shipping. Overall program management for irradiation testing in JOYO, however, resided with NRPCT. A summary of PNNL involvement as well as the NRPCT perspective in JOYO contracting is included in Reference (13).

NRPCT concluded that the "Peaceful Uses Agreement"1 and "Nuclear Technologies Agreement ${ }^{\text {22 }}$ (or NTA) were applicable to this work and that proposed JOYO-1 irradiation experiments needed to be performed within the bounds of these existing agreements. An outline of these and other required agreements is shown in Figure 2. To accomplish the JOYO1 test program, NRPCT identified that a Specific Memorandum of Agreement (SMA) needed to be established between Naval Reactors (DOE) and JAEA under the NTA. The NTA was set to expire on August 22, 2005; however, any SMA signed prior to the NTA expiration would remain active for the duration of proposed testing. JAEA and NR had reached agreement on the SMA wording, but MEXT would not allow JAEA to sign without establishing a new DOE/MEXT agreement to replace the expiring NTA. Therefore, the NTA expired without DOE/JAEA

\footnotetext{
1 "Agreement for Cooperation between the Government of the United States of America and the Government of Japan concerning peaceful uses of Nuclear Energy (Nov. 4, 1987)"

2 "Agreement between of the Department of Energy of the United States of America and the Japan Nuclear Cycle Development Institute in the fieid of Nuclear Technologies"
} 
renewal. NRPCT had identified options on how to proceed without the NTA, but were not acted upon before the project restructuring occurred. Additional details on international agreements are included in Reference (13).

\section{Capsule Design and Structural Material Irradiation Rig (SMIR) Design Summary}

Based on the analysis performed up to the time of project restructuring, NRPCT has shown that a viable capsule design capable of housing various material specimens could be fabricated for use in the JOYO reactor. The capsule design report included as Enclosure 7, defines the requirements the NRPCT planned to invoke for JOYO-1 capsule design, fabrication and shipping. Following a Design for Permission presentation [Reference (14)] for the capsule design, JAEA agreed that the design basis, materials, welding, quality control, and shipping requirements as stated in the Design for Permission report [Reference (15)] were acceptable. Specific structural, thermal and nuclear analyses in conjunction with a robust quality assurance program were demonstrated to JAEA at the design review meeting. This assured JAEA that NRPCT understood operational dynamics and testing parameters for JOYO and that capsule integrity would be maintained.

The NRPCT JOYO-1 capsule design was based on limited information from JAEA regarding the reactor operating environment and expected capsule requirements. Due to the lack of a signed SMA and communication difficulties, technical exchange of information was limited. Most information was gathered by the NRPCT at the four face-to-face meetings held in Japan with JAEA. Capsule design goals included establishing test temperatures for encapsulated materials, while maintaining a structurally sound pressure boundary. NRPCT reviewed and compared American and Japanese design standards and received JAEA concurrence on the proposed materials and design methods. The capsule design consisted of a stainless steel $(316 \mathrm{H})$ boundary with various internal specimen holder/insulator arrangements. Each capsule was to be filled with one of three predetermined mixtures of helium and argon gases, each with a different thermal conductivity. Specific gas mixtures were used along with various gap sizes between specimens, holders, and capsule bodies to achieve desired specimen temperatures.

The SMIR is a structural assembly designed to allow capsule/test specimen placement in specific flux ranges within JOYO. Each SMIR has seven compartments; six spaced as a hexagonal ring encompassing a center compartment. Each compartment has five tiers. Each tier represents a capsule location (see Figure 1). The NRPCT planned to utilize the six hexagonal ring compartments with the center compartment remaining empty. JAEA planned to use the empty compartment for structural stability and potentially additional reactor monitoring. This arrangement allowed for thirty capsules in each rig for a total of sixty capsules to be tested simultaneously using two SMIRs. Since JOYO-1 was intended to be an uninstrumented test, passive temperature monitors were chosen to indicate capsule temperatures. For JOYO-1 testing, the SMIRs were to be placed in the reflector region just outside the reactor fuel region. This area provided the necessary gamma and neutron flux to achieve desired material specimen temperatures. Additional details on the SMIR design are included in Enclosure 7.

\section{Structural Material Irradiation Riq Disposition Summary}

JAEA was fabricating parts for the JOYO-1 Structural Material Irradiation Rig (SMIR) to meet the June 2006 test commencement. The contract for these parts provided that they were considered contract 'consumables' and JAEA property. 
The Manager, SNR

The Manager, PNR

\author{
MDO-723-0044 \\ B-MT(SRME)-52 \\ Page 11 of 16
}

PNNL, on behalf of NRPCT, facilitated contract placement of the SMIR design with JAEA in May, 2005. JAEA fabricated sufficient parts such as handling heads, wrapper tubes, nozzles, and compartments to support SMIR assembly by March 2006. This would have allowed test capsule installation supporting a June 2006 test commencement. SMIR design incorporated previous JAEA designs and dimensions needed by NRPCT for capsule interface. Further, JAEA planned to submit a license request to Japan's Ministry of Education, Culture, Sports, Science and Technology (MEXT) to use the two SMIRs. JAEA was to disposition them following irradiation.

Following the announcement of project restructuring, JAEA agreed to consider an alternate U.S. use for the SMIRs in the event that the NRPCT would not be using them, if identified by January 20,2006 . NRPCT was unable to find a U.S. group that could benefit, so the SMIRs were turned over to JAEA for their unconditional use.

\title{
Shipping and Waste Summary
}

Many compliance issues exist with shipment and burial/disposal of irradiated materials both domestically and internationally. International shipments require obtaining import/export licenses, shipping approvals, cask licenses from authorities cognizant of the shipping/receiving country, and identifying importers (i.e., Nippon Express) and exporters (i.e., JAEA). Identifying waste streams and a final burial location is also a major issue that requires upfront planning. Aside from specimens that may be returned and stored at the PIE Contractor's site, a substantial amount of irradiated waste material was to be generated. These waste items would have included SMIRs owned by JAEA, NRPCT test capsules, and residual waste from any PIE operations. Japan does not have a centralized waste disposal program and can only store waste temporarily. JAEA stated that they would not dispose of stainless steel capsule material and any internals because these parts were owned by the NRPCT. JAEA agreed that they would dispose of the SMIRs, and would evaluate storing waste on an interim basis. Reference (16) discusses these and other logistical issues pertaining to international shipping and waste handling as they relate to JOYO-1 testing.

\section{Post-Irradiation Examination (PIE) Plans Summary}

PIE plans for JOYO-1 were being developed at the time of project restructuring. Several facilities were being evaluated for the JOYO-1 post irradiation examinations (PIE) including Oak Ridge National Laboratory (ORNL), Pacific Northwest National Laboratory (PNNL), Japan Atomic Energy Agency (JAEA), or any combination of the three. The final PIE location(s) would have been chosen based on several factors, including costs (including current facility capabilities and required upgrades), potential shipping issues and waste acceptance/burial requirements at the forefront of that decision. PIE testing was delineated into two sections by material type, ceramic or metallic. The large number of sub-sized specimens, multiple types of specimens and materials, and strict testing conditions (e.g., high temperature and high vacuum) necessitated that careful assessments be made of the candidate facilities. The PIE campaign was envisioned to be conducted in at least three phases such that high priority test data would have been obtained in the most rapid and cost-effective manner to provide input to reactor design studies.

Enclosure 9 summarizes the PIE plans that were under development and a limited assessment of PIE capabilities of candidate facilities. The NRPCT presented the PIE technical requirements for JOYO-1 irradiation test specimens to JAEA in order to discuss and clarify the testing needs 
[Reference (17)]. Further meetings with JAEA defined key testing issues, provided an understanding of current JAEA capabilities, and identified upgrades required in order to perform the examinations. Detailed cost analyses of performing PIE at the candidate locations had not been completed. NRPCT considered the most cost effective location to be either ORNL or PNNL; however, performing PIE at JAEA had advantages from a timing standpoint, if facility capabilities and waste stream issues could be overcome.

\section{Beyond JOYO-1 Testing}

JOYO-1 testing was planned as an initial screening test for candidate materials for Prometheus in a fast reactor. JOYO-1 was to be followed with two additional structural material test phases (JOYO-2 and JOYO-3) providing needed material data for Prometheus design applications. To be run simultaneously with JOYO-3 test phase in 2008 , a follow-on fuel materials test phase (JOYO-4) was being considered to better understand fuel performance in a fast reactor. 


\section{Concurrences}

This document has been reviewed and concurred with by the Managers of KAPL- Space Materials and Bettis- Advanced Materials Technology.

Prepared by:

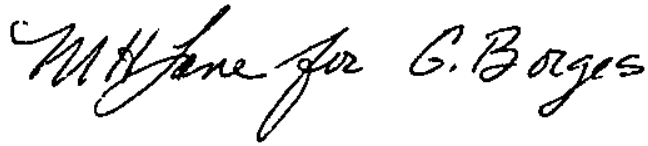

Guillermo Borges, Engineer Space Structural Materials

KAPL-Materials Development Operation

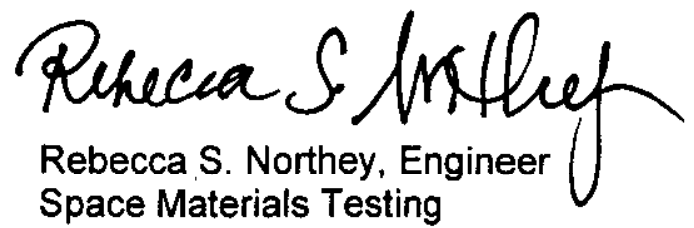

KAPL-Materials Development Operation

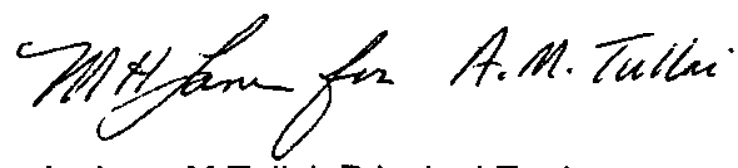

Andrew. M Tullai, Principal Engineer

Space Materials Irradiations

Bettis-Materials Technology

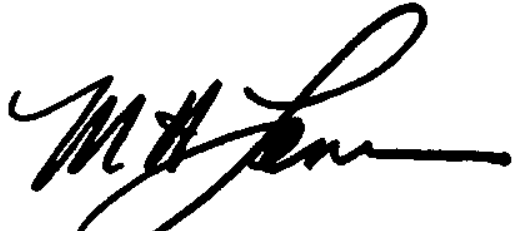

Midhaer H. Lane, Principal Engineer Space Materials Testing KAPL-Materials Development Operation

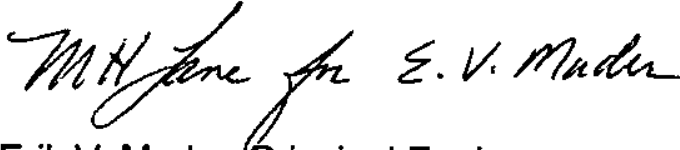
Erik V. Mader, Principal Engineer Space Reactor Materials Engineering Bettis-Materials Technology

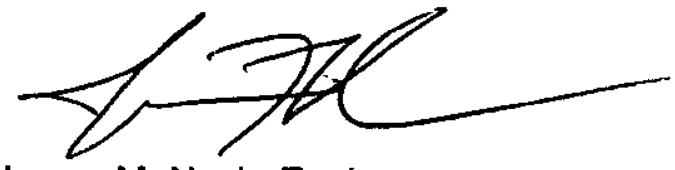
James M. Nash, Engineer Fuel and Shield Technology KAPL-Materials Development Operation

Approved by:

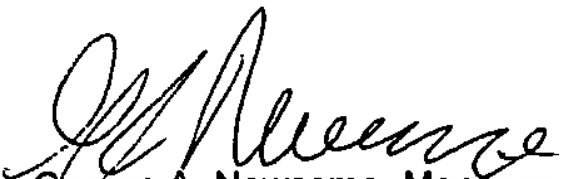

George A. Newsome, Manager Space Materials Testing

KAPL-Materials Development Operation

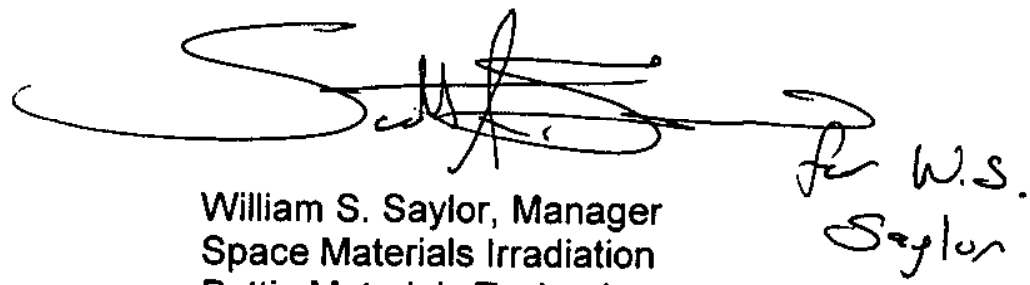

Space Materials Irradiation

Bettis-Materials Technology

Saglon

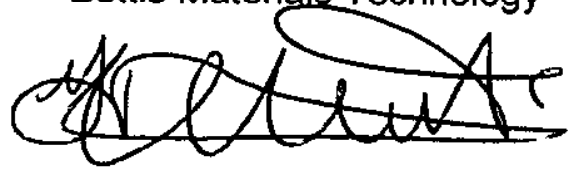

Youssef A. Ballout, Manager

Space Structural Materials KAPL-Materials Development Operation

Rita Baranwal, Acting Manager

Space Reactor Materials Engineering

Bettis-Materials Technology

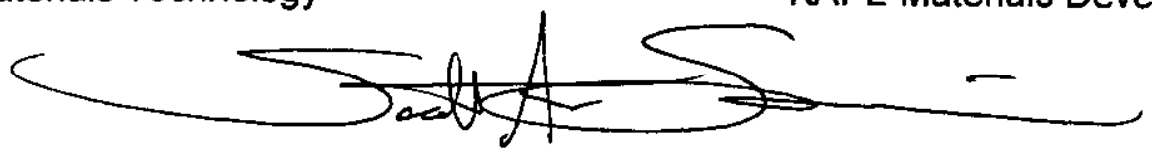

PRE-DECISIONAL - For planning and discussion purposes only 


\section{References}

(1) MDO-723-0021/B-MT(SRME)-21, "Request For Technical And Funding Approval Of The Test Matrix and Associated Changes For The JOYO-1 Irradiation Test of Structural Materials in the JOYO Experimental Fast Reactor to Support Space Reactor Development", April 2005.

(2) Senor, D., et. al. "Feasibility Study for an Overseas High Fluence Irradiation Test in Support of Project Prometheus: Final Assessment of Candidate Reactor Facilities. PNNL Report PNNL-15124, March 2005.

(3) B-MT(SRME)-32/MDO-723-0041, "Material Properties and Specimen Dimensions for Capsule Design to Support the JOYO-1 Irradiation Test, Revision 0", November 2005

(4) Telecon, B-MT(SRME)-29T, Telecon to Finalize Test Matrix for JOYO-1, June 2005.

(5) MDO-723-0043, "Assessing the Effects of Radiation Damage on Ni-base Alloys for the Prometheus Space Reactor System", January 2006.

(6) SM-7231-0009/B-MT(SPME)-3, "Beryllium Oxide and Beryllium Irradiation Data and JOYO reflector Material Test Plan Summary, for Information", April 2005.

(7) MDO 720-1137, "Design Review \# 1996, Design Review of JOYO-1 Test Matrix" September 2005.

(8) B-MT(SRME)-51, "Basic Processing Methods of T-111, Ta-10W, FS-85, Mo-47.5Re, and ASTAR-811C Sample Production", To Be Issued.

(9) B-MT(SRME)-50, "Biaxial Creep Specimen Fabrication," To Be Issued.

(10) B-MT(SRME)-49, "Closeout of JOYO-1 Specimen Fabrication Efforts", October 2005.

(11) B.P.Bewlay, C.L. Briant, and M.L. Murray, "Molybdenum-Tungsten Interdeffusion and Influence on Potassium Bubbles in Tungsten Lamp Wire", Metallurgical and Materials Transactions, vol 29A, (Dec1998) page 2933.

(12) MDO-723-0046/B-MT(SPME)-23, "Space Reflector Materials for a Prometheus Application", January 2006.

(13) MDO-723-0057, "Experiences and Considerations with Irradiation Test Performance in an International Environment", To Be Issued.

(14) B-MT(EDT)S-018, “JOYO-1 Design for Permission Report Presentation to the Japan Nuclear Cycle Development Institute (JNC) for Testing of Space Structural Materials in the JOYO reactor", June 2005 .

(15) B-MT(EDT)-014, "JOYO-1 Design for Permission Report", June 2005.

(16) MDO-723-0036/Bettis Letter B-MT(SRME)-37, "Overview of Domestic and International Shipping of Irradiated Structural Materials and Handling Associated Waste, for NR Information", January 2006.

(17) SM-7234-0010, "Review Status of JOYO-1 Project", November 2005. 
The Manager, SNR

The Manager, PNR

MDO-723-0044

B-MT(SRME)-52

Figure 1

Page 15 of 16

NRPCT Irradiation Rig Stackup JOYO-1
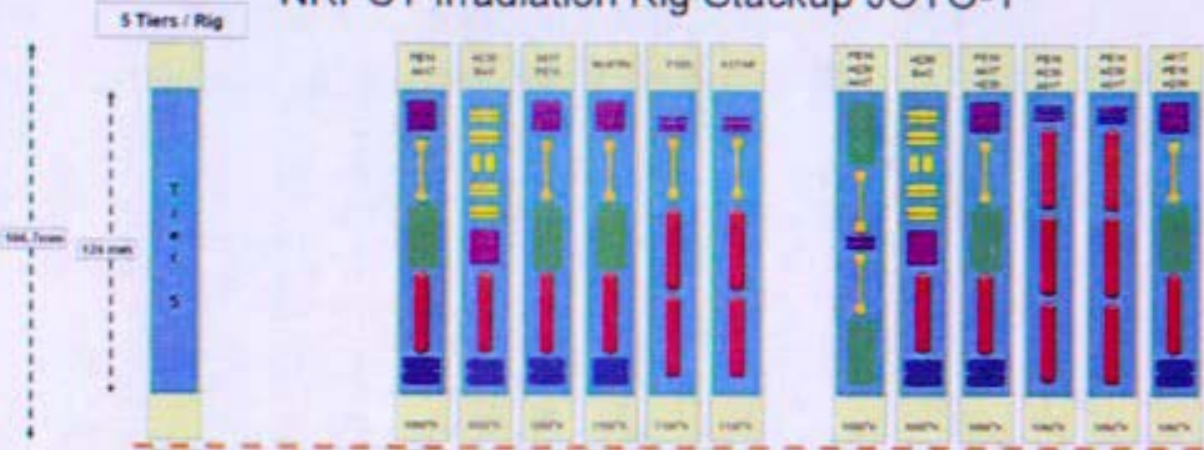

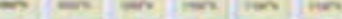

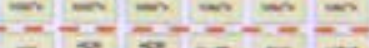

insun
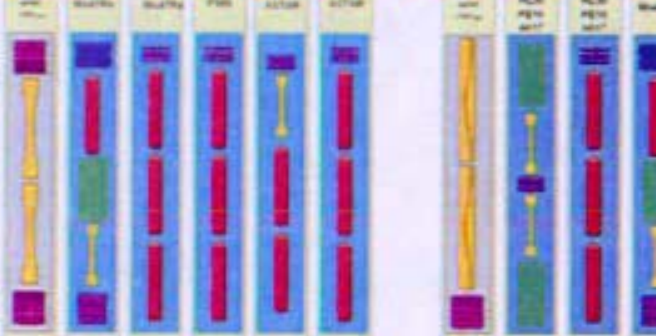

nowint-
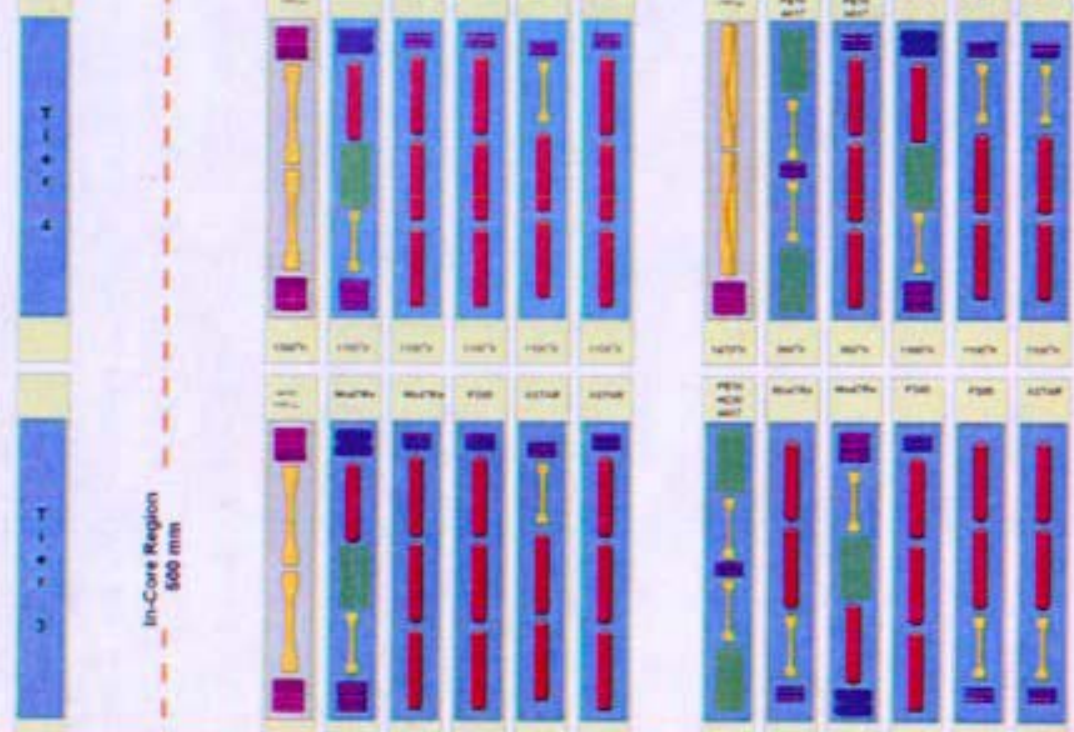

Coramik Speciment
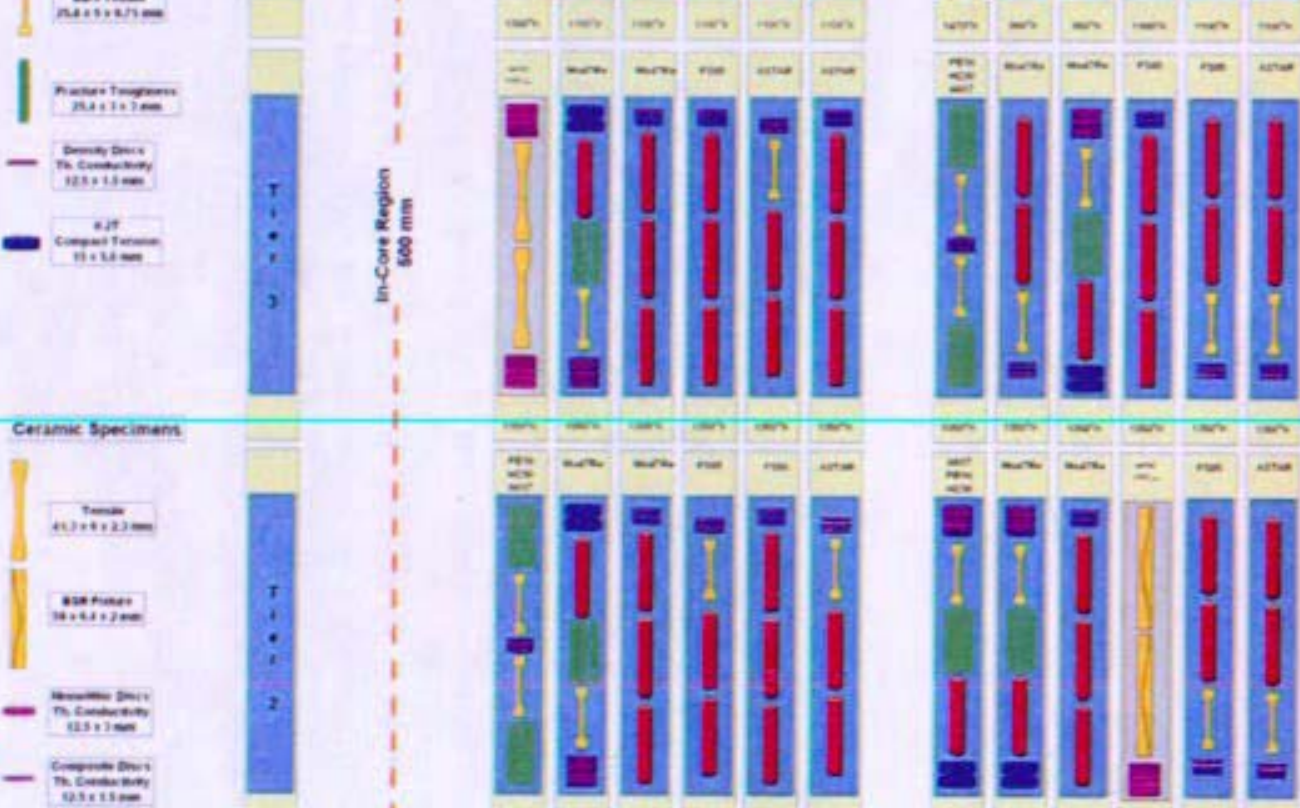

$-\underset{\cos }{\operatorname{and}}$

$y+\frac{m}{n-1}$
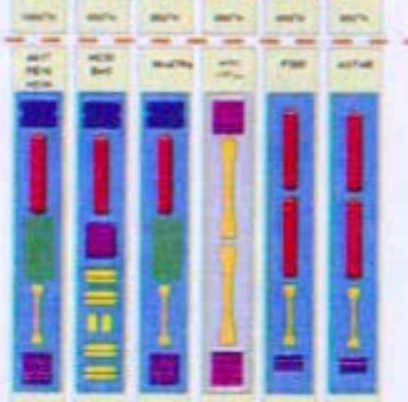

$\underset{m i n}{m} \frac{m}{n} \frac{m}{n} \frac{m}{n}$

Rig A (2 Cycles)

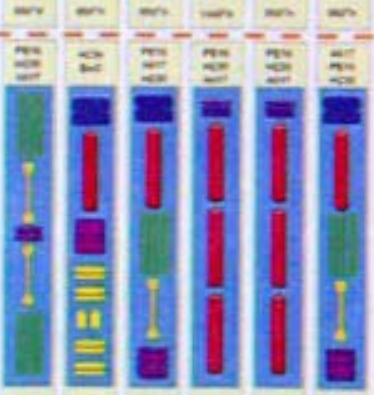

$\frac{m}{n 2} \frac{m}{n} \frac{m}{n_{4}} \frac{m}{n_{6}}$

Rig B (1 Cycle) 


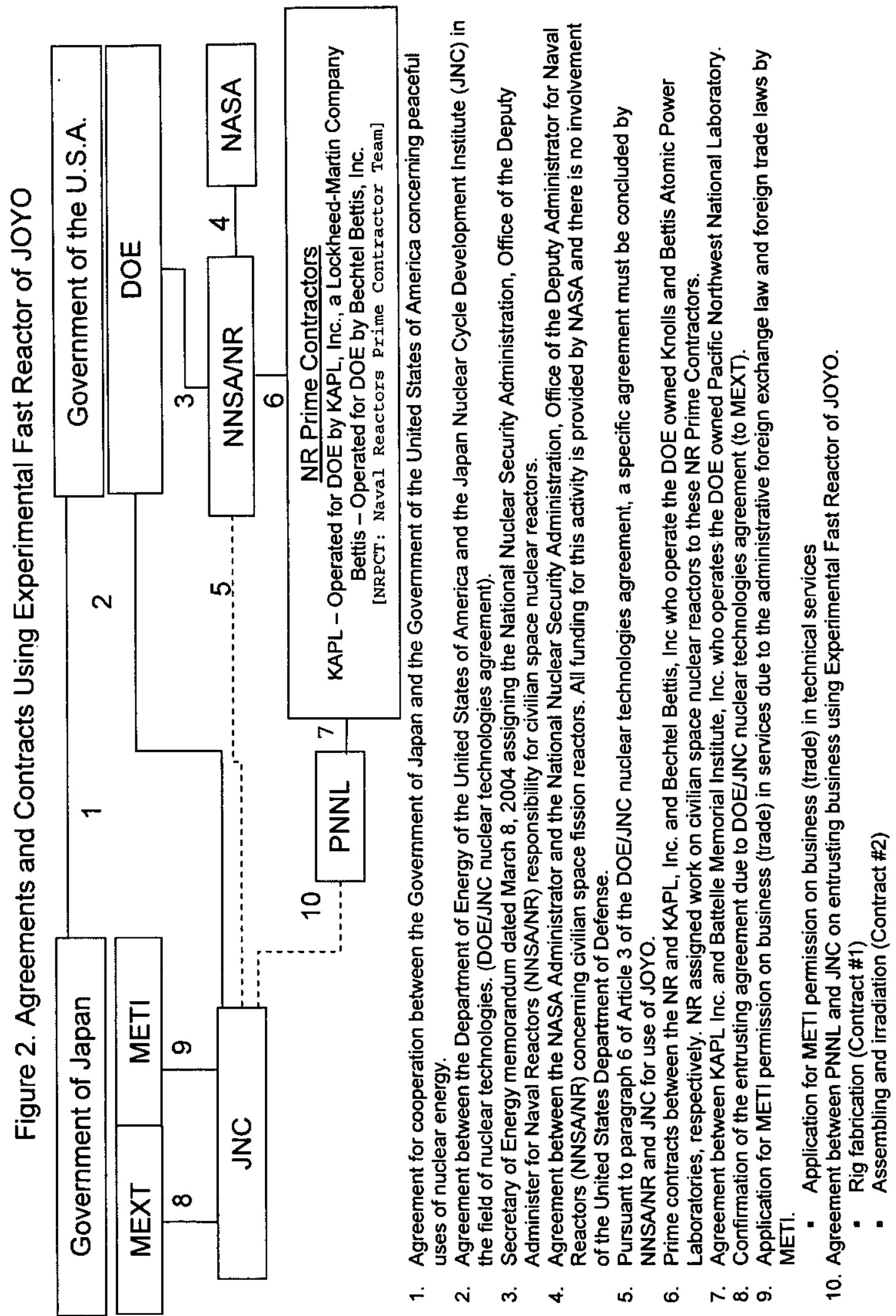


Enclosure 1 to MDO-723-0044 /

B-MT(SRME)-52

Page 1

Enclosure 1

to

MD0-723-0044 / B-MT(SRME)-52

JOYO-1 Structural Refractory Metal Materials Specimen Fabrication Efforts

\author{
J. Bump
}

R. Luther

January 2006 
Enclosure 1 to

MDO-723-0044 /

B-MT(SRME)-52

Page 2

\section{Summary}

Because of JOYO-1 space limitations, three metal refractory alloys (ASTAR-811C, FS- 85 and Mo-47.5Re) representing different alloy classes, were selected for the JOYO-1 test matrix. These alloys were candidates for cladding and core applications for Prometheus-1. Including refractory metal alloys as Prometheus- 1 structural material candidates provided several challenges, ranging from low commercial availability (requiring significant development efforts) to embrittlement concerns during fabrication and testing (by interstitial contamination) to long term microstructural and property stability.

Upon restructuring of the Prometheus Project, tensile specimens of all three metal alloys were in the fabrication process. Biaxial creep specimens required the most significant time investment because of the multi-step fabrication process. Biaxial creep specimen parts were completed for ASTAR-811C and FS-85. Material delivery for Mo-47.5Re was behind schedule, and tubing was still being formed at Rhenium Alloys (Cleveland, $\mathrm{OH}$ ). Processing of these specimen parts and other specimens is detailed in Reference 1. Although the specimen parts were completed, a welding process for ASTAR-811C and FS-85 was being developed at Pacific Northwest National Laboratory (PNNL). Electron beam welding of end-caps to tubes was successfully developed for both materials, but laser-seal welding of the pressurization hole in the top end-cap was not successfully developed and required further work. Details of biaxial creep specimen fabrication efforts are provided in Reference 2.

\section{Introduction}

Refractory metal alloys under consideration for cladding and core applications for JOYO-1 included tantalum-base, niobium-base, and molybdenum-base alloys. Due to limited space available for test specimens in the JOYO irradiation rigs, one alloy from each of these classes was chosen for the JOYO-1 test campaign. Alloys were chosen based on their individual attributes and their ability to represent the class of material. Refractory metal materials tend to have lower production availability and greater fabrication constraints. Attachments 1, 2 and 3 provide fabrication flow charts for each of the three refractory metal alloy categories chosen for testing in JOYO-1.

While there is a significant database of basic mechanical properties for the majority of the refractory materials under investigation, there was insufficient data to support an adequate design basis. This was especially true for irradiated materials at prototypical temperatures and fluence levels, as summarized in Figure 1. The special nature of refractory metals has led to limited commercial production and a considerable material database gap. This includes fabrication and weiding know-how, mechanical properties, fatigue data, chemical compatibility and radiation effects. Many of these materials have had a long dormant period of fabrication. Therefore, limited material property and behavior data will need to be reproduced to verify the reliability of the resurrected fabrication process and provide a credible and robust baseline for potential minor compositional and/or processing changes that may be necessary. Gaps in the data will also need to be filled for conventional materials.

Refractory metal alloys that were considered for the space reactor become susceptible to irradiation damage below $1000 \mathrm{~K}$. This damage can lead to irradiation creep of components duting active irradiation that can rival thermal creep rates observed at much higher 
temperatures. In addition, irradiation embrittlement can severely reduce ductility during postirradiation and transient loading conditions. Because it was not planned to restart the reactor if it shuts down, post-irradiation embrittlement is not a significant issue for the flight units, but may have a significant impact on the prototype designs. However, transient conditions can lead to rapid stress excursions when the rate of irradiation creep is slower than the rate of loading. That essentially reduces the transient excursion to the equivalent of a post-irradiation loading condition. Irradiation creep is viewed as a positive phenomenon in typical pressurized water reactor (PWR) and boiling water reactor (BWR) systems that helps maintain low stresses without damaging the zirconium alloys used in fuel elements. The zirconium alloys retain sufficient post-irradiation ductility to protect against failure during typical transients. Little is known about irradiation creep in refractory metal alloys.

Figure 1

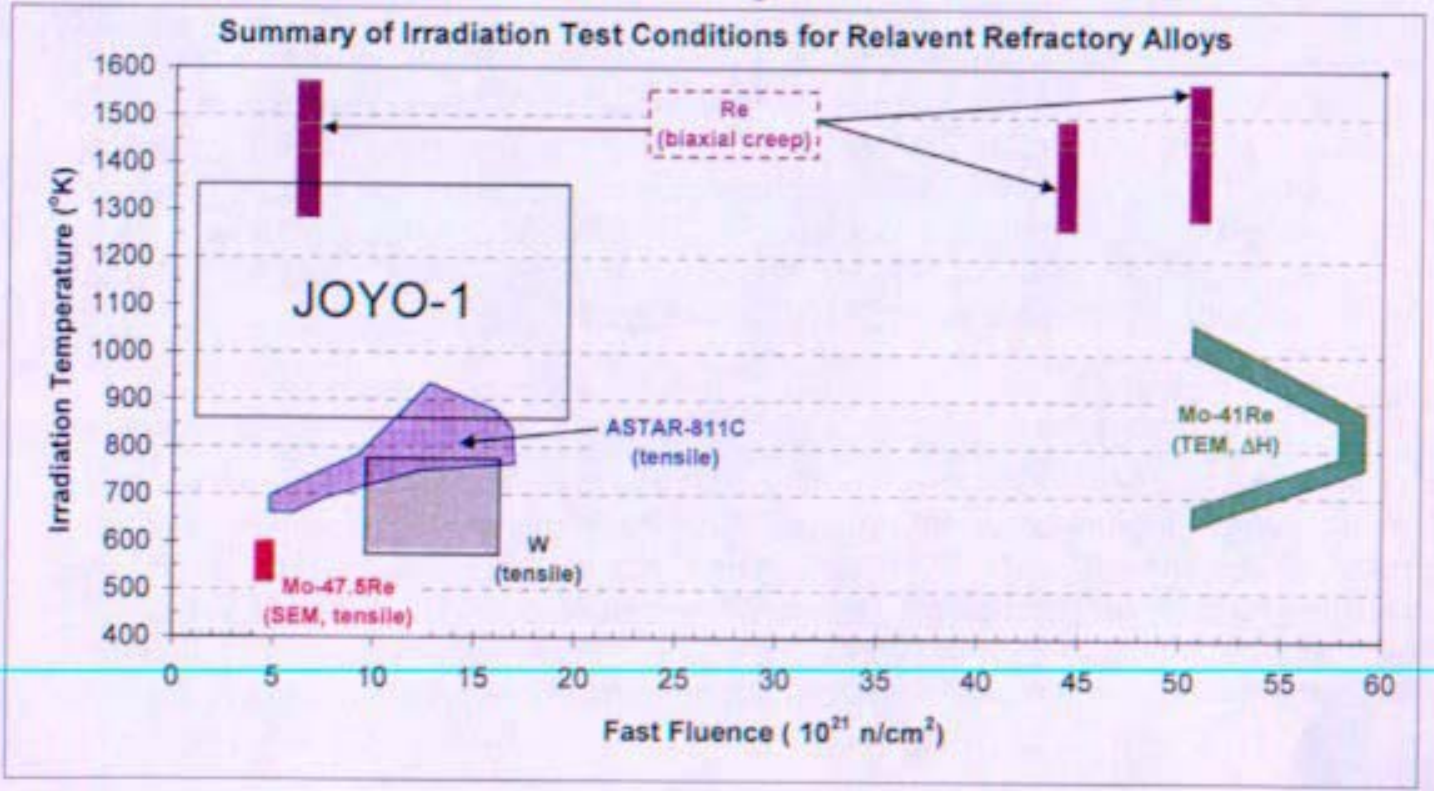

Three tantalum alloys were considered for structural space reactor materials for the JOYO-1 test including ASTAR-811 C (Ta-8W-1Re-0.7Hf-0.025C), T-111 (Ta-8W-2Hf), and Ta-10W. Only ASTAR-811C was chosen for JOYO-1. The selection was primarily based on its exceptional high-temperature creep strength, which is attributed partially to the presence of small carbide precipitates. T-111 represents a slightly lower strength alloy, but one that is less complex metallurgically because the major alloying constituents are mutually soluble. Ta-10W is the lowest strength tantalum-base alloy candidate of the three, but it is the only one commercially available. For the proper perspective on commercial experience, note that the last commercial size ingot of ASTAR-811C was melted over 30 years ago. ASTAR-811C test specimens for JOYO-1 were to include biaxial creep specimens, tensile specimens, and density and conductivity discs. From these specimens, information would have been gained on irradiation creep properties, strength, micro-structural stability, swelling, and thermal conductivity. However, due to the high residual activity of irradiated tantalum alloys, it would not have been possible to perform transmission electron microscopy (TEM) during post-irradiation 
Enclosure 1 to

MDO-723-0044 /

B-MT(SRME)-52

Page 4

examinations (PIE). This would eliminate obtaining a microstructural understanding of radiation induced changes to physical and mechanical behavior.

Only one niobium-base alloy, FS-85 (Nb-10W-27Ta-1Zr), was considered for JOYO-1 materials. Previous efforts on SP-100 (Reference 3 ) focused on Nb-1Zr alloy with PWC-11 ( Nb-1Zr $+1000 \mathrm{ppm} \mathrm{C}$ ) as a backup. However, NRPCT evaluations of SP-100 efforts concluded that the strength of $\mathrm{Nb}-1 \mathrm{Zr}$ alloy was too low to merit consideration for JOYO-1. Furthermore, it appeared that the alloy lacked reproducibility since there was a wide variation in creep behavior generated from various $\mathrm{Nb}-1 \mathrm{Zr}$ ingots melted either during or before the SP-100 era. This creep variation occurred despite similar chemistry levels among ingots. A subsequent Naval Reactors Prime Contractor Team (NRPCT) review of niobium alloys developed during the last 30 years concluded that FS-85 solid-solution-strengthened alloy was the best choice to represent niobium alloys for JOYO-1 testing. This alloy provided the best blend of high-temperature strength and fabricability. FS-85 specimens that were to be tested in JOYO- 1 included biaxial creep specimens, tensile specimens, density discs and conductivity discs. Since the FS-85 alloy contains tantalum, the anticipated high activation of irradiated samples prevents microstructural evaluations via TEM.

A major concern with the three candidate tantalum-base alloys and the single niobium-base alloy is their propensity to become contaminated by oxygen, nitrogen and other interstitials. Contamination can occur during processing operations, including vacuum annealing or welding. It could also occur during space reactor operations as a result of contact with oxide- or nitridebased fuels, or as a result of exposure to an impure gas coolant. If contamination of these alloys is severe enough, the alloys can become significantly embrittled.

Molybdenum alloys exhibit a significantly lower tendency to become contaminated (with oxygen, nitrogen and interstitials) during fabrication, testing, or usage compared to niobium and tantalum base alloys. However, fabricability of molybdenum and most of its alloys is appreciably poorer than that of niobium and tantalum alloys. Weldments made within pure molybdenum and most of its alloys typically exhibit a Ductile-Brittle-Transition-Temperature (DBTT) that is well above room temperature. Simple forming operations with pure molybdenum or most of its alloys are more problematic than those for niobium and tantalum alloys. Molybdenum base materials require high temperatures for success rather than the room temperature or slightly elevated temperatures required for forming niobium and tantalum.

Rhenium additions to molybdenum at levels approaching 50 weight percent (w/o) results in a significant increase in ductility with respect to both forming and welding operations. These high rhenium levels; however, can result in the formation of rhenium-rich phases during processing or thermal exposures. Furthermore, in-reactor exposures apparently cause additional rheniumrich precipitates to form. These precipitates may contain a significant amount of osmium, a transmutation product of rhenium. As a consequence, microstructural phase instability of the Mo-Re alloys is a major concern. While increases in thenium content may cause rhenium-rich precipitates to form, decreasing rhenium content decreases fabricability. The optimum level needed to achieve adequate fabricabilty while minimizing formation of rhenium-rich precipitates is not certain. This level of alloying was under active NRPCT evaluation during initial formulation of the JOYO-1 test matrix and through test specimen fabrication prior to the Prometheus Project restructuring. 
Consequences of this uncertainty resulted in consideration of a variety of molybdenum-rhenium alloys for JOYO-1. Rhenium levels for candidate molybdenum-rhenium alloys ranged from 41 w/o to 47.5 w/o rhenium. Mo-47.5Re was ultimately chosen for JOYO-1 because it exhibited the highest level of ductility. However, molybdenum- 44.5 w/o rhenium was included as a backup alloy. Oxide dispersion strengthened molybdenum (ODS-Mo) was also being evaluated as a possible cladding material, but is still considered developmental. JOYO-1 specimens of MO47.5Re to be tested included biaxial creep specimens, fracture toughness bars, compact tension discs, tensile specimens, density discs and conductivity discs.

\section{Discussion}

All candidate refractory metal alloys, with one exception, were melted as small laboratory-size (3-inch diameter) ingots at Pittsburgh Materials Technology (PMTI, Large, PA), a small vendor with extensive experience processing refractory metal alloys. This exception was Mo-44.5Re, which was obtained as a small powder metallurgy (PM) billet from Rhenium Alloys, Inc. (Elyria, $\mathrm{OH}$ ). Three-inch-diameter melted ingots and the single PM billet were then to be processed through a series of fabrication steps that began with ingot extrusion at Wright Patterson Air Force base (Dayton, $\mathrm{OH}$ ). Flat extrusions were to be processed into sheet product from which tensile specimens and other flat specimen types were to be machined. Similarly, round extrusions were to be processed into tubing and smaller-diameter rod. Tubing and rod were then intended to be used for formation of biaxial creep specimen components.

When processing was terminated upon project restructuring, all ingots had undergone some portion of their intended processing. Ta-10 W and Mo-44.5Re had only been processed through extrusion. T-111, ASTAR-811C, FS-85, and Mo-47.5Re had been extruded and flat extrusions had been processed into thin sheet at PMTI. Thin sheets had been sent to Kin-Tech Mfg., Inc. (North Huntingdon, PA) where JOYO-1 tensile specimens were machined for each alloy.

In addition, thin-walled $6.35 \mathrm{~mm}(0.250$-inch) diameter tubing had been successfully drawn by True Tube, Inc. (Paso Robles, CA) from T-111, ASTAR-811C, and FS-85 round extrusions. Tubing from these three alloys was cut into $31.75 \mathrm{~mm}(1.25$-inch) sections for biaxial creep specimens. Swaged rods from the same alloys were machined into end-caps for biaxial creep specimens at Vangura Tool, Inc. (Clairton, PA). Tube and end-cap components had been delivered to Pacific Northwest National Laboratory (PNNL) in Hanford, Washington where they were used in an abbreviated weld qualification for final assembly of biaxial creep specimens. Biaxial creep specimen assembly involved electron beam (EB) welding two end-caps to each tube, and laser-seal welding a small hole in the end-cap used for specimen pressurization with helium. Abbreviated qualification efforts had successfully developed EB welding parameters for all refractory metal alloys. However, development of laser welding parameters was not successful and required further effort. Details of the biaxial creep specimen fabrication effort at PNNL are documented in Reference 2.

Mo-47.5Re round extrusions were processed into thin-wall tubing at Rhenium Alloys, Inc. This is believed to be the first time that arc cast Mo-47.5Re has been formed into thin walled tubing. However, this processing took longer than tube formation of other materials and therefore, Mo47.5Re biaxial creep specimen components were never sent to PNNL for specimen assembly. Detailed information on processing of JOYO-1 refractory metal alloy specimens is documented in Reference 1. 
Enclosure 1 to MDO-723-0044 /

B-MT(SRME)-52

Page 6

\section{References}

1. B-MT(SRME)-51, "Basic Processing Methods of T-111, Ta-10W, FS-85, Mo-47.5Re, and ASTAR-811C Sample Production", To Be Issued.

2. B-MT(SRME)-50, "Biaxial Creep Specimen Fabrication," To Be Issued.

3. Peter Ring, "SP-100 Materials and Fabrication Technology Lessons learned," SP-100 Program Information Release \#1199, March 1994. 
Enclosure 1 to MDO-723-0044 /

B-MT(SRME)-52

Page 7

\section{Attachments}

Attachment 1 Processing of Mo-47.5Re Flow Chart

Attachment 2 Processing of ASTAR 811C Flow Chart

Attachment 3 Processing of FS-85 Flow Chart

Enclosure 1 to MDO-723-0044/B-MT(SRME)-52 
Attachment 1 to

Enclosure 1 to MDO-723-0044/

B-MT(SRME)-52

Page 1

\author{
Attachment 1 \\ Processing of Mo-47.5Re Flow Chart \\ Enclosure 1 to MDO-723-0044/B-MT(SRME)-52
}


Attachment 1 to

Enclosure 1 to

MDO-723-0044I

B-MT(SRME)-52

Page 2

\section{Flow Chat 1. Processing of MoRe Alloy}

Processing of Mo-47.5Re Flat Stock

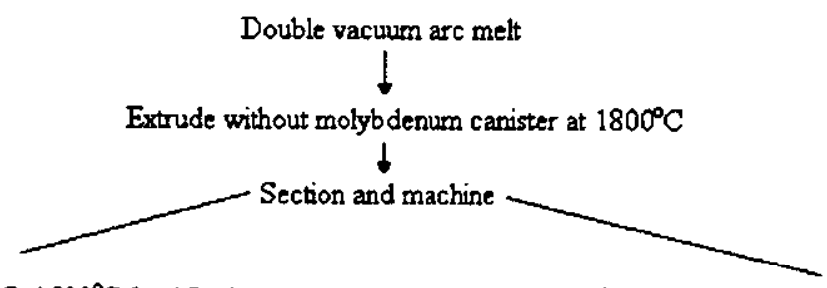

1M. Anneal in H2@1600 $\mathrm{C}$ for $15 \mathrm{mins}$

2M. Preheat at $1425^{\circ} \mathrm{C}$ and reduce the material by rolling $10-20 \%$ each reduction. Heat before each roll and recuce material to a total reduction of $50 \%$

3M Anneal in H2 furnace at $1600^{\circ} \mathrm{C}$ for 15 minutes

4M. Repeat steps $2 \mathrm{M}$ and $3 \mathrm{M}$ for another $50 \%$ reduction

1R Anneal in H2@1600 $\mathrm{C}$ for 15 mins

$2 R$ Cold Roll 5-10\% passes until a total of $20 \%$ reduction is reached.

3R Anneal in $\mathrm{H} 2$ furnace at $1600^{\circ} \mathrm{C}$ for 15 minutes

4R. Repeat cold toll and anneal process until a thickness of 0.080 inches is reached.

5R. Roll to 0.040 inches

SM Roll to 0.040 inches at $1425^{\circ} \mathrm{C}$

6M. Machine into tensiles

- These two paths, $M(1-6)$ and $R(1-6)$ were used during rolling of a 3-inch diameter Mo$47.5 \mathrm{Re}$ ingot.

- A third path was added by the NRPCT that combined portions of both recommended paths. This third variant began with high temperature rolling using path $\mathrm{M}$ parameters which then switched to the cold rolling process of path $R$ when a thickness of $\sim 0.080$-inches had been obtained. Cold rolling for this final path continued until a thickness of 0.040 -inches was reached.

- Materials representing all three paths were successfully processed to a final thickness of 0.040 -inches on the STANAT mill at PMTI. 
Attachment 2 to

Enclosure 1 to

MDO-723-0044/

B-MT(SRME)-52

Page 1

Attachment 2

Processing of ASTAR 811C Flow Chart

Enclosure 1 to MDO-723-0044/B-MT(SRME)-52

PRE- DECISIONAL - For Planning and Discussion Purposes Only 


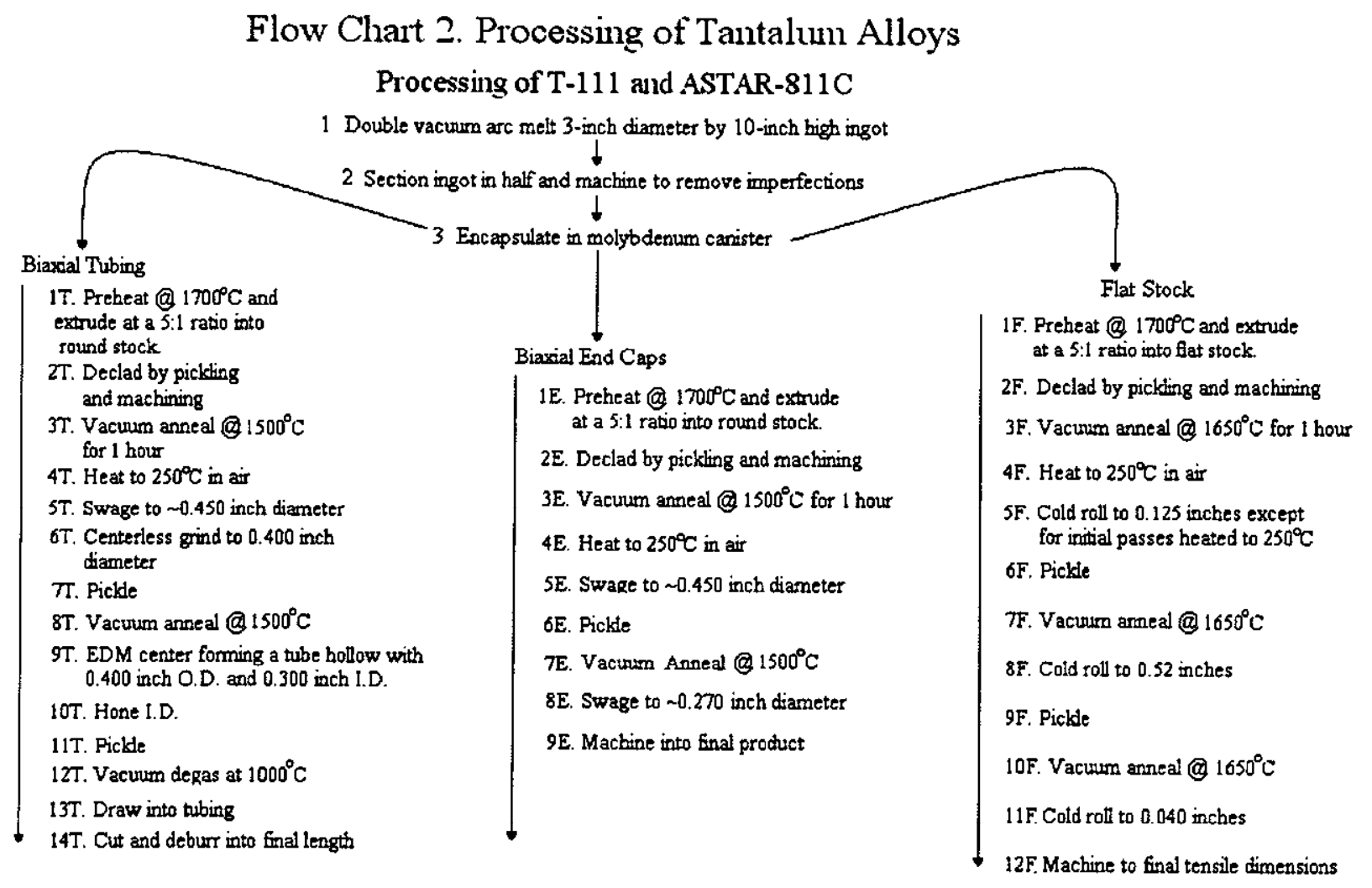


Attachment 3 to Enclosure 1 to MDO-723-0044/ B-MT(SRME)-52 Page 1

\section{Attachment 3}

Processing of FS-85 Flow Chart

Enclosure 1 to MDO-723-0044/B-MT(SRME)-52 
Attachment 3 to

Enclosure 1 to

MDO-723-0044/

B-MT(SRME)-52

Page 2

\section{Flow Chart 3. Processing of Niobium Alloy}

Plocessing of FS-85

\begin{tabular}{|c|c|c|}
\hline 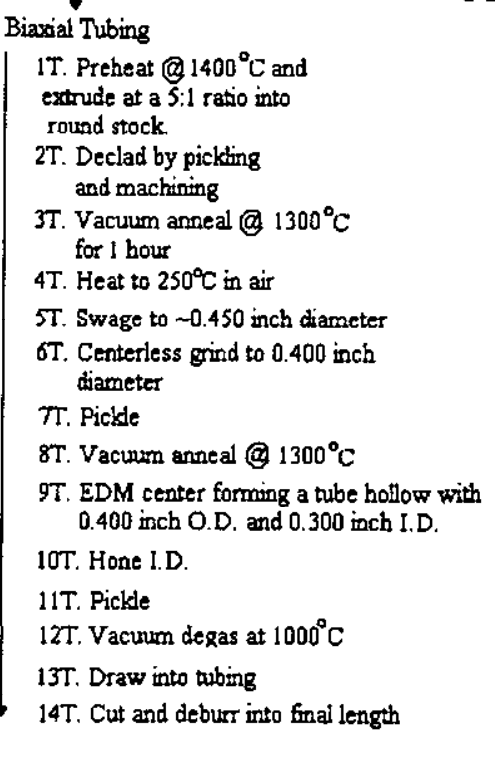 & $\begin{array}{l}\text { Biaxial End Caps } \\
\text { 1E. Preheat } 01400^{\circ} \mathrm{C} \text { and extrude } \\
\text { at a s:1 ratio into routad stock } \\
\text { 2E. Declad by pickding and machining } \\
\text { 3E. Vacuum anneal @ } 1300^{\circ} \mathrm{C} \text { for } 1 \text { hour } \\
\text { 4E. Heat to } 250^{\circ} \mathrm{C} \text { in air } \\
\text { 5E. Swage to } \sim 0.450 \text { inch diameter } \\
\text { 6E. Pickte } \\
\text { 7E. Vacuura Anneal } 1300^{\circ} \mathrm{C} \\
\text { 8E. Swage to } \sim 0.270 \text { inch diameter } \\
\text { 9E. Machine into final procuct }\end{array}$ & $\begin{array}{l}\text { Flat Stock } \\
\text { 1F. Preheat } 1400^{\circ} \mathrm{C} \text { and extrude } \\
\text { at a s:1 ratio into lat stocik } \\
\text { 2F. Declad by pickling and machining } \\
\text { 3F. Vacuum anneal @ } 1400^{\circ} \mathrm{C} \text { for } 1 \text { hour } \\
\text { 4F. Heat to } 250^{\circ} \mathrm{C} \text { in air } \\
\text { 5F. Cold rotl to } 0.125 \text { inches except } \\
\text { for initial passes beated to } 250^{\circ} \mathrm{C} \\
\text { 6F. Picke } \\
\text { TF. Vacuum anneal @ } 1400^{\circ} \mathrm{C} \\
\text { 8F. Cold roll to } 0.52 \text { inches } \\
\text { 9F. Pickie } \\
\text { 10F. Vacuum anneal @ } 1400^{\circ} \mathrm{C} \\
\text { 11F. Cold roll to } 0.040 \text { inches } \\
\text { 12F. Machine to final tensile dimensions }\end{array}$ \\
\hline
\end{tabular}


Enclosure 2 to MDO-723-0044 /

B-MT(SRME)-52

Page 1

\author{
Enclosure 2 \\ to \\ MDO-723-0044 / B-MT(SRME)-52 \\ JOYO-1 Superalloy Specimen Fabrication Efforts
}

\author{
S.J. Buresh \\ G. Borges
}

January 2006 
Enclosure 2 to

MDO-723-0044/

B-MT(SRME)-52

Page 2

\section{Summary}

Three structural nickel-based superalloys were chosen for the first JOYO irradiation test, two solid solution strengthened alloys (Alloy 617, Haynes 230), and a precipitation hardened alloy (Nimonic PE-16). These materials were primarily chosen for structural plant and core applications for Prometheus-1. Procurement of stock nickel-base superalloys and subsequent machining into specimens was successfully progressing. These alloys were readily available in sufficient stock supplies of several product forms to promptly begin specimen machining processes. Hence, except for a new developmental specimen type, superalloy specimen fabrication was on schedule. A large number of superalloy specimens were fabricated for material properties testing. This included planned and active fabrication of over 500 various specimens for the JOYO-1 irradiation test, and a similar number of specimens for out-of-pile testing to differentiate thermal degradation from radiation effects. Based on a few stock limitations, plans to procure larger quantities of the materials and product forms chosen for eventual Prometheus-1 structures required longer lead purchase orders.

Biaxial creep specimen fabrication was progressing toward a full qualification procedure. This included welding end caps, pressurized seal welding, and hermeticity verification. Development of weld parameters was performed at Pacific Northwest National Laboratory (PNNL) for the different alloy classes. Machining of superalloy tensile, fracture toughness and density disc specimens were completed without significant difficulty. Development of precracking parameters for superalloy subsized fracture toughness bars was initiated in-house. This was an important gain for the Prometheus Project since fracture toughness data is not available for these materials and there was no NRPCT or vendor experience with precracking these subsized specimens. Drawings for $0.2 T$ compact tension disc specimens and the fixtures necessary to bolt-load them (for in-pile crack growth rate testing) were created. Development of a precracking and bolt-loading procedure was planned for these first-of-a-kind specimens.

\section{Introduction}

Specimen types for the first round of testing (JOYO-1) were chosen based on data that was most critical and/or lacking for Prometheus-1 structural applications. Superalloys of interest were reduced to three because of limited volume capacity available in JOYO: Two rigs, each accommodating 30 capsules. Within these capsules, an appropriate number of materials and specimen types were allocated to obtain the best mix of maximum breadth and depth of data. The primary properties that superalloys were chosen for included their high temperature strength and creep resistance. Hence, it was first necessary to ascertain the effect of irradiation on these properties. This was particularly necessary in JOYO, since it is capable of providing the most prototypical fluence and temperature range for Prometheus-1 design concepts.

A relatively larger number of Nickel-base alloys have been selected for study because unlike tantalum, niobium and molybdenum-rhenium alloys, no single Nickel-base superalloy stands out as a clear choice or can be expected to fully represent this rather broad material class. The strengthening nature makes superalloys behave differently under irradiation as the phase stability can be very sensitive to composition. ASTAR-81 1C, FS-85 and Mo-47.5Re have three or four constituents each, while these Nickel-base superalloys each have eight or more constituents. This significantly complicates the phase stability, both thermally and under irradiation. 
Precipitation strengthened alloys, such as PE-16, have higher strengths than the solid-solution strengthened alloys due to precipitates of $\gamma^{\prime}\left(\mathrm{Ni}_{3}(\mathrm{Al}, \mathrm{Ti})\right)$ and/or $\gamma^{\prime \prime}\left(\mathrm{Ni}_{3}(\mathrm{Nb}, \mathrm{Ti})\right)$. These precipitates strengthen the material by imposing a large degree of coherency or semi-coherency strain within the matrix. However, under neutron irradiation, these precipitates are unstable and the $\eta$ phase, $\mathrm{Ni}_{3} \mathrm{Ti}$, are formed in $\gamma^{\prime}$-containing alloys and $\delta$ phase, $\mathrm{Ni}_{3} \mathrm{Nb}$ are formed in $\gamma^{\prime \prime}-$ containing alloys. When $\eta$ or $\tilde{\delta}$ phases form on grain boundaries, the ductility is severely decreased (Reference 1). Conversely, solid-solution strengthened superalloys contain little to no titanium or niobium and are believed not to suffer from this embrittlement mechanism. Solid solution strengthened superalloys Alloy 617 and Haynes 230 show promise for Prometheus-1 applications, but there is much more irradiation data for precipitation hardened Nickel-base alloys, such as Nimonic PE-16 (see plot below). Nimonic PE-16 was successfully used as fuel cladding in the PFR at Dounreay, UK without a single failure at similar temperature and higher fluence ranges than those of Prometheus-1.

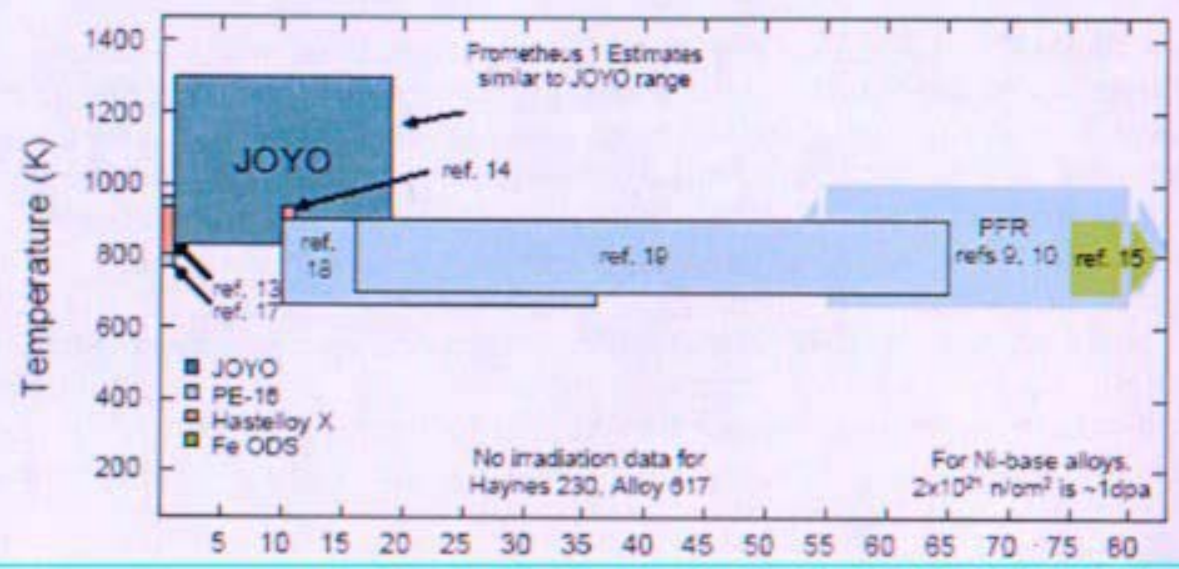

Fluence $\left(\times 10^{21} \mathrm{n} / \mathrm{cm}^{2}\right)$

Figure 1: Summary of neutron irradiation data for Ni-base superalloy PE16 relative to the proposed JOYO experiments. No irradiation data is available for Haynes 230 or Alloy 617 (References in chart are located in MDO-723-0021/B-MT(SRME)-21).

This enclosure describes the progress accomplished and the status of superalloy specimen fabrication. Table 1 contains a count of superalloy specimens that were planned for JOYO-1 insertion. All fabricated specimens are inventoried at Bettis, while superalloy product forms (e.g., plates, rods), from which specimens were machined, are inventoried at KAPL.

Table 1: JOYO-1 Superalloy Specimen Count

\begin{tabular}{|l|c|c|c|c|c|c|}
\hline Material & $\begin{array}{c}\text { Biaxial } \\
\text { Creep }\end{array}$ & $\begin{array}{c}\text { Fracture } \\
\text { Toughness } \\
\text { Bars }\end{array}$ & $\begin{array}{c}\text { Compact } \\
\text { Tension } \\
\text { Discs }\end{array}$ & $\begin{array}{c}\text { Tensile } \\
\text { Bars }\end{array}$ & $\begin{array}{c}\text { Density \& } \\
\text { Conductivity } \\
\text { Discs }\end{array}$ & $\begin{array}{c}\text { Total } \\
\text { Specimen }\end{array}$ \\
\hline Alloy 617 & 9 & 54 & 8 & 65 & 40 & 176 \\
\hline Haynes 230 & 9 & 54 & 8 & 54 & 41 & 166 \\
\hline Nimonic PE-16 & 9 & 54 & 8 & 61 & 41 & 173 \\
\hline
\end{tabular}




\section{Discussion}

An early material selection criterion was established to make every effort possible to maintain a minimum of 10 grains across the thickness of all specimens. This is based on a rule of thumb principle (by experienced testing experts) of, at least, 7 to 10 through-wall grains for miniature or so called subsized specimens. Below this range, excessive variation in test data can result. Using this material requirement, original plans were to fabricate biaxial creep specimens from seamless tubing with end-caps machined from rod stock, while tensile, fracture toughness, compact tension and density specimens were to be machined from plate material. JOYO-1 specimen fabrication efforts began with a focus on longer lead specimens (biaxial creep), and specifications were developed to produce seamless tubing from other gun drilled rods or existing oversize tubing.

\section{Biaxial Creep}

Attachment 1 is an example of a specification developed for seamless tubing. Several vendors were consulted (Superior Tube Co., True Tube, Inc., Tube Methods, Inc., and Vindum Engineering, Inc.) during the specification development phase to determine capabilities and industry standards for this type of tubing. Following these consultations, requests for quote were issued for seamless tubing. An alternative approach that was ultimately chosen to pursue after consideration of both technical and schedule concerns was to machine biaxial tubing parts out of raw plate or bar material form. Seamless tubing was pursued in parallel. Specimens fabricated from seamless tubing would be used for comparison testing out-of-pile and future irradiation tests.

Fabrication of Haynes 230 biaxial creep specimens was accomplished using stock of appropriate sized Haynes 230 welded tubing, produced by Tube Methods, Inc. This tubing had sufficient cold work during processing to make welds indistinguishable, although as seen in Figure 2, some grain size variations across the wall thickness were evident.

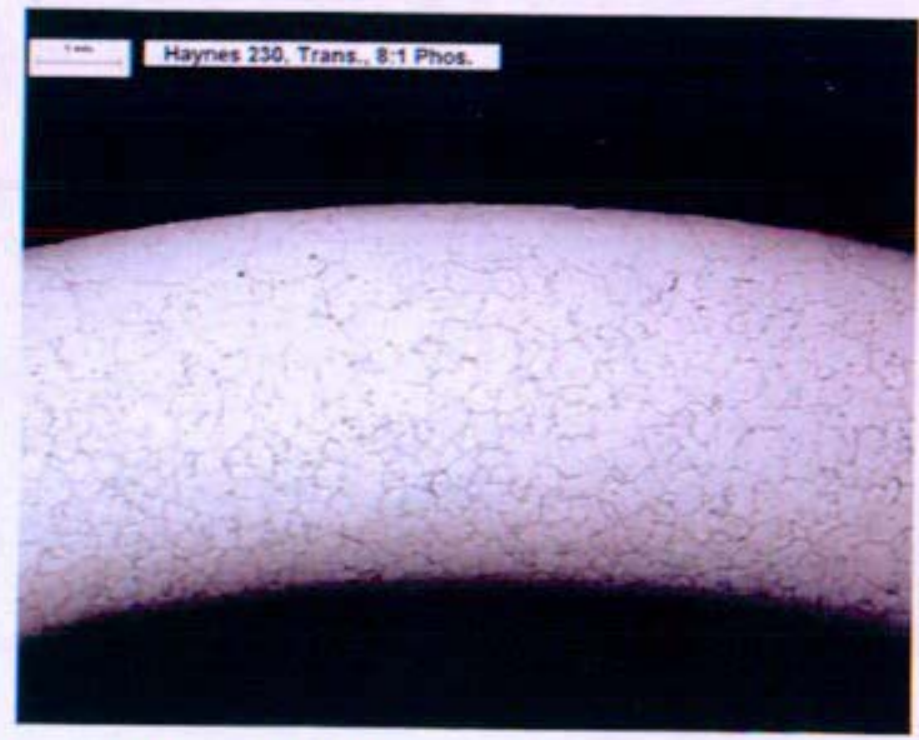

Figure 2: Haynes 230 welded tubing cross section showing grain size variation. 
Since no current production Nimonic PE-16 material was found in the US, some rod stock (45 $\mathrm{mm}$ and $65 \mathrm{~mm}$ diameter) was purchased from Enpar Special Alloys Limited (a division of Firth Rixson located in England). Additional stock of Nimonic PE-16 was located Oak Ridge National Laboratory (ORNL), although with a limited pedigree. Inquiries to Special Alloys confirmed that the ORNL historical stock PE-16 heat/lot number related its origin to one of their plants in England. Samples of ORNL historical Nimonic PE-16 were subsequently obtained, tested for chemistry and metallographically evaluated. Metallography of this material showed some small, but acceptable variation in grain size longitudinally in the rod. Figures 3 and 4 show precipitate banding that is typically found in the longitudinal direction and a grain size range of approximately ASTM 7.8 to $6(\sim 0.64 \mu \mathrm{m}$ to $1.1 \mu \mathrm{m})$. In parallel with evaluating the Nimonic PE16 rod from ORNL, an order was placed through Northern Specialty Metals to obtain $45 \mathrm{~mm}$ and $65 \mathrm{~mm}$ diameter rod from Enpar Special Alloys Limited. Use of ORNL historical Nimonic PE-16 was judged to be acceptable to produce biaxial creep specimens after the chemical and microstructural evaluation was completed. Out-of-pile testing was planned to correlate current production Nimonic PE-16 to historical ORNL material. An image from a metallographic mount of Enpar Nimonic PE-16 $45 \mathrm{~mm}$ diameter rod is shown in Figure 5. This image shows precipitates that appear more like stringers than the dotted bands visible in Figures 3 and 4 . These variations are not unusual; however, as PE-16 material has a tendency to reveal different microstructures for identical fabrication routines (Reference 2 ).

Alloy 617 biaxial creep specimens were fabricated out of available plate stock having the required grain size.

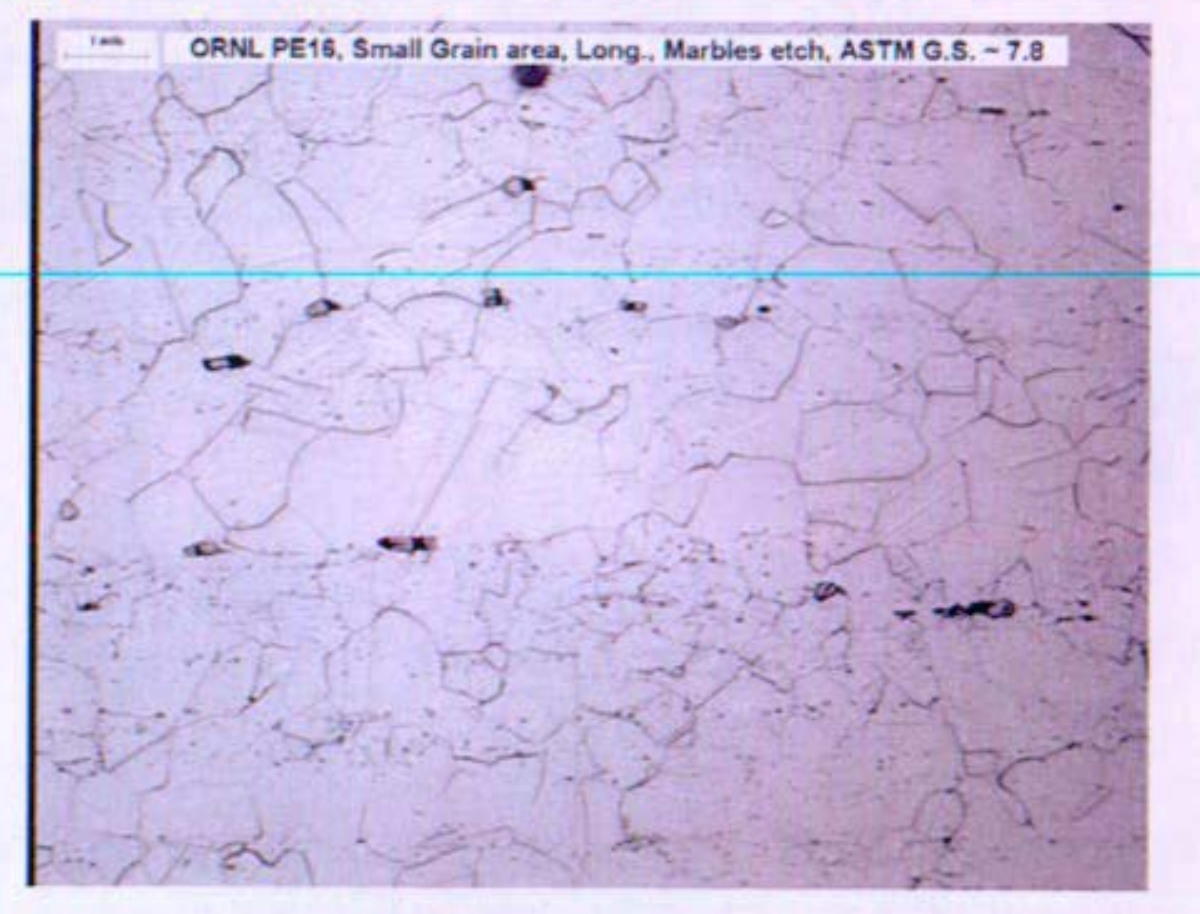

Figure 3: Nimonic PE-16 from ORNL historical stock (TC1747), longitudinal view showing precipitate banding in primarily small grain area. 


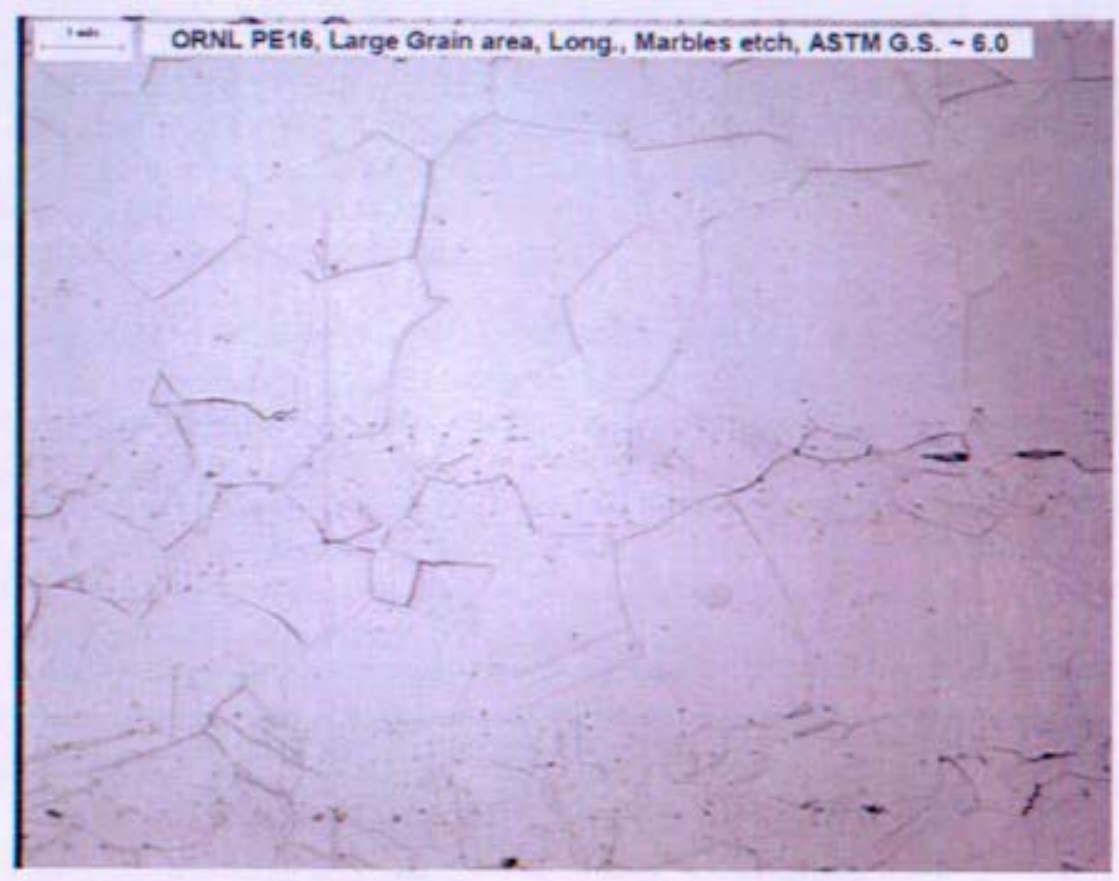

Figure 4: Nimonic PE-16 from ORNL historical stock (TC1747), longitudinal view showing precipitate banding in primarily large grain area.

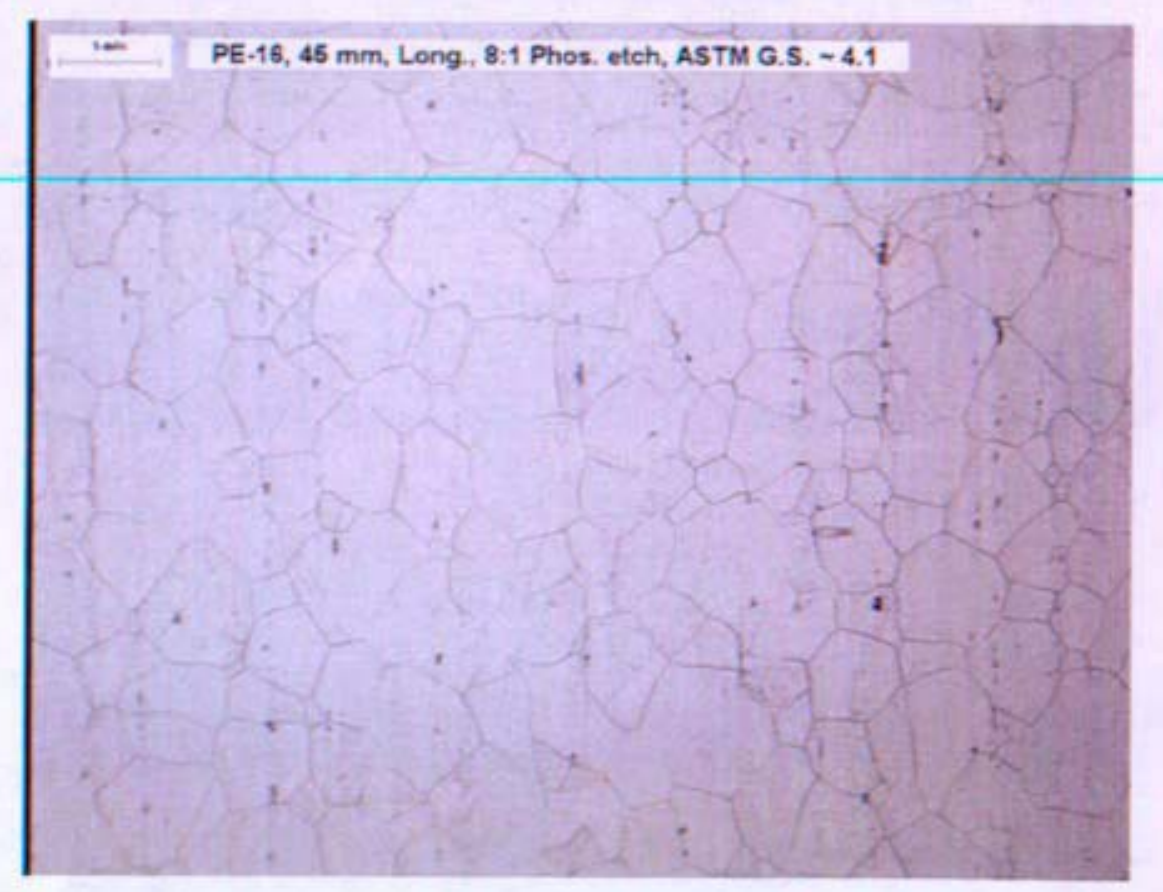

Figure 5: Nimonic PE-16 from Enpar, $45 \mathrm{~mm}$ diameter bar showing longitudinal view with some precipitate banding/stringers. 
Enclosure 2 to

MDO-723-0044 I

B-MT(SRME)-52

Page 7

Specifications for machining Haynes 230 and Nimonic PE-16 biaxial creep specimens were also developed and, in parallel, final drawings of specimen designs were completed. Attachment 2 provides machining specifications developed to produce Haynes 230 biaxial creep specimens from tubing and rod stock for end-caps, Alloy 617 specimens from plate stock, and Nimonic PE16 specimens from ORNL rod stock. To expedite specimen fabrication, vendors were directed to procure raw materials and provide an additional piece of starting raw material for future testing and evaluation. Initial specifications for creating the radjography groove geometry on biaxial creep specimen end-caps specified a $0.127 \mathrm{~mm}(0.005 \mathrm{inch})$ root radius on the groove (inside corners). This was insufficient for radiography resolution limits, which required, at least, a $0.0762 \mathrm{~mm}(0.003 \mathrm{inch})$ root radius. A resolution was established to machine the groove bottom radius based on previous fabrication experience of these specimens. NRPCT contracted with Dependable Tool and Die to make Haynes 230 biaxial creep specimens and Vangura Tool was contracted to machine PE-16 and Alloy 617 biaxial creep specimens. A brief receipt inspection performed on a sampling of Haynes 230 specimens showed these initial samples were within specification limits. A second order was also placed for more Haynes 230 biaxial creep specimens with Dependable Tool and Die to provide backup specimens.

Manufacturing plans were to have superalloy biaxial creep specimen parts marked, cleaned and baked out at NRPCT prior to being shipped to PNNL, where they would be assembled, welded, inspected, pressurized, and thermally treated. PNNL would then ship completed samples back to NRPCT for JOYO capsule insertion. Sample work instructions for marking, cleaning, and baking out of biaxial creep specimen parts are provided in Attachment 3 . Final specimen parts were to be wrapped in low lint paper wipes and put into small glass vials with screw on tops for shipping to PNNL. The specimens designated for QA qualification and final fabrication were never used and all specimen parts have been inventoried at Bettis.

Specifications for Alloy 617 required specimens to have a maximum of ASTM grain size $4(\sim 2.3$ $\mu \mathrm{m})$ to maintain the recommended minimum number of through-wall grains. Vangura used grain size 3 material for initial biaxial creep specimens with a surface finish estimated at $\sim 0.76$ to $0.89 \mathrm{\mu m}(0.41 \mathrm{\mu m}$ was requested). These specimens were accepted for the purpose of welding development since NRPCT did not expect significant differences in welding characteristics between material of grain size 3 or 4 , or with a slightly rougher surface finish.

NRPCT evaluated effects of different end-cap weld concavities via finite element analysis (FEA) to reassess original $0.076 \mathrm{~mm}(0.003$ inch) concavity requirements. Weld concavity FEA showed that a maximum concavity of $0.228 \mathrm{~mm}$ (0.009inch) for all materials did not cause any significant changes in local stresses at tube weld end-caps. Following this analysis the weld concavity requirement was modified to a maximum of $0.228 \mathrm{~mm}(0.009 \mathrm{inch})$.

Development of superalloy biaxial creep specimens was successfully achieved. Assessment of the work completed to date indicated that fully qualifying specimens for testing was on schedule. Development of weld parameters and associated issues are detailed in Reference (3).

\section{Tensile, Fracture Toughness, and Density Disc Specimens}

Specifications for machining other specimens. needed for JOYO-1 were developed and are provided in Attachment 4. Alloy 617 and Haynes 230 specimens were machined from plate 
material and Nimonic PE-16 specimens were machined from $45 \mathrm{~mm}$ diameter bar material procured from Enpar Special Alloys Limited. No significant difficulties were encountered in machining tensile, fracture toughness, or density disc specimens. These specimens were planned to be marked, cleaned, and baked out by NRPCT. However, after project restructuring, most specimens have been left in an as-received condition from the machining vendors. Standard pre-packaging cleaning processes for specimens from Vangura Tool is an acetone rinse while Dependable Tool uses an alcohol rinse.

At project restructuring, NRPCT was considering various precracking options. Since vendors with experience in precracking sub-size fracture toughness specimens were not found, an inhouse effort was initiated to develop a precracking procedure. Limited in-house work was performed using Haynes 230 specimens to develop precracking parameters, resulting in an a $W$ $=0.5$ on a second trial and error attempt. This was a timely success as NRPCT had no previous experience precracking subsized fracture toughness bars and literature data was not available on the fracture toughness $\left(K_{1 c}\right)$ values for these alloys. Some initial calculations were made estimating $\mathrm{K}_{1 \mathrm{c}}$ and load requirements for precracking bars. Attachments 6.1 through 6.4 provide experimental setup details and the precracking parameters obtained for Haynes 230 .

\section{Compact Tension Specimens}

In a test of fabricability and to evaluate bolt or wedge loading techniques, some miniature circular disc compact tension (CT) specimens were machined from historical Haynes 230 bar (Heat 47105). Figure 6 shows a microstructure photo of bar stock, a sketch of specimen orientation in the bar and a picture of a CT specimen. Stringers are clearly visible in the photo and coincide with rolling direction, but a preferred grain orientation was not detected. Four development specimens were machined to the drawing in Figure 7, with the exceptions that the developmental specimen had a straight notch (vice chevron) and did not have the clip gage notches machined. A subsequent drawing was created (Figure 8 ) to include a bolt as an initial loading technique. Plans were in place to develop a precracking and bolt-loading procedure for $0.2 T$ CT specimens. A vendor with experience in precracking (ORNL) $0.2 T C T$ specimens was available. However, the proposed in-pile crack growth rate test required a loading technique that could work within the physical constraints of the JOYO capsules. Since experienced labs/vendors were not available, continued (future) development would have to be performed inhouse. 
Enclosure 2 to

MDO-723-0044 I

B-MT(SRME)-52

Page 9

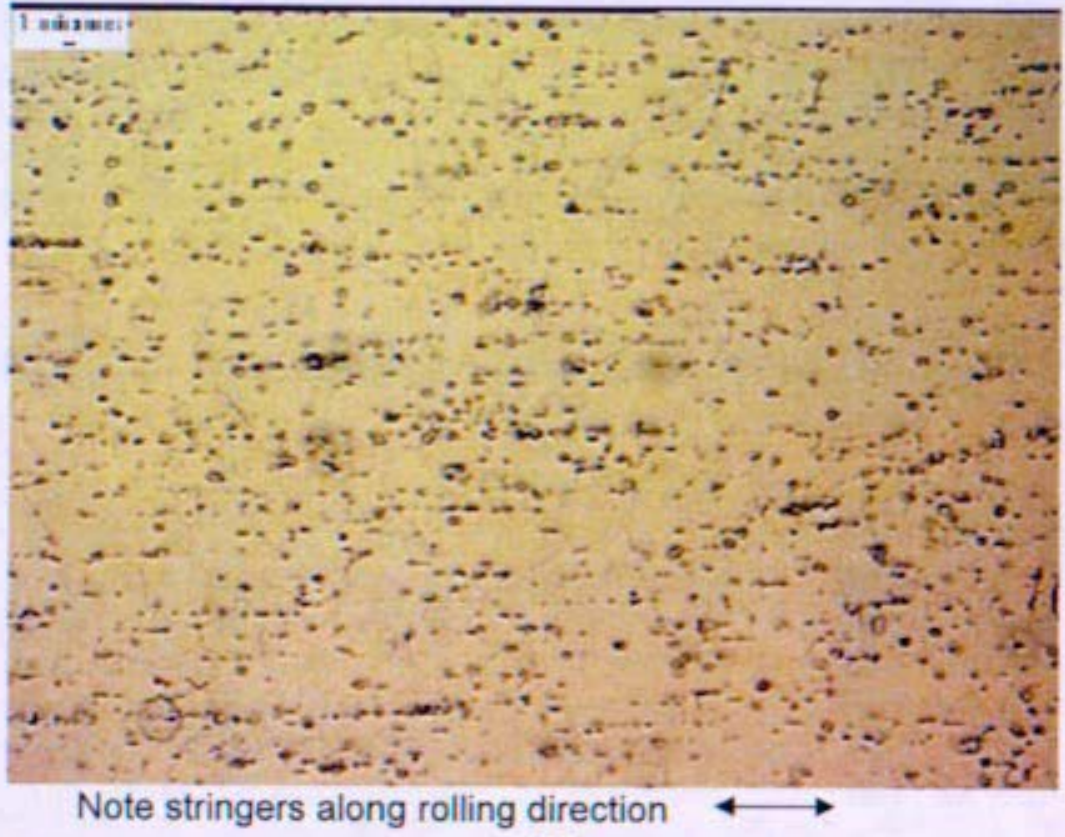

(A)

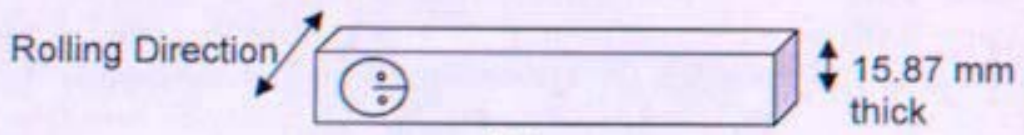

(B)

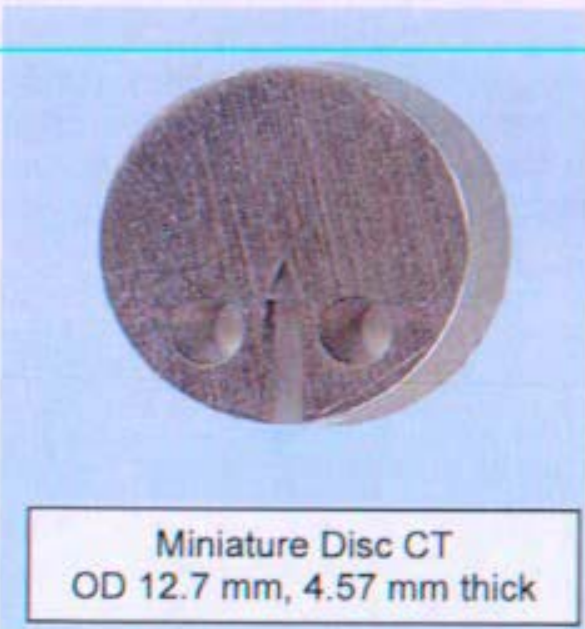

(C)

Figure 6: (A) Micrograph, (B) Machining Orientation Sketch, and (C) Photo of Haynes 230 (Heat 47105) Circular Disc Compact Tension Specimen. Visible stringers coincide with rolling direction, but a preferred grain orientation was not detected. 
Enclosure 2 to

MDO-723-0044 I

B-MT(SRME)-52

Page 10

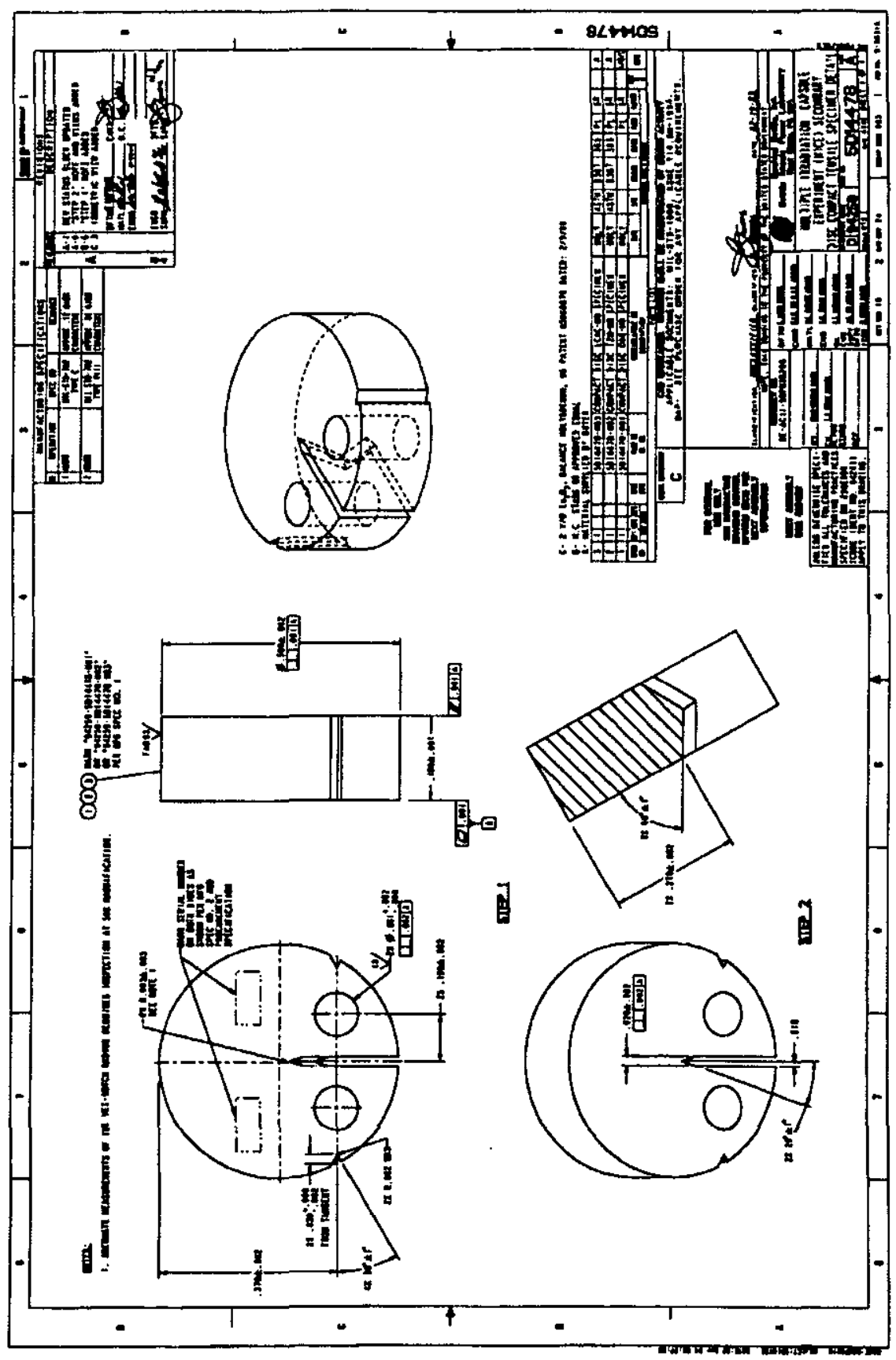

Figure 7: NRPCT issued CAD specifications for the compact tension disc. 
Enclosure 2 to

MDO-723-0044 /

B-MT(SRME)-52

Page 11

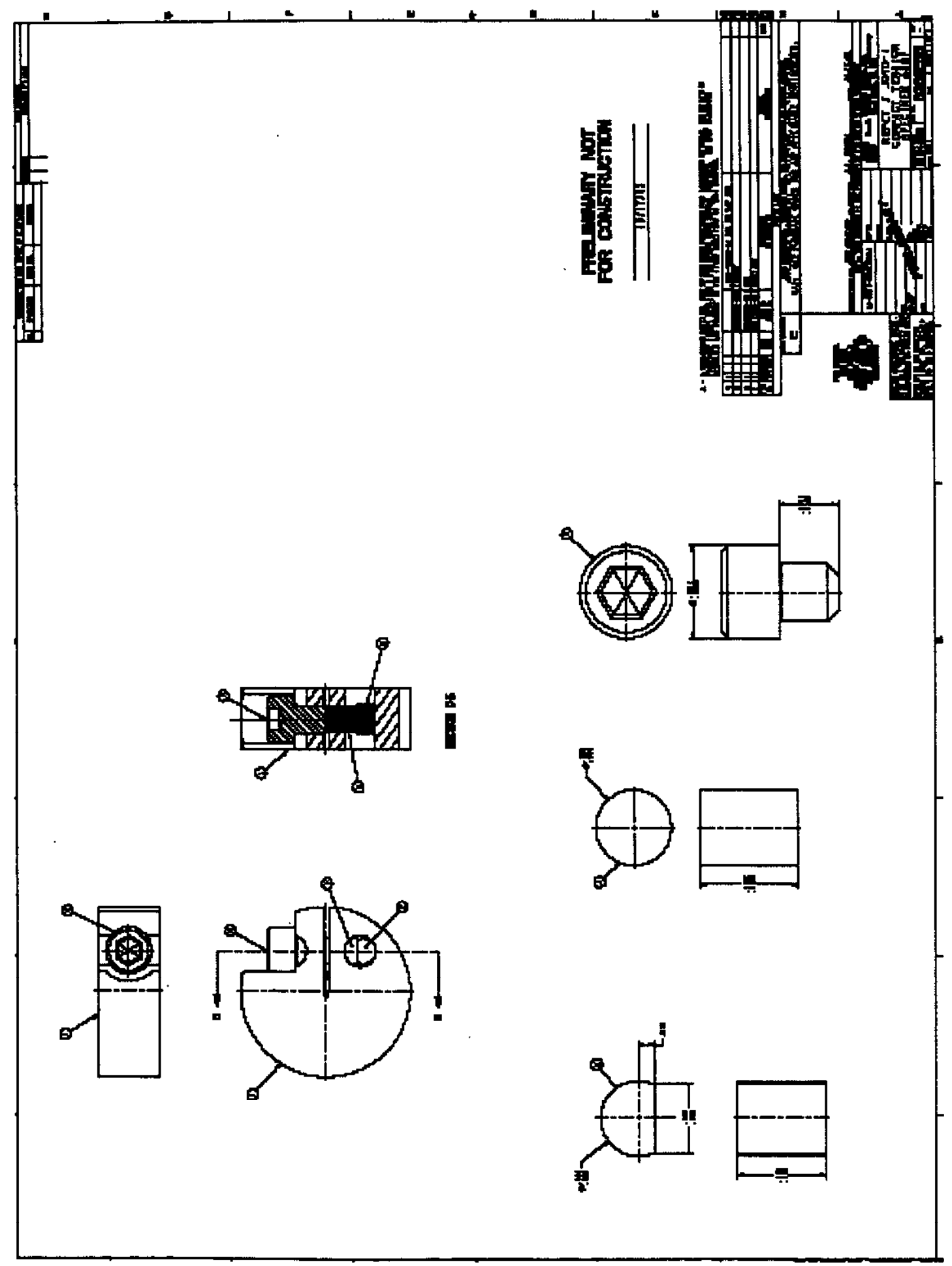

Figure 8: NRPCT issued CAD specifications for the compact tension disc, showing bolt-loading method. 
Enclosure 2 to

MDO-723-0044 I

B-MT(SRME)-52

Page 12

A listing of the materials obtained/procured for the superalloy specimens is provided in Table 2.

Table 2: Summary of Raw Superalloy Materials

\begin{tabular}{|c|c|c|c|c|}
\hline $\begin{array}{c}\text { Raw } \\
\text { Material }\end{array}$ & Source & Material Size & Cost & Status/Comment \\
\hline \multirow{3}{*}{$\begin{array}{l}\text { Alloy } \\
617 \\
\text { Plate }\end{array}$} & All Metal Sales & $0.5^{\prime \prime}$ thick plate, $12^{\prime \prime} \times 24^{\prime \prime}$ & $\$ 2,860$ & Rcvd. Remnant \\
\hline & High Temp Metals & $0.52^{n}$ thick plate $12^{n} \times 24^{n}$ & $\$ 3,120$ & Rcvd. Remnant \\
\hline & Haynes Int. & $0.5^{\prime \prime}$ thick plate, $12^{\prime \prime} \times 24^{\prime \prime}$ & $\$ 2,910$ & Rcvd. Remnant \\
\hline $\begin{array}{c}\text { Haynes } \\
230 \\
\text { Plate }\end{array}$ & Haynes Int. & $0.5^{\prime \prime}$ thick plate, $12^{\prime \prime} \times 24 "$ & $\$ 2,280$ & Rcvd. Remnant \\
\hline Tube & Bettis & $\begin{array}{c}0.25 \times 0.025 \text { welded } \sim 4 \text { pieces } \\
12 " \text { long remain at } \mathrm{KAPL} \text {, Bettis } \\
\text { has stock remaining }\end{array}$ & N/A & Revd. \\
\hline \multirow{3}{*}{$\begin{array}{l}\text { Nimonic } \\
\text { PE-16 } \\
\text { Rod }\end{array}$} & ORNL Archive & $\begin{array}{c}0.625^{\prime \prime} \text { OD, 2" remnant and } \\
0.375^{\prime \prime} \times 8 \text { " remnant }\end{array}$ & N/A & $\begin{array}{c}\text { Returned Extra to } \\
\text { ORNL }\end{array}$ \\
\hline & \multirow{2}{*}{$\begin{array}{l}\text { Rod from ENPAR } \\
\text { in UK, }\end{array}$} & $45 \mathrm{~mm}$ dia., $5 \mathrm{ft}$ & $\$ 3,910$ & \multirow[b]{2}{*}{ Revd. } \\
\hline & & $65 \mathrm{~mm}$ dia., $5 \mathrm{ft}-2$ pieces. & $\$ 7,690$ & \\
\hline
\end{tabular}

Chemistry and some metallography results for the superalloy materials are provided in Attachment 5. 


\section{Enclosure 2 to \\ MDO-723-0044 / \\ B-MT(SRME)-52 \\ Page 13}

\section{References}

1. S. Zinkle et al., "Critical Assessment of Structural Materials for Space Nuclear Applications," ORNL/LTR/NR-JIMO/04-08, Oct. 2004.

2. Partidge, A. et al., Journal of Nuclear Materials Volume 186, 1992. pp. 100-116.

3. Bettis Letter B-MT(SRME)-50, "Biaxial Creep Specimen Fabrication Close-out Report (U)" To Be Issued. 
Enclosure 2 to

MDO-723-0044 I

B-MT(SRME)-52

Page 14

\author{
Attachments \\ Attachment 1: Specification for Alloy 617 Tubing \\ Attachment 2: Specifications for Machining Biaxial Creep Specimens Parts \\ Attachment 2.1 Haynes $230\left(1^{\text {st }}\right.$ Order $)$ \\ Attachment 2.2 Haynes 230 ( $2^{\text {nd }}$ Order) \\ Attachment 2.3 Alloy 617 and Nimonic PE-16
}

Attachment 3: Marking / Cleaning / Bakeout Instructions

Attachment 4: Specifications for Machining Tensile. Fracture Toughness and Density Disc Specimens

Attachment 4.1 Haynes 230

Attachment 4.2 Alloy 617

Attachment 4.3 Nimonic PE-16

Attachment 5: Superalloy Material Details (Chemistry, Order / Inspection Info, Metallography)

Attachment 6: Fracture Toughness Precracking Experimentation

Attachment 6.1 Fracture Toughness Estimation

Attachment 6.2 Load \& Cycling Data for S/N 1

Attachment 6.3 Plotted Load Data

Attachment 6.4 Projected Cycles for a $W=0.5$

Enclosure 2 to MDO-723-0044 / B-MT(SRME)-52

PRE-DECISIONAL - For Planning and Discussion Purposes Only 
Attachment 1 to

Enclosure 2 to

MDO-723-0044/

B-MT(SRME)-52

Page 1

Attachment 1

Specification for Alloy 617 Tubing

Enclosure 2 to MDO-723-0044/B-MT(SRME)-52 
Attachment 1 to

Enclosure 2 to

MDO-723-0044/

B-MT(SRME)-52

Page 2

\section{SPECIFICATION COVER SHEET}

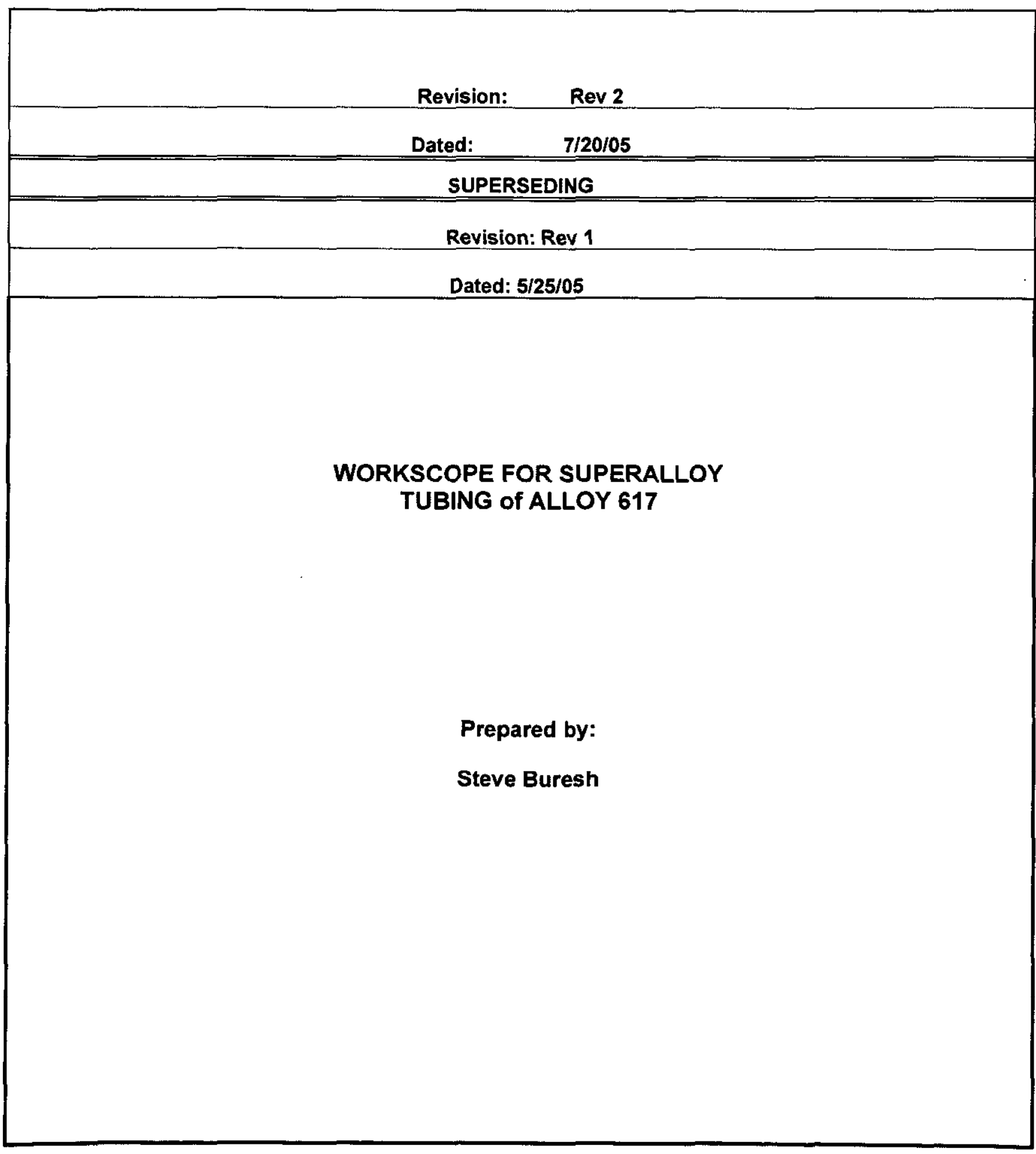




\section{WORKSCOPE FOR SUPERALLOY TUBING OF ALLOY 617}

1.0 Scope. The Selier shall provide Superalloy Tubing of Alloy 617 as specified herein.

\subsection{Applicable Documents}

2.1 ASTM B167 - 01 "Standard Specification of Nickel-Chromium-iron Alloys and Nickel-ChromiumCobalt -Molybdenum Alloy Seamless Pipe and Tube".

2.2 ASTM B829 - 04a "Standard Specification for General Requirements for Nickel and Nickel Alloys Seamless Pipe and Tube".

\subsection{Requirements}

3.1 Classification. The Alloy 617 shall meet the composition, type, form, condition, finish, and dimensions listed in ASTM B167 and ASTM B829.

\subsubsection{Composition.}

3.1.1.1 The Alloy 617 material shall meet the chemistry requirements of ASTM B 167 Table 1 for Alloy (UNS) N06617. Elements not specified shall not be deliberately employed as alloying or refining additions without approval.

3.1.2 Ingot Type. Starting stock shall originate from VIM or VAR or ESR melted material. Chemistry analysis of the starting stock shall be supplied.

\subsubsection{Form. Tubing shall be seamless.}

3.1.4 Condition. The tubing shall be provided in the solution annealed condition unless approved by the Buyer. Final tubing shall not be reannealed after the final heat treatment and straightening without obtaining prior approval.

3.1.5 Finish. Final tubing surfaces shall be free of scratches and defects deeper than $5 \%$ of the nominal tubing wall thickness. The tubing inside and outside surface finishes shall not be rougher than 16 microinches and shall be free of any scale or oxide. Tubing shall have a final bright hydrogen anneal and a final light grind or polish (which minimizes material cold work) may be performed to obtain the required surface finish, acid cleaning subsequent to the final bright hydrogen anneal is not permitted Light imperfections resulting from material handling are not considered cause for reject.

3.1.6 Dimensions. Final tubing shall be $0.25^{\prime \prime} \mathrm{OD}+/-0.004^{\prime \prime} / 0.000$ " with a wall thickness of $0.025^{\prime \prime}+1-0.0025^{\prime \prime}$. Ovality shall be held within $1 \%$ of the theoretical average outside diameter.

3.1.7 Straightness. The tubing shall be straight and free of kinks and bends. No straightening shall be performed after the final heat treatment without prior approval by the Buyer, except to meet straightness requirements and conduct ultrasonic testing. The straightening operation after the final anneal shall be performed in a manner which minimizes both the cold work due to straightening and the tube-to-tube variation in tensile 
Attachment 1 to

Enclosure 2 to

MDO-723-0044I

B-MT(SRME)-52

Page 4

properties due to this cold work.

3.1.8 Quantity. The Seller shall furnish a minimum of 100 feet in minimum $24^{\text {" }}$ pieces.

\subsection{Chemical analysis.}

3.2.1 Sampling. Chemical samples shall be taken when the material has been reduced to the final nominal size and shape. One sample shall be taken from each heat or lot and analyzed for the elements of $\mathrm{Ni}, \mathrm{Cr}, \mathrm{Fe}, \mathrm{Ti}, \mathrm{Al}, \mathrm{C}, \mathrm{Nb}, \mathrm{Mo}, \mathrm{Mg}, \mathrm{Mn}, \mathrm{Si}, \mathrm{Cu}, \mathrm{Co}, \mathrm{B}, \mathrm{S}, \mathrm{P}, \mathrm{Zr}, \mathrm{O}, \mathrm{N}, \mathrm{H}$.

3.2.2 Procedure. Chemical analysis shall be performed using instruments and techniques with the demonstrated capability to provide the precision, accuracy and sensitivity adequate to certify conformance of the material to the specified compositional requirements.

3.2.3 Acceptance criteria. When any sample fails to conform to the requirements listed in Item 3.1 .1 , the lot represented by that sample shall be rejected. At the option of the Seller, each piece in the rejected lot may be analyzed at the Seller's expense; if the individual piece results are acceptable, no further action is required. The results of the original test, along with the individual retest results shall then be included in the material certification.

3.3 Mechanical requirements. Testing shall be performed on the final tubing (after straightening, if performed) for each material lot. The tensile strength, $0.2 \%$ offset yield strength, and $\%$ elongation shall be reported. Hydrostatic testing will not be required, Eddy current testing will be performed instead (para. 3.4). Replacement or discarding of test specimens and retests need to be reviewed by the Buyer and are subject to reject/approval by the Buyer with the following exceptions:

A test specimen may be discarded and a replacement specimen selected from the same lot of material under the following conditions:

Where the specimen is incorrectly machined.

Where the test procedure is incorrect.

Where there is a malfunction of the testing equipment.

(d) Where a flaw that is not indicative of an inferior or defective lot of material develops during the test.

However, internal flaws such as cracks, ruptures and porosity are not reasons for the selection of a replacement test specimen.

3.4 Inspection. Testing shall be performed on all of the final tubing (after the final heat treatment) using Eddy current to detect defects that are $10 \%$ of the tubing wall thickness. A final tubing grain size shall be measured and reported, the grain size shall be measured per ASTM E112 on a transverse sample representing full wall thickness. The grain size shall be an average ASTM grain size of 4.5 to 7. The tubing shall not exhibit a fine grain layer on the inside diameter surface. A fine grain layer is a continuous band of grains characterized by an average grain size of approximately two or more ASTM grain sizes finer than the average bulk tube grain size and the band may contain heavy precipitates.

3.4.1 Removal of Defects. Defects may be removed by filing, machining, or grinding provided such removal does not exceed 5 percent of the nominal wall thickness. Defect removal method shall not deposit iron on the surface. Repaired areas shall be faired smoothly into the surrounding material so that the bottom radius of the repaired area equals at least three times 
Attachment 1 to

Enclosure 2 to

MDO-723-0044/

B-MT(SRME)-52

Page 5

the depth of the repair. The removal of a defect shall be verified by the method originally used to detect the defect.

\subsubsection{Weld Repairs. Weld repair of the material is prohibited.}

3.5 Marking. Individual pieces shall each be identification marked on one end. Identification marking shall include the manufacturer, the alloy descriptor (Alloy 617) and the material lot number. The marking shall be performed using, electrochemical etch, vibratool, laser, or other Buyer approved method with the intent to minimize mechanical marking damage to the tubing.

\subsection{Cleanliness}

3.6.1 Cleaning. Prior to any heat treatment material shall be cleaned free of chips, dirt, dust, oils, oxides, scale, weld spatter, and other foreign matter by wiping or washing with soft brushes or cloths. If shot, grit or vapor blasting is used, the process shall not deposit iron on the surfaces.

3.6.2 Descaling. Descaling shall be accomplished by chemical descaling or by machining, grinding or other mechanical means so that the inside and outside surfaces are free from scale and other foreign matter. If chemical descaling is used, the inside and outside diameter surfaces of the tube or pipe shall be thoroughly neutralized and rinsed in water to remove the descaling solution. Descaling or pickling shall not be performed after the final anneal.

3.6.3 Detrimental Materials. Contact with antimony, arsenic, bismuth, cadmium, lead, tin and zinc, and consumable products, such as lubricants and marking materials, containing these low melting point materials in excess of 250 parts per million (ppm) each is prohibited. This prohibition on low melting point materials applies during thermal treatments and to the finished or cleaned surfaces of tubes offered for acceptance. Products do not need to be analyzed when product manufacturers certify that low melting point materials in excess of $250 \mathrm{ppm}$ are not present in the products. Contact with mercury and consumable products that contain more than $10 \mathrm{ppm}$ mercury is prohibited. Products do not need to be analyzed when product manufacturers certify that mercury in excess of $10 \mathrm{ppm}$ is not present in the product, or certify that mercury and mercury compounds have not been added to the product and have not come in contact with the product during processing. Marking materials containing phosphorus or sulfur in excess of $250 \mathrm{ppm}$ each shall not be used during thermal treatments and on finished or cleaned surfaces of tubes offered for acceptance.

3.7 Certification of quality conformance. Two copies of a certification of quality conformance shall be furnished with the lot of material offered for acceptance. One copy will be provided to the Buyer and one copy shall be shipped with the material. The certification shall include:

1.) Metallurgical condition of finished tubing.

2.) Thermal/Mechanical Processing History Outline from original tube hollow (extrusion or drilled hollow).

3.) Ingot/ Heat or lot identification numbers and the piece identification numbers associated with the Ingot/Heat or lot.

4.) Quantitative results of specified chemical tests at final product thickness

5.) Results of dimensional inspection to confirm that the dimensional requirements have been met.

6.) Results of visual, Eddy current and mechanical tests. 
Attachment 1 to

Enclosure 2 to

MDO-723-0044/

B-MT(SRME)-52

Page 6

3.8 Process Outline, Schedule and Buyer Witnessing. Within one week after order placement, the Seller shall provide a detailed process outline of the manufacturing/fabrication processes and the planned schedule for the key processing steps. As a minimum, the process outline shall identify all intermediate and final percent reduction, and any in process thermal treatments. Buyer has the option to witness processing steps and to inspect the final product prior to shipment at the Seller's facility.

\subsection{Preparation for Delivery}

\subsection{Shipping Release.}

Seller is required to obtain formal QA shipping release prior to shipment of the equipment to Buyer. Following completion of the Seller processing and testing, Seller shall formally request shipping release, which will be addressed by separate correspondence. The Seller's request should include:

-Purchase order line item identification;

-Listing of Degradation of Specification Requirements, hardware or software discrepancy reports (including status (open or closed) and indication whether critical or non-critical);

-Copy of completed compliance documentation (e.g., final inspection and test reports, certifications, etc.) verifying compliance by signature and/or initial. The documentation should be aligned with

(and traceable to) each element of the specification compliance checklist;

-Identification of Open Items ("Punch List");

-Statement that all required submittals have been made and accepted by BUYER;

-Statement that BUYER has been notified of all Unusual Incidents;

-Statement that all Corrective Action Requests (CAR) have been accepted by BUYER;

-Identification of any items "short-shipped", with justification.

\subsection{Preparation for packaging and packing. Preparation for packaging and packing shall be as} follows:

(a) Tubes shall be clean and free of chips, dirt, dust, oils, oxides, scale, weld spatter, and other foreign matter, mechanical cleaning may be used when approved.

(b) Contact preservative shall not be used.

(c) Tubes shall be segregated as to material type.

4.3 Packaging, packing, and marking for shipment. Unless additional requirements are specified by the Buyer, material shall be prepared for shipment in accordance with commercial practice to ensure delivery of product in full compliance with this specification. The level of packaging and marking for shipment shall meet the requirements of carrier rules and regulations applicable to the mode of transportation.

As a minimum, the tubes shall be placed in shipping containers with packing material placed between the box and the tubes to prevent movement during shipping. The outside of the box shall be clearly labeled with the words "Superalloy Tubing" the heat or lot number. Individual heats or lots shall be packaged in separate shipping containers. Paperwork indicating tube quantities and heat shall be enclosed within each shipping container. A copy of the certification of quality conformance shall be enclosed with the tubes as outlined in 3.7 .

\subsection{Data Submittals}

5.1 Summary Table of data required for submittal: 
Attachment 1 to

Enclosure 2 to

MDO-723-0044/

B-MT(SRME)-52

Page 7

\begin{tabular}{|l|c|l|l|}
\hline Description (Reference) & $\begin{array}{l}\text { No. of } \\
\text { Copies }\end{array}$ & Date Due & Type \\
\hline Process Outline and Schedule (par. 3.8) & 2 & $\begin{array}{l}\text { One week after date of } \\
\text { order }\end{array}$ & Information \\
\hline Material Chemical Analysis (par. 3.1.2 and 3.2) & 2 & Prior to Lot Shipment & Information \\
\hline Mechanical Testing Data (par. 3.3) & 2 & Prior to Lot Shipment & Information \\
\hline Inspection Data (3.4) & 2 & Prior to Lot Shipment & Information \\
\hline Request for Shipping Release (par. 4.1) & 2 & Prior To Lot Shipment & Information \\
\hline Certificate of Compliance (par. 3.7) & 2 & Prior to Lot Shipment & Information \\
\hline
\end{tabular}

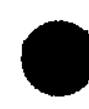


Attachment 2.1 to

Enclosure 2 to

MDO-723-0044/

B-MT(SRME)-52

Page 1

Attachment 2.1

Specifications for Machining Biaxial Creep Specimens Parts

Haynes $230\left(1^{\text {st }}\right.$ Order $)$

Enclosure 2 to MDO-723-0044/B-MT(SRME)-52 
Attachment 2.1 to

Enclosure 2 to

MDO-723-0044/

B-MT(SRME)-52

Page 2

\section{Haynes 230 Biaxial Creep Specimens Machining Details \\ $\operatorname{Rev} 1(6 / 30 / 05)$ \\ WA05PFG104}

Supply 50 sets of matched end caps and tube per drws. 5D15996 and SK-DPM3051705. Each specimen consists of a tube (5D15996 ITEM 17) and two endcaps, one with a center hole (SKDPM3051705) and one solid (5D15996 ITEM 11). Each specimen consists of a tube and two endcaps, one with a center hole and one solid.

End caps shall be made from Haynes 230 annealed rod material per AMS 5891 with material certifications supplied.

End caps shall be machined to match fit inside the supplied tubing with a clearance of $0.0005^{\prime \prime}$ to $0.0015^{n}$.

$\sim 0.5$ " diameter Haynes 230 rod to AMS 5891 can be obtained from stock at:

Haynes International

Karen Spencer

8004261963

Hi Temp Metals

8005002141

Attached is some vendor information on Haynes $\mathbf{2 3 0}$ materials for your reference. 


\section{MACHINING}

HAYNES* $230^{*}$ alloy is similar in machining characterlstics to other solid-solution-strengthoned nickel-base alloys. These alloys as a group are classified as moderate to difficult to machine; however, it should bo emphasized that they can be machined using conventional methods at satisfactory rotes. As these alloys will work-harden tapidly, the keys to successfu machining are to use slower speeds and teeds, and to take heavier cuts than would be used for machining stainiess oteels. See Haynes Intemational publication $\mathrm{H}-3159$ for more detalled information.

\section{Nomal Roughing (Tuming/Facing)}

Use carbide $C-2 / C-3$ grode too!

Speed: Go surface feetminute

Feed: 0.010 in /revolution

Depth of Cut: 0.150 in.

Negative rake oquare Insert, $45^{\circ}$ SCEA'

1/32 in. nose radius. Tool holder: $5^{\prime}$ negative back and side rakes.

Lubricant: Dryt, Oil or water-base*t

\section{F-inushing (fuming/Facing)}

Use carbide C-2/C-3 grade tool

Speed: 95-1 to surface teot/minute

Feed 0.005-0.007 in.:́revolution

Eepth of Cut: 0.040 in.

\section{Dilling}

Use high speed steel M-33M-40 series $\%$

or T-15 grades"

Speed: 10-15 surface foedminute 1200

RPM maximum for $1 / 4 \mathrm{in}$. diameiet or smaller)

Lubricant: O:l or watel-base. Use cooiant feed drils if possible
Positive rake square insert, if possible, 45. SCEA, 1/32 in. nose radius. Tool halder $5=$ positive beck and gide rakes.

Lubrican:; Dry or water-base

- Carbide drils no: recommended, but may be used in some set-ups. See Haynes inithational plbioation H-3:5e for cetails.

Notes: 1 SCEA-Side cutting edge angle, or fead angle of the tool.

2 At any point where dry cuting is recommended, en air jet directed on the tool nuay provide substantial tool life increases. A water-base coolant mist may also bo effective.

- Oil coolant should be a premium qualty, sulfochlorinated oil with extreme presoure additives. A visecsity at 100"F trom 50 to 125 SSU is atandard.

- Water-base cooiant should be a 15:1 mix of water with either a premium quality, sulfoehloninated water soluble oil or c chemical emulsion with extreme pressure additives.

s Water-base coolants may cause chipping or rapid failure of carbide toois in interrupted cuts.

- M-40 series High Speed Steels include M-41 through M-45 at time of wnting, others may be added, and should be equaly suitable.

Acknowledgements:

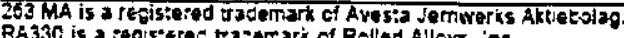

RA330 is a iegrieres tojerraft of Rolled Alloys, 'ne

INCONEL and ZDEGT are ceqistered trafemarks of Inco Fam'ly co Companies. 
Attachment 2.1 to

Enclosure 2 to

MDO-723-0044/

B-MT(SRME)-52

Page 4

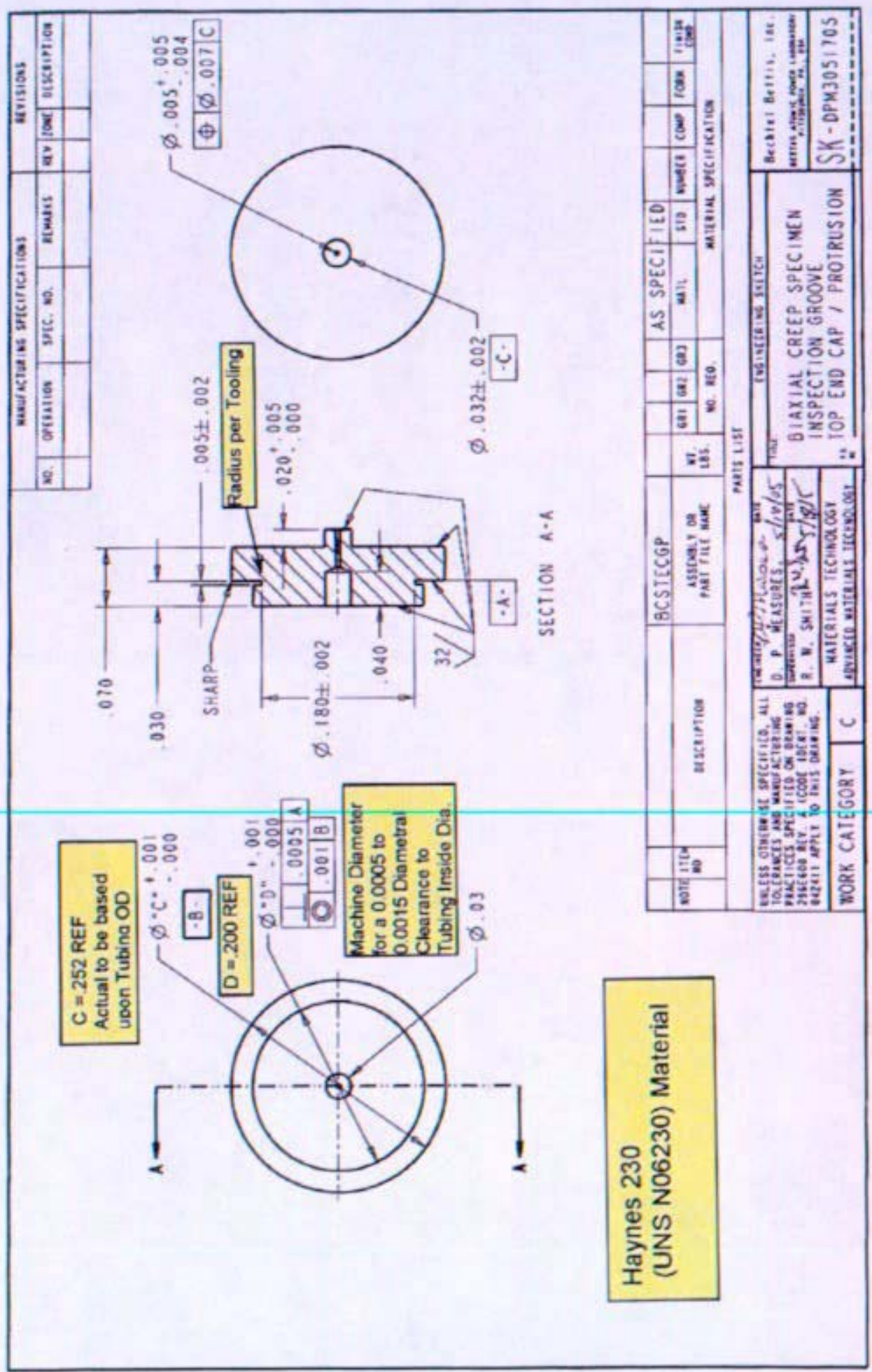

PRE-DECISIONAL - For Planning and Discussion Purposes Only 


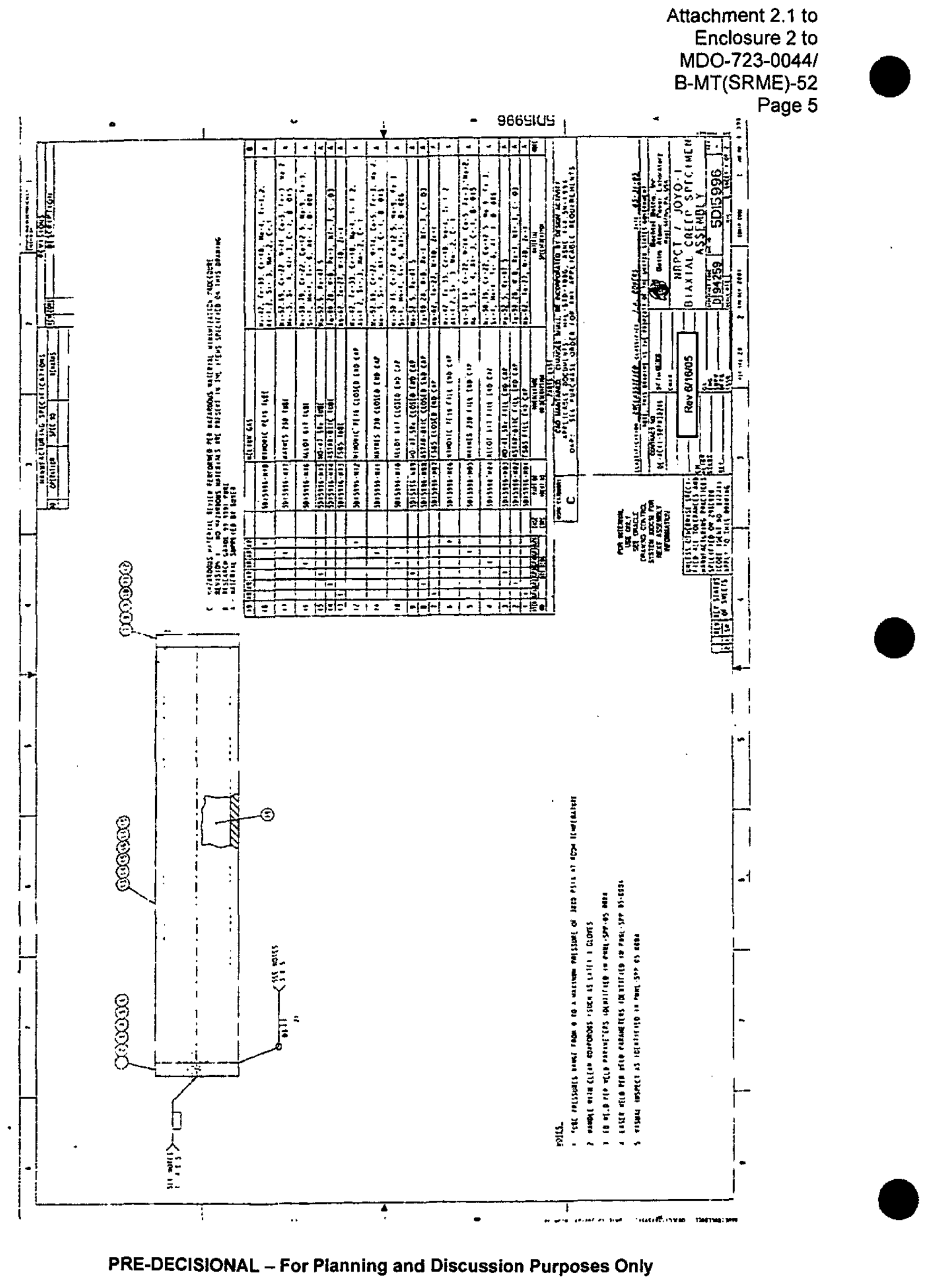


Attachment 2.1 to

Enclosure 2 to

MDO-723-0044I

B-MT(SRME)-52

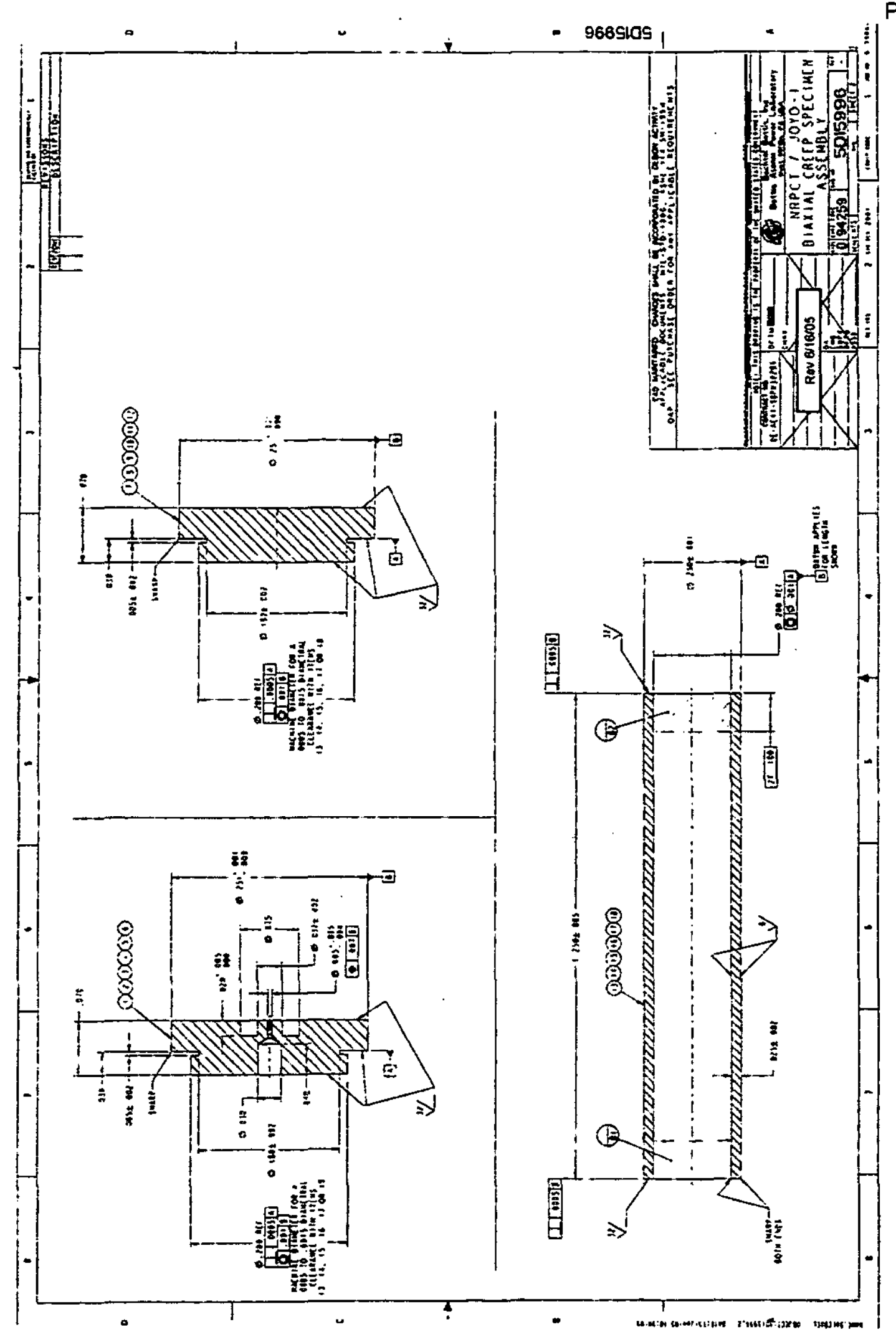

Page 6
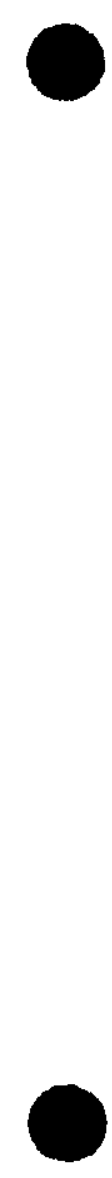

PRE-DECISIONAL - For Planning and Discussion Purposes Only 
Attachment 2.2 to

Enclosure 2 to

MDO-723-0044/

B-MT(SRME)-52

Page 1

Attachment 2.2

Specifications for Machining Biaxial Creep Specimens Parts

Haynes 230 ( $2^{\text {nd }}$ Order)

Enclosure 2 to MDO-723-0044/B-MT(SRME)-52 
Attachment 2.2 to

Enclosure 2 to

MDO-723-0044I

B-MT(SRME)-52

Page 2

\section{Haynes 230 Biaxial Creep Specimens Machining Details}

Supply 30 sets of Haynes 230 matched end caps and tubes per drw. SK-DPM3051705, and 5D15996. Each specimen consists of a tube and two endcaps, one with a center hole (SKDPM3051705) and one solid (5D15996 ITEM11). The protrusion style endcap shall be used for the one with the hole in it will per SK-DPM3051705. End caps shall be made from Haynes 230 annealed rod material per AMS 5891 with material certifications supplied.

The dimensions " $C$ " and " $D$ " on SK-DPM3051705 and equivalent dimensions on Item 11 of 5D15996 shall be reported for each endcap and supplied with the parts. The dimensions shall be traceable to the individual endcap by identification on the endcap packaging.

End caps shall be machined to match fit inside the fabricated tubing with a clearance of $0.0005^{\prime \prime}$ to $0.0015^{n}$.

If EDM is used to cut the specimens a minimum of $0.005^{\prime \prime}$ per surface shall be removed to eliminate any EDM recast layer.

0.5 inch diameter Haynes 230 rod to AMS 5891 can be obtained from stock at:

Haynes International

Karen Spencer

8004261963

Hi Temp Metals

8005002141

Delivery of the 30 sets shall be broken into two shipments of 15 sets. 
Attachment 2.3 to

Enclosure 2 to

MDO-723-0044/

B-MT(SRME)-52

Page 1

Attachment 2.3

Specifications for Machining Biaxial Creep Specimens Parts

Alloy 617 and Nimonic PE 16

Enclosure 2 to MDO-723-0044/B-MT(SRME)-52 
Attachment 2.3 to

Enclosure 2 to

MDO-723-0044/

B-MT(SRME)-52

Page 2

\title{
Alloy 617 and Nimonic PE16 Biaxial Creep Specimens Machining Details Rev1 7/28/05
}

\begin{abstract}
Alloy 617
Supply 34 sets of Alloy 617 matched end caps and tubes with a maximum ASTM grain size of 4.0 (max average grain diameter $0.0035^{\prime \prime}$ ) and 36 sets of Alloy 617 matched end caps and tubes with a maximum ASTM grain size of 3.0 (max average grain diameter $0.005^{\prime \prime}$ ). The endcaps and tubes will be per drw. SK-DPM3051705, and 5D15996. Each specimen consists of a tube and two endcaps, one with a center hole (SK-DPM3051705) and one solid(5D15996 ITEM10). The protrusion style endcap shall be used for the one with the hole in it will per SK-DPM3051705.

The tube shall be machined from plate stock with the axis aligned with the plate rolling direction and the surface finish shall be 16 microinches (or better) on the OD and ID. The dimensions " $C$ " and " $D$ " on SK-DPM3051705 and equivalent dimensions on Item 12 of 5D15996 shall be reported for each endcap and supplied with the parts. The dimensions shall be traceable to the individual endcap by identification on the endcap packaging
\end{abstract}

End caps and tubes shall be made from Alloy 617 annealed plate material per AMS 5888 or ASTM B168 with material certifications supplied.

End caps shall be machined to match fit inside the supplied tubing with a clearance of $0.0005^{\prime \prime}$ to $0.0015 "$.

If EDM is used to cut the specimens a minimum of $0.005^{n}$ per surface shall be removed to eliminate any EDM recast layer.

$\sim 0.5^{n}$ thick plate of Alloy 617 to AMS 5888/ASTM B168 with the required grain size can be obtained from stock at:

Haynes International

Karen Spencer

8004261963

Altemp Alloys Inc.

Tony Baldridge

7149380601

An additional piece of all the identical heat Alloy 617 plate(s) used to produce the specimens shall also be supplied to the customer with minimum dimensions of $12^{\prime \prime} \times 24^{\prime \prime}$.

Attached is some vendor information on Alloy 617 material for your reference. 
Attachment 2.3 to

Enclosure 2 to

MDO-723-0044/

B-MT(SRME)-52

Page 3

\section{Machining}

Information on machining of alloy 617 can be obtained from Special Metals.

Cutting tools should be sharp and have positive cutting angles to minimize work hardening of the material. Cutting feed and depth of cut must be sufficient to prevent burnishing of the workpiece surface. Additional information on machining is available in the Special Metals publication 'Machining' on the company website, www.specialmetals.com. 
Attachment 2.3 to

Enclosure 2 to

MDO-723-0044/

B-MT(SRME)-52

Page 4

\section{Nimonic PE16}

Supply 50 sets of Nimonic PE16 matched end caps and tube per drw. SK-DPM3051705, and 5D15996. Each specimen consists of a tube and two endcaps, one with a center hole (SKDPM3051705) and one solid (5D15996 ITEM12). The protrusion style endcap shall be used for the one with the hole in it will per SK-DPM3051705. The tube shall be machined from rod stock with the axis aligned with the rod axis and the surface finish shall be 16 microinches or better on the OD and ID. The dimensions " $\mathrm{C}$ " and "D" on SK-DPM3051705 and equivalent dimensions on Item 12 of 5D15996 shall be reported for each endcap and supplied with the parts. The dimensions shall be traceable to the individual endcap by identification on the endcap packaging.

End caps shall be machined to match fit inside the fabricated tubing with a clearance of $0.0005^{\prime \prime}$ to $0.0015^{\prime \prime}$.

If EDM is used to cut the specimens a minimum of $0.005^{\prime \prime}$ per surface shall be removed to eliminate any EDM recast layer.

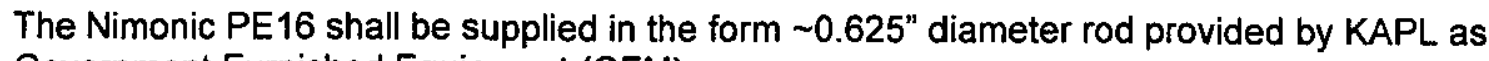
Government Furnished Equipment (GFM). 
Attachment 3 to

Enclosure 2 to

MDO-723-0044I

B-MT(SRME)-52

Page 1

Attachment 3

Marking / Cleaning / Bakeout Instructions

Enclosure 2 to MDO-723-0044/B-MT(SRME)-52 
Attachment 3 to

Enclosure 2 to

MDO-723-0044I

B-MT(SRME)-52

\section{MARKING INSTRUCTIONS}

Page 2

Laser ID on the larger face of the solid end plug using:

0.05 " font ( $~ 10-15$ passes full power) Taylor Hobson font

Alloy 617 A-AAR thru A-ABF;

Haynes 230 A-ADX thru A-AEK;

PE16 A-AHK thru A-AHZ

(ONLY PUT THE FOUR LETTER and DASH ID on the parts DO NOT USE LETTERS Q and U)

After marking clean per below instructions keeping parts matched. After cleaning handle with white gloves only,

Vacuum Bake parts at $475 \mathrm{~F}$ for $30 \mathrm{~min}$ at min vac $5 \times 10^{-5}$ torr (cover with new SS or Inconel house)

Wrap parts in a white low lint KIM wipes and place in glass vials (both provided).

\section{CLEANING INSTRUCTIONS}

0 . Keep end caps and tubes paired up as supplied.

1. Starting bath of each cleaning agent shall be new. The same bath may be used to clean different materials without replacing the cleaning agent provided that the following requirements are met:

- Parts made of the same material may be placed simultaneously into the same bath.

- Different material parts are not allowed to be placed simultaneously into the same bath.

- A cleaning agent must be replaced after one marterial set of components are cleaned in the bath before being used to clean other materials.

2. Immerse each part in iso-propanol bath for $3-5$ minutes (longer is permitted). Parts made of same material may be placed into same bath all at once or in several loads.

3. Select one of the following options:

- Clean the part(s) ultrasonically for 2 - 3 minutes, or

- Clean the part(s) using nylon or equivalent brush, lightly scrub each part in the bath. A new unique brush shall be used for each type of material

4. Remove from bath (observe material contact restrictions) and drip dry for minimum 15 seconds.

5. Immerse each part in another clean iso-propanol bath for $3-5$ minutes (longer is permitted).

6. Remove from bath (observe material contact restrictions) and rinse each part with fresh isopropanol. Drip dry for $\sim 30$ seconds,

7. Allow to air dry on lint free talc free cloth or paper towel (observe material contact restrictions).

8. After components are completely dry, package individual parts (or similar parts together) into separate new clear poly proceed to low temp bakeout. Exercise caution to avoid damages of parts due to mutual contact. 
Attachment 4.1 to

Enclosure 2 to MDO-723-0044/

B-MT(SRME)-52

Page 1

\author{
Attachment 4.1 \\ Specifications for Machining \\ Tensile, Fracture Toughness and Density Disk Specimens \\ Haynes 230 \\ Enclosure 2 to MDO-723-0044/B-MT(SRME)-52
}


Attachment 4.1 to

Enclosure 2 to

MDO-723-0044/

B-MT(SRME)-52

Page 2

\section{Haynes 230 Test Specimens Machining Details Rev 3 $8 / 4 / 05$}

1). Supply 100 fracture toughness specimens per drawing 5D16339.

The width, nominal $0.118^{\prime \prime}$, and height, nominal $0.118^{\prime \prime}$, dimensions shall be reported for each specimen and supplied with the parts. The dimensions shall be traceable to the individual specimen by identification on the specimen packaging

2). Supply 100 tensile specimens per drawing $5 D 16338$

The gage section width, nominal 0.060 ", and gage section thickness, nominal 0.030 ", dimensions shatl be reported for each specimen and supplied with the parts. The dimensions shall be traceable to the individual specimen by identification on the specimen packaging.

3). Supply 75 density disk specimens per drawing 5D16357

The diameter, nominal $0.492^{\prime \prime}$, and thickness, nominal $0.059^{\prime \prime}$, dimensions shall be reported for each specimen and supplied with the parts. The dimensions shall be traceable to the individual specimen by identification on the specimen packaging.

The specimens shall be machined from minimum $\sim 0.25^{n}$ thick plate. If the specimens are cut using EDM, a minimum of 0.005 " per surface shall be removed by grinding or conventional machining to eliminate any EDM recast layer. The disks surfaces shall be parallel to the starting plate surfaces. The tensile and fracture toughness specimen axis shall be aligned with the rolling direction of the plate. Additionally, the tensile specimen face (one with hole through it) shall be oriented parallel to the plate edge (width) surfaces and the fracture toughness specimen groove shall be oriented perpendicular to the plate edge (width) surfaces (See Figure 1 Below). The specimens shall be taken from a minimum depth of $0.06^{\prime \prime}$ from the starting stock surface. The Haynes 230 plate shall be to ASTM-B-435-03 / AMS 5878B with material certifications supplied and shall have a maximum grain size of ASTM 4.5 (max average grain diameter $0.003^{\text {") }}$ ). Plate material that meets these requirements should be available at:
Hi Temp Metals
8005002141
Haynes International
Karen Spencer
8004261963

An additional piece of the same heat Haynes 230 plate used to produce the specimens shall also be supplied to the customer with minimum dimensions of 12 " $\times 24$ ".

Attached is some vendor information on Haynes 230 material for your reference. 
Attachment 4.1 to

Enclosure 2 to

MDO-723-0044/

B-MT(SRME)-52

Page 3

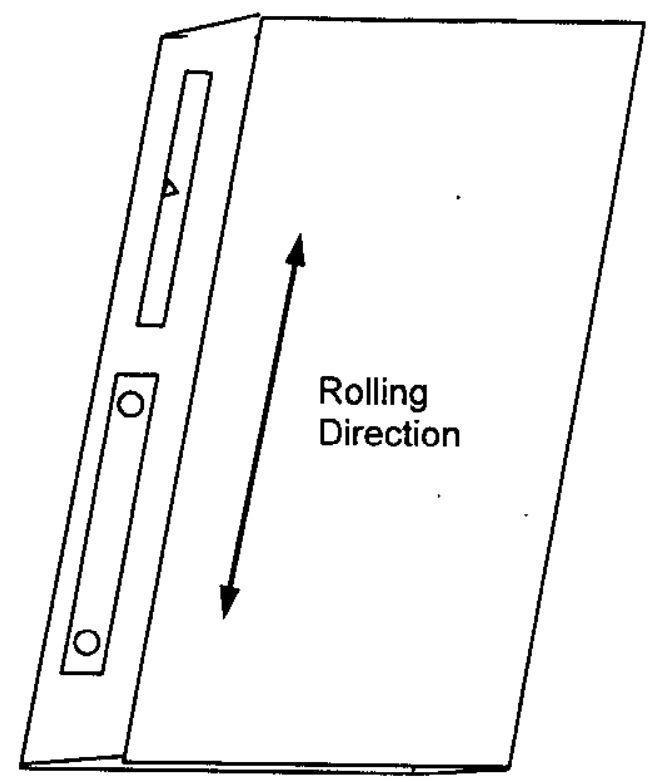

Figure 1. Orientation for Tensile and Fracture Toughness Specimens and Specimen Features 


\section{MACHINING}

HAYNES* $230^{\circ}$ alloy is similar in machining characteristics to other solid-solution-strengthened nickel-base alloys. These alloys as a group are classified as moderate to difficult to machine; however, it should be emphasized that they can be machined using convertional methods at satistactory rates. As these alloys will work-harden papidly, the keys to succeseful machining are to use slower speeds and feeds, and to take heavier cuts than would be used for machining stainles: oteels. Soe Haynes Intemational pubfication $\mathrm{H}-3159$ for more detailed information.

\section{Nomal Roughing (Tuming/Facing)}

Use carbide C-2/C-3 grade tool

Speed: 80 suriace foet/minute

Feed: 0.010 in./revolution

Depth of Cut: $0.150 \mathrm{in}$.

Negative rake square ineert, $45^{\circ}$ SCEA'

$1 / 32$ in. nose radius. Tool holder: $5^{*}$ negative

back and side rakes.

Lubricant: Ory ${ }^{2}$, Oil' or water-basea"

\section{Finsturng (Tuming/Facing)}

Use carbide $\mathrm{C}-2 / \mathrm{C}-3$ grade tool

Speed: 95-110 surface feetminute

Feed: 0.005-0.007 in.frevolution

Depth of Cut: 0.040 in.

Positive rake square insert, if possible, 45' SCEA, I/32 in. nose radius. Tool holder: 5" positive back and side rakes.

Lubricant: Dry or water-base

\section{Drilling}

Use high speed steel M-33M-40 seres"/ or T-15 grades"

Short, heavy-web drills with $135^{*}$ crank shat point. Thinning af web at point may reduce thrust.

Speed: $10-15$ ourface feetminute 200 RPM maximum for $1 / 4$ in. diameter or smalier)

Lubricent: Oil or water-base. Use coolant feed dnils if possible

Feed (per revolution):

0.001 in. rev. $1 / 3$ in. dia. 0.002 in. rev. $1 / 4$ in dia.

0.003 in. rev. $1 / 2$ in. dia. 0.005 in. rev. $3 / 4$ in. da. 0.007 in. rev, 1 in. dia.

- Carbide dizis nes recommended, but may be used in sorre set-ups. See fiaynes tnepmarional publioation li-3:5e lor details

Notes: I SCEA-Side cutting edge angle, or iead angle of the tool.

2 At any point where dry cutting is recommended, an air jet directed on the tool may provide substantial tool l.fo increases. A water-base coolarit mist may also be effective.

- Ol coalant should be a premium quality, sulfochlorina:ed oil with extrene prossure additives. A viscosity at $100^{*} \mathrm{~F}$ hom 50 to $125 \mathrm{SSU}$ is standard.

- Water-base cooiant shouid be a 15:1 mix of water with either a premitm quality, sulfoctlorinated water soluble oil or a chemical emulsion with extreme pressure additives.

5 Water-base coolants may cause chipping of rapid fai'ure of carbide tools in interrupied cuts.

- M-40 serieg High Speed Steels include M-41 through M-46 at time of writing, others may be added, and should be equally suitablo.

Acknowledgements:

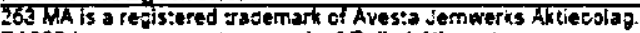

RA330 is a regisiered tracerr ark of Rolled Aloys ine

INCONEL and zDOi:T are repistered trademarks of Inะo Farn ly of Corrparies. 
Attachment 4.2 to

Enclosure 2 to MDO-723-0044I

B-MT(SRME)-52

Page 1

Attachment 4.2

Specifications for Machining

Tensile, Fracture Toughness and Density Disk Specimens

Alloy 617

Enclosure 2 to MDO-723-0044/B-MT(SRME)-52 
Attachment 4.2 to

Enclosure 2 to

MDO-723-0044I

B-MT(SRME)-52

Page 2

\section{Alloy 617 Test Specimens \\ Machining Details 05PFGxxx}

\section{Rev 3}

7/28/05

1). Supply 75 (Option 1A 100 pcs) fracture toughness specimens per drawing 5D16339

The width, nominal $0.118^{\prime \prime}$, and height, nominal $0.118^{\prime \prime}$, dimensions shall be reported for each specimen and supplied with the parts. The dimensions shall be traceable to the individual specimen by identification on the specimen packaging

2). Supply 85 (Option $2 A 100$ pcs) tensile specimens per drawing 5D16338

The gage section width, nominal $0.060^{\prime \prime}$, and gage section thickness, nominal 0.030 ", dimensions shall be reported for each specimen and supplied with the parts. The dimensions shall be traceable to the individual specimen by identification on the specimen packaging.

3). Supply 60 (Option $3 A 75$ pcs) density disk specimens per drawing 5D16357

The diameter, nominal 0.492 ", and thickness, nominal 0.059 ", dimensions shall be reported for each specimen and supplied with the parts. The dimensions shall be traceable to the individual specimen by identification on the specimen packaging.

The specimens shall be machined from minimum $\sim 0.25$ " thick plate. If the specimens are cut using EDM, a minimum of 0.005 " per surface shall be removed by grinding or conventional machining to eliminate any EDM recast layer. The disks surfaces shall be parallel to the starting plate surfaces. The tensile and fracture toughness specimen axis shall be aligned with the rolling direction of the plate. Additionally, the tensile specimen face (one with hole through it) shall be oriented parallel to the plate edge (width) surfaces and the fracture toughness specimen groove shall be oriented perpendicular to the plate edge (width) surfaces (See Figure 1 Below). The specimens shall be taken from a minimum depth of $0.06^{\text {n }}$ from the starting stock surface. The Alloy 617 plate shall be to ASTM-B-168 / AMS 5888 with material certifications supplied and shall have a maximum grain size of ASTM 4.5 (max average grain diameter 0.003 "). No engraving on the specimen (laser marking) is required. Plate material that meets these requirements should be available at:

$$
\begin{aligned}
& \text { Hi Temp Metals } \\
& 8005002141 \\
& \text { Haynes International } \\
& \text { Karen Spencer } \\
& 8004261963
\end{aligned}
$$

\section{Altemp Alloys Inc. Tony Baldridge 7149380601}

Special Metals

Debbie Fende

3045265023

An additional piece of the same heat Alloy 617 plate used to produce the specimens shall also be supplied to the customer with minimum dimensions of $12^{\prime \prime} \times 24^{\prime \prime}$.

Attached is some vendor information on Alloy 617 material for your reference. 
Attachment 4.2 to

Enclosure 2 to

MDO-723-0044/

B-MT(SRME)-52

Page 3

\section{Machining}

Informutior or mabining of alio 617 can be ontaned fon Specixl Meizls. Clttic tools stould je suarp end iare go:tive rabe acgles to minimize rotk berdening of the mater2]. Cuting foed and deph o: cut must be slificiect to present oupising of ite rotspiace 5urace. Additans] informition or machinine st axisole in the Special Meta: pablication 'Mrebining on the compary nebsite. พทพ.Epecialretali.cort.

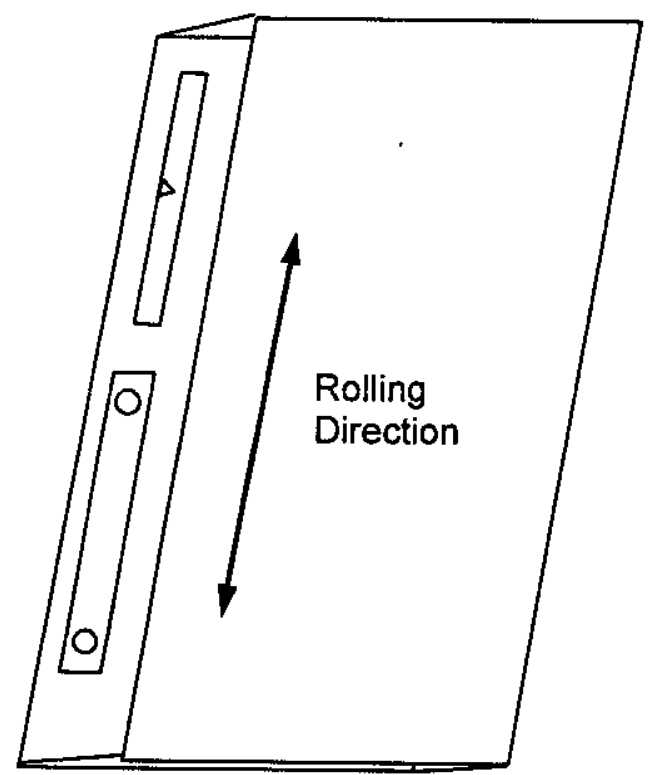

Figure 1. Orientation for Tensile and Fracture Toughness Specimens and Specimen Features 
Attachment 4.3 to

Enclosure 2 to MDO-723-0044I

B-MT(SRME)-52

Page 1

\section{Attachment 4.3}

Specifications for Machining

Tensile, Fracture Toughness and Density Disk Specimens

Nimonic PE16

Enclosure 2 to MDO-723-0044/B-MT(SRME)-52 


\section{Nimonic PE16 (1.75" dia rod Supplied) Test Specimens Machining Details Rev 2 $8 / 10 / 05$}

1). Supply 100 fracture toughness specimens per drawing $5 D 16339$

The width, nominal $0.118^{\prime \prime}$, and height, nominal $0.118^{\prime \prime}$, dimensions shall be reported for each specimen and supplied with the parts. The dimensions shall be traceable to the individual specimen by identification on the specimen packaging

2). Supply 100 tensile specimens per drawing 5D16338

The gage section width, nominal $0.060^{\prime \prime}$, and gage section thickness, nominal $0.030^{\prime \prime}$, dimensions shall be reported for each specimen and supplied with the parts. The dimensions shall be traceable to the individual specimen by identification on the specimen packaging.

3). Supply 75 density disk specimens per drawing 5D16357

The diameter, nominal 0.492 ", and thickness, nominal 0.059 ", dimensions shall be reported for each specimen and supplied with the parts. The dimensions shall be traceable to the individual specimen by identification on the specimen packaging.

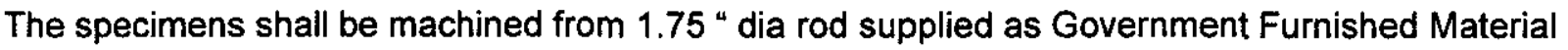

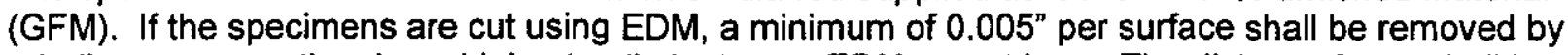
grinding or conventional machining to eliminate any EDM recast layer. The disks surfaces shall be parallel to the axis of the rod. The tensile and fracture toughness specimen axis shall be aligned with the axis of the rod. The notch on the fracture toughness specimens shall be made facing the surface of the rod (tangent to the rod surface). The specimens shall be cut out/laid out of the material to minimize material usage, however the specimens shall be taken from a minimum depth of $0.06^{\prime \prime}$ from the starting stock surface. 
Attachment 4.3 to

Enclosure 2 to

MDO-723-0044/

B-MT(SRME)-52

Page 3

\section{Nimonic PE16 Specimen Machining Orientation}

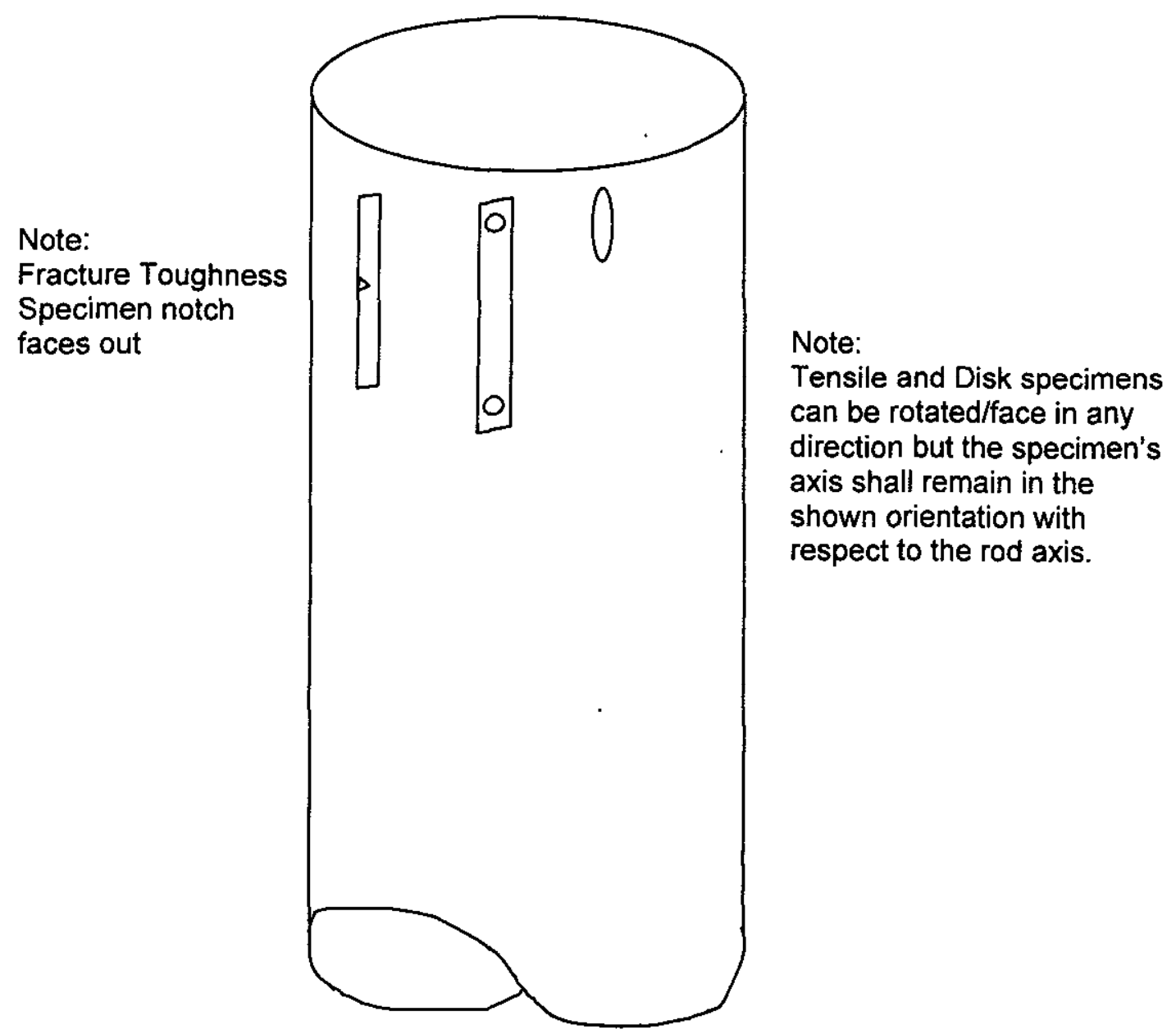


Attachment 5 to Enclosure 2 to MDO-723-0044I B-MT(SRME)-52

Page 1

Attachment 5

Superalloy Material Details

(Chemistry, Order / Inspection Info, Metallography)

Enclosure 2 to MDO-723-0044/B-MT(SRME)-52 
Attachment 5 to

Enclosure 2 to

MDO-723-0044/

B-MT(SRME)-52

Page 2

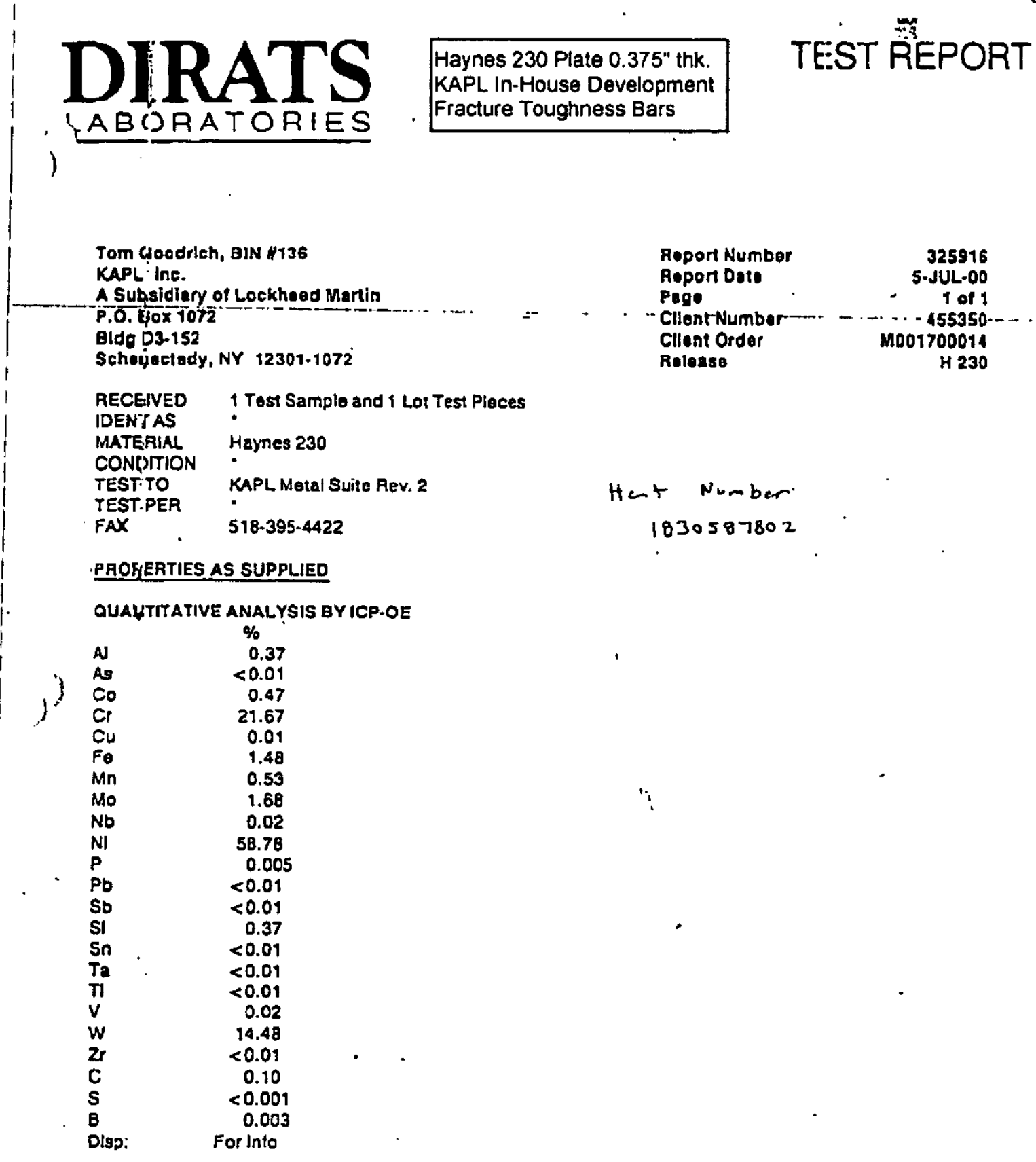

The symbol < slgnifies not detected at the detectabltiry limit indicated.

WAFGP WE CERTIFY THIS IS A TRUE COPY OF OUR RECORDS Signed for J. Dirats and Co. by Eric E. Dirats, Audit Manager

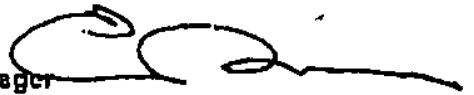

NOTE: The recording of false, ficllllous or fraudulent stetements or entries on this documont may be punished as a felony under federal law.

4) AlliPOAT ROAD P.O. 8OX 39 WESTFIELD, MA 01086-0039 FAX 413-\$68-1453 4i3-558-157; 
Attachment 5 to

Enclosure 2 to

MDO-723-0044/

B-MT(SRME)-52

Page 3

Alloy 617 Plate $0.5^{\prime \prime}$ thk.Gr $\mathrm{Sz} 3$

Biaxial Creep Specimens for Weld

Development and Grain Size

Sensitivity Study(36 specimens)

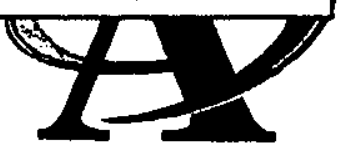

All Metal Sales, Inc.

\section{Material Certification}

\author{
29260 Clemens Road - Westlake, Ohio 44145 \\ P: 888-333-0101 - F: 888-333-0017 \\ sales@alimetalsalesinc.com \\ www.allmetalsalesinc.com
}

Distributors of Every Size, Type and Form

\begin{tabular}{|c|c|}
\hline SOLD TO: & \\
\hline $\begin{array}{l}\text { Vangura Tool } \\
440 \text { Waddell Avenue } \\
\text { Clairton, PA } 15025\end{array}$ & $\begin{array}{l}\text { Tool } \\
\text { dell Avenus } \\
\text { PA } 15025\end{array}$ \\
\hline $\begin{array}{l}\text { Customer POA: } \\
4745-8068 \\
\end{array}$ & $\begin{array}{l}\text { Ans Ordor: } \\
050507-10\end{array}$ \\
\hline $\begin{array}{l}\text { Matorial bescripton: } \\
\text { Alloy } 617\end{array}$ & $\begin{array}{l}\text { Stze of Materla Shlpped: } \\
1 \mathrm{pc}-.500^{\circ} \times 15.00^{*} \times 24.00^{\prime \prime} \\
\text { Grain in } 15^{\prime \prime} \text { Direction }\end{array}$ \\
\hline $\begin{array}{l}\text { Heat Number: } \\
861738810\end{array}$ & \\
\hline
\end{tabular}

Specifications: AMS 5888: ASTM-B-168

Chemical Composition (in weight percent)

\begin{tabular}{|c|c|c|c|c|c|c|c|c|c|}
\hline $\mathrm{Al}$ & $\mathrm{B}$ & $\mathrm{C}$ & $\mathrm{Co}$ & $\mathrm{Cr}$ & $\mathrm{Cu}$ & $\mathrm{Fe}$ & $\mathrm{Mn}$ & $\mathrm{Mo}$ & $\mathrm{Nl}$ \\
1.09 & .003 & .080 & 12.24 & 21.99 & .01 & 1.08 & .03 & 9.60 & 52.88 \\
\hline $\mathrm{P}$ & $\mathrm{S}$ & $\mathrm{SI}$ & $\pi$ & $\mathrm{V}$ & $\mathrm{W}$ & $\mathrm{Cb}(\mathrm{Nb})$ & $\mathrm{Ta}$ & $\mathrm{Zr}$ & $\mathrm{Mg}$ \\
$<.005$ & .002 & .08 & .44 & $<.10$ & $<.10$ & .080 & $<.100$ & $<.020$ & $<.002$ \\
\hline
\end{tabular}

Physical Analysis

\begin{tabular}{|c|c|c|c|c|c|c|}
\hline $\begin{array}{l}\text { Ultimale } \\
\text { Tensile }\end{array}$ & $\begin{array}{c}.2 \% \text { Yield } \\
\text { PSt }\end{array}$ & \% Elong & $\% R A$ & Stress & Hardness & Grain Size \\
\hline PSI & & & & Rupiure & & \\
\hline 113.000 & 48,600 & $56 \%$ & $58 \%$ & $\overline{O K}$ & 89 & 3 \\
\hline
\end{tabular}

I hereby certify that the heat numbers and anapyes detailed hereon are correct as containad in the records of this corporation and are extracted from the data fumished to us by the producing mill. (Or any alternativo source as acquired) Because All Metal Seles has no control over the subsequent processing or product application, All Motal Sales expressly disctains any and all expressed or implled warranty other than tha! of the warranty herein above set forth. Such disclaimer inctudes without limitation, warranty of fitness for particular purpose and warranty of merchantability.

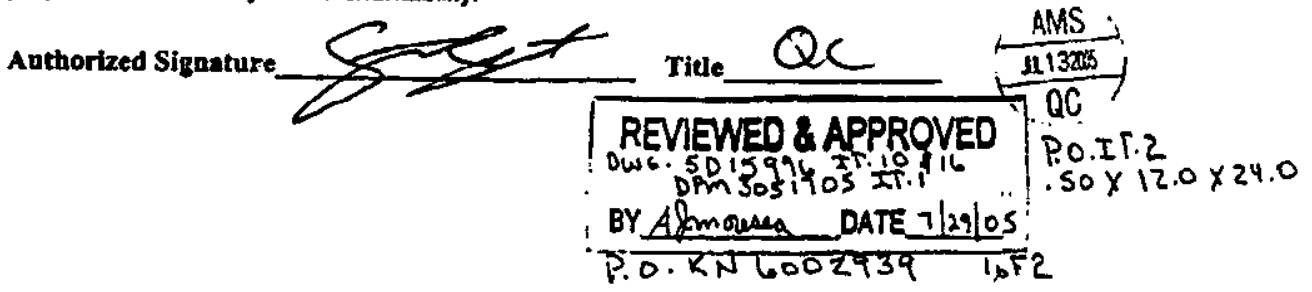


Attachment 5 to

Enclosure 2 to

MD0-723-0044I

B-MT(SRME)-52

Page 4

Alloy 617 Plate $0.5^{\prime \prime}$ thk.Gr $\mathrm{Sz} 3$

Biaxial Creep Specimens for Weld

Development and Grain Size

Sensitivity Study(36 specimens)

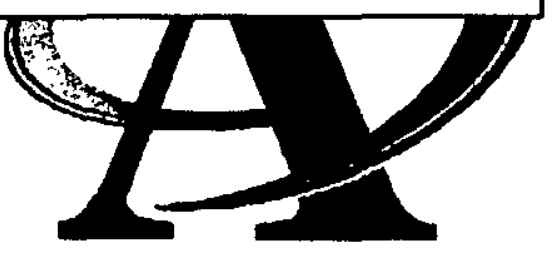

All Metal Sales, Inc.

Distributor of Every Size, Type and Form.

29260 Clemens Road - Wertlake, OH 44145

Ph: (888) 333-0101 - Fax: (888) 333-0017

www.allmetalsaledic.com

\section{CERT OF CONFORMANCE I PACKING SLIP}

\begin{tabular}{|l|l|l|l|}
\hline DATE & $7 / 13 / 2005$ & INVOICE \# & 050507-10 \\
\hline \hline SHIP TO: & Vangura Tool, Inc. \\
& 440 Waddell Ave. \\
& Clairton, PA 15025 \\
& & \\
& \\
\hline
\end{tabular}

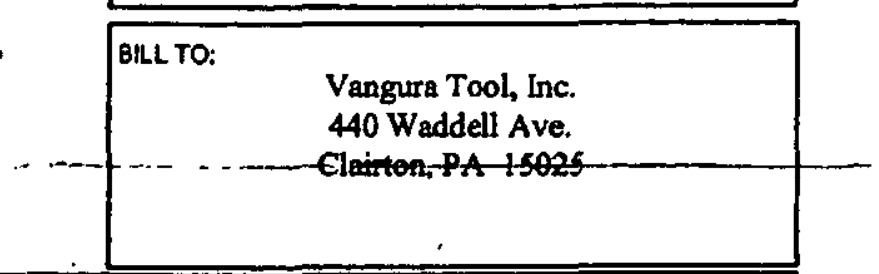

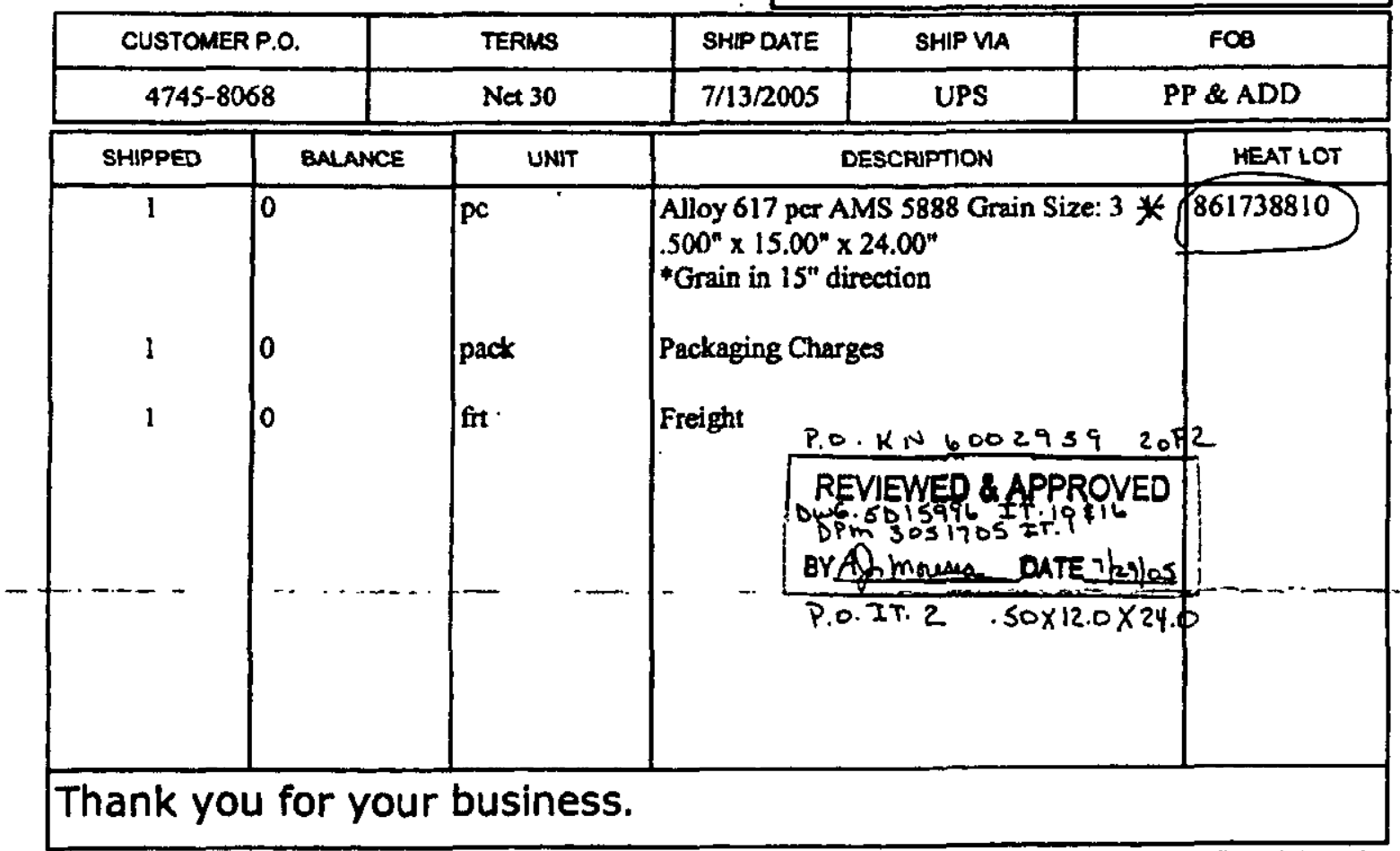

Wo hereby certify that the manufactured items stated above are in conformance with the applicable specifications as required by the purchase order and supporting documents.

Date: $7 / B / S S$

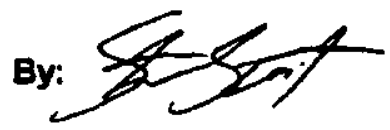

$\frac{\text { AMS }}{\frac{\text { III } 1306}{. Q C}}$ 


\section{Nimonic PE16 0.625" Dia.}

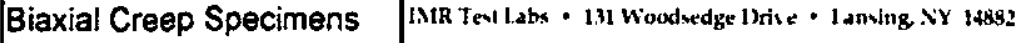

Reference No.: 05PFG116

Material ID: ORNL \#[C1747

Material Type: Nickel-base Alloy

\section{CHFMISTRY}

\begin{tabular}{|c|c|c|}
\hline Element & Sample & Expected Values \\
\hline $\mathrm{C}^{1}$ & 0.05 & $0.04 \cdot 0.08$ \\
\hline Sì & 0.27 & 0.5 Maximum \\
\hline $\mathrm{Mn}$ & 0.07 & 0.2 Maximum \\
\hline Si & 0.001 & 0.015 Maximum \\
\hline $\mathrm{Ag}$ & $<0.0005$ & 0.0005 Maximum \\
\hline Al & 1.79 & $1.1 \cdot 1.3$ \\
\hline B & $<0.005$ & O.n05 Maximum \\
\hline $\mathrm{Bi}^{2}$ & $<0.0001$ & 0,0001 Naximum \\
\hline $\mathrm{Co}$ & 0.25 & 2.0 Naximum \\
\hline $\mathrm{Cr}$ & 16.66 & $15.5 \cdot 17.5$ \\
\hline $\mathrm{Cu}$ & 0.09 & 0.5 Aaximum \\
\hline Mo & 3.16 & $2.8-3.8$ \\
\hline $\mathrm{Ni}+\mathrm{Co}^{3}$ & +3.84 & $+2.0-45.0$ \\
\hline $\mathrm{Pb} \mathrm{b}^{2}$ & $<0.0001$ & 0.0015 Maximum \\
\hline $\mathrm{Ti}$ & 1.10 & $1.1 \cdot 1.3$ \\
\hline $\mathrm{Zr}$ & $0.01 *$ & $0.02=0.04$ \\
\hline Fe & 33.50 & Balance \\
\hline OT & 0.06 & - \\
\hline $\mathrm{Nb}$ & 0.01 & $\cdots$ \\
\hline$P$ & 0.01 & - \\
\hline Ta & 0.02 & $\cdots$ \\
\hline V & 0.02 & -- \\
\hline
\end{tabular}

- Out of specification.

'Determined by combustion-infrared atsorbance.

2Determined by GFAA.

'Determined by' difference.

Other elements tested $(<0.01 \%)$ were $\mathrm{Cd}, \mathrm{HI}, \mathrm{Mg}, \mathrm{Se}, \mathrm{Sn}$, and $\mathrm{Zn}$

Results in weight percent unless otherwise indicated.

Methods: CAP-046A (ICP-AES), CAP-017E (ICP-AES), CAP-009A (GFAA), and ASTM E 1019-03. 
Attachment 5 to

Enclosure 2 to

MDO-723-0044I

B-MT(SRME)-52

Page 6

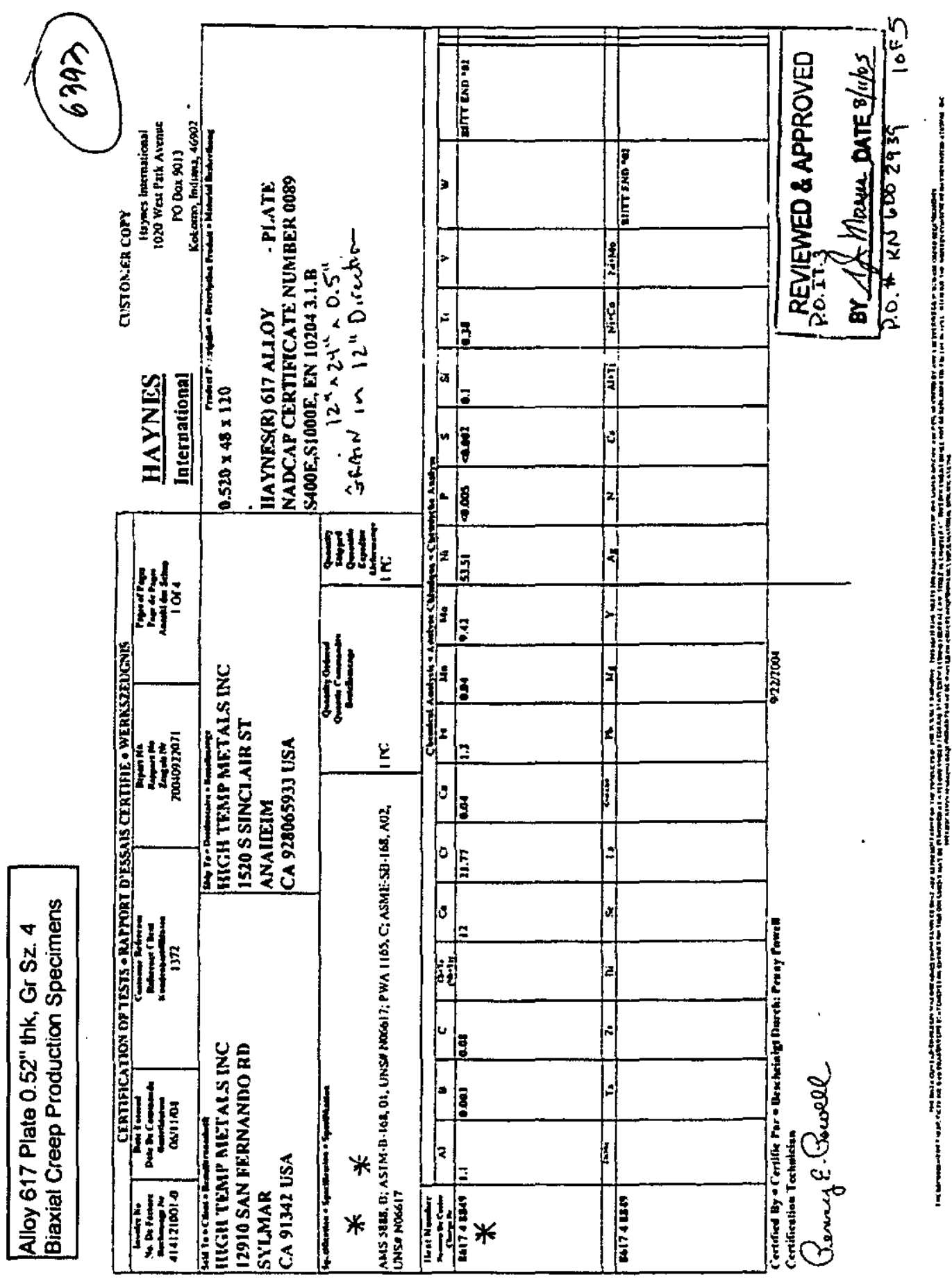


Attachment 5 to

Enclosure 2 to

MDO-723-0044/

B-MT(SRME)-52

Page 7

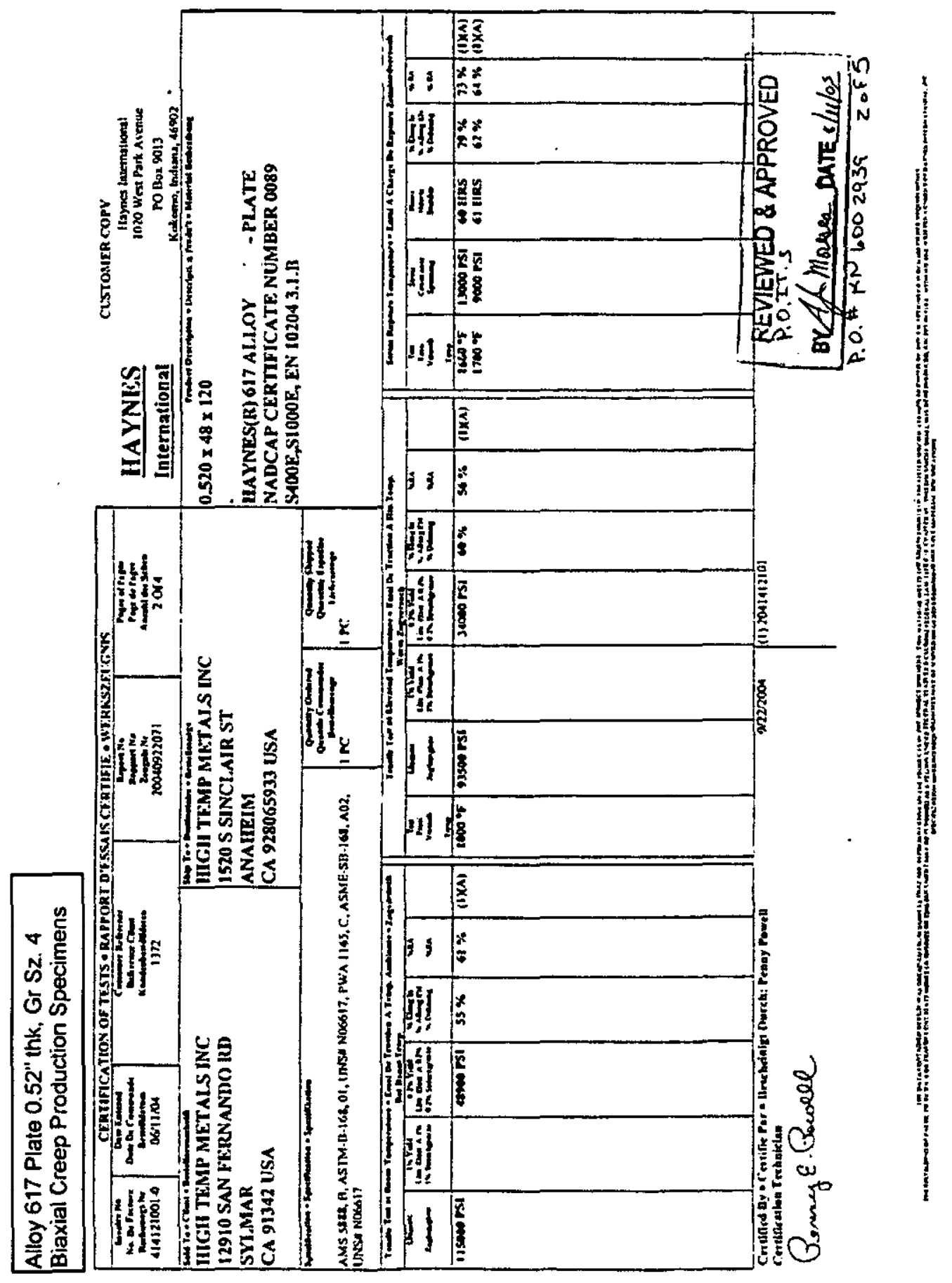


Attachment 5 to

Enclosure 2 to

MDO-723-0044/

B-MT(SRME)-52

Page 8

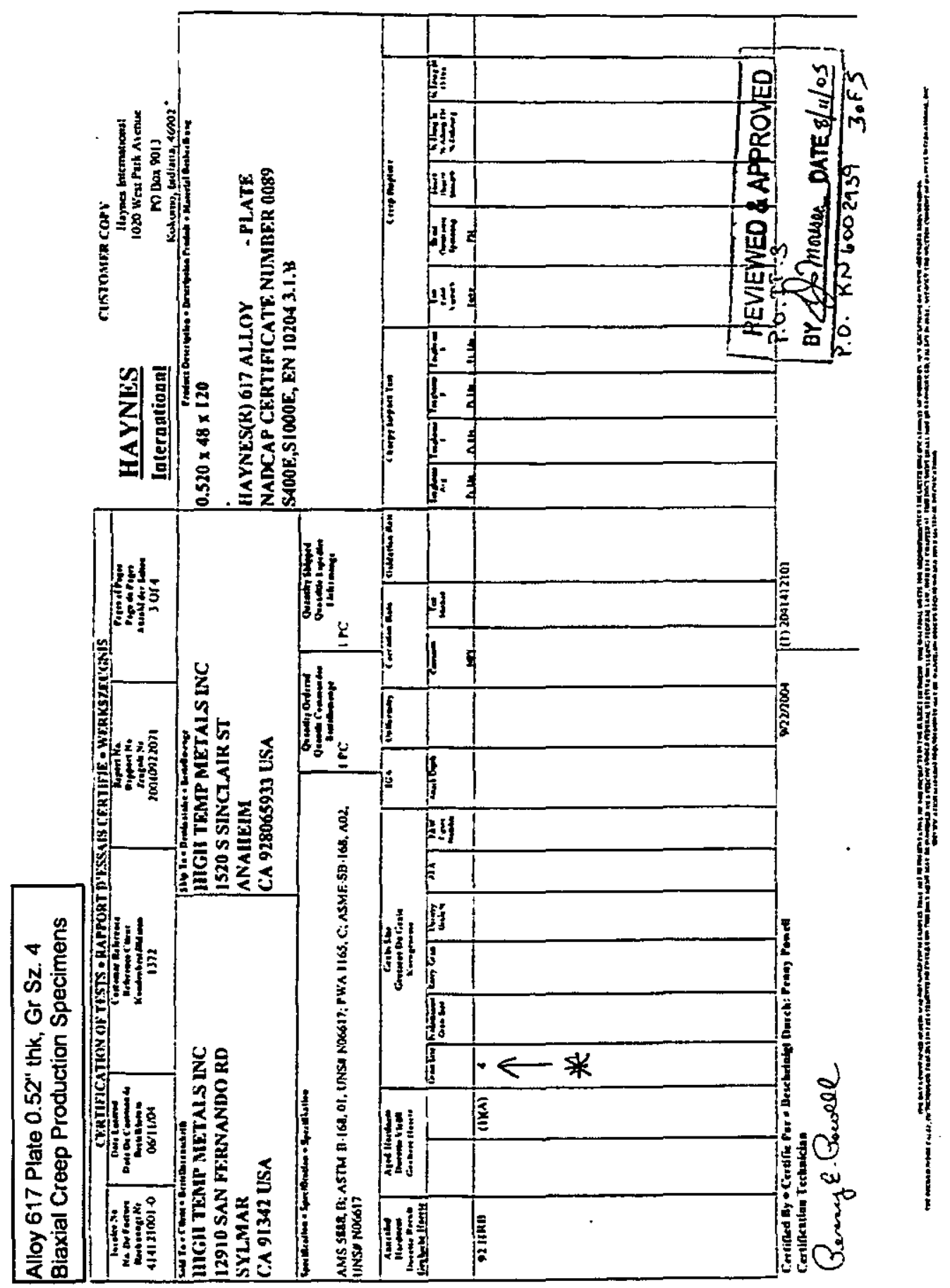




\section{Attachment 5 to \\ Enclosure 2 to \\ MDO-723-0044/ \\ B-MT(SRME)-52 \\ Page 9}
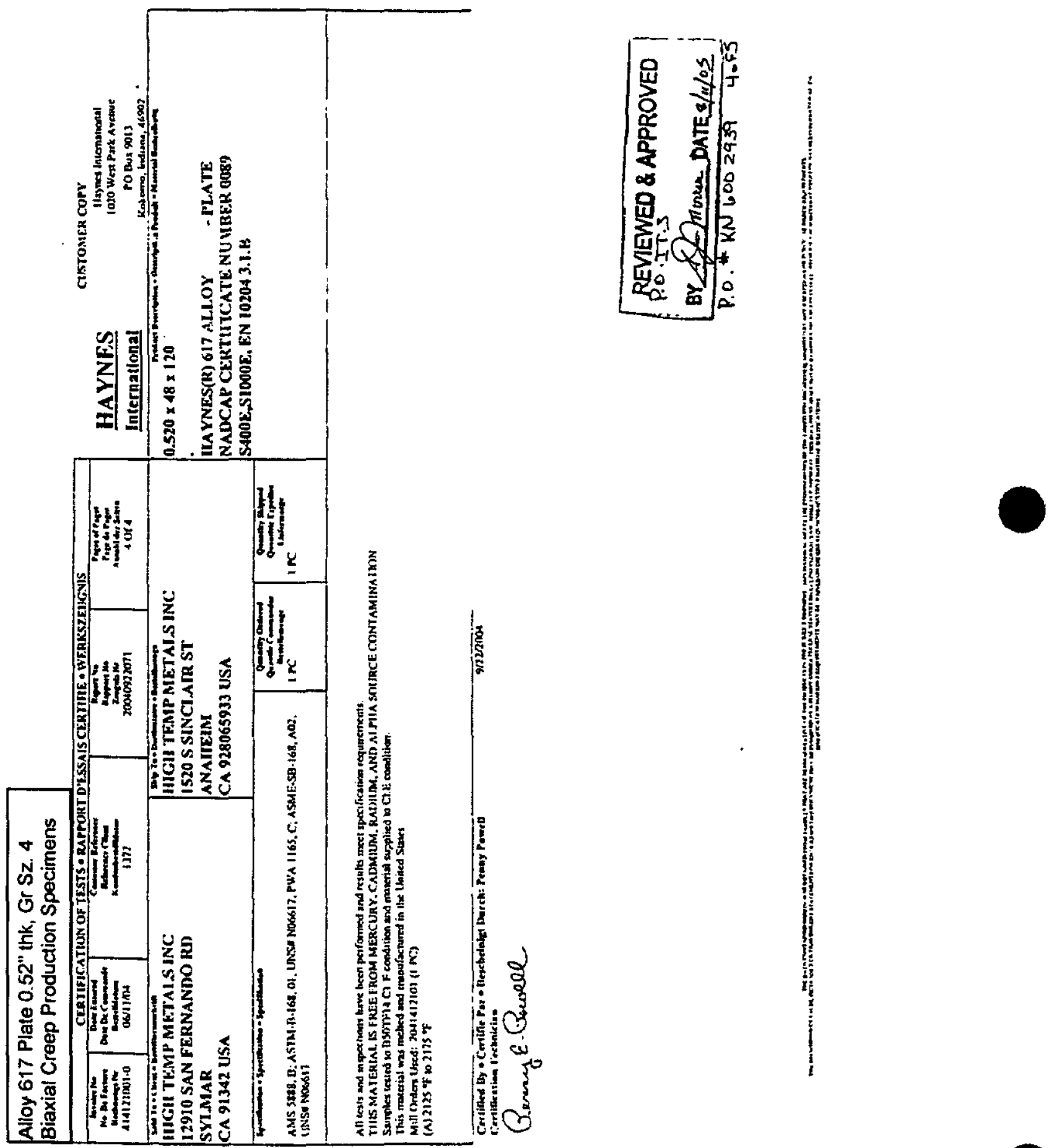
Attachment 5 to

Enclosure 2 to

MDO-723-0044I

B-MT(SRME)-52

Page 10

97/29/2905 14:95 3397948833

n

MASTERSQLRCEDA

PAGE

$3: / 25$

Alloy 617 Plate 0.52" thk, Gr Sz. 4

Biaxial Creep Production Specimens

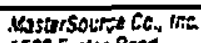

ISts Exeter Road

Alrem, Ohio disa

9907947100

for. 794 gast

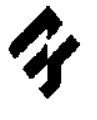

MasterSOURCE

souctany Mease hothe

600964 i7f

\section{CERTIFICATE OF CONFORMANCE}

TO: Vanguza Tool

440 waddeli st.

DATE: $07 / 29 / 05$

clairton, $\mathrm{Pa} 15025$

PURCHASE ORDER: 4797-8068

OUR ORDER: 21759

MATERIAL DESCRIPTION:

Haypos 617 Alloy Plate Armenled .520" Th $\times 15-1 / 2^{n} \times 24-1 / 2^{\prime \prime}$

out in $15-1 / 2^{\prime \prime}$ grain direction

QUANTTY: 1 EC

SPECIFICATION: ASTM B168-01

HEAT/LOT NUMBER: 861748849

We hereby certify that the above material/s meet the applicable specification. This is besed on evidence in our liles ox in those of our suppliers and is true to the best of our knowledge.

We further certify that to the best of our knowledge, lead and other low melting point metals, theix compounds or materials containing low melting point metals as a baie chemical constituent were not used in direct contact with surfaces of the product at any time.

Note: The recording of false, fictitious of fraudulent statements or entries on this document may be punished as a felony under Federal statutes including Federal Law, Title 18, Chapter 47.
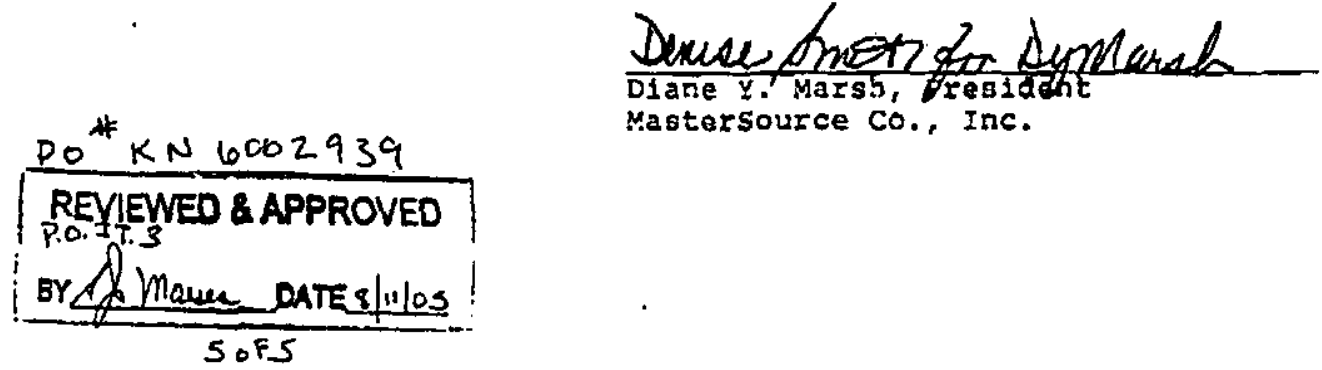

Diane y. Marsh, Fresidat Mastersource Co., Inc. 
Attachment 5 to

Enclosure 2 to

MDO-723-0044/

B-MT(SRME)-52

Page 11

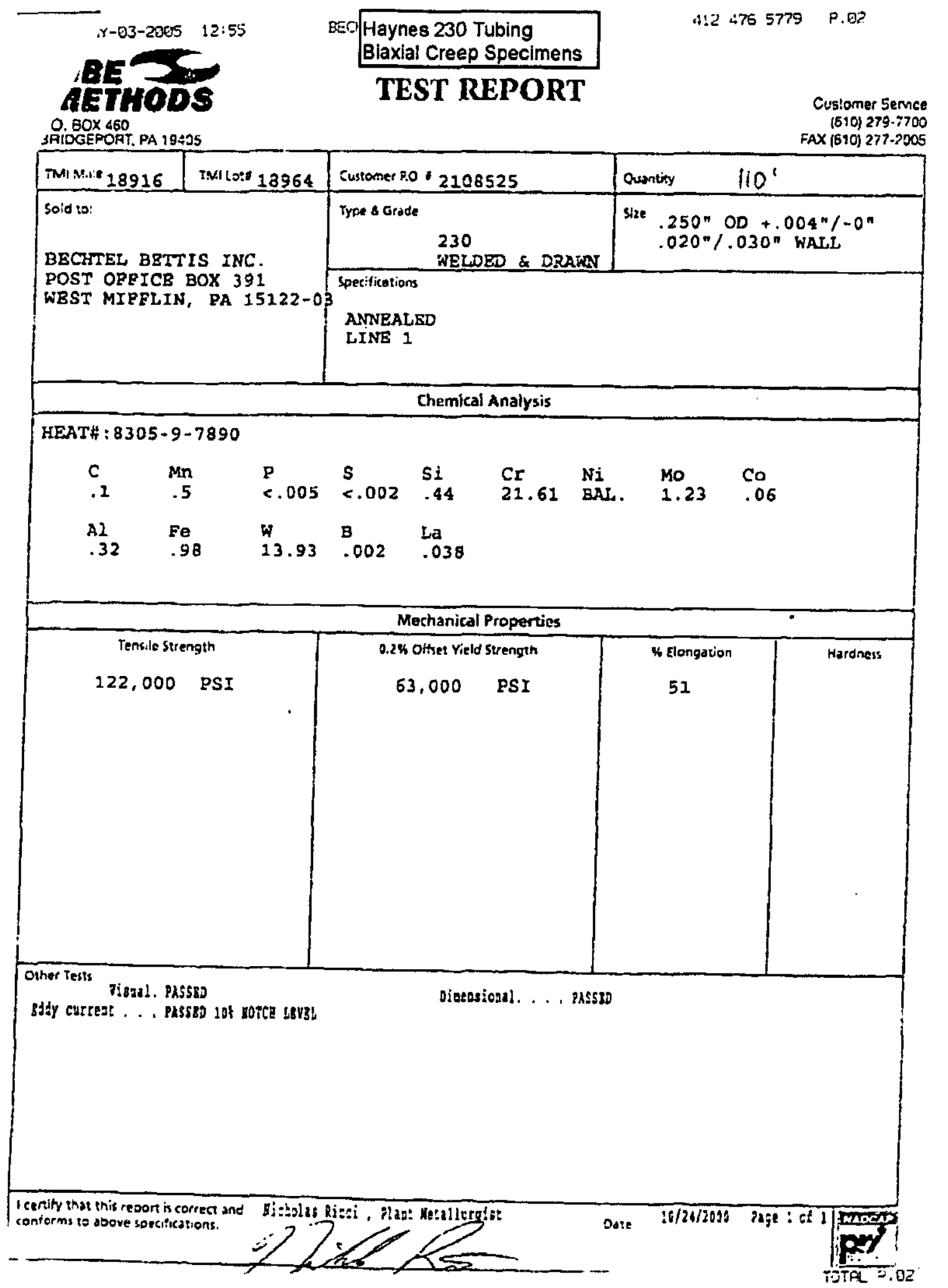


Attachment 5 to

Enclosure 2 to

MDO-723-0044/

B-MT(SRME)-52

Page 12
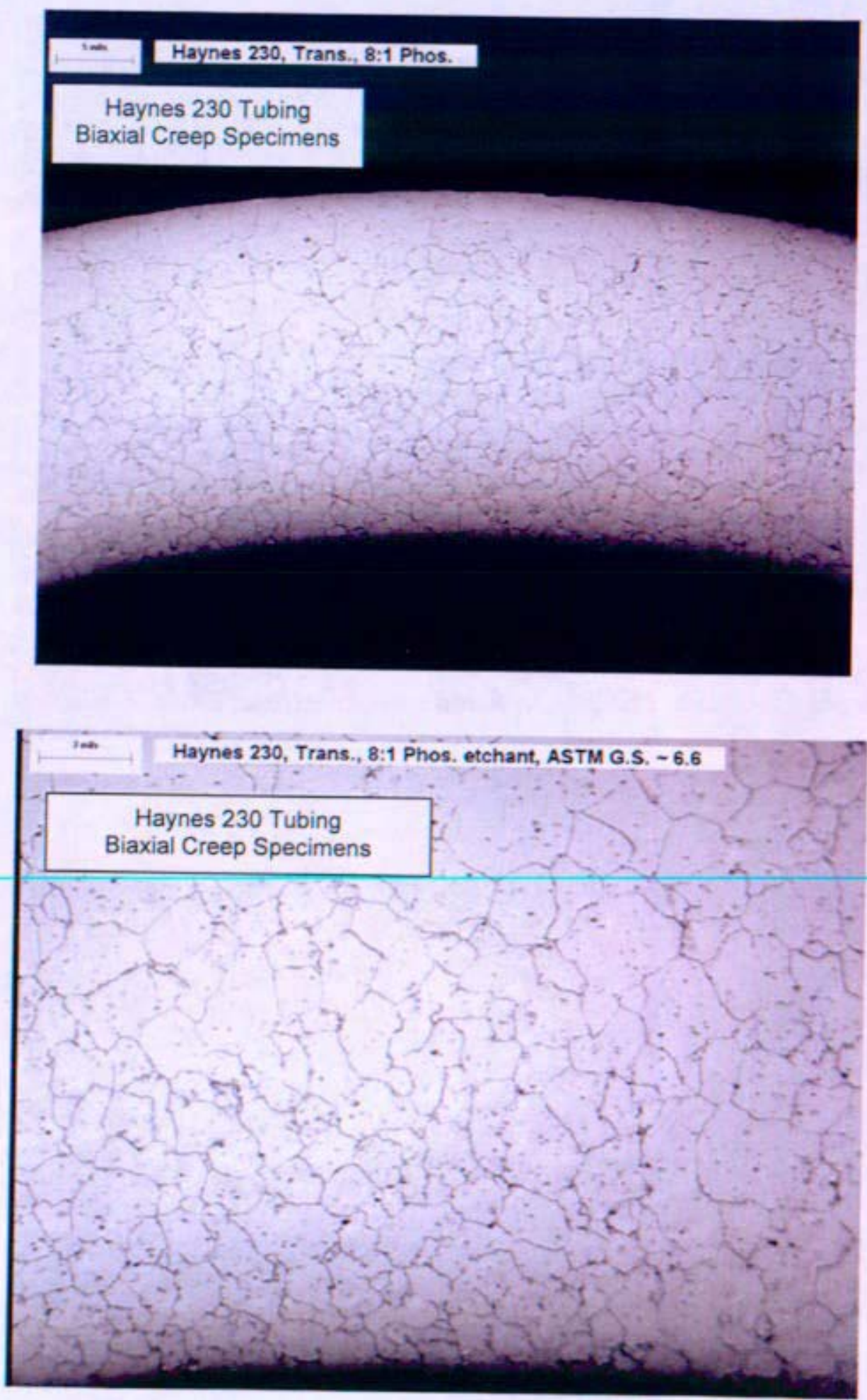

PRE-DECISIONAL - For Planning and Discussion Purposes Only 
Attachment 5 to

Enclosure 2 to

MDO-723-0044/

B-MT(SRME)-52

Page 13
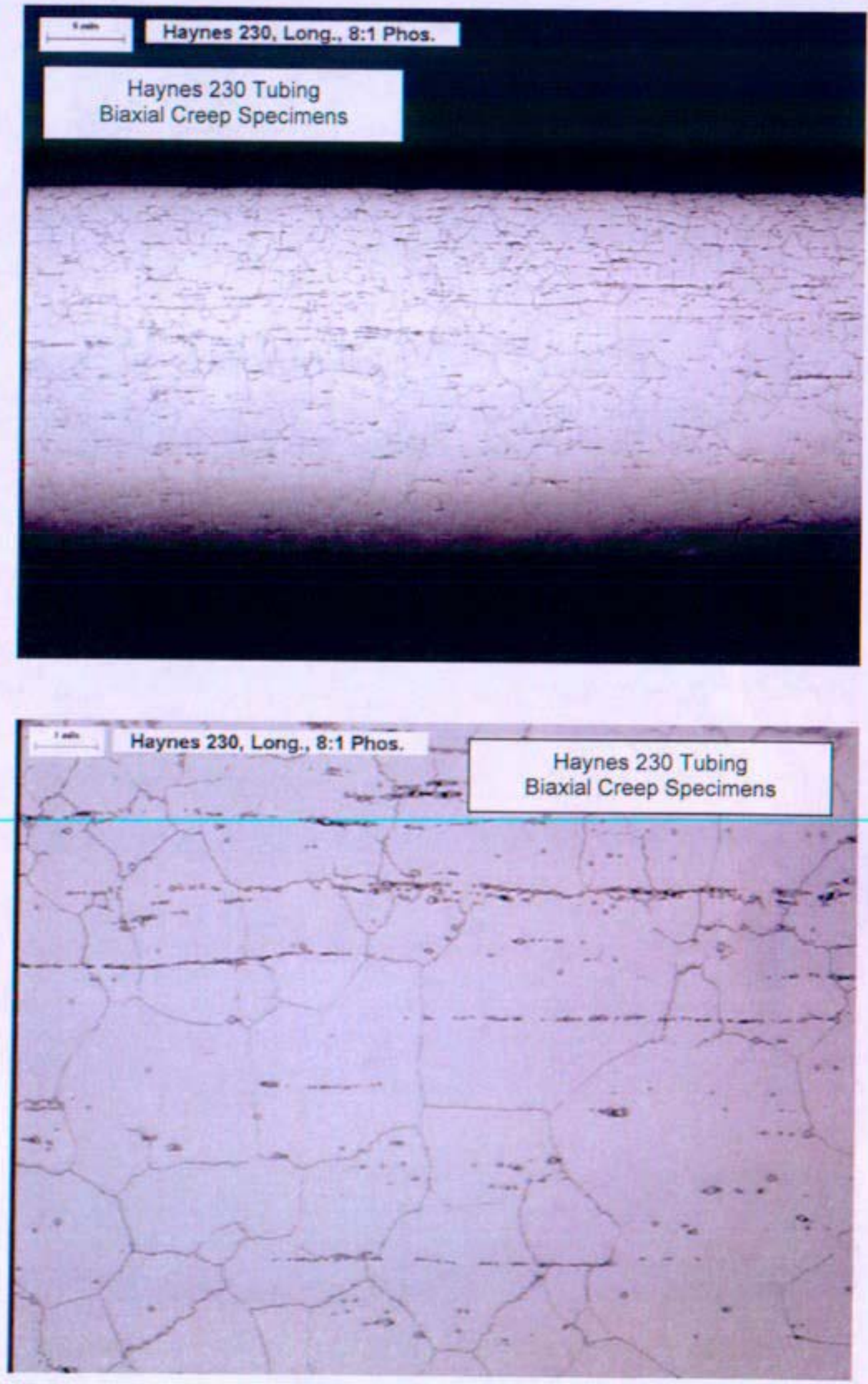
Attachment 5 to

Enclosure 2 to

MDO-723-0044/

B-MT(SRME)-52

Page 14

है

$E$
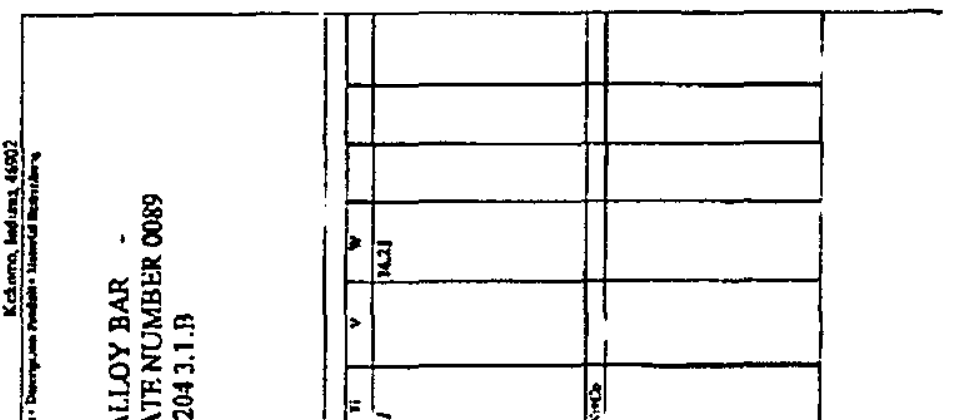

$\int \frac{1}{3}$

突

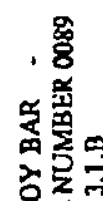

要然

1 월

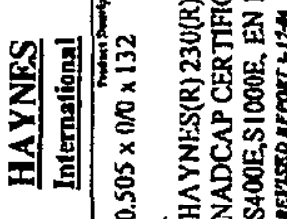

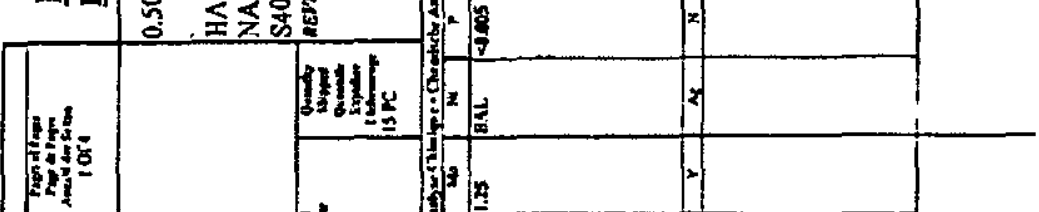

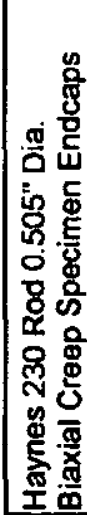

7

(2)

z

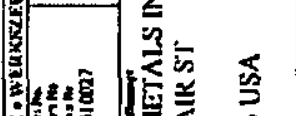

瀂

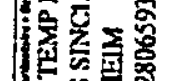

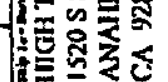

促

울

部

i

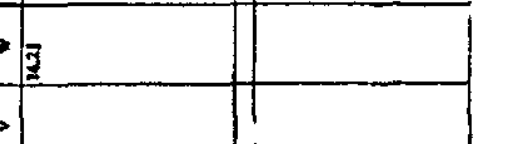

s

3
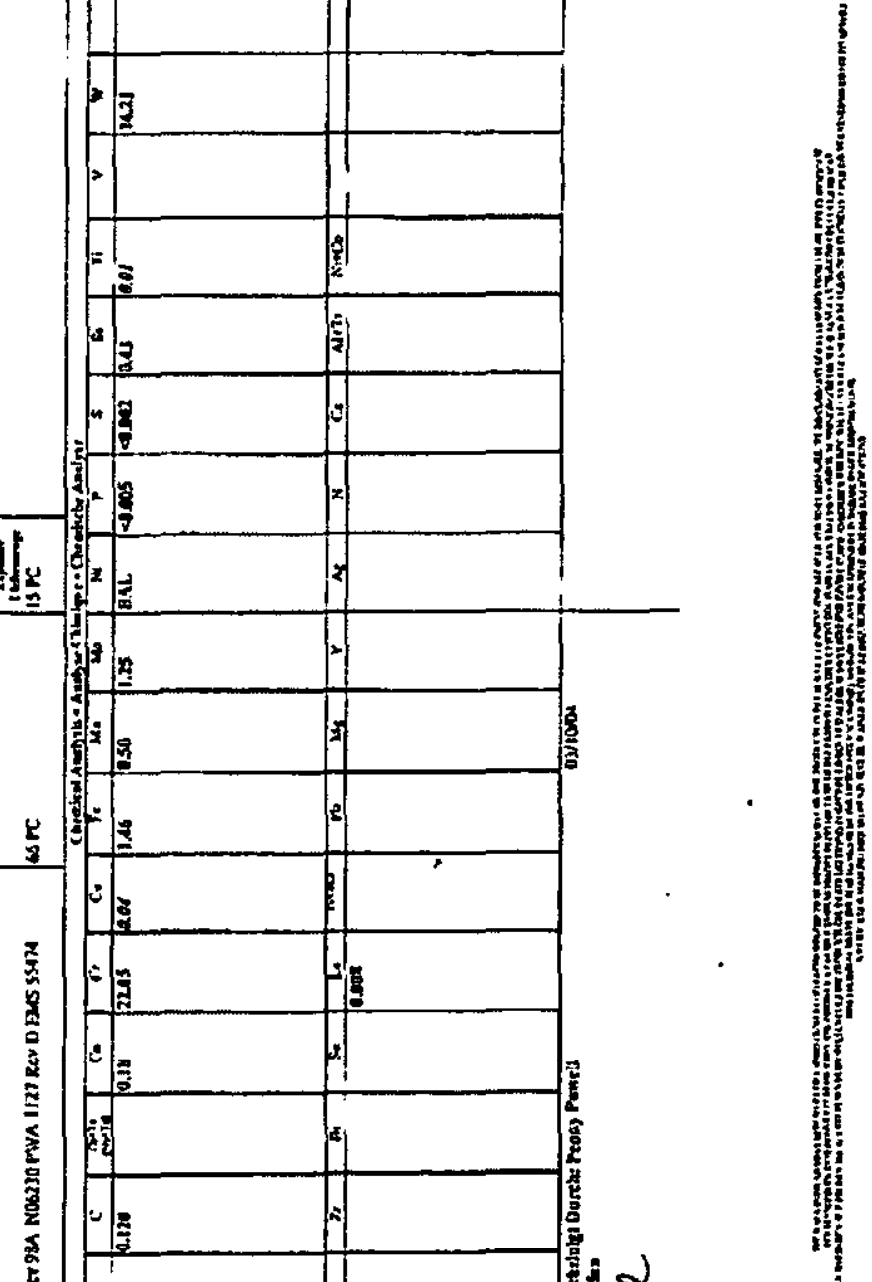

PRE-DECISIONAL - For Planning and Discussion Purposes Only 
Attachment 5 to

Enclosure 2 to

MDO-723-0044I

B-MT(SRME)-52

Page 15

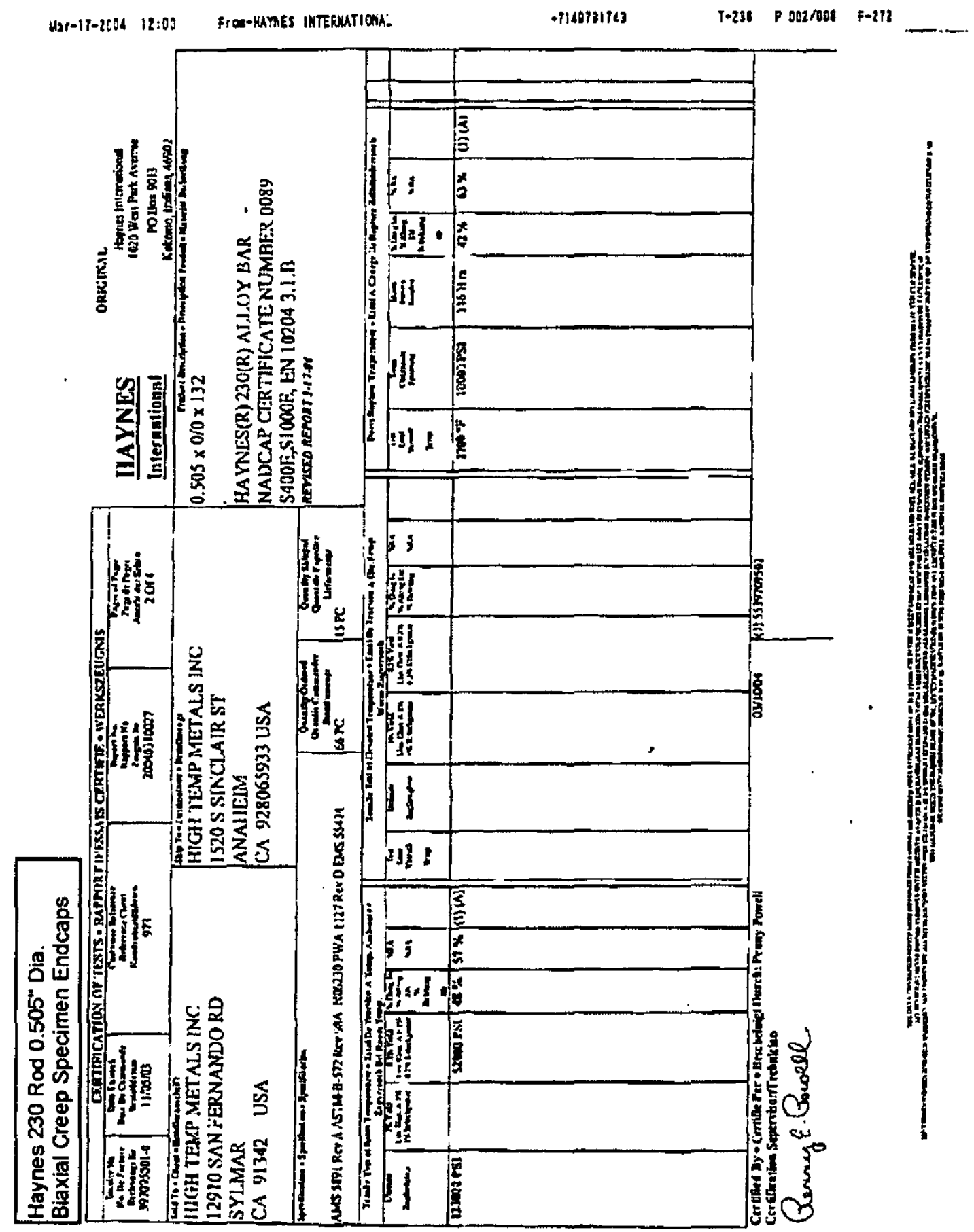


Attachment 5 to

Enclosure 2 to

MDO-723-0044/

B-MT(SRME)-52

Page 16

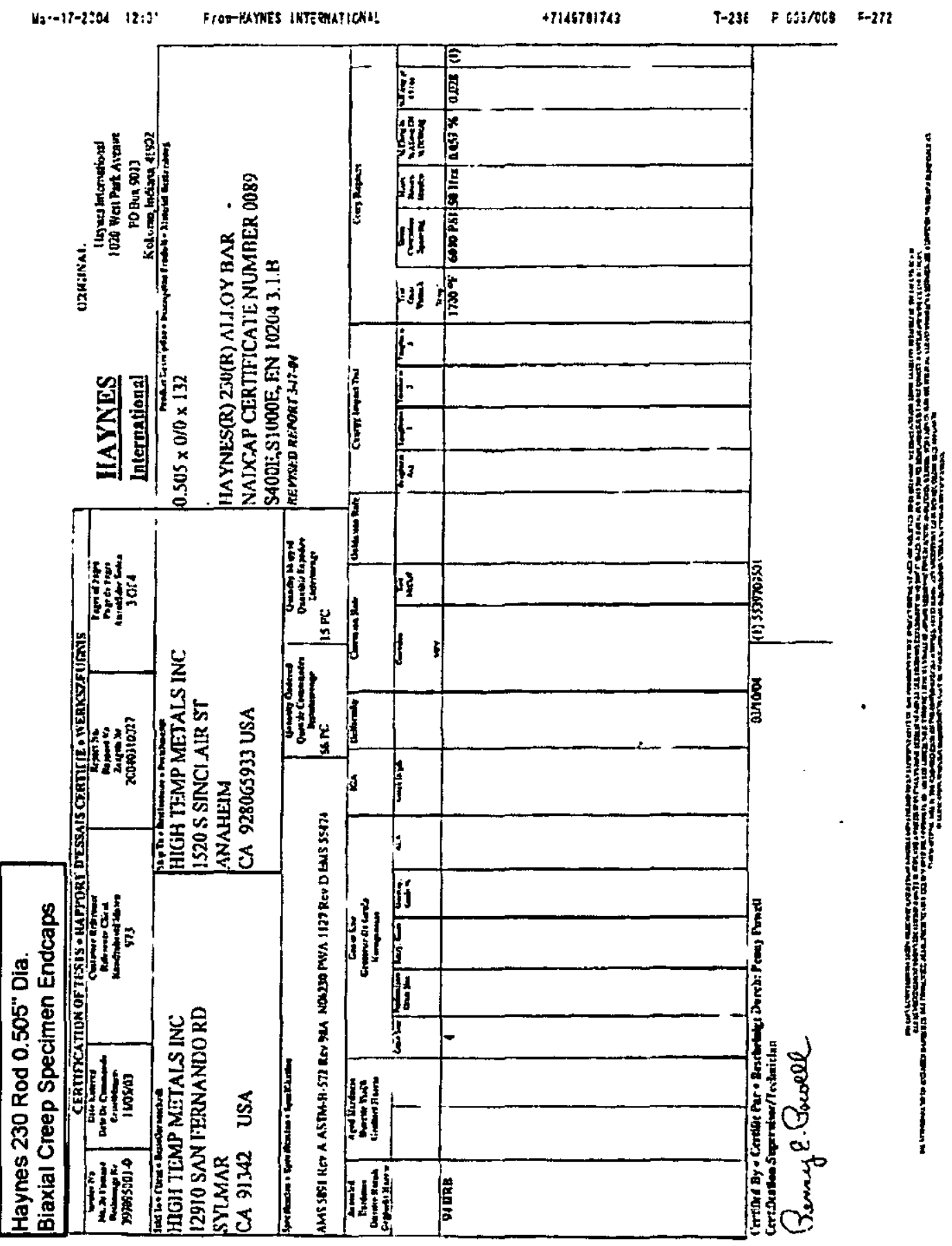

PRE-DECISIONAL - For Planning and Discussion Purposes Only 
Attachment 5 to

Enclosure 2 to

MDO-723-0044/

B-MT(SRME)-52

Page 17
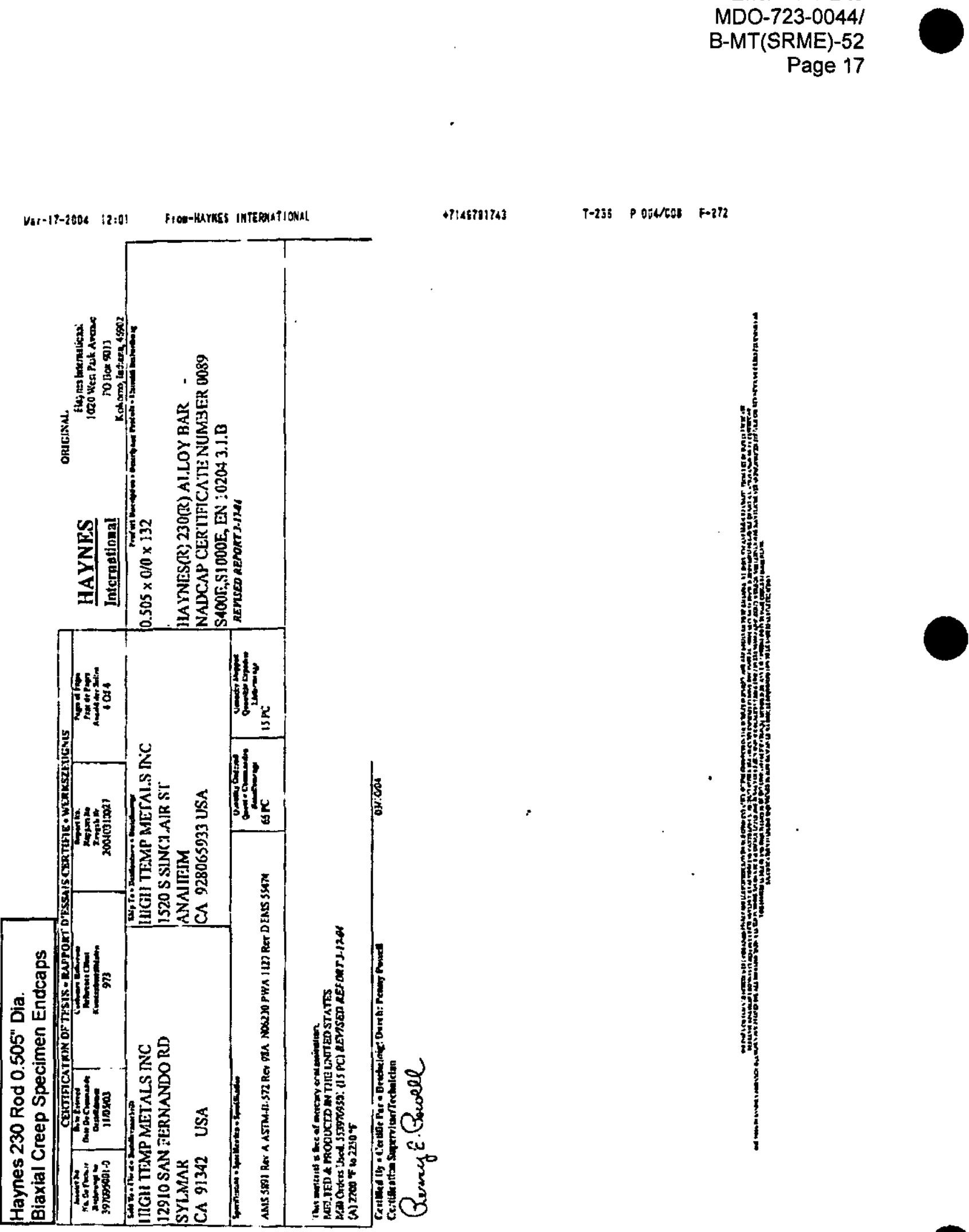

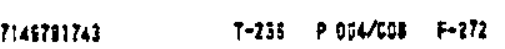


Attachment 5 to

Enclosure 2 to

MDO-723-0044!

B-MT(SRME)-52

Page 18

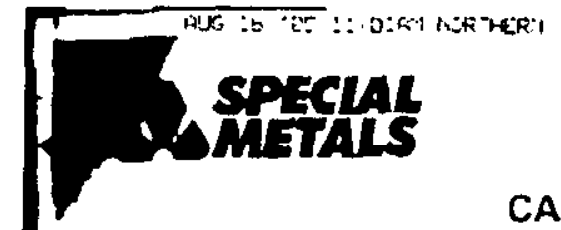

CAA APPROVED CERTIFICATE

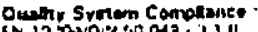

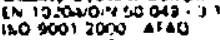

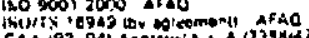

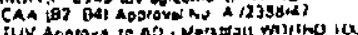

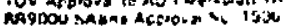

For 109t portarimed by lace Toll
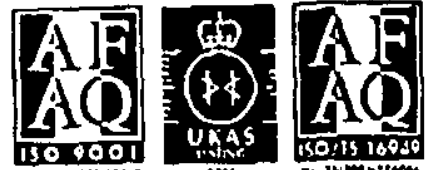

1261783

Curbunaw

ENPAR SPECIAL ALLOYS LTD.

STATION ROAU. ECCLESFIEI D

SHEFAELD.

S35 gYR

SPECIAL METALS WIGGIN LINYTED AL

F

fax +44 (0)11432 26403? 382200 far: 38121

Nimonic PE16 2.5" (65mm) Dia

Customer's Under Mo. I Contugal No. P20662

numior

Product Oesciption \& Uipimelan

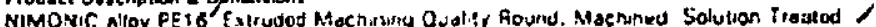

$65001 A \times 26384142672$ MAN NOMANAL

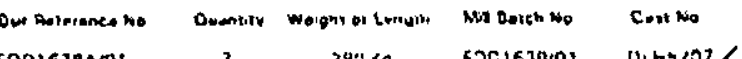

ENPAR SPECIAL ALLOYS LTD.

STATHON AOAD, ECCLESFIELD

SHEFFIELD.

\$35 9rR

RA MSHL ISSz ISS,

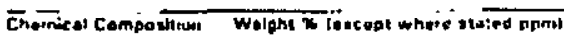

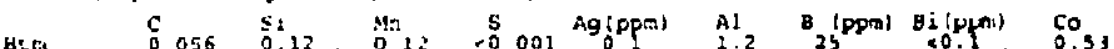

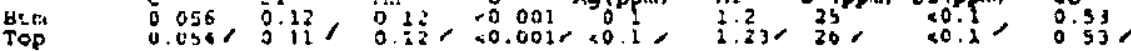

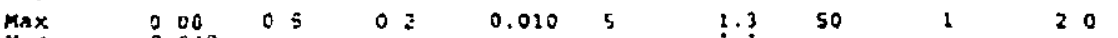

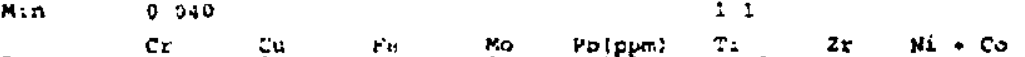

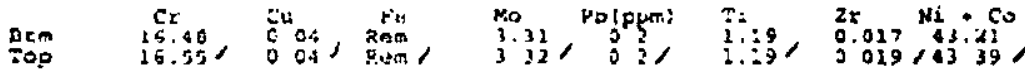

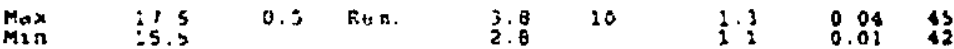

Toti Hoinurt Mat

7302034

Moxhinew tivis

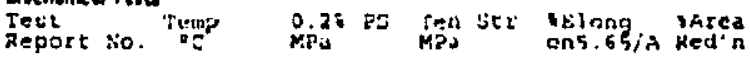

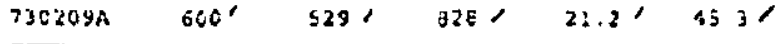

Cioep Tout:

Teat

Aeport Ho. MPo

7302094

Mati veringem

4 10 .

Tens. $\quad$ i TPS

it $100 \mathrm{~h}$

WE CEATIFY THE: THSS IS A

entcti in 1040 C AC

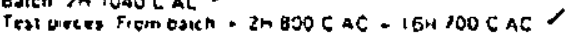

Othei iexts - remaiks

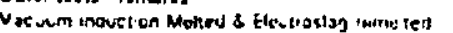

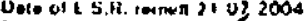

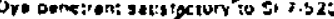

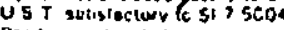

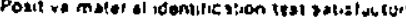

4.220

COINY OF T- 5 OANGINAL

CEATIF: OI:

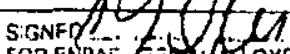

FCAENDA: Fin/

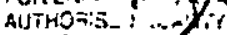

arten Nors

A57241

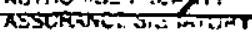

Pintary cj:1 mutions ; OKL345

MMUS 33270I/RANO ISTO

Fnd aten cons savilugtory.

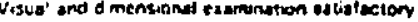

Eno of cortinkato

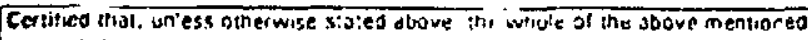

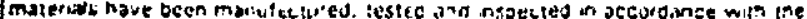

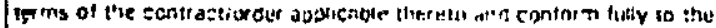

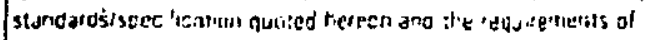

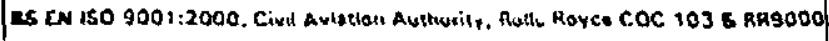

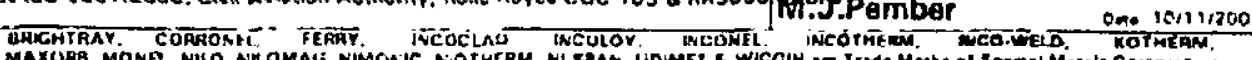

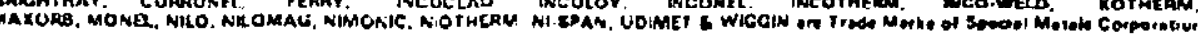


Attachment 5 to

Enclosure 2 to

MDO-723-0044/

B-MT(SRME)-52

Page 19

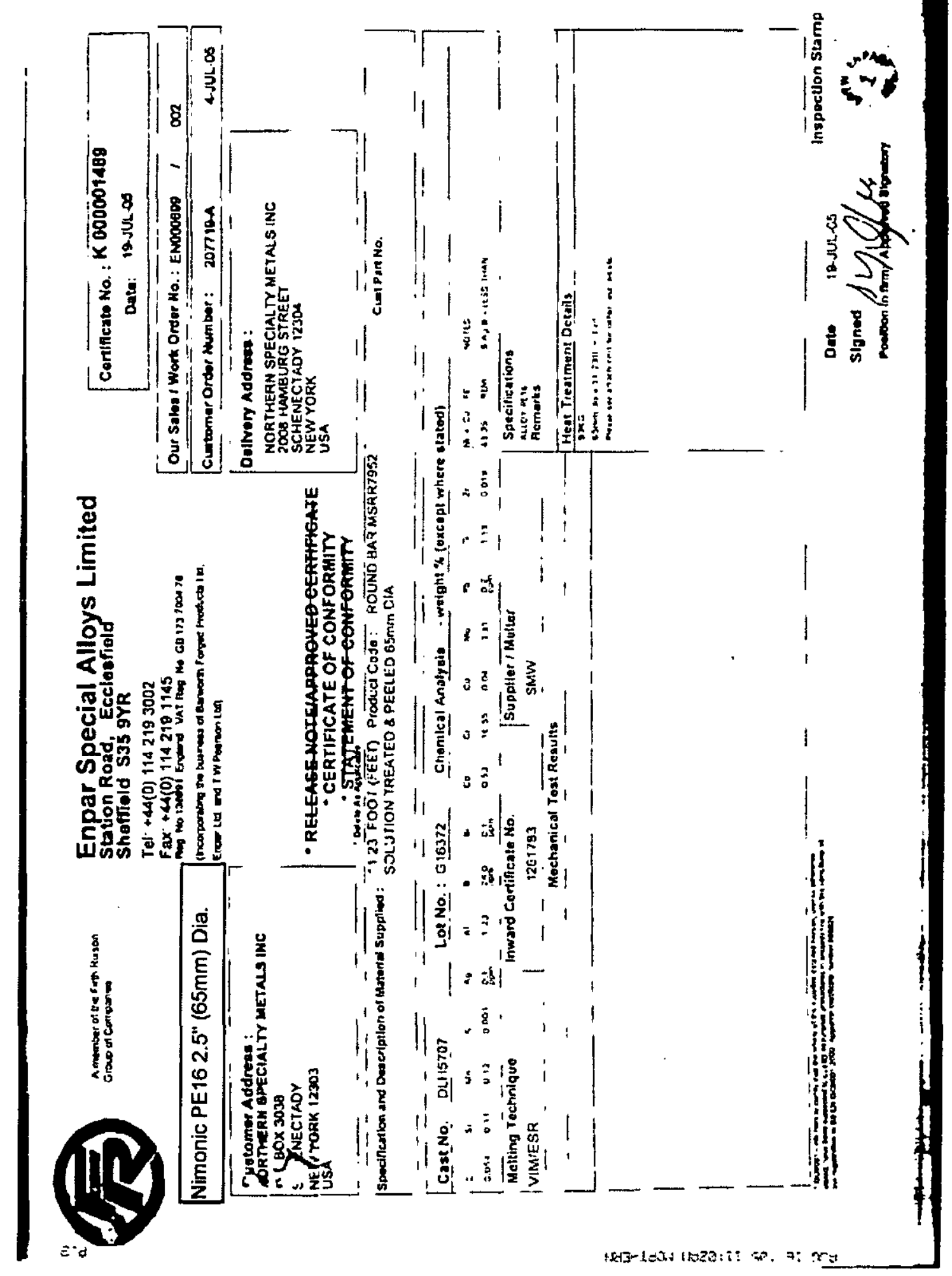

PRE-DECISIONAL - For Planning and Discussion Purposes Only 
Attachment 5 to

Enclosure 2 to

MDO-723-0044/

B-MT(SRME)-52

Page 20
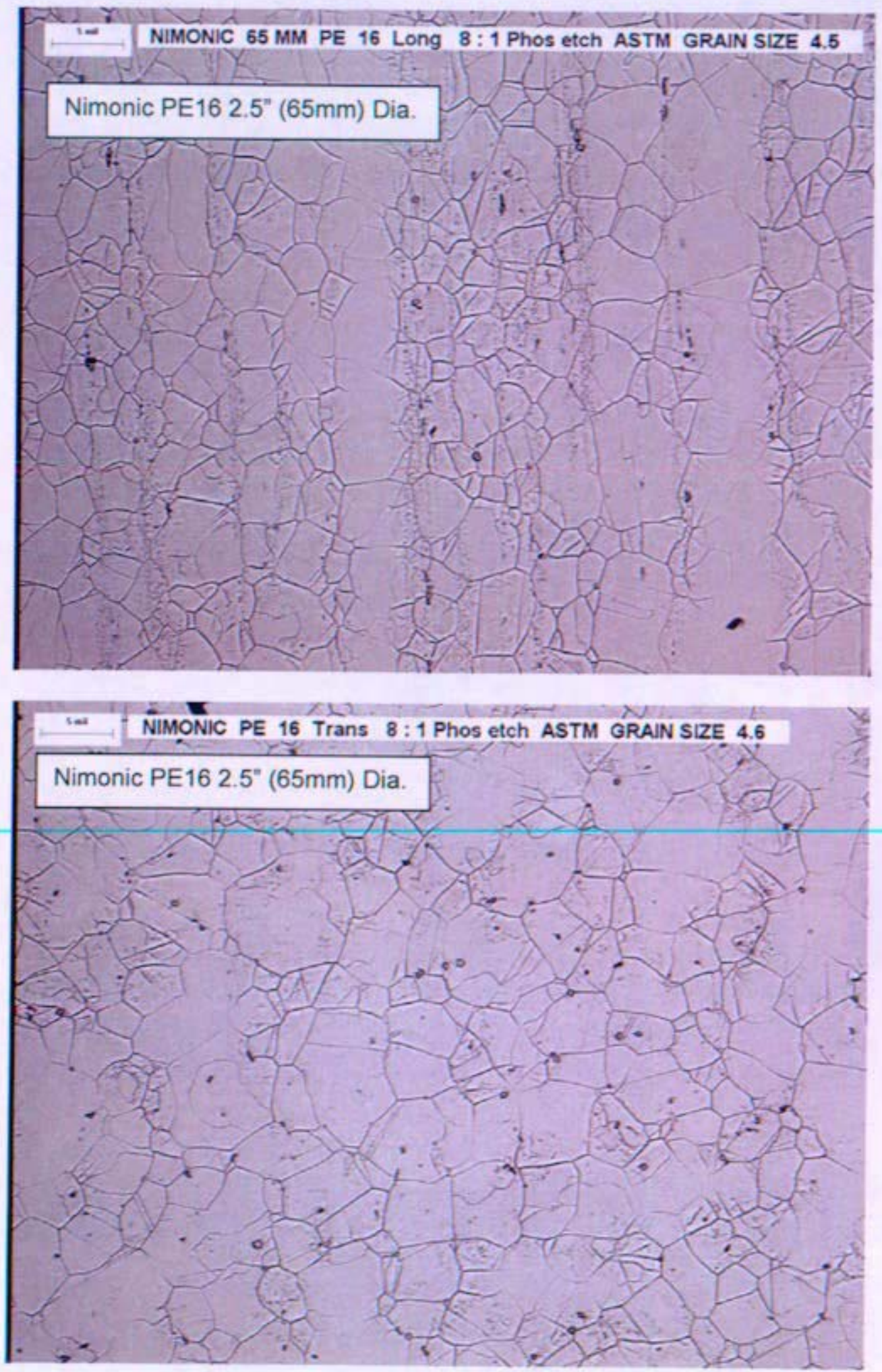

PRE-DECISIONAL - For Planning and Discussion Purposes Only 
Attachment 5 to

Enclosure 2 to

MDO-723-0044/

B-MT(SRME)-52

Page 21

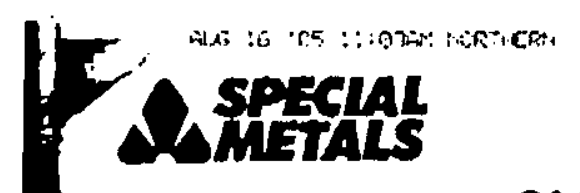

CAA APPROVED CERTIFICATE

SPECIAL METALS WIGGIN LIMITED Holmei hood, Hewaford. Enobang Mas gsl.

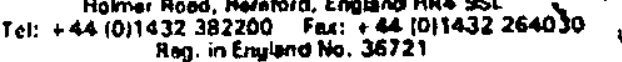

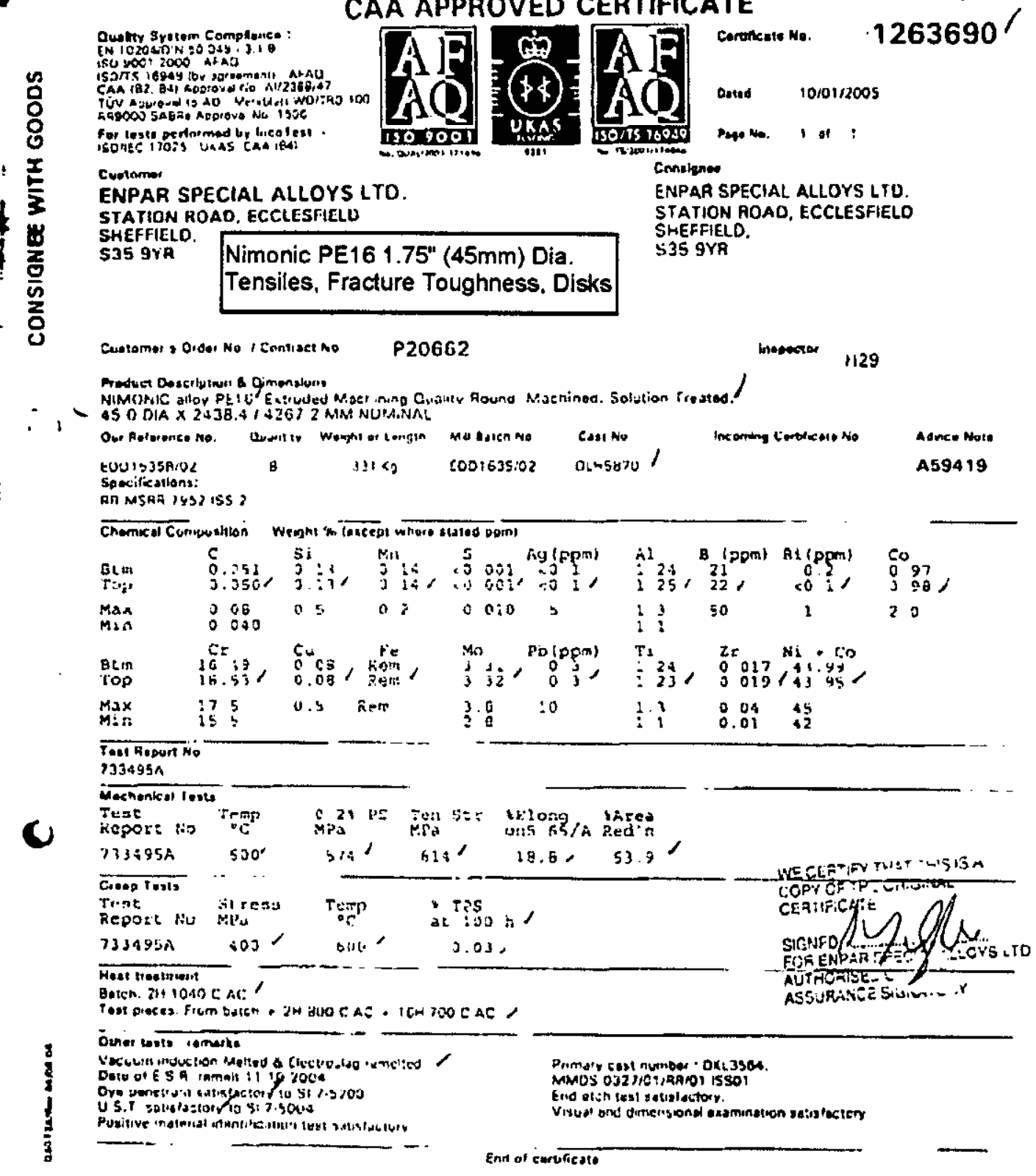

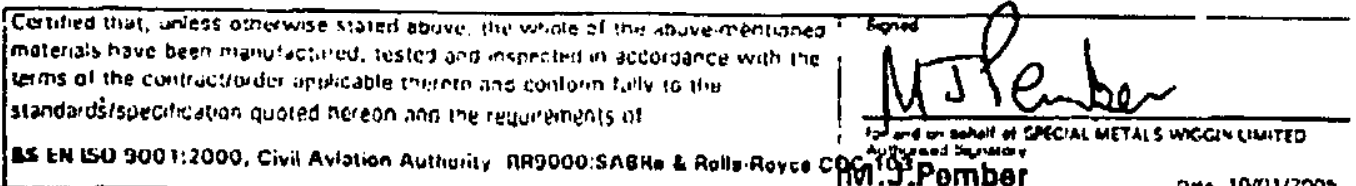

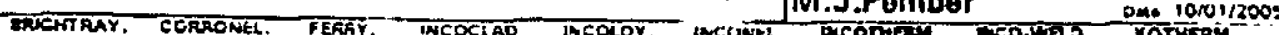

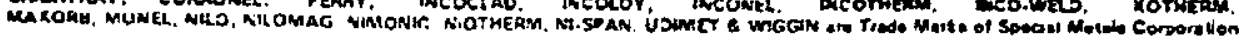


Attachment 5 to

Enclosure 2 to

MDO-723-0044I

B-MT(SRME)-52

Page 22

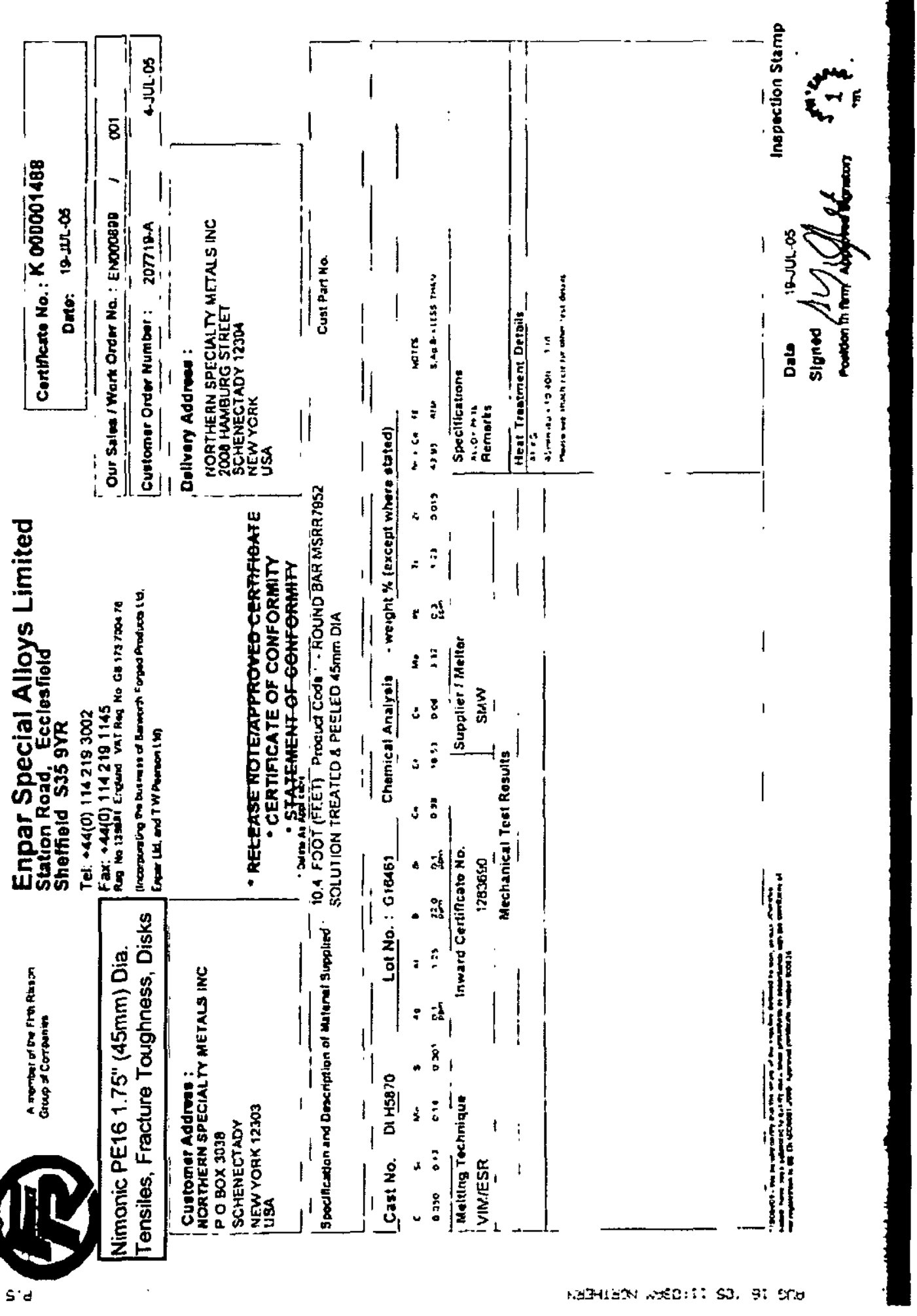

PRE-DECISIONAL - For Planning and Discussion Purposes Only 
Attachment 5 to

Enclosure 2 to

MDO-723-0044/

B-MT(SRME)-52

Page 23

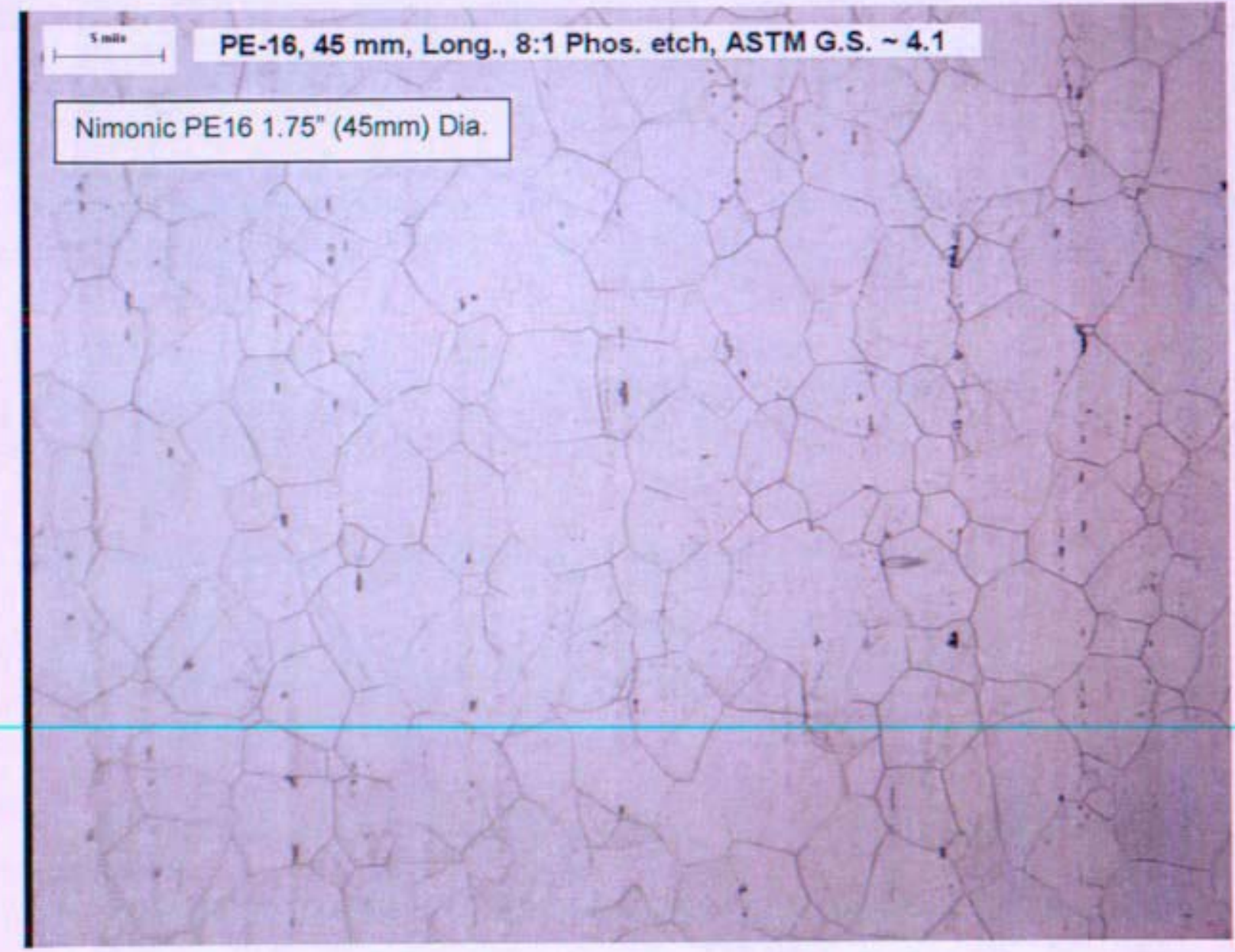




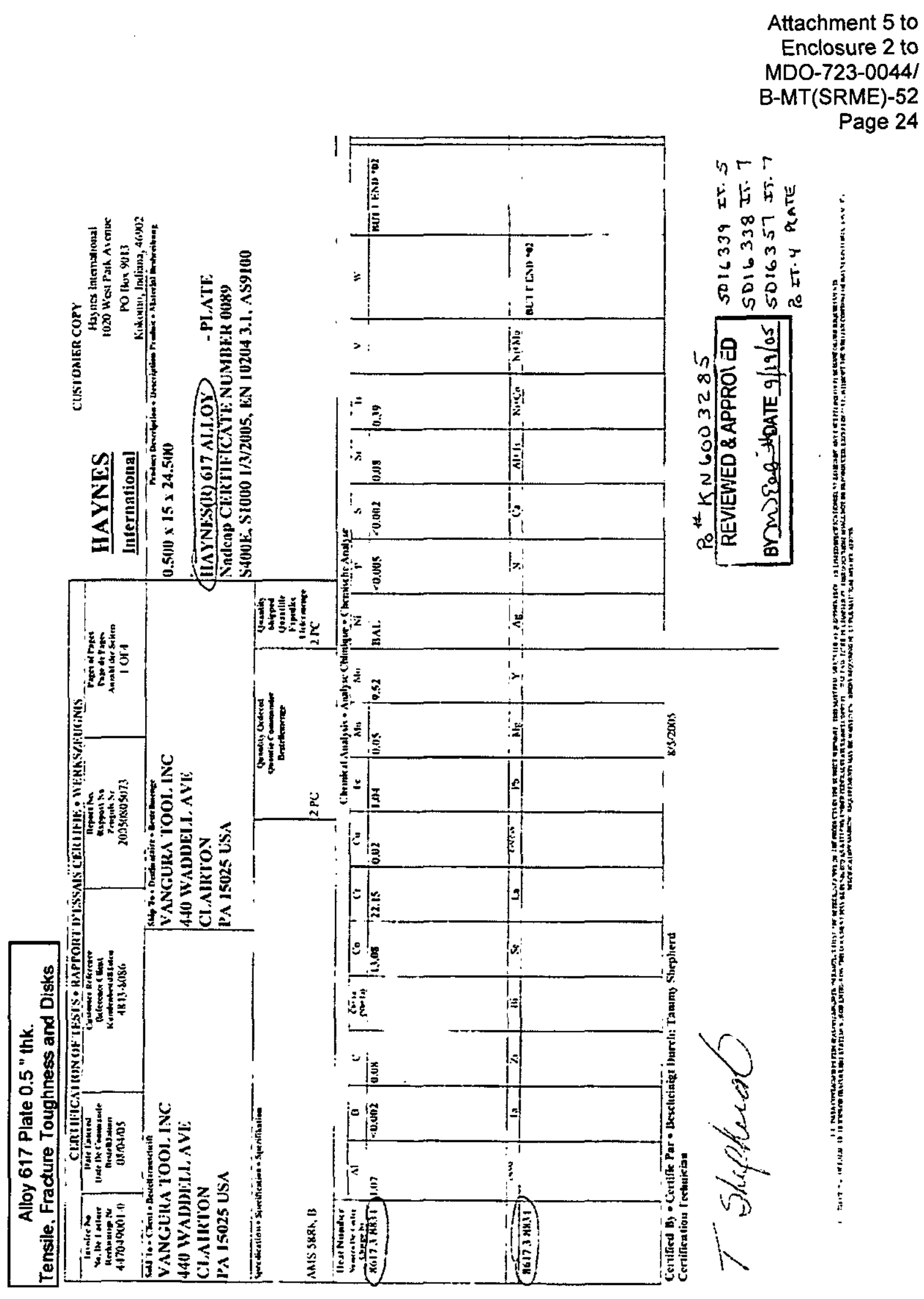

PRE-DECISIONAL - For Planning and Discussion Purposes Only 


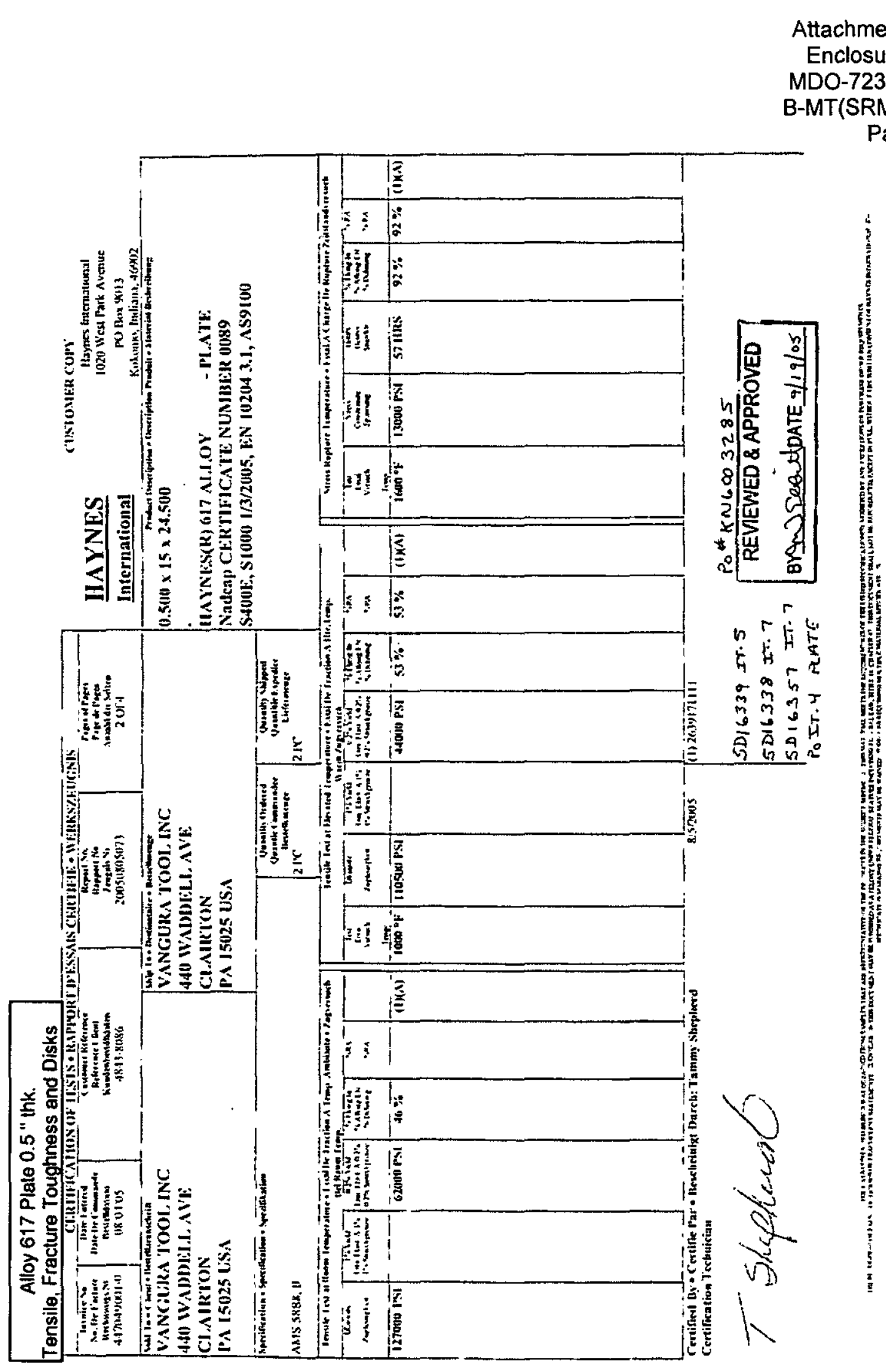

Page 25 
Attachment 5 to

Enclosure 2 to

MDO-723-0044/

B-MT(SRME)-52

Page 26

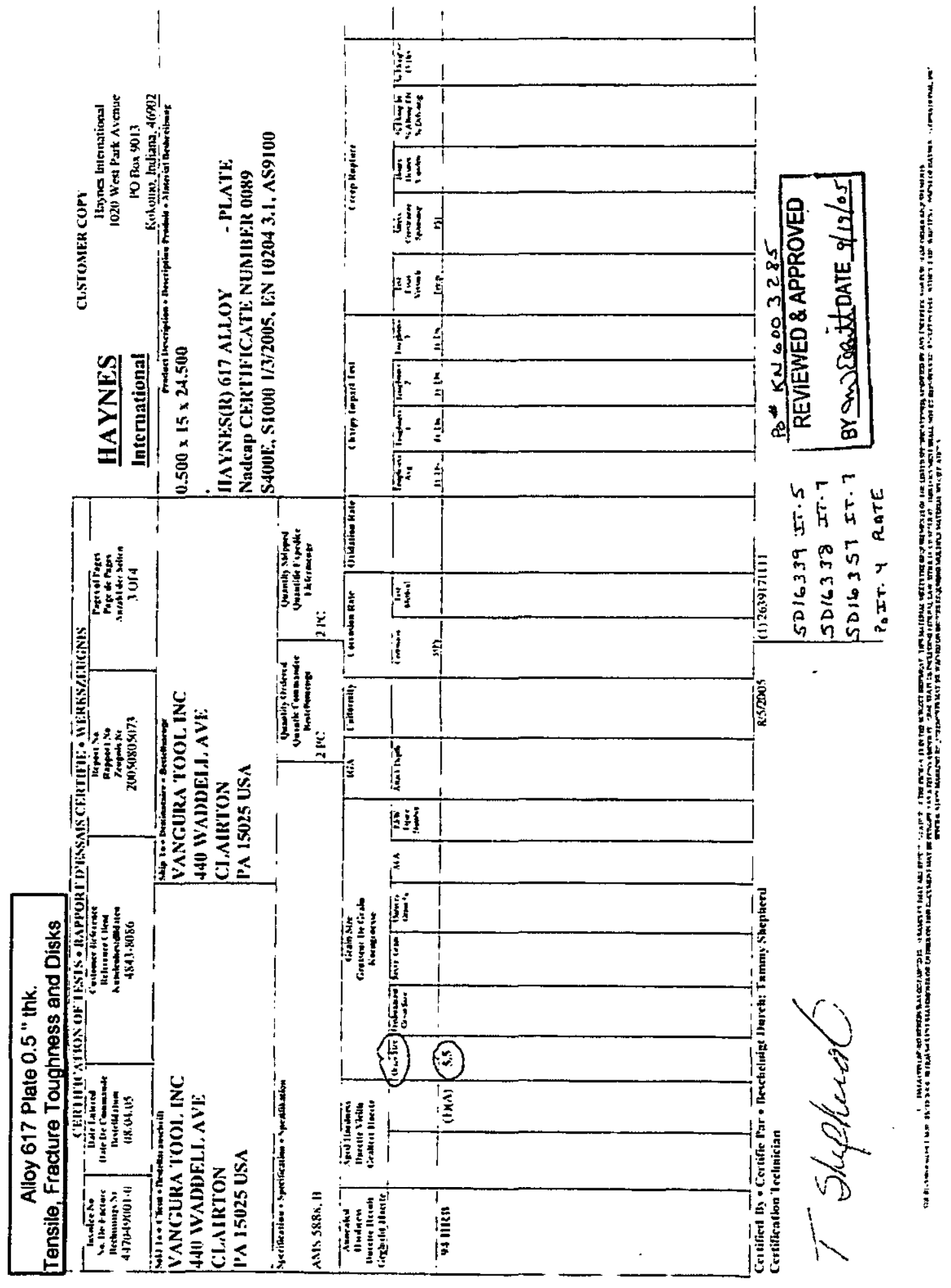




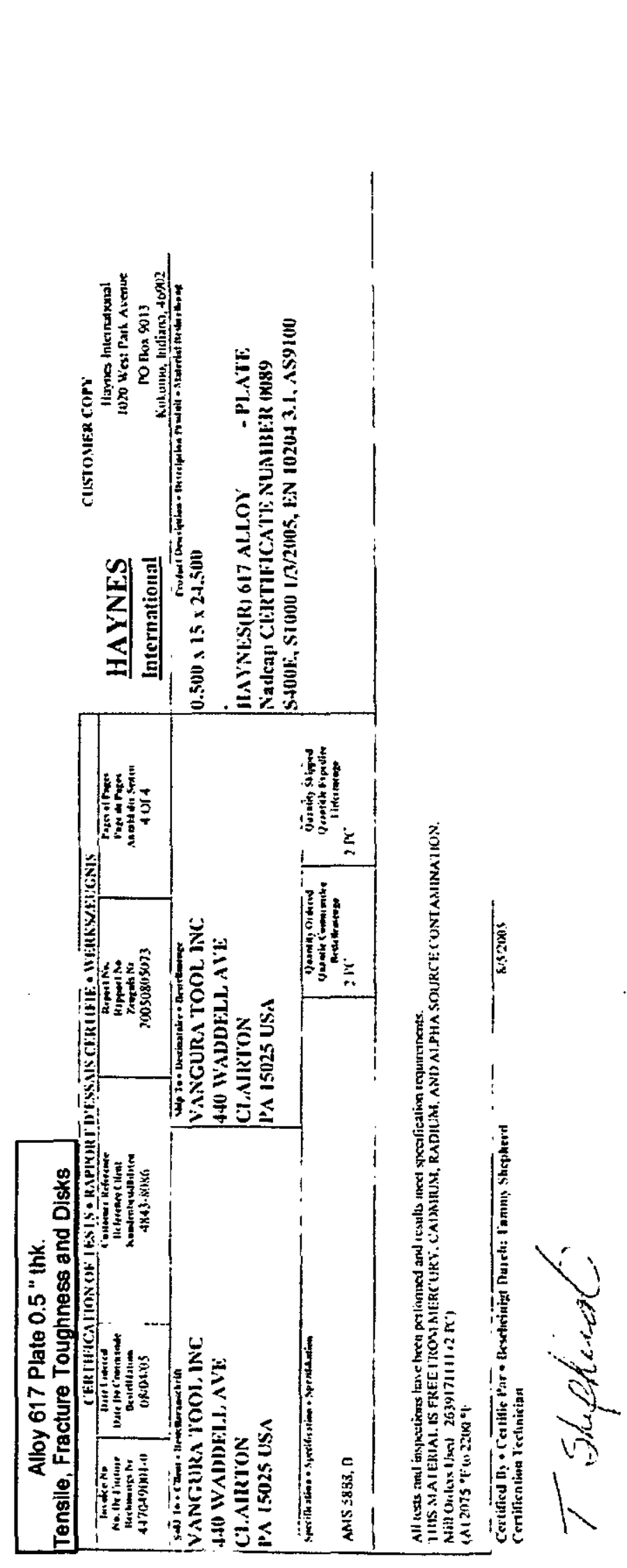

Attachment 5 to

Enclosure 2 to

MDO-723-0044/

B-MT(SRME)-52

Page 27

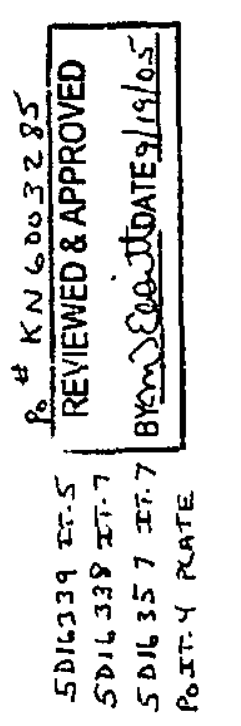

בל 
Attachment 5 to

Enclosure 2 to

MDO-723-0044/

B-MT(SRME)-52

Page 28

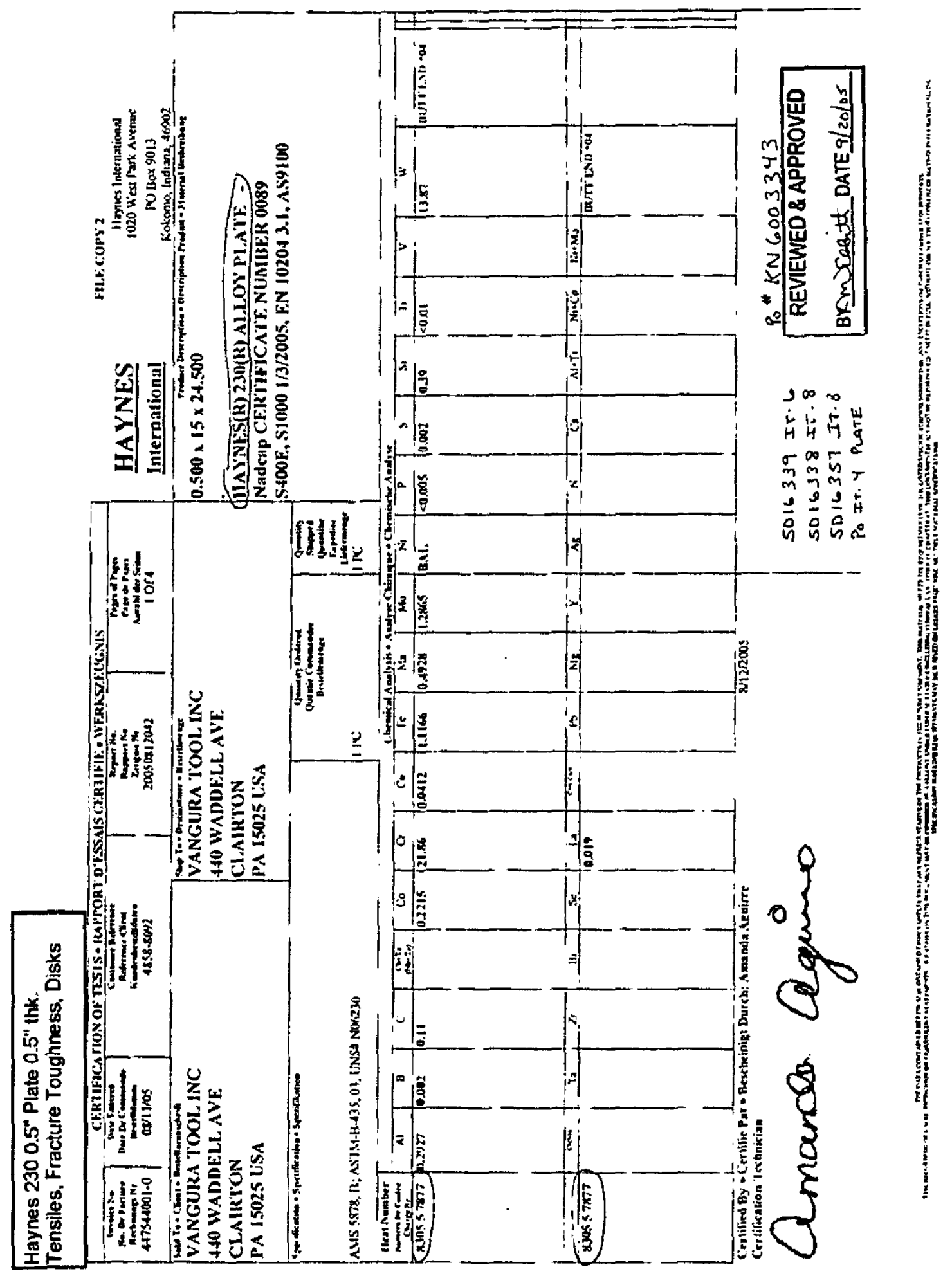


Attachment 5 to

Enclosure 2 to

MDO-723-0044/

B-MT(SRME)-52

Page 29

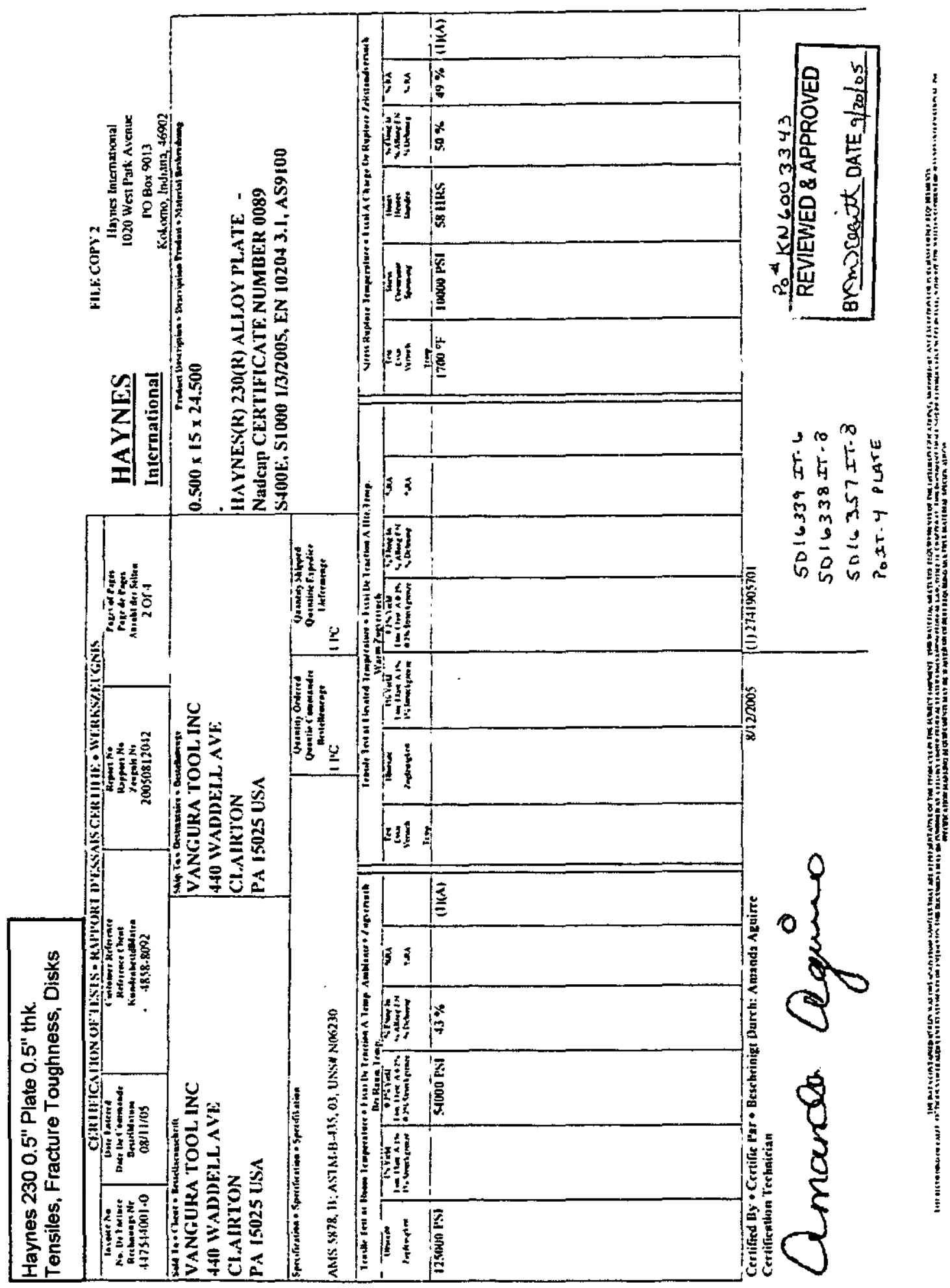


Attachment 5 to

Enclosure 2 to

MDO-723-0044I

B-MT(SRME)-52

Page 30

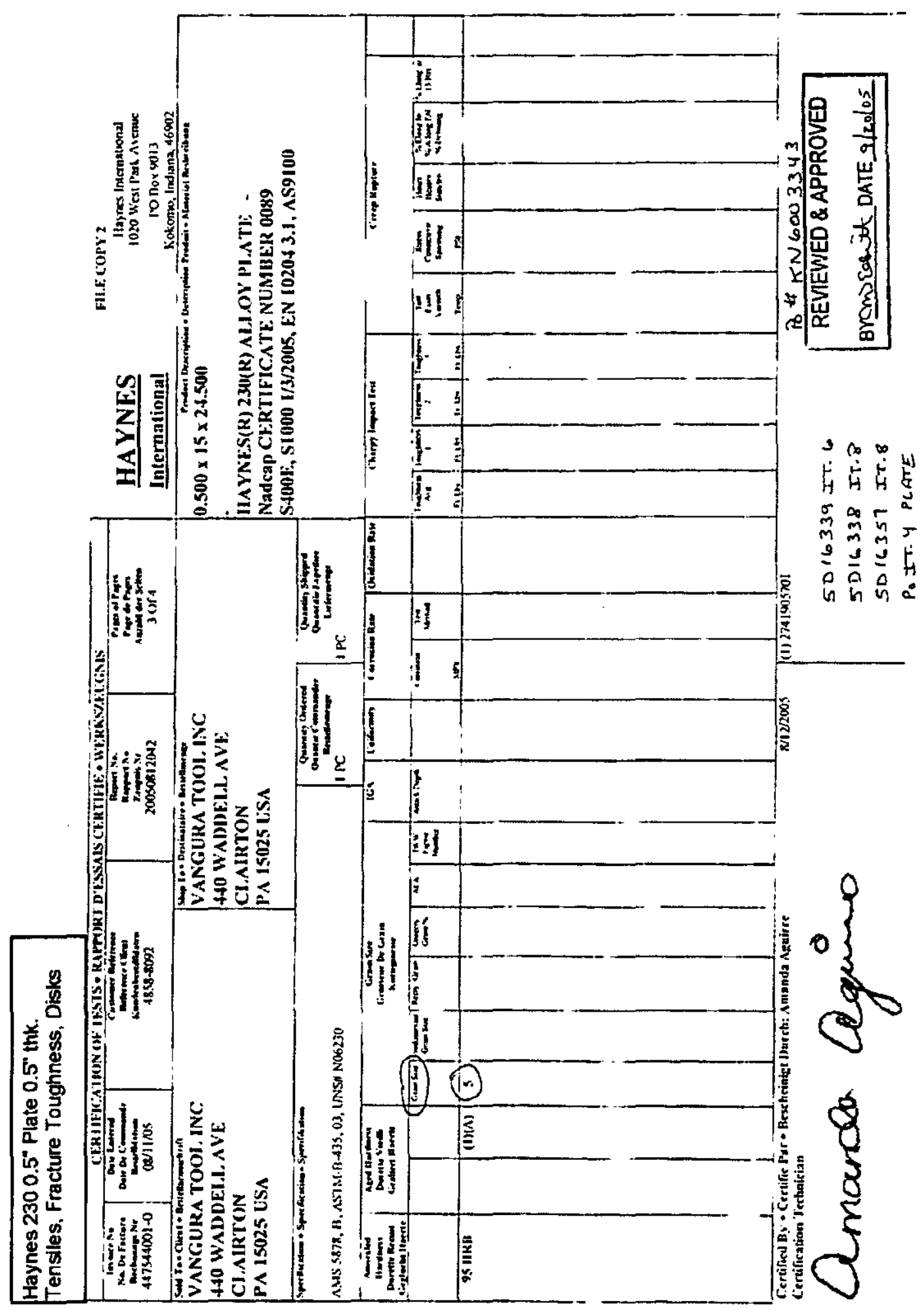

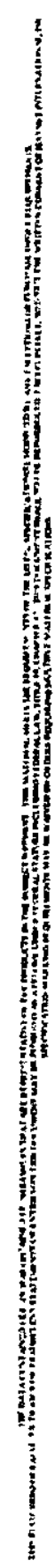


Attachment 5 to

Enclosure 2 to

MDO-723-0044/

B-MT(SRME)-52

Page 31
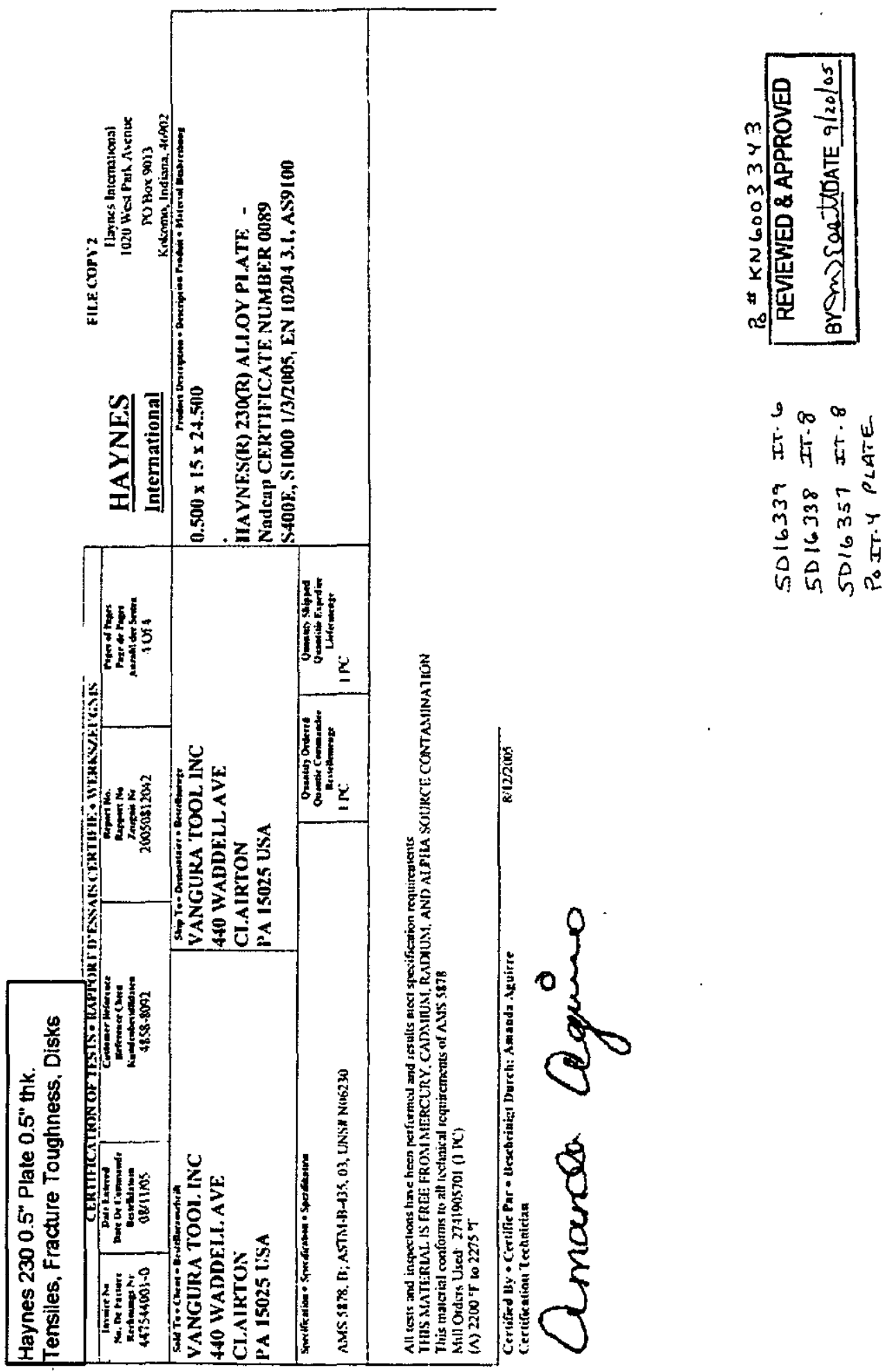

A 
Attachment 5 to

Enclosure 2 to

MDO-723-0044/

B-MT(SRME)-52

Page 32

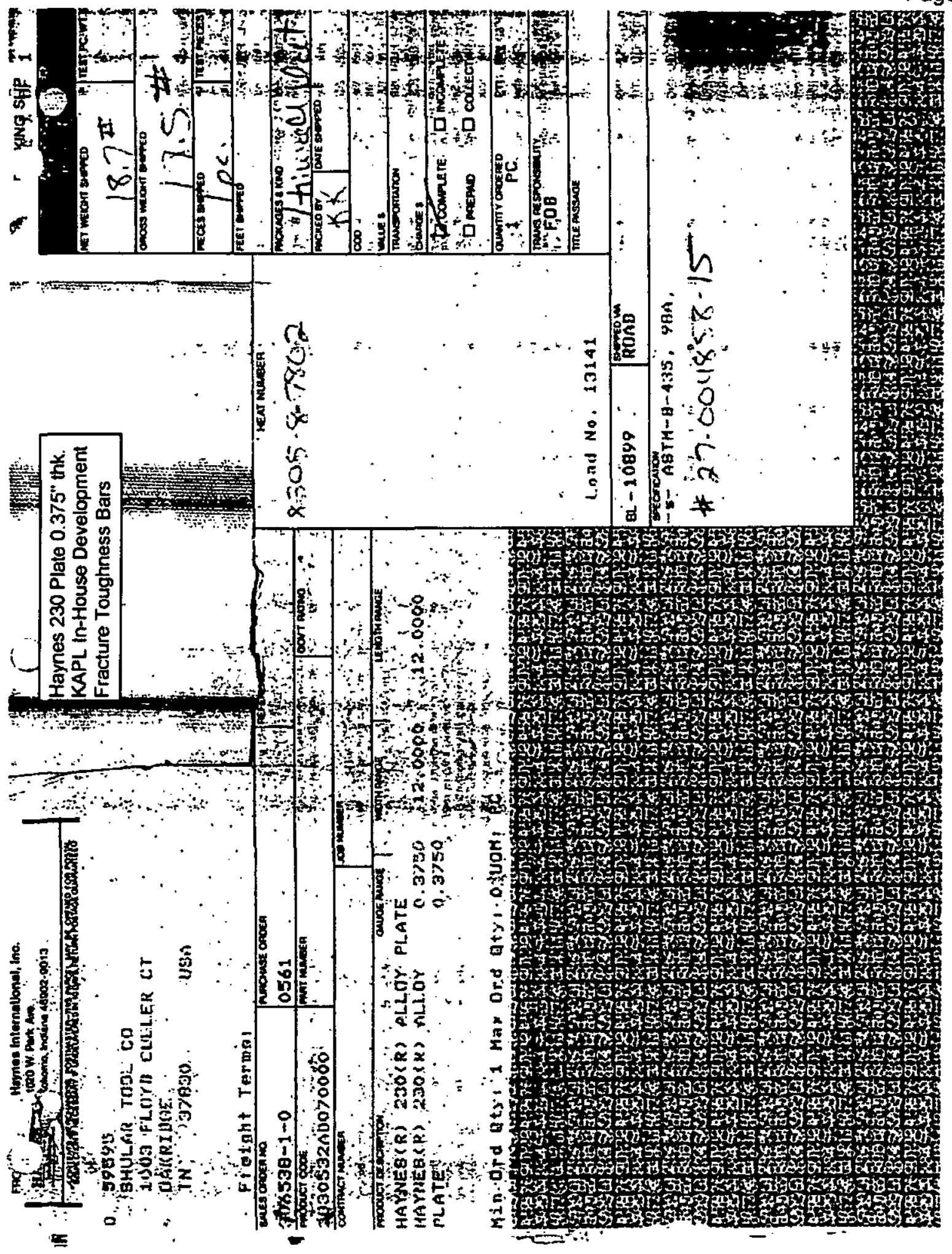


Attachment 5 to

Enclosure 2 to

MDO-723-0044/

B-MT(SRME)-52

Page 33

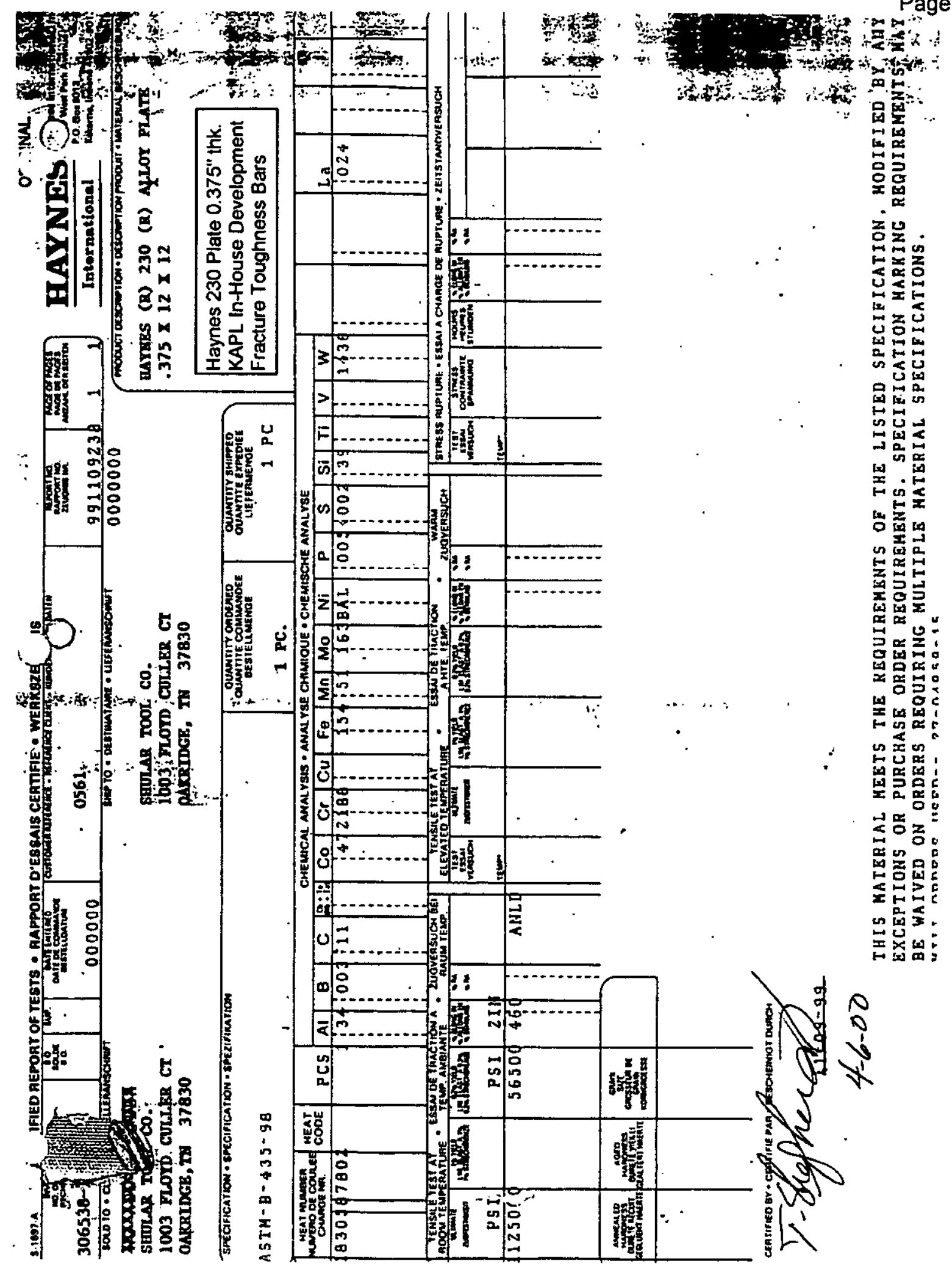


Attachment 5 to

Enclosure 2 to

MDO-723-0044/

B-MT(SRME)-52

Page 34

DIRATS

LABORATORIES

)

Haynes 230 Plate 0.375" thk. KAPL In-House Development Fracture Toughness Bars

\section{TESST REPORT}

Tom Goodrich, BIN HT36

Report Number

Asport Date

A Subsidiary of Lockheod Martin

Pogo

325916

P.0. box 1072

5.JUL.00

Bldo D3-152

Schajoctady, NY 12301-1072

Cllont Ordar

1 of 1

Release

M001700014

RECENVED 1 Tost Sample and 1 Lo: Test Pleces

IDENT AS

MATERIAL

CONPITION

TESTTO

TEST.PEA

-

Haynes 230

KAPL Motal Suite Aev. 2

FAX

518.395 .4422

Het Nrober

1830587802

\section{PROFERTIES AS SUPPLIED}

QUAUTTIATINE ANALYSIS BYICP.OE

$\%$

0.37

$<0.01$

0.47

21.67

0.01

1.48

0.53

1.68

0.02

58.78

0.005

$<0.01$

$<0.01$

0.37

$<0.01$

$<0.01$

$<0.01$

0.02

14.48

$<0.01$

0.10

$<0.001$

0.003

Dlsp:

For Into

Tho symbol < signifies not detected at the detectablity limit indicated.

$x$

$j$

WATED WE CERTIFY THIS IS A TRUE COPY OF OUR RECOROS

Signed for J. Dirats and Co. by Eric E. Dirats, Audit Manager

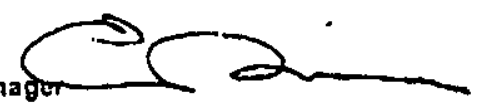

NOTE: The recording of false, fictlilous or fraudulent statements or entrias on this document may be punished as a folony under foderal law.

$\therefore$

41 AliłPOFT ROAD P.O. BOX 39 WESTFIELD, MA 0i086-0039 FAX 4i3-5j68-1453 413-568-157; 
Attachment 6.1 to

Enclosure 2 to

MDO-723-0044/

B-MT(SRME)-52

Page 1

Attachment 6.1

Fracture Toughness Estimation

Enclosure 2 to MDO-723-0044/B-MT(SRME)-52 


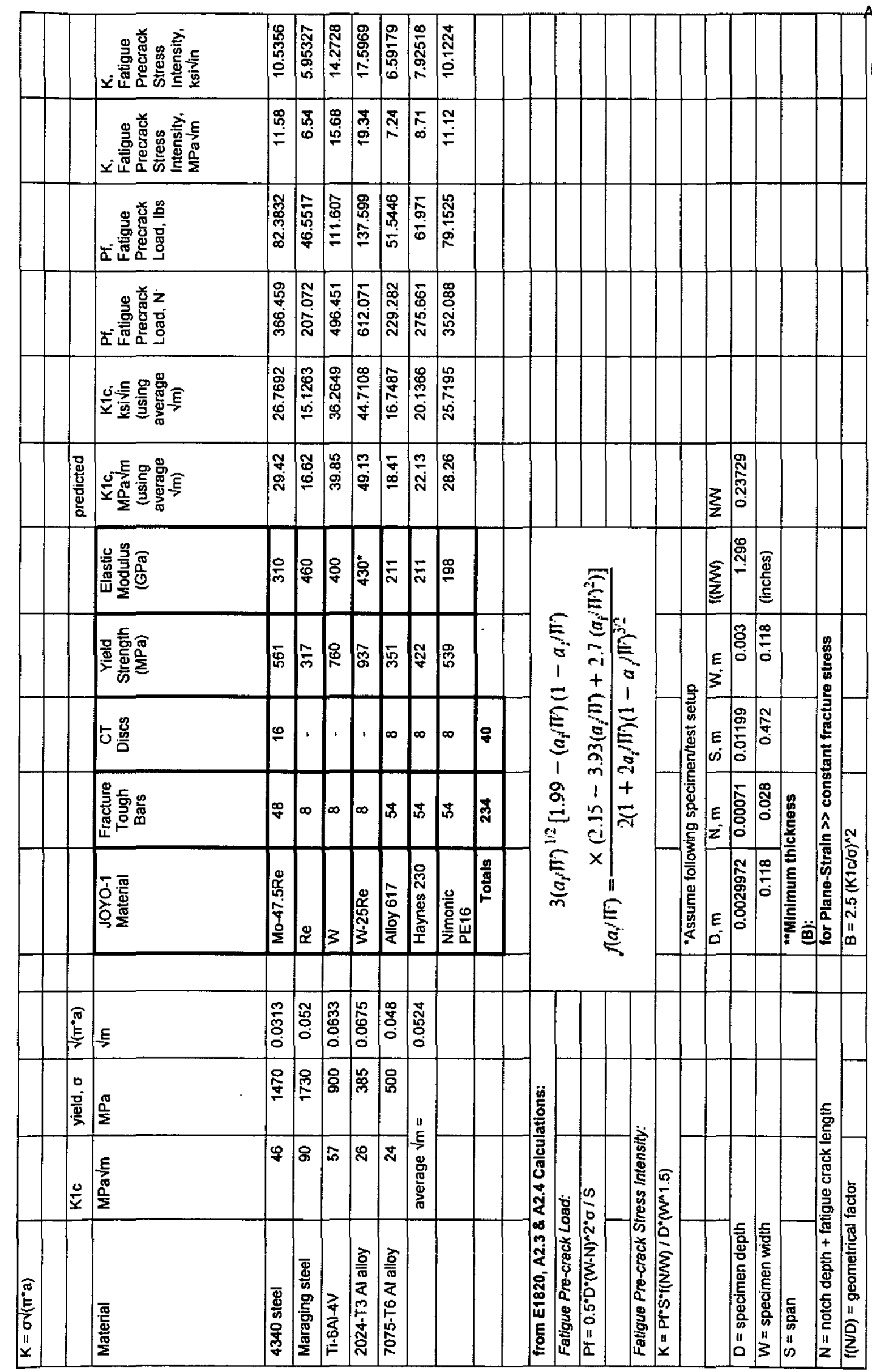

Attachment 6.1 to Enclosure 2 to MDO-723-0044/ B-MT(SRME)-52 Page 2

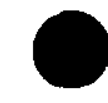


Table for Materials Characterization Lab:

\begin{tabular}{|l|c|r|l|l|l|l|}
\hline $\begin{array}{l}\text { JOYO-1 } \\
\text { Material }\end{array}$ & $\begin{array}{c}\text { Yield } \\
\text { Strength } \\
\text { (MPa) }\end{array}$ & $\begin{array}{c}\text { K1c, } \\
\text { MPaVm } \\
\text { estimate }\end{array}$ & $\begin{array}{l}\text { Pf, } \\
\text { Fatigue } \\
\text { Precrack } \\
\text { Load, N }\end{array}$ & $\begin{array}{l}\text { Pf, } \\
\text { Fatigue } \\
\text { Precrack } \\
\text { Load, } \\
\text { lbs }\end{array}$ & $\begin{array}{l}\text { K, } \\
\text { Fatigue } \\
\text { Precrack } \\
\text { Stress } \\
\text { Intensity, } \\
\text { MPaVm }\end{array}$ & $\begin{array}{l}\text { K, } \\
\text { Fatigue } \\
\text { Precrack } \\
\text { Stress } \\
\text { Intensity, } \\
\text { ksivin }\end{array}$ \\
\hline W-25Re & 937 & 49.13 & 612.07 & 137.60 & 19.34 & 17.60 \\
\hline W & 760 & 39.85 & 496.45 & 111.61 & 15.68 & 14.27 \\
\hline Alloy 617 & 351 & 18.41 & 229.28 & 51.54 & 7.24 & 6.59 \\
\hline Haynes 230 & 422 & 22.13 & 275.66 & 61.97 & 8.71 & 7.93 \\
\hline PE16, SA* & 539 & 28.26 & 352.09 & 79.15 & 11.12 & 10.12 \\
\hline PE16, HT a* & 450 & 23.60 & 293.95 & 66.08 & 9.29 & 8.45 \\
\hline PE16, HT b* & 525 & 27.53 & 342.94 & 77.10 & 10.83 & 9.86 \\
\hline
\end{tabular}

"SA = Solution Annealed, $15 \mathrm{~min} / 1040^{\circ} \mathrm{C} / \mathrm{AC}$

${ }^{*} \mathrm{HT} \mathrm{a}=4 \mathrm{~h} / 1040^{\circ} \mathrm{C} / \mathrm{AC}+1 \mathrm{~h} / 900^{\circ} \mathrm{C} / \mathrm{AC}+8 \mathrm{~h} / 750^{\circ} \mathrm{C} / \mathrm{AC}$

"HT $b=2 h / 1040^{\circ} \mathrm{C} / \mathrm{AC}+2 \mathrm{~h} / 800^{\circ} \mathrm{C} / \mathrm{AC}+16 \mathrm{~h} / 700^{\circ} \mathrm{C} / \mathrm{AC}$
Attachment 6.1 to Enclosure 2 to MDO-723-0044/ B-MT(SRME)-52

Page 3 
Attachment 6.2 to

Enclosure 2 to MDO-723-0044/

B-MT(SRME)-52

Page 1

Attachment 6.2

Load \& Cycling Data \& Results for S/N 1

Enclosure 2 to MD0-723-0044/B-MT(SRME)-52 
Attachment 6.2 to

Enclosure 2 to

MDO-723-0044/

B-MT(SRME)-52

Page 2

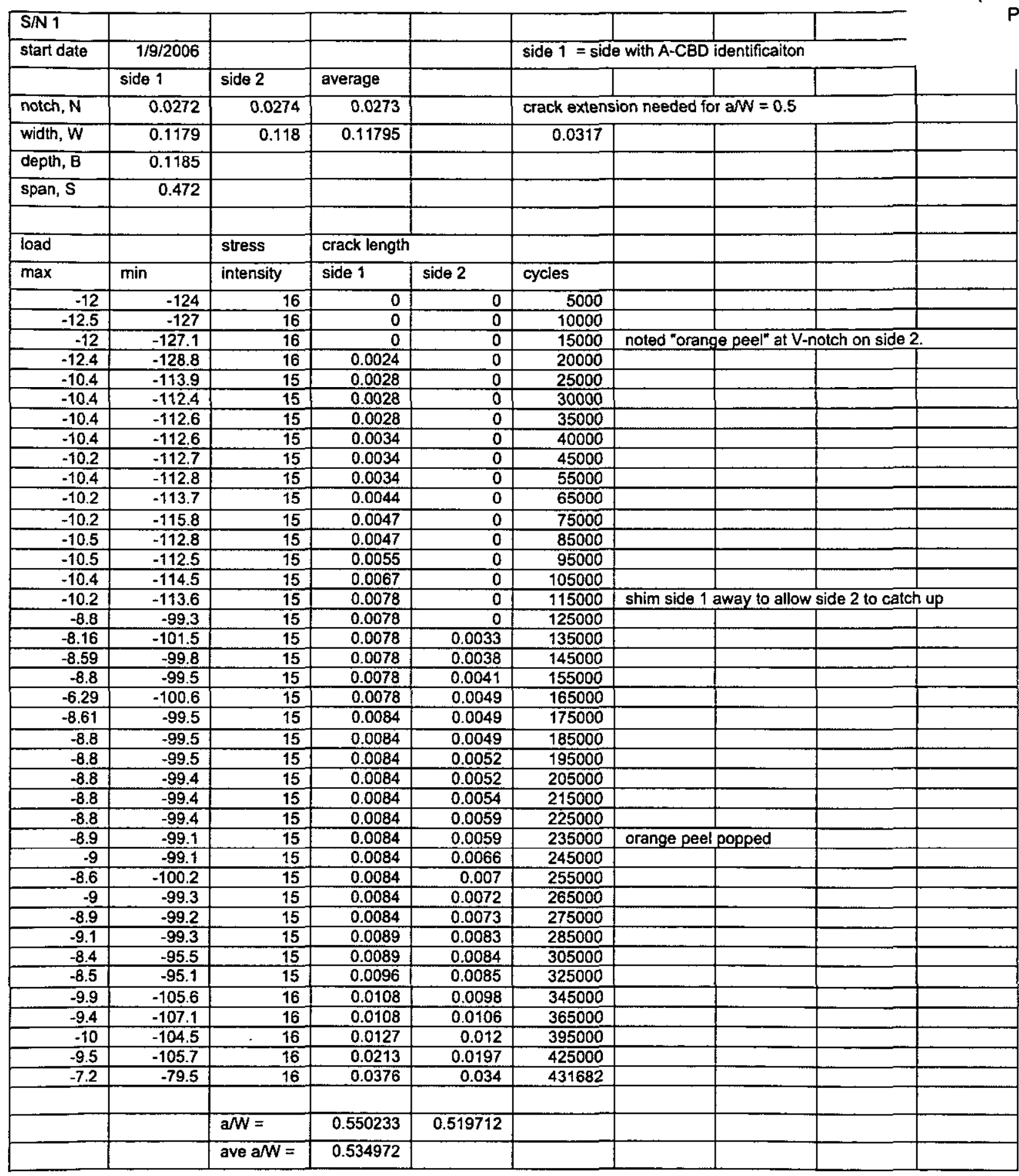


Two replicate specimens were precracked:

- Both specimens cycled in compression with a sine wave between 5 and 20 hertz. This information is captured on the individual data sheets. The material was Haynes 230 .

- The specimens were lightly sanded with 600 grit paper and then polished with diamond paste. This provided a smooth enough surface to enable the crack extension to be measured.

- Fixtures and specimens were not handled as "clean" that means that care was taken to not contaminate the polished surfaces of the specimen. But the fixture was not cleaned and the specimen was but not handled with gloves. Specimens were only handled at the ends, not near the notch or fatigue crack. That said, there were unidentified impurities that appeared on the surfaces of both specimens during the fatiguing that kept reappearing after wiping with cotton.

- First one precracked

- Used the $50 \mathrm{Lb}$ load cell and the $500 \mathrm{Lb}$ load cell

- Initially cycled between -43 and -3 pounds after 300,000 cycles after no crack growth the specimen was incrementally cycled at larger loads and load ranges.

- $23,069 \mathrm{cycles}$ into a $16 \mathrm{ksi} \sqrt{\mathrm{in}}$ at the notch fatiguing period the specimen experienced crack growth and ultimately reached a final stress intensity of $41.6 \mathrm{ksi}$ in at a crack length of .066 inches. At this point the specimen was bent.

$\mathbf{S} / \mathrm{N} 1$

- Used the $500 \mathrm{Lb}$ load cell

- Initially cycled between -124 and -12 pounds which was equivalent to $16 \mathrm{ksi}$ in

- As the crack grew the stress intensity was maintained between 14 and $16 \mathrm{ksi}$ in for the majority of the crack extension.

- Side 2 was not cracking up to 125,000 cycles, when a shim was place on Side 1 to allow Side 2 to "catch up" (see data sheets).

- Near the end the stress intensity was increased above 16 by approximately a maximum of 14 pounds. This was done to achieve the final desired $a / W(0.54)$ in the allowable amount of time.

- No noticeable/gross plastic deformation was observed. However, "sweating" was observed and wiped several times during the cycling process. Also, "orange peeling" can be observed in the precracked specimen.

- The final crack length (including notch) was 0.063 (ave).

- The data sheet contains the test details, both parameters and results.

Authors / Performers

Bruce Kallenburg $\quad 4584$

Charlene Vice $\quad 7785$ 
Attachment 6.2 to

Enclosure 2 to

MDO-723-0044/

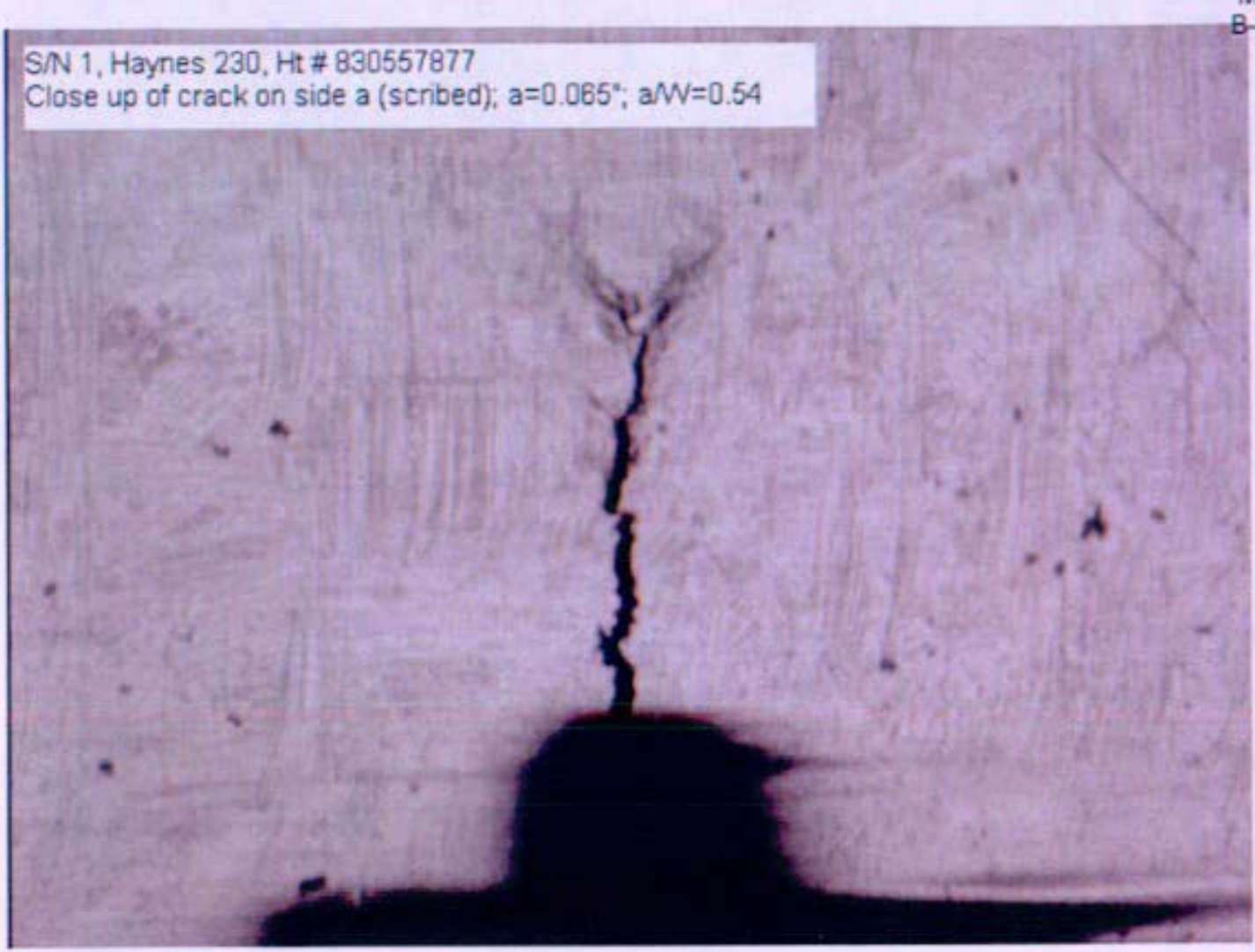

Page 4

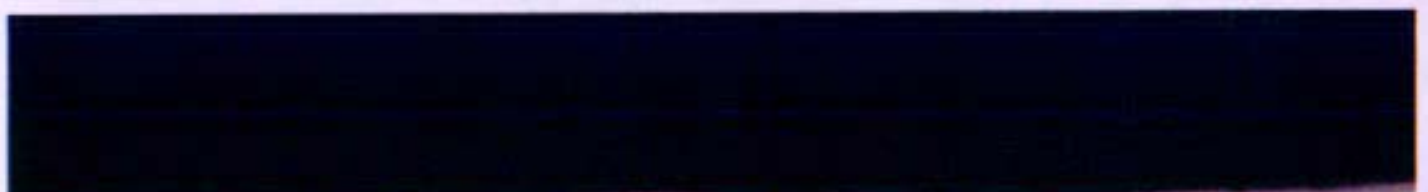

SiN 1. Haynes 230, Ht \# 830557877

Macroview of side a fatigue precrack $a=0.065^{\circ}, a / W=0.54$

FT bar dimensions

$0.118^{*} \times 0.118^{\prime \prime} \times 1^{\prime \prime}$ 
Attachment 6.2 to

Enclosure 2 to

MDO-723-0044

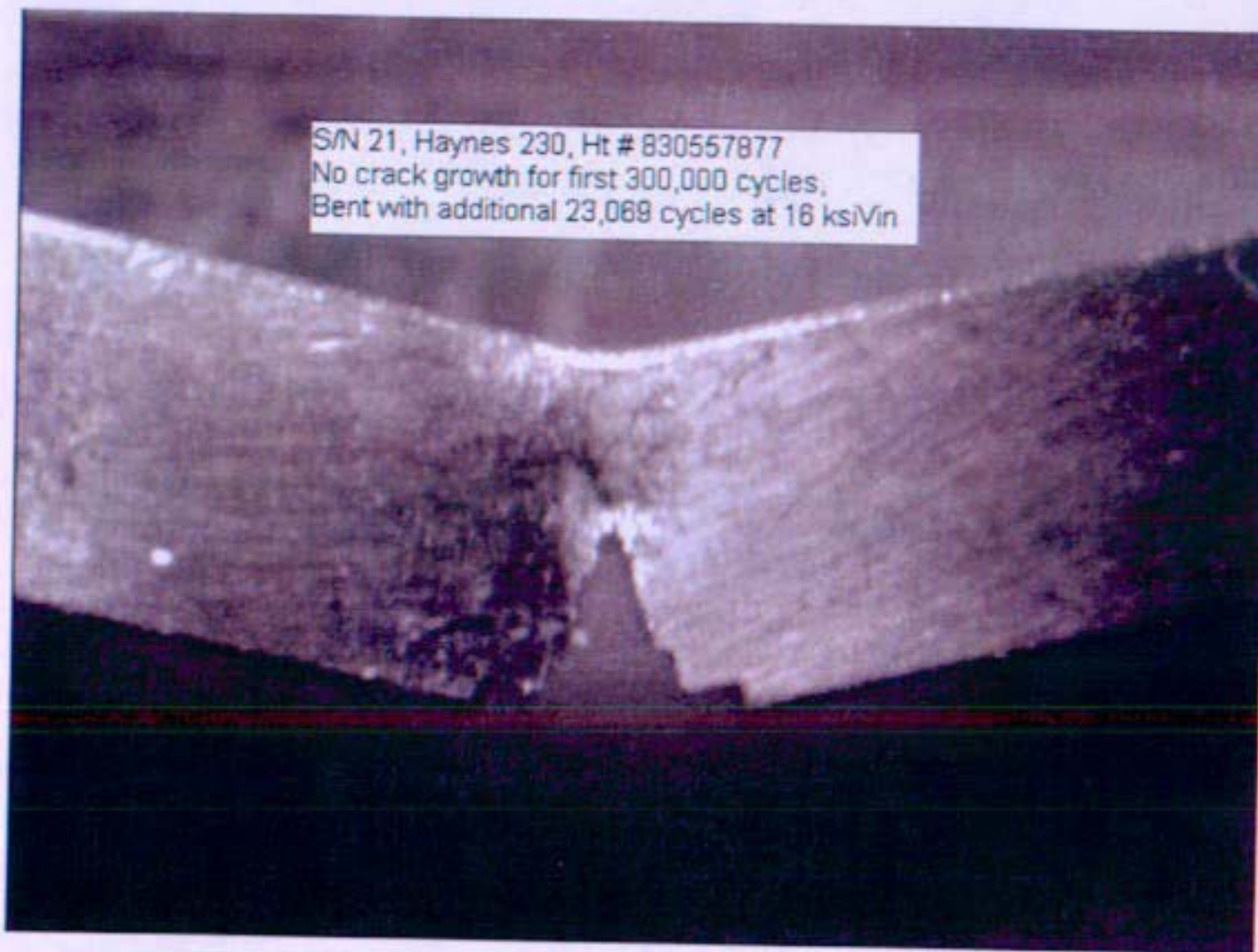

Page 5
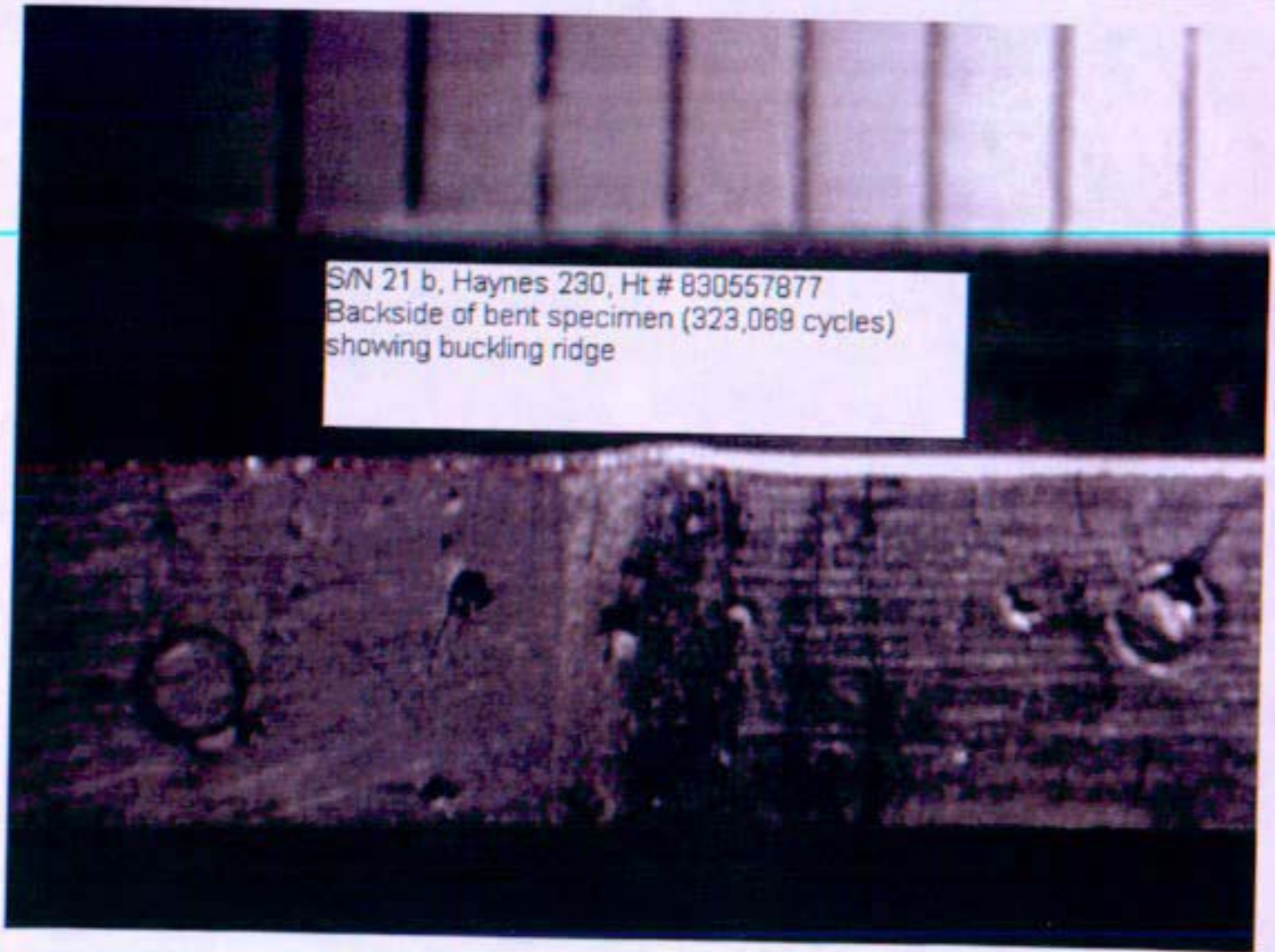
Attachment 6.3 to

Enclosure 2 to

MDO-723-0044!

B-MT(SRME)-52

Page 1

Attachment 6.3

Projected Cycles for $\mathrm{a} M=0.5$

Enclosure 2 to MDO-723-0044/B-MT(SRME)-52

PRE-DECISIONAL - For Planning and Discussion Purposes Only 
Attachment 6.3 to

Enclosure 2 to MDO-723-0044/

B-MT(SRME)-52

Page 2.
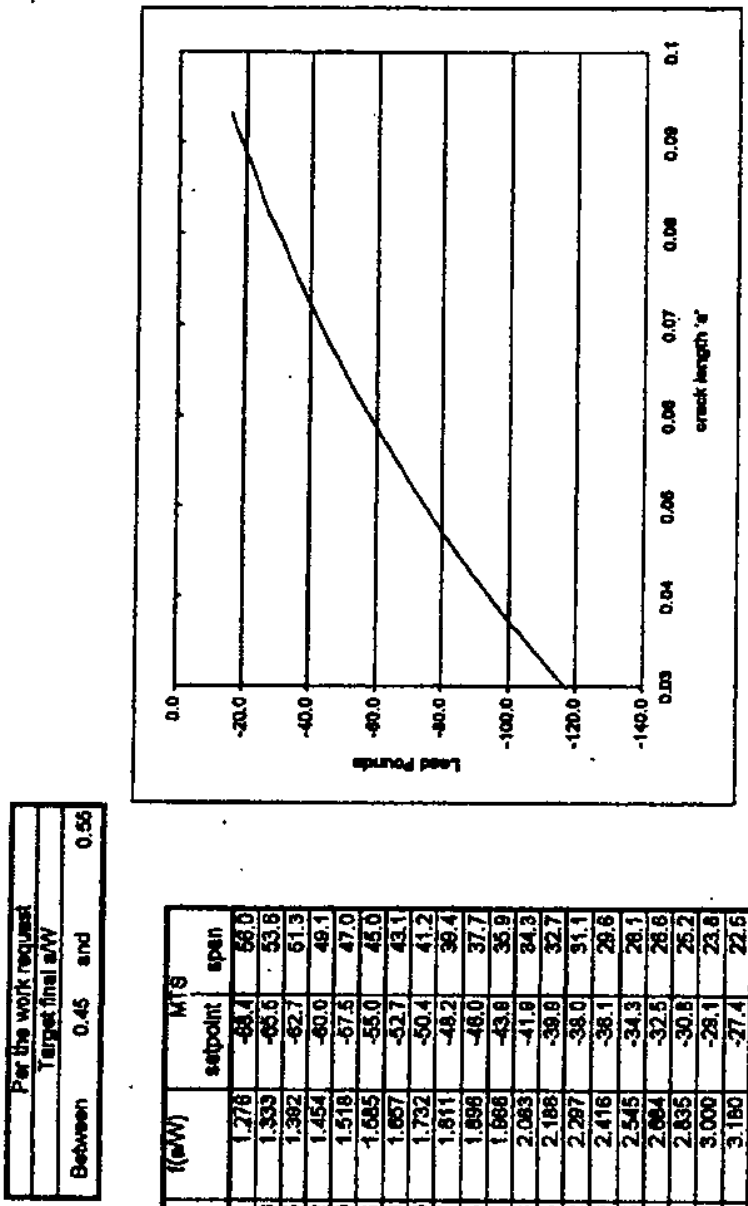

率点
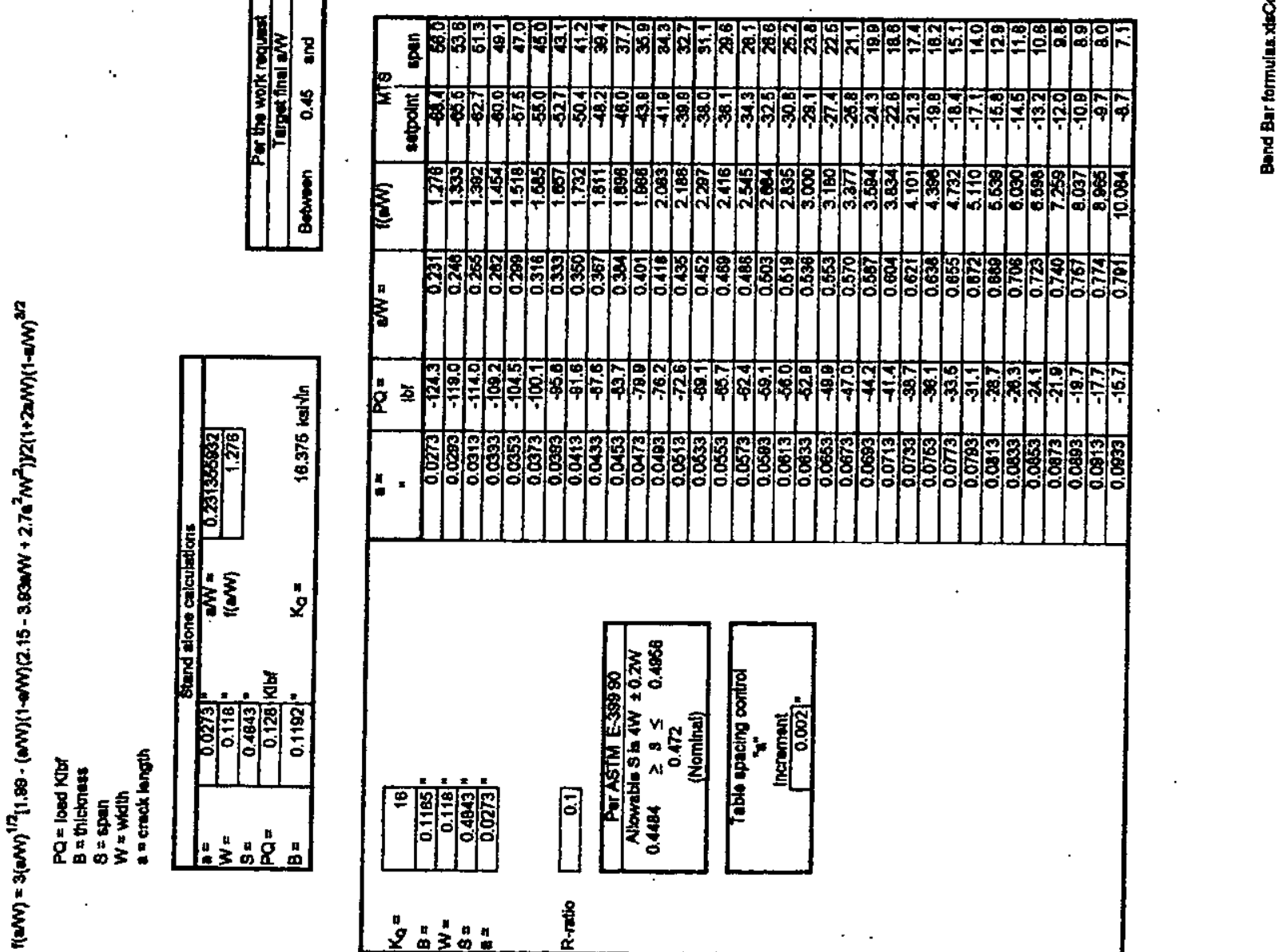

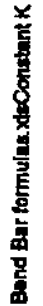

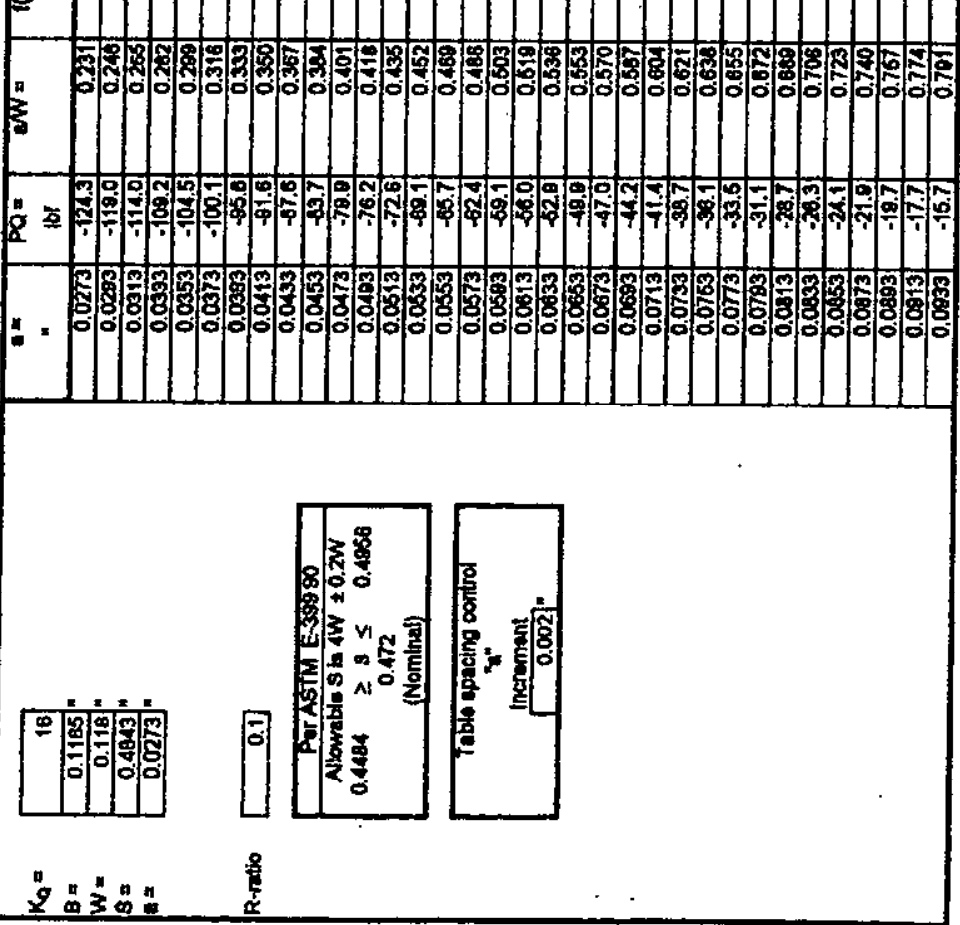

PRE-DECISIONAL - For Planning and Discussion Purposes Only 


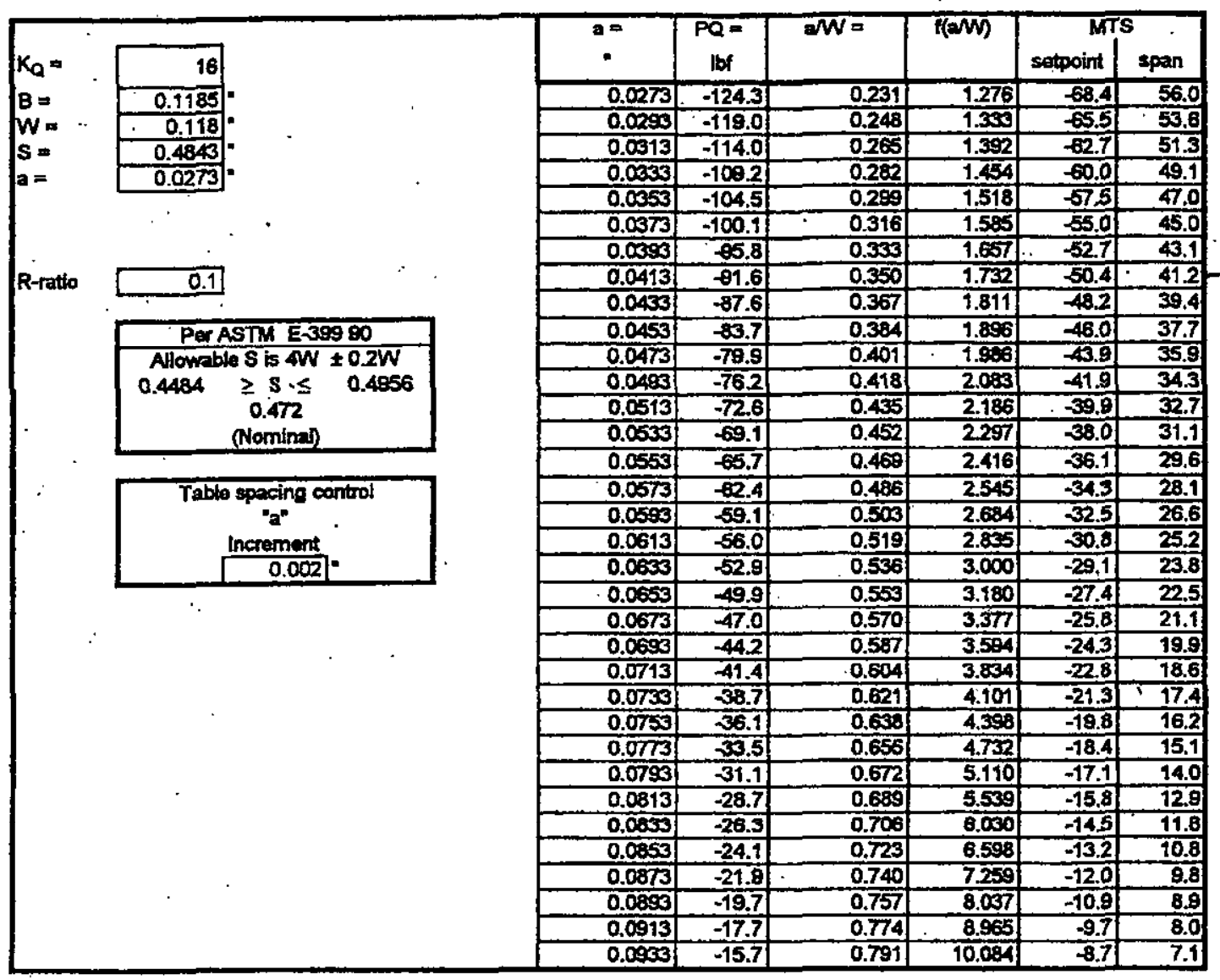

Attachment 6.3 to

Enclosure 2 to

MDO-723-0044I

B-MT(SRME)-52

Page 3

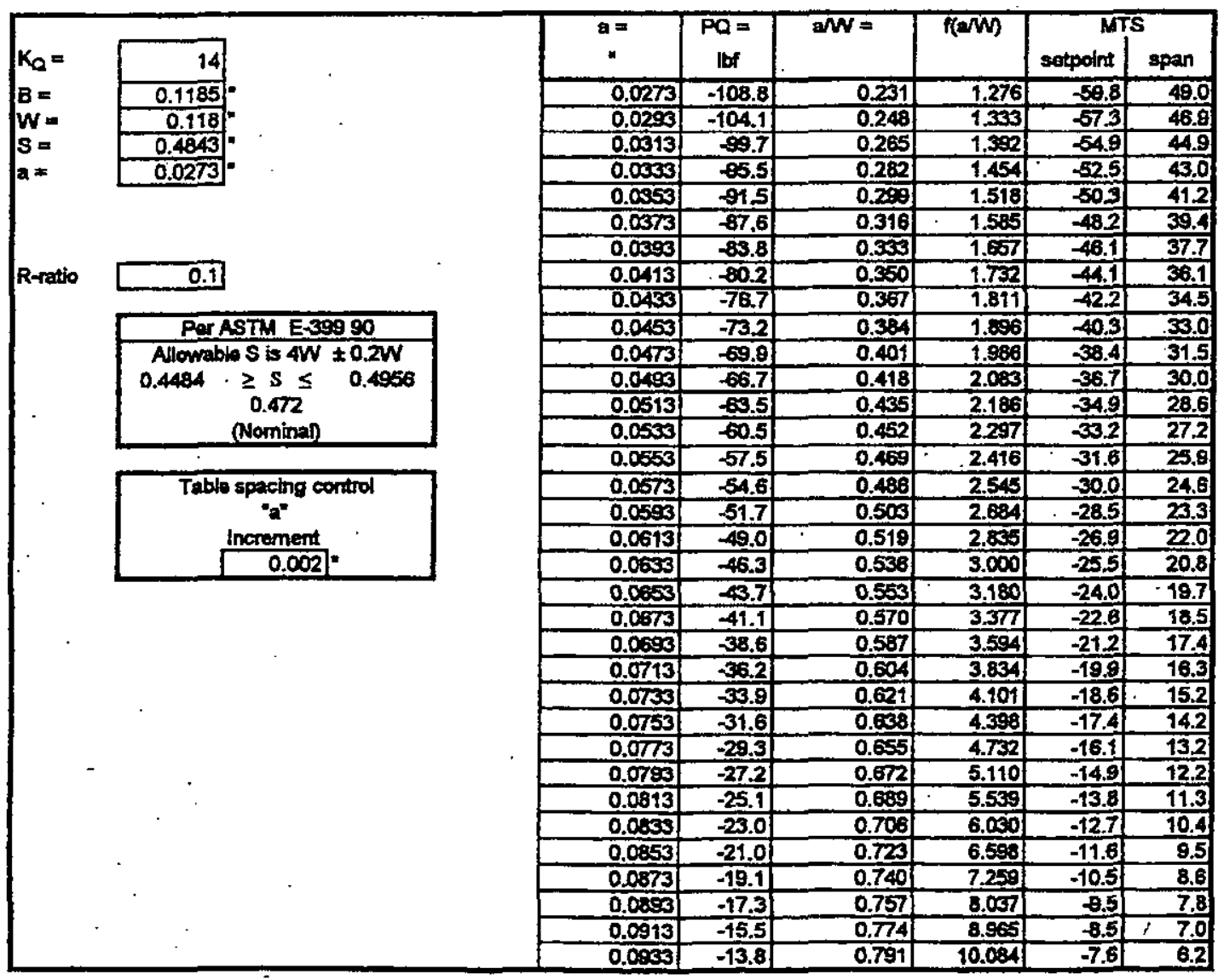

Bend Bar fonmulas xjsconstant $K$

PRE-DECISIONAL - For Planning and Discussion Purposes Only 
Attachment 6.3 to

Enclosure 2 to

MDO-723-0044I

B-MT(SRME)-52

Page 4

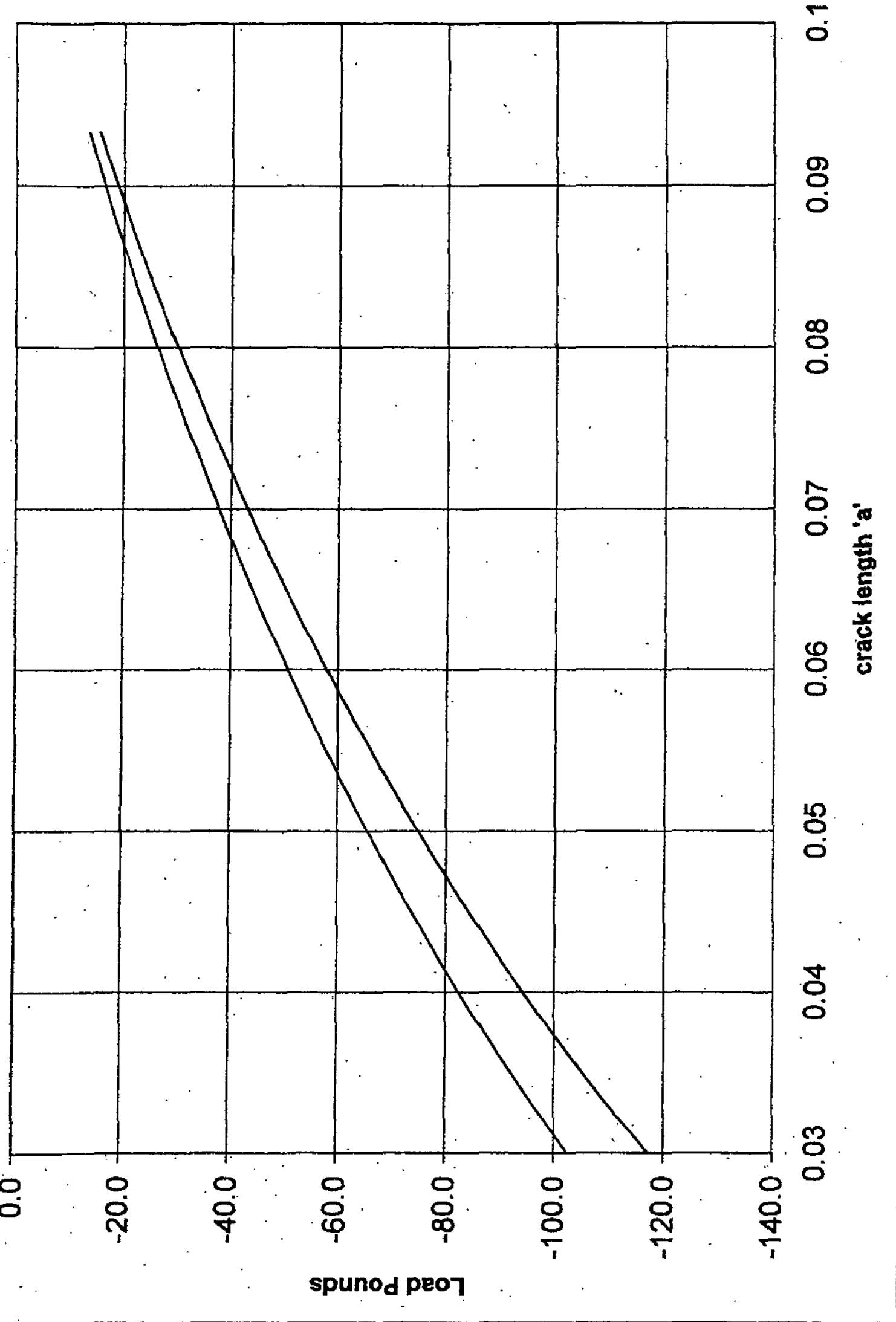

PRE-DECISIONAL - For Planning and Discussion Purposes Only 
Enclosure 3 to MDO-723-0044 /

B-MT(SRME)-52

Page 1

Enclosure 3

to

MD0-723-0044/B-MT-(SRME)-52

JOYO-1 Refractory Metal (Liner Materials) Specimen Fabrication Efforts

G. Borges

S.J. Buresh

January 2006 


\section{Summary}

Refractory metal liner materials were candidates for a diffusion barrier between the fueled region and cladding in Prometheus-1 designs. Efforts were in progress to fabricate a large number of refractory metal liner specimens for material property testing. Over 150 specimens planned for fabrication for JOYO-1 irradiation testing and for out-of-pile (replicate) testing to evaluate thermal degradation with respect to radiation effects. Specimen types included tensile, fracture toughness, and density discs. Refractory metal metals and alloys of interest included tungsten $(W)$, tungsten-25 weight percent $(w / 0)$ rhenium (W-25Re), and rhenium (Re). Table 1 contains a count of refractory liner specimens that were planned for JOYO-1 insertion. No refractory metal liner specimens were fabricated. Plates from which specimens were to be machined have been inventoried. Materials received included rhenium sheets, potassiumdoped tungsten rod, and W-25Re sheet and rod.

Table 1: JOYO-1 Refractory Metal Liner Specimen Count

\begin{tabular}{|l|c|c|c|c|}
\hline Material & $\begin{array}{c}\text { Fracture } \\
\text { Toughness } \\
\text { Bars }\end{array}$ & $\begin{array}{c}\text { Tensile } \\
\text { Bars }\end{array}$ & $\begin{array}{c}\text { Density \& } \\
\text { Conductivity } \\
\text { Discs }\end{array}$ & $\begin{array}{c}\text { Total } \\
\text { Specimen }\end{array}$ \\
\hline Re & 8 & 24 & 22 & 54 \\
\hline W & 8 & 23 & 21 & 52 \\
\hline W-25Re & 8 & 24 & 21 & 53 \\
\hline
\end{tabular}

\section{Introduction}

The use of refractory metal alloy cladding will likely require the use of a liner material to minimize clad-fuel interactions. Various springs, spacers, etc. will also be required to hold the fuel in place during launch and maintain its position during operation. Stress relaxation and/or embrittlement could have deleterious effects on the ability of these components to function as intended. Rhenium, tungsten, and W-25Re are the primary candidates for these materials.

Experience indicates that significant damage occurs from interactions between nitrogen and Molybdenum-based alloys used with uranium nitride (UN) fuel, although the interpretation of these observations is not clear. Rhenium is a candidate for fuel assembly components but can react with nitrogen depleted UN fuel to produce URe $\mathrm{U}_{2}$ in the presence of Niobium-based cladding. There is controversy concerning an observed ductility drop in rhenium, in both the irradiated and unirradiated conditions, that needs to be resolved prior to application of thick sections of rhenium in fuel assemblies. Rhenium may serve best as a diffusion barrier between the cladding and a tungsten or W-Re liner in rodded reactor concepts. Tungsten is chemically compatible with both $\mathrm{UN}$ and $\mathrm{UO}_{2}$ fuels and could serve as a matrix and/or cladding material for cermet fuel elements.

\section{Discussion}

Refractory metal liner materials included in the JOYO-1 test matrix are well-known materials. However, they are not abundant, not manufactured in large quantities, or generally not kept instock at suppliers. Initial plans were to fabricate specimens out of material forms that would be prototypic of those anticipated for production liners. Because of differences in material availability, cost, ductility, and welding, different starting material liner product forms were 
required for specimen fabrication. Thus, ductility and costs of rhenium metal made it logical to produce specimens from thin sheet since rhenium is typically produced in thin sheets and production methods were well established.

Conversely, W-Re sheet is not regularly produced, and its ductile-to-brittle-transitiontemperature (DBTT) is above room temperature. Consequently, forming $W$-Re at room temperature is not possible and its welds are usually brittle. Therefore, $W-25 R e$ specimens were machined from rod stock. This form of the material is readily available, likely because rod is a precursor to wire, a standard tungsten-rhenium product (e.g., weld filler, heater elements).

\section{Tungsten}

Tungsten sheet is usually not ductile above a thickness of $\sim 0.05 \mathrm{~mm}(0.002$ inches $)$ and has poor welding/joining characteristics. Pure tungsten in a stress relieved or recrystallized state also has a DBTT above room temperature, which severely affects fracture toughness. One alternative to alleviate the poor fracture toughness of pure tungsten is to use a more ductile, potassium doped ( $-50-100 \mathrm{ppm})$ tungsten material for specimen fabrication. Potassium $(\mathrm{K})$ doped material also has some improved high temperature properties and is sometimes referred to as non-sag (NS) tungsten. Moreover, a potassium dopant level $<100 \mathrm{ppm}$ did not pose a significantly concern with activation or neutronics (References 1 and 2). Based on the above issues and available product forms, $\sim 12.7 \mathrm{~mm}(0.5 \mathrm{inch})$ diameter rod was purchased from Schwarzkopf Technologies LLC, (US distributor of Plansee in Franklin, MA), according to the requirements provided in Attachment 1.

\section{Rhenium}

Because of their production capabilities, quality assurance (QA) experience, and proximity, rhenium Alloys was chosen as a bulk refractory metal source for thenium and W-25Re. To minimize machining and rhenium scrap material, NRPCT procured sheet stock similar to the thicknesses required for the test specimens (i.e. $0.762 \mathrm{~mm}(0.030 \mathrm{inch}), 1.524 \mathrm{~mm}$ (0.060 inch) and $3.00 \mathrm{~mm}(0.118 \mathrm{inch})$ ).

\section{Tungsten-25 Rhenium}

Small diameter (6.32 mm / 0.25 inch) W-25Re rod (sinter-swage), initially procured for fuel capsule work, was re-designated as backup material for JOYO-1 samples. W-25Re rod was sintered and hot isostatically pressed (HIP'ed), followed by a hot swaging to final dimensions. This process provided a very dense and homogenous material (Figure 1). NRPCT also procured some historical stock of $\sim 2.03 \mathrm{~mm}(0.080$ inch) thick W-25Re sheet material from Rhenium Alloys as backup material for specimens, in case schedule issues arose with production of the sinter-HIP-swage rod. This historical sheet material was also planned to be evaluated out-of-pile against the more standard rod material.

\section{Material Disposition}

Specifications developed for machining refractory liner material specimens are provided in Attachment 2. Of the planned specimens in Table 1, only three fracture toughness bars of $W$ 25Re and Potassium-doped Tungsten were machined in-house using Electro-Discharge 
Machining (EDM) with a $0.1 \mathrm{~mm}$ (0.004 inch) brass wire. W-25Re rod used for these development specimens was historical stock from Rhenium Alloys $(6.35 \mathrm{~mm} / 0.25$ inch diameter) with limited traceability beyond the original lot number (WR 1460) and the Potassiumdoped Tungsten was from material listed in Table 2. Images from metallographic mounts of these materials are provided in Figures 1 and 2, confirming a dense, homogeneous microstructure with an ASTM grain size of $\sim 6.6$. Chemistry and additional metallographic results from the materials are provided in Attachment 3.

\section{Suggestions for Future Work}

Due to the small dimensions of the fracture toughness notch and difficulties with machining refractory materials, the notch was EDM'd. Electro-discharge machining these specimens uses molybdenum wire and a cleaning/pickling process to remove contaminants. However, cleaning and pickling could not etch away the recast layer and still meet drawing requirements. Recommended alternatives include:

(1) Using an EDM machine that provides non-electrolysis and uses alternating current skim passes to reduce both material damage and recast layer thickness.

(2) Using submerged EDM

(3) Investigating Metal Samples (Munford, AL), which has developed a special cleaning process for refractory metal samples.

Table 2: Summary of Raw Refractory Metal Liner Materials

\begin{tabular}{|c|c|c|c|c|}
\hline Raw Material & Source & Material Size & $\begin{array}{c}\text { Cost }^{\star} \\
\text { (in 2005) }\end{array}$ & Status/Comment \\
\hline W-25Re Sheet & Rhenium Alloys & $\begin{array}{l}\sim 2.03 \mathrm{~mm} \text { thick sheet, } \\
10.1 \mathrm{~cm} \times 20.3 \mathrm{~cm}, 2 \mathrm{pcs}\end{array}$ & $\$ 6708$ & Received \\
\hline W-25Re Rod & Rhenium Alloys & $6.35 \mathrm{~mm}$ dia. rod, $1.8 \mathrm{~m}$. & $\$ 2760$ & Received \\
\hline K-Doped W Rod & Schwarkopf & $12.7 \mathrm{~mm}$ dia. rod, $2.4 \mathrm{~m}$ & $\$ 1479$ & Received \\
\hline Re Sheet & Rhenium Alloys & $\begin{array}{c}3.0 \mathrm{~mm} \text { thick sheet, } \\
5.1 \mathrm{~cm} \times 10.2 \mathrm{~cm} \\
1.52 \mathrm{~mm} \text { thick sheet, } \\
10.2 \mathrm{~cm} \times 17.8 \mathrm{~cm} \\
0.76 \mathrm{~mm} \text { thick sheet, } \\
10.2 \mathrm{~cm} \times 15.2 \mathrm{~cm}\end{array}$ & $\begin{array}{l}\$ 3141 \\
\$ 5538 \\
\$ 2598\end{array}$ & Received \\
\hline
\end{tabular}

"Cost is listed for these materials as a notice of their expense relative to other JOYO-1 materials. 
Enclosure 3 to MDO-723-0044 I

B-MT(SRME)-52

Page 5

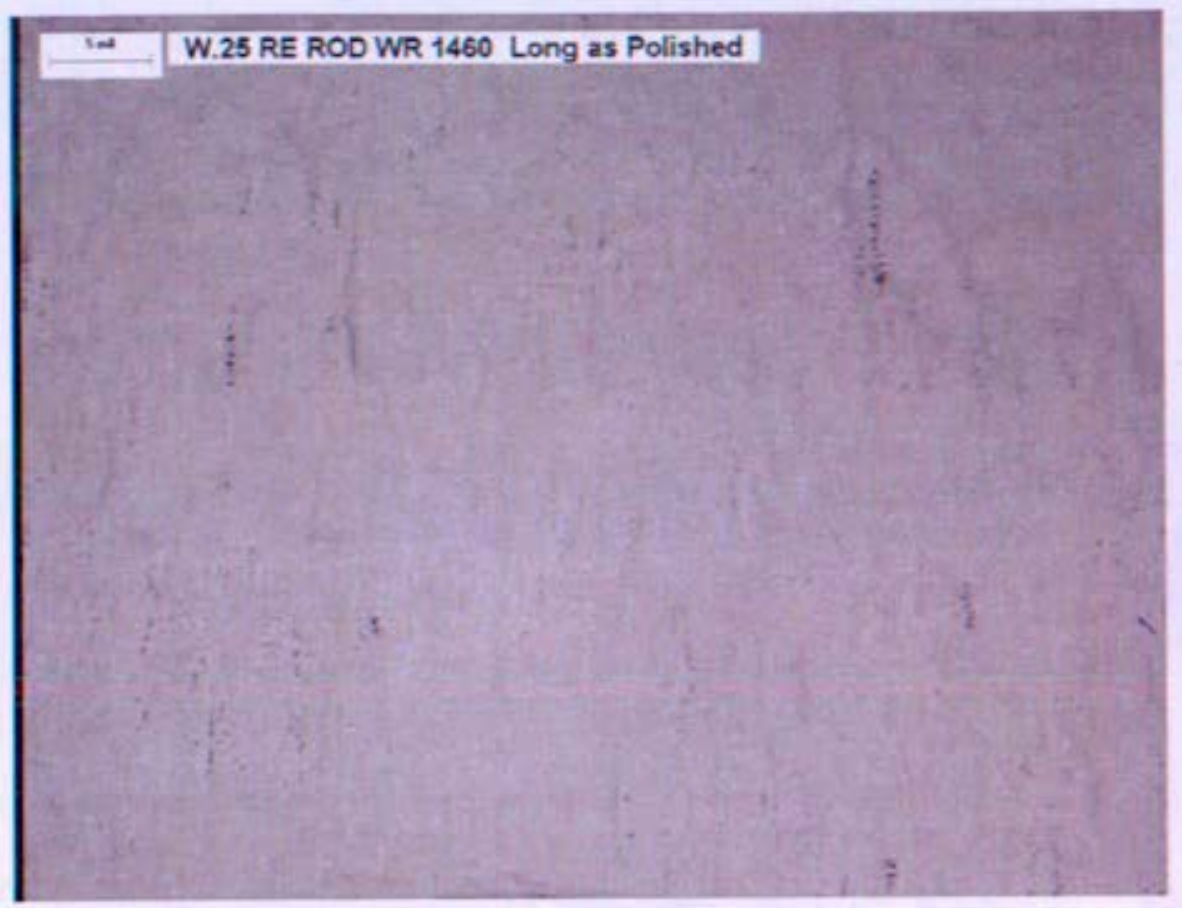

Figure 1: Development W-25Re fracture toughness bar material (Rhenium Alloys WR-1460 rod)

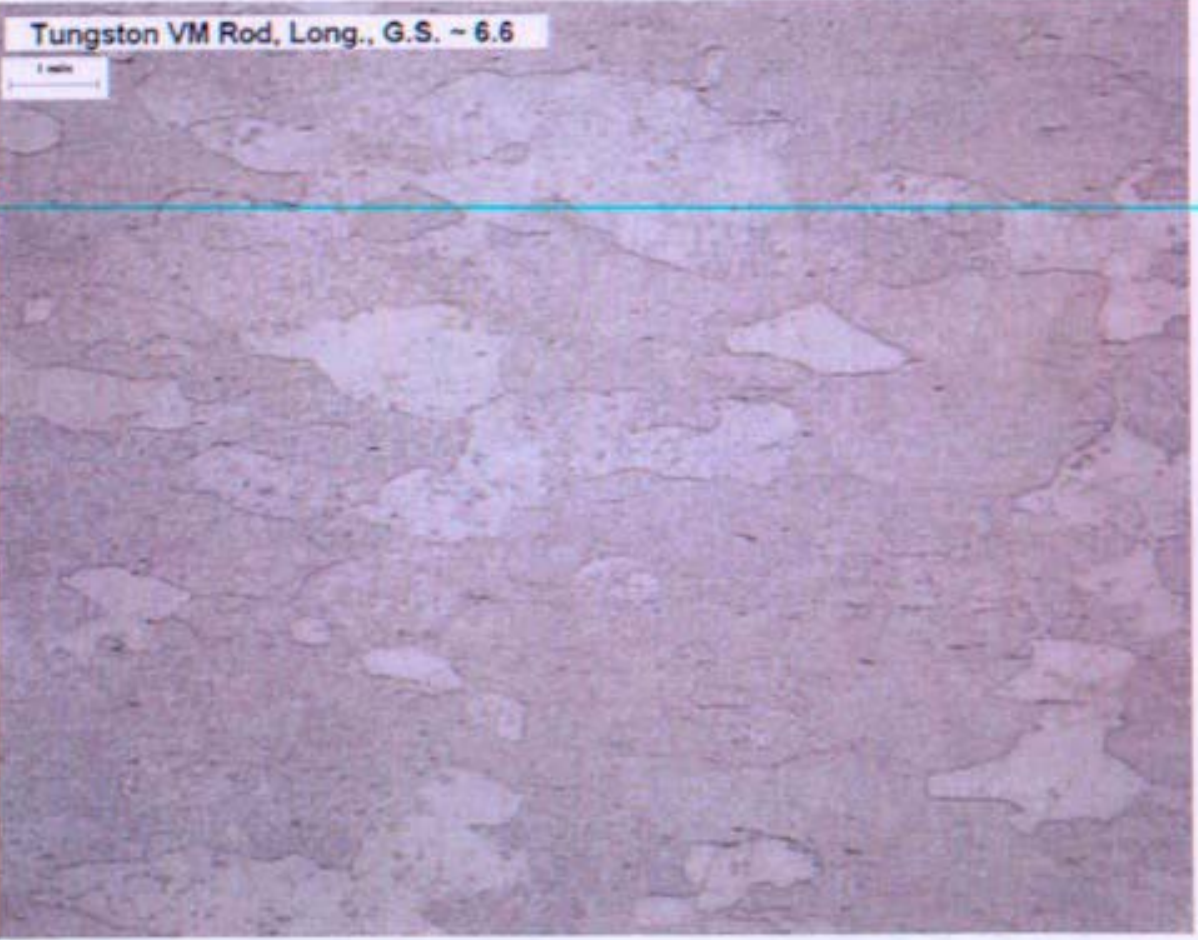

Figure 2: Development Potassium-doped Tungsten fracture toughness bar material, longitudinal section. (Schwarzkopf [Plansee] WVM rod) 
Enclosure 3 to

MDO-723-0044 /

B-MT(SRME)-52

Page 6

\section{Reference}

1. B.P. Bewlay, C.L. Briant, and M.L. Murray, "Molybdenum-Tungsten Interdiffusion and Influence on Potassium Bubbles in Tungsten Lamp Wire", Metallurgical and Materials Transactions, vol 29A, (Dec. 1998) page 2933.

2. J.L. Walter and C.L. Briant, "Microstructure and Properties of Doped Tungsten Wire for Incandescent Lamps", Tungsten and Tungsten Alloys: Recent Advances, TMS, pp. 187194, 1991. 
Enclosure 3 to

MDO-723-0044 /

B-MT(SRME)-52

Page 7

\section{Attachments}

\section{Attachment 1: Refractory Liner Material Ordering Requirements}

Attachment 1.1 Rhenium Requirements

Attachment 1.2 Tungsten-25\% Rhenium Requirements Attachment 1.3 Tungsten (Potassium Doped) Requirements

Attachment 2: Refractory Liner Materials Machining Requirements

Attachment 2.1 Rhenium Specimen Requirements Attachment 2.2 Tungsten 25 Rhenium Specimen Requirements Attachment 2.3 Tungsten (Potassium Doped) Specimen Requirements

Attachment 3: Refractory Metal Liner Material Details (Chemistry, Order Info, Metallography)

Enclosure 3 to MDO-723-0044 / B-MT(SRME)-52 
Attachment 1.1 to Enclosure 3 to MDO-723-0044 B-MT(SRME)-52

Page 1

Attachment 1.1

Rhenium Requirements

Enclosure 3 to MDO-723-0044/B-MT(SRME)-52 
Attachment 1.1 to

Enclosure 3 to

MDO-723-0044

B-MT(SRME)-52

Page 2

\title{
Rhenium Sheet Requirements
}

\author{
Line Item \\ $10.030^{\prime \prime}$ thick rhenium sheet $+0.005 /-0.000^{\prime \prime}, 4.00^{\prime \prime} \times 6.00^{\prime \prime}+0.06 /-0.00^{\prime \prime}$ \\ $20.060^{\prime \prime}$ thick rhenium sheet $+0.010 /-0.000^{\prime \prime}, 4.00^{\prime \prime} \times 7.00^{\prime \prime}+0.06 / 0.00$ " \\ 3. $0.118 "$ thick rhenium sheet $+.010 /-.000^{\prime \prime}, 2.00^{\prime \prime} \times 4.00 "+0.06 / 0.00 "$
}

Attachment to Material Request for Rhenium Sheets

The Seller shall supply the minimum quantities of sheet material in the hydrogen annealed condition. The material shall all be from the same lot of material/powder and the rolling direction shall be aligned with the sheets longest dimension. No cross rolling allowed. The material shall be $99.97 \% \mathrm{Re}$ with a maximum Oxygen content of $50 \mathrm{ppm}$ and a total all other element content to be no greater than 250 ppm. Density shall be $99.9 \%$ of theoretical density $(21.04 \mathrm{~g} / \mathrm{cc})$. Flatness for the sheets shall be a maximum of $4 \%\left[(H / L)^{\star} 100\right.$, as defined per para 6.4 , ASTM B760-86]. The Seller shall supply a full chemical analysis using glow discharge mass spectrometry (GDMS) of each sheet at the final nominal thickness and final heat treat condition with a minimum element list of $\mathrm{Ag}, \mathrm{Al}, \mathrm{As}, \mathrm{B}, \mathrm{Ba}, \mathrm{Be}, \mathrm{Bi}, \mathrm{C}, \mathrm{Ca}$, $\mathrm{Cd}, \mathrm{Co}, \mathrm{Cr}, \mathrm{Cu}, \mathrm{Fe}, \mathrm{Ge}, \mathrm{H}, \mathrm{In}, \mathrm{K}, \mathrm{Li}, \mathrm{Mg}, \mathrm{Mn}, \mathrm{Mo}, \mathrm{N}, \mathrm{Na}, \mathrm{Nb}, \mathrm{Ni}, \mathrm{O}, \mathrm{P}, \mathrm{Pb}, \mathrm{Sb}, \mathrm{Si}, \mathrm{Sn}, \mathrm{Sr}, \mathrm{Ti}, \mathrm{V}, \mathrm{W}, \mathrm{Zn}$, and $\mathrm{Zr}$. The analysis shall also indicate the total of all other elements (TAO). The $0.030^{\prime \prime}$ thick material shall have a maximum ASTM grain size of 4.0 and the maximum grain size on the 0.060 " thick and $0.118^{n}$ thick material shall be ASTM grain size 4.5. Grain size measurement shall be per ASTM E112-96.

\section{Summary}

- All material shall be from the same material/powder lot.

- Hydrogen annealed condition.

- Rolling direction shall be in the sheet's longest dimension.

- No cross rolling allowed.

- $99.97 \%$ Re with maximum 50 ppm Oxygen and all other elements maximum 250 ppm.

- $99.9 \%$ dense.

- Flatness $4 \%$ (ASTM B760-86, para 6.4).

- Full chemistry using Glow Discharge Mass Spectrometry (GDMS) on each final sheet.

- Maximum ASTM Grain Size 4.0 for 0.030" thick sheet and ASTM Grain size 4.5 for $0.060^{\prime \prime}$ and 0.118 " thick material (per ASTM E112-96) 
Attachment 1.2 to

Enclosure 3 to MDO-723-0044

B-MT(SRME)-52

Page 1

Attachment 1.2

Tungsten-25\% Rhenium Requirements

Enclosure 3 to MDO-723-0044/B-MT(SRME)-52 
Attachment 1.2 to

Enclosure 3 to

MDO-723-0044

B-MT(SRME)-52

Page 2

\title{
Tungsten 25\% Rhenium Sheet and Rod Requirements
}

\author{
Tungsten $25 \%$ Rhenium (W25Re) Sheet \\ W25Re sheet $0.075^{n}$ thick $+/-10 \%, 4^{\prime \prime} \times 8^{n}+0.06^{\prime \prime} /-0.00$ (two pieces) \\ Material shall be in the stress relieved condition and shall be clean, free of oxide and scale. Actual \\ chemistry of the final size/heat treatment shall be provided. All material shall be from the same lot \\ (WR-1466). Flatness shall be maximum of $4 \%$ per ASTM B760-86 paragraph 6.4 .
}

Tungsten $25 \%$ Rhenium (W25Re) Rod (Order Cancelled)

W25Re rod $0.5^{\prime \prime}$ diameter $+0.010^{\prime \prime} /-0.000,48$ inches long, minimum length if supplied as multiples pieces is 12 ".

Material shall be supplied from one material lot ( $99.95 \%$ pure W25Re) and shall be HIP'ed and swaged (99.9\% theoretical density). Final material condition shall be stress relieved with a ground surface. A full chemistry analysis of the final rod/final heat treatment shall be supplied. Final material shall be inspected for cracks/defects using dye penetrant testing. 
Attachment 1.3 to

Enclosure 3 to MDO-723-0044

B-MT(SRME)-52

Page 1

Attachment 1.3

Tungsten (Potassium Doped) Requirements

Enclosure 3 to MDO-723-0044/B-MT(SRME)-52 
Attachment 1.3 to

Enclosure 3 to

MDO-723-0044

B-MT(SRME)-52

Tungsten (Potassium Doped) Requirements

Page 2

Tungsten Rod (Potassium Doped)

WM Tungsten Rod $0.5^{\prime \prime}$ nominal diameter ( $\left.+/-0.005^{\prime \prime}\right) 96$ inches long, minimum length if supplied as multiples pieces is $12^{n}$ and the tolerance on the total length supplied is $1^{n}$. Material shall be fabricated per Plansee WWM rod specification supplied below. 


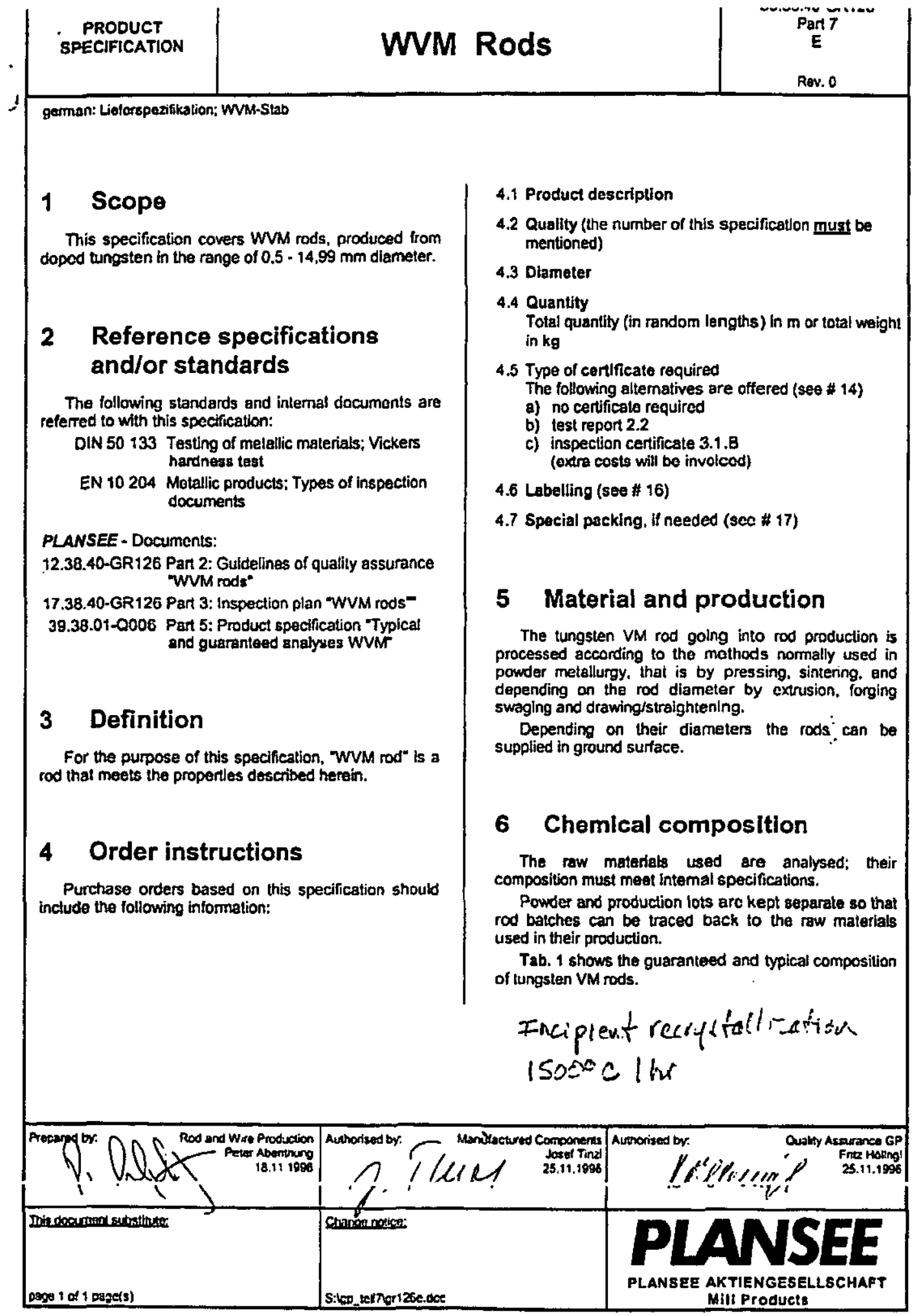


Attachment 1.3 to

Enclosure 3 to

MDO-723-0044

B-MT(SRME)-52

Page 4

Tab. 1: Guaranteed and typleal enalyses values

\begin{tabular}{|c|c|c|}
\hline Element & $\begin{array}{c}\text { Guarantood analyses } \\
\text { max. } \\
\text { [uglg] }\end{array}$ & $\begin{array}{c}\text { Typlesl enslyses } \\
\text { [ugg] }\end{array}$ \\
\hline $\begin{array}{l}\text { Ag } \\
\text { As } \\
\text { Ba }\end{array}$ & $\begin{array}{r}5 \\
5 \\
10 \\
\end{array}$ & $\begin{array}{l}<5 \\
<1 \\
<2 \\
\end{array}$ \\
\hline $\begin{array}{l}\mathrm{C} \\
\mathrm{Ca} \\
\mathrm{Cd}\end{array}$ & $\begin{array}{l}30 \\
30 \\
10 \\
\end{array}$ & $\begin{array}{r}15 \\
5 \\
<1 \\
\end{array}$ \\
\hline $\begin{array}{l}\mathrm{Co} \\
\mathrm{Cr} \\
\mathrm{Cu}\end{array}$ & $\begin{array}{l}30 \\
10 \\
20 \\
\end{array}$ & $\begin{array}{r}5 \\
<10 \\
5 \\
\end{array}$ \\
\hline $\begin{array}{l}\text { Fe } \\
\mathrm{H} \\
\mathrm{Mg}\end{array}$ & $\begin{array}{r}50 \\
5 \\
5 \\
\end{array}$ & $\begin{array}{r}20 \\
<1 \\
<5 \\
\end{array}$ \\
\hline $\begin{array}{l}\text { Mn } \\
\text { Mo } \\
\mathrm{N}\end{array}$ & $\begin{array}{r}5 \\
100 \\
10 \\
\end{array}$ & $\begin{array}{r}<5 \\
<20 \\
<5\end{array}$ \\
\hline $\begin{array}{l}\mathrm{Na} \\
\mathrm{Nb} \\
\mathrm{Ni}\end{array}$ & $\begin{array}{l}10 \\
10 \\
20 \\
\end{array}$ & $\begin{array}{r}<5 \\
<5 \\
5 \\
\end{array}$ \\
\hline $\begin{array}{l}\mathrm{P} \\
\mathrm{Pb} \\
\mathrm{S} \\
\end{array}$ & $\begin{array}{r}50 \\
10 \\
5 \\
\end{array}$ & $\begin{array}{r}20 \\
<5 \\
\times 2 \\
\end{array}$ \\
\hline $\begin{array}{l}T a \\
T \\
Z n\end{array}$ & $\begin{array}{l}20 \\
10 \\
10 \\
\end{array}$ & $\begin{array}{l}<5 \\
<5 \\
<5 \\
\end{array}$ \\
\hline$z$ & 20 & 65 \\
\hline
\end{tabular}

\begin{tabular}{|c|c|c|}
\hline$A$ & $\max .20$ & 10 \\
\hline$K$ & $30-70$ & 50 \\
\hline $5 i$ & $\max .30$ & 5 \\
\hline 0 & $\max .90$ & 10 \\
\hline$W$ & min. $99,95 \%$ & $99,98 \%$ \\
\hline
\end{tabular}

7 Physical and mechanical properties

\subsection{Density}

WVM rods have a guaranteed densily of: For diameters of $0,50-14,99 \mathrm{~mm}$
$0,50-6,00 \mathrm{~mm}=\min .19,20 \mathrm{~g} / \mathrm{cm}^{3}$

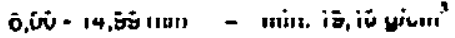

\subsection{Hardness}

Tab. 2: Hardness

\begin{tabular}{|c|c|}
\hline $\begin{array}{c}\text { Dlameter } \\
{[\mathrm{mm}]}\end{array}$ & $\begin{array}{c}\text { Hardnass } \\
\text { min. } \\
H \mathrm{~V}\end{array}$ \\
\hline $0.5-2,99$ & - \\
$3,0-9.99$ & $420 \cdot 500$ \\
$10.0-14.99$ & $420 \cdot 400$ \\
\hline
\end{tabular}

\section{Heat treatment}

The rods are delivered in stregs relieved condition.

\section{Dimensions and tolerances}

\subsection{Diameier}

Tab. 3: Diameter and

guarantoed production tolerance

\begin{tabular}{|c|c|}
\hline $\begin{array}{c}\text { Diameter } \\
{[\mathrm{mm}]}\end{array}$ & $\begin{array}{c}\text { Diemeter tolerence } \\
{[\mathrm{mm}]}\end{array}$ \\
\hline $0,50-1,00$ & $\begin{array}{c}\text { Qualtit } \\
\text { finc ground }\end{array}$ \\
\hline $1,01-3,00$ & $\pm 0,005$ \\
$3,01-6,00$ & $\pm 0,010$ \\
$6,01-14,99$ & $\pm 0,015$ \\
\end{tabular}

\subsection{Production lengths}

Tab. 4: Production length and

guaranteed length tolerance.

\begin{tabular}{|c|c|c|}
\hline $\begin{array}{c}\text { Diamotor } \\
{[\mathrm{mm}]}\end{array}$ & Production tength & $\begin{array}{c}\text { Tolerance } \\
\text { per } \mathrm{m} \\
{[\mathrm{mm}]}\end{array}$ \\
\hline $0.50 \cdot 1,00$ & $500-1000$ & $\pm 1,2$ \\
$1,01-4,00$ & $1000-3000$ & $\pm 1,2$ \\
$4,01-8,00$ & $1000-2500$ & \pm 1.2 \\
$6.01-10,00$ & $1000-2000$ & $\pm 1,2$ \\
$10.01-14,99$ & $500-1500$ & \pm 1.2 \\
\hline
\end{tabular}

Greater lengths upon request

\subsection{Straightness}

Tab. 5: Stralghtness measured par $m$

\begin{tabular}{|c|c|}
\hline Dlameter & $\begin{array}{c}\text { Stratghtnes: } \\
\text { per m } \\
\text { [mm] }\end{array}$ \\
{$[$ [mm] } & $<2,5$ \\
$0,5 \cdot 1,00$ & $<2,0$ \\
\hline $1,00 \cdot 14,99$ & \\
\hline
\end{tabular}

10 Quality, surface condition and appearance 
The rods are free of kinks, overlaps, splits and cracks which wuld be a disadvantage in further processing.

Depending on their oliameters the rods can be supplied in a ground surfaca.

\section{1 "Ground" surface}

For diameters of $0,50=14,99 \mathrm{~mm}$

The rods can also be offered with a centeriess circulor ground surface.

\subsubsection{Roughness}

$R_{1}=\max .1 \mu \mathrm{m}$

\section{Sampling}

\subsection{Non destructive test}

\subsubsection{Eddy current test}

Eddy current tests are made on ground WVM rods for diamelers of 0,50 - $14,99 \mathrm{~mm}$.

Details of test methods, conditions and frequency can be found in the internal documents 12.38.40-GR126 Pan 2 and $17,38,40-$ GR126 Part 3.

\subsubsection{Uitrasonic testing}

Ultrasonic testing is made accorting to MIL-STD. 2154 for diameters of $>12 \mathrm{~mm}$

\section{Methods of chemical analyses}

Rendom chemical analyses of the individual production batches are carried oul according to internal quality control procedures and insinuctions. Details of these analyses methods can be made known upon request.

\section{Claims}

A claim is warranted should the customer find a deviation from the guaranteed quality at the time of the incoming quality control.

Should cause for a claim arise, the customer is requested to notify PLANSEE in writing, stating the reason for this claim.

It is also requested that the technical control data of the incoming quality control be mede avaliable to PLANSEE together with the defective WVM rods.

Clatms based on limit values or samples not yet established cen only be handles after mutual agreement

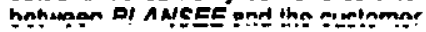

Amendments to the quality requirements will only be valid from the time of witten notification received by the contraclual patner.

\section{Certification}

Upon request the following certificates according to EN 10204 can be supplied

\section{1 "Test report 2.2"}

PLANSEE confirms with this test repon that the WVM rods supplied meet the present specification and gives details of the malerial properties according to ongoing production surveiliance, not directly related to the particular production lot.

This test report includes the following data:

Order no.

Lot no.

Rod diameter

Rod length

Type of surface

Total weight

Number of rods

Guaranteed chemical analyses

Guaranteed physical and mechanical properties

\section{2 "Inspection certificate 3.1. B"}

A works inspector from PLANSEE confirms with this inspection centificate that the supplies WVM rods meet this present specification and gives test results related to the particular production lot.

This test report includos the following data:

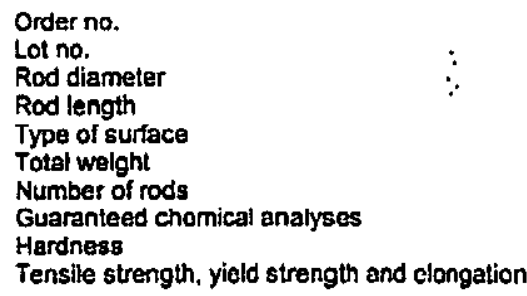

\section{Packaglng}

Rods up to $6 \mathrm{~mm}$ diameter will be defivered in bundles, larger sizes will be individually packed.

The cods with be packed so as to avaid mechanical damage, moisture, oxidation and other sources of contamination during transport and handling.

Should the material nevertheless be damaged in transport a claim is to be lodged with the forwarder within a reasonzble time lepse.

\section{Labelling}

A label will be enclosed with each package showing the following informatlon:

Producer's name: PLANSEE PLANSEE Order no. 


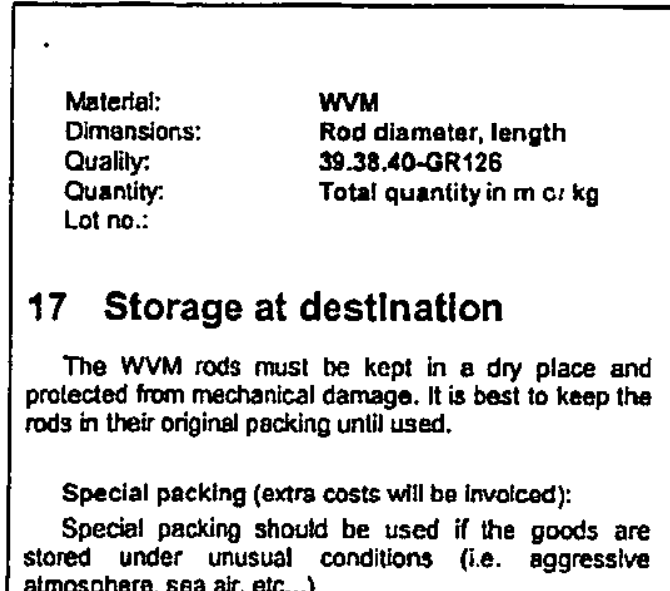
atmosphere, sea air, etc...)

\section{Appendix A}

Rulet for the administration of this spectication.

\section{Doeumentation}

This produc specifieation is bound by the rutes of documestarion. Should smendmente be made to it, o copy of the obsolece version is to be kept for at leas 5 years with the quality marngement department. A sorting out can then only be done after re-examination by the head of the department.

\section{Ameadment service}

Amendments to this specification can only be carried out by the quality management departmenc.

\section{Distribution}

Distribution of this document is made tpon demond and not repistered. 
Attachment 2.1 to

Enclosure 3 to

MDO-723-0044

B-MT(SRME)-52

Page 1

Attachment 2.1

Rhenium Specimen Requirements

Enclosure 3 to MDO-723-0044/B-MT(SRME)-52

PRE-DECISIONAL - For Planning and Discussion Purposes Only 
Attachment 2.1 to

Enclosure 3 to

MDO-723-0044

B-MT(SRME)-52

Page 2

\title{
Rhenium Test Specimens Machining Details \\ $\operatorname{Rev} 1$ \\ $8 / 15 / 05$
}

\begin{abstract}
1). Supply 30 fracture toughness specimens per drawing 5D 16339
The width, nominal $0.118^{\prime \prime}$, and height, nominal 0.118 ", dimensions shall be reported for each specimen and supplied with the parts. The dimensions shall be traceable to the individual specimen by identification on the specimen packaging. The specimens shall be laid out with the long axis of the specimen parallel with the long dimension of the sheet supplied.
\end{abstract}

2). Supply 50 tensile specimens per drawing $5 D 16338$

The gage section width, nominal $0.060^{\prime \prime}$, and gage section thickness, nominal 0.030 ", dimensions shall be reported for each specimen and supplied with the parts. The dimensions shall be traceable to the individual specimen by identification on the specimen packaging. The specimens shall be laid out with the long axis of the specimen parallel with the long dimension of the sheet supplied.

3). Supply 50 density disk specimens per drawing $5 \mathrm{D} 16357$

The diameter, nominal $0.492^{\prime \prime}$, and thickness, nominal $0.059^{\prime \prime}$, dimensions shall be reported for each specimen and supplied with the parts. The dimensions shall be traceable to the individual specimen by identification on the specimen packaging.

The specimens shall be machined from the appropriate sheet thickness for the specimen $\left(0.030^{\prime \prime}\right.$, $0.060^{\prime \prime}$, and $0.118^{\prime \prime}$ nominal thicknesses) supplied as GFM by KAPL. The specimens shall be EDM cut out and a minimum of $0.005^{\prime \prime}$ per surface shall be removed by grinding or conventional machining to eliminate any EDM recast layer. The specimens shall be cut out/laid out of the material to minimize material usage. No engraving or laser marking is required on the specimen.

\section{Vendors}

Sylhan LLC

Low Stress Grind

Rhenium Alloys

Rembar

HC Starck (Latrobe, PA)

Vangura Tool

Metal Samples (Alabama) 2563584202

Kin-Tech Manufacturing (PA) 
Attachment 2.2 to

Enclosure 3 to

MDO-723-0044

B-MT(SRME)-52

Page 1

Attachment 2.2

Tungsten 25 Rhenium Specimen Requirements

Enclosure 3 to MDO-723-0044/B-MT(SRME)-52

PRE-DECISIONAL - For Planning and Discussion Purposes Only 
Attachment 2.2 to

Enclosure 3 to

MDO-723-0044

B-MT(SRME)-52

\section{Tungsten Rhenium Alloy Test Specimens Machining Details Rev - $7 / 14 / 05$}

Page 2

1). Supply 30 fracture toughness specimens per drawing $5 D 16339$

The width, nominal $0.118^{\prime \prime}$, and height, nominal $0.118^{n}$, dimensions shall be reported for each specimen and supplied with the parts. The dimensions shall be traceable to the individual specimen by identification on the specimen packaging.

2). Supply 50 tensile specimens per drawing 5D16338

The gage section width, nominal $0.060^{\prime \prime}$, and gage section thickness, nominal $0.030^{\prime \prime}$, dimensions shall be reported for each specimen and supplied with the parts. The dimensions shall be traceable to the individual specimen by identification on the specimen packaging.

3). Supply 50 density disk specimens per drawing 5016357

The diameter, nominal $0.492^{\prime \prime}$, and thickness, nominal 0.059 ", dimensions shall be reported for each specimen and supplied with the parts. The dimensions shall be traceable to the individual specimen by identification on the specimen packaging.

The specimens shall be machined from nominal $0.5^{\prime \prime}$ rod supplied as GFM by KAPL. The specimens shall be EDM cut out and a minimum of $0.005^{\prime \prime}$ per surface shall be removed by grinding or conventional machining to eliminate any EDM recast layer. The specimens shall be cut out/aid out of the material to minimize material usage (i.e. 4 fracture toughness specimens will fit into the cross section of the $0.5^{\prime \prime}$ diameter rod). No engraving or laser marking is required on the specimen.

Vendors

Sylhan LLC

Low Stress Grind

Rhenium Alloys

Rembar

HC Starck (Latrobe, PA)

Vangura Tool

Metal Samples (Alabama) 2563584202

Kin-Tech Manufacturing (PA) 
Attachment 2.3 to

Enclosure 3 to

MDO-723-0044

B-MT(SRME)-52

Page 1

Attachment 2.3

Tungsten (Potassium Doped) Specimen Requirements

Enclosure 3 to MDO-723-0044/B-MT(SRME)-52

PRE-DECISIONAL - For Planning and Discussion Purposes Only 
Attachment 2.3 to

Enclosure 3 to

MDO-723-0044

B-MT(SRME)-52

Page 2

\section{Potassium Doped Tungsten Test Specimens Machining Details Rev1 $8 / 1 / 05$}

1). Supply 30 fracture toughness specimens per drawing 5D16339

The width, nominal $0.118^{n}$, and height, nominal $0.118^{\prime \prime}$, dimensions shall be reported for each specimen and supplied with the parts. The dimensions shall be traceable to the individual specimen by identification on the specimen packaging

2). Supply 50 tensile specimens per drawing 5D16338 The gage section width, nominal $0.060^{\prime \prime}$, and gage section thickness, nominal $0.030^{\prime \prime}$, dimensions shall be reported for each specimen and supplied with the parts. The dimensions shall be traceable to the individual specimen by identification on the specimen packaging.

3). Supply 50 density disk specimens per drawing 5D16357

The diameter, nominal $0.492^{\prime \prime}$, and thickness, nominal $0.059^{\prime \prime}$, dimensions shall be reported for each specimen and supplied with the parts. The dimensions shall be traceable to the individual specimen by identification on the specimen packaging.

The specimens shall be machined from $\sim 0.5^{\prime \prime}$ diameter rod supplied as GFM by KAPL. The specimens shall be EDM cut out and a minimum of $0.005^{\prime \prime}$ per surface shall be removed by grinding or conventional machining to eliminate any EDM recast layer. The specimens shall be cut out/laid out of the material to minimize material usage (i.e. 4 fracture toughness specimens will fit into the cross section of the 0.5 " diameter rod). No engraving or laser marking is required on the specimen.

\section{Vendors}

Sylhan LLC

Low Stress Grind

Rhenium Alloys

Rembar

HC Starck (Latrobe, PA)

Vangura Tool

Metal Samples (Alabama) 2563584202 
Attachment 3 to

Enclosure 3 to

MDO-723-0044

B-MT(SRME)-52

Page 1

\section{Attachment 3}

Refractory Metal Liner Material Details

(Chemistry, Order Info, Metallography)

Enclosure 3 to MDO-723-0044/B-MT(SRME)-52 
Attachment 3 to

Enclosure 3 to

MDO-723-0044

B-MT(SRME)-52

Page 2

\section{PACKING SLIP}

\section{R RHENIUM ALLOYS, INC.}

Rhenium Alloys, Inc.

P.O. BOX 245

1329 TAYLOR ST

ELYRLA OH $\quad 4036-0245$

Phane: $40-365-7388$ * 1-898-743-6486 * Fax: 440-368-5831

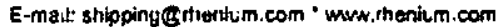

BIII To:

KAPL INC

ACCOUNTS PAYABLE

PO BOX 1072

SCHENECTADY NY 12301-1072

USA

\begin{tabular}{|l|l|}
\hline Packing Sllp & 0013826 \\
\hline Date & $11 / 4 / 2005$ \\
\hline Pagh & 1 \\
\hline
\end{tabular}

Rhenium Sheet Order Information

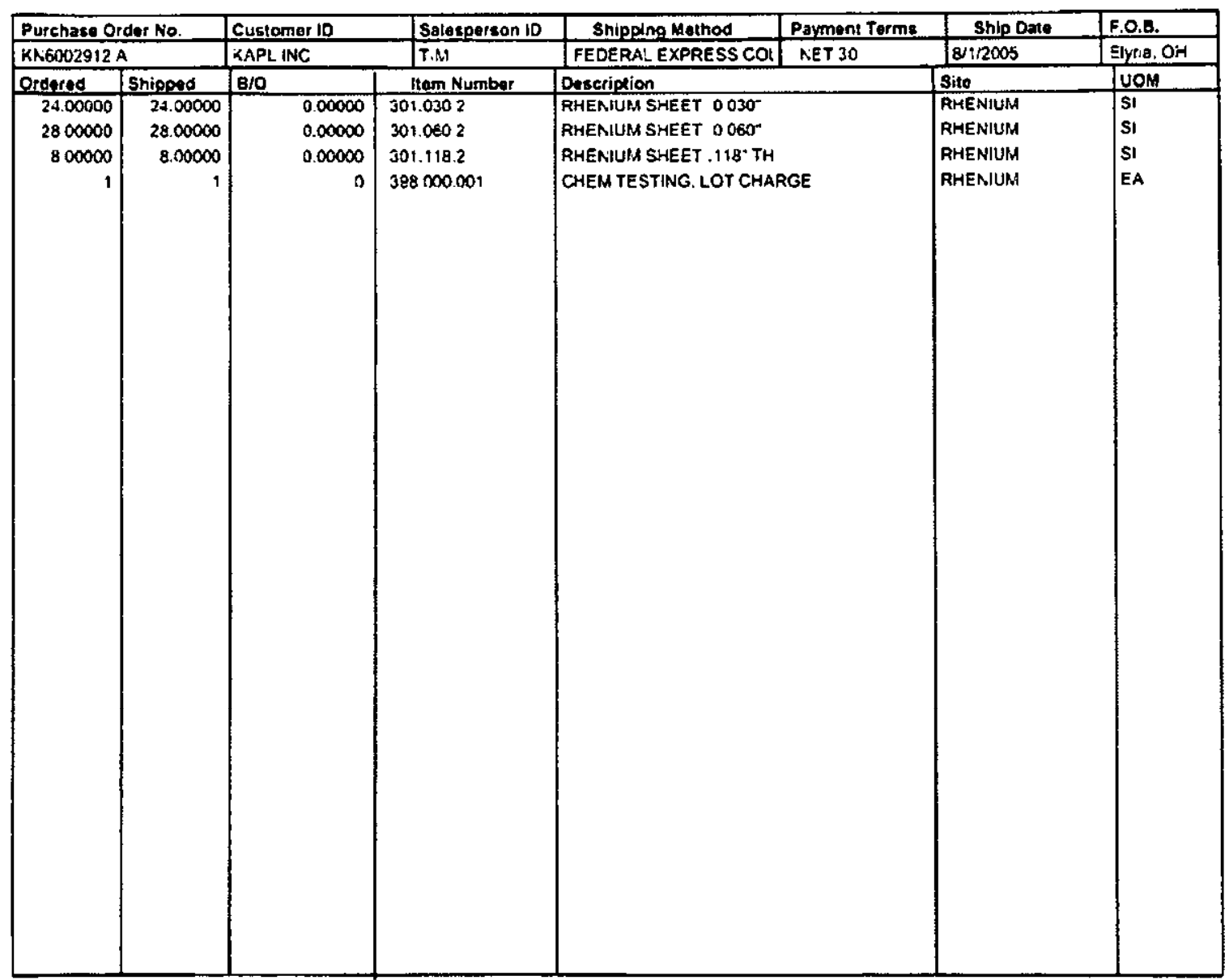

CERTIFICATION INCLUDED START POWDER AMALYSIS

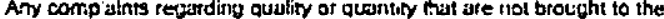
attention ol Renenisth Alloys. Ine. Within 72 hours after shippong will not be accefted. Retum Matorial Authorization Requirad 
Attachment 3 to

Enclosure 3 to

MDO-723-0044

B-MT(SRME)-52

Page 3

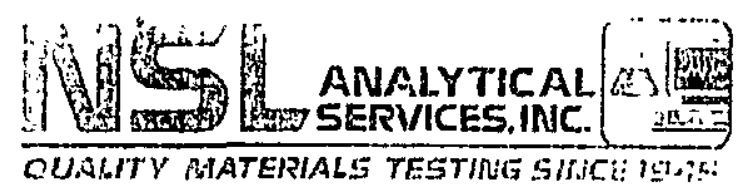

Rhenium Alloy, linc.

P. 0. Box 245

El'ria, Olin 440313

Alit:Fiank Danck

\section{Rhenium Sheet Original Starting Powder Chemistry}

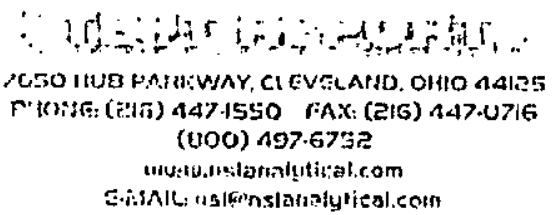

P.O. No: 7100

Page 1 oi 1

Clleue Descrintion: Rhoniun Powder

NSL Lab No Sample 10

010002500 Lot $12-1369,-200 H G S$

Test

Spec

Rezults/units

Impurilies

Elements looked lor and lout! to se <16pan

E, K. Ni, Na. Mo, F, Si, W

Elements looked for and tound to be $<5$

Al. As, Ba, Ba, Bi, Ca, Cd. Co, Cr. Cs, Cu, Ga, in, Li, Mg, Mn, Po. Rb, Se, Ag, St, Ti, V. U, Zn, B, Ge, Nv. Ta. Ti, Zr, Alt, Ir, Pd. Pt, Rh, Ru, Sil. Ie. Ht, sb, Th

$\mathrm{Re}=$ lawjor

$\therefore$

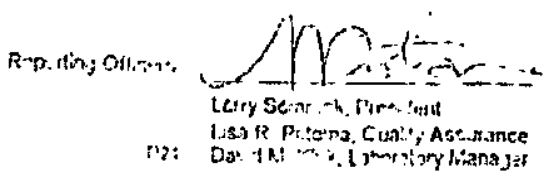




\section{MATERIAL CERTIFICATION}

DATE: October 31. 2005

SOI.D TO: Knolls Atonic Power Latoratory.

P.O. BOA 1072

Sthenectudy. NY 12301-1072

PURCHASE

ORDER:

$\mathrm{KN} 6002912 \mathrm{~A}$

LINE ITEM: $: ; 3$

\section{MATERIAL: Rhenium shecl}

This is a shipment against your order $\# \mathrm{KN} 6002912 \mathrm{~A}$ which completes the requirement for rhenium shect. The thenium. $1 \mathrm{~g} "$ shee mets the requirements of the purchase order and the attachunent specitication. "This material was manufuctured in compliance with KAPL's quality assuranee specification KIBPQAR-1 daced March 2004 and the revisted amendment for thickness.

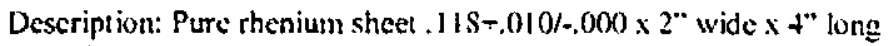
Quantily: 1

Lot number R-1499

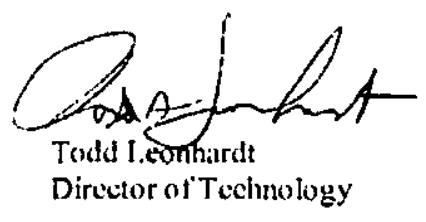

Rhtorium flllogs, Inc 
Attachment 3 to

Enclosure 3 to

MDO-723-0044

B-MT(SRME)-52

Page 5

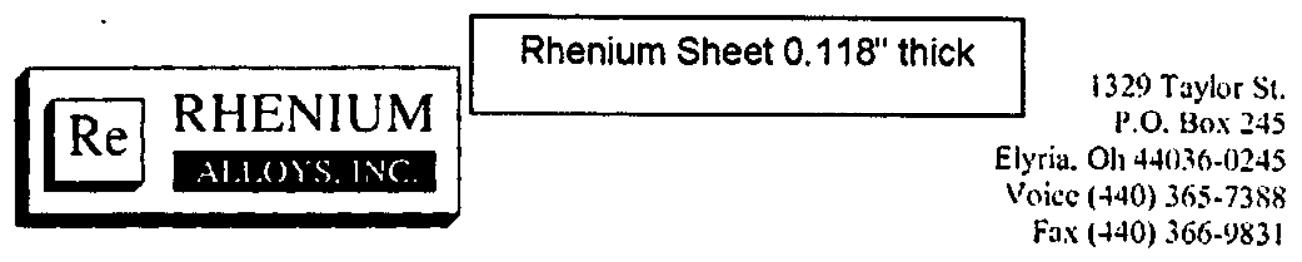

Repont No. $13-882$

KAPL. Ine

P.O. Box 1072

Schenectady. New York 12301-1072

$10 / 31 / 05$

Subject

Rhenium shect

$4 " \times 2 " x .118$

Subrnitled to Our Laboratory ofl Order Nunber $\mathrm{POH}$ KN6002912A 


\section{Rhenium Sheet $0.118^{\prime \prime}$ thick}

KAPL

REPORT NO B-882

\section{BACKGROUND}

One sample was submitted for testing which is representative of the rhentum sheet identified as Lot numbur R-1499. . I s thenium sheet and compared to the KAPL. purchase order and the attachment specification.

\section{IEST RHOLESTED}

Grain sizc. Density. Dinensionals. Chemical Amalysis, and microscopic exumination.

\section{GRAIN SIZF EXAUINATION}

The submittol specimen was prepared in accordance with ASTM 1:3-95 for grain size examination. it was cxanined at $100 \mathrm{X}$ tor grain size determination. The average grain size was rated in accordanee with the ASTM EII 2-96 comparison method for determining grain size.

The average ASTM grain size is 5.0

\section{DENSITY}

Density is metsured using ASTM B.3!1-93: Test Method for Densily Determination for Powder Mctallurgy (P/M) Malcrials Containing I ess Than Two Petcent Porosity.

- The density of the 118 shee is $99.92 \%$ or $21.02 \mathrm{~g} / \mathrm{ec}$

\section{DI.MENSIONAIS}
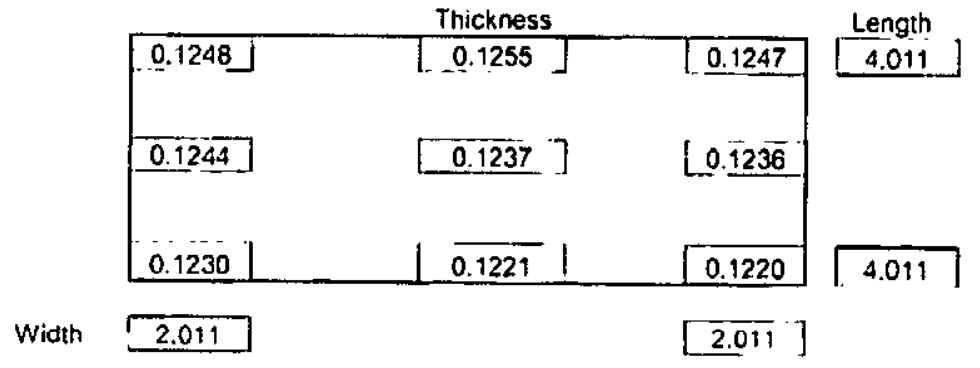

\section{CHFMICAI.ANALYSIS}

See attachment 
Attachment 3 to

Enclosure 3 to

MDO-723-0044

B-MT(SRME)-52

Page 7

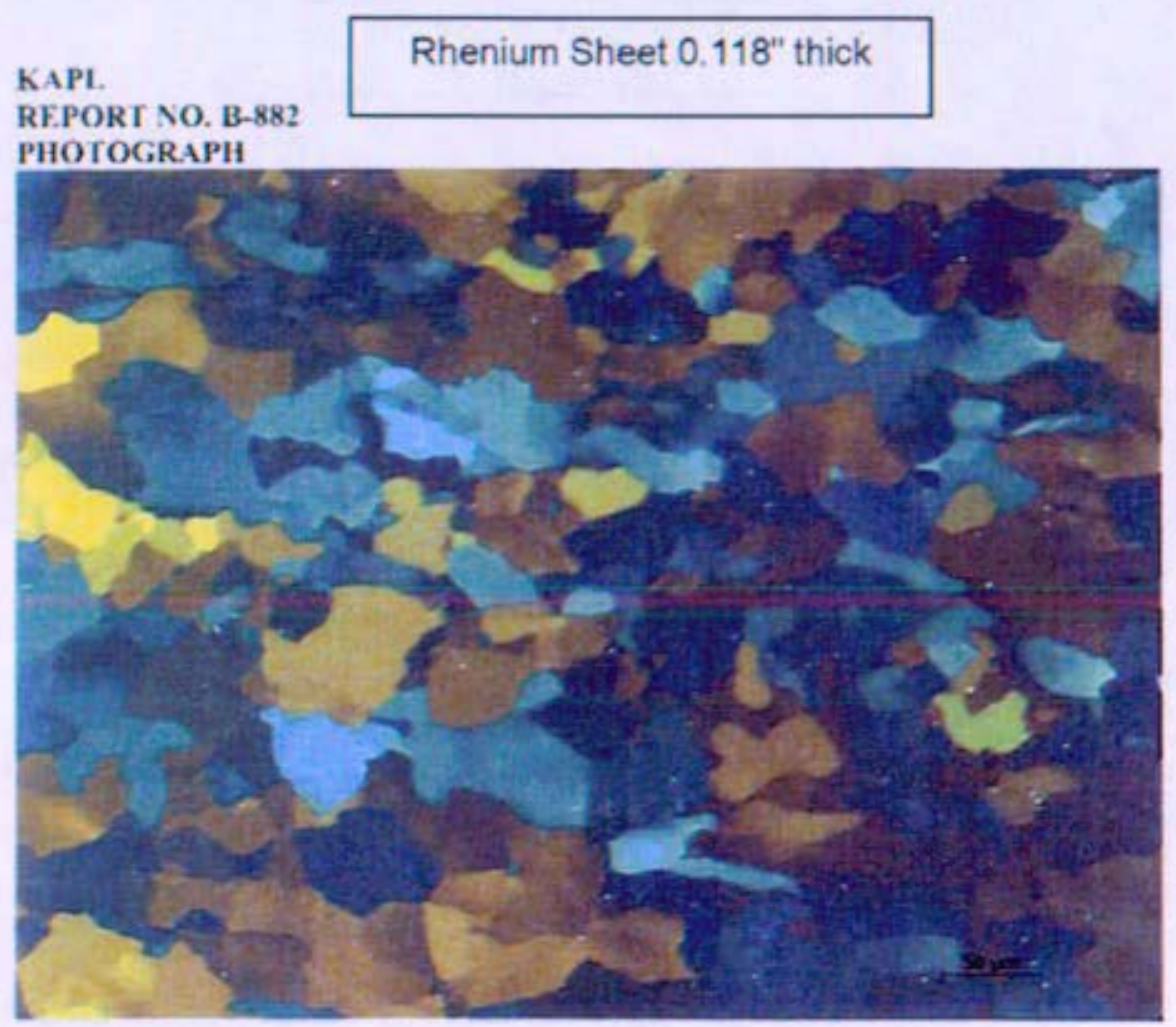

This is a representatuve view of the microstructure from the submitted sample.

Magnification: 260X

Etchant: Modifiod Murakami's Reagent

Polarized Light

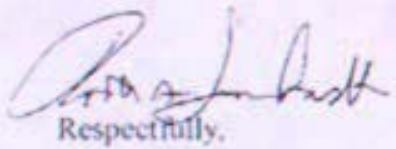

Todd Leonhardt 
Attachment 3 to

Enclosure 3 to

MDO-723-0044

B-MT(SRME)-52

Page 8

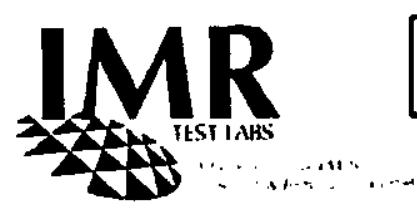

Rhenium Sheet 0.118 " thick

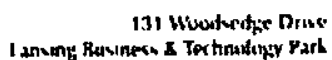

tansing wr iftill

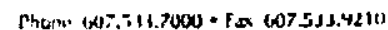

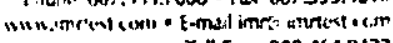
tidl free ano.46-4.11422

Octobuer 23, 2005

Clili Cutiman

CERTIFIED MATERIAL ANALYSIS

Rhonium dilary lac

PO Bor 7.5

Elyr:i, OH $40103-112-45$

USA

IMR Report Number 200509821

PONuntor

13064

1 are Reacived

Octaber 25. 2005

Ioti"

$R=1+99$

Deorition

.115 thich Re Ghest

Sprcification

NiN6002012

\section{SUMMARY}

The sample meets the chemical requirements of KNG002912 as suppliad by Uhe customer. The GDMS purtion of the andlysis was outsourced by IMR

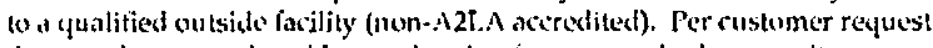
the sumple was analyzed by comlustion tor oxygen, hydrogen, nitrogen and cirbon.

The results are given on the tollowing Fages.

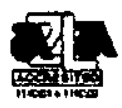

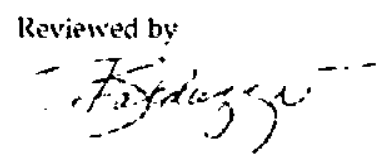

Reviewed by

\section{Hank Bals lucxi Chemist \\ Melissa lirst \\ Quality Assistant \\ Nadcap}

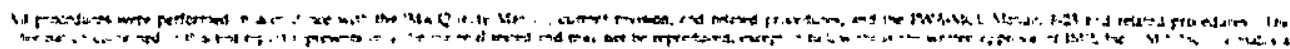

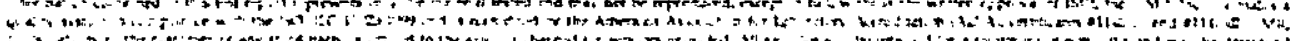

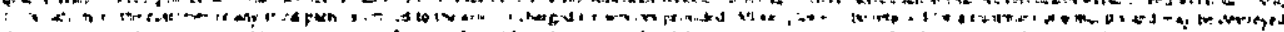

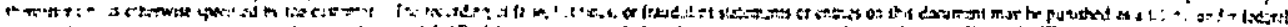

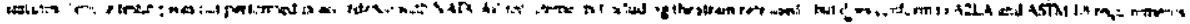

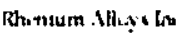

linger 1 of

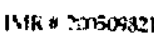


Attachment 3 to

Enclosure 3 to

MDO-723-0044

B-MT(SRME)-52

Page 9

Rhenium Sheet 0.118 " thick

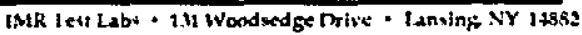

CHEMISTRY

\begin{tabular}{|c|c|c|}
\hline Element & R-I499 & Specifteation \\
\hline $\mathrm{Ri}:$ & $>99,990$ & $90.97 \%$ \\
\hline $\mathrm{Ol}$ & 27 & 50 prm Maximum \\
\hline Or & 40 & 250 ppin Mavimum \\
\hline $\mathrm{Ci}$ & 14 & $=$ \\
\hline $\mathrm{H}^{2}$ & 1.9 & $\cdots$ \\
\hline$x^{2}$ & 1 & $=$ \\
\hline Li & 0.01 & $=$ \\
\hline $\mathrm{Be}$ & $<0.005$ & $=$ \\
\hline 13 & $\leq 0,010$ & $\cdots$ \\
\hline$F$ & $<0.05$ & -- \\
\hline Na & 0.19 & $\cdots$ \\
\hline$M / 8$ & 50.01 & $\ldots$ \\
\hline A! & 0.69 & $\cdots$ \\
\hline $\mathrm{Si}$ & 0.61 & - \\
\hline [ & 0.02 & - \\
\hline 5 & 0.09 & $\cdots$ \\
\hline CI & 0,05 & $=$ \\
\hline$k$ & 0.35 & $+\infty$ \\
\hline Ca & 0.21 & $\cdots$ \\
\hline 5 & $<0.005$ & $\ldots$ \\
\hline$T i$ & 0.09 & $\cdots$ \\
\hline$\underline{V}$ & $<0.005$ & $\cdots$ \\
\hline $\mathrm{Cr}$ & 7.9 & $m$ \\
\hline Wn & 0.39 & $\cdots$ \\
\hline Fe & 10 & 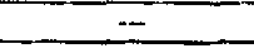 \\
\hline $\mathrm{Co}$ & 0.01 & $\cdots$ \\
\hline $\mathrm{Ni}$ & $\overline{5}$ & $=$ \\
\hline $\mathrm{Cu}$ & 0.1 & $\cdots$ \\
\hline $2 n$ & $<0.05$ & $+\infty$ \\
\hline Ga & $\angle 0.05$ & $=$ \\
\hline $\mathrm{Ge}$ & $<0.05$ & 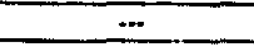 \\
\hline As & $<0.05$ & $\ldots$ \\
\hline Se & $<0.05$ & - \\
\hline Br & $<0.05$ & $\ldots$ \\
\hline $\mathrm{RG}$ & $<0.01$ & $\ldots$ \\
\hline $5 r$ & $<0.01$ & - \\
\hline$Y$ & 50,0015 & $\cdots$ \\
\hline$\angle r$ & 0.03 & - \\
\hline $\mathrm{Nb}$ & 0.02 & $\rightarrow$ \\
\hline Mo & 0.33 & $=$ \\
\hline Ru & $<0.01$ & - \\
\hline $\mathrm{Rh}$ & $<0.0 !$ & $\ldots$ \\
\hline $\mathrm{Pd}$ & $<0.01$ & $\cdots$ \\
\hline
\end{tabular}

Fhothtt a dllews inc

[inge 2a1] 3

iste +20050993

PRE-DECISIONAL - For Planning and Discussion Purposes Only 
Attachment 3 to

Enclosure 3 to

MDO-723-0044

B-MT(SRME)-52

Page 10

Rhenium Sheet $0.118^{\prime \prime}$ thick

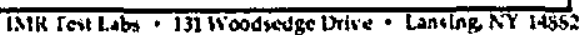

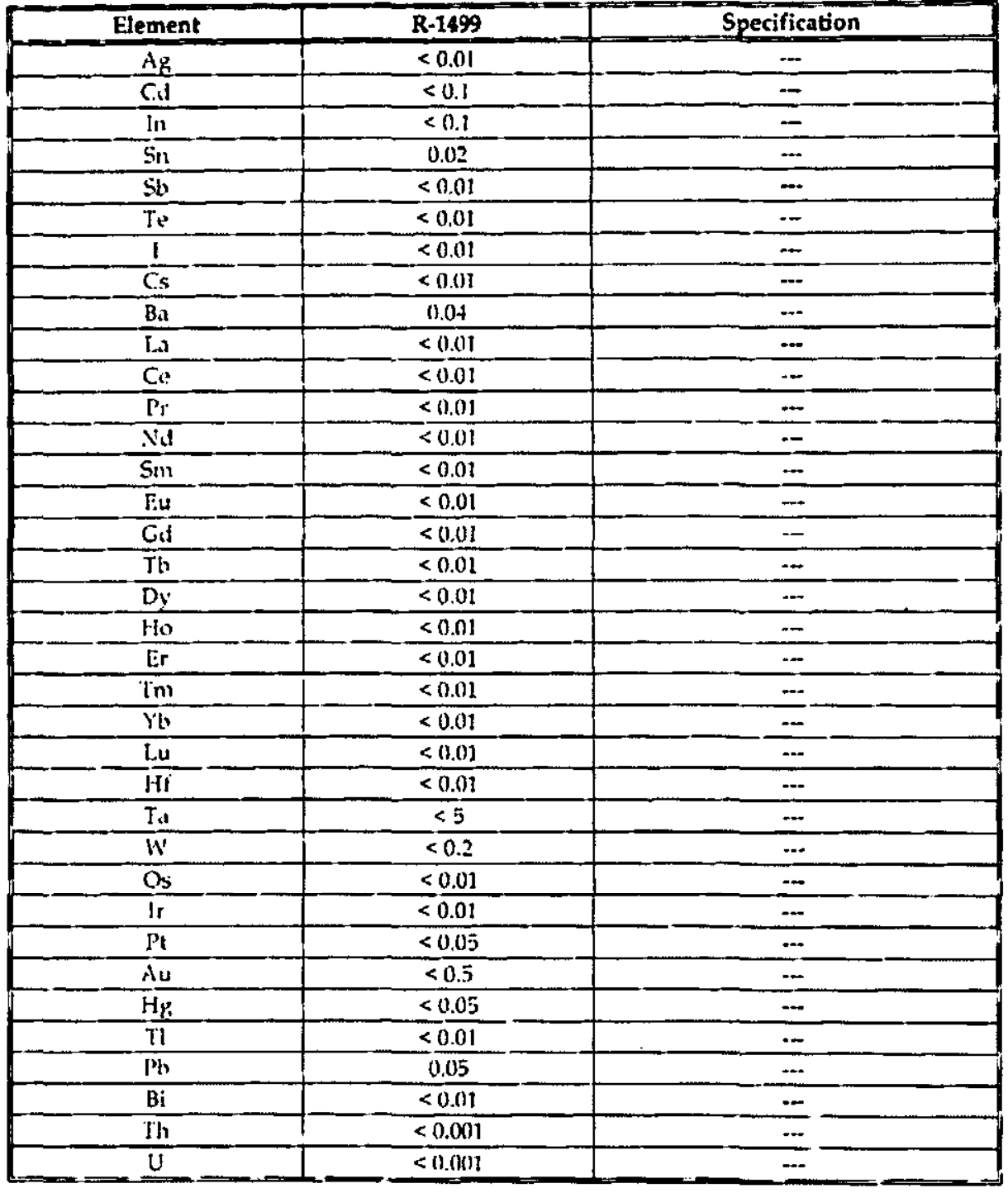

+ Dotermined by combustion-infrated absorbance.

inelermines by inerl gas fusion-thermal conductivity.

Ol' - Other clements, toul.

Unless atherwise indicated, elements were malyzed by GDWIS.

Results in ppm (ppm = parts per nillion $=n y / \mathrm{Kg}): 0.1001 \%$ by $w \mathrm{t}=1000 \mathrm{ppm}$ unless otherwise indicaled.

Nethods: CDNS, ASTM E 1019-03, and CAP-32A. 
Attachment 3 to

Enclosure 3 to

MDO-723-0044

B-MT(SRME)-52

Page 11

Rhenium Sheet 0.030 " thick

1329 Taylot St.

P.O. Box 245

Re RHENIUM

Voice $(+40)$ 365-7388

Far (4t1) 366-9831

\section{MATERIAL CERTIFICATION}

DATE: Octoher 31.2005

SOLD TO: Knolls Alamic Power Laboratory.

P.O. Bnx 1072

Schenectauy. NY 12301-1072

PURCHASE

ORDFR: KN600291?A

LINF ITEW: $\#$

MATERIAL: Rhenium slieet

Ttus is a shipment against jour order $4 K \times 6002912 \mathrm{~A}$ which completes the requirement tor thenium sheet. The rhonium $.030^{\prime \prime}$ sheet meets the requirements of the purchase order and the attactument specification. This material was manu factured in compliance with KAPL's guality assurance specification KBPQAR-1 dated March 2004 and the revised amendment for thickness.

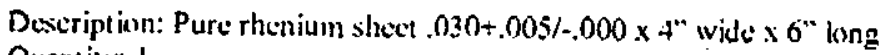

Quantity: I

Lot number R- $\$ 799$

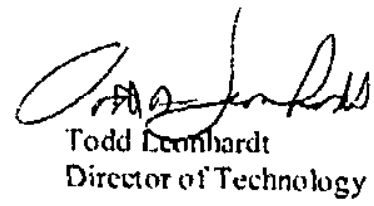

\footnotetext{
Rhonium Alloys, Inc

Yesterday's Pioneer. Today's leader

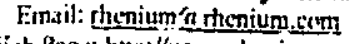

Web Page: hilp://wnw:rhenium.cons
} 
Attachment 3 to

Enclosure 3 to

MD0-723-0044

B-MT(SRME)-52

Page 12

\section{$\operatorname{Re}$ RHENIUM}

A.LOYS. INC.

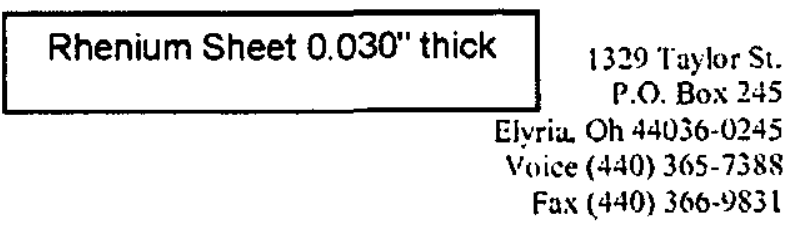

Repon No. B-8so

KAPL, Inc

P.O. Box 1072

Schenectady. New York $12301-1072$

$10 / 31 / 05$

Subject

Rhenium shest

$6^{\prime \prime} \times 4 " \times .030$

Submitted to Our Laboratory on Order Number

POH KNG002912A

Rheniam Alloy. Inc

Ensuil: thenium igrteniurn.conm

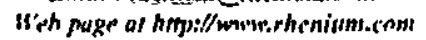

PRE-DECISIONAL - For Planning and Discussion Purposes Only 
Attachment 3 to

Enclosure 3 to

MDO-723-0044

B-MT(SRME)-52

Page 13

\section{Rhenium Sheet 0.030 " thick}

KAPI.

REPORT YO B-880

BACKGROUND

Onc sample was submited for testing which is represcntative of the rhenium shect idemtified as lol number R-1499. 030 thenium sheet and compared to the KAPL. purchasc order and the attachment specitication.

\section{TEST REQLESTED}

Grain sisc, Density, Dimensionals. Chemieal Analysis, and microseopic cxamination.

\section{GRAIN SI7.E EXANIINATION}

The submitced specincen was prepared in accordance with ASIM l:3-95 for grain size examination. It was examined at $100 X$ for grain size deternination. The average grain size was rated in accordunce with the ASTM E $112-96$ comparison method for detemining grain size.

The averase ASTM grain side is 4.0

\section{DEXSITY}

Densily is measured using AST.M B311-93: Tesl Muthod for Density Deterinination for powder Melallurgy (P/M) Materials Containing less Than Two Percent Porosty.

The density of the .030 sheet is $99.97 \%$ or $21,03 \mathrm{~g} / \mathrm{co}$

\section{DIMENSIONALS}

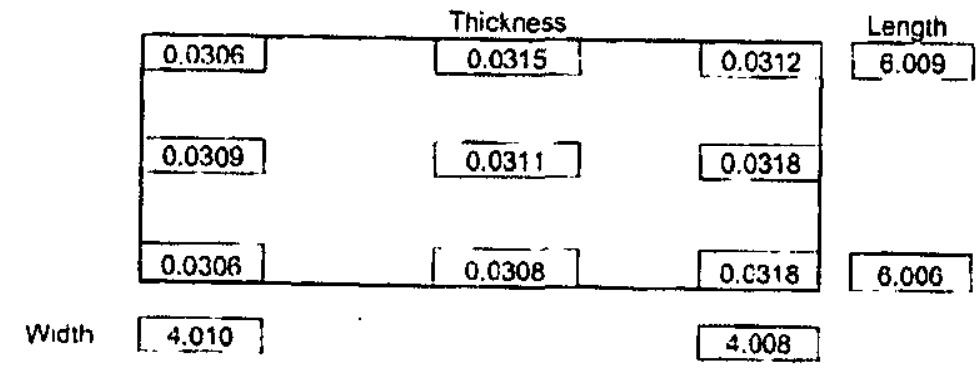

\section{CHE.MICAL, ANALISIS}

See altuchment

Rhe'nium slloys, In

Etsail: thenumiantacrium.com

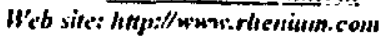


Attachment 3 to

Enclosure 3 to

MDO-723-0044

B-MT(SRME)-52

Page 14

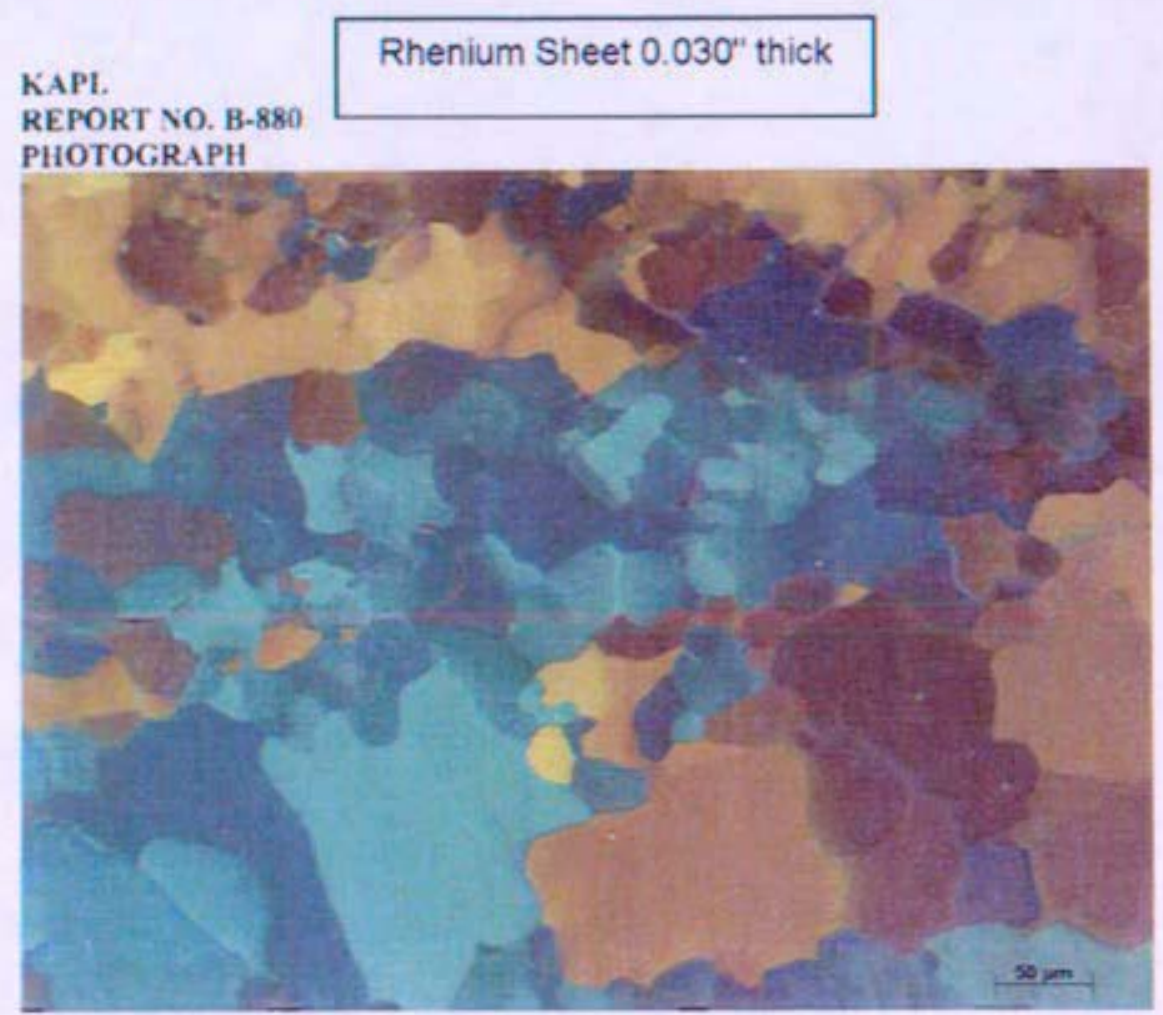

This is a representative view of the mierostructure from the submitted sample.

Magnification: 260X

Etchant: Modified Murakami's Reagent

Polarized Light

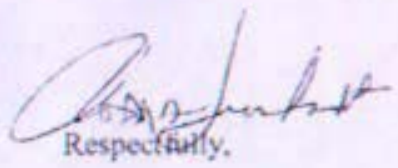

Todd Leonhandt

Rhenium Alloys, Inc

Email: dhanium arhonivan gert

Web sile: hapt/ wow rhenium. enm 


\title{
CERTIFIED MATERIAL ANALYSIS
}

Clifir Cuthman

Rhenium Alloys lme

PO Box $2+5$

Flyra, OH + $+n 36-0345$

USA

\author{
IMR Report Nunaber 200505734-Revision 1 (Corrected sample \\ description per customer request)
}

\section{roNumbor}

$12 \times 56$

\section{SUMMARY}

Itreketint

September 21, 2005

The sample meets the clunical requirentents of KN6002912 as supplied by the customer. The GDMS portion of the analysis was ou tsource! by INR

Iax: to a ywalifiest outside facility (non-A2LA accretited). Per customer request

R.1 199 the sumple was analyzed by combustion for oyygen, hydergen, nitrogen

Dessription and carbon.

.030 Re Sheet

The results are given on the following pages.

Specification

Kivtologl2

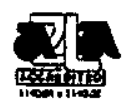

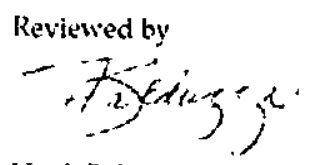

Reviened by,

\section{Hank Balduzzi \\ Chemist \\ Melissa First \\ Quality Assistant \\ Faidcap}

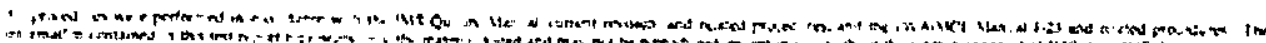

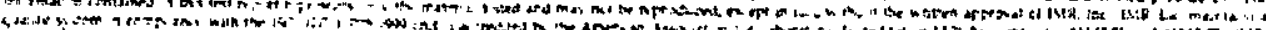

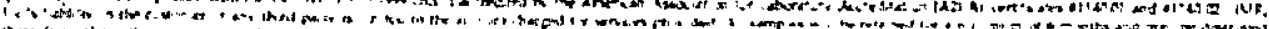

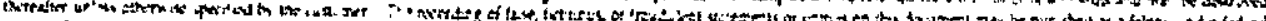

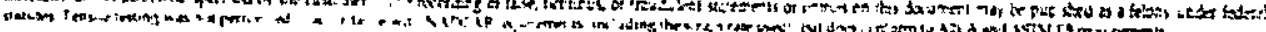

Riteraium Allows ine 
Attachment 3 to

Enclosure 3 to

MDO-723-0044

B-MT(SRME)-52

Page 16

Rhenium Sheet $0.030^{\prime \prime}$ thick

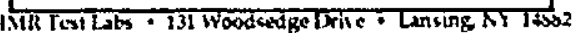

CHEMISTRY

\begin{tabular}{|c|c|c|}
\hline Element & R-1499 & Specification \\
\hline Ike & $>99.99$ & $99.97 \%$ \\
\hline $\mathrm{OH}$ & $<250$ ppin & $250 \mathrm{pem}$ Mlavinum \\
\hline 0 & .13 Ppm & $\cdots$ \\
\hline$C$ & $19 \mathrm{ppn}$ & $\cdots$ \\
\hline H: & A.8 ppin & - \\
\hline .12 & 3 p p & 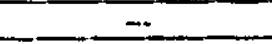 \\
\hline $\mathrm{Li}$ & $<0.0115$ & - \\
\hline Be & $<01,0175$ & $=$ \\
\hline$\dot{B}$ & $\{1,0\}$ & $\ldots$ \\
\hline F & $<0.05$ & - \\
\hline$N a$ & 0.22 & + \\
\hline$\overline{\mathrm{N}}$ & $<0.01$ & $=$ \\
\hline AI & 0.25 & $\cdots$ \\
\hline$\overline{\mathrm{Si}}$ & 23 & 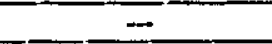 \\
\hline$P$ & $0.0 \overline{5}$ & 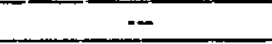 \\
\hline$S$ & 0.09 & $\cdots$ \\
\hline CI & 0.04 & $=$ \\
\hline $\bar{K}$ & 0.25 & - \\
\hline$\overline{\mathrm{Ca}}$ & 0.2 & -- \\
\hline$S^{-}$ & $<0,005$ & - \\
\hline Ti & 0.114 & - \\
\hline$V$ & 0.008 & -- \\
\hline $\mathrm{Cr}$ & 2.7 & $-\infty$ \\
\hline $\mathrm{Min}$ & $0 .+1$ & -- \\
\hline $\mathrm{Es}^{2}$ & 3.5 & $=$ \\
\hline Co & 0.01 & $\cdots$ \\
\hline $\mathrm{Ni}$ & 2.7 & -- \\
\hline $\mathrm{Cu}$ & 0.05 & $-\infty$ \\
\hline 211 & $<0.05$ & $\ldots$ \\
\hline Ga & $<0.105$ & - \\
\hline Gt & $<0.115$ & $\cdots$ \\
\hline$A s$ & $<0,05$ & 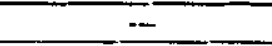 \\
\hline $5 \mathbf{x}^{3}$ & $<0.05$ & $\cdots$ \\
\hline $13 \%$ & $<0.05$ & $\cdots$ \\
\hline $\mathrm{R} \mathbf{b}$ & $<0.01$ & $=$ \\
\hline Sr & $<0.0)$ & $\ldots$ \\
\hline$Y$ & $<0.005$ & - \\
\hline $2 r$ & 0.07 & $\cdots$ \\
\hline $\mathrm{Sb}$ & 0.01 & - \\
\hline Ma & 0.09 & $\therefore$ \\
\hline IRu & $<0.01$ & $\cdots$ \\
\hline $\mathrm{Rh}$ & $<0.01$ & $=$ \\
\hline$P a t$ & $<0.01$ & $\cdots$ \\
\hline
\end{tabular}

Rinium Allens br

Inys 2 ui?

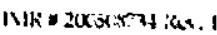

PRE-DECISIONAL - For Planning and Discussion Purposes Only 
Attachment 3 to

Enclosure 3 to

MDO-723-0044

B-MT(SRME)-52

Page 17

Rhenium Sheet $0.030^{\prime \prime}$ thick

\begin{tabular}{|c|c|c|}
\hline Element & R-1499 & Specification \\
\hline$\Delta y$ & $<0.01$ & $=$ \\
\hline $\mathrm{Cd}$ & $<0.1$ & $\ldots$ \\
\hline in & $<0.1$ & 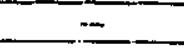 \\
\hline Sn & 0.07 & - \\
\hline Sh & $\leq 0.01$ & $\cdots$ \\
\hline Te & $<0.01$ & $\cdots$ \\
\hline 1 & $<0.01$ & 4et \\
\hline $\mathrm{Cs}$ & $<0.01$ & $\cdots$ \\
\hline 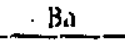 & 0.03 & $\cdots$ \\
\hline La & $<0.01$ & $\cdots$ \\
\hline Co & $<0.01$ & 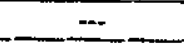 \\
\hline $\mathrm{Pr}$ & $<0.01$ & $\cdots$ \\
\hline Nd & $<0,01$ & $\because$ \\
\hline $5 \mathrm{sm}$ & 50.01 & $\cdots$ \\
\hline Eu & $<0.01$ & $\cdots$ \\
\hline Gil & $\leq 0.01$ & $=$ \\
\hline $\mathrm{Tb}$ & $\leq 0.01$ & $m$ \\
\hline Dy & $<0.01$ & - \\
\hline Ho & $<0.01$ & 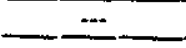 \\
\hline E: & $<0.01$ & $=$ \\
\hline $\mathrm{Tm}$ & $<0.01$ & $\cdots$ \\
\hline Yb. & $<0.01$ & - \\
\hline Lut & $\leq 0.01$ & $\ldots$ \\
\hline IfI & $<0.01$ & $\ldots$ \\
\hline$T a$ & $<\overline{5}$ & $=$ \\
\hline$W$ & $<0.2$ & - \\
\hline Ke & Matrix & $=$ \\
\hline Os & $<0.01$ & $=$ \\
\hline $1 \mathrm{r}$ & $<0.01$ & $=$ \\
\hline $\mathrm{Pt}$ & $<0.05$ & $\cdots$ \\
\hline Al & $<0.5$ & -- \\
\hline $\mathrm{H}_{\mathrm{B}}$ & $<0.05$ & $\cdots$ \\
\hline 11 & $<0.01$ & $=$ \\
\hline \% & 0.09 & 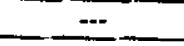 \\
\hline 83 & $<0.01$ & $m$ \\
\hline Ih & $<0.001$ & $\ldots$ \\
\hline $\mathrm{U}$ & $<0 .(0) 1$ & $\ldots$ \\
\hline
\end{tabular}

Determined by combustion-infrared alsorbance.

- Dutermined by inert gas fusion-ilemal conductivity.

Or $=$ Other clements, to tal

Unless otherwise indicated, elenents were analyad by GDMS.

Resuils in ppon (ppm - parts par million $\left.=m g / K_{b}\right) ; 0.1000^{\circ}$ by wt + $1000 \mathrm{ppm}$ unless otherwise indicated.

Wethods: GDMS and ASTM E 1019-03, CAP'-32N.

Nhunum Allogs ltu .

rupr 3 nit

INK 20050874.RM. I 


\section{MATERIAL CERTIFICATION}

DATE: Oclobet \$1, 2005

SOLD TO: Kinolls Alomic l'ower Laboratory. P.O. Bo. 1072

Sejencelady. NY $12301-1072$

PURCHASE

ORDER: KNGU02912A

LINE ITEM: $\$ 2$

MATERIAL: Rhenium shcet

This is a shipmert against your order $4 K N 6002912 \mathrm{~A}$ which completes the requirement for rhenium shee. The thenium $.060^{\prime \prime}$ sheet meets the requirements of the purchase order and the attachment specification. This raterial was nanufactured in compliance with KAPL's qualiyy assurance specifieation KBPQAR-I dated March 2004 and the revised antendment for thickness.

Deseription: Pure thenium shest .060+.010/-.000 x +" wide $\times 7$ long

Quant ity: I

Lot number R-1 $\$ 99$

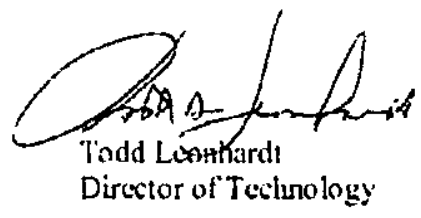

Rhenium . Alloys. Int

Feverdaj's Pioncer. Todug's Letader

Emal: thenitmin a thomuln Lom

Web Page hup://unw,rieniunticon 
Attachment 3 to

Enclosure 3 to

MDO-723-0044

B-MT(SRME)-52

Page 19

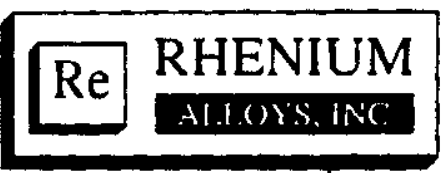

\title{
Rhenium Sheet $0.030^{\prime \prime}$ thick
}

1329 Taylor St.

P.O. Box 245

Elyria. Oh $+4036-02+5$

Voice (440) $365-7388$

Fax (440) 366-9831

Report No. B-8s!

KAPL, Ine

P.O. Box 1072

Sihenectady. New York 12301-1072

\begin{abstract}
$10 / 31 / 05$
Subject

Rhenium sheet

$7 " \times 4 " \times .060$

Submitted to Our l.alurstory on Order Number

POit KN6002912A
\end{abstract}

Rhenium dlloy: Ins

Fmail: thmiugnghonium covm

Whb page at hipp://wn'wirheniuntioum 


\section{Rhenium Sheet $0.030^{\prime \prime}$ thick}

KAPL.

\section{REPORT NO B-881}

BACKGROLIND

One sample was subminted for tosting which is representalive of the thenium sheet identified as Lut nutnber R-1499, .060 rhenium sheet and compared to the KAPL. purchasc order and attachnent specificat ion.

\section{TEST REOUESTED}

Gran size. Density. Dimensionals, Chemical Analysis, and microscopic examination.

\section{GRAR SIZF. EXAMIINATIOX}

The submitted specinen was prepared in accordance with ASTM E3-95 for grain size examination. It was examined at $100 x$ for grain sire deternination. The arerige grain size was rated in accordance with the ASTM E112-96 comparison method tor detemmining grain si\%:

The average $A S T M$ grain sire is 4.5

\section{DEXSITY}

Density is mensured using ASTM B311-93; Fest Method for Dessity Determinution for Powder Metallurgy (P/M) Materials Containing Less Than Two Percent Porosity.

The density of the 060 shet is $99.95 \%$ or $21.03 \mathrm{~g} / \mathrm{ce}$

\section{DIMENSIONALS}
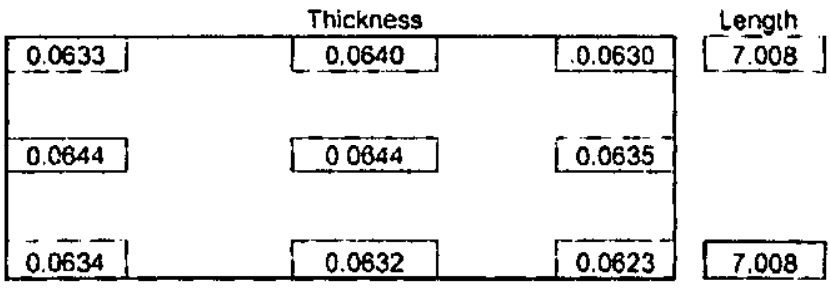

Width [ 40000

\section{CHEMICALANAIASIS}

Sce attachment 
Attachment 3 to Enclosure 3 to

MDO-723-0044

B-MT(SRME)-52

Page 21

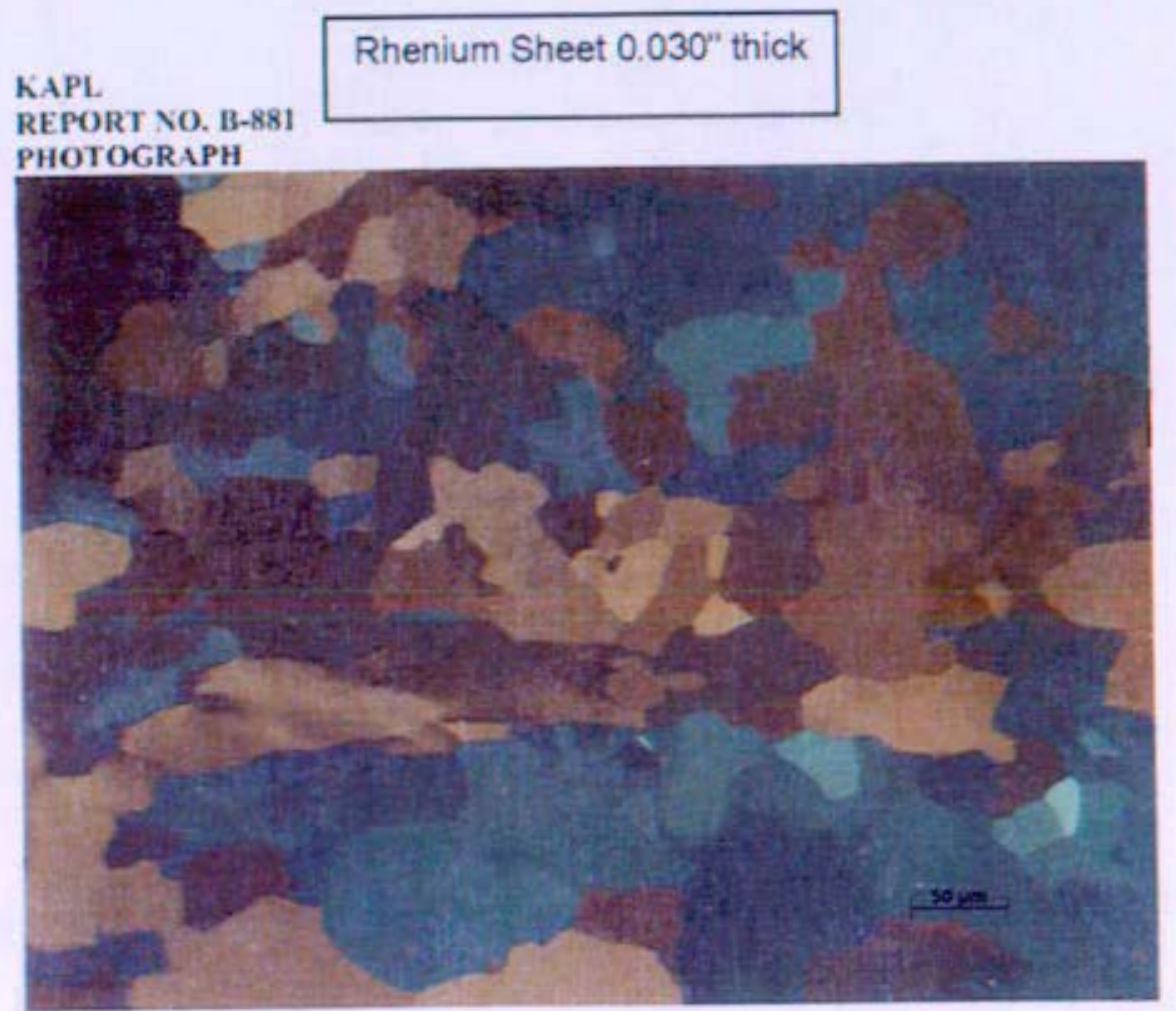

This is a representative view of the microstructure from the submitted sumple.

Magnification:260X

Etchant: Moditied Murakami's Reagent

Polarized Light

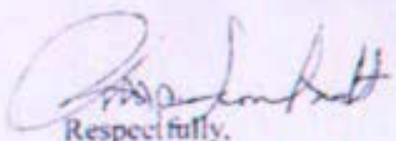

Todd Leonhardt

Rhersiaw Alloys, Ine

Email: rhainam is rhenium sem

Web site: hrtps/ irwwirhrnium. com

PRE-DECISIONAL - For Planning and Discussion Purposes Only 
Attachment 3 to

Enclosure 3 to

MDO-723-0044

B-MT(SRME)-52

Page 22

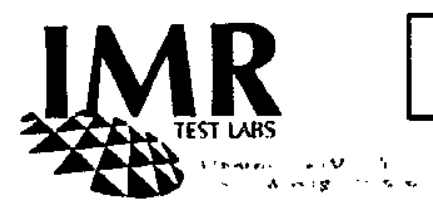

Rhenium Sheet $0.030^{\prime \prime}$ thick

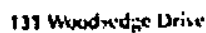

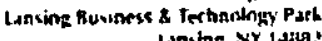

Lunving Nr 1 +3ig:

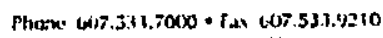

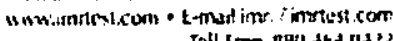

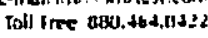

October 28, 2005

Clitif Guthnuan

CERTIFIED MATERIAL ANALYSIS

Rhenitin: Alloys Inc.

5O Bon 245

Biyrad OH $+4030-02+5$

USA

IMR Report Number 204509814

roNuniter

13065

Derr Raticd

Octobur 25, 20105

1at"

R-1490)

Decoiption

.060 thick ho shicet

Spucifintion

KN6002912

\section{SUMMARY}

The sample meets the chemicol requirenents of KN6002912 as supplied by the customer. The GDNS portion of the andysis was outsoutod by IMIR to a qualified outside facility (non-A2L,A accredind). Per customer request the sample wds andyed by combustion fir oxyen, hydrogen, nitrogen and carbon.

The results are given on the following payes.

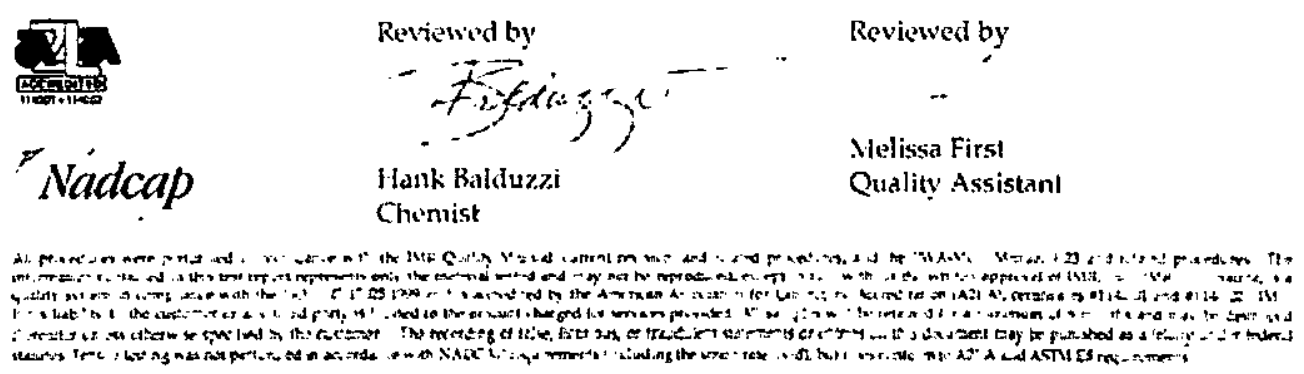

Rhonam . Nhan's its

Pagn 1 of 3

$13 \mathrm{AR}=20030 \% 1+$ 
Attachment 3 to

Enclosure 3 to

MDO-723-0044

B-MT(SRME)-52

Page 23

\section{Rhenium Sheet $0.030^{\prime \prime}$ thick}

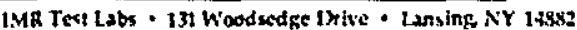

CIIEMISTRY

\begin{tabular}{|c|c|c|}
\hline Element & R-1499 & Specification \\
\hline Rt: & $>99.99 \%$ & $99,97 \%$ \\
\hline $\mathrm{O}$ & 23 & $50 \mathrm{ppm}$ Maximum \\
\hline OT & 34 & $250 \mathrm{p}$ pm Maximum \\
\hline Ci & 16 & $\cdots$ \\
\hline HI & 2.7 & $\cdots$ \\
\hline 12 & 1 & - \\
\hline $\mathrm{Li}$ & 40.005 & $\ldots$ \\
\hline Be & $<0.005$ & $=$ \\
\hline B & $<0.005$ & 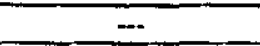 \\
\hline$F$ & $<0.05$ & $\pi$ \\
\hline Na & 0.22 & $=-$ \\
\hline $\mathrm{N} / \mathrm{g}$ & 50.01 & $=$ \\
\hline$A !$ & 1.4 & $\because$ \\
\hline $5 i$ & 0.67 & $\cdots$ \\
\hline$\vec{p}$ & 0.02 & -- \\
\hline 5 & 0.03 & $\therefore$ \\
\hline Cl & 0.01 & - \\
\hline$K$ & 0.73 & $\cdots$ \\
\hline Ca & 0.16 & $=$ \\
\hline $5 x$ & $\leqslant 0.0 \times 15$ & - \\
\hline $\mathrm{Ti}$ & 0.04 & $\cdots$ \\
\hline V & <ndus & $\therefore$ \\
\hline$\overline{C r}$ & 3.5 & $=$ \\
\hline $\mathrm{Mn}$ & 0.14 & $\cdots$ \\
\hline It & 4.0 & 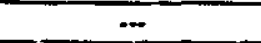 \\
\hline $\overrightarrow{C O}$ & 0.009 & - \\
\hline $\mathrm{Ni}$ & 3.2 & $\rightarrow$ \\
\hline $\mathrm{Cu}$ & $<0.05$ & $\cdots$ \\
\hline$\overline{Z n}$ & $\leqslant 0.05$ & $=$ \\
\hline $\mathrm{G}$ & $<0.05$ & - \\
\hline $\mathrm{Cu}$ & $<0.05$ & $=$ \\
\hline As & $<0.05$ & $\ldots$ \\
\hline 50 & $<0.05$ & - \\
\hline $\mathrm{Br}$ & $<0,05$ & - \\
\hline $\mathrm{Rb}$ & $<0.01$ & $\cdots$ \\
\hline $5 r$ & $<0.01$ & $=$ \\
\hline 1 & $<0.005$ & $m$ \\
\hline $\mathrm{Zr}$ & 0.04 & - \\
\hline $\mathrm{Nb}$ & 0.02 & $\cdots$ \\
\hline Mn & 0.20 & $=$ \\
\hline $\bar{R} u$ & $<0.01$ & $\ldots$ \\
\hline $\mathrm{Bh}$ & $<0.01$ & $\cdots$ \\
\hline$P_{4}$ & $<0.01$ & - \\
\hline
\end{tabular}


Attachment 3 to

Enclosure 3 to

MDO-723-0044

B-MT(SRME)-52

Page 24

\section{Rhenium Sheet $0.030^{\prime \prime}$ thick}

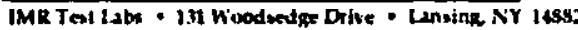

\begin{tabular}{|c|c|c|}
\hline Element & R-1499 & Specification \\
\hline Ag & $<0.01$ & $=$ \\
\hline Cd & $\leq 0.1$ & $\therefore$ \\
\hline In & $<0.1$ & $\cdots$ \\
\hline 511 & $<0.111$ & $\cdots$ \\
\hline $5 \mathrm{~b}$ & $<0.01$ & $\cdots$ \\
\hline Ie & $<0.01$ & $\because$ \\
\hline I & $<0.01$ & $\cdots$ \\
\hline$\overline{C s}$ & $<0.01$ & 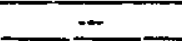 \\
\hline Ba & 0.04 & $=$ \\
\hline Ld & $<0.01$ & $\cdots$ \\
\hline Co & $<0.01$ & $=$ \\
\hline$P^{\prime}$ & $<0.01$ & $\cdots$ \\
\hline $\mathrm{Nd}$ & $<0.01$ & $\ldots$ \\
\hline Sm & $<0.01$ & - \\
\hline Fu & $<0.01$ & - \\
\hline Cd & $<0.01$ & 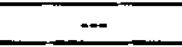 \\
\hline $\mathrm{Th}$ & $<0.01$ & - \\
\hline Dy & $<0.01$ & $=$ \\
\hline Ha & $<0.01$ & $\ldots$ \\
\hline$E_{r}$ & $<0,01$ & $-\infty$ \\
\hline $\mathrm{Tm}$ & $<0.01$ & $\cdots$ \\
\hline $\mathrm{Yb}$ & $<0.01$ & $=-$ \\
\hline Lu & $<0.01$ & $\cdots$ \\
\hline $\mathrm{Hit}$ & $<0.01$ & - \\
\hline Ta & $<5$ & + \\
\hline$W$ & $<0.2$ & -- \\
\hline Os & $<0.01$ & $\approx$ \\
\hline II & $<0.01$ & $m$ \\
\hline $\mathbf{P t}_{\mathrm{t}}$ & $<0.05$ & - \\
\hline A11 & $<0.5$ & $=$ \\
\hline $1 \mathrm{tg}$ & $<0.05$ & - \\
\hline 71 & $<0,01$ & $\approx$ \\
\hline $\mathrm{Pb}$ & 0.03 & $=$ \\
\hline Bi & $<0.01$ & \\
\hline Th & $<0001$ & \\
\hline $\bar{U}$ & $<0.001$ & \\
\hline
\end{tabular}

"Determined by combustion-infrared absorbance.

2Determinel by inert gas fusion-thermal conductivity.

$\mathrm{OT}=$ Othur elentents, total.

Unless othe'wise indicatel, elemonts were analyzat by CADMS

Results in ppm (ppm = parts per million $=m g / K g$ ): $0.1000 \%$ by $w t=1000 \mathrm{ppm}$ unless otherwise indicated.

Methods: GDMS, ASTM I: 1019-03, and CAP-32A. 
Attachment 3 to

Enclosure 3 to

MDO-723-0044

B-MT(SRME)-52

Page 25

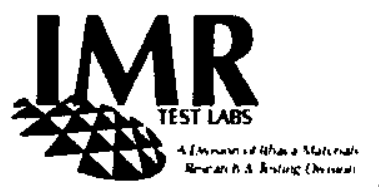
Tungaten 25\% Rhenium Plate
$\sim 0.080^{\prime \prime}$ thk, $4^{n} \times 8^{n}$

131 wanthed Ore

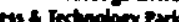

Insing or 14682

Phene 607.533.7000 + Fax 607.53J.9210

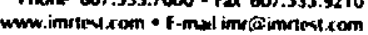
rall free nestersu

\section{CERTIFIED MATERIAL ANALYSIS}

IMR Report Number 20050685s

\section{SUMALARY}

The sample was analyzed for the elements listed in the table below.

Jvly 28, 2005

\section{CHEMISTRY}

Todd Leonhardt

Rhenium Altoys Inc.

PO Box 245

Elyrin, OH 44036-0245

USA

TONumber

12581

DurRowind

July 26, 2005

Iat

WR1466

\begin{tabular}{|c|c|}
\hline Elemeat & Sample \\
\hline B & $<0.010$ \\
\hline $\mathrm{C}^{1}$ & 0.002 \\
\hline Co & 0.01 \\
\hline Fe & 0.03 \\
\hline $\mathrm{H}^{2}$ & 0.003 \\
\hline $\mathbf{M g}_{\mathbf{g}}$ & $<0.01$ \\
\hline Mn & $<0.01$ \\
\hline Mo & $<0.01$ \\
\hline $\mathbf{N}$ & 0.0002 \\
\hline$O^{\prime}$ & 0,0009 \\
\hline Re & 25.12 \\
\hline $\mathrm{Si}$ & $<0.01$ \\
\hline Sn & $<0.01$ \\
\hline$w^{3}$ & 74.83 \\
\hline
\end{tabular}

Determined by combustion-infrared absorbance.

2Determined by inert gas fusion-thermal conductivity.

Determined by difference.

Results in weight percent unless otherwise indicated.

Methods: ASTM E 1019-03, CAP-046B (ICP-AES), CAP-017F (ICP-AES),

and CAP-032A (Hydrogen).

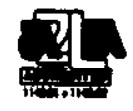

$r_{\text {Nadcap }}$
Reviewed by

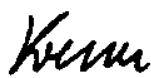

Kevin Kosty

Chemical Technician
Reviewed by

Teza<smiles>C1CCC2(C1)C1CCC(C1)C1C3CCC2C31</smiles>

Peter Damian, Manager Chemistry Department

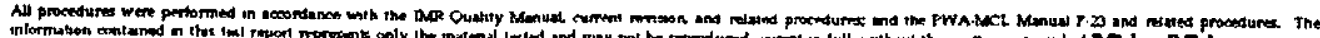

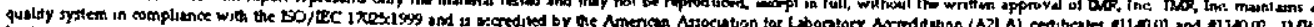

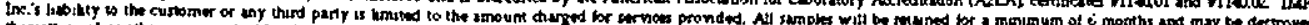

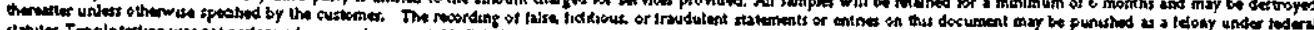

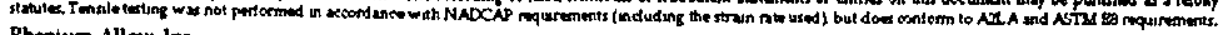

Rheriun Alloy, in:

Page 1 of 1

IMR $\#$ 200506058 
Attachment 3 to

Enclosure 3 to

MDO-723-0044

B-MT(SRME)-52

Page 26

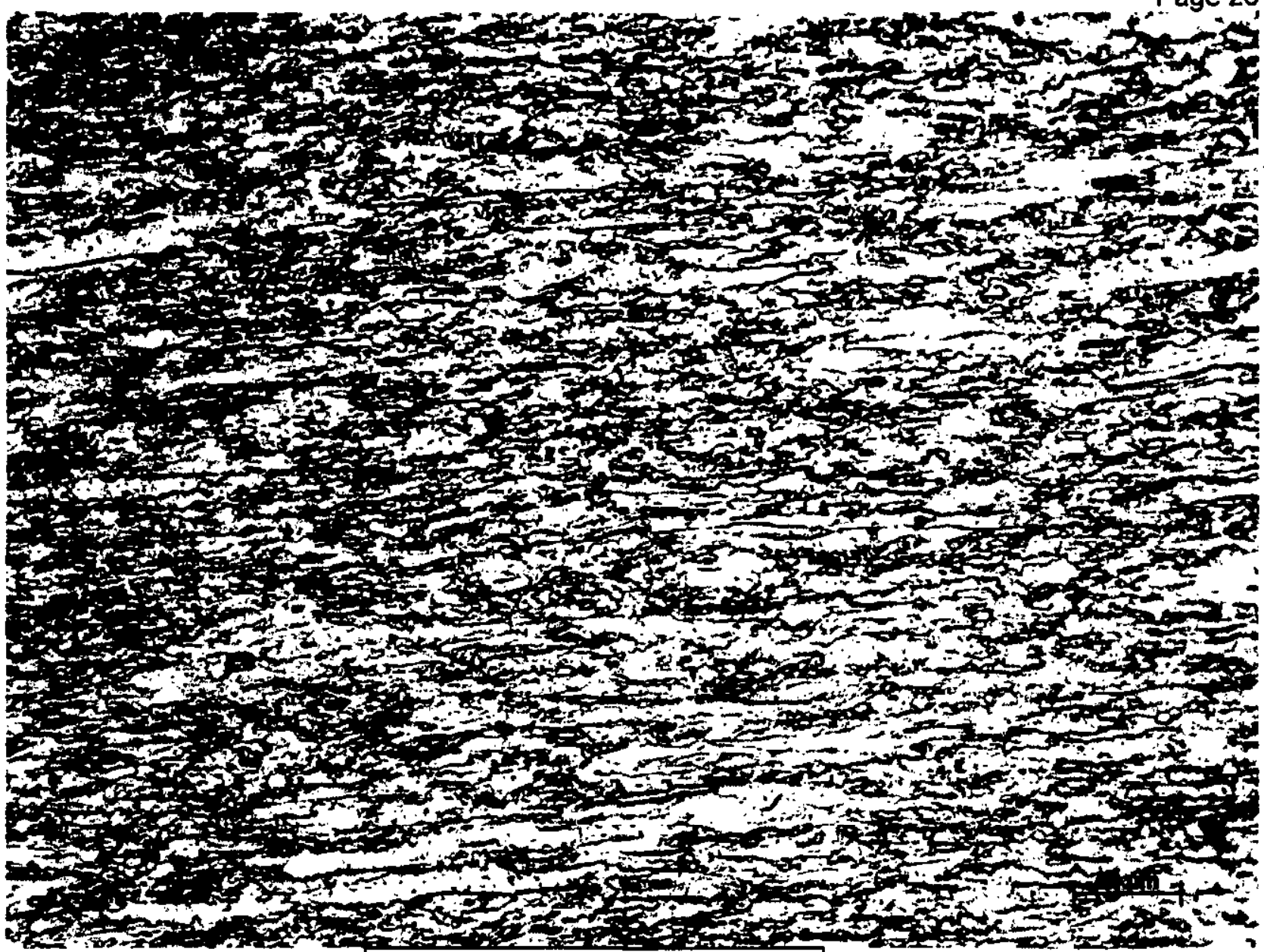

Tungsten $25 \%$ Rhenium Plate

$\sim 0.080^{\prime \prime}$ thk, $4^{\prime \prime} \times 8^{\prime \prime}$ 
Attachment 3 to

Enclosure 3 to

MDO-723-0044

B-MT(SRME)-52

Page 27

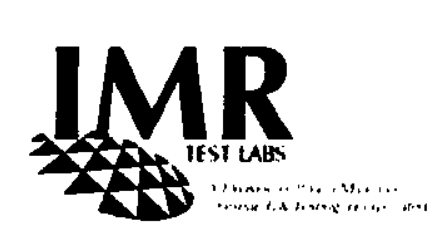

Tungsten 25\% Rhenium Rod (0.25* Dia)

Backup Refructory Liner Raw Material

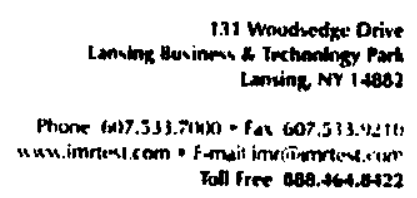

June 23, 2005

Cliff Cuthman

Rhenium Alloys inc.

PO) Box 245

Elyria, OII +4036-0245

USA

\section{CERTIFIED MATERIALANALYSIS}

IMR Report Number 200505572

\section{SUMMARY}

NoNumber

124 to

The sample was analyzed for the clements listed on the following page.

\section{Dete Reained}

June 16.2005

LotNumber

WR-1518

Decoiption

W/ 25: Re-rod

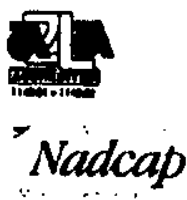

\section{Reviewed by}

$$
\text { itix. } i \text {, e. }
$$

Robert Hali

Chemist
Reviewed by

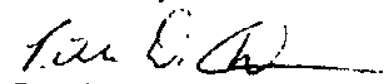

Peter Damian

Chem. Dept. Manager

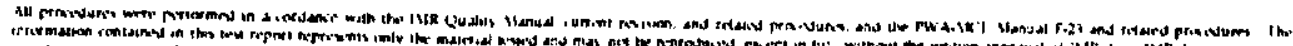

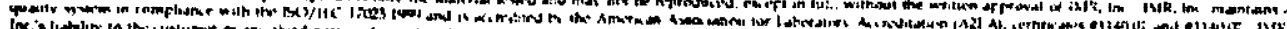

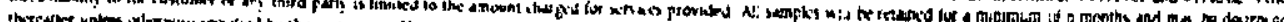

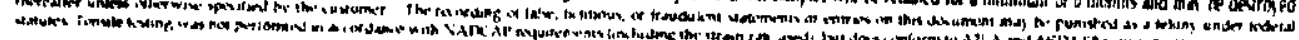
Fisurtiatin Allings Im

Page 1 at 2

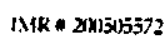


Attachment 3 to

Enclosure 3 to

MDO-723-0044

B-MT(SRME)-52

Page 28

Tungsten 25\% Rhenium Rod (0.25" Dla)

Backup Retractory Liner Raw Material

1MR Tes! 1 2bm - 13] Woodsedge Drive - I Insing NY 1432

\section{CHEMISTRY}

\begin{tabular}{|c|c|}
\hline Element & Sumple \\
\hline Al & $<0.01$ \\
\hline $\mathbf{C a}$ & $<0.01$ \\
\hline $\mathrm{Cd}$ & $<0.01$ \\
\hline Co & $<0.01$ \\
\hline $\mathrm{Cr}$ & $<0.01$ \\
\hline $\mathrm{Cu}$ & $<0.01$ \\
\hline $\mathrm{Fe}$ & 0.01 \\
\hline $\mathbf{K}$ & 0.01 \\
\hline $\mathrm{Mg}$ & $<0.01$ \\
\hline $\mathrm{Mn}$ & $<0.01$ \\
\hline Mo & 0.05 \\
\hline via & $<0.01$ \\
\hline $\mathrm{Nb}$ & $<0.01$ \\
\hline $\mathrm{Ni}$ & 0.03 \\
\hline$P$ & 0.030 \\
\hline $\mathrm{Re}$ & 25.23 \\
\hline Si & $<0.01$ \\
\hline Sn & $<0.01$ \\
\hline $\mathrm{Ti}$ & $<0.01$ \\
\hline$W_{1}$ & 74.62 \\
\hline$Z n$ & 0.02 \\
\hline $\mathbf{Z r}$ & $<0.01$ \\
\hline
\end{tabular}

Determined by difference.

Resuls in weight percent unless otherwise indicated.

Other elements tested $(<0.01 \%)$ werd $\mathrm{Pb}$, Ta and $\mathrm{V}$.

Method: CAP-046A (ICP-AES). 
Attachment 3 to

Enclosure 3 to

MDO-723-0044

B-MT(SRME)-52

Page 29

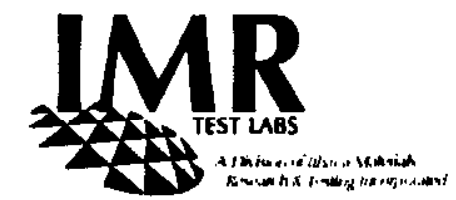

K Doped Tungsten Rod 0.5" Dia.

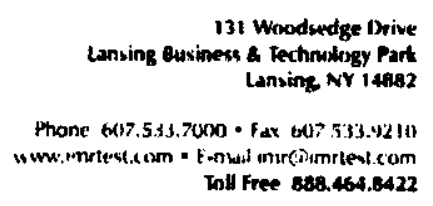

August 19, 2005

Shij Gifford

Knolls Atumic Power Lab

P.O. Hon 11072

Sherketudy, N) 123)1-1072

USA

Fonumber

KNGon?34s

CERTIFIED MATERIAL ANALYSIS

Dar Rexived

August 12, 2(h)5

IMR Report Number 200507463

Padting list

C006958

Mtrial D

Shlowershopi WVW tungsten rors!

Material Name

Potassium doped tungstern

\section{SUMMARY}

\section{Material Type}

Other

Reference No.

1)5I'FGJ36

The sample was analyad for the clements tisted on the following page.

(5)
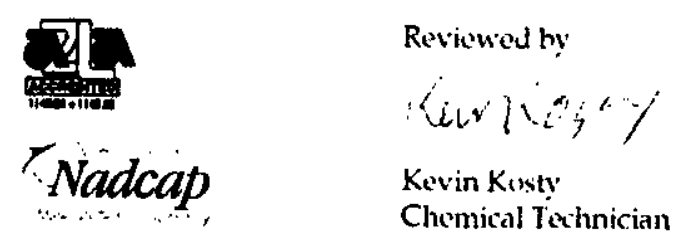

Kevin Kesty

Chemical Terhnician

Review'd by

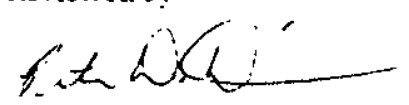

Peter Danian, Mlanager Chemistry Department

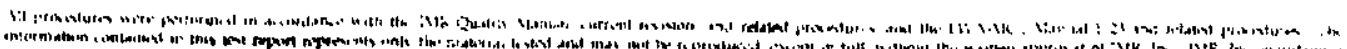

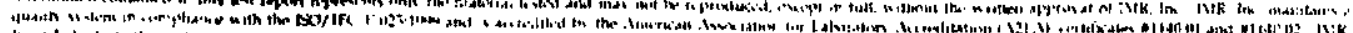

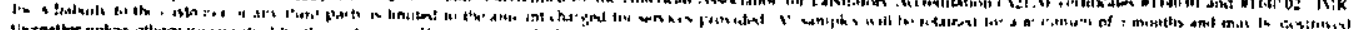

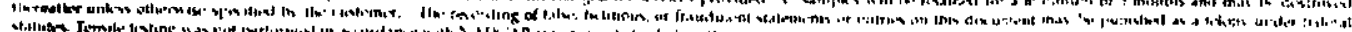

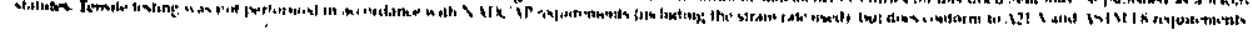


K Doped Tungsten Rod 0.5" Dia. st Labs - 131 Woodsedge Drive - Lanving NY 14062 Material ID: Schwarzkopf WVM tunsten rod Material Name: potassium doped tungsten Reference No.: 05PFG136

Material Type: Other

\section{CHEMISTRY}

\begin{tabular}{|c|c|}
\hline Element & Sa \\
\hline$A y$ & $<0.01$ \\
\hline$-1-1$ & 0.01 \\
\hline As & $<0.01$ \\
\hline$C$ & 0.01 \\
\hline$-\ldots$ & $<0,(0)$ \\
\hline$--\overline{C_{1}}$ & -1 \\
\hline$--\overline{C_{0}}$ & $0.0]$ \\
\hline$\overline{C_{I}}$ & $<0.01$ \\
\hline$[-1$ & - \\
\hline$F_{0}$ & $<0.0$ \\
\hline$-1-1$ & -1 \\
\hline$-1-\ldots$ & $86 \pm 10, \ldots$ \\
\hline Mg & - \\
\hline $\mathrm{Nn}$ & - \\
\hline$[-$ & -1 \\
\hline$N=$ & - \\
\hline- & --1 \\
\hline$-1-\ldots$ & $-1-1<0$ \\
\hline$-\ldots$ & $-\ldots-\leq 0.01$ \\
\hline$-\ldots-1$ & $---0,0 ! 1$ \\
\hline$-\ldots$ & $-1 \leq$ \\
\hline- & $<0.01$ \\
\hline si & 0.001 \\
\hline $\mathrm{Si}$ & 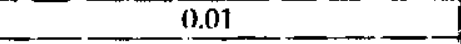 \\
\hline$T a$ & $-\ldots$ \\
\hline - . & -1 \\
\hline- & Remainder \\
\hline$-\ldots$ & $<0.01$ \\
\hline $\mathrm{Z}_{\mathrm{T}}$ & $<0.01$ \\
\hline
\end{tabular}

Determined by combustion-infrarcd absorbance.

2Determined by inert gas fusion-thermal conductivity.

Results in weight percent unless otherwise inclicatod.

Wethods: CAP-017T (ICP-AES). CAP-146B (ICP-AFS) and ASTM F 1019-0,3. 
Attachment 3 to

Enclosure 3 to

MDO-723-0044

B-MT(SRME)-52

Page 31

SCHWARZKOPF

A PLANSEE Alfiliated Company

K Doped Tungsten Rod 0.5" Dia.

KNOLLS ATOMIC POWER LABORATORY

Central Receiving Bidg M2

2401 River Road

SCHENECTADY NY 12309

USA

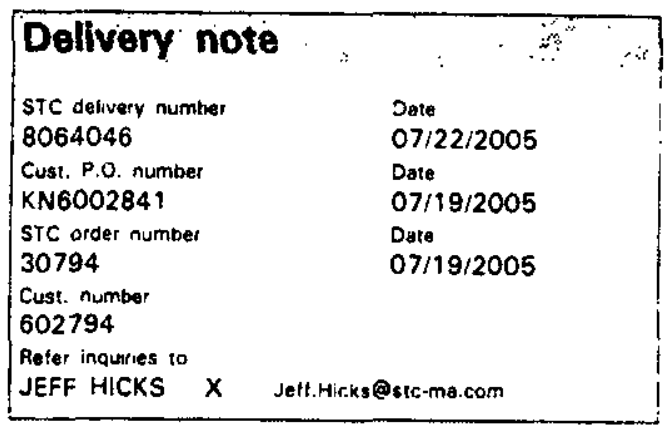

Shipping instructions: Fed $X$ Collecs $\$ 0122-0027.7$

Shipping conditions Normal

Terms of delivery: FOB Franklin, MA

Gross weight

$6.660 \mathrm{KG}$ Net weight

$6.660 \mathrm{KG}$

Shipping instructions: Fed $X$ Collect

Regionat Sales Manager: JIM PALOMBO/REGIONAL SALES MANAGER

Telephone: $617 \cdot 306-3564$

MATERIAL CERTS REQUIRED

\begin{tabular}{|c|c|c|c|}
\hline Itom & $\begin{array}{l}\text { Material } \\
\text { Description }\end{array}$ & $a_{t y}$ & Welght \\
\hline 01 & $\begin{array}{l}220838 \\
\text { PLANSEE WVM TUNGSTEN ROD } \\
\text { O.500" dia } \times \text { ri } \\
\text { tolernace on total length } 1+1-9\end{array}$ & $6.660 \mathrm{KG}$ & $6.660 \mathrm{KG}$ \\
\hline
\end{tabular}

Schwarzkopf Technologies LLC

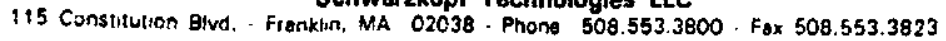

......... - Www.stcmatols.com

PRE-DECISIONAL - For Planning and Discussion Purposes Only 
Attachment 3 to

Enclosure 3 to

MDO-723-0044

B-MT(SRME)-52

Page 32

K Doped Tungsten Rod 0.5" Dia

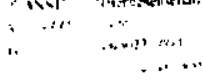

n... . . nisin

\section{INSPECTION CERTIFICATE}

a:r IO DIN EN $10204 \quad 3.1 \mathrm{~B}$

\section{PLANSEE}

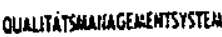

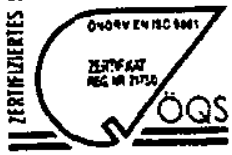

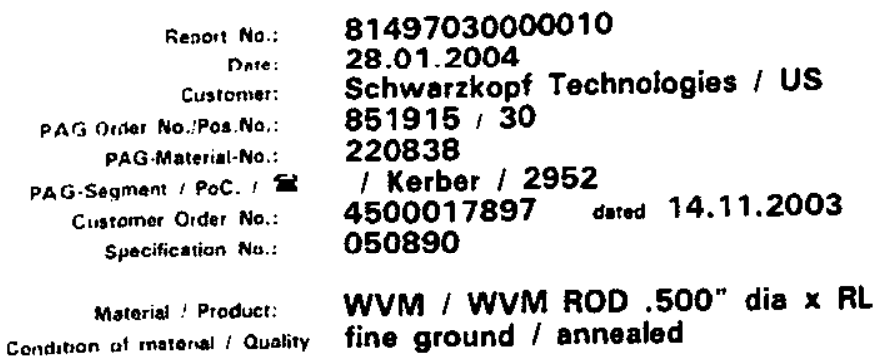

GUARANTEED CHEMICAL ANALYSIS: acc. to PAG.Spoc. A052

$W \min 99.95 \%$ *

An $\max 10 \mu \mathrm{g} / \mathrm{g}$

Ca max. $5 \mu g^{/ g}$

C. $\max .20 \quad \mu g / g$

hig $\max 5 \mu g / g$

if max. $10 \quad \mu g / g$

Piv max. $5 \mu g / g$

Zn $\max .5 \mu / g$

Al max. $20 \mu g / g$

G max. $30 \quad \mu g / g$

C max. $30 \mu g / g$

$\max .20 \quad \mu g / 9$

$\begin{array}{llrl}\text { As } & \max . & 5 & \mu g / g \\ \text { Cd } & \max . & 5 & \mu g / g \\ \text { Cu } & \max .10 & \mu g / g \\ \mathrm{Mn} & \max . & 5 & \mu g / g \\ \mathrm{Nb} & \max .10 & \mu g / g \\ \mathrm{Ta} & \max .20 & \mu g / g \\ \mathrm{Zl} & \max . & 5 & \mu g / g \\ \mathrm{~K} & \mathbf{3 0}-70 & \mu g / g \\ & & & \\ \mathrm{H} & \max . & 5 & \mu g / g \\ \mathrm{~S} & \max . & 5 & \mu g / g\end{array}$

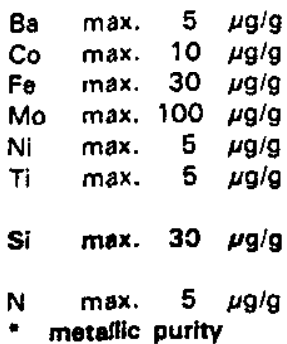

Density : $=>18,90 \mathrm{~g} / \mathrm{cm}^{3}$

Grain size : ASTM No, finer $\mathbf{3 . 0}$

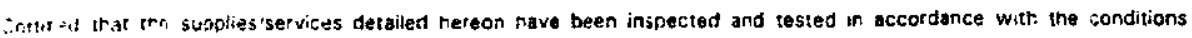

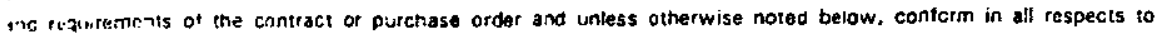

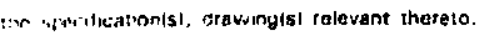

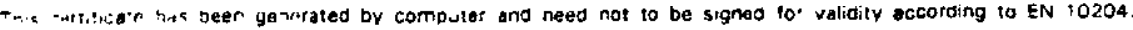

Writ linspector

101 ithusnerl
PLANSEE Aktiongoeollsehent

Migh Purformance Materiale.

Intpection Departmant GMSZ

(Partnar )

(Kerber ) 
Enclosure 4 to MDO-723-0044 /

B-MT(SRME)-52

Page 1

\author{
Enclosure 4 \\ to \\ MD0-723-0044/B-MT(SRME)-52 \\ JOYO-1 Silicon Carbide Specimens
}

R. S. Northey

January 2006 


\section{Summary}

Silicon carbide was considered in various forms by the Naval Reactors Prime Contractor Team (NRPCT) for Prometheus-1 core and cladding structural materials. Prior to Project restructuring, fabrication of SiC specimens for the JOYO-1 irradiation test campaign was completed.

Specimens were machined from plate stock of monolithic and composite silicon carbide (SiC). Chemical vapor deposited (CVD) monolithic SiC was chosen for JOYO-1 as a baseline material to demonstrate basic radiation effects on silicon carbide. The SiC composite selected for irradiation testing in JOYO-1 consisted of Hi-Nicalon Type S fibers in a Chemical Vapor Infiltration (CVI) SiC matrix. For these materials, miniature tensile specimens and thermal diffusivity discs were fabricated. Use of a miniature tensile specimen design permitted inclusion of sufficient replicates to produce statistically reliable results. This is important for $\mathrm{SiC}$, a classically brittle material, which typically has a large strength variability.

Bend Stress Relaxation (BSR) specimens for (irradiation) creep estimation were also procured for JOYO-1 testing, for monolithic SiC only. In this technique, monolithic SiC thin strip specimens are held at a defined radius during irradiation through the use of a fixture. Creep estimation is determined by comparing the specimen radius before and after irradiation. The BSR specimens included 50, 100 and $200 \mu \mathrm{m}$-thick strips corresponding to an applied flexural strength of 100,200 and $400 \mathrm{MPa}$, respectively. The BSR technique allows for detection of small creep rates $\left(\sim 10^{-11} \mathrm{~s}^{-1}\right)$, expected in SiC.

Initial thermal diffusivity and mechanical testing was completed to characterize the baseline material properties of the monolithic and composite SiC. Thermal diffusivity results were within range of published data. Tensile testing of the composite material was successful resulting in an average ultimate strength of $41.5 \pm 3.58 \mathrm{ksi}$ and percent elongation of $0.27 \pm 0.09$. Initial tensile testing of the monolithic material was unsuccessful. The specimens broke in the clamps, therefore data was unattainable. Additional testing would be needed to determine appropriate clamping devices.

\section{Introduction}

The NRPCT considered silicon carbide in various forms for core and cladding structural materials for the Prometheus-1 reactor. Prometheus-1 required a tough, hermetic, seal-less clad that was mechanically, but not thermally de-bonded from the fuel to contain fission products and avoid brittle failure in the event of fuel swelling. This was particularly true for direct gas-cooled concepts, such as Prometheus-1, where the primary coolant flows behind the reactor shield. Silicon carbide composites have been developed for high-temperature reactor applications demonstrating that an attempt to fabricate a hermetic composite is a difficult, but potentially solvable challenge. An additional concern is losing hermeticity through matrix cracking. The composite derives its improved toughness from its ability to crack along fiber/matrix interfaces, in turn creating additional paths for fission product migration. Therefore, the component must be designed to keep stresses below the matrix cracking stress for normal operation. A potential solution is the laminate structure (duplex/triplex systems). In this structure, the addition of an outer layer of monolithic material may act as the actual fission product containment barrier while the inner composite and monolithic layers accommodate stresses arising from fission product generation. 
Enclosure 4 to

MDO-723-0044 /

B-MT(SRME)-52

Page 3

\begin{tabular}{|c|c|c|c|c|c|c|c|c|}
\hline \multirow[b]{2}{*}{ Capsule } & \multirow{2}{*}{$\begin{array}{c}\text { Dose } \\
\left(\mathrm{n} / \mathrm{cm}^{2} \times 10^{20}\right)\end{array}$} & \multirow[b]{2}{*}{ Color } & \multirow{2}{*}{$\begin{array}{c}\text { Temp. } \\
\text { (K) }\end{array}$} & \multicolumn{5}{|c|}{ Number of Specimens } \\
\hline & & & & $\begin{array}{c}\text { Tensile } \\
\text { (SiC) }\end{array}$ & $\begin{array}{l}\text { Tensile } \\
\text { SiC/SiC } \\
\end{array}$ & $\begin{array}{l}\text { Disc } \\
\text { SiC }\end{array}$ & $\begin{array}{c}\text { Disc } \\
\text { SiC/Sic }\end{array}$ & BSR \\
\hline $\begin{array}{l}\text { Rig A, } \\
\text { Tier } 1\end{array}$ & $40-110$ & & 1100 & & 12 & 6 & 6 & \\
\hline $\begin{array}{l}\text { Rig A, } \\
\text { Tier } 3\end{array}$ & $180-220$ & $\mathbb{W}$ & 1350 & 6 & 6 & 6 & 6 & \\
\hline $\begin{array}{l}\text { Rig A, } \\
\text { Tier } 4\end{array}$ & $92-180$ & & 1350 & 6 & 6 & 6 & 6 & \\
\hline $\begin{array}{l}\text { Rig B, } \\
\text { Tier } 2\end{array}$ & $40-71$ & & 1100 & & & 3 & 3 & 8 \\
\hline $\begin{array}{l}\text { Rig B, } \\
\text { Tier } 4\end{array}$ & $46-89$ & 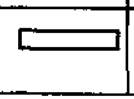 & 1470 & & & 3 & 3 & 8 \\
\hline
\end{tabular}

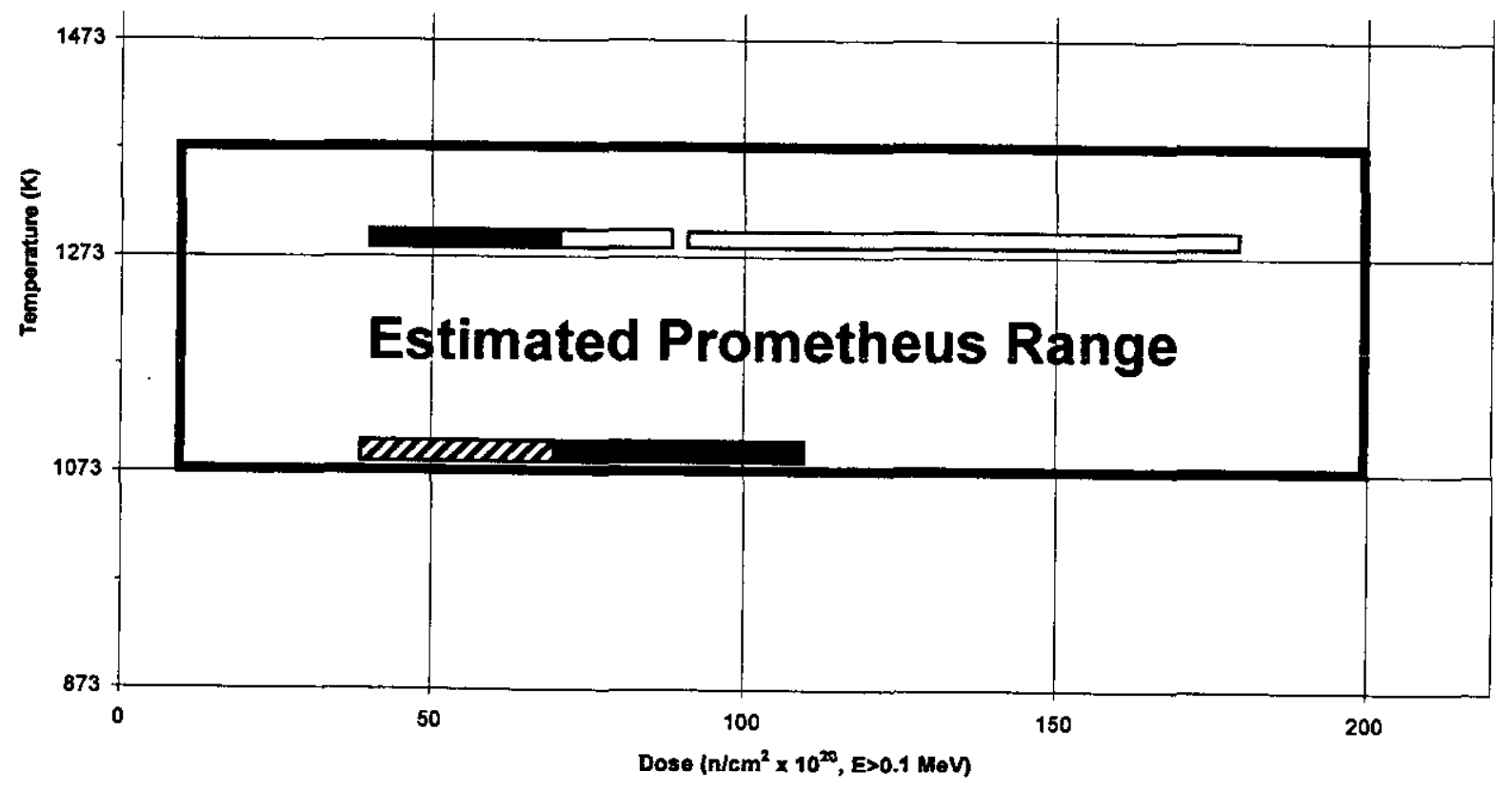

Figure 1: Estimated temperature and fluence range for the Prometheus-1 reactor. Within this range five SiC capsules were to be irradiated in JOYO-1.

JOYO-1 was the NRPCT's initial step to understanding SiC radiation performance in the Prometheus-1 design space (Figure 1). For Prometheus-1, the NRPCT pursued the option of a hermetic ceramic clad design consisting of monolithic and composite SiC materials in a duplex and/or triplex configuration. In order to understand the material and mechanical properties of the laminate cladding, it was necessary to collect data in the Prometheus-1 temperature/dose range. Although the NRPCT would have preferred to irradiate laminate (duplex/triplex) structures in addition to the constituent materials, lamination fabrication efforts were not possible due to the JOYO-1 insertion time-frame. Therefore, the basic constituent materials, monolithic and composite SiC specimens, were prepared for irradiation. The materials included 
a beta-phase monolithic CVD SiC and a composite $\mathrm{SiC}$ consisting of consisted of Hi-Nicalon Type S fibers in a CVI-derived SiC matrix (SiC/SiC). The Hi-Nicalon Type S fibers are nanocrystalline and nearly stoichoimetric. These intrinsic material properties provide stability at extreme conditions, including high temperatures and severe neutron irradiation (Reference 1). Figure 1 shows the estimated Prometheus-1 temperature and fluence ranges and corresponding capsules in the JOYO-1 test matrix. This irradiation test would have provided data for preliminary design and material selection development.

Monolithic $\mathrm{SiC}$ was chosen as a potential cladding material because it has been shown to provide excellent high-temperature irradiation stability and has a high strength/weight ratio. Monolithic $\mathrm{SiC}$ is advantageous in that it can provide a hermetic seal; however, it is brittle and has low fracture toughness. Composite $\mathrm{SiC}$ has significantly improved fracture toughness, although it is challenging to produce hermetic pieces, especially thin sections. Hermetic composites have been fabricated using several techniques: improved or optimized matrix infiltration (e.g., melt infiltration), addition of a hermetic monolithic seal coating, or addition of monolithic layer(s) onto the composite material (e.g., duplex or triplex).

\section{Discussion}

Prior to project restructuring, monolithic and composite SiC specimens were fabricated for the JOYO-1 irradiation test campaign. Miniature tensile specimens, thermal diffusivity discs, and bend stress relaxation specimens were machined from plates of these materials. Design of the miniature tensile specimen permitted the inclusion of a sufficient number of replicates to ensure statistically reliable results for $\mathrm{SiC}$, which may have a large strength variability. Table 1 contains a count of silicon carbide specimens planned for the JOYO-1 insertion. All fabricated specimens and bulk silicon carbide plates have been stored at KAPL.

Table 1: JOYO-1 Silicon Carbide Specimen Count

\begin{tabular}{|c|c|c|c|c|}
\hline Material & $\begin{array}{c}\text { Tensile } \\
\text { Bars }\end{array}$ & $\begin{array}{c}\text { Density \& } \\
\text { Conductivity } \\
\text { Discs }\end{array}$ & $\begin{array}{c}\text { Bend } \\
\text { Stress } \\
\text { Relaxation }\end{array}$ & $\begin{array}{c}\text { Total } \\
\text { Specimen }\end{array}$ \\
\hline Monolithic SiC & 12 & 24 & 16 & 52 \\
\hline Composite SiC & 24 & 24 & - & 48 \\
\hline
\end{tabular}

Monolithic Tensile Specimens and Thermal Diffusivity Discs

Tensile specimens and thermal diffusivity discs were machined from monolithic CVD beta phase $\mathrm{SiC}$ plates (6 in. $\times 6$ in. $\times 1 / 4$ in.) purchased from Rohm and Haas Advanced Materials, Woburn, MA. These plates were delivered with a Certificate of Analysis (Attachment 1.1) providing the intrinsic material properties listed in Table 2.

An independent chemical analysis, via glow discharge mass spectroscopy (GDMS), was performed on the bulk material at Shiva Technologies, Inc, Syracuse, NY. GDMS testing certified that the bulk material did not contain impurities above the Rohm and Haas Advanced Materials material certifications (Specimen A-CAG was consumed in this testing). In addition,

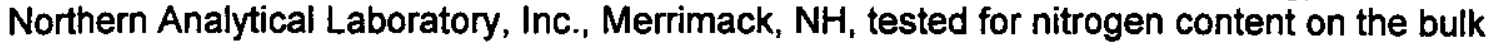
material, resulting in a reading of $0.0114 \pm 0.0006 \%$ (Attachment 1.1 , Specimen A-CAF). Documenting the nitrogen content is required for end-of-life accounting purposes for $\mathrm{SiC}$ specimens and their respective quantities of Carbon-14 produced during irradiation. 
Enclosure 4 to

MDO-723-0044 I

B-MT(SRME)-52

Page 5

Table 2: Bulk material properties provided by Rohm and Haas Advanced Materials for monolithic SiC specimens in the JOYO-1 irradiation test campaign.

\begin{tabular}{|c|c|}
\hline Property & Average Value \\
\hline Grain Size $(\mu \mathrm{m})$ & $7.1-9.4$ \\
\hline Chemical Purity $(\mathrm{ppmw})$ & $<5.74$ \\
Glow discharge mass spectroscopy $(\mathrm{GDMS})$ & \\
\hline Thermal Conductivity $\left(\mathrm{W} \cdot \mathrm{m}^{-1} \cdot \mathrm{K}^{-1}\right)$ & $310 \pm 19$ \\
\hline Flexural Strength $(\mathrm{MPa})$ & $417 \pm 48$ \\
\hline
\end{tabular}

Monolithic SiC thermal diffusivity discs and miniature tensile specimens were machined at PremaTech Advanced Ceramics, Worcester, MA, in accordance with NRPCT technical specification 05PFG093 ${ }_{k}{ }^{1}$. The technical specification included plate identifications, machining instructions, NRPCT issued drawings, requirements for a first piece inspection, and a list of detrimental materials. The machining requirements were based on ASTM E1461-01 for thermal diffusivity testing and ASTM C1275-00 for tensile specimens. Overall, this technical specification included the manufacture of 294 monolithic SiC tensile specimens and 120 thermal diffusivity discs (Attachments 1.2 and 1.3), from which 44 tensile specimens and 30 discs were to be used for the JOYO-1 irradiation test campaign. Specimens not included in JOYO- 1 were intended for future irradiation tests, in addition to out-of-pile and compatibility testing. Prior to machining, engineers from NRPCT and PremaTech Advanced Ceramics completed a readiness review to ensure machining details were understood. Upon agreement of the requirements, a first piece inspection was required to ensure dimensional accuracy within the specified tolerances for two discs and two tensile specimens. Upon receipt of a positive inspection, the balance of the order was completed and delivered. Specimen receipt was accompanied by a dimensional report, and a Certificate of Compliance to provide strict compliance with dimensions and detrimental materials (Attachment 1.3). Photographs of monolithic tensile specimens and thermal diffusivity discs for JOYO-1 irradiation testing are included in Attachment 1.4.

Initial thermal diffusivity and mechanical testing was performed to characterize the baseline properties of the monolithic material. Initial tensile testing was unsuccessful. The specimens broke in the clamps, and data was unattainable. Additional testing would be needed to determine appropriate clamping devices.

Initial thermal diffusivity testing was completed for monolithic $\mathrm{SiC}$ and is included in Figure 2 and Attachment 1.5. Thermal diffusivity was measured under a flowing argon atmosphere in an ANTER FL5000 laser flash unit. The unit was equipped with an Nd-glass laser having a wavelength of $1.06 \mu \mathrm{m}$ and a pulse width of $0.3 \mathrm{~ms}$. Each test run included five $\mathrm{SiC}$ and one graphite reference specimen. Test temperature sequencing in $100^{\circ} \mathrm{C}$ increments included testing from $100^{\circ} \mathrm{C}$ to $1300^{\circ} \mathrm{C}$. Thermal diffusivity of the specimens was analyzed using the Koski method which corrects for heat loss due to radiation (Results are listed in Attachment 1.5). Preliminary thermal diffusivity curves were collected for the JOYO-1 test specimens (Figure 2). Monolithic thermal diffusivity measurements consisted of a negative slope starting with 0.694 $\mathrm{cm}^{2} / \mathrm{sec}$ at $100^{\circ} \mathrm{C}$ and ending with $0.111 \mathrm{~cm}^{2} / \mathrm{sec}$ at $1300^{\circ} \mathrm{C}$. This data is within error of published diffusivity curves in References 2 and 3.

\footnotetext{
${ }^{1}$ The symbol $\mathrm{K}$ indicates a KAPL specific issued document.
} 


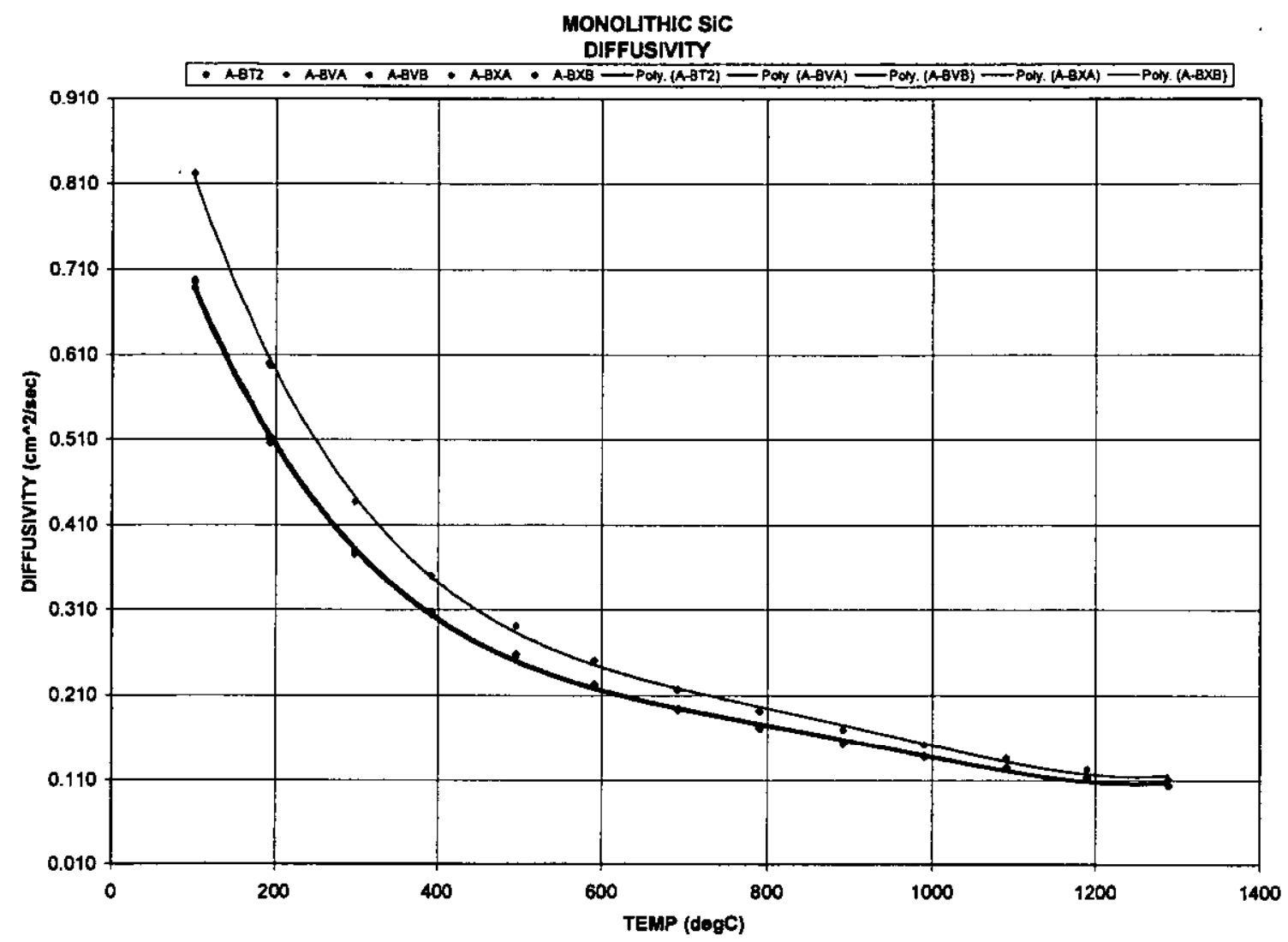

Figure 2: Thermal diffusivity measurements were collected for five monolithic $\mathrm{SiC}$ test specimens from $100^{\circ} \mathrm{C}$ to $1300^{\circ} \mathrm{C}$ at $100^{\circ} \mathrm{C}$ intervals.

\section{Monolithic Bend Stress Relaxation (BSR) Technique}

In addition to miniature tensile specimens and thermal diffusivity discs, Bend Stress Relaxation (BSR) specimens for in-situ estimates of irradiation creep for monolithic SiC were machined for the JOYO-1 irradiation test campaign. It is hypothesized that stress relief occurs during irradiation, thus improving the performance of SiC. Oak Ridge National Laboratory (ORNL) developed the BSR technique to test this theory and estimate the irradiation creep of monolithic $\mathrm{SiC}$ (Reference 4). In this technique, monolithic SiC thin strip specimens are held at a defined radius during irradiation through the use of a fixture which applies stress by holding the specimens at a specified radius during irradiation (Figure 3 ). The holder consisted of 3 plates and 4 pins, and the specimens had a thickness of 50,100, and $200 \mu \mathrm{m}$, corresponding to an applied flexural strength of 100,200 , and $400 \mathrm{MPa}$, respectively. Monolithic SiC was chosen as the bulk material for both holders and specimens to avoid potential chemical interactions. Composite thin strip specimens could not be fabricated into the thin strips needed due to the thickness of the specimens. 
Irradiation creep measurements using the BSR technique is based on the following explanation. Residual stress after relaxation $\left(\sigma_{\mathrm{a}}\right)$ can be determined through measurements of the bend radius before and after irradiation, as shown in the following equation:

$$
\sigma_{a}=\frac{E t\left(\varphi_{Q}-\varphi_{g}\right)}{2 L}
$$

where $E$ is the Young's Modulus, $t$ is the specimen thickness, $L$ the specimen length, $\varphi_{0}$ the initial bend angle and $\varphi_{a}$ the residual bend angle in the freed sample and the initial stress in the sample $\left(\sigma_{0}\right)$. Thus the BSR ratio $(m)$ can be determined using the following equation:

$$
m=\frac{\sigma_{a}}{\sigma_{0}}=1-\frac{\varphi_{a}}{\varphi_{0}}
$$

In this equation, a BSR ratio $(m)$ of zero indicates that no creep occurred, whereas a value greater than zero indicates irradiation induced creep has occurred. An advantage of the BSR technique is its ability to detect small creep rates $\left(\sim 10^{-11} \mathrm{~s}^{-1}\right)$, which are expected for $\mathrm{SiC}$ (Reference 4).
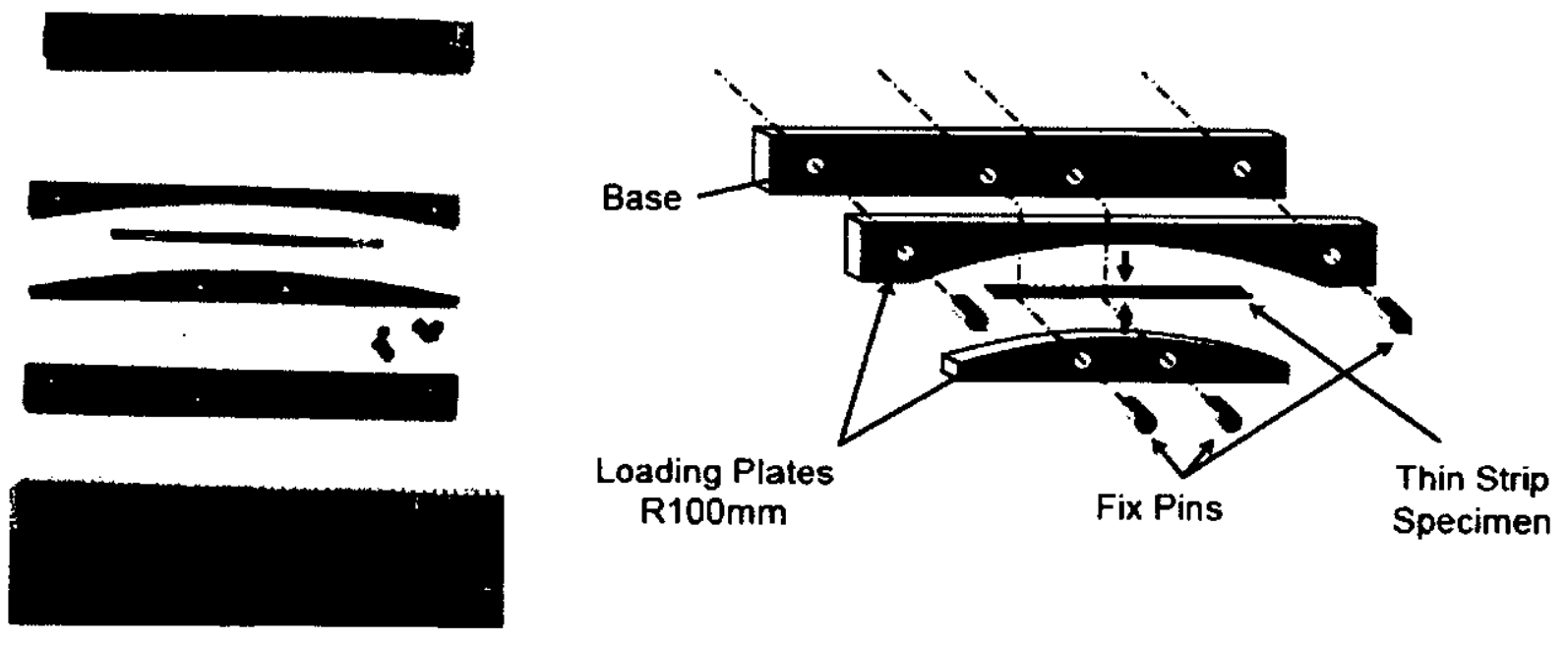

Figure 3: Images of the BSR fixture and thin strip specimens (Reference 4)

The specimens and holders were machined from two separate lots of SiC plates (6 in. $x 6$ in. $x$ $1 / 4$ in.), purchased from Rohm and Haas Advanced Materials Woburn, MA. Holders were machined from a lot of material purchased May 2005 (Table 3 and Attachment 2.1); whereas the thin strip specimens were machined from a plate provided by ORNL (Reference 5).

The BSR technique was tested at ORNL in a series of thermal studies (Reference 4). A portion of the original SiC plate used in the ORNL thermal studies was consumed in the fabrication of the JOYO-1 thin strip specimens. Using this monolithic SiC plate for JOYO-1 specimens allows for a direct material comparison between the historic thermal and future irradiation studies, whereby plate to plate variations are eliminated. 
Enclosure 4 to

MDO-723-0044 I

B-MT(SRME)-52

Page 8

Table 3. Bulk material properties for JOYO-1 monolithic SiC BSR Holders (Procured May 2005).

\begin{tabular}{|c|c|}
\hline Property & Average Value \\
\hline Grain Size $(\mu \mathrm{m})$ & $8.9 \pm 1.2$ \\
\hline $\begin{array}{c}\text { Chemical Purity }(\mathrm{ppmw}) \\
\text { Glow discharge mass spectroscopy }(\mathrm{GDMS})\end{array}$ & $<1.35$ \\
\hline Thermal Conductivity $\left(\mathrm{W} \cdot \mathrm{m}^{-1} \cdot \mathrm{K}^{-1}\right)$ & $295 \pm 3$ \\
\hline Flexural Strength $(\mathrm{MPa})$ & $457 \pm 47$ \\
\hline
\end{tabular}

Prior to machining thin strip specimens, the bulk material plate was annealed for one hour at $1900^{\circ} \mathrm{C}$ in an ultra-high purity argon flow to reduce the concentration of as-deposited defects. These defects are reportedly responsible for anelastic deformation of CVD SiC in the $1000^{\circ} \mathrm{C}$ $1500^{\circ} \mathrm{C}$ range (References 6 and 7). This was a concerning factor because BSR specimens in the JOYO-1 test campaign would have been subjected to irradiation temperatures up to $1100^{\circ} \mathrm{C}$. An $\mathrm{x}$-ray diffraction analysis after annealing confirmed that phase transformation did not occur as no $\alpha-\mathrm{SiC}$ was detected in the plate. This machining required precision, including maintaining a gap of $400 \mu \mathrm{m}+20 /-0 \mu \mathrm{m}$ wide between the curved surfaces of the assembled top and bottom holder plates and machining 200,100 and $50 \mu \mathrm{m}$ thick SiC thin strip samples. Via ORNL-SPP-05-0054 (Reference 5), ORNL contracted the machining work to Morise Sieko Company, Yokohama, Japan, the only known company capable of machining to these specifications with guaranteed accuracy. A total of forty holder assemblies and 180 thin strip specimens were machined. Reference 5 is a final report issued by ORNL (ORNL/LTR/NRPROM 1/05-39) "Preparation of Silicon Carbide Irradiation Creep Specimens and Holders" that provides photographs, dimensions, material description, drawings and inspection results.

\section{Composite SiC Tensile Specimens and Thermal Diffusivity Discs}

Miniature tensile specimens and thermal diffusivity discs were machined from four silicon carbide composite plates $\left(7.25^{\prime \prime} \times 7.25^{\prime \prime} \times 0.125^{\prime \prime}\right)$, produced by Hyper-Therm High-Temperature Composites, Inc., Huntington Beach, CA. The SiC composite consisted of eleven plies of 8harness satin (8HS) weave Hi-Nicalon Type $S$ fiber fabric in a cross-ply $\left(0^{\circ} / 90^{\circ}\right)$ orientation. SiC fiber preforms were coated with a $0.4 \mu \mathrm{m}$ thick multilayer coating system consisting of four alternating layers of pyrolytic carbon (PyC) and $\mathrm{SiC}$. Upon completion of the fiber perform coating the resultant panels were chemical vapor infiltrated to produce a SiC composite (Attachment 3.1).

An independent GDMS chemical analysis was performed on the bulk composite material at Shiva Technologies, Inc., Syracuse, NY (Specimen A-ANW). Testing confirmed that high levels of impurities did not exist in the bulk material. Results were similar to the monolithic specimens. In addition, Northern Analytical Laboratory, Inc., Merrimack, NH, measured the nitrogen content of the bulk material to be $0.0057 \pm 0.0002 \%$ (Specimen A-ANX, Attachment 3.1).

Thermal diffusivity discs and miniature tensile specimens were machined at Machined Ceramics, Inc., Bowling Green, KY to the NRPCT requirements in technical specification 05PFG071 . The document included plate identifications, machining instructions, NRPCT issued drawings, first piece inspection requirements, and a list of detrimental materials. Machining requirements were based on ASTM E1461-01 for thermal diffusivity testing and ASTM C1275-00 for tensile testing. Overall, 288 composite tensile specimens and 120 thermal diffusivity discs were fabricated 
(Attachment 3.2 and 3.3). JOYO-1 irradiation and pre-irradiation specimens, 48 tensile and 30 discs, are listed in Attachment 3.3 along with their unique identification. Prior to machining, engineers from NRPCT and Machined Ceramics, Inc. performed a readiness review to ensure the technical specification details were understood. This review identified that a first piece inspection was required to ensure dimensional accuracy within specified tolerances for two specimens of each type. Upon receipt of a positive inspection, the balance of the order was completed and delivered. Early in the machining process, Machined Ceramics, Inc. contacted the NRPCT technical lead to indicate that the mechanical stop had not been set properly prior to grinding the thickness of plate one. This resulted in a thickness of $1.359 \mathrm{~mm}(0.0535$ inches) for 18 thermal diffusivity discs, rather than the specified thickness of 1.499 to $1.549 \mathrm{~mm}(0.059$ to 0.061 inches). Although this is less than the required dimensions, thermal diffusivity testing is not hindered by this thickness variation; therefore, the specimens were marked accordingly and machining was completed with no other complications. Specimen receipt was accompanied by a dimensional report and a Certificate of Compliance (Attachment 3.3) to ensure strict compliance with dimensions and detrimental materials. Photographs of the composite tensile specimens and thermal diffusivity discs for JOYO-1 irradiation testing are included in Attachment 3.4.

Initial tensile and thermal diffusivity testing of the silicon carbide composite specimens was conducted to characterize the baseline material and mechanical properties (Attachment 3.5). Tensile testing results provided an average ultimate tensile strength of $41.5 \pm 5.38 \mathrm{ksi}$ and elongation of $0.27 \%$ (Table 4). Reference 7 contains published tensile results for a Hi-Nicalon Type S composite, which indicates that the initial NRPCT composite tensile data is within the range of published data. Thermal diffusivity was measured from $100^{\circ} \mathrm{C}$ to $1300^{\circ} \mathrm{C}$, resulting in a thermal diffusivity verses temperature plot (Figure 4). The thermal diffusivity of the composite material resulted in a plate-to-plate difference, within error. Plate 3 measurements consisted of a negative slope starting at $0.063 \mathrm{~cm}^{2} / \mathrm{sec}$ at $100^{\circ} \mathrm{C}$ and ending with $0.019 \mathrm{~cm}^{2} / \mathrm{sec}$ at $1300^{\circ} \mathrm{C}$. Plate 2 diffusivity measurements consisted of a negative slope starting with $0.048 \mathrm{~cm}^{2} / \mathrm{sec}$ at $100^{\circ} \mathrm{C}$ and ending with 0.015 at $1300^{\circ} \mathrm{C}$.

Table 4: Tensile Testing Results for SiC Composite

\begin{tabular}{|c|c|c|c|c|c|}
\hline \multicolumn{5}{|c|}{ MATERIAL: SIC COMPOSITE, HI-NICALON TYPE S FIBERS CVI MATRIX } \\
\hline $\begin{array}{c}\text { SPECIMEN } \\
\text { IDENTITY }\end{array}$ & $\begin{array}{c}\text { MAXIMUM } \\
\text { LOAD (Ibs) }\end{array}$ & $\begin{array}{c}\text { ULT. STRENGTH } \\
\text { (ksi) }\end{array}$ & $\begin{array}{c}\% \text { ELONG } \\
0.5 \text { " G.L. }\end{array}$ & $\begin{array}{c}\text { ORIGINAL } \\
\text { AREA (in }{ }^{2} \text { ) }\end{array}$ & $\begin{array}{c}\text { Elastic } \\
\text { Modulus } \\
\text { (ksi) }\end{array}$ \\
\hline AVERAGE & 463.1 & 41.5 & 0.27 & 0.011 & 25300.84 \\
\hline StDev & 60.4 & 5.38 & 0.09 & $3.83 E-05$ & 4015.86 \\
\hline
\end{tabular}


Enclosure 4 to

MDO-723-0044 /

B-MT(SRME)-52

Page 10

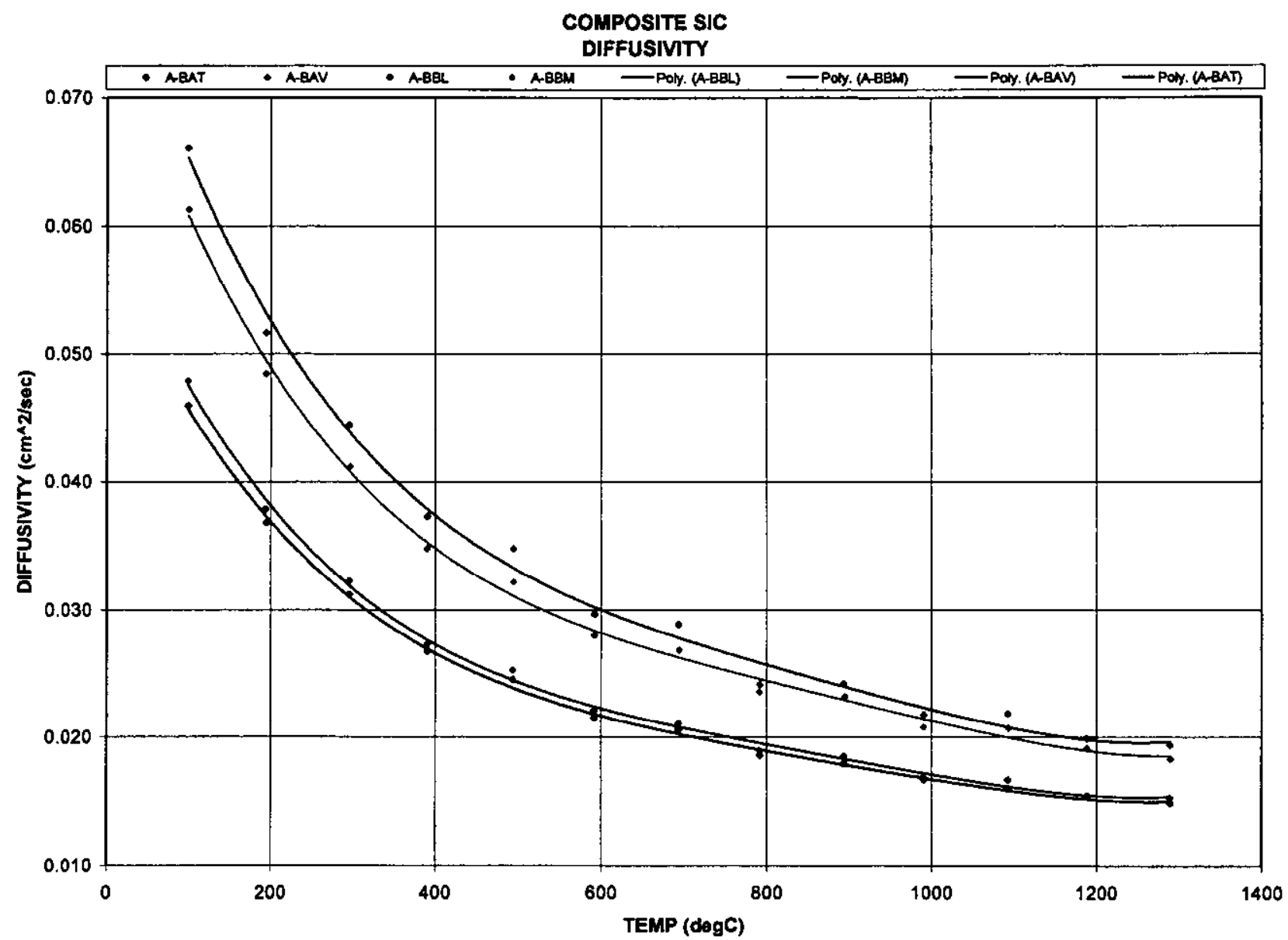

Figure 4: Thermal diffusivity measurements for four composite SiC test specimens were collected from $100^{\circ} \mathrm{C}$ to $1300^{\circ} \mathrm{C}$ at $100^{\circ} \mathrm{C}$ intervals. Specimen A-BBL and A-BBM were from plate 3 and Specimens ABAV and A-BAT were from plate 2. 


\section{References}

1. T. Nozawa, K. Hironaka, T. Taguchi et al., "Tensile Properties of Stoichiometric Silicon Carbide Fiber Reinforced FCVI Derived Silicon Carbide Matrix Composites, "Semi Annual Progress Report Department of Energy Fusion Materials, 31, (2001), 40-46.

2. Advanced Materials Rohm and Haas Co., "CVD Silicon Carbide," Technical Bulletin \#107, (1994).

3. D. Senor, G. Youngblood, L. Greenwood, D. Archer, D. Alexander, M. Chen, and G. Newsome, "Defect Structure and Evolution in Silicon Carbide Irradiated to $1 \mathrm{dpa}-\mathrm{SiC}$ at $1100^{\circ} \mathrm{C}, "$ Joumal of Nuclear Materials, 317, (2003), 145-159.

4. Y. Katoh and L. L. Snead, "Bend Stress Relaxation Creep of CVD Silicon Carbide," Ceram. Eng. Sci. Proc., 26, 2, (2005), 265-272.

5. L. Snead and Y. Katoh, "Preparation of Silicon Carbide Irradiation Creep Specimens and Holders", ORNLLTR/NR-PROM 1/05-391/05, (10/2005).

6. J.A. DiCarlo, "Creep of Chemically Vapour Deposited SiC Fibers," Joumal of Material Science, 21, (1986), 217-224.

7. C.H. Carter, Jr., R.F. Davis, and J. Bentley, "Kinetics and Mechanisms of High Temperature Creep in Silicon Carbide: II, Chemically Vapor Deposited," Joumal of American Ceramics Society, 67, (1984), 732-740.

8. Y. Katoh, T. Nozawa and L. L. Snead, "Mechanical Properties of Thin Pyrolitic Carbon Interphase SiC-Matrix Composites Reinforced with Near Stoichiometric SiC Fibers," Joumal of American Ceramics Society, 88, 11, (2005) 3088-3095. 
Attachment to

Enclosure 4 to

MDO-723-0044 I

B-MT(SRME)-52

Page 1

\section{Attachments}

Machining Documentation for

Monolithic Silicon Carbide Tensile Specimens and Thermal Diffusivity Discs

1.1 Material Purchase Documentation and Certification

1.2 Technical Specification for Machining

1.3 Machining Documentation

1.4 Specimen Photographs

1.5 Thermal Diffusivity Data

\section{Machining Documentation for Bend Stress Relaxation Technique}

2.1 Material Purchase Documentation and Certification

Machining Documentation for

Composite Silicon Carbide Tensile Specimens and Thermal Diffusivity Discs

3.1 Material Purchase Documentation and Certification

3.2 Technical Specification for Machining

3.3 Machining Documentation

3.4 Specimen Photographs

3.5 Tensile Specimens and Thermal Diffusivity Discs

Enclosure 4 to MDO-723-0044/B-MT(SRME)-52 
Attachment 1.1 to Enclosure 4 to MDO-723-0044/ B-MT(SRME)-52

Page 1

Attachment 1.1

Material Purchase Documentation and Certification

Monolithic SiC Tensile Specimens and Thermal Diffusivity Discs

Enclosure 4 to MD0-723-0044/B-MT(SRME)-52 


\section{KAPL, Inc.}

O. Box 1072

chenectady, New York 12301-1072
PAGE 1 OF 1

PURCHASE ORDER NUMBER

KN6002216

PURCHASE ORDER DATE

29-APR-2005

IMPORTANT: Show Purchase Order Number on all correspondence. Complete packing fist must accompany each ahlpment.

Effective Date: Expiratlon Date:

KAPL, INC., A LOCKHEEO MARTIN COMPANY, OPERATES THE KNOLLS ATOMIC POWER LABORATORY UNDER GOVERNMENT CONTRACT DE-AC-12-OOSN39357.

5 ROHM \& HAAS ADVANCED MATERIALS

185 NEW BOSTON STREET

P WOBURN, MA 01810

United Statos

Confirmed BY:

5 KNOLLS RECEVNG BLDG M2

H CENTRAL RECENING - KNO

P 2401 RIVER ROAD

SCHENECTADY,NY 12309

I

\begin{tabular}{|l|l}
\hline ine & \multicolumn{1}{|c}{ Description } \\
\hline 1 & MORTON CVO PLATES 6" $x$ \\
& $6^{\prime \prime} \times 1 / 8^{*}$ THICK \\
& NNSTRUCTIONS TO \\
& PURCHASE ORDER \\
KNGO02216 SHALL APPLY.
\end{tabular}

MATERIAL CERTIFICATIONS

FOR ITEM 1

INSTRUCTIONS TO

PURCHASE ORDER

KN6002216 SHALL APPLY.

PRICE INCLUDED IN PRICE

OF ITEM 1

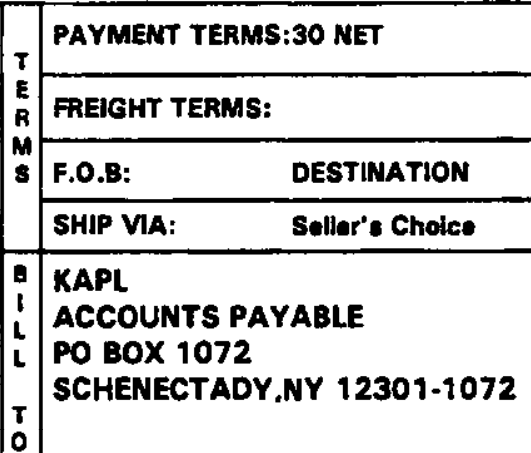

Promised Date

Unh Price

1727

$17,270.00$

Total Price

.

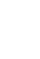

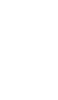




\begin{tabular}{|l|l|l|r}
\hline $\begin{array}{l}\text { Purchase } \\
\text { Order }\end{array}$ & $\begin{array}{l}\text { Document No. } \\
\text { KN6002216 }\end{array}$ & Document Tite & $\begin{array}{r}\text { Attachment } 1.1 \text { to } \\
\text { Enclosure } 4 \text { to } \\
\text { MDO-723-0044/ } \\
\text { B-MT(SRME)-52 } \\
\text { Page 3 }\end{array}$ \\
\cline { 2 - 4 } & $\begin{array}{r}\text { Buyer Name } \\
\text { CJ THOMAS }\end{array}$ & & Pag97 \\
\hline
\end{tabular}

INSTRUCTIONS TO PURCHASE ORDER KN6002216

\section{THIS IS A FIRM FIXED PRICE ORDER}

I. Material andor Service Required:

Provide the line items in accordance with the instructions listed below. The specifications, standards, drawings and documents listed and referred to in these instructions are incorporated by reference and made a part hereof.

II. Delivery Requirements:

As set forth below:

A. Item 1 - two (2) to four (4) each with Item 2 on or before two (2) weeks after date of order.

B. Balance of Item 1 and with Item 2 - on or before four (4) weeks after date of order.

III. Applicable Documents:

The following documents with all documents referenced therein form a part of this order:

A. Commercial Terms and Conditions, dated 8/97.

IV. Modifications to Instruction III:

A. Commercial Terms and Conditions is hereby modified as follows:

1. With respect to the "Changes" Article, only the individuals identified below are authorized to issue and sign change orders and amendments for Buyer. You will be notified in writing by the Manager, Supply Chain Management of any changes of named individuals:

Position

Name

Manager, Supply Chain Management

Manager, Technology Sourcing

E.M. Audi

Senior Contract Administrator, Technology Sourcing

Telephone

D.R. Devenpeck

Fax

(518) $395-6649$

(518) 395-7579

V. The IV System:

Contractual communications shall be accomplished by means of the following documents:

A. Inquiry to Vendor (IV) - used for requested for quotations covering additional work.

B. Information to Vendor (IV) - used for drawing or process approval, transmittal of minutes of meeting, confirmation of phone conversations and other general subjects of communication.

One copy of the applicable IV must be signed and returned to the cognizant Contract Administrator within five (S) working days after receipt. 
ROHM AND HAAS COMPANY

185 NEW BOSTON STREET. WOBUAN, MA Q1801-6278 USA

TELEPHONE: [781] 933-9243 FAX: (781) 933-5142

\title{
PART INSPECTION REPORT
}

\author{
CUSTOMER ORDER \#: KN6002216 O \\ DATE: 04/30/05 \\ R\&H W.O. \#: \\ 1024702 \\ PART NAME: PLATE \\ DRAWING \#: \\ NA \\ MATERIAL: CYD SILICON CARBIDE ${ }^{T M}$ INSPECTOR: C. BEDARD
}

\begin{tabular}{|c|c|c|}
\hline \multicolumn{2}{|c|}{$\mathbf{S} / \mathbf{N}:$} & NA \\
\hline \multicolumn{2}{|c|}{ Source Lot: } & 9112 \\
\hline \multicolumn{2}{|c|}{ Specincations: } & $\begin{array}{c}\text { SPECIFICATIONS IN } \\
\text { MILLIMETERS }\end{array}$ \\
\hline & Speciflestion & ACCEPT \\
\hline & 6.00 & $\mathrm{ACC}$ \\
\hline $\mathbf{B}$ & 6.00 & $\mathrm{ACC}$ \\
\hline C & .125 & $\mathrm{ACC}$ \\
\hline D & VISUAL & $\mathrm{ACC}$ \\
\hline
\end{tabular}

\section{COMMENTS:}




\section{Certificate of Analysis}

Material: CVD SILICON CARBIDE@

Lot No.:9112

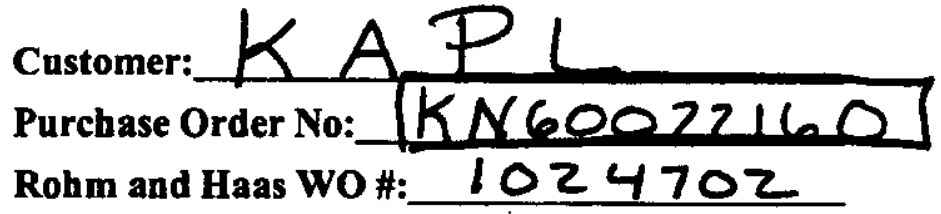

Run No_9112 was performed in our Weeks Island, LA, SiC facility.

One sample each was tested for grain size, microstructure and total trace impurities, six samples were tested for thermal conductivity, and thirty samples were prepared for flexural strength (modulus of rupture) testing. Measured results are listed below.

\section{Property}

Grain size ( $\mu \mathrm{m})$ :

Chemical purity (ppmw): (glow discharge mass spectroscopy)

Thermal Conductivity $\left(\mathbf{W} \cdot \mathbf{m}^{-1} \cdot K^{-1}\right)$

Flexural Strength (MPa)

\section{Average Value}

7.1-9.4

$<5.74$

$310 \pm 19$

$417 \pm 48$

\section{Conclusions}

CVD SILICON CARBIDE@ from lot Run No. 9112 is a typical high purity, polycrystalline, pure $\beta$-phase $\mathrm{SiC}$ produced at Rohm and Haas Advanced Materials. It has been processed in accordance with the manufacturing procedures for CVD SILICON CARBIDEB maintained by Rohm and Haas Advanced Materials, and was produced to meet the nominal material properties for CVD SILICON CARBIDEß as listed on our data sheet.

\section{MATERIAL FROM THIS LOT HAS BEEN ACCEPTED FOR USE TOWARDS ALL CUSTOMER ORDERS.}

Roman Baran

QA/QC Department

Date: January 12, 2004 
FA Artachment 1.1 to

Customer: Knolls Atomic Power Laboratory

Date:

2-Nov-05

Customer ID: SIC

\section{A-CAG}

Element Concentration [pom wt ]

\begin{tabular}{|c|c|c|c|}
\hline & L pom wt & & [ppm wt \\
\hline $\mathrm{Li}$ & $<0.01$ & $\overline{P d}$ & $<0.01$ \\
\hline $\mathrm{Be}$ & $<0.005$ & $\overline{A g}$ & $<0.5$ \\
\hline $\bar{B}$ & 0.48 & $\mathrm{Cd}$ & $<0.5$ \\
\hline $\bar{c}$ & Matrix & $\ln$ & $<1$ \\
\hline $\mathrm{N}$ & -100 & sn & $<0.05$ \\
\hline 0 & $\sim 35$ & $\mathrm{Sb}$ & $<0.05$ \\
\hline $\mathrm{F}$ & $<0.5$ & $\mathrm{Te}$ & $<0.05$ \\
\hline $\mathrm{Na}$ & $<0.01$ & 1 & $<0.01$ \\
\hline$\overline{\mathrm{Mg}}$ & $<0.01$ & $\overline{\mathrm{Cs}}$ & $<0.005$ \\
\hline A) & 0.32 & $\mathrm{Ba}$ & $<0.005$ \\
\hline$\overline{S i}$ & Matrix & La & $<0.005$ \\
\hline$P$ & 0.02 & $\mathrm{Co}$ & $<0.01$ \\
\hline S & 0.05 & $\mathrm{Pr}$ & $<0.01$ \\
\hline $\mathrm{Cl}$ & 0.06 & Nd & $<0.005$ \\
\hline $\mathrm{K}$ & $<0.05$ & $\mathrm{Sm}$ & $<0.005$ \\
\hline$\overline{\mathrm{C} a}$ & 0.07 & $E u$ & $<0.005$ \\
\hline $\mathrm{sc}$ & $<0.01$ & $\mathrm{Gd}$ & $<0.005$ \\
\hline $\mathrm{Ti}$ & $<0.01$ & $\mathrm{~Tb}$ & $<0.005$ \\
\hline $\mathrm{V}$ & $<0.005$ & Dy & $<0.005$ \\
\hline $\mathrm{Cr}_{r}$ & $<0.5$ & $\mathrm{Ho}$ & $<0.005$ \\
\hline $\mathrm{Mn}$ & $<0.01$ & $\mathrm{Er}$ & $<0.005$ \\
\hline $\mathrm{Fe}$ & 0.05 & $\mathrm{Tm}$ & $<0.005$ \\
\hline Co & $<0.005$ & $Y b$ & $<0.005$ \\
\hline$\overline{\mathrm{Ni}}$ & 0.22 & Lu & $<0.005$ \\
\hline $\mathrm{Cu}$ & $<0.05$ & $\mathrm{Hf}$ & $<0.01$ \\
\hline $\mathrm{Zn}$ & $<0.05$ & $\mathrm{Ta}$ & Source \\
\hline $\mathrm{Ga}$ & $<0.05$ & $\bar{W}$ & $<0.01$ \\
\hline $\mathrm{Ge}$ & $<0.05$ & $R_{e}$ & $<0.01$ \\
\hline As & $<0.05$ & Os & $<0.01$ \\
\hline $\mathrm{Se}$ & $<0.5$ & Ir & $<0.01$ \\
\hline $\mathrm{Br}$ & $<0.5$ & $\overline{\mathrm{Pt}}$ & $<0.01$ \\
\hline$\overline{R b}$ & $<0.1$ & $\overline{A u}$ & $<0.05$ \\
\hline $\mathrm{Sr}$ & $<0.01$ & $\mathrm{Hg}$ & $<0.05$ \\
\hline$Y$ & $<0.01$ & TI & $<0.01$ \\
\hline $\mathrm{Zr}$ & $<0.01$ & $\mathrm{~Pb}$ & $<0.01$ \\
\hline $\mathrm{Nb}$ & $<0.01$ & $\mathrm{Bi}$ & $<0.01$ \\
\hline Mo & $<0.05$ & Th & $<0.001$ \\
\hline$\overline{R u}$ & $<0.01$ & U & $<0.001$ \\
\hline $\mathrm{Rh}$ & $<0.005$ & & \\
\hline
\end{tabular}

NOTE: $\sim$ Semiquantitative Values

$\begin{array}{ll}\text { P.O.\# } & \text { KN6004044 } \\ \text { Job \# } & \text { U04798 }\end{array}$

Shiva ID: $\quad$ U051026148 


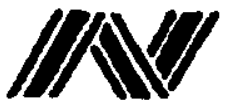

NORTHERN ANALYTICAL LABORATORY, INC.

\section{TEST REPORT}

23 Depot St., Merrimack, NH 03054

Phone: (603) 429-9500

FAX: (603) 429-9471

Ms. Rebecal Northey

KAPL, Ine.

PO Box 1072

Schenectady, New York 1230!-1072
OPRE

my! ? mos

4 i.

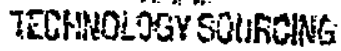

$\begin{array}{llll}\text { RECEIVED } & \text { IO/2S/OS } & \text { SAMPLE NUMBER } & \text { L30129 } \\ \text { IDENT AS } & \text { SER below } & \text { REPORT DATE } & 11 / 7105 \\ \text { MATERIAL } & \text { SiC } & \text { PAGE } & \text { I Of } 1 \\ \text { CONDITION } & \text { Solid } & \text { CLIENT ORDER } & \text { KN6004010 }\end{array}$

TEST TO

TEST PER

TP-LECOI

Method:

Stmple ID.

A-ANX

A-CAP

$0.0114 \pm 0.0006$

\section{Lesco}

N(\%)

$0.0057 \pm 0.0002$
Analysic 8y:

Peter S. Dieksort Analytical Chemi?

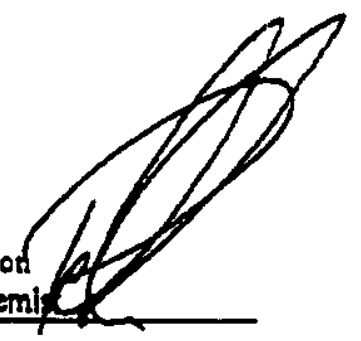

Approved By: Willizan. Guidoboni

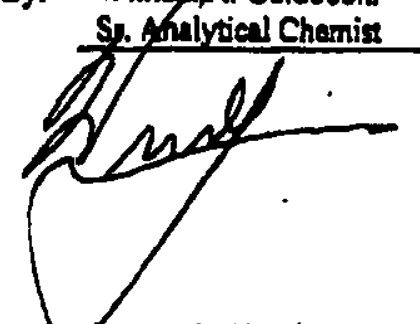

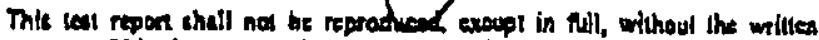

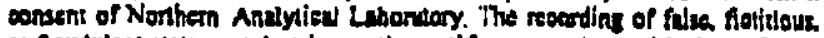

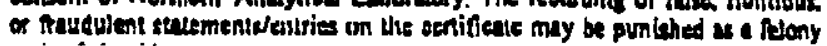


Attachment 1.2 to

Enclosure 4 to MDO-723-0044I

B-MT(SRME)-52

Page 1

Attachment 1.2

Technical Specifications for Machining

Monolithic SiC Tensile Specimens and Thermal Diffusivity Discs

Enclosure 4 to MDO-723-0044/B-MT(SRME)-52 


\title{
SPECIFICATION FOR 05PFG093
}

\author{
Rev. -
}

DATED 5/23/05

1. KAPL will supply the monolithic plates as described below:

\begin{tabular}{|l|l|l|l|l|l|}
\hline NO. & PLATE ID & $\begin{array}{l}\text { PLATE } \\
\text { TYPE }\end{array}$ & $\begin{array}{l}\text { LENGTH } \\
\text { (IN) }\end{array}$ & $\begin{array}{l}\text { WIDTH } \\
\text { (IN) }\end{array}$ & $\begin{array}{l}\text { THICKNESS } \\
\text { (IN) }\end{array}$ \\
\hline 1 & KN6002216-1 & Morton & 6 & 6 & -0.125 \\
(6 Plates) & KN6002216-2 & CVD Plates & & & \\
& KN6002216-3 & & & \\
& KN6002216-4 & & & & \\
& KN6002216-5 & & & & \\
& KN6002216-6 & & & & \\
\hline
\end{tabular}

\section{APPLICABLE DRAWINGS AND SKETCHES}

- KAPL Drawing 216C6883 Rev, C Diffusivity ITEM NO. 2 and 6.

- KAPL Drawing SK239B9368 Rev. - Miniature Tensile Specimen

- KAPL Drawing 108E7300 Rev. - Default Manufacturing Practices.

- KAPL Orientation Sketch NO. 1

- KAPL Specification, Attachment 1 "Detrimental Materials Prohibitions and Restriction for Silicon Carbide Composite Materials" Dated 3/6/03.

- KAPL supplied Table 1 'Machining Instruction Sheet.

\section{MACHINING INSTRUCTION}

- For each piece of GFM plate, extract and machine the required number of specimens as specified in the attached table 1 "Machining Instruction".

- The location and number of specimens per plate are shown in orientation sketch NO. 1 and were specified to maximize the available GFM material. Before extracting samples, the seller shall layout its cuts to verify the quantity of each plate and report to KAPL its layout for approval.

- Samples shall not be machined from the outer $1 / 8$ " perimeter of the GFM plate.

- Stock removal rate shall not exceed $0.03 \mathrm{~mm}$ per pass to the last $0.06 \mathrm{~mm}$. Final finishing shall be preformed with diamond tools that have between 320 and 600 grit. No less than $0.06 \mathrm{~mm}$ per face shall be removed during the final finishing phase and at a rate not more than $0.002 \mathrm{~mm}$ per pass. 
- Remove approximately equal stock from opposite faces for both thermal diffusivity and miniature tensile specimens.

- The miniature tensile specimen shall have chamfered edges with the following dimensions; $0.0035-0.0059 \times 40^{\circ}-50^{\circ}$.

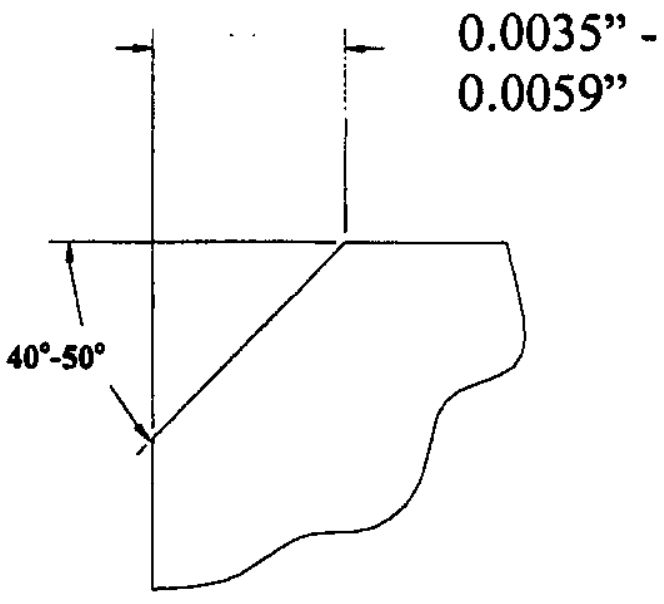

- The surface finish for the monolithic miniature tensile specimen shall be $16 \mu$ in all over except end faces which may be $32 \mu$ in.

- Final grind of gage section shall be longitudinal.

- Compliance with KAPL Specification on detrimental materials is required during . all aspects of this fabrication.

- The seller shall use dedicated resin-bonded diamond tooling for all machining of silicon carbide specimens. If other than resin-bonded tooling is need the seller shall obtain KAPL's approval prior to fabrication.

- The seller shall also use new coolant for this work scope.

- All specimens shall be identified by a bag and tag method with plate ID, specimen type, specimen ID and drawing number.

- All specimens from each plate shall be packaged and labeled together.

- The seller shall optimize GFM material usage and return all excess GFM greater than $0.5^{\prime \prime} \times 0.5^{\prime \prime}$.

- There shall be a verbal briefing prior to the start of machining.

\section{FIRST PIECE INSPECTION}

Two specimens of each type shall be provided for a first piece inspection to assure that they meet all of the requirements of KAPL Drawings prior to proceeding to the next piece. The seller shall notify KAPL with results of the first piece inspection prior to completing the fabrication on the balance of the order. KAPL requests the seller to provide to KAPL the 
TABLE 1

Machining Instruction Sheet 05PFG093

\begin{tabular}{|c|c|c|c|c|c|}
\hline \multicolumn{2}{|l|}{ PLATE ID } & \multirow{2}{*}{$\begin{array}{l}\text { SPECIMEN } \\
\text { TYPE } \\
\text { Miniature } \\
\text { Tensile }\end{array}$} & \multirow{2}{*}{$\begin{array}{l}\text { SPECIMEN } \\
\text { ID's } \\
1-56\end{array}$} & \multirow{2}{*}{$\begin{array}{l}\text { QUANTITY } \\
56\end{array}$} & \multirow{2}{*}{$\begin{array}{l}\text { DRAWING } \\
\text { NO. } \\
\text { Dwg. } \\
\text { SK239B9368 } \\
\text { Rev. - } \\
\end{array}$} \\
\hline \begin{tabular}{|l} 
KN6002216 \\
-1 \\
Plate \# 1 \\
\end{tabular} & $\begin{array}{l}\text { Morton CVD } \\
\text { Plates }\end{array}$ & & & & \\
\hline & & $\begin{array}{l}\text { Thermal } \\
\text { Diffusivity }\end{array}$ & $1-25$ & 25 & \begin{tabular}{|l|} 
Dwg. \\
216C6883 \\
ITEM 2 \\
\end{tabular} \\
\hline & & \begin{tabular}{|l|} 
Thermal \\
Diffusivity
\end{tabular} & $1-3$ & 3 & \begin{tabular}{|l|} 
Dwg. \\
216C6883 \\
ITEM 6 \\
\end{tabular} \\
\hline \multirow[t]{3}{*}{$\begin{array}{l}\text { KN6002216 } \\
-2 \\
\text { Plate \# } 2 \\
\end{array}$} & $\begin{array}{l}\text { Morton CVD } \\
\text { Plates }\end{array}$ & \begin{tabular}{|l|} 
Miniature \\
Tensile
\end{tabular} & $1-56$ & 56 & $\begin{array}{l}\text { Dwg. } \\
\text { SK239B9368 } \\
\text { Rev. - } \\
\end{array}$ \\
\hline & & $\begin{array}{l}\text { Thermal } \\
\text { Diffusivity }\end{array}$ & $1-25$ & 25 & \begin{tabular}{|l|} 
Dwg. \\
216C6883 \\
ITEM 2 \\
\end{tabular} \\
\hline & & $\begin{array}{l}\text { Thermal } \\
\text { Diffusivity }\end{array}$ & $1-3$ & 3 & \begin{tabular}{|l|} 
Dwg. \\
216C6883 \\
ITEM 6 \\
\end{tabular} \\
\hline \multirow[t]{3}{*}{\begin{tabular}{|l} 
KN6002216 \\
-3 \\
Plate \# 3 \\
\end{tabular}} & $\begin{array}{l}\text { Morton CVD } \\
\text { Plates }\end{array}$ & $\begin{array}{l}\text { Miniature } \\
\text { Tensile }\end{array}$ & $1-56$ & 56 & $\begin{array}{l}\text { Dwg. } \\
\text { SK239B9368 } \\
\text { Rev. - } \\
\end{array}$ \\
\hline & & $\begin{array}{l}\text { Thermal } \\
\text { Diffusivity }\end{array}$ & $1-25$ & 25 & $\begin{array}{l}\text { Dwg. } \\
\text { 216C6883 } \\
\text { ITEM } 2 \\
\end{array}$ \\
\hline & & $\begin{array}{l}\text { Thermal } \\
\text { Diffusivity }\end{array}$ & $1-3$ & 3 & $\begin{array}{l}\text { Dwg. } \\
216 \mathrm{C} 6883 \\
\text { ITEM } 6 \\
\end{array}$ \\
\hline \multirow[t]{3}{*}{\begin{tabular}{|l}
$\mathrm{KN} 6002216$ \\
-4 \\
Plate \# 4 \\
\end{tabular}} & $\begin{array}{l}\text { Morton CVD } \\
\text { Plates }\end{array}$ & $\begin{array}{l}\text { Miniature } \\
\text { Tensile. }\end{array}$ & $1-56$ & 56 & $\begin{array}{l}\text { Dwg. } \\
\text { SK239B9368 } \\
\text { Rev. - } \\
\end{array}$ \\
\hline & & $\begin{array}{l}\text { Thermal } \\
\text { Diffusivity }\end{array}$ & $1-25$ & 25 & $\begin{array}{l}\text { Dwg. } \\
\text { 216C6883 } \\
\text { ITEM 2 } \\
\end{array}$ \\
\hline & & $\begin{array}{l}\text { Thermal } \\
\text { Diffusivity }\end{array}$ & $1-3$ & 3 & $\begin{array}{l}\text { Dwg. } \\
\text { 216C6883 } \\
\text { ITEM } 6\end{array}$ \\
\hline
\end{tabular}




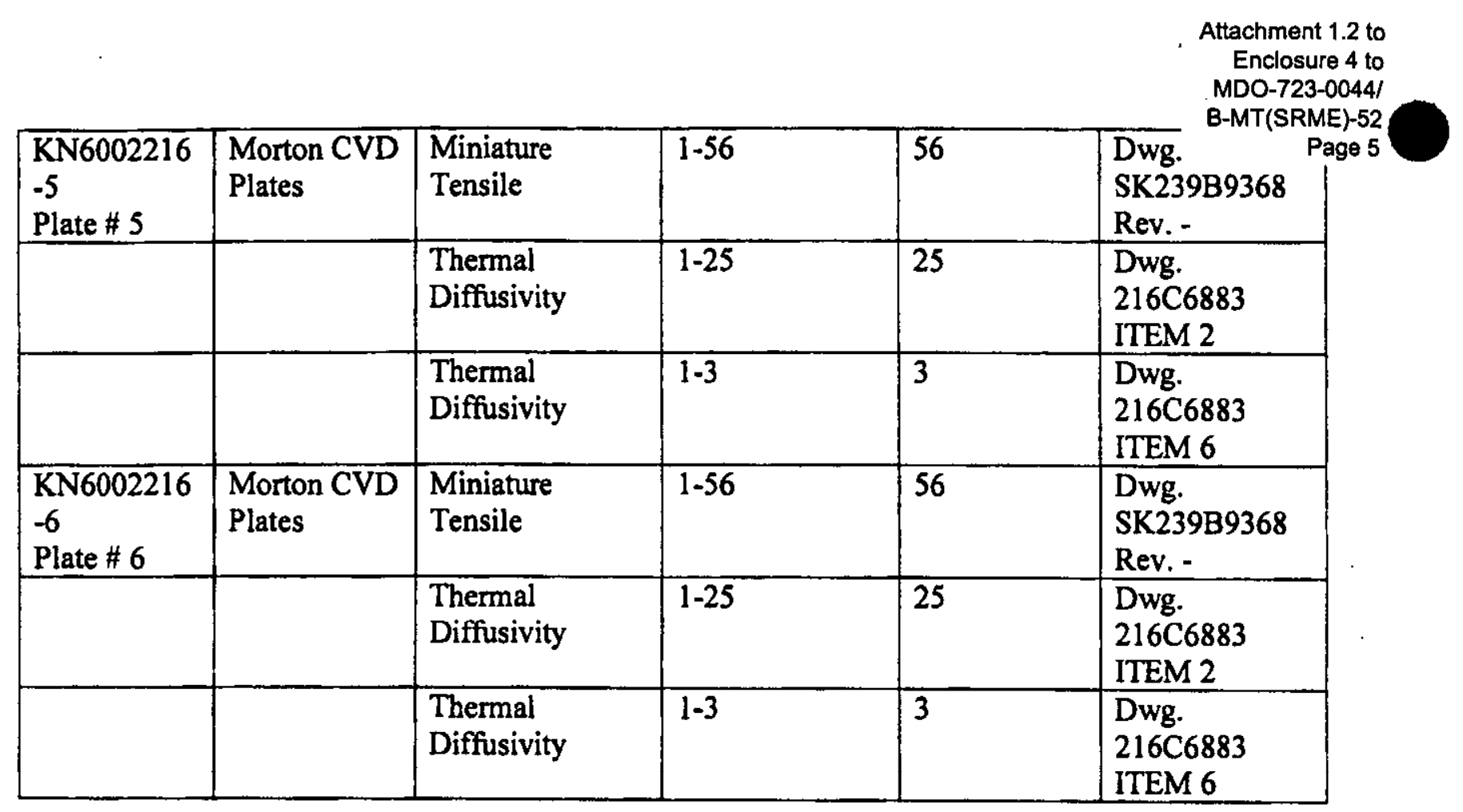

\section{DATA REQUIREMENTS}

- Complete As-Built data for all specimens (Miniature Tensile and Diffusivity).

- Certs of Compliance with KAPL Specification on detrimental materials (Attachment 1 dated 3/6/03).

- Orientation layout.

- First piece inspection notification.

Prepared By

Reviewed By 
Attachment 1.2 to

Enclosure 4 to

MDO-723-0044/

B-MT(SRME)-52

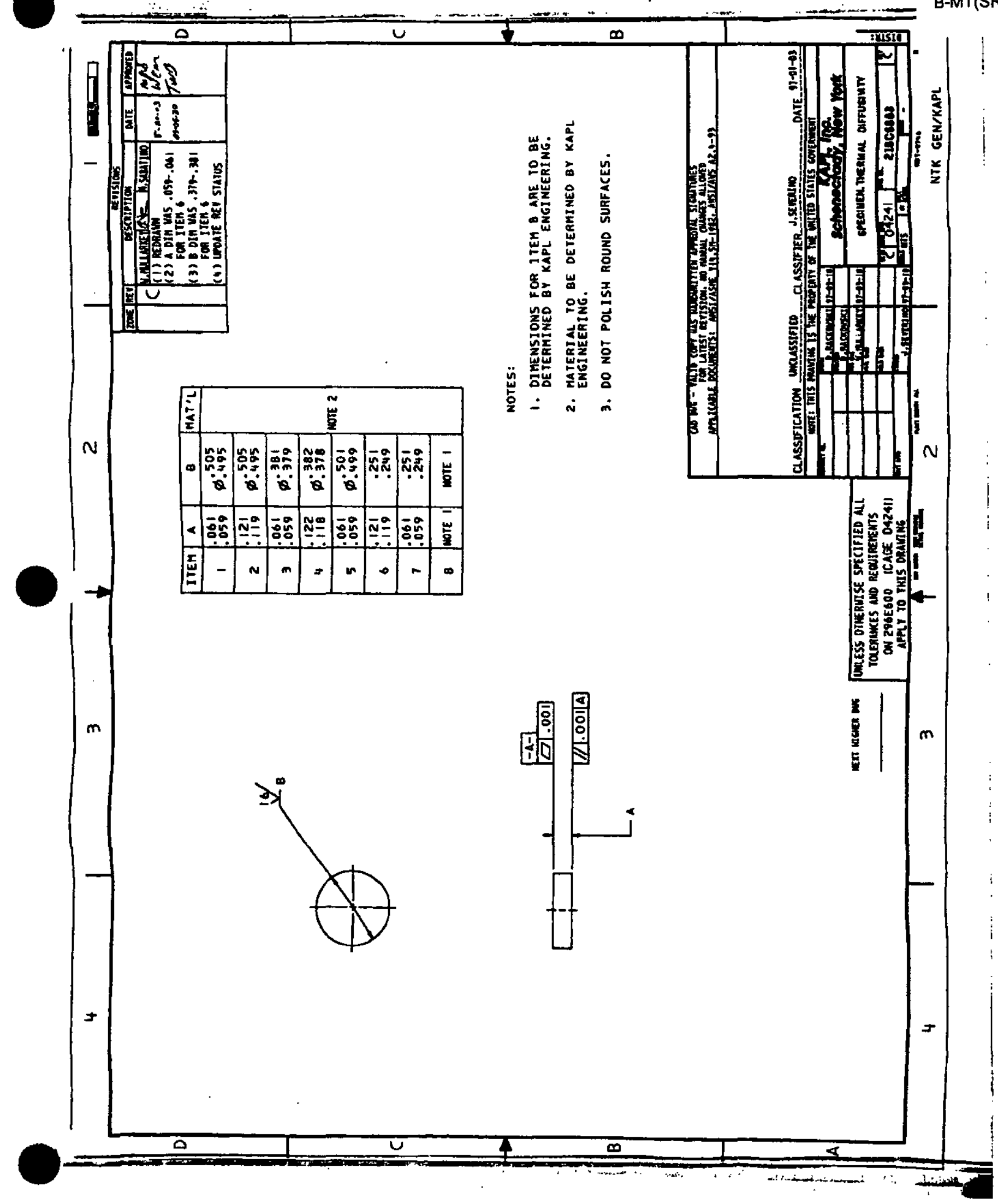

Page 6 


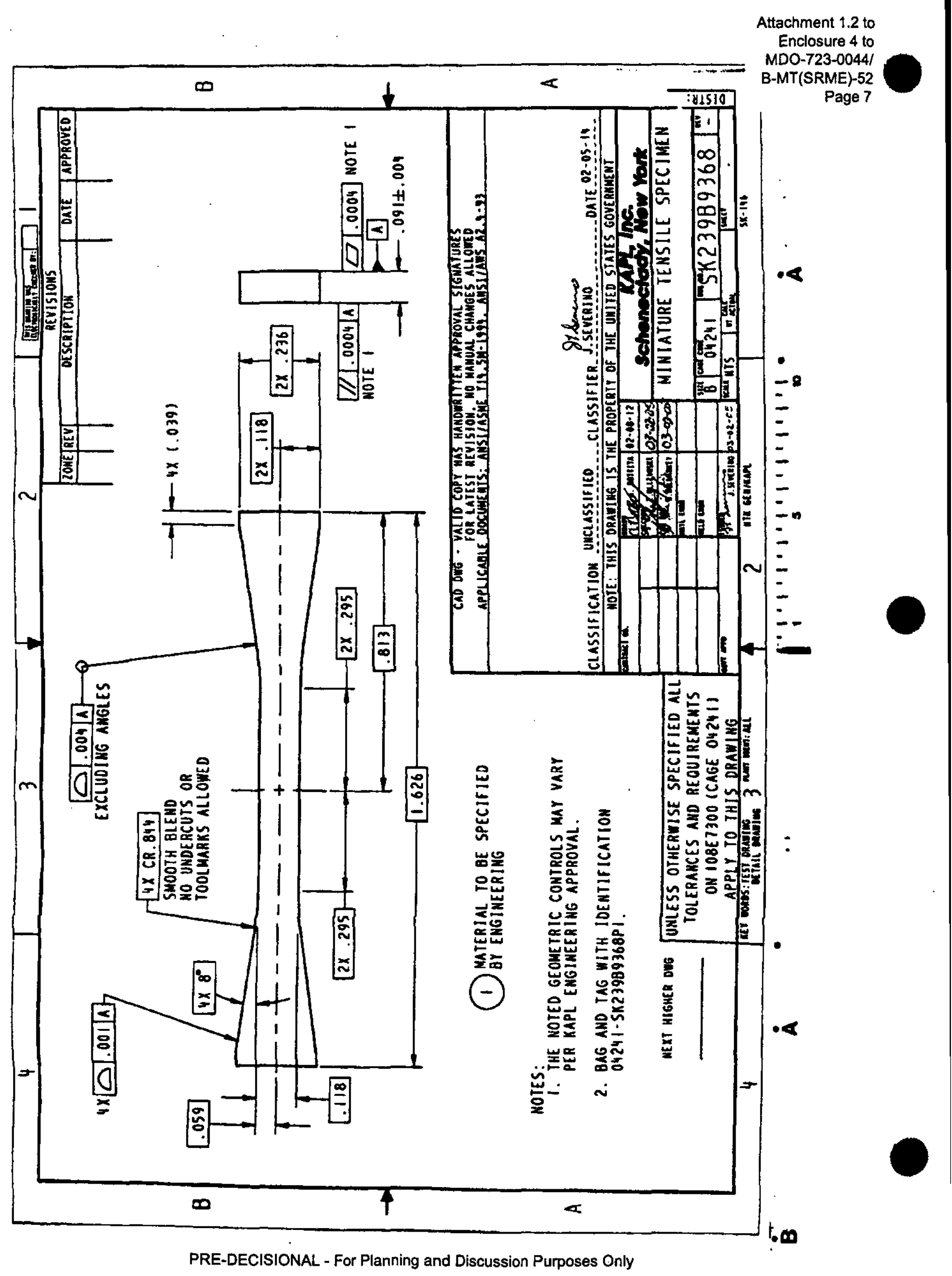


Attachment 1.2 to

Enclosure 4 to

MDO-723-0044I

B-MT(SRME)-52

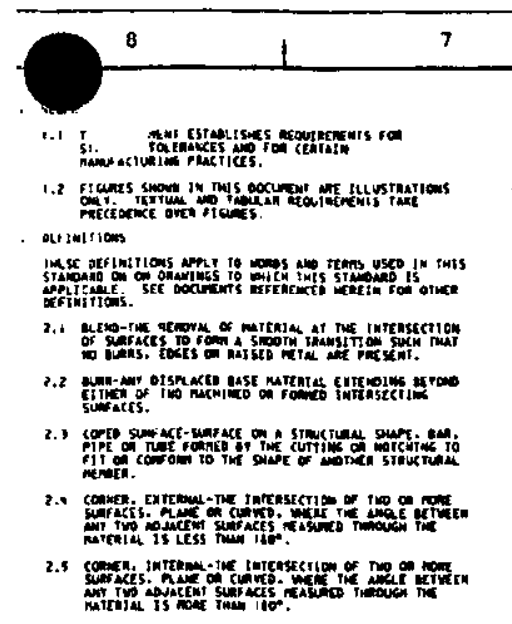

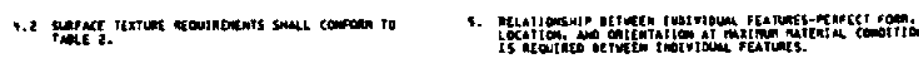

\begin{tabular}{|c|c|}
\hline 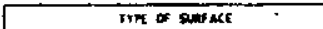 & 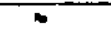 \\
\hline 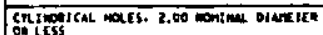 & $\overline{\mathbf{z a}}$ \\
\hline 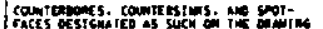 & ise \\
\hline Cotos sumates & $E$ \\
\hline 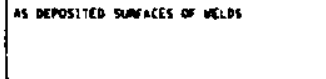 & 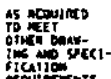 \\
\hline siocin materians & 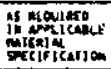 \\
\hline Lotuth & $\mathrm{Im}$ \\
\hline
\end{tabular}

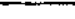

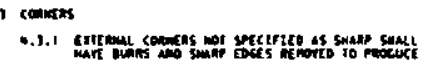

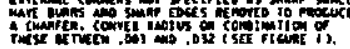

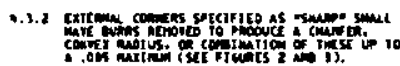

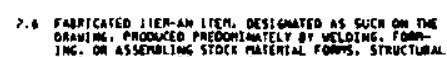

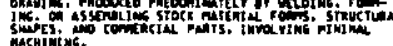

nask moutherems

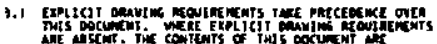

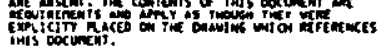

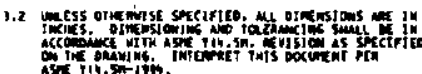

D.) marrues ane contruss

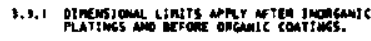

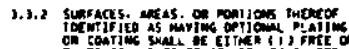
soint

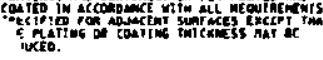

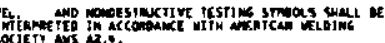

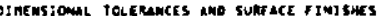

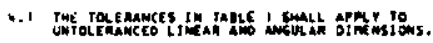

\begin{tabular}{|c|c|c|c|c|}
\hline \\
\hline Dingasiom & upen & $4-14$ & onet 11-14 & own \\
\hline muge ptotimbs & - .5095 & .0 .19 & \multirow[b]{2}{*}{ 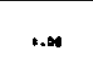 } & \multirow[b]{2}{*}{4.11} \\
\hline $2 \operatorname{mak} x \times 1 m_{1}$ & 1,08 & ".on & & \\
\hline tmals's & \\
\hline 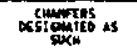 & \multicolumn{4}{|c|}{ Lew } \\
\hline
\end{tabular}
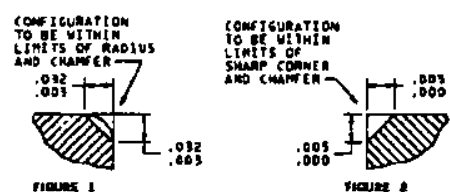

กorex !

$$
\text { isenting }
$$

smane *

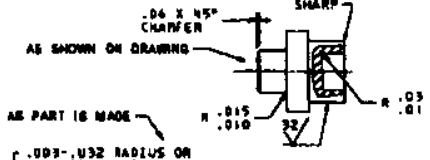

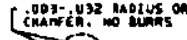
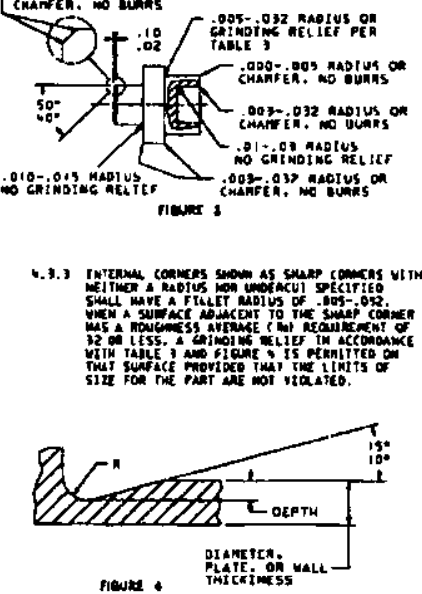

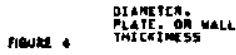

\begin{tabular}{|c|c|c|c|}
\hline \multicolumn{2}{|c|}{ 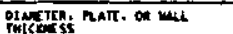 } & \multirow[t]{2}{*}{ ofpTH } & \multirow[t]{2}{*}{ 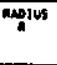 } \\
\hline onn & MELWoi in & & \\
\hline 㳗桑 & ; & 淟 & $.01-.02$ \\
\hline
\end{tabular}

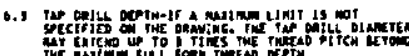

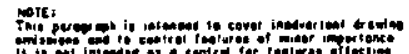
int

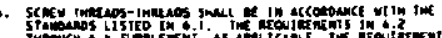

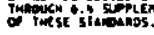

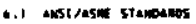

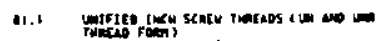

1::

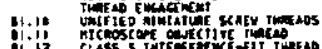

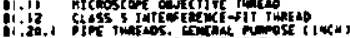

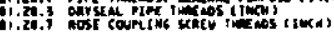

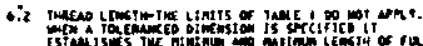

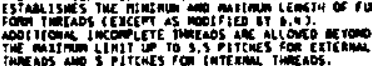

Page 8

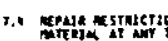

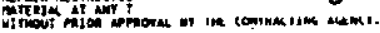

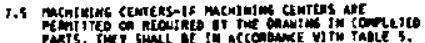
giscien

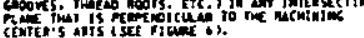

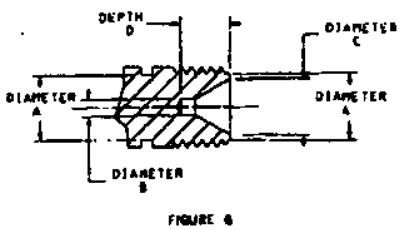

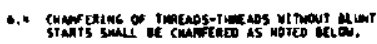

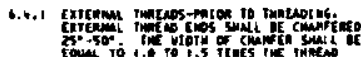

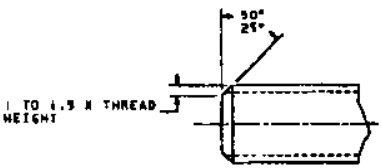

nIOAE

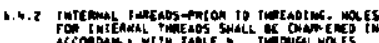

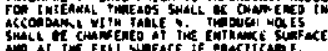

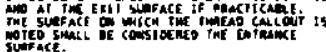

\begin{tabular}{|c|c|c|c|c|c|}
\hline \multicolumn{6}{|c|}{1040} \\
\hline \multicolumn{2}{|c|}{ Dtaneresh a } & \multirow{2}{*}{ 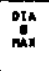 } & \multirow{2}{*}{ of: } & \multirow{2}{*}{ 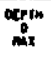 } & \multirow{2}{*}{ 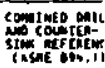 } \\
\hline orent & Tícluosing & & & & \\
\hline 溃 & : & 㗊 & 萿 & 畾 & : \\
\hline : & i: & 满 & 78 & 絮 & 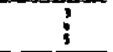 \\
\hline 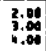 & 80 & 㴶 & 㳻 & 清 & $i$ \\
\hline
\end{tabular}

1. Fammisateb itiens

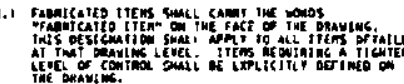

Tene .

\begin{tabular}{|c|c|c|c|}
\hline \multirow{2}{*}{\multicolumn{2}{|c|}{ Thetedo sits }} & \multicolumn{2}{|c|}{ 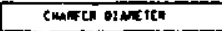 } \\
\hline & & 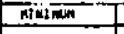 & $\min$ \\
\hline 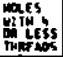 & 46 sizrs & 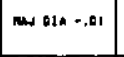 & 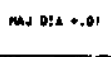 \\
\hline 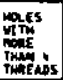 & 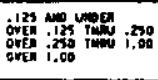 & 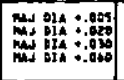 & 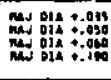 \\
\hline $\mathrm{Cmang}_{4}$ & 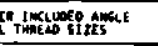 & & 100 \\
\hline
\end{tabular}

1.2

12 semitions

:1,

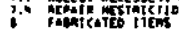

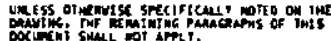

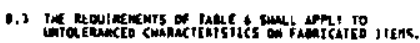

\begin{tabular}{|c|c|c|}
\hline chenactiretsits & \multicolumn{2}{|c|}{ mearingerst } \\
\hline 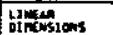 & Imprin : * & 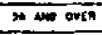 \\
\hline & 4.18 &..$- x$ \\
\hline mans & \multicolumn{2}{|c|}{5} \\
\hline 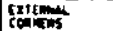 & \multicolumn{2}{|c|}{ 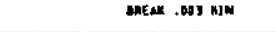 } \\
\hline $\operatorname{mig}_{x \rightarrow 0}$ & \multicolumn{2}{|c|}{ A. $005 \mathrm{n110}$} \\
\hline Gurace & 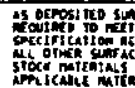 & 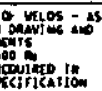 \\
\hline
\end{tabular}

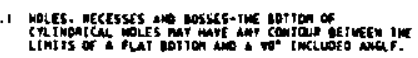

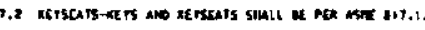

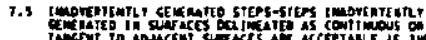 等

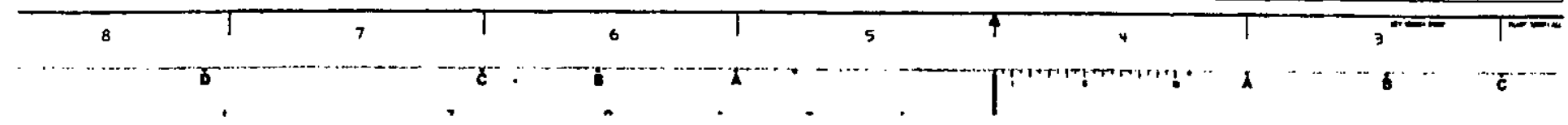




\section{Monolithic SiC}

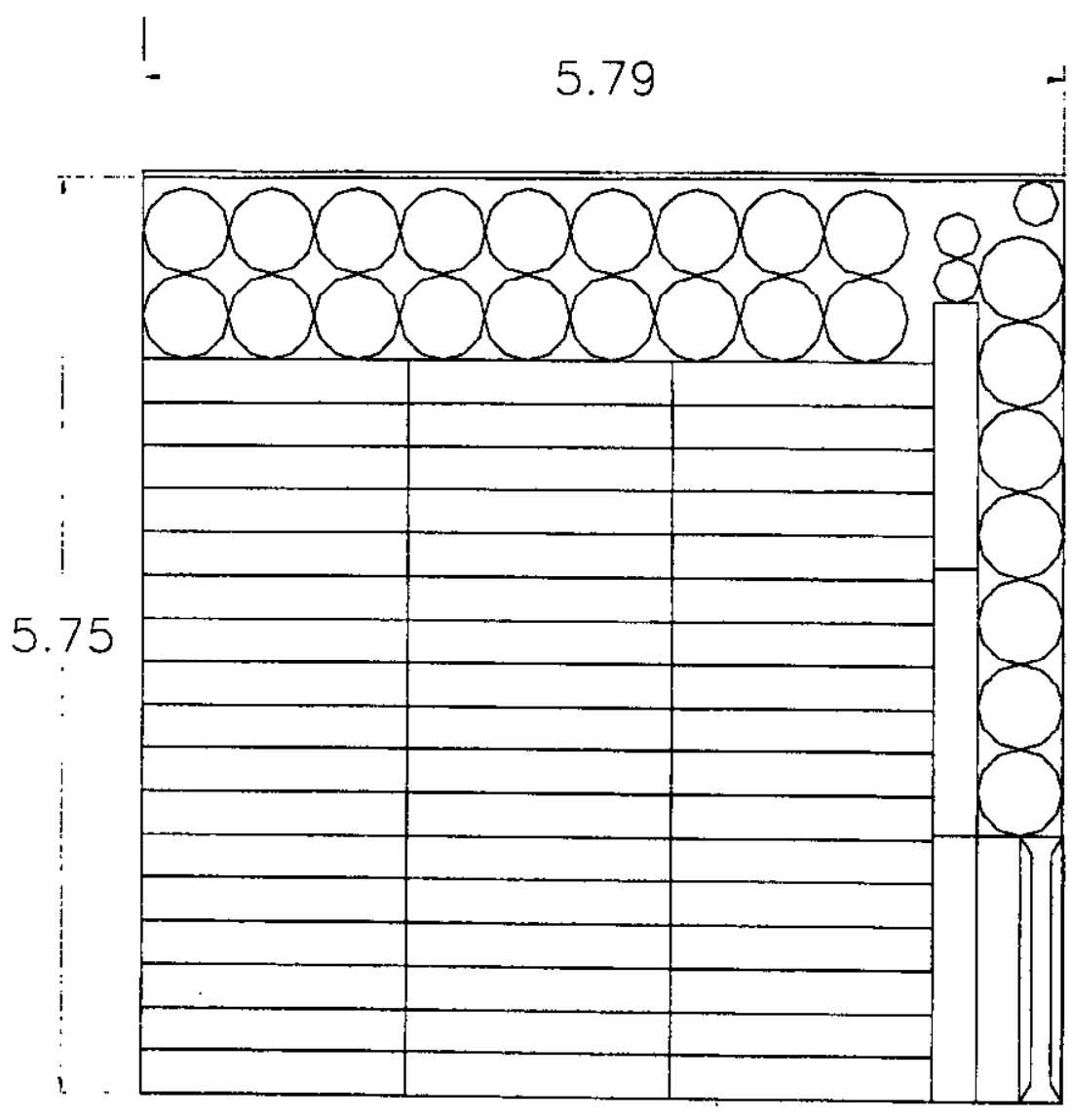

Specimen dimensions:

Nominal Dimension + Max Tolerance

+ 0.030" For Tooling

All Dimensions Shown in inches
6 Plates

56 Tensile

25 Thermal Diff

$3 \mathrm{Sm}$. Therm. Diff.

Specimen Totals

336 Tensile

150 Th. Diff. $\left(0.5^{\prime \prime}\right)$

$18 \mathrm{Sm}$. Therm. Diff.

\section{Tensile Specimen}

Shown for Orientation

\section{.}




\section{$3 / 6 / 03$}

\section{Detrimental Materials Prohibitions and Restrictions for Silicon Carbide Composite Materials}

Certain chemical elements in contact with a test specimen may cause premature failure of the specimen under test. Those chemical elements known as detrimental materials must not be allowed to contaminate the specimens at any time before or during the test. Therefore, the specimens must be guarded against contamination from detrimental materials during such activities as manufacturing, packaging, shipping, inspection, or testing.

1.0 During the machining, handling, storage, or shipping of specimens, the specimens shall not come in direct contact with:

1.1 Mercury, compounds containing mercury in excess of $10 \mathrm{ppm}$, or any mercury-containing device employing a single boundary of containment.

1.2 Lead or compounds containing lead in excess of $250 \mathrm{ppm}$.

1.3 Cadmium, particularly cadmium plated tools or hardware, or alloys containing more than one percent cadmium, or compounds containing more than $250 \mathrm{ppm}$ cadmium.

1.4 Compounds containing halogens in excess of $250 \mathrm{ppm}$ (total where fluorine is restricted to $25 \mathrm{ppm}$ ) except lubricants used in machining.

1.5 Salt, or saline solutions, or any solid surface that may introduce alkali metals (e.g., sodium, potassium, lithium).

1.6 Compounds with molybdenum disulfide in excess of $250 \mathrm{ppm}$.

2.0 Compounds with compositions listed below shall not directly contact spocimen surfaces after final machining.

2.1 Compounds containing more than $250 \mathrm{ppm}$ of any of the following antimony, bismuth, copper, phosphorus, sulfur, tin, zinc, total halogens (except that total fluorine shall be restricted to a maximum of $25 \mathrm{ppm}$ ).

2.2 Nuclear poisons (e.g., boron) in excess of $100 \mathrm{ppm}$.

2.3 Liquids which are not water-soluble except as allowed in Paragraphs 6.1 and 6.2 /milldew/mp/DMP\&R 
3.0 Thread sealants, such as teflon tape, tefion jam nuts, or epoxy resins shall not be used to affix or secure specimens to fixtures.

4.0 Aluminum, copper, nickel, and alloys of these materials containing more than 50 percent aluminum, copper, or nickel shall not be used as soft pads, hammers, or tools which contact the specimens during or subsequent to final machining.

5.0 Only dedicated resin-bonded diamond tooling shall be used for all machining of silicon carbide composite materials.

\subsection{Solvent and Cleaning Solution Requirements:}

6.1 Solutions having a pH of less than 6 at the temperature at which they are to be used shall not be used to clean parts within accessible areas.

6.2 All halogenated cleaning solutions (250 ppm or more halogens, with fluorine restricted to $25 \mathrm{ppm}$ ) are prohibited from use.

7.0 Temperature indicating crayons, marking materials (including white-out) lubricants and low melting alloys shall not contact the final machined surfaces of the specimens if they contain:

7.1 More than $250 \mathrm{ppm}$ of any of the following antimony, arsenic, bismuth, copper, phosphorus, sulfur, tin, zinc, and total halogens (except that fluorine shall be restricted to a maximum of $25 \mathrm{ppm}$ ).

\subsection{Metals or alloys which melt at 1000 degrees $F$ or less (e.g.,} Corrolow, Kirksite, Cerrobend, and cadmium).

8.0 Test and machining fixtures and other components, which directly contact the final machined surfaces of the specimens shall not come in direct contact with items specified in Paragraph 1.0. If fixtures have previously contacted the items specified in Paragraph 1.0, then rigorous cleaning (such as by acid pickling) is required prior to use on the specimens.

9.0 Bench tops and work surfaces upon which the final machined specimens will be placed shall be covered by KimPak creped wadding paper (Kimberly-Clark Co.) or a similar product prior to use.

10.0 Seller shall provide a statement of conformance with the requirements of this specification. 
Attachment 1.3 to

Enclosure 4 to

MDO-723-0044/

B-MT(SRME)-52

Page 1

Attachment 1.3

Machining Documentation for Monolithic SiC Thermal Diffusivity Discs

Enclosure 4 to MDO-723-0044/B-MT(SRME)-52 
Rebeca Northey $\times 6708$

Space Chavge Code D2317 D3 808

lease complete the following Dimensional overcheck for the following Mini

Tensite Specimens, Drwg SK239B9368.

Composite Mini Tensile Specimens

M002360004AG

Plate No. 1

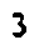

18

26

53

71

Plate No. 2

73

98

Plate No. 3

146

164

154

153

151

145

\begin{tabular}{|l|l|l|l|l|l|l|l|l|l|}
\hline \multicolumn{3}{|c|}{$4 \times 8^{\circ}$} & 0.118 & 1.626 & \multicolumn{2}{|c|}{$2 \times 0.236$} & 0.0004 & $0.091 \pm 0.004$ \\
\hline $8^{\circ}$ & $8^{0}$ & $8^{0}$ & $8^{0}$ & 121 & 1.625 & 237 & .237 & .0002 & .093 \\
\hline $8^{0}$ & $8^{0}$ & $8^{0}$ & $8^{0}$ & .121 & 1.626 & .237 & .237 & .0002 & .094 \\
\hline $8^{0}$ & $8^{0}$ & $8^{\circ}$ & $8^{0}$ & 121 & 1.628 & .237 & .237 & .0003 & .094 \\
\hline $8^{\circ}$ & $8^{0}$ & $8^{0}$ & $8^{0}$ & 120 & 1.627 & .237 & .237 & .0003 & .094 \\
\hline $8^{0}$ & $8^{\circ}$ & $8^{\circ}$ & $8^{0}$ & .120 & 1.627 & .237 & .237 & .0001 & .094 \\
\hline
\end{tabular}

\begin{tabular}{|c|c|c|c|c|c|c|c|c|c|}
\hline \multicolumn{3}{|c|}{$4 \times 8^{\circ}$} & 0.118 & 1.626 & $2 \times 0.236$ & 0.0004 & $0.091 \pm 0.004$ \\
\hline $8^{\circ}$ & $8^{0}$ & $8^{0}$ & $8^{0}$ & 120 & 1.628 & .236 & .236 & .0002 & .093 \\
\hline $8^{0}$ & $8^{0}$ & $8^{0}$ & $8^{\circ}$ & 120 & 1.628 & .237 & .236 & .0003 & .093 \\
\hline
\end{tabular}

\begin{tabular}{|c|c|c|c|c|c|c|c|c|c|}
\hline \multicolumn{4}{|c|}{$4 \times 8^{\circ}$} & 0.118 & 1.626 & \multicolumn{2}{|c|}{$2 \times 0.236$} & 10.0004 & $0.091 \pm 0.004$ \\
\hline & $8^{0}$ & $8^{\circ}$ & $8^{\circ}$ & 120 & 7.628 & 237 & .237 & 1,0003 & 093 \\
\hline & $8^{\circ}$ & $8^{\circ}$ & $8^{\circ}$ & 120 & 1.628 & 237 & 237 & .0003 & .094 \\
\hline & $8^{\circ}$ & $8^{0}$ & $8^{\circ}$ & 120 & 1.629 & 236 & 236 & 22 & 1093 \\
\hline & $\rho^{0}$ & $8^{\circ}$ & $18^{\circ}$ & 119 & 1.628 & .236 & .236 & .0002 & 0 \\
\hline & $8^{0}$ & $8^{\circ}$ & $8^{\circ}$ & 120 & 1.6 & .237 & .236 & 002 & .093 \\
\hline 70 & $8^{\circ}$ & $8^{\circ}$ & 80 & .120 & 7.628 & .237 & 237 & $000 /$ & .093 \\
\hline
\end{tabular}

Monolithic Mini Tensile Specimens

KN6002216-2

(Plate 2)

41

37

25

\begin{tabular}{|c|c|c|c|c|c|c|c|c|c|}
\hline \multicolumn{4}{|c|}{$4 \times 8^{\circ}$} & 0.118 & 1.626 & $2 \times 0.236$ & 0.0004 & $0.091 \pm 0.004$ \\
\hline $8^{0}$ & 80 & $8^{0}$ & $8^{0}$ & .118 & 1.626 & 236 & .236 & 0000 & .094 \\
\hline $8^{0}$ & $8^{0}$ & $8^{0}$ & $8^{0}$ & .119 & 1.626 & .236 & .236 & .0000 & .094 \\
\hline $8^{0}$ & $8^{0}$ & $8^{0}$ & $8^{0}$ & .118 & 1.626 & .236 & .236 & 0000 & .054 \\
\hline
\end{tabular}

KN6002216-4

(Plate 4)

8

45

28

\begin{tabular}{|c|c|c|c|c|c|c|c|c|c|}
\hline \multicolumn{3}{|c|}{$4 \times 8^{\circ}$} & 0.118 & 1.626 & $2 \times 0.236$ & 0.0004 & $0.091 \pm 0.004$ \\
\hline $8^{\circ}$ & 80 & $8^{\circ}$ & 80 & .118 & 1.626 & 236 & 236 & 0001 & .094 \\
\hline 80 & 80 & 80 & 80 & .116 & 1626 & .236 & .236 & .0001 & .094 \\
\hline $8^{\circ}$ & 80 & 80 & 80 & .118 & 1.626 & 236 & .236 & .0001 & 024 \\
\hline
\end{tabular}

KN6002216-5

(Plate 5)

22

2

39

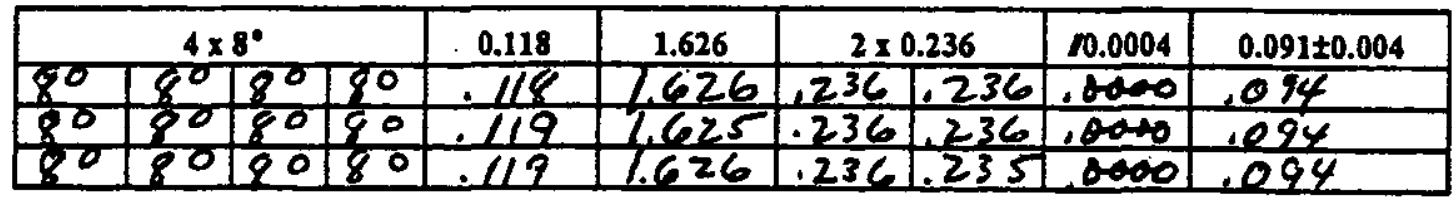

KN6002216-6

(Plate 6)

5

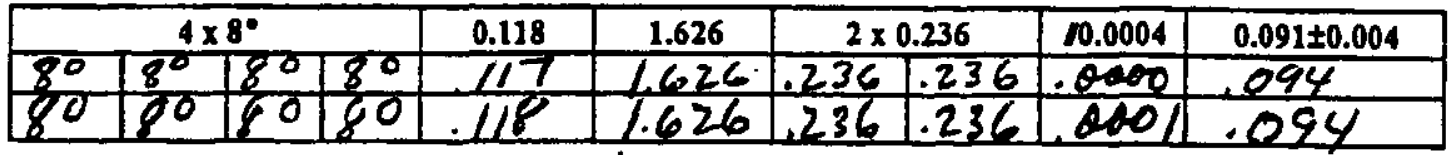

$\Rightarrow A_{\text {belengo AUO } 5} 2005$

PRE-DECISIONAL - For Planning and Discussion Purposes Only 
Attachment 1.3 to

Enclosure 4 to MDO-723-0044/ B-MT(SRME)-52

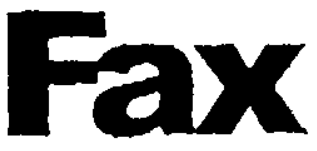

ADVANCED CERAMICS.

\begin{tabular}{lll} 
To: & SKIP GIFFORD & From: \\
\hline Fax: $\quad 518-395-6848$ & Pages: 2 \\
\hline Phone: 518-395-6187 & Date: G/9/2005 \\
\hline Re: $\quad$ O5PFG093 & CC: $\quad$ FILE \\
\hline
\end{tabular}

SKIP,

PLEASE SEE THE ATTACHED CUTtTING MAP. (DRAWING 33580 REV A)

THE YIELD PER PLATE IS SLIGHTLY LOWER THAN WHAT YOU HAVE IN YOUR SPECIFICATION. PLEASE LET ME KNOW IF THIS MAP IS ACCEPTABLE SO THAT WE MAY PROCEEO WITH THE MACHINING.

I ESTIMATE THAT IF WE COULD UTILIZE THE 1/8" PERIMETER OF THE PLATE, THE YIELD PER PLATE COULD GO UP SLIGHTLY (FROM 49 TO 54 ON THE TENSILE SPECIMENS AND FROM 20 TO 22 ON THE LARGE THERMAL DIFFUSIVITY DISCS). IF THIS IS FEASABLE. LET ME KNOW AND I WILL FORWARD A REVISED CUTTING MAP.

IN REVIEWING THE SPECIFICATION, I DISCOVERED THAT THE PLATE ID IN THE SPEC (KN6002216-1, -2, -3, -4, -5, -6) DOES NOT MATCH THE ID ON THE PLATES (KN60022160-1, $-2,-3,-4,-5,-6)$. PLEASE LET ME KNOW WHICH ID YOU WOULD LIKE US TO TRACK THROUGHOUT THE PROCESS.

THANK YOU FOR YOUR HELP,

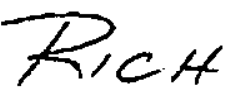

$\mathrm{RICH}$

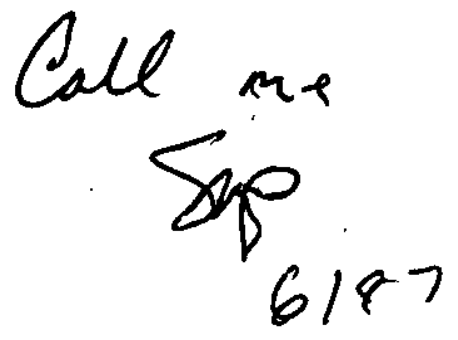

PremaTech Advanced Ceramics 2 Coppage Dr. Worcester, MA 01603 unuw.PremaFechAC.com (508) $791-9549(\mathrm{~T})$ (508) $793-9814$ (F) 


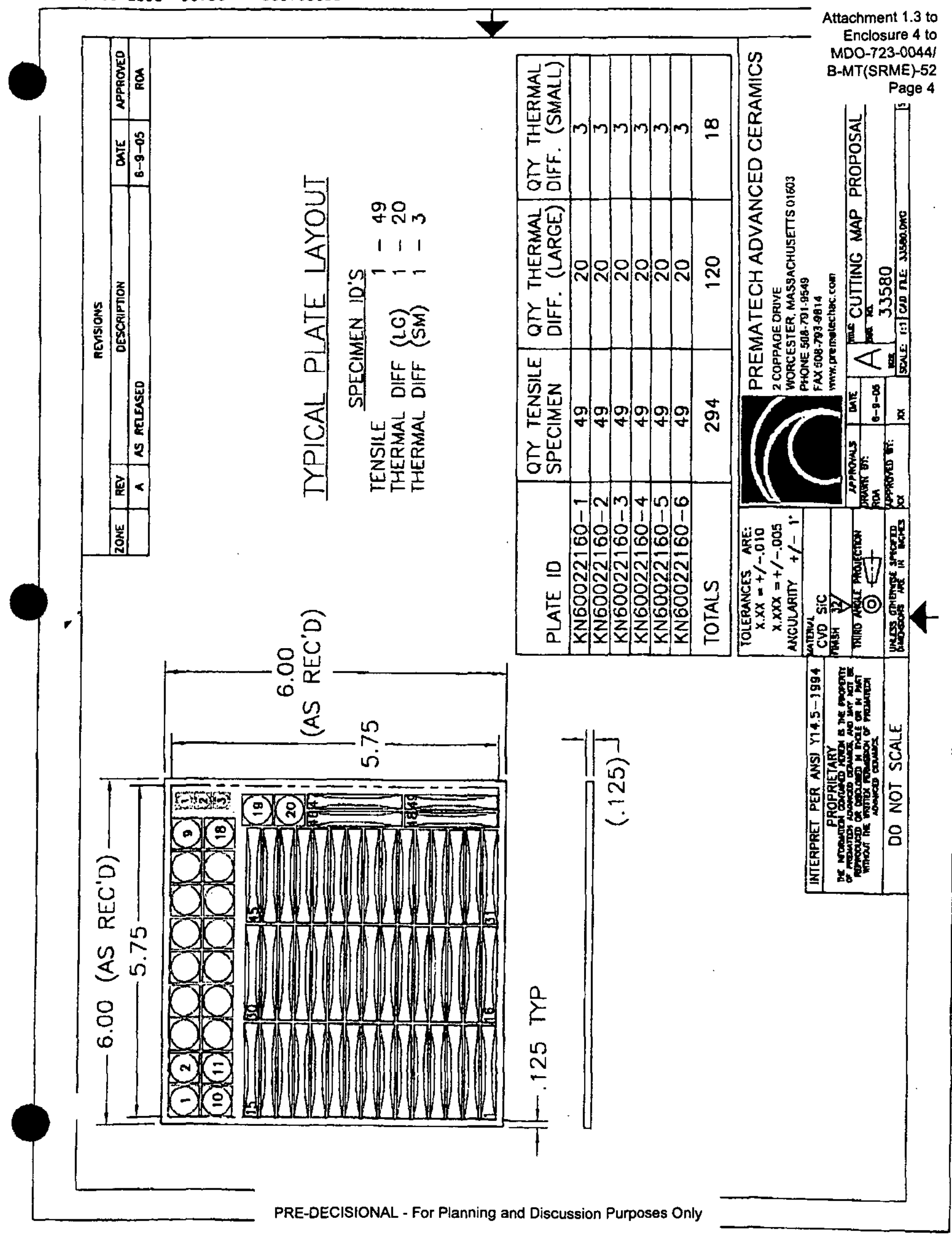




\section{CERTIFICATE OF COMPLIANCE}

To:

Knolls Receiving BLDG M2

Central Receiving - KNO

2401 River Road

Schnectady, NY 12309

USA

PCACJob

No.: C33580

Dwg. No.: SK239B9368
P.O. No.: KN6002619

PCACPIL No.: 46511

P/N: Miniature Tensile Specimen

Rov.: -

It is hereby certified that material and manufacturing processes utilized in the production of the above order conform to customer supplied specification 05PFG093، Rev-, Dated 05/23/05 as well as drawing SK23989368, Rev -

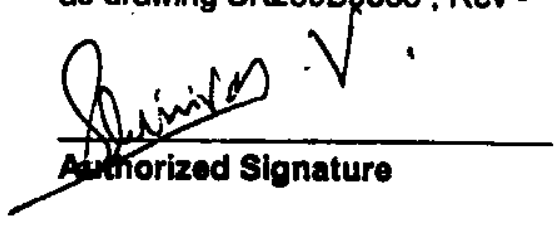

Material: CVD SiC (Morton)

Srinivas Vishweswaraiah

Name

$6 / 24 / 05$

Date

Quality Engineer

Titio

Authorized Signature 
INSPECTION REPORT

EUST. NO: KNOATD P.O. NO: KN 6002619 INSPECTED ar:

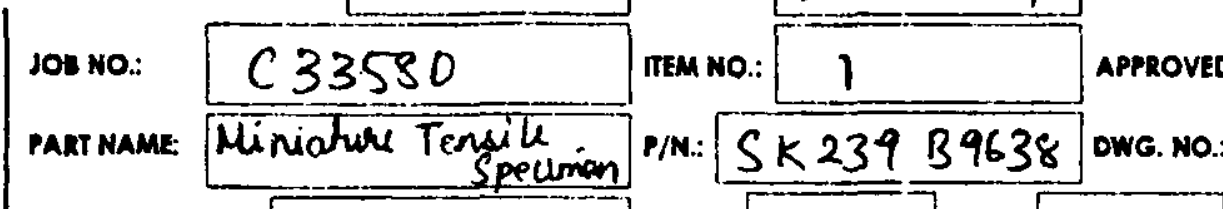

PI Attachment 1.3 to Enclosure 4 to MDO-723-0044/ B-MT(SRME)-52

Page 6

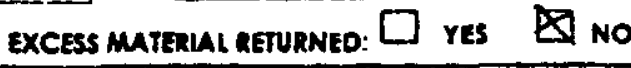

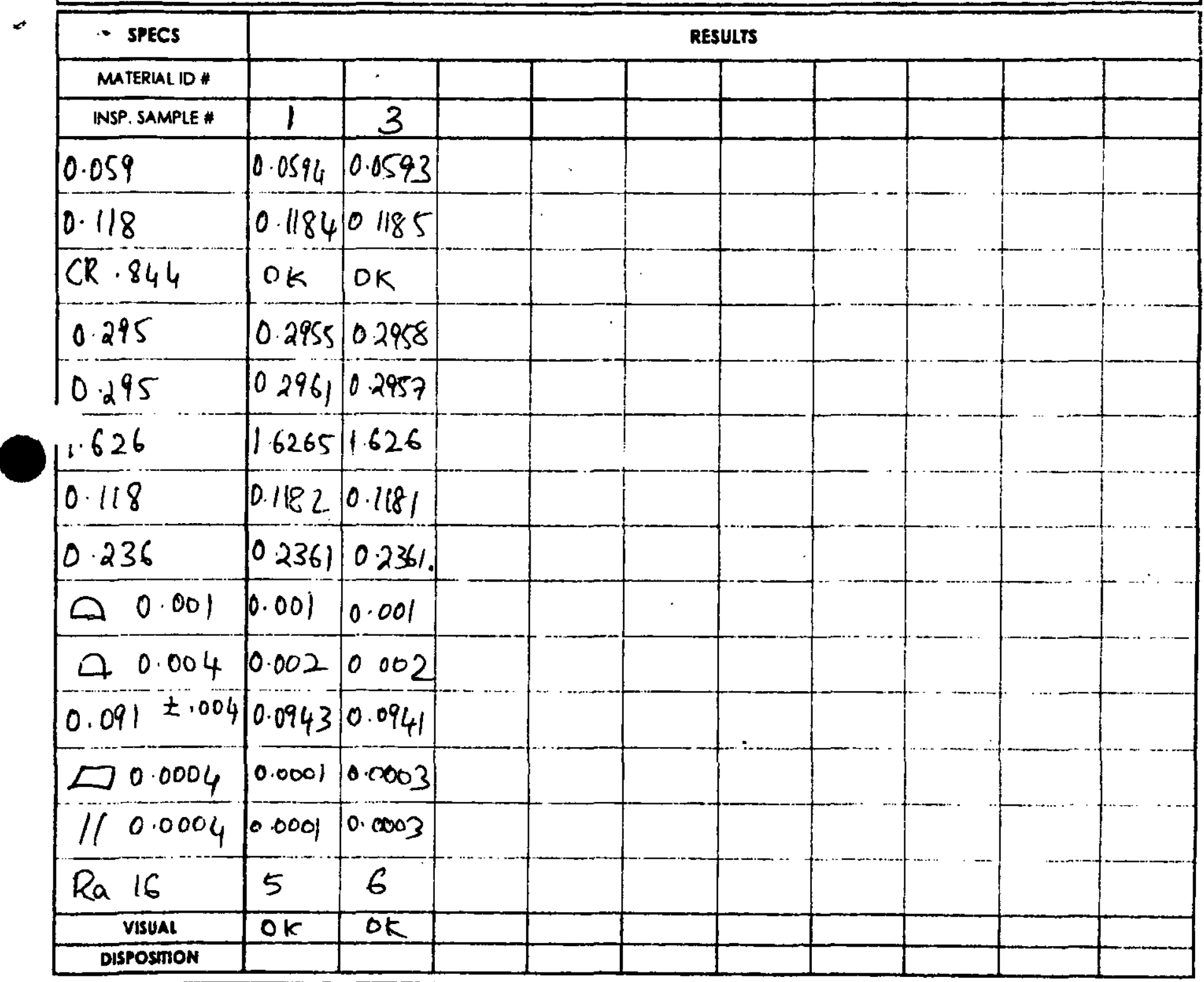

comments: Longitudinal grinding incorporated. CR 844 has been generated by CNC. There is a Smooth blend and no undercuts of tool marks 0.295 dimension has been measured using $0 G P$ flare Scope (Generated by PremaTech Advanced Ceramics TWO COPPAGE DRIVE, WORCESTER, MA 01603-1252 Ph: (508)791-9549 Fax: (508)793-9814 http:/ $/$ www.premachand.com

PRE-DECISIONAL - For Planning and Discussion Purposes Only

glistered Company

Dorser 


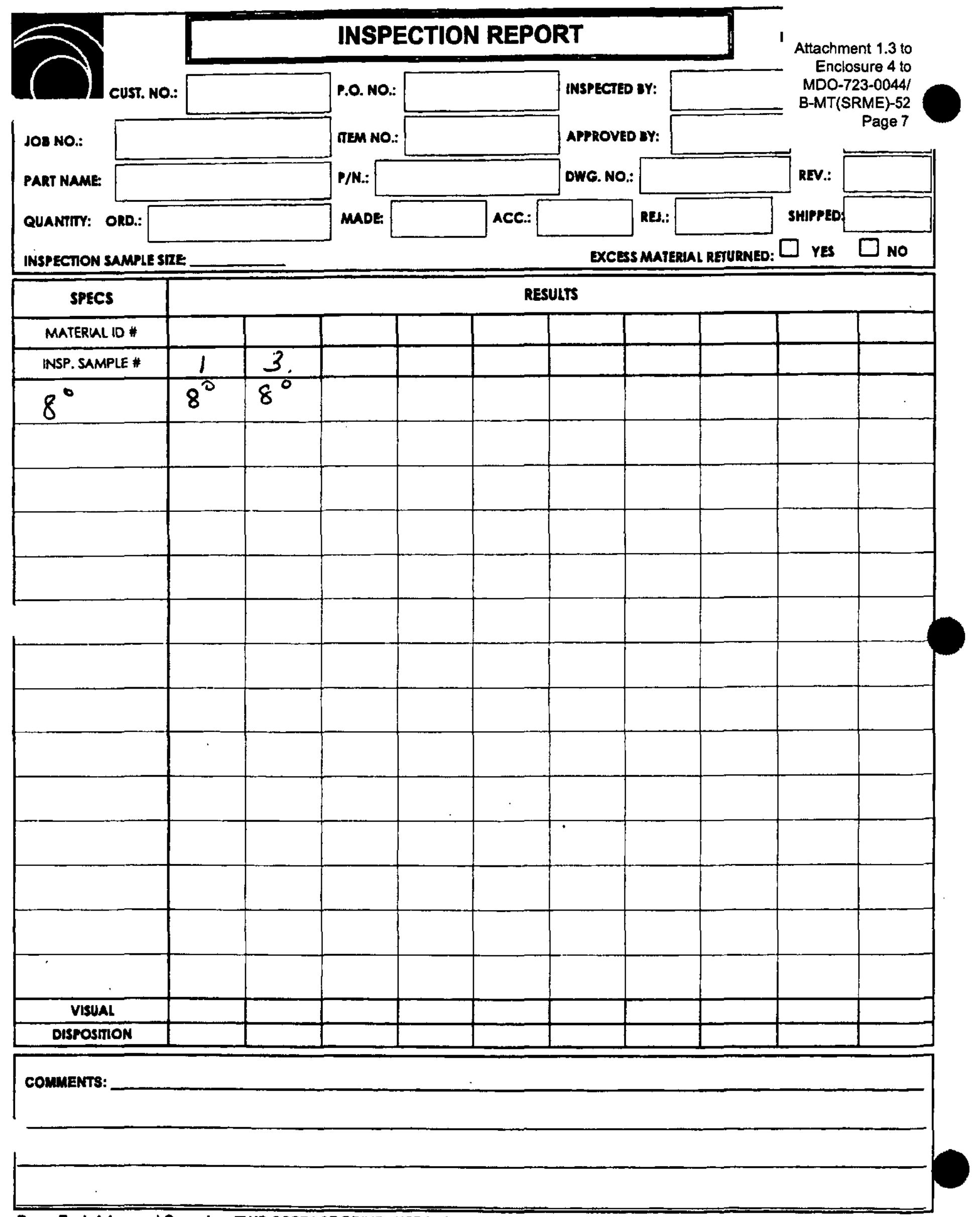

PremaTech Advanced Coramics TWO COPPAGE DRIVE, WORCESTER, MA 01603-1252 Ph: (508)791-9549 Fax.: (508)793-9814 http://www.premachand.con 

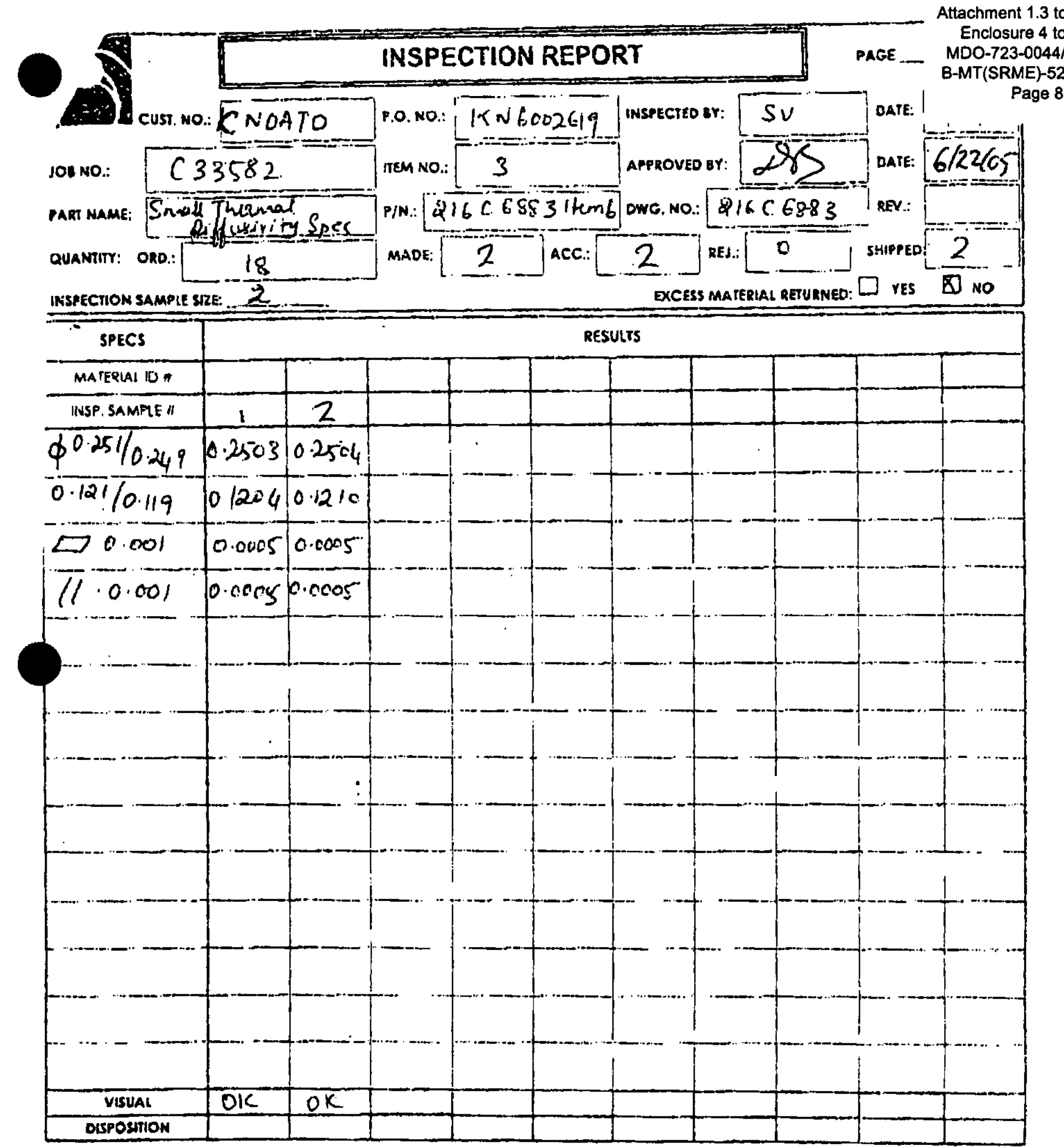

COMMENTS: $M R 189497$

KN60:22160=1 

MDO-723-0044/

B-MT(SRME)-52

Page 1

\section{Attachment 1.4}

Specimen Photographs

Monolithic SiC Tensile Specimens and Thermal Diffusivity Discs

Enclosure 4 to MDO-723-0044/B-MT(SRME)-52 


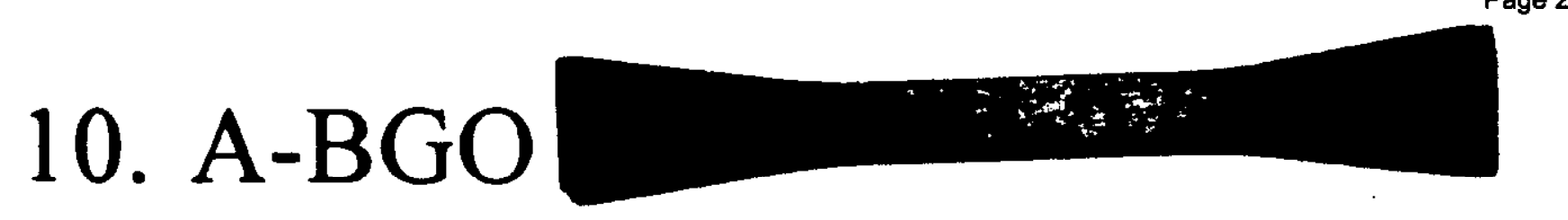

11. A-BGP

12. A-BGR

10. A-BGO

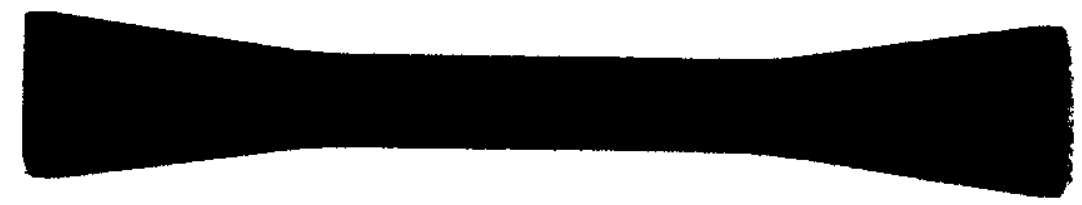

11. A-BGP

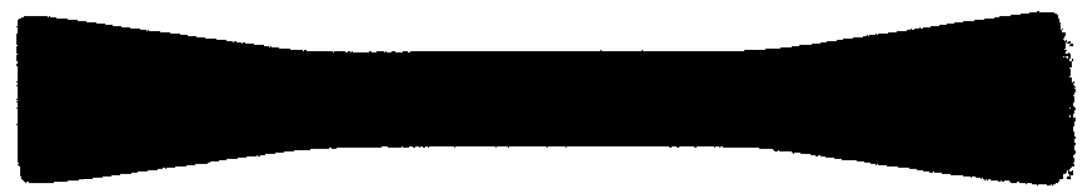

12. A-BGR

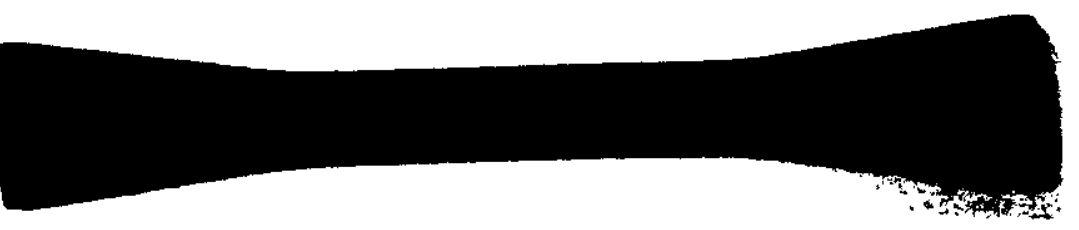


Attachment 1.4 to

Enclosure 4 to

MDO-723-0044/

B-MT(SRME)-52

Page 3

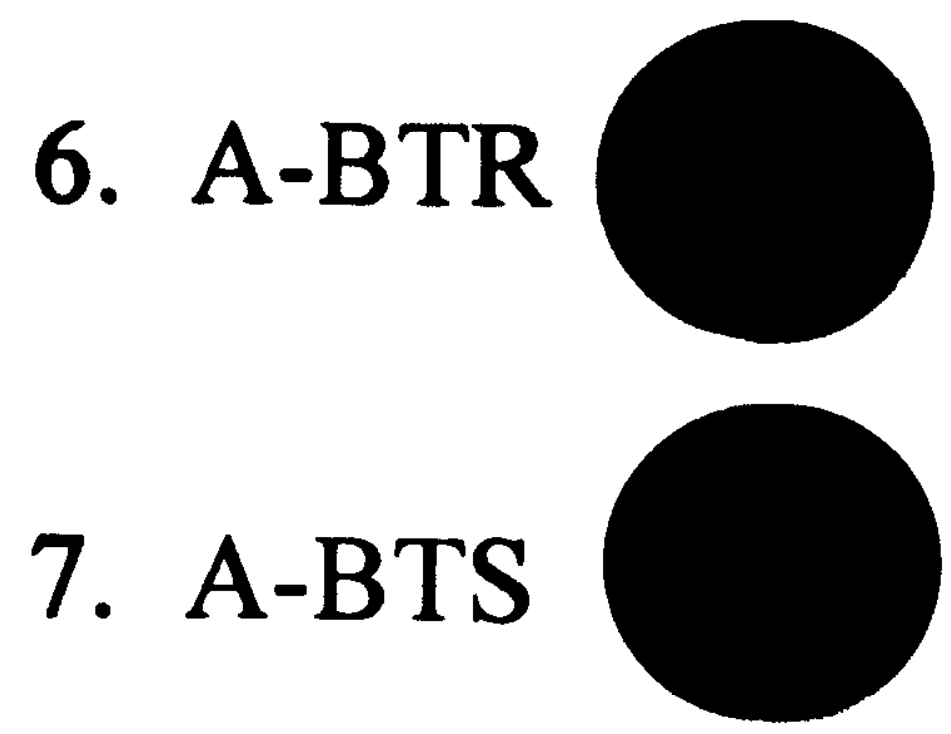

8. A-BTT

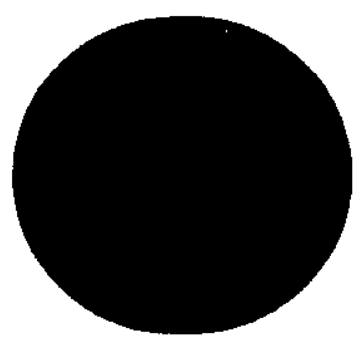

9. A-BTV

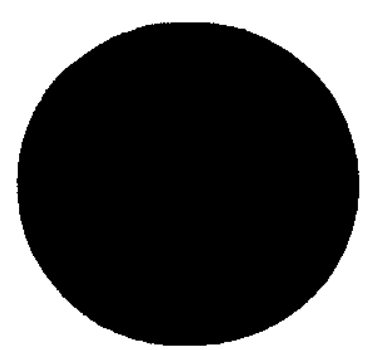


Attachment 1.5 to

Enclosure 4 to MDO-723-0044I

B-MT(SRME)-52

Page 1

Attachment 1.5

Thermal Diffusivity Data for Monolithic SiC Discs

Enclosure 4 to MDO-723-0044/B-MT(SRME)-52 
KAPL, Ine.

Knolls Atomic Power Laburatory

Post Office Box 1072 Schienectady, NY 12301-1072

Telephone (518) 395-4000 Facsimile (518) 395-4422

TO-7934-06-02

January 13, 2006

To:

R. Northey

Subject: $\quad$ Diffusivity Testing - Space Reactor Monolithic / Composite SiC

MCL JOB \#: $\quad 4782$

QA Category: C

Test Performer: R.A. Griesau L

Reviewed By:

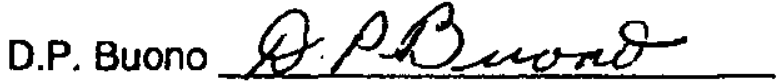

\section{INTRODUCTION AND TEST EQUIPMENT}

Thermal diffusivity was measured using an Anter Corp "Flashline 5000" laser-pulsed diffusivity measurement system. This system uses a solid state (Xenon flash pumped Neodymium glass rod) infrared laser pulsed at $1053 \mathrm{nM}$ through a fiber-optic cable onto a small disk specimen. The conducted thermal wave through the specimen is detected on the opposing face by an $\mathrm{LN}_{2}$-cooled Indium Antimonide crystal infrared detector. High speed data acquisition records the temperature rise curve, which is then analyzed by the software using algorithms by Koski, Clark and Taylor, Parker, Cowan, etc. The most accepted analysis is Koski which corrects for non-axial conduction and laser pulse width errors. The furnace system is comprised of a SS vertical water-cooled cylindrical chamber with graphite heating elements, insulation and specimen support carousel. A rotating carousel allows testing of up to six specimens per test run.

\section{SPECIMENS}

Two specimen types were tested, monolithic beta phase Silicon Carbide (SiC) and composite SiC consisting of Hi-Nicalon Type S fibers in a Chemical Vapor Infiltration (CVI) SiC matrix. Specimens were right cylindrical disks $0,5^{n}$ dia with a thickness of $0.120^{\prime \prime}$ and $0.061^{\prime \prime}$ for the monolithic and composite specimens, respectively. These specimens were placed directly into the $1 / 2^{\prime \prime}$ dia graphite carousel positions in the furnace without adapter holders. The reference specimen NIST-2 ( $12^{x}$ dia Stainless 
Steel) was placed in carousel positions 6 and 2 for the composite and monolithic runs Page 3 respectively. Specimen thickness measurements were provided by the test sponsor.

\section{TEST PROCEDURE AND CONDITIONS}

Testing was performed in conformance to MCL Operating Procedure No. 100 and ASTM E $1461-01$.

Monolithic SiC specimens will often display translucence in the infrared spectrum (wavelength of test system laser) requiring an opaque, high emissivity coating, typically graphite to be applied to prevent "shine-through" of the laser pulse. That was not the case with the specimens in this program. No coating was applied to the monolithic or composite specimens for this testing.

Five test specimens were loaded in each of the two runs with a graphite reference specimen loaded in position 6 for the composites and position 2 for the monolithics. The composite specimens were loaded with the ID marked face down, toward the IR detector. No specific specimen face orientation was utilized relative to the edge ID marking for the monolithic $\mathrm{SiC}$ specimens. Three vacuum pumpdown and argon backfill cycles to atmosphere were performed, with the last pumpdown to $-100 \mathrm{mT}$ torr. A continuous argon purge was established at $-3-4$ bubbles per second through an oilfilled bubbler vent.

Soak points were programmed from $200^{\circ} \mathrm{C}-1200^{\circ} \mathrm{C}$ and $1300^{\circ} \mathrm{C}-100^{\circ} \mathrm{C}$ at $200^{\circ} \mathrm{C}$ increments. This up/down sequence was requested to reveal potential hysteresis due to structural or properties changes through the heating cycle. The soak period is determined by the software based on programmed criteria that ensure specimen temperature stability. Laser shots were fired at each specimen at the programmed soak temperatures in a 1-2-3..., 1-2-3... order; three shots per specimen per soak temperature. The laser flash-lamp capacitor charge voltage (laser power) was set to $1.4,1.2 \& 1.0 \mathrm{kVdc}$ from the low test temperatures to the highest respectively. Auxiliary laser incident apertures were not used, in that, the carousel cover provides sized penetrations for the default $1 / 2^{\prime \prime}$ dia specimen size.

\section{GALIBRATION}

Independent pyrometer and IR detector (data sources) calibrations are not performed on this test instrument. Data validity is verified by testing of a reference material with comparison to known book values (see charts 3-6). The reference specimen is run as the sixth specimen with each test run loading. The reference specimen error values were within acceptable limits. 


\section{RESULTS}

Note: Caution should always be used when evaluating diffusivity data from a composite test specimen. A composite is a "structural system" and hence, the specimen measurement does not represent the bulk diffusivity value for the inter-fiber matrix or the fibers.

Upon completion of a test run a "results.all" file is generated by the software which contains diffusivity values calculated for all of the analysis types (i.e. Parker, Koskl, Clark and Taylor ...). Tabulated values are provided by the software for each of the three laser shots per specimen, fired at each soak temperature. These files were copied to an Excel spreadsheet. The temperature and Koski diffusivity values were averaged for the three laser shots at each soak point and plotted (see charts 1 \& 2). The averaged values were sorted by ascending temperature (the test program had upgoing and down-going soak sequences). The averaged values were collected in the attached table for each specimen.

No anomalies were detected in testing. The specimen-to-specimen spread in the data between the two groups (composites / monolithics) is roughly within the same order of magnitude. The overall comparative ranges between the two groups are offset as might be expected with the homogeneous monolithics being higher in value.

The Excel file with processed data will be forwarded in parallel with this report. The Excel file with processed data and the raw test system files will be electronically transferred to "O:MCLJobs WMCL 4782 - SPACE SIC - DIFFUSIVITY - NORTHEY - RG" for record keeping. Archive copies of raw data will be maintained in the Materials Characterization Lab on optical disk.

\section{REFERENCES}

- ASTM E1461-01 Standard Test Method for Thermal Diffusivity by the Flash Method

- Test Community Work Request - MC Job\# 4782 Dated: 10-12-05

- Readiness Review Checklist Dated 11/3/05

- MCL Operating Procedure No. 100 - Anter Flashline 5000 
MCL TEST PROGRAM \# 4782

PROMETHEUS SPACE REACTOR

COMPOSITE and MONOLITHIC SIC

KOSKI DIFFUSIVITY - AVERAGE of 3 LASER SHOTS
Attachment 1.5 to

Enclosure 4 to

MDO-723-0044/

B-MT(SRME)-52

Page 5

\begin{tabular}{|c|c|c|c|c|c|c|c|c|c|}
\hline \multicolumn{10}{|c|}{ Monolithic SiC } \\
\hline \multicolumn{2}{|c|}{ A-BT2 } & \multicolumn{2}{|c|}{ A-BVA } & \multicolumn{2}{|c|}{$\overline{A-B V B}$} & \multicolumn{2}{|c|}{$A-B \times A$} & \multicolumn{2}{|c|}{$A-B \times B$} \\
\hline TEMP & Diffusivity & TEMP & Diffusivity & TEMP & Diffusivity & TEMP & Diffusivity & TEMP & Diffusivity \\
\hline (degF) & $\left(\mathrm{cm}^{\wedge} 2 / \mathrm{sec}\right)$ & (degf) & $\left(\mathrm{cm}^{\wedge} 2 / \mathrm{sec}\right)$ & (degF) & $\left(\mathrm{cm}^{\wedge} 2 / \mathrm{sec}\right)$ & $(\operatorname{deg} \mathrm{F})$ & $\left(\mathrm{cm}^{\wedge} 2 / \mathrm{sec}\right)$ & $(\operatorname{deg} F)$ & $\left(\mathrm{cm}^{\wedge} 2 / \mathrm{sec}\right)$ \\
\hline 212 & 0.6969 & 214 & 0.6942 & 212 & 0.6881 & 212 & 0.8210 & 212 & 0.8217 \\
\hline 379 & 0.5119 & 378 & 0.5128 & 379 & 0.5052 & 378 & 0.5998 & 379 & 0.5964 \\
\hline 568 & 0.3793 & 570 & 0.3780 & 567 & 0.3748 & 568 & 0.4365 & 568 & 0.4371 \\
\hline 738 & 0.3069 & 736 & 0.3079 & $73 \overline{8}$ & $\overline{0.3035}$ & 736 & 0.3501 & 738 & 0.3494 \\
\hline$\overline{924}$ & 0.2578 & 925 & 0.2579 & 923 & 0.2549 & 924 & 0.2906 & 924 & 0.2913 \\
\hline 1096 & 0.2222 & 1095 & 0.2233 & 1096 & 0.2200 & 1095 & 0.2500 & 1096 & 0.2502 \\
\hline 1277 & 0.1940 & 1278 & 0.1942 & 1276 & 0.1923 & 1276 & 0.2161 & $12 \overline{76}$ & 0.2169 \\
\hline 1455 & 0.1718 & 1455 & 0.1727 & 1456 & 0.1702 & 1455 & 0.1911 & $\overline{1455}$ & 0.1905 \\
\hline 1637 & 0.1532 & 1638 & 0.1545 & 1636 & 0.1519 & 1636 & 0.1693 & 1636 & 0.1687 \\
\hline 1814 & 0.1378 & 1814 & 0.1387 & 1814 & 0.1367 & 1813 & 0.1518 & 1813 & 0.1513 \\
\hline 1995 & 0.1248 & 1996 & 0.1253 & 1994 & 0.1235 & 1995 & 0.1361 & 1994 & 0.1364 \\
\hline 2172 & 0.1130 & 2172 & 0.1141 & 2173 & 0.1121 & 2172 & 0.1228 & 2172 & 0.1229 \\
\hline 2351 & 0.1033 & 2350 & 0.1031 & 2351 & 0.1026 & 2351 & 0.1109 & 2350 & 0.1111 \\
\hline
\end{tabular}

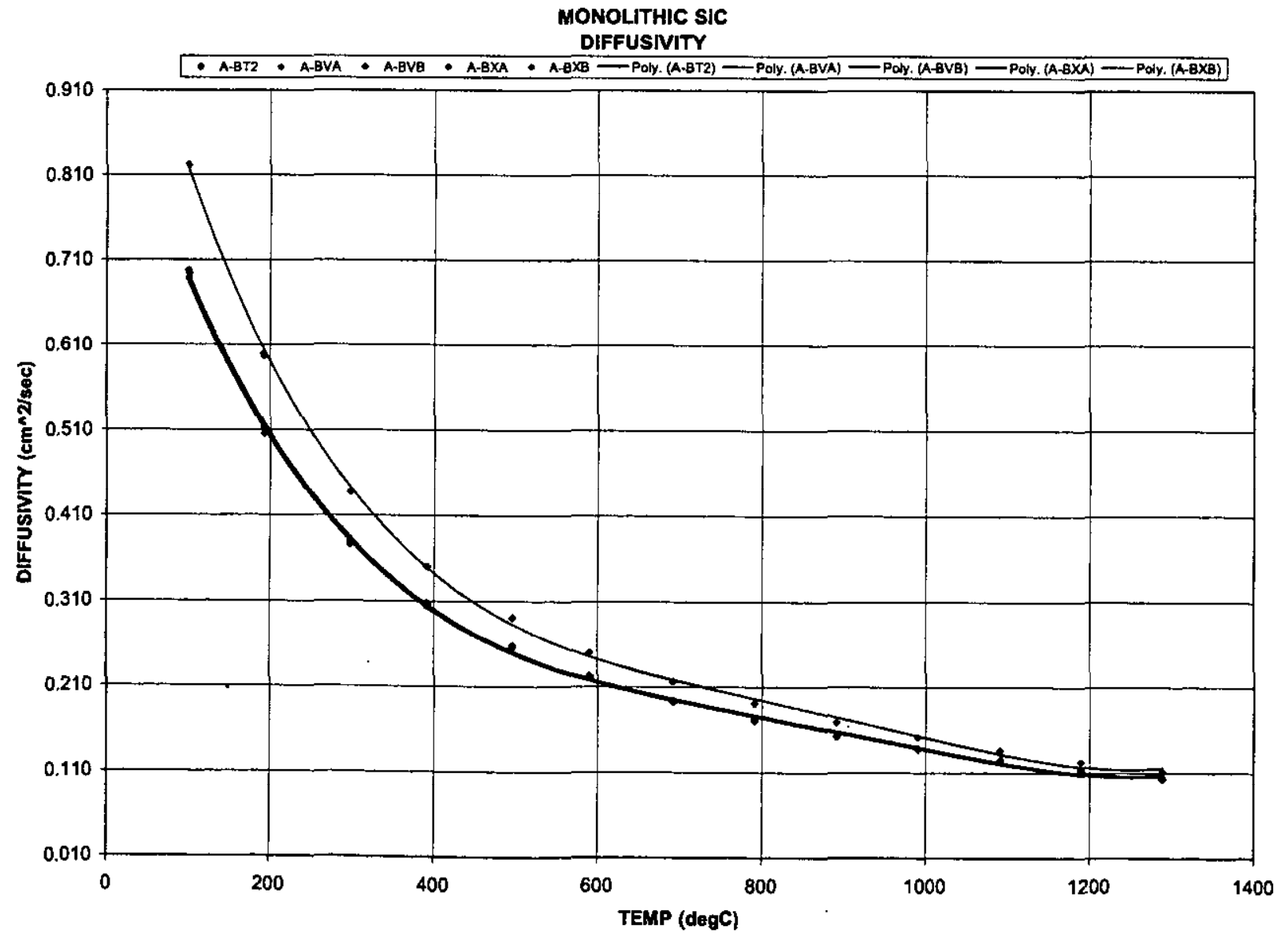


Attachment 2.1 to

Enclosure 4 to

MDQ-723-0044I

B-MT(SRME)-52

Page 1

Attachment 2.1

Material Purchase Documentation and Certification

Enclosure 2 to MDO-723-0044/B-MT(SRME)-52 
PACKING LIST

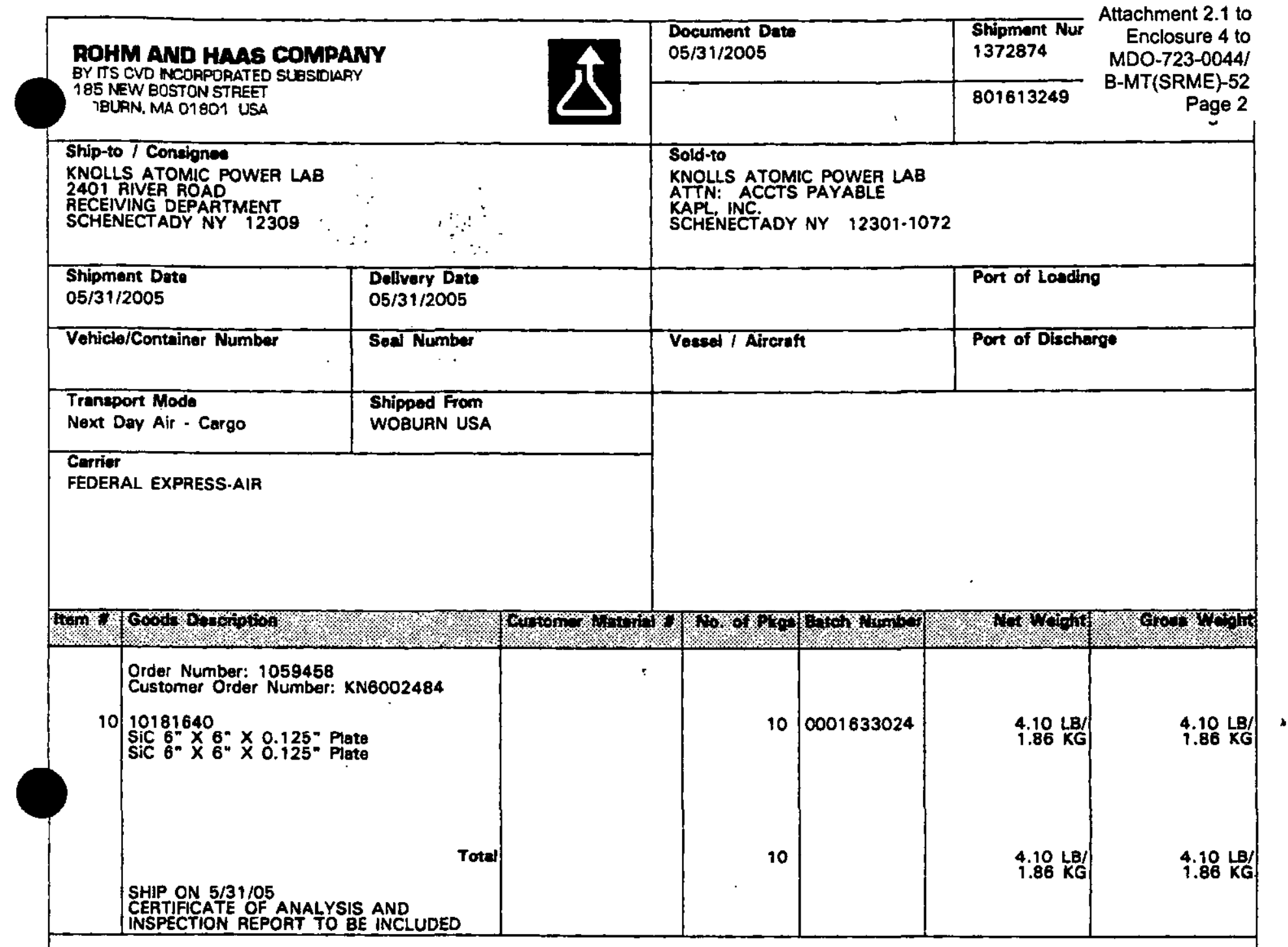




\section{PART INSPECTION REPORT}

CUSTOMER ORDER \#: KN6002484

DATE: MAY 31, 2005

R\&H W.O. \#:1059458

Part Name:PLATE

DRAWING \#: N/A

\section{MATERLAL: $\quad$ CHDSTICONCAR:IDE}

\begin{tabular}{|c|c|c|}
\hline QTY & & \\
\hline Soure & & 9139 \\
\hline Spectr & & SPECIFICATTONS IN \\
\hline & Spedifeadion & Acrual \\
\hline 는 & $6 \times 6 \times 0.125$ PLATE & $10 \mathrm{ACC}$ \\
\hline & & \\
\hline & & \\
\hline & & \\
\hline & & \\
\hline & & \\
\hline & & \\
\hline & & \\
\hline & & \\
\hline & & \\
\hline & & \\
\hline & & \\
\hline Vibual & & \\
\hline Diepor & & \\
\hline
\end{tabular}

\author{
INSPECTOR: C. BEDARD
}

\section{COMMENTS:}




\section{Certificate of Analysis}

ROHM AND HAAS COMPANY

Page 4

185 NEW BCSTON STAET, WOBUAN, MA 01801-627B USA

TELEPHONE: (781) 933-9243 FAX: [781] 933-5142

\section{Material: CVD SILICON CARBIDE_(8)}

Lot No.:9139

Customer: KAPL
Purchase Order No: KN6.002484
Rohm and Haas WO \#: 1059458

Run No_9139 was performed in our Weeks Island, LA, SiC facility.

Two samples were tested for crystallographic structure, grain size and microstructure, one sample was tested for total trace impurities, a minimum of six samples were tested for thermal conductivity, and thirty samples were prepared for flexural strength (modulus of rupture) testing. Measured results are listed below.

\section{Property}

Grain size ( $\mu \mathrm{m})$ :

Chemical purity (ppmw):

(glow discharge mass spectroscopy)

Thermal Conductivity $\left(\mathrm{W} \cdot \mathrm{m}^{-1} \cdot \mathrm{K}^{-1}\right)$

Flexural Strength (MPa)
Average Value

$8.9 \pm 1.2$

$<1.35$

$295 \pm 3$

$457 \pm 47$

\section{Conclusions}

CVD SILICON CARBIDE® from lot Run No. _9139 is a typical high purity, polycrystalline, pure $\beta$-phase $\mathrm{SiC}$ produced at Rohm and Haas Advanced Materials. It has been processed in accordance with the manufacturing procedures for CVD SLICON CARBIDEB maintained by Rohm and Haas Advanced Materials, and was produced to meet the nominal material properties for CVD SILICON CARBIDE® as listed on our data sheet.

MATERIAL FROM THUS LOT HAS BEEN ACCEPTED FOR USE TOWARDS ALL CUSTOMER ORDERS.

Roman Baran QA/QC Department Date $2 / 18 / 05$

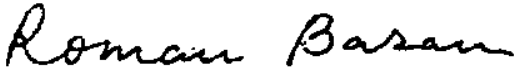


Attachment 3.1 to

Enclosure 4 to MDO-723-0044/

B-MT(SRME)-52

Page 1

Attachment 3.1

Material Purchase Documentation and Certification Composite SiC Tensile Specimens and Thermal Diffusivity Discs

Enclosure 4 to MDO-723-0044/B-MT(SRME)-52 
Hyper-Therm High-Temperature Composites, Inc.

Attachment 3.1 to

Enclosure 4 to

MDO-723-0044I

B-MT(SRME)-52

Page 2

18411 Gothard Street, Units A, B \& C

Tel: (714) 375-4085

Huntington Beach, CA 92648

Fax: (714) 375-4087

September 30,2003

Mr. Bill Mullarkey

Knolls Atomic Power Laboratory, Inc.

P.O. Box 1072

Schenectady, NY 12301

Dear Bill:

The following is the technical description for the fabrication of Hi-Nicalon Type S 8-HS, HiNicalon Type S PW, Tyranno SA 8-HS, and Tyranno SA.PW SiC-matrix composite panels shipped 10/1/03. This work was performed under KAPL Purchase Order No. M003260004AG.

Four (4) laminated Hi-Nicalon Type $S$ fiber preforms, nominally 6-inches $x$ 6-inches in planform $x$ a target 0.125 -inches in thickness, were each fabricated by stacking 11 plies of 8 harness satin weave (8HS) SiC fabric in a cross-ply $(0 / 90)$ orientation. The areal weight of the fabric used (provided by KAPL) was given as $355 \mathrm{~g} / \mathrm{m}^{2}$, and the fiber density was given as $3.0 \mathrm{~g} / \mathrm{cm}^{3}$. The optimum target " as-tooled" fiber volume fraction employed for the fabrication of Hi-Nicalon-based $\mathrm{SiC}$ matrix composites is $\sim 40 \%$. However, because of the particular fabric weave physical characteristics (i.e.. $8 \mathrm{HS}$ ) used in the fabrication of these plates, $40 \%$ was not exactly achievable. The two (2) closest fiber volume fractions obtainable were $38 \%$ using 11 plies and $42 \%$ using 12 plies. The $38 \%$ as-tooled fiber volume fraction was selected for two reasons, namely: (1) the lower volume fraction, although not optimum, will yield a better balance of mechanical properties (i.e., matrix cracking strength, ultimate strength and modulus) than too high a volume fraction (i.e., $>40 \%$ ); and (2) initial as-tooled volume fractions in excess of $40 \%$ result in producing composites with dimpled surfaces due to the excessive required compaction in the perforated fixturing tool. Thus, the $11-\mathrm{ply} / 38 \mathrm{~V}_{\mathrm{f}} \%$ was selected for fabrication. The dry laminated preforms were fixtured and compacted in graphite holding tools to maintain an initial fiber volume fraction of $38 \%$ by controlling the preform thickness to 0.125 -inches prior to subsequent composite processing. No resinous binders were used in the layup/preforming process.

Four (4) laminated Hi-Nicalon Type S fiber preforms, also nominally 6 -inches $\times 6$-inches in planform $x$ a target 0.125 -inches in thickness, were each fabricated by stacking 13 plies of plain weave (PW) SiC fabric in a cross-ply (0/90) orientation. The areal weight of the fabric used (provided by KAPL) was given as $271 \mathrm{~g} / \mathrm{m}^{2}$, and again the fiber density was 3.0 $\mathrm{g} / \mathrm{cm}^{3}$. The optimum target " as-tooled" fiber volume fraction employed for the fabrication of Hi-Nicalon-based $\mathrm{SiC}$ matrix composites is $-40 \%$. However, because of the particular fabric weave physical characteristics (i.e., PW) used in the fabrication of these plates, $40 \%$ was not exactly achievable. The two (2) closest fiber volume fractions obtainable 
were $38 \%$ using 13 plies and $.42 \%$ using 14 plies. The $38 \%$ as-tooled fiber volume fraction

was selected for two reasons, namely: (1) the lower volume fraction, although not optimum, will yield a better balance of mechanical properties (i.e., matrix cracking strength, ultimate strength and modulus) than too high a volume fraction (i.e., $>40 \%$ ); and (2) initial as-tooled volume fractions in excess of $40 \%$ result in producing composites with dimpled surfaces due to the excessive required compaction in the perforated fixturing tool. Thus, the 13-ply $/ 38 \mathrm{~V}_{\mathrm{f}} \%$ was selected for fabrication. The dry laminated preforms were fixtured and compacted in graphite holding tools to maintain an initial fiber volume fraction of $38 \%$ by controlling the preform thickness to 0.125 -inches prior to subsequent composite processing. No resinous binders were used in the lay-up/preforming process.

Four (4) laminated Tyranno SA fiber preforms, nominally 6 -inches $\times 6$-inches in planform $x$ a target 0.125 -inches in thickness, were each fabricated by stacking 12 plies of $8 \mathrm{HS}$ SiC fabric in a cross-ply $(0 / 90)$ orientation. The areal weight of the fabric used (provided by $\mathrm{KAPL}$ ) was measured to be $305 \mathrm{~g} / \mathrm{m}^{2}$, and the fiber density was given as $3.1 \mathrm{~g} / \mathrm{cm}^{3}$. The optimum target " as-tooled" fiber volume fraction employed for the fabrication of Tyrannobased SiC matrix composites is not currently known. However, this fiber was assumed to be similar to that of Hi-Nicalon Type S. Because of the particular fabric weave physical characteristics, a $40 \%$ fiber volume fraction was not achievable. The two (2) closest fiber volume fractions obtainable were $38 \%$ using 12 plies and $41 \%$ using 13 plies. The $38 \%$ as-tooled fiber volume fraction was selected for reasons previously given for that of $\mathrm{Hi}$ Nicalon Type S. Thus, the $12-\mathrm{ply} / 38 \mathrm{~V} / \%$ was selected for fabrication. The dry laminated preforms were fixtured and compacted in graphite holding tools to maintain an initial fiber volume fraction of $38 \%$ by controlling the preform thickness to 0.125 -inches prior to subsequent composite processing. No resinous binders were used in the layup/preforming process.

Four (4) laminated Tyranno SA fiber preforms, nominally 6 -inches $\times 8$-inches in planform $x$ a target 0.125 -inches in thickness, were each fabricated by stacking 14 plies of plain weave (PW) SiC fabric in a cross-ply $(0 / 90)$ orientation. The areal weight of the fabric used (provided by KAPL) was measured to be $262 \mathrm{~g} / \mathrm{m}^{2}$, and the fiber density was given as 3.1 $\mathrm{g} / \mathrm{cm}^{3}$. Again, the optimum target " as-tooled" fiber volume fraction employed by HyperTherm for the fabrication of Tyranno-based SiC matrix composites is not currently known. However, this fiber was assumed to be similar to that of Hi-Nicalon Type S. Because of the particular fabric weave physical characteristics, a $40 \%$ fiber volume fraction was not achievable. The two (2) closest fiber volume fractions obtainable were $38 \%$ using 14 plies and $41 \%$ using 15 plies. The $38 \%$ as-tooled fiber volume fraction was selected for the same reasons previously given. Thus, the $14-\mathrm{ply} / 38 \mathrm{~V}_{\mathrm{f}} \%$ was selected for fabrication. The dry laminated preforms were fixtured and compacted in graphite hoiding tools to maintain an initial fiber volume fraction of $38 \%$ by controlling the preform thickness to 0.125 -inches prior to subsequent composite processing. No resinous binders were used in the layup/preforming process.

It is well known that in order to exploit the benefits of fiber-reinforced ceramics, a relatively weak fiber/matrix interfacial bond strength is necessary to prevent catastrophic failure from propagating matrix cracks. The interface must provide sufficient fiber/matrix bonding for effective load transfer, but must be weak enough to debond and slip in the wake of matrix cracking while leaving the fibers to bridge the cracks and support the far-field applied load. Currently available fiber coatings such as pyrolytic carbon and BN have demonstrated the 
desired mechanical characteristics necessary to enhance the composite strength and Page 4 toughness, however the utility of these coating materials are severely limited by their susceptibility to mechanical degradation when exposed to high-flux neutron irradiation. This limitation is due in part to the dimensional and microstructural instability (volumetric shrinkage and densification) of the fiber coating resulting from radiation exposure. Since toughness is the primary advantage of fiber-reinforced ceramic composites, stable fiber coating systems are therefore critical to the resulting performance of these materials.

In effort to enhance the durability of ceramic composites, Hyper-Therm HTC has developed an engineered multilayer fiber coating system based on CVI SiC as an alternative to $\mathrm{PyC}$ and $\mathrm{BN}$. All $\mathrm{SiC} / \mathrm{SiC}$ composites fabricated under this effort were processed with Hyper-Therm's patented multilayer SiC fiber coating system. This coating system consists of alternating layers of PyC and SiC produced by CVI. The very thin PyC secondary layers were deposited to a target thickness of $<25 \mathrm{~nm}$, and the SiC primary layers were deposited to a target thickness of about $100 \mathrm{~nm}$. The resulting 4 layer (SiC) fiber coating system was deposited onto the laminated preforms with a total thickness of about $0.4 \mu \mathrm{m}$. Figure 1 shows a high magnification backscattered electron image of the multilayer $\mathrm{SiC}$ fiber coating system.

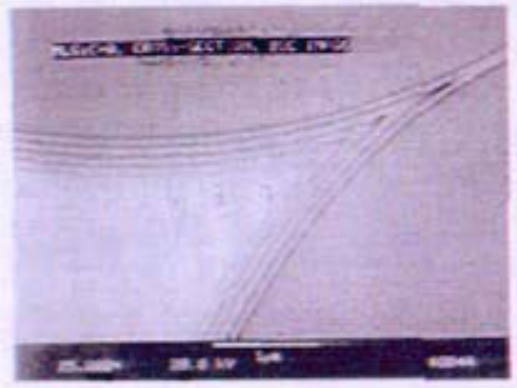

Figure 1. Fiber Coating Microstructure

Following the application of the fiber coating, the panels were consolidated with a CVIbased SiC matrix to a target density level of $2.7 \mathrm{~g} / \mathrm{cm}^{3}$. However, final densities were less than that anticipated. Tables 1-4 summarize the physical properties measured on the various $\mathrm{SiC} / \mathrm{SiC}$ composite panels produced under this effort. Using CVI processing methods that typically yield densities of at least $2.7 \mathrm{~g} / \mathrm{cm}^{3}$ in Hi-Nicalon and Hi-Nicalon Type S PW laminates, these composites fabricated from $8 \mathrm{HS}$ fabric and Tyranno $8 \mathrm{HS} / \mathrm{PW}$ had lower than expected final densities. The reason for the lower final densities for these composites is attributed to: (1) the higher areal weight Hi-Nicalon Type S satin fabric, as compared to that of plain weave, has a more tortuous initial porosity structure than the "open" plain weave. This increased tortuosity results in closing off accessible surface porosity sooner than that of more open plain weave fabric architectures, thus resulting in lower theoretical densities and higher residual porosities for these composites. The best solution to this problem is to relax the number of tow ends per linear inch in the fabric weave such that the final areal weight is about the same as that for plain weave fabric (e.g., $<300 \mathrm{~g} / \mathrm{m}^{2}$ ). (2) the Tyranno fiber has a diameter $(\sim 11 \mu \mathrm{m})$ nearty half that of HiNicalon $(-13 \mu \mathrm{m})$. And unlike that of Hi-Nicalon, Tyranno fibers are highly- uniform in diameter, which allows them to pack more intimately in their tow bundle. The combination 
of small fiber diameter and intimate filament packing increases the level of difficulty for CVI-densification of the woven preform. As a result, the filament bundles and planform surfaces seal off prematurely, thus limiting the final theoretical density level of the finished composite.

Final fiber volume fractions were calculated based on the measurements of preform fabric areal weight, fiber density (manufacturers data) and final panel thickness in accordance with Equation 1. The Hi-Nicalon Type $\mathrm{S} 8$-harness satin weave fabric used in this effort had a measured areal weight of $355 \mathrm{~g} / \mathrm{m}^{2}$ and a reported density of $3.00 \mathrm{~g} / \mathrm{cm}^{3}$, and the Hi-Nicalon Type S plain weave had a measured areal weight of $271 \mathrm{~g} / \mathrm{m}^{2}$. The Tyranno SA $8 \mathrm{HS}$ had a measured areal weight of $305 \mathrm{~g} / \mathrm{m}^{2}$ and a reported fiber density of $3.1 \mathrm{~g} / \mathrm{cm}^{3}$. and the Tyranno plain weave had a measured areal weight of $262 \mathrm{~g} / \mathrm{m}^{2}$. Composite bulk densities were thus calculated from mass and dimensional measurements taken on each panel produced.

$$
V_{f}=\frac{n A_{w}}{\rho_{f} h}
$$

where $\boldsymbol{n}$ is the number of plies comprising the laminate, $\mathbf{A}_{\mathbf{w}}$ is the areal weight of the fabric reinforcement, $\rho_{f}$ is the fiber density, and $h$ is the overall laminate thickness.

Residual porosity was determined in accordance to Equation 2. The value for porosity is a total value, which includes the addition of internal (closed) and external (open) porosity components.

$$
v_{p}=1+\left[V_{f}\left(\frac{\rho_{f}}{\rho_{m}}-1\right)+V_{i}\left(\frac{\rho_{i}}{\rho_{m}}-1\right)-\left(\frac{\rho_{c}}{\rho_{m}}\right)\right]
$$

where $\rho_{\mathrm{c}}$ is the measured composite bulk density, $\rho_{\mathrm{m}}$ is the matrix density, $\rho_{\mathrm{l}}$ is the interphase (i.e., fiber coating) density, and $V_{1}$ is the interphase volume fraction given by:

$$
V_{i}=V_{f}\left[\left(\frac{d_{f}+2 t_{i}}{d_{f}}\right)^{2}-1\right]
$$

where $d_{f}$ is the nominal fiber diameter and $t_{t}$ is the total fiber coating thickness. 
Attachment 3.1 to

Enclosure 4 to

MDO-723-0044/

B-MT(SRME)-52

Page 6

\section{Table 1. Physical Properties of Hi-Nicalon-S 8-HS $/ \mathrm{MLSiC}_{i} / \mathrm{CVI}^{-S_{i} \mathrm{C}_{m}}$ Composites}

11.Plles HI-Nicalon Typo-S 8-HS/CVL-SIC (Fabric Areal Weight=355g/mi', Fiber Density=3.0g/ec)

\begin{tabular}{|c|c|c|c|c|c|c|c|c|}
\hline Panel tD & Length (in) & Width (in) & Thickness (in) & Mass (q) & Density $(g / c c)$ & Fiber Volume (\%) & Interphase $\{\%\}$ & Porosily (\%) \\
\hline 1 & 7.188 & 7.188 & 0.161 & 337.91 & 2.48 & 31.8 & 4.0 & 20.4 \\
\hline 2 & 7.25 & 7.125 & 0.154 & 338.69 & 2.60 & 33.3 & 4.2 & 16.6 \\
\hline 3 & 7.188 & 7.125 & 0.158 & 342.07 & 2.58 & 32.4 & 4.1 & 17.2 \\
\hline 4 & 7.25 & 7.188 & 0.141 & 325.09 & 2.70 & 36.3 & 4.6 & 13.2 \\
\hline Average & - & - & 0.154 & 335.94 & 2.59 & 33.5 & 4.2 & 16.9 \\
\hline Std. Deviation & - & $=$ & 0.009 & 7.46 & 0.09 & 2.0 & 0.3 & 3.0 \\
\hline
\end{tabular}

Table 2. Physical Properties of Tyranno SA 8-HS $/ \mathrm{HLSiC}_{\mathrm{i}} / \mathrm{CVI}-\mathrm{SiC}_{\mathrm{m}}$ Composites 12-Plles Tyranno SA 8-HS/CVI-SiC (Fabric Areal Weight=305g/m, Fiber Density=3.1g/cc)

\begin{tabular}{|c|c|c|c|c|c|c|c|c|}
\hline Panel ID & Length (in) & Width (in) & Thickness (in) & Mass (g) & Density ( $g / c c)$ & Fiber Volume (\%) & interphase (\%) & Porosity (\%) \\
\hline 1 & 7.125 & 7.125 & 0.145 & 326.11 & 2.70 & 32.1 & 4.8 & 14.5 \\
\hline 2 & 7.125 & 7.063 & 0.141 & 294.82 & 2.54 & 33.0 & 5.0 & 19.7 \\
\hline 3 & 7.125 & 7.063 & 0.143 & 316.03 & 2.68 & 32.5 & 4.9 & 15.2 \\
\hline 4 & 7.125 & 7.063 & 0.142 & 316.14 & 2.70 & 32.7 & 4.9 & 14.6 \\
\hline Average & - & - & 0.143 & 313.28 & 2.65 & 32.6 & 4.9 & 16.0 \\
\hline Sid. Deviation & - & - & 0.002 & 13.18 & 0.08 & 0.4 & 0.1 & 2,5 \\
\hline
\end{tabular}

Table 3. Physical Properties of Hi-Nicalon-S PW $/$ MLSiC $/$ CVII-SiC $_{m}$ Composites 13-Plies HI-Nicalon Type-S Plain Weave/CVI-SiC (Fabric Areal Weighta27 $1 \mathrm{~g} / \mathrm{m}^{6}$, Fiber Denslty=3.0g/cc)

\begin{tabular}{|c|c|c|c|c|c|c|c|c|}
\hline Panel ID & Length (in) & Width (in) & Thickness (in) & Mass (g) & Density $(\mathrm{g} / \mathrm{cc})$ & Fiber Volume (\%) & Inlephase (\%) & Porosity $(\%)$ \\
\hline 1 & 7.875 & 6.25 & 0.16 & 343.24 & 2.66 & 32.5 & 4.1 & 14.7 \\
\hline 2 & 7.938 & 6.313 & 0.148 & 325.36 & 2.68 & 35.2 & 4.5 & 14.0 \\
\hline 3 & 8.063 & 6.25 & 0.157 & 360.77 & 2.78 & 33.1 & 4.2 & 10.9 \\
\hline 4 & 7.875 & 6.313 & 0.144 & 319.52 & 2.72 & 36.1 & 4.6 & 12.5 \\
\hline Average & $=$ & - & 0.152 & 337.22 & 2.71 & 34.2 & 4.3 & $13 . \overline{0}$ \\
\hline Sid. Deviation & - & $=$ & 0.008 & 18.66 & 0.05 & 1.7 & 0.2 & 1.7 \\
\hline
\end{tabular}


Attachment 3.1 to

Enclosure 4 to

MDO-723-0044/

B-MT(SRME)-52

Page 7

Table 4. Physical Properties of Tyranno SA PW/MLSiC/CVI-SiC $\mathrm{C}_{m}$ Composites

14-Plies Tyranno SA Plain Weave/CV/-SIC, (Fabric Areal Weight=262g/mi, Fiber Dens $/ \mathrm{ty}=3.1 \mathrm{~g} / \mathrm{cc}$ )

\begin{tabular}{|c|c|c|c|c|c|c|c|c|}
\hline Panel ID & Length (in) & Width (in) & Thickness (in) & Mass (g) & Density (g/cc) & Fiber Volume $(\%)$ & Interphase (\%) & Porosity (\%) \\
\hline 1 & 7.125 & 7.125 & 0.146 & 321.36 & 2.65 & 31.9 & 4.8 & 16.3 \\
\hline 2 & 7.125 & 7.125 & 0.145 & 308.11 & 2.55 & 32.1 & 4.8 & 19.2 \\
\hline 3 & 7.125 & 7.063 & 0.142 & 300.04 & 2.56 & 32.8 & 4.9 & t8.9 \\
\hline 4 & 7.125 & 7.063 & 0.138 & 304.92 & 2.68 & 33.8 & 5.1 & 15.2 \\
\hline Average & - & $\overline{5}$ & 0.143 & 308.61 & 2.61 & 32.6 & 4.9 & 17.4 \\
\hline Std. Deviation & - & - & 0.004 & 9.13 & 0.06 & 0.8 & 0.1 & 1.9 \\
\hline
\end{tabular}




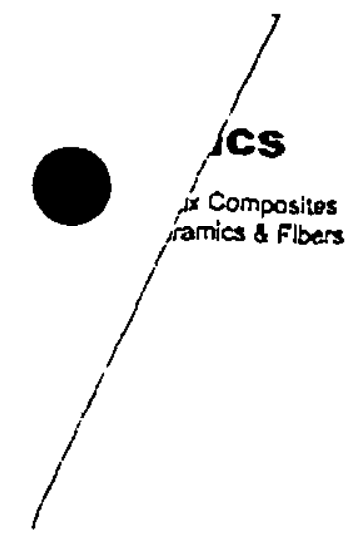

$$
\begin{array}{r}
B \times x \\
129
\end{array}
$$

Attachment 3.1 to Enclosure 4 to MD0-723-0044/ B-MT(SRME)-52

Page 8

To:

Product:

Material Number:
Mr. George Newsome or Bill Mullarkey KAPL

HI-NICALON Silicon Carbide Cloth

None

1. Product Identification

HNSP1717

2. Quantity $\left(\mathrm{m}^{2}\right)$

6.0

3. Manufacture Date

January, 2002

4. Shipment Date

January 24,2002

5. Customer PO Number

PL00105413AJ, Line \#3

6. Cloth Specifications

$\begin{array}{ll}\text { Weaving Construction } & \text { Plain Weave } \\ \text { Sizing Resin Type } & \text { PVA (polyvinyl alcohol) } \\ \text { Tread Count (/inch) } & \text { Warp }=17 ; \text { Weft }=17 \\ \text { Width } x \text { Length }(\mathrm{m}) & 0.3 \times 20.0 \\ \text { Lot Number } & \text { AP3154-01-IS-01 } \\ \text { Pieces } & 1\end{array}$

7. Cloth Test Data

Cloth Weight $\left(\mathrm{g} / \mathrm{m}^{2}\right)$

271

Thickness (mm)

0.30

8. Specifications of Yarn Used

$\begin{array}{ll}\text { Yarn Grade } & \text { HI-NICALON Type S } \\ \text { Filaments/Yarn } & 500\end{array}$

7. Test Results of Yarr:

Yan Lot PG319201

Tex Count ( $\mathrm{g} / 1000 \mathrm{~m})$

Density $(\mathrm{g} / \mathrm{cc})$

Tensile Strength (GPa)

Tensile Modulus (GPa)

Sizing (wt.\%)

Oxygen Content (wt. \%)

188

3.0

2.8

380

1.7

0.7

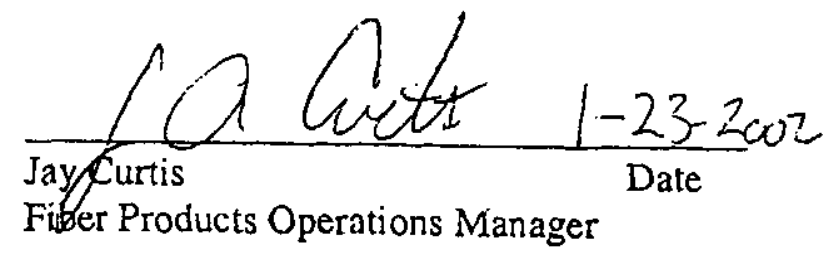

- Phone +1801 PRE-DECISIONAL - For Planning and Discussion Purposes Only .. 


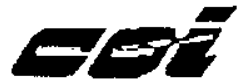

\section{Ceramics}

Coramic Martix Composiles

Speciaity Ceramics A Fibers

January 24,2002

Mr. George Newsome or Bill Mullarkey

KAPL

P. O. Box 1072

Schenectady, NY 12301-1072

Dear George or Bill,

Please find enclosed 20 linear meters $(30 \mathrm{~cm}$ wide $x 20$ meters long) of HI-NCALON Type STM $\mathrm{SiC}$ cloth, plain weave, PVA sizing. Detailed information on this cloth is provided in the following certification sheet. This shipment is sent in fulfillment of Line \#3 of KAPL PO \#PLO0105413AJ.

If you have any qucstions, please contact me anytime at the number/email below. Thank you.

Sincerely,

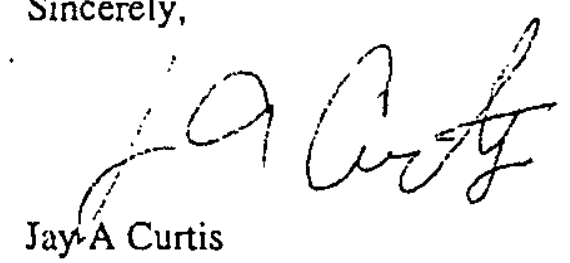

Fiber Producis Operations Manager

Enclosures 
23 Depot St., Merrimack, NH 03054

Phone: (603) 429-9500

FAX: (603) 429-9471

Ms. Rebecca Northey

KAPL, Inc.

PO Box 1072

Schenectady, New York 12301-1072
Withon

Hi: $\because 46$

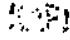

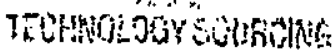

\begin{tabular}{ll} 
RECEIVED & IO/2S/OS \\
IDENT AS & SeE below \\
MATERIAL & SiC \\
CONOITION & Solid \\
TEST TO & \\
\hline
\end{tabular}

SAMPLE NUMBER L30829

REPORT DATE .11/7/105

PAGE I Of I

CLIENT ORDER KN6004010

TEST PER

TP.LECOI

Method:

Snaole I.D.

A-ANX

A.CAF

\section{LECO}

$\mathrm{N}(\%)$

$0.0057 \pm 0.0002$ Cinposite Soximion?

$0.0114 \pm 0.0006$
Anelysis By:

Peter S. Dickuon Analvical Chemi

Approved By: Williapata. Guidoboni 


\section{Customer: Knolls Atomic Power Laboratory \\ Date: \\ 3-Nov-05}

Customer ID: SiC

\section{A-ANW}

\begin{tabular}{|c|c|c|c|}
\hline Element & $\begin{array}{c}\text { Concentration } \\
\text { (gpm wt }\end{array}$ & Element & $\begin{array}{l}\text { Concentration } \\
\text { [pgen wt] }\end{array}$ \\
\hline $\mathrm{Li}$ & $<0.01$ & $P d$ & $<0.01$ \\
\hline Be & $<0.005$ & $\mathrm{Ag}$ & $\leq 0.5$ \\
\hline $\mathrm{B}$ & 1.3 & $\mathrm{Cd}$ & $<0.5$ \\
\hline $\mathrm{C}$ & Matrix & $\ln$ & $<1$ \\
\hline $\mathrm{N}$ & -80 & $\mathrm{sn}$ & $<0.05$ \\
\hline 0 & $-0.3 w \%$ & $S b$ & $<0.05$ \\
\hline$F$ & $<0.5$ & $\mathrm{Te}$ & $<0.05$ \\
\hline $\mathrm{Na}$ & 0.29 & 1 & $\leq 0.01$ \\
\hline $\mathrm{Mg}$ & 0.29 & $\overline{c s}$ & $<0.005$ \\
\hline Al & 2.5 & $\mathrm{Ba}$ & 2.3 \\
\hline Si & Matrix & La & $<0.005$ \\
\hline$P$ & 0.38 & $\mathrm{Ce}$ & $<0.01$ \\
\hline $\bar{s}$ & 14 & $\overline{P_{f}}$ & $<0.01$ \\
\hline C! & 120 & Nd & $<0.005$ \\
\hline $\mathrm{K}$ & $<0.05$ & $\mathrm{sm}$ & $<0.005$ \\
\hline $\mathrm{Ca}$ & 1.2 & $E \mathrm{E}$ & $<0.005$ \\
\hline$\overline{S e}$ & $<0.01$ & $\overline{G d}$ & $<0.005$ \\
\hline$T i$ & 0.07 & $T b$ & $<0.005$ \\
\hline V & 0.06 & Dy & $<0.005$ \\
\hline $\mathrm{Cr}$ & $\angle 0.5$ & Ho & $<0.005$ \\
\hline $\mathrm{Mn}$ & $\leq 0.01$ & Er & $<0.005$ \\
\hline $\mathrm{Fe}$ & 8.4 & $\mathrm{Im}$ & $<0.005$ \\
\hline $\mathrm{Co}$ & 0.05 & $\overline{\mathrm{Yb}}$ & $<0.005$ \\
\hline $\mathrm{Ni}$ & 0.57 & Lu & $<0.005$ \\
\hline $\mathrm{CU}$ & $<0.05$ & $\mathrm{HF}$ & $\leq 0.01$ \\
\hline$Z n$ & 0.06 & Ta & Source \\
\hline Ga & $\leq 0.05$ & $\bar{W}$ & 0.18 \\
\hline$\underline{G e}$ & $<0.05$ & $\mathrm{Re}$ & $<0.01$ \\
\hline$\overline{A S}$ & $\leq 0.05$ & Os & $=0.01$ \\
\hline Se & $<0.5$ & Ir & $<0.01$ \\
\hline Br & $<0.5$ & $\overline{P t}$ & $\leq 0.01$ \\
\hline $\mathrm{Rb}$ & $<0.1$ & $\mathrm{Au}$ & $<0.05$ \\
\hline Sr & $\leq 0.01$ & $\mathrm{Hg}$ & $<0.05$ \\
\hline$Y$ & $<0.01$ & $T I$ & $<0.01$ \\
\hline $\mathrm{Zr}$ & $\leq 0.01$ & $\mathrm{~Pb}$ & $=0.01$ \\
\hline $\mathrm{Nb}$ & $<0.01$ & Bi & $<0.01$ \\
\hline Mo & 0.07 & $T h$ & 50.001 \\
\hline$R U$ & $<0.01$ & $\bar{U}$ & $\leq 0.001$ \\
\hline$R h$ & $<0.005$ & & \\
\hline
\end{tabular}
P.O.\#
KN6004044
Job \#
UO4798

Shiva 1D: $\quad$ U051026149 
Attachment 3.2 to

Enclosure 4 to

MDO-723-0044/

B-MT(SRME)-52

Page 1

\section{Attachment 3.2}

Technical Specifications for Machining

Composite SiC Tensile Specimens and Thermal Diffusivity Discs

Enclosure 4 to MDO-723-0044/B-MT(SRME)-52 


\section{SPECIFICATION FOR 05PFG071}

Rev. -

\section{DATED $5 / 4 / 05$}

1. KAPL will supply composite plate as described below:

\begin{tabular}{|l|l|l|l|l|l|}
\hline NO. & PLATE ID & $\begin{array}{l}\text { PLATE } \\
\text { TYPE }\end{array}$ & $\begin{array}{l}\text { LENGTH } \\
(\mathrm{IN})\end{array}$ & $\begin{array}{l}\text { WIDTH } \\
\text { (IN) }\end{array}$ & $\begin{array}{l}\text { THICKNESS } \\
\text { (IN) }\end{array}$ \\
\hline 1 & M00236000 & Hyper- & 7.25 & 7.25 & $\sim 0.125$ \\
(4 Plates) & $4 A G-1$ & Therm & & & \\
& M00236000 & Hi-Nicalon & & & \\
& $4 A G-2$ & Type S & & & \\
& M00236000 & CVI/SiC \\
& $4 A G-3$ & Composite & & & \\
& M00236000 & & & & \\
& $4 A G-4$ & & & & \\
& & & & & \\
\hline
\end{tabular}

\section{APPLICABLE DRAWINGS AND SKETCHES}

- KAPL Drawing 216C6883 Rev. C Diffusivity ITEM NO. 1 and 7.

- KAPL Drawing SK239B9368 Rev. - Miniature Tensile Specimen

- KAPL Drawing 108 E7300 Rev. - Default Manufacturing Practices.

- KAPL Orientation Sketch NO. 1

- KAPL Specification, Attachment 1 "Detrimental Materials Prohibitions and Restriction for Silicon Carbide Composite Materials".

- KAPL supplied Table 1 'Machining Instruction Sheet.

\section{MACHINING INSTRUCTION}

- For each piece of GFM plate, extract and machine the required number of specimens as specified in the attached table 1 "Machining Instruction".

- The location and number of specimens per plate are shown in orientation sketch NO. 1 and were specified to maximize the available GFM material. Before extracting samples, the seller shall layout its cuts to verify the quantity of each plate and report to KAPL its layout for approval.

- Samples shall not be machined from the outer $1 / 4$ " perimeter of the GFM plate.

- Stock removal rate shall be on the order of $0.03 \mathrm{~mm}$ per pass using diamond tools that have between 320 and 600 grit. 
- Remove approximately equal stock from opposite faces for both thermal diffusivity and miniature tensile specimens.

- Fibers in a composite miniature tensile specimen shall be parallel and perpendicular to the length direction.

- The surface finish for the composite miniature tensile specimen shall be within the range of $22 \mu$ in $-40 \mu$ in all over except end faces which may be $40 \mu$ in $-80 \mu$ in.

- Compliance with KAPL Specification on detrimental materials is required during all aspects of this fabrication.

- The seller shall use dedicated resin-bonded diamond tooling for all machining of silicon carbide composite specimens. If other than resin-bonded tooling is need the seller shall obtain KAPL's approval prior to fabrication.

- The seller shall also use new coolant for this work scope.

- All specimens shall be identified by a bag and tag method with plate ID, specimen type, specimen ID and drawing number.

- All specimens from each plate shall be packaged and labeled together.

- The seller shall optimize GFM material usage and return all excess GFM greater than 0.5 " $x 0.5$ ".

- There shall be a verbal briefing prior to the start of machining.

4.

\section{FIRST PIECE INSPECTION}

Two specimens of each type shall be provided for a first piece inspection to assure that they meet all of the requirements of KAPL Drawings prior to proceeding to the next piece. The seller shall notify KAPL with results of the first piece inspection prior to completing the fabrication on the balance of the order. KAPL requests the seller to provide to KAPL the specimens used for first piece inspection prior to completion of the balance of the order.

TABLE 1

Machining Instruction Sheet 05PFG071

\begin{tabular}{|l|l|l|l|l|l|}
\hline PLATE ID & $\begin{array}{l}\text { SPECIMEN } \\
\text { TYPE }\end{array}$ & $\begin{array}{l}\text { SPECIMEN } \\
\text { D's }\end{array}$ & QUANTITY & $\begin{array}{l}\text { DRAWING } \\
\text { NO. }\end{array}$ \\
\hline $\begin{array}{l}\text { M00236000 } \\
\text { 4AG } \\
\text { Plate \# 1 }\end{array}$ & $\begin{array}{l}\text { Hyper- } \\
\text { Therm } \\
\text { Hi-Nicalon } \\
\text { Type S/CVI } \\
\text { SiC } \\
\text { Composite }\end{array}$ & $\begin{array}{l}\text { Miniature } \\
\text { Tensile }\end{array}$ & $1-76$ & 76 & Dwg. \\
& & & & SK239B9368 \\
Rev. - & & & \\
\hline
\end{tabular}


Attachment 3.2 to

Enclosure 4 to

MDO-723-0044/

\begin{tabular}{|c|c|c|c|c|c|}
\hline & & $\begin{array}{l}\text { Thermal } \\
\text { Diffusivity }\end{array}$ & $1-33$ & 33 & $\begin{array}{l}\text { Dwg. } \\
\text { 216C6883 } \\
\text { ITEM } 1\end{array}$ \\
\hline & & $\begin{array}{l}\text { Thermal } \\
\text { Diffusivity }\end{array}$ & 1.15 & 15 & \begin{tabular}{|l|} 
Dwg. \\
$216 \mathrm{C} 6883$ \\
ITEM 7 \\
\end{tabular} \\
\hline \multirow[t]{3}{*}{$\begin{array}{l}\text { M00236000 } \\
\text { 4AG } \\
\text { Plate \# 2 }\end{array}$} & $\begin{array}{l}\text { Hyper- } \\
\text { Therm } \\
\text { Hi-Nicalon } \\
\text { Type S/CVI } \\
\text { SiC } \\
\text { Composite }\end{array}$ & $\begin{array}{l}\text { Miniature } \\
\text { Tensile }\end{array}$ & 1.76 & 76 & $\begin{array}{l}\text { Dwg. } \\
\text { SK239B9368 } \\
\text { Rev. - }\end{array}$ \\
\hline & & $\begin{array}{l}\text { Thermal } \\
\text { Diffusivity }\end{array}$ & $1-33$ & $\overline{33}$ & \begin{tabular}{|l} 
Dwg. \\
216C6883 \\
ITEM 1 \\
\end{tabular} \\
\hline & & $\begin{array}{l}\text { Thermal } \\
\text { Diffusivity }\end{array}$ & $1-15$ & 15 & \begin{tabular}{|l|} 
Dwg. \\
216C6883 \\
ITEM 7 \\
\end{tabular} \\
\hline \multirow[t]{3}{*}{$\begin{array}{l}\text { M00236000 } \\
\text { 4AG } \\
\text { Plate \# } 3\end{array}$} & $\begin{array}{l}\text { Hyper- } \\
\text { Therm } \\
\text { Hi-Nicalon } \\
\text { Type S/CVI } \\
\text { SiC } \\
\text { Composite }\end{array}$ & $\begin{array}{l}\text { Miniature } \\
\text { Tensile }\end{array}$ & $1-76$ & 76 & $\begin{array}{l}\text { Dwg. } \\
\text { SK239B9368 } \\
\text { Rev. - }\end{array}$ \\
\hline & & $\begin{array}{l}\text { Thermal } \\
\text { Diffusivity }\end{array}$ & $1-33$ & 33 & \begin{tabular}{|l|} 
Dwg. \\
216C6883 \\
ITEM 1 \\
\end{tabular} \\
\hline & & $\begin{array}{l}\text { Thermal } \\
\text { Diffusivity }\end{array}$ & $1-15$ & 15 & $\begin{array}{l}\text { Dwg. } \\
\text { 216C6883 } \\
\text { ITEM } 7\end{array}$ \\
\hline \multirow[t]{3}{*}{$\begin{array}{l}\text { M00236000 } \\
\text { 4AG } \\
\text { Plate \# } 4\end{array}$} & $\begin{array}{l}\text { Hyper- } \\
\text { Therm } \\
\text { Hi-Nicalon } \\
\text { Type S/CVI } \\
\text { SiC } \\
\text { Composite } \\
\end{array}$ & $\begin{array}{l}\text { Miniature } \\
\text { Tensile }\end{array}$ & $1-76$ & 76 & $\begin{array}{l}\text { Dwg. } \\
\text { SK239B9368 } \\
\text { Rev. - }\end{array}$ \\
\hline & & $\begin{array}{l}\text { Thermal } \\
\text { Diffusivity }\end{array}$ & $1-33$ & 33 & $\begin{array}{l}\text { Dwg. } \\
\text { 216C6883 } \\
\text { ITEM 1 }\end{array}$ \\
\hline & & $\begin{array}{l}\text { Thermal } \\
\text { Diffusivity }\end{array}$ & $1-15$ & 15 & \begin{tabular}{|l|} 
Dwg. \\
$216 \mathrm{C} 6883$ \\
ITEM 7 \\
\end{tabular} \\
\hline
\end{tabular}

\section{DATA REQUIREMENTS}


- Complete As-Built data for all specimens (Miniature Tensile and Diffusivity).

- Certs of Compliance with KAPL Specification on detrimental materials (Attachment 1 dated 3/6/03).

- Orientation layout.

- First piece inspection notification.

Prepared By

Reviewed By 
Attachment 3.2 to

Enclosure 4 to

MDO-723-0044/

B-MT(SRME)-52

Page 6

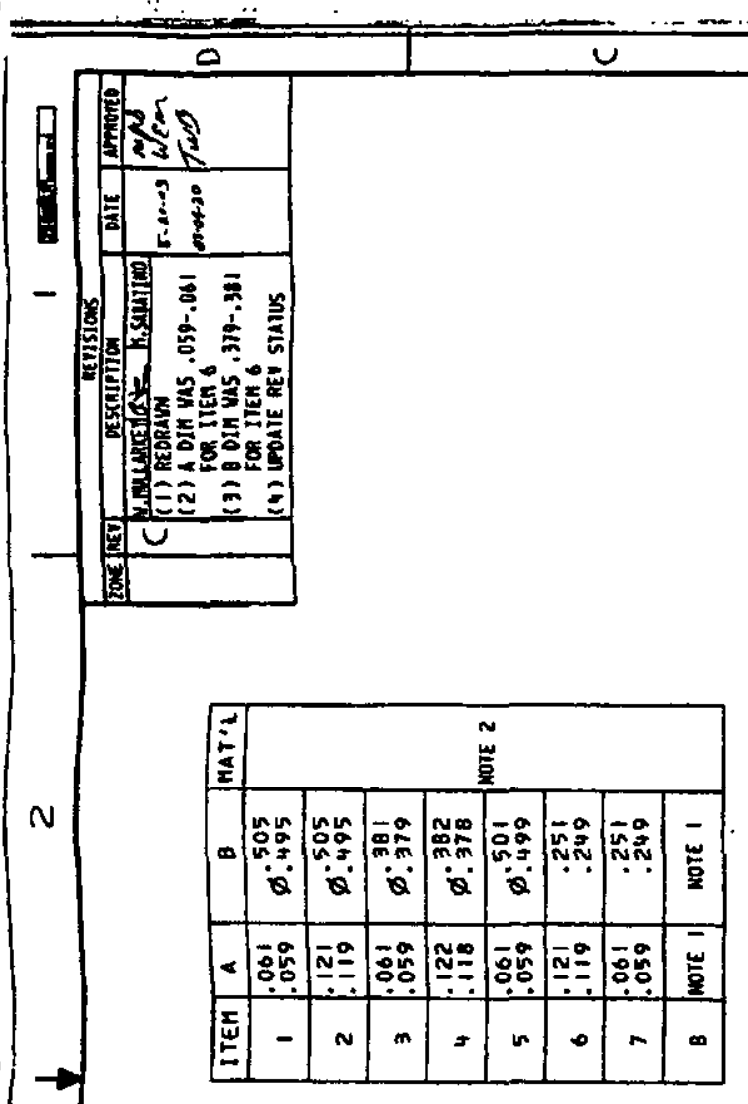

$\infty$
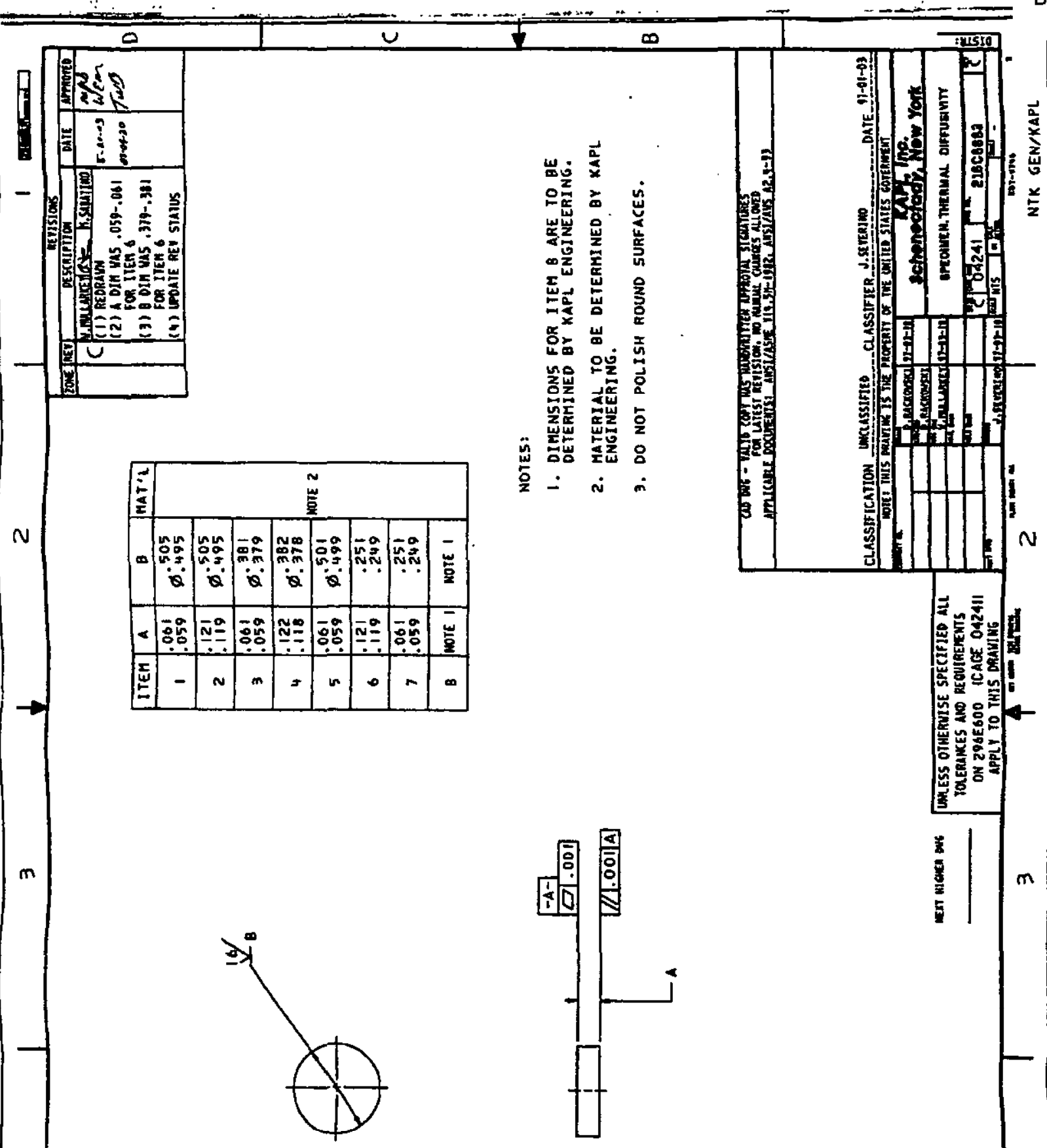

$\exists$

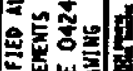

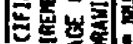

总害语

岁司:

宸咲

준

岁

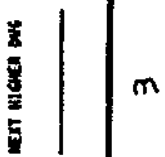


Attachment 3.2 to

Enclosure 4 to

MDO-723-0044/

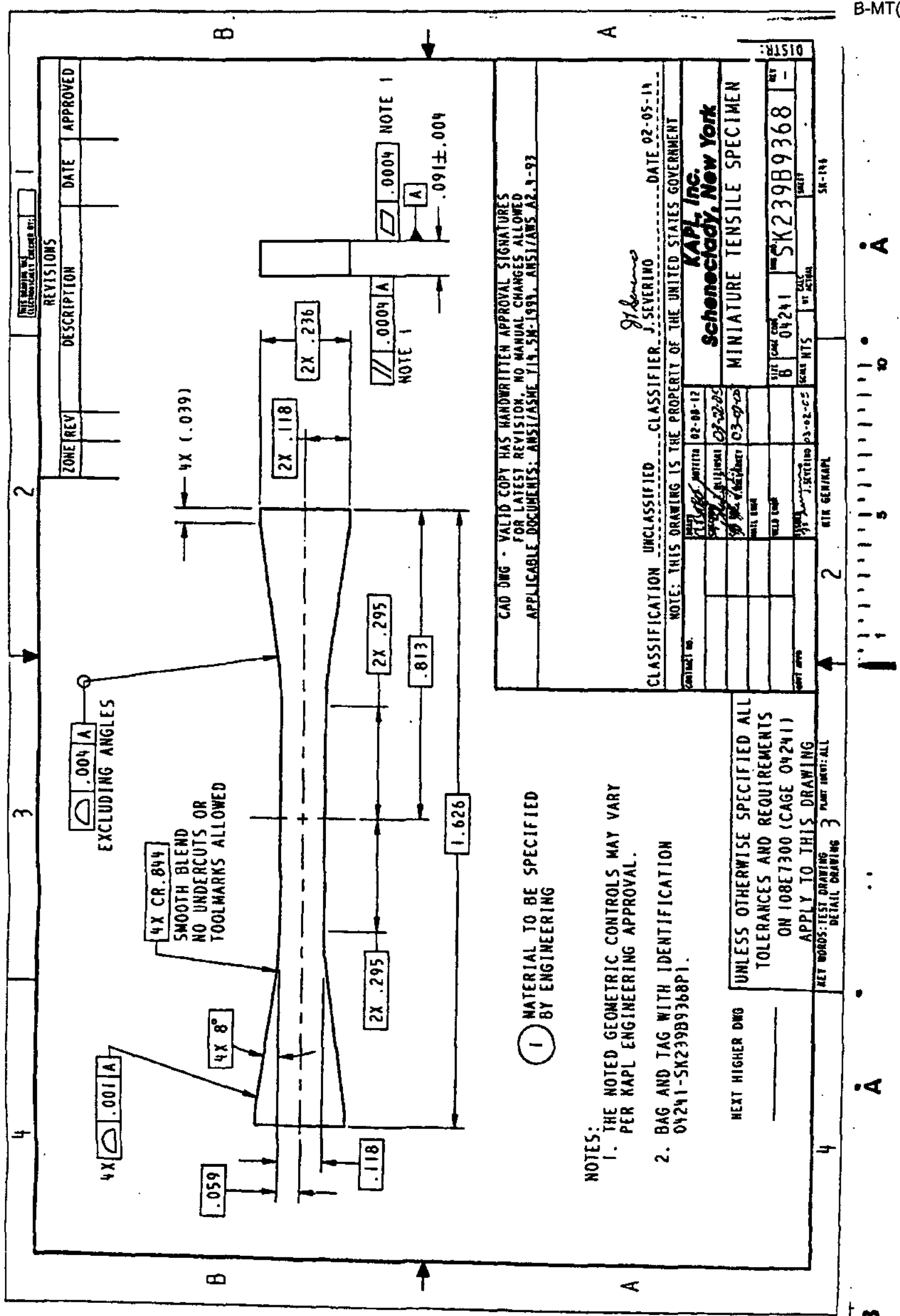

Page 7 
Attachment 3.2 to

Enclosure 4 to

MDO-723-0044/

B-MT(SRME)-52

Page 8

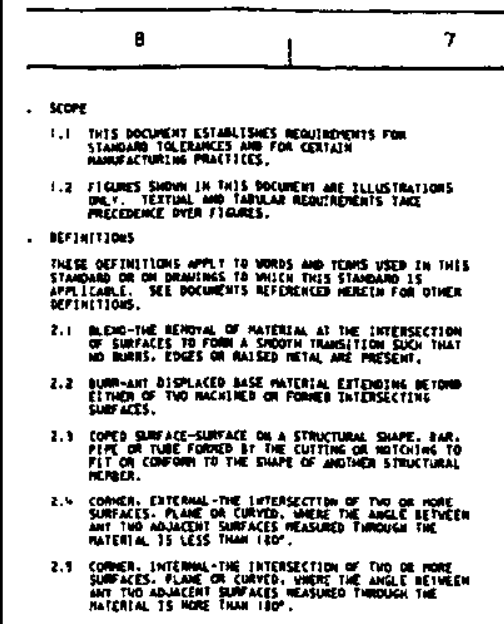

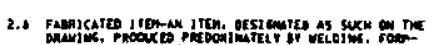
等

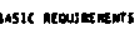

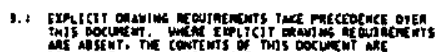

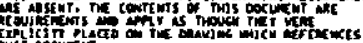

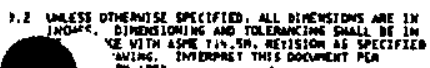
Las wo conituss

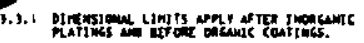

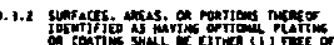

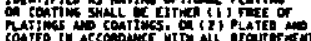

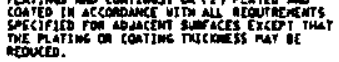

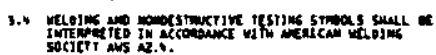

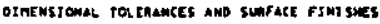

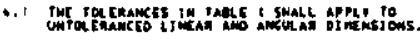

\begin{tabular}{|c|c|c|c|c|}
\hline ostrusion & meten & $t-11$ & enca $+1-14$ & orth \\
\hline - PaAte Beciras & 0.005 & 1.110 & \multirow{2}{*}{0.00} & \multirow{2}{*}{2.14} \\
\hline 2 rigefore!nuts & $t, 02$ & $5+84$ & & \\
\hline प्राES & \multirow{2}{*}{\multicolumn{4}{|c|}{ 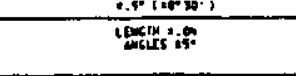 }} \\
\hline 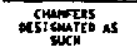 & & & & \\
\hline
\end{tabular}

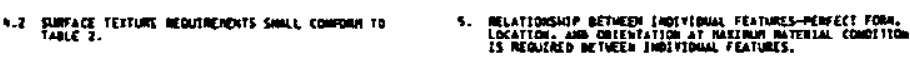

\begin{tabular}{|c|c|}
\hline topt of waskd & $\omega$ \\
\hline 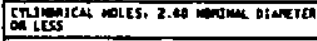 & 250 \\
\hline 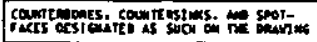 & ma \\
\hline $\cos \operatorname{sen} x+4 s^{\circ}$ & $\$ 0$ \\
\hline 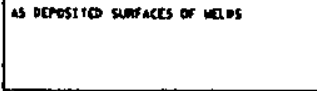 & 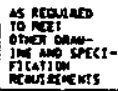 \\
\hline stock artaits & 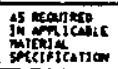 \\
\hline$\Delta 1$ arkich & 189 \\
\hline
\end{tabular}

1,1 romers

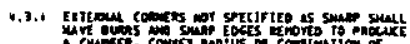

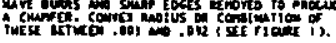

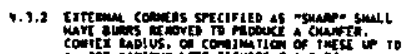
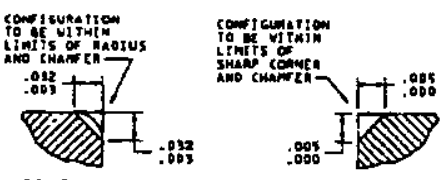

Frowat 1

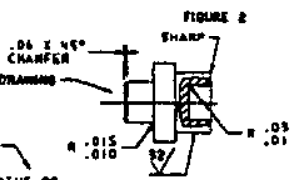

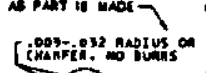

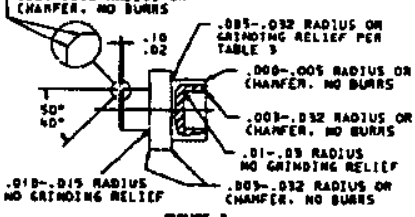

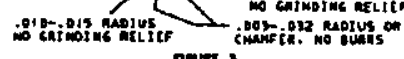

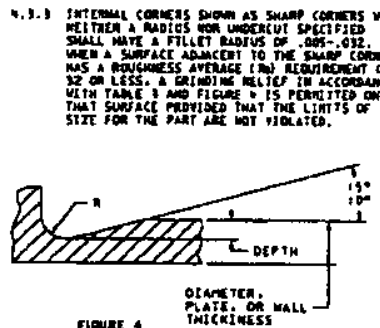

Trin

\begin{tabular}{|c|c|c|c|}
\hline \multicolumn{2}{|c|}{ 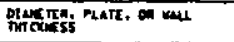 } & \multirow[t]{2}{*}{ Aepry } & \multirow[t]{2}{*}{ mesjus } \\
\hline ans & Incício & & \\
\hline$\frac{-11}{78}$ & $\pi$ & in & $+\infty$ \\
\hline
\end{tabular}
rite:

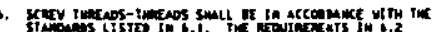

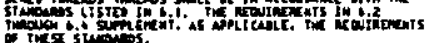

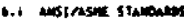

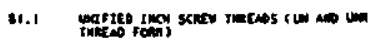

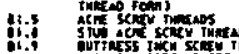

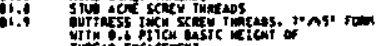

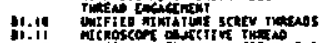

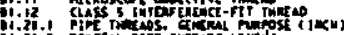

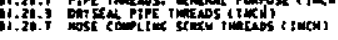

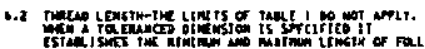

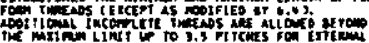

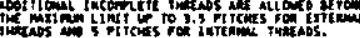

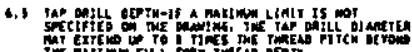

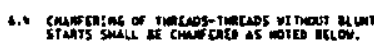

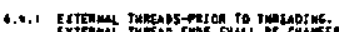

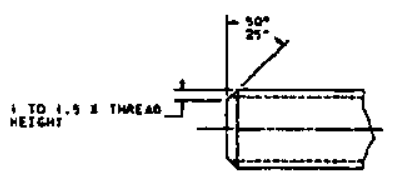

nane

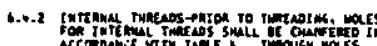

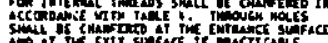

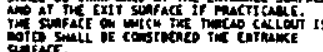

thous racinmes do Phaciless

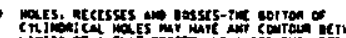

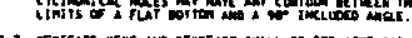

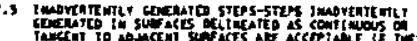
The

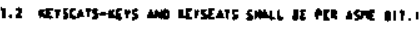

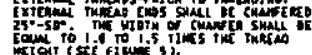

nouke -

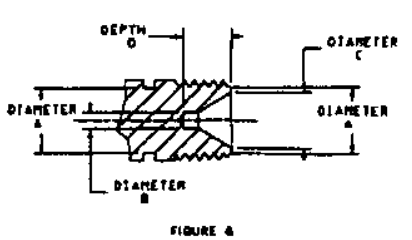

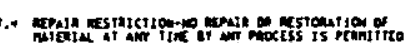

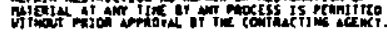

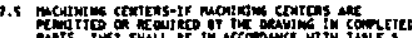

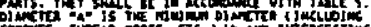

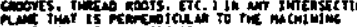

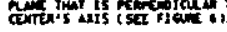

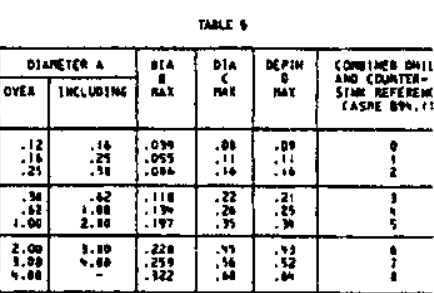

Canicafed trems

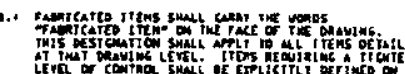

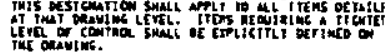

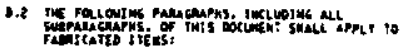

\begin{tabular}{|c|c|c|c|}
\hline \multirow{2}{*}{\multicolumn{2}{|c|}{ Tuktent 537}} & \multicolumn{2}{|c|}{ 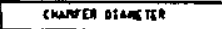 } \\
\hline & & 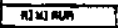 & molinum \\
\hline 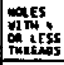 & Als thats & mos a1s r.t. & 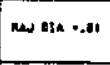 \\
\hline mots & 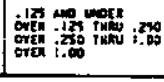 & mot of ot & 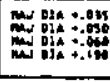 \\
\hline \multicolumn{2}{|c|}{ 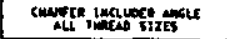 } & \multicolumn{2}{|c|}{ not - } \\
\hline
\end{tabular}

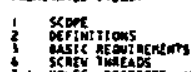

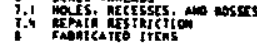

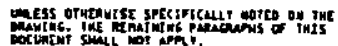

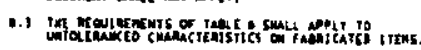

\begin{tabular}{|c|c|c|}
\hline 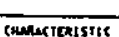 & \multicolumn{2}{|c|}{ arowisngint } \\
\hline \multirow{2}{*}{$\begin{array}{l}\text { Limean } \\
\text { Dinnusions }\end{array}$} & $\operatorname{Loth} 7$ & the wen \\
\hline & $F .11$ & $x, 8$ \\
\hline Axars & \multicolumn{2}{|c|}{.4} \\
\hline Exiphas & \multicolumn{2}{|c|}{ 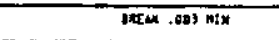 } \\
\hline tontents & \multicolumn{2}{|c|}{. } \\
\hline 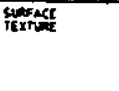 & \multicolumn{2}{|c|}{ 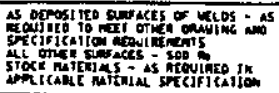 } \\
\hline
\end{tabular}

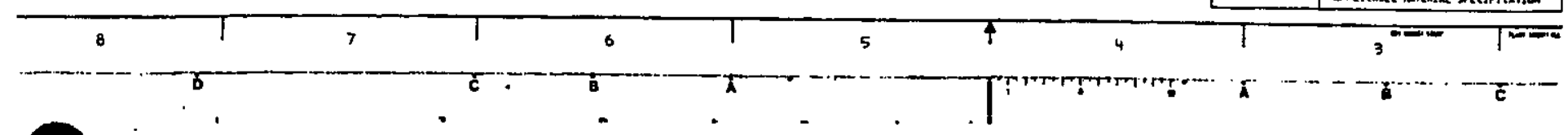


Attachment 3.2 to

Enclosure 4 to MDO-723-0044

B-MT(SRME)-52

Page 9

\section{Composite SiC}

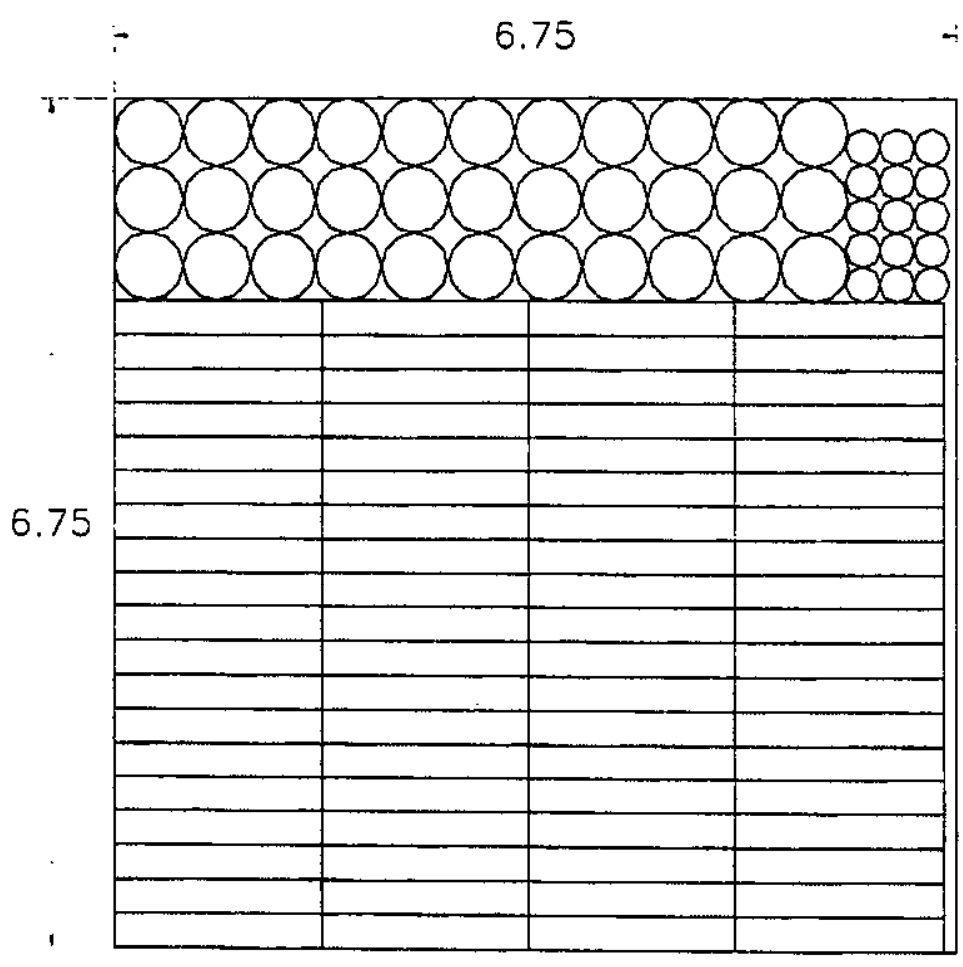

Four Plates

76 Tensile

33 Thermol Diff.

$15 \mathrm{Sm}$. Therm. Diff.

Specimen Totals

304 Tensile

132 Th. Diff. (0.5")

$60 \mathrm{Sm}$. Therm. Diff.

Specimen dimensions:

Nominal Dimension + Mox

Tolerance + 0.030" For Tooling 


\section{ATTACHMENT 1}

Attachment 3.2 to

Enclosure 4 to

MDO-723-0044/

B-MT(SRME)-52

Page 10

$3 / 6 / 03$

\section{Detrimental Materials Prohibitions and}

Restrictions for Silicon Carbide Composite

Materials

Certain chemical elements in contact with a test specimen may cause premature failure of the specimen under test. Those chemical elements known as detrimental materials must not be allowed to contaminate the specimens at any time before or during the test. Therefore, the specimens must be guarded against contamination from detrimental materials during such activities as manufacturing, packaging, shipping, inspection, or testing.

1.0 During the machining, handling, storage, or shipping of specimens, the specimens shall not come in direct contact with:

1.1 Mercury, compounds containing mercury in excess of $10 \mathrm{ppm}$, or any mercury-containing device employing a single boundary of containment.

1.2 Lead or compounds containing lead in excess of $250 \mathrm{ppm}$.

1.3 Cadmium, particularty cadmium plated tools or hardware, or alloys containing more than one percent cadmium, or compounds containing more than $250 \mathrm{ppm}$ cadmium.

1.4 Compounds containing halogens in excess of $250 \mathrm{ppm}$ (total where fluorine is restricted to $25 \mathrm{ppm}$ ) except lubricants used in machining.

1.5 Salt, or saline solutions, or any solid surface that may introduce alkali metals (e.g., sodium, potassium, lithium).

1.6 Compounds with molybdenum disulfide in excess of $250 \mathrm{ppm}$.

2.0 Compounds with compositions listed below shall not directly contact specimen surfaces after final machining.

2.1 Compounds containing more than $250 \mathrm{ppm}$ of any of the following antimony, bismuth, copper, phosphorus, sulfur, tin, zinc, total halogens (except that total fluorine shall be restricted to a maximum of $25 \mathrm{ppm}$ ).

2.2 Nuclear poisons (e.g., boron) in excess of $100 \mathrm{ppm}$.

2.3 Liquids which are not water-soluble except as allowed in Paragraphs 6.1 and 6.2 /millder/ump/DMP\&R 
3.0 Thread sealants, such as teflon tape, teflon jam nuts, or epoxy resins shal! MDO-723-0044 not be used to affix or secure specimens to fixtures.

4.0 Aluminum, copper, nickel, and alloys of these materials containing more than 50 percent aluminum, copper, or nickel shall not be used as soft pads, hammers, or tools which contact the specimens during or subsequent to final machining.

5.0 Only dedicated resin-bonded diamond tooling shall be used for all machining of silicon carbide composite materials.

\subsection{Solvent and Cleaning Solution Requirements:}

6.1 Solutions having a pH of less than 6 at the temperature at which they are to be used shall not be used to clean parts within accessible areas.

\subsection{All halogenated cleaning solutions (250 ppm or more halogens, with} fluorine restricted to $25 \mathrm{ppm}$ ) are prohibited from use.

7.0 Temperature indicating crayons, marking materials (including white-out) lubricants and low melting alloys shall not contact the final machined surfaces of the specimens if they contain:

7.1 More than $250 \mathrm{ppm}$ of any of the following antimony, arsenic, bismuth, copper, phosphorus, sulfur, tin, zinc, and total halogens (except that fluorine shall be restricted to a maximum of $25 \mathrm{ppm}$ ).

7.2 Metals or alloys which melt at 1000 degrees $F$ or less (e.g., Cerrolow, Kirksite, Cerrobend, and cadmium).

8.0 Test and machining fixtures and other components, which directly contact the final machined surfaces of the specimens shall not come in direct contact with items specified in Paragraph 1.0. If fixtures have previously contacted the items specified in Paragraph 1.0, then rigorous cleaning (such as by acid pickling) is required prior to use on the specimens.

9.0 Bench tops and work surfaces upon which the final machined specimens will be placed shall be covered by KimPak creped wadding paper (Kimberly-Clark Co.) or a similar product prior to use.

10.0 Seller shall provide a statement of conformance with the requirements of this specification. 
Attachment 3.3 to

Enclosure 4 to

MDO-723-0044/

B-MT(SRME)-52

Page 1

Attachment 3.3

Machining Documentation for

Composite SiC Tensile Specimens and Thermal Diffusivity Discs

Enclosure 4 to MDO-723-0044/B-MT(SRME)-52 
- JUN-13-2005 MON 11:44 AM MACHINED CERAMICS INC

FAX NO. 2707819361

P. 01

Attachment 3.3 to

Enclosure 4 to

MDO-723-0044/

MACHINED CERAMICS, INC. $\mathrm{B}-\mathrm{MT}$ (SRME)-52

North Industrial Park - 629 North Graham Street - Bowling Green, KY 42101 Phone: 270-781-0512 - Fax: 270-7e1-936

mall: mercer opremlernet.net

FROM: KEN MARSHALL

PHONE : $270-781-0512$

FAX: $270-781-9361$

EMAIL = 2MACCEREINSIGHTBB. COM

WEB = WWW.CERAMTCS.COM/MNCCER

$1 / 2^{\text {Page } 2}$

DATE: $4 / 13 / 05$

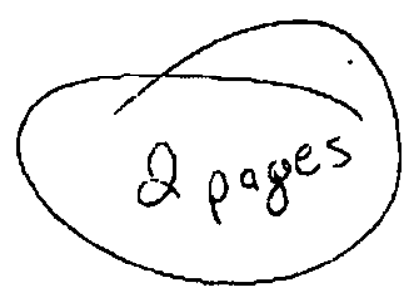

mo: skip Giffond

PHONE:

FAX: $518 \quad 395-6848$

Update for Tensile and Disk Specimens:

(1) Altered cutting sketch to allow for appropriate saw kerf. (see attached sketch)

This sketch can be altered for plates 3 and 4.

(2) Finishing thickness on all specimens first.

(3) will send out first article samples on Tuesday 6/i4 for inspection.

Please advise ASAP!

Kan Marshall

PRE-DECISIONAL - For Planning and Discussion Purposes Only

Developmental Engineering Precision Machining 
6 pes. $=.550 \times 6=3.30$

4 pes. $=2.20$

seuts .05 $\times 5=.25$

3.55

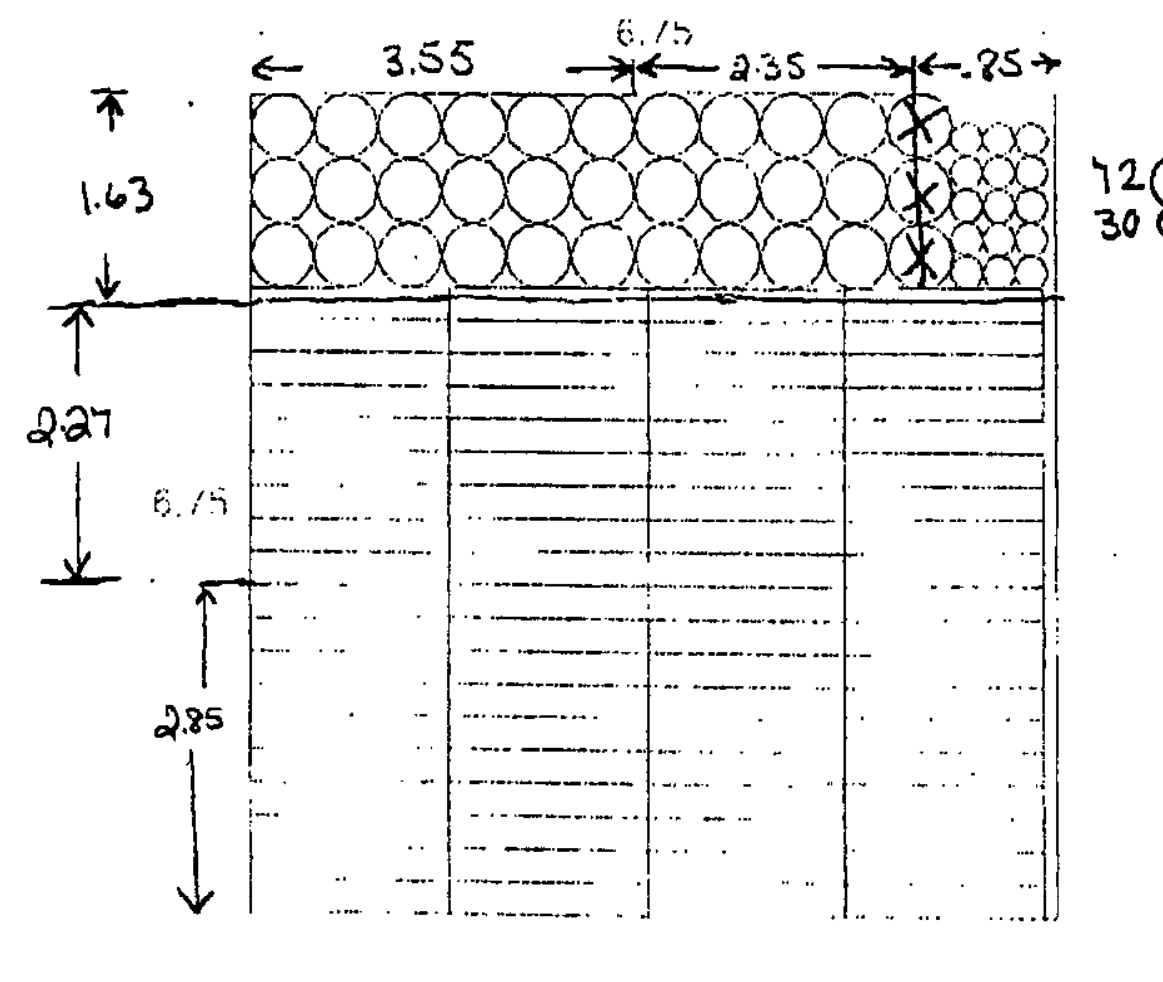

labr ploles

120 lansile

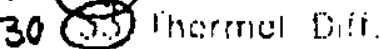

is Sim. inerm. 1h.

grementabas

sol lensile

1.3) Th. Dill. (0.3)

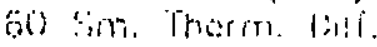

Sperimen dimersions:

Nomnul Dirnension + Max

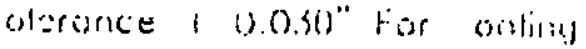

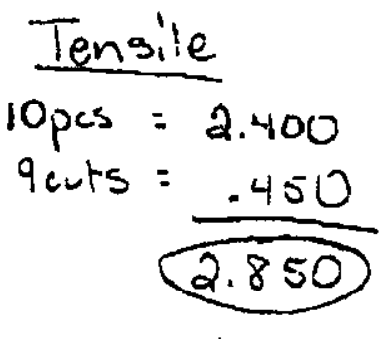

8 pes. $=1.92$

Touts $=.35$

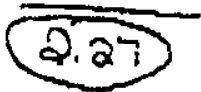




\section{Rebecca Northey $\times 6708$}

Attachment 3.3 to

Enclosure 4 to

MDO-723-0044/

Space Charge Code D2317 D3 808

B-MT(SRME)-52

lease complete the following Dimensional overcheck for the following Mini

Page 4

Tensile Specimens, Drwg SK239B9368.

Composite Mini Tensile Specimens

M002360004AG

Plate No. 1

3

18

26

53

71

\begin{tabular}{|l|l|l|l|l|l|l|l|l|l|}
\hline \multicolumn{4}{|c|}{$4 \times 8^{\circ}$} & 0.118 & 1.626 & $2 \times 0.236$ & 10.0004 & $0.091 \pm 0.004$ \\
\hline $8^{0}$ & $8^{0}$ & $8^{0}$ & $8^{0}$ & 121 & $1.628^{\circ}$ & 237 & .237 & .0002 & .093 \\
\hline $8^{0}$ & $8^{0}$ & $8^{0}$ & $8^{0}$ & 121 & 1.626 & .237 & .237 & .0002 & .094 \\
\hline $8^{0}$ & $8^{0}$ & $8^{0}$ & $8^{0}$ & 121 & 1.628 & .237 & .237 & .0003 & .094 \\
\hline $8^{0}$ & $8^{0}$ & $8^{0}$ & $8^{0}$ & .120 & 1.627 & .237 & .237 & .0003 & .094 \\
\hline $8^{\circ}$ & $8^{0}$ & $8^{\circ}$ & $8^{0}$ & .120 & 1.627 & .237 & .237 & .0001 & .094 \\
\hline
\end{tabular}

Plate No. 2

73

\begin{tabular}{|c|c|c|c|c|c|c|c|c|c|}
\hline \multicolumn{4}{|c|}{$4 \times 8^{\circ}$} & 0.118 & 1.626 & \multicolumn{2}{|c|}{$2 \times 0.236$} & 10.0004 & $0.091 \pm 0.004$ \\
\hline $8^{\circ}$ & $8^{\circ}$ & 90 & 80 & 120 & 1.628 & .236 & .236 & .00002 & .093 \\
\hline 80 & 80 & $8^{\circ}$ & 80 & 120 & 1.628 & .237 & .236 & .0003 & .093 \\
\hline
\end{tabular}

Plate No. 3

146

164

154

153

151

145

\begin{tabular}{|l|l|l|l|l|l|l|l|l|l|}
\hline \multicolumn{3}{|c|}{$4 \times 8^{\circ}$} & 0.118 & 1.626 & \multicolumn{2}{|c|}{$2 \times 0.236$} & 10.0004 & $0.091 \pm 0.004$ \\
\hline $8^{0}$ & $8^{0}$ & $8^{0}$ & $8^{0}$ & 120 & 1.628 & 237 & .237 & .0003 & 1093 \\
\hline $8^{0}$ & $8^{0}$ & $8^{0}$ & $8^{0}$ & .120 & 1.628 & .237 & .237 & .0003 & .094 \\
\hline $8^{0}$ & $8^{0}$ & $8^{0}$ & $8^{\circ}$ & 120 & 1.629 & .236 & .236 & 0002 & .093 \\
\hline $8^{\circ}$ & $8^{0}$ & $8^{0}$ & $8^{0}$ & .119 & 1.628 & .236 & .236 & .0002 & .093 \\
\hline $8^{0}$ & $8^{0}$ & $8^{0}$ & $8^{0}$ & 120 & 1.629 & .237 & .236 & .0002 & .093 \\
\hline $8^{0}$ & $8^{0}$ & $8^{0}$ & $8^{0}$ & .120 & 1.628 & .237 & .237 & $.000 /$ & .093 \\
\hline
\end{tabular}

Monolithic Mini Tensile Specimens

KN6002216-2

(Plate 2)

41

37

25

\begin{tabular}{|l|l|l|l|l|l|l|l|l|l|}
\hline \multicolumn{4}{|c|}{$4 \times 8^{\circ}$} & 0.118 & 1.626 & $2 \times 0.236$ & 0.0004 & $0.091 \pm 0.004$ \\
\hline $8^{0}$ & $8^{0}$ & $8^{0}$ & $8^{0}$ & .118 & 1.626 & .236 & .236 & 0000 & .094 \\
\hline $8^{0}$ & $3^{0}$ & $8^{0}$ & $8^{0}$ & .119 & 1.626 & .236 & .236 & .0000 & .094 \\
\hline $8^{0}$ & $8^{0}$ & $8^{0}$ & $8^{0}$ & .118 & 1.626 & .236 & .236 & 0000 & .054 \\
\hline
\end{tabular}

KN6002216.4

(Plate 4)

\begin{tabular}{|c|c|c|c|c|c|c|c|c|c|}
\hline \multicolumn{3}{|c|}{$4 \times 8^{\circ}$} & 0.118 & 1.626 & $2 \times 0.236$ & 10.0004 & $0.091 \pm 0.004$ \\
\hline $8^{0}$ & $8^{0}$ & $8^{\circ}$ & $8^{\circ}$ & 118 & 1.626 & 236 & .236 & .0001 & .094 \\
\hline $8^{\circ}$ & $7^{0}$ & $8^{0}$ & $8^{\circ}$ & .118 & 1.626 & .236 & .236 & .0001 & .094 \\
\hline $8^{0}$ & $8^{0}$ & $8^{\circ}$ & $8^{\circ}$ & .118 & 1.626 & .236 & .236 & .0001 & 094 \\
\hline
\end{tabular}

KN6002216-5

(Plate 5)

22

2

39

\begin{tabular}{|l|l|l|l|l|l|l|l|l|l|}
\hline \multicolumn{4}{|c|}{$4 \times 8^{\circ}$} & 0.118 & 1.626 & $2 \times 0.236$ & 10.0004 & $0.091 \pm 0.004$ \\
\hline $8^{\circ}$ & $8^{0}$ & $8^{0}$ & 80 & .118 & 7.626 & .236 & .236 & .0000 & .094 \\
\hline 80 & $8^{0}$ & $8^{0}$ & 80 & .119 & 1.625 & .236 & 236 & .0000 & .094 \\
\hline 80 & 80 & 80 & 80 & .119 & 1.626 & .236 & .235 & 0000 & .094 \\
\hline
\end{tabular}

KN6002216-6

(Plate 6)

5

32

\begin{tabular}{|c|c|c|c|c|c|c|c|c|c|}
\hline \multicolumn{3}{|c|}{$4 \times 8^{\circ}$} & 0.118 & 1.626 & $2 \times 0.236$ & 10.0004 & $0.091 \pm 0.004$ \\
\hline $8^{\circ}$ & $8^{\circ}$ & 80 & 30 & 117 & 1.026 & .236 & .236 & .0000 & .094 \\
\hline $8^{0}$ & $8^{\circ}$ & 80 & $8^{\circ}$ & .118 & 1.626 & .236 & .236 & .000 & .094 \\
\hline
\end{tabular}

$\Rightarrow f$ felleis? AUG 52005

PRE-DECISIONAL - For Planning and Discussion Purposes Only 


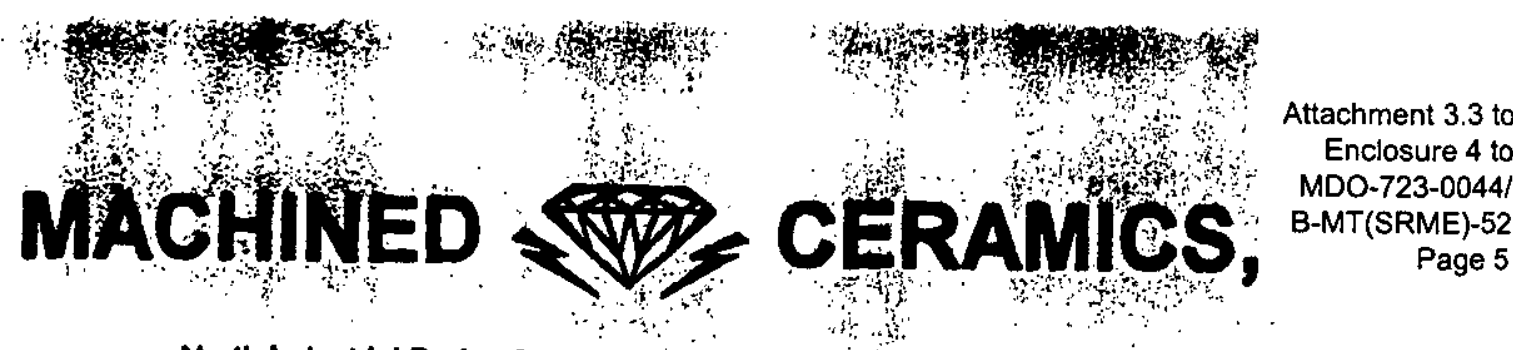

North Industrial Pork - 629 North Oraham Streol \& Bowling Greon, Kr 42101 Phona: 502-761-0512 • Fax: \$02-781-0361

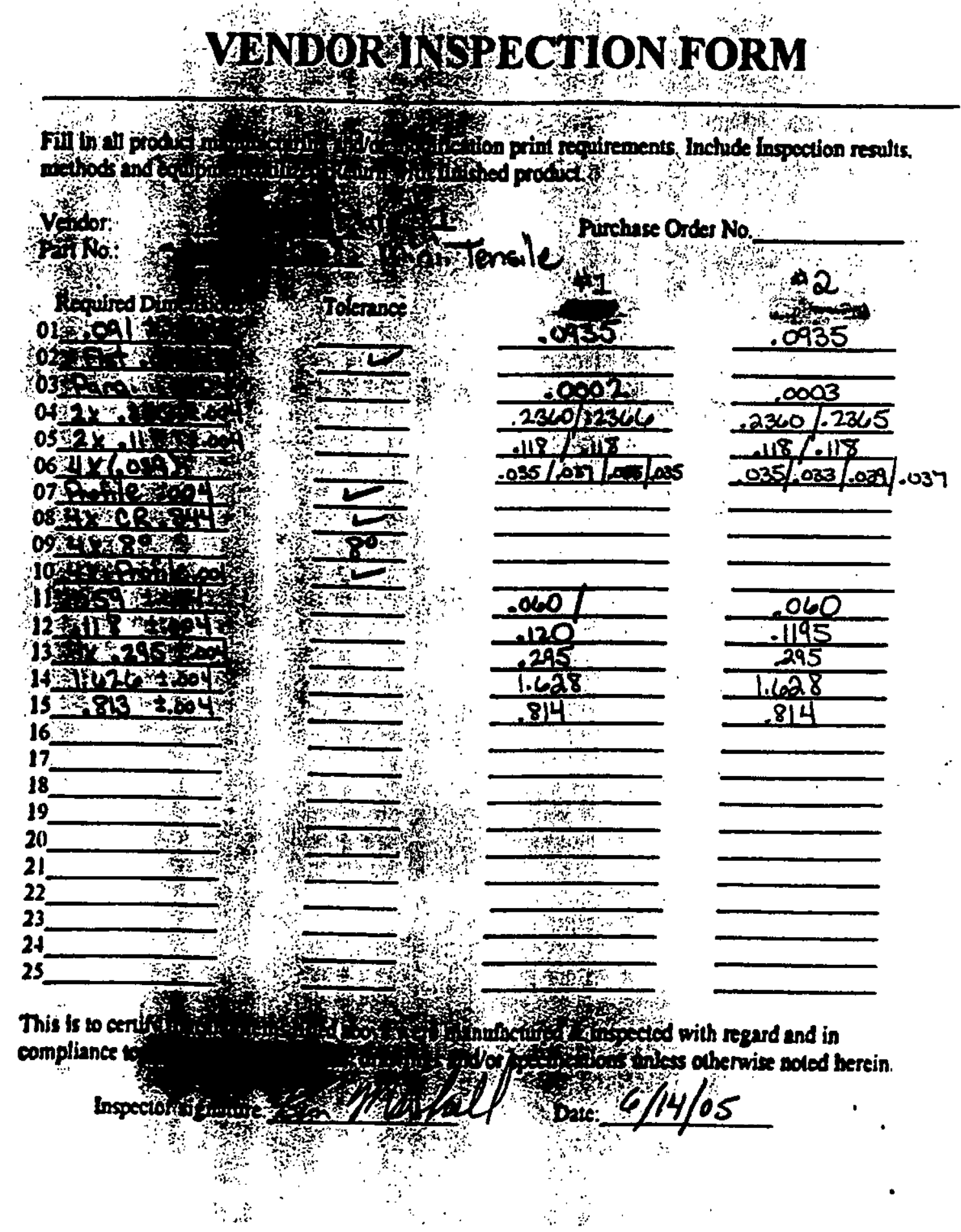


JUN-14-2005 TUE 11:59 AM MACHINED CERAMICS INC

FAX NO. 2707819381

P. 01

Attachment 3.3 to

Enclosure 4 to

MDO-723-0044/

B-MT(SRME)-52

MACHINED CERAMICS, INC.

Page 6

North Indusinal Park * 629 North Graham Street - Bowing Green, KV 42101 Phone: 270-781-0512 - Fac 270-784-8361 email: acer premiemet.net

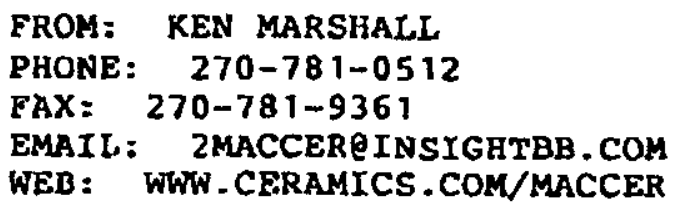

DATE: $6 / 14 / 05$

to: Skip Gifford $K A P L$

PHONE:

$\max =(518) 395-6848$

Skip, The Blanks for the disks were cut to: $\left\{\begin{array}{l}\approx 1.63 \times 3.55 \\ \text { (Plate } 1 \text { oNLY) }\end{array} \quad \begin{array}{l}\approx 1.63 \times 3.20\end{array}\right.$

when grinding the thickness, the mechanical stop was not
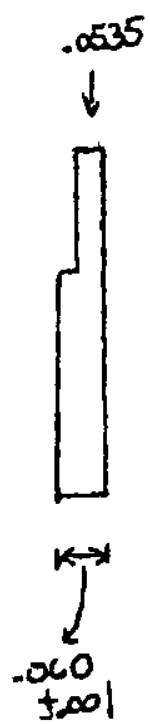
properly set and the thickness of one section is at .0535 on both pieces.

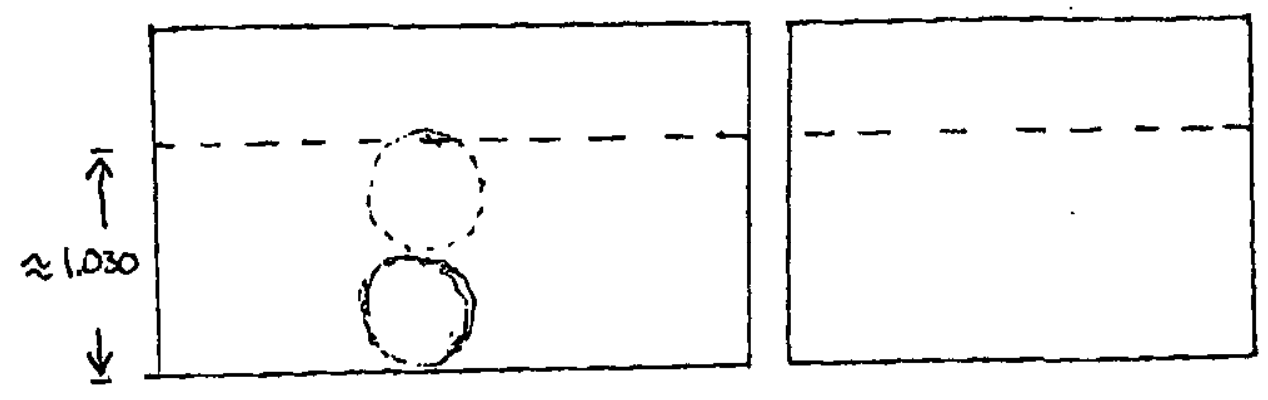

Please advise, Kaon Marshall

PRE-DECISIONAL - For Planning and Discussion Purposes Only

Developmental Englnooring Precision Machining 
MACHINED CERAMICS, INC.

North Industrial Park 629 North Graham Street

Bowling Green, KY 42101

270-781-0512 • Fax: 270-781-9361

e-mail: machinedceramics $₫$ insightbb.com
Attachment 3.3 to Enclosure 4 to MDO-723-0044/ B-MT(SRME)-52 Page 7

KAPL PO\# KN60002443

DETRIMENTAL MATERIALS REPORTS

June 24, 2005

This is to certify that Machined Ceramics, Inc. is in conformance to the Detrimental Materials Prohibitions per Q.A. M-6

Manager:<smiles>C#CCCC(C)CC(C)C</smiles> 
MACHINED CERAMICS, INC.

North Industrial Park

629 North Graham Street

Bowling Green, KY 42101

270-781-0512 - Fax: 270-781-9361

Attachment 3.3 to

Enclosure 4 to

MDO-723-0044/

8-MT(SRME)-52

Page 8

e-mail: machinedceramics $\Theta$ insightbb.com

KAPL PO\# KN60002443

GOVERNMENT FURNISHED MATERIAL REPORT

June 24, 2005

This is to certify that Machined Ceramics, Inc. used government furnished material that was supplied for this purchase order. All furnished material was consumed on this order.

Manager:<smiles>C=CCCCCCCCCCC</smiles> 
Attachment 3.3 to

Enclosure 4 to

MDO-723-0044/

B-MT(SRME)-52

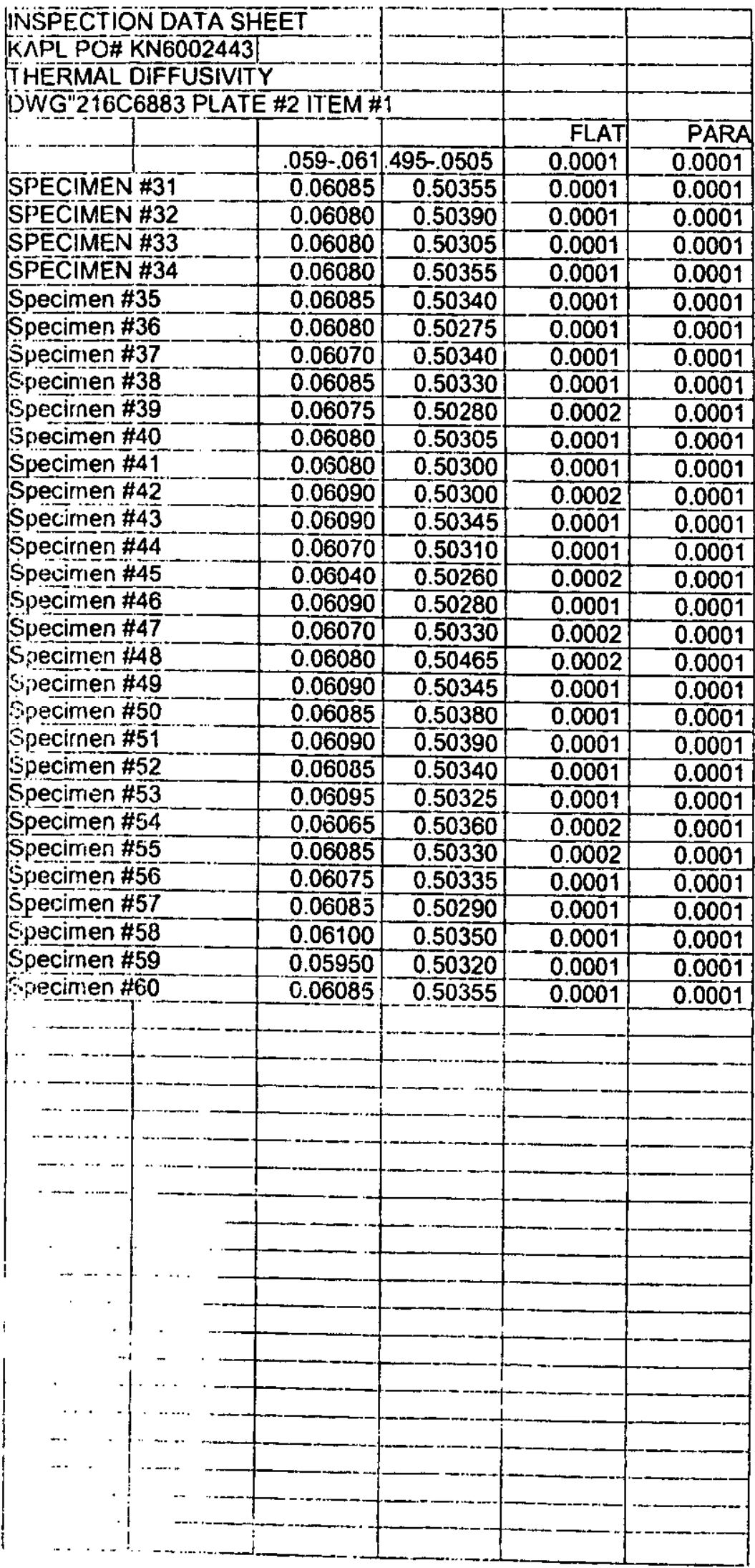

Page 9 
Attachment 3.3 to

Enclosure 4 to

MDO-723-0044/

B-MT(SRME)-52

Page 10

IINSPECTION DATA SHEET

KAPL PO\# KN6002443

MINIATURE TENSILE M00236000 4AG DWG SK23989368 REV. PLATE \#3

\begin{tabular}{|c|c|c|c|c|c|c|c|c|}
\hline & & & FLAT & PARA & & & $4 \mathrm{X}$ & $2 x$ \\
\hline & & $.091-.004$ & 0.0004 & 0.0004 & $1.626-.004$ & $.813-.004$ & $.295-.004$ & $.236-.004$ \\
\hline \multicolumn{2}{|c|}{ SPECIMEN \#145 } & 0.09240 & 0.0002 & 0.0003 & 1.6280 & 0.814 & 0.295 & $23675 / .23675$ \\
\hline \multicolumn{2}{|c|}{ SPECIMEN $\# 146$} & 0.09275 & 0.0002 & 0.0001 & 1.6280 & 0.814 & 0.295 & $23665 / .23715$ \\
\hline \multicolumn{2}{|c|}{ SPECIMEN \#147 } & 0.09250 & 0.0002 & 0.0003 & 1.6285 & 0.814 & 0.295 & $23640 / .23665$ \\
\hline \multicolumn{2}{|c|}{ SPECIMEN \#148 } & 0.09250 & 0.0001 & 0.0002 & 1.6285 & 0.814 & 0.295 & $.23710 / .23610$ \\
\hline \multicolumn{2}{|c|}{ SPECIMEN \#149 } & 0.09230 & 0.0001 & 0.0001 & 1.6285 & 0.814 & 0.295 & $23655 / .23650$ \\
\hline \multicolumn{2}{|c|}{ SPECIMEN \#150 } & 0.09275 & 0.0001 & 0.0001 & 1.6285 & 0.814 & 0.295 & $.23640 / .23660$ \\
\hline \multicolumn{2}{|c|}{ SPECIMEN \#151 } & 0.09280 & 0.0002 & 0.0001 & 1.6285 & 0.814 & 0.295 & $.23715 / .23570$ \\
\hline \multicolumn{2}{|c|}{ SPECIMEN \#152 } & 0.09285 & 0.0002 & 0.0001 & 1.6280 & 0.814 & 0.295 & $23650 / .23640$ \\
\hline \multicolumn{2}{|c|}{ SPECIMEN \#153 } & 0.09260 & 0.0002 & 0.0001 & 1.6285 & 0.814 & 0.295 & $23670 / .23675$ \\
\hline \multicolumn{2}{|c|}{ SPECIMEN \#154 } & 0.09250 & 0.0002 & 0.0002 & 1.6290 & 0.814 & 0.295 & $23660 / .23655$ \\
\hline \multicolumn{2}{|c|}{ SPECIMEN \#155 } & 0.09285 & 0.0001 & 0.0001 & 1.6285 & 0.814 & 0.295 & $23650 / .23645$ \\
\hline \multicolumn{2}{|c|}{ SPECIMEN \#156 } & 0.09275 & 0.0002 & 0.0001 & 1.6285 & 0.814 & 0.295 & $.23730 / .23550$ \\
\hline \multicolumn{2}{|c|}{ SPECIMEN \#157 } & 0.09265 & 0.0003 & 0.0002 & 1.6285 & 0.814 & 0.295 & $23655 / .23660$ \\
\hline \multicolumn{2}{|c|}{ SPECIMEN \#158 } & 0.09230 & 0.0004 & 0.0001 & 1.6290 & 0.814 & 0.295 & $23645 / .23640$ \\
\hline \multicolumn{2}{|c|}{ SPECIMEN \#159 } & 0.09240 & 0.0002 & 0.0001 & 1.6290 & 0.814 & 0.295 & $23680 / .23650$ \\
\hline \multicolumn{2}{|c|}{ SPECIMEN \#160 } & 0.09215 & 0.0003 & 0.0001 & 1.6290 & 0.814 & 0.295 & $23680 / .23680$ \\
\hline \multicolumn{2}{|c|}{ SPECIMEN \#161 } & 0.09250 & 0.0003 & 0.0002 & 1.6290 & 0.814 & 0.295 & $.23685 / .23685$ \\
\hline \multicolumn{2}{|c|}{ SPECIMEN \#162 } & 0.09240 & 0.0003 & 0.0001 & 9.6290 & 0.814 & 0.295 & $.23615 / .23725$ \\
\hline \multicolumn{2}{|c|}{ ŜPCIMEN \#163 } & 0.02700 & 0.0002 & 0.0001 & 1.6280 & 0.814 & 0.295 & $.23675 / .23670$ \\
\hline DECIMEN & $\# 164$ & 0.09285 & 0.0002 & 0.0001 & 1.6285 & 0.814 & 0.295 & $.23720 / .23680$ \\
\hline PECIMEN & \#165 & 0.09235 & 0.0002 & 0.0001 & 1.6290 & 0.814 & 0.295 & $.23570 / .23625$ \\
\hline SPECIMEN & $\# 166$ & 0.09235 & 0.0004 & 0.0001 & 1.6285 & 0.814 & 0.295 & $23660 / .23655$ \\
\hline SPECIMEN & \#167 & 0.09330 & 0.0003 & 0.0002 & 1.6285 & 0.814 & 0.295 & $.23690 / .23695$ \\
\hline SPECIMEN & \#168 & 0.09270 & 0.0002 & 0.0001 & 1.6290 & 0.814 & 0.295 & $.23655 / .23640$ \\
\hline SPECIMEN & $\# 169$ & 0.09335 & 0.0002 & 0.0001 & 1.6290 & 0.814 & 0.295 & $23720 / .23680$ \\
\hline SPECIMEN & $\$ 170$ & 0.09290 & 0.0002 & 0.0001 & 1.6285 & 0.814 & 0.295 & $.23660 / .23720$ \\
\hline SPECIMEN & $\# 171$ & 0.09245 & 0.0002 & 0.0001 & $\$ .6290$ & 0.814 & 0.295 & $23675 / .23650$ \\
\hline SPECIMEN & $\# 172$ & 0.09290 & 0.0003 & 0.0001 & 1.6280 & 0.814 & 0.295 & $.23695 / .23660$ \\
\hline SPECIMEN & $\# 173$ & 0.09305 & 0.0001 & 0.0001 & 1.6285 & 0.814 & 0.295 & $.23670 / .23680$ \\
\hline SPECIMEN & $\# 174$ & 0.09225 & 0.0002 & 0.0001 & 1.6280 & 0.814 & 0.295 & $.23650 / .23655$ \\
\hline & & & & & & & & \\
\hline & & & & & & & & \\
\hline & & & & & & & & \\
\hline & & & & & & & & \\
\hline & & & & & & & & \\
\hline & & & & & & & & \\
\hline & & & & & & & & \\
\hline & & & & & & & & \\
\hline & & & & & & & & \\
\hline & & & & & & & & \\
\hline & & & & & & & & \\
\hline & & & & & & & & \\
\hline & & & & & & & & \\
\hline & & & & & & & & \\
\hline & & & & & & & & \\
\hline & & & & & & & & \\
\hline & & & & & & & & \\
\hline & & & & & & & & \\
\hline & & & & & & & & \\
\hline
\end{tabular}

PRE-DECISIONAL - For Planning and Discussion Purposes Only 
Attachment 3.3 to

Enclosure 4 to

MDO-723-0044/

B-MT(SRME)-52

\begin{tabular}{|c|c|c|c|c|c|c|c|c|}
\hline & & & & & & & & \\
\hline & & & & & & & & \\
\hline & & & & & & & & \\
\hline $2 x$ & & & PROFILE & 4X PROFILE & $4 \times C R$ & & & \\
\hline $.118-.004$ & $4 \times(.039)$ & & 0.004 & 0.001 & 0.844 & $4 \times 8^{*}$ & $.059-.004$ & $.118-.004$ \\
\hline 0.118 & $.042 / .037$ & $.041 / .036$ & OK & $\mathrm{OK}$ & 0.844 & 8 & 0.060 & 0.12015 \\
\hline 0.118 & $.040 / .040$ & $.038 / .034$ & OK & OK & 0.844 & 8 & 0.060 & 0.11990 \\
\hline 0.118 & $.033 / .043$ & $.037 / .040$ & $\overline{\mathrm{OK}}$ & $\overline{O K}$ & 0.844 & 8 & 0.060 & 0.11920 \\
\hline 0.118 & $.039 / .033$ & .0451 .036 & OK & OK & $0 . \overline{844}$ & 8 & 0.060 & 0.11965 \\
\hline 0.118 & $.036 / .040$ & $.036 / .042$ & OK & OK & 0.844 & 8 & 0.060 & 0.12005 \\
\hline 0.118 & $.033 / .039$ & $.038 / .039$ & $\mathrm{OK}$ & OK & 0.844 & 8 & 0.060 & 0.11990 \\
\hline 0.118 & .0371 .040 & $.033 / .046$ & OK & OK & 0.844 & 8 & 0.060 & 0.12000 \\
\hline 0.118 & $.044 / .037$ & $.043 / .036$ & $\mathrm{OK}$ & OK & $0 . \overline{844}$ & 8 & 0.060 & 0.12005 \\
\hline 0.118 & $.042 / .034$ & $.041 / .036$ & OK & OK & 0.844 & 8 & 0.060 & 0.11995 \\
\hline 0.118 & .0361 .039 & $.037 / .042$ & OK & OK & 0.844 & 8 & 0.060 & 0.12005 \\
\hline 0.118 & $.042 / .035$ & $.045 / .041$ & OK & OK & 0.844 & 8 & 0.060 & 0.12015 \\
\hline 0.118 & $.046 / .043$ & $.039 / .032$ & OK & OK & 0.844 & 8 & 0.060 & 0.12010 \\
\hline 0.118 & $.033 / .041$ & $.035 / .040$ & $\overline{O K}$ & OK & 0.844 & 8 & 0.060 & 0.11950 \\
\hline 0.118 & $.039 / .034$ & $.045 / .045$ & OK & OK & 0.844 & 8 & 0.060 & 0.12040 \\
\hline 0.118 & $.035 / .039$ & $.035 / .041$ & OK & $\overline{O K}$ & 0.844 & 8 & 0.060 & $0 . \overline{12030}$ \\
\hline 0.118 & $.035 / .040$ & $.035 / .040$ & OK & OK & 0.844 & 8 & 0.060 & 0.11975 \\
\hline 0.118 & .0401 .042 & $.033 / .040$ & OK & $\mathrm{OK}$ & 0.844 & 8 & 0.060 & 0.11970 \\
\hline 0.118 & $.040 / .031$ & $.040 / .039$ & $\overline{O K}$ & OK & 0.844 & 8 & 0.060 & 0.12000 \\
\hline 0.118 & $.035 / .036$ & $.039 / .040$ & $\overline{\mathrm{OK}}$ & $\mathrm{OK}$ & 0.844 & 8 & 0.060 & 0.11975 \\
\hline 0.118 & $.045 / .034$ & $.041 / .034$ & OK & OK & 0.844 & 8 & 0.060 & 0.12015 \\
\hline 0.118 & $.039 / .038$ & $.044 / .035$ & OK & OK & 0.844 & 8 & 0.060 & 0.11900 \\
\hline 0.118 & $.034 / .039$ & $.039 / .040$ & $\overline{\mathrm{OK}}$ & OK & 0.844 & 8 & 0.060 & 0.12020 \\
\hline 0.118 & $.042 / .035$ & $.044 / .037$ & $\overline{O K}$ & OK & 0.844 & 8 & 0.060 & 0.12015 \\
\hline 0.118 & $.032 / .039$ & $.035 / .042$ & OK & OK & 0.844 & 8 & 0.060 & 0.11980 \\
\hline 0.118 & $.034 / .042$ & .0321 .037 & OK & OK & 0.844 & 8 & 0.060 & 0.11985 \\
\hline 0.118 & .0421 .032 & $.044 / .036$ & OK & OK & 0.844 & 8 & 0.060 & 0.11950 \\
\hline 0.118 & .0421 .032 & $.044 / .036$ & $\overline{O K}$ & OK & 0.844 & 8 & 0.060 & 0.11970 \\
\hline 0.118 & $.039 / .031$ & $.039 / .035$ & OK & $\overline{\mathrm{OK}}$ & 0.844 & 8 & 0.060 & 0.11920 \\
\hline $0.11 \overline{8}$ & $.031 / .042$ & $.033 / .041$ & $\overline{O K}$ & OK & 0.844 & 8 & 0.060 & 0.11935 \\
\hline 0.118 & $.033 / .042$ & $.034 / .043$ & OK & OK & 0.844 & 8 & 0.060 & 0.11945 \\
\hline & & & & & & & & \\
\hline & & & & & & & & \\
\hline & & & & & & & & \\
\hline & & & & & & & & \\
\hline & & & & & & & & \\
\hline & & & & & & & & \\
\hline & & & & & & & & \\
\hline & & & & & & & & \\
\hline$\therefore$ & & & & & & & & \\
\hline & & & & & & & & \\
\hline & & & & & & & & \\
\hline & & & & & & & & \\
\hline & & & & & & & & \\
\hline & & & & & & & & \\
\hline & & & & & & & & \\
\hline & & & & & & & & \\
\hline & & & & & & & & \\
\hline & & & & & & & & \\
\hline & & & & & & & & \\
\hline & & & & & & & & \\
\hline
\end{tabular}

Page 11 
Attachment 3.4 to Enclosure 4 to MDO-723-0044/ B-MT(SRME)-52

Page 1

Attachment 3.4

Specimen Photographs

Composite SiC Tensile Specimens and Thermal Diffusivity Discs Enclosure 4 to MDO-723-0044/B-MT(SRME)-52 
Attachment 3.4 to

Enclosure 4 to

MDO-723-0044/

B-MT(SRME)-52
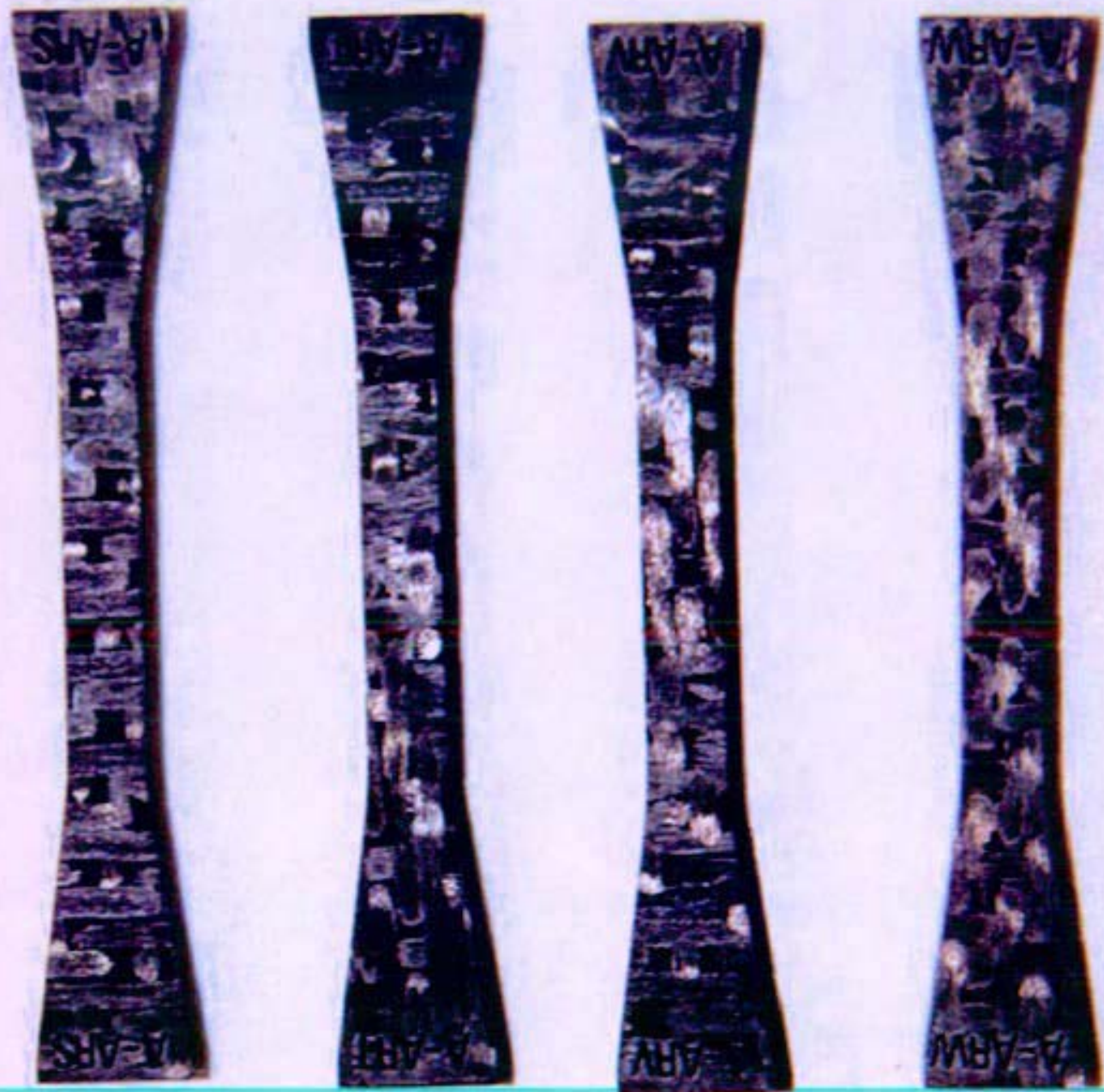

Page 2
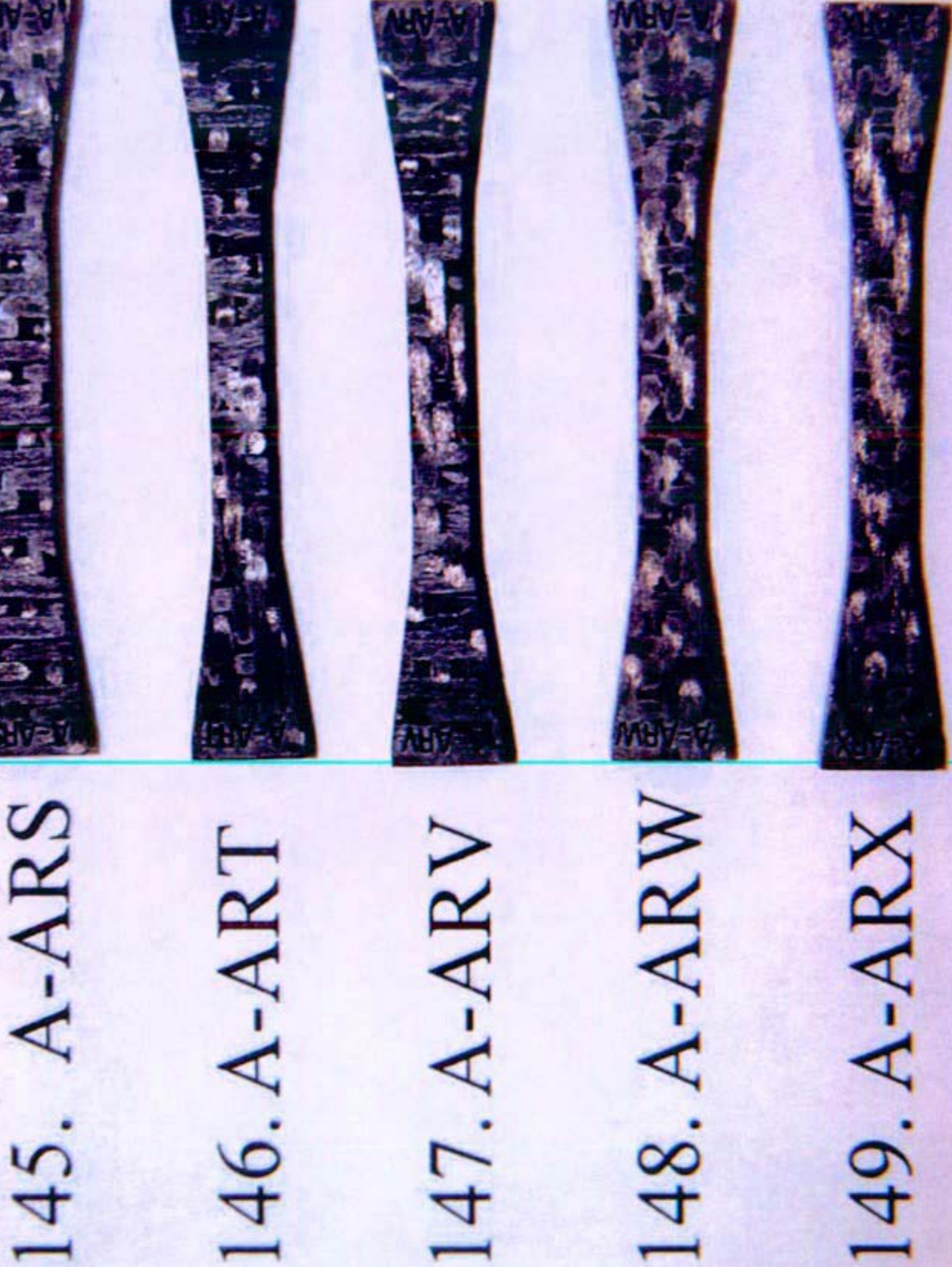

Attachment 3.4 to Enclosure 4 to MDO-723-0044/ B-MT(SRME)-52

\section{A-BAF}

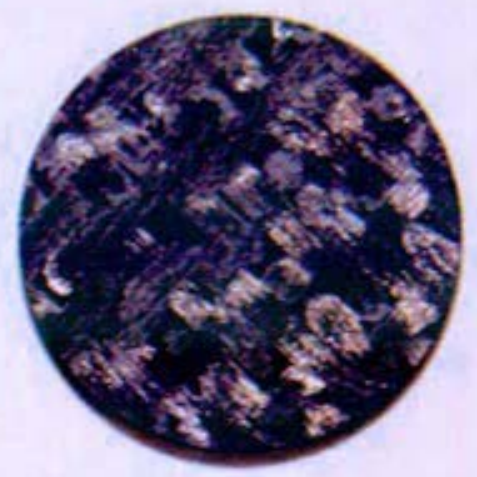

$$
\text { Page } 4
$$

38. A-BAG

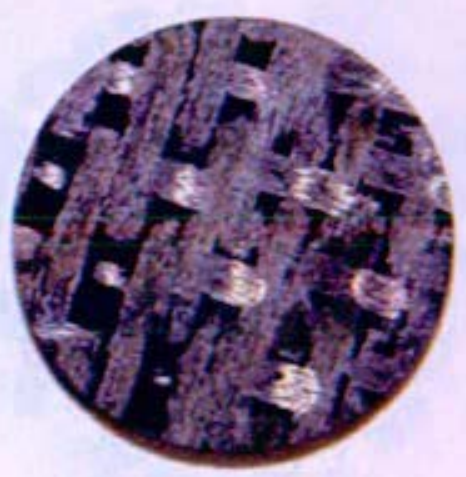

39. A-BAH 
Attachment 3.4 to

Enclosure 4 to

MDO-723-0044/

B-MT(SRME)-52

Page 5

37. A-BAF
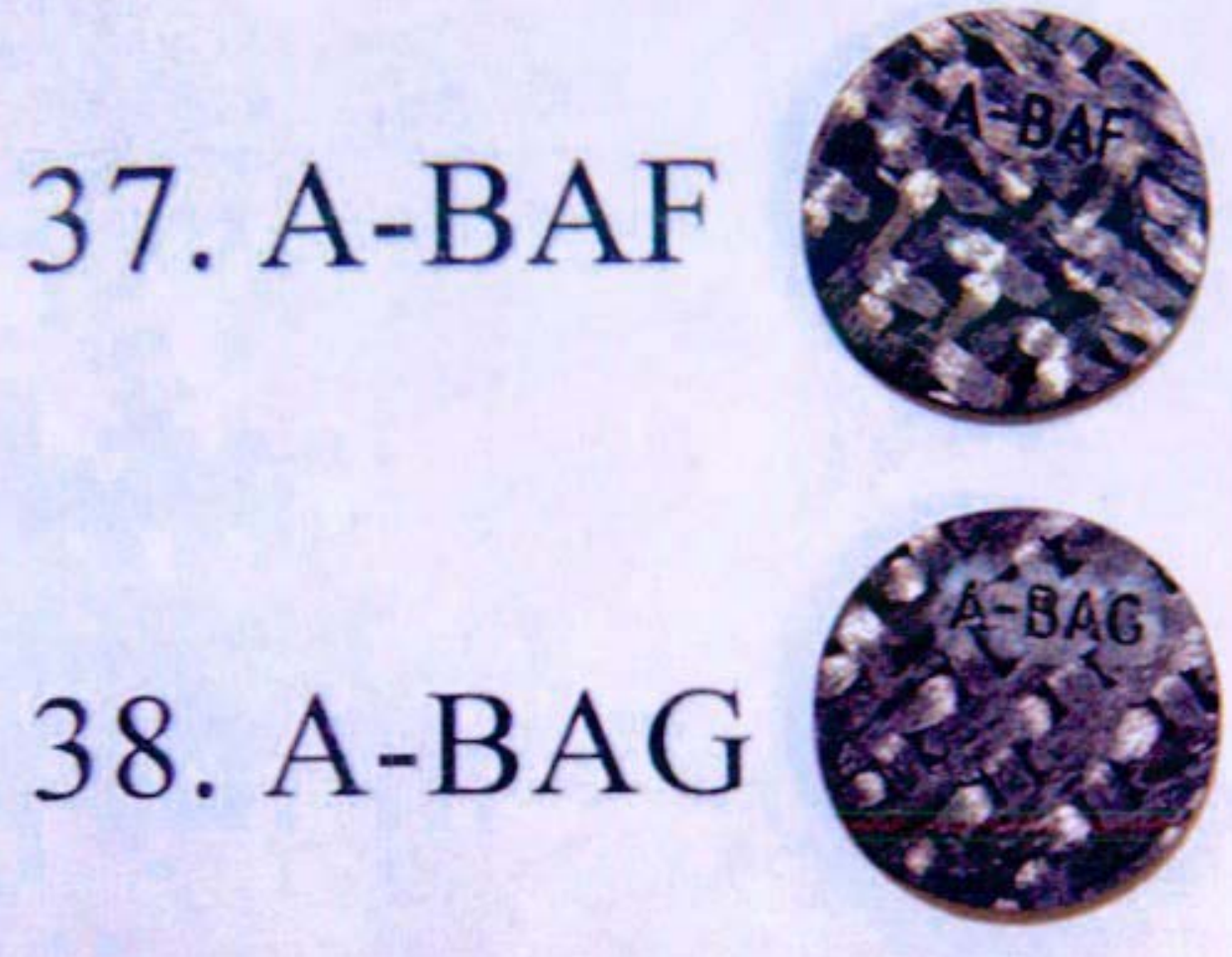

39. A-BAH 
Attachment 3.5 to Enclosure 4 to MDO-723-0044/ B-MT(SRME)-52

Page 1

Attachment 3.5

Tensile and Thermal Diffusivity Data for SiC Composite Tensile Specimens and Thermal Diffusivity Discs

Enclosure 4 to MDO-723-0044/B-MT(SRME)-52 
Attachment 3.5 to

Enclosure 4 to

MATERIAL: SIC COMPOSITE, HI-NICALON TYPE S FIBERS CVI MATRIX

MDO-723-0044/

\begin{tabular}{|c|c|c|c|c|c|}
\hline \multicolumn{6}{|c|}{ MATERIAL: SIC COMPOSITE, HI-NICALON TYPE S FIBERS CVI MATRIX } \\
\hline $\begin{array}{l}\text { SPECIMEN } \\
\text { IDENTITY }\end{array}$ & $\begin{array}{l}\text { MAXIMUM } \\
\text { LOAD (lbs) }\end{array}$ & $\begin{array}{c}\text { ULT. } \\
\text { STRENGTH } \\
\text { (ksi) }\end{array}$ & $\begin{array}{c}\text { \% ELONG } \\
\text { 0.5" G.L. }\end{array}$ & $\begin{array}{l}\text { ORIGINAL } \\
\text { AREA }\left(\mathrm{in}^{2}\right)\end{array}$ & $\begin{array}{l}\text { Modulus } \\
\text { (Msi) }\end{array}$ \\
\hline A-AOS (1) & 573 & 51.3 & 0.41 & 0.01116 & 21.32 \\
\hline A-AOT (1) & 457 & 41.1 & 0.13 & 0.01111 & 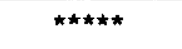 \\
\hline $\begin{array}{l}\text { A-AOV } \\
\text { (1)(2) }\end{array}$ & 486 & 43.4 & 0.3 & 0.0112 & 21.19 \\
\hline A-AOW (1) & 482 & 42.9 & 0.34 & 0.01123 & 29.62 \\
\hline A-AOX (1) & 302 & 27.1 & 0.05 & 0.01113 & $\star \star * * * *$ \\
\hline A-AOY & 421 & 37.8 & 0.2 & 0.01113 & 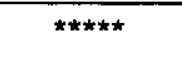 \\
\hline A-AOZ & 437 & 39.2 & 0.28 & 0.01114 & 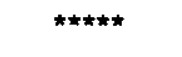 \\
\hline A-APA & 548 & 49.1 & 0.38 & 0.01116 & 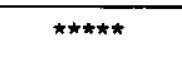 \\
\hline A-APB & 439 & 39.1 & 0.33 & 0.01123 & 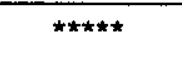 \\
\hline A-APC & 473 & 42.5 & 0.33 & 0.01114 & 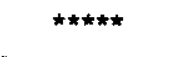 \\
\hline A-APD & 436 & 39.2 & 0.22 & 0.01112 & 29.41 \\
\hline A-APE & 494 & 44.3 & 0.25 & 0.01114 & 29.53 \\
\hline A-APF & 464 & 41.5 & 0.29 & 0.01117 & 21.72 \\
\hline A-APG & 470 & 42.3 & 0.23 & 0.01112 & 27.42 \\
\hline A-APH & 464 & 41.6 & 0.32 & 0.01114 & 22.21 \\
\hline AVERAGE & 463.1 & 41.5 & 0.27 & 0.011 & 25.30 \\
\hline StDev & 60.4 & 5.38 & 0.09 & 3.83E-05 & 4.02 \\
\hline
\end{tabular}

Page 2

NOTES: (1) STRAIN GAGED WITH ED-03-015DJ-120 G.F. $=2.07$

(2) SPECIMEN FAILED IN GRIP END

MCL NUMBER 4787, BRUCE FURBECK

PRE-DECISIONAL - For Planning and Discussion Purposes Only 
Attachment 3.5 to

Enclosure 4 to

MDO-723-0044/

B-MT(SRME)-52

Page 3

Example of Stress vs Strain Curves for

Composite SiC Specimens

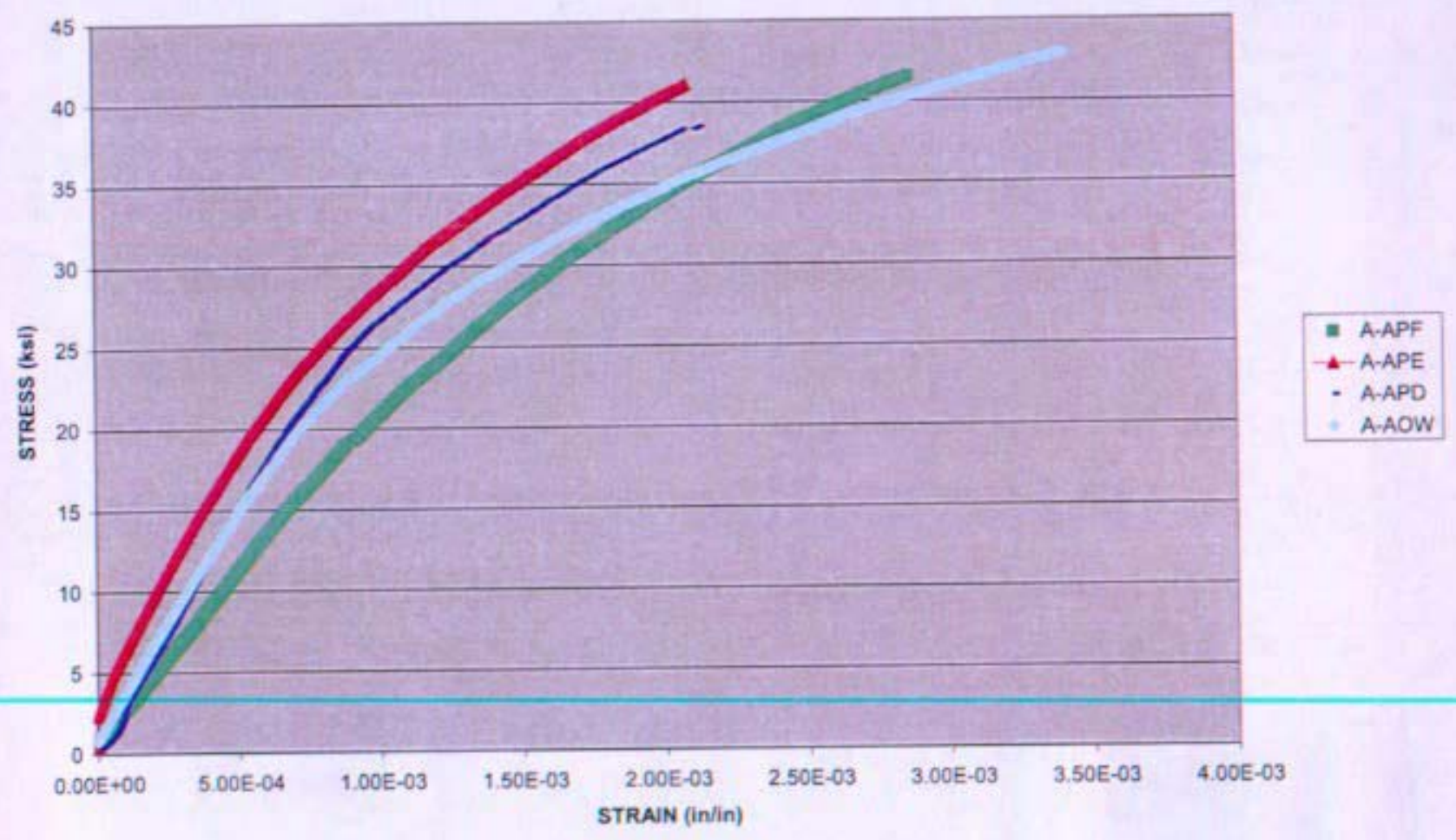

PRE-DECISIONAL - For Planning and Discussion Purposes Only

PRE-DECISIONAL - For Planning and Discussion Purposes Only 
KAPL, Inc.

Knolls Atonic Power Labyyatory

Post Office Box 1072 Schénectady, NY 12301-1072

Telephone (518) 395-4000 Facsimile (518) 395-4422

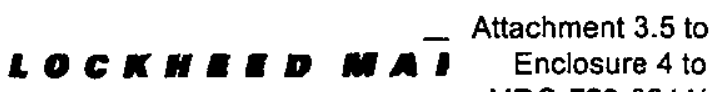

MDO-723-0044/

B-MT(SRME)-52

Page 4

TO-7934-06-02

January 13, 2006

To:

R. Northey

Subject: $\quad$ Diffusivity Testing - Space Reactor Monolithic / Composite SiC

MCL JOB \#: $\quad 4782$

QA Category: $\quad$ C

Test Performer: R.A. Griesau

Reviewed By:

D.P. Buono \&.PR wore

\section{INTRODUCTION AND TEST EQUIPMENT}

Thermal diffusivity was measured using an Anter Corp "Flashline 5000" laser-pulsed diffusivity measurement system. This system uses a solid state (Xenon flash pumped Neodymium glass rod) infrared laser pulsed at $1053 \mathrm{nM}$ through a fiber-optic cable onto a small disk specimen. The conducted thermal wave through the specimen is detected on the opposing face by an $\mathrm{LN}_{2}$-cooled Indium Antimonide crystal infrared detector. High speed data acquisition records the temperature rise curve, which is then analyzed by the software using algorithms by Koski, Clark and Taylor, Parker, Cowan, etc. The most accepted analysis is Koski which corrects for non-axial conduction and laser pulse width errors. The furnace system is comprised of a SS vertical water-cooled cylindrical chamber with graphite heating elements, insulation and specimen support carousel. A rotating carousel allows testing of up to six specimens per test run.

\section{SPECIMENS}

Two specimen types were tested, monolithic beta phase Silicon Carbide (SiC) and composite SiC consisting of Hi-Nicalon Type S fibers in a Chemical Vapor Infiltration (CVI) SiC matrix. Specimens were right cylindrical disks $0.5^{n}$ dia with a thickness of $0.120^{\prime \prime}$ and $0.061^{\prime \prime}$ for the monolithic and composite specimens, respectively. These specimens were placed directly into the $12^{n}$ dia graphite carousel positions in the furnace without adapter holders. The reference specimen NIST-2 (1/2 dia Stainless 
Steel) was placed in carousel positions 6 and 2 for the composite and monolithic runs Page 5 respectively. Specimen thickness measurements were provided by the test sponsor.

\section{IEST PROCEDURE AND CONDITIONS}

Testing was performed in conformance to MCL Operating Procedure No. 100 and ASTM E $1461-01$.

Monolithic SiC specimens will often display translucence in the infrared spectrum (wavelength of test system laser) requiring an opaque, high emissivity coating, typically graphite to be applied to prevent "shine-through" of the laser pulse. That was not the case with the specimens in this program. No coating was applied to the monolithic or composite specimens for this testing.

Five test specimens were loaded in each of the two runs with a graphite reference specimen loaded in position 6 for the composites and position 2 for the monolithics. The composite specimens were loaded with the ID marked face down, toward the IR detector. No specific specimen face orientation was utilized relative to the edge ID marking for the monolithic SiC specimens. Three vacuum pumpdown and argon backfill cycles to atmosphere were performed, with the last pumpdown to $-100 \mathrm{mTorr}$. A continuous argon purge was established at $-3-4$ bubbles per second through an oilfilled bubbler vent.

Soak points were programmed from $200^{\circ} \mathrm{C}-1200^{\circ} \mathrm{C}$ and $1300^{\circ} \mathrm{C}-100^{\circ} \mathrm{C}$ at $200^{\circ} \mathrm{C}$ increments. This up/down sequence was requested to reveal potential hysteresis due to structural or properties changes through the heating cycle. The soak period is determined by the software based on programmed criteria that ensure specimen temperature stability. Laser shots were fired at each specimen at the programmed soak temperatures in a $1-2-3 \ldots, 1-2-3 \ldots$ order, three shots per specimen per soak temperature. The laser flash-lamp capacitor charge voltage (laser power) was set to $1.4,1.2 \& 1.0 \mathrm{kVdc}$ from the low test temperatures to the highest respectively. Auxiliary laser incident apertures were not used, in that, the carousel cover provides sized penetrations for the default $1 / 2^{u}$ dia specimen size.

\section{Calibration}

Independent pyrometer and IR detector (data sources) calibrations are not performed on this test instrument. Data validity is verified by testing of a reference material with comparison to known book values (see charts 3-6). The reference specimen is run as the sixth specimen with each test run loading. The reference specimen error values were within acceptable limits. 


\section{RESULTS}

Note: Caution should always be used when evaluating diffusivity data from a composite test specimen. A composite is a "structural system" and hence, the specimen measurement does not represent the bulk diffusivity value for the inter-fiber matrix or the fibers.

Upon completion of a test run a "results.all" file is generated by the software which contains diffusivity values calculated for all of the analysis types (i.e. Parker, Koski, Clark and Taylor ...). Tabulated values are provided by the software for each of the three laser shots per specimen, fired at each soak temperature. These files were copied to an Excel spreadsheet. The temperature and Koski diffusivity values were averaged for the three laser shots at each soak point and plotted (see charts $1 \& 2$ ). The averaged values were sorted by ascending temperature (the test program had upgoing and down-going soak sequences). The averaged values were collected in the attached table for each specimen.

No anomalies were detected in testing. The specimen-to-specimen spread in the data between the two groups (composites / monolithics) is roughly within the same order of magnitude. The overall comparative ranges between the two groups are offset as might be expected with the homogeneous monolithics being higher in value.

The Excel file with processed data will be forwarded in parallel with this report. The Excel file with processed data and the raw test system files will be electronically transferred to "O:MCLJobsWCL 4782 - SPACE SIC - DIFFUSIVITY - NORTHEY - RG" for record keeping. Archive copies of raw data will be maintained in the Materials Characterization Lab on optical disk.

\section{REFERENCES}

- ASTM E1461-01 Standard Test Method for Thermal Diffusivity by the Flash Method

- Test Community Work Request - MC Job\# 4782 Dated: 10-12-05

- Readiness Review Checklist Dated 11/3/05

- MCL Operating Procedure No. 100 - Anter Flashline 5000 
MCL TEST PROGRAM \# 4782

PROMETHEUS SPACE REACTOR

COMPOSITE and MONOLITHIC SIC

KOSKI DIFFUSIVITY - AVERAGE of 3 LASER SHOTS
Attachment 3.5 to

Enclosure 4 to

MDO-723-0044

B-MT(SRME)-52

Page 7

\begin{tabular}{|c|c|c|c|c|c|c|c|c|c|}
\hline \multicolumn{10}{|c|}{ Composite SiC } \\
\hline \multicolumn{2}{|c|}{ A-BAT } & \multicolumn{2}{|c|}{ A-BAV } & \multicolumn{2}{|c|}{$\mathrm{A}-\mathrm{BBL}$} & \multicolumn{2}{|c|}{ A-BBM } & \multicolumn{2}{|c|}{ A-BBN } \\
\hline TEMP & Diffusivity & TEMP & Diffusivity & TEMP & Diffusivity & TEMP & Diffusivity & TEMP & Diffusivity \\
\hline (degF) & $\left(\mathrm{cm}^{\wedge} 2 / \mathrm{sec}\right)$ & $(\operatorname{deg} F)$ & $\left(\mathrm{cm}^{\wedge} 2 / \mathrm{sec}\right)$ & (degF) & $\left(\mathrm{cm}^{\wedge} 2 / \mathrm{sec}\right)$ & (degF) & $\left(\mathrm{cm}^{\wedge} 2 / \mathrm{sec}\right)$ & (degF) & $\left(\mathrm{cm}^{\wedge} 2 / \mathrm{sec}\right)$ \\
\hline 210 & 0.0459 & 210 & 0.0479 & 210 & 0.0661 & 211 & 0.0613 & 210 & 0.0884 \\
\hline 382 & 0.0368 & 381 & 0.0379 & 382 & 0.0516 & 381 & 0.0484 & 381 & 0.0696 \\
\hline 565 & 0.0312 & 565 & 0.0323 & 565 & 0.0444 & 567 & 0.0412 & 565 & 0.0602 \\
\hline 736 & 0.0267 & 735 & 0.0273 & 736 & 0.0373 & 735 & 0.0348 & 735 & 0.0515 \\
\hline 920 & 0.0245 & 919 & 0.0253 & 921 & 0.0348 & 922 & 0.0322 & 921 & 0.0478 \\
\hline 1097 & 0.0215 & 1096 & 0.0221 & 1098 & 0.0297 & 1098 & 0.0280 & 1096 & 0.0415 \\
\hline 1279 & 0.0206 & 1279 & 0.0211 & 1279 & 0.0288 & 1281 & 0.0268 & 1280 & 0.0395 \\
\hline 1456 & 0.0186 & 1455 & 0.0190 & 1456 & 0.0241 & 1456 & 0.0235 & 1455 & 0.0349 \\
\hline 1640 & 0.0179 & 1640 & 0.0185 & 1640 & 0.0242 & 1642 & 0.0232 & 1641 & 0.0341 \\
\hline 1815 & 0.0167 & 1813 & 0.0169 & 1815 & 0.0218 & 1814 & 0.0208 & 1815 & 0.0309 \\
\hline 1998 & 0.0160 & 1998 & 0.0167 & 1998 & 0.0218 & 2000 & 0.0207 & 1999 & 0.0303 \\
\hline 2170 & 0.0154 & 2170 & 0.0154 & 2170 & 0.0199 & 2170 & 0.0191 & 2170 & 0.0283 \\
\hline 2352 & 0.0149 & 2351 & 0.0153 & 2352 & 0.0194 & 2352 & 0.0183 & 2352 & 0.0265 \\
\hline
\end{tabular}

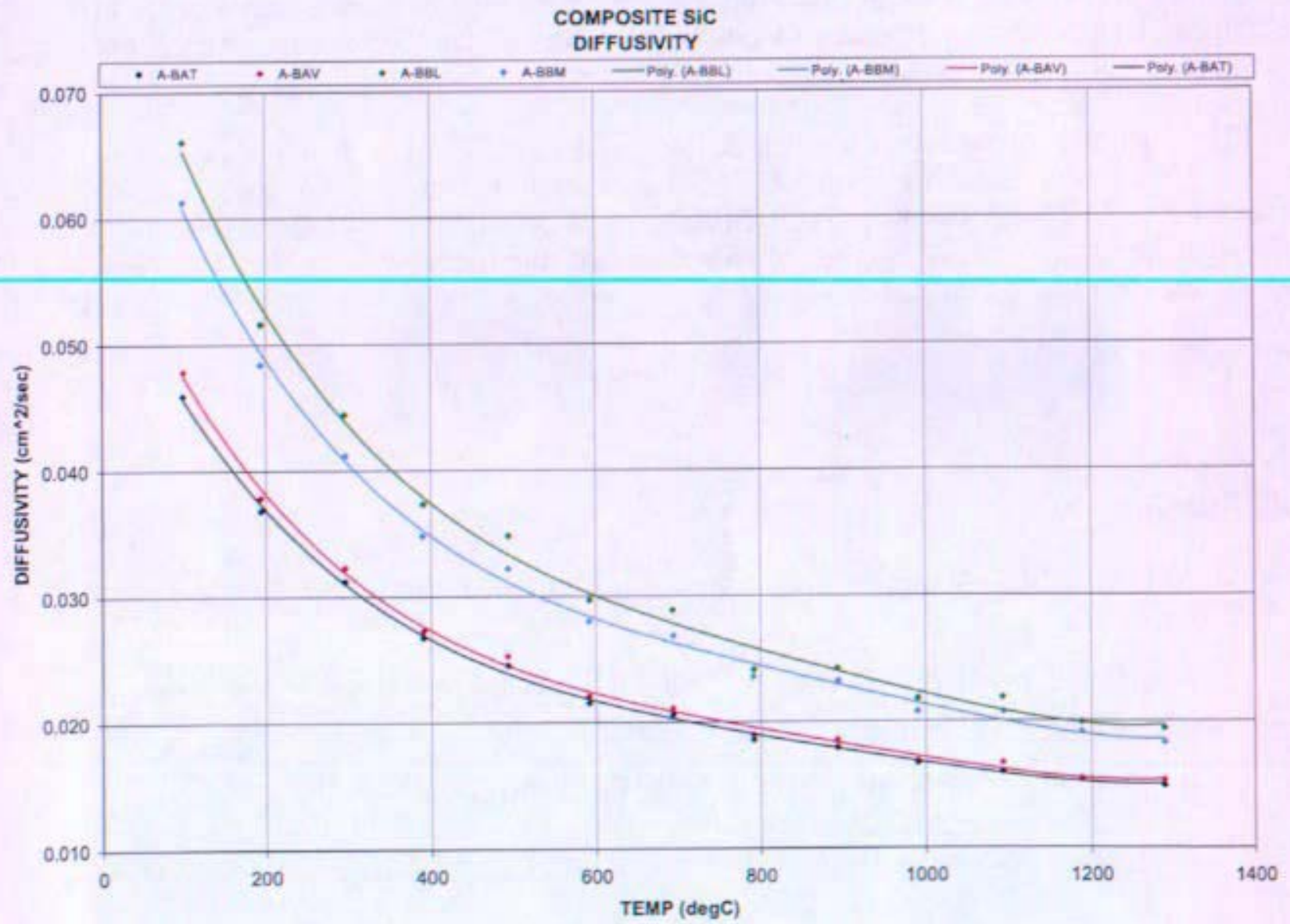


Enclosure 5 to MDO-723-0044/

B-MT(SRME)-52

Page 1

\author{
Enclosure 5 \\ to \\ MDO-723-0044/B-MT(SRME)-52: \\ JOYO-1 Beryllium Oxide Specimen Fabrication Efforts
}

James Nash

January 2006 


\section{Summary}

Beryllium oxide $(\mathrm{BeO})$ and beryllium $(\mathrm{Be})$ were being considered for reflectors in the Prometheus- 1 reactor. Upon restructuring of the Prometheus Project, it had not been concluded that $\mathrm{BeO}$ was more desirable than beryllium as a reflector material in lower temperature applications (radial reflector) from either a mass, cost, fabricability or simplicity standpoint. However, beryllium oxide was considered a leading material candidate for high temperature reflector applications (axial reflectors).

For JOYO-1 irradiation testing, a single grade of BeO was selected: BW-1000 manufactured by Brush Ceramic Products. This grade was selected because of the material's fine grain size (10 $\mu \mathrm{m}$ nominal) and historical documentation implying improved irradiation performance of fine grain sized BeO. Beryllium oxide discs were proposed for thermal diffusivity, density and swelling measurements while cylinders were proposed for compression, density and swelling. Material property testing of unirradiated BeO was completed for baseline purposes.

\section{Introduction}

Most space reactor studies since the 1950's have assumed that BeO would be used in situations where neutron reflection was necessary. Beryllium oxide has very low neutron absorption and high number density, is excellent at moderating fast flux neutrons [both due to Jow mass of beryllium and the inelastic scattering by oxygen], and has high temperature capability $\left(T_{m}=2840 \mathrm{~K}\right)$ due to its ceramic nature. Beryllium oxide actually improves upon the moderating capabilities of beryllium with a higher neutron scattering cross section and lower absorption cross section, at the expense of density. In addition to its moderating capability, previous nuclear design studies favored the high thermal conductivity of $\mathrm{BeO}$. For example, at room temperature, the thermal conductivity of $\mathrm{BeO}$ is similar to aluminum.

Beryllium oxide has been sporadically studied for several decades. Irradiated material performance of BeO (specifically swelling) has shown sensitivity to grain size, composition and processing techniques. This is significant because much of the Prometheus-1 design space remains uncharacterized and most of the historical testing was conducted on different grades and purity levels of BeO that are no longer commercially available.

Upon project restructuring, it had not been concluded that BeO was more desirable than beryllium as a reflector material from either a mass, cost, fabricability or simplicity standpoint. Although, the NRPCT had judged that if estimated Prometheus- 1 temperatures were within beryllium material limits (not determined at this time), beryllium may be the best reflector material (radial reflector). The NRPCT was planning BeO irradiation testing in both the JOYO test reactor and the High Flux Isotope Reactor (HFIR). The parallel testing would have filled in gaps in the irradiated material property database for BeO. This scheme was necessary because any testing delays would likely have eliminated $\mathrm{BeO}$ from consideration due to Prometheus-1 schedular pressures. 
Enclosure 5 to

MDO-723-0044/

B-MT(SRME)-52

Page 3

\section{Discussion}

\section{JOYO Test Matrix}

Details of the proposed JOYO-1 irradiation test and BeO test matrix are described in Reference (1). Beryllium oxide samples selected for irradiation testing in JOYO consisted of discs and cylinders. BeO discs were proposed for thermal diffusivity testing (ASTM E-1461-01), density (ASTM C-373-88) and swelling measurements, while cylinders were considered for compression testing (ASTM C-773-88), density (ASTM C-373-88) and swelling measurements. References (2) and (3) are drawings for the BeO disc and cylinder specimens, respectively. A schematic view of the capsules containing $\mathrm{BeO}$ specimens is shown in Figure 1 (see Enclosure 7 for detailed capsule design drawings). This schematic, however, does not show sample holders and dividers for all specimens that prevent the BeO specimens from contacting other specimens in the shared capsule. Beryllium oxide specimens (yellow specimens in schematic) are located in Tier 1 and Tier 5 (reflector region) of both Irradiation Rig A (120 day irradiation exposure) and Irradiation Rig B (60 day irradiation exposure).

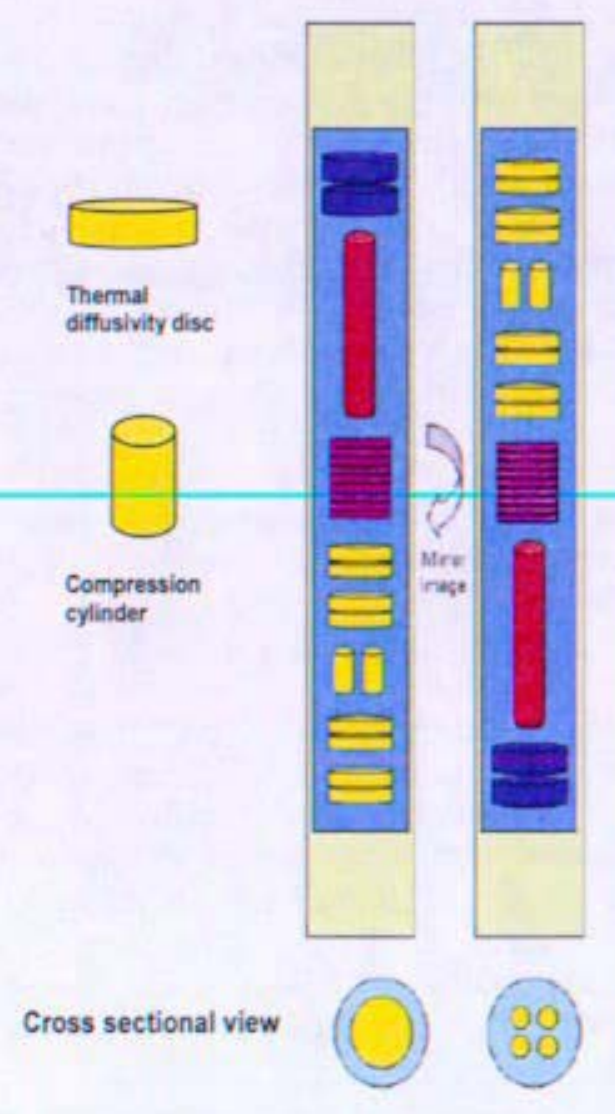

Figure 1: View of Irradiation Capsules with BeO Specimens 
Table 1 provides the test matrix for planned irradiation testing of BeO specimens in the JOYO test reactor. All planned JOYO-1 test samples had the same grain size, percent theoretical density and fabrication method. Japan Nuclear Cycle Development Institute (JNC) indicated in the March 2005 NRPCT/JNC meeting that excessive amounts of materials capable of reflecting back moderated neutrons (thereby causing local power peaking in adjacent fuel rigs) could require additional approval requirements. As such, the volume of materials with low atomic number elements ( $\mathrm{SiC}$ and $\mathrm{BeO}$ ) was minimized without sacrificing test objectives (Reference 4).

Table 1: JOYO Test Matrix for BeO Specimens

\begin{tabular}{|c|c|c|c|c|}
\hline $\begin{array}{c}\text { Density and } \\
\text { Thermal } \\
\text { Diffusivity } \\
\text { Discs }\end{array}$ & $\begin{array}{c}\text { Compression } \\
\text { Cylinders }\end{array}$ & $\begin{array}{c}\text { - Irradiation } \\
\text { Temperat } \\
\text { ure }\end{array}$ & $\begin{array}{c}\text { Nominal } \\
\text { Testing } \\
\text { Fluence } \\
\left(\mathrm{n} / \mathrm{cm}^{2}\right) \\
(\mathrm{E}>0.1 \mathrm{MeV})\end{array}$ & - Location \\
\hline - 8 & - 4 & - $850 \mathrm{~K}$ & $\begin{array}{c}1.3-2.2 \\
\times 10^{21} \\
\end{array}$ & $\begin{array}{c}\text { - } \quad \text { Tier } 1 \text { Rig B (60 } \\
\text { days) }\end{array}$ \\
\hline - 8 & - 4 & - $1050 \mathrm{~K}$ & $\begin{array}{c}1.3-2.6 \\
\times 10^{21} \\
\end{array}$ & $\begin{array}{c}\text { - Tier } 5 \text { Rig B (60 } \\
\text { days) }\end{array}$ \\
\hline - 8 & - 4 & - $850 \mathrm{~K}$ & $\begin{array}{c}2.6-4.5 \\
\times 10^{21} \\
\end{array}$ & $\begin{array}{c}\text { - } \quad \text { Tier } 1 \text { Rig } A \\
\text { (120 days) }\end{array}$ \\
\hline - 8 & - 4 & - $1050 \mathrm{~K}$ & $\begin{array}{c}2.7-5.1 \\
\times 10^{21} \\
\end{array}$ & $\begin{array}{l}\text { - Tier 5 Rig A } \\
\text { (120 days) }\end{array}$ \\
\hline
\end{tabular}

\section{BeO Test Specimens/Brush Ceramic Products}

NRPCT initiated a contract with Brush Ceramic Products, a subsidiary of Brush Wellman, for test specimen fabrication in support of irradiation testing in JOYO. Details of the specification provided to Brush Ceramics are provided in Reference (4). In creating this specification, numerous variables were considered, including sample fabrication, general fabrication processes, safety and handling concerns, experimental efforts and documentation.

Specimen fabrication was the focus of this technical specification, which resulted in decisions made on processing routes, quality control, use of chamfers and sample identification methods (Reference 4). Brush Ceramic Products utilizes multiple processing methods for BeO parts; isopressing and dry pressing are most common. Of these processing routes, isopressed BW$1000 \mathrm{BeO}$ was selected because it provided the finest grain size material available (see Reference 4 for grain size discussion). Typical properties of BW-1000 BeO are summarized in Table 2 (Reference 5). Chamfers were specified for all BeO test specimens in an attempt to prevent cracking and inaccurate property test data. Specimen identifications were required for all irradiated test specimens. Based on requirements for irradiation test specimens, laser scribing was selected as the best option for identifying BeO specimens (Reference 4). A small- 
scale study at Brush Ceramic Products was planned to establish the effect of laser scribing on material properties.

Table 2: Material Properties of BW-1000 BeO (Reference 5).

\begin{tabular}{|c|c|c|c|}
\hline - Property & - Unit & - Test Method & - BW-1000 \\
\hline - BeO content & - Weight $\%$ & $\begin{array}{c}\text { Spectrographic } \\
\text { by difference }\end{array}$ & - $\quad 99.5(\mathrm{~min})$ \\
\hline - Nominal Density & - $\mathrm{g} / \mathrm{cm}^{3}$ & - ASTM C-373 & - 2.85 \\
\hline - Hardness & - & - ASTM E-18 & $\begin{array}{c}60 \mathrm{~min} \\
\text { - } \quad \text { Rockwell } \\
45 \mathrm{~N})\end{array}$ \\
\hline - Grain Size & - Microns & - ASTM E-112 & $\begin{array}{c}9-12(10 \\
\text { typical) }\end{array}$ \\
\hline - Flexural Strength & - $\mathrm{MPa}$ & - $\quad$ ASTM F-417 & - $260(\min )$ \\
\hline $\begin{array}{c}\text { Thermal } \\
\text { Conductivity }\end{array}$ & $\begin{array}{c}\text { - } W / m-K \text { (at } \\
\left.T_{\text {room }}\right)\end{array}$ & - Laser Flash & - 275 \\
\hline $\begin{array}{l}\text { - Mean Coefficient } \\
\text { of Thermal } \\
\text { Expansion }\end{array}$ & - $10^{-6} /{ }^{\circ} \mathrm{C}$ & - ASTM E-228 & $\begin{array}{l}9.0(R T- \\
\left.1000^{\circ} \mathrm{C}\right)\end{array}$ \\
\hline
\end{tabular}

Small-scale research efforts were included in the test specimen fabrication specification. These include grain size reduction runs and material property testing (Reference 4). As discussed in Reference (4), grain size has a strong effect on the irradiated properties of BeO. This research effort was an attempt to reduce the grain size of standard BW-1000 material from $10 \mu \mathrm{m}$ to 5 $\mu \mathrm{m}$ (nominally). Material property testing of $\mathrm{BeO}$ specimens was completed for unitradiated standard BW-1000 material and was originally planned for targeted BeO with a reduced grain size (referred to as BW-1000K). Unirradiated material properties including thermal expansion, specific heat, thermal diffusivity, density and compressive strength testing were evaluated over a range of temperatures. Results are reported in Reference (4).

Brush Ceramic Products was contracted to provide manufacturing process documentation and material reports on BW-1000 and BW-1000K material. Manufacturing process documentation included chemical analysis, starting grain size, processing parameters, sintering protocol and machining instructions. Material reports for $\mathrm{BW}-1000$ and $\mathrm{BW}-1000 \mathrm{~K}$ were originally planned to include material properties and developmental efforts associated with $\mathrm{BW}-1000 \mathrm{~K}$ material.

Following project restructuring, NRPCT downsized the work on $\mathrm{BeO}$ test specimens. A limited amount of work continued on grain size reduction runs and unirradiated material property testing, as reported in Reference (4).

BeO Health and Safety

Beryllium oxide as a particulate is a hazardous material causing lung diseases and skin irritations. Inhaling particulate beryllium may cause a serious lung disease called chronic 
beryllium disease (CBD). Additionally, the international agency on cancer research lists beryllium as a known human carcinogen. Brush Ceramic Products, as well as other DOE facilities investigated (BWXT/ Y-12 and Los Alamos National Laboratory) have taken extensive measures to control this hazard, including protective equipment, engineered ventilation controls at source points and throughout buildings including interlocked air showers, dressing rooms, training, and administrative controls. To avoid potential health hazards at NRPCT facilities, formal cleaning, handling and packaging requirements were established for all BeO specimens prior to shipment. Because loose BeO particulate was of greatest concern from a health and safety standpoint, all processes generating powder (e.g. machining, grinding etc.) were completed by Brush Ceramic Products at their facility.

Current OSHA, DOE and KAPL airborne and surface control limits for beryllium work areas are summarized in Table 3 (References 6 and 7). Based on these guidelines, the NRPCT planned to establish a surface contamination limit of $0.25 \mu \mathrm{g} / 100 \mathrm{~cm}^{2}$ for all BeO specimens. A detailed cleaning procedure was established at Brush Ceramics to ensure test specimens were practically BeO particulate free prior to shipment to the NRPCT. Figure 2 outlines this post machining process, including the cleaning procedure, and more details can be found in Reference (8). It should be noted that the cleaning process may introduce chemical contamination, and therefore requires verification to ensure that material performance is not adversely affected.

Pre-irradiation specimen requirements were included in the post-machining process at Brush Ceramics. Specimen dimensions and mass measurements were required prior to shipment. Specimens were to be cleaned, baked out in an inert environment, and then stored in an inert atmosphere to minimize specimen contamination. Brush Ceramics planned to individually package each specimen in an inert atmosphere and shipped to NRPCT facilities. Following delivery, samples were planned to be placed directly into irradiation capsules with minimal handling.

As illustrated in Figure 3, a selected number of specimens were to be wet swipe tested following cleaning to verify specimens were below the proposed airborne and surface contamination limit. Wet-swipe testing of BeO specimens is a destructive test. Therefore, a statistically determined number of "sister" samples (samples cleaned and prepared identically to the final delivered samples) were to be destructively tested to verify that the entire batch of cleaned specimens were free of loose BeO. Brush Ceramic Products currently uses a wet benzylkonium chloride wipe to detect loose BeO. Benzylkonium chloride wipes are analyzed using the inductively coupled plasma (ICP) method with a detection limit of $0.2 \mu \mathrm{g}$ of beryllium. Other surface swipe testing methods were examined for use at NRPCT facilities (e.g., ChemTest ${ }^{\circ}$ beryllium Swipes).

Table 3: Airborne and Surface Contamination Limits for beryllium

\begin{tabular}{|c|c|}
\hline \multicolumn{2}{|c|}{ - Airborne Limits } \\
\hline$-2 \mu \mathrm{g} / \mathrm{m}^{3}$ & $\bullet$ OSHA limit \\
\hline $0.2 \mu \mathrm{g} / \mathrm{m}^{3}$ & - DOE limit \\
\hline & $\bullet$ BCP limit \\
\hline $0.2 \mu \mathrm{g} / 100 \mathrm{~cm}^{2}$ & $\bullet$ DOE release criteria \\
\hline
\end{tabular}


Enclosure 5 to MDO-723-0044I

B-MT(SRME)-52

Page 7

\begin{tabular}{|c|c|}
\hline - $0.25 \mu \mathrm{g} / 100 \mathrm{~cm}^{2}$ & - Planned NRPCT control level \\
\hline - $\quad 0.5 \mu \mathrm{g} / 100 \mathrm{~cm}^{2}$ & $\begin{array}{l}\text { - KAPL administrative control } \\
\text { level }\end{array}$ \\
\hline - $3.0 \mu \mathrm{g} / 100 \mathrm{~cm}^{2}$ & - DOE "housekeeping" limit \\
\hline
\end{tabular}


Enclosure 5 to

MDO-723-0044/

B-MT(SRME)-52

Page 8

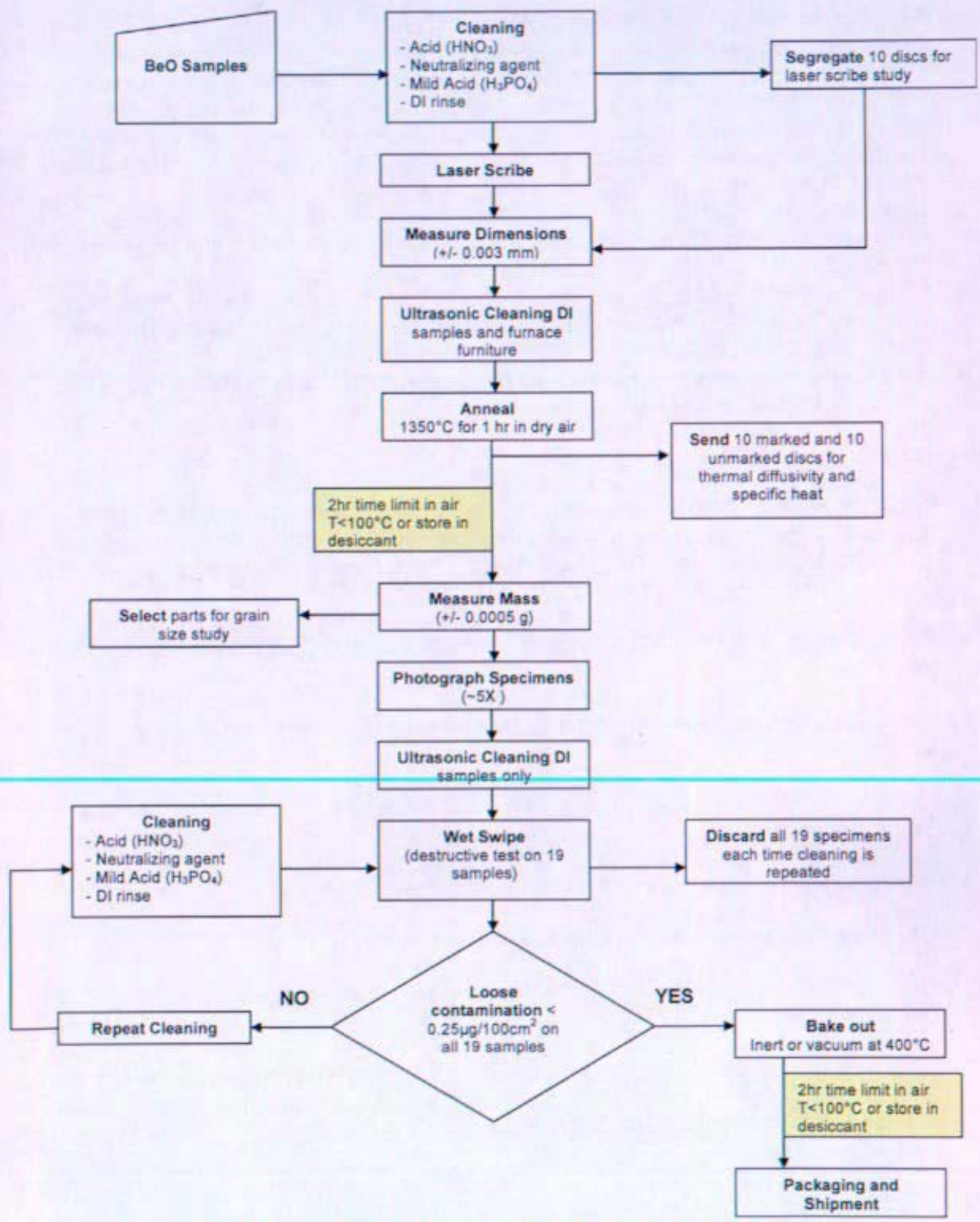

Figure 2: Post Machining Process for BeO Specimens at Brush Ceramics

\section{Conclusions}


- Beryllium oxide is a candidate reffector material requiring testing to determine irradiated material properties.

- Critical properties required to assess the viability of BeO in space nuclear applications, include irradiation-induced swelling, thermal conductivity and compressive strength.

- Based on the limited number of test specimens that were to be allowed in the first JOYO insertion, a single grade of BeO was selected for irradiation testing (BW-1000 BeO, Brush Ceramic Products), and future testing likely would have been required.

- Controls are required to handle BeO. However, if dust/loose particle generation processes (i.e. machining or grinding) are avoided, the controls are judged to be readily achievable.

- If design conditions (e.g., lower temperatures) allowed the use of beryllium as a reflector material it would be considered over $\mathrm{BeO}$ for the following reasons: potentially lower mass; likely lower cost overall; simpler to design with and fabricate.

- BeO was considered a leading material candidate for high temperature reflector applications (axial reflectors).

\section{References}

1. NRPCT Letter MDO-723-0021/B-MT(SRME)-21, "Request for Technical and Funding Approval of the Test Matrix and Associated Changes for the JOYO-1 Irradiation Test of Structural Materials in the JOYO Experimental Fast Reactor to Support Space Reactor Development", dated April 18, 2005.

2. KAPL Drawing SK216C9453, "Sample, Thermal Diffusivity Disc, JOYO," dated October $26,2005$.

3. KAPL Drawing SK216C9454, "Sample, Compression Cylinder, JOYO," dated October $26,2005$.

4. KAPL Letter MDO-723-0046, "Space Reflector Materials for a Prometheus Application," To Be Issued.

5. Brush Ceramic Products, Product Guide, Isopressed Ceramics CDI-20, Rev. E (2001).

6. OSHA 29 CFR 1910.1000, "Air Contaminants," May 1971.

7. DOE 10 CFR 850 , "Chronic Beryllium Disease Prevention Program; Final Rule," December 1999.

8. KAPL Letter SM-7232-0004, "Discuss Cleaning, Handling and Packaging Procedures at BCP to Ensure Delivery of BeO Specimens with a Surface Contamination Level Below NRPCT Limits", dated September 27, 2005. 
Enclosure 6 to MDO-723-0044 I

B-MT(SRME)-52

Page 1

\title{
Enclosure 6 \\ to \\ MDO-723-0044/B-MT(SRME)-52: \\ JOYO-1 Temperature Monitors and \\ Dosimetry Fabrication Efforts
}

\author{
R. S. Northey \\ A. Tullai \\ G. C. Carpenter
}

January 2006 


\section{Summary}

Passive temperature monitors and dosimeters were being fabricated for use in the JOYO-1 irradiation test. Temperature monitors were machined from silicon carbide (for temperatures below $1250 \mathrm{~K}$ ) and zirconium carbide (for temperatures above $1250 \mathrm{~K}$ ). Machining of the silicon carbide ( $\mathrm{SiC}$ ) passive temperature monitors was completed, while machining of the zirconium carbide $(\mathrm{ZrC})$ monitors was unfinished. The machining vendor was having difficulty fabricating the $\mathrm{ZrC}$ monitors because the base material ( $\mathrm{ZrC}$ rod stock) was breaking into many pieces during the machining operation. A limited investigation did not identify reasons for the unexpected breaking; therefore, a corrective action could not be identified. Machining was stopped, and the remaining $\mathrm{ZrC}$ was returned.

A limited neutron flux dosimetry set (also called flux wire assemblies) consisting of iron, nickel, and titanium wires was specified for use with selected JOYO capsules. These wires were a sub-set of a "full" flux dosimetry package sometimes used in JOYO by the Japan Atomic Energy Agency (JAEA). The sub-set was selected because the full JOYO package required use of the space between capsules in the Structural Materials Irradiation Rigs (SMIR), thus reducing the space available for specimens. Since the limited dosimetry set required less space, it allowed the dosimetry to be located closer to the test specimens. This would produce results more representative of the specimen exposures. The iron, nickel, and titanium wires were judged to provide sufficient neutron spectral sensitivity to confirm (or adjust) calculated neutron fluence values. The capsule designers planned to install a flux wire assembly into five of the ten JOYO1 capsule types. The flux wire assemblies were ordered, delivered, inspected, and accepted. The assemblies were subsequently certified to be true and correct per the purchase order and sketch requirements.

\section{Temperature Monitors}

\section{Introduction}

For the JOYO-1 test campaign, silicon carbide and zirconium carbide passive temperature monitors were procured. These base materials were selected because neutron irradiationinduced lattice expansions begin to anneal out when post-irradiation annealing temperatures exceed irradiation temperatures. Using this material behavior, irradiation temperatures can be determined through post-irradiation examination (PIE) procedures which include recording electrical resistivity changes and/or dimensional changes following sequential isochronal anneals (References 1 and 2). These two techniques may be used alone or together for additional measurement certainty. Examples of these PIE techniques are illustrated in Figure 1.

The irradiation temperature can be determined using dimensional changes as demonstrated in Figure 1a. This technique involves the use of a series of anneals, starting at temperatures well below the estimated irradiation temperature. Between each sequential anneal, the sample length is measured. As shown in Figure 1a, the irradiation temperature can be identified by drawing intersecting lines though the data points. Subsequently, Figure $1 \mathrm{~b}$ demonstrates the effect of an isochronal anneal on electrical resistivity. The irradiation temperature is identified by a divergence above the scatter (outlined in grey).

$\mathrm{SiC}$ passive temperature monitors were machined for capsules intended for irradiation temperatures below $1250 \mathrm{~K}$. Historically, $\mathrm{SiC}$ has been used effectively $( \pm 20 \mathrm{~K}$ ) within this 
temperature regime (Reference 1). Above $1250 \mathrm{~K}$, SiC temperature monitors are not effective as the radiation damage mechanism over long irradiation exposures changes from point defect formation to void formation. As a result of this limitation, $\mathrm{ZrC}$ was selected as the bulk material for temperature monitors above $1250 \mathrm{~K} . \mathrm{ZrC}$ is an experimental material for this application.

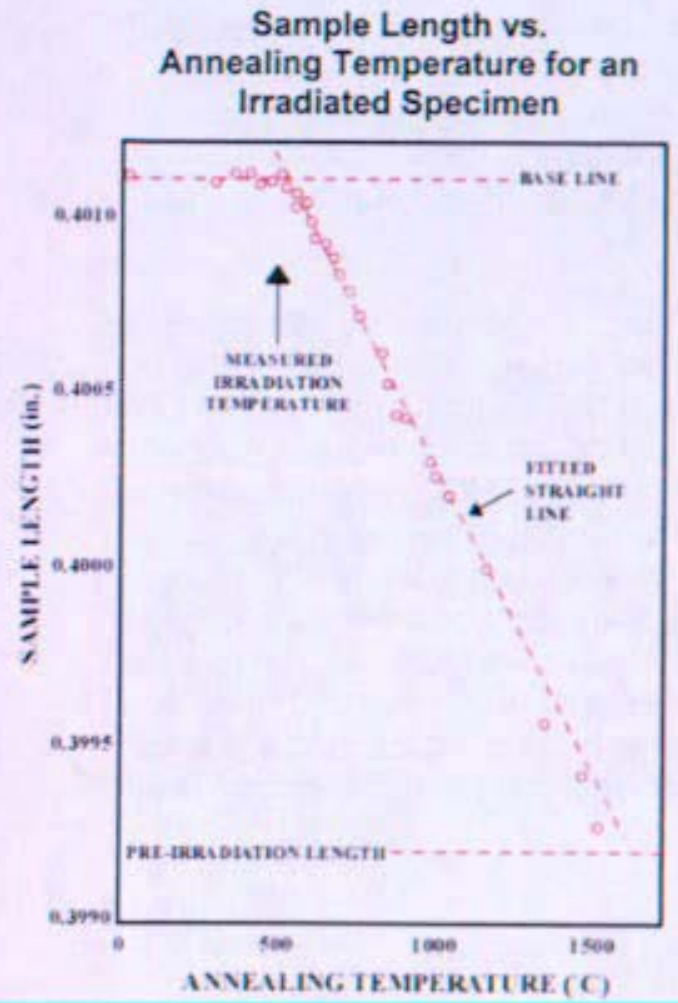

a.

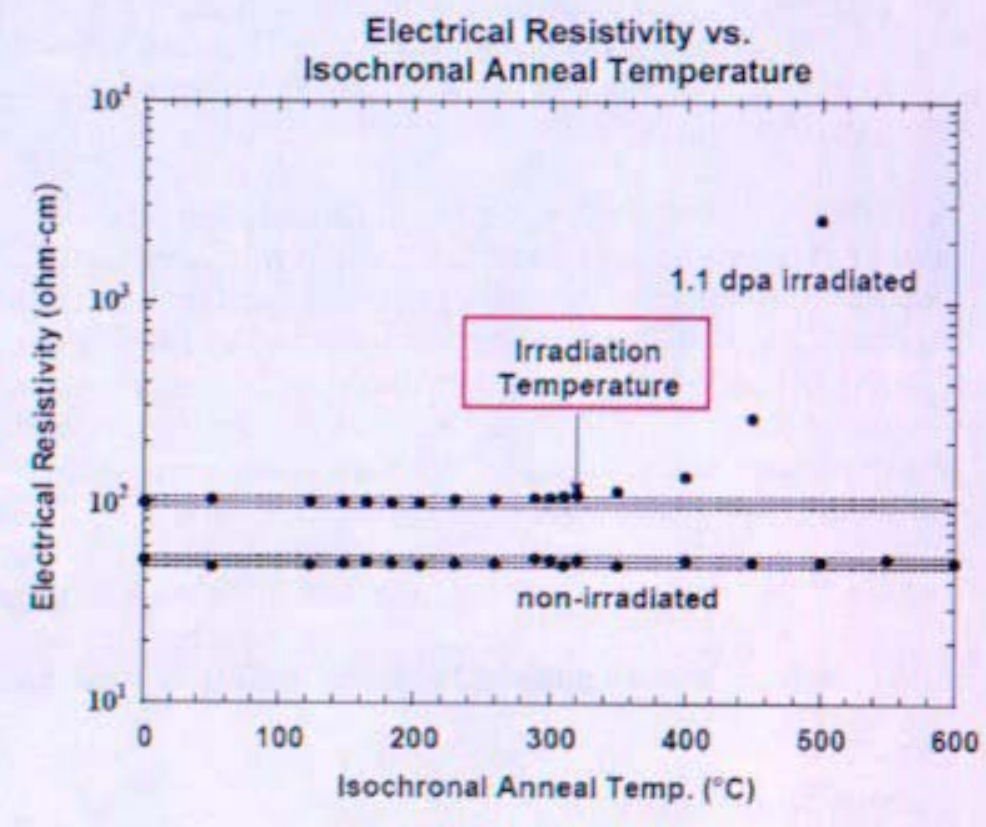

b.

Figure 1: a. Annealing temperature effect on sample length. Through the application of intersecting isochronal annealing lines the irradiation temperature can be determined.

b. Annealing temperature effect on electrical resistivity for irradiated monolithic SiC (Ref. 2).

Measurement techniques are dependent upon the concentration of simple point defects produced during irradiation, a process which is a function of the homologous irradiation temperature. Point defect concentrations can be directly correlated to swelling in $\mathrm{ZrC}$ in much the same way they are in $\mathrm{SiC}$, at least in theory. Because $\mathrm{ZrC}$ has a higher melting point than $\mathrm{SiC}$, theoretically it should be effective to higher temperatures (i.e., the temperature at which point defects effectively anneal in pile should occur above $1250 \mathrm{~K}$ ). Potential complicating factors for comparing temperature determinations using $\mathrm{ZrC}$ and $\mathrm{SiC}$ include: differing $\mathrm{SiC} / \mathrm{ZrC}$ crystal structures, variable $\mathrm{ZrC}$ stoichiometry, and limited basic irradiation data on $\mathrm{ZrC}$.

One study to date has included $\mathrm{ZrC}$ as a passive temperature monitor. The Pathfinder Irradiation Program was initiated in the High Flux Isotope Reactor (HFIR) at Oak Ridge National Laboratory (ORNL) to support Naval Reactor Prime Contractor Team (NRPCT) efforts to generate rapid, low-dose information on irradiation effects at low fluences in refractory metal alloys. The program used $\mathrm{ZrC}$ to gain data for its use as a temperature monitor. At project 
restructuring, $\mathrm{ZrC}$ monitors had been irradiated at $1223 \mathrm{~K}$ for one and two cycles (24 irradiation days in HFIR) alongside SiC temperature monitors. This side-by-side comparison would benchmark $\mathrm{ZrC}$ against the better understood $\mathrm{SiC}$. In addition, $\mathrm{ZrC}$ monitors were irradiated at $1373 \mathrm{~K}$ without the inclusion of $\mathrm{SiC}$. Examination of SiC monitors is currently underway, although analysis of $\mathrm{ZrC}$ thermometry has been delayed due to high radioactivity levels. This residual activity is expected to decay within acceptable limits by March 2006 (Reference 3).

\section{Discussion}

$\mathrm{SiC}$ temperature monitors were machined from monolithic beta phase silicon carbide plates ( 6 in. $x 6$ in. $\times 1 / 4$ in.) purchased from Rohm and Haas Advanced Materials, Woburn, MA (Attachment 1.1). These SiC plates were delivered with a Certificate of Analysis (Attachment 1.1) providing the following material properties:

Table 1: Bulk material properties for bulk monolithic SiC plates.

\begin{tabular}{|c|c|}
\hline Property & Average Value \\
\hline Grain Size $(\mu \mathrm{m})$ & $7.1-9.4$ \\
\hline Chemical Purity $(\mathrm{ppmw})$ & $<5.74$ \\
Glow discharge mass spectroscopy $(\mathrm{GDMS})$ & \\
\hline Thermal Conductivity $\left(\mathrm{W} \cdot \mathrm{m}^{-1} \cdot \mathrm{K}^{-1}\right)$ & $310 \pm 19$ \\
\hline Flexural Strength $(\mathrm{MPa})$ & $\mathbf{4 1 7 \pm 4 8}$ \\
\hline
\end{tabular}

An independent chemical analysis, via glow discharge mass spectroscopy (GDMS), was performed on the bulk material at Shiva Technologies, Inc, Syracuse, NY. GDMS testing certified that the bulk material did not contain impurities above the Rohm and Haas Advanced materials certifications (Provided in Table 1 and Attachment 1.1). In addition, Northern Analytical Laboratory, Inc., Merrimack, $\mathrm{NH}$, tested for nitrogen content on the bulk material, resulting in a reading of $0.0114 \pm 0.0006 \%$ (Attachment 1.1 , Specimen A-CAF). Documenting the nitrogen content is required for end-of-life accounting purposes of SiC specimens and their respective quantities of Carbon-14 produced during irradiation.

Bomas Machine Specialties, Inc., Somerville, MA, machined the monolithic SiC monitors according to NRPCT technical specification, 05PFG094 ${ }^{1}{ }^{1}$. The technical specification included plate identifications, machining instructions, NRPCT issued drawings, requirements for a first piece inspection, and a list of detrimental materials. Overall, the technical specification (Attachment 1.2 and 1.3) provided instruction for the fabrication of approximately 180 monitors. These monitors consisted of three length configurations, corresponding to the possible fixture sizes within a capsule. Prior to machining, engineers from Bomas Machine Specialties and NRPCT completed a readiness review to ensure details of the issued technical specification were understood. Upon agreement of the requirements, a first piece inspection was required to ensure dimensional accuracy within specified tolerances for two temperature monitors of each size (Attachment 1.3). Upon receipt of a positive inspection, the balance of the order was completed and delivered. The specimen receipt was accompanied by a dimensional report and a Certificate of Compliance (Attachment 1.3) to ensure strict compliance with issued machining instructions (dimensions and detrimental materials). Photographs of the silicon carbide temperature monitors are included in Attachment 1.4.

\footnotetext{
${ }^{\mathfrak{1}}$ The symbol ${ }_{K}$ indicates a KAPL specific issued document.
} 
For temperature monitors in capsules irradiated above $1250 \mathrm{~K}, \mathrm{ZrC}$ temperature monitors were procured. The $\mathrm{ZrC}$ bulk material was polycrystalline, zone-refined zirconium carbide. Eighty rods were purchased from Applied Physics Technology, Inc., McMinnville, OR (Attachment 2.1). This material was then sent to PremaTech Advanced Ceramics, Worchester, MA, for machining according to the NRPCT technical specification 05PFG115 ${ }_{\mathrm{K}}$ (Attachment 2.2). At project restructuring, PremaTech Advanced Ceramics was having difficulty machining the ZrC temperature monitors as the $\mathrm{ZrC}$ rods were breaking into many pieces during the machining operation. A limited investigation did not identify reasons for the unexpected breaking; therefore, a corrective action could not be identified. Machining was stopped, and the remaining $\mathrm{ZrC}$ was returned (Attachment 2.3).

\section{Dosimetry}

\section{Introduction}

Nominal axial fast flux profiles for each of the six SMIR radial compartment positions within JOYO core position $6 \mathrm{A4}$ are shown in Figure 2. The insert at the upper right corner of Figure 2 shows the compartment locations relative to the core centerline. The graph also indicates the tier locations for all compartment positions. Additional capsule design details are found in Enclosure 7. The 6A4 position is in the JOYO reflector in the first row outside the fuel rows. The reflector positions tend to have relatively large radial flux gradients. Specimens in the Tier 1 and 5 capsules near the core top and bottom are also subject to large axial flux gradients. As a result of these gradients, fluxes vary significantly as a result of the compartment and tier position. The presence of these large gradients justifies use of at least some neutron flux dosimetry to confirm nuclear calculations and best characterize neutron fluence exposures of test specimens.

A limited neutron flux dosimetry set (also called flux wire assemblies) consisting of iron, nickel, and titanium wires was specified for use with selected JOYO capsules. These wires are a subset of a "full" flux dosimetry package sometimes used in JOYO by JAEA. The subset was selected because the full JOYO package required use of the space between the capsules in the $S M I R$, thus reducing the space available for specimens. Since the limited dosimetry set required less space, it allowed the dosimetry to be located closer to the test specimens. This would produce results more representative of the specimen exposures. The iron (Fe), nickel (Ni), and titanium (Ti) wires were judged to provide sufficient neutron spectral sensitivity to confirm (or adjust) calculated neutron fluence values.

Flux dosimetry analysis typically begins with an acid dissolution step of the irradiated monitors. The monitors are weighed prior to irradiation to provide the most accurate weight basis for the analysis. The dilution is typically on the order of a million to reduce the activity of the sample to acceptable levels for removal from the hot cell and counting of the radioactive isotopes. The gamma ray spectrometry data are converted into fluence values using standard calculation techniques. When accounting for the dilution, equipment accuracy and calibration, and the duration from reactor shutdown to the actual counting time, the measured flux wire fluence uncertainty can be within $5 \%$ to $7 \%$. The uncertainty can be reduced to approximately $3 \%$ to $5 \%$ by calibration at the energies of interest and adjusting count times to minimize any error due to statistical fluctuation. 


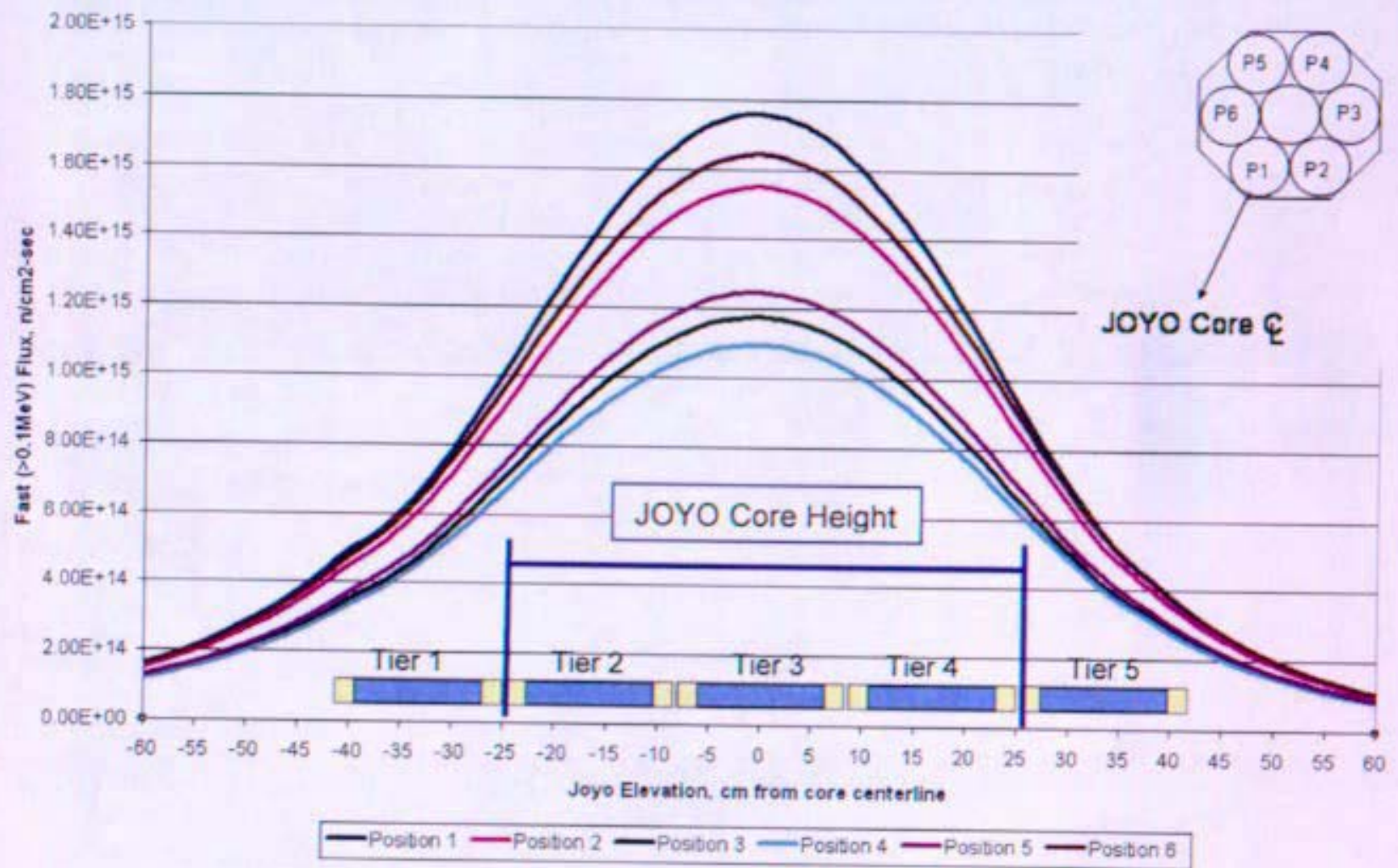

Figure 2: Nominal Fast Flux Profiles for JOYO Core Position 6A4 and Compartment Positions P1-P6

\section{Discussion}

The capsule designers planned to install a flux wire assembly (see Figure 3 and Figure 4) into five of the ten JOYO-1 capsule types: TFT, DBU/DBD, and A5U/A5D. Figure 5 shows a cutaway of a TFT Capsule. A description of the JOYO-1 capsule types is found in Enclosure 7. The material specimen holders in the capsules were designed with sufficient radial and axial space to accept the flux wire (and temperature monitor) assemblies. The flux wire assemblies (Table 2) included wires made from $0.25 \mathrm{~mm}$ diameter titanium, nickel and iron in decreasing lengths in order to distinguish the wires apart from each other when analyzed in a hot cell. The titanium was wrapped in $0.025 \mathrm{~mm}$ thick molybdenum foil to prevent a Ti-Ni eutectic at $1215 \mathrm{~K}$ and a Fe-Ti eutectic at $1358 \mathrm{~K}$. In addition, it was likely that substantial interdiffusion would occur at points of contact, effectively diffusion bonding the wires into the holders. The wires were encapsulated in a molybdenum tube with tapered end plugs. A hole was drilled in the side of the molybdenum tube to prevent pressure buildup. The molybdenum tube and wires were directly purchased in the proper form, and cut to length by a manufacturing company contracted by the NRPCT. 
Eighty-seven flux wires assemblies were ordered on PO $3007724_{B}{ }^{2}$ per Sketch SK-RRM1011, Group 1. These assemblies were delivered, inspected, and accepted. The assemblies were subsequently certified to be true and correct per the purchase order and sketch requirements as documented in Reference 4.

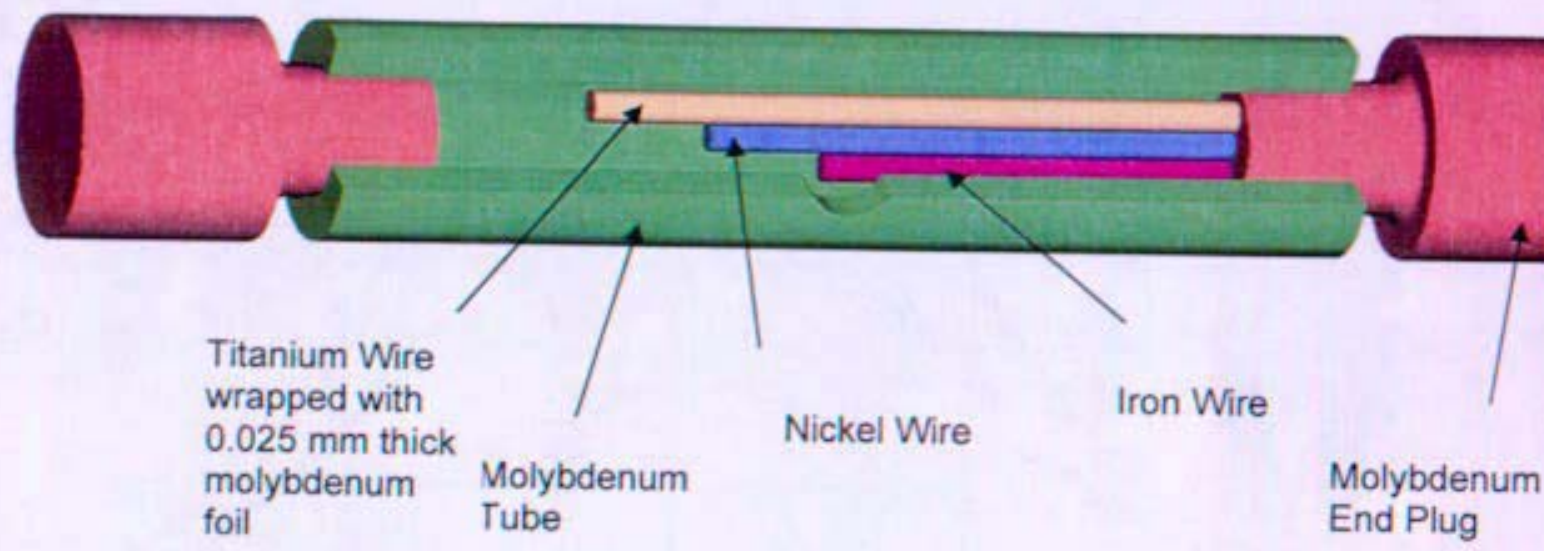

Figure 3: Flux Wire Assembly

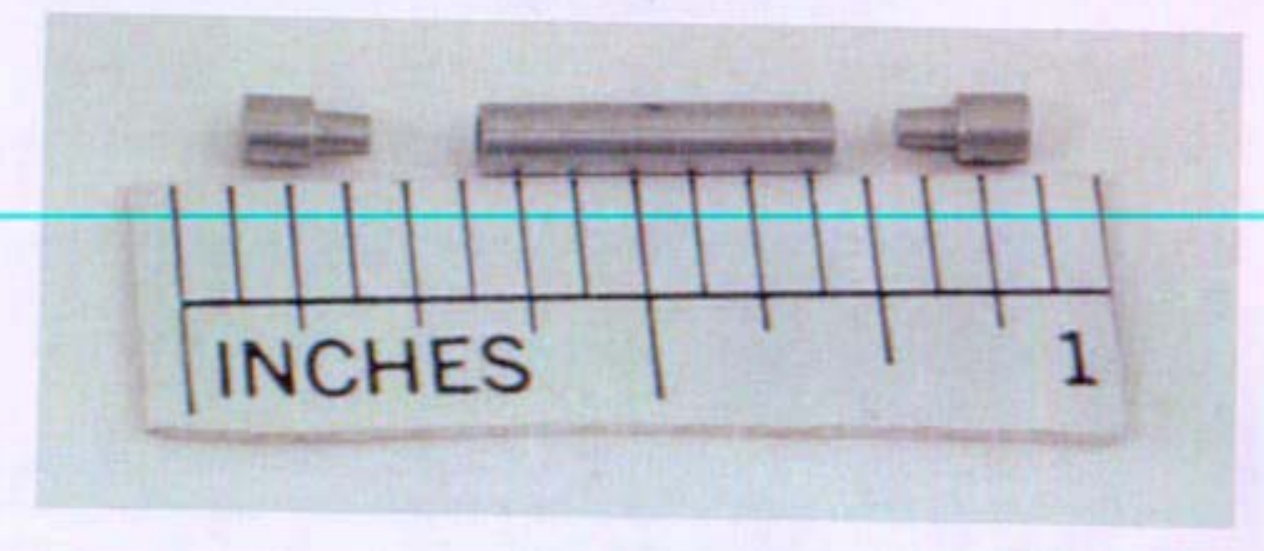

Figure 4: Molybdenum Components for Flux Wire Assembly

\footnotetext{
${ }^{2}$ The symbol ${ }_{B}$ indicates a Bettis specific issued document.
} 


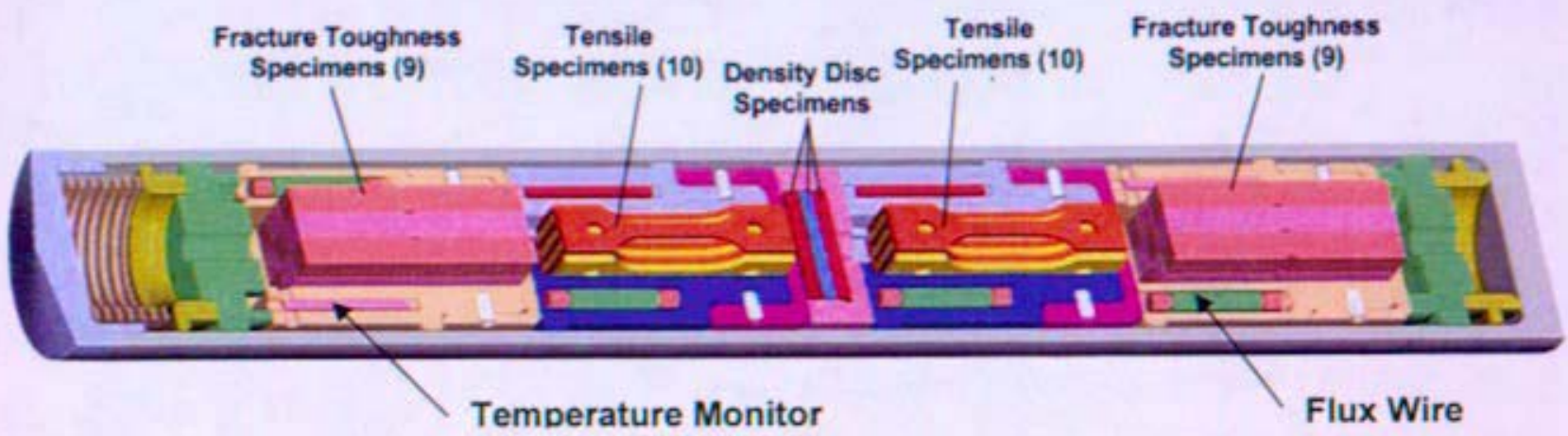

Figure 5: TFT Capsule showing Flux wires and Temperature Monitors

\begin{tabular}{|c|c|c|c|c|}
\hline \multicolumn{5}{|c|}{ Table 2: Flux Wire Assembly Components } \\
\hline $\begin{array}{c}\text { Flux Wire Assembly } \\
\text { Component }\end{array}$ & $\begin{array}{c}\text { Sketch } \\
\text { SK-RRM-1011 }\end{array}$ & Material & $\begin{array}{c}\text { Composition } \\
\text { Weight \% }\end{array}$ & Notes \\
\hline Molybdenum tube & Item 2 & Molybdenum & $99.99 \mathrm{Mo}$ & 1 \\
\hline Molybdenum end plug & Item 1 & Molybdenum & $99.99 \mathrm{Mo}$ & 2 \\
\hline Nickel wire & Item 4 & Nickel & $99.99 \mathrm{Ni}$ & 3 \\
\hline Titanium wire & Item 3 & Titanium & $99.99 \mathrm{Ti}$ & 4 \\
\hline Iron wire & Item 5 & Iron & $99.99 \mathrm{Fe}$ & 5 \\
\hline Molybdenum Foil & Item 6 & Molybdenum & $99.95 \mathrm{Mo}$ & 6 \\
\hline
\end{tabular}

1. Catalog Number MO007301, Goodfellow Corp, Berwyn, PA

2. Catalog Number MO007901, Goodfellow Corp, Berwyn, PA

3. Catalog Number 10931, Alfa Aesar, Word Hill, MA

4. Catalog Number 10392, Alfa Aesar, Word Hill, MA

5. Catalog Number 10937, Alfa Aesar, Word Hill, MA

6. Catalog Number 10042, Alfa Aesar, Word Hill, MA

\section{Conclusion}

Eighty-seven flux wire assemblies consisting of iron, titanium and nickel wire components were procured, manufactured, and delivered per NRPCT Purchase Order $3007724_{\mathrm{B}}$, Line Item 3 , in accordance with Sketch SK-RRM1011, Group 1. The components were packaged separately such that they could be cleaned and vacuum-baked to remove moisture. The flux wire assemblies were to be inserted into JOYO-1 Capsule types TFT, DBU/DBD, and A5U/A5D. The assemblies represented a smaller subset of the dosimetry package typically used in JOYO testing. The smaller size allowed the assemblies to be placed within individual capsules closer to the material specimens. This is advantageous when considering the larger gradients predicted in the reflector region where the SMIRs were to be located. 
Enclosure 6 to

MDO-723-0044 /

B-MT(SRME)-52

Page 9

\section{References}

1. R. J. Price, "Annealing Behavior of Neutron-Irradiated Silicon Carbide Temperature Monitors, Journal of Nuclear Technology Volume 16, Dec. 1972.

2. L.L. Snead, A.M. Williams, and A.L. Qualls, "Revisiting the Use of $\mathrm{SiC}$ as a Post Irradiation Temperature Monitor," The Effects of Radiation on Material: $21^{\text {st }}$ Int. Symposium, ASTM STP 1447, M.L. Grossbeck, T. R. Allen, R.G. Lott, and A.S. Kumar, Eds., ASTM International, West Conshoshocken, PA, 2004.

3. J. T. Busby, K.J. Leonard, and S. J. Zinkle, Effects of Neutron Irradiation on Refractory Metal Alloys, ORNL/LTR/NR-PROM1/05-31, Oak Ridge National Laboratory, Oak Ridge. Tenn., June 2005.

4. "Certification of Review for Irradiations Test Hardware - JOYO Flux wire assembly," B-MT(EDT)R-1773, To Be Issued. 


\begin{abstract}
Attachments
Machining Documentation for Silicon Carbide Temperature Monitors

1.1 Material Purchase Documentation and Certification 1.2 Technical Specification for Machining 1.3 Machining Certificates of Compliance and Quality Assurance 1.4 Specimen Photographs
\end{abstract}

Machining Documentation for Zirconium Carbide Temperature Monitors

2.1 Material Purchase Documentation and Certification

2.2 Technical Specification for Machining 2.3 Closeout Documentation

Enclosure 6 to MDO-723-0044/B-MT(SRME)-52 
Attachment 1.1 to Enclosure 6 to MDO-723-0044/

B-MT(SRME)-52

Page 1

Attachment 1.1

Material Purchase Documentation and Certification

Enclosure 6 to MDO-723-0044/B-MT(SRME)-52 


\section{Certificate of Analysis}

Material: CVD SILICON CARBIDE® Lot No.:9112

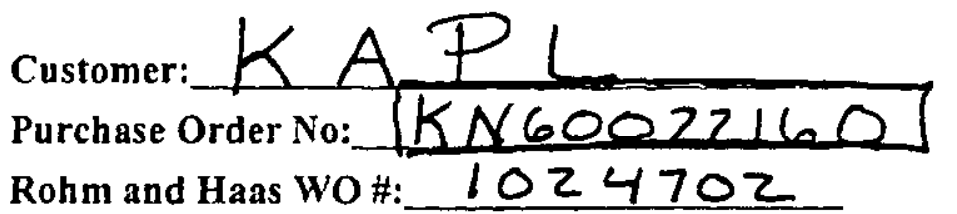

Run No 9112 was performed in our Weeks Island, LA, SiC facility.

One sample each was tested for grain size, microstructure and tota! trace impurities, six samples were tested for thermal conductivity, and thirty samples were prepared for flexural strength (modulus of nupture) testing. Measured results are listed below.

$\begin{array}{cc}\text { Property } & \text { Average Value } \\ \text { Grain size }(\mu \mathrm{m}): & 7.1-9.4 \\ \begin{array}{c}\text { Chemical purity }(\mathrm{ppmw}): \\ \text { (glow discharge mass spectroscopv) }\end{array} & <5.74 \\ \text { Thermal Conductivity }\left(\mathrm{W} \cdot \mathrm{m}^{-1} \cdot \mathrm{K}^{-1}\right) & 310 \pm 19 \\ \text { Flexural Strength }(\mathrm{MPa}) & 417 \pm 48\end{array}$

\section{Conclusions}

CVD SILICON CARBIDE® from lot Run No. 9112 is a typical high purity, polycrystalline. pure $\beta$-phase SiC produced at Rohn and Huas Advanced Materials. It has been processed in accordance with the manufacturing procedures for CVD SILICON CARBIDEB maintained by Rohm and Haas Advanced Materials, and was produced to meet the nominal material properties for CVD SILICON CARBIDE@ as listed on our data sheet.

\section{MATERIAL FROM THIS LOT HAS BEEN ACCEPTED FOR LSE TOWARDS ALL CLSTOMER ORDERS.}

Roman Baran

QA/QC Department

Date: January 12, 2004 
ROHM AND HAAS

ADVANCED MATERIALS

PDH:H AN" HAAS COMPANY

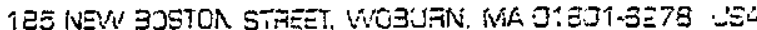

E_P:HONE: (781) 933-9943 FAX: (78:: S33-5:42

Attachment 1.1 to

Enclosure 6 to

MDO-723-0044/

B-MT(SRME)-52

Page 3

\section{PART INSPECTION REPORT}

Clstomer ORder H: KN60022160

R\&H W.O. \#:

PART NAME:

DRAWIYG \#:

Material:

MATER

SN:

Source Lot:

\begin{tabular}{|l|r|r|}
\hline Specifications: & $\begin{array}{r}\text { SPECIFICATIONS } \\
\text { MILLMMETERS }\end{array}$ \\
\hline & Specificntion & ACCFPTIOAC \\
\hline $\mathrm{B}$ & 6.00 & $\mathrm{ACC}$ \\
\hline $\mathrm{C}$ & 6.00 & $\mathrm{ACC}$ \\
\hline $\mathrm{D}$ & .125 & $\mathrm{ACC}$ \\
\hline
\end{tabular}

1024702

PLATE

NA

CVDSILICON CARBIDE ${ }^{T H}$

$\mathbf{Y A}$

9112
DATE: 04/30/05

IXSPECTOR: C. BEDARD 


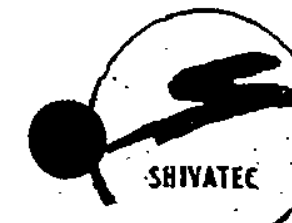

IYTICAL SOLUTIONS

WORIDWIDE

Date $11 / 8 / 2005$

C J Thomas

Knolls Atomic Power Laborator

PO Box 1072 MS 148

Schenectady, NY 12301-1072

Enclosed are the analytical reports for the following samples.

PO\#_Shiva Job \#

KN6004044

UO4798

Includes Your Id \#

A-CAG
Attachment 1.1 to $?$

Enclosure 6 to MDO-723-0044/ B-MT(SRME)-52

Syracuse, New York - 13; $\quad$ Page 4

Tel: (315) 431-9900

Fax: (315) $431-9800$

Web: www.shivatec.com

Email: info shivatec.com
Account KAP 


\section{FAX TRANSMITTAL INFORMATION}

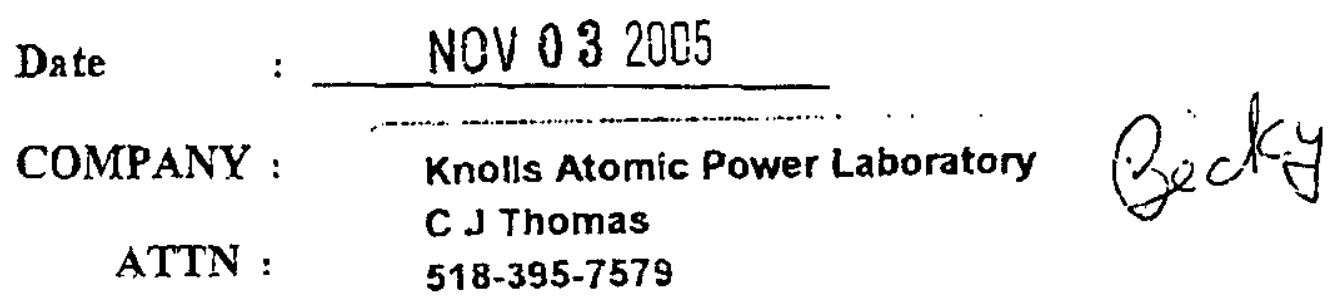

FAX :

PO \# KN6004044

JOB \#

\section{8}

Your Client Services Team is here to help you with any inquiries you may have:
Sam Murthy
Vice President,
Richard Balamut
Director,
Matthew IePage
Client Services
Client Services
Director,
Business Development

NOTES:

Sample return policy: Analyzed samples will be stored in our facility for one year and will be destroved. The samples can be returned on request at additional cost or using vour account courier number.

Original report will he mailed to voutuaur Company within one week from the dafe of completion of

analysis

Job Status: CfFully compIcted $\square$ Partially completed $\square$ Other test resulte follows

Total pages (including cover sheet):

Time Sent:

If yo'd do not receive all pages or if there are any questions, please le: us know.

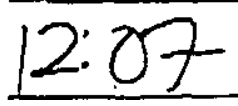

THANK YOU for your continued support

- Trace and Ulira-trace determinations

- Bulk and Near-surface Analysis

- Depth Profile Elemental Evaluation

- Material Analysis from ZI to U

- ISO 9001-2000, Nade
- Compositional Analysis by ICP-OES

- Metallography

- $H, C, O, N$ and $S$ by Inert Gas Fusion

- Mitimal sample requirements

i, ELROPE and ASLA 
FA Attachment 1.1 to ANALYTICAL REPORT

Customer: Knolls Atomic Power Laboratory

Date:

2-Nov-05

Customer ID: SiC

\section{A-CAG}

\section{Element Concentration} [ppm wt]

\begin{tabular}{|r|}
\hline \\
\hline \\
\hline \\
\hline
\end{tabular}

Li

$\mathrm{Be}$

B

$<0.01$

$<0.005$

C Matrix

\begin{tabular}{l|l}
$\mathrm{N}$ & -100 \\
\hline
\end{tabular}

$\mathrm{F}$

$\mathrm{Na}$

-

$-35$

M

A

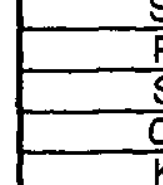

\begin{tabular}{l|l}
$\mathrm{Al}$ & \\
\hline
\end{tabular}

$<0.5$

$<0.01$

$<0.01$

0.32

Matrix

0.02

\begin{tabular}{l|l}
$\mathrm{S}$ & 0.02 \\
\hline
\end{tabular}

\begin{tabular}{l|l}
$\mathrm{Cl}$ & 0.06
\end{tabular}

$<0.05$

\begin{tabular}{l|l}
$\mathrm{Ca}$ & 0.07 \\
$\mathrm{Sc}$ & $<0.01$ \\
\hline
\end{tabular}

\begin{tabular}{l|l}
$\mathrm{Ti}$ & $<0.01$ \\
\hline $\mathrm{V}$ & $<0.005$
\end{tabular}

\begin{tabular}{l|l}
$\mathrm{Cr}$ & $<0.5$ \\
$\mathrm{Mr}$ & $<0.01$
\end{tabular}

\begin{tabular}{c|c}
$\mathrm{Mn}$ & $<0.01$ \\
\hline $\mathrm{Fe}$ & 0.05
\end{tabular}

\begin{tabular}{l|r}
$\mathrm{Fe}$ & 0.05 \\
\hline $\mathrm{Co}$ & $<0.005$ \\
\hline
\end{tabular}

\begin{tabular}{l|l}
$\mathrm{Ni}$ & 0.22 \\
\hline
\end{tabular}

\begin{tabular}{l|l}
$\mathrm{Cu}$ & $<0.05$ \\
\hline $\mathrm{Zn}$ & $<0.05$
\end{tabular}

\begin{tabular}{l|l}
$\mathrm{Ga}$ & $<0.05$ \\
\hline $\mathrm{Ga}$ & $<0.05$
\end{tabular}

\begin{tabular}{l|l}
$\mathrm{Ge}$ & $<0.05$ \\
\hline
\end{tabular}

\begin{tabular}{l|l} 
As & $<0.05$ \\
\hline
\end{tabular}

\begin{tabular}{l|l}
$\mathrm{Se}$ & $<0.5$
\end{tabular}

\begin{tabular}{l|l}
$\mathrm{Br}$ & $<0.5$ \\
\hline
\end{tabular}

\begin{tabular}{l|l}
$\mathrm{Rb}$ & $\leq 0.1$ \\
\hline
\end{tabular}

\begin{tabular}{l|l}
$\mathrm{Sr}$ & $<0.01$ \\
\hline $\mathrm{Y}$ & $<0.01$
\end{tabular}

\begin{tabular}{l|l}
\hline $\mathrm{Y}$ & $<0.0$ \\
\hline $\mathrm{Zr}$ & $<0.0$ \\
\hline
\end{tabular}

\begin{tabular}{l|l}
$\mathrm{Nb}$ & $<0.01$
\end{tabular}

\begin{tabular}{l|l} 
Mo & $<0.05$
\end{tabular}

RU $<0.01$

$\mathrm{Rh}$

NOTE: $\sim$ Semiquantitative Values

Te

Pr

S

D

P.O.\# KN6004044

Job \# UO4798

Shiva ID: $\quad$ U051026148

Element Concentration

\begin{tabular}{|c|c|}
\hline $\mathrm{Pd}$ & [ppm wt] \\
\hline $\mathrm{Ag}$ & $<0.01$ \\
\hline $\mathrm{Cd}$ & $<0.5$ \\
\hline $\mathrm{In}$ & $<0.5$ \\
\hline $\mathrm{Sn}$ & $<1$ \\
\hline $\mathrm{Sg}$ & $<0.05$ \\
\hline
\end{tabular}

\begin{tabular}{l|l}
\hline $\mathrm{S}$ & $<0.05$ \\
\hline & $<0.05$
\end{tabular}

Te $\quad<0.05$

\begin{tabular}{l|l}
\hline & $<0.01$ \\
\hline
\end{tabular}

\begin{tabular}{l|l}
\hline & $<0.005$ \\
\hline
\end{tabular}

\begin{tabular}{|c|c|}
\hline $\mathrm{Ba}$ & $<0.005$ \\
\hline $\mathrm{La}$ & $<0.005$ \\
\hline $\mathrm{Ce}$ & $<0.01$ \\
\hline
\end{tabular}

\begin{tabular}{l|l}
\hline Co & $<0.01$ \\
\hline
\end{tabular}

\begin{tabular}{l|l}
$\mathrm{Nd}$ & $<0.005$ \\
\hline $\mathrm{Sm}$ & $<0.005$
\end{tabular}

\begin{tabular}{l|l}
$\mathrm{Sm}$ & $<0.005$ \\
\hline $\mathrm{Eu}$ & $<0.005$ \\
\hline
\end{tabular}

\begin{tabular}{l|l} 
EU & $<0.005$ \\
\hline Gd & $<0.005$ \\
\hline Tb & $<0.005$
\end{tabular}

\begin{tabular}{l|l}
$\mathrm{Tb}$ & $<0.005$ \\
\hline
\end{tabular}

\begin{tabular}{l|l} 
Dy & $<0.005$ \\
\hline
\end{tabular}

\begin{tabular}{ll} 
Ho & $<0.005$ \\
\hline
\end{tabular}

\begin{tabular}{l|l} 
Er & $<0.005$ \\
\hline
\end{tabular}

\begin{tabular}{|c|c|}
\hline$T m$ & $<0.005$ \\
\hline$Y b$ & $<0.005$ \\
\hline $\mathrm{Lu}$ & $<0.005$ \\
\hline
\end{tabular}

Th

\begin{tabular}{|c|c|}
\hline$T a$ & Source \\
\hline$W$ & $<0.01$ \\
\hline$R e$ & $<0.01$ \\
\hline $\mathrm{Os}$ & $<0.01$ \\
\hline $\mathrm{Ir}$ & $<0.01$ \\
\hline $\mathrm{Pt}$ & $<0.01$ \\
\hline $\mathrm{AU}$ & $<0.05$ \\
\hline $\mathrm{Hg}$ & $<0.05$ \\
\hline $\mathrm{Tl}$ & $<0.01$ \\
\hline $\mathrm{Pb}$ & $<0.01$ \\
\hline $\mathrm{Bi}$ & $<0.01$ \\
\hline $\mathrm{Th}$ & $<0.001$ \\
\hline$U$ & $<0.001$ \\
\hline
\end{tabular}




\section{1}

NORTHERN ANALYTICAL LABORATORY, INC.

\section{TEST REPORT}

23 Depot St., Merrimack, NH 03054

Phone: (603) 429-9500

FAX: (603) 429.9471

\section{Ms. Rebecca Northey}

KAPL, Inc.

PO Box 1072

Schenectady, New York 1230!-1072
Prom

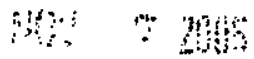

$\therefore i$

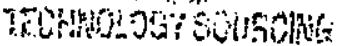

$\begin{array}{llll}\text { RECEIVED } & \text { 10/2S/OS } & \text { SAMPLE NUMBER } & \text { L,30829 } \\ \text { IDENT AS } & \text { Sei below } & \text { REPORT DATE } & 11 / 7105 \\ \text { MATERIAL } & \text { SiC } & \text { PAGE } & \text { 1 of } 1 \\ \text { CONDITION } & \text { SOlid } & \text { CLIBNT ORDER } & \text { XN6004010 } \\ \text { TEST TO } & & & \\ \text { TEST PER } & \text { TP-LECOI } & & \end{array}$

Method:

\section{LECO}

Semple in.

N(\%)

A-ANX

$0.0057 \pm 0.0002$

$A-C A F$

$0.0114 \pm 0.0006$

Analysis ay:

Pater S. Dickson Analotical Chemit

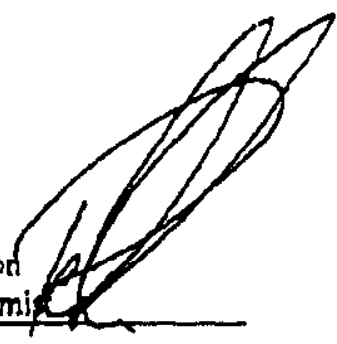

Approved By: Wiliapta, Guidoboni

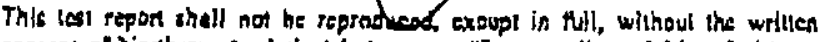
oonsent of Northern Analyiew Luhurewry. Jhe resording of Bibe. Holitious. 
Attachment 1.2 to

Enclosure 6 to

MDO-723-0044/

B-MT(SRME)-52

Page 1

Attachment 1.2

Technical Specification for Machining

Enclosure 6 to MDO-723-0044/B-MT(SRME)-52 


\section{SPECIFICATION FOR 05PFG094}

Rev. -

\section{DATED 6/1/05}

1. KAPI. will supply compositc plate as described below:

\begin{tabular}{|l|l|l|l|l|l|}
\hline NO. & PLATE ID & $\begin{array}{l}\text { PI.ATE } \\
\text { TYPE }\end{array}$ & $\begin{array}{l}\text { IENGTH } \\
\text { (IN) }\end{array}$ & $\begin{array}{l}\text { WIDTH } \\
(\text { IN })\end{array}$ & $\begin{array}{l}\text { THICKNESS } \\
(\mathrm{NN})\end{array}$ \\
\hline 1 (1 Plate) & KN6002216-7 & $\begin{array}{l}\text { Morton } \\
\text { CVD Plates }\end{array}$ & 6 & 6 & $\sim 0.125$ \\
\hline
\end{tabular}

2. APPI.IC $\triangle B L E$ DRAWINGS AND SKETCHES

- Betis Drawing 5D]4442 Rev. [Modified] Multiple Irradiation Capsule Fxperiment (MICE) Secondary Passive Temperature Indicator Detail.

- KAPL Drawing 296E600 Rev. A Standard Tolerances and Manufacturing Practices.

- KAPL Spccification, Attachment I "Detrimental Materials Prohibitions and Restriction for Silicon Carbide Composite Materials" Dated 3/6/03.

- KAPL supplied Table 1 'Machining Instruction Sheet".

\section{MACHINING INSTRUCTION}

- For each piece of (IFM plate, extact and machine the required number of specimens as specified in the attached table I "Machining Instrtction".

- Before extracting samples, the seller shall layout its cuts to verify the quantity of each plate and report to KAPL its layout for approval.

- Samples shall not be machined from the outer $1 / 8$ " perimeter of the GFM plate.

- Stock removal rate shall not exceed $0.03 \mathrm{~mm}$ per pass to the last $0.06 \mathrm{~mm}$. Final finishing shall be preformed with diamond tools that have between 320 and 600 grit. No less than $0.06 \mathrm{~mm}$ per face shall be removed during the final finishing phase and at a rate not more than $0.002 \mathrm{~mm}$ per pass.

- Remove approximately equal stock from opposite faces.

- The surface finish for the monolithic passive temperature indicator shall be $16 \mu$ in.

- Compliance with KAPL Specification on detrimental materials is required during all aspects of this fabrication.

- The seller shall use dedicated resin-bonded diamond tooling for all machining of silicon carbide specimens. 11" other than resin-bonded tooling is need the seller shall obain KAPL's approval prior to fabrication. 
- The seller shall also use new coolant for this work scope.

- All specimens shall be jdentified by a bag and tag method with plate ID, specimen type. specimen ID and drawing number.

- There shall be no markings inscribed onto the surface of the part. Disregard note on drawing.

- The seller shall optimize GFM material usage and relurn all excess GFM greater than $0.5^{\prime \prime} \times 0.5^{\prime \prime}$.

- There shall be a verbal bricting prior to the start of machining.

4.

\section{FIRST PIECE INSPECTION}

Two specimens of each type shall be provided for a first piece inspection to assure that they meet all of the requirtments of KAPL Drawings prior to proceeding to the next piece. The seller slatl notify KAPL with results of the first piece inspection prior to completing the fabrication on the balance of the order. KAPL requests the seller to provide to KAPL the specimens used for lirst piece inspection prior to completion of the balance of the order.

TABI.E।

Machining Instruction Shcet $05 P \mathrm{PCO} 04$

\begin{tabular}{|c|c|c|c|c|c|}
\hline PIATEID & & SPECINEN TYPE & $\begin{array}{l}\text { SPECIMEN } \\
\text { ID's }\end{array}$ & QUANTITY & $\begin{array}{l}\text { DRAWNG } \\
\text { NO. }\end{array}$ \\
\hline $\begin{array}{l}\text { KN6002216-7 } \\
\text { Plate \# ! }\end{array}$ & $\begin{array}{l}\text { Morton } \\
\text { CVD } \\
\text { Plates }\end{array}$ & $\begin{array}{l}\text { Item } A \\
\text { SiC Temperature } \\
\text { Monitor (Length } \\
=1.299^{\prime \prime} \pm 0.030 \text { ) }\end{array}$ & $1-10$ & 10 & $\begin{array}{l}\text { Modified } \\
\text { Dwg. } \\
\text { SD } 14442 \\
\text { Rev.- }\end{array}$ \\
\hline $\begin{array}{l}\text { KN6002216-7 } \\
\text { Plate \#1 }\end{array}$ & $\begin{array}{l}\text { Mortol] } \\
\text { CVD } \\
\text { Plates }\end{array}$ & $\begin{array}{l}\text { Itcm B } \\
\text { SiC. Temperaure } \\
\text { Monitor (Length } \\
=1.102 "=0.030 \text { ) }\end{array}$ & 1.154 & 154 & $\begin{array}{l}\text { Modified } \\
\text { Dwg. } \\
\text { SD14442 } \\
\text { Rev.- }\end{array}$ \\
\hline $\begin{array}{l}\text { KN6002216-7 } \\
\text { Plate \# }\end{array}$ & $\begin{array}{l}\text { Morton } \\
\text { CVD } \\
\text { Plates }\end{array}$ & $\begin{array}{l}\text { Item C } \\
\text { SiC Temperature } \\
\text { Monitor }(\text { l.ength } \\
\left.=0.551^{*}=0.030\right)\end{array}$ & $1-108$ & 108 & $\begin{array}{l}\text { Modified } \\
\text { Dwg. } \\
\text { SD } 4442 \\
\text { Rev..- }\end{array}$ \\
\hline $\begin{array}{l}\text { KN6002216-7 } \\
\text { Plate \#1 }\end{array}$ & $\begin{array}{l}\text { Morton } \\
\text { CVD } \\
\text { Plates }\end{array}$ & $\begin{array}{l}\text { Item D } \\
\text { SiC Temperature } \\
\text { Monitor (Lengh } \\
=0.276^{\prime \prime}=0.030 \text { ) }\end{array}$ & $1-10$ & 10 & $\begin{array}{l}\text { Modified } \\
\text { Dwg. } \\
\text { 5D } 4442 \\
\text { Rev.- }\end{array}$ \\
\hline
\end{tabular}


- Complece As-Buill data for all specimens.

- Certs. of Compliance with KAPL Specification on detrimental materials (Altachment l dated 3/6/03).

- Oricntation layout.

- First piece inspection notification.

Prepared $\mathrm{B}_{3}$

Revietved By 


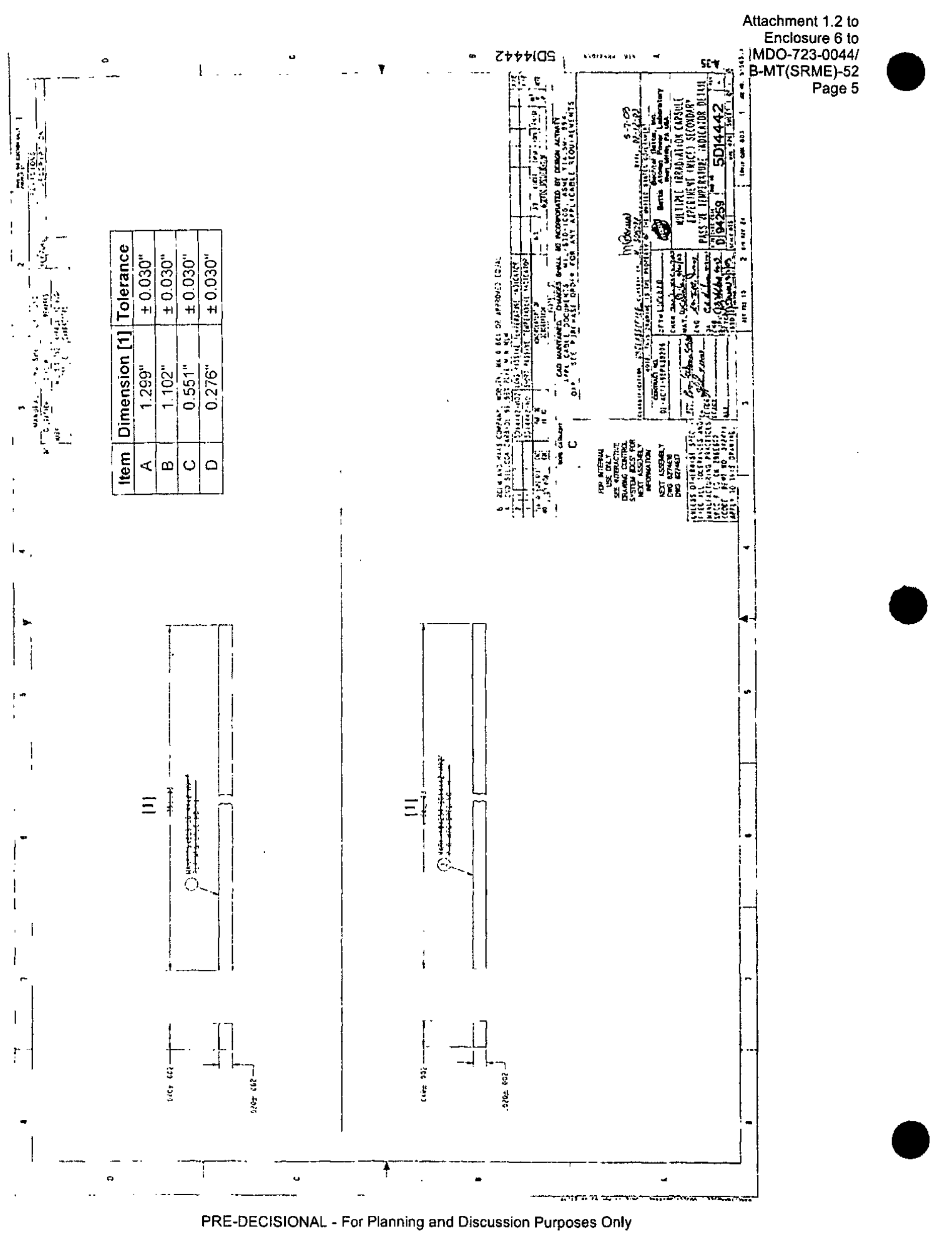




\section{Attachment 1 dated $3 / 6 / 03$ \\ Detrimental Materials \\ Prohibitions and \\ Restrictions for Silicon \\ Carbide Composite \\ Materials}

Attachment 1.2 to

Enclosure 6 to

MDO-723-0044I

B-MT(SRME)-52

Page 6

Certain chemical elements in contact with a test specimen may cause premature failure of the specimen under test. Those chemical elements known as detrimental materials must not be allowed to contaminate the specimens at any time before or during the test. Therefore, the specimens must be guarded against contamination from detrimental materials during such activities as manufacturing, packaging, shipping, inspection, or testing.

1.0 During the machining, handling, storage, or shipping of specimens, the specimens shall not come in direct contact with:

1.1 Mercury, compounds containing mercury in excess of $10 \mathrm{ppm}$, or any mercury-containing device employing a single boundary of containment.

1.2 Lead or compounds containing lead in excess of $250 \mathrm{ppm}$.

1.3 Cadmium, particularly cadmium plated tools or hardware, or alloys containing more than one percent cadmium, or compounds containing more than $250 \mathrm{ppm}$ cadmium.

1.4 Compounds containing halogens in excess of $250 \mathrm{ppm}$ (total where fluorine is restricted to $25 \mathrm{ppm}$ ) except lubricants used in machining.

1.5 Salt, or saline solutions, or any solid surface that may introduce alkali metals (e.g., sodium, potassium, lithium).

1.6 Compounds with molybdenum disulfide in excess of $250 \mathrm{ppm}$.

2.0 Compounds with compositions listed below shall not directly contact specimen surfaces after final machining.

2.1 Compounds containing more than $250 \mathrm{ppm}$ of any of the following antimony, bismuth, copper, phosphorus, sulfur, tin, zinc, total halogens (except that total fluorine shall be restricted to a maximum of $25 \mathrm{ppm}$ ).

2.2 Nuclear poisons (e.g., boron) in excess of $100 \mathrm{ppm}$.

2.3 Liquids which are not water-soluble except as allowed in

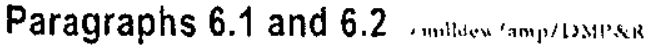


3.0 Thread sealants, such as teflon tape, teflon jam nuts, or epoxy resins shall not be used to affix or secure specimens to fixtures.

4.0 Aluminum, copper, nickel, and alloys of these materials containing more than 50 percent aluminum, copper, or nickel shall not be used as soft pads, hammers, or tools which contact the specimens during or subsequent to final machining.

5.0 Only dedicated resin-bonded diamond tooling shall be used for all machining of silicon carbide composite materials.

6.0 Solvent and Cleaning Solution Requirements:

6. $\uparrow$ Solutions having a pH of less than 6 at the temperature at which they are to be used shall not be used to clean parts within accessible areas.

\subsection{All halogenated cleaning solutions (250 ppm or more halogens, with} fluorine restricted to $25 \mathrm{ppm}$ ) are prohibited from use.

7.0 Temperature indicating crayons, marking materials (including white-out) lubricants and low melting alloys shall not contact the final machined surfaces of the specimens if they contain:

7.1 More than $250 \mathrm{ppm}$ of any of the following antimony, arsenic, bismuth, copper, phosphorus, sulfur, tin, zinc, and total halogens (except that fluorine shall be restricted to a maximum of $25 \mathrm{ppm}$ ).

7.2 Metals or alloys which melt at 1000 degrees $F$ or less (e.g., Cerrolow, Kirksite, Cerrobend, and cadmium).

8.0 Test and machining fixtures and other components, which directly contact the final machined surfaces of the specimens shall not come in direct contact with items specified in Paragraph 1.0. If fixtures have previously contacted the items

specified in Paragraph 1.0, then rigorous cleaning (such as by acid pickling) is required prior to use on the specimens.

9.0 Bench tops and work surfaces upon which the final machined specimens will be placed shall be covered by KimPak creped wadding paper (Kimberly-Clark Co.) or a similar product prior to use.

10.0 Seller shall provide a statement of conformance with the requirements of this specification. 
Attachment 1.3

Machining Certificates of Compliance and Quality Assurance Enclosure 6 to MDO-723-0044/B-MT(SRME)-52 


\section{KAPL, Inc.}

PURCHASE ORDER

O. Box 7072

henectady, New York 12301-1072
PAGE 1 OF 2

PURCHASE ORDER NUMBER

KN6002789

Page 2

PURCHASE ORDER DATE AMENDMENT DATE

16-JUN-2005 $\quad 35$ PFGOGS

IMPORTANT: Show Purchase Order Number on all correspondence. Complete packing list must accompany each shipment.

Effective Date:

KAPL. INC. A LOCKHEEO MAHTIN COMHANY, OPERAIES IHE KNOLLS ATOMIC POWER LABOFATCAY UNDEF GOVERNMENT CONTAACT DE-AC.12-COSN39357.

S BOMAS MACHINE SPECIALTIES, INC.

334 WASHINGTON ST.

P SOMERVILLE, MA 02143

(L)

E

A Confirmed By:

8 KNOLLS RECEIVWG BLDG M2

CENTRAL RECEIVING - KNO

2401 RIVER ROAO

SCHENECTADY,NY 12309

o

บOM Unit Price

TEMPERATUAE MONITOAS IN

10.00

EACH

17.5

$\$ 175.00$

SPECIEICATC WITH

OSPFGO94, ITEM A.

INSTRUCTIONS TO

PURCHASE ORDEA

KN6002789 SHALL APPLY.

This PO line reterences cortract POH: 6002075

TEMPEAATURE MONITORS IN

ACCORDANCE WITH

154.00

EACH

17.5

$\$ 2,695.00$

SPECIFICATION FCR

.

INSTRUCTIONS TO
PURCHASE ORDER

KN6002789 SHALL APPLY.

This PO line referchces contract ${ }^{\circ} \mathrm{OA:} 6002075$

OSPFGO94. ITEM C.

INSTRUCTIONS TO

PURCHASE ORDER

KN6002789 SHALL APPLY.

This PO line references contract POH: 6002075

TEMPERATURE MONITGAS IN

ACCORDANCE WHTH

SPECIFICATION FOR

O5PFGO94, ITEM D.

INSTAUCTIONS TO

PURCHASE ORDER

KN6002789 SHALL A PPLY

This $P O$ line referances contuac $P O *: 6002075$ 


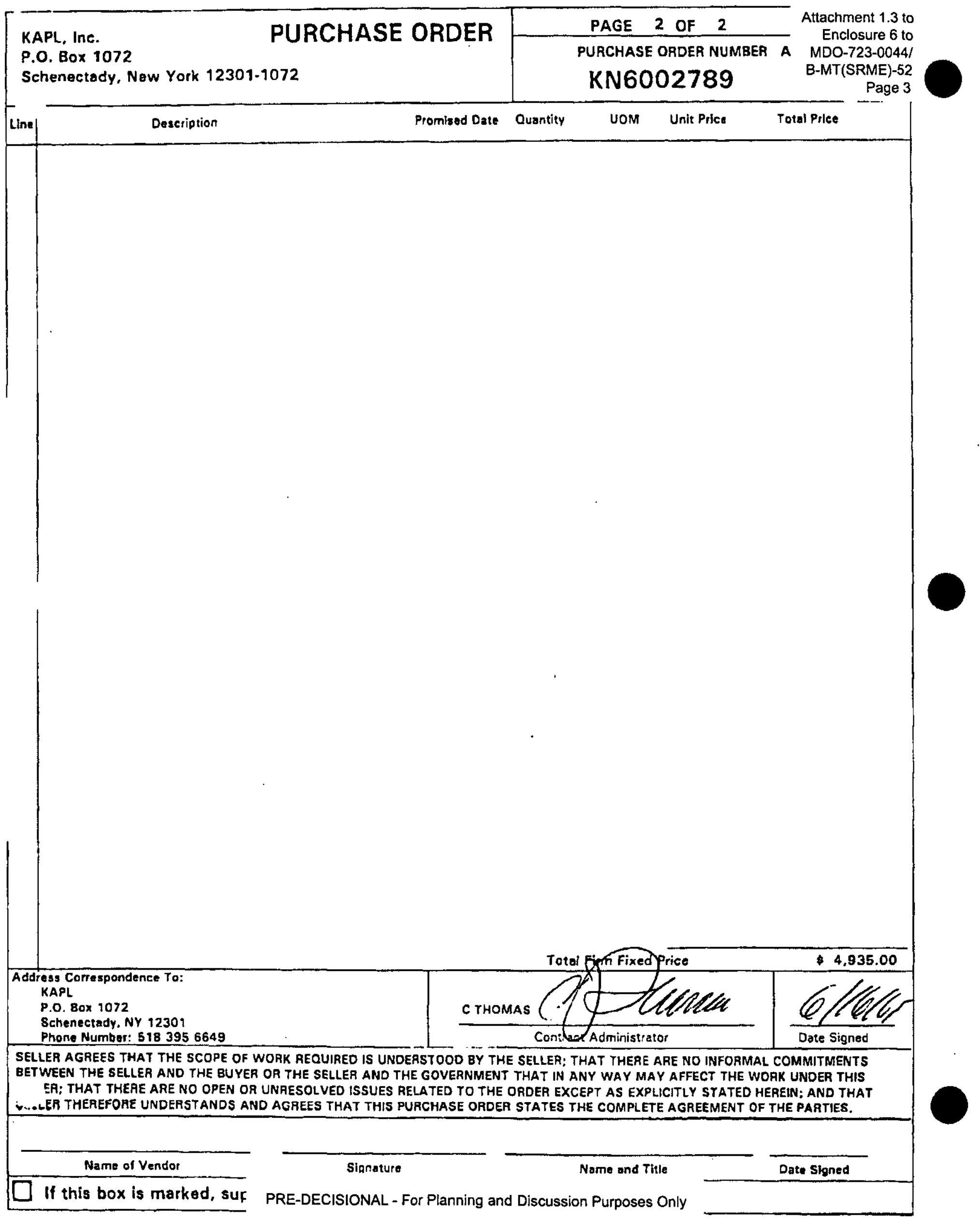




\begin{tabular}{|c|c|c|c|}
\hline $\begin{array}{l}\text { Delivery } \\
\text { Order }\end{array}$ & $\begin{array}{l}\text { Document No. } \\
\text { K. } 6002789\end{array}$ & $\begin{array}{l}\text { Document Title } \\
\text { 05PrG094 }\end{array}$ & Page 1 of I \\
\hline & $\begin{array}{l}\text { Buyer Name } \\
\text { CJ THOMAS }\end{array}$ & & \\
\hline
\end{tabular}

INSTRLCTIONS TO PURCHASE ORDER K. 6002789

$\star * * * * * * * * * * * * *$

\section{PI.EASENOTE}

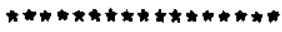

\section{PLEASE FORWALD ACKNOWLEDGEMENT COPY AND AVY OTHER CORRESPONDENCE TO: C.J THOMAS, MAIL STOP 148}

\section{REFEKENCE NLMBER: 05PFG094}

Seller is herehy authorized 10 perform the work set forth in Paragraph A. in accordance with the reyuirtments or Paragraphs $B$, and $C$. of the above referenced number.

\section{DELIVERY REOUIRFMEYTS}

Time is of the essence - Buyer requires and it is essential that delivery be made, as set forth below:

A. Half of ltens I through 4 - On or before twenty (20) work days after delivery of nrder and receipt of Government Furnished Material.

B. Balance of Items 1 thrnugh 4 - On or before forty (40) work days after delivery of order and receipt of Government Fumished Material.

\section{APRL,ICAIBIE DOCLMFNTS}

The following documents with all doeuments referenced therein form a part of this nrder:

A. Specification For 0SPFG094, Rev. -, dated 6/1/05.

\section{GOVERVMENT FIJRNISHED MATERIAL}

A. One (1) Each - Mornon CVD Plate

Buyer estimates the Government Furnished Material will be shipped on or before one (I) week after date of order. 
KNOLLS ATOMIC POWER LABORATORY

KAPL Specification \# 05PFG094
Attachment 1.3 to

Enclosure 6 to MDO-723-0044

B-MT(SRME)-52 Page 5

DIMENSIONAL REPORT IN INCHES

Item A

$\# 1$

$\begin{array}{lll}0.02 & 0.0404 & 1.299\end{array}$

\#2

0.02

0.0404

1.299

Item $\mathbf{B}$

\#3

0.02

0.0404

1.102

\#4

0.02

0.0404

1.102

Item C

\#5

\#8

0.02

0.0404

0.02

0.0404

0,551

0.551

Item D

$\# 7$

\#

0.02

0.0404

0.2765

0.02

0.0404

0.2765

PO\# KN6002789

Dwg. No. 5014442

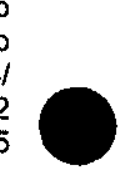




\section{BOMAS MACHINE SPECIALTIES, INC.}

: $\quad 334$ Washington Street

Somerville, Massachusetts 02143

(617)628-3831 - FAX (617)628-6108

e-mail-jannese@bomas.com

\section{CERTIFICATE OF COMPLIANCE}

Date : $\quad 07-12-05$

Dwg. No: 5D14442

Company : Knolls Atomic Power Lab 2401 River Road

Schenectady , NY 12309

Purchase Order \# : $\quad$ KN6002789

Job No : 050783

Quantity : (8) TOTAL, 2 of Item A

2 of Item $B$

Invoice \# : 12530

2 of Iten $C$

Specification 05PFG094

Bomas certifies that all materials and/or machining procedures used in the manufacturing under this purchase order is in strict compliance with all company specifications.

FIRST PIECE INSPECTION

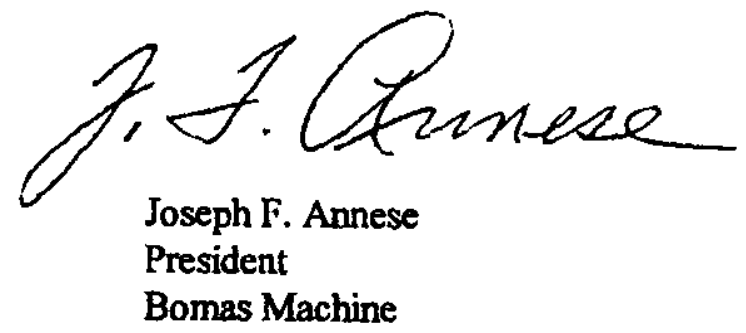




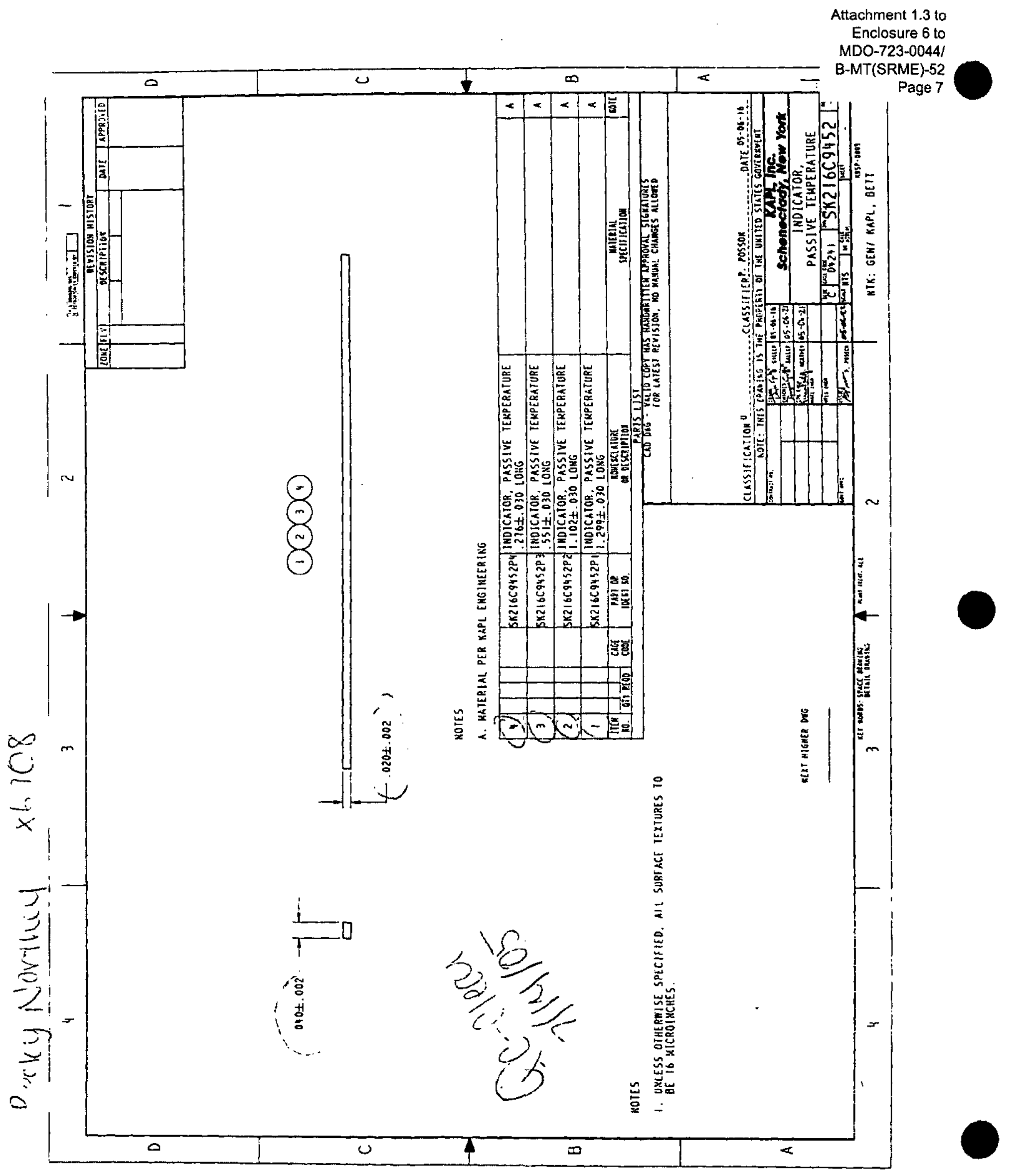


Bomas Machine Specialties, Inc.

\section{Washington Street}

merville, Massacfusetts 02143

Phone 617-628-3831 / fax 617-628-6108

Email jannese@6omas.com

Sold to: $\quad$ Knolls Atomic Power Lab

Accounts Payable ( PO\# KN6002789)

P. O. Box 1072

Schenectady, NY 12301-1072
Invoice No. 12

Attachment 1.3 to

Enclosure 6 to MDO-723-0044/ B-MT(SRME)-52

Date $07-19-05$
Ship to: Knolls Atomic Power Lab Central Receiving - KNO 2401 River Road

Schenectady, NY 12309

Attn: Becky Northey

PO\# KN6002789

KN6002789

ups/red

Qrder No. Via Terms Net 30

Item No. Quantity Description Unit Cost Amount

Specification 05PFG094

8

152

106

8

dwg. no. 5D14442, ltem A

dwg. no. 5D14442, Item B 154

3

4

ORDER COMPLETE 
BOMAS MACHINE SPECIALTIES, INC.

Attachment 1.3 to

Enclosure 6 to MDO-723-0044/

B-MT(SRME)-52

334 Washington Street

Somerville, Massachusetts 02143

(617)628-383I - FAX (617)628-6108

e-mail-jannese@bomas.com

\section{CERTIFICATE OF COMPLIANCE}

Date : 07-19-05

Company : Knolis Atomic Power Lab 2401 River Road

Schenectady , NY 12309

Purchase Order \# : $\quad$ KN6002789

Specification 05PFG094
Dwg. No : 5D14442

Job No : 050783

Quantity : (274), (8) of Item A (152) of Item B (106) of Item $C$

Invoice \# : 12537

Bomas certifies that all materials and/or machining procedures used in the manufacturing under this purchase order is in strict compliance with all company specifications.

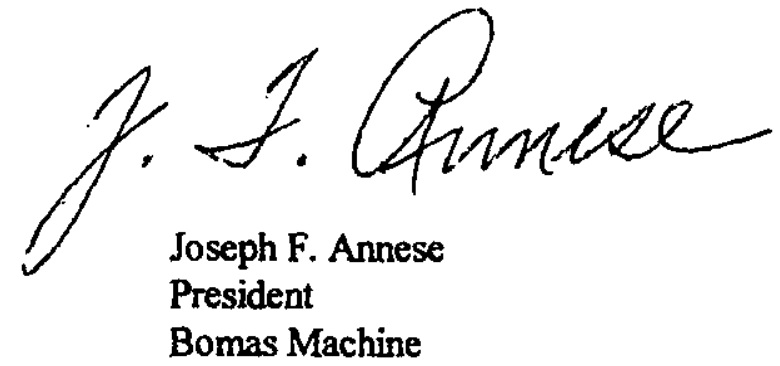


KNOLLS ATOMIC POWER LABORATORY

PO\# KN6002789

KAPL Specification \# 06PFG094
Dwg. No. 5D14442

\section{DIMENSIONAL REPORT IN INCHES}

\section{Item A}

$\begin{array}{llll}\# 3 & 0.021^{\prime \prime} & 0.040^{\prime \prime} & 1.299^{\prime \prime} \\ \# 4 & 0.021^{\prime \prime} & 0.040^{*} & 1.299^{\prime \prime} \\ \# 5 & 0.021^{\prime \prime} & 0.040^{\prime \prime} & 1.299^{\prime \prime} \\ \# 6 & 0.021^{\prime \prime} & 0.040^{\prime \prime} & 1.299^{\prime \prime} \\ \# 7 & 0.021^{\prime \prime} & 0.040^{*} & 1.299^{\prime \prime} \\ \# 8 & 0.021^{\prime \prime} & 0.040^{\prime \prime} & 1.299^{\prime \prime} \\ \# 9 & 0.021^{\prime \prime} & 0.040^{*} & 1.299^{\prime \prime} \\ \# 10 & 0.021^{\prime \prime} & 0.040^{\prime \prime} & 1.299^{\prime \prime}\end{array}$

Note: 1 \& 2 were sent to KAPL on 07-12-05 as "FIRST PIECE INSPECTION" specimens

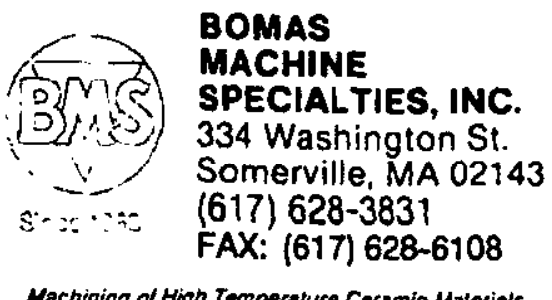


KNOLLS ATOMIC POWER LABORATORY

KAPL Specification \# 06PFG094
Attachment 1.3 to

Enclosure 6 to MDO-723-0044/ B-MT(SRME)-52 Page 11

\section{DIMENSIONAL REPORT IN IMCHES}

\begin{tabular}{|c|c|c|c|c|c|c|c|}
\hline$\# 3$ & $0.021^{\prime \prime}$ & $0.040^{\circ}$ & $1.102^{n}$ & $\$ 43$ & $0.021^{4}$ & $0.040^{n}$ & $1.102^{n}$ \\
\hline$* 4$ & $0.021^{\prime \prime}$ & $0.040^{n}$ & $1.102^{\prime \prime}$ & $\# 44$ & $0.021 "$ & $0.040^{\prime \prime}$ & $1.102^{\prime \prime}$ \\
\hline$* 5$ & $0.021^{\prime \prime}$ & $0.040^{\prime \prime}$ & $1.102^{\pi}$ & $\# 45$ & $0.021^{*}$ & $0.040^{\prime \prime}$ & $1.102^{n}$ \\
\hline 10 & $0.021^{\prime \prime}$ & $0.040^{n}$ & $1.102^{\prime \prime}$ & $\$ 46$ & $0.021^{\prime \prime}$ & $0.040^{\circ}$ & $1.102^{n}$ \\
\hline$\$ 7$ & $0.021^{\prime \prime}$ & $0.040^{\circ}$ & $1.102^{\prime \prime}$ & $\$ 47$ & $0.021^{\prime \prime}$ & $0.040^{*}$ & $1.102^{n}$ \\
\hline$\# 8$ & $0.021^{\prime \prime}$ & $0.040^{\circ}$ & $1.102^{\prime \prime}$ & $\# 48$ & $0.021^{\prime \prime}$ & $0.040^{n}$ & $1.102^{n}$ \\
\hline \#9 & $0.021^{*}$ & $0.040^{n}$ & $1.102^{n}$ & $\$ 49$ & $0.021^{\prime \prime}$ & $0.040^{\circ}$ & $1.102^{\prime \prime}$ \\
\hline$\$ 10$ & $0.021^{\prime \prime}$ & $0.040^{\prime \prime}$ & $1.102^{n}$ & $\$ 50$ & $0.021 "$ & $0.040^{n}$ & $1.102^{n \prime}$ \\
\hline$\# 11$ & $0.021^{*}$ & $0.040^{\prime \prime}$ & $1.102^{\circ}$ & $\# 51$ & $0.021^{\prime \prime}$ & $0.040^{\circ}$ & $1.102^{n}$ \\
\hline \#12 & $0.021^{\prime \prime}$ & $0.040^{\prime \prime}$ & $1.102^{n}$ & 152 & $0.021^{n}$ & $0.040^{n}$ & 1.102 \\
\hline$\# 13$ & $0.021^{\prime \prime}$ & $0.040^{\prime \prime}$ & $1.102^{n}$ & 453 & $0.021^{\circ}$ & $0.040^{n}$ & $1.102^{\circ}$ \\
\hline$\# 14$ & $0.021^{\prime \prime}$ & $0.040^{\prime \prime}$ & $1.102^{\prime \prime}$ & \#54 & $0.021^{\prime \prime}$ & $0.040^{n}$ & $1.102^{\prime \prime}$ \\
\hline$\# 15$ & $0.021^{\circ}$ & $0.040^{\prime \prime}$ & $1.102^{n}$ & · $\$ 55$ & $0.021^{\prime \prime}$ & $0.040^{\mathrm{n}}$ & $1.102^{\circ}$ \\
\hline$\# 16$ & $0.021^{\circ}$ & $0.040^{\circ}$ & $1.102^{n}$ & $\$ 56$ & $0.021^{\prime \prime}$ & $0.040^{n}$ & $1.102^{n}$ \\
\hline$\# 17$ & $0.021 "$ & $0.040^{*}$ & $1.102^{\prime \prime}$ & $\div 67$ & $0.021^{\prime \prime}$ & $0.040^{n}$ & $1.102^{\prime \prime}$ \\
\hline$\# 18$ & $0.021^{\prime \prime}$ & $0.040^{\circ}$ & $1.102^{\circ}$ & \#58 & $0.021^{\prime \prime}$ & $0.040^{\prime \prime}$ & $1.102^{n}$ \\
\hline$\#+9$ & $0.021^{\prime \prime}$ & $0.040^{4}$ & $1.102^{\prime \prime}$ & $\$ 59$ & $0.021^{\prime \prime}$ & $0.040^{n}$ & $1.102^{\mathrm{n}}$ \\
\hline$\$ 20$ & $0.021^{\prime \prime}$ & $0.040^{\prime \prime}$ & $1.102^{\mathrm{g}}$ & $\# 60$ & $0.021^{n}$ & $0.040^{\prime \prime}$ & $1.102^{n}$ \\
\hline$\# 21$ & $0.021^{\prime \prime}$ & $0.040^{\circ}$ & $1.102^{\prime \prime}$ & $\# 61$ & $0.021^{\prime \prime}$ & $0.040^{4}$ & $1.102^{4}$ \\
\hline$\$ 22$ & $0.021^{\prime \prime}$ & $0.040^{\prime \prime}$ & $1.102^{\prime \prime}$ & $\$ 62$ & $0.021^{\prime \prime}$ & $0.040^{\prime \prime}$ & $1.102^{n}$ \\
\hline \#23 & $0.021^{\prime \prime}$ & $0.040^{\prime \prime}$ & $1.102^{m}$ & $\# 63$ & $0.021^{\prime \prime}$ & $0.040^{n}$ & $1.102^{\prime \prime}$ \\
\hline \#24 & $0.021^{\prime \prime}$ & $0.040^{n}$ & $1.102^{\circ}$ & $\$ 64$ & $0.021 "$ & $0.040^{\circ}$ & 1.102 \\
\hline \#25 & $0.021^{\prime \prime}$ & $0.040^{\circ}$ & $1.102^{n}$ & \#65 & $0.021^{n}$ & $0.040^{\circ}$ & $1.102^{\prime \prime}$ \\
\hline \#26 & $0.021^{\circ \prime}$ & $0.040^{n}$ & $1.102^{n}$ & $\# 86$ & $0.021^{n}$ & $0.040^{\prime \prime}$ & 1.102 \\
\hline+27 & $0.021^{\prime \prime}$ & $0.040^{\circ}$ & $1.102^{n}$ & 柏 67 & $0.021^{n}$ & $0.040^{\prime \prime}$ & 1.102 \\
\hline \#28 & $0.021^{\prime \prime}$ & $0.040^{\circ}$ & $1.102^{\prime \prime}$ & \#88 & $0.021^{n}$ & $0.040^{\prime \prime}$ & 1.102 \\
\hline \#29 & $0.021^{\prime \prime}$ & $0.040^{\circ}$ & $1.102^{\prime \prime}$ & "\#99 & $0.021^{\prime \prime}$ & $0.040^{4}$ & $1.102^{\prime \prime}$ \\
\hline$\# 30$ & $0.021^{\prime \prime}$ & $0.040^{\circ}$ & $1.102^{\circ}$ & $\$ 70$ & $0.021^{\prime \prime}$ & $0.040^{\prime \prime}$ & $1.102^{\prime \prime}$ \\
\hline \#31 & $0.021^{\prime \prime}$ & $0.040^{\circ}$ & $1.102^{\prime \prime}$ & $\# 71$ & $0.021^{n}$ & $0.040^{*}$ & $1.102^{\prime \prime}$ \\
\hline$\# 32$ & $0.021 "$ & $0.040^{\prime \prime}$ & $1.102^{\prime \prime}$ & $\# 72$ & $0.021 "$ & $0.040^{\circ}$ & $1.102^{m}$ \\
\hline \#33 & $0.021^{\prime \prime}$ & $0.040^{n}$ & $1.102^{*}$ & $\$ 73$ & $0.021^{\prime \prime}$ & $0.040^{*}$ & $1.102^{n}$ \\
\hline \#34 & $0.021^{\prime \prime}$ & $0.040^{\circ}$ & $1.102^{\prime \prime}$ & $\$ 74$ & $0.021^{\prime \prime}$ & $0.040^{\prime \prime}$ & $1.102^{n}$ \\
\hline \#35 & $0.021^{\prime \prime}$ & $0.040^{\prime \prime}$ & $1.102^{\circ}$ & $\$ 75$ & $0.021^{\prime \prime}$ & $0.040^{\prime \prime}$ & $1.102^{\prime \prime}$ \\
\hline \#36 & $0.021^{\prime \prime}$ & $0.040^{*}$ & $1.102^{n}$ & $\$ 78$ & $0.021^{\prime \prime}$ & $0.040^{n}$ & $1.102^{p}$ \\
\hline \#37 & $0.021^{\prime \prime}$ & $0.040^{\prime \prime}$ & $1.102^{4}$ & $\$ 77$ & $0.021^{\prime \prime}$ & $0.040^{\prime \prime}$ & $1.102^{n}$ \\
\hline$\$ 38$ & $0.021^{\circ}$ & $0.040^{n}$ & $1.102^{\prime \prime}$ & $\$ 78$ & $0.021^{\prime \prime}$ & $0.040^{\circ}$ & $1.102^{\prime \prime}$ \\
\hline$\$ 39$ & $0.021^{\prime \prime}$ & $0.040^{\prime \prime}$ & $1.102^{\prime \prime}$ & $\$ 79$ & $0.021^{\prime \prime}$ & $0.040^{n}$ & $1.102^{\prime \prime}$ \\
\hline$\$ 40$ & $0.021^{\prime \prime}$ & $0.040^{\prime \prime}$ & $1.102^{\prime \prime}$ & 480 & $0.021^{\prime \prime}$ & $0.040^{\circ}$ & $1.102^{\prime \prime}$ \\
\hline \#41 & $0.021^{\prime \prime}$ & $0.040^{*}$ & $1.102^{\prime \prime}$ & $\$ 81$ & $0.021^{\prime \prime}$ & $0.040^{\prime \prime}$ & $1.102^{n}$ \\
\hline$\$ 42$ & $0.021^{\prime \prime}$ & $0.040^{n}$ & $1.102^{\prime \prime}$ & $\$ 82$ & $0.021 "$ & $0.040^{\prime \prime}$ & $1.102^{\prime \prime}$ \\
\hline
\end{tabular}

BOMAS

MACHINE

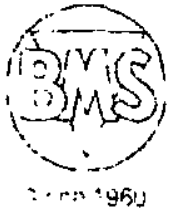

SPECIALTIES, INC.

334 Washington St.

Somerville, MA 02143

(617) 628-3831

Dwg. No. 6D14442

\section{(}


KAPL

PO\# KN6002789

KAPL Specification \# 05PFG094
Attachment 1.3 to

Enclosure 6 to MDO-723-0044/

B-MT(SRME)-52

Page 12

DWENSIONAL REPORT W WCHES

\begin{tabular}{|c|c|c|c|}
\hline$\# 83$ & $0.021^{\prime \prime}$ & $0.040^{\prime \prime}$ & $1.102^{n}$ \\
\hline$\# 84$ & $0.021^{\prime \prime}$ & $0.040^{\prime \prime}$ & $1.102^{n}$ \\
\hline$\# 65$ & $0.021^{\prime \prime}$ & $0.040^{\circ}$ & 1.102 \\
\hline$\# 88$ & $0.021^{\prime \prime}$ & $0.040^{\circ}$ & 1.10 \\
\hline$\# 87$ & $0.021^{\prime \prime}$ & $0.040^{\prime \prime}$ & .102 \\
\hline$\# 88$ & $0.021^{\prime \prime}$ & $0.040^{\prime \prime}$ & 1.102 \\
\hline$\$ 89$ & $0.021^{n}$ & $0.040^{n}$ & 1.102 \\
\hline$\# 90$ & $0.021^{\prime \prime}$ & $0.040^{\prime \prime}$ & $1.102^{\prime \prime}$ \\
\hline$\# 91$ & $0.021^{\prime \prime}$ & $0.040^{n}$ & $1.102^{n}$ \\
\hline \#92 & $0.021^{\prime \prime}$ & $0.040^{\prime \prime}$ & 1.102 \\
\hline$\# 93$ & $0.021^{n}$ & $0.040^{n}$ & 1.102 \\
\hline \#94 & $0.021^{\prime \prime}$ & $0.040^{\circ}$ & $1.102^{n}$ \\
\hline$\# 95$ & $0.021^{*}$ & $0.040^{\circ}$ & $1.102^{n}$ \\
\hline$\# 96$ & $0.021^{\prime \prime}$ & $0.040^{\circ}$ & 1.102 \\
\hline$\# 97$ & $0.021^{\prime \prime}$ & $0.040^{n}$ & 1.102 \\
\hline \#98 & $0.021^{*}$ & $0.040^{\prime \prime}$ & 1.102 \\
\hline$\# 99$ & $0.021^{\prime \prime}$ & $0.040^{n}$ & $1.102^{n}$ \\
\hline$\# 100$ & $0.021^{\prime \prime}$ & $0.040^{n}$ & $1.102^{m}$ \\
\hline$\# 101$ & $0.021^{\prime \prime}$ & $0.040^{\mathrm{N}}$ & 1.102 \\
\hline$\# 102$ & $0.021^{\prime \prime}$ & $0.040^{n}$ & $1.102^{\circ}$ \\
\hline$\# 103$ & $0.021^{\prime \prime}$ & $0.040^{n}$ & $1.102^{n}$ \\
\hline$\# 104$ & $0.021^{*}$ & $0.040^{n}$ & $1.102^{n}$ \\
\hline$\# 105$ & $0.021^{\prime \prime}$ & $0.040^{\prime \prime}$ & $1.102^{n}$ \\
\hline$\because 106$ & $0.021^{\prime \prime}$ & $0.040^{\circ}$ & 1.102 \\
\hline$\# 107$ & $0.021^{\prime \prime}$ & $0.040^{n}$ & 1.102 \\
\hline$\# 108$ & $0.021^{\prime \prime}$ & $0.040^{\prime \prime}$ & 1.10 \\
\hline$\# 100$ & $0.021^{\prime \prime}$ & $0.040^{n}$ & $1.102^{n}$ \\
\hline$\# 110$ & $0.021^{\prime \prime}$ & $0.040^{\circ}$ & 1.10 \\
\hline$\# 111$ & $0.021^{\prime \prime}$ & $0.040^{n}$ & $1.102^{n}$ \\
\hline$\# 112$ & $0.021^{\prime \prime}$ & $0.040^{n}$ & $1.102^{n}$ \\
\hline$\# 1+3$ & $0.021^{\prime \prime}$ & $0.040^{n}$ & $1.102^{\circ}$ \\
\hline$\# 114$ & $0.021^{n}$ & $0.040^{\circ}$ & $1.102^{\prime \prime}$ \\
\hline$\# 115$ & $0.0211^{\prime \prime}$ & $0.040^{\mathrm{n}}$ & $1.102^{n}$ \\
\hline$\# 116$ & $0.021^{\prime \prime}$ & $0.040^{n}$ & $1.102^{\prime \prime}$ \\
\hline$\# 117$ & $0.021^{*}$ & $0.040^{\prime \prime}$ & $1.102^{-1}$ \\
\hline$\# 118$ & $0.021^{\prime \prime}$ & $0.040^{n}$ & $1.102^{\circ}$ \\
\hline \#119 & $0.021^{\prime \prime}$ & $0.040^{\circ}$ & $1.102^{\prime \prime}$ \\
\hline$\# 120$ & $0.021^{\prime \prime}$ & $0.040^{\prime \prime}$ & $1.102^{\circ}$ \\
\hline$\# 121$ & $0.021^{\prime \prime}$ & $0.040^{*}$ & $1.102^{m}$ \\
\hline "122 & $0.021^{*}$ & $0.040^{\circ}$ & $1.102^{*}$ \\
\hline \#123 & $0.021^{*}$ & $0.040^{\circ}$ & $1.102^{4}$ \\
\hline$\$ 124$ & $0.021^{\prime \prime}$ & $0.040^{\circ}$ & $1.102^{\prime \prime}$ \\
\hline$\$ 125$ & $0.021^{\prime \prime}$ & $0.040^{4}$ & $1.102^{\circ}$ \\
\hline
\end{tabular}

\begin{tabular}{|c|c|c|c|}
\hline$\# 128$ & $0.021^{\prime \prime}$ & $0.040^{\mathrm{N}}$ & $1.102^{n}$ \\
\hline \$127 & $0.021^{n t}$ & $0.040^{*}$ & $1.102^{n}$ \\
\hline$\# 128$ & $0.021^{\prime \prime}$ & $0.040^{n}$ & $1.102^{\prime \prime}$ \\
\hline$\# 129$ & $0.021^{\circ}$ & $0.040^{n}$ & $1.102^{n}$ \\
\hline$\# 130$ & $0.021^{\prime \prime}$ & $0.040^{\circ}$ & $1.102^{\prime \prime}$ \\
\hline$\# 131$ & $0.021^{\prime \prime}$ & $0.040^{\circ}$ & $1.102^{n}$ \\
\hline$\# 132$ & $0.021^{\prime \prime}$ & $0.040^{n}$ & $1.102^{\prime \prime}$ \\
\hline *133 & $0.021^{\prime \prime}$ & $0.040^{\prime \prime}$ & $1.102^{n}$ \\
\hline$\# 134$ & $0.021^{\prime \prime}$ & $0.040^{\prime \prime}$ & $1.102^{n}$ \\
\hline \#135 & $0.021^{\prime \prime}$ & $0.040^{n}$ & $1.102^{n}$ \\
\hline$\because 136$ & $0.021^{\prime \prime}$ & $0.040^{\circ}$ & $1.102^{n}$ \\
\hline$\# 137$ & $0.021 "$ & $0.040^{\mathrm{n}}$ & $1.102^{a t}$ \\
\hline$\# 138$ & $0.021^{\prime \prime}$ & $0.040^{\circ}$ & $1.102^{n}$ \\
\hline$\# 139$ & $0.021^{\prime \prime}$ & $0.040^{\prime \prime}$ & $1.102^{\prime \prime}$ \\
\hline 140 & $0.021^{\prime \prime}$ & $0,040^{\circ}$ & $1.102^{\prime \prime}$ \\
\hline$\# 141$ & $0.021^{n}$ & $0.040^{\circ}$ & $1.102^{\prime \prime}$ \\
\hline$\# 142$ & $0.021^{\prime \prime}$ & $0.040^{\circ}$ & $1.102^{n}$ \\
\hline$\# 143$ & $0.021^{\prime \prime}$ & $0.040^{\circ}$ & $1.102^{*}$ \\
\hline "144 & $0.021^{\prime \prime}$ & $0.040^{\circ}$ & $1.102^{n}$ \\
\hline$\# 45$ & $0.021^{\prime \prime}$ & $0.040^{\prime \prime}$ & $1.102^{n}$ \\
\hline$\# 148$ & $0.021^{\prime \prime}$ & $0.040^{\prime \prime}$ & $1.102^{n}$ \\
\hline$\$ 147$ & $0.021^{\prime \prime}$ & $0.040^{\mathrm{n}}$ & $1.102^{n}$ \\
\hline$\# 148$ & $0.021^{\prime \prime}$ & $0.040^{\circ}$ & $1.102^{\circ}$ \\
\hline$\# 149$ & $0.021^{7}$ & $0.040^{\prime \prime}$ & $1.102^{\prime \prime}$ \\
\hline$\# 150$ & $0.021^{n}$ & $0.040^{\prime \prime}$ & $1.102^{n}$ \\
\hline \#151 & $0.021^{\prime \prime}$ & $0.040^{*}$ & $1.102^{n}$ \\
\hline$\# 152$ & $0.021^{n}$ & $0.040^{\prime \prime}$ & $1.102^{n}$ \\
\hline \#153 & $0.021^{\prime \prime}$ & $0.040^{n}$ & $1.102^{n}$ \\
\hline$\# 154$ & $0.021^{\prime \prime}$ & $0.040^{n}$ & $1.102^{\circ}$ \\
\hline Note: & \multicolumn{3}{|c|}{$\begin{array}{l}\text { \#s } 1 \text { \& } 2 \text { were sent to KAPL on } \\
\text { O7-12-05 as "FIRST PIECE } \\
\text { INSPECTION" specimens }\end{array}$} \\
\hline
\end{tabular}

BOMAS

MACHINE

SPECIALTIES, INC.

334 Washington St.

Somerville, MA 02143

(617) 628-3831

FAX: (617) 628-6108

Machining of High Tomperature Ceramic Malorials 
KNOLLS ATOMIC POWER LABORATORY

KAPL Specification \# 05PFG094
Attachment 1.3 to

Enclosure 6 to MDO-723-0044/

PO\# KN6002789

B-MT(SRME)-52

Page 13

Dwg. No. 5D14442

DIMENSIOMAL REPORT IN WCHES

\begin{tabular}{|c|c|c|c|c|c|c|c|}
\hline$\#$ & $0.021^{\prime \prime}$ & $0.040^{n}$ & $0.551^{*}$ & $\$ 43$ & $0.021^{\prime \prime}$ & $0.040^{n}$ & $0.551^{*}$ \\
\hline$\# 4$ & $0.021^{\prime \prime}$ & $0.040^{n}$ & $0.551^{\prime \prime}$ & $\$ 44$ & $0.021^{n}$ & $0.040^{*}$ & $0.554^{\prime \prime}$ \\
\hline$\# 5$ & $0.021^{\prime \prime}$ & $0.040^{\circ}$ & $0.551^{\prime \prime}$ & $\$ 45$ & $0.021^{\prime \prime}$ & $0.040^{\prime \prime}$ & $0.551^{\prime \prime}$ \\
\hline$\# 6$ & $0.021^{\prime \prime}$ & $0.040^{n}$ & $0.551^{\prime \prime}$ & $\$ 46$ & $0.021^{\prime \prime}$ & $0.040^{n}$ & $0.551^{\prime \prime}$ \\
\hline$\$ 7$ & $0.021^{\prime \prime}$ & $0.040^{\circ}$ & $0.551^{\prime \prime}$ & $\$ 47$ & $0.021^{\prime \prime}$ & $0.040^{* \prime}$ & $0.551^{*}$ \\
\hline$\# 8$ & $0.021^{\prime \prime}$ & $0.040^{n}$ & $0.551^{n}$ & $\# 48$ & $0.021 "$ & $0.040^{\prime \prime \prime}$ & $0.551^{\prime \prime}$ \\
\hline \#9 & $0.021^{\prime \prime}$ & $0.040^{\mathrm{m}}$ & $0.551^{* \prime}$ & $\$ 49$ & $0.021^{\prime \prime}$ & $0.040^{\prime \prime}$ & $0.551^{\prime \prime}$ \\
\hline$\$ 10$ & $0.021^{*}$ & $0.040^{n}$ & $0.559^{\prime \prime}$ & $\# 50$ & $0.021^{\circ}$ & $0.040^{n}$ & $0.551^{\prime \prime}$ \\
\hline$\$ 11$ & $0.021^{\prime \prime}$ & $0.040^{\mathrm{a}}$ & $0.551 "$ & $\$ 51$ & $0.021^{\prime \prime}$ & $0.040^{\prime \prime}$ & $0.551^{\prime \prime}$ \\
\hline$* 12$ & $0.02 t^{n}$ & $0.040^{n}$ & $0.551^{\prime \prime}$ & "152 & $0.021^{\prime \prime}$ & $0.040^{\prime \prime}$ & $0.551^{\prime \prime}$ \\
\hline$\# 13$ & $0.021^{\prime \prime}$ & $0.040^{n}$ & $0.551^{\prime \prime}$ & $\$ 53$ & $0.021^{\prime \prime}$ & $0.040^{\prime \prime}$ & $0.551^{\prime \prime}$ \\
\hline$\# 14$ & $0.021^{\prime \prime}$ & $0.040^{n}$ & $0.551^{\prime \prime}$ & 154 & $0.021^{\prime \prime}$ & $0.040^{\circ}$ & $0.551^{\prime \prime}$ \\
\hline$\# 15$ & $0.021^{n}$ & $0.040^{\prime \prime}$ & $0.55 t^{\prime \prime}$ & *55 & $0.021^{\prime \prime}$ & $0.040^{\prime \prime}$ & $0.551^{*}$ \\
\hline$\# 16$ & $0.021^{\prime \prime}$ & $0.040^{n}$ & $0.551^{\prime \prime}$ & $\$ 56$ & $0.021^{*}$ & $0.040^{\circ}$ & $0.551^{\prime \prime}$ \\
\hline$\# 17$ & $0.021^{\prime \prime}$ & $0.040^{\prime \prime}$ & $0.551^{\prime \prime}$ & $\$ 57$ & $0.021^{\prime \prime}$ & $0.040^{n}$ & $0.551^{*}$ \\
\hline 48 & $0.021^{\prime \prime}$ & $0.040^{n}$ & $0.551^{\prime \prime}$ & 458 & $0.021^{*}$ & $0.040^{\prime \prime}$ & $0.551^{\prime \prime}$ \\
\hline$\# 19$ & $0.021^{\prime \prime}$ & $0.040^{\prime \prime}$ & $0.551^{\prime \prime}$ & \#59 & $0.021^{\prime \prime}$ & $0.040^{\circ}$ & $0.55 t^{\prime \prime}$ \\
\hline$\# 20$ & $0.021^{\prime \prime}$ & $0.040^{n}$ & $0.551^{\prime \prime}$ & $\$ 60$ & $0.021^{\prime \prime}$ & $0.040^{*}$ & $0.551^{n}$ \\
\hline$\# 21$ & $0.021^{\prime \prime}$ & $0.040^{n}$ & $0.551^{n}$ & \#1 & $0.021^{4}$ & $0.040^{\prime \prime}$ & $0.551 "$ \\
\hline$\# 22$ & $0.021^{*}$ & $0.040^{m}$ & $0.551^{\prime \prime}$ & $\# 62$ & $0.021^{n}$ & $0.040^{\circ}$ & $0.551^{\prime \prime}$ \\
\hline$\# 23$ & $0.021^{\prime \prime}$ & $0.040^{\circ}$ & $0.551^{\prime \prime}$ & $\# 63$ & $0.021^{\prime \prime}$ & $0.040^{n}$ & $0.551^{\prime \prime}$ \\
\hline \#24 & $0.021^{*}$ & $0.040^{\prime \prime}$ & $0.551^{*}$ & $\# 64$ & $0.021^{\prime \prime}$ & $0.040^{n}$ & $0.551^{\prime \prime}$ \\
\hline$\# 25$ & $0.021^{*}$ & $0.040^{n}$ & $0.551^{*}$ & $\mathbf{1 6 5}$ & $0.021^{\prime \prime}$ & $0.040^{\circ}$ & $0.551^{\prime \prime}$ \\
\hline \#26 & $0.021^{\circ}$ & $0.040^{\circ}$ & $0.551^{* \prime}$ & $\# 66$ & $0.021^{\prime \prime}$ & $0.040^{\circ}$ & $0.551^{n}$ \\
\hline$\# 27$ & $0.021^{\prime \prime}$ & $0.040^{\mathrm{m}}$ & $0.551^{n}$ & $\# 67$ & $0.021^{\prime \prime}$ & $0.040^{\circ}$ & $0.551^{\prime \prime}$ \\
\hline$\$ 28$ & $0.021^{\prime \prime}$ & $0.040^{*}$ & $0.551^{\prime \prime}$ & $\because 68$ & $0.021^{\prime \prime}$ & $0.040^{*}$ & $0.551^{\prime \prime}$ \\
\hline$\# 29$ & $0.021^{\prime \prime}$ & $0.040^{\mathrm{n}}$ & $0.551^{n}$ & *69 & $0.021^{\mathrm{N}}$ & $0.040^{\circ}$ & $0.551^{n}$ \\
\hline$\$ 30$ & $0.021^{\prime \prime}$ & $0.040^{*}$ & $0.551^{\prime \prime}$ & $\$ 70$ & $0.021^{\prime \prime}$ & $0.040^{\prime \prime}$ & $0.551^{\prime \prime}$ \\
\hline \#1 & $0.021^{*}$ & $0.040^{\circ \prime}$ & $0.551^{\prime \prime}$ & $\# 71$ & $0.021^{\prime \prime}$ & $0.040^{\circ}$ & $0.551^{n}$ \\
\hline$\# 32$ & $0.021^{*}$ & $0.040^{\circ}$ & $0.551^{\prime \prime}$ & $\$ 72$ & $0.021^{\prime \prime}$ & $0.040^{*}$ & $0.551^{n}$ \\
\hline$\# 33$ & $0.021^{\prime \prime}$ & $0.040^{*}$ & $0.551^{\prime \prime}$ & $\$ 73$ & $0.021^{\prime \prime}$ & $0.040^{\prime \prime}$ & $0.551^{\prime \prime}$ \\
\hline$\$ 34$ & $0.021^{n}$ & $0.040^{\circ}$ & $0.551^{\prime \prime}$ & $\$ 74$ & $0.021^{n}$ & $0.040^{*}$ & $0.551^{\prime \prime}$ \\
\hline$\# 35$ & $0.021^{\prime \prime}$ & $0.040^{\circ \prime}$ & $0.55 t^{\prime \prime}$ & $\$ 75$ & $0.021^{\prime \prime}$ & $0.040^{\prime \prime}$ & $0.551^{\prime \prime}$ \\
\hline$\$ 36$ & $0.021^{\prime \prime}$ & $0.040^{*}$ & $0.55 t^{\prime \prime}$ & \pm 70 & $0.021^{\prime \prime}$ & $0.040^{n}$ & $0.551^{\prime \prime}$ \\
\hline$\$ 37$ & $0.021^{4}$ & $0.040^{n}$ & $0.551^{*}$ & $\# 77$ & $0.021^{\prime \prime}$ & $0.040^{n}$ & $0.551^{\prime \prime}$ \\
\hline$\$ 38$ & $0.021^{\star \prime}$ & $0.040^{-}$ & $0.551^{* \prime}$ & \#78 & $0.021^{n}$ & $0.040^{\circ}$ & $0.551^{n}$ \\
\hline$\# 39$ & $0.021^{\prime \prime}$ & $0.040^{\circ}$ & $0.551^{\prime \prime}$ & $\# 79$ & $0.021^{4}$ & $0.040^{\prime \prime}$ & $0.551^{n}$ \\
\hline$\# 40$ & $0.021^{\prime \prime}$ & $0.040^{\prime \prime}$ & $0.551^{\circ}$ & $\# 80$ & $0.021^{\prime \prime}$ & $0.040^{\prime \prime}$ & $0.551^{\prime \prime}$ \\
\hline$\# 41$ & $0.021^{\prime \prime}$ & $0.040^{\circ}$ & $0.551^{\prime \prime}$ & $\$ 81$ & $0.021^{n}$ & $0.040^{\prime \prime}$ & $0.551^{\prime \prime}$ \\
\hline$\# 42$ & $0.021^{n}$ & $0.040^{\prime \prime}$ & $0.551^{n}$ & $\$ 182$ & $0.021^{\mathrm{m}}$ & $0.040^{\circ}$ & $0.551^{n}$ \\
\hline \multicolumn{8}{|c|}{$\begin{array}{l}\text { BOMAS } \\
\text { MACHINE } \\
\text { SPECIALTIES, INC. } \\
334 \text { Washington St. } \\
\text { Somerville, MA } 02143 \\
\text { (617) 628-3831 } \\
\text { EAV. IS17) G39.a1na }\end{array}$} \\
\hline
\end{tabular}

PRE-DECISIONAL - For Planning and Discussion Purposes Only 
KAPL

PO\# KN6002789

Attachment 1.3 to

Enclosure 6 to MDO-723-0044/ B-MT(SRME)-52 Page 14

KAPL Specificntion \# 05PFG094

Dwg. No. 5D+4442

\section{DIMENSIONAL REPORT IN INCHES}

\begin{tabular}{|c|c|c|c|}
\hline$\# 83$ & $0.021^{\prime \prime}$ & $0.040^{n}$ & $0.551^{\circ}$ \\
\hline$\# 84$ & $0.021^{n}$ & $0.040^{*}$ & $0.551^{\prime \prime}$ \\
\hline$\# 85$ & $0.021^{\prime \prime}$ & $0.040^{\circ}$ & $0.551 "$ \\
\hline$\# 86$ & $0.021^{\prime \prime}$ & $0.040^{*}$ & $0.551^{-}$ \\
\hline *87 & $0.021^{\prime \prime}$ & $0.040^{\prime \prime}$ & $0.551^{\text {" }}$ \\
\hline$\# 88$ & $0.021^{*}$ & $0.040^{*}$ & $0.551^{\prime \prime}$ \\
\hline$\$ 89$ & 0.021" & $0.040^{\circ}$ & $0.551^{n}$ \\
\hline$\# 90$ & $0.021^{\prime \prime}$ & $0.040^{n}$ & $0.551^{\pi}$ \\
\hline \#1 & $0.021^{\prime \prime}$ & $0.040^{n}$ & $0.55 t^{\prime \prime}$ \\
\hline$\# 92$ & $0.021^{\prime \prime}$ & $0.040^{n}$ & $0.551^{\prime \prime}$ \\
\hline$\# 93$ & $0.021^{\prime \prime}$ & $0.040^{n}$ & $0.551^{\mathrm{m}}$ \\
\hline 194 & $0.021^{*}$ & $0.040^{\circ}$ & 0.551" \\
\hline \#95 & $0.021^{\prime \prime}$ & $0.040^{\mathrm{m}}$ & $0.551^{\prime \prime}$ \\
\hline$\# 96$ & $0.021 "$ & $0.040^{n}$ & $0.551^{\circ}$ \\
\hline$\$ 97$ & $0.021^{\prime \prime}$ & $0.040^{\prime \prime}$ & $0.551^{\prime \prime}$ \\
\hline \#98 & $0.021^{\prime \prime}$ & $0.040^{*}$ & $0.551^{\prime \prime}$ \\
\hline$\$ 99$ & $0.021^{n}$ & $0.040^{\circ}$ & $0.551^{*}$ \\
\hline$\because 100$ & $0.021^{\prime \prime}$ & $0.040^{\circ}$ & $0.551^{\circ "}$ \\
\hline$\$ 101$ & $0.021^{\prime \prime}$ & $0.040^{\prime \prime}$ & $0.551^{\prime \prime}$ \\
\hline$\$ 102$ & $0.021^{\prime \prime}$ & $0.040^{\circ}$ & $0.551^{\prime \prime}$ \\
\hline$\$ 103$ & $0.021^{\prime \prime}$ & $0.040^{\prime \prime}$ & $0.551^{\prime \prime}$ \\
\hline$* 104$ & $0.021^{\prime \prime}$ & $0.040^{\circ}$ & $0.551^{\prime \prime}$ \\
\hline$\# 105$ & $0.021^{\prime \prime}$ & $0.040^{n}$ & $0.551^{\prime \prime}$ \\
\hline$\# 108$ & $0.021^{\prime \prime}$ & $0.040^{n}$ & $0.551^{\prime \prime}$ \\
\hline$\# 107$ & 0.021" & $0.040^{\circ}$ & $0.551^{n}$ \\
\hline$\# 108$ & $0.021^{\prime \prime}$ & $0.040^{n}$ & $0.551^{-}$ \\
\hline
\end{tabular}

Note: 1 \& 2 were sent to KAPL on 07-12-05 as "FIRST PIECE INSPECTION" specimens

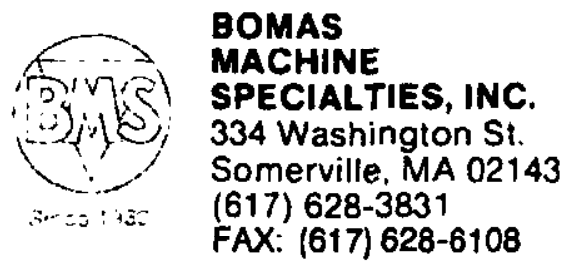

Mechining of High Temperature Ceramic Materials 
Attachment 1.3 to

Enclosure 6 to

KNOLLS ATOMIC POWER LABORATORY

POA KN6002789 MDO-723-0044/ B-MT(SRME)-52 Page 15

KAPL Specification \# 06PFC094

Dwg. No. 6014442

\section{DIMENSIONAL REPORT IN IMCHES}

Item D

\begin{tabular}{|c|c|c|c|}
\hline$\#$ & $0.021^{\circ}$ & $0.040^{\circ}$ & $0.276^{\circ}$ \\
\hline$\$ 4$ & $0.021^{\circ}$ & $0.040^{n}$ & $0.276^{\circ}$ \\
\hline 45 & $0.021^{\prime \prime}$ & $0.040^{n}$ & $0.27 \theta^{n}$ \\
\hline 16 & $0.021^{\circ}$ & $0.040^{n}$ & $0.278^{\prime \prime}$ \\
\hline$\# 7$ & $0.021^{\circ}$ & $0.040^{\prime \prime}$ & $0.276^{n}$ \\
\hline 40 & $0.021^{\prime \prime}$ & $0.040^{*}$ & $0.276^{n}$ \\
\hline & $0.021^{\prime \prime}$ & $0.040^{\prime \prime}$ & $0.276^{\prime \prime}$ \\
\hline 10 & $0.021^{n}$ & $0,040^{\circ}$ & $0.276^{n}$ \\
\hline
\end{tabular}

Note: 1 \& 2 were sent to KAPL on 07-12-05 as "FIRST PIECE INSPECTION" specimens

BOMAS

MACHINE

SPECIALTIES, INC.

334 Washington St.

Somerville. MA 02143

(617) 628-3831

FAX: (617) 628-6108

Machinng of High Temperalure Ceramuc Malerials 
Attachment 1.4 to

Enclosure 6 to

MDO-723-0044i

B-MT(SRME)-52

Page 1

\section{Attachment 1.4}

\section{Specimen Photographs}

Enclosure 6 to MDO-723-0044/B-MT(SRME)-52 
Attachment 1.4 to Enclosure 6 to MDO-723-0044/

B-MT(SRME)-52

Page 2

\section{IIIIIIIII IIII|IIII| $\mid$ IIIIIIII| CENTIMETERS}

Image 1: The following image shows the three lengths of Monolithic Silicon Carbide Temperature Monitors for the JOYO-1 Irradiation Test Campaign. 
Attachment 2.1 to

Enclosure 6 to MDO-723-0044/

B-MT(SRME)-52

Page 1

Attachment 2.1

Material Purchase Documentation and Certification

Enclosure 6 to MDO-723-0044/B-MT(SRME)-52 
70: BeELY NoRtyE:

KAPL, Inc. PURCHASE ORDER

Box 1072

Swianectady, New York 12301-1072

PAGE 1 OF 1

Attachment 2.1 to

Enclosure 6 to

PURCHASE ORDER NUMBER MDO-723-0044I

KN6002827

B-MT(SRME)-52

Page 2

PURCHASE ORDER DATE AMENDMENT DATE

23-JUN-2005

IMPORTANT: Show Purchase Order Number on all correspondence. Complete packing list must accompany each shipment.

Effective Date: $\quad$ Expiration Date:

KAPL, INC.. A LOCKHEEO MAATIN COMPANY, OPERATES THE KNOLLS ATOMIC POWER LABORATORY UNDER GOVERNMENT CONTRACT DE.AC-12.COSN39357.

S APPLIED PHYSICS TECHNOLOGIES INC

U. 1600 N.E. MILLER STREET

MCMINIVILLE, OR 97128

United States

Confirmed By:

s. KNOLLS RECEIVING BLDG M2

H CENTRAL RECEIVING - KNO

P 2401 RIVER ROAD

SCHENECTADY,NY 12309

T)

Line

1 POLYCRYSTALLINE ZrC

ZONE REFINED AS-IS RODS

1.4" LENGTH: $0.080^{n}$

0.090" DIAMETER.

INSTRUCTIONS TO

PURCHASE ORDER

KN6002827 SHALL APPLY

2

MATERIAL CERTIFICATIONS

FOR ITEM 1.

$\$ .00$

EACH

0

$\$ 0.00$

INSTRUCTIONS TO

PURCHASE ORDER

KN6002827 SHALL APPLY.

PRICE INCLUDED IN PRICE

OF ITEM 1

Address Carrespondence To

Total Firm Fixed Price

\$ 28.520 .00

KAPL

P.0. $80 \times 1072$

Schenectady, NY 12301

Phone Number: 5183956649

IR AGREES THAT THE SCOPE OF WORK REOUIRED IS UNDERSTOOD BY THE SELLER

SELER; THAT THERE ARE NO INFORMAL COMMITMENTS

DER; THAT THERE ARE NO OPEN OR UNRESOLVED ISSUES REATED TO THE ORDER EXCE WAY MAY AFFECT THE WORK UNDER THIS

THE ORDER EXCEPY AS EXPLCITLY STATED HEREN; AND THAT

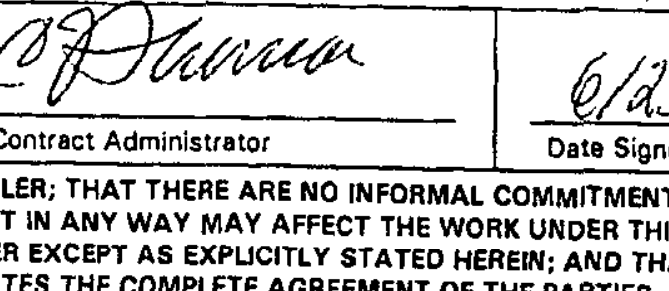

Name of Vendor

(女)

If this box is marked, su 


\begin{tabular}{|c|c|c|c|c|}
\hline \multirow[t]{2}{*}{$\begin{array}{l}\text { Purchase } \\
\text { Order }\end{array}$} & $\begin{array}{l}\text { Document No. } \\
\text { KN6002827 }\end{array}$ & $\begin{array}{l}\text { Document Title } \\
\text { MR!92201 }\end{array}$ & \multirow[t]{2}{*}{$P_{a c}$} & $\begin{array}{l}\text { Attachment } 2.1 \text { to } \\
\text { Enclosure } 6 \text { to } \\
\text { MDO-723-0044/ } \\
\text { B-MT(SRME)-52 }\end{array}$ \\
\hline & $\begin{array}{l}\text { Buyer Name } \\
\text { C.J. Thomas }\end{array}$ & & & \\
\hline
\end{tabular}

INSTRUCTIONS TO PURCHASE ORDER KN6002827

THIS IS A FIRM FIXED PRICE ORDER

1. Material and/or Service Required:

Provide the line items in accordance with the instructions listed below. The specifications, standards, drawings and documents listed and referred to in these instructions are incorporated by reference and made a part hereof.

II. Delivery Requirements:

As set forth below:

A. Partial of liem I with ltem 2 - On or before five (5) weeks after date of order.

B. Balance of Item I with Item 2 - On or before eight (8) weeks after date of order.

III. Applicable Documents:

The following documents with all documents referenced therein form a part of this order:

A. Commercial Terms and Conditions, dated 8/97.

IV. Modifications to Instruction III:

A. Commercial Terms and Conditions is hereby modified as follows:

1. With respect to the "Changes" Article, only the individuals identified below are authorized to issue and sign change orders and amendments for Buyer. You will be notified in writing by the Manager, Supply Chain Management of any changes of named individuals:

Position

Manager, Supply Chain Management

Manager, Technology Sourcing

Contract Administrator, Technology Sourcing

Telephone

Fax
Name

E.M. Audi

D.R. Devenpeck

C.J. Thomas

(518) 395-6649

(518) 395-7579

V. The IV System:

Contractual communications shall be accomplished by means of the following documents:

A. Inquiry to Vendor (IV) - used for requested for quotations covering additional work.

B. Information to Vendor (IV) - used for drawing or process approval, transmittal of minutes of meeting, confirmation of phone conversations and other general subjects of comnunication.

One copy of the applicable IV must be signed and returned to the cognizant Contract Administrator within five (5) working days after receipt. 
Attachment 2.2 to Enclosure 6 to MDO-723-0044/

B-MT(SRME)-52

Page 1

Attachment 2.2

Technical Specification for Machining

Enclosure 6 to MDO-723-0044/B-MT(SRME)-52

PRE-DECISIONAL - For Planning and Discussion Purposes Only 


\title{
SPECIFICATION FOR 0SPFG115
}

\author{
Rev. -
}

\section{DATED 6/29/05}

1. KAPL will supply $\mathrm{ZrC}$ rods as described below:

\begin{tabular}{|l|l|l|l|l|}
\hline NO. & ROD & PLATE TYPE & $\begin{array}{l}\text { DIAMETER } \\
\text { (IN) }\end{array}$ & $\begin{array}{l}\text { LENGTH } \\
(\mathrm{N})\end{array}$ \\
\hline 80-(Rods) & $\begin{array}{l}\text { MR192201-1 } \\
\text { through } \\
\text { MR192201-80 }\end{array}$ & $\begin{array}{l}\text { Zone Refined, } \\
\text { As-Is, } \\
\text { Polycrystalline } \\
\text { ZrC Rods }\end{array}$ & $\begin{array}{l}0.080-0.090 \\
1.4\end{array}$ & \\
\hline
\end{tabular}

\section{APPLICABLE DRAWINGS AND SKETCHES}

- KAPL Drawing SK216C9452 Passive Temperature Indicator.

- KAPL Specification, Attachment 1 "Detrimental Materials Prohibitions and Restriction for Silicon Carbide Composite Materials" Dated 3/6/03.

- KAPL supplied Table 1 'Machining Instruction Shect'.

\section{MACHINING INSTRUCTION}

- For each piece of Government Furnished Material (GFM) rod, extract and machine the required number of specimens as specified in the attached table 1 "Machining Instruction".

- Stock removal rate shall not exceed $0.03 \mathrm{~mm}$ per pass to the last $0.06 \mathrm{~mm}$. Final finishing shall be preformed with diamond tools that have between 320 and 600 grit. No less than $0.06 \mathrm{~mm}$ per face shall be removed during the final finishing phase and at a rate not more than $0.002 \mathrm{~mm}$ per pass.

- Remove approximately equal stock from opposite faces.

- The surface finish for the passive temperature indicator shall be $16 \mu$ in.

- Compliance with KAPL Specification on detrimental materials is required during all aspects of this fabrication.

- The seller shall use dedicated resin-bonded diamond tooling for all machining of $\mathrm{ZrC}$ specimens. If other than resin-bonded tooling is need the seller shall obtain KAPL's approval prior to fabrication.

- The seller shall also use new coolant for this work scope.

- All specimens shall be identified by a bag and tag method with rod ID, specimen type, specimen ID and drawing number.

- There shall be a verbal briefing prior to the start of machining. 
Two specimens of each type shall be provided for a first piece inspection to assure that they meet all of the requirements of KAPL Drawings prior to proceeding to the next piece. The seller shall notify KAPL with results of the first piece inspection prior to completing the fabrication on the balance of the order. KAPL requests the seller to provide to KAPL the specimens used for first piece inspection prior to completion of the balance of the order.

TABLE 1

Machining Instruction Sheet

05PFG115

\begin{tabular}{|c|c|c|c|c|c|}
\hline ROD ID & & $\begin{array}{l}\text { SPECIMEN } \\
\text { TYPE }\end{array}$ & $\begin{array}{l}\text { SPECIMEN } \\
\text { ID's }\end{array}$ & QUANTITY & $\begin{array}{l}\text { DRAWING } \\
\text { NO. }\end{array}$ \\
\hline $\begin{array}{l}\text { MR192201-1 } \\
\text { Through } \\
\text { MR192201- } \\
12\end{array}$ & $\begin{array}{l}\text { Zone Refined, } \\
\text { As-ls, } \\
\text { Polycrystalline } \\
\text { ZrC Rods }\end{array}$ & $\begin{array}{l}\text { Item } 1 \\
\text { ZrC Temperature } \\
\text { Monitor (Length } \\
=1.299^{\prime \prime} \pm 0.030 \text { ) }\end{array}$ & $1-12$ & 12 & $\begin{array}{l}\text { SK216C9452 } \\
\text { Item 1 }\end{array}$ \\
\hline $\begin{array}{l}\text { MRI92201- } \\
13 \\
\text { Through } \\
\text { MR192201- } \\
57\end{array}$ & $\begin{array}{l}\text { Zone Refined, } \\
\text { As-Is, } \\
\text { Polycrystalline } \\
\text { ZrC Rods }\end{array}$ & $\begin{array}{l}\text { Item } 2 \\
\text { ZrC Temperature } \\
\text { Monitor (Length } \\
\left.=1.102^{\prime \prime} \pm 0.030\right)\end{array}$ & $1-44$ & 44 & $\begin{array}{l}\text { SK216C9452 } \\
\text { Item } 2\end{array}$ \\
\hline $\begin{array}{l}\text { MR192201- } \\
58 \\
\text { Through } \\
\text { MR192201- } \\
66\end{array}$ & $\begin{array}{l}\text { Zone Refined, } \\
\text { As-Is, } \\
\text { Polycrystalline } \\
\text { ZrC Rods }\end{array}$ & $\begin{array}{l}\text { Item } 3 \\
\text { ZrC Temperature } \\
\text { Monitor (Length } \\
=0.551 " \pm 0.030 \text { ) }\end{array}$ & $1-16$ & 16 & $\begin{array}{l}\text { SK216C9452 } \\
\text { ltem } 3\end{array}$ \\
\hline
\end{tabular}

\section{DATA REQUIREMENTS}

- Complete As-Built data for all specimens.

- Certs. of Compliance with KAPL Specification on detrimental materials (Attachment I dated 3/6/03).

- First piece inspection notification. 
Attachment 2.2 to

Enclosure 6 to MDO-723-0044/

B-MT(SRME)-52

Page 4

Prepared By

Reviewed By 


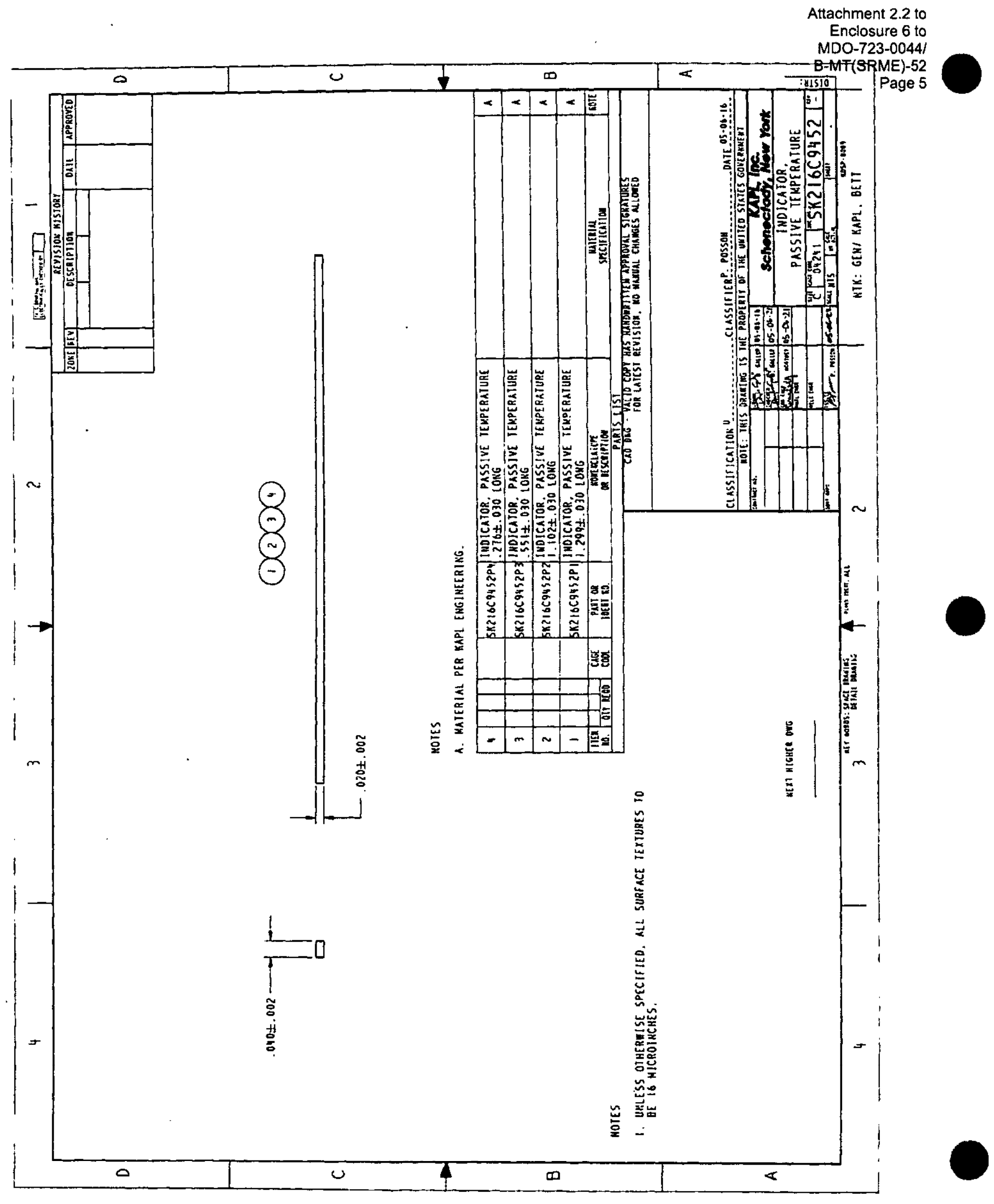




\section{KAPL, Inc.}

\section{PURCHASE ORDER}

P. $0.80 \times 1072$

Thenectady, New York 12301-1072
PAGE 1 OF 1

PURCHASE ORDER NUMBER

KN6003137

Enclosure 6 to

MDO-723-0044/

B-MT(SRME)-52

Page 6

PURCHASE ORDER DATE AMENUMENI UAJE

20.JUL-2005

OSPFG / 15

IMPORTANT: Show Purchase Order Number on all cortespondence. Complete packing list must accompany each shipment.

Effective Date: $\quad$ Expiration Date:

KAPL, INC., A: OCKHECD MAATIN COMIDANY, OPERATES THE KNOLLS ATOMIC POWER LABCRATCAY UNDER GOVERNMENT CONTRACT DE-AC-12.00SN39357.

5 PAEMATECH ADVANCED CERAMICS

U A DIVISION OF PREMATECH LLC

2 COPPAGE DRIVE

L WORCESTER, MA $01603-1252$

1

E

ค Confirmed By:

\begin{tabular}{|c|c|c|c|}
\hline \multirow{4}{*}{$\begin{array}{l}\text { AMICS } \\
\text { LC }\end{array}$} & \multirow{4}{*}{$\begin{array}{l}T \\
\mathbf{E} \\
\mathbf{R} \\
\mathbf{M} \\
\mathbf{s}\end{array}$} & \multicolumn{2}{|c|}{ PAYMENT TERMS: 30 NET } \\
\hline & & \multicolumn{2}{|c|}{ FAEIGHT TEAMS: } \\
\hline & & F.O.Bi & DESTINATION \\
\hline & & SHIP VIA: & Saller's Choico \\
\hline & $\begin{array}{l}0 \\
1 \\
L \\
L \\
T \\
0\end{array}$ & \multicolumn{2}{|c|}{$\begin{array}{l}\text { KAPL } \\
\text { ACCOUNTS PAYABLE } \\
\text { PO BOX } 1072 \\
\text { SCHENECTADY,NY } 12301-1072\end{array}$} \\
\hline
\end{tabular}

(1)

KNOLLS RECEIVING BLDG M2

CENTRAL RECEIVING - KNO

2401 RIVER ROAD

SCHENECTADY.NY 12309

Description

Promlsed Dato Quantity

UOM Unit Price

Total Price

TEMPEAATURE MONITORS IN

12.00

EACH

48.9

$\$ 586.80$

5K216C9452 1 AND

SPECIFICATION FOP

OSPFG115. INSTALCTIONS

TO PURCHASE ORCER

KN6003137 SHALL APPLY.

This PO line refarences contract POF: 6000621

2 TEMPERATURE MONITORS IN

ACCORDANCE WITH

SK2 $16 C 9452$ P2 ANO

SPECIFICATION FOA

O5PFG 115. INSTRUCTIONS

TO PURCHASE ORDEA

KN6003137 S'IALL APPLY.

This PO line references contract POH: 6000521

3 TEMPERATURE MONTTORS IN

ACCORDANCE WITH

SK2 16C9452 P3 AND

SPECIFICATION FOR

O5PFG 115. INSTAUC:ONS

TO PURCHASE ORDER

KNSO003137 SHALL APPLY.

This PO line fcterences contract POH: 6000621

44.00

EACH

40

$\$ 1.760 .00$

EACH

45.85

$\$ 733.60$

Address Corrospondence To:

KAPL

P.O. Box 1072

Schenectady. NY 12301

Phone Number: 5183956649

Total Fing-fixed Price

$\$ 3.080 .40$

SELLER AGREES THAT THE SCOPE OF WORK REOUIRED IS UNDERSTOOD BY THE SELLER; THAT THERE ARE NO INFORMAL COMMITMENTS BETWEEN THE SELLER AND THE BUYER OR THE SELLER AND THE GOVERNMENT THAT IN ANY WAY MAY AFFECT THE WORK UNDER THIS TER; THAT THERE ARE NO OPEN OR UNRESOLVED ISSUES RELATED TO THE ORDER EXCEPT AS EXPLICITLY STATED HEAEIN; AND THAT -ER THEREFORE UNDERSTANDS AND AGREES THAT THIS PURCHASE ORDER STATES THE COMPLETE AGREEMENT OF THE PARTIES 


\begin{tabular}{|l|l|l|l|}
\hline $\begin{array}{l}\text { Delivery } \\
\text { Order }\end{array}$ & $\begin{array}{l}\text { Document No. } \\
\text { KN6003137 }\end{array}$ & $\begin{array}{l}\text { Document Title } \\
\text { 05PFG115 }\end{array}$ & Page 1 of 1 \\
\hline & $\begin{array}{l}\text { Buyer Yiame } \\
\text { CJ THOMAS }\end{array}$ & & \\
\hline
\end{tabular}

INST'RLCTIONS TO PURCHASE ORDER KN6003137

\section{PIEASE FORIVARD ACKNOWLEDGEMENT COPY AND AYY OTHER CORRESPONDENCF} TO: C.I TIIOMAS, MALL STOP 148

\section{RI:FERENCE NUMBER: OSPFGIIS}

Scller is herehy authorjzed to pertorm the work set forth in Paragraph A. in accordance with the tequirements of $P$ alagraphs $B$. and $C$. of the above referenced number.

\section{DEI,IVERY REOUMREMENTS}

Tine is of the essence - Buyer requires and it js cssential that delivery be made, as sel forth below:

A. Ilems 1 through 3 . One-half . On or before twenty (20) work days after delivery of order and reccipt of Government Furnished Material.

A. Items 1 through 3 - Balance - On or before forty (40) work days a fter delivery of order and receipt of Government Furnished Material.

\section{APPLICABLE DOCIJMENTS}

The following documents with all documents referenced therein form a part of this order:

A. Specification For 05PFG1 15, Rev. -, dated 6/29/05.

\section{GOVERYMIENT FURYISHED MATERIAL}

A. Eighty (80) each - Polycrystalline Rods

Buyer estimates the Government Fumished Material will be shipped on or before nwo (2) weeks after datc of order. 
Attachment 2.3 to

Enclosure 6 to MDO-723-0044/

B-MT(SRME)-52

Page 1

Attachment 2.3

Closeout Documentation

Enclosure 6 to MDO-723-0044/B-MT(SRME)-52 
Attachment 2.3 to

Enclosure 6 to MDO-723-0044/

\section{KNOLLS ATOMIC POWER LABORATORY}

B-MT(SRME)-52

Page 2

Date 12/1/2005

Page 1 of 3

\section{LNSTRUCTION TO VENDOR}

(for internal use only)

TO: PremaTech Advanced Ceramics

Subject: $\quad$ Order No. KN6003137

I.V. No.

Aetion Required By SCM

Type Contract

Source of Funds

Charge Code

Dollars, This Change

Dollars, Presently Committed

Dollars, Accumulative
MDO/SM

D23I7D3808

$-\$ 2480,40$

$\mathbf{S 3}, 080.40$

$\$ 600.00$
(Signatures as Required ${ }^{\star}$ )

Programming

Cost \& Budget Contro!*

Quality Control

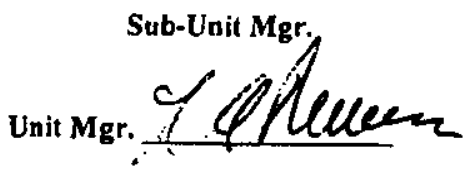

Sub-Section Mgr.*

Section Mgr.

General Mgr

NR APPROVAL REQUIRED? Yes ; No X

NR APPROVAL DOCUMENT NO. DATE

\section{FOR COST-TYPE ORDERS ONLY}

Estimate for incorporating IV

Man Hours

Overall Effect on

Order Amount
Estimate Prepared By

Estimate Approved By SUB-SECTION MGR,

INSTRUCTIONS:

See Attached Technical Specification for 05PFG115 Rev. 2.

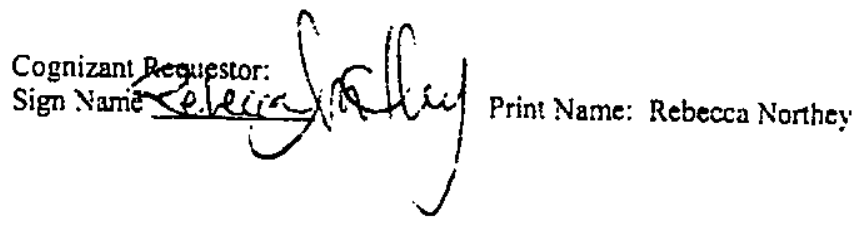




\title{
SPECIFICATION FOR 05PFG115
}

\author{
Rev. - 2
}

DATED 10/10/05

1. KAPL has supplied the Government Furnished Material (GFM) in the form of ZrC rods as described below:

\begin{tabular}{|l|l|l|l|l|}
\hline NO. & ROD & PLATE TYPE & $\begin{array}{l}\text { DIAMETER } \\
\text { (IN) }\end{array}$ & $\begin{array}{l}\text { LENGTH } \\
\text { (IN) }\end{array}$ \\
\hline 80 -(Rods) & $\begin{array}{l}\text { MR192201-1 } \\
\text { through } \\
\text { MR192201-80 }\end{array}$ & $\begin{array}{l}\text { Zone Refined, } \\
\text { As-Is, } \\
\text { Polycrystalline } \\
\text { ZrC Rods }\end{array}$ & $0.080-0.090$ & 1.4 \\
\hline
\end{tabular}

\section{APPLICABLE DRAWINGS AND SKETCHES}

- KAPL Drawing SK216C9452 Passive Temperature Indicator.

- KAPL Specification, Attachment 1 "Detrimental Materials Prohibitions and Restriction for Silicon Carbide Composite Materials" Dated 3/6/03.

- KAPL supplied Table 1 'Machining Instruction Sheet'.

\section{MACHINING INSTRUCTION}

- PremaTech Advanced Ceramics has completed partial grinding on the six test specimens listed in Table $I$.

- The remaining 74 pieces of GFM and the 6 partially machined temperature monitors have been delivered to KAPL.

- This machining has been stopped due to close-out of the space program. 
Attachment 2.3 to

Enclosure 6 to

MDO-723-0044/

TABLE ]

B-MT(SRME)-52

Machining Instruction Sheet

Page 4

05PFG115

\begin{tabular}{|l|l|l|l|l|l|}
\hline ROD ID & $\begin{array}{l}\text { SPECIMEN } \\
\text { TYPE }\end{array}$ & $\begin{array}{l}\text { SPECLMEN } \\
\text { ID's }\end{array}$ & QUANTITY & $\begin{array}{l}\text { DRAWING } \\
\text { NO. }\end{array}$ \\
\hline $\begin{array}{l}\text { MR 192201-1 } \\
\text { MR 192201-2 }\end{array}$ & $\begin{array}{l}\text { Zone Refined, } \\
\text { As-Is, } \\
\text { Polycrystalline } \\
\text { ZrC Rods }\end{array}$ & $\begin{array}{l}\text { ZrC Temperature } \\
\text { Monitor (Length } \\
=1.299 " \pm 0.030)\end{array}$ & $1-2$ & 2 & SK216C9452 \\
\hline $\begin{array}{l}\text { MR192201-3 } \\
\text { MR 192201-4 }\end{array}$ & $\begin{array}{l}\text { Zone Refined, } \\
\text { As-Is. } \\
\text { Polycrystalline } \\
\text { ZrC Rods }\end{array}$ & $\begin{array}{l}\text { ZrC Temperature } \\
\text { Monitor (Length } \\
=1.102 " \pm 0.030)\end{array}$ & $3-4$ & 2 & SK216C9452 \\
\hline $\begin{array}{l}\text { MR192201-5 } \\
\text { MR192201-6 }\end{array}$ & $\begin{array}{l}\text { Zone Refined, } \\
\text { As-Is, } \\
\text { Polycrystalline } \\
\text { ZrC Rods }\end{array}$ & $\begin{array}{l}\text { ZrC Tenperature } \\
\text { Monitor (Length } \\
=0.551 " \pm 0.030)\end{array}$ & $5-6$ & 2 & SK216C9452 \\
\hline
\end{tabular}

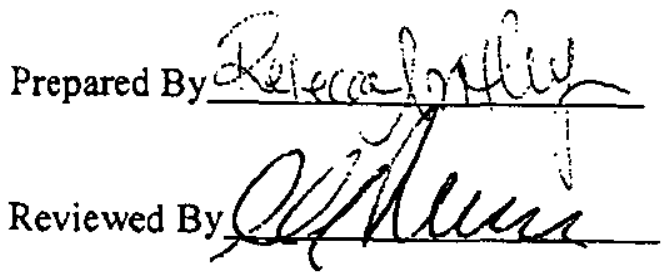




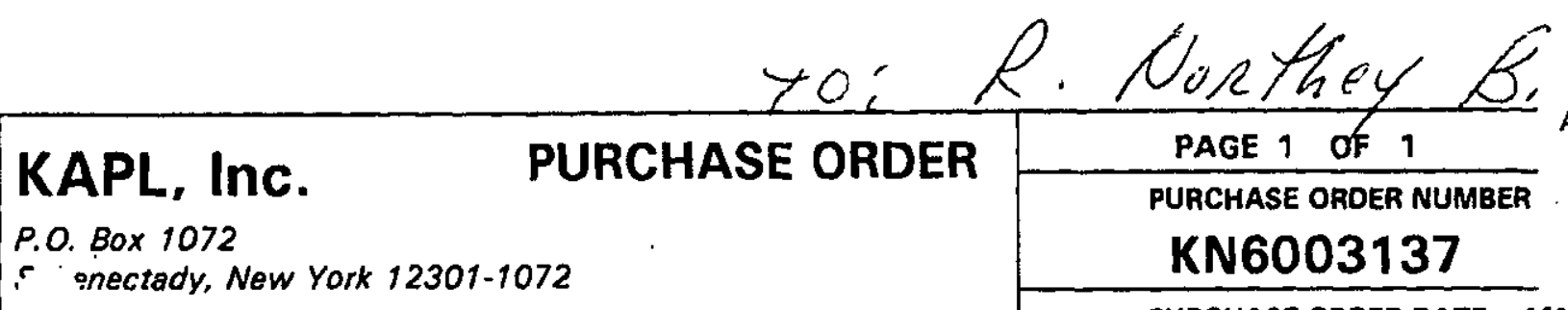

PURCHASE ORDER DATE AMENDMENT DATE 20.JUL-2005
Attachment 2.3 to Enclosure 6 to MDO-723-0044/ B-MT(SRME)-52

Page 5

IMPORTANT: Show Purchase Order Number on all correspondence. Complete packing list must accompany each shipment.

\begin{tabular}{l|l|l} 
Effective Date: & Expiration Date: \\
\hline
\end{tabular}

KAPL, INC., A LOCKHEED MARTIN COMPANY, OPERATES THE KNOLLS ATOMIC POWER LABORATORY UNDER GOVERNMENT CONTRACT DE-AC-12-0OSN39357.

s PREMATECH ADVANCED CERAMICS

U A DIVISION OF PREMATECH LLC

P 2 COPPAGE DRIVE

L WORCESTER, MA 01603-1252

E

R Confirmad By:

S KNOLLS RECEIVING BLDG MZ

H CENTRAL RECEIVINO - KNO

P 2401 RIVER ROAD

SCHENECTADY.NY 12309

T.

\begin{tabular}{|c|c|c|}
\hline \multirow{4}{*}{$\begin{array}{c}T \\
E \\
\text { R } \\
M \\
\text { S }\end{array}$} & \multirow{2}{*}{\multicolumn{2}{|c|}{\begin{tabular}{|l} 
PAYMENT TERMS:30 NET \\
FREJGHT TERMS:
\end{tabular}}} \\
\hline & & \\
\hline & F.O.B: & DESTINATION \\
\hline & SHIP VIA: & Seller': Choice \\
\hline $\begin{array}{l}B \\
i \\
L\end{array}$ & \multicolumn{2}{|c|}{$\begin{array}{l}\text { KAPL } \\
\text { ACCOUNTS PAYABLE } \\
\text { PO BOX } 1072 \\
\text { SCHENECTADY,NY } 12301-1072\end{array}$} \\
\hline
\end{tabular}

\begin{tabular}{|c|c}
\hline Line & Description \\
\hline 1 & TEMPERATURE MONITORS IN \\
\hline
\end{tabular}

TEMPERATURE MONITORS IN

ACCORDANCE WITH

SK2 16C9452 P1 AND

SPECIFICATION FOR

OEPFG 115. INSTRUCTIONS

TO PURCHASE ORDER

KN6003137 SHALL APPLY.

This PO line references contract POH: 6000621

2 TEMPERATURE MONITORS IN

ACCORDANCE WITH

SK216C9452 P2 AND

SPECIFICATION FOR

OSPFG $\uparrow 15$. INSTRUCTIONS

TO PURCHASE ORDER

KN6003 137 SHALL APPLY.

This PO line references contract POH: 6000621

3 TEMPERATURE MONITORS IN

ACCORDANCE WITH

SK216C9452 P3 AND

SPECIFICATION FOR

O5PFG 115 . INSTRUCTIONS

TO PURCHASE ORDER

KN6003137 SHALL APPLY.

This PO line relerences contract PON: 6000621

$\mathrm{EACH}$

100

$\$ 200.00$

$\$ 200.00$

2.00

$\mathrm{EACH}$

100

$\$ 200.00$

Address Correspondence To:

KAPL

P.O. Box 1072

Schenectady, NY 12301

Phone Number: 5183956649

SELLER AGREES THAT THE SCOPE OF WORK REQUIRED IS UNDERSTOOD BY THE SEULE. THAT THERE ARE NO INFORMAL COMMITMENTS BETWEEN THE SELLER AND THE BUYER OR THE SELLER AND THE GOVERNMENT THAT IN ANY WAY MAY AFFECT THE WORK UNDER THIS

' :R; THAT THERE ARE NO OPEN OR UNRESOLVED ISSUES RELATED TO THE ORDER EXCEPT AS EXPLICITLY STATED HEREN; AND THAT 2 .ER THEREFORE UNDERSTANDS AND AGREES THAT THIS PURCHASE ORDER STATES THE COMPLETE AGREEMENT OF THE PARTIES.

Name of Vendor

If this box is marked, su
Sinnoture

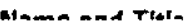

PRE-DECISIONAL - For Planning and Discussion Purposes Only
8600.00

C THOMA'S

Total Firm Fixed Price

\section{-}

NO INFORMAL COMMITMENTS
Date Signed
Y STATED HEREN; AND TH
REEMENT OF THE PARTIES.

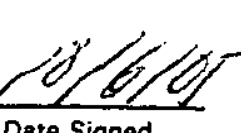




\begin{tabular}{|l|l|l|l|}
\hline $\begin{array}{l}\text { Delivery } \\
\text { Order } \\
\text { Modification }\end{array}$ & Document No. & Document Title & Page 1 of I \\
\hline & $\begin{array}{l}\text { Buyer Name } \\
\text { CJ THOMAS }\end{array}$ & O5PG 115 & \\
\hline
\end{tabular}

INSTRUCTIONS TO PURCHASE ORDER KN6003137

\section{Amendment No.1}

Amendment 1 is issued to reduce the quantities of Items 1 through 3 from 12 each for Item 1 , from 44 each for Item 2 and from 16 each for Item 3 to 2 each for each of ltems 1 through 3 , and change the unit price for these items to $\$ 100 /$ each. The total order amount is reduced by $\$ 2480.40$, from $\$ 3,080.40$ to $\$ 600.00$.

\section{PLEASE NOTE \\ PLEASE FORWARD ACKNOWLEDGEMENT COPY AND ANY OTHER CORRESPONDENCE TO: CJ THOMAS, MAIL STOP 148}

\section{REFERENCE NUMBER: OSPFG115}

Seller is hereby authorized to perform the work set forth in Paragraph A, in accordance with the requirements of Paragraphs $B$. and $C$. of the above referenced number.

\section{DELIVERY REOUIREMENTS}

Time is of the essence - Buyer requires and it is essential that delivery be made, as set forth below:

A. Items 1 through 3 - One-half - On or before twenty (20) work days after delivery of order and receipt of Government Furnished Material.

A. Itenss 1 through 3 - Balance - On or before forty (40) work days after delivery of order and receipt of Government Furnished Material,

\section{APPLICABLE DOCUMENTS}

The following documents with all documents referenced therein form a part of this order:

A. Specification For 05PFG115, Rev. 1, dated 10/6/05.

\section{GOVERNMENT FURNISHED MATERIAL}

A. Eighty (80) each - Polyerystalline Rods Buyer estimates the Government Furnished Material will be shipped on or before two (2) weeks after date
of order. 
Enclosure (7) to

MDO-723-0044I

B-MT(SRME)-52

Page i

\title{
Enclosure 7
}

to

MDO-723-0044/B-MT(SRME)-52

JOYO-1 Capsule Design

\author{
A.M. Tullai, Design Lead \\ D.C. Stambolis, Thermal Analysis Lead \\ G.D. Carpenter, Nuclear Analysis Lead \\ R.A. Clapp \\ J.L. Davis \\ J.P. Hill \\ E.V. Mader \\ R.R. Metzger \\ J.A. Mills \\ C.M. Rodenbush
}


Enclosure (7) to

MDO-723-0044/

B-MT(SRME)-52

Page ii

\section{TABLE OF CONTENTS}

I. Description of Test .......................................................................................

A. Statement of the Design ...................................................................... 1

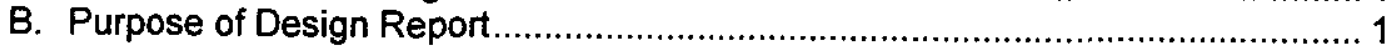

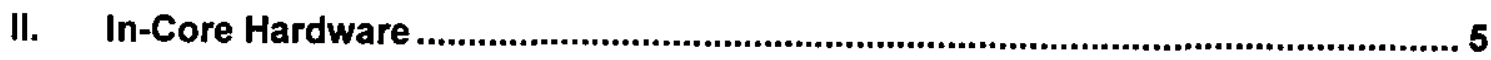

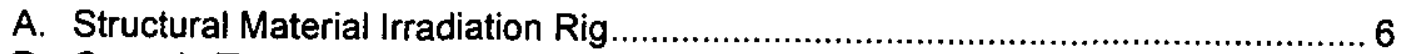

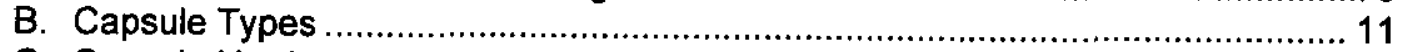

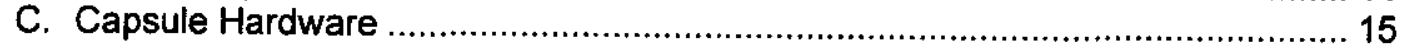

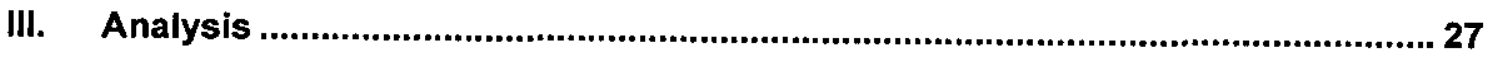

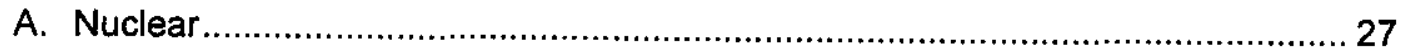

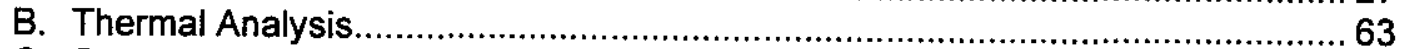

C. Structural Analysis of JOYO-1 Capsule Pressure Boundary …........................... 93

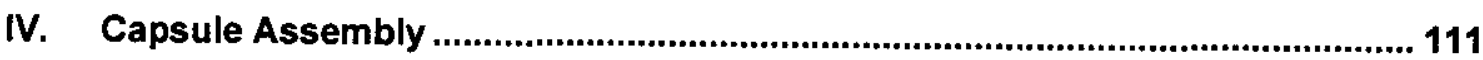

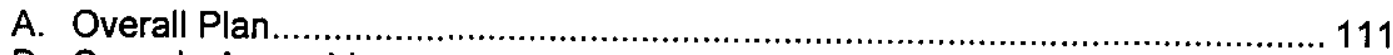

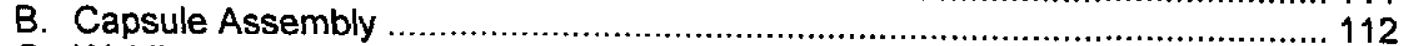

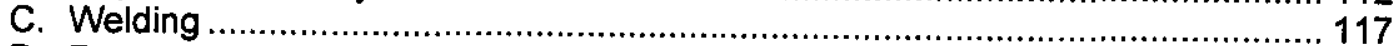

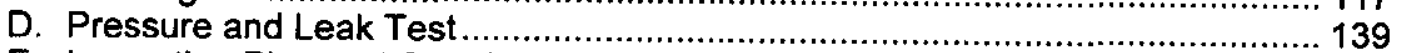

E. Inspection Plan and Standards or Procedures............................................. 140

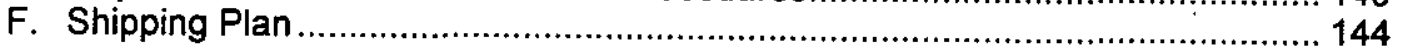

V. Future Considerations and Open Items ..................................................... 149

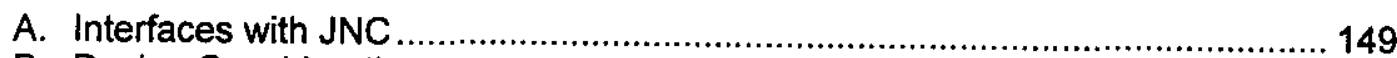

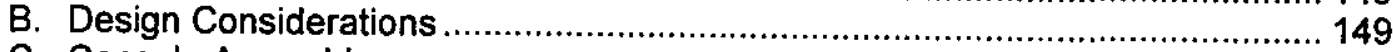

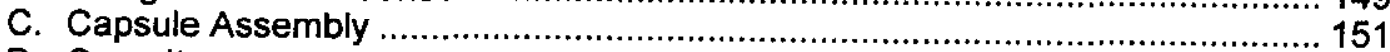

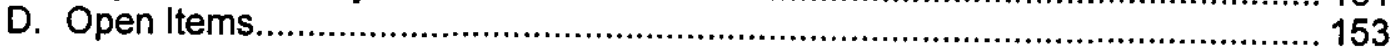

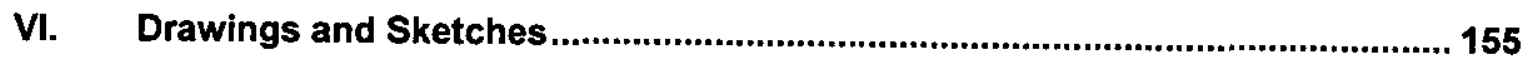

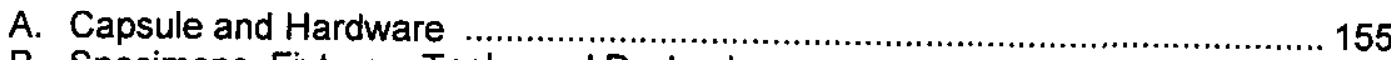

B. Specimens, Fixtures, Tools, and Packaging ............................................... 209

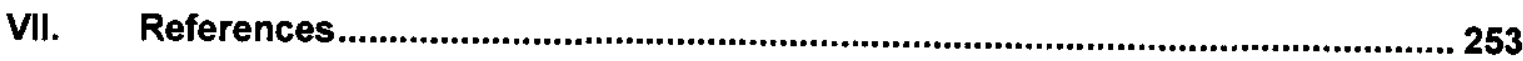

Attachments to Enclosure (7)
A - Adjacent Fuel Affects for JOYO-1 Irradiation Test
B - Review of NRPCT Interpretation of JNC Supplied Gamma Heating Rates
C - Review of NRPCT Interpretation of JNC Supplied Gamma Heating Rates
D - Initial JOYO Initial Design Conditions
E - JOYO-1 Hardware Cleaning Procedure
F - JOYO-1 Hardware Bake-Out Procedure
G - JOYO-1 Pressure and Helium Leak Test Procedure
H - NRPCT JOYO Pressure Boundary Weld Qualification Process 


\section{Description of Test}

\section{A. Statement of the Design}

Based on the analysis performed up to project restructuring, the NRPCT has shown that a viable capsule design capable of housing various material specimens could be fabricated for use in the JOYO reactor. The specific structural, thermal and nuclear analysis in conjunction with a robust quality assurance program would assure capable integrity while maintaining specimen flexibility to support the wide range of material data needs for Project Prometheus.

\section{B. Purpose of Design Report}

This capsule design enclosure defines the design basis, materials (pressure boundary and test specimens), welding requirements, quality control requirements, and shipping requirements that the Naval Reactor Prime Contractor Team (NRPCT) planned to invoke on the NRPCT/JOYO-1 capsule (herein after called the JOYO-1 Capsule). The JOYO-1 capsules were to be installed into the Experimental Fast Flux Reactor JOYO located at the O-arai Engineering Center of the Japan Nuclear Cycle Development Institute (JNC). JNC agreed following a Design for Permission presentation (Reference l-1) that the design basis, materials, and welding, quality control, and shipping requirements as stated in the Design for permission report and summarized below were acceptable.

The capsule design conditions include the various capsule Service Level Conditions. The most limiting case is presented in this report. The limiting case is a simultaneous failure of the three biaxial creep specimens in a BCD capsule at the highest reactor power with corresponding highest sodium temperature and flow conditions and releasing the biaxial creep specimen internal pressure to the capsule internal gas volume. The BCD capsule is illustrated in Figure I.B-1 and shown on Sketch SK-AMT-2214 in Section VI.A of this Enclosure. The limiting case includes:

- A combination of three pressurized biaxial specimens that numerically add up to 41.0 MPa (6,000 psi) absolute.

- Pressure boundary wall temperatures calculated using:

- A Compartment 2, Tier 3 BCD capsule with a JOYO gamma heat rate 1.64 times higher than normal operation. The 1.64 factor includes a 1.25 gamma heating factor including uncertainty times a 1.05 hot spot factor times a 1.25 Loss of Flow factor $(1.25 \times 1.05 \times 1.25=1.64)$. Both the gamma heating uncertainty and the safety factor were provided to NRPCT following the design for permission meeting in June 2005 NRPCT/JNC/PNNL meeting.

- The gamma heating rates, called Revision 2, were used in the thermal analysis.

- Bulk sodium temperatures using a 1.64 factor: $353^{\circ} \mathrm{C}$ entering compartment 2 and $452^{\circ} \mathrm{C}$ existing.

Section III.A documents the nuclear heat rate calculation basis used for the last capsule design iteration. Nuclear heat rates are sometimes called "gamma heat" even though the calculated values include all nuclear heating contributions (neutrons and gammas). Three sets of nuclear heat rate calculations were used in the design process. The initial scoping 
basis was designated Revision 0 and the two design iteration sets were designated Revisions 1 and 2. Only the Revision 2 heat rate values are included in this report.

A report from PNNL (Attachment A to this enclosure) identifies that neither the test specimen materials described in Enclosures 1 through 5 nor the capsule configuration within the SMIR will create adverse reactions with adjacent JOYO modules. The evaluations demonstrate that the main effect on the JOYO core of the SMIR structural material irradiation tests, including the small amounts of low atomic weight materials, will be a small decrease in adjacent fuel pin powers compared to the reference radial reflector subassemblies. This small negative effect should satisfy concerns over potential JNC licensing issues related to the impact on the core for these tests. Further evaluation by JNC core management will determine if the small negative reactivity effect of the tests will need to be compensated by adjustment of the Cycle 5-6 core loadings.

Section III.B describes the final thermal analysis that was performed. The thermal analysis used the Revision 2 gamma heat values described in Section III.A. This analysis represents the $2^{\text {nd }}$ of two major design iterations beyond the initial scoping basis design. An iteration was completed once a design was established that met specimen temperature functional requirements. Since gamma heat rates are perturbed by changes in either dimensions or materials, each design required a new nuclear calculation to feedback into the thermal and mechanical design.

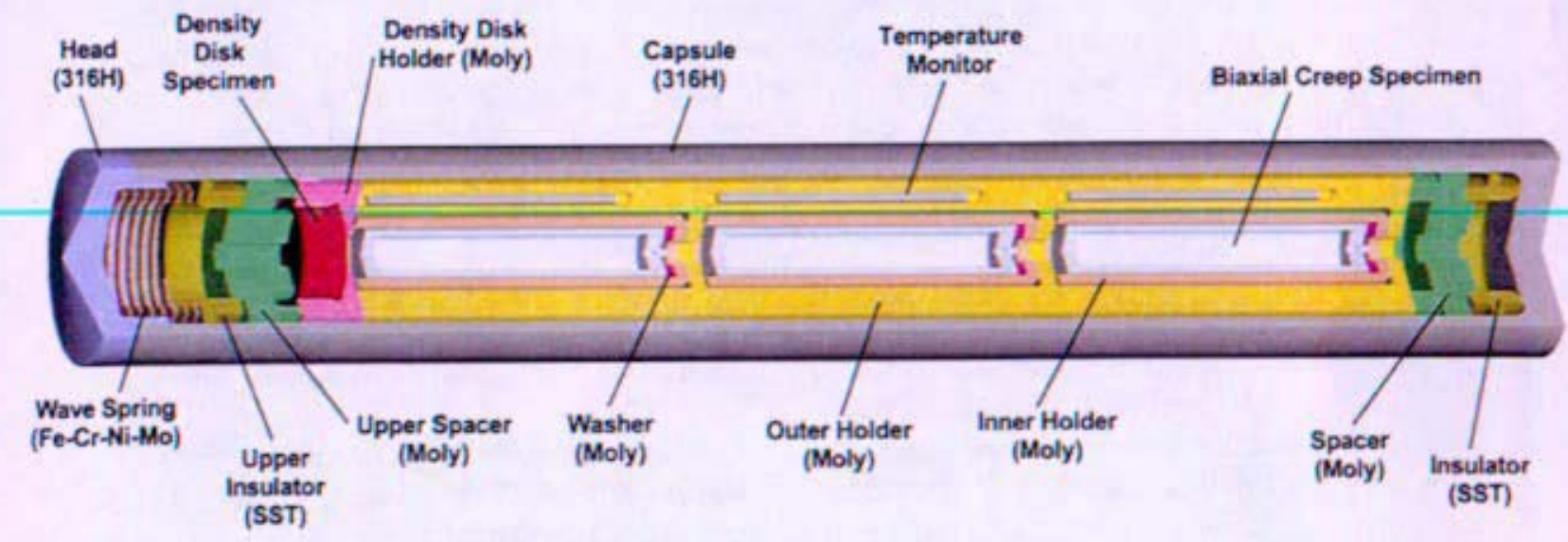

Figure I.B-1 JOYO BCD Capsule

Section III.C describes the structural analysis performed on the limiting capsule design. The design basis for pressure boundary adequacy is the American Society of Mechanical Engineers (ASME) Boiler and Pressure Vessel Code, Code Case N-253-13 that provides rules for an ASME Section III, Class 3 component at elevated temperatures. The ASME Code classification selection and exceptions with calculations are provided in Section III.C. The capsule is designed for an internal pressure of $6.55 \mathrm{MPa}$ at $455^{\circ} \mathrm{C}\left(950 \mathrm{psig}\right.$ at $\left.850^{\circ} \mathrm{F}\right)$.

Since the ASME Code Case N-253-13 will be used as the design basis, the material must conform to the specification, grade, class, and type requirements per Tables $\mathrm{C}-1.0$ of Code Case N-253-13. Therefore, ASME SA-479, Type $316 \mathrm{H}$ or Type 316 (which meets 
supplemental requirements of ASME SA-479) was chosen. Capsule pressure boundary material is discussed in Section II.C.1 and III.C.3.

Section IV describes the capsule assembly, welding, post weld inspections, quality control plan, and shipping requirements. The assembly strategy was developed for all 60 of the JOYO capsules plus ten spares. A rig worth of capsules were to be assembled in order of the type of gas entrained in the capsule. Other elements for capsule assembly included cleaning, bake-out and temporarily storing capsule components and specimens. Final assembly and welding of the capsules were to be performed in a glove box with stringent moisture and oxygen controls. The capsule welding qualification is performed per ASME Section IX and ASME Section III and is described Section IV.C. Capsule assembly would be documented in a route card recording serial numbers of the capsule, internal components and specimens as well as all the post weld inspections.

The quality control plan includes requirements and defines specific personnel and qualifications to perform reviews of material certifications, welding inspections (including visual, dimension, liquid penetrant, partial radiographs on selected capsules, pressure testing and leak testing) and capsule weighing. Section IV.F covers the shipping requirements of the unirradiated capsules from NRPCT through PNNL to JNC which are per Title 49 Code of Federal Regulations. The final destination of the capsules after JOYO irradiation has not been finalized at this time. All considerations from cradle to grave must be considered prior to the start of irradiation in order to ensure that all of Japan's shipping, handling, and disposal laws are followed. In order to ensure Japan's rules are followed, JNC must confirm that the:

- Irradiated materials can be shipped from the JOYO reactor to hot cell facilities within Japan in compliance with Japan's rules.

- Irradiated materials can be properly and safely handled in Japan's hot cells per Japan's rules.

- Irradiated materials can be properly and safely disposed of per Japan's rules.

If the specimens are to be shipped back to the United States, all agreements must be in place to ship the irradiated material per Japan's and United States' rules. 
Enclosure (7) to

MDO-723-0044/

B-MT(SRME)-52

Page 4

THIS PAGE INTENTIONALLY BLANK 


\section{In-Core Hardware}

The JOYO MK-III core (Figure II-1) with a fuel height of $50 \mathrm{~cm}$ contains a maximum of 85 hexagonal shaped fuel assemblies, 6 control rods, and various irradiation test rigs. The fuel is surrounded by reflectors and shielding. NRPCT would have used two Structural Material Irradiation Rigs (SMIR) located in JOYO Core, Cycle 5, positions 6A4 and 6D4 as shown in Figure II-2. Within each SMIR are seven cylindrical compartments with six compartments located in a hexagonal ring surrounding the center compartment. Within each compartment are 5 capsules arranged vertically which are called tiers with the tier 1 capsule on the bottom and the tier 5 capsule on top. The tier 3 capsule is centered with the core fuel midplane. The 5 capsule tiers and 6 compartments allowed for thirty capsules in each rig for a total of sixty capsules to be tested simultaneously using two SMIRs.

The six compartments were classified into radial positions within the SMIR in decreasing flux levels to assign capsules for the specimen matrix. The filled triangle near the lower left compartment C1 in Figure II-2 represents the SMIR hex key which properly orients the SMIR in the JOYO core. There are ten capsule types to accommodate the various specimen material and geometric forms.

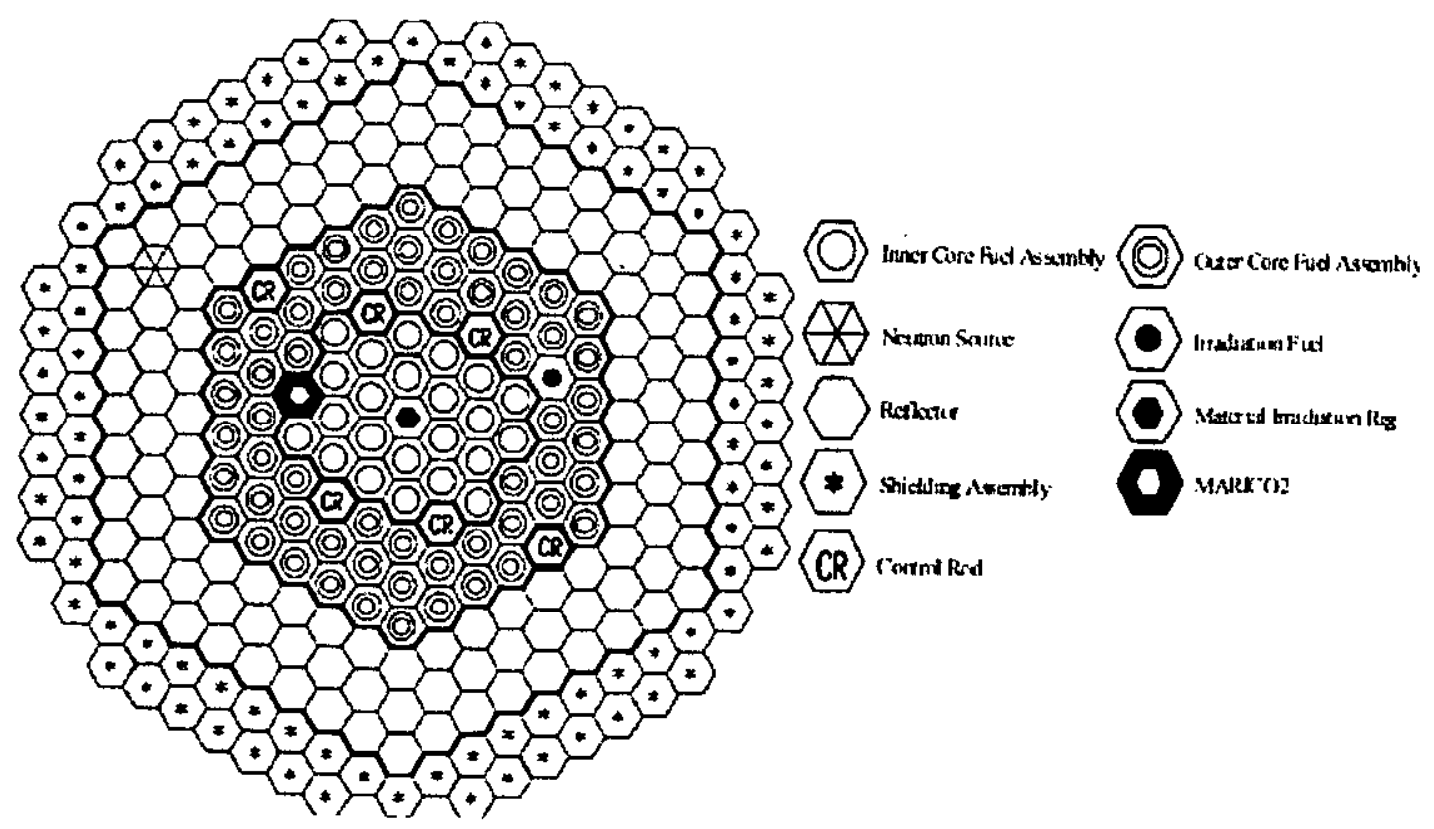

Figure II-1 JOYO Core Configuration, Cycle 5 
Enclosure (7) to

MDO-723-0044/

B-MT(SRME)-52

Page 6

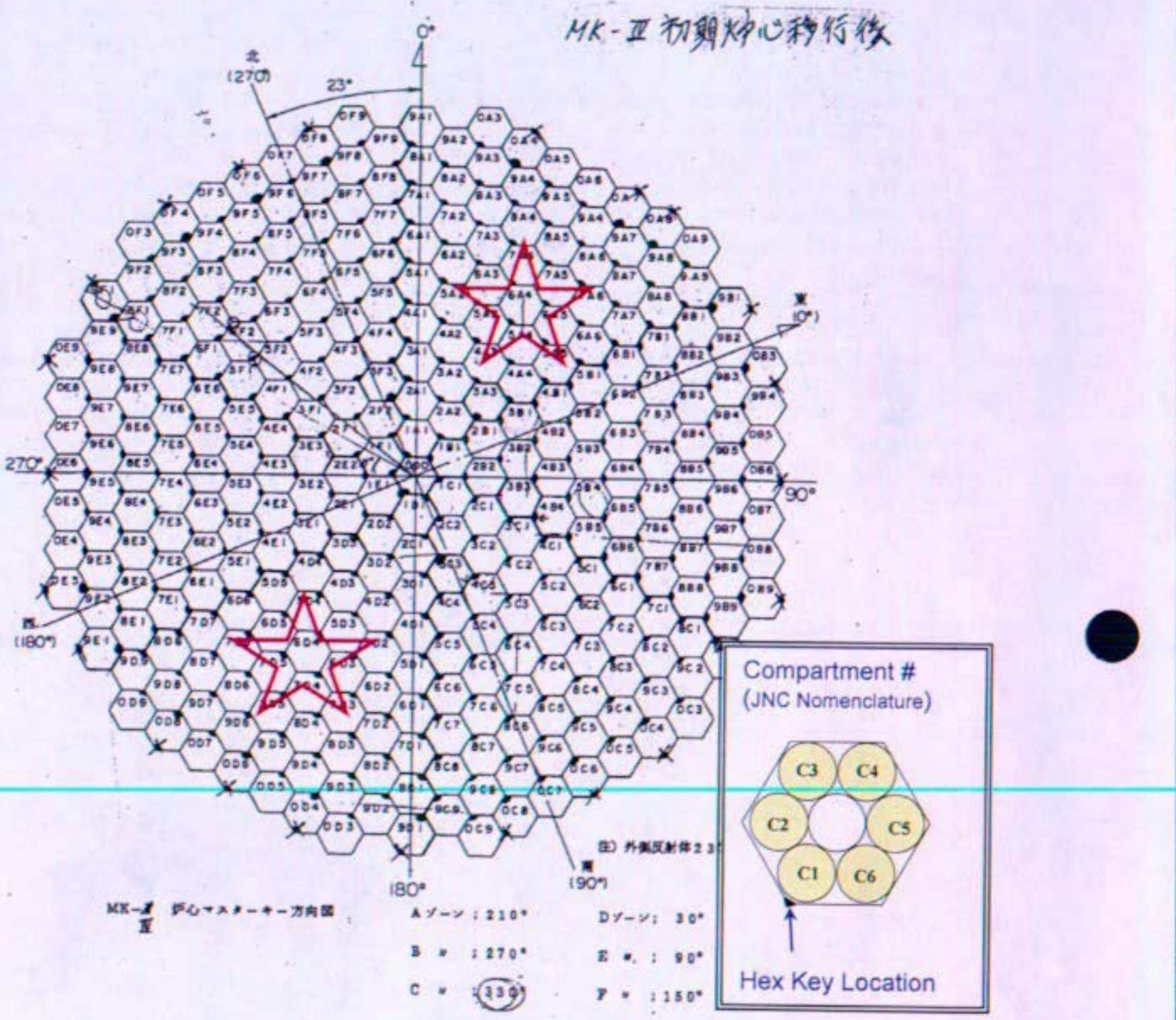

Figure II-2 - JOYO Core locations for JOYO-1, 6A4 \& 6D4

\section{A. Structural Material Irradiation Rig}

The SMIR is a standard JOYO hexagonal shaped stainless steel assembly (Figure II.A-1) designed to allow test capsule placement in specific flux ranges within JOYO. Each SMIR has seven compartments with six spaced as a hexagonal ring encompassing a center compartment. JNC identified that the center compartment would be either a tie bolt to secure the rig or would be hollow to allow for additional test capsules. Based on the estimated weights provided by NRPCT, the weight was low enough that JNC could use the center compartment for additional capsule tests and possibly some dosimetry.

The compartment ( $24.7 \mathrm{~mm}$ inside diameter) provided an available test length of $1857 \mathrm{~mm}$. The 5 capsule lengths sum to $833 \mathrm{~mm}$. Although more capsules could have been included 
Enclosure (7) to

MDO-723-0044/

B-MT(SRME)-52

Page 7

for testing, the flux drops sharply beyond the core height of $500 \mathrm{~mm}$ thus the total fluence for capsules beyond what the NRPCT established would have been lower than desired. The length of the capsule was chosen to 1) allow the capsules to be machinable (minimize the length to diameter ratio to ease inside capsule machining), 2) maximize the number of specimens to fit within the capsules, and 3 ) minimize the fixed lengths. Fixed lengths include the pressure boundary thickness in the capsule head/body and include space for insulators, spacers, and a spring. The insulators and spacers help reduce thermal end losses thus reducing the thermal gradient over the specimens. To position the capsules vertically inside the compartment and meet the requirement that the tier 3 capsule be centered with the core midplane, spacers would be required above and below the NRPCT capsules as shown in Figure (II.A-1).

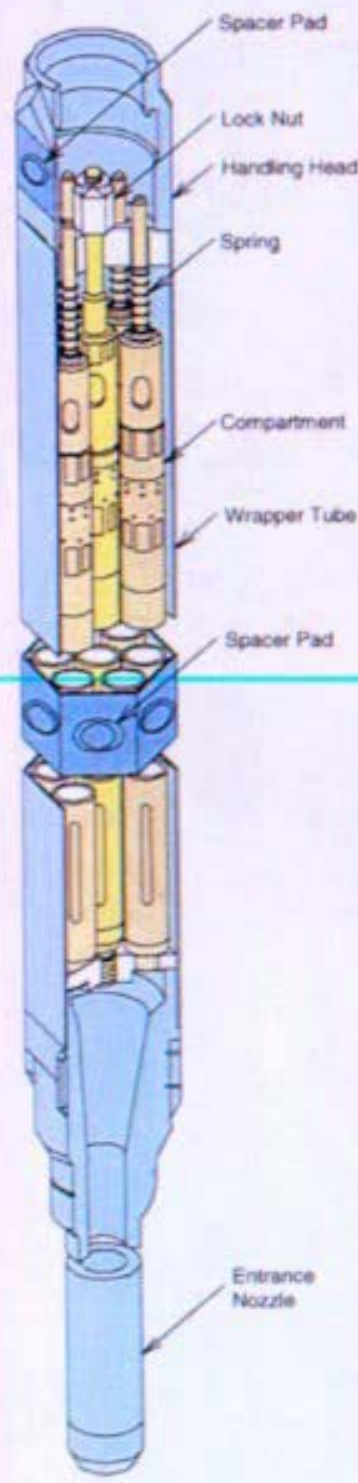

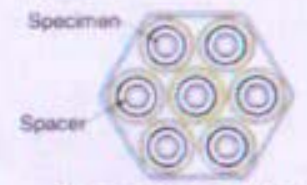

Cross section of Structure Materials Irradiation Rig (SMIR)

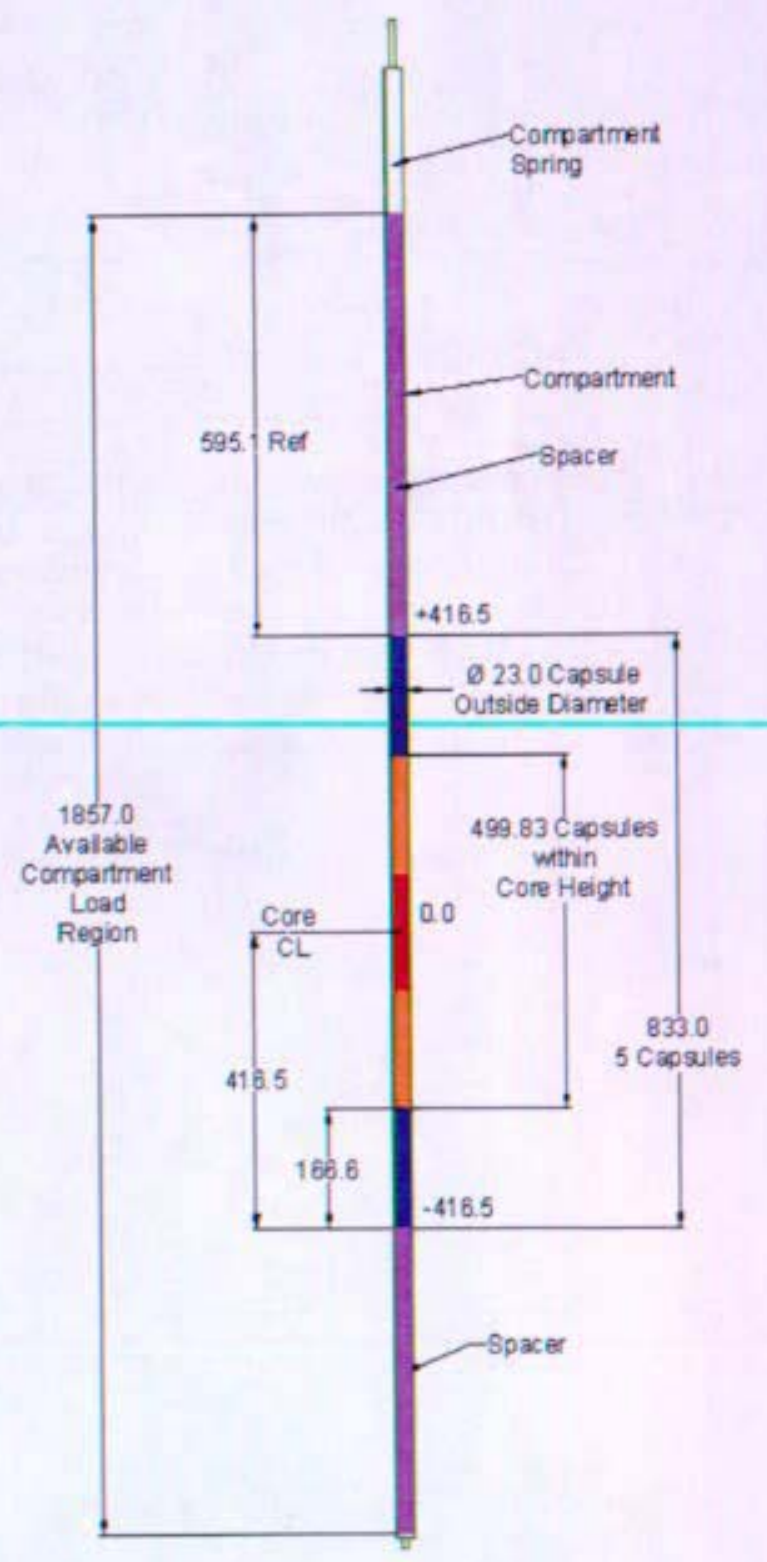

Figure II.A-1 SMIR and Compartment 
Enclosure (7) to

MDO-723-0044/

B-MT(SRME)-52

Page 8

The six compartments were classified into radial positions within the SMIR in decreasing flux levels as shown in Table II-1. For example, compartment "C1" will contain the capsules that are closest to the JOYO core to achieve the highest fluence within the rig. Similarly, compartment "C4" will contain the capsules furthest from the core to achieve the lowest fluence within the rig. Four zones exist of similar flux levels within each rig/tier combination. Hence compartment "C2" and "C6" will be very similar in flux/fluence as will compartments $\mathrm{C} 3$ and $\mathrm{C} 5$. This compartment designation corresponds to the JNC compartment designations. NRCT interpretation of JNC-supplied (March 2005) neutron flux and gamma heating data was confirmed by PNNL as documented in Attachment B to this enclosure.

\begin{tabular}{|c|}
\hline Table II-1 Compartment Nomenclature \\
\hline C1 (highest flux) \\
\hline $\mathrm{C} 6$ \\
\hline $\mathrm{C} 2$ \\
\hline $\mathrm{C} 5$ \\
\hline $\mathrm{C} 3$ \\
\hline C4 (lowest flux) \\
\hline
\end{tabular}

Figures of the SMIR (Figure II.A-2) and compartment (Figure II.A-3) were provided by JNC to NRPCT. SMIR fabrication was contracted to JNC on May 19, 2005. PNNL facilitated contract placement with JNC on behalf of NRPCT. JNC was to fabricate sufficient parts such as handling heads, wrapper tubes, nozzles, compartments, and spacers to support SMIR assembly by March 2006 . This would allow test capsule installation supporting a June 2006 test commencement. 
Enclosure (7) to

MDO-723-0044/

B-MT(SRME)-52

Page 9

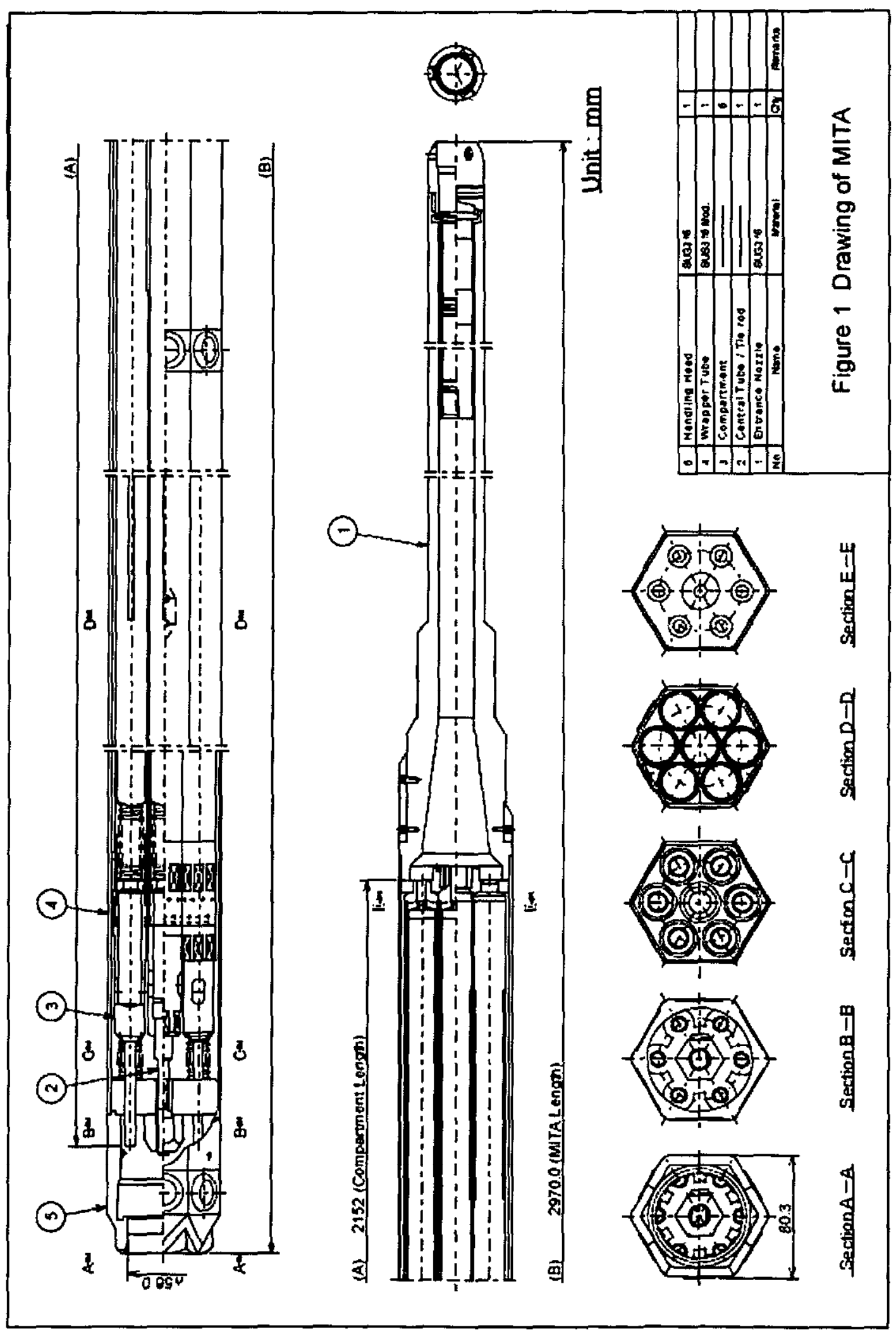

Figure II.A-2 Sketch of Rig provided by JNC 
Enclosure (7) to

MDO-723-0044/

B-MT(SRME)-52

Page 10

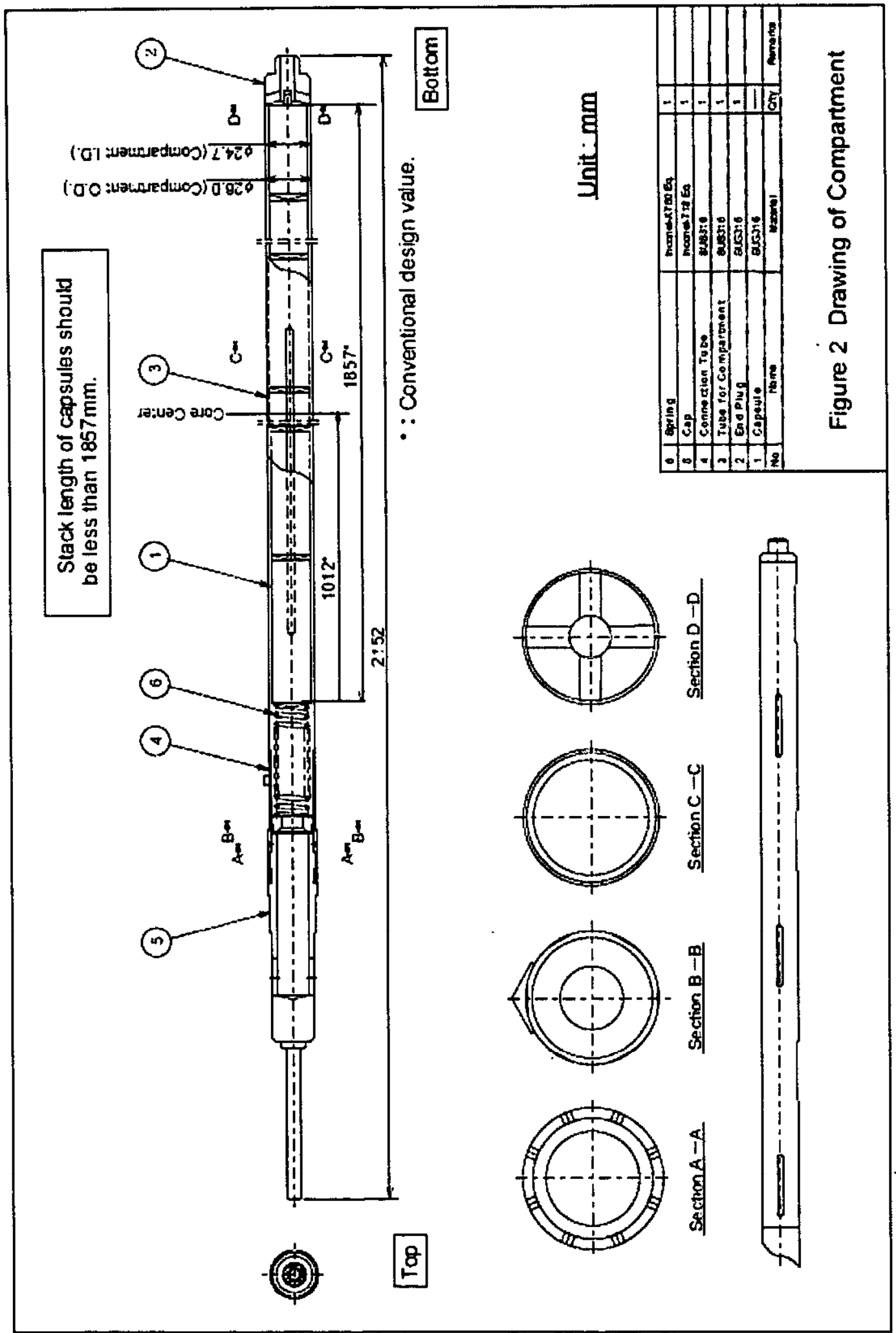

Figure II.A-3 Sketch of Compartment provided by JNC 


\section{B. Capsule Types}

The number of specimen types, the material compatibility requirements within a particular capsule, and the numerous desired temperature/fluence combinations necessitated utilization of several capsule types. A total of ten different capsule types were selected to balance the material radiation effects needs and the desire to minimize capsule design, analyses, and assembly logistics. The ten capsule types are presented in Figure II.B-1 through II.B-10 of which 3 are mirror images of other capsules. The mirror images are included to effectively use the flux profile with respect to the specimen types within the capsule (e.g., biaxial creep samples are of primary interest for most of the metals) within the lowest and highest capsule axial elevations within the SMIR). This is done because of the strong axial gradients of neutron flux (fluence) in the out-of-core elevations.

The capsule types are designated with three digit mnemonic codes $(B C D, D B U, D B D$, A5U, A5D, SCA, NBU, NBD, BSR, and TFT) and the arrangement of each capsule within each of two rigs is indicated in Tables II.B-1 and II.B-2.

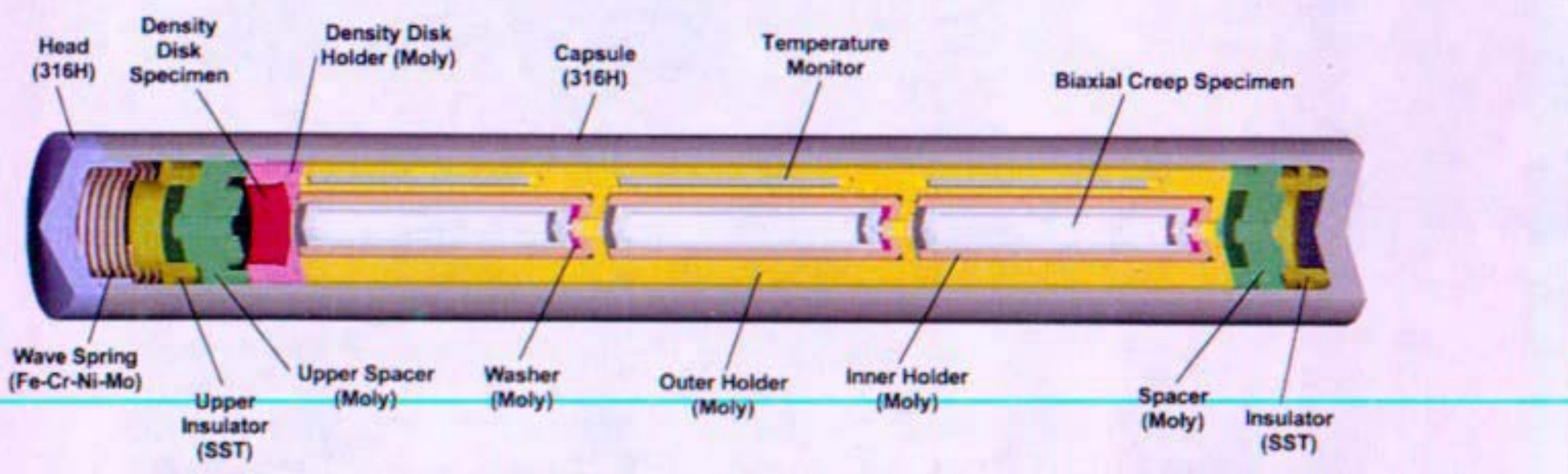

Figure II.B-1 BCD Capsule

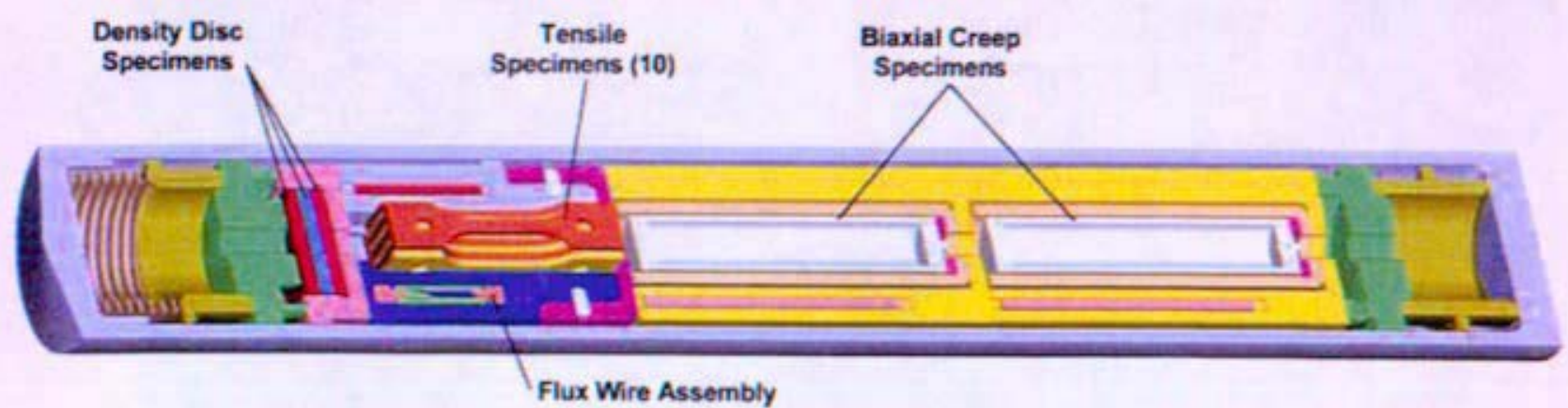

Figure II.B-2 DBU Capsule 
Enclosure (7) to

MDO-723-0044/

B-MT(SRME)-52

Page 12

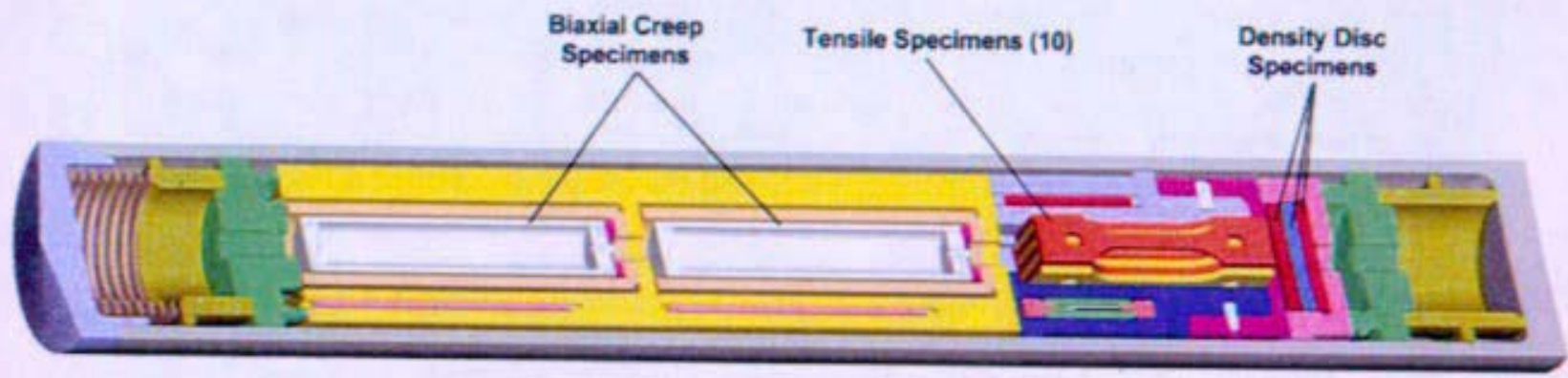

Figure II.B-3 DBD Capsule

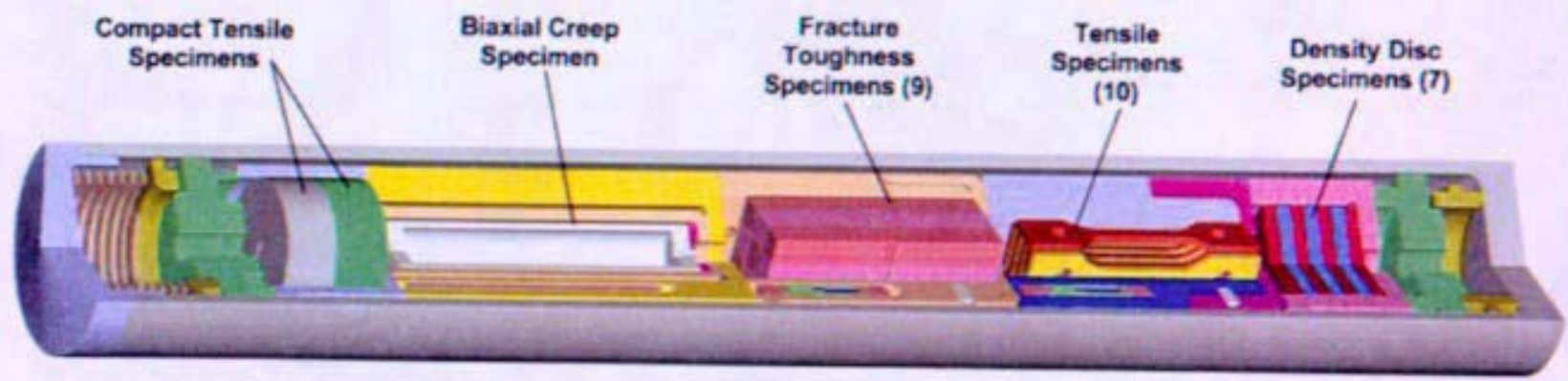

Figure II.B-4 A5U Capsule

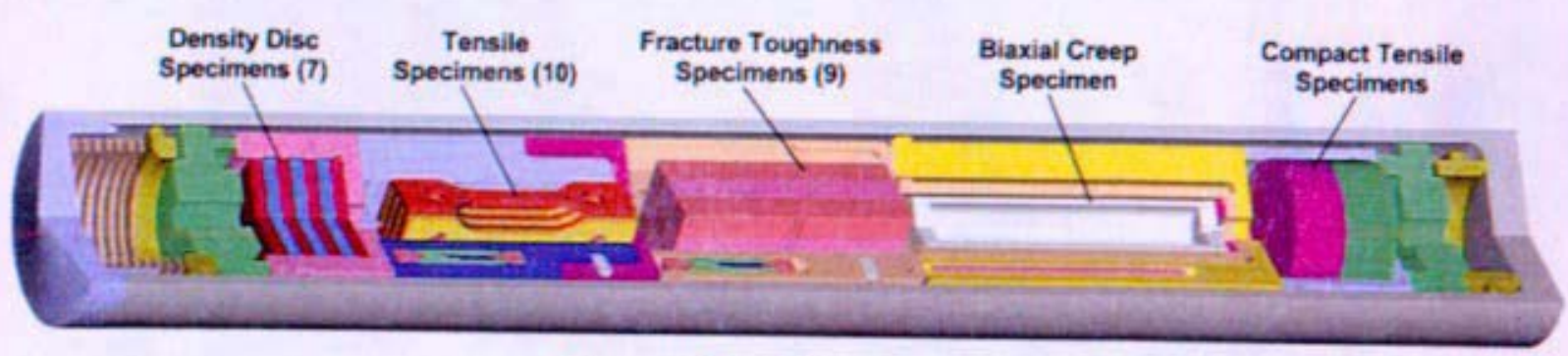

Figure II.B-5 A5D Capsule 


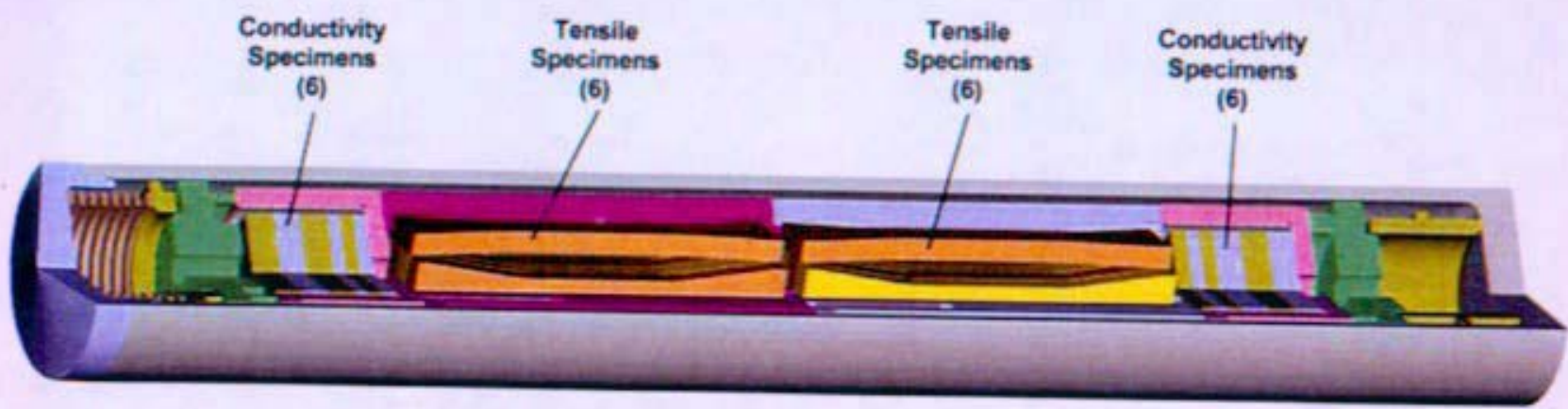

Figure II.B-6 SCA Capsule

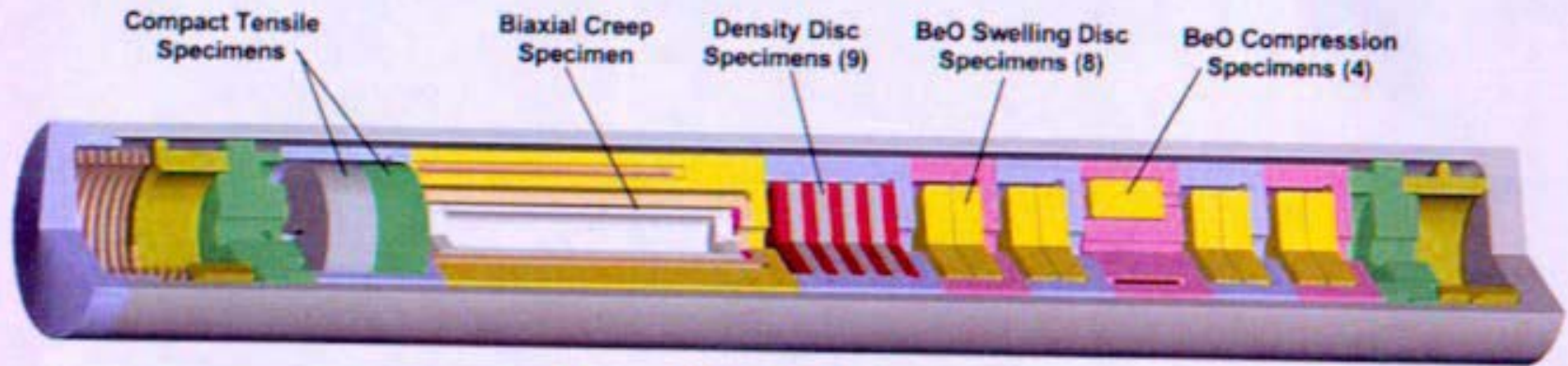

Figure II.B-7 NBU Capsule

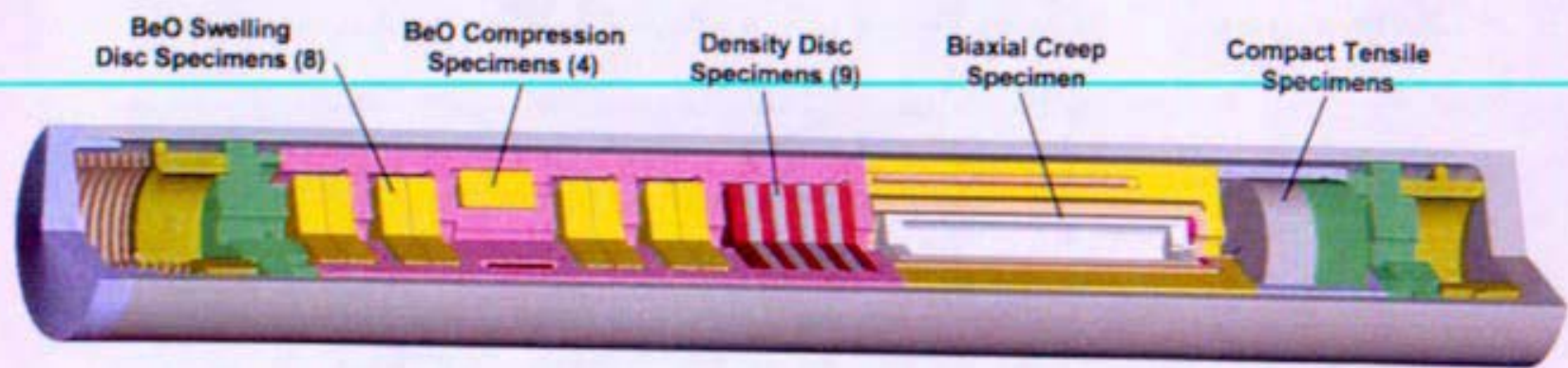

Figure II.B-8 NBD Capsule

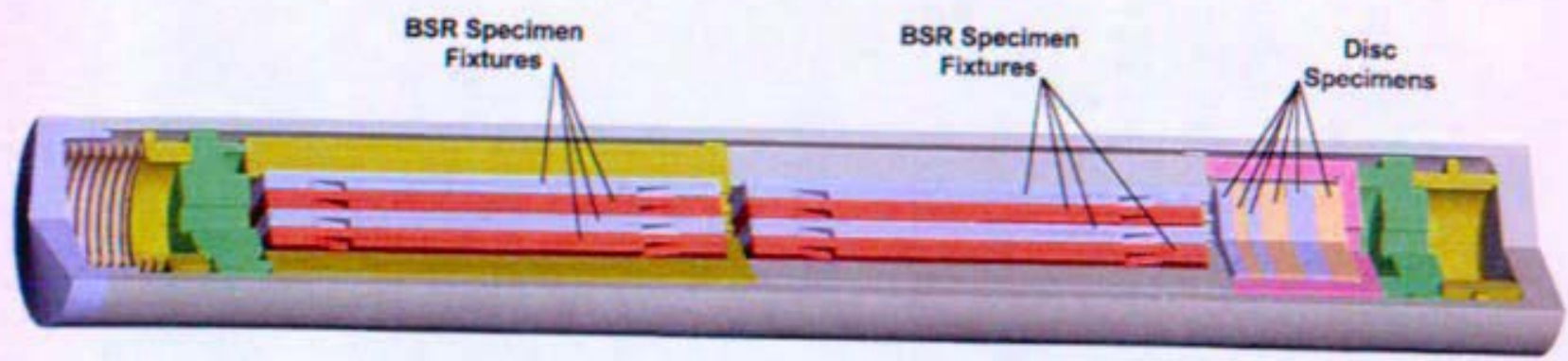

Figure II.B-9 BSR Capsule 
Enclosure (7) to

MDO-723-0044/

B-MT(SRME)-52

Page 14

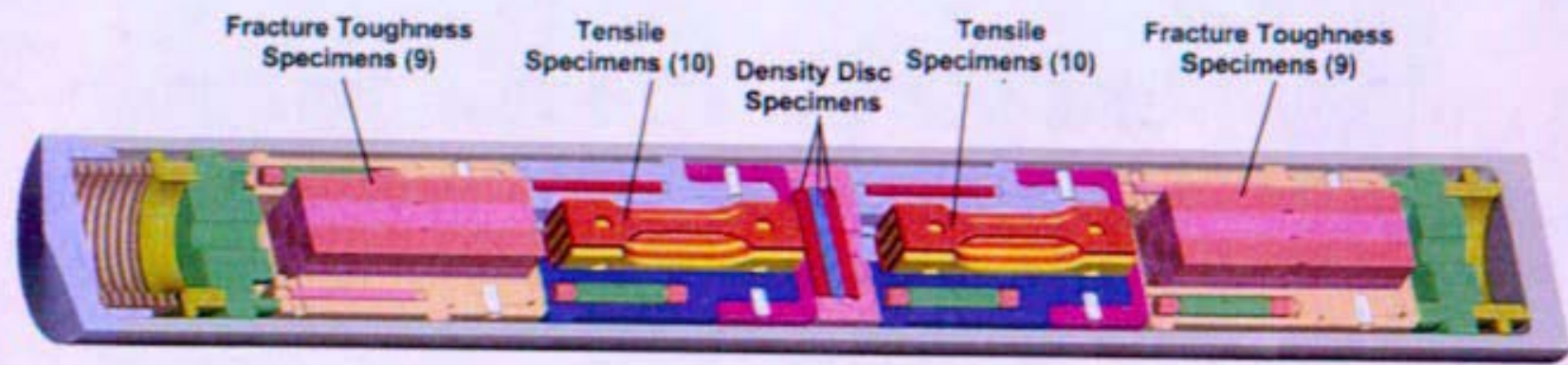

Figure II.B-10 TFT Capsule

\begin{tabular}{|c|c|c|c|c|c|c|}
\hline \multicolumn{7}{|c|}{ Table II.B-1 } \\
\hline \multirow{2}{*}{$\begin{array}{l}\text { Capsule } \\
\text { Elevation }\end{array}$} & \multicolumn{6}{|c|}{ Compartment $^{1}$} \\
\hline & C1 & $\mathrm{C} 6$ & C2 & C5 & C3 & C4 \\
\hline Tier 5 & A5D & NBD & A5D & $A 5 D$ & DBU & DBU \\
\hline Tier 4 & SCA & $\mathrm{A} 5 \mathrm{U}$ & $B C D$ & $B C D$ & DBU & $\mathrm{BCD}$ \\
\hline Tier 3 & SCA & A5U & $B C D$ & $B C D$ & DBU & $\mathrm{BCD}$ \\
\hline Tier 2 & TFT & A5U & $B C D$ & DBU & $\mathrm{BCD}$ & DBU \\
\hline Tier 1 & $\mathrm{~A} 5 \mathrm{U}$ & NBU & A5U & SCA & DBD & DBD \\
\hline
\end{tabular}

${ }^{1}$ Arranged in order of decreasing neutron flux (i.e., C1 has highest flux and C4 has lowest flux)

\begin{tabular}{|c|c|c|c|c|c|c|}
\hline \multicolumn{7}{|c|}{ Table II.B-2 } \\
\hline \multirow{2}{*}{$\begin{array}{l}\text { Capsule } \\
\text { Elevation }\end{array}$} & \multicolumn{6}{|c|}{ Compartment ${ }^{2}$} \\
\hline & C1 & C6 & $\mathrm{C} 2$ & C5 & $\mathrm{C} 3$ & C4 \\
\hline Tier 5 & TFT & NBU & A5D & $B C D$ & $\mathrm{BCD}$ & A5D \\
\hline Tier 4 & BSR & TFT & $B C D$ & A5U & DBU & DBU \\
\hline Tier 3 & TFT & DBD & A5D & $B C D$ & DBD & DBD \\
\hline Tier 2 & A5D & A5D & $B C D$ & BSR & DBD & DBD \\
\hline Tier 1 & TFT & NBU & A5U & $B C D$ & $B C D$ & A5U \\
\hline
\end{tabular}

${ }^{2}$ Arranged in order of decreasing neutron flux (i.e., C1 has highest flux and C4 has lowest flux). 


\section{Capsule Hardware}

\section{Description}

The typical capsule hardware is shown in Figure C.1-1. Each of the ten capsule types has this same basic hardware. What differentiates each capsule is the type of specimens and specimen holders that each capsule has.

Figure II.C.1-1 Typical Capsule Hardware

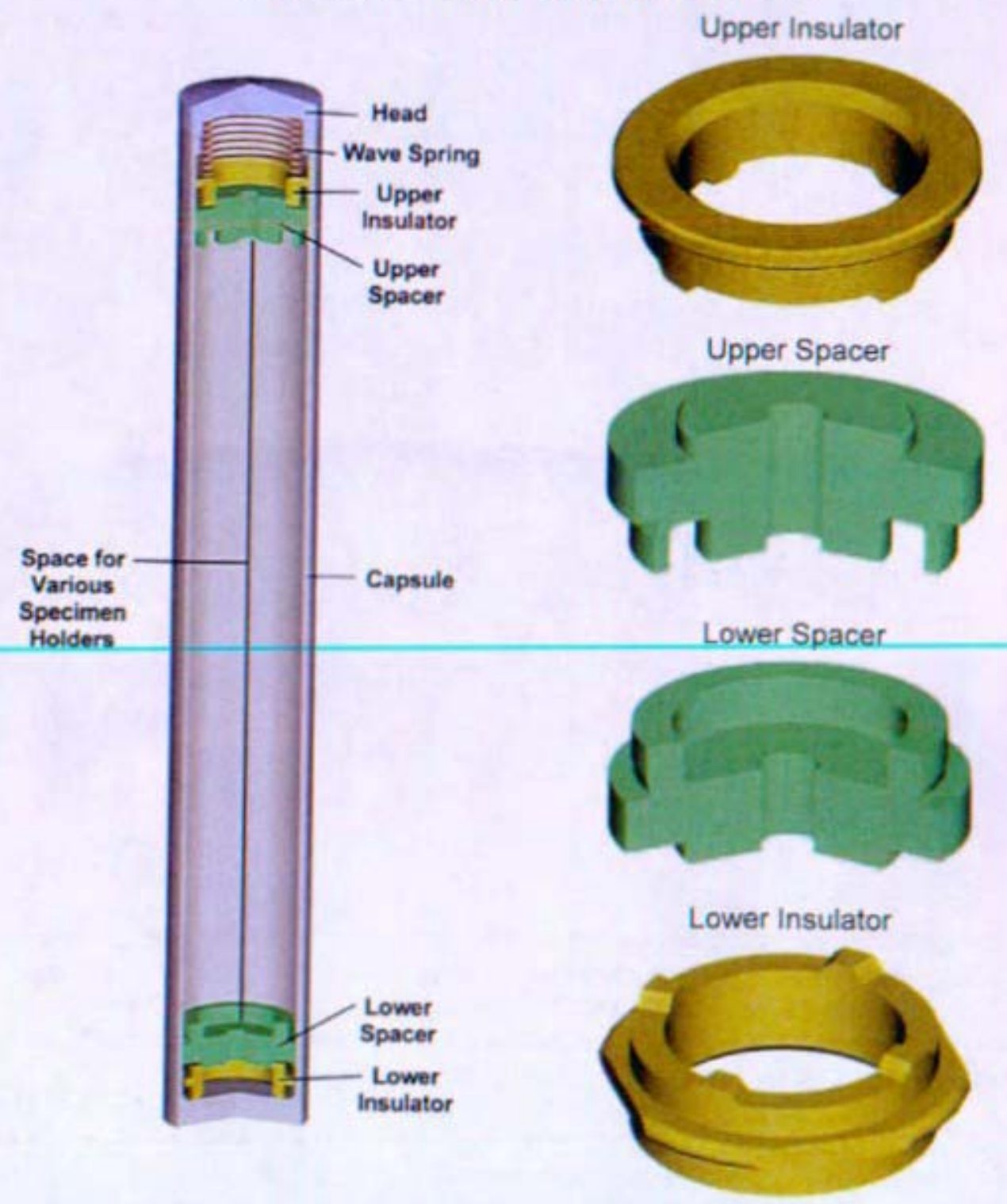

Shown in Figure II.C. 1-2 is the JOYO-1 capsule that consists of a cylindrical shaped Stainless Steel shell and is capped by a Stainless Steel head (Figure II.C.1-3). Both the capsule and head are manufactured from ASME SA479, Type $316 \mathrm{H}$, Annealed, Bar. The capsule has a fillet radius between the shell and base to minimize stress concentrations. The capsule head is cone shaped to provide shedding of the sodium coolant. The head 
also has a fillet radius between the side wall and head to minimize stress concentrations. An integral backing ring is included in the head to provide alignment between the head and capsule during welding. The capsule and head are welded together in an inert atmosphere and inspected as described in Section IV.

Figure II.C.1-2 Capsule Shell Shell

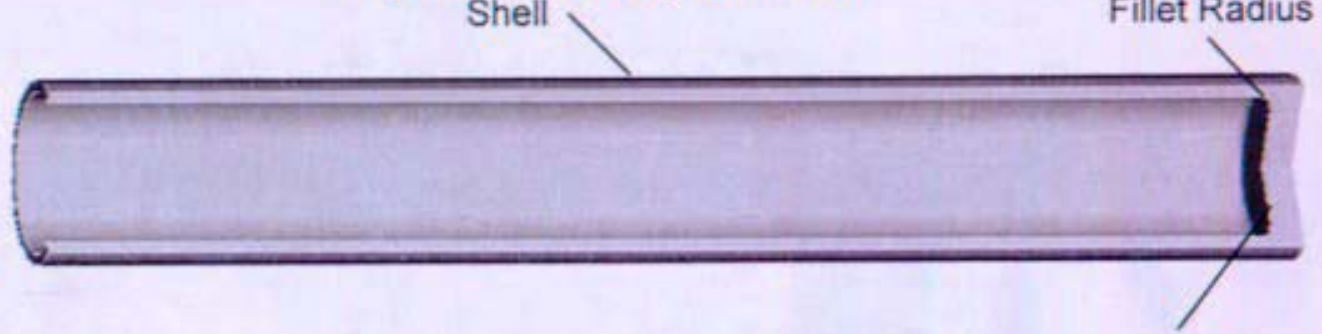

Base

Figure II.C.1-3 Capsule Head

Fillet Radius
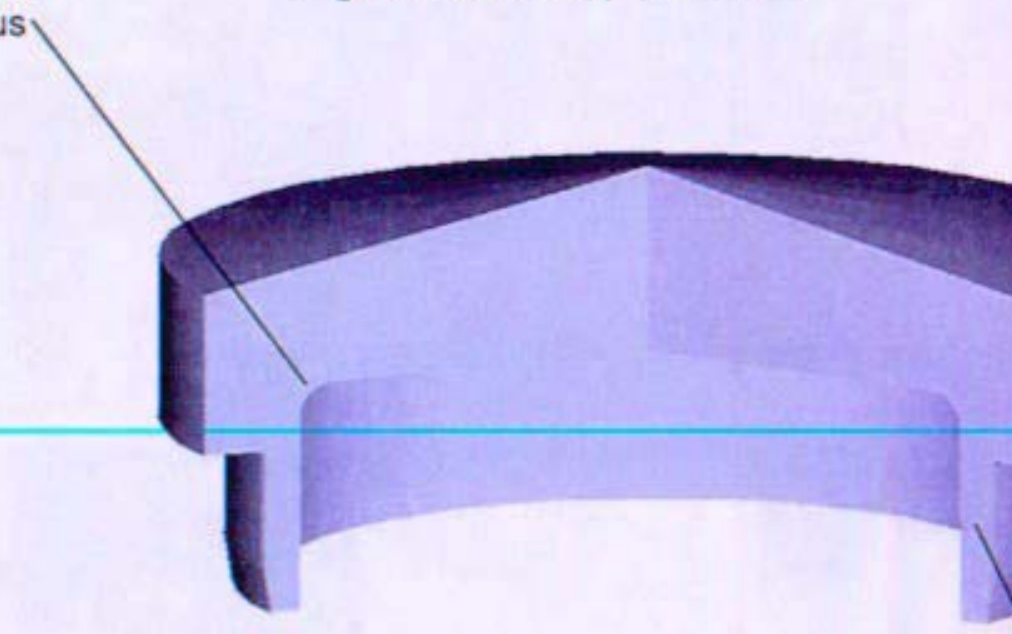
Enclosure (7) to

MDO-723-0044/

B-MT(SRME)-52

Page 17

Similar to the bottom of the capsule, an upper spacer (made from ASTM B387, Type 361 , unalloyed powdered metallurgy molybdenum bar) and an upper insulator (made from ASTM A276, Type 316, Condition A, Bar) are used. Several different upper insulator lengths are used to accommodate the various holder lengths. To complete the assembly, a wave spring is used to provide for thermal expansion differences between the inner components and the capsule.

To accommodate the various specimen geometries, the JOYO-1 arrangement uses an assortment of holders. For the metallic specimens, the holders are manufactured from ASTM B387, Type 361, unalloyed powdered metallurgy molybdenum bar. Tables II.C.1-1 through II.C.1.-7 illustrates the different metallic specimen holders. Molybdenum material was chosen for these components since it has a high gamma heating rate which is needed to obtain specimen temperatures, it is also one of the allowable contact materials for specimens, and it is readily machinable. Three tabs on the exterior top and bottom of the holders are used to center the holder with respect to the capsule. The gap between the holder and capsule is adjusted such that the specimen temperature can be controlled. Temperature refinement is possible since heat transfer is a function of the thermal conductivity and distance.

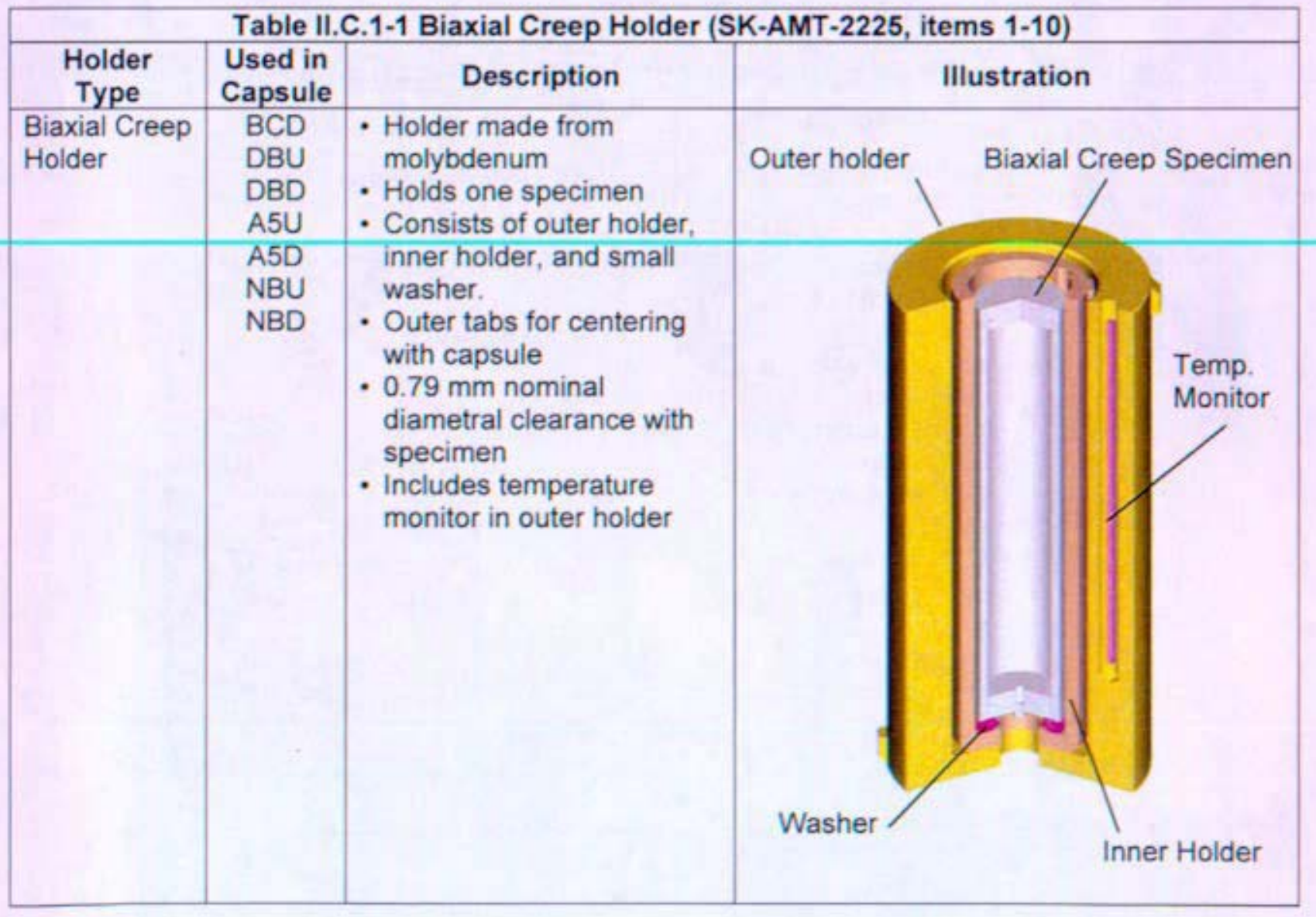


Enclosure (7) to

MDO-723-0044/

B-MT(SRME)-52

Page 18

\begin{tabular}{|c|c|c|c|c|}
\hline \multicolumn{5}{|c|}{ Table II.C.1-2 Tensile Holder (SK-AMT-2226, groups 1-5) } \\
\hline $\begin{array}{c}\text { Holder } \\
\text { Type }\end{array}$ & $\begin{array}{l}\text { Used in } \\
\text { Capsule }\end{array}$ & Description & Illustration & \\
\hline $\begin{array}{l}\text { Tensile } \\
\text { Holder }\end{array}$ & $\begin{array}{l}\text { DBU } \\
\text { DBD } \\
\text { A5U } \\
\text { A5D } \\
\text { TFT }\end{array}$ & $\begin{array}{l}\text { - Holder made from } \\
\text { molybdenum } \\
\text { - Holds ten specimens } \\
\text { - Three part construction } \\
\text { - Outer tabs for centering } \\
\text { with capsule } \\
\text { - } 1.06 \mathrm{~mm} \text { nominal total } \\
\text { clearance across } \\
\text { thickness for } 8 \text { specimens } \\
\text { - } 0.55 \text { mm nominal width } \\
\text { clearance } \\
\text { - One temperature monitor } \\
\text { - One flux wire assembly }\end{array}$ & Metallic Tensile Specimens & $\begin{array}{l}\text { Flux } \\
\text { Monitor }\end{array}$ \\
\hline
\end{tabular}

\begin{tabular}{|c|c|c|c|}
\hline Holder & Used in & 1-3 Fracture Toughness & Holder (SK-AMT-2227, groups 1-5 \\
\hline Type & Capsule & Description & Illustration \\
\hline $\begin{array}{l}\text { Tensile } \\
\text { Holder }\end{array}$ & $\begin{array}{l}\text { A5U } \\
\text { A5D } \\
\text { TFT }\end{array}$ & $\begin{array}{l}\text { - Holder made from } \\
\text { molybdenum } \\
\text { - Holds nine specimens }\end{array}$ & Fracture Toughness Specimen \\
\hline & & $\begin{array}{l}\text { - Three part } \\
\text { construction } \\
\text { - Outer tabs for } \\
\text { centering with capsule } \\
\text { - } 0.6 \text { mm nominal total } \\
\text { clearance across } 3 \\
\text { specimens } \\
\text { - One temperature } \\
\text { monitor } \\
\text { - One flux wire } \\
\text { assembly }\end{array}$ & \\
\hline
\end{tabular}




\begin{tabular}{|c|c|c|c|}
\hline \multicolumn{4}{|c|}{ Table II.C.1-4 Disc Holder (SK-AMT-2228, items 1-16) } \\
\hline $\begin{array}{l}\text { Holder } \\
\text { Type }\end{array}$ & $\begin{array}{l}\text { Used in } \\
\text { Capsule }\end{array}$ & Description & Illustration \\
\hline Disc Holder & $\begin{array}{l}\text { BCD } \\
\text { DBU } \\
\text { DBD } \\
\text { A5U } \\
\text { A5D } \\
\text { NBU } \\
\text { NBD } \\
\text { TFT }\end{array}$ & $\begin{array}{l}\text { - Holder made from } \\
\text { molybdenum } \\
\text { - Holder has three different } \\
\text { lengths to accommodate } \\
\text { three, seven, or nine } \\
\text { specimens } \\
0.43 \mathrm{~mm} \text { nominal } \\
\text { diametral clearance } \\
0.5 \mathrm{~mm} \text { nominal axial } \\
\text { clearance } \\
\text { Outer tabs for centering } \\
\text { with capsule }\end{array}$ & Metallic Disc Specimen \\
\hline
\end{tabular}

\begin{tabular}{|c|c|c|c|}
\hline \multicolumn{4}{|c|}{ Table II.C.1-5 Compact Tensile Holder (SK-AMT-2235, items 1-4) } \\
\hline $\begin{array}{l}\text { Holder } \\
\text { Type }\end{array}$ & $\begin{array}{l}\text { Used in } \\
\text { Capsule }\end{array}$ & Description & Illustration \\
\hline $\begin{array}{l}\text { Compact } \\
\text { Tensile } \\
\text { Holder }\end{array}$ & $\begin{array}{l}\text { A5U } \\
\text { A5D } \\
\text { NBU } \\
\text { NBD }\end{array}$ & $\begin{array}{l}\text { - Holder made from } \\
\text { molybdenum } \\
\text { - Holds two specimens } \\
\text { - } 1.0 \mathrm{~mm} \text { nominal diametral } \\
\text { clearance } \\
\text { - } 0.7 \text { mm nominal axial } \\
\text { clearance } \\
\text { - Outer-tabs for-centering } \\
\text { with capsule }\end{array}$ & Compact Tens \\
\hline & Table II.C. & $1-6 \mathrm{BeO}$ & SK \\
\hline $\begin{array}{l}\text { Holder } \\
\text { Type }\end{array}$ & $\begin{array}{l}\text { Used in } \\
\text { Capsule }\end{array}$ & Description & Illustration \\
\hline $\begin{array}{l}\mathrm{BeO} \\
\text { Compression } \\
\text { Holder }\end{array}$ & $\begin{array}{l}\text { NBU } \\
\text { NBD }\end{array}$ & $\begin{array}{l}\text { - Holder made from } \\
\text { molybdenum } \\
\text { - Holds four specimens } \\
\text { - } 0.5 \mathrm{~mm} \text { nominal diametral } \\
\text { clearance } \\
\text { - } 1 \text { mm nominal axial } \\
\text { clearance } \\
\text { - Outer tabs for centering } \\
\text { with capsule } \\
\text { - One temperature monitor }\end{array}$ & BeO Compressic \\
\hline
\end{tabular}


Enclosure (7) to

MDO-723-0044/

B-MT(SRME)-52

Page 20

Table II.C.1-7 BeO Disc Holder (SK-AMT-2228, items 17-20)

\begin{tabular}{|l|c|l|l|}
\hline $\begin{array}{c}\text { Holder } \\
\text { Type }\end{array}$ & $\begin{array}{c}\text { Used in } \\
\text { Capsule }\end{array}$ & \multicolumn{1}{c|}{ Description } \\
\hline $\begin{array}{l}\text { BeO Disc } \\
\text { Holder }\end{array}$ & $\begin{array}{l}\text { NBU } \\
\text { NBD }\end{array}$ & $\begin{array}{l}\text { - Holder made from } \\
\text { molybdenum } \\
\text { - Holds two specimens } \\
\text { - Outer tabs for centering } \\
\text { with capsule } \\
\text { - Thin sheet of moly } \\
\text { between specimens }\end{array}$ & BeO Disc Specimen \\
\hline
\end{tabular}

For the Ceramic Specimens, tungsten hoiders with graphite between the specimens and the holders are used. The tungsten holders are made from $99.95 \%$ pure tungsten and the graphite liner is .005 thick grafoil. The grafoil liner ensures that the tungsten does not contaminate the ceramic specimens. The ceramic specimen holders are depicted in Tables II.C.1-8 through II.C.1-10 below.

\begin{tabular}{|l|c|c|c|c|}
\hline \multicolumn{4}{|c|}{ Table II.C.1-8 SiC Tensile Holder (SK-RRM1023, item 1,2,3) } \\
\hline $\begin{array}{c}\text { Holder } \\
\text { Type }\end{array}$ & $\begin{array}{c}\text { Used in } \\
\text { Capsule }\end{array}$ & \multicolumn{1}{c|}{ Description } \\
\hline $\begin{array}{l}\text { SiC Tensile } \\
\text { Holder }\end{array}$ & SCA & $\begin{array}{l}\text { - Holder made from } \\
\text { tungsten } \\
\text { - Holds six specimens } \\
\text { - One temperature monitor }\end{array}$ \\
\hline
\end{tabular}




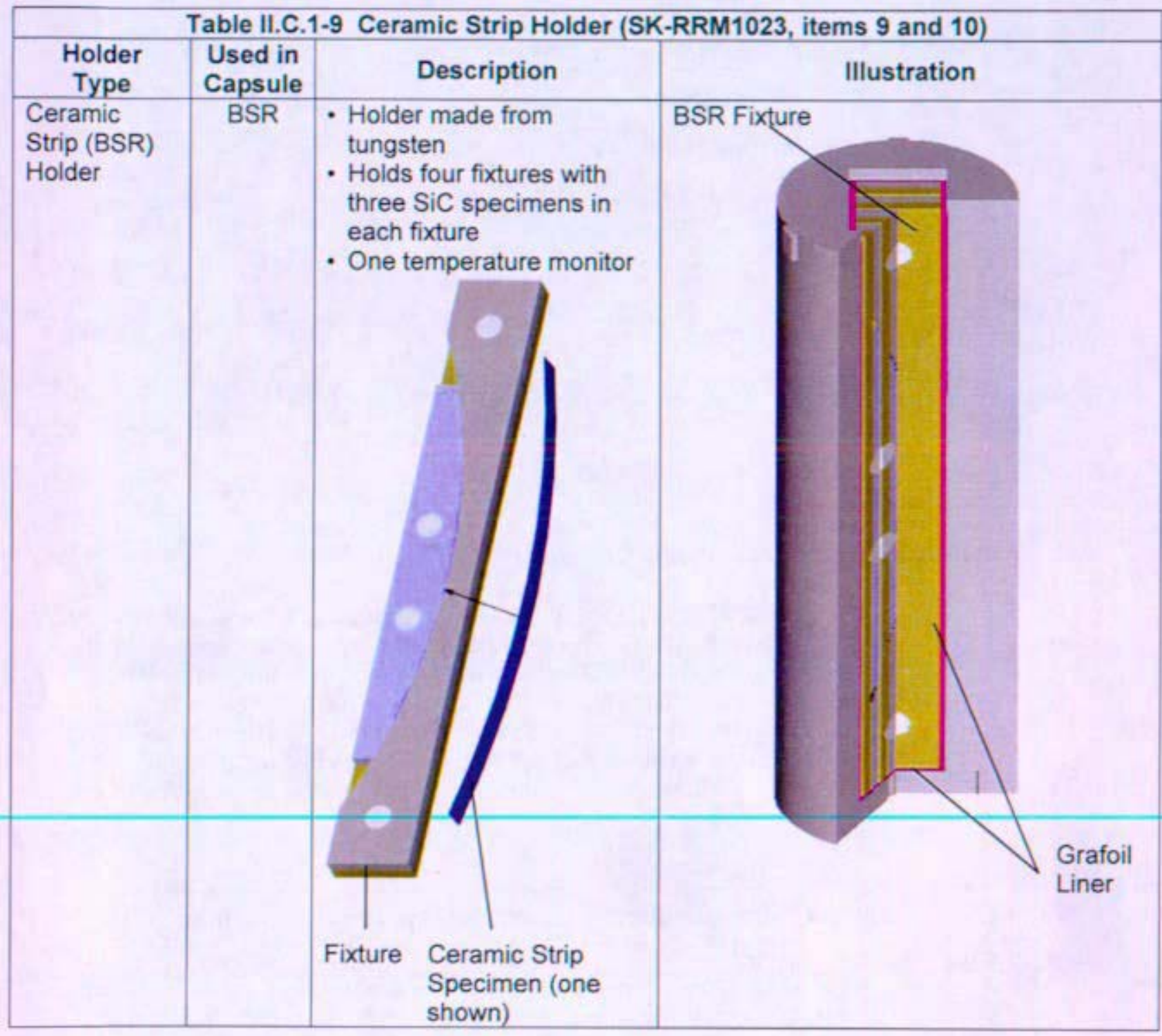


Enclosure (7) to

MDO-723-0044/

B-MT(SRME)-52

Page 22

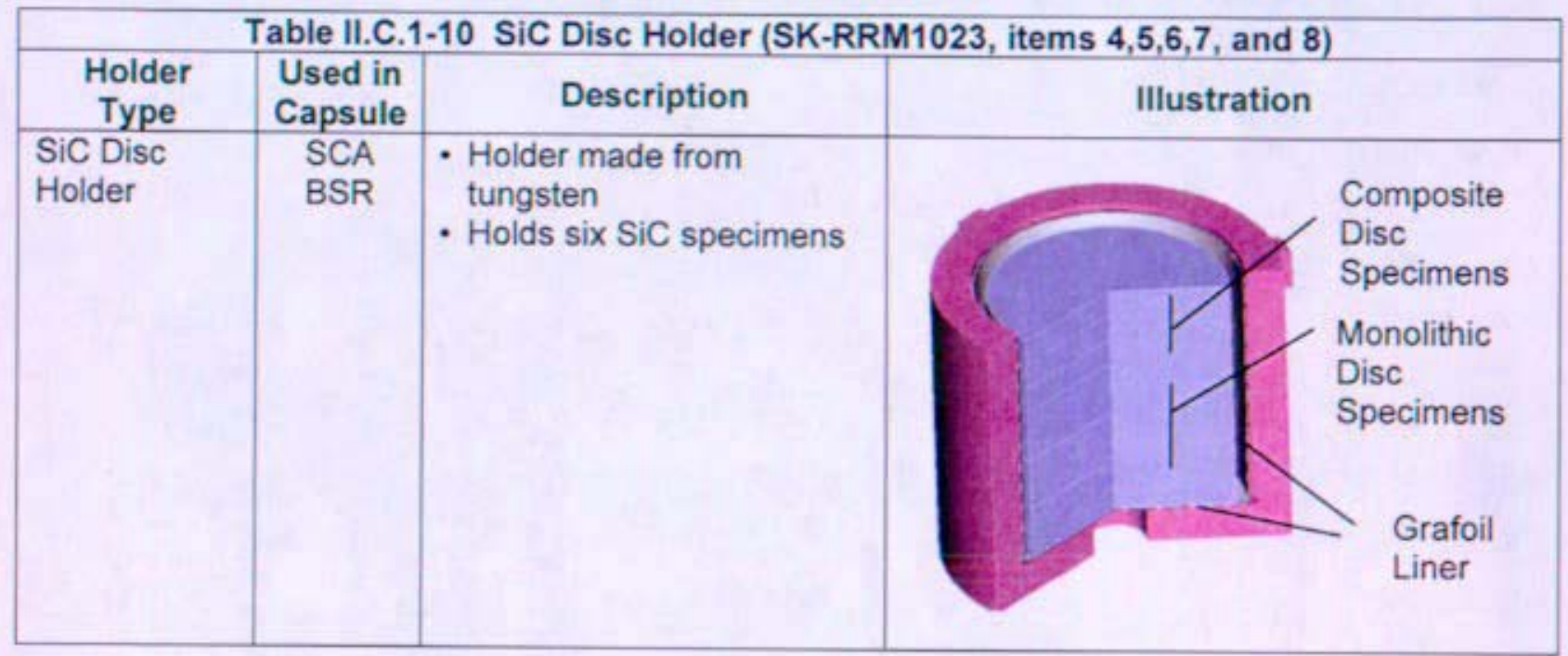

2. Material Descriptions Including Composition.

The JOYO-1 test components exposed to the reactor coolant are comprised of ASME SA 479, Type $316 \mathrm{H}$ stainless steel. The capsule internal hardware components are fabricated from POCO graphite, 316 Stainless Steel, or molybdenum. Several flux wire assemblies that include a Titanium, Nickel, and Iron flux wires are installed in each capsule assembly. Also, in several capsule assemblies are CVD silicon carbide and zirconium carbide rectangular pieces that will be used as passive temperature monitors. Table II.B-2.1 delineates the material specification and composition of each hardware-component

Table II.C.2-1 JOYO-1 Hardware Material Specifications and Composition

\begin{tabular}{|c|c|c|c|c|}
\hline Component & Sketch & Material & Composition $\%$ & Note \\
\hline Capsule & SK-AMT-2221 & \multirow{2}{*}{$\begin{array}{l}\text { Type } 316 \mathrm{H} \\
\text { Stainless Steel }\end{array}$} & \multirow{2}{*}{$\begin{array}{l}0.08 \mathrm{C}, 2 \mathrm{Mn}, 04 \mathrm{P} \\
.03 \mathrm{~S}, 1 \mathrm{Si}, 17 \mathrm{Cr}, 12 \mathrm{Ni} \text {, } \\
2.5 \mathrm{Mo}, 65.35 \mathrm{Fe}\end{array}$} & \multirow[t]{2}{*}{1} \\
\hline Head & SK-AMT-2222 & & & \\
\hline Insulators & $\begin{array}{c}\text { SK-AMT-2224 } \\
\text { Items } 1-7\end{array}$ & $\begin{array}{l}\text { Type } 316 \text { Stainless } \\
\text { Steel }\end{array}$ & $\begin{array}{c}0.08 \mathrm{C}, 2 \mathrm{Mn}, .045 \mathrm{P} \\
.03 \mathrm{~S}, 1 \mathrm{Si}, 17 \mathrm{Cr}, 12 \mathrm{Ni} \text {, } \\
2.5 \mathrm{Mo}, 65.345 \mathrm{Fe}\end{array}$ & 2 \\
\hline Spacers & $\begin{array}{l}\text { SK-AMT-2223 } \\
\text { Items } 1-2\end{array}$ & \multirow[t]{5}{*}{$\begin{array}{l}\text { Molybdenum, Type } \\
365\end{array}$} & \multirow{5}{*}{$\begin{array}{c}.01 \mathrm{C}, .0015 \mathrm{O}, .002 \mathrm{~N} \\
.01 \mathrm{Fe}, .002 \mathrm{Ni}, .01 \mathrm{Si} \\
\text { balance } \mathrm{Mo}\end{array}$} & \multirow[t]{5}{*}{3} \\
\hline $\begin{array}{l}\text { Biaxial Creep Outer \& } \\
\text { Inner Holder, \& washer }\end{array}$ & $\begin{array}{l}\text { SK-AMT-2225 } \\
\text { Items } 1-10\end{array}$ & & & \\
\hline Tensile Holder & $\begin{array}{l}\text { SK-AMT-2226 } \\
\text { Group 1-5 }\end{array}$ & & & \\
\hline $\begin{array}{l}\text { Fracture Toughness } \\
\text { Holder }\end{array}$ & $\begin{array}{l}\text { SK-AMT-2227 } \\
\text { Group } 1-5\end{array}$ & & & \\
\hline BeO Compression Holder & $\begin{array}{l}\text { SK-AMT-2241, } \\
\text { Items } 1-4\end{array}$ & & & \\
\hline
\end{tabular}




\begin{tabular}{|c|c|c|c|c|}
\hline Component & Sketch & Material & Composition \% & Note \\
\hline Disc Holder & $\begin{array}{l}\text { SK-AMT-2228 } \\
\text { Items } 1-20\end{array}$ & & & \\
\hline Compact Tensile Holder & $\begin{array}{l}\text { SK-AMT-2235, } \\
\text { Items 1-4 }\end{array}$ & & & \\
\hline $\begin{array}{l}\text { Tungsten Holders } \\
\text { Tensile } \\
\text { Disc } \\
\text { Ceramic Strip }\end{array}$ & $\begin{array}{l}\text { SK-RRM } 1023 \\
\text { Items } 1-10\end{array}$ & $\begin{array}{l}99.95 \% \text { Pure } \\
\text { Tungsten }\end{array}$ & $99.95 \mathrm{C}$ & 4 \\
\hline Wave Spring & SK-AMT-2239 & Cr-Ni-Mo, Wire & $\begin{array}{l}.7 \mathrm{Mo}, 24.32 \mathrm{Ni}, \\
16.15 \mathrm{Cr}, 56.02 \mathrm{Fe}, \\
2.45 \mathrm{Ti}, .33 \mathrm{~V}, .03 \mathrm{~B}\end{array}$ & 5 \\
\hline Flux Wire Assemblies & SK-RRM-1011 & & & \\
\hline Moly tube & Item 2 & Molybdenum & $99.99 \mathrm{Mo}$ & 6 \\
\hline Moly end plug & Item 1 & Molybdenum & $99.99 \mathrm{Mo}$ & 7 \\
\hline Nickel wire & Item 4 & Nickel & $99.99 \mathrm{Ni}$ & 8 \\
\hline Titanium wire & Item 3 & Titanium & $99.99 \mathrm{Ti}$ & 9 \\
\hline Iron wire & Item 5 & Iron & 99.99Fe & 10 \\
\hline Moly Foil & Item 6 & Moly & $99.95 \mathrm{Mo}$ & 11 \\
\hline $\begin{array}{l}\text { Passive Temperature } \\
\text { Monitor }\end{array}$ & $\begin{array}{c}\text { SK216C9452 } \\
\text { Lengths of } \\
1.299 \text { inch, } \\
1.102 \text { inch, } \\
0.551 \text { inch, } \\
\text { and } 0.276 \text { inch }\end{array}$ & $\begin{array}{l}\text { CVD Silicon } \\
\text { Carbide }\end{array}$ & $99.99 \mathrm{Si}$ & 12 \\
\hline $\begin{array}{l}\text { Passive Temperature } \\
\text { Monitor }\end{array}$ & $\begin{array}{c}\text { SK216C9452 } \\
\text { Lengths of } \\
1.299 \text { inch, } \\
1.102 \text { inch, } \\
0.551 \text { inch, } \\
\text { and } 0.276 \text { inch }\end{array}$ & $\mathrm{ZrC}$ & $99.99 \mathrm{ZrC}$ & 12 \\
\hline $\begin{array}{l}\text { Grafoil Liner for tungsten } \\
\text { holders }\end{array}$ & $\begin{array}{c}\text { SK-RRM1025, } \\
\text { Items 1-7 }\end{array}$ & Graphite & & 13 \\
\hline
\end{tabular}

Table Notes:

1. ASME SA479, BAR, Annealed. The alternate material, ASME SA479, Type 316, Annealed is permitted providing the material meets the ASME SA 479 supplementary requirements.

2. ASTM A 276, 316, BAR, Annealed

3. ASTM B387, Stress Relieved, Bar. The alternate material ASTM B387 Composition 360, 361, 363, or 364 are permitted.

4. $99.95 \%$ Pure Tungsten

5. AMS 5810, Composition UNS S66286, Wire

6. Catalog Number MO007301, Goodfellow Corp, Berwyn, PA

7. Catalog Number MO007901, Goodfellow Corp, Berwyn, PA

8. Catalog Number 10931, Alfa Aesar, Word Hill, MA

9. Catalog Number 10392, Alfa Aesar, Word Hill, MA

10. Catalog Number 10937, Alfa Aesar, Word Hill, MA

11. Catalog Number 10042, Alfa Aesar, Word Hill, MA

12. TBD

13. Grade GTA (nuclear grade) grafoil sheet. $127 \mathrm{~mm}$ (.005 in) thick, Graftech International, Lakewood, $\mathrm{OH}$. 
Enclosure (7) to

MDO-723-0044l

B-MT(SRME)-52

Page 24

\section{Closeout Information}

The following table summarizes the state of the capsule parts that were in the process of being machined as of the time of project closeout.

\begin{tabular}{|c|c|c|c|}
\hline \multicolumn{4}{|c|}{ Table II.C.3-1 JOYO-1 Hardware Material Specifications and Composition } \\
\hline DRAWING NUMBER & PART & PO & Status \\
\hline SK-AMT-2221-H01 & NRPCT Joyo Capsule & 3007447 & Complete \\
\hline SK-AMT-2222-H01 & NRPCT Joyo Head & 3007447 & Complete \\
\hline SK-AMT-2223-H01 & Lower Spacer & 3007724 & t \\
\hline SK-AMT-2223-HO2 & Upper Spacer & 3007724 & $*$ \\
\hline SK-AMT-2224-H01 & Upper Insulator - Short & 3007447 & Complete \\
\hline SK-AMT-2224-H02 & Upper Insulator - Medium & 3007447 & Complete \\
\hline SK-AMT-2224-H03 & Upper Insulator - Long & 3007447 & Complete \\
\hline SK-AMT-2224-H04 & Upper Insulator - Xlong & 3007447 & Complete \\
\hline SK-AMT-2224-H05 & Lower Insulator - Short & 3007447 & Complete \\
\hline SK-AMT-2224-H06 & Lower Insulator - Medium & 3007447 & Complete \\
\hline SK-AMT-2224-H07 & Lower Insulator - Long & 3007447 & Complete \\
\hline SK-AMT-2225-H01 & Biaxial Creep Outer Holder $(0.4)$ & 3007457 & Machining Incomplete \\
\hline SK-AMT-2225-HO2 & Biaxial Creep Outer Holder $(0.5)$ & 3007457 & Machining Incomplete \\
\hline SK-AMT-2225-H03 & Biaxial Creep Outer Holder (0.6) & 3007457 & Machining Incomplete \\
\hline SK-AMT-2225-H04 & Biaxial Creep Outer Holder $(0.7)$ & 3007457 & Machining Incomplete \\
\hline SK-AMT-2225-H05 & Biaxial Creep Outer Holder $(0.8)$ & 3007457 & Machining Incomplete \\
\hline SK-AMT-2225-H06 & Biaxial Creep Outer Holder (0.9) & 3007457 & Machining Incomplete \\
\hline SK-AMT-2225-H07 & Biaxial Creep Outer Holder (1.0) & 3007457 & Machining Incomplete \\
\hline SK-AMT-2225-H09 & Biaxial Creep Inner Holder & 3007450 & * \\
\hline SK-AMT-2225-H10 & Biaxial Creep Washer & 3007450 & * \\
\hline SK-AMT-2226-H01 & Tensile Holder Rh (0.4) & 3007453 & Machining Incomplete \\
\hline SK-AMT-2226-H02 & Tensile Holder Rh (0.5) & 3007453 & Machining Incomplete \\
\hline SK-AMT-2226-HO3 & Tensile Holder Rh (0.6) & 3007453 & Machining Incomplete \\
\hline SK-AMT-2226-H04 & Tensile Holder Rh (0.7) & 3007453 & Machining Incomplete \\
\hline SK-AMT-2226-H05 & Tensile Holder Rh (0.8) & 3007453 & Machining Incomplete \\
\hline SK-AMT-2226-H06 & Tensile Holder Rh (0.9) & 3007453 & Machining Incomplete \\
\hline SK-AMT-2226-HO7 & Tensile Holder Rh (1.0) & 3007453 & Machining Incomplete \\
\hline SK-AMT-2226-H09 & Tensile Holder Lh (0.4) & 3007453 & Machining Incomplete \\
\hline SK-AMT-2226-H10 & Tensile Hotder Lh (0.5) & 3007453 & Machining Incomplete \\
\hline SK-AMT-2226-H11 & Tensile Holder Lh (0.6) & 3007453 & Machining incomplete \\
\hline SK-AMT-2226-H12 & Tensile Holder Lh (0.7) & 3007453 & Machining Incomplete \\
\hline SK-AMT-2226-H13 & Tensile Holder Lh (0.8) & 3007453 & Machining Incomplete \\
\hline SK-AMT-2226-H14 & Tensile Holder Lh (0.9) & 3007453 & Machining Incomplete \\
\hline SK-AMT-2226-H15 & Tensile Holder Lh (1.0) & 3007453 & Machining Incomplete \\
\hline SK-AMT-2226-H17 & Tensile Holder Base $(0.4)$ & 3007453 & 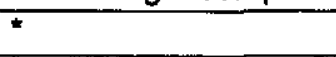 \\
\hline SK-AMT-2226-H18 & Tensile Holder Base (0.5) & 3007453 & \# \\
\hline SK-AMT-2226-H19 & Tensile Holder Base $(0.6)$ & 3007453 & $\star$ \\
\hline SK-AMT-2226-H2O & Tensile Holder Base $(0.7)$ & 3007453 & * \\
\hline SK-AMT-2226-H21 & Tensile Holder Base $(0.8)$ & 3007453 & * \\
\hline SK-AMT-2226-H22 & Tensile Holder Base (0.9) & 3007453 & 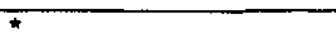 \\
\hline
\end{tabular}


Enclosure (7) to

MDO-723-0044/

B-MT(SRME)-52

Page 25

Table II.C.3-1 JOYO-1 Hardware Material Specifications and Composition

\begin{tabular}{|c|c|c|c|}
\hline DRAWING NUMBER & PART & PO & Status \\
\hline SK-AMT-2226-H23 & Tensile Holder Base (1.0) & 3007453 & 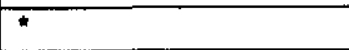 \\
\hline SK-AMT-2227-H01 & Fracture Toughness Holder Rh (0.4) & 3007453 & Machining Incomplete \\
\hline SK-AMT-2227-H02 & Fracture Toughness Holder $\mathrm{Rh}(0.5)$ & 3007453 & Machining Incomplete \\
\hline SK-AMT-2227-HO3 & Fracture Toughness Holder Rh (0.6) & 3007453 & Machining Incomplete \\
\hline SK-AMT-2227-HO4 & Fracture Toughness Holder Rh (0.7) & 3007453 & Machining Incomplete \\
\hline SK-AMT-2227-H05 & Fracture Toughness Holder Rh (0.8) & 3007453 & Machining Incomplete \\
\hline SK-AMT-2227-H06 & Fracture Toughness Holder Lh (0.4) & 3007453 & Machining Incomplete \\
\hline SK-AMT-2227-H07 & Fracture Toughness Holder Lh (0.5) & 3007453 & Machining Incomplete \\
\hline SK-AMT-2227-H08 & Fracture Toughness Holder Lh (0.6) & 3007453 & Machining Incomplete \\
\hline SK-AMT-2227-H09 & Fracture Toughness Holder Lh (0.7) & 3007453 & Machining Incomplete \\
\hline SK-AMT-2227-H10 & Fracture Toughness Holder Lh (0.8) & 3007453 & Machining Incomplete \\
\hline SK-AMT-2227-H11 & Fracture Toughness Holder Base (0.4) & 3007453 & * \\
\hline SK-AMT-2227-H12 & Fracture Toughness Holder Base (0.5) & 3007453 & $\div$ \\
\hline SK-AMT-2227-H13 & Fracture Toughness Holder Base (0.6) & 3007453 & $*$ \\
\hline SK-AMT-2227-H14 & Fracture Toughness Holder Base (0.7) & 3007453 & * \\
\hline SK-AMT-2227-H15 & Fracture Toughness Holder Base $(0.8)$ & 3007453 & $\star$ \\
\hline $\begin{array}{l}\text { SK-AMT-2228-H01 } \\
\text { thru H12 }\end{array}$ & Density Disc Holder - Short & 3007450 & Machining Not Started \\
\hline $\begin{array}{l}\text { SK-AMT-2228-H15 \& } \\
\text { H19 }\end{array}$ & Density Disc Holder & 3007450 & Machining Not Started \\
\hline $\begin{array}{l}\text { SK-AMT-2235-HO1 } \\
\text { thru H04 }\end{array}$ & Ct Specimen Hotder & 3007450 & Machining Not Started \\
\hline SK-AMT-2239-HO1 & Wave Spring & 3006712 & Complete \\
\hline SK-AMT-2241-H03 & BeO Compression Holder $(0.7)$ & 3007453 & * \\
\hline SK-AMMT-2243-G01 & Joyo Capsule Clamp Assembly & 3007456 & Complete \\
\hline SK-JPH-1110-H01 & Joyo Capsule Adapter Holder & 3007456 & Complete \\
\hline SK-JPH-1110-H02 & Joyo Capsule Adapter Holder & 3007456 & Complete \\
\hline $\begin{array}{l}\text { SK-RRM-1011-H01 \& } \\
\text { H02 }\end{array}$ & Moly Holder & 3007724 & Complete \\
\hline $\begin{array}{l}\text { SK-RRM-1011-H03 \& } \\
\text { HO6 }\end{array}$ & Titanium Wire Wrapped In Moly Foil & 3007724 & Complete \\
\hline SK-RRM-1013-H01 & NRPCT Capsule Mockup & 3007445 & Complete \\
\hline SK-RRM-1013-H01 & NRPCT Capsule Mockup & 3006936 & Complete \\
\hline SK-RRM-1014-H01 & NRPCT Head Mockup & $300744 \overline{5}$ & Complete \\
\hline SK-RRM-1014-H01 & NRPCT Head Mockup & 3006936 & Complete \\
\hline SK-RRM-1015-H01 & NRPCT Volume Spacer & 3007445 & Complete \\
\hline SK-RRM-1017-H01 & NRPCT Capsule Mockup Threaded & 3006936 & Complete \\
\hline SK-RRM-1018-HOI & Joyo Weld Cooling Tube & 3007445 & Complete \\
\hline SK-RRM-1019-H01 & NRPCT Joyo Solid Capsule & 3007454 & Complete \\
\hline SK-RRM-1023-H01 & Tungsten Tensile Holder $(0 . \overline{6})$ & $3007 \overline{18}$ & Machining Incomplete \\
\hline SK-RRM-1023-HO2 & Tungsten Tensile Holder $(0.75)$ & 3007718 & Machining Incomplete \\
\hline SK-RRM-1023-HO3 & Tungsten Tensile Holder (1.1) & 3007718 & Machining Incomplete \\
\hline SK-RRM-1023-H04 & Tungsten Disc Holder (0.6) & 3007718 & Machining Incomplete \\
\hline SK-RRM-1023-H05 & Tungsten Disc Hoider (0.75) & 3007718 & Machining Incomplete \\
\hline SK-RRM-1023-H07 & Tungsten Disc Holder (1.1) & 3007718 & Machining Incomplete \\
\hline SK-RRM-1023-HO9 & Tungsten Ceramic Strip Holder (0.6) & 3007718 & Machining Incomplete \\
\hline SK-RRM-1023-H10 & Tungsten Ceramic Strip Holder $(0.75)$ & 3007718 & Machining Incomplete \\
\hline
\end{tabular}


Enclosure (7) to

MOO-723-0044/

B-MT(SRME)-52

Page 26

\begin{tabular}{|l|l|l|l|}
\hline \multicolumn{4}{|c|}{ Table II.C.3-1 JOYO-1 Hardware Material Specifications and Composition } \\
\hline DRAWING NUMBER & PART & PO & Status \\
\hline SK-WJU-1000-H01 & Head Mockup Long & 3007445 & Complete \\
\hline SK-WJU-1004-H01 & Capsule Mockup Long & 3007445 & Complete \\
\hline & & & \\
\hline * Machining complete, part still needs heat treating, cleaning, and pickling \\
\hline
\end{tabular}

The following is a list of issues with the capsule hardware that were not resolved as of the project restructuring:

a. Silicon carbide temperature monitors may diffusion bond to the molybdenum holders. One solution for this problem may be to use grafoil as an interface material between the silicon carbide temperature monitors and the molybdenum holders.

b. Thermal analysis with silicon carbide specimens and tungsten holders shows that some of the specimens may not be within the targeted temperature range.

c. The specimens in the fracture toughness holder may have too large of a gap between the specimen and the holder. This will cause there to be a large thermal resistance between the specimens and the holder causing the specimens to exceed their target temperature in the reactor. It also causes there to be greater uncertainty in the temperature of the specimen since the exact position of the specimen in the holder would be unknown.

\section{Certification Information}

All hardware for JOYO-1 was to be certified by Engineers with Management oversight as described in Section IV.E.3 to confirm that the hardware was procured, assembled, and inspected to established technical requirements. The following table lists the items that have been certified for insertion into a reactor:

\begin{tabular}{|l|l|l|l|}
\hline \multicolumn{4}{|c|}{ Table II.C.4-1, Material Certifications } \\
\hline Item Description & PO Number & Part Number & Certification Document \\
\hline ASME SA479 316H Bar Stock & 3006713 & N/A & B-MT(EDT)R-1759 \\
\hline $\begin{array}{l}\text { Molybdenum Bar Stock } \\
\text { (Type 361 \& Type 365) }\end{array}$ & 3005772 & N/A & B-MT(EDT)R-1760 \\
\hline NRPCT JOYO Capsule & 3007447 & SK-AMT-2221-H01 & B-MT(EDT)R-1767 \\
\hline NRPCT JOYO Head & 3007447 & SK-AMT-2222-H01 & B-MT(EDT)R-1767 \\
\hline NRPCT JOYO Insulators & 3007447 & SK-AMT-2224-H01 thru H07 & B-MT(EDT)R-1767 \\
\hline Grafoil Nuclear Grade GTA & 3007720 & N/A & B-MT(EDT)R-1768 \\
\hline NRPCT Wavespring & 3006712 & SK-AMT-2239-H01 & B-MT(EDT)R-1772 \\
\hline JOYO Flux Wire Assembly & 3007724 & SK-RRM1011-G01 & B-MT(EDT)R-1773 \\
\hline
\end{tabular}

The remaining items were not certified because fabrication was not completed as of project closeout. 
III. Analysis

A. Nuclear

\section{Background}

The design of irradiation tests for any reactor requires the calculation of heat generation rates and neutron flux. These calculations require detailed models of the reactor facility with a nuclear analysis code. Nuclear models of any given facility are generally owned only by the operators of the facility. Although, the NRPCT has nuclear analysts on its staff, no nuclear model of the JOYO reactor was available for our use. JNC would not release such a model to the NRPCT, because of Japanese government imposed release of information issues.

JNC initially provided nuclear calculation results for use in design scoping that included neutron fluxes and stainless steel heat rates from a previous JOYO cycle run. Those results were adequate to establish the initial feasibility of testing in JOYO and as a basis for selecting the JOYO core locations for the two test assemblies. The NRPCT was limited to locations outside of the core in the reflector region beginning with JOYO Row 6. The NRPCT chose JOYO locations 6A4 and 6D4 because those locations offered the highest neutron flux available. Figures III.A.1 and III.A.2 show the initial scoping values of fast flux at the Row $6 A 4$ and 6D4 locations, respectively.

Once preliminary designs for the test capsules were established and core locations were selected, it was necessary to obtain heat generation and neutron flux calculations specific to these capsules and JOYO positions. The typical irradiation test design process requires several design iterations between nuclear analysts and thermalmechanical designers. JNC nuclear analysts would normally provide these calculations.

Because the schedule for these tests to meet the targeted JOYO cycle was extremely aggressive, it was obvious that it would be difficult to get JNC nuclear analysts to support several design iterations on the required schedule. Fortuitously, a PNNL nuclear analyst, Dr. David Wooten, was working at the JOYO reactor site on a special fellowship assignment during the NRPCT initial inquiries on the feasibility of performing materials irradiation tests in JOYO. Part of his assignment with JNL included the development and qualification of a nuclear model for heat rate and flux calculations. With JNC's agreement, the NRPCT was able to place a contract with PNNL to have Dr. Wooten provide the required nuclear calculations.

NRPCT nuclear analysts interfaced with Dr. Wooten on the calculations, and provided interpretation and correlation of the calculation results for the NRPCT capsule designers. Dr. Wooten had just completed calculations for the $2^{\text {nd }}$ design iteration at the time of the Prometheus program termination.

\section{Heat Rate Calculation Basis}

The heat generation rate calculations used for the capsule thermal analyses were performed by PNNL and supplied to the NRPCT in Excel spreadsheets in September 2005. The calculations were performed with the MCNP code. A description of the MCNP model is in the attached report titled Heating Rates and Neutron Flux for JOYO- 
Enclosure (7) to

MDO-723-0044/

B-MT(SRME)-52

Page 28

1 Irradiation Tests - Revision 2 by David Wootan. This set of heat rate calculations have been designated Revision 2 and provided in Attachment $C$ to this enclosure.

These are some of the important assumptions inherent to the calculated Revision 2 heat rates.

- The MCNP calculations were made using a JOYO whole core model that was based on a preliminary Cycle 5 core loading.

- Heat rates are not expected to be substantially different for the cycles with the NRPCT tests.

- The MCNP heat rate calculations primarily used ENDF/B-VI cross sections.

- The MCNP code does not explicitly include gamma heating contributions from delayed gamma and beta rays. But a $\sim+6 \%$ correction was made to the total reactor power normalization to take this into account.

Notable changes from the previous calculation set include:

- The model represented separate capsule, holder, and specimen regions rather than homogenized capsules.

- The holder material used for SiC and Be specimens was changed from graphite to tungsten in capsules containing these specimen types.

- JOYO control rods were represented at the cycle average position rather than the beginning of cycle position.

\section{NRPCT Interpretation and Use of PNNL Revision 2 Heat Rate Calculations}

This section describes the NRPCT interpretation and use of the Revision 2 MCNP heat rate calculations. The NRPCT previously selected core locations 6A4 and 6D4 because they provide the highest fast flux levels available in JOYO Row 6.

The two NRPCT test rigs consist of 5 tiers with 6 compartments per tier for a total of 60 capsules. The PNNL MCNP calculations provided total heat rates (watts/gram) for 21 elements (He, B, Be, C, O, Al, Si, Ti, V, Cr, Mn, Fe, Ni, Co, Zr, Nb, Mo, Hf, Ta, W, Re), in 3 radial regions (outside capsule steel, middle holder, and specimens), with 8 axial segments (approximately $2 \mathrm{~cm}$ ) per capsule.

The total heat rates for the given elements were used to determine the heat rates for the materials in the NRPCT test rigs. Table III.A-1 gives the compositions and densities used convert the element total heat rates into alloy total heat rates based on weight fraction. It was assumed that a value given for an axial segment was the midpoint value of that segment. Linear interpolation was used to determine a value elsewhere in the segment. Table III.A-2 provides the total heat rates determined for a specific material per compartment and elevation for the JOYO $6 a 4$ and 6d4 positions. These values were used for the thermal analysis. 
Enclosure (7) to

MDO-723-0044/

B-MT(SRME)-52

Page 29

Figure III.A.1

JOYO Irradiation Test Fast Flux Estimates, 6A4

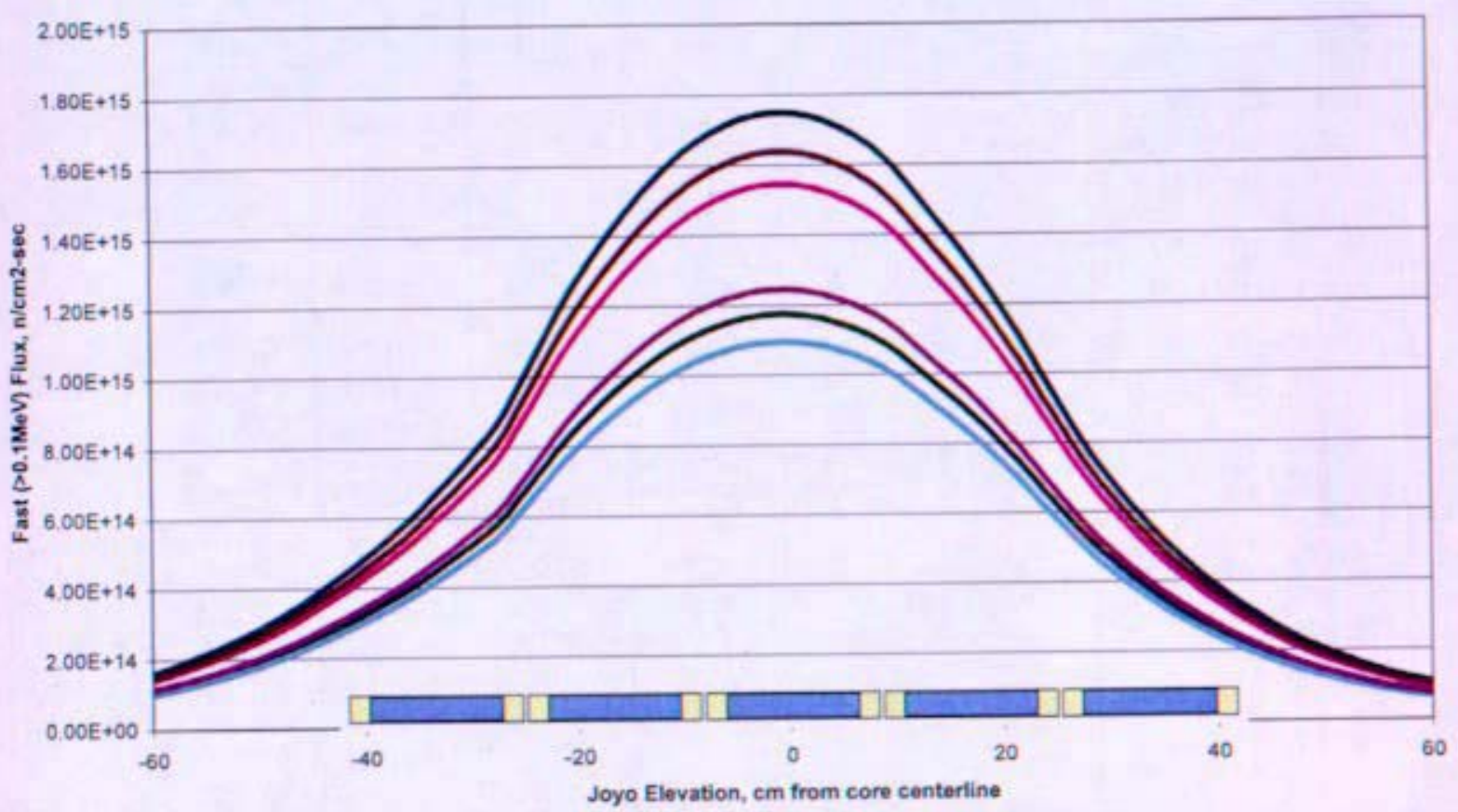

- Compartment 1 - Compartment 2 - Compartment 3 - Compartment 4 - Compartment 5 - Compartment 5

Figure III.A.2

JOYO Irradiation Test Fast Flux Estimates, 6D4

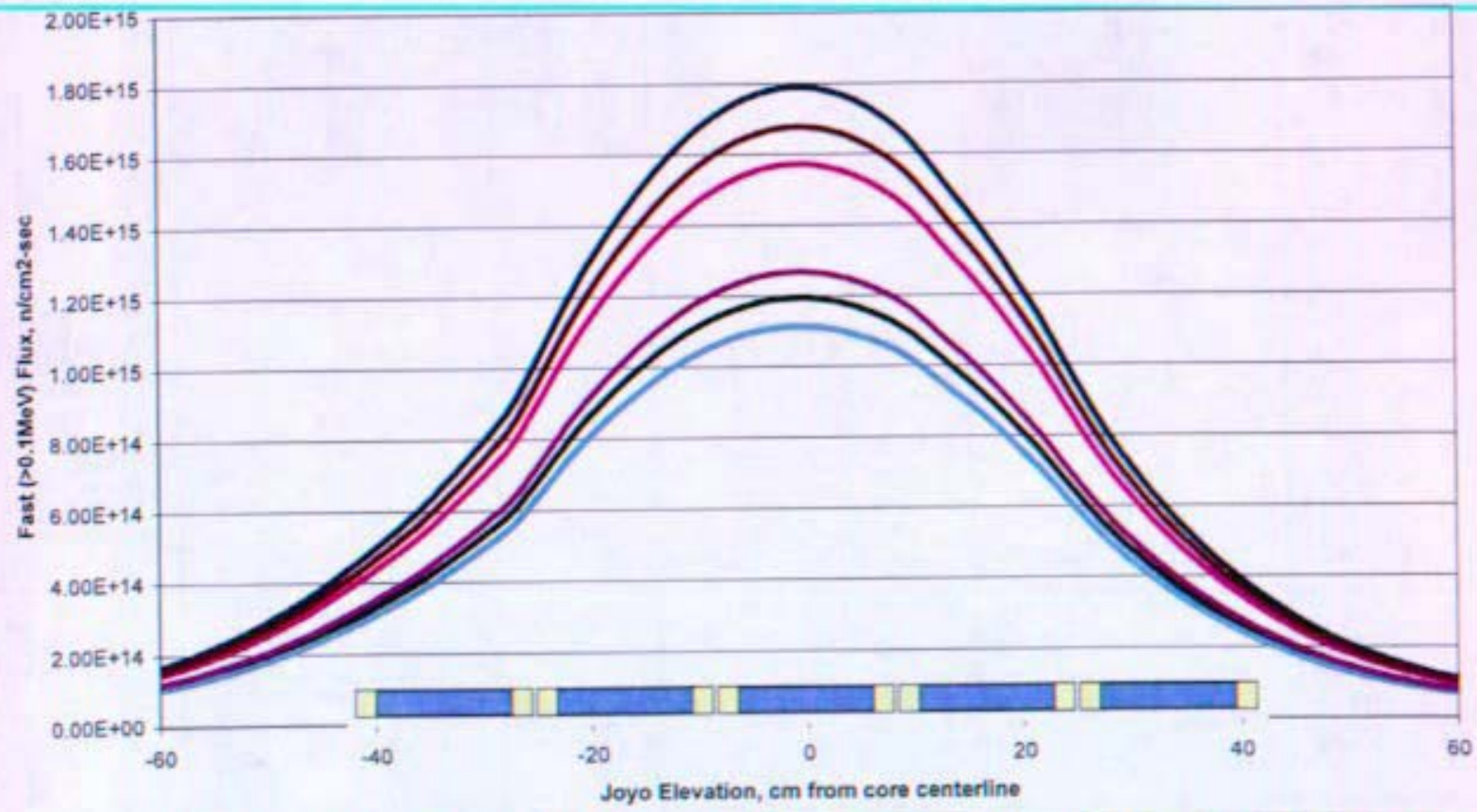

- Compartment 1 - Compartment $2-$ Compartment 3 - Compartment 4 - Compartment 5 - Cornpartment 6 
Enclosure (7) to

MDO-723-0044/

B-MT(SRME)-52

Page 30

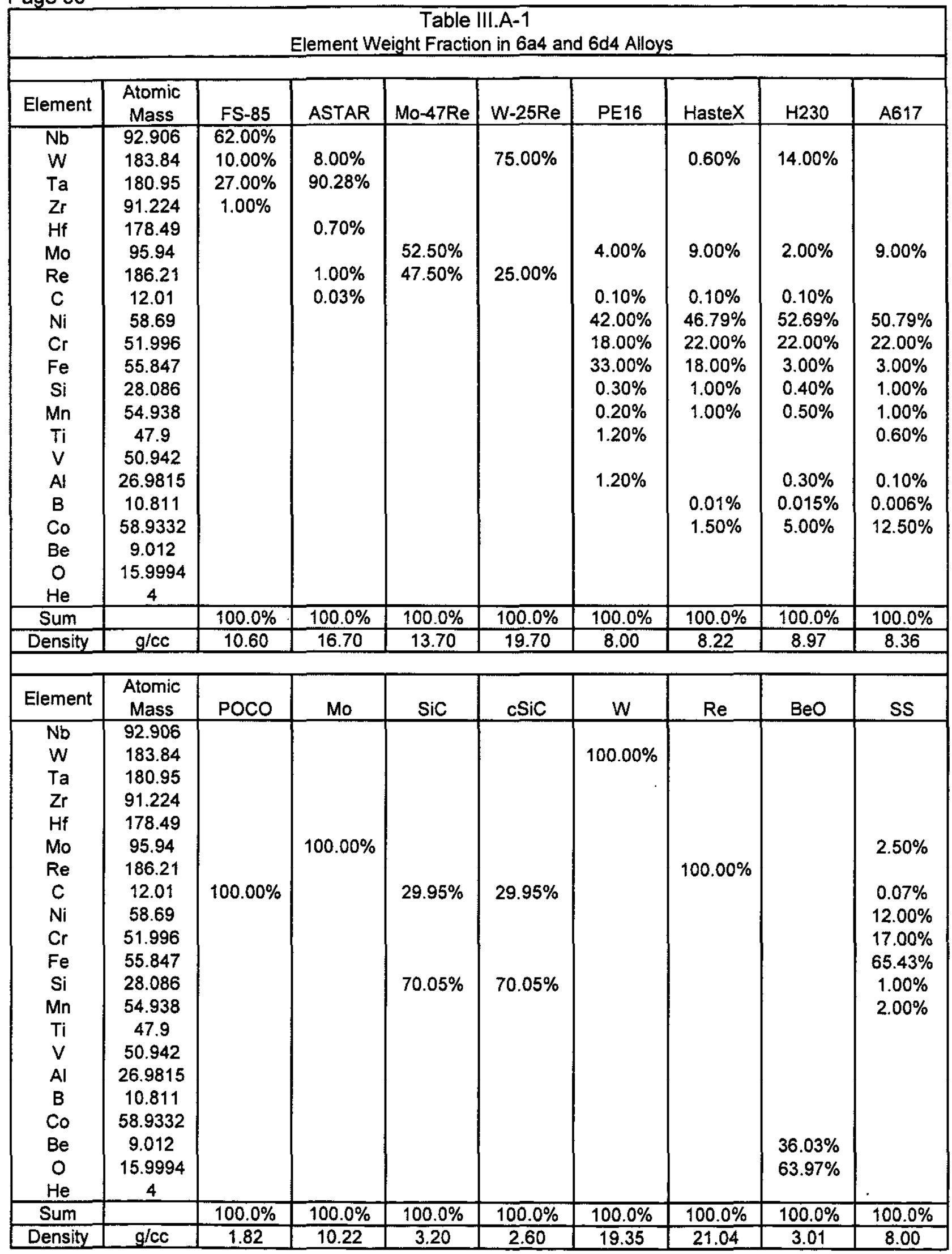


Enclosure (7) to MDO-723-0044I

B-MT(SRME)-52

Page 31

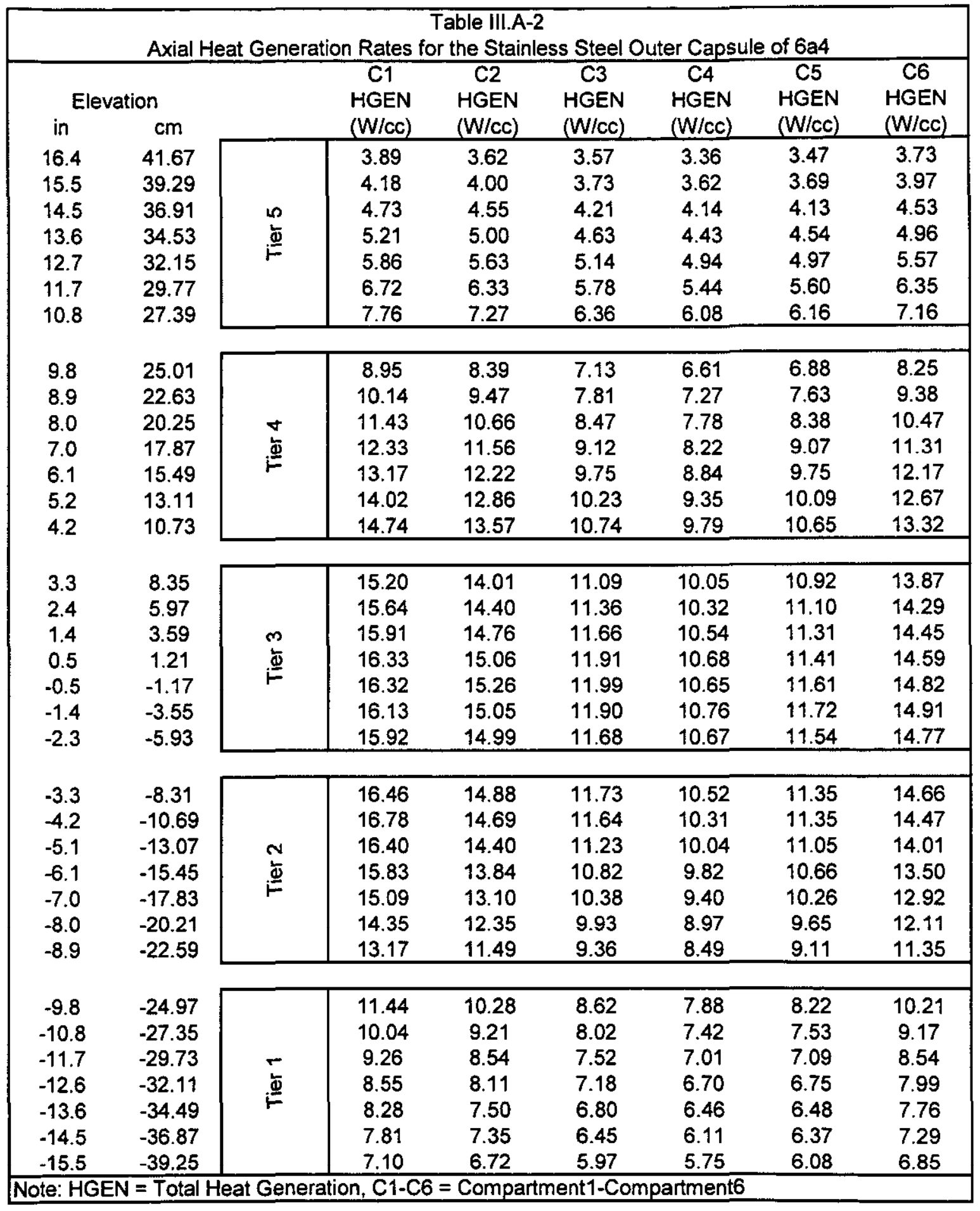




\begin{tabular}{|c|c|c|c|c|c|c|c|c|}
\hline \multicolumn{9}{|c|}{$\begin{array}{l}\text { Enclosure (7) to } \\
\text { MDO-723-0044/ } \\
\text { B-MT(SRME)-52 } \\
\text { Page } 32\end{array}$} \\
\hline \multicolumn{9}{|c|}{$\begin{array}{c}\text { Table III.A-2 } \\
\text { Axial Heat Generation Rates for the Stainless Steel Outer Capsule of } 6 \mathrm{~d} 4\end{array}$} \\
\hline \multirow{2}{*}{\multicolumn{2}{|c|}{ Elevation }} & & $\mathrm{C1}$ & $\mathrm{C} 2$ & $\mathrm{C} 3$ & $\mathrm{C4}$ & $\mathrm{C5}$ & $\mathrm{C6}$ \\
\hline & & & HGEN & HGEN & HGEN & HGEN & HGEN & HGEN \\
\hline in & $\mathrm{cm}$ & & (W/cc) & $(W / c c)$ & (W/cc) & (W/Cc) & (W/cc) & $(W / C c)$ \\
\hline 16.4 & 41.67 & & 3.85 & 3.79 & 3.38 & 3.57 & 3.54 & 3.92 \\
\hline 15.5 & 39.29 & & 4.21 & 4.06 & 3.74 & 3.67 & 3.79 & 4.16 \\
\hline 14.5 & 36.91 & 10 & 4.66 & 4.51 & 4.23 & 4.02 & 4.25 & 4.72 \\
\hline 13.6 & 34.53 & $\frac{1}{0}$ & 5.39 & 4.98 & 4.67 & 4.40 & 4.68 & 5.05 \\
\hline 12.7 & 32.15 & $\mathrm{i}$ & 6.01 & 5.78 & 5.21 & 4.93 & 5.16 & 5.77 \\
\hline 11.7 & 29.77 & & 6.78 & 6.36 & 5.78 & 5.27 & 5.54 & 6.39 \\
\hline 10.8 & 27.39 & & 7.85 & 7.13 & 6.32 & 5.83 & 6.32 & 7.12 \\
\hline 9.8 & 25.01 & & 8.88 & 8.29 & 7.01 & 6.46 & 6.94 & 8.23 \\
\hline 8.9 & 22.63 & & 10.15 & 9.49 & 7.65 & 7.29 & 7.63 & 9.48 \\
\hline 8.0 & 20.25 & $\nabla$ & 11.31 & 10.61 & 8.53 & 7.86 & 8.11 & 10.59 \\
\hline 7.0 & 17.87 & 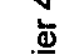 & 12.28 & 11.51 & 9.30 & 8.39 & 8.76 & 11.54 \\
\hline 6.1 & 15.49 & $i=$ & 13.17 & 12.35 & 9.79 & 8.81 & 9.44 & 12.05 \\
\hline 5.2 & 13.11 & & 13.88 & 13.15 & 10.40 & 9.43 & 9.88 & 12.92 \\
\hline 4.2 & 10.73 & & 14.49 & 13.46 & 10.69 & 9.70 & 10.47 & 13.74 \\
\hline 3.3 & 8.35 & & 15.65 & 14.06 & 11.08 & 10.09 & 10.95 & 14.28 \\
\hline 2.4 & 5.97 & & 16.88 & 14.40 & 11.38 & 10.41 & 11.38 & 14.80 \\
\hline 1.4 & 3.59 & $m$ & 17.56 & 14.99 & 11.89 & 10.56 & 11.70 & 15.19 \\
\hline 0.5 & 1.21 & $\underline{\mathbf{a}}$ & 17.80 & 15.26 & 11.83 & 10.77 & 11.99 & 15.54 \\
\hline-0.5 & -1.17 & $\stackrel{\mathscr{N}}{=}$ & 17.86 & 15.58 & 11.90 & 10.91 & 12.16 & 15.75 \\
\hline-1.4 & -3.55 & & 17.93 & 15.43 & 11.89 & 11.03 & 11.96 & 15.46 \\
\hline-2.3 & -5.93 & & 17.82 & 15.18 & 11.93 & 10.77 & 11.90 & 15.35 \\
\hline & & & & & & & & \\
\hline-3.3 & -8.31 & & 17.45 & 15.15 & 11.89 & 10.57 & 14.09 & 14.89 \\
\hline-4.2 & -10.69 & & 16.90 & 14.72 & 11.45 & 10.19 & 10.33 & 14.33 \\
\hline-5.1 & -13.07 & c & 16.52 & 14.43 & 11.04 & 9.80 & 10.12 & 14.27 \\
\hline-6.1 & -15.45 & $\Phi$ & 15.82 & 14.07 & 10.69 & 9.37 & 9.94 & 13.73 \\
\hline-7.0 & -17.83 & $F$ & 15.02 & 13.16 & 10.03 & 9.20 & 9.26 & 12.90 \\
\hline-8.0 & -20.21 & & 14.23 & 12.23 & 9.87 & 8.64 & 9.00 & 11.94 \\
\hline-8.9 & -22.59 & & 13.13 & 11.38 & 9.30 & 8.33 & 8.48 & 11.34 \\
\hline-9.8 & -24.97 & & 11.61 & 10.25 & 872 & & 816 & 1056 \\
\hline-10.8 & -27.35 & & 10.21 & 9.43 & 8.16 & 7.61 & 7.94 & 9.53 \\
\hline-11.7 & -29.73 & $\sigma$ & 9.25 & 8.84 & 7.53 & 7.16 & 7.57 & 8.67 \\
\hline-12.6 & -32.11 & 乌. & 9.04 & 8.24 & 7.34 & 6.88 & 7.05 & 8.18 \\
\hline-13.6 & -34.49 & $=$ & 8.41 & 8.02 & 6.98 & 6.37 & 6.87 & 7.45 \\
\hline-14.5 & -36.87 & & 7.88 & 7.20 & 6.51 & 6.20 & 6.34 & 7.38 \\
\hline-15.5 & -39.25 & & 7.36 & 6.80 & 6.10 & 5.63 & 6.11 & 6.71 \\
\hline
\end{tabular}


Enclosure (7) to

MDO-723-0044I

B-MT(SRME)-52

Page 33

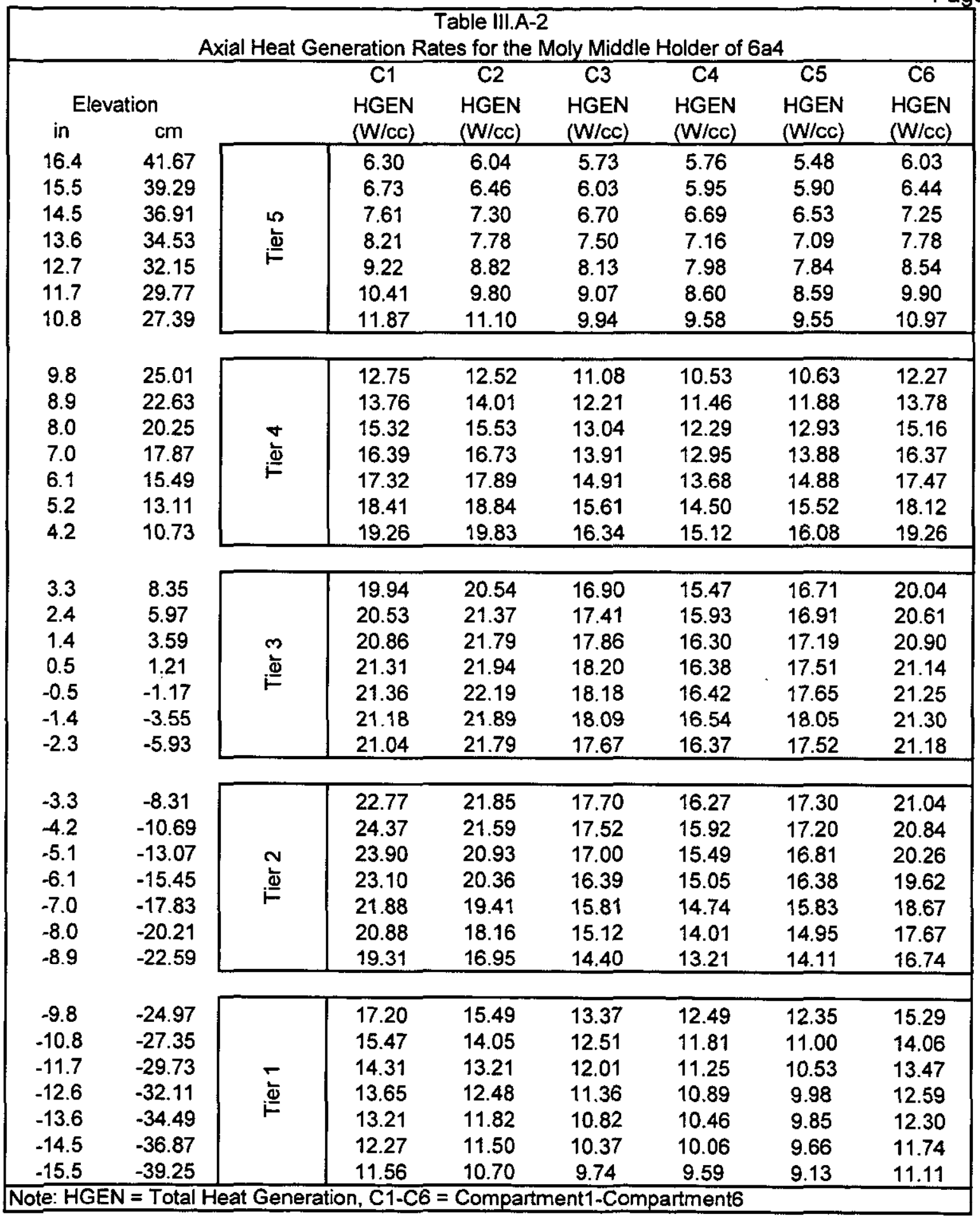


Enclosure (7) to

MDO-723-0044/

B-MT(SRME)-52

Page 34

Table III.A-2

Axial Heat Generation Rates for the Moly Middle Holder of $6 \mathrm{~d} 4$

\begin{tabular}{|c|c|c|c|c|c|c|c|c|}
\hline \multicolumn{2}{|c|}{ Elevation } & & $\begin{array}{c}\text { C1 } \\
\text { HGEN }\end{array}$ & $\begin{array}{c}\text { C2 } \\
\text { HGEN }\end{array}$ & $\begin{array}{c}\text { C3 } \\
\text { HGEN }\end{array}$ & $\begin{array}{c}\text { C4 } \\
\text { HGEN }\end{array}$ & $\begin{array}{c}\text { C5 } \\
\text { HGEN }\end{array}$ & $\begin{array}{c}\text { C6 } \\
\text { HGEN }\end{array}$ \\
\hline in & $\mathrm{cm}$ & & $(\mathrm{W} / \mathrm{cc})$ & $(\mathrm{W} / \mathrm{cc})$ & & $(W / c c)$ & $(W / c c)$ & $(W / c c)$ \\
\hline 16.4 & 41.67 & & 6.34 & 6.13 & 5.46 & 5.57 & 5.59 & 6.34 \\
\hline 15.5 & 39.29 & & 6.74 & 6.58 & 6.07 & 5.81 & 6.05 & 6.58 \\
\hline 14.5 & 36.91 & 10 & 7.59 & 7.15 & 6.73 & 6.61 & 6.63 & 7.28 \\
\hline 13.6 & 34.53 & $\underline{\underline{\omega}}$ & 8.84 & 8.01 & 7.41 & 7.21 & 7.55 & 8.02 \\
\hline 12.7 & 32.15 & 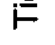 & 9.42 & 8.88 & 8.06 & 7.47 & 8.03 & 8.94 \\
\hline 11.7 & 29.77 & & 10.34 & 10.10 & 9.17 & 8.29 & 8.85 & 9.78 \\
\hline 10.8 & 27.39 & & 12.01 & 11.09 & 9.74 & 9.27 & 9.81 & 10.93 \\
\hline 9.8 & 25.01 & & 12.44 & 12.62 & 10.79 & 10.23 & 10.62 & 12.52 \\
\hline 8.9 & 22.63 & & 13.19 & 14.31 & 12.00 & 11.32 & 11.29 & 14.00 \\
\hline 8.0 & 20.25 & $\nabla$ & 14.48 & 15.78 & 13.34 & 12.10 & 12.03 & 15.61 \\
\hline 7.0 & 17.87 & $\underline{\underline{\Phi}}$ & 15.74 & 17.27 & 14.10 & 12.73 & 12.92 & 16.80 \\
\hline 6.1 & 15.49 & $\bar{F}$ & 17.04 & 18.44 & 14.79 & 13.80 & 13.98 & 17.69 \\
\hline 5.2 & 13.11 & & 17.54 & 19.57 & 15.70 & 14.31 & 14.82 & 19.24 \\
\hline 4.2 & 10.73 & & 18.73 & 20.25 & 16.50 & 14.77 & 15.49 & 20.10 \\
\hline 3.3 & 8.35 & & 21.45 & 20.64 & 17.00 & 15.76 & 16.61 & 21.06 \\
\hline 2.4 & 5.97 & & 24.44 & 20.86 & 17.56 & 16.16 & 17.32 & 21.62 \\
\hline 1.4 & 3.59 & $m$ & 25.79 & 21.68 & 18.17 & 16.32 & 17.94 & 22.04 \\
\hline 0.5 & 1.21 & 过 & 25.59 & 21.99 & 17.91 & 16.94 & 18.20 & 22.58 \\
\hline-0.5 & -1.17 & $i$ & 25.86 & 22.30 & 18.28 & 16.77 & 18.35 & 22.77 \\
\hline-1.4 & -3.55 & & 25.94 & 22.22 & 17.97 & 17.00 & 18.36 & 22.69 \\
\hline-2.3 & -5.93 & & 25.72 & 21.91 & 17.76 & 16.77 & 18.06 & 22.42 \\
\hline-3.3 & -8.31 & & 25.22 & 22.26 & 17.82 & 16.22 & 15.92 & 21.52 \\
\hline-4.2 & -10.69 & & 24.84 & 21.74 & 17.34 & 15.51 & 14.32 & 20.58 \\
\hline-5.1 & -13.07 & $N$ & 24.03 & 21.23 & 17.04 & 14.94 & 13.69 & 20.52 \\
\hline-6.1 & -15.45 & 烏 & 23.06 & 20.68 & 16.01 & 14.62 & 13.48 & 19.77 \\
\hline-7.0 & -17.83 & 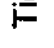 & 22.12 & 19.42 & 15.56 & 14.12 & 12.80 & 18.80 \\
\hline-8.0 & -20.21 & & 20.69 & 18.21 & 15.22 & 13.85 & 12.25 & 17.48 \\
\hline-8.9 & -22.59 & & 19.16 & 16.96 & 14.42 & 13.04 & 11.71 & 16.62 \\
\hline-9.8 & -24.97 & & 17.46 & 15.36 & 13.57 & 12.50 & 12.04 & 15.66 \\
\hline-10.8 & -27.35 & & 15.47 & 14.55 & 12.85 & 12.00 & 12.54 & 14.64 \\
\hline-11.7 & -29.73 & $\sigma$ & 14.49 & 13.92 & 11.89 & 11.15 & 11.98 & 13.47 \\
\hline-12.6 & -32.11 & 黑 & 14.39 & 13.26 & 11.21 & 10.74 & 11.28 & 12.67 \\
\hline-13.6 & -34.49 & $\bar{F}$ & 13.24 & 12.58 & 10.88 & 10.12 & 10.92 & 12.12 \\
\hline-14.5 & -36.87 & & 12.69 & 11.95 & 10.48 & 9.65 & 10.32 & 11.77 \\
\hline-15.5 & -39.25 & & 12.08 & 11.00 & 9.94 & 9.21 & 9.83 & 10.84 \\
\hline
\end{tabular}


Enclosure (7) to

MDO-723-0044/

B-MT(SRME)-52

Page 35

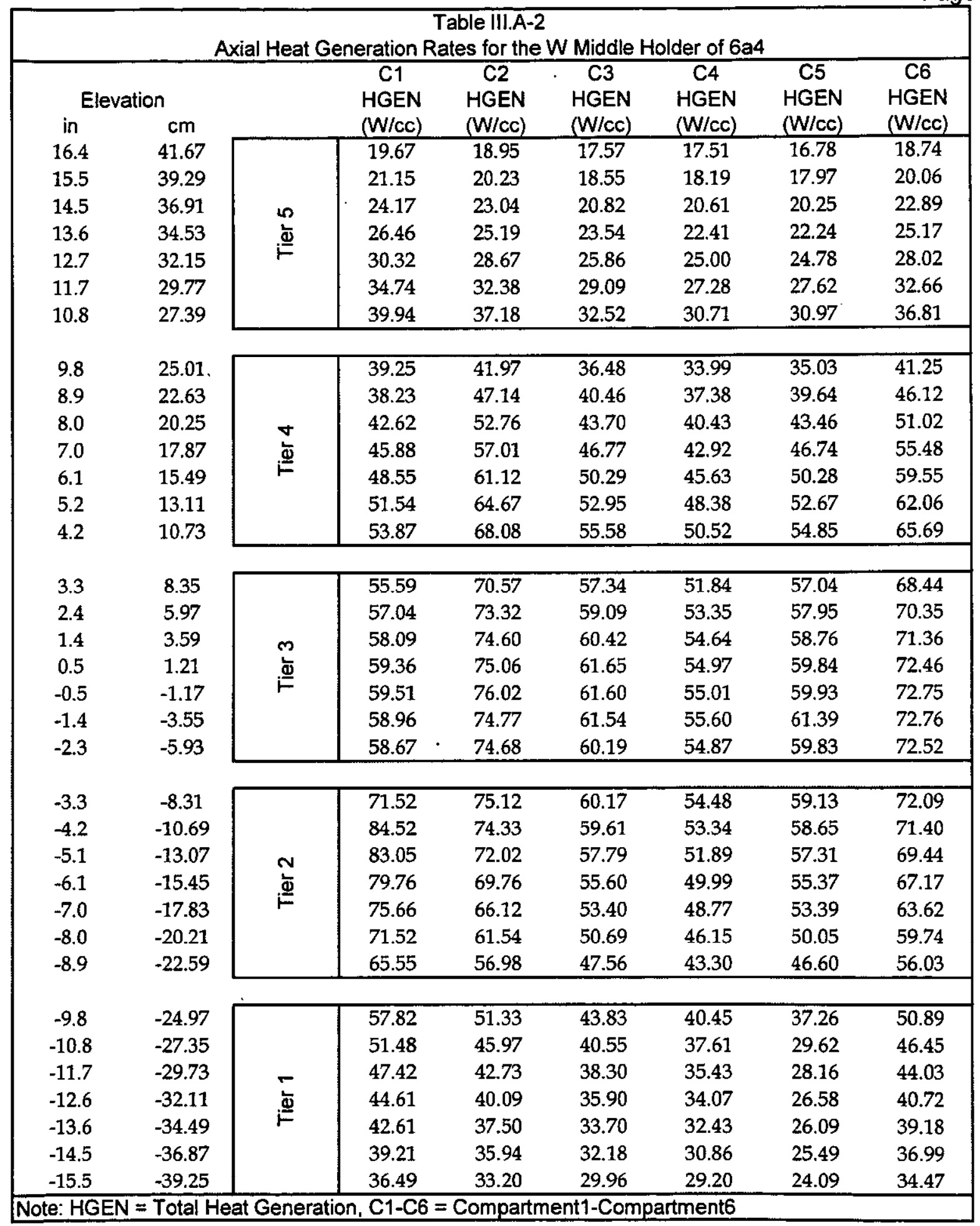


Enclosure (7) to

MDO-723-0044/

B-MT(SRME)-52

Page 36

\begin{tabular}{|c|c|c|c|c|c|c|c|c|}
\hline \multicolumn{9}{|c|}{$\begin{array}{r}\text { Table III.A-2 } \\
\text { Axial Heat Generation Rates for the }\end{array}$} \\
\hline \multicolumn{2}{|c|}{ Elevation } & & $\begin{array}{c}\text { C1 } \\
\text { HGEN }\end{array}$ & $\begin{array}{c}\text { C2 } \\
\text { HGEN }\end{array}$ & $\begin{array}{c}\text { C3 } \\
\text { HGEN }\end{array}$ & $\begin{array}{c}\text { C4 } \\
\text { HGEN }\end{array}$ & $\begin{array}{c}\text { C5 } \\
\text { HGEN }\end{array}$ & $\begin{array}{c}\text { C6 } \\
\text { HGEN }\end{array}$ \\
\hline in & $\mathrm{cm}$ & & $(\mathrm{W} / \mathrm{cc})$ & (W/Cc) & (W/cc) & $(W / c c)$ & (W/cc) & (W/cc) \\
\hline 16.4 & 41.67 & \multirow{7}{*}{$\stackrel{\llcorner 0}{\stackrel{\omega}{\Phi}}$} & 19.82 & 19.15 & 16.87 & 17.32 & 17.18 & 19.64 \\
\hline 15.5 & 39.29 & & 21.09 & 20.60 & 18.80 & 17.91 & 18.80 & 20.58 \\
\hline 14.5 & 36.91 & & 24.05 & 22.71 & 21.12 & 20.42 & 20.77 & 23.14 \\
\hline 13.6 & 34.53 & & 28.30 & 25.70 & 23.52 & 22.66 & 23.78 & 25.71 \\
\hline 12.7 & 32.15 & & 30.71 & 28.93 & 25.76 & 23.79 & 25.63 & 28.96 \\
\hline 11.7 & 29.77 & & 34.13 & 33.12 & 29.63 & 26.83 & 28.62 & 32.09 \\
\hline 10.8 & 27.39 & & 40.07 & 37.00 & 32.06 & 30.25 & 32.26 & 36.42 \\
\hline 9.8 & 25.01 & \multirow{7}{*}{$\begin{array}{l}\nabla \\
\stackrel{\Xi}{\Xi}\end{array}$} & 37.86 & 42.53 & 35.88 & 33.29 & 35.14 & 42.04 \\
\hline 8.9 & 22.63 & & 35.92 & 48.25 & 39.87 & 37.18 & 37.64 & 47.56 \\
\hline 8.0 & 20.25 & & 39.48 & 53.53 & 44.61 & 40.24 & 40.67 & 53.05 \\
\hline 7.0 & 17.87 & & 43.02 & 59.06 & 47.59 & 42.47 & 43.92 & 57.59 \\
\hline 6.1 & 15.49 & & 46.53 & 63.24 & 49.87 & 46.00 & 47.48 & 61.84 \\
\hline 5.2 & 13.11 & & 48.15 & 67.41 & 53.35 & 47.75 & 50.37 & 66.64 \\
\hline 4.2 & 10.73 & & 51.53 & 70.34 & 56.08 & 49.53 & 52.83 & 69.43 \\
\hline 3.3 & 8.35 & \multirow{7}{*}{$\stackrel{m}{\stackrel{m}{\omega}}$} & 67.30 & 71.09 & 58.05 & 52.71 & 56.78 & 72.36 \\
\hline 2.4 & 5.97 & & 85.18 & 71.69 & 60.13 & 54.14 & 59.29 & 74.56 \\
\hline 1.4 & 3.59 & & 89.86 & 74.59 & 62.04 & 54.83 & 61.58 & 75.99 \\
\hline 0.5 & 1.21 & & 89.26 & 75.71 & 61.42 & 56.38 & 62.20 & 77.65 \\
\hline-0.5 & -1.17 & & 90.51 & 76.69 & 62.56 & 56.57 & 63.03 & 78.58 \\
\hline-1.4 & -3.55 & & 90.48 & 76.52 & 61.32 & 57.34 & 62.79 & 78.45 \\
\hline-2.3 & -5.93 & & 89.69 & 75.95 & 60.80 & 56.48 & 61,47 & 77.60 \\
\hline-3.3 & -8.31 & \multirow{7}{*}{ 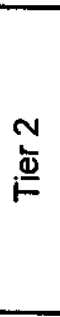 } & 87.93 & 76.74 & 60.71 & 54.31 & 49.55 & 74.27 \\
\hline-4.2 & -10.69 & & 86.30 & 74.98 & 58.99 & 51.95 & 39.23 & 70.94 \\
\hline-5.1 & -13.07 & & 83.36 & 73.08 & 57.82 & 50.38 & 37.48 & 70.23 \\
\hline-6.1 & -15.45 & & 80.35 & 70.75 & 54.38 & 48.85 & 36.85 & 67.42 \\
\hline-7.0 & -17.83 & & 76.82 & 66.14 & 52.34 & 46.38 & 34.96 & 63.73 \\
\hline-8.0 & -20.21 & & 71.32 & 61.94 & 50.81 & 45.13 & 33.21 & 58.92 \\
\hline-8.9 & -22.59 & & 65.20 & 57.45 & 47.69 & 42.64 & 31.68 & 55.17 \\
\hline-9.8 & -24.97 & \multirow{7}{*}{$\stackrel{\underline{\omega}}{-\infty}$} & 58.66 & 51.66 & 44.43 & 40.65 & 35.91 & 51.91 \\
\hline-10.8 & -27.35 & & 51.61 & 48.27 & 41.83 & 38.61 & 40.63 & 48.50 \\
\hline-11.7 & -29.73 & & 47.74 & 45.50 & 38.92 & 35.64 & 38.55 & 44.41 \\
\hline-12.6 & -32.11 & & 46.76 & 42.91 & 36.09 & 33.96 & 35.83 & 41.33 \\
\hline-13.6 & -34.49 & & 42.55 & 40.17 & 34.27 & 31.96 & 34.55 & 39.08 \\
\hline-14.5 & -36.87 & & 40.38 & 37.80 & 32.49 & 30.04 & 32.14 & 37.20 \\
\hline-15.5 & -39.25 & & 37.86 & 34.69 & 30.60 & 28.31 & 30.34 & 34.00 \\
\hline
\end{tabular}


Enclosure (7) to

MDO-723-0044I

B-MT(SRME)-52

Page 37

\begin{tabular}{|c|c|c|c|c|c|c|c|c|}
\hline \multicolumn{9}{|c|}{$\begin{array}{l}\text { Table } 1 \mathrm{II} . \mathrm{A}-2 \\
\text { Axial Heat Generation Rates for the Alloy }\end{array}$} \\
\hline \multirow{2}{*}{\multicolumn{2}{|c|}{ Elevation }} & & \multirow{3}{*}{$\begin{array}{c}\text { C1 } \\
\text { HGEN } \\
\text { (W/CC) }\end{array}$} & \multirow{3}{*}{$\begin{array}{c}\text { C2 } \\
\text { HGEN } \\
\text { (W/cc) }\end{array}$} & \multirow{3}{*}{$\begin{array}{c}\text { C3 } \\
\text { HGEN } \\
\text { (W/CC) }\end{array}$} & \multirow{3}{*}{$\begin{array}{c}\text { C4 } \\
\text { HGEN } \\
\text { (W/CC) }\end{array}$} & \multirow{3}{*}{$\begin{array}{c}\text { C5 } \\
\text { HGEN } \\
\text { (W/CC) }\end{array}$} & \multirow{3}{*}{$\begin{array}{c}\text { C6 } \\
\text { HGEN } \\
\text { (W/Cc) }\end{array}$} \\
\hline & & & & & & & & \\
\hline in & $\mathrm{cm}$ & & & & & & & \\
\hline 16.4 & 41.67 & & 4.52 & 4.58 & 5.10 & 6.05 & 4.09 & 4.79 \\
\hline 15.5 & 39.29 & & 5.05 & 5.03 & 5.26 & 6.02 & 4.55 & 5.04 \\
\hline 14.5 & 36.91 & مه & 5.55 & 5.69 & 5.73 & 6.66 & 5.17 & 5.51 \\
\hline 13.6 & 34.53 & $\underline{\underline{\underline{\underline{Q}}}}$ & 6.24 & 5.86 & 6.38 & 6.84 & 5.34 & 6.02 \\
\hline 12.7 & 32.15 & & 6.92 & 6.74 & 6.87 & 7.73 & 5.79 & 6.64 \\
\hline 11.7 & 29.77 & & 7.39 & 7.35 & 7.60 & 8.14 & 6.50 & 7.57 \\
\hline 10.8 & 27.39 & & 8.86 & 8.59 & 8.41 & 8.99 & 7.03 & 8.40 \\
\hline 9.8 & 25.01 & & 9.82 & 9.47 & 9.68 & 9.66 & 8.47 & 8.97 \\
\hline 8.9 & 22.63 & & 10.51 & 10.63 & 11.14 & 10.48 & 9.55 & 9.93 \\
\hline 8.0 & 20.25 & v & 11.76 & 11.56 & 11.50 & 11.18 & 10.58 & 10.78 \\
\hline 7.0 & 17.87 & $\stackrel{\grave{I}}{=}$ & 12.71 & 12.39 & 12.29 & 11.55 & 11.45 & 11.60 \\
\hline 6.1 & 15.49 & & 13.64 & 13.53 & 12.74 & 12.12 & 12.19 & 12.40 \\
\hline 5.2 & 13.11 & & 14.00 & 14.07 & 13.96 & 12.94 & 12.80 & 12.74 \\
\hline 4.2 & 10.73 & & 14.48 & 15.04 & 14.30 & 13.47 & 13.29 & 13.29 \\
\hline 3.3 & 8.35 & & 15.52 & 15.72 & 14.54 & 13.56 & 13.94 & 14.00 \\
\hline 2.4 & 5.97 & & 15.83 & 16.02 & 14.88 & 14.03 & 13.95 & 14.50 \\
\hline 1.4 & 3.59 & $n$ & 16.19 & 16.54 & 15.60 & 14.11 & 13.97 & 14.41 \\
\hline 0.5 & 1.21 & $\underline{\underline{\Phi}}$ & 16.28 & 16.64 & 15.94 & 14.06 & 14.34 & 14.74 \\
\hline-0.5 & -1.17 & & 16.15 & 16.80 & 15.38 & 14.10 & 14.20 & 14.79 \\
\hline-1.4 & -3.55 & & 16.18 & 16.78 & 15.68 & $\uparrow 4.42$ & 14.81 & $14: 95$ \\
\hline-2.3 & -5.93 & & 16.26 & 16.63 & 15.32 & 14.23 & 14.26 & 14.98 \\
\hline-3.3 & -8.31 & & 17.30 & 16.80 & 15.04 & 14.30 & 14.23 & 14.63 \\
\hline-4.2 & -10.69 & & 18.33 & 16.36 & 14.20 & 14.12 & 14.15 & 14.26 \\
\hline-5.1 & -13.07 & $w$ & 18.16 & 15.89 & 13.91 & 14.08 & 13.61 & 13.81 \\
\hline-6.1 & -15.45 & 产 & 17.73 & 15.30 & 12.78 & 13.73 & 13.70 & 13.66 \\
\hline-7.0 & -17.83 & & 16.23 & 14.84 & 13.02 & 13.45 & 12.99 & 12.94 \\
\hline-8.0 & -20.21 & & 16.00 & 13.47 & 12.25 & 12.74 & 12.37 & 11.88 \\
\hline-8.9 & -22.59 & & 14.73 & 12.99 & 11.73 & 12.26 & 12.02 & 11.45 \\
\hline-9.8 & -24.97 & & 13.05 & 11.68 & 10.88 & 11.63 & 9.98 & 11.11 \\
\hline-10.8 & -27.35 & & 11.81 & 10.16 & 10.53 & 11.16 & 8.05 & 10.80 \\
\hline-11.7 & -29.73 & $\sigma$ & 10.78 & 9.55 & 10.41 & 10.80 & 7.77 & 10.22 \\
\hline-12.6 & -32.11 & 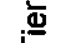 & 10.56 & 9.21 & 9.98 & 10.75 & 7.43 & 9.83 \\
\hline-13.6 & -34.49 & & 10.37 & 8.70 & 9.18 & 10.23 & 7.33 & 9.61 \\
\hline-14.5 & -36.87 & & 9.18 & 8.45 & 8.96 & 10.33 & 7.20 & 9.15 \\
\hline-15.5 & -39.25 & & 8.94 & 8.20 & 8.42 & 9.74 & 6.63 & 8.62 \\
\hline
\end{tabular}


Enclosure (7) to

MDO-723-0044/

B-MT(SRME)-52

Page 38

Table III.A-2

Axial Heat Generation Rates for the Alloy 617 Center Specimen of 6d4

\begin{tabular}{|c|c|c|c|c|c|c|c|c|}
\hline \multicolumn{2}{|c|}{ Elevation } & & $\begin{array}{c}\text { C1 } \\
\text { HGEN }\end{array}$ & $\begin{array}{c}\mathrm{C} 2 \\
\text { HGEN }\end{array}$ & $\begin{array}{c}\text { C3 } \\
\text { HGEN }\end{array}$ & $\begin{array}{c}\text { C4 } \\
\text { HGEN }\end{array}$ & $\begin{array}{c}\text { C5 } \\
\text { HGEN }\end{array}$ & $\begin{array}{c}\text { C6 } \\
\text { HGEN }\end{array}$ \\
\hline in & $\mathrm{cm}$ & & (W/cc) & (W/cc) & $(\mathrm{W} / \mathrm{cc})$ & $(W / c c)$ & (W/cc) & $(W / c c)$ \\
\hline 16.4 & 41.67 & & 5.01 & 5.24 & 3.83 & 4.33 & 4.39 & 5.12 \\
\hline 15.5 & 39.29 & & 5.20 & 5.10 & 4.39 & 4.60 & 4.56 & 5.12 \\
\hline 14.5 & 36.91 & 10 & 5.86 & 5.50 & 5.05 & 5.32 & 5.19 & 5.52 \\
\hline 13.6 & 34.53 & $\underline{\underline{\omega}}$ & 6.78 & 5.97 & 5.87 & 5.64 & 5.83 & 6.47 \\
\hline 12.7 & 32.15 & $i=$ & 7.08 & 6.67 & 6.26 & 5.86 & 6.10 & 6.89 \\
\hline 11.7 & 29.77 & & 7.86 & 7.58 & 6.86 & 6.42 & 6.93 & 7.41 \\
\hline 10.8 & 27.39 & & 9.21 & 7.92 & 7.47 & 6.64 & 7.57 & 8.36 \\
\hline 9.8 & 25.01 & & 9.74 & 9.73 & 8.54 & 8.67 & 7.83 & 9.29 \\
\hline 8.9 & 22.63 & & 10.03 & 11.26 & 9.87 & 10.20 & 7.87 & 10.51 \\
\hline 8.0 & 20.25 & $\nabla$ & 11.22 & 12.04 & 11.18 & 11.02 & 8.42 & 11.94 \\
\hline 7.0 & 17.87 & $\underline{\underline{\omega}}$ & 12.21 & 13.21 & 11.27 & 11.16 & 9.09 & 12.65 \\
\hline 6.1 & 15.49 & $F$ & 13.41 & 14.41 & 11.90 & 12.14 & 10.23 & 13.30 \\
\hline 5.2 & 13.11 & & 13.64 & 14.90 & 12.44 & 12.49 & 10.23 & 14.57 \\
\hline 4.2 & 10.73 & & 13.58 & 15.39 & 13.03 & 13.02 & 10.78 & 14.98 \\
\hline 3.3 & 8.35 & & 16.23 & 14.84 & 13.60 & 13.85 & 12.75 & 16.23 \\
\hline 2.4 & 5.97 & & 18.72 & 14.37 & 13.92 & 14.38 & 14.04 & 16.75 \\
\hline 1.4 & 3.59 & $m$ & 19.35 & 14.94 & 14.21 & 14.66 & 14.24 & 17.01 \\
\hline 0.5 & 1.21 & 过 & 19.11 & 15.47 & 14.42 & 15.35 & 14.57 & 17.26 \\
\hline-0.5 & -1.17 & $F$ & 19.33 & 14.93 & 14.06 & 14.69 & 14.90 & 16.13 \\
\hline-1.4 & -3.55 & & 19.33 & 15.91 & 14.37 & 15.31 & 15.25 & 17.03 \\
\hline-2.3 & -5.93 & & 19.39 & 14.85 & 13.73 & 14.55 & 14.28 & 16.64 \\
\hline-3.3 & -8.31 & & 19.18 & 16.14 & 14.44 & 14.10 & 12.57 & 15.03 \\
\hline-4.2 & -10.69 & & 18.94 & 16.57 & 14.07 & 13.66 & 10.79 & 13.86 \\
\hline-5.1 & -13.07 & $N$ & 17.93 & 15.94 & 13.75 & 13.82 & 10.35 & 13.76 \\
\hline-6.1 & -15.45 & $\underline{\underline{J}}$ & 17.08 & 15.40 & 13.22 & 12.62 & 10.36 & 13.60 \\
\hline-7.0 & -17.83 & F & 16.76 & 14.72 & 13.04 & 12.90 & 10.03 & 12.99 \\
\hline-8.0 & -20.21 & & 15.74 & 14.17 & 12.50 & 12.87 & 9.14 & 12.09 \\
\hline-8.9 & -22.59 & & 14.36 & 12.78 & 12.29 & 11.75 & 9.09 & 11.85 \\
\hline-9.8 & -24.97 & & 13.24 & 11.78 & 11.57 & 10.49 & 9.14 & 11.44 \\
\hline-10.8 & -27.35 & & 11.92 & 11.01 & 10.11 & 9.17 & 9.55 & 11.70 \\
\hline-11.7 & -29.73 & - & 11.13 & 10.25 & 9.09 & 8.87 & 9.44 & 10.50 \\
\hline-12.6 & -32.11 & $\underline{\Phi}$ & 11.02 & 10.03 & 8.76 & 8.24 & 8.70 & 9.53 \\
\hline-13.6 & -34.49 & $F$ & 10.05 & 9.58 & 8.62 & 8.05 & 8.42 & 9.72 \\
\hline-14.5 & -36.87 & & 9.67 & 9.05 & 7.99 & 7.66 & 7.17 & 9.19 \\
\hline-15.5 & -39.25 & & 9.43 & 8.61 & 7.46 & 7.06 & 7.62 & 8.44 \\
\hline
\end{tabular}


Enclosure (7) to MDO-723-0044I

B-MT(SRME)-52

Page 39

\begin{tabular}{|c|c|c|c|c|c|c|c|c|}
\hline & & & on $\mathrm{Re}$ & $\begin{array}{l}\text { able III.A } \\
\text { or the A }\end{array}$ & Cent & Jecime & $6 a 4$ & \\
\hline in & ion & & $\begin{array}{c}\text { C1 } \\
\text { HGEN } \\
\text { (W/CC) }\end{array}$ & $\begin{array}{c}\mathrm{C2} \\
\text { HGEN } \\
(\mathrm{W} / \mathrm{CC})\end{array}$ & $\begin{array}{c}\text { C3 } \\
\text { HGEN } \\
\text { (W/cc) }\end{array}$ & $\begin{array}{c}\mathrm{C4} \\
\text { HGEN } \\
\text { (W/CC) }\end{array}$ & $\begin{array}{c}\text { C5 } \\
\text { HGEN } \\
\text { (W/cc) }\end{array}$ & $\begin{array}{c}\text { C6 } \\
\text { HGEN } \\
\text { (W/CC) }\end{array}$ \\
\hline $\begin{array}{l}16.4 \\
15.5 \\
14.5 \\
13.6 \\
12.7 \\
11.7 \\
10.8\end{array}$ & $\begin{array}{l}41.67 \\
39.29 \\
36.91 \\
34.53 \\
32.15 \\
29.77 \\
27.39\end{array}$ & $\stackrel{\omega}{\frac{\omega}{\omega}}$ & $\begin{array}{l}16.44 \\
18.30 \\
20.44 \\
23.48 \\
26.03 \\
28.54 \\
34.19 \\
\end{array}$ & $\begin{array}{l}16.20 \\
17.81 \\
20.06 \\
20.99 \\
24.54 \\
26.77 \\
31.66 \\
\end{array}$ & $\begin{array}{l}16.38 \\
17.13 \\
18.58 \\
20.67 \\
22.45 \\
24.78 \\
28.08 \\
\end{array}$ & $\begin{array}{l}19.51 \\
19.32 \\
21.16 \\
21.78 \\
24.06 \\
25.64 \\
28.34 \\
\end{array}$ & $\begin{array}{l}11.81 \\
13.25 \\
15.13 \\
15.62 \\
17.01 \\
19.04 \\
20.84 \\
\end{array}$ & $\begin{array}{l}16.57 \\
17.73 \\
19.40 \\
21.61 \\
23.92 \\
27.44 \\
30.83 \\
\end{array}$ \\
\hline $\begin{array}{l}9.8 \\
8.9 \\
8.0 \\
7.0 \\
6.1 \\
5.2 \\
4.2\end{array}$ & $\begin{array}{l}25.01 \\
22.63 \\
20.25 \\
17.87 \\
15.49 \\
13.11 \\
10.73\end{array}$ & 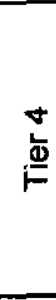 & $\begin{array}{l}34.31 \\
32.65 \\
36.32 \\
39.81 \\
42.58 \\
43.41 \\
45.00 \\
\end{array}$ & $\begin{array}{l}32.70 \\
34.58 \\
37.93 \\
40.46 \\
43.94 \\
45.90 \\
49.29 \\
\end{array}$ & $\begin{array}{l}31.31 \\
35.07 \\
36.61 \\
38.74 \\
40.71 \\
44.34 \\
45.30 \\
\end{array}$ & $\begin{array}{l}30.63 \\
33.21 \\
35.53 \\
36.74 \\
38.91 \\
41.30 \\
42.78 \\
\end{array}$ & $\begin{array}{l}26.91 \\
31.95 \\
35.70 \\
38.40 \\
40.64 \\
43.15 \\
44.61 \\
\end{array}$ & $\begin{array}{l}30.01 \\
29.58 \\
32.14 \\
34.62 \\
37.01 \\
38.46 \\
40.29 \\
\end{array}$ \\
\hline $\begin{array}{c}3.3 \\
2.4 \\
1.4 \\
0.5 \\
-0.5 \\
-1.4 \\
-2.3\end{array}$ & $\begin{array}{c}8.35 \\
5.97 \\
3.59 \\
1.21 \\
-1.17 \\
-3.55 \\
-5.93\end{array}$ & $\stackrel{2}{\underline{2}}$ & $\begin{array}{l}48.02 \\
49.09 \\
49.78 \\
50.12 \\
49.28 \\
49.53 \\
50.36 \\
\end{array}$ & $\begin{array}{l}51.70 \\
52.76 \\
54.25 \\
54.48 \\
55.46 \\
54.94 \\
55.12 \\
\end{array}$ & $\begin{array}{l}46.21 \\
47.42 \\
49.49 \\
50.56 \\
49.13 \\
49.90 \\
49.03 \\
\end{array}$ & $\begin{array}{l}43.49 \\
44.59 \\
45.07 \\
45.31 \\
44.74 \\
45.98 \\
45.52 \\
\end{array}$ & $\begin{array}{l}46.54 \\
47.20 \\
47.05 \\
48.81 \\
47.66 \\
49.50 \\
47.95 \\
\end{array}$ & $\begin{array}{l}42.28 \\
43.46 \\
43.30 \\
44.22 \\
44.46 \\
45.09 \\
44.98 \\
\end{array}$ \\
\hline $\begin{array}{l}-3.3 \\
-4.2 \\
-5.1 \\
-6.1 \\
-7.0 \\
-8.0 \\
-8.9\end{array}$ & $\begin{array}{c}-8.31 \\
-10.69 \\
-13.07 \\
-15.45 \\
-17.83 \\
-20.21 \\
-22.59\end{array}$ & $\stackrel{N}{\stackrel{N}{E}}$ & $\begin{array}{l}60.19 \\
70.20 \\
68.72 \\
67.97 \\
62.34 \\
60.56 \\
55.34 \\
\end{array}$ & $\begin{array}{l}55.53 \\
54.23 \\
52.55 \\
50.86 \\
48.78 \\
43.98 \\
42.22 \\
\end{array}$ & $\begin{array}{l}48.85 \\
47.39 \\
46.74 \\
43.13 \\
43.22 \\
40.51 \\
38.70 \\
\end{array}$ & $\begin{array}{l}45.70 \\
45.20 \\
45.02 \\
43.49 \\
42.83 \\
40.63 \\
39.15 \\
\end{array}$ & $\begin{array}{l}47.98 \\
47.77 \\
46.26 \\
46.05 \\
43.35 \\
41.56 \\
39.70 \\
\end{array}$ & $\begin{array}{l}43.99 \\
42.95 \\
41.56 \\
41.14 \\
38.94 \\
36.01 \\
34.56 \\
\end{array}$ \\
\hline $\begin{array}{r}-9.8 \\
-10.8 \\
-11.7 \\
-12.6 \\
-13.6 \\
-14.5 \\
-15.5 \\
\end{array}$ & $\begin{array}{l}-24.97 \\
-27.35 \\
-29.73 \\
-32.11 \\
-34.49 \\
-36.87 \\
-39.25 \\
\end{array}$ & $i$ & $\begin{array}{l}49.00 \\
43.73 \\
40.38 \\
38.80 \\
37.80 \\
33.47 \\
32.52 \\
\end{array}$ & $\begin{array}{l}36.50 \\
30.44 \\
28.40 \\
27.41 \\
25.79 \\
24.99 \\
24.06 \\
\end{array}$ & $\begin{array}{l}36.03 \\
34.51 \\
33.67 \\
32.21 \\
29.78 \\
28.90 \\
27.44 \\
\end{array}$ & $\begin{array}{l}37.09 \\
35.35 \\
34.32 \\
34.16 \\
32.45 \\
33.04 \\
31.08\end{array}$ & $\begin{array}{l}32.08 \\
24.57 \\
23.19 \\
22.23 \\
21.98 \\
21.63 \\
19.86\end{array}$ & $\begin{array}{l}36.80 \\
38.80 \\
36.92 \\
35.05 \\
33.94 \\
32.41 \\
30.26\end{array}$ \\
\hline te: $\mathrm{HC}$ & Total & & $\overline{\mathrm{C}} 1-$ & Compa & 19 & tment6 & & \\
\hline
\end{tabular}


Enclosure (7) to

MDO-723-0044/

B-MT(SRME)-52

Page 40

\begin{tabular}{|c|c|c|c|c|c|c|c|c|}
\hline \multicolumn{9}{|c|}{$\begin{array}{r}\text { Table III.A-2 } \\
\text { Axial Heat Generation Rates for the ASTA }\end{array}$} \\
\hline \multicolumn{2}{|c|}{ Elevation } & \multirow{9}{*}{$\stackrel{n}{\mathbf{\Phi}}$} & \multirow{2}{*}{$\begin{array}{c}\text { C1 } \\
\text { HGEN } \\
\text { (W/CC) }\end{array}$} & \multirow{2}{*}{$\begin{array}{l}\text { C2 } \\
\text { HGEN } \\
(W / C c)\end{array}$} & \multirow{2}{*}{$\begin{array}{c}\text { C3 } \\
\text { HGEN } \\
(\text { W/CC) }\end{array}$} & \multirow{2}{*}{$\begin{array}{c}\text { C4 } \\
\text { HGEN } \\
\text { (W/Cc) }\end{array}$} & \multirow{2}{*}{$\begin{array}{c}\text { C5 } \\
\text { HGEN } \\
(W / C c)\end{array}$} & \multirow{2}{*}{$\begin{array}{c}\text { C6 } \\
\text { HGEN } \\
\text { (W/CC) }\end{array}$} \\
\hline in & $\mathrm{cm}$ & & & & & & & \\
\hline 16.4 & 41.67 & & 17.90 & 17.74 & 13.06 & 15.42 & 14.96 & 17.77 \\
\hline 15.5 & 39.29 & & 18.29 & 17.94 & 14.97 & 16.13 & 15.43 & 18.00 \\
\hline 14.5 & 36.91 & & 20.60 & 19.77 & 17.46 & 18.94 & 17.97 & 19.98 \\
\hline 13.6 & 34.53 & & 24.22 & 21.73 & 20.99 & 20.56 & 19.93 & 23.29 \\
\hline 12.7 & 32.15 & & 25.51 & 24.34 & 22.70 & 20.87 & 21.62 & 24.94 \\
\hline 11.7 & 29.77 & & 28.14 & 28.58 & 24.09 & 24.38 & 24.68 & 26.47 \\
\hline 10.8 & 27.39 & & 33.56 & 30.14 & 27.04 & 24.59 & 27.20 & 30.78 \\
\hline 9.8 & 25.01 & & 32.79 & 36.09 & 29.95 & 29.27 & 25.87 & 34.62 \\
\hline 8.9 & 22.63 & & 30.76 & 40.95 & 32.70 & 32.45 & 23.43 & 39.17 \\
\hline 8.0 & 20.25 & 寸 & 33.47 & 43.60 & 37.21 & 35.07 & 25.21 & 44.60 \\
\hline 7.0 & 17.87 & I & 36.36 & 49.52 & 37.58 & 35.69 & 27.33 & 48.21 \\
\hline 6.1 & 15.49 & $i$ & 40.21 & 52.79 & 39.44 & 38.60 & 30.70 & 51.23 \\
\hline 5.2 & 13.11 & & 40.14 & 55.76 & 41.86 & 39.99 & 30.75 & 55.35 \\
\hline 4.2 & 10.73 & & 41.03 & 58.53 & 43.86 & 41.72 & 32.28 & 56.17 \\
\hline 3.3 & 8.35 & & 56.70 & 50.86 & 45.39 & 44.24 & 40.52 & 56.93 \\
\hline 2.4 & 5.97 & & 70.97 & 43.41 & 46.01 & 45.67 & 47.16 & 54.29 \\
\hline 1.4 & 3.59 & $m$ & 73.16 & 44.83 & 46.97 & 46.30 & 48.51 & 55.02 \\
\hline 0.5 & 1.21 & $\Phi$ & 73.54 & 46.62 & 48.00 & 48.38 & 50.17 & 55.49 \\
\hline-0.5 & -1.17 & & 74.94 & 45.97 & 47.31 & 47.22 & 51.11 & 52.79 \\
\hline-1.4 & -3.55 & & 74.75 & 48.79 & 47.51 & 48.92 & 51.65 & 55.61 \\
\hline-2.3 & -5.93 & & 75.72 & 45.30 & 45.92 & 46.21 & 48.49 & 54.43 \\
\hline-3.3 & -8.31 & & 73.60 & 50.99 & 48.33 & 44.90 & 40.83 & 47.27 \\
\hline-4.2 & -10.69 & & 73.30 & 54.35 & 48.07 & 43.32 & 32.37 & 41.83 \\
\hline-5.1 & -13.07 & $N$ & 69.87 & 52.67 & 46.29 & 44.23 & 31.08 & 41.30 \\
\hline-6.1 & -15.45 & 产 & 66.11 & 51.10 & 45.20 & 40.46 & 31.22 & 40.75 \\
\hline-7.0 & -17.83 & $\vdash$ & 64.70 & 47.25 & 43.63 & 41.05 & 30.01 & 38.79 \\
\hline-8.0 & -20.21 & & 60.79 & 45.90 & 41.84 & 40.48 & 27.57 & 36.29 \\
\hline-8.9 & -22.59 & & 54.95 & 41.79 & 40.64 & 37.21 & 27.40 & 35.51 \\
\hline-9.8 & -24.97 & & 49.29 & 41.48 & 39.74 & 35.71 & 31.07 & 37.77 \\
\hline-10.8 & -27.35 & & 43.86 & 41.33 & 36.48 & 32.99 & 34.64 & 42.03 \\
\hline-11.7 & -29.73 & - & 41.71 & 37.42 & 33.05 & 31.09 & 33.31 & 38.59 \\
\hline-12.6 & -32.11 & à & 39.98 & 37.51 & 31.57 & 29.40 & 30.60 & 34.98 \\
\hline-13.6 & -34.49 & $F$ & 35.95 & 35.29 & 30.02 & 28.36 & 29.73 & 34.84 \\
\hline-14.5 & -36.87 & & 34.54 & $32.5 \uparrow$ & 28.05 & 27.09 & 24.73 & 33.20 \\
\hline-15.5 & -39.25 & & 33.50 & 30.97 & 26.02 & 24.84 & 25.86 & 29.97 \\
\hline
\end{tabular}


Enclosure (7) to MDO-723-0044/

B-MT(SRME)-52

Page 41

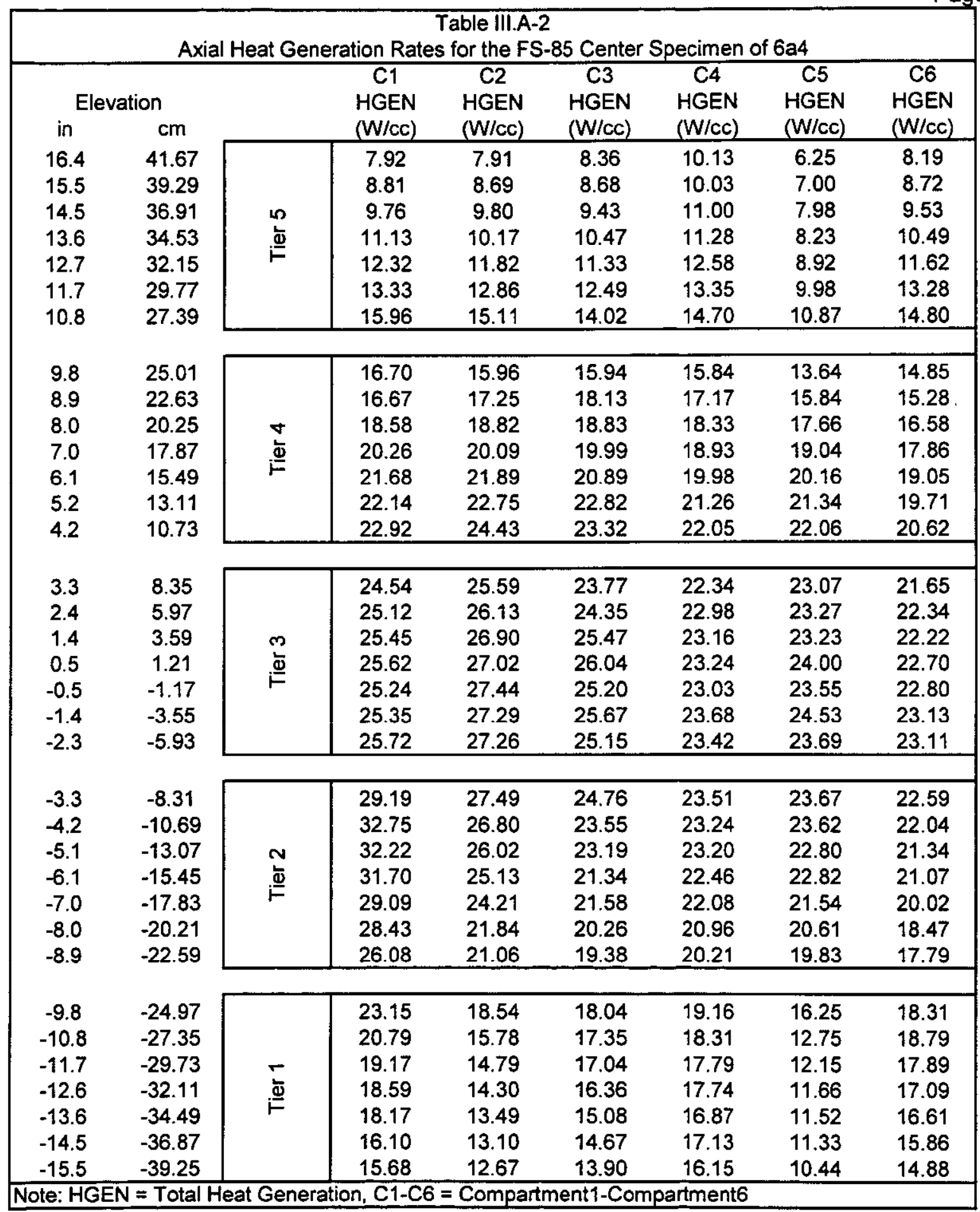


Enclosure (7) to

MDO-723-0044/

B-MT(SRME)-52

Page 42

\begin{tabular}{|c|c|c|c|c|c|c|c|c|}
\hline \multicolumn{9}{|c|}{$\begin{array}{l}\text { Table III.A-2 } \\
\text { Axial Heat Generation Rates for the FS-85 Center Specimen of } 6 \mathrm{~d} 44\end{array}$} \\
\hline \multirow{2}{*}{\multicolumn{2}{|c|}{ Elevation }} & & C1 & C2 & C3 & $\mathrm{C4}$ & C5 & $\overline{\mathrm{C} 6}$ \\
\hline & & & HGEN & HGEN & HGEN & HGEN & HGEN & HGEN \\
\hline in & $\mathrm{cm}$ & & $(W / c c)$ & $(W / c c)$ & (W/cc) & $(W / c c)$ & $(W / c c)$ & (W/cc) \\
\hline 16.4 & 41.67 & \multirow{7}{*}{$\stackrel{n}{\underline{\omega} \underline{\underline{\alpha}}}$} & 8.73 & 8.83 & 6.44 & 7.53 & 7.38 & 8.78 \\
\hline 15.5 & 39.29 & & 8.94 & 8.76 & 7.36 & 7.90 & 7.64 & 8.84 \\
\hline 14.5 & 36.91 & & 10.07 & 9.56 & 8.56 & 9.21 & 8.81 & 9.69 \\
\hline 13.6 & 34.53 & & 11.76 & 10.43 & 10.16 & 9.92 & 9.81 & 11.33 \\
\hline 12.7 & 32.15 & & 12.33 & 11.68 & 10.93 & 10.13 & 10.48 & 12.12 \\
\hline 11.7 & 29.77 & & 13.59 & 13.54 & 11.72 & 11.55 & 11.96 & 12.83 \\
\hline 10.8 & 27.39 & & 16.05 & 14.18 & 13.00 & 11.71 & 13.08 & 14.72 \\
\hline 9.8 & 25.01 & \multirow{7}{*}{$\stackrel{\stackrel{+}{\Phi}}{\stackrel{+}{=}}$} & 16.24 & 17.12 & 14.58 & 14.64 & 12.85 & 16.43 \\
\hline 8.9 & 22.63 & & 15.95 & 19.55 & 16.32 & 16.74 & 12.15 & 18.52 \\
\hline 8.0 & 20.25 & & 17.40 & 20.75 & 18.53 & 18.08 & 13.05 & 21.08 \\
\hline 7.0 & 17.87 & & 18.83 & 23.31 & 18.64 & 18.38 & 14.09 & 22.57 \\
\hline 6.1 & 15.49 & & 20.83 & 25.00 & 19.65 & 19.92 & 15.83 & 23.90 \\
\hline 5.2 & 13.11 & & 20.96 & 26.24 & 20.68 & 20.54 & 15.83 & 25.97 \\
\hline 4.2 & 10.73 & & 21.10 & 27.38 & 21.66 & 21.41 & 16.65 & 26.44 \\
\hline 3.3 & 8.35 & \multirow{7}{*}{$\stackrel{m}{\stackrel{m}{\circ}}$} & 27.46 & 24.73 & 22.53 & 22.75 & 20.48 & 27.51 \\
\hline 2.4 & 5.97 & & 33.20 & 22.24 & 22.98 & 23.55 & 23.34 & 27.16 \\
\hline 1.4 & 3.59 & & 34.29 & 23.02 & 23.47 & 23.88 & 23.83 & 27.58 \\
\hline 0.5 & 1.21 & & 34.31 & 23.90 & 23.89 & 25.05 & 24.57 & 27.83 \\
\hline-0.5 & -1.17 & & 34.89 & 23.37 & 23.43 & 24.19 & 25.07 & 26.26 \\
\hline-1.4 & -3.55 & & 34.72 & 24.87 & 23.69 & 25.19 & 25.47 & 27.69 \\
\hline-2.3 & -5.93 & & 35.06 & 23.07 & 22.75 & 23.84 & 23.86 & 27.10 \\
\hline-3.3 & -8.31 & \multirow{7}{*}{$\stackrel{N}{\stackrel{N}{*}}$} & 34.28 & 25.66 & 23.95 & 23.11 & 20.49 & 23.83 \\
\hline-4.2 & -10.69 & & 33.99 & 27.04 & 23.63 & 22.34 & 16.84 & 21.41 \\
\hline-5.1 & -13.07 & & 32.34 & 26.13 & 22.88 & 22.73 & 16.18 & 21.19 \\
\hline-6.1 & -15.45 & & 30.73 & 25.33 & 22.26 & 20.82 & 16.18 & 20.96 \\
\hline-7.0 & -17.83 & & 30.13 & 23.67 & 21.66 & 21.16 & 15.57 & 19.99 \\
\hline-8.0 & -20.21 & & 28.25 & 23.01 & 20.80 & 20.99 & 14.39 & 18.68 \\
\hline-8.9 & -22.59 & & 25.69 & 20.83 & 20.29 & 19.23 & 14.29 & 18.33 \\
\hline-9.8 & -24.97 & \multirow{7}{*}{$\bar{\Phi}$} & $23 . \overline{7}$ & 2011 & 1953 & 1778 & 1533 & $18 \overline{83}$ \\
\hline-10.8 & -27.35 & & 20.96 & 19.56 & 17.55 & 15.91 & 16.64 & 20.36 \\
\hline-11.7 & -29.73 & & 19.84 & 17.89 & 15.91 & 15.17 & 16.18 & 18.55 \\
\hline-12.6 & -32.11 & & 19.29 & 17.86 & 15.25 & 14.29 & 14.93 & 16.87 \\
\hline-13.6 & -34.49 & & 17.42 & 16.93 & 14.70 & 13.84 & 14.50 & 16.97 \\
\hline-14.5 & -36.87 & & 16.76 & 15.76 & 13.72 & 13.22 & 12.13 & 16.17 \\
\hline-15.5 & -39.25 & & 16.33 & 15.04 & 12.77 & 12.15 & 12.81 & 14.69 \\
\hline
\end{tabular}


Enclosure (7) to

MDO-723-0044/

B-MT(SRME)-52

Page 43

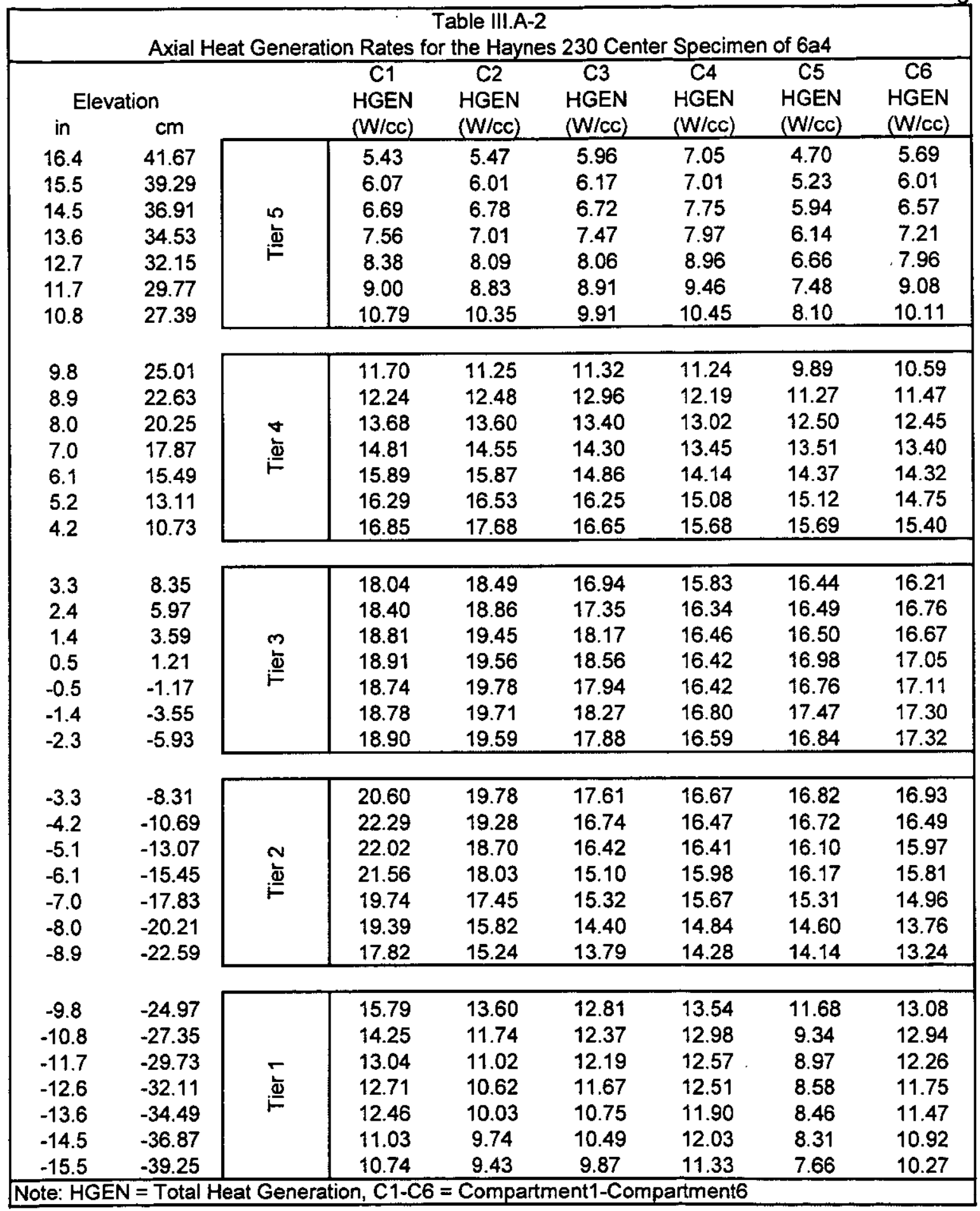


Enclosure (7) to

MDO-723-0044I

B-MT(SRME)-52

Page 44

Table III.A-2

Axial Heat Generation Rates for the Haynes 230 Center Specimen of $6 \mathrm{~d} 4$

\begin{tabular}{|c|c|c|c|c|c|c|c|c|}
\hline \multicolumn{2}{|c|}{ Elevation } & & $\begin{array}{c}\text { C1 } \\
\text { HGEN }\end{array}$ & $\begin{array}{c}\text { C2 } \\
\text { HGEN }\end{array}$ & $\begin{array}{c}\text { C3 } \\
\text { HGEN }\end{array}$ & $\begin{array}{c}\text { C4 } \\
\text { HGEN }\end{array}$ & $\begin{array}{c}\text { C5 } \\
\text { HGEN }\end{array}$ & $\begin{array}{c}\text { C6 } \\
\text { HGEN }\end{array}$ \\
\hline in & $\mathrm{cm}$ & & (W/cc) & (W/cc) & (W/Cc) & (W/Cc) & (W/CC) & (W/cc) \\
\hline 16.4 & 41.67 & & 6.00 & 6.20 & 4.56 & 5.18 & 5.21 & 6.08 \\
\hline 15.5 & 39.29 & & 6.20 & 6.09 & 5.21 & 5.48 & 5.40 & 6.11 \\
\hline 14.5 & 36.91 & $\omega$ & 6.98 & 6.59 & 6.01 & 6.36 & 6.17 & 6.62 \\
\hline 13.6 & 34.53 & L & 8.11 & 7.18 & 7.03 & 6.77 & 6.91 & 7.75 \\
\hline 12.7 & 32.15 & $F$ & 8.49 & 8.01 & 7.52 & 7.01 & 7.29 & 8.25 \\
\hline 11.7 & 29.77 & & 9.40 & 9.17 & 8.18 & 7.78 & 8.29 & 8.86 \\
\hline 10.8 & 27.39 & & 11.06 & 9.62 & 8.97 & 8.01 & 9.08 & 10.06 \\
\hline 9.8 & 25.01 & & 11.50 & 11.75 & 10.18 & 10.24 & 9.23 & 11.22 \\
\hline 8.9 & 22.63 & & 11.63 & 13.53 & 11.62 & 11.88 & 9.09 & 12.70 \\
\hline 8.0 & 20.25 & $\forall$ & 12.96 & 14.46 & 13.17 & 12.83 & 9.73 & 14.43 \\
\hline 7.0 & 17.87 & $\underline{\Phi}$ & 14.10 & 15.98 & 13.28 & 13.01 & 10.51 & 15.36 \\
\hline 6.1 & 15.49 & $F$ & 15.50 & 17.34 & 14.01 & 14.13 & 11.82 & 16.19 \\
\hline 5.2 & 13.11 & & 15.71 & 18.02 & 14.70 & 14.57 & 11.83 & 17.68 \\
\hline 4.2 & 10.73 & & 15.73 & 18.68 & 15.39 & 15.19 & 12.45 & 18.13 \\
\hline 3.3 & 8.35 & & 19.34 & 17.62 & 16.03 & 16.15 & 1489 & 1935 \\
\hline 2.4 & 5.97 & & 22.71 & 16.64 & 16.37 & 16.75 & 16.57 & 19.63 \\
\hline 1.4 & 3.59 & $m$ & 23.46 & 17.27 & 16.71 & 17.06 & 16.86 & 19.93 \\
\hline 0.5 & 1.21 & 包 & 23.26 & 17.90 & 16.99 & 17.84 & 17.29 & 20.20 \\
\hline-0.5 & -1.17 & & 23.56 & 17.34 & 16.60 & 17.15 & 17.67 & 18.95 \\
\hline-1.4 & -3.55 & & 23.56 & 18.47 & 16.91 & 17.85 & 18.03 & 20.01 \\
\hline-2.3 & -5.93 & & 23.68 & 17.23 & 16.20 & 16.94 & 16.90 & 19.55 \\
\hline-3.3 & -8.31 & & 23.32 & 18.84 & 17.03 & 16.43 & $14 . \overline{74}$ & 17.53 \\
\hline-4.2 & -10.69 & & 23.09 & 19.47 & 16.67 & 15.90 & 12.47 & 16.04 \\
\hline-5.1 & -13.07 & $N$ & 21.89 & 18.76 & 16.24 & 16.11 & 11.96 & 15.91 \\
\hline-6.1 & -15.45 & $\underline{\underline{\Phi}}$ & 20.82 & 18.14 & 15.66 & 14.72 & 11.97 & 15.72 \\
\hline-7.0 & -17.83 & $E$ & 20.41 & 17.22 & 15.38 & 15.02 & 11.59 & 15.00 \\
\hline-8.0 & -20.21 & & 19.17 & 16.60 & 14.74 & 14.95 & 10.57 & 13.97 \\
\hline-8.9 & -22.59 & & 17.46 & 15.02 & 14.46 & 13.67 & 10.50 & 13.68 \\
\hline-9.8 & -24.97 & & 15.99 & 14.06 & 13.72 & 12.41 & 10.83 & 13.47 \\
\hline-10.8 & -27.35 & & 14.36 & 13.34 & 12.14 & 10.99 & 11.48 & 14.02 \\
\hline-11.7 & -29.73 & $r$ & 13.46 & 12.34 & 10.93 & 10.58 & 11.28 & 12.65 \\
\hline-12.6 & -32.11 & $\underline{\Phi}$ & 13.23 & 12.12 & 10.51 & 9.85 & 10.38 & 11.47 \\
\hline-13.6 & -34.49 & $F$ & 12.04 & 11.54 & 10.27 & 9.60 & 10.06 & 11.63 \\
\hline-14.5 & -36.87 & & 11.58 & 10.85 & 9.53 & 9.15 & 8.53 & 11.01 \\
\hline-15.5 & -39.25 & & 11.27 & 10.32 & 8.89 & 8.43 & 9.03 & 10.08 \\
\hline
\end{tabular}


Enclosure (7) to

MDO-723-0044/

B-MT(SRME)-52

Page 45

\begin{tabular}{|c|c|c|c|c|c|c|c|c|}
\hline \multicolumn{9}{|c|}{$\begin{array}{c}\text { Table III.A-2 } \\
\text { Axial Heat Generation Rates for the PE16 Center Specimen of } 6 a 4\end{array}$} \\
\hline \multicolumn{2}{|c|}{ Elevation } & & $\begin{array}{c}\text { C1 } \\
\text { HGEN } \\
\text { (W/CC) }\end{array}$ & $\begin{array}{c}\text { C2 } \\
\text { HGEN } \\
\text { (W/CC) }\end{array}$ & $\begin{array}{c}\text { C3 } \\
\text { HGEN } \\
\text { (W/CC) }\end{array}$ & $\begin{array}{c}\text { C4 } \\
\text { HGEN } \\
\text { (W/CC) }\end{array}$ & $\begin{array}{c}\text { C5 } \\
\text { HGEN } \\
\text { (W/cc) }\end{array}$ & $\begin{array}{c}\text { C6 } \\
\text { HGEN } \\
\text { (W/Cc) }\end{array}$ \\
\hline $\begin{array}{l}16.4 \\
15.5 \\
14.5 \\
13.6 \\
12.7 \\
11.7 \\
10.8\end{array}$ & $\begin{array}{l}41.67 \\
39.29 \\
36.91 \\
34.53 \\
32.15 \\
29.77 \\
27.39\end{array}$ & $\stackrel{\sim}{\infty}$ & $\begin{array}{l}4.26 \\
4.77 \\
5.24 \\
5.89 \\
6.53 \\
6.98 \\
8.37 \\
\end{array}$ & $\begin{array}{l}4.32 \\
4.75 \\
5.37 \\
5.53 \\
6.36 \\
6.94 \\
8.12 \\
\end{array}$ & $\begin{array}{l}4.81 \\
4.97 \\
5.42 \\
6.03 \\
6.49 \\
7.18 \\
7.95 \\
\end{array}$ & $\begin{array}{l}5.70 \\
5.67 \\
6.28 \\
6.46 \\
7.29 \\
7.68 \\
8.49 \\
\end{array}$ & $\begin{array}{l}3.87 \\
4.31 \\
4.89 \\
5.06 \\
5.49 \\
6.16 \\
6.66 \\
\end{array}$ & $\begin{array}{l}4.52 \\
4.75 \\
5.20 \\
5.68 \\
6.27 \\
7.15 \\
7.93 \\
\end{array}$ \\
\hline $\begin{array}{l}9.8 \\
8.9 \\
8.0 \\
7.0 \\
6.1 \\
5.2 \\
4.2\end{array}$ & $\begin{array}{l}25.01 \\
22.63 \\
20.25 \\
17.87 \\
15.49 \\
13.11 \\
10.73\end{array}$ & $\stackrel{+}{\underline{\Phi}}$ & $\begin{array}{c}9.29 \\
9.96 \\
11.15 \\
12.05 \\
12.94 \\
13.28 \\
13.74 \\
\end{array}$ & $\begin{array}{c}8.96 \\
10.08 \\
10.96 \\
11.74 \\
12.83 \\
13.34 \\
14.26 \\
\end{array}$ & $\begin{array}{c}9.15 \\
10.52 \\
10.86 \\
11.62 \\
12.04 \\
13.19 \\
13.52 \\
\end{array}$ & $\begin{array}{c}9.12 \\
9.89 \\
10.56 \\
10.91 \\
11.45 \\
12.23 \\
12.73 \\
\end{array}$ & $\begin{array}{c}8.02 \\
9.03 \\
10.01 \\
10.83 \\
11.54 \\
12.11 \\
12.58\end{array}$ & $\begin{array}{c}8.49 \\
9.43 \\
10.23 \\
11.02 \\
11.79 \\
12.10 \\
12.63 \\
\end{array}$ \\
\hline $\begin{array}{r}3.3 \\
2.4 \\
1.4 \\
0.5 \\
-0.5 \\
-1.4 \\
-2.3\end{array}$ & $\begin{array}{r}8.35 \\
5.97 \\
3.59 \\
1.21 \\
-1.17 \\
-3.55 \\
-5.93\end{array}$ & $\stackrel{n}{e}$ & $\begin{array}{l}14.72 \\
15.00 \\
15.36 \\
15.45 \\
15.32 \\
15.36 \\
15.43 \\
\end{array}$ & $\begin{array}{l}14.90 \\
15.19 \\
15.68 \\
15.77 \\
15.93 \\
15.91 \\
15.76\end{array}$ & $\begin{array}{l}13.75 \\
14.06 \\
14.75 \\
15.07 \\
14.53 \\
14.82 \\
14.48\end{array}$ & $\begin{array}{l}12.82 \\
13.25 \\
13.34 \\
13.29 \\
13.33 \\
13.62 \\
13.44\end{array}$ & $\begin{array}{l}13.19 \\
13.19 \\
13.22 \\
13.58 \\
13.44 \\
14.02 \\
13.50 \\
\end{array}$ & $\begin{array}{l}13.30 \\
13.78 \\
13.70 \\
14.01 \\
14.05 \\
14.21 \\
14.23\end{array}$ \\
\hline $\begin{array}{l}-3.3 \\
-4.2 \\
-5.1 \\
-6.1 \\
-7.0 \\
-8.0 \\
-8.9\end{array}$ & $\begin{array}{c}-8.31 \\
-10.69 \\
-13.07 \\
-15.45 \\
-17.83 \\
-20.21 \\
-22.59\end{array}$ & $\stackrel{N}{\stackrel{N}{\omega}}$ & $\begin{array}{l}16.39 \\
17.34 \\
17.18 \\
16.77 \\
15.36 \\
15.14 \\
13.94 \\
\end{array}$ & $\begin{array}{l}15.92 \\
15.51 \\
15.05 \\
14.50 \\
14.06 \\
12.76 \\
12.30 \\
\end{array}$ & $\begin{array}{l}14.23 \\
13.44 \\
13.16 \\
12.09 \\
12.31 \\
11.59 \\
11.10 \\
\end{array}$ & $\begin{array}{l}13.51 \\
13.34 \\
13.30 \\
12.97 \\
12.71 \\
12.03 \\
11.58 \\
\end{array}$ & $\begin{array}{l}13.46 \\
13.39 \\
12.87 \\
12.96 \\
12.29 \\
11.71 \\
11.37 \\
\end{array}$ & $\begin{array}{l}13.91 \\
13.54 \\
13.12 \\
12.97 \\
12.29 \\
11.28 \\
10.86 \\
\end{array}$ \\
\hline $\begin{array}{l}-9.8 \\
-10.8 \\
-11.7 \\
-12.6 \\
-13.6 \\
-14.5 \\
-15.5\end{array}$ & $\begin{array}{l}-24.97 \\
-27.35 \\
-29.73 \\
-32.11 \\
-34.49 \\
-36.87 \\
-39.25\end{array}$ & 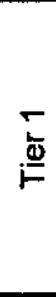 & $\begin{array}{c}12.33 \\
11.15 \\
10.17 \\
9.97 \\
9.78 \\
8.66 \\
8.42 \\
\end{array}$ & $\begin{array}{l}11.07 \\
9.64 \\
9.05 \\
8.73 \\
8.24 \\
8.01 \\
7.77 \\
\end{array}$ & $\begin{array}{c}10.29 \\
9.95 \\
9.84 \\
9.43 \\
8.67 \\
8.46 \\
7.94\end{array}$ & $\begin{array}{c}10.97 \\
10.53 \\
10.19 \\
10.14 \\
9.65 \\
9.74 \\
9.18 \\
\end{array}$ & $\begin{array}{l}9.44 \\
7.61 \\
7.35 \\
7.02 \\
6.93 \\
6.80 \\
6.27 \\
\end{array}$ & $\begin{array}{c}10.51 \\
10.19 \\
9.64 \\
9.28 \\
9.06 \\
8.62 \\
8.13 \\
\end{array}$ \\
\hline$\overline{\mathrm{e} H}$ & $=$ Total & & $1, \mathrm{Cl}-\mathrm{C}$ & 6 & ant $1-C$ & itmen & & \\
\hline
\end{tabular}


Enciosure (7) to

MDO-723-0044/

B-MT(SRME)-52

Page 46

\begin{tabular}{|c|c|c|c|c|c|c|c|c|}
\hline \multicolumn{9}{|c|}{$\begin{array}{r}\text { Table III.A-2 } \\
\text { Axial Heat Generation Rates for the PE }\end{array}$} \\
\hline \multicolumn{2}{|c|}{ Elevation } & & $\begin{array}{c}\text { C1 } \\
\text { HGEN }\end{array}$ & $\begin{array}{l}\text { C2 } \\
\text { HGEN }\end{array}$ & $\begin{array}{c}\text { C3 } \\
\text { HGEN }\end{array}$ & $\begin{array}{c}\mathrm{C4} \\
\text { HGEN }\end{array}$ & $\begin{array}{c}\text { C5 } \\
\text { HGEN }\end{array}$ & $\begin{array}{c}\text { C6 } \\
\text { HGEN }\end{array}$ \\
\hline in & $\mathrm{cm}$ & & (W/cc) & $(W / c c)$ & $(W / c c)$ & $(W / C c)$ & $(W / c c)$ & $(W / c c)$ \\
\hline 16.4 & 41.67 & & 4.73 & 4.94 & 3.62 & 4.08 & 4.14 & 4.82 \\
\hline 15.5 & 39.29 & & 4.91 & 4.81 & 4.15 & 4.34 & 4.30 & 4.83 \\
\hline 14.5 & 36.91 & no & 5.53 & 5.19 & 4.77 & 5.02 & 4.90 & 5.20 \\
\hline 13.6 & 34.53 & 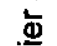 & 6.39 & 5.63 & 5.54 & 5.31 & 5.50 & 6.10 \\
\hline 12.7 & 32.15 & $F$ & 6.69 & 6.29 & 5.90 & 5.54 & 5.76 & 6.49 \\
\hline 11.7 & 29.77 & & 7.43 & 7.15 & 6.48 & 6.05 & 6.55 & 7.00 \\
\hline 10.8 & 27.39 & & 8.70 & 7.48 & 7.06 & 6.26 & 7.16 & 7.89 \\
\hline 9.8 & 25.01 & & 9.21 & 9.20 & 8.07 & 8.19 & 7.41 & 8.78 \\
\hline 8.9 & 22.63 & & 9.50 & 10.65 & 9.34 & 9.63 & 7.47 & 9.94 \\
\hline 8.0 & 20.25 & $\checkmark$ & 10.65 & 11.40 & 10.58 & 10.40 & 7.99 & 11.29 \\
\hline 7.0 & 17.87 & 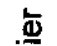 & 11.59 & 12.50 & 10.66 & 10.53 & 8.62 & 11.96 \\
\hline 6.1 & 15.49 & $i=$ & 12.72 & 13.65 & 11.26 & 11.46 & 9.70 & 12.58 \\
\hline 5.2 & 13.11 & & 12.95 & 14.10 & 11.77 & 11.80 & 9.71 & 13.78 \\
\hline 4.2 & 10.73 & & 12.90 & 14.56 & 12.33 & 12.30 & 10.23 & 14.18 \\
\hline 3.3 & 8.35 & & 15.37 & 14.07 & 12.87 & 13.09 & 12.09 & 15.37 \\
\hline 2.4 & 5.97 & & 17.72 & 13.65 & 13.17 & 13.59 & 13.29 & 15.87 \\
\hline 1.4 & 3.59 & $\infty$ & 18.32 & 14.19 & 13.45 & 13.86 & 13.47 & 16.12 \\
\hline 0.5 & 1.21 & $\stackrel{\underline{\underline{\omega}}}{=}$ & 18.09 & 14.70 & 13.65 & 14.51 & 13.79 & 16.37 \\
\hline-0.5 & -1.17 & $F$ & 18.28 & 14.18 & 13.30 & 13.88 & 14.10 & 15.28 \\
\hline-1.4 & -3.55 & & 18.29 & 15.11 & 13.60 & 14.47 & 14.43 & 16.14 \\
\hline-2.3 & -5.93 & & 18.34 & 14.11 & 12.99 & 13.75 & 13.51 & 15.77 \\
\hline-3.3 & -8.31 & & 18.15 & 15.31 & 13.66 & 13.32 & 11.90 & 14.26 \\
\hline-4.2 & -10.69 & & 17.92 & 15.70 & 13.31 & 12.91 & 10.23 & 13.16 \\
\hline-5.1 & -13.07 & $N$ & 16.96 & 15.11 & 13.01 & 13.05 & 9.80 & 13.07 \\
\hline-6.1 & -15.45 & $\stackrel{\Phi}{\Phi}$ & 16.16 & 14.59 & 12.50 & 11.92 & 9.82 & 12.92 \\
\hline-7.0 & -17.83 & $F$ & 15.85 & 13.96 & 12.34 & 12.19 & 9.52 & 12.34 \\
\hline-8.0 & -20.21 & & 14.89 & 13.42 & 11.82 & 12.15 & 8.66 & 11.48 \\
\hline-8.9 & -22.59 & & 13.59 & 12.11 & 11.63 & 11.10 & 8.60 & 11.24 \\
\hline-9.8 & -24.97 & & 12.51 & 11.14 & 10.94 & 9.90 & 8.64 & 10.82 \\
\hline-10.8 & -27.35 & & 11.25 & 10.39 & 9.55 & 8.65 & 9.01 & 11.04 \\
\hline-11.7 & -29.73 & $\leftarrow$ & 10.49 & 9.67 & 8.57 & 8.37 & 8.92 & 9.90 \\
\hline-12.6 & -32.11 & 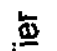 & 10.41 & 9.45 & 8.27 & 7.77 & 8.21 & 8.98 \\
\hline$=13.6$ & -34.49 & $F$ & 9.48 & 9.03 & 8.14 & 7.60 & 7.94 & 9.17 \\
\hline-14.5 & -36.87 & & 9.13 & 8.53 & 7.53 & 7.22 & 6.77 & 8.65 \\
\hline-15.5 & -39.25 & & 8.89 & 8.11 & 7.04 & 6.66 & 7.19 & 7.95 \\
\hline
\end{tabular}


Enclosure (7) to

MDO-723-0044/

B-MT(SRME)-52

Page 47

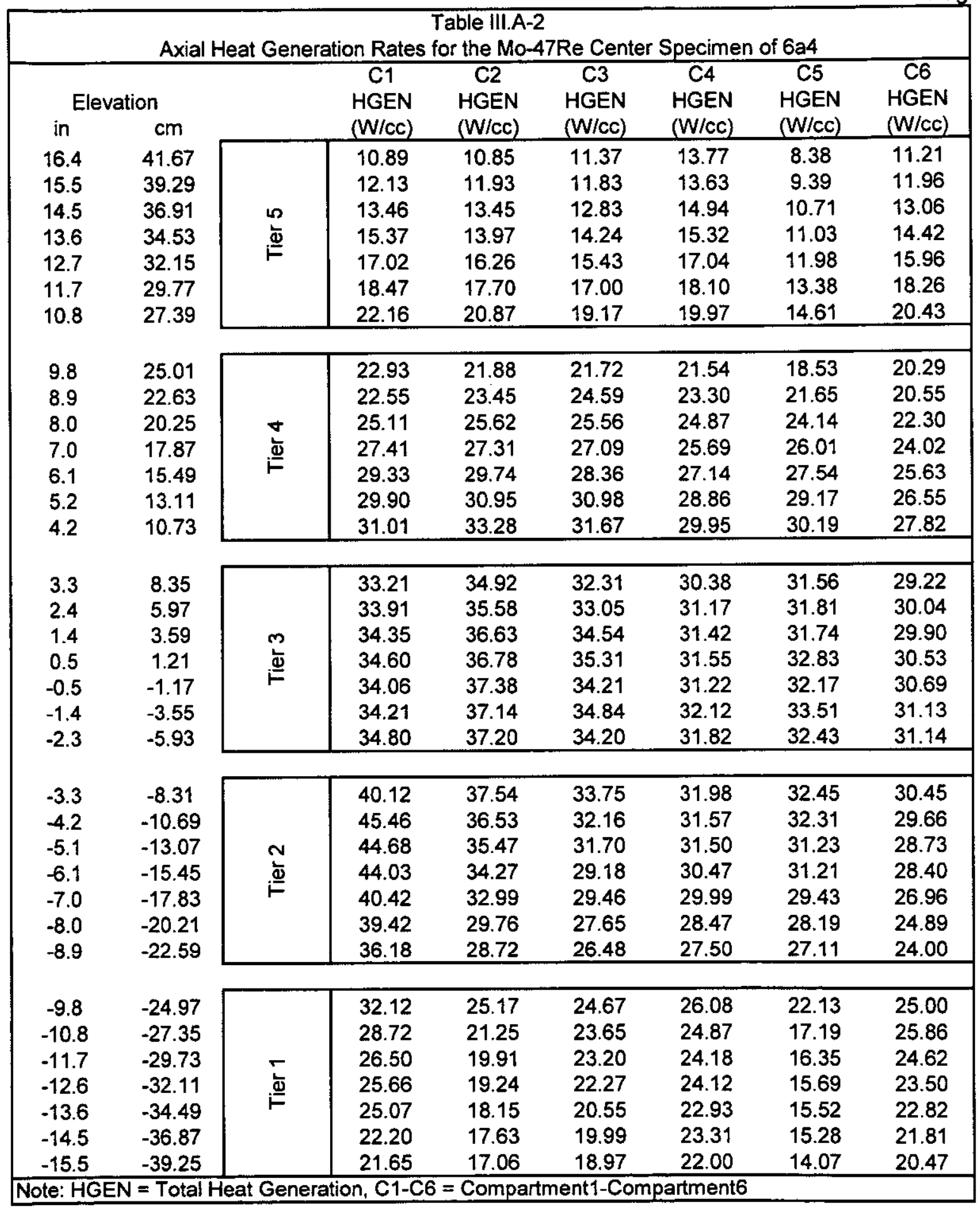


Enclosure (7) to

MDO-723-0044/

B-MT(SRME)-52

Page 48

\begin{tabular}{|c|c|c|c|c|c|c|c|c|}
\hline & Axial & & on Rat & $\begin{array}{l}\text { able III.A } \\
\text { r the Mo }\end{array}$ & $\mathrm{Re} \mathrm{Ce}$ & Specim & $6 \mathrm{~d} 4$ & \\
\hline & & & $\mathrm{C1}$ & $\mathrm{C} 2$ & C3 & $\mathrm{C4}$ & C5 & C6 \\
\hline & ion & & HGEN & HGEN & HGEN & HGEN & HGEN & HGEN \\
\hline in & $\mathrm{cm}$ & & (W/Cc) & (W/Cc) & $(W / c c)$ & (W/cc) & (W/cc) & (W/cc) \\
\hline 16.4 & 41.67 & & 11.99 & 12.05 & 8.80 & 10.34 & 10.10 & 12.03 \\
\hline 15.5 & 39.29 & & 12.27 & 12.02 & 10.08 & 10.83 & 10.44 & 12.12 \\
\hline 14.5 & 36.91 & 10 & 13.81 & 13.14 & 11.72 & 12.66 & 12.07 & 13.33 \\
\hline 13.6 & 34.53 & $\underline{\Phi}$ & 16.16 & 14.36 & 13.97 & 13.67 & 13.42 & 15.57 \\
\hline 12.7 & 32.15 & $F$ & 16.95 & 16.08 & 15.05 & 13.91 & 14.40 & 16.66 \\
\hline 11.7 & 29.77 & & 18.67 & 18.71 & 16.08 & 15.97 & 16.44 & 17.62 \\
\hline 10.8 & 27.39 & & 22.15 & 19.64 & 17.91 & 16.18 & 18.03 & 20.33 \\
\hline 9.8 & 25.01 & & 22.20 & 23.69 & 20.06 & 20.04 & 17.54 & 22.75 \\
\hline 8.9 & 22.63 & & 21.48 & 26.97 & 22.27 & 22.73 & 16.32 & 25.60 \\
\hline 8.0 & 20.25 & $\nabla$ & 23.38 & 28.63 & 25.30 & 24.54 & 17.52 & 29.15 \\
\hline 7.0 & 17.87 & 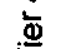 & 25.30 & 32.26 & 25.46 & 24.95 & 18.96 & 31.28 \\
\hline 6.1 & 15.49 & $F$ & 28.02 & 34.54 & 26.81 & 27.02 & 21.30 & 33.13 \\
\hline 5.2 & 13.11 & & 28.13 & 36.33 & 28.27 & 27.88 & 21.29 & 35.99 \\
\hline 4.2 & 10.73 & & 28.46 & 38.03 & 29.65 & 29.11 & 22.42 & 36.64 \\
\hline 3.3 & 8.35 & & 37.74 & 33.94 & 30.81 & 30.94 & 27.80 & 37.86 \\
\hline 2.4 & 5.97 & & 46.05 & 29.94 & 31.31 & 31.94 & 31.89 & 36.93 \\
\hline 1.4 & 3.59 & $m$ & 47.53 & 30.98 & 31,98 & 32.38 & 32.62 & 37.46 \\
\hline 0.5 & 1.21 & 逗 & 47.63 & 32.18 & 32.59 & 33.95 & 33.69 & 37.81 \\
\hline-0.5 & -1.17 & $F$ & 48.48 & 31.55 & 32.00 & 32.86 & 34.34 & 35.72 \\
\hline-1.4 & -3.55 & & 48.28 & 33.57 & 32.29 & 34.20 & 34.84 & 37.69 \\
\hline-2.3 & -5.93 & & 48.86 & 31.16 & 31.11 & 32.38 & 32.68 & 36.95 \\
\hline-3.3 & -8.31 & & 47.71 & 34.87 & 32.79 & 31.41 & 27.90 & 32.33 \\
\hline-4.2 & -10.69 & & 47.27 & 36.83 & 32.38 & 30.29 & 22.62 & 28.81 \\
\hline-5.1 & -13.07 & $N$ & 45.00 & 35.63 & 31.30 & 30.88 & 21.71 & 28.51 \\
\hline-6.1 & -15.45 & 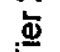 & 42.72 & 34.56 & 30.49 & 28.26 & 21.73 & 28.20 \\
\hline-7.0 & -17.83 & $\mathrm{~F}$ & 41.86 & 32.19 & 29.61 & 28.72 & 20.92 & 26.90 \\
\hline-8.0 & -20.21 & & 39.28 & 31.29 & 28.43 & 28.45 & 19.33 & 25.14 \\
\hline-8.9 & -22.59 & & 35.71 & 28.41 & 27.75 & 26.14 & 19.22 & 24.72 \\
\hline-9.8 & -24.97 & & 32.40 & 27.68 & 26.84 & 24.37 & 20.97 & 25.71 \\
\hline-10.8 & -27.35 & & 28.93 & 27.07 & 24.19 & 21.90 & 22.94 & 28.04 \\
\hline-11.7 & -29.73 & $r$ & 27.44 & 24.69 & 21.92 & 20.83 & 22.24 & 25.61 \\
\hline-12.6 & -32.11 & 亦 & 26.59 & 24.71 & 21.01 & 19.65 & 20.51 & 23.27 \\
\hline-13.6 & -34.49 & $j$ & 23.98 & 23.37 & 20.19 & 19.02 & 19.91 & 23.36 \\
\hline-14.5 & -36.87 & & 23.08 & 21.71 & 18.86 & 18.17 & 16.64 & 22.26 \\
\hline-15.5 & -39.25 & & 22.50 & 20.73 & 17.55 & 16.72 & 17.57 & 20.22 \\
\hline
\end{tabular}


Enclosure (7) to MDO-723-0044/

B-MT(SRME)-52

Page 49

\begin{tabular}{|c|c|c|c|c|c|c|c|c|}
\hline \multicolumn{9}{|c|}{$\begin{array}{c}\text { Table III.A-2 } \\
\text { Axial Heat Generation Rates for the Re Center Specimen of } 6 a 4\end{array}$} \\
\hline \multicolumn{2}{|c|}{ Elevation } & & $\begin{array}{c}\text { C1 } \\
\text { HGEN } \\
\text { (W/cc) }\end{array}$ & $\begin{array}{c}\text { C2 } \\
\text { HGEN } \\
(W / c c)\end{array}$ & $\begin{array}{c}\text { C3 } \\
\text { HGEN } \\
\text { (W/cc) }\end{array}$ & $\begin{array}{c}\text { C4 } \\
\text { HGEN } \\
\text { (W/CC) }\end{array}$ & $\begin{array}{c}\text { C5 } \\
\text { HGEN } \\
\text { (W/CC) }\end{array}$ & $\begin{array}{c}\text { C6 } \\
\text { HGEN } \\
\text { (W/cC) }\end{array}$ \\
\hline $\begin{array}{l}16.4 \\
15.5 \\
14.5 \\
13.6 \\
12.7 \\
11.7 \\
10.8\end{array}$ & $\begin{array}{l}41.67 \\
39.29 \\
36.91 \\
34.53 \\
32.15 \\
29.77 \\
27.39\end{array}$ & $\stackrel{n}{\stackrel{n}{*}}$ & $\begin{array}{l}21.35 \\
23.78 \\
26.60 \\
30.57 \\
33.90 \\
37.21 \\
44.69 \\
\end{array}$ & $\begin{array}{l}21.07 \\
23.16 \\
26.08 \\
27.32 \\
31.95 \\
34.87 \\
41.38 \\
\end{array}$ & $\begin{array}{l}21.25 \\
22.24 \\
24.14 \\
26.85 \\
29.19 \\
32.21 \\
36.63 \\
\end{array}$ & $\begin{array}{l}25.37 \\
25.13 \\
27.53 \\
28.35 \\
31.29 \\
33.37 \\
36.99 \\
\end{array}$ & $\begin{array}{l}15.27 \\
17.12 \\
19.57 \\
20.20 \\
22.01 \\
24.64 \\
27.05 \\
\end{array}$ & $\begin{array}{l}21.53 \\
23.05 \\
25.24 \\
28.14 \\
31.16 \\
35.75 \\
40.32 \\
\end{array}$ \\
\hline $\begin{array}{l}9.8 \\
8.9 \\
8.0 \\
7.0 \\
6.1 \\
5.2 \\
4.2\end{array}$ & $\begin{array}{l}25.01 \\
22.63 \\
20.25 \\
17.87 \\
15.49 \\
13.11 \\
10.73\end{array}$ & $\stackrel{+}{\stackrel{+}{\alpha}}$ & $\begin{array}{l}44.88 \\
42.53 \\
47.28 \\
51.80 \\
55.46 \\
56.49 \\
58.72 \\
\end{array}$ & $\begin{array}{l}42.70 \\
44.92 \\
49.30 \\
52.51 \\
57.04 \\
59.65 \\
64.16 \\
\end{array}$ & $\begin{array}{l}40.90 \\
45.69 \\
47.73 \\
50.49 \\
53.09 \\
57.80 \\
59.19 \\
\end{array}$ & $\begin{array}{l}40.04 \\
43.28 \\
46.30 \\
47.89 \\
50.74 \\
53.84 \\
55.90 \\
\end{array}$ & $\begin{array}{l}35.05 \\
41.62 \\
46.50 \\
50.02 \\
52.94 \\
56.25 \\
58.28 \\
\end{array}$ & $\begin{array}{l}39.20 \\
38.39 \\
41.72 \\
44.93 \\
48.04 \\
49.96 \\
52.44 \\
\end{array}$ \\
\hline $\begin{array}{r}3.3 \\
2.4 \\
1.4 \\
0.5 \\
-0.5 \\
-1.4 \\
-2.3\end{array}$ & $\begin{array}{r}8.35 \\
5.97 \\
3.59 \\
1.21 \\
-1.17 \\
-3.55 \\
-5.93\end{array}$ & $\stackrel{m}{\underline{\underline{a}}}$ & $\begin{array}{l}62.72 \\
63.89 \\
64.82 \\
65.27 \\
64.13 \\
64.44 \\
65.73 \\
\end{array}$ & $\begin{array}{l}67.41 \\
68.63 \\
70.60 \\
70.87 \\
72.13 \\
71.43 \\
71.86 \\
\end{array}$ & $\begin{array}{l}60.47 \\
61.86 \\
64.53 \\
65.93 \\
64.09 \\
65.11 \\
64.12 \\
\end{array}$ & $\begin{array}{l}56.89 \\
58.12 \\
58.75 \\
59.10 \\
58.32 \\
59.95 \\
59.51 \\
\end{array}$ & $\begin{array}{l}60.83 \\
61.50 \\
61.31 \\
63.59 \\
62.13 \\
64.52 \\
62.64 \\
\end{array}$ & $\begin{array}{l}55.08 \\
56.40 \\
56.23 \\
57.39 \\
57.72 \\
58.54 \\
58.51 \\
\end{array}$ \\
\hline $\begin{array}{l}-3.3 \\
-4.2 \\
-5.1 \\
-6.1 \\
-7.0 \\
-8.0 \\
-8.9\end{array}$ & $\begin{array}{c}-8.31 \\
-10.69 \\
-13.07 \\
-15.45 \\
-17.83 \\
-20.21 \\
-22.59\end{array}$ & $\stackrel{N}{\stackrel{N}{\Phi}}$ & $\begin{array}{l}78.77 \\
91.75 \\
89.78 \\
88.81 \\
81.49 \\
79.09 \\
72.39 \\
\end{array}$ & $\begin{array}{l}72.47 \\
70.53 \\
68.33 \\
66.13 \\
63.42 \\
57.22 \\
55.00 \\
\end{array}$ & $\begin{array}{l}63.89 \\
61.77 \\
60.93 \\
56.24 \\
56.30 \\
52.76 \\
50.50 \\
\end{array}$ & $\begin{array}{l}59.79 \\
58.94 \\
58.71 \\
56.69 \\
55.83 \\
52.95 \\
51.14 \\
\end{array}$ & $\begin{array}{l}62.78 \\
62.30 \\
60.35 \\
60.04 \\
56.49 \\
54.15 \\
51.80 \\
\end{array}$ & $\begin{array}{l}57.30 \\
55.74 \\
53.95 \\
53.40 \\
50.52 \\
46.73 \\
44.93 \\
\end{array}$ \\
\hline $\begin{array}{c}-9.8 \\
-10.8 \\
-11.7 \\
-12.6 \\
-13.6 \\
-14.5 \\
-15.5 \\
\end{array}$ & $\begin{array}{l}-24.97 \\
-27.35 \\
-29.73 \\
-32.11 \\
-34.49 \\
-36.87 \\
-39.25 \\
\end{array}$ & $\overline{\frac{5}{9}}$ & $\begin{array}{l}64.15 \\
56.99 \\
52.61 \\
50.52 \\
49.19 \\
43.54 \\
42.39 \\
\end{array}$ & $\begin{array}{l}47.54 \\
39.44 \\
36.78 \\
35.46 \\
33.37 \\
32.33 \\
31.19 \\
\end{array}$ & $\begin{array}{l}47.06 \\
44.86 \\
43.72 \\
41.85 \\
38.68 \\
37.53 \\
35.70 \\
\end{array}$ & $\begin{array}{l}48.49 \\
46.02 \\
44.68 \\
44.45 \\
42.20 \\
42.97 \\
40.51 \\
\end{array}$ & $\begin{array}{l}41.90 \\
31.90 \\
30.09 \\
28.83 \\
28.49 \\
28.03 \\
25.74 \\
\end{array}$ & $\begin{array}{l}48.00 \\
50.56 \\
48.09 \\
45.64 \\
44.16 \\
42.17 \\
39.44 \\
\end{array}$ \\
\hline te: $H \bar{G}$ & Total & & $\mathrm{C1-C}$ & Compa & $1+1-C$ & & & \\
\hline
\end{tabular}


Enclosure (7) to

MDO-723-0044/

B-MT(SRME)-52

Page 50

Table III.A-2

Axial Heat Generation Rates for the Re Center Specimen of $6 \mathrm{~d} 4$

\begin{tabular}{|c|c|c|c|c|c|c|c|c|}
\hline \multicolumn{2}{|c|}{ Elevation } & & $\begin{array}{c}\text { C1 } \\
\text { HGEN }\end{array}$ & $\begin{array}{c}\text { C2 } \\
\text { HGEN }\end{array}$ & $\begin{array}{c}\text { C3 } \\
\text { HGEN }\end{array}$ & $\begin{array}{c}\text { C4 } \\
\text { HGEN }\end{array}$ & $\begin{array}{c}\text { C5 } \\
\text { HGEN }\end{array}$ & $\begin{array}{c}\text { C6 } \\
\text { HGEN }\end{array}$ \\
\hline in & $\mathrm{cm}$ & & $(W / c c)$ & (W/cc) & $(W / c c)$ & $(W / C c)$ & (W/cc) & $(W / c c)$ \\
\hline 16.4 & 41.67 & \multirow{7}{*}{ 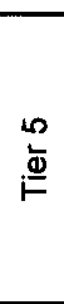 } & 23.28 & 23.03 & 16.98 & 20.04 & 19.42 & 23.09 \\
\hline 15.5 & 39.29 & & 23.80 & 23.32 & 19.47 & 20.96 & 20.05 & 23.40 \\
\hline 14.5 & 36.91 & & 26.78 & 25.71 & 22.72 & 24.62 & 23.36 & 26.00 \\
\hline 13.6 & 34.53 & & 31.52 & 28.30 & 27.30 & 26.74 & 25.90 & 30.29 \\
\hline 12.7 & 32.15 & & 33.20 & 31.69 & 29.57 & 27.13 & 28.15 & 32.46 \\
\hline 11.7 & 29.77 & & 36.64 & 37.25 & 31.36 & 31.76 & 32.10 & 34.44 \\
\hline 10.8 & 27.39 & & 43.83 & 39.42 & 35.31 & 32.13 & 35.53 & 40.22 \\
\hline 9.8 & 25.01 & \multirow{7}{*}{ 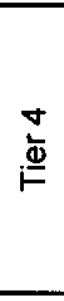 } & 42.86 & 47.23 & 39.16 & 38.25 & 33.78 & 45.31 \\
\hline 8.9 & 22.63 & & 40.04 & 53.43 & 42.60 & 42.26 & 30.39 & 51.12 \\
\hline 8.0 & 20.25 & & 43.52 & 56.92 & 48.43 & 45.67 & 32.67 & 58.20 \\
\hline 7.0 & 17.87 & & 47.23 & 64.63 & 48.97 & 46.54 & 35.48 & 62.97 \\
\hline 6.1 & 15.49 & & 52.29 & 68.89 & 51.38 & 50.33 & 39.85 & 66.89 \\
\hline 5.2 & 13.11 & & 52.21 & 72.83 & 54.60 & 52.15 & 39.91 & 72.32 \\
\hline \multirow[t]{2}{*}{4.2} & 10.73 & & 53.58 & 76.69 & 57.31 & 54.52 & 42.00 & 73.56 \\
\hline & & & & & & $\cdot$ & & \\
\hline 3.3 & 8.35 & \multirow{7}{*}{$\stackrel{m}{\stackrel{m}{\underline{\Phi}}}$} & 74.23 & 66.56 & 59.35 & 57.87 & 52.84 & 74.51 \\
\hline 2.4 & 5.97 & & 92.77 & 56.36 & 59.93 & 59.53 & 61.45 & 70.63 \\
\hline 1.4 & 3.59 & & 95.58 & 58.21 & 61.18 & 60.36 & 63.25 & 71.46 \\
\hline 0.5 & 1.21 & & 96.13 & 60.55 & 62.56 & 63.04 & 65.46 & 72.16 \\
\hline-0.5 & -1.17 & & 97.98 & 59.77 & 61.70 & 61.59 & 66.65 & 68.71 \\
\hline-1.4 & -3.55 & & 97.76 & 63.37 & 61.93 & 63.78 & 67.33 & 72.39 \\
\hline-2.3 & -5.93 & & 99.24 & 59.01 & 60.02 & 60.39 & 63.35 & 71.04 \\
\hline-3.3 & -8.31 & \multirow{7}{*}{$\stackrel{N}{\stackrel{N}{\omega}}$} & 96.50 & 66.52 & 63.23 & 58.72 & 53.36 & 61.65 \\
\hline-4.2 & -10.69 & & 95.75 & 70.72 & 62.75 & 56.46 & 42.11 & 54.27 \\
\hline-5.1 & -13.07 & & 91.32 & 68.57 & 60.34 & 57.65 & 40.37 & 53.57 \\
\hline-6.1 & -15.45 & & 86.39 & 66.56 & 58.96 & 52.76 & 40.48 & 52.86 \\
\hline-7.0 & -17.83 & & 84.52 & 61.42 & 56.88 & 53.50 & 38.94 & 50.34 \\
\hline-8.0 & -20.21 & & 79.43 & 59.64 & 54.52 & 52.71 & 35.77 & 47.08 \\
\hline-8.9 & -22.59 & & 71.91 & 54.50 & 53.06 & 48.59 & 35.62 & 46.16 \\
\hline-9.8 & -24.97 & \multirow{7}{*}{ 产 } & 64.51 & 54.20 & 51.94 & $4 \overline{6.66}$ & 40.55 & 4925 \\
\hline-10.8 & -27.35 & & 57.15 & 53.88 & 47.51 & 42.93 & 45.13 & 54.77 \\
\hline-11.7 & -29.73 & & 54.33 & 48.76 & 43.05 & 40.45 & 43.33 & 50.30 \\
\hline-12.6 & -32.11 & & 52.02 & 48.87 & 41.09 & 38.23 & 39.81 & 45.59 \\
\hline-13.6 & -34.49 & & 46.81 & 45.94 & 39.05 & 36.89 & 38.65 & 45.35 \\
\hline-14.5 & -36.87 & & 44.95 & 42.28 & 36.51 & 35.22 & 32.17 & 43.17 \\
\hline-15.5 & -39.25 & & 43.66 & 40.33 & 33.89 & 32.34 & 33.67 & 39.06 \\
\hline
\end{tabular}


Enclosure (7) to

MDO-723-0044/

B-MT(SRME)-52

Page 51

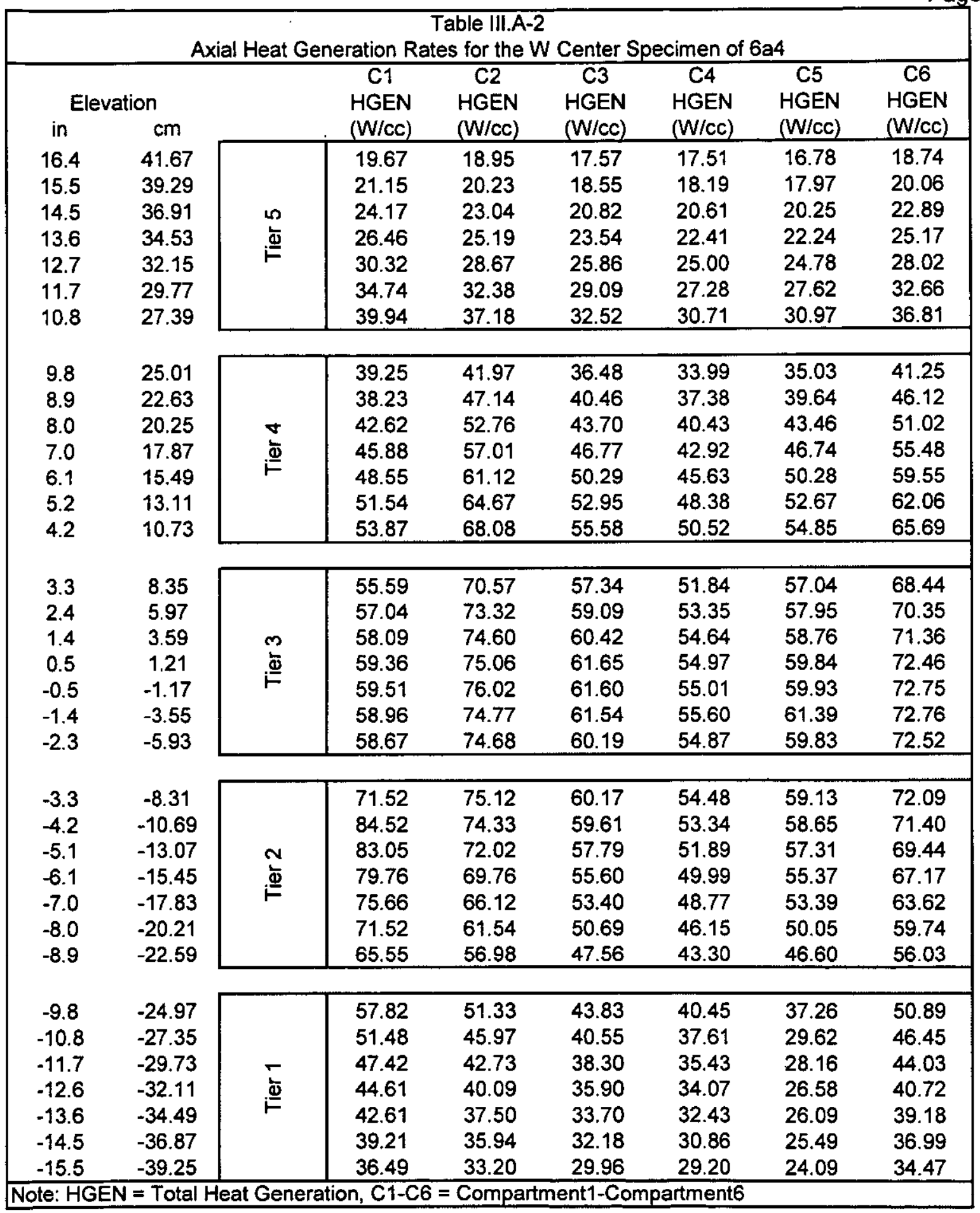


Enclosure (7) to

MDO-723-0044/

B-MT(SRME)-52

Page 52

\begin{tabular}{|c|c|c|c|c|c|c|c|c|}
\hline \multicolumn{9}{|c|}{$\begin{array}{r}\text { Table III.A-2 } \\
\text { Axial Heat Generation Rates for the W }\end{array}$} \\
\hline \multirow{2}{*}{\multicolumn{2}{|c|}{ Elevation }} & & $\mathrm{C1}$ & $\mathrm{C} 2$ & $\mathrm{C3}$ & $\mathrm{C} 4$ & $\overline{C 5}$ & C6 \\
\hline & & & HGEN & HGEN & HGEN & HGEN & HGEN & HGEN \\
\hline in & $\mathrm{cm}$ & & $(W / c c)$ & $(\mathrm{W} / \mathrm{cc})$ & $(W / c c)$ & $(W / c c)$ & $(W / c c)$ & (W/cc) \\
\hline 16.4 & 41.67 & & 21.03 & 20.82 & 15.34 & 18.10 & 17.55 & 20.87 \\
\hline 15.5 & 39.29 & & 21.49 & 21.07 & 17.58 & 18.94 & 18.11 & 21.14 \\
\hline 14.5 & 36.91 & n & 24.19 & 23.22 & 20.52 & 22.24 & 21.10 & 23.47 \\
\hline 13.6 & 34.53 & $\stackrel{4}{=}$ & 28.46 & 25.54 & 24.66 & 24.15 & 23.40 & 27.35 \\
\hline 12.7 & 32.15 & $F$ & 29.96 & 28.61 & 26.69 & 24.51 & 25.41 & 29.31 \\
\hline 11.7 & 29.77 & & 33.06 & 33.60 & 28.31 & 28.66 & 28.98 & 31.07 \\
\hline 10.8 & 27.39 & & 39.45 & 35.45 & 31.79 & 28.91 & 31.98 & 36.18 \\
\hline 9.8 & 25.01 & & 38.53 & 42.46 & 35.21 & 34.40 & 30.38 & 40.72 \\
\hline 8.9 & 22.63 & & 36.14 & 48.17 & 38.43 & 38.13 & 27.45 & 46.08 \\
\hline 8.0 & 20.25 & $\nabla$ & 39.29 & 51.31 & 43.70 & 41.21 & 29.50 & 52.46 \\
\hline 7.0 & 17.87 & 包 & 42.65 & 58.25 & 44.16 & 41.96 & 32.03 & 56.74 \\
\hline 6.1 & 15.49 & $F$ & 47.19 & 62.11 & 46.35 & 45.38 & 35.99 & 60.26 \\
\hline 5.2 & 13.11 & & 47.12 & 65.63 & 49.21 & 47.01 & 36.03 & 65.15 \\
\hline 4.2 & 10.73 & & 48.22 & 68.92 & 51.54 & 49.03 & 37.83 & 66.12 \\
\hline 3.3 & 8.35 & & 66.72 & 59.77 & 53.33 & 52.00 & 47.55 & 66.93 \\
\hline 2.4 & 5.97 & & 83.56 & 50.86 & 54.04 & 53.68 & 55.42 & 63.70 \\
\hline 1.4 & 3.59 & $m$ & 86.11 & 52.53 & 55.18 & 54.42 & 57.02 & 64.44 \\
\hline 0.5 & 1.21 & $\stackrel{\underline{\Phi}}{\underline{\Phi}}$ & 86.58 & 54.64 & 56.40 & 56.85 & 58.99 & 65.06 \\
\hline-0.5 & -1.17 & $F$ & 88.24 & 53.92 & 55.61 & 55.51 & 60.06 & 61.93 \\
\hline-1.4 & -3.55 & & 88.03 & 57.20 & 55.83 & 57.51 & 60.70 & 65.26 \\
\hline-2.3 & -5.93 & & 89.16 & 53.11 & 53.97 & 54,32 & 56.99 & 63.89 \\
\hline-3.3 & -8.31 & & 86.64 & 59.80 & 56.81 & 52.77 & 47.98 & 55.40 \\
\hline-4.2 & -10.69 & & 86.27 & 63.74 & 56.55 & 50.91 & 38.03 & 48.98 \\
\hline-5.1 & -13.07 & $N$ & 82.27 & 61.80 & 54.40 & 51.98 & 36.45 & 48.36 \\
\hline-6.1 & -15.45 & 高 & 77.83 & 59.99 & 53.14 & 47.57 & 36.57 & 47.72 \\
\hline-7.0 & -17.83 & $F$ & 76.16 & 55.40 & 51.29 & 48.24 & 35.19 & 45.46 \\
\hline-8.0 & -20.21 & & 71.56 & 53.77 & 49.17 & 47.55 & 32.30 & 42.51 \\
\hline-8.9 & -22.59 & & 64.68 & 49.04 & 47.76 & 43.73 & 32.11 & 41.60 \\
\hline-9.8 & -24.97 & & 57.97 & 48.72 & 46.70 & 41.95 & 36.49 & 44.32 \\
\hline-10.8 & -27.35 & & 51.56 & 48.59 & 42.88 & 38.76 & 40.72 & 49.42 \\
\hline-11.7 & -29.73 & - & 49.02 & 43.99 & 38.85 & 36.53 & 39.13 & 45.38 \\
\hline-12.6 & -32.11 & 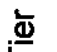 & 46.97 & 44.10 & 37.09 & 34.52 & 35.95 & 41.12 \\
\hline-13.6 & -34.49 & $F$ & 42.25 & 41.48 & 35.27 & 33.32 & 34.93 & 40.94 \\
\hline-14.5 & -36.87 & & 40.58 & 38.19 & 32.96 & 31.82 & 29.05 & 38.98 \\
\hline-15.5 & -39.25 & & 39.35 & 36.37 & 30.56 & 29.15 & 30.36 & 35.20 \\
\hline
\end{tabular}


Enclosure (7) to

MDO-723-0044/

B-MT(SRME)-52

Page 53

\begin{tabular}{|c|c|c|c|c|c|c|c|c|}
\hline \multicolumn{9}{|c|}{$\begin{array}{r}\text { Table III.A-2 } \\
\text { Axial Heat Generation Rates for the W-25 }\end{array}$} \\
\hline \multirow{2}{*}{\multicolumn{2}{|c|}{ Elevation }} & & \multirow{3}{*}{$\begin{array}{c}\text { C1 } \\
\text { HGEN } \\
\text { (W/CC) }\end{array}$} & \multirow{3}{*}{$\begin{array}{c}\text { C2 } \\
\text { HGEN } \\
\text { (W/CC) }\end{array}$} & \multirow{3}{*}{$\begin{array}{c}\text { C3 } \\
\text { HGEN } \\
(W / C C)\end{array}$} & \multirow{3}{*}{$\begin{array}{c}\text { C4 } \\
\text { HGEN } \\
\text { (W/CC) }\end{array}$} & \multirow{3}{*}{$\begin{array}{c}\text { C5 } \\
\text { HGEN } \\
(W / C c)\end{array}$} & \multirow{3}{*}{$\begin{array}{c}\text { C6 } \\
\text { HGEN } \\
(W / C c)\end{array}$} \\
\hline & & & & & & & & \\
\hline in & $\mathrm{cm}$ & & & & & & & \\
\hline 16.4 & 41.67 & & 19.73 & 19.47 & 19.64 & 23.42 & 14.13 & 19.89 \\
\hline 15.5 & 39.29 & & 21.97 & 21.40 & 20.55 & 23.21 & 15.84 & 21.29 \\
\hline 14.5 & 36.91 & $n$ & 24.57 & 24.10 & 22.30 & 25.42 & 18.10 & 23.31 \\
\hline 13.6 & 34.53 & 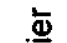 & 28.23 & 25.23 & 24.80 & 26.17 & 18.68 & 25.97 \\
\hline 12.7 & 32.15 & & 31.30 & 29.49 & 26.95 & 28.90 & 20.36 & 28.76 \\
\hline 11.7 & 29.77 & & 34.34 & 32.19 & 29.75 & 30.81 & 22.78 & 32.99 \\
\hline 10.8 & 27.39 & & 41.17 & 38.12 & 33.75 & 34.08 & 24.96 & 37.12 \\
\hline 9.8 & 25.01 & & 41.30 & 39.30 & 37.66 & 36.86 & 32.31 & 36.08 \\
\hline 8.9 & 22.63 & & 39.25 & 41.46 & 42.16 & 39.93 & 38.41 & 35.45 \\
\hline 8.0 & 20.25 & $\nabla$ & 43.64 & 45.49 & 44.03 & 42.72 & 42.90 & 38.53 \\
\hline 7.0 & 17.87 & 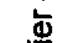 & 47.81 & 48.46 & 46.59 & 44.18 & 46.16 & 41.49 \\
\hline 6.1 & 15.49 & & 51.19 & 52.66 & 48.96 & 46.80 & 48.84 & 44.35 \\
\hline 5.2 & 13.11 & & 52.14 & 55.05 & 53.32 & 49.68 & 51.89 & 46.12 \\
\hline 4.2 & 10.73 & & 54.09 & 59.10 & 54.51 & 51.48 & 53.68 & 48.33 \\
\hline 3.3 & 8.35 & & 57.74 & 62.04 & 55.64 & 52.35 & 56.00 & 50.72 \\
\hline 2.4 & 5.97 & & 58.96 & 63.30 & 57.05 & 53.62 & 56.74 & 52.07 \\
\hline 1.4 & 3.59 & $m$ & 59.83 & 65.14 & 59.53 & 54.19 & 56.56 & 51.91 \\
\hline 0.5 & 1.21 & $\underline{\Phi}$ & 60.23 & 65.38 & 60.82 & 54.51 & 58.67 & 52.98 \\
\hline-0.5 & -1.17 & & 59.18 & 66.55 & 59.11 & 53.80 & 57.31 & 53.28 \\
\hline-1.4 & -3.55 & & 59.49 & 65.89 & 60.05 & 55.30 & 59.52 & 54.04 \\
\hline-2.3 & -5.93 & & 60.56 & 66.18 & 59.04 & 54.79 & 57.69 & 53.93 \\
\hline-3.3 & -8.31 & & 72.49 & 66.69 & 58.79 & 55.02 & 57.76 & 52.77 \\
\hline-4.2 & -10.69 & & 84.58 & 65.07 & 56.99 & 54.37 & 57.46 & 51.47 \\
\hline-5.1 & -13.07 & $N$ & 82.78 & 63.04 & 56.20 & 54.16 & 55.66 & 49.81 \\
\hline-6.1 & -15.45 & $\underline{\underline{\omega}}$ & 81.87 & 61.01 & 51.87 & 52,30 & 55.39 & 49.30 \\
\hline-7.0 & -17.83 & & 75.12 & 58.51 & 51.95 & 51.51 & 52.12 & 46.65 \\
\hline-8.0 & -20.21 & & 72.93 & 52.79 & 48.69 & 48.86 & 49.96 & 43.15 \\
\hline-8.9 & -22.59 & & 66.66 & 50.68 & 46.53 & 47.11 & 47.73 & 41.43 \\
\hline-9.8 & -24.97 & & 59.03 & 43.78 & 43.33 & 44.63 & 38.57 & 44.21 \\
\hline-10.8 & -27.35 & & 52.60 & 36.45 & 41.42 & 42.48 & 29.48 & 46.66 \\
\hline-11.7 & -29.73 & $\sigma$ & 48.56 & 34.00 & 40.39 & 41.24 & 27.81 & 44.39 \\
\hline-12.6 & -32.11 & 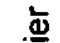 & 46.64 & 32.79 & 38.66 & 41.04 & 26.66 & 42.13 \\
\hline-13.6 & -34.49 & & 45.42 & 30.85 & 35.74 & 38.98 & 26.34 & 40.78 \\
\hline-14.5 & -36.87 & & 40.21 & 29.90 & 34.67 & 39.69 & 25.92 & 38.94 \\
\hline-15.5 & -39.25 & & 39.09 & 28.80 & 32.94 & 37.35 & 23.77 & 36.37 \\
\hline
\end{tabular}


Enclosure (7) to

MDO-723-0044/

B-MT(SRME)-52

Page 54

\begin{tabular}{|c|c|c|c|c|c|c|c|c|}
\hline & Axia & & tion Rate & $\begin{array}{l}\text { ble III.A } \\
\text { ir the W }\end{array}$ & Re Cent & pecime & $6 \mathrm{~d} 4$ & \\
\hline & & & $\mathrm{C} 1$ & $\mathrm{C2}$ & $\mathrm{C3}$ & $\mathrm{C4}$ & $\overline{C 5}$ & C6 \\
\hline & ion & & HGEN & HGEN & HGEN & HGEN & HGEN & HGEN \\
\hline in & $\mathrm{cm}$ & & $(W / c c)$ & (W/cc) & (W/Cc) & $(W / C c)$ & $(W / C c)$ & $\langle W / c c\rangle$ \\
\hline 16.4 & 41.67 & & 21.51 & 21.29 & 15.69 & 18.51 & 17.95 & 21.34 \\
\hline 15.5 & 39.29 & & 21.98 & 21.55 & 17.98 & 19.37 & 18.52 & 21.62 \\
\hline 14.5 & 36.91 & $\omega$ & 24.74 & 23.75 & 20.98 & 22.75 & 21.58 & 24.01 \\
\hline 13.6 & 34.53 & $\underline{\underline{a}}$ & 29.11 & 26.13 & 25.22 & 24.70 & 23.93 & 27.97 \\
\hline 12.7 & 32.15 & $F$ & 30.65 & 29.26 & 27.30 & 25.07 & 25.99 & 29.98 \\
\hline 11.7 & 29.77 & & 33.82 & 34.38 & 28.96 & 29.32 & 29.64 & 31.79 \\
\hline 10.8 & 27.39 & & 40.39 & 36.30 & 32.54 & 29.60 & 32.73 & 37.04 \\
\hline 9.8 & 25.01 & & 39.46 & 43.48 & 36.05 & 35.22 & 31.10 & 41.70 \\
\hline 8.9 & 22.63 & & 36.97 & 49.28 & 39.32 & 39.01 & 28.07 & 47.15 \\
\hline 8.0 & 20.25 & $\nabla$ & 40.19 & 52.50 & 44.70 & 42.16 & 30.17 & 53.68 \\
\hline 7.0 & 17.87 & 这 & 43.62 & 59.61 & 45.18 & 42.93 & 32.76 & 58.06 \\
\hline 6.1 & 15.49 & $F$ & 48.27 & 63.55 & 47.42 & 46.43 & 36.81 & 61.67 \\
\hline 5.2 & 13.11 & & 48.20 & 67.16 & 50.36 & 48.10 & 36.85 & 66.67 \\
\hline 4.2 & 10.73 & & 49.36 & 70.57 & 52.77 & 50.20 & 38.72 & 67.71 \\
\hline 3.3 & 8.35 & & 68.32 & 61.22 & 54.61 & 53.25 & 48.68 & 68.54 \\
\hline 2.4 & 5.97 & & 85.52 & 52.03 & 55.29 & 54.92 & 56.70 & 65.17 \\
\hline 1.4 & 3.59 & $m$ & 88.12 & 53.74 & 56.46 & 55.69 & 58.35 & 65.93 \\
\hline 0.5 & 1.21 & $\stackrel{ \pm}{ \pm}$ & 88.61 & 55.90 & 57.71 & 58.17 & 60.36 & 66.57 \\
\hline-0.5 & -1.17 & $E$ & 90.31 & 55.16 & 56.90 & 56.81 & 61.46 & 63.37 \\
\hline-1.4 & -3.55 & & 90.10 & 58.51 & 57.12 & 58.84 & 62.11 & 66.77 \\
\hline-2.3 & -5.93 & $\therefore$ & 91.31 & 54.37 & 55.26 & 55.62 & 58.34 & 65.42 \\
\hline-3.3 & -8.31 & & 88.74 & 61.23 & 58.18 & 54.04 & 49.12 & 56.73 \\
\hline-4.2 & -10.69 & & 88.29 & 65.22 & 57.87 & 52.09 & 38.90 & 50.10 \\
\hline-5.1 & -13.07 & $\mathrm{CV}$ & 84.20 & 63.24 & 55.66 & 53.19 & 37.28 & 49.46 \\
\hline-6.1 & -15.45 & $\stackrel{\grave{\Phi}}{\underline{\underline{\omega}}}$ & 79.65 & 61.39 & 54.38 & 48.67 & 37.40 & 48.81 \\
\hline-7.0 & -17.83 & $F$ & 77.93 & 56.68 & 52.48 & 49.36 & 35.99 & 46.49 \\
\hline-8.0 & -20.21 & & 73.24 & 55.02 & 50.31 & 48.64 & 33.04 & 43.48 \\
\hline-8.9 & -22.59 & & 66.22 & 50.20 & 48.89 & 44.76 & 32.85 & 42.57 \\
\hline-9.8 & -24.97 & & 59.37 & $4 \overline{9.89}$ & 47.82 & 42.96 & 37.35 & 45.37 \\
\hline-10.8 & -27.35 & & 52.75 & 49.72 & 43.86 & 39.64 & 41.66 & 50.55 \\
\hline-11.7 & -29.73 & $r$ & 50.15 & 45.00 & 39.75 & 37.36 & 40.02 & 46.43 \\
\hline-12.6 & -32.11 & 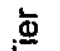 & 48.04 & 45.12 & 37.94 & 35.31 & 36.77 & 42.07 \\
\hline-13.6 & -34.49 & $=$ & 43.22 & 42.42 & 36.07 & 34.07 & 35.71 & 41.88 \\
\hline-14.5 & -36.87 & & 41.51 & 39.05 & 33.72 & 32.54 & 29.71 & 39.87 \\
\hline-15.5 & -39.25 & & 40.27 & 37.21 & 31.26 & 29.83 & 31.06 & 36.02 \\
\hline
\end{tabular}


Enclosure (7) to

MDO-723-0044/

B-MT(SRME)-52

Page 55

\begin{tabular}{|c|c|c|c|c|c|c|c|c|}
\hline \multicolumn{9}{|c|}{$\begin{array}{c}\text { Table III.A-2 } \\
\text { Axial Heat Generation Rates for the HasteloyX Center Specimen of } 6 a 4\end{array}$} \\
\hline \multicolumn{2}{|c|}{ Elevation } & & $\begin{array}{c}\text { C1 } \\
\text { HGEN } \\
\text { (W/CC) }\end{array}$ & $\begin{array}{c}\text { C2 } \\
\text { HGEN } \\
\text { (W/CC) }\end{array}$ & $\begin{array}{c}\text { C3 } \\
\text { HGEN } \\
\text { (W/CC) }\end{array}$ & $\begin{array}{c}\text { C4 } \\
\text { HGEN } \\
\text { (W/CC) }\end{array}$ & $\begin{array}{c}\text { C5 } \\
\text { HGEN } \\
\text { (W/CC) }\end{array}$ & $\begin{array}{c}\text { C6 } \\
\text { HGEN } \\
\text { (W/CC) }\end{array}$ \\
\hline $\begin{array}{l}16.4 \\
15.5 \\
14.5 \\
13.6 \\
12.7 \\
11.7 \\
10.8\end{array}$ & $\begin{array}{l}41.67 \\
39.29 \\
36.91 \\
34.53 \\
32.15 \\
29.77 \\
27.39\end{array}$ & $\stackrel{i n}{i \frac{\mathfrak{\omega}}{E}}$ & $\begin{array}{l}4.46 \\
4.99 \\
5.48 \\
6.16 \\
6.83 \\
7.30 \\
8.75 \\
\end{array}$ & $\begin{array}{l}4.52 \\
4.97 \\
5.61 \\
5.78 \\
6.65 \\
7.25 \\
8.47 \\
\end{array}$ & $\begin{array}{l}5.03 \\
5.19 \\
5.65 \\
6.29 \\
6.77 \\
7.48 \\
8.29\end{array}$ & $\begin{array}{l}5.96 \\
5.93 \\
6.56 \\
6.74 \\
7.62 \\
8.02 \\
8.85\end{array}$ & $\begin{array}{l}4.03 \\
4.49 \\
5.09 \\
5.26 \\
5.70 \\
6.40 \\
6.92\end{array}$ & $\begin{array}{l}4.73 \\
4.97 \\
5.44 \\
5.94 \\
6.55 \\
7.47 \\
8.29 \\
\end{array}$ \\
\hline $\begin{array}{l}9.8 \\
8.9 \\
8.0 \\
7.0 \\
6.1 \\
5.2 \\
4.2\end{array}$ & $\begin{array}{l}25.01 \\
22.63 \\
20.25 \\
17.87 \\
15.49 \\
13.11 \\
10.73\end{array}$ & 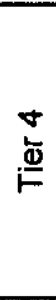 & $\begin{array}{c}9.68 \\
10.35 \\
11.58 \\
12.52 \\
13.44 \\
13.79 \\
14.26 \\
\end{array}$ & $\begin{array}{c}9.34 \\
10.48 \\
11.39 \\
12.20 \\
13.33 \\
13.86 \\
14.82 \\
\end{array}$ & $\begin{array}{c}9.54 \\
10.97 \\
11.32 \\
12.10 \\
12.55 \\
13.74 \\
14.08 \\
\end{array}$ & $\begin{array}{c}9.51 \\
10.32 \\
11.01 \\
11.37 \\
11.93 \\
12.75 \\
13.26 \\
\end{array}$ & $\begin{array}{c}8.35 \\
9.41 \\
10.43 \\
11.28 \\
12.01 \\
12.62 \\
13.09 \\
\end{array}$ & $\begin{array}{c}8.84 \\
9.78 \\
10.61 \\
11.43 \\
12.21 \\
12.54 \\
13.09 \\
\end{array}$ \\
\hline $\begin{array}{r}3.3 \\
2.4 \\
1.4 \\
0.5 \\
-0.5 \\
-1.4 \\
-2.3\end{array}$ & $\begin{array}{r}8.35 \\
5.97 \\
3.59 \\
1.21 \\
-1.17 \\
-3.55 \\
-5.93\end{array}$ & 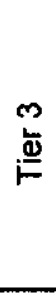 & $\begin{array}{l}15.28 \\
15.58 \\
15.94 \\
16.03 \\
15.89 \\
15.93 \\
16.02 \\
\end{array}$ & $\begin{array}{l}15.48 \\
15.79 \\
16.29 \\
16.39 \\
16.56 \\
16.53 \\
16.38 \\
\end{array}$ & $\begin{array}{l}14.32 \\
14.65 \\
15.36 \\
15.70 \\
15.14 \\
15.44 \\
15.09 \\
\end{array}$ & $\begin{array}{l}13.36 \\
13.81 \\
13.90 \\
13.85 \\
13.88 \\
14.20 \\
14.02 \\
\end{array}$ & $\begin{array}{l}13.73 \\
13.74 \\
13.76 \\
14.14 \\
13.99 \\
14.60 \\
14.05\end{array}$ & $\begin{array}{l}13.79 \\
14.28 \\
14.19 \\
14.52 \\
14.56 \\
14.72 \\
14.75 \\
\end{array}$ \\
\hline $\begin{array}{l}-3.3 \\
-4.2 \\
-5.1 \\
-6.1 \\
-7.0 \\
-8.0 \\
-8.9\end{array}$ & $\begin{array}{c}-8.31 \\
-10.69 \\
-13.07 \\
-15.45 \\
-17.83 \\
-20.21 \\
-22.59\end{array}$ & $\stackrel{N}{\stackrel{N}{\underline{\alpha}}}$ & $\begin{array}{l}17.06 \\
18.10 \\
17.92 \\
17.50 \\
16.02 \\
15.79 \\
14.54\end{array}$ & $\begin{array}{l}16.55 \\
16.12 \\
15.65 \\
15.07 \\
14.62 \\
13.27 \\
12.79 \\
\end{array}$ & $\begin{array}{l}14.82 \\
13.99 \\
13.71 \\
12.59 \\
12.82 \\
12.07 \\
11.56\end{array}$ & $\begin{array}{l}14.08 \\
13.91 \\
13.87 \\
13.52 \\
13.25 \\
12.55 \\
12.08 \\
\end{array}$ & $\begin{array}{l}14.02 \\
13.95 \\
13.41 \\
13.50 \\
12.80 \\
12.20 \\
11.85\end{array}$ & $\begin{array}{l}14.41 \\
14.04 \\
13.60 \\
13.45 \\
12.74 \\
11.70 \\
11.27\end{array}$ \\
\hline $\begin{array}{c}-9.8 \\
-10.8 \\
-11.7 \\
-12.6 \\
-13.6 \\
-14.5 \\
-15.5\end{array}$ & $\begin{array}{l}-24.97 \\
-27.35 \\
-29.73 \\
-32.11 \\
-34.49 \\
-36.87 \\
-39.25\end{array}$ & 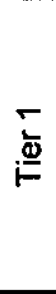 & $\begin{array}{c}12.88 \\
11.66 \\
10.64 \\
10.42 \\
10.23 \\
9.06 \\
8.82 \\
\end{array}$ & $\begin{array}{c}11.50 \\
10.01 \\
9.41 \\
9.07 \\
8.57 \\
8.33 \\
8.08\end{array}$ & $\begin{array}{c}10.73 \\
10.38 \\
10.27 \\
9.83 \\
9.05 \\
8.84 \\
8.30 \\
\end{array}$ & $\begin{array}{c}11.45 \\
10.99 \\
10.64 \\
10.59 \\
10.08 \\
10.18 \\
9.59\end{array}$ & $\begin{array}{l}9.83 \\
7.93 \\
7.65 \\
7.32 \\
7.22 \\
7.09 \\
6.54 \\
\end{array}$ & $\begin{array}{c}10.95 \\
10.66 \\
10.09 \\
9.70 \\
9.48 \\
9.03 \\
8.51\end{array}$ \\
\hline te: $\mathrm{HG}$ & $=$ Total & & $\overline{C 1-C}$ & & $n+1-C$ & tment & & \\
\hline
\end{tabular}


Enclosure (7) to

MDO-723-0044/

B-MT(SRME)-52

Page 56

\begin{tabular}{|c|c|c|c|c|c|c|c|c|}
\hline \multicolumn{9}{|c|}{$\begin{array}{l}\text { Table III.A-2 } \\
\text { Axial Heat Generation Rates for the POCO Specimen of } 6 \mathrm{~d} 4\end{array}$} \\
\hline \multirow{2}{*}{\multicolumn{2}{|c|}{ Elevation }} & & $\mathrm{C} 1$ & $\mathrm{C2}$ & C3 & $\mathrm{C} 4$ & $\mathrm{C5}$ & C6 \\
\hline & & & HGEN & HGEN & HGEN & HGEN & HGEN & HGEN \\
\hline in & $\mathrm{cm}$ & & (W/Cc) & $(W / c c)$ & $(W / c c)$ & (W/Cc) & (W/cc) & (W/Cc) \\
\hline 16.4 & 41.67 & \multirow{7}{*}{$\stackrel{\omega}{\mathscr{E}}$} & 1.93 & 1.95 & 1.61 & 1.57 & 1.65 & 1.84 \\
\hline 15.5 & 39.29 & & 2.06 & 2.01 & 1.77 & 1.68 & 1.75 & 1.94 \\
\hline 14.5 & 36.91 & & 2.40 & 2.26 & 2.04 & 1.95 & 2.03 & 2.19 \\
\hline 13.6 & 34.53 & & 2.76 & 2.59 & 2.33 & 2.15 & 2.32 & 2.53 \\
\hline 12.7 & 32.15 & & 3.06 & 2.94 & 2.70 & 2.39 & 2.60 & 2.83 \\
\hline 11.7 & 29.77 & & 3.62 & 3.40 & 3.01 & 2.72 & 2.91 & 3.34 \\
\hline 10.8 & 27.39 & & 4.37 & 3.86 & 3.39 & 3.01 & 3.33 & 3.86 \\
\hline 9.8 & 25.01 & \multirow{7}{*}{$\underset{⿱ 亠 凶}{ \pm}$} & 4.92 & 4.63 & 3.88 & 3.66 & 3.69 & 4.42 \\
\hline 8.9 & 22.63 & & 5.41 & 5.32 & 4.43 & 4.18 & 4.03 & 5.13 \\
\hline 8.0 & 20.25 & & 6.22 & 5.90 & 4.87 & 4.55 & 4.42 & 5.84 \\
\hline 7.0 & 17.87 & & 6.85 & 6.50 & 5.25 & 4.86 & 4.78 & 6.34 \\
\hline 6.1 & 15.49 & & 7.46 & 7.02 & 5.53 & 5.23 & 5.24 & 6.92 \\
\hline 5.2 & 13.11 & & 7.72 & 7.40 & 5.97 & 5.55 & 5.55 & 7.32 \\
\hline 4.2 & 10.73 & & 7.96 & 7.78 & 6.19 & 5.79 & 5.84 & 7.71 \\
\hline 3.3 & 8.35 & \multirow{7}{*}{$\stackrel{m}{\stackrel{m}{\Phi}}$} & 8.76 & 7.86 & 6.51 & 6.09 & 6.36 & 8.19 \\
\hline 2.4 & 5.97 & & 9.57 & 7.93 & 6.70 & 6.36 & 6.65 & 8.44 \\
\hline 1.4 & 3.59 & & 9.78 & 8.14 & 6.84 & 6.47 & 6.79 & 8.53 \\
\hline 0.5 & 1.21 & & 9.85 & 8.46 & 7.00 & 6.53 & 6.93 & 8.72 \\
\hline-0.5 & -1.17 & & 9.73 & 8.24 & 6.97 & 6.64 & 7.06 & 8.55 \\
\hline-1.4 & -3.55 & & 9.81 & 8.44 & 7.03 & 6.59 & 7.11 & 8.69 \\
\hline-2.3 & -5.93 & & 9.83 & 8.25 & 6.82 & 6.43 & 6.85 & 8.49 \\
\hline-3.3 & -8.31 & \multirow{7}{*}{$\stackrel{N}{i=}$} & 9.64 & 8.35 & 6.88 & 6.29 & 6.40 & 8.18 \\
\hline-4.2 & -10.69 & & 9.44 & 8.19 & 6.71 & 6.06 & 5.94 & 7.80 \\
\hline-5.1 & -13.07 & & 8.96 & 7.90 & 6.38 & 5.90 & 5.71 & 7.50 \\
\hline-6.1 & -15.45 & & 8.42 & 7.55 & 6.08 & 5.58 & 5.48 & 7.22 \\
\hline-7.0 & -17.83 & & 8.14 & 7.14 & 5.79 & 5.47 & 5.29 & 6.82 \\
\hline-8.0 & -20.21 & & 7.57 & 6.72 & 5.47 & 5.20 & 4.77 & 6.24 \\
\hline-8.9 & -22.59 & & 6.73 & 6.12 & 5.19 & 4.75 & 4.48 & 5.80 \\
\hline-9.8 & -24.97 & \multirow{7}{*}{$\underset{i}{\stackrel{ \pm \omega}{=}}$} & 5.91 & 5.33 & 4.75 & 4.26 & 4.23 & 5.25 \\
\hline-10.8 & -27.35 & & 5.17 & 4.82 & 4.19 & 3.74 & 4.02 & 4.87 \\
\hline-11.7 & -29.73 & & 4.60 & 4.41 & 3.74 & 3.49 & 3.76 & 4.30 \\
\hline-12.6 & -32.11 & & 4.25 & 3.98 & 3.45 & 3.13 & 3.41 & 3.83 \\
\hline-13.6 & -34.49 & & 3.86 & 3.61 & 3.18 & 2.93 & 3.22 & 3.60 \\
\hline-14.5 & -36.87 & & 3.57 & 3.30 & 2.85 & 2.69 & 2.74 & 3.27 \\
\hline-15.5 & -39.25 & & 3.26 & 2.98 & 2.57 & 2.39 & 2.61 & 2.92 \\
\hline
\end{tabular}


Enclosure (7) to

MDO-723-0044/

B-MT(SRME)-52

Page 57

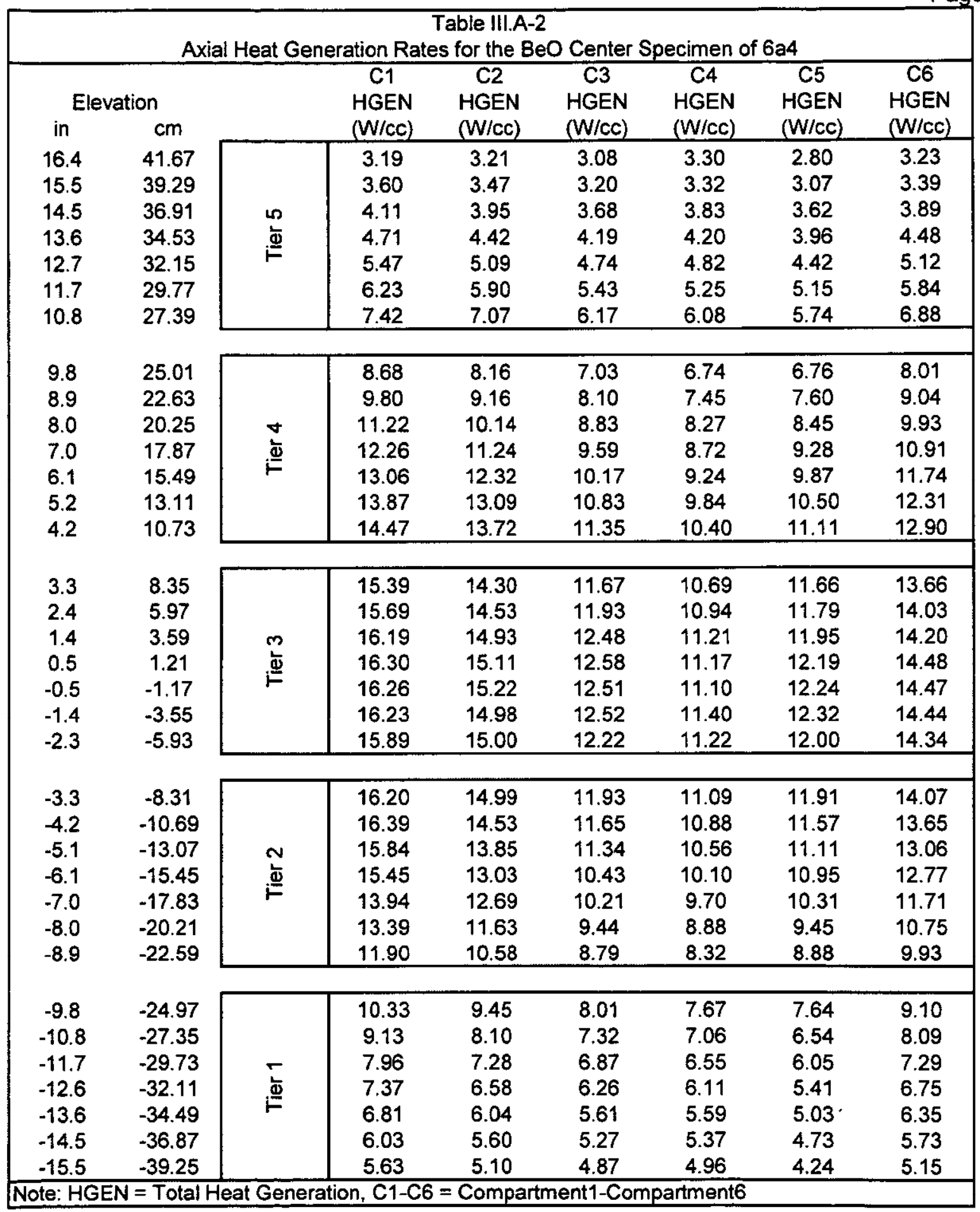


Enclosure (7) to

MDO-723-0044/

B-MT(SRME)-52

Page 58

\begin{tabular}{|c|c|c|c|c|c|c|c|c|}
\hline \multicolumn{9}{|c|}{ Table III.A-2 } \\
\hline \multirow{2}{*}{\multicolumn{2}{|c|}{ Elevation }} & & C1 & $\mathrm{C2}$ & C3 & $\mathrm{C4}$ & $\mathrm{C5}$ & C6 \\
\hline & & & HGEN & HGEN & HGEN & HGEN & HGEN & HGEN \\
\hline in & $\mathrm{cm}$ & & (W/cc) & (W/cc) & (W/cc) & (W/Cc) & (W/Cc) & $(W / c c)$ \\
\hline 16.4 & 41.67 & \multirow{7}{*}{ 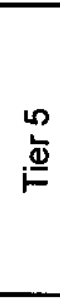 } & 3.37 & 3.39 & 2.83 & 2.77 & 2.88 & 3.19 \\
\hline 15.5 & 39.29 & & 3.60 & 3.50 & 3.11 & 2.94 & 3.07 & 3.39 \\
\hline 14.5 & 36.91 & & 4.20 & 3.98 & 3.56 & 3.39 & 3.54 & 3.85 \\
\hline 13.6 & 34.53 & & 4.83 & 4.54 & 4.11 & 3.75 & 4.04 & 4.42 \\
\hline 12.7 & 32.15 & & 5.38 & 5.18 & 4.74 & 4.20 & 4.56 & 4.96 \\
\hline 11.7 & 29.77 & & 6.41 & 6.01 & 5.27 & 4.77 & 5.11 & 5.90 \\
\hline 10.8 & 27.39 & & 7.71 & 6.82 & 5.98 & 5.33 & 5.87 & 6.81 \\
\hline 9.8 & 25.01 & \multirow{7}{*}{$\underset{+}{ \pm}$} & 8.70 & 8.18 & 6.84 & 6.45 & 6.53 & 7.83 \\
\hline 8.9 & 22.63 & & 9.60 & 9.37 & 7.77 & 7.33 & 7.15 & 9.06 \\
\hline 8.0 & 20.25 & & 11.01 & 10.43 & 8.55 & 7.98 & 7.83 & 10.32 \\
\hline 7.0 & 17.87 & & 12.16 & 11.45 & 9.26 & 8.54 & 8.54 & 11.15 \\
\hline 6.1 & 15.49 & & 13.24 & 12.42 & 9.80 & 9.20 & 9.26 & 12.23 \\
\hline 5.2 & 13.11 & & 13.73 & 13.06 & 10.55 & 9.79 & 9.85 & 12.92 \\
\hline 4.2 & 10.73 & & 14.17 & 13.73 & 10.94 & 10.22 & 10.36 & 13.69 \\
\hline 3.3 & 8.35 & \multirow{7}{*}{$\stackrel{m}{\stackrel{\infty}{\Phi}}$} & 15.54 & 13.90 & 11.52 & 10.75 & 11.22 & 14.51 \\
\hline 2.4 & 5.97 & & 16.85 & 14.06 & 11.84 & 11.24 & 11.72 & 14.91 \\
\hline 1.4 & 3.59 & & 17.25 & 14.43 & 12.11 & 11.39 & 12.03 & 15.03 \\
\hline 0.5 & 1.21 & & 17.39 & 14.97 & 12.36 & 11.50 & 12.22 & 15.39 \\
\hline-0.5 & -1.17 & & 17.19 & 14.63 & 12.33 & 11.69 & 12.41 & 15.14 \\
\hline-1.4 & -3.55 & & 17.32 & 14.96 & 12.43 & 11.61 & 12.54 & 15.40 \\
\hline-2.3 & -5.93 & & 17.37 & 14.64 & 12.09 & 11.31 & 12.11 & 14.95 \\
\hline-3.3 & -8.31 & \multirow{7}{*}{$\stackrel{N}{\stackrel{N}{10}}$} & 16.98 & 14.79 & 12.18 & 11.08 & 11.33 & 14.48 \\
\hline-4.2 & -10.69 & & 16.63 & 14.44 & $\$ 1.89$ & 10.67 & 10.55 & 13.81 \\
\hline-5.1 & -13.07 & & 15.77 & 13.86 & 11.32 & 10.37 & 10.15 & 13.25 \\
\hline-6.1 & -15.45 & & 14.83 & 13.24 & 10.73 & 9.84 & 9.71 & 12.75 \\
\hline-7.0 & -17.83 & & 14.35 & 12.65 & 10.18 & 9.59 & 9.38 & 12.07 \\
\hline-8.0 & -20.21 & & 13.40 & 11.85 & 9.62 & 9.03 & 8.45 & 11.02 \\
\hline-8.9 & -22.59 & & 11.88 & 10.76 & 9.13 & 8.31 & 7.94 & 10.19 \\
\hline-9.8 & -24.97 & \multirow{7}{*}{$\stackrel{\bar{\Phi}}{\underline{\Phi}}$} & 10.39 & 9.40 & 8.34 & 7.49 & 7.47 & 9.24 \\
\hline-10.8 & -27.35 & & 9.07 & 8.47 & 7.36 & 6.56 & 7.06 & 8.52 \\
\hline-11.7 & -29.73 & & 8.05 & 7.73 & 6.57 & 6.07 & 6.57 & 7.55 \\
\hline-12.6 & -32.11 & & 7.43 & 6.94 & 6.04 & 5.48 & 5.97 & 6.71 \\
\hline-13.6 & -34.49 & & 6.73 & 6.31 & 5.53 & 5.07 & 5.61 & 6.25 \\
\hline-14.5 & -36.87 & & 6.23 & 5.73 & 4.94 & 4.68 & 4.77 & 5.66 \\
\hline-15.5 & -39.25 & & 5.66 & 5.17 & 4.43 & 4.14 & 4.51 & 5.05 \\
\hline
\end{tabular}


Enclosure (7) to MDO-723-0044/

B-MT(SRME)-52

Page 59

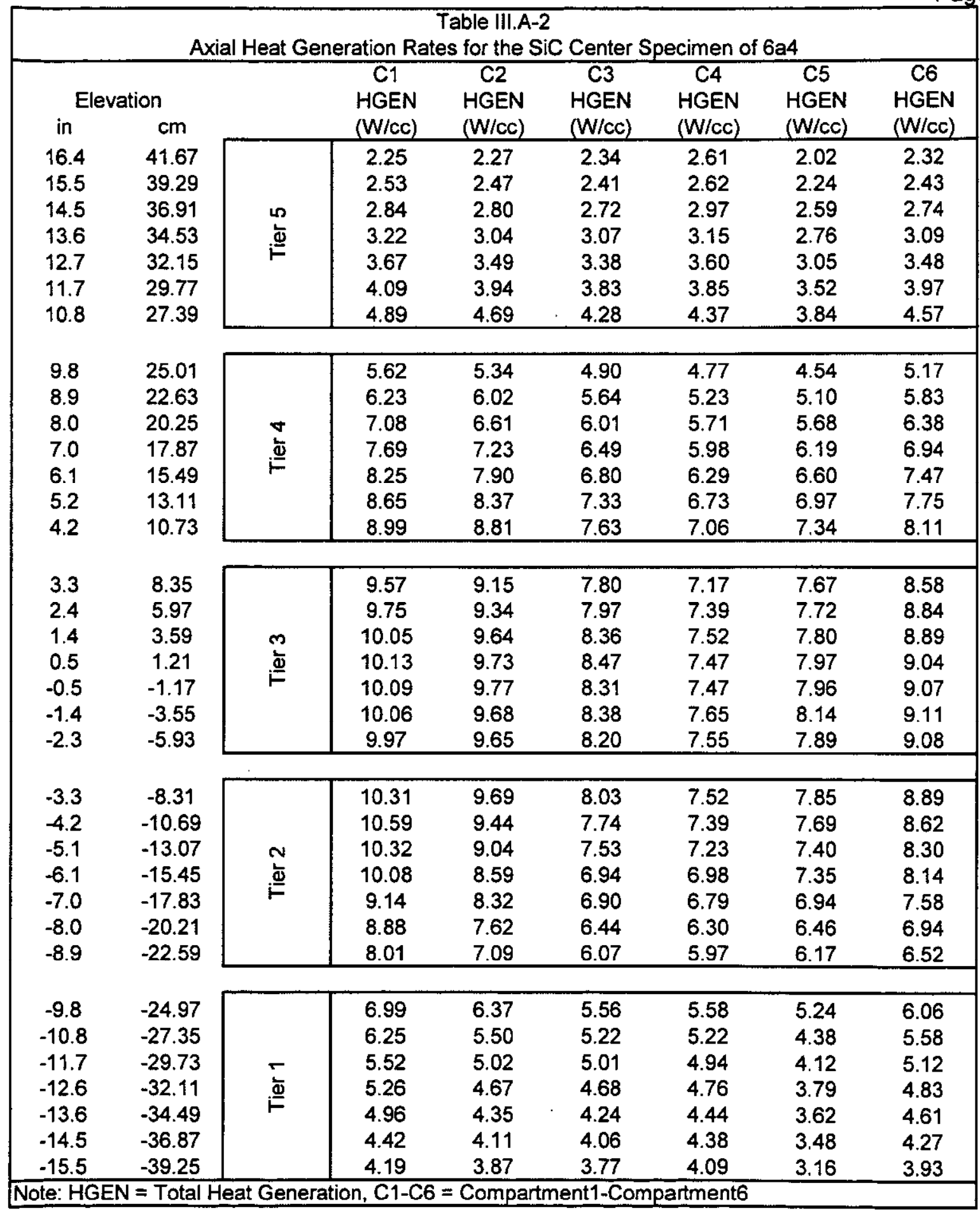


Enclosure (7) to

MDO-723-0044/

B-MT(SRME)-52

Page 60

Table III.A-2

Axial Heat Generation Rates for the SiC Center Specimen of $6 \mathrm{~d} 4$

\begin{tabular}{|c|c|c|c|c|c|c|c|c|}
\hline \multicolumn{2}{|c|}{ Elevation } & & $\begin{array}{c}\text { C1 } \\
\text { HGEN }\end{array}$ & $\begin{array}{c}\text { C2 } \\
\text { HGEN }\end{array}$ & $\begin{array}{c}\text { C3 } \\
\text { HGEN }\end{array}$ & $\begin{array}{c}\mathrm{C} 4 \\
\mathrm{HGEN}\end{array}$ & $\begin{array}{c}\text { C5 } \\
\text { HGEN }\end{array}$ & $\begin{array}{c}\text { C6 } \\
\text { HGEN }\end{array}$ \\
\hline in & $\mathrm{cm}$ & & & & & & & \\
\hline 16.4 & 41.67 & & 2.42 & 2.49 & 1.97 & 2.03 & 2.11 & 2.38 \\
\hline 15.5 & 39.29 & & 2.57 & 2.50 & 2.21 & 2.17 & 2.22 & 2.46 \\
\hline 14.5 & 36.91 & $n$ & 2.95 & 2.78 & 2.54 & 2.52 & 2.55 & 2.71 \\
\hline 13.6 & 34.53 & $\underline{\underline{\underline{\omega}}}$ & 3.41 & 3.12 & 2.91 & 2.71 & 2.89 & 3.16 \\
\hline 12.7 & 32.15 & F & 3.69 & 3.53 & 3.26 & 2.94 & 3.15 & 3.46 \\
\hline 11.7 & 29.77 & & 4.26 & 4.05 & 3.61 & 3.28 & 3.55 & 3.96 \\
\hline 10.8 & 27.39 & & 5.07 & 4.45 & 4.02 & 3.56 & 4.00 & 4.52 \\
\hline 9.8 & 25.01 & & 5.60 & 5.40 & 4.59 & 4.44 & $\overline{4.34}$ & 5.13 \\
\hline 8.9 & 22.63 & & 6.03 & 6.23 & 5.25 & 5.12 & 4.59 & 5.94 \\
\hline 8.0 & 20.25 & $\nabla$ & 6.89 & 6.83 & 5.84 & 5.55 & 4.99 & 6.74 \\
\hline 7.0 & 17.87 & 訇 & 7.55 & 7.49 & 6.16 & 5.79 & 5.40 & 7.24 \\
\hline 6.1 & 15.49 & 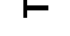 & 8.25 & 8.16 & 6.48 & 6.28 & 5.98 & 7.81 \\
\hline 5.2 & 13.11 & & 8.51 & 8.50 & 6.94 & 6.59 & 6.20 & 8.35 \\
\hline 4.2 & 10.73 & & 8.69 & 8.88 & 7.20 & 6.87 & 6.53 & 8.76 \\
\hline 3.3 & 8.35 & & 9.79 & 8.85 & 7.55 & 7.25 & 7.28 & 9.38 \\
\hline 2.4 & 5.97 & & 10.88 & 8.82 & 7.77 & 7.55 & 7.72 & 9.65 \\
\hline 1.4 & 3.59 & $m$ & 11.19 & 9.11 & 7.93 & 7.71 & 7.89 & 9.75 \\
\hline 0.5 & 1.21 &.$\underline{\underline{D}}$ & 11.14 & 9.47 & 8.06 & 7.90 & 8.05 & 9.97 \\
\hline-0.5 & -1.17 & $F$ & 11.11 & 9.19 & 7.97 & 7.83 & 8.21 & 9.58 \\
\hline-1.4 & -3.55 & & 11.18 & 9.51 & 8.11 & 7.92 & 8.33 & 9.90 \\
\hline-2.3 & -5.93 & & 11.21 & 9.16 & 7.81 & 7.65 & 7.94 & 9.65 \\
\hline-3.3 & -8.31 & & 11.05 & 9.47 & 7.99 & 7.46 & 7.26 & 9.12 \\
\hline-4.2 & -10.69 & & 10.84 & 9.42 & 7.79 & 7.21 & 6.58 & 8.62 \\
\hline-5.1 & -13.07 & $N$ & 10.25 & 9.08 & 7.47 & 7.11 & 6.31 & 8.38 \\
\hline-6.1 & -15.45 & $\underline{\underline{\Phi}}$ & 9.68 & 8.71 & 7.14 & 6.62 & 6.14 & 8.13 \\
\hline-7.0 & -17.83 & F & 9.41 & 8.30 & 6.89 & 6.62 & 5.97 & 7.75 \\
\hline-8.0 & -20.21 & & 8.82 & 7.84 & 6.56 & 6.40 & 5.37 & 7.11 \\
\hline-8.9 & -22.59 & & 7.91 & 7.11 & 6.33 & 5.87 & 5.15 & 6.72 \\
\hline-9.8 & -24.97 & & 7.06 & 6.32 & 5.85 & 5.25 & 4.97 & 6.21 \\
\hline-10.8 & -27.35 & & 6.22 & 5.78 & 5.15 & 4.62 & 4.88 & 5.94 \\
\hline-11.7 & -29.73 & $\tau$ & 5.63 & 5.32 & 4.59 & 4.38 & 4.69 & 5.27 \\
\hline-12.6 & -32.11 & $\underline{\underline{\Phi}}$ & 5.35 & 4.97 & 4.31 & 3.97 & 4.28 & 4.73 \\
\hline-13.6 & -34.49 & $F$ & 4.89 & 4.59 & 4.08 & 3.78 & 4.07 & 4.60 \\
\hline-14.5 & -36.87 & & 4.60 & 4.26 & 3.71 & 3.53 & 3.48 & 4.24 \\
\hline-15.5 & -39.25 & & 4.30 & 3.92 & 3.40 & 3.19 & 3.47 & 3.84 \\
\hline
\end{tabular}


Enclosure (7) to

MDO-723-0044/

B-MT(SRME)-52

Page 61

\begin{tabular}{|c|c|c|c|c|c|c|c|c|}
\hline & & & $\operatorname{tion} \mathrm{Ra}$ & $\begin{array}{l}\text { able III.A } \\
\text { for the }\end{array}$ & Center & ecimen & & \\
\hline & ion & & $\begin{array}{c}\text { C1 } \\
\text { HGEN } \\
\text { (W/CC) }\end{array}$ & $\begin{array}{c}\text { C2 } \\
\text { HGEN } \\
\text { (W/CC) }\end{array}$ & $\begin{array}{c}\text { C3 } \\
\text { HGEN } \\
\text { (W/cC) }\end{array}$ & $\begin{array}{c}\text { C4 } \\
\text { HGEN } \\
(W / C C)\end{array}$ & $\begin{array}{c}\text { C5 } \\
\text { HGEN } \\
\text { (W/CC) }\end{array}$ & $\begin{array}{c}\text { C6 } \\
\text { HGEN } \\
\text { (W/CC) }\end{array}$ \\
\hline $\begin{array}{l}16.4 \\
15.5 \\
14.5 \\
13.6 \\
12.7 \\
11.7 \\
10.8\end{array}$ & $\begin{array}{l}41.67 \\
39.29 \\
36.91 \\
34.53 \\
32.15 \\
29.77 \\
27.39\end{array}$ & 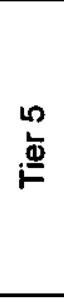 & $\begin{array}{l}1.83 \\
2.06 \\
2.31 \\
2.62 \\
2.98 \\
3.33 \\
3.98 \\
\end{array}$ & $\begin{array}{l}1.84 \\
2.01 \\
2.28 \\
2.47 \\
2.84 \\
3.20 \\
3.81 \\
\end{array}$ & $\begin{array}{l}1.90 \\
1.96 \\
2.21 \\
2.50 \\
2.75 \\
3.11 \\
3.48 \\
\end{array}$ & $\begin{array}{l}2.12 \\
2.13 \\
2.41 \\
2.56 \\
2.92 \\
3.13 \\
3.55 \\
\end{array}$ & $\begin{array}{l}1.64 \\
1.82 \\
2.10 \\
2.24 \\
2.48 \\
2.86 \\
3.12 \\
\end{array}$ & $\begin{array}{l}1.89 \\
1.98 \\
2.22 \\
2.51 \\
2.83 \\
3.22 \\
3.71 \\
\end{array}$ \\
\hline $\begin{array}{l}9.8 \\
8.9 \\
8.0 \\
7.0 \\
6.1 \\
5.2 \\
4.2\end{array}$ & $\begin{array}{l}25.01 \\
22.63 \\
20.25 \\
17.87 \\
15.49 \\
13.11 \\
10.73\end{array}$ & 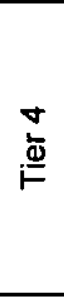 & $\begin{array}{l}4.56 \\
5.06 \\
5.75 \\
6.25 \\
6.70 \\
7.03 \\
7.30 \\
\end{array}$ & $\begin{array}{l}4.34 \\
4.89 \\
5.37 \\
5.87 \\
6.42 \\
6.80 \\
7.15 \\
\end{array}$ & $\begin{array}{l}3.98 \\
4.58 \\
4.88 \\
5.27 \\
5.52 \\
5.96 \\
6.20 \\
\end{array}$ & $\begin{array}{l}3.88 \\
4.25 \\
4.64 \\
4.86 \\
5.11 \\
5.47 \\
5.73 \\
\end{array}$ & $\begin{array}{l}3.69 \\
4.15 \\
4.61 \\
5.03 \\
5.36 \\
5.66 \\
5.96 \\
\end{array}$ & $\begin{array}{l}4.20 \\
4.74 \\
5.19 \\
5.64 \\
6.07 \\
6.29 \\
6.59 \\
\end{array}$ \\
\hline $\begin{array}{l}3.3 \\
2.4 \\
1.4 \\
0.5 \\
-0.5 \\
-1.4 \\
-2.3\end{array}$ & $\begin{array}{c}8.35 \\
5.97 \\
3.59 \\
1.21 \\
-1.17 \\
-3.55 \\
-5.93\end{array}$ & $\stackrel{m}{\stackrel{2}{\varrho}}$ & $\begin{array}{l}7.77 \\
7.92 \\
8.17 \\
8.23 \\
8.20 \\
8.17 \\
8.10 \\
\end{array}$ & $\begin{array}{l}7.44 \\
7.59 \\
7.83 \\
7.90 \\
7.94 \\
7.87 \\
7.84 \\
\end{array}$ & $\begin{array}{l}6.33 \\
6.48 \\
6.79 \\
6.89 \\
6.75 \\
6.81 \\
6.66 \\
\end{array}$ & $\begin{array}{l}5.82 \\
6.01 \\
6.11 \\
6.07 \\
6.07 \\
6.22 \\
6.13 \\
\end{array}$ & $\begin{array}{l}6.23 \\
6.27 \\
6.34 \\
6.48 \\
6.46 \\
6.62 \\
6.41 \\
\end{array}$ & $\begin{array}{l}6.97 \\
7.19 \\
7.22 \\
7.35 \\
7.37 \\
7.40 \\
7.38 \\
\end{array}$ \\
\hline $\begin{array}{l}-3.3 \\
-4.2 \\
-5.1 \\
-6.1 \\
-7.0 \\
-8.0 \\
-8.9\end{array}$ & $\begin{array}{c}-8.31 \\
-10.69 \\
-13.07 \\
-15.45 \\
-17.83 \\
-20.21 \\
-22.59\end{array}$ & $\stackrel{\sim}{\stackrel{N}{\Xi}}$ & $\begin{array}{l}8.38 \\
8.61 \\
8.38 \\
8.19 \\
7.43 \\
7.21 \\
6.51 \\
\end{array}$ & $\begin{array}{l}7.87 \\
7.67 \\
7.34 \\
6.98 \\
6.76 \\
6.19 \\
5.76 \\
\end{array}$ & $\begin{array}{l}6.53 \\
6.29 \\
6.12 \\
5.64 \\
5.60 \\
5.23 \\
4.93 \\
\end{array}$ & $\begin{array}{l}6.11 \\
6.00 \\
5.88 \\
5.67 \\
5.51 \\
5.12 \\
4.85 \\
\end{array}$ & $\begin{array}{l}6.38 \\
6.25 \\
6.01 \\
5.97 \\
5.64 \\
5.25 \\
5.01 \\
\end{array}$ & $\begin{array}{l}7.22 \\
7.01 \\
6.74 \\
6.62 \\
6.16 \\
5.64 \\
5.30 \\
\end{array}$ \\
\hline $\begin{array}{c}-9.8 \\
-10.8 \\
-11.7 \\
-12.6 \\
-13.6 \\
-14.5 \\
-15.5 \\
\end{array}$ & $\begin{array}{l}-24.97 \\
-27.35 \\
-29.73 \\
-32.11 \\
-34.49 \\
-36.87 \\
-39.25 \\
\end{array}$ & $\stackrel{\overline{\underline{\underline{\alpha}}}}{F}$ & $\begin{array}{l}5.68 \\
5.08 \\
4.49 \\
4.27 \\
4.03 \\
3.59 \\
3.40 \\
\end{array}$ & $\begin{array}{l}5.17 \\
4.47 \\
4.08 \\
3.79 \\
3.54 \\
3.34 \\
3.15 \\
\end{array}$ & $\begin{array}{l}4.52 \\
4.24 \\
4.07 \\
3.81 \\
3.45 \\
3.30 \\
3.06 \\
\end{array}$ & $\begin{array}{l}4.53 \\
4.25 \\
4.02 \\
3.87 \\
3.61 \\
3.56 \\
3.32 \\
\end{array}$ & $\begin{array}{l}4.25 \\
3.56 \\
3.35 \\
3.08 \\
2.94 \\
2.83 \\
2.57 \\
\end{array}$ & $\begin{array}{l}4.93 \\
4.53 \\
4.16 \\
3.93 \\
3.75 \\
3.47 \\
3.20 \\
\end{array}$ \\
\hline te: $\mathrm{HC}$ & Total & & $\mathrm{Cl}$ & Comp & 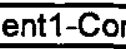 & $7 m$ & & \\
\hline
\end{tabular}


Enclosure (7) to

MDO-723-0044/

B-MT(SRME)-52

Page 62

\begin{tabular}{|c|c|c|c|c|c|c|c|c|}
\hline \multicolumn{9}{|c|}{$\begin{array}{l}\text { Table III.A-2 } \\
\text { Axial Heat Generation Rates for the cSi }\end{array}$} \\
\hline \multicolumn{2}{|c|}{ Elevation } & & $\begin{array}{c}\text { C1 } \\
\text { HGEN }\end{array}$ & $\begin{array}{l}\text { C2 } \\
\text { HGEN }\end{array}$ & $\begin{array}{c}\text { C3 } \\
\text { HGEN }\end{array}$ & $\begin{array}{c}\text { C4 } \\
\text { HGEN }\end{array}$ & $\begin{array}{c}\text { C5 } \\
\text { HGEN }\end{array}$ & $\begin{array}{c}\text { C6 } \\
\text { HGEN }\end{array}$ \\
\hline in & $\mathrm{cm}$ & & (W/cc) & $(W / c c)$ & $(W / c c)$ & (W/Cc) & (W/Cc) & $(W / c c)$ \\
\hline 16.4 & 41.67 & & 1.97 & 2.02 & 1.60 & 1.65 & 1.71 & 1.94 \\
\hline 15.5 & 39.29 & & 2.08 & 2.03 & 1.79 & 1.76 & 1.81 & 2.00 \\
\hline 14.5 & 36.91 & $n$ & 2.40 & 2.26 & 2.06 & 2.04 & 2.07 & 2.20 \\
\hline 13.6 & 34.53 & 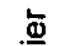 & 2.77 & 2.53 & 2.36 & 2.20 & 2,35 & 2.56 \\
\hline 12.7 & 32.15 & $F$ & 2.99 & 2.86 & 2.64 & 2.39 & 2.56 & 2.81 \\
\hline 11.7 & 29.77 & & 3.46 & 3.29 & 2.94 & 2.67 & 2.89 & 3.21 \\
\hline 10.8 & 27.39 & & 4.12 & 3.61 & 3.26 & 2.89 & 3.25 & 3.67 \\
\hline 9.8 & 25.01 & & 4.55 & 4.38 & 3.73 & 3.61 & 3.52 & 4.17 \\
\hline 8.9 & 22.63 & & 4.90 & 5.06 & 4.27 & 4.16 & 3.73 & 4.83 \\
\hline 8.0 & 20.25 & 8 & 5.60 & 5.55 & 4.75 & 4.51 & 4.05 & 5.48 \\
\hline 7.0 & 17.87 & 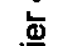 & 6.13 & 6.09 & 5.01 & 4.70 & 4.39 & 5.88 \\
\hline 6.1 & 15.49 & $F$ & 6.70 & 6.63 & 5.26 & 5.10 & 4.86 & 6.34 \\
\hline 5.2 & 13.11 & & 6.92 & 6.91 & 5.64 & 5.36 & 5.03 & 6.78 \\
\hline 4.2 & 10.73 & & 7.06 & 7.22 & 5.85 & 5.58 & 5.30 & 7.12 \\
\hline 3.3 & 8.35 & & 7.96 & 7.19 & 6.13 & 5.89 & 5.92 & 7.62 \\
\hline 2.4 & 5.97 & & 8.84 & 7.16 & 6.31 & 6.14 & 6.28 & 7.84 \\
\hline 1.4 & 3.59 & $m$ & 9.09 & 7.40 & 6.45 & 6.27 & 6.41 & 7.92 \\
\hline 0.5 & 1.21 & $\underline{\underline{\Phi}}$ & 9.05 & 7.70 & 6.55 & 6.42 & 6.54 & 8.10 \\
\hline-0.5 & -1.17 & $i$ & 9.03 & 7.47 & 6.47 & 6.37 & 6.67 & 7.78 \\
\hline-1.4 & -3.55 & & 9.08 & 7.72 & 6.59 & 6.43 & 6.76 & 8.04 \\
\hline-2.3 & -5.93 & & 9.11 & 7.44 & 6.34 & 6.21 & 6.45 & 7.84 \\
\hline-3.3 & -8.31 & & 8.98 & 7.70 & 6.49 & 6.06 & 5.90 & 7.41 \\
\hline-4.2 & -10.69 & & 8.81 & 7.66 & 6.33 & 5.86 & 5.35 & 7.00 \\
\hline-5.1 & -13.07 & $N$ & 8.33 & 7.38 & 6.07 & 5.78 & 5.13 & 6.81 \\
\hline-6.1 & -15.45 & $\underline{\underline{\Phi}}$ & 7.87 & 7.07 & 5.80 & 5.38 & 4.99 & 6.61 \\
\hline-7.0 & -17.83 & $F$ & 7.65 & 6.74 & 5.60 & 5.38 & 4.85 & 6.29 \\
\hline-8.0 & -20.21 & & 7.17 & 6.37 & 5.33 & 5.20 & 4.36 & 5.77 \\
\hline-8.9 & -22.59 & & 6.43 & 5.78 & 5.15 & 4.77 & 4.18 & 5.46 \\
\hline-9.8 & -24.97 & & 5.74 & 5.13 & 4.76 & 4.27 & 4.03 & 5.04 \\
\hline-10.8 & -27.35 & & 5.06 & 4.69 & 4.19 & 3.75 & 3.97 & 4.83 \\
\hline-11.7 & -29.73 & $\sigma$ & 4.57 & 4.32 & 3.73 & 3.56 & 3.81 & 4.28 \\
\hline-12.6 & -32.11 & $\underline{\Phi}$ & 4.35 & 4.04 & 3.50 & 3.23 & 3.47 & 3.84 \\
\hline-13.6 & -34.49 & $F$ & 3.97 & 3.73 & 3.32 & 3.07 & 3.31 & 3.74 \\
\hline-14.5 & -36.87 & & 3.74 & 3.46 & 3.02 & 2.87 & 2.83 & 3.44 \\
\hline-15.5 & -39.25 & & 3.50 & 3.19 & 2.76 & 2.59 & 2.82 & 3.12 \\
\hline
\end{tabular}




\section{B. Thermal Analysis}

\section{Model Description}

\section{a. Analysis Tool}

In order to achieve the stated objectives for the JOYO irradiation test program, a test matrix consisting of sixty unique test capsules has been developed. The need to perform sixty unique thermal analyses within a brief timeframe demanded the use of an analysis tool which would provide sufficient accuracy and detail coupled with fast solution time to allow the necessary design iterations and sensitivity studies to be completed. For these reasons, the commercially available ANSYS finite element analysis computer program was chosen as the thermal design tool for the JOYO test program. Additionally, the ANSYS code allows thermal stress results to be quickly and easily calculated from the thermal results.

ANSYS has been successfully employed as an analysis tool for NRPCT sponsored irradiation testing conducted in the DOE operated Advanced Test Reactor (ATR) in Idaho. The sixty capsules in the JOYO test matrix are similar in construction to the capsules which have been tested in the ATR. The ATR capsules have been assembled both with and without thermocouple instrumentation. Measurements from the ATR instrumented capsules have validated the accuracy of the ANSYS design models.

\section{b. Model Geometry}

There are ten different specimen stackup arrangements. The capsule shell design for all of the specimen stackups is the same. As illustrated in the overall Test Matrix, the ten different specimen arrangements have been strategically placed into the sixty JOYO SMIR (Rig A and Rig B) positions. Specimen materials vary from capsule to capsule. Three of the ten designs are vertical geometric mirror images of the base designs.

The ANSYS models were constructed from engineering component and assembly drawings. The models were all prepared as $2 \mathrm{D}$ axisymmetric representations. As previously discussed, prior experience has shown that, for these types of capsules, axisymmetric representation altows adequate detail to be accurately depicted while quick solution and convergence is provided. Figure III.B- 1 shows the axisymmetric model profile for a tensile specimer in a JOYO-1 capsule. Test specimen and test capsule thermal profile results are shown for each specimen arrangement type. The specific capsule Rig, Tier, and position is labeled on each figure. 


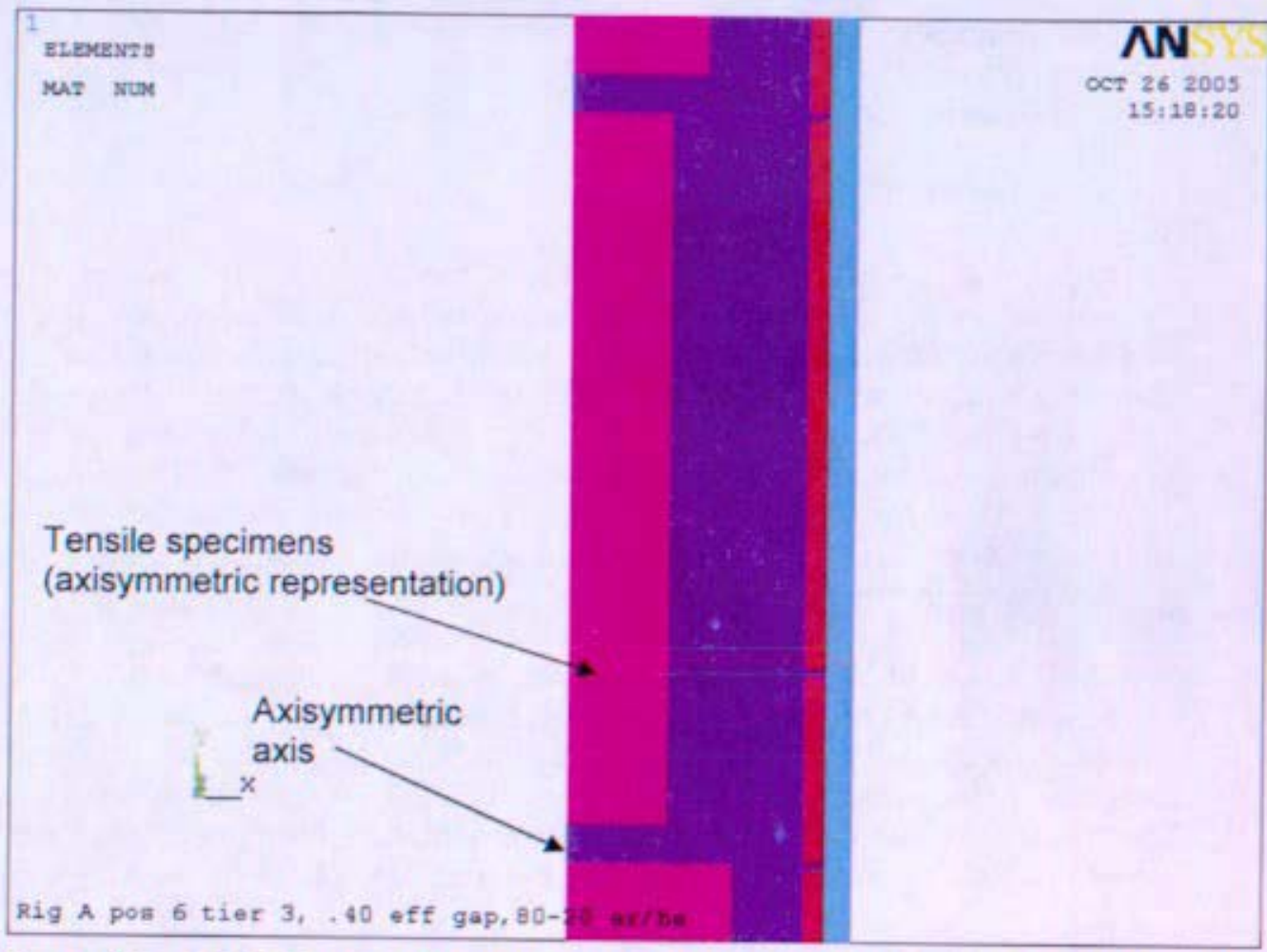

Figure III.B-1 ANSYS axisymmetric finite element representation of the JOYO-1 capsules.

\section{c. Model Features}

The capsule shell and capsule internals (i.e., spacers, insulators, wave springs, specimens, and specimen holders) were all represented as material regions in the ANSYS models, along with the gas regions. All dimensions used were nominal dimensions. The effects of thermal expansion were not included in the thermal problem calculations. Past experience has shown that thermal expansion of the specimen and capsule materials will not significantly affect thermal profile results. However, for the mechanical design of the capsule assembly, thermal expansions of the specimen materials and of the internal capsule component materials were assessed in order to preclude any fitup interferences during irradiation.

Minor modifications to the models were introduced to simplify the analysis where appropriate. In some instances geometric adjustments were made to allow axisymmetric representation of non-axisymmetric features. Contact interfaces were simulated as $0.03 \mathrm{~mm}$ gas gaps.

\section{d. Finite Elements}

The basic ANSYS finite element chosen for these analyses was the PLANE35 element. The PLANE35 element is a 6-node triangular element which, due to its triangular shape, is well suited to mesh irregularly shaped regions. The element has one degree of freedom, temperature, at each node. The 6-noded thermal element is applicable to a 2D steady state or transient thermal analysis, and can be used as a 
planar element or an axisymmetric element. This element can readily be replaced by an equivalent structural element, PLANE2, for cases in which structural analyses are to be performed. Figure III.B-2 shows typical PLANE35 mesh elements in a capsule model. PLANE35 element sizes in the capsule models were maintained in the range of $0.03 \mathrm{~mm}$ to $0.5 \mathrm{~mm}$. This range was found to provide adequate accuracy with fast solution convergence.

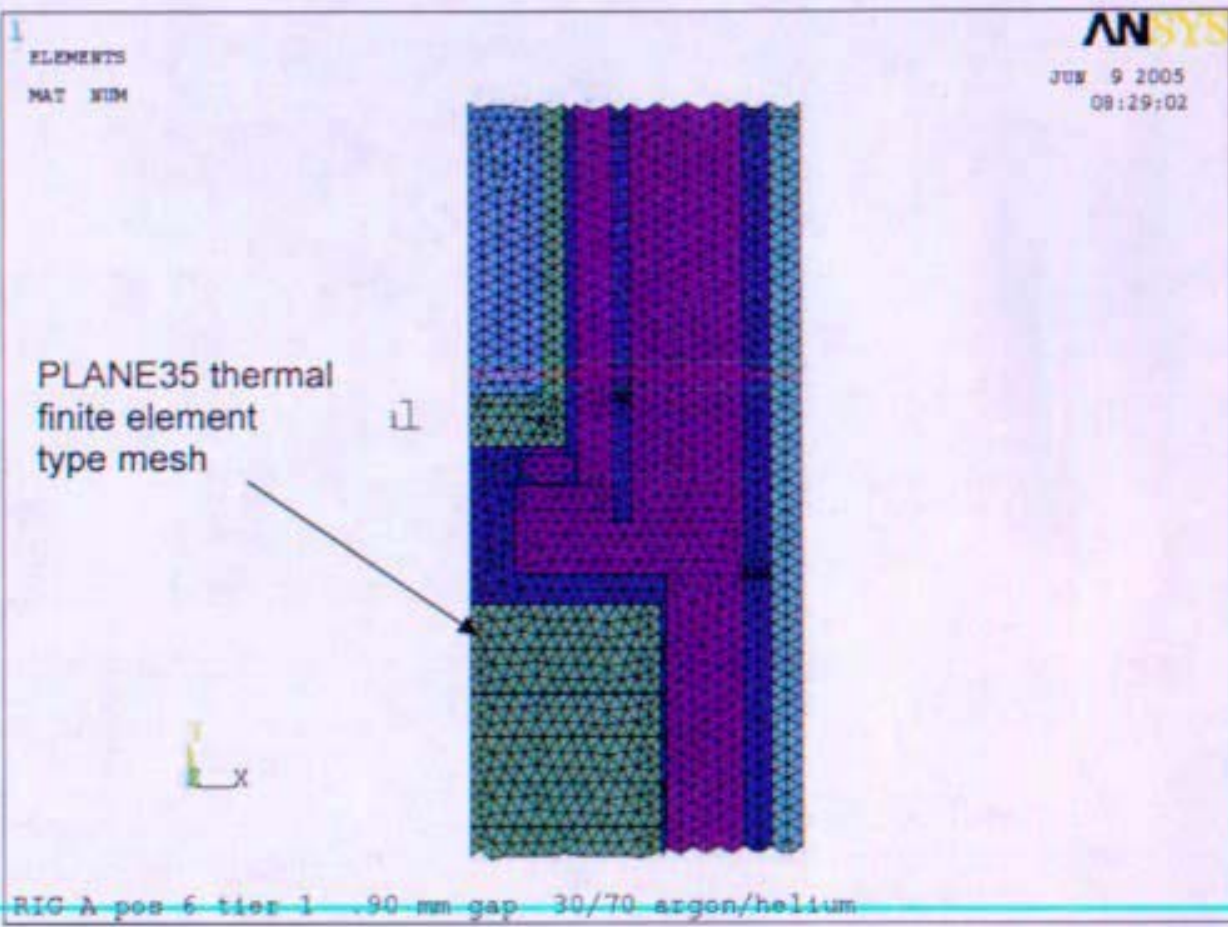

Figure III.B-2 PLANE35 mesh elements in a segment of the capsule finite element model.

Other element types used in the thermal analysis were ANSYS LINK elements. LINK31 elements were used to represent radiation heat flow in the problems. LINK31 is a uniaxial element which models the radiation heat flow between two points in space. The link has a single degree of freedom, temperature, at each node. This link element is suitable to a $2 \mathrm{D}$ planar or axisymmetric steady state thermal analysis. Each thermal link element is defined via "real constants" characterizing radiating surface area, geometric form factors, and temperature dependent radiative emissivity. In the ANSYS models, the LINK31 elements were included where needed to overlay the continuum PLANE35 finite gas elements in order to provide parallel heat transfer paths between surfaces. Figure III.B-3 illustrates how radiation link elements are defined across a gas gap region. The gas mesh elements were removed from the figure so that the radiation links can be readily viewed. 


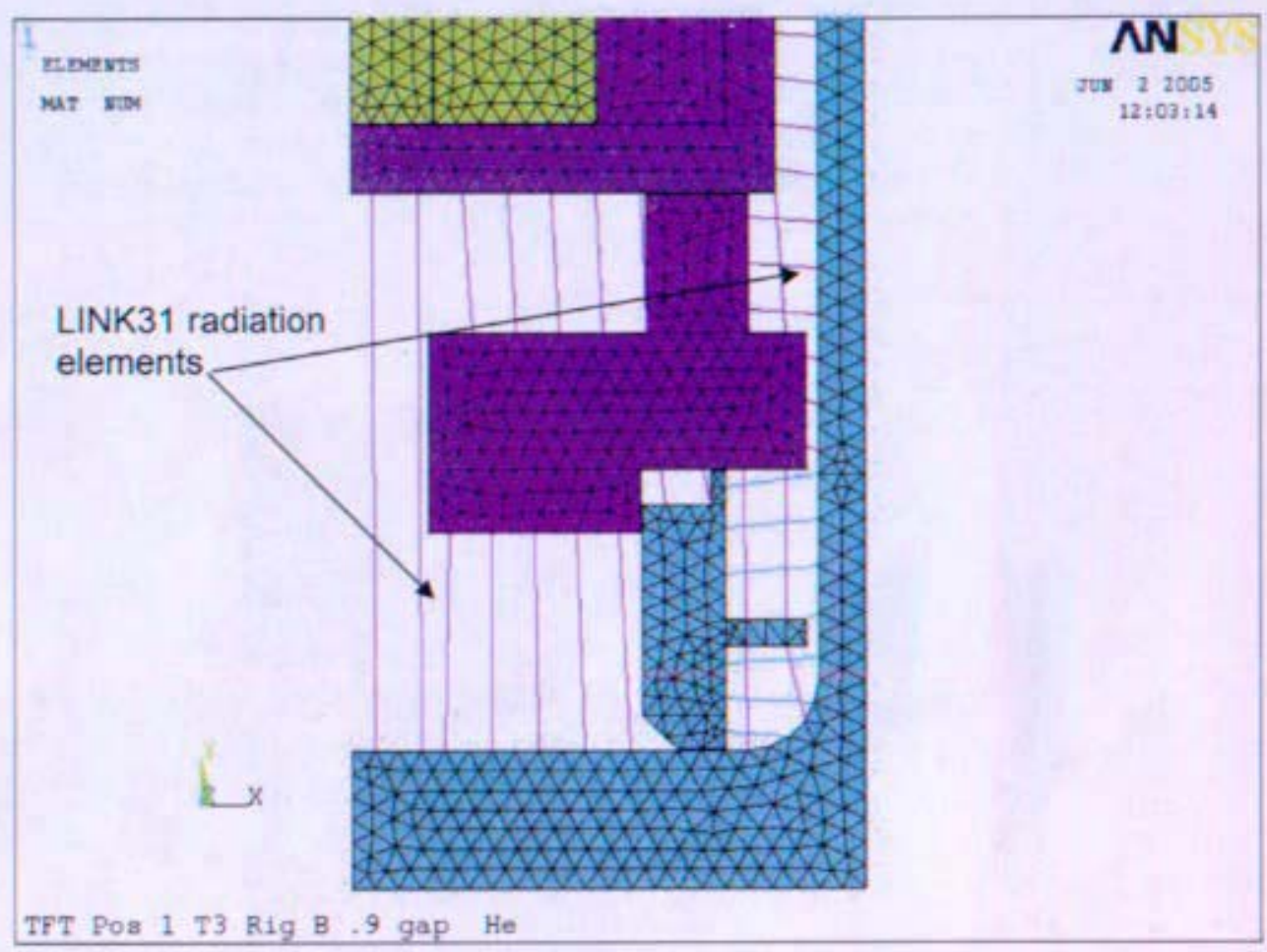

Figure III.B-3 Radiation LINK32 elements used to model radiative heat transfer.

The finite element model of the NBU capsule utilized LINK32 uniaxial elements to represent the non-axisymmetric geometry of the BeO cylindrical specimens. LINK32 is a uniaxial element with the ability to conduct heat between its nodes. The element has a single degree of freedom, temperature, at each node point and is defined by two nodes, a cross-sectional area, and material properties. Adjustments to the actual specimen material dimensions and configurations along with the use of LINK32 elements provided acceptable modeling. Total heat generation and heat transfer were preserved.

\section{e. Boundary Conditions / Loading Conditions}

Calculating the loading conditions that the capsules are subjected to was an iterative process that required multiple steps. Also, the specimen temperature predictions are a relatively strong function of both the loading and boundary conditions. Therefore, it is important to complete the iterations before a final design is locked in to minimize deviations from target temperatures. Initial JOYO sodium conditions were provided to NRPCT and shown in Attachment D to this enclosure.

A convective film coefficient and liquid sodium sink temperatures were applied as boundary conditions to the capsule outer surface wetted by the coolant. Unloaded external surfaces were treated as adiabatic. Liquid metal heat transfer is very efficient. Accordingly, a large film coefficient was used in the models. The combination of efficient surface heat transfer with the relatively low capsule external surface heat flux results in a very low or essentially negligible calculated film drop. Sensitivity calculations show that the use of a fixed boundary temperature, as opposed to the use 
of an applied film coefficient, provides the same thermal profile as when convective surface heat transfer is used.

The liquid sodium boundary conditions were provided in two iterations. The first provided by JNC, gave only a single reactor coolant temperature for each tier. The second iteration was received in August 2005 and provided much more detailed information. The sodium temperatures were given as a function of elevation by centimeter for 54 sub-channels in the test rig as shown in Figure III.B-4. The effects of eight different operating conditions including; nominal flow and combinations of $25 \%$ over power, hot spot factor, transient overpower, and loss of flow conditions were also given so that a worst case analysis could be done.

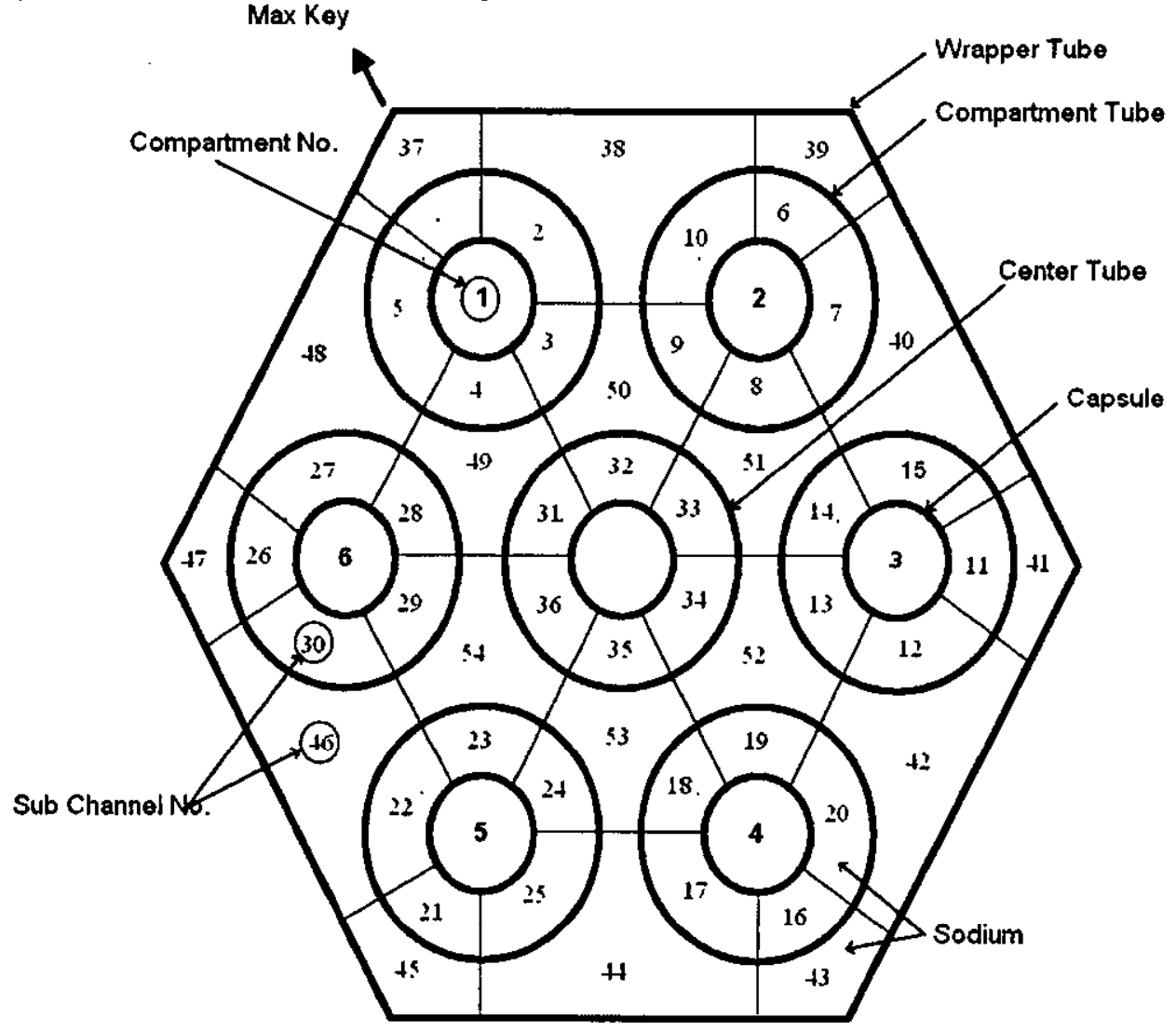

Figure III.B-4 JOYO SMIR sub-channel positions used to analyze sodium coolant temperatures for boundary conditions to the JOYO-1 Capsules.

Sub-channel temperatures were averaged around their respective compartments and applied as an axial linear gradient to each capsule, specific to their tier and compartment location. It was assumed that boundary conditions for both rigs were similar given their symmetric locations with respect to the core. Sink temperatures for the sodium coolant are given in ${ }^{\circ} \mathrm{C}$ for the nominal and worst case condition (loss of flow, $25 \%$ overpower, hot spot factor combination) in Figure III.B-5. 

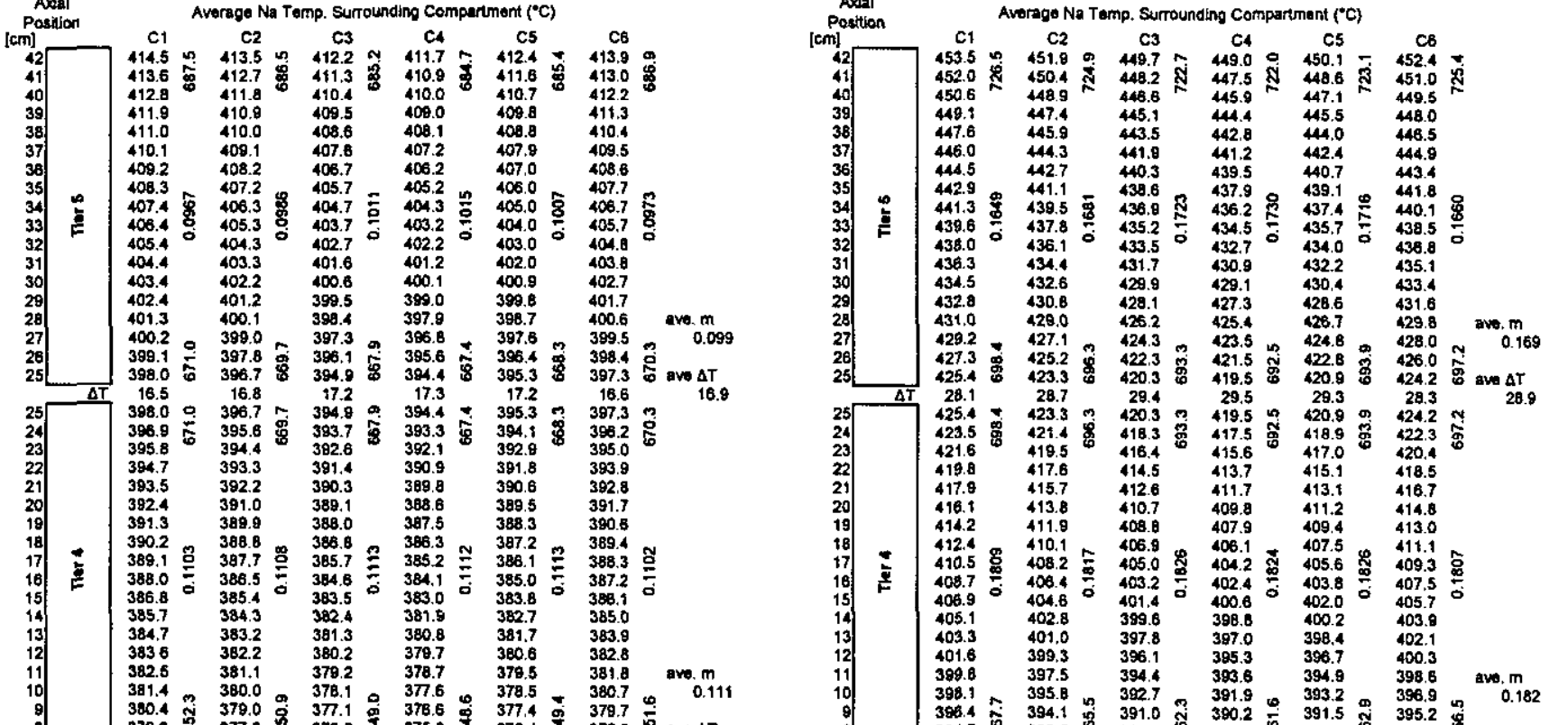

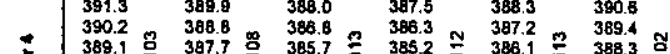

F 388.1 .0

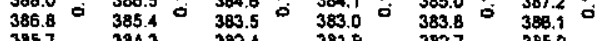

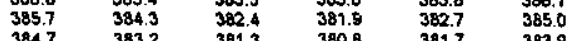

$\begin{array}{llllll}384.7 & 383.2 & 351.3 & 380.8 & 331.7 & 383.9 \\ 3838 & 382.2 & 380.2 & 379.7 & 3006 & 3328\end{array}$

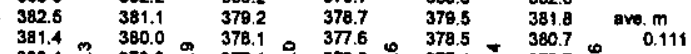

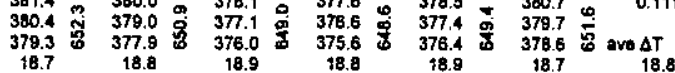

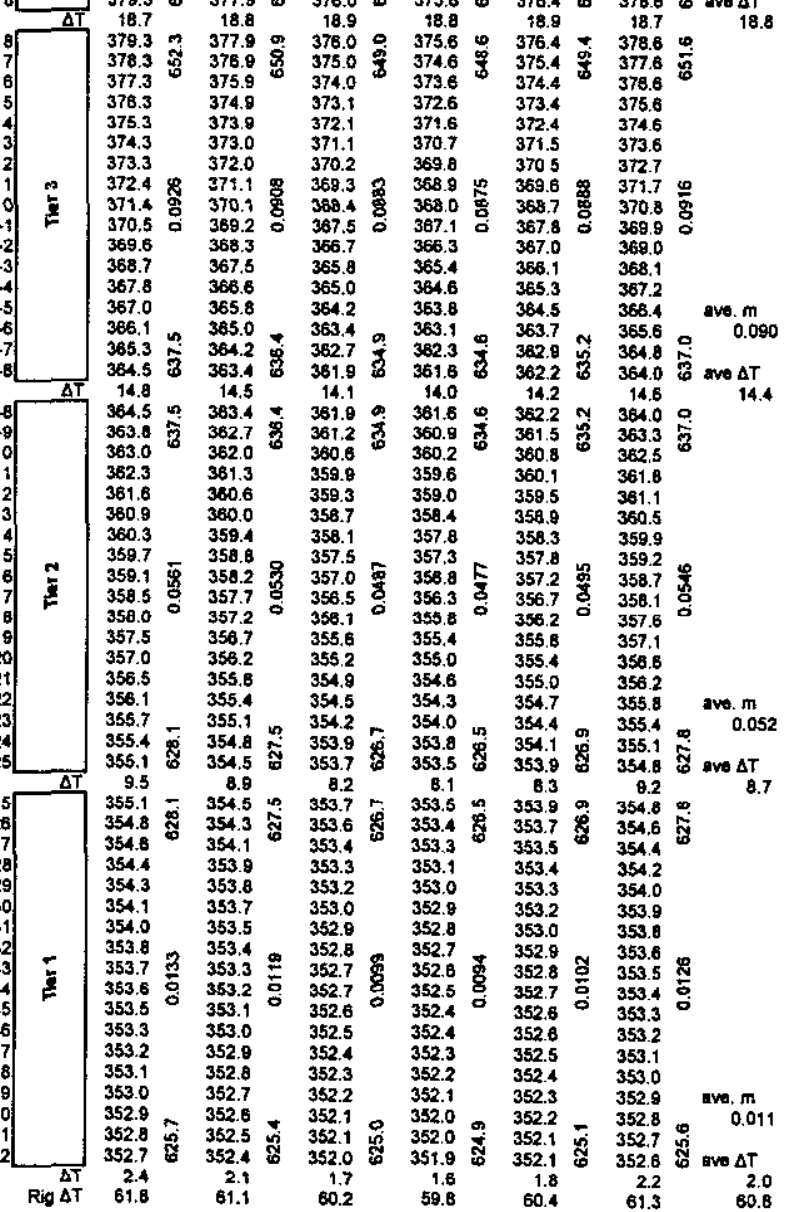

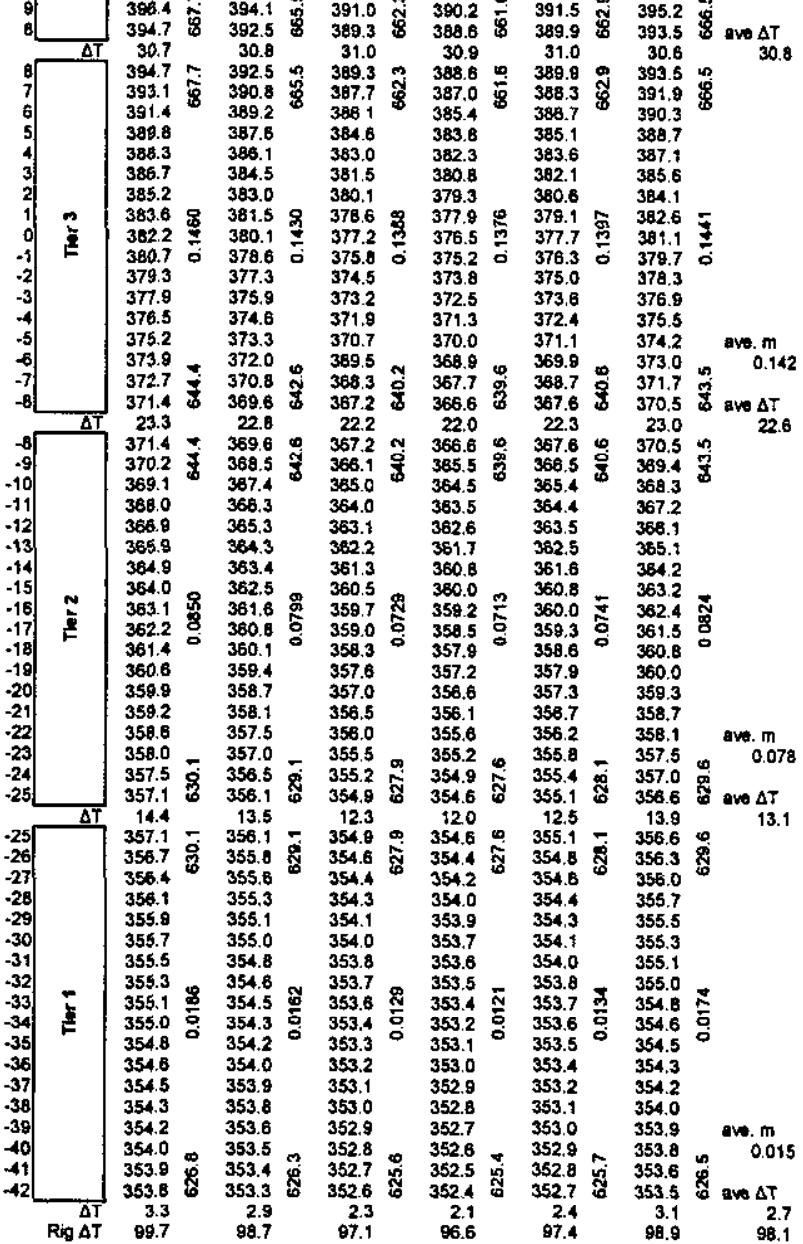

Figure III.B-5 Reactor sodium coolant temperatures used for boundary condition on the JOYO-1 capsules for both the nominal and worst case conditions. 
Volumetric heat generation rates were given in four iterations; revision 0 , revision 1 preliminary, revision 1 final, and revision 2. Detailed thermal calculation where performed for each heat rate evolution. Final capsule design adjustments, i.e. gas mixture and/or gap thickness based on the final revision 2 heat rate and sink temperatures, have not been specified.

The volumetric heat generation rates were applied to the model material regions. Determination of the input heat generation rates is described in Section III.A of this report. Section III.A also describes the axial heat generation rate variations as a function of capsule tier position. For the ANSYS thermal models, each capsule was divided into seven axial increments of approximately $24 \mathrm{~mm}$ each. This is judged to be adequate for describing and accounting for the axial heat generation variations.

\section{Material Properties}

A list of all of the materials which are being used in the JOYO irradiation test is presented in Table III.B- 1.

Table III.B- 1 List of materials to be used in the JOYO-1 test

\begin{tabular}{|c|c|c|}
\hline \multicolumn{3}{|c|}{ List of Materials } \\
\hline Material & Material Code & Application \\
\hline Stainless Steel 316 & SS & Capsule shell / internals \\
\hline FanSteel 85 & FS-85 & Specimen \\
\hline Astar $811 \mathrm{C}$ & ASTAR & Specimen \\
\hline Mo-47.5Re & Mo-47Re & Specimen \\
\hline Composite SiC & $\mathrm{cSiC}$ & Specimen \\
\hline Monolithic SiC & $\mathrm{mSiC}$ & Specimen \\
\hline Rhenium & $\operatorname{Re}$ & Specimen \\
\hline Tungsten & $W$ & Specimen/holder \\
\hline W-25Re & W-25Re & Specimen \\
\hline Beryllia & $\mathrm{BeO}$ & Specimen \\
\hline Unalloyed Moly & Mo & $\begin{array}{l}\text { Specimen holders } \\
\text { Capsule spacers }\end{array}$ \\
\hline POCO Graphite & POCO & Specimen holders \\
\hline Alloy 617 & $\mathrm{~A} 617$ & Specimen \\
\hline Nimonic PE-16 & PE16 & Specimen \\
\hline Haynes230 & $\mathrm{H} 230$ & Specimen \\
\hline SpringSteel & AMS5810 & Capsule wave spring \\
\hline
\end{tabular}

For the thermal analyeis being discussed herein, the only two material properties of consequence are thermal conductivity and radiative-emissivity.

It is noted that the JOYO-1 test objectives require specific specimen temperatures to be achieved for specific test specimen materials at indicated capsule positions. All of the capsules are designed such that the largest radial gas gap will be between the 
Enclosure (7) to

MDO-723-0044/

B-MT(SRME)-52

Page 70

specimen holder outside diameter and the capsule wall inside diameter. Essentially all of the heat generated by the specimen and specimen holders is transferred across this outermost gap. The specimen design temperatures were obtained by performing design iterations in which the outermost radial gap thickness was varied. Additionally, three separate gas compositions were available to select from to further adjust the predicted specimen temperatures. Table III.B- 2 lists the three gases and the thermal conductivity values that were used for these gases in the ANSYS problems. The properties for Argon are included for comparison even though no capsule will be filled with $100 \%$ Argon. The conductivities for the pure Argon and Helium are standard literature values. The gas mixture conductivities were calculated using accepted calculational techniques for gas mixtures.

Table III.B- 2 Capsule gas thermal conductivities for the gases used in the JOYO-1 capsules

\begin{tabular}{|c|c|c|c|c|}
\hline \multicolumn{5}{|c|}{ Capsule Gas Thermal Conductivities } \\
\hline $\operatorname{Temp.,~}_{\mathrm{K}}^{\circ}$ & $\begin{array}{c}\text { Helium } \\
\text { thermal } \\
\text { conductivity, } \\
\text { W/mm }{ }^{\circ} \mathrm{K}\end{array}$ & $\begin{array}{l}\text { Argon thermal } \\
\text { conductivity, } \\
\text { W/mm } \mathrm{m}^{\circ} \mathrm{K}\end{array}$ & $\begin{array}{c}80 / 20 \text { v/o } \\
\text { Argon / Helium } \\
\text { thermal } \\
\text { conductivity, } \\
\text { W/mm }{ }^{\circ} \mathrm{K}\end{array}$ & $\begin{array}{l}30 / 70 \text { v/o } \\
\text { Argon / Helium } \\
\text { thermal } \\
\text { conductivity, } \\
\text { W/mm }{ }^{\circ} \mathrm{K}\end{array}$ \\
\hline 300 & $.1505 \times 10^{-3}$ & $.1776 \times 10^{-4}$ & $.2865 \times 10^{-4}$ & $.7828 \times 10^{-4}$ \\
\hline 600 & $.2470 \times 10^{-3}$ & $.3019 \times 10^{-4}$ & $.4798 \times 10^{-4}$ & $.1290 \times 10^{-3}$ \\
\hline 1000 & $.3633 \times 10^{-3}$ & $.4288 \times 10^{-4}$ & $.6919 \times 10^{-4}$ & $.1890 \times 10^{-3}$ \\
\hline 1200 & $.4152 \times 10^{-3}$ & $.4830 \times 10^{4}$ & $.7842 \times 10^{-4}$ & $.2156 \times 10^{-3}$ \\
\hline 1400 & $.4691 \times 10^{-3}$ & $.5372 \times 10^{-4}$ & $.8783 \times 10^{-4}$ & $.2432 \times 10^{-3}$ \\
\hline 1600 & $.5210 \times 10^{-3}$ & $.5893 \times 10^{-4}$ & $.9688 \times 10^{-4}$ & $.2697 \times 10^{-3}$ \\
\hline
\end{tabular}

The emissivity and thermal conductivity values used in the analysis for capsule and specimen materials are given in reference III.B-1, "Material Properties and Specimen Dimensions for Capsule Design to Support the JOYO-1 Irradiation Test, Revesion 0".

As mentioned previously, the temperature drop across the specimen holder outside diameter and the capsule wall inside diameter is significant. For all of the capsules, the specimen holders are fabricated from unalloyed Moly except for two designs in which they are fabricated from Tungsten. In both instances, significant radiative heat transfer occurs between the holder outside diameter surface and the capsule wall inside diameter surface. All capsules are fabricated from Type $316 \mathrm{H}$ or 316 Stainless Steel as discussed in Sections II.B and III.C, hereafter referred to 316 SS. Use of the ANSYS LINK31 element as previously discussed in this report requires the input of a single emissivity value for each link. In order to accurately specify the link emissivity value, a coupled emissivity between the holder outside diameter surface and the capsule wall inside diameter surface had to be calculated. The coupled emissivity was calculated using the following equation for cylindrical geometry. 
Enclosure (7) to

MDO-723-0044/

B-MT(SRME)-52

Page 71

$$
\begin{aligned}
& \varepsilon=\frac{1}{\left[\frac{1}{\varepsilon_{i}}+\frac{1-\varepsilon_{o}}{\varepsilon_{o}}\left(\frac{r_{i}}{r_{o}}\right)\right]} \\
& \varepsilon_{i}=\text { emissivity of specimen holder material } \\
& \varepsilon_{o}=\text { emissivity of } 316 \mathrm{ss} \\
& r_{i}=\text { outer radius of specimen holder } \\
& r_{o}=\text { inside radius of capsule wall }
\end{aligned}
$$

\begin{tabular}{|c|c|c|c|c|c|c|c|c|}
\hline \multicolumn{3}{|c|}{ Molybdenum } & \multicolumn{3}{|c|}{ Tungsten } & \multicolumn{3}{|c|}{ 316H Stainless Steel } \\
\hline$T(K)$ & emis & $k(\mathrm{~W} / \mathrm{mmK})$ & $T(K)$ & emis & $\mathrm{k}(\mathrm{W} / \mathrm{mmK})$ & $T(K)$ & emis & $\mathrm{k}(\mathrm{W} / \mathrm{mmK})$ \\
\hline 600 & 0.11018 & 0.128456 & 600 & 0.067364 & 0.142628 & 600 & 0.11152 & 0.018322 \\
\hline 800 & $0.136 \overline{32}$ & $0.1 \overline{6024}$ & 800 & 0.099436 & 0.130672 & $\overline{800}$ & 0.15064 & 0.021328 \\
\hline 1000 & 0.1633 & 0.1218 & 1000 & $0.12 \overline{97}$ & $0.1 \overline{2} 13$ & 1000 & 0.304 & 0.02409 \\
\hline 1200 & $\overline{0.19112}$ & 0.115784 & $\overline{1200}$ & $0.15 \overline{8156}$ & $0 . \overline{114512}$ & 1200 & & $0.0 \overline{26608}$ \\
\hline$\overline{1400}$ & 0.21978 & 0.107976 & $\overline{1400}$ & $\overline{0} . \overline{84} \overline{804}$ & 0.110308 & $\overline{1400}$ & & $\overline{0.02888 \overline{2}}$ \\
\hline$\overline{1600}$ & $0 \overline{24928}$ & 0.098376 & 1600 & 0.209644 & 0.108688 & 1600 & & 0.030912 \\
\hline
\end{tabular}

Table III.B- 3 lists the emissivities and thermal conductivities which were used for the three materials of concern.

Table III.B- 3 Material Properties for JOYO-1 capsule pressure boundany and specimen holders.

\section{Results}

Thermal analysis results depicting specimen temperatures are provided for nominal operating conditions. The result for the worst case capsule under the limiting condition is also provided.

\section{a. Nominal}

To represent the matrix of all sixty capsules two charts are given (one for each rig) in Figure III.B- 6 and Figure III.B- 7. These charts illustrate each capsule type by location and provide specimen temperature results as well as capsule gas and gap information. Figure III.B- 8 through Figure III.B- 17 show the finite element temperature plots of the specimens in the capsule. One figure for each of the ten capsule types is given. Positions of these capsules were chosen so that one capsule from each tier for both rig $A$ and rig $B$ is represented, as well as one capsule from each compartment. 
Enclosure (7) to

MDO-723-0044/

B-MT(SRME)-52

Page 72

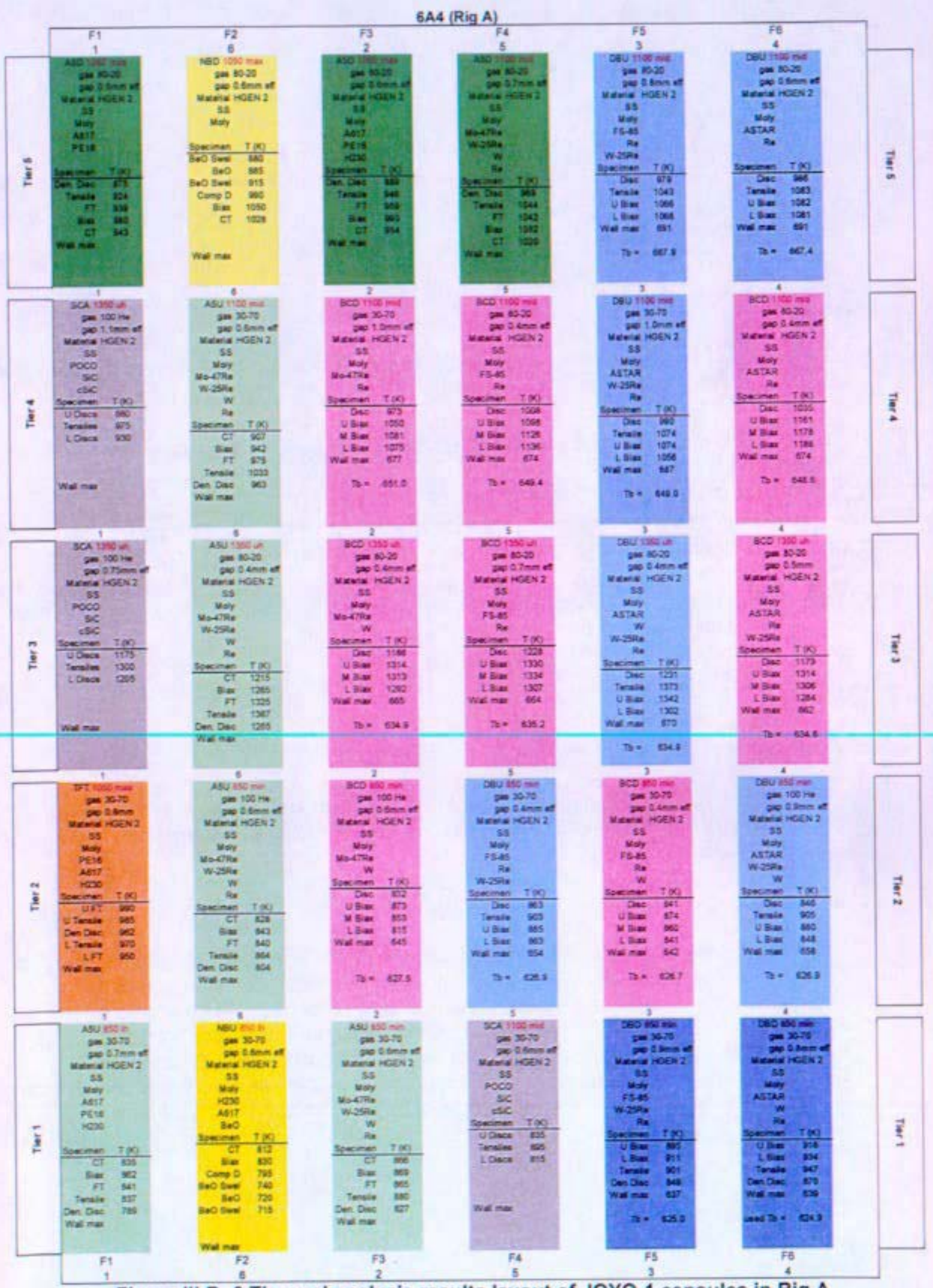

Figure III.B-6 Thermal analysis results layout of JOYO-1 capsules in Rig A 
Enclosure (7) to

MDO-723-0044/

B-MT(SRME)-52

Page 73

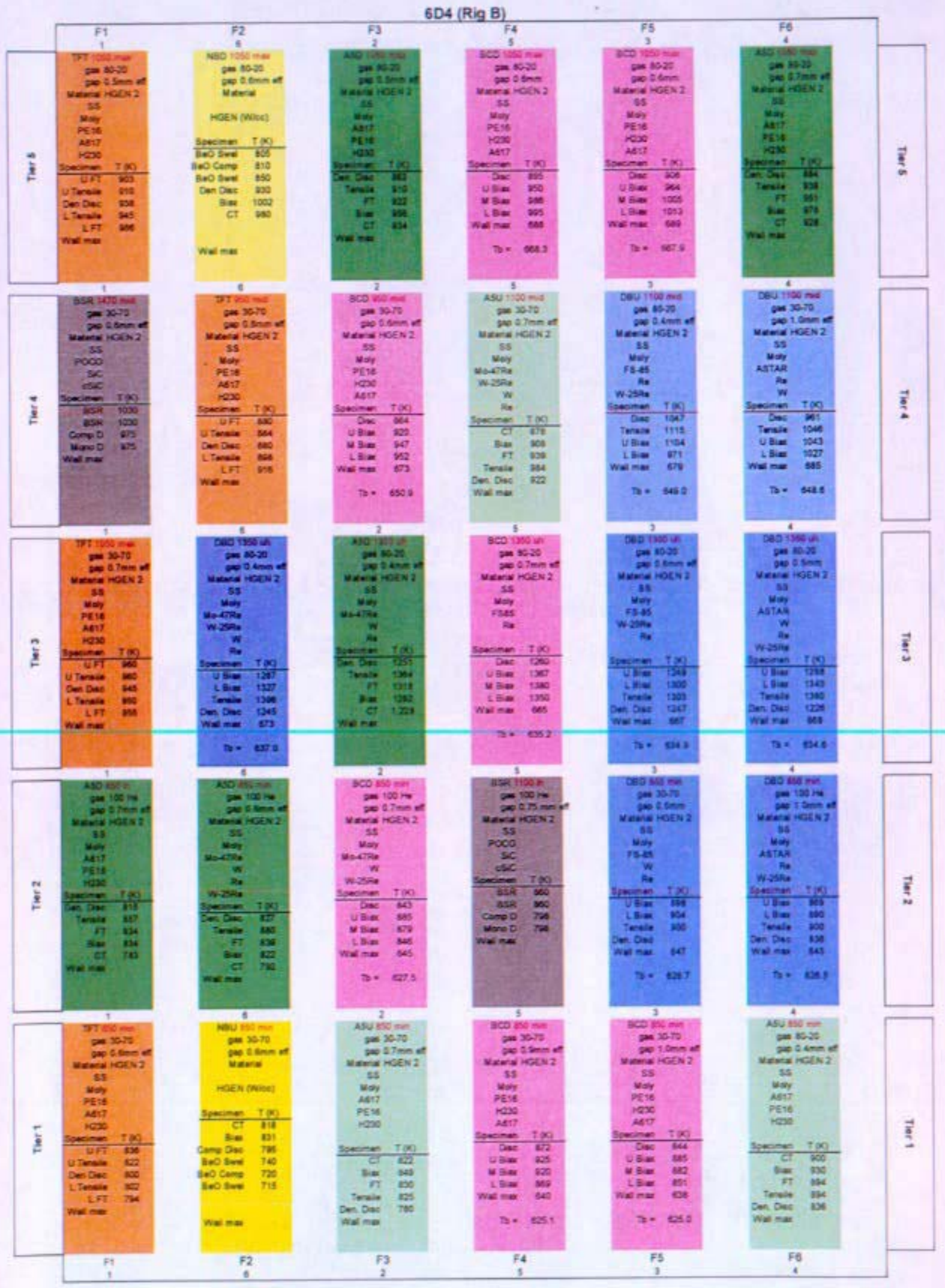

Figure III.B- 7 Thermal analysis results layout of JOYO-1 capsules in Rig B 
Enclosure (7) to

MDO-723-0044/

B-MT(SRME)-52

Page 74

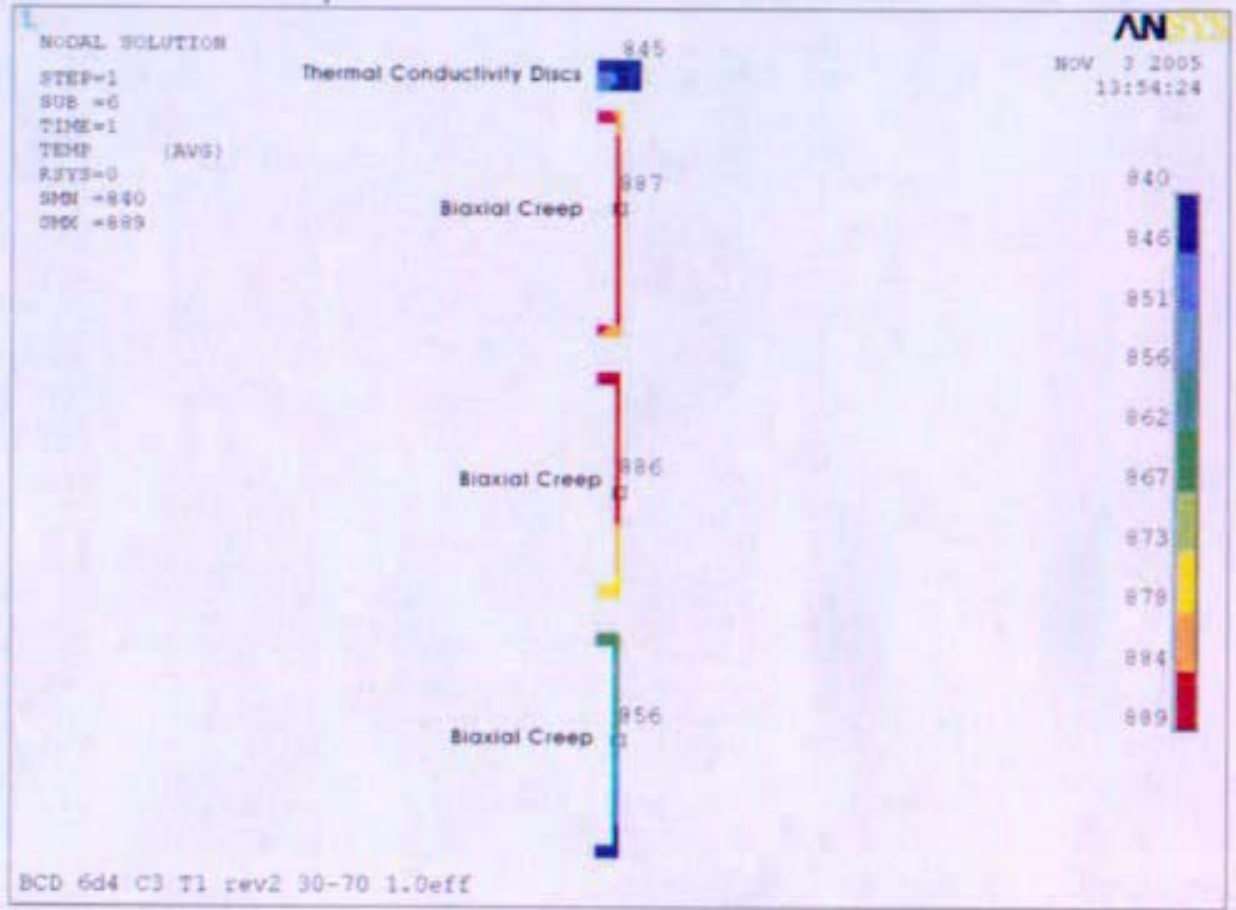

Figure III.B- 8 Specimen temperature profile of a BCD capsule located in rig B, tier 1 , compartment 3 under nominal conditions.

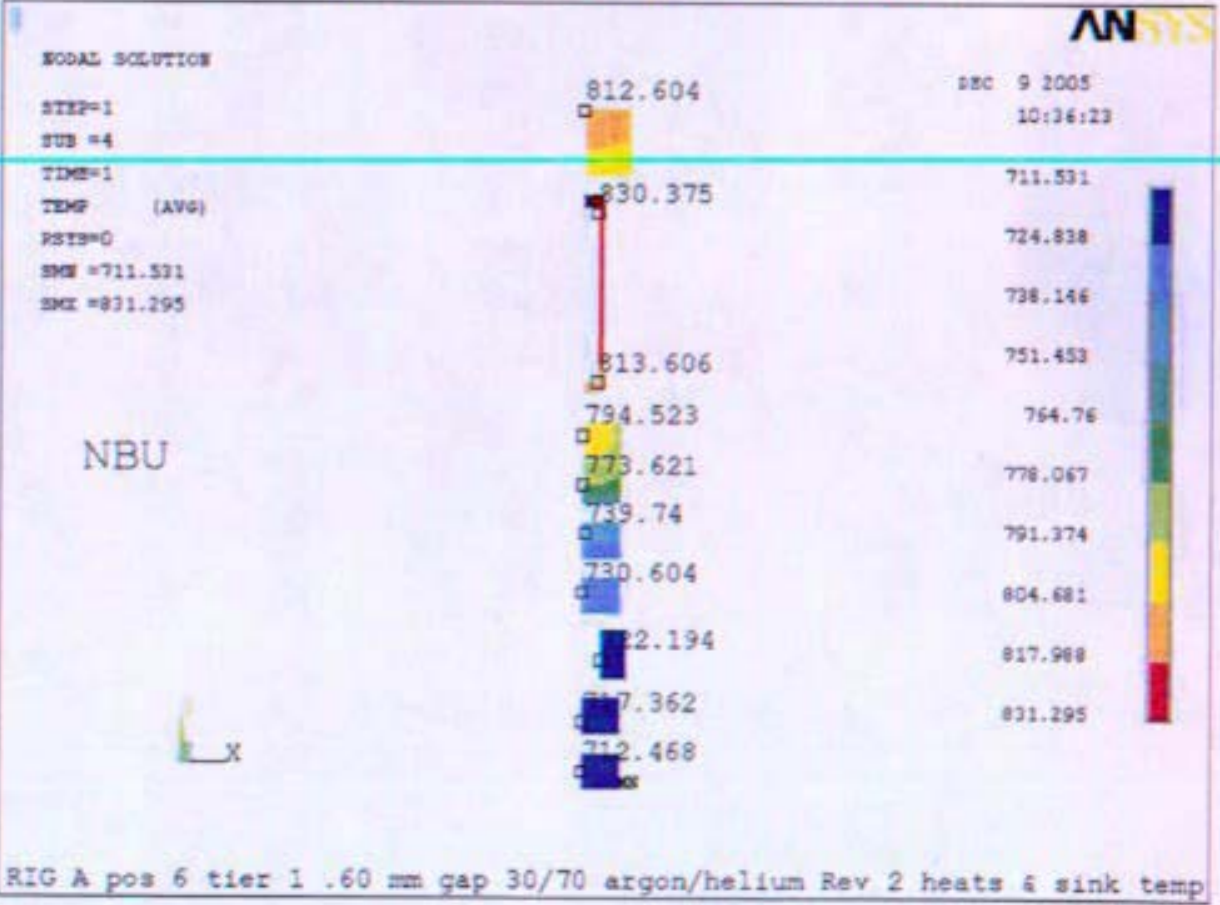

Figure III.B- 9 Specimen temperature profile of a NBU capsule located in rig A, tier 1, compartment 6 under nominal conditions. 


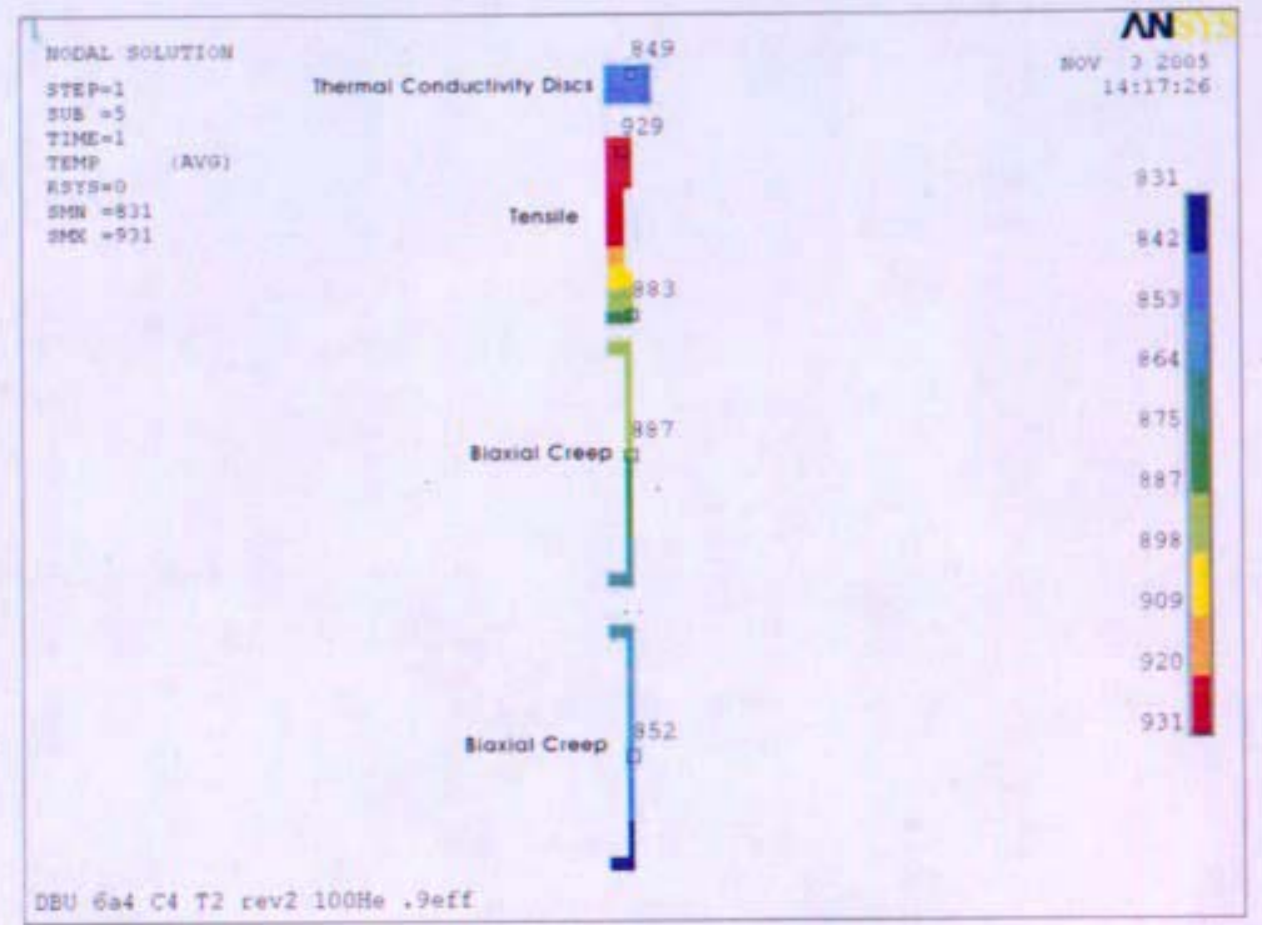

Figure III.B- 10 Specimen temperature profile of a DBU capsule located in rig A, tier 2, compartment 4 under nominal conditions.

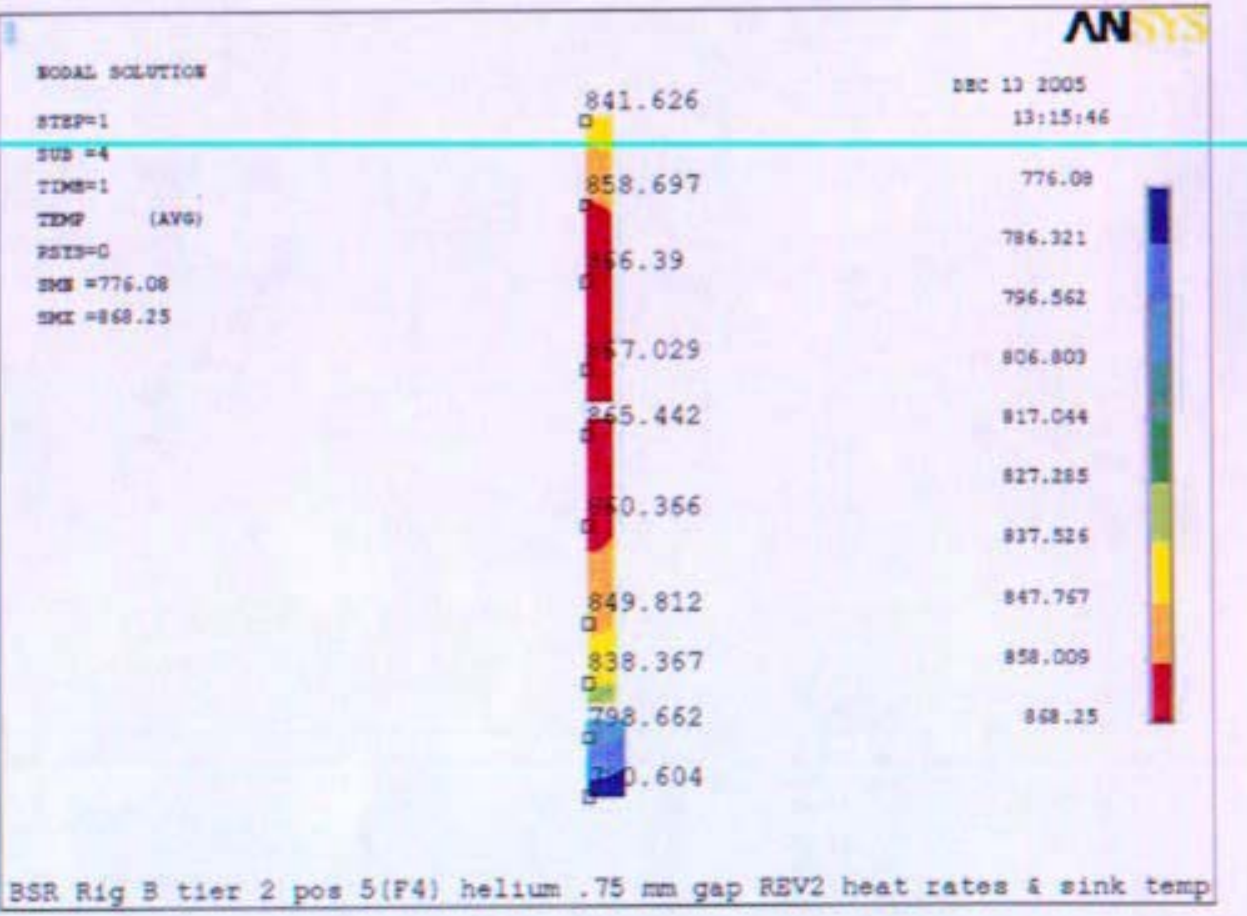

Figure III.B-11 Specimen temperature profile of a BSR capsule located in rig B, tier 2, compartment 5 under nominal conditions. 
Enclosure (7) to

MDO-723-0044/

B-MT(SRME)-52

Page 76

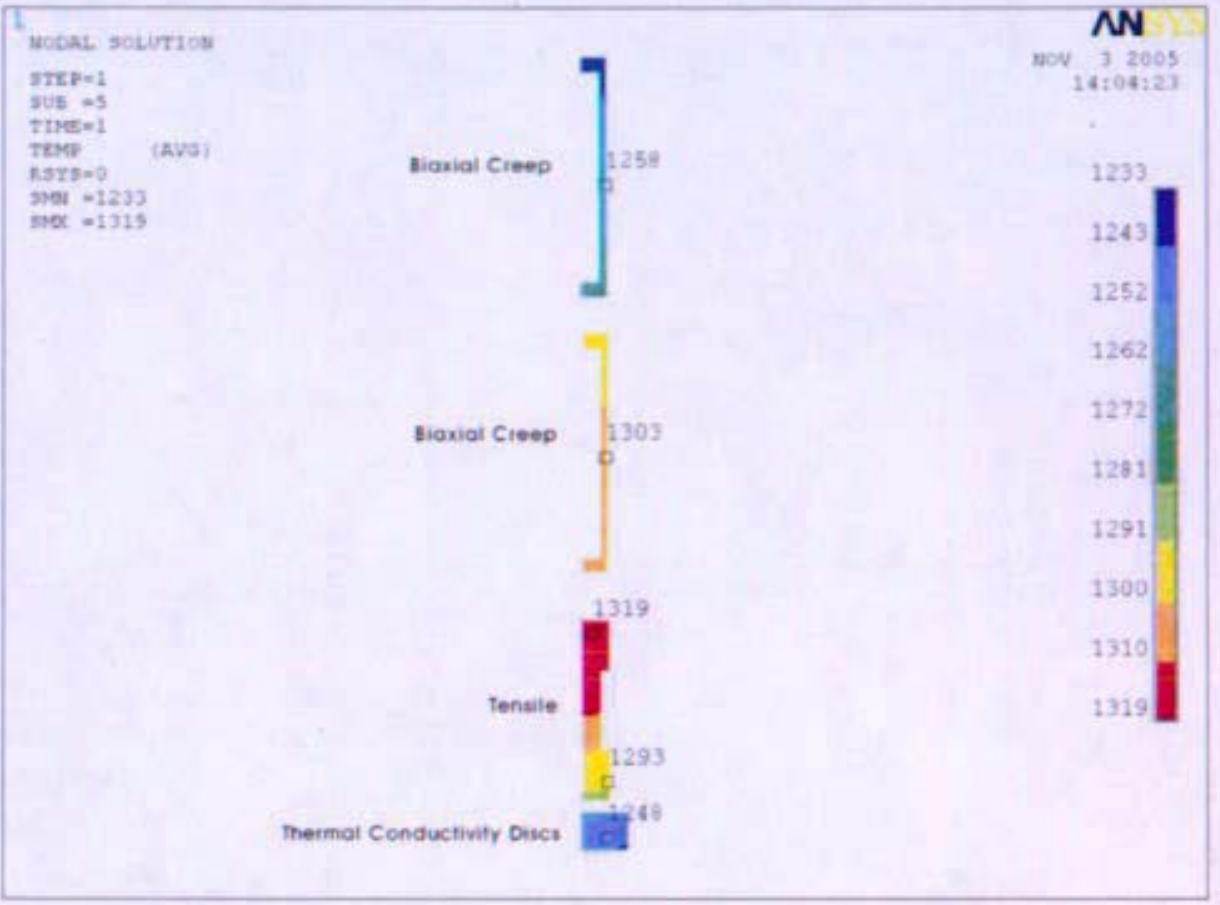

Figure III.B- 12 Specimen temperature profile of a DBD capsule located in rig B, tier 3 , compartment 3 under nominal conditions.

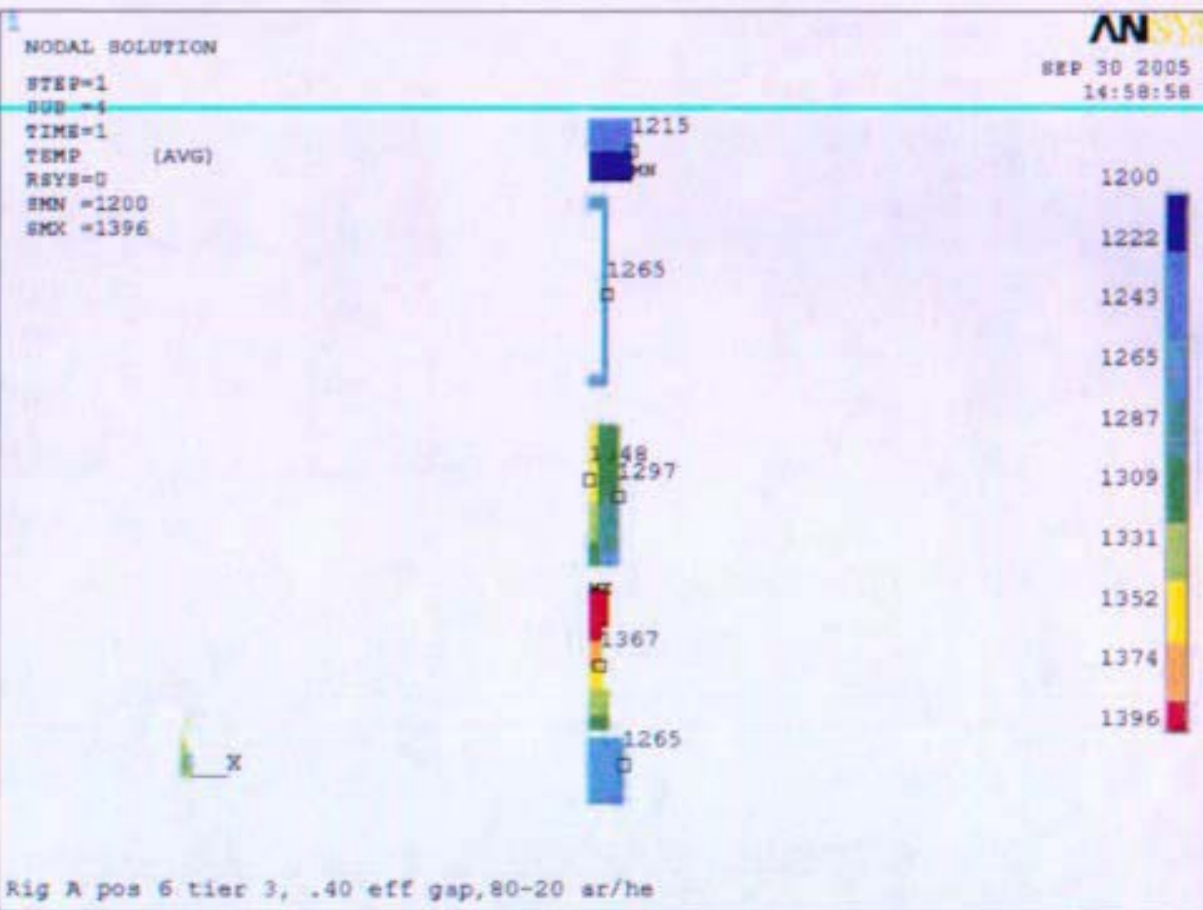

Figure III.B- 13 Specimen temperature profile of a A5U capsule located in rig A, tier 3 , compartment 6 under nominal conditions. 
Enclosure (7) to

MDO-723-0044/

B-MT(SRME)-52

Page 77

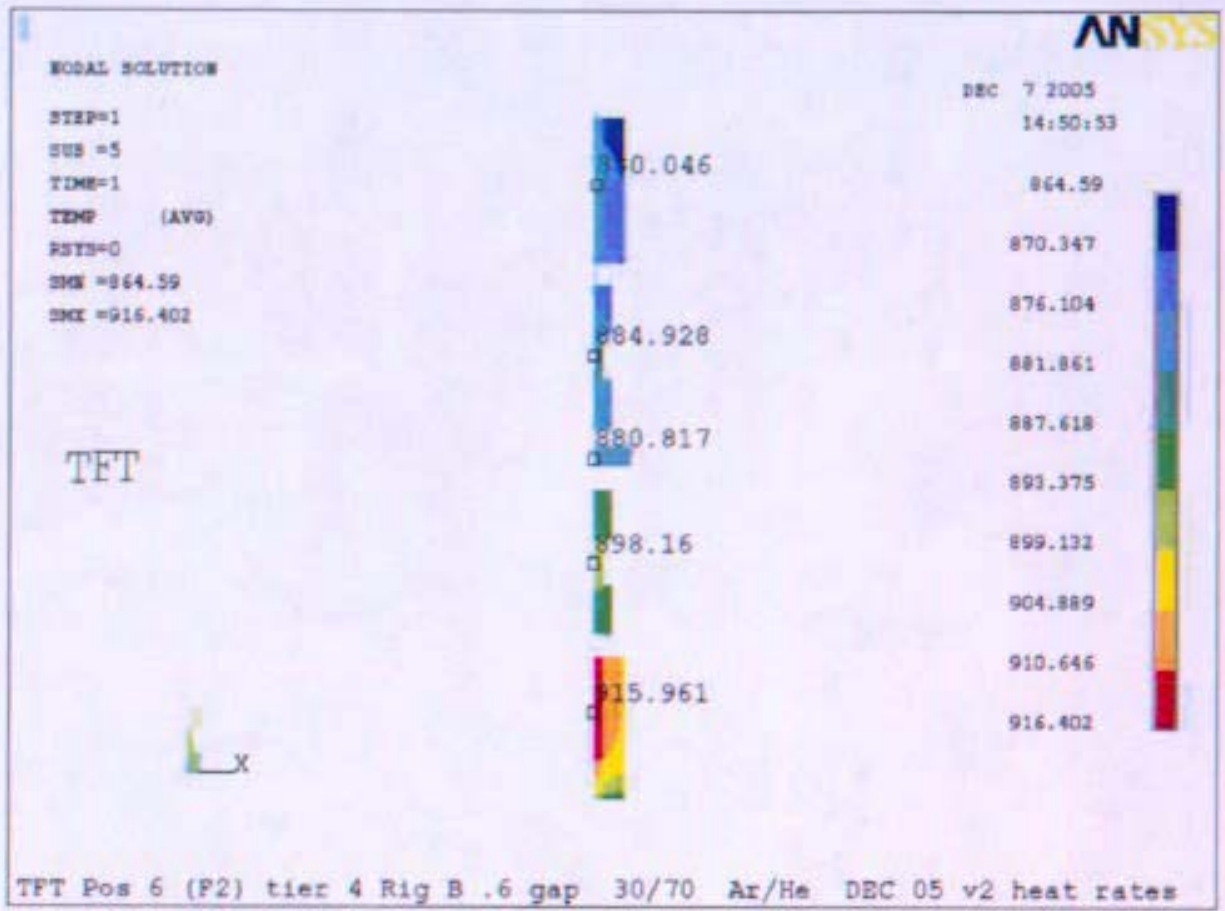

Figure III.B-14 Specimen temperature profile of a TFT capsule located in rig B, tier 4, compartment 6 under nominal conditions.

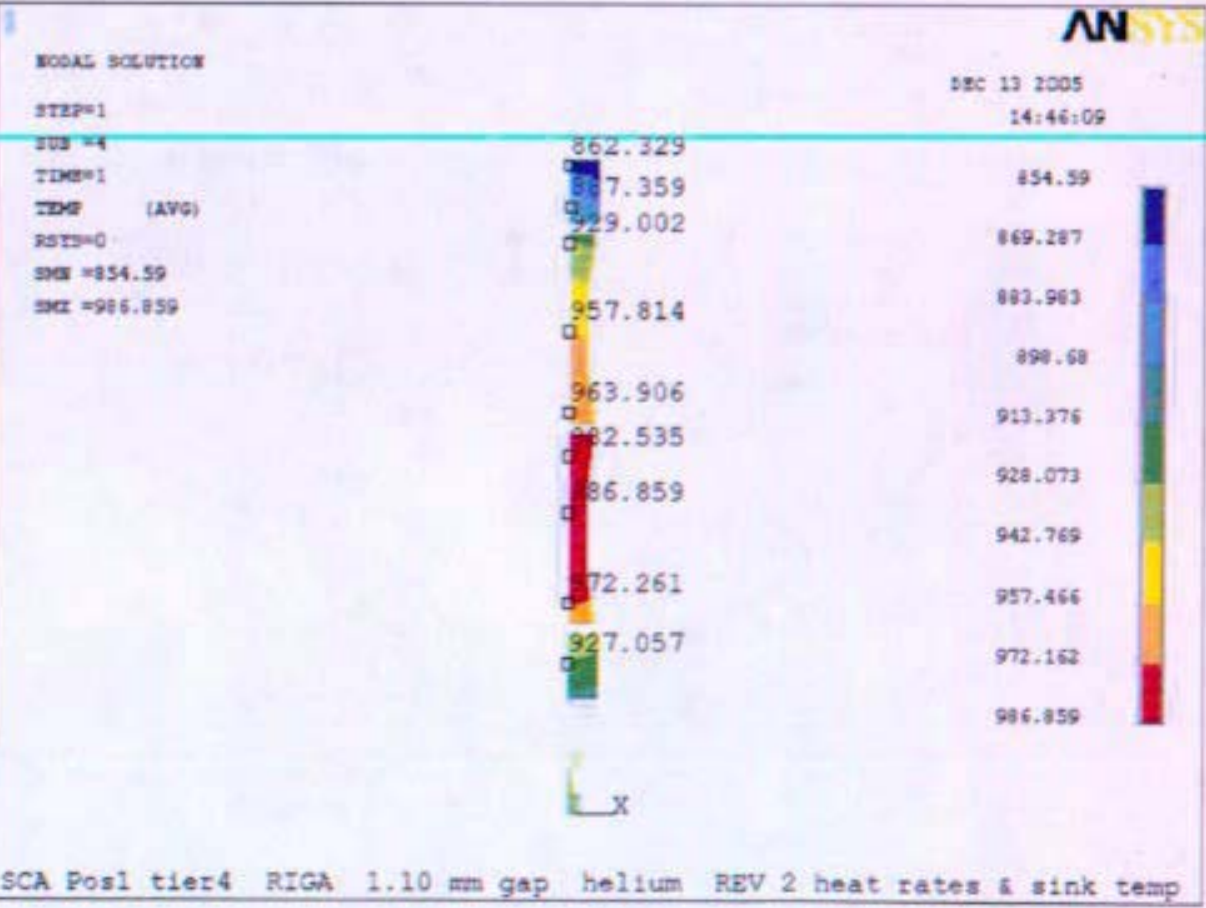

Figure III.B-15 Specimen temperature profile of a SCA capsule located in rig A, tier 4, compartment 1 under nominal conditions. 
Enclosure (7) to

MDO-723-0044/

B-MT(SRME)-52

Page 78

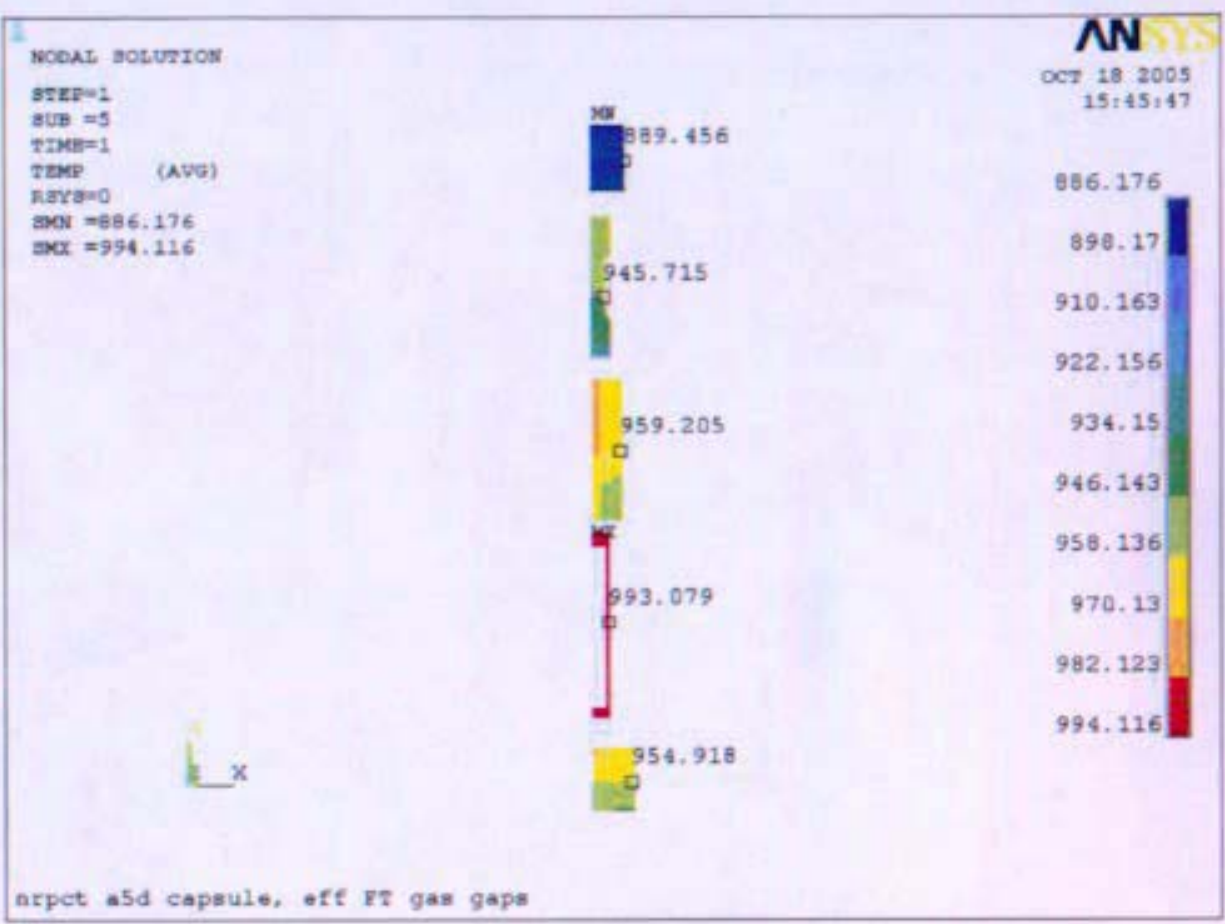

Figure III.B- 16 Specimen temperature profile of an A5D capsule located in rig A, tier 5 , compartment 2 under nominal conditions.

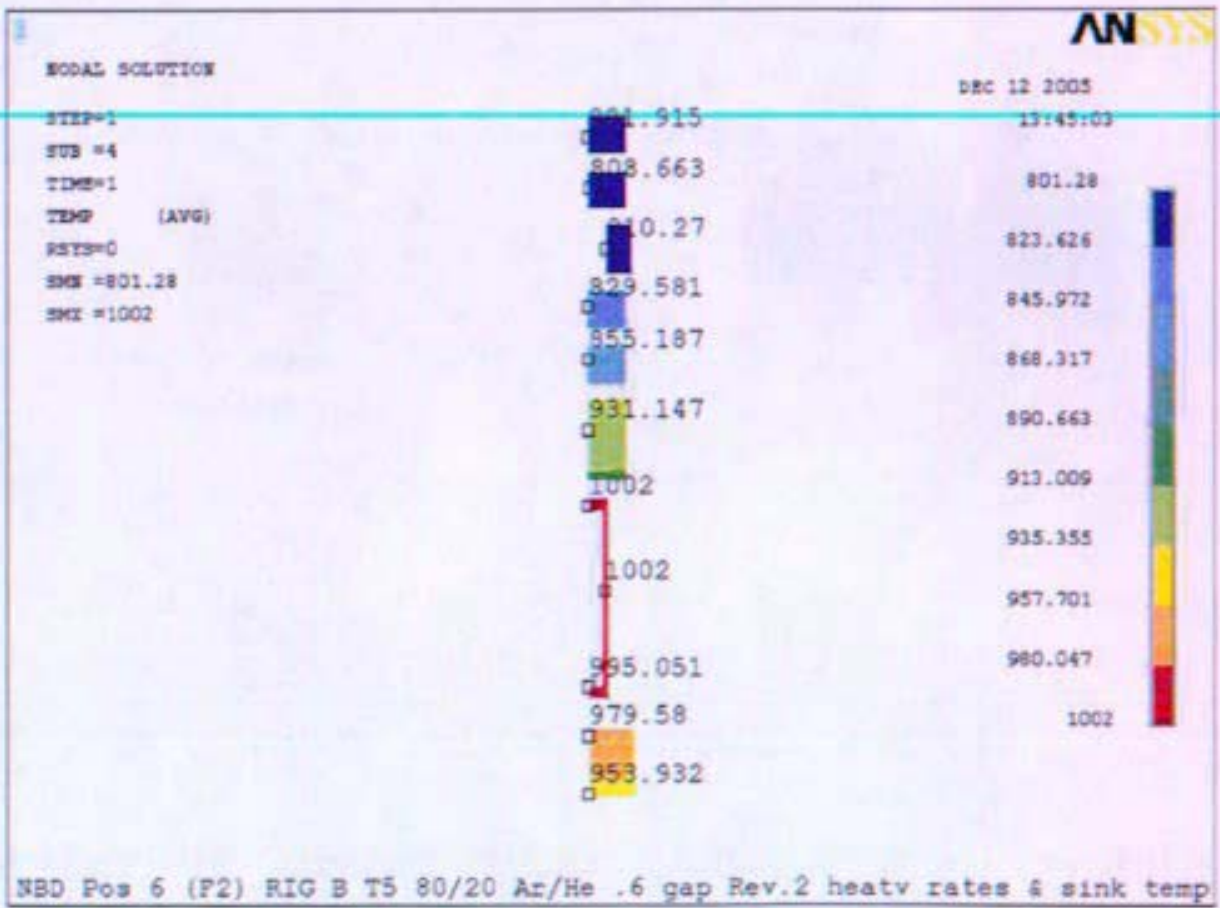

Figure III.B-17 Specimen temperature profile of a NBD capsule located in rig B, tier 5 , compartment 6 under nominal conditions. 


\section{b. Worst Case}

A worst case analysis was done on the most limiting capsules for both a maximum temperature and a maximum pressure case. The limiting condition includes a combination of loss of flow (1.25), a hot spot factor (1.05), and an overpower condition (1.25). This condition was applied to the model by increasing the heat generation rate by a factor of 1.64 and applying the boundary conditions given in the right hand column of Figure III.B-5.

The capsule subjected to the greatest pressure is the BCD capsule closest to the center of the core in tier 3. Figure III.B-18 and Figure III.B- 19 show thermal profiles of the capsule assembly and pressure boundary, respectively. The thermal results from this limiting pressure case capsule were used for subsequent structural analysis.

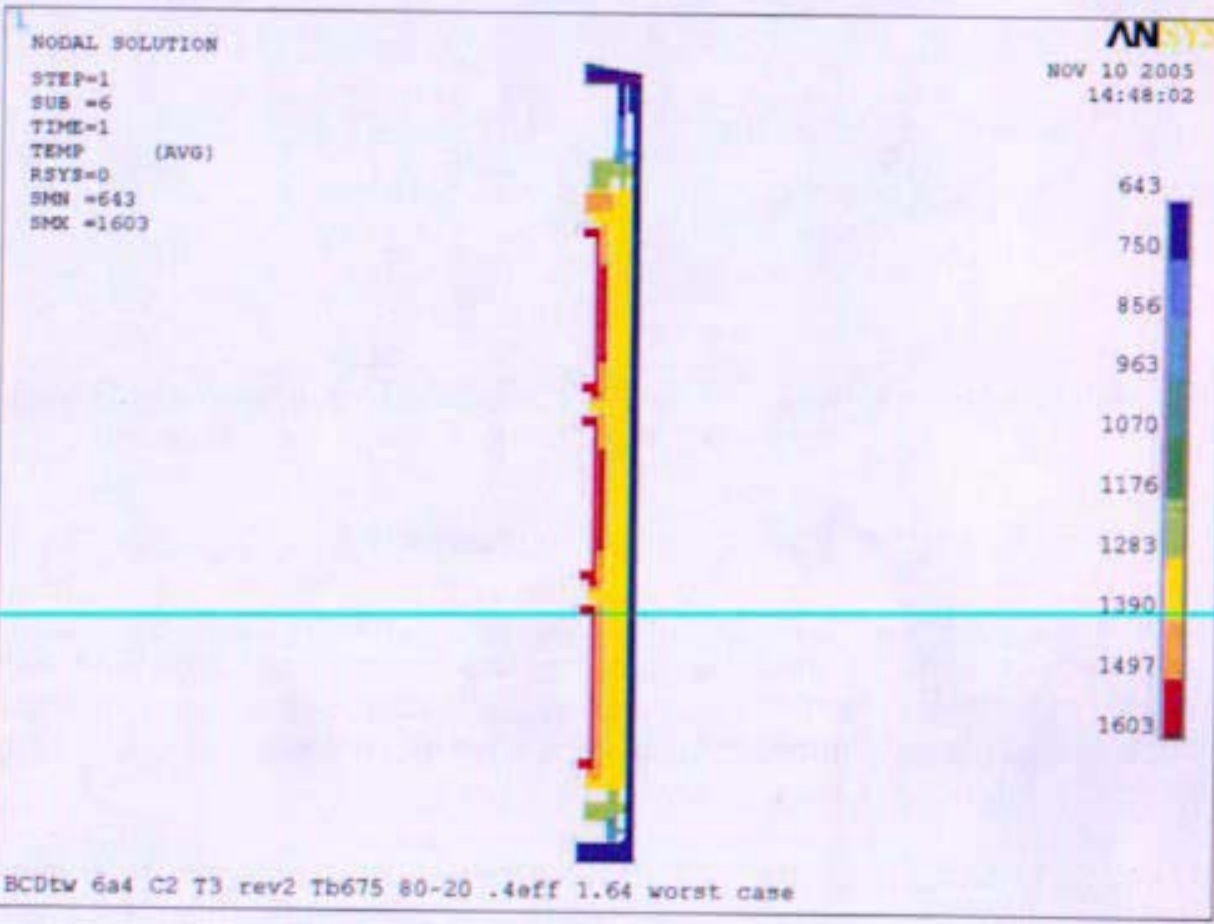

Figure III.B- 18 Capsule assembly temperature profile of the limiting capsule (BCD capsule located in rig A, tier 3 , compartment 2 ). 
Enclosure (7) to

MDO-723-0044/

B-MT(SRME)-52

Page 80

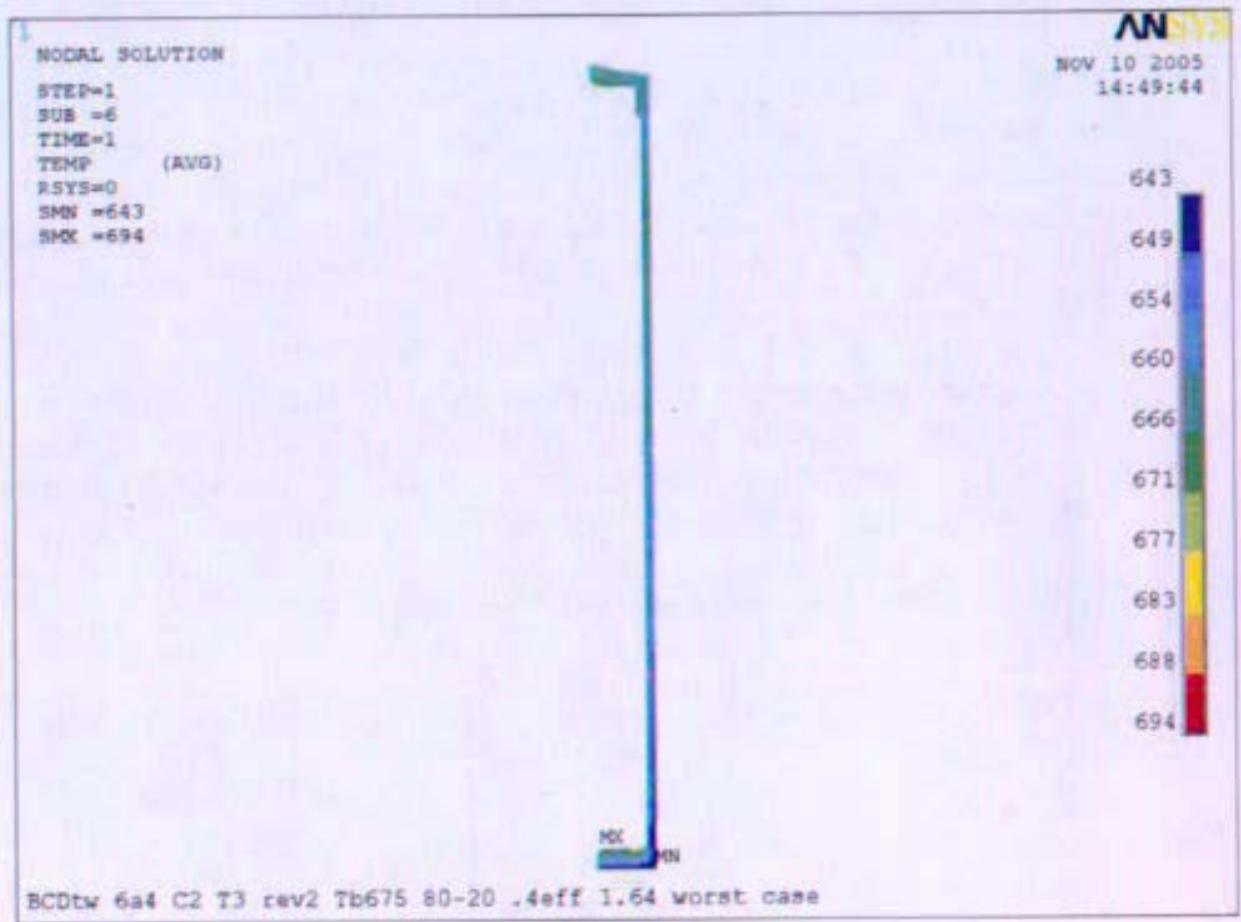

Figure III.B- 19 Pressure boundary temperature profile of the limiting capsule (BCD capsule located in rig A, tier 3, compartment 2).

\section{Revision Effects on Specimen Temperatures}

The design of the JOYO-1 capsules was very much an iterative process. The design affected the loading conditions, and the loading conditions affected the design. With that in mind every effort should be taken to finalize loading conditions before a final gas type and gap size is decided upon. This will ensure a capsule design capable of producing the desired specimen temperatures.

The JOYO-1 capsules gas type and gap size was based on thermal analysis results from revision 1 gamma heating and boundary conditions. Unforeseen conditions were present in the revision 2 analysis that had adverse effects on specimen temperature predictions. Notable changes from the previous revision 1 calculations are described in section III.A. Also, a more detailed analysis of the sodium temperature surrounding the capsules was provided by JNC in revision 2 . This change from revision 1 also reduced the predicted specimen temperatures.

In order to document how the multiple revisions of gamma heating and boundary conditions affected the temperature profile of the JOYO-1 capsules, comparative data between revision 1 and revision 2 is provided in Table III.B- 4 . The first column of the table shows the maximum temperature change $\left({ }^{\circ} \mathrm{K}\right)$ between revision 1 final and revision 2 for, the average specimen temperature, maximum wall temperature, and maximum bulk temperature. The second column gives the average temperature change $\left({ }^{\circ} \mathrm{K}\right)$ between revision 1 final and revision 2 for; the average specimen temperature, maximum wall temperature, and maximum bulk temperature. The data 
was averaged across the six compartments for each tier and is shown for Rig A, Rig B, and the average of both rigs together.

Table III.B- 4 Temperature changes incurred in the JOYO-1 capsules between revision 1 and revision 2.

\begin{tabular}{|c|c|c|c|c|c|c|c|c|c|c|}
\hline$\Delta T(K)$ & $\begin{array}{l}\text { 6D4 } \\
\text { REV2 } \\
\max \\
\end{array}$ & $\begin{array}{l}\text { av1 } \\
\text { ave }\end{array}$ & & $\Delta T(K)$ & $\begin{aligned} & 6 \mathrm{~A} 4 \\
&= \mathrm{REV} 2 \\
& \max \\
&\end{aligned}$ & ave & & $\begin{array}{r}B \\
\Delta T(K)\end{array}$ & $\begin{array}{l}\text { th } \mathrm{Ri} \\
\mathrm{REV} 2 \\
\max \\
\end{array}$ & $\begin{array}{l}\text { V1 } \\
\text { ave }\end{array}$ \\
\hline specimen & -84 & -62 & $n$ & specimen & -89 & -51 & $\Rightarrow$ & specimen & -89 & -57 \\
\hline wall & -73 & -48 & $E$ & wall & -71 & -47 & $\vec{G}$ & wall & -73 & -48 \\
\hline tbulk & -106 & -96 & & tbulk & -111 & -97 & & tbulk & -111 & -97 \\
\hline & $\max$ & ave & & & $\max$ & ave & & & $\max$ & ave \\
\hline specimen & -410 & -90 & $\forall$ & specimen & -344 & -80 & $\Rightarrow$ & specimen & -410 & -85 \\
\hline wall & -48 & -41 & $F$ & wall & -49 & -43 & $A$ & wall & -49 & -42 \\
\hline tbulk & -84 & -66 & & tbulk & -81 & -61 & & tbulk & -84 & -64 \\
\hline & $\max$ & ave & & & $\max$ & ave & & & $\max$ & ave \\
\hline specimen & -111 & -54 & m & specimen & -103 & -45 & $\Rightarrow 1$ & specimen & -111 & -49 \\
\hline wall & -37 & -25 & $E$ & wall & -38 & -35 & $\omega$ & wall & -38 & -30 \\
\hline tbulk & -63 & -47 & & tbulk & 61 & -47 & & tbulk & -63 & -47 \\
\hline & $\max$ & ave & & & $\max$ & ave & & & $\max$ & ave \\
\hline specimen & -255 & -54 & N & specimen & -56 & -31 & $\overrightarrow{-1}$ & specimen & -255 & -42 \\
\hline wall & -23 & -17 & $E$ & wall & -22 & -19 & $N$ & wall & -23 & -18 \\
\hline tbulk & -44 & -33 & & tbulk & -43 & -30 & & tbulk & -44 & -31 \\
\hline & $\max$ & ave & & & $\max$ & ave & & & $\max$ & ave \\
\hline specimen & -33 & -12 & $=$ & specimen & -228 & -39 & $\frac{-1}{6}$ & specimen & -228 & -25 \\
\hline wall & -1 & 1 & 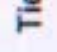 & wall & -54 & -27 & $\rightarrow$ & wall & .54 & -13 \\
\hline tbuik & -19 & -12 & & tbulk & -19 & -12 & & tbulk & -19 & -12 \\
\hline REV 1: rew & Inal ga & $\begin{array}{l}\text { ulk }=c \\
\text { heat }\end{array}$ & $c e$ & $\begin{array}{l}\text { in the av } \\
\text { in the max } \\
\text { average } s \\
d \text { bulk tem }\end{array}$ & $\begin{array}{l}\text { age } T \\
\text { num } \\
\text { lium } b\end{array}$ & $\begin{array}{l}\text { every } \\
\text { the p } \\
\text { k) at t } \\
\text { REV }\end{array}$ & $\operatorname{nen} t$ & $\begin{array}{l}\text { each tier } \\
\text { Jary } \\
\text { ch capsule } \\
\text { a heat rates. }\end{array}$ & 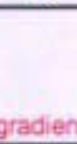 & \\
\hline
\end{tabular}


Enclosure (7) to

MDO-723-0044/

B-MT(SRME)-52

Page 82

Figure III.B- 20 through Figure III.B- 28 illustrate specimen temperatures with respect to their target temperatures. Results are given for each capsule by compartment and tier location. Specimen temperatures are shown by the specimen's corresponding elevation within the capsule.

The charts show that generally the predicted temperatures are below the target temperature as quantified in Table III.B- 4, this due to changes between revision 1 and revision 2. This is especially true for the BSA and SCA capsules where large selfshielding effects of the tungsten holders had not been anticipated.

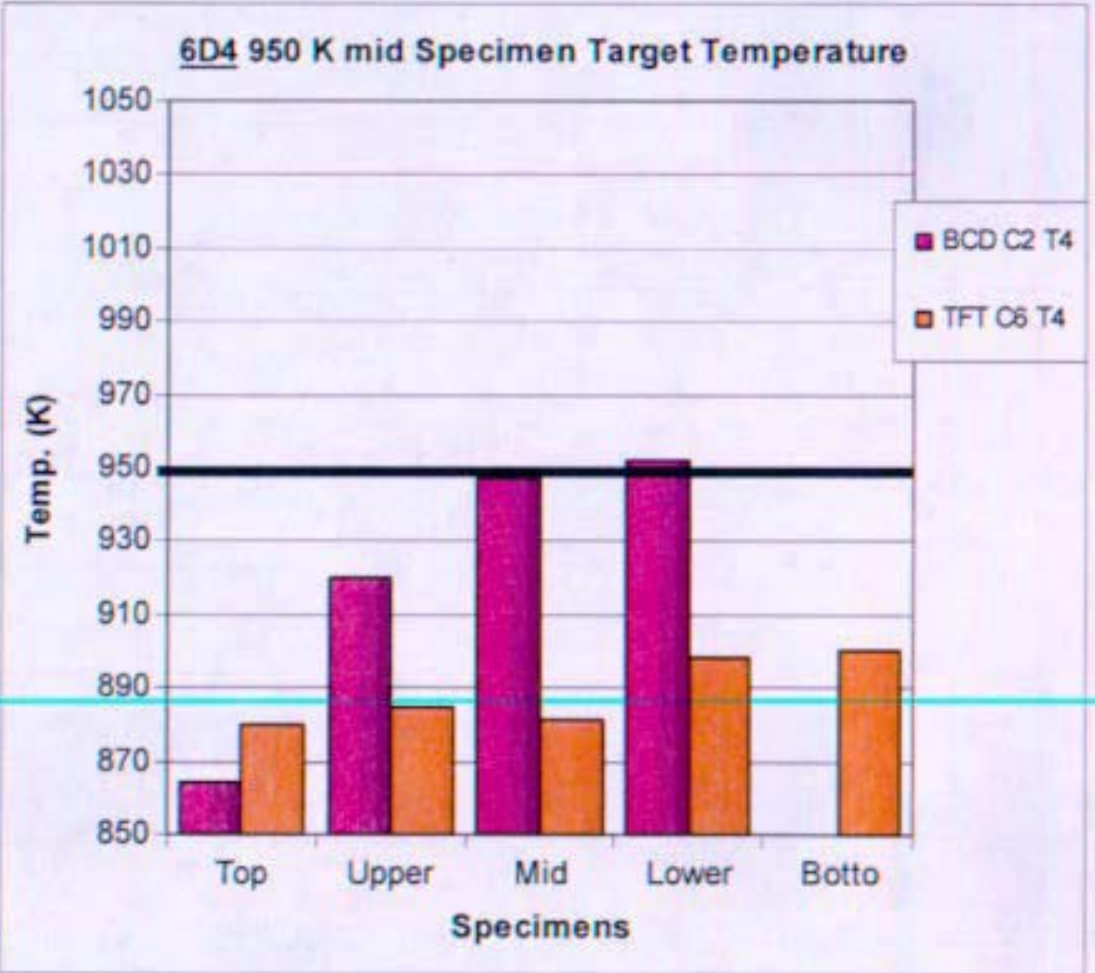

Figure III.B- 20 Specimen temperatures in Rig A with a target temperature of $950 \mathrm{~K}$ 


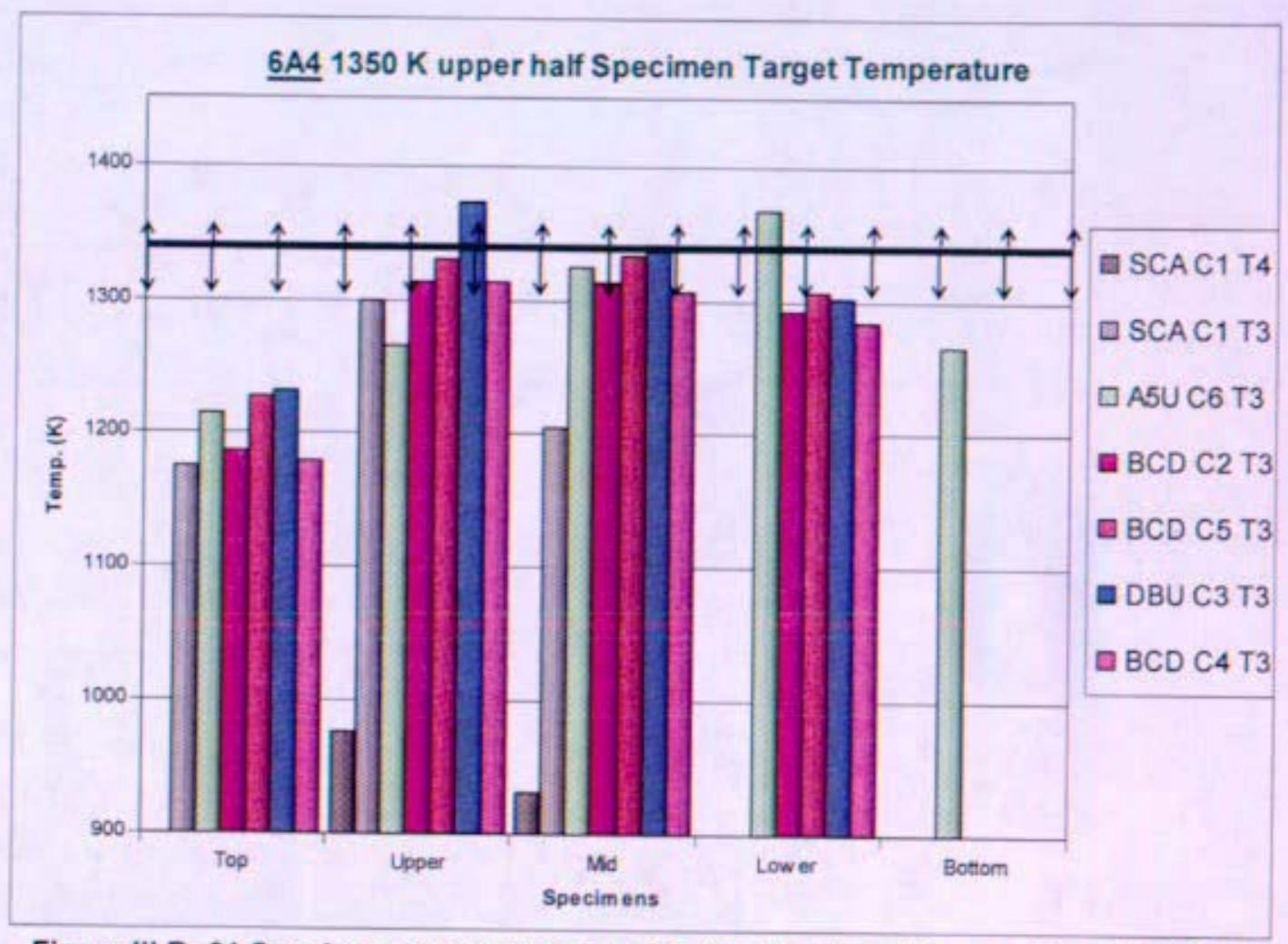

Figure III.B- 21 Specimen temperatures in Rig A with a target temperature of $1350 \mathrm{~K}$.

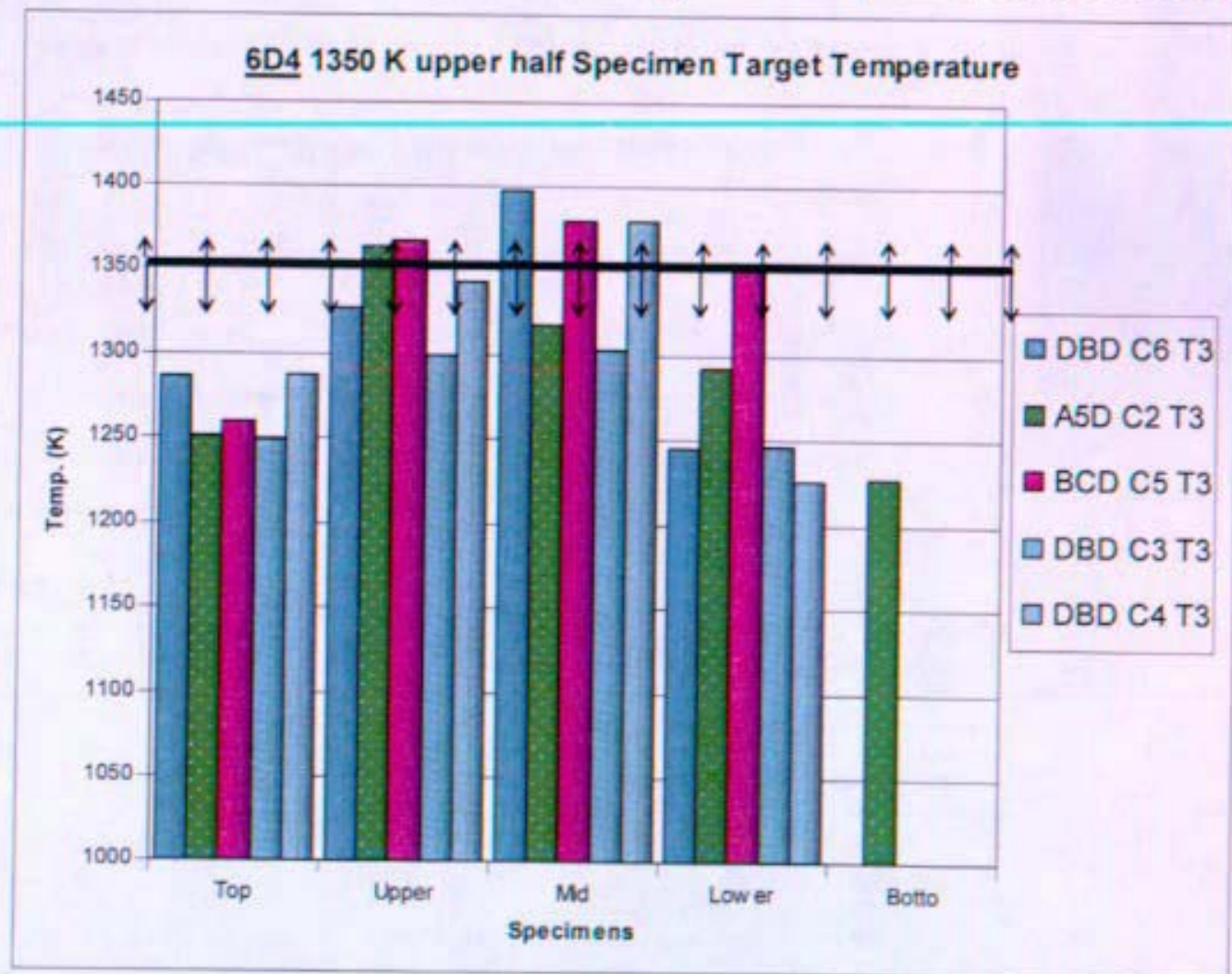

Figure III.B-22 Specimen temperatures in Rig B with a target temperature of $1350 \mathrm{~K}$. 
Enclosure (7) to

MDO-723-0044/

B-MT(SRME)-52

Page 84

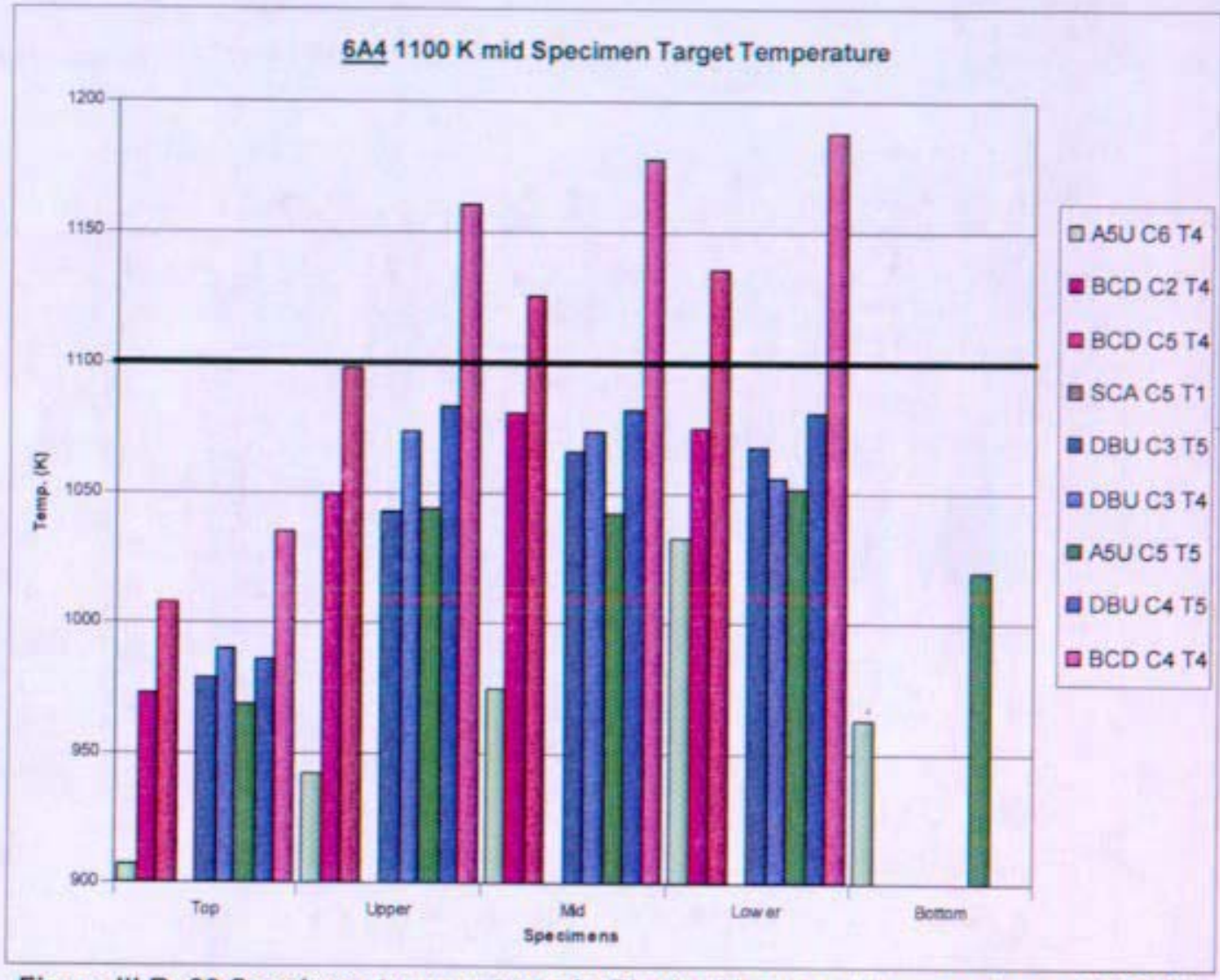

Figure III.B- 23 Specimen temperatures in Rig A with a target temperature of $1100 \mathrm{~K}$.

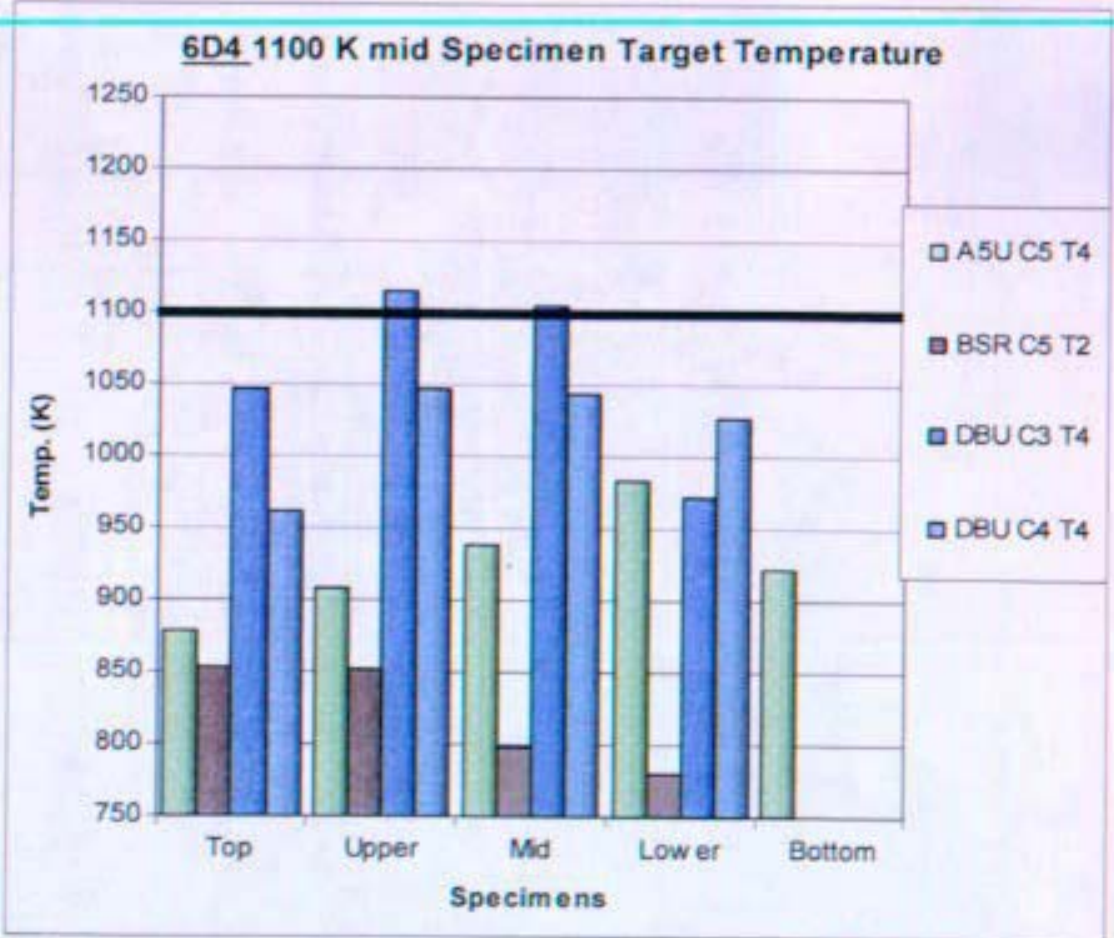

Figure III.B- 24 Specimen temperatures in Rig B with a target temperature of $1100 \mathrm{~K}$. 
Enclosure (7) to

MDO-723-0044/

B-MT(SRME)-52

Page 85

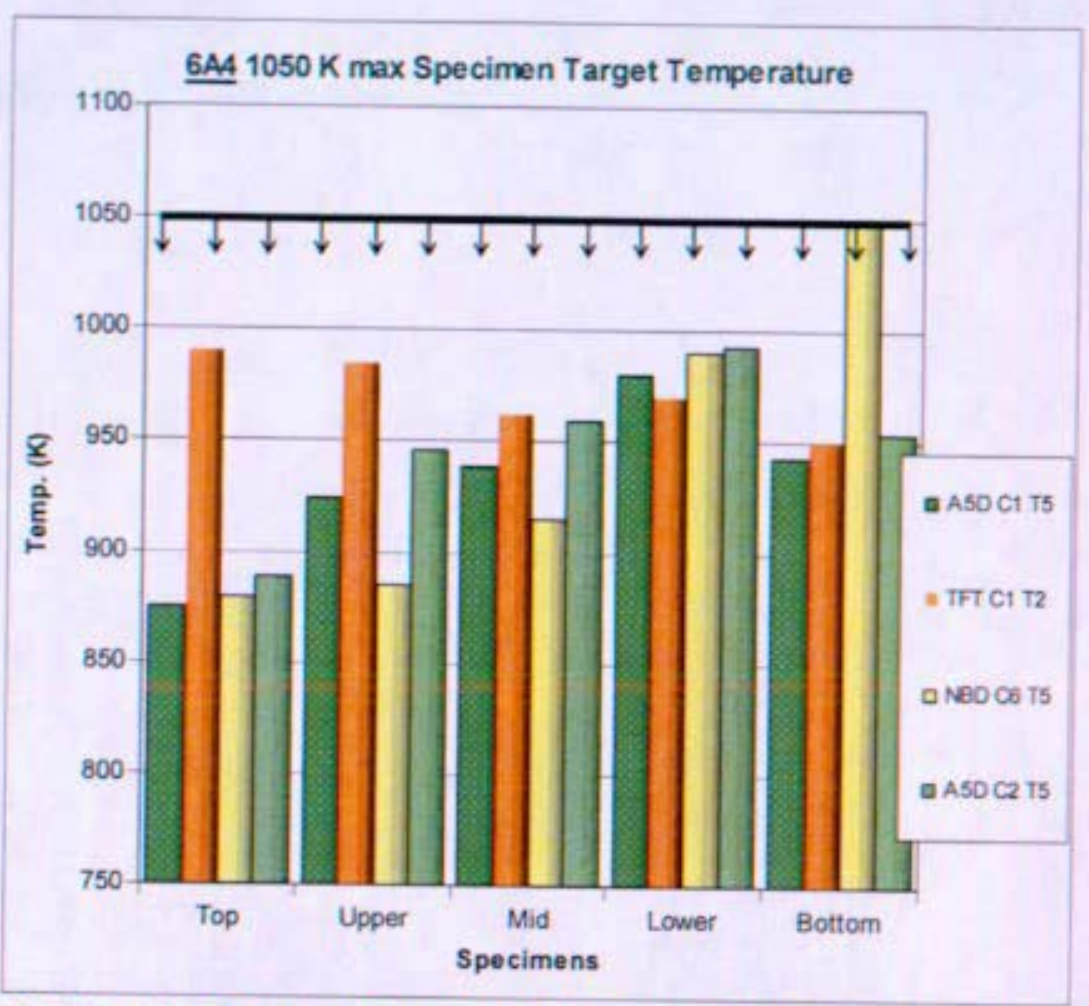

Figure III.B- 25 Specimen temperatures in Rig A with a maximum temperature of $1050 \mathrm{~K}$.

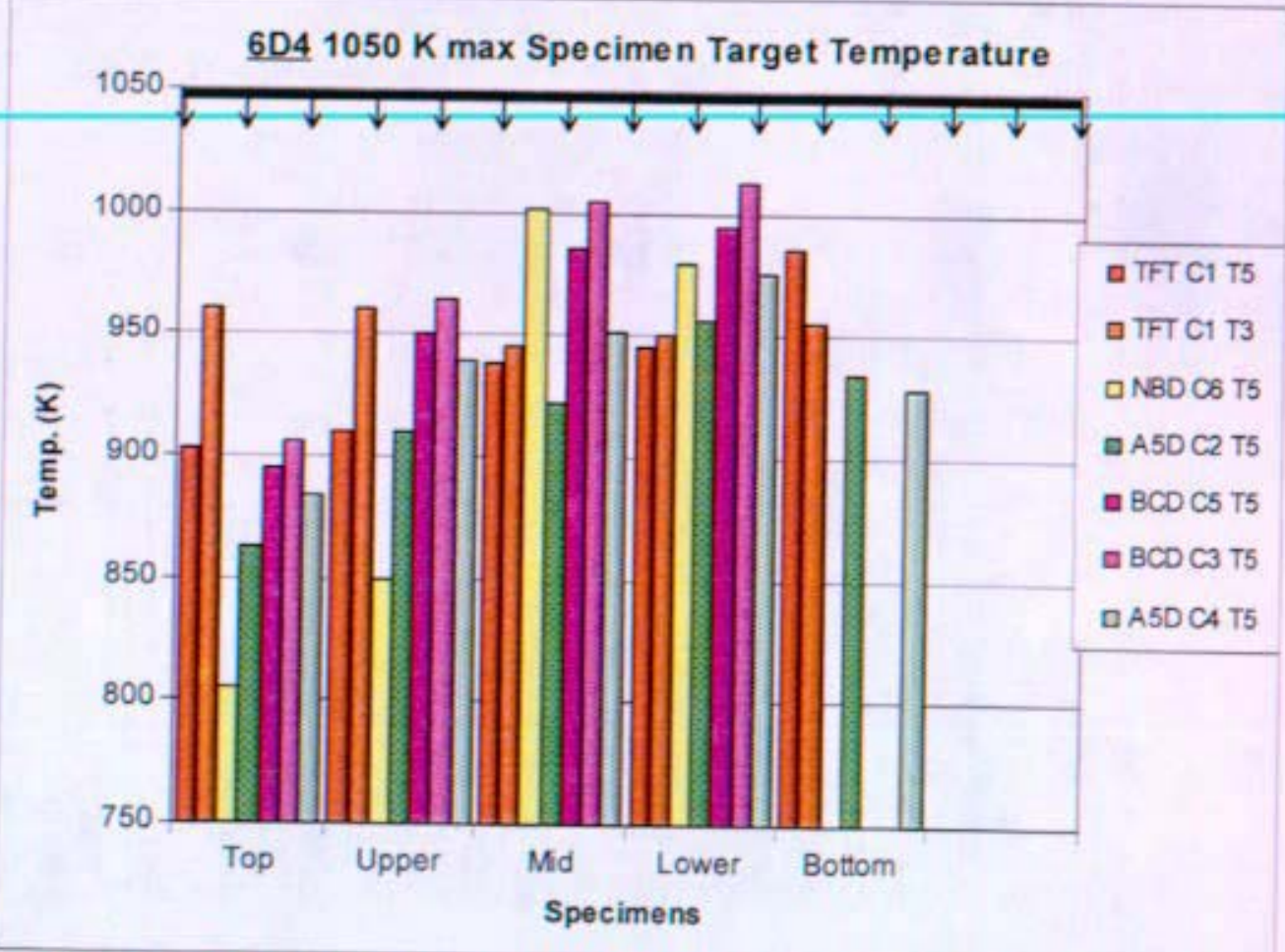

Figure III.B- 26 Specimen temperatures in Rig B with a maximum temperature of $1050 \mathrm{~K}$. 
Enclosure (7) to

MDO-723-0044/

B-MT(SRME)-52

Page 86

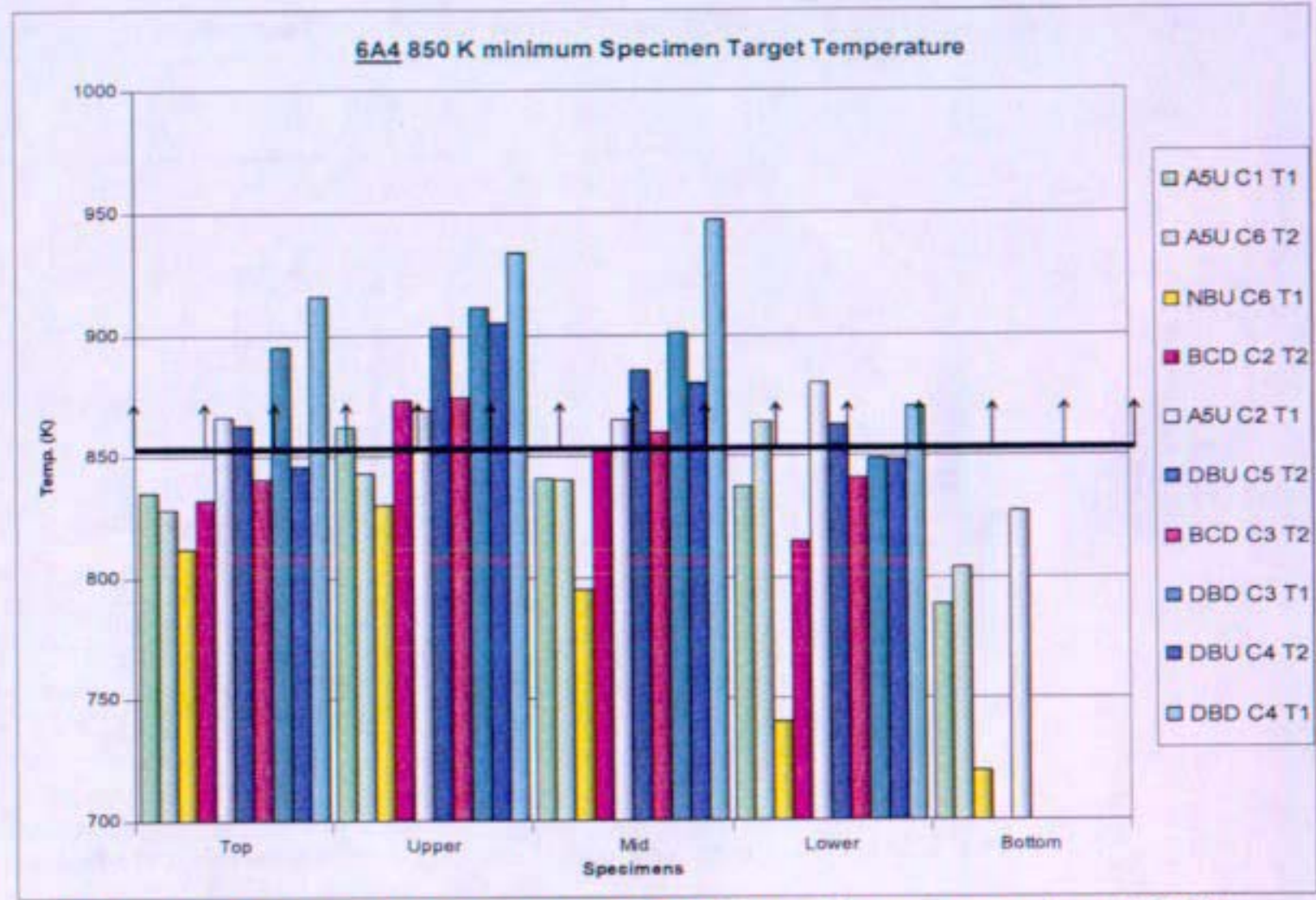

Figure III.B- 27 Specimen temperatures in Rig A with a minimum temperature of $850 \mathrm{~K}$.

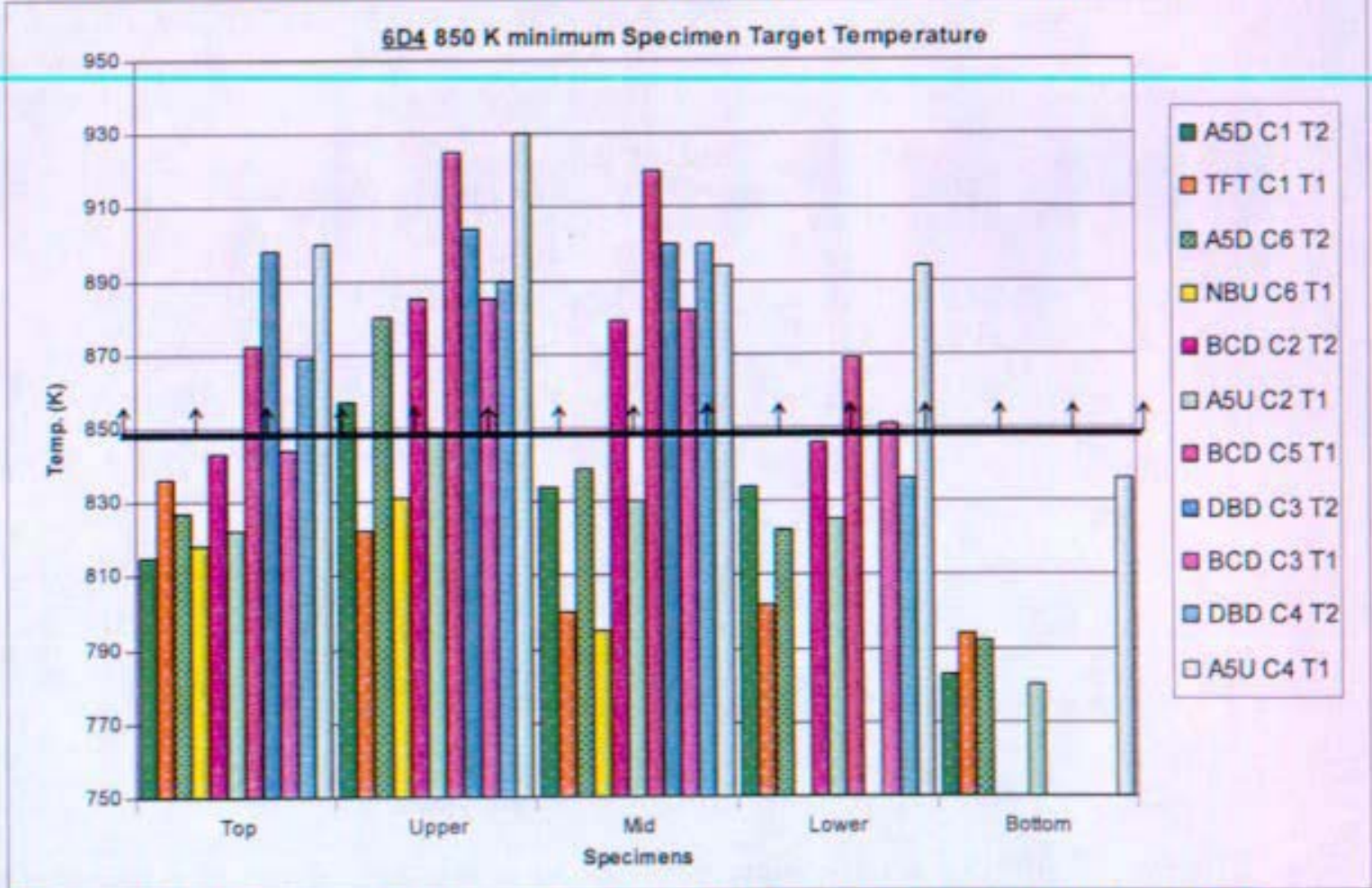

Figure III.B- 28 Specimen temperatures in Rig B with a minimum temperature of $850 \mathrm{~K}$. 


\section{Confirmatory Hand Calculations}

Two dimensional planer hand calculations where done to support the finite element models. These calculations were performed on the BCD capsule located in Rig A, Tier 3 , Compartment 2 which is considered to be a worst case capsule. The hand calculations evaluate the thermal model at a plane cutting through the mid-section of the middle biaxial creep specimen. The effects of radiation, conduction, and convection were considered in the analysis, however convection was found to be negligible as shown below.

The temperature difference across the specimen, holders, and capsule due to the heat generation was calculated by the basic heat transfer equation for a hollow cylinder with internal heat generation

$$
\Delta T=\frac{Q}{4 k}\left[\left(r_{0}^{2}-r_{1}^{2}\right)-2 r_{i}^{2} \ln \left(\frac{r_{o}}{r_{i}}\right)\right]
$$

where

$$
\begin{array}{ll}
r_{0}=\text { outer radius of the cylinder } & k=\text { conductivity } \\
r_{i}=\text { inner radius of the cylinder } & Q=\text { volumetric heat generation }
\end{array}
$$

The effect of conduction through the specimen, holders, and capsule was calculated using the relationship

$$
\Delta T=\frac{q}{2 \pi k} \ln \left(\frac{r_{o}}{r_{i}}\right)
$$

Heat transfer through the gas inside the capsule due to radiation and conduction was found by using the equation

$$
\Delta T=\frac{q}{2 \pi r\left(h_{r}+h_{c}\right)}
$$

with

$$
h_{c}=\frac{k}{r_{i} \ln \left(\frac{r_{o}}{r_{i}}\right)}
$$

and

$$
h_{r}=\frac{q_{r} "}{\Delta T}
$$

where

$$
q_{r}{ }^{\prime \prime}=\sigma\left(T_{h}^{4}-T_{c}^{4}\right) \varepsilon \quad \text { and } \quad \varepsilon=\frac{1}{\left[\frac{1}{\varepsilon_{i}}+\frac{1-\varepsilon_{o}}{\varepsilon_{o}}\left(\frac{r_{i}}{r_{o}}\right)\right]}
$$

where

$$
\mathrm{T}_{\mathrm{h}}=\text { temperature of the hot side } \quad \varepsilon=\text { coupled emissivity }
$$


Enclosure (7) to

MDO-723-0044/

B-MT(SRME)-52

Page 88

$T_{c}=$ temperature of the cold side

$h_{r}=$ radiation heat transfer coefficient

$h_{c}=$ conduction heat transfer coefficient

$q=$ heat transfer rate $\varepsilon_{\mathrm{i}}=$ emissivity of the inner surface

$\varepsilon_{0}=$ emissivity of the outer surface

$\mathrm{q}_{r}^{\prime \prime}=$ radiative heat flux

The effect of convective heat transfer by means of convection currents was considered because of the large temperature difference in the area filled by gas between the inner radius of the capsule and the outer radjus of the specimen holder. Convection affects were considered for both Argon and Helium gases and in both cases found to be negligible by using data that correlates the Nusselt number $(\mathrm{Nu})$ to the Rayleigh number (Ra) [Reference (III.B-2)] through the following equations.

The convective heat transfer relationship relates the heat flux $\left(q^{\prime \prime}\right)$ from a hot junction $\left(T_{h}\right)$, to a colder junction $\left(T_{c}\right)$ by the relationship

$$
q^{\prime \prime}=h_{c o m}\left(T_{h}-T_{c}\right)
$$

where $h_{\text {conv }}$ is the convection heat transfer coefficient. This relationship can also be calculated from the form

$$
q^{\prime \prime}=\frac{k_{e f f}}{z}\left(T_{h}-T_{c}\right)
$$

where $k_{\text {eff }}$ is the effective thermal conductivity of the gas in the capsule and $z$ is the gap distance between the inner capsule radius and the outer specimen radius. These relationships are solved for $k_{\text {eff }}$.

$$
k_{e f f}=\frac{q^{\prime \prime} z}{\left(T_{h}-T_{c}\right)}=h z
$$

Defining the Nusselt number as

$$
N u=\frac{h z}{k}
$$

where $k$ is the normal molecular thermal conductivity of the gas. Then

$$
N u=\frac{k_{e f f}}{k}
$$

The Nusselt number is correlated to the Rayleigh number (Ra) [Reference (III.B1)].The Rayleigh number is a product of Grashof number (Gr) and the PrandtI number (Pr). 


$$
\begin{aligned}
& R a=G r \operatorname{Pr} \\
& G r=\frac{\rho^{2} g \beta z^{3} \Delta T}{\mu^{2}} \\
& \operatorname{Pr}=\frac{\mu C_{p}}{k}
\end{aligned}
$$

where

$$
\begin{aligned}
& \mu=\text { viscosity } \\
& \beta=\text { volumetric expansion coefficient } \\
& g=\text { acceleration of gravity } \\
& k=\text { molecular conductivity }
\end{aligned}
$$

$\rho=$ density

$C_{p}=$ specific heat

$\Delta T=$ temperature difference

$z=$ separation distance

By definition a Nusselt number greater than unity, implies that convection currents may have an effect on the overall heat transfer. Reference (III.8-2) shows that Nu increases as Ra increases, so for a given fluid, convection currents depend on the temperature difference and the separation distance. While the temperature difference between the capsule and holder is large $(500 \mathrm{~K})$ the gap between the capsule and holder is sufficiently small $(0.4 \mathrm{~mm}$ to $1.5 \mathrm{~mm})$ to eliminate the convection currents, thus convective heat transfer was neglected.

The hand calculations agree with the results produced from the finite element analysis, except in the gas region between the outer holder and inner holder. In this region the 3D models show greater heat flux in the axial direction due a path of less resistance created by the centering tabs on the holders. The $2 \mathrm{D}$ planar calculations do not model the axial flow and this is the probable cause for the disagreement between the two models.

Table III.B- 5 shows the comparison between the results from the finite element axisymmetric model and the 2D planer hand calculations done on the $\mathrm{BCD}$ capsule located in Rig A, Tier 3, Compartment 2. Figure III.B-29 compares the temperature results as a function of the radial distance from the center of the capsule. 
Enclosure (7) to

MDO-723-0044/

B-MT(SRME)-52

Page 90

Table III.B- 5 Comparison of axisymmetric finite element and 2D planer hand calculation temperature results.

\begin{tabular}{|c|c|c|c|c|c|c|c|c|c|}
\hline & Biax & $\begin{array}{c}\text { Biax } \\
\text { OD }\end{array}$ & $\begin{array}{c}\text { Inner Holder } \\
\text { ID }\end{array}$ & $\begin{array}{c}\text { Inner Holder } \\
\text { OD }\end{array}$ & $\begin{array}{l}\text { Outer Holder } \\
\text { ID }\end{array}$ & $\begin{array}{c}\text { Outer Holder } \\
\text { OD }\end{array}$ & Capsule & Capsule & \\
\hline & 2.54 & 3.175 & 3.57 & 4.725 & 5.36 & 9.60 & 10 & 11 & $r(\mathrm{~mm})$ \\
\hline $\begin{array}{c}\text { Hand } \\
\text { Calculation }\end{array}$ & & 1456 & 1368 & 1368 & 1135 & 1128 & 649 & 644 & \multirow{3}{*}{$T(K)$} \\
\hline $\begin{array}{c}\text { ANSYS } \\
\text { Axisymmetric }\end{array}$ & 1316 & 1315 & 1242 & 1242 & 1127 & 1125 & 649 & 644 & \\
\hline difference & & 141 & 126 & 126 & 8 & 3 & 0 & 0 & \\
\hline
\end{tabular}

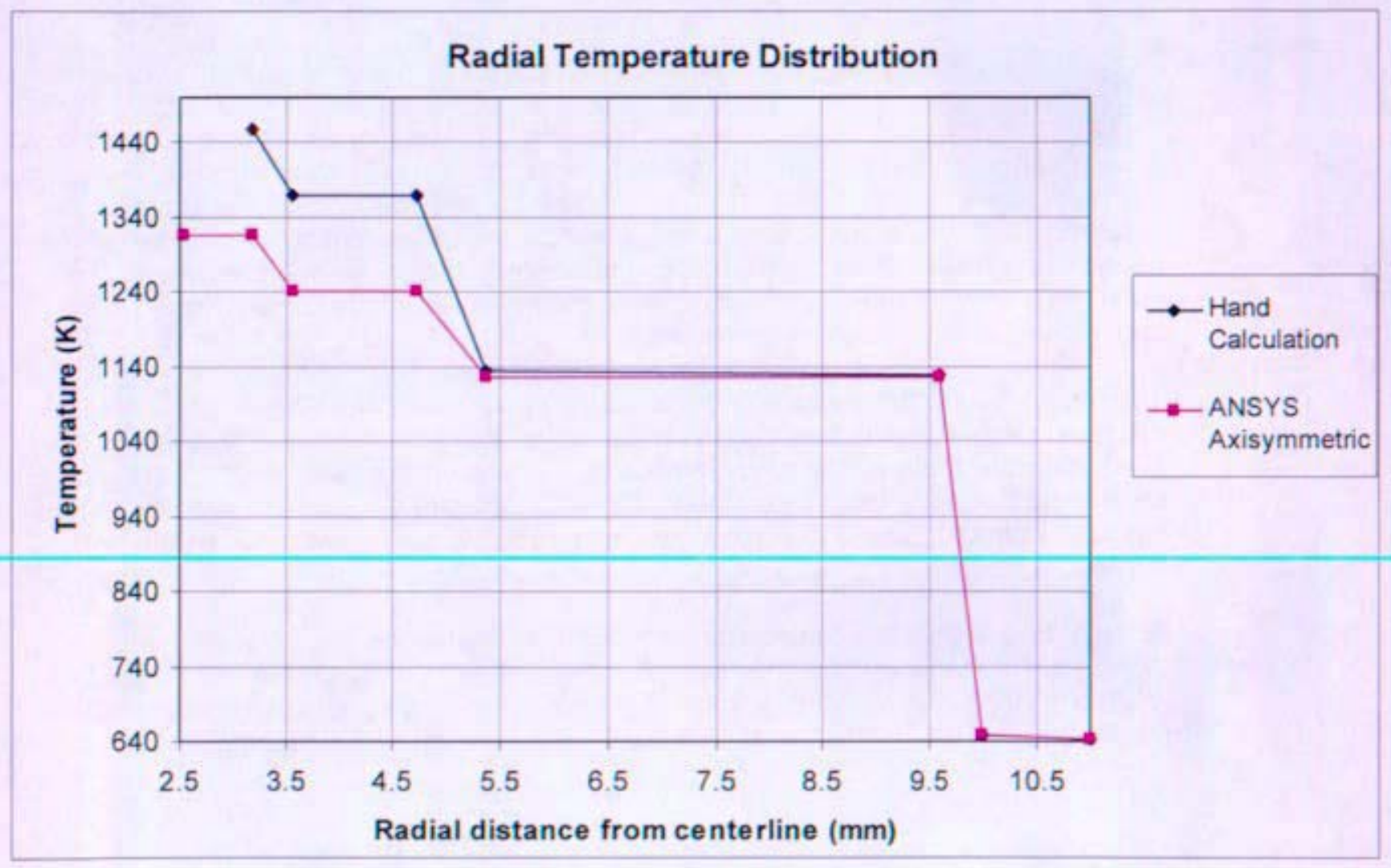

Figure III.B-29 Temperature results from finite element model and hand calculations. 
Enclosure (7) to

MDO-723-0044/

B-MT(SRME)-52

Page 91

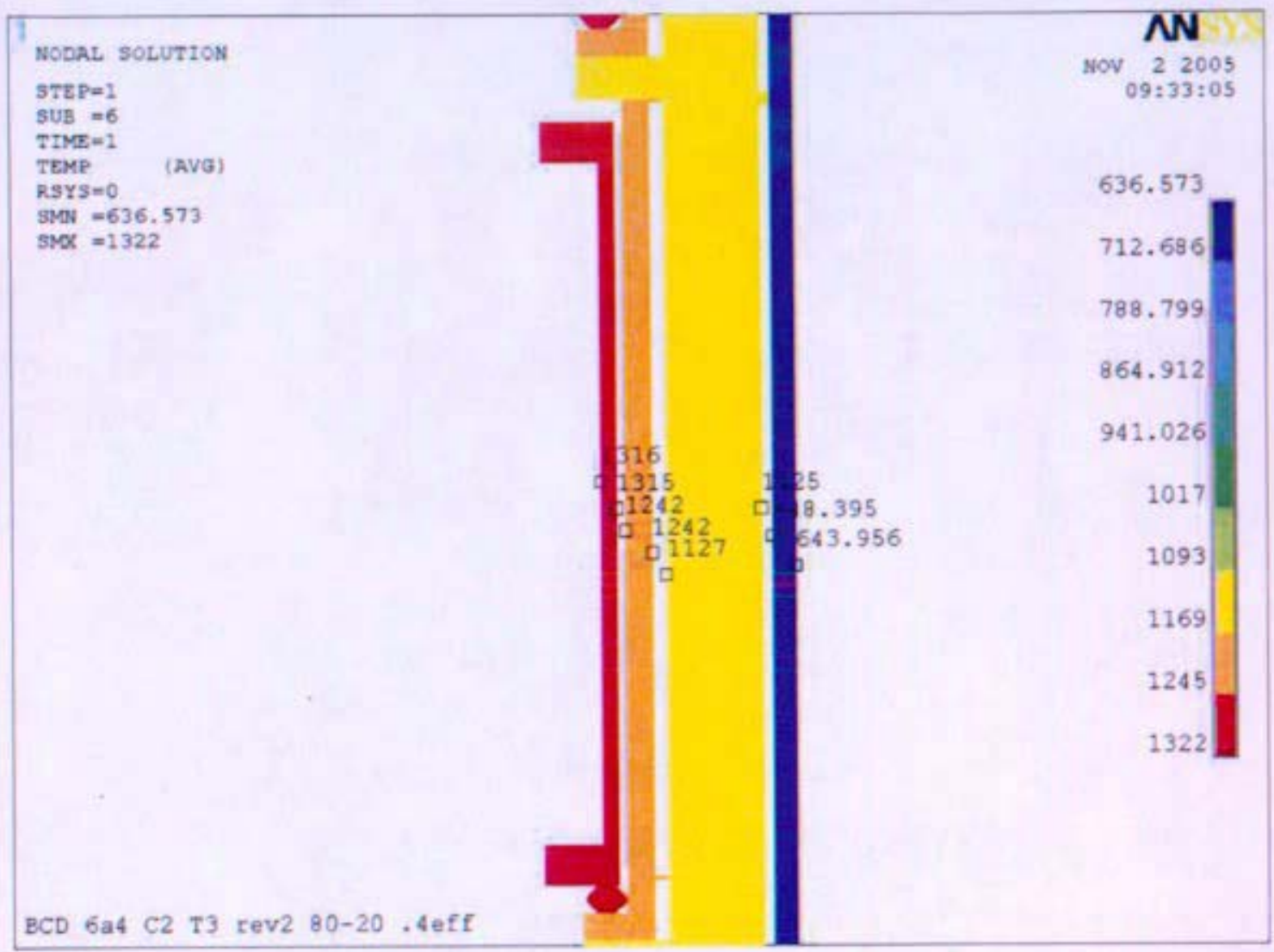

Figure III.B- 30 Thermal results from the finite element model of the BCD capsule in rig A, tier 3, compartment 2 . 
Enclosure (7) to

MDO-723-0044/

B-MT(SRME)-52

Page 92

THIS PAGE IS INTENTIONALLY BLANK 


\section{Structural Analysis of JOYO-1 Capsule Pressure Boundary}

\section{Classification}

NRPCT requested and received agreement (Reference 1-1) that the pressure boundary will be designed, constructed, and inspected in accordance with American Society of Mechanical Engineers (ASME) Boiler and Pressure Vessel Code, Code case N-253-13 and Section III, Class 3. Adhering to the rules of the ASME Boiler and Pressure Vessel Code (hereafter referred to as the Code) will satisfy JNC for capsule insertion into JOYO.

The Code, adopted throughout industry in the United States, provides rules of safety governing the design, fabrication and inspection during construction of pressure vessels. Section III, Division 1 of the Code specifies rules for nuclear power plant components. Section III, Division 1 is further broken down into five classes, designated Code Classes 1, 2, 3, MC and CS. A nuclear power plant component is assigned a class by the owner of the plant based on the level of importance associated with the function of the item relative to the safe operation of the plant. For test capsules irradiated in the Advanced Test Reactor (ATR) in Idaho the capsule pressure boundary is assigned Class 3 . To be consistent, the JOYO-1 capsule pressure boundary were assigned Class 3.

Class 3 pressure boundaries are required to be designed, constructed and inspected per the rules of Section III, Division 1, Subsection ND of the Code. However, Class 3 pressure boundaries only apply to design temperatures up to $8008 \mathrm{~F}(4278 \mathrm{C})$. Since the JOYO- 1 capsules have a design temperature of $8518 \mathrm{~F}(4558 \mathrm{C})$, the Code requires that the supplementary design and material selection requirements of Code Case $\mathrm{N}$ 253-13 be followed. In summary, the JOYO-1 capsule pressure boundary will be designed to the supplementary requirements of Code Case N-253-13 and Section III, Division 1, Subsection ND of the Code. However, three exceptions to these requirements are identified by the NRPCT, as listed below:

a. The JOYO-1 pressure vessel will not be Code stamped. The NRPCT will be fabricating and inspecting the capsules but does not hold an ASME Certification and Code Stamp.

b. An authorized nuclear inspector will not be used for inspection of the capsule pressure boundary. Instead, qualified NRPCT Quality Assurance (QA) and engineering personnel will be used for nondestructive examination of the pressure boundary, including pressure and leak testing. Specific inspections throughout the capsule construction are described in IV.C.

c. Full radiography of the head to capsule weld will not be performed. Full radiography is not feasible due to the small capsule diameter and location of the internal components relative to the weld joint. Note that the sidewall of selected capsules will be radiographed by NRPCT QA personnel to confirm complete weld penetration. 
Enclosure (7) to

MDO-723-0044/

B-MT(SRME)-52

Page 94

\section{Design Conditions}

Each component establishing the JOYO-1 stainless steel capsule pressure boundary (Capsule, SK-AMT-2221 and Head, SK-AMT-2222) must show compliance to the Code for the service conditions listed in Table III.C.2-1. The design temperature and pressure encompasses all Service Level Conditions. Pressures listed in Table III.C.2-1 are gage pressures (i.e., 0 psig $=14.7$ psia).

Table III.C.2-1 Design and Service Conditions

\begin{tabular}{|c|c|}
\hline \multicolumn{2}{|l|}{ Design Conditions } \\
\hline - Design Life & $\begin{array}{l}2 \text { Reactor Cycles at } \\
60 \text { days/Reactor Cycle }\end{array}$ \\
\hline - Design Temperature, Capsule Pressure Boundary & $455^{\circ} \mathrm{C}\left(850^{\circ} \mathrm{F}\right)$ \\
\hline Reactor coolant temperature in compartment 2. & $\begin{array}{l}353 \text { to } 452^{\circ} \mathrm{C} \\
\left(667 \text { to } 846^{\circ} \mathrm{F}\right)\end{array}$ \\
\hline $\begin{array}{l}\text { Capsule Pressure Boundary Temperature with biaxial } \\
\text { creep specimen. }\end{array}$ & $\max : 443^{\circ} \mathrm{C}\left(829.4^{\circ} \mathrm{F}\right)$ \\
\hline \multicolumn{2}{|l|}{ - Design Pressure, Capsule Pressure Boundary } \\
\hline $\begin{array}{l}\text { Normal Internal at Room Temperature } \\
\text { Due to assembly of capsule in glovebox }\end{array}$ & $\begin{array}{l}\max : 623 \mathrm{~Pa}(0.09 \mathrm{psi}) \\
\min :-345 \mathrm{~Pa}(-0.05 \mathrm{psi})\end{array}$ \\
\hline $\begin{array}{l}\text { Maximum Internal if Creep Specimens Fail } \\
\text { Assume failure of three pressurized biaxial creep } \\
\text { specimens whose combined pressures do no } \\
\text { exceed } 41.4 \mathrm{MPa}(6,000 \text { psi) absolute. In } \\
\text { addition, the maximum pressure of any one biaxial } \\
\text { creep specimen shall not exceed } 3000 \text { psi. }\end{array}$ & $6.55 \mathrm{MPa}(950 \mathrm{psi})$ \\
\hline $\begin{array}{l}\text { Normal External } \\
\text { Joyo reactor pressure plus weight of sodium } \\
\text { above lowest capsule }\end{array}$ & $\begin{array}{l}\max : 50.3 \mathrm{KPa}(7.3 \mathrm{psi}) \\
\min : 0 \mathrm{kPa}(0 \mathrm{psi})\end{array}$ \\
\hline External during Pneumatic Pressure Test & $7.86 \mathrm{MPa}(1140 \mathrm{psi})$ \\
\hline - Test Gas Mixtures & $\begin{array}{l}100 \% \mathrm{He} \\
30 \% \mathrm{Ar} 70 \% \mathrm{He} \\
80 \% \mathrm{Ar} 20 \% \mathrm{He} \\
\end{array}$ \\
\hline \multicolumn{2}{|l|}{ - Other External Loadings } \\
\hline SMIR Compartment Spring Force & $60 \mathrm{~N}(13.5 \mathrm{lbf})$ \\
\hline $\begin{array}{l}\text { Component Weights } \\
\text { Includes four capsules and spacer }\end{array}$ & $38.6 \mathrm{~N}(8.68 \mathrm{lbf})$ \\
\hline \multicolumn{2}{|l|}{ - Other Internal Loadings } \\
\hline Capsule Internal Wave Spring & $15.5 \mathrm{~N}(3.5 \mathrm{lbf})$ \\
\hline $\begin{array}{l}\text { - JOYO Reactor Power (Note: } 1.25 \text { design factor applied to } \\
\text { all gamma heat rate values) }\end{array}$ & $140 \mathrm{MW}$ \\
\hline - Reactor Coolant Flow & $\max : 32.81 \mathrm{~m} / \mathrm{sec}(10 \mathrm{ft} / \mathrm{sec})$ \\
\hline - Corrosion Allowance & Zero (negligible) \\
\hline
\end{tabular}


Table III.C.2-1 Design and Service Conditions (cont.)

\begin{tabular}{|l|l|}
\hline \multicolumn{3}{|c|}{ Level A (Normal) Service Conditions } \\
\hline - Reactor Startup, Shutdowns, Scram Recoveries & 3/Cycle, Total 6 Cycles \\
\hline
\end{tabular}

\begin{tabular}{|c|c|c|}
\hline \multicolumn{3}{|c|}{$\begin{array}{l}\text { Level B (Upset) Service Conditions } \\
\text { or deviations from normal (Level A) conditions which are } \\
\text { ough that the design shall be capable to withstand these } \\
\text { al impairment. }\end{array}$} \\
\hline $\begin{array}{c}\text { Transient Overpower } \\
-\quad \text { Miss Withdrawal } \\
-\quad \text { Increase gas flow }\end{array}$ & $\begin{array}{l}1.07 \text { overpower ratio } \\
120 \text { seconds/incident } \\
5 / \text { cycle }\end{array}$ & $\begin{array}{l}\text { max calculated sodium } \\
\text { temperature } 422^{\circ} \mathrm{C}\end{array}$ \\
\hline $\begin{array}{c}\text { Loss of flow } \\
-\quad \text { Loss of Power } \\
-\quad \text { Primary Pump Trip }\end{array}$ & $\begin{array}{l}1.25 \text { overpower ratio } \\
5 \text { seconds/incident } \\
8 / \text { cycle }\end{array}$ & $\begin{array}{l}\text { max calculated sodium } \\
\text { temperature } 434^{\circ} \mathrm{C}\end{array}$ \\
\hline
\end{tabular}

\section{Level C (Emergency) Service Conditions}

Loadings which have a low probability of occurring (unlikely) but are included to provide assurance that no gross loss of structural integrity will result should the event occur

\begin{tabular}{|l|l|l|}
\hline $\begin{array}{c}\text { Transient Overpower } \\
-\quad \text { Fuel Slumping }\end{array}$ & $\begin{array}{l}1.07 \text { overpower ratio } \\
120 \text { seconds/incident } \\
5 / \text { cycle }\end{array}$ & $\begin{array}{l}\text { max calculated sodium } \\
\text { temperature } 439^{\circ} \mathrm{C}\end{array}$ \\
\hline $\begin{aligned} \text { Loss of flow } \\
-\quad \text { Primary Pump Stick }\end{aligned}$ & $\begin{array}{l}1.25 \text { overpower ratio } \\
5 \text { seconds/incident } \\
8 / \mathrm{cycle}\end{array}$ & $\begin{array}{l}\text { max calculated sodium } \\
\text { temperature } 454^{\circ} \mathrm{C}\end{array}$ \\
\hline $\begin{array}{l}\text { To envelope Level C power and flow transients, analysis performed at: } \\
820 \text { psi internal and O psi external. }\end{array}$
\end{tabular}

\section{Level D (Faulted) Service Conditions:}

Those events that have an extremely low probability (Extremely Unlikely) of occurring. These limits permit gross general deformations with some consequent loss of dimensional stability and damage requiring repair or component removal

- Seismic Conditions:

JNC support required for this service condition.

\section{a. Design Conditions Discussion}

\section{(1) Temperature}

JOYO uses sodium coolant. JNC identified the nominal and worse case coolant temperatures shown in the previous section, Figure III.B-5. 
Enclosure (7) to

MDO-723-0044I

B-MT(SRME)-52

Page 96

The sodium coolant temperature and capsule component gamma heating rates dictate the temperature of each capsule pressure boundary (as determined by ANSYS models). For conservatism a Level $\mathrm{C}$ service condition was considered to determine the capsule wall maximum temperature. This Level $\mathrm{C}$ condition includes gamma heating rates 1.64 times greater than nominal (Power $\times 1.25$ overpower $\times 1.25$ Loss of flow $\times 1.05$ loss of flow factor $=1.64$ design factor) to determine the maximum capsule wall temperature. The highest BCD capsule wall temperature at the 1.64 design factor exists in Tier 5, Compartment 5, Rig $6 \mathrm{D} 4$ and was calculated to be $829.48 \mathrm{~F}(4438 \mathrm{C})$.

A design temperature was set slightly higher at $8508 \mathrm{~F}(454.48 \mathrm{C})$ which exceeds any of the Service Level Conditions.

(2) Internal Pressure

The JOYO-1 capsules are assembled in a glove box backfilled with $100 \% \mathrm{He}$, $30 \% \mathrm{Ar}-70 \% \mathrm{He}$, or $80 \% \mathrm{Ar}-20 \% \mathrm{He}$ at a pressure of -345 to $623 \mathrm{~Pa}$ gage $(-0.05$ to $0.09 \mathrm{psig}$ ). The BCD Type capsules house three pressurized biaxial creep specimens and, should the creep specimen fail in service, a significant increase in internal capsule pressure will result.

The $B C D$ capsule that experiences the highest internal pressure gas during operation (should the biaxial creep specimens fail) is the Tier 3, compartment 2 , rig $6 \mathrm{A4}$. For a mean capsule gas temperature of $1085^{\circ} \mathrm{K}$, the resulting internal capsule pressure is $6.55 \mathrm{MPa}$ (950 psi) gage. The highest internal pressure exists with the restriction of the sum of the three biaxial creep specimens to be no higher than $41.4 \mathrm{MPa}$ absolute during the initial creep specimen fill. The Tier 3 is limiting because these capsules have the highest mean capsule gas temperature as compared to any other tier due to the gamma heating rates.

Listed below is the derivation of this value, which defines the internal design pressure of the capsule pressure boundary. Note that the mean capsule gas temperature was calculated at design conditions, 1.64 design factor.

To calculate the capsule internal pressure after three pressurized biaxial creep specimens have failed, the ideal gas law will be followed where:

$$
\frac{\mathrm{P}_{1} V_{1}}{\mathrm{nRT}_{1}}=\frac{\mathrm{P}_{2} V_{2}}{\mathrm{nRT}_{2}}
$$

which simplifies to:

$$
\frac{P_{1} V_{1}}{T_{1}}=\frac{P_{2} V_{2}}{T_{2}}
$$


Solving for $P_{2}$ :

$$
P_{2}=\frac{P_{1} V_{1} T_{2}}{V_{2} T_{1}}
$$

The total capsule internal gas pressure is the sum of the pressure due to the capsule backfill gas at temperature, plus the pressure due to the gas release from each of three creep specimens:

$$
P_{2}=P_{2-\text { capsule }}+P_{2 \text {-top_specimen }}+P_{2 \text {-middle_specimen }}+P_{2 \text {-bottom_specimen }}
$$

where

$$
P_{\text {2-capsule }}=\frac{P_{1 C} V_{1 C} T_{2}}{V_{2} T_{1 C}}
$$

and

$$
P_{2 \text {-top specimen }}=P_{2 \text {-middle specimen }}=P_{2 \text {-bottom specimen }}=\frac{P_{1 S} V_{1 S} T_{2}}{V_{2} T_{1 S}}
$$

$\mathrm{P}_{1 \mathrm{C}}=$ Maximum initial capsule gas pressure during glovebox assembly, $\mathrm{Pa}$

$$
=101,235 \mathrm{~Pa}+623 \mathrm{~Pa}
$$$$
=101,900 \mathrm{~Pa}
$$

$P_{1 \mathrm{~S}}=$ Biaxial creep specimen gas pressure at room temperature, $\mathrm{Pa}$

$=20.7 \mathrm{MPa}$ for bottom specimen

$=20.7 \mathrm{Mpa}$ for the middle specimen

$=0 \mathrm{MPa}$ for the top specimen

$\mathrm{P}_{2}=$ Final capsule gas pressure during operation assuming all biaxial creep specimens have failed, $\mathrm{Pa}$ absolute

$=6.55 \mathrm{MPa}$

$V_{\text {IC }}=$ Initial capsule volume per Table III.C.2-2, $\mathrm{mm}^{3}$. Calculated with holders at the minimum gap sizes.

$=13,021 \mathrm{~mm}^{3}$

$V_{1 \mathrm{~s}}=$ Initial biaxial creep specimen internal volume, $\mathrm{mm}^{3}$

$$
=613.1 \mathrm{~mm}^{3}
$$

$\mathrm{V}_{2}=$ Final capsule volume which includes the initial capsule volume plus the internal volume of the failed creep specimens, $\mathrm{mm}^{3}$

$$
=\mathrm{V}_{1 \mathrm{c}}+3 \mathrm{~V}_{\mathrm{is}}
$$


Enclosure (7) to

MDO-723-0044I

B-MT(SRME)-52

Page 98

$$
\begin{aligned}
& =14,860 \mathrm{~mm}^{3} \\
\mathrm{~T}_{1 \mathrm{C}} & =\text { Capsule fill gas temperature during glovebox assembly, }{ }^{\circ} \mathrm{K} \\
& =295^{\circ} \mathrm{K} \\
\mathrm{T}_{1 \mathrm{~S}} & =\text { Biaxial creep fill gas temperature during assembly, }{ }^{\circ} \mathrm{K} \\
& =295^{\circ} \mathrm{K} \\
\mathrm{T}_{2} & =\text { Calculated mean capsule gas temperature during operation, }{ }^{\circ} \mathrm{K} \\
& =1085^{\circ} \mathrm{K}
\end{aligned}
$$

Table III.C.2-2 Capsule Internal Gas Volume $V_{1 C}$

Component volumes were calculated using the drawings of Section $V$ using the holders that provide the minimum gas gaps.

\begin{tabular}{|c|c|c|}
\hline & $\begin{array}{c}\text { Capsule Internal Volume } \\
\text { without internal } \\
\text { components } \\
\text { mm }^{3}\end{array}$ & $\begin{array}{c}\text { Capsule } \\
\text { Gas Volume } \\
\mathbf{m m}^{\mathbf{3}}\end{array}$ \\
\hline BCD & \multirow{3}{*}{49,340} & 13,021 \\
\hline DBU \& DBD & & 14,793 \\
\hline A5U \& A5D & & 14,445 \\
\hline NBU \& NBD & & 17,538 \\
\hline
\end{tabular}

(3) External Pressure

The Code requires pressure testing of the pressure boundary vessel at 1.2 times the design pressure. Since the capsules cannot be internally pressure tested since there are no boundary penetrations, an external pressure test would be performed. For the JOYO capsules, the test pressure would be 1.2 times $950 \mathrm{psig}=1140 \mathrm{psig}$. This test pressure is above the Code requirements of no less than 1.1 times the design pressure to allow for test pressure tolerances.

\section{Analysis Method}

All calculations were performed using the capsule and head dimensions conforming to drawing tolerances that provided for the most conservative analyses. For example, to show compliance to the Code, the required head thickness was compared to the minimum (not nominal) head thickness specified in Sketch SK-AMT-2222.

The units used in the following calculations are in the U.S. Customary units of measurement. The Code identifies (NCA-1150) that the SI units are for information only and are not to be interpreted as specific requirements or limits.

Material Properties for ASME SA 479, Type $316 \mathrm{H}$ or Type 316 (if the material meets ASME SA 479 Supplementary Requirements) are shown in Table III.C.3-1. 
The General requirements for design (Code Case N-235-13) are based on the acceptable component design which meets the following requirements and are calculated in sections III.C.4 and III.C.5:

a. The design satisfies the general design requirements of ND-3100.

b. The design satisfies the component rules in ND-3300 (vessel design by formula).

c. The design shall guard against failure from low energy fracture.

Time-Temperature limits for creep and stress-rupture are satisfied since the pressure boundary metal temperature for the $316 \mathrm{H}$ capsule is in service (120 days or 2,880 hours) less than the allowable time ( $10^{6}$ hours) and has less than 25 Service Level $C$ events as permitted in Appendix E, Code Case N-253-13.

\begin{tabular}{|l|c|c|c|c|c|c|c|c|c|}
\hline \multicolumn{7}{|c|}{ Table III.C.3-1 ASME SA 479, Type 316H Stress Limits } \\
\hline \multicolumn{1}{|c|}{ Temperature ${ }^{\circ} \mathrm{F}$} & 800 & 850 & 900 & 950 & 1000 & 1050 & 1100 & 1150 & 1200 \\
\hline $\begin{array}{l}\text { Allowable Stress (ksi) } \\
\text { [Code Case N-253-11, } \\
\text { Table C-1.1(c)] }\end{array}$ & 15.9 & 15.7 & 15.6 & 15.4 & 15.3 & 15.1 & 14.7 & 13.6 & 12.8 \\
\hline $\begin{array}{l}\text { Allowable Stress } \\
\text { Intensity (ksi) } \\
\text { [Subsection NH, Class1 } \\
\text { Table I-14.2] }\end{array}$ & 15.9 & 157 & 15.6 & 15.5 & 15.3 & 14.5 & 12.4 & 9.8 & 7.4 \\
\hline $\begin{array}{l}\text { Yield Strength } \\
\text { (ksi) } \\
\text { [Subsection NH, Class1 } \\
\text { Table I-14.5] }\end{array}$ & 17.6 & 17.4 & 17.3 & 17.1 & 17.0 & 16.7 & 16.5 & 16.4 & 16.2 \\
\hline $\begin{array}{l}\text { Stress-To-Rupture for } \\
\text { 1,000 hours (ksi) } \\
\text { [Subsection NH, Class1 } \\
\text { Table l-14.6B] }\end{array}$ & 64.5 & 63.3 & 62 & 51.6 & 42.1 & 34.4 & 28.1 & 23.0 & 18.8 \\
\hline $\begin{array}{l}\text { Stress-To-Rupture for } \\
\text { 3,000 hours (ksi) } \\
\text { [Subsection NH, Class1 } \\
\text { Table l-14.6B] }\end{array}$ & 64.5 & 63.3 & 58 & 46.5 & 37.5 & 30.2 & 24.2 & 19.5 & 15.6 \\
\hline
\end{tabular}

\section{Subsection ND Code Calculations - Capsule Shell}

The Code calculations in this subsection apply to the JOYO-1 capsule body, SK-AMT2221, and Head, SK-AMT-2222. See Figure III.C.4-1. The capsule body is a cylindrical shape with slight counter-bore to accept the head. The shell thickness is 0.059 in. 
Enclosure (7) to MDO-723-0044/

B-MT(SRME)-52

Page 100

Figure III.C.4-1, JOYO-1 Pressure Boundary
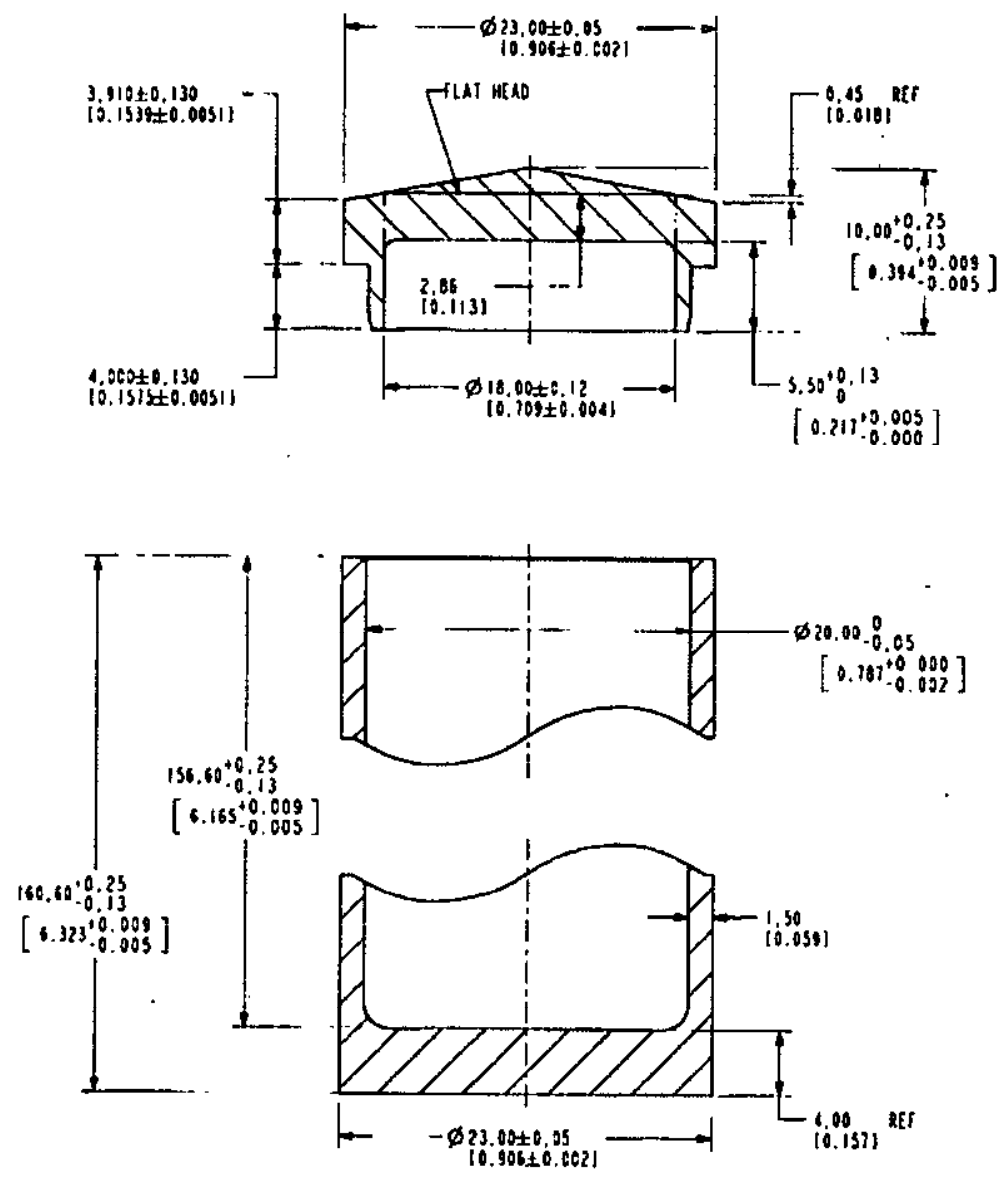

a. Capsule Shell Thickness Calculation for External Pressure

The maximum allowable external pressure $P_{a}$ for the capsule shell is calculated using ND-3133.3(a):

$D_{0} \quad=$ capsule shell outside dimension $=0.906$ inch

$\mathrm{T} \quad=$ capsule shell thickness $=.059$ inch

$\mathrm{L} \quad=$ capsule length $=6.22$ inch

$D_{0} / T=0.906 / .059=15.347$

$L D_{0}=6.22 / 0.906=6.869$

Determine Factor A from the Code Section II, Part D, Subpart 3, Table G using $D_{\mathrm{o}} / \mathrm{T}$ and $\mathrm{L} / \mathrm{D}_{\mathrm{o}}$

$A=0.00496$

Determine Factor B from the Code Section II, Part D, Subpart 3, Table HA-2 using the pressure boundary temperature and Factor $A$. Two limiting cases must be analyzed: 
Case 1 - Capsule operating in reactor subjected to design conditions Allowable pressure, $P_{a}$, must exceed external pressure due to Joyo reactor pressure plus weight of sodium above lowest capsule ( $7.3 \mathrm{psi})$

The capsule maximum temperature subjected to design conditions is 8508F. At $8508 \mathrm{~F}$ and $A=0.00496: B=9820$

$$
\begin{aligned}
& P_{a}=4 B /\left(3 D_{o} / T\right) \\
& P_{a}=4 \times 9820 /(3 \times 15.347)=853 p s i g>7.3 p s i g
\end{aligned}
$$

Case 2 - Capsule externally pressurized during pneumatic pressure test Allowable pressure, $P_{a}$, must exceed pneumatic test pressure (1140 psi)

The capsule temperature during pneumatic testing is $1008 \mathrm{~F}$ maximum. At $1008 \mathrm{~F}$ and $\mathrm{A}=\mathrm{A}=0.00496: \mathrm{B}=13,385$

$$
\begin{aligned}
& P_{a}=4 B /\left(3 D_{o} / T\right) \\
& P_{a}=4 \times 13,385 /(3 \times 15.347)=1163 p s i g>1140 \text { psig }
\end{aligned}
$$

\section{b. Cylinders Under Axial Compression}

The maximum allowable compressive area to be used in the design of cylindrical shells and tubular products subject to loadings that produce longitudinal compressive stresses in the wall shall be the lesser of the values given in (a) and (b) below:

(a) the value of $S$ for the Type 316 stainless steel at design temperature given in Table C-1.1(c), Code Case N-253-11 Case 1 - Design Conditions, $8508 \mathrm{~F}$ operating
$\mathrm{S}_{850^{\circ} \mathrm{F}}=15,700 \mathrm{psi}$

Case 2 - Pressure Test, 1008F $\mathrm{S}_{100^{\circ} \mathrm{F}}=20,000 \mathrm{psi}$

(b) the value of factor B determined from the Tabulated Data, ASME Section II, Part D, Subpart 3, HA-2 which is determined as follows:

$$
A=\frac{0.125}{(R / T)}
$$

where

$R=$ inside radius of the cylindrical shell $=0.393$ inch

$\mathrm{T}=$ minimum thickness $=0.059$ inch

$$
A=\frac{0.125}{(0.393 / 0.059)}
$$


Case 1 - The capsule maximum temperature subjected to design conditions is 8508F. At $8508 \mathrm{~F}$ and $A=0.0188: B=10,865$

Case 2 - The capsule temperature is $1008 \mathrm{~F}$. At $1008 \mathrm{~F}$ and $\mathrm{A}=0.0188$ : $B=14,592$

Lesser value of (a) and (b)

Case 1 - The lesser value for design conditions was found in (b), therefore, the maximum allowable axial compression will be compared to $10,865 \mathrm{psi}$.

Case 2 - The lesser value for the pressure testing condition was found in (b), therefore, the maximum allowable axial compression will be compared to 14,592 psi.

Case 1 - Compute the longitudinal stress at design conditions:

$\sigma_{\mathrm{C}}=\frac{\mathrm{P}_{\mathrm{C}}}{\mathrm{A}_{\mathrm{C}}}$

where

$P_{c}=P_{p}+P_{s w}+P_{c s}$

where

$$
\begin{aligned}
P_{p}= & \text { axial load due to normal external pressure (reactor pressure plus } \\
& \text { sodium weight) }=7.3 \text { psi (Table III.C. } 2-1 \text { ) } \\
P_{s w}= & \text { axial load due to weight of the stacked components }=8.68 \mathrm{lbf} \text { (Table } \\
& \text { III.C. } 2-1) \text {. }
\end{aligned}
$$

Calculate $P_{p}$ : Total load from normal external pressure:

Axial load due to pressure

$$
\begin{aligned}
& P_{p}=\left(7.3 \times \frac{\pi}{4} \times\left(0.906^{2}\right)\right) \\
& \text { where } 0.906 \text { inch is the capsule outside diameter. } \\
& P_{p}= 4.7 \mathrm{lbf} \\
& P_{C}=(4.7+8.68+13.5) \mathrm{lbf} \\
& P_{C}=26.9 \mathrm{lbf} \\
& \sigma_{C}=\frac{P_{C}}{A_{C}}
\end{aligned}
$$




$$
=\frac{26.9}{\frac{\pi}{4} \times\left(0.906^{2}-0.785^{2}\right)}
$$

where 0.906 inch $=$ outside diameter and $0.785=$ inside diameter.

Thus

$$
\sigma_{\mathrm{c}}=174 p s i
$$

Since $\sigma_{\mathrm{c}}<10,865 \mathrm{psi}$, the longitudinal stress is acceptable

Case 2 - Compute the longitudinal stress during pressure test

$$
\begin{aligned}
\sigma_{C} & =\frac{P_{C}}{A_{C}} \\
& =\frac{1025}{\frac{\pi}{4} \times\left(0.906^{2}-0.785^{2}\right)}
\end{aligned}
$$

Thus

$$
\sigma_{\mathrm{C}}=6480 p s i
$$

Since $\sigma_{C}<14,592 \mathrm{psi}$, the longitudinal stress is acceptable 
Enclosure (7) to

MDO-723-0044/

B-MT(SRME)-52

Page 104

c. Internal Pressure Capsule Shell Calculation

The maximum allowable internal pressure of the JOYO-1 capsule shell given $950 \mathrm{psi}$ internal pressure and $850^{\circ} \mathrm{F}$ metal temperature is:

Per ND-3324.3(a), Circumferential Stress (Longitudinal Joints)

$$
\begin{aligned}
& P=\frac{\text { SEt }}{R+0.6 t} \text { where } \\
& \mathrm{t}=\quad \text { shell thickness }=0.059 \text { inch } \\
& \mathrm{P} \quad=\quad \text { design pressure (internal pressure) }=950 \mathrm{psi} \\
& \mathrm{R}=\quad \text { inside radius of the capsule }=0.393 \text { inch } \\
& \mathrm{S}=\text { maximum allowable stress of the Type } 316 \mathrm{H} \text { Stainless Steel } \\
& \text { Capsule at } 850^{\circ} \mathrm{F}=15,700 \text { psi } \\
& \mathrm{E}=\text { joint efficiency }=0.65 \text { for a category } \mathrm{B} \text { joint that is neither fully } \\
& \text { radiographed, partially radiographed, nor spot radiographed. } \\
& \text { Where an } E \text { of } 0.65 \text { applies to a type } 2 \text { weld for a single welded } \\
& \text { butt joint with backing strip per ND-4245. } \\
& P_{850 F}=\frac{15,700 \times 0.65 \times 0.059}{0.393+(0.6 \times 0.059)}=1,405 p s i>950 \mathrm{psi}
\end{aligned}
$$

Per ND-3324.3(b), Longitudinal Stress (Circumferential Joints)

$$
\begin{aligned}
& P=\frac{2 \times S E t}{R-0.4 t} \\
& P_{850 F}=\frac{2 \times 15,700 \times 0.65 \times 0.059}{0.393-(0.4 \times 0.059)}=3,260 \mathrm{psi}>950 \mathrm{psi}
\end{aligned}
$$

Since the maximum allowable internal pressure exceeds the design pressure of $950 \mathrm{psi}$, the capsule shell thickness requirements are satisfied.

\section{Subsection ND Code Calculations - Capsule Head and Capsule Base}

The Code calculations in this subsection apply to the Capsule Head (SK-AMT-2222) and Capsule Base (SK-AMT-2221). All heads have the same thickness, outside diameter and inside diameter. The head is conically shaped for sodium shedding. The head is assumed to be flat with a minimum head thickness of $0.115 \pm .010$ inch. This thickness is determined by projecting the head inside diameter 0.709 inch up to the conical surface. The head has a fillet radius to the inside diameter. The capsule base is flat with a fillet radius to the shell. The capsule base thickness is dimensional controlled to $0.113 \pm .014$ inch. No penetrations extend through the head or capsule base. 
Required thickness calculations will be shown for the design conditions since it consists of the highest temperature and therefore has the lowest allowable stress. The metal temperature of this head and base is $850^{\circ} \mathrm{F}$.

\section{a. Head Thickness Calculations}

Per ND-3325.2(b), the minimum required thickness of the head is:

where

$$
t=d \sqrt{\frac{C P}{S}}
$$

$t=$ required thickness of the head, inch

$d=$ inside diameter of head $=0.709$ inch

$\mathrm{C}=\mathrm{a}$ factor depending on construction. From Figure ND-3325-1, the head most closely resembles illustration $(h)$. Thus, $C=0.33$

$P=$ design pressure $=950$ psi

$S=$ maximum allowable stress of the Type $316 \mathrm{H}$ Stainless Steel Capsule

$$
\text { at } 850^{\circ} \mathrm{F}, \mathrm{S}=15,700 \mathrm{psi}
$$

Thus,

$$
\begin{gathered}
t_{850}=0.709 \sqrt{\frac{0.33 \times 950}{15,700}} \\
t_{850 F}=0.100 \text { inch }<\begin{array}{l}
\text { the actual }(0.105 \text { in. min) head thickness } \\
\text { so the requirements of ND-3325.2 are } \\
\text { met. }
\end{array}
\end{gathered}
$$

\section{b. Capsule Base Thickness Calculation}

As stated at the beginning of this section, the thickness of the capsule base is dimensionally controlled to $0.157 \pm .014$ inch.

Per ND-3325.2(b), the minimum required thickness of the bottom plate is:

$$
t=d \sqrt{\frac{C P}{S}}
$$

where:

$d=$ inside diameter of capsule $=0.787$ inch

$P=$ external pressure plus external load $=950$ psi

$\mathrm{S}=$ maximum allowable stress of the Type $316 \mathrm{H}$ Stainless Steel Capsule at $850^{\circ} \mathrm{F}=15,700 \mathrm{psi}$

$\mathrm{C}=\mathrm{a}$ factor depending on construction. From Figure ND-3325-1, the capsule base most closely resembles illustration (d). Thus, $C=0.13$ 
Enclosure (7) to

MDO-723-0044/

B-MT(SRME)-52

Page 106

$$
\begin{aligned}
& t_{850 F}=0.787 \sqrt{\frac{0.13(950)}{15,700}} \\
& t_{850 F}=0.070 \text { inch }<\text { the actual }(0.157) \text { base thickness so the capsule base } \\
& \text { meets the requirements of ND-3325.2 }
\end{aligned}
$$

\section{Additional Analysis}

Code Case $\mathrm{N}-254-13$ requires that the design guard against failure form low energy fracture. This analysis would have been performed.

\section{Finite Element Structural Model}

\section{a. Structural Model Description}

A finite element structural analysis was done on the most limiting capsule at worst case conditions (BCD capsule Rig 6A4, Tier 3, Compartment 2) to ensure structural adequacy of the capsule. The capsule's internals were stripped away in the structural analysis and only the pressure boundary was analyzed.

The analysis was done using the thermal finite element model described in the thermal analysis section. The one degree of freedom (temperature) PLANE35 elements were converted to the analogous structural six-node PLANE2 elements that have two degrees of freedom at each node (translation in $x$ and $y$ ). Resultant nodal temperatures from the thermal analysis were applied as temperature loads to the pressure boundary nodes.

In order to satisfy equilibrium, model constraints were applied to the $2 \mathrm{D}$ axisymmetric model. The centerline was fixed in the global $x$-direction to prevent spurious displacements. To simulate the confinement of the surrounding capsules, the pressure boundary was fixed locally in the y-direction at the base.

External forces applied to the model are; a force of $88.17 \mathrm{~N}$ applied to the top of the capsule accounting for the weight of the heaviest $(521 \mathrm{~g})$ capsules stacked in Tier 4 and 5 above it, the weight of the compartment spacer $(1.83 \mathrm{~kg})$, and the force of the compartment spring $(60 \mathrm{~N})$.

Internal forces applied to the model are; the pressure that results from the failure of all three biaxial creep specimens at the highest gamma heating and coolant temperature. This pressure was applied to the internal surfaces of the pressure boundary in the model. Also the force of the capsule wave spring (15.5 N) was applied to the top and bottom of the corresponding internal surface of the model.

b. Localized Stress Results

The ASME B \& PV Code, Section III, Division I, paragraph NB-3220 requires that stress intensity limits must be satisfied for the design loadings. The stresses induced as a result of the defined loadings are considered relative to the code.

(1) NB-3221.1 General Primary Membrane Stress Intensity

(2) NB-3221.2 Local Membrane Stress Intensity 
This stress intensity is derived from the average value across the thickness of a section of the primary stresses produced by design mechanical loads but excluding all thermal loads. These stresses are limited to $S_{m}$ at the design temperatures in general regions removed from any discontinuities, or $1.5 \mathrm{~S}_{\mathrm{m}}$ at the discontinuities. In all cases of the limiting capsule, the primary membrane stresses $(P, m)$ as illustrated by Table III.C.7-1 are much less than the allowable stress.

\begin{tabular}{|c|c|c|c|}
\hline \multicolumn{4}{|c|}{$\begin{array}{c}\text { Table III.C.7- } 1 \\
\text { NB-3221.1 General Primary Membrane Stress } \\
\text { Intensity } \\
\text { Worst Case BCD capsule }\end{array}$} \\
\hline Path & $\begin{array}{c}\text { Temp } \\
\text { (F) }\end{array}$ & $\begin{array}{c}\text { Sm } \\
\text { (psi) }\end{array}$ & $\begin{array}{l}P \\
m\end{array}$ \\
\hline Base 1 & 737.6 & 16,074 & 1,044 \\
\hline Base 2 & 766.4 & 15,902 & 1,751 \\
\hline Base 3 & 710.6 & 16,236 & 1,265 \\
\hline Wall 1 & 708.8 & 16,247 & 3,044 \\
\hline Wall 2 & 735.8 & 16,085 & 5,849 \\
\hline Wall 3 & 728.6 & 16,128 & 3,569 \\
\hline Cap 1 & 737.6 & 16,074 & 74 \\
\hline Cap 2 & 732.2 & 16,107 & 457 \\
\hline Cap 3 & 743 & 16,042 & 1,476 \\
\hline Joint & 734 & 16,096 & 2,296 \\
\hline \multicolumn{4}{|c|}{$S_{m}=$ allowable stress intensity $P=$ Primary } \\
\hline
\end{tabular}

(3) NB-3221.3 Primary Membrane plus Primary Bending Stress Intensity

This stress intensity is derived from the highest value of membrane stresses plus bending stresses produced by the design mechanical loads, but excluding all thermal loads. These stresses are limited to $1.5 \mathrm{~S}_{\mathrm{m}}$. As shown in Table III.C.7-2, the primary membrane plus bending stresses $(P, m+b)$ are much less than the allowable stress. 
Enclosure (7) to

MDO-723-0044I

B-MT(SRME)-52

Page 108

\begin{tabular}{|c|c|c|c|}
\hline \multicolumn{4}{|c|}{$\begin{array}{c}\text { Table III.C.7- } 2 \\
\text { NB-3221.3 Primary Membrane plus Primary Bending } \\
\text { Stress Intensity } \\
\text { Worst Case BCD capsule }\end{array}$} \\
\hline Path & $\begin{array}{c}\text { Temp } \\
\text { (F) }\end{array}$ & $\begin{array}{c}1.5 \mathrm{sm} \\
(\mathrm{psi})\end{array}$ & $\begin{array}{c}P \\
m+b\end{array}$ \\
\hline Base 1 & 737.6 & 24,112 & 3,320 \\
\hline Base 2 & 766.4 & 23,852 & 2,296 \\
\hline Base 3 & 710.6 & $24, \overline{355}$ & 4,257 \\
\hline Wall 1 & 708.8 & 24,371 & 8,292 \\
\hline Wall 2 & 735.8 & 24,128 & 6,664 \\
\hline Wall 3 & 728.6 & 24,193 & 4,084 \\
\hline Cap 1 & 737.6 & 24,112 & 299 \\
\hline Cap 2 & 732.2 & 24,160 & 3,403 \\
\hline Cap 3 & 743 & 24,063 & 3,935 \\
\hline Joint & 734 & 24,144 & 3,696 \\
\hline \multicolumn{2}{|c|}{$S_{m}=$ allowable stress intensi } & \multicolumn{2}{|c|}{$\mathrm{m}=$ membrane $\mathrm{b}=$ bending } \\
\hline
\end{tabular}

(4) NB-3222.2 Primary Plus Secondary Stress Intensity

This stress intensity is derived from the highest value of the primary membrane, plus bending, plus secondary stresses induced by the design loading conditions, mechanical and thermal. These stresses are limited to $3 \mathrm{~S}_{\mathrm{m}}$. Again, as illustrated by Table III.C.7-3 the primary membrane, plus bending, plus secondary $(P+Q, m+b)$ are less than the allowable stress.

\begin{tabular}{|c|c|c|c|}
\hline \multicolumn{4}{|c|}{ Table III.C.7-3 } \\
NB-3222.2 Primary plus Secondary Stress Intensity \\
Worst Case BCD capsule \\
\hline Path & $\begin{array}{c}\text { Temp } \\
\text { (F) }\end{array}$ & $\begin{array}{c}\text { 3 Sm } \\
\text { (psi) }\end{array}$ & $\begin{array}{c}\mathrm{P}+\mathrm{Q} \\
\mathrm{m}+\mathrm{b}\end{array}$ \\
\hline Base 1 & 737.6 & 48,223 & 9,990 \\
\hline Base 2 & 766.4 & 47,705 & 11,319 \\
\hline Base 3 & 710.6 & 48,709 & 5,855 \\
\hline Wall 1 & 708.8 & 48,742 & 9,742 \\
\hline Wall 2 & 735.8 & 48,256 & 10,633 \\
\hline Wall 3 & 728.6 & 48,385 & 4,711 \\
\hline Cap 1 & 737.6 & 48,223 & 1,033 \\
\hline Cap 2 & 732.2 & 48,320 & 3,479 \\
\hline Cap 3 & 743 & 48,126 & 5,648 \\
\hline Joint & 734 & 48,288 & 2,999 \\
\hline $\mathrm{S}_{\mathrm{m}}$ a allowable stress intensity & $\mathrm{P}=$ Primary & $\mathrm{Q}=$ Secondary \\
& & $\mathrm{m}=$ membrane b $=$ bending \\
\hline
\end{tabular}


Enclosure (7) to

MDO-723-0044/

B-MT(SRME)-52

Page 109

Tables III.C.7-4 provides linearized stress intensity values in both metric and English units for all loading cases on the bounding case under the identified operating conditions, at the specified locations. In the tables $\mathrm{P}$ denotes primary loading, $Q$ denotes secondary loading, $m$ denotes membrane stress, and $m+b$ denotes membrane plus bending stress. Path locations are shown on the pressure boundary in Figure III.C.7-1.

Table III.C.7-4

BCD Capsule Categorized Stresses

Rig A, Tier 3, Compartment 2 Metric Units

Worst case 1.64 Factor (loss of flow (1.25), hot spot (1.05), and an overpower condition (1.25)

\begin{tabular}{|c|c|c|c|c|c|c|c|c|c|}
\hline Path & $\begin{array}{c}\text { Temp } \\
(\mathrm{K})\end{array}$ & $\begin{array}{l}\mathrm{Sm} \\
(\mathrm{Pa})\end{array}$ & $\begin{array}{l}3 \mathrm{Sm} \\
(\mathrm{Pa}) \\
\end{array}$ & $\begin{array}{c}P+Q \\
m \\
\end{array}$ & $\begin{array}{l}P+Q \\
m+b\end{array}$ & $\begin{array}{l}P \\
m\end{array}$ & $\begin{array}{c}P \\
m+b\end{array}$ & $\begin{array}{l}\mathrm{Q} \\
\mathrm{m}\end{array}$ & $\begin{array}{c}a \\
m+b\end{array}$ \\
\hline Base 1 & 665 & $1.11 E+08$ & $3.32 \mathrm{E}+08$ & $8.50 \mathrm{E}+06$ & $6.89 \mathrm{E}+07$ & $7.20 \mathrm{E}+06$ & $2.29 \mathrm{E}+07$ & $1.48 E+07$ & $5.94 \mathrm{E}+07$ \\
\hline Base 2 & 681 & $1.10 \mathrm{E}+08$ & $3.29 \mathrm{E}+08$ & 1.42E+07 & $7.80 \mathrm{E}+07$ & $1.21 \mathrm{E}+07$ & $1.58 \mathrm{E}+07$ & $1.79 \mathrm{E}+07$ & $7.79 E+07$ \\
\hline Base 3 & 650 & $1.12 E+08$ & $3.36 E+08$ & $3.90 \mathrm{E}+07$ & $4.04 \mathrm{E}+07$ & 8.73E+06 & $2.94 E+07$ & $3.52 \mathrm{E}+07$ & $5.61 E+07$ \\
\hline Wall 1 & 649 & $1.12 E+08$ & $3.36 \mathrm{E}+08$ & $5.47 \mathrm{E}+07$ & $6.72 \mathrm{E}+07$ & $2.10 \mathrm{E}+07$ & $5.72 \mathrm{E}+07$ & $5.97 \mathrm{E}+07$ & $9.60 E+07$ \\
\hline Wall 2 & 664 & $1.11 E+08$ & $3.33 E+08$ & 4.30E+07 & $7.33 \mathrm{E}+07$ & $4.03 E+07$ & $4.60 \mathrm{E}+07$ & $3.99 \mathrm{E}+06$ & $4.11 \mathrm{E}+07$ \\
\hline Wall 3 & 660 & $1.11 \mathrm{E}+08$ & $3.34 E+08$ & $2.88 \mathrm{E}+07$ & $3.25 E+07$ & $2.46 \mathrm{E}+07$ & $2.82 \mathrm{E}+07$ & $5.27 \mathrm{E}+06$ & 1.17E+07 \\
\hline Cap 1 & 665 & $1.11 \mathrm{E}+08$ & $3.32 E+08$ & $4.24 E+06$ & $7.12 \mathrm{E}+06$ & $5.11 \mathrm{E}+05$ & 2.06E+06 & $4.27 \mathrm{E}+06$ & $6.01 E+06$ \\
\hline $\mathrm{Cap} 2$ & 662 & $1.11 E+08$ & $3.33 E+08$ & 1.17E+07 & $2.40 \mathrm{E}+07$ & $3.15 \mathrm{E}+06$ & $2.35 \mathrm{E}+07$ & $1.23 \mathrm{E}+07$ & 1.80E+07 \\
\hline Cap 3 & 668 & $1.11 E+08$ & $3.32 E+08$ & $6.42 \mathrm{E}+06$ & $3.89 \mathrm{E}+07$ & $1.02 \mathrm{E}+07$ & $2.71 E+07$ & $7.84 E+06$ & $2.73 \mathrm{E}+07$ \\
\hline Joint & 663 & $1.11 \mathrm{E}+08$ & $3.33 E+08$ & 1.31E+07 & $2.07 E+07$ & $1.58 E+07$ & $2.55 \mathrm{E}+07$ & $9.39 E+06$ & $1.75 \mathrm{E}+07$ \\
\hline
\end{tabular}

English Units

\begin{tabular}{|c|c|c|c|c|c|c|c|c|c|}
\hline Path & $\begin{array}{c}\text { Temp } \\
(F)\end{array}$ & $\begin{array}{c}\mathrm{sm} \\
\text { (psi) }\end{array}$ & $\begin{array}{l}3 \mathrm{Sm} \\
\text { (psi) }\end{array}$ & $\begin{array}{c}\mathrm{P}+\mathrm{Q} \\
\mathrm{m}\end{array}$ & $\begin{array}{l}P+Q \\
m+b\end{array}$ & $\begin{array}{l}P \\
m\end{array}$ & $\begin{array}{c}P \\
m+b\end{array}$ & $\begin{array}{l}Q \\
m\end{array}$ & $\begin{array}{c}Q \\
m+b\end{array}$ \\
\hline Base 1 & 737.6 & 16,074 & 48,223 & 1,233 & 9,990 & 1,044 & 3,320 & 2,147 & 8,612 \\
\hline Base 2 & 766.4 & 15,902 & 47,705 & 2,062 & 91,319 & 1,751 & 2,296 & 2,596 & 11,295 \\
\hline Base 3 & 710.6 & 16,236 & 48,709 & 5,659 & 5,855 & 1,265 & 4,257 & 5,098 & 8,141 \\
\hline Wall 1 & 708.8 & 16,247 & 48,742 & 7,926 & 9,742 & 3,044 & 8,292 & 8,651 & 13,924 \\
\hline Wall 2 & 735.8 & 16,085 & 48,256 & 6,238 & 10,633 & 5,849 & 6,664 & 578 & 5,965 \\
\hline Wall 3 & 728.6 & 16,128 & 48,385 & 4,181 & 4,711 & 3,569 & 4,084 & 764 & 1,691 \\
\hline Cap 1 & 737.6 & 16,074 & 48,223 & 616 & 1.033 & 74 & 299 & 619 & 872 \\
\hline Cap 2 & 732.2 & 16,107 & 48,320 & 1.703 & 3,479 & 457 & 3,403 & 1,785 & 2,615 \\
\hline Cap 3 & 743 & 16,042 & $48, \overline{126}$ & 931 & 5,648 & 1,476 & 3,935 & 1,137 & 3,961 \\
\hline Joint & 734 & 16,096 & 48,288 & 1,894 & 2,999 & 2,296 & 3,696 & 1,361 & 2,534 \\
\hline
\end{tabular}
$P=$ Primary
$Q=$ Secondary
$m=$ membrane
$b=$ bending
$S_{m}=$ allowable stress intensity 
Enclosure (7) to

MDO-723-0044/

B-MT(SRME)-52

Page 110
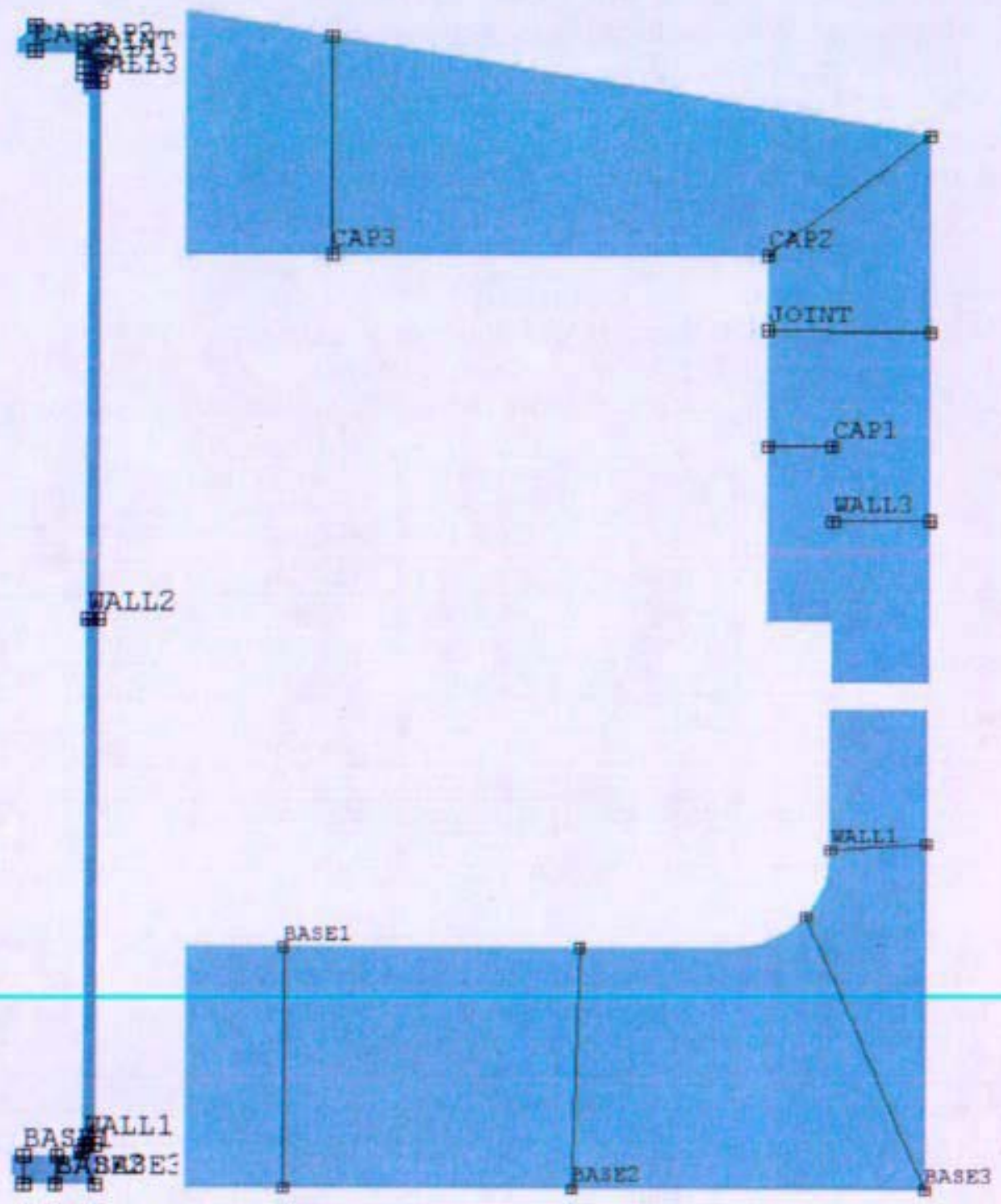

Figure III.C.7-1 BCD capsule stress linearization cut line locations. 


\title{
IV. Capsule Assembly
}

Page 111

\section{A. Overall Plan}

An assembly strategy was developed for all 60 of the JOYO capsules plus ten spares. The sequence of assembly initially focused on the fabricating the capsules based on the type of gas to be entrained in each capsule. This arrangement would allow for the most efficient welding process whereby the transitions and setup time from one gas combination to another were minimized. This strategy was the basis for planning the work effort with all the support groups and for providing delivery schedules for PNNL and JNC.

As the development and fabrication of the material test specimens progressed and more information on the status of their delivery was received, the assembly sequence strategy for the capsules was modified to accommodate the assembly of capsules with the material specimens that would be received first. At the time of the restructuring of the Prometheus Program, the NRPCT was starting to rearrange the capsule assembly work plan to accommodate partial shipments of biaxial creep specimens. In addition, the planning for the initial welding configuration was being determined in order to establish the most efficient process to minimize the number of transitions in gas supply. Note that the design of the capsules used three combinations of gas to obtain the desired test temperatures of the material test specimens.

Early in the program, a process was established at Bettis for the assembly of the JOYO capsules. Various location or labs at Bettis were committed for the stages of the assembly process such as inspection, cleaning, assembly and welding. Equipment was procured and the assembly process was established as of the project closeout. The assembly of the capsules at Bettis allowed for the most control of the end product capsule and more control of the assembly resources and assembly time. Control of the assembly process was considered important due to the expedited schedule that was invoked in order to get the capsules installed into JOYO in June 2006. Bettis considered the use of outside vendors for the assembly process and/or portions of the process, but Bettis has program experience in the design, assembly, and delivery of these types of capsules. Therefore the Bettis process was considered the most efficient process for the JOYO need.

The assembly process was a series of operations as shown in Figure IV.A-1 and as described in the following sections. The basic operations involved are:

\author{
- Hardware and Specimen Cleaning \\ - Bakeout of all Moisture \\ - Subassembly of all Specimens in holders \\ - Capsule Assembly \\ - Capsule Welding \\ - Capsule Inspection \\ - Capsule Leak Testing
}




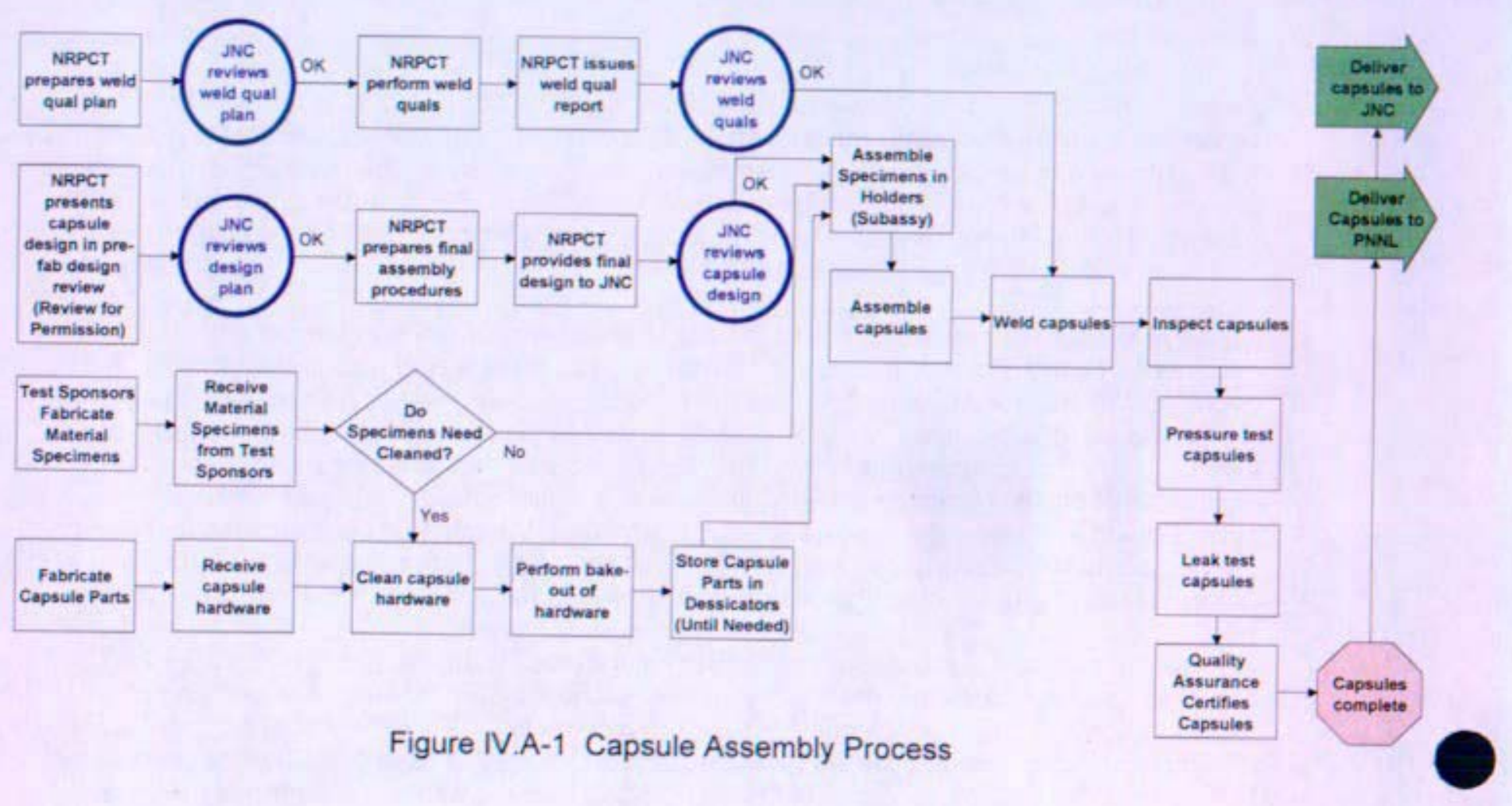

\section{B. Capsule Assembly}

\section{Cleaning Process}

All internal capsule components were to be cleaned to prevent contaminating test specimens which could alter test results. The final cleaning procedure (Attachment $E$ to this Enclosure) was drafted detailing the JOYO parts cleaning and handling to meet this objective. This cleaning procedure focused on the removal of all greases and particles that may have accumulated on the parts during manufacturing and shipping. Dissimilar material types were not to be cleaned together. For instance, all stainless steel parts should be cleaned together and all graphite parts should be cleaned together. It was necessary to drain the tank after cleaning all graphite parts because graphite tends to leave a residue in the bath that could deposit on any non graphite parts.

When molybdenum parts are put through an ultrasonic cleaning of deionized water, the parts tend to form an oxide layer. This oxide layer would be detrimental to the reactor and to the performance and accuracy of the test. Therefore, the procedure notes that all molybdenum parts would be put through the 2-propanol and acetone baths only.

\section{Bake-out Process}

The bake-out process ensures that the surfaces of the parts are free of moisture and trace gases. The bake-out procedure (Attachment $F$ to this Enclosure) outlines the steps necessary to complete this task. The process requires the parts to be in a vacuum 
environment at about $752^{\circ} \mathrm{C}$ and $5 \times 10^{-5}$ torr for 2 hours. Due to the considerable quantity of parts, a large capacity furnace was planned.

To prevent cross contamination, the furnace would receive a pre-bake. The furnace would be heated to $1000^{\circ} \mathrm{F}$ and held for at least one hour. Any contaminants in the furnace from previous heat treat runs would diffuse out before the new parts were placed into the furnace.

To enhance vacuum performance, a getter material would be used during the bake-out to remove traces of gas. A getter of molybdenum foil would be bent into a triangular shape and placed over all of the parts like a tent in lieu of wrapping parts individually.

It was estimated that 4 to 5 runs would be necessary to complete the bake out of all of the parts. One run would take approximately 2 days including ramping up and down time of the furnace. All of the parts would be baked out within 3 weeks. In the event that the planned furnace would not be available, smaller furnaces at Bettis were available.

\section{Temporary Storage}

After all the parts have been cleaned and dried they were to be placed into desiccators to keep all parts clean, dry, and well organized until they were needed for assembly. In order to avoid any confusion among parts and to ensure that adequate space is available, six desiccators were to be used. This allowed each of the three gas mixtures for both Rig $6 \mathrm{A4}$ and Rig 6D4 to have its own desiccators so that all of the parts could be appropriately arranged in a way that facilitates the assembly process.

Since it is crucial to have good airflow through the dessicators, spacing of the parts within the dessicators must be considered. Table IV.A-1 and Table IV.A-2 show the total area necessary for storing all the parts and specimens when everything is lying down in its most stable position. The table is separated into Rig 6A4 and Rig 6D4, both of which are then broken down into their three respective gas mixtures. This shows the total number of pieces in each gas mixture along with the total area of these pieces. The total area for both rigs of about 1240 in $^{2}$ with all the pieces packed tightly together. Increasing this area by $25 \%$ brings the total area to $1550 \mathrm{in}^{2}$ and allows the pieces to be slightly spaced-out, providing better airflow throughout the desiccators. 
Enclosure (7) to

MDO-723-0044/

B-MT(SRME)-52

Page 114

\begin{tabular}{|c|c|c|c|c|c|c|c|c|c|}
\hline \multicolumn{10}{|c|}{ Table IV.A-1: JOYO-1 Rig A Gas Mixture Hardware List } \\
\hline DRAWING NO & PART & $80-20$ & $\frac{\text { AREA }}{\text { in }^{2}}$ & $30-70$ & $\frac{\text { AREA }}{\text { in }^{2}}$ & $100 \mathrm{He}$ & $\frac{\text { AREA }}{\mathrm{in}^{2}}$ & TOTAL & $\frac{\text { T. AREA }}{\text { in }^{2}}$ \\
\hline SK-AMT-2221-H01 & CAPSULE & 13 & 74.43 & 12 & 68.70 & 5 & 28.63 & 30 & 171.75 \\
\hline SK-AMT-2222-HO1 & HEAD & 13 & 10.66 & 12 & 9.84 & 5 & 4.10 & 30 & 24.60 \\
\hline SK-AMT-2239-H01 & WAVE SPRING & 13 & 6.24 & 12 & 5.76 & 5 & 2.40 & 30 & 14.40 \\
\hline SK-AMT-2223-H01 & LOWER SPACER & 13 & 7.28 & 12 & 6.72 & 5 & 2.80 & 30 & 16.80 \\
\hline SK-AMT-2224-H05 & LOWER INSULATOR - SHORT & 9 & 5.31 & 6 & 3.54 & 2 & 1.18 & 17 & 10.03 \\
\hline SK-AMT-22224-H06 & LOWER INSULATOR - MEDIUM & 1 & 0.59 & 1 & 0.59 & 0 & 0.00 & 2 & 1.18 \\
\hline SK-AMT-22224-H07 & LOWER INSULATOR - LONG & 3 & 1.77 & 5 & 2.95 & 3 & 1.77 & 11 & 6.49 \\
\hline SK-AMT-2223-H02 & UPPER SPACER & 13 & 7.28 & 12 & 6.72 & 5 & 2.80 & 30 & 16.80 \\
\hline SK-AMT-2224-H01 & UPPER INSULATOR - SHORT & 4 & 2.36 & 4 & 2.36 & 3 & 1.77 & 11 & 6.49 \\
\hline SK-AMT-22224-H02 & UPPER INSULATOR - MEDIUM & 0 & 0.00 & 1 & 0.59 & 0 & 0.00 & 1 & 0.59 \\
\hline SK-AMT-2224-H03 & UPPER INSULATOR - LONG & 5 & 295 & 2 & 1.18 & 1 & 0.59 & 8 & 4.72 \\
\hline SK-AMT-2224-H04 & UPPER INSULATOR - XLONG & 4 & 2.36 & 5 & 2.95 & 1 & 0.59 & 10 & 5.90 \\
\hline SK-AMT-2220-H01 & THERMAL SHEILD - SHORT & 0 & 0.00 & 1 & 3.70 & 2 & 7.40 & 3 & 11.11 \\
\hline SK-AMT-2220-H02 & THERMAL SHIELD - LONG & 0 & 0.00 & 0 & 0.00 & 0 & 0.00 & 0 & 0.00 \\
\hline SK-RRM1011-G01 & FLUX WIRE ASSEMBLY & 11 & 0.50 & 14 & 0.63 & 3 & 0.14 & 28 & 1.26 \\
\hline SK216C9452 & TEMPERATURE MONITOR (7) & 1 & 0.01 & 1 & 0.01 & 0 & 0.00 & 2 & 0.02 \\
\hline SK216C9452 & TEMPERATURE MONITOR (14) & 11 & 0.24 & 14 & 0.31 & 3 & 0.07 & 28 & 0.62 \\
\hline SK216C9452 & TEMPERATURE MONITOR (28) & 26 & 1.14 & 18 & 0.79 & 6 & 0.26 & 50 & 2.20 \\
\hline SK216C9452 & TEMPERATURE MONITOR (33) & 0 & 0.00 & 2 & 0.10 & 4 & 0.21 & 6 & 0.31 \\
\hline SK-AMT-2228-H01 & DENSITY DISK HOLDER - SHORT & 8 & 4.72 & 7 & 4.13 & 2 & 1.18 & 17 & 10.03 \\
\hline SK-AMT-2228-HO2 & DENSITY DISK HOLDER - MEDIUM & 4 & 2.36 & 3 & 1.77 & 1 & 0.59 & 8 & 4.72 \\
\hline SK-AMT-2228-H03 & DENSITY DISK HOLDER - LONG & 1 & 0.59 & 1 & 0.59 & 0 & 0.00 & 2 & 1.18 \\
\hline SK-AMT-2225-H01 & BIAXIAL CREEP OUTER HOLDER & 26 & 30.52 & 18 & 21.13 & 6 & 7.04 & 50 & 58.70 \\
\hline SK-AMT-2225-HO2 & BIAXIAL CREEP INNER HOLDER & 26 & 15.60 & 18 & 10.80 & 6 & 3.60 & 50 & 30.00 \\
\hline SK-AMT-2225-HO3 & BIAXIAL CREEP WASHER & 26 & 1.87 & 18 & 1.30 & 6 & 0.43 & 50 & 3.60 \\
\hline SK-AMT-2227-G01 & FRACTURE TOUGHNESS HOLDER & 4 & 9,10 & 5 & 11.38 & 1 & 2.28 & 10 & 22.75 \\
\hline SK-AMT-2226-G01 & TENSILE HOLDER & 7 & 15.93 & 9 & 20.48 & 2 & 4.55 & 18 & 40.95 \\
\hline SK-AMT-2235-HO1 & CT HOLDER & 5 & 2.95 & 4 & 2.36 & 1 & 0.59 & 10 & 5.90 \\
\hline SK-AMT-2228-HO4 & DISK HOLDER - BEO & 4 & 2.36 & 4 & 2.36 & 0 & 0.00 & 8 & 4.72 \\
\hline SK-AMT-2241-H01 & COMPRESSION HOLDER - BEO & 1 & 0.59 & 1 & 0.59 & 0 & 0.00 & 2 & 1.18 \\
\hline SK-RRM1024 & BEO SPACER & 4 & 0.98 & 4 & 0.98 & 0 & 0.00 & 8 & 1.97 \\
\hline SK-AMT-2240-H02 & DISK GRAPHITE HOLDER & 0 & 0.00 & 2 & 0.81 & 4 & 1.63 & 6 & 2.44 \\
\hline SK-AMT-2240-H03 & CERAMIC STRIP GRAPHITE HOLDER & 0 & 0.00 & 0 & 0.00 & 0 & 0.00 & 0 & 0.00 \\
\hline SK-AMT-2240-H01 & TENSILE GRAPHITE HOLDER & 0 & 0.00 & 2 & 2.25 & 4 & 4.49 & 6 & 6.74 \\
\hline 5D16357 & DENSITY DISK SPECIMEN & 61 & 14.70 & 51 & 12.29 & 13 & 3.13 & 125 & 30.13 \\
\hline DD15996 & BIAXIAL CREEP SPECIMEN & 26 & 8.13 & 18 & 5.63 & 6 & 1.88 & 50 & 15.63 \\
\hline 5016338 & TENSILE SPECIMEN & 70 & 13.65 & 90 & 17.55 & 20 & 3.90 & 180 & 35.10 \\
\hline $5 D 16339$ & FRACTURE TOUGHNESS SPECIMEN & 36 & 4.25 & 45 & 5.31 & 9 & 1.06 & 90 & 10.62 \\
\hline SK216C9453 & BEO DISK SPECIMEN & 8 & 1.94 & 8 & 1.94 & 0 & 0.00 & 16 & 3.87 \\
\hline $5 D 16356$ & CT SPECIMEN & 10 & 3.77 & 8 & 3.02 & 2 & 0.75 & 20 & 7.54 \\
\hline SK216C9454 & BEO COMPRESSION SPECIMEN & 4 & 0.20 & 4 & 0.20 & 0 & 0.00 & 8 & 0.40 \\
\hline $216 C 6883$ & MONOLITHIC DISK SPECIMEN & 0 & 0.00 & 6 & 1.53 & 12 & 3.06 & 18 & 4.59 \\
\hline $216 C 6883$ & COMPOSITE DISK SPECIMEN & 0 & 0.00 & 6 & 1.53 & 12 & 3.06 & 18 & 4.59 \\
\hline SK110E3981 & CERAMIC STRIP SPECIMEN & 0 & 0.00 & 0 & 0.00 & 0 & 0.00 & 0 & 0.00 \\
\hline SK239B9368 & CERAMIC TENSILE SPECIMEN & 0 & 0.00 & 12 & 4.61 & 24 & 9.22 & 36 & 13.82 \\
\hline & Total & 471 & 257.32 & 478 & 251.97 & 184 & 107.14 & 1133 & 616.43 \\
\hline
\end{tabular}


Enclosure (7) to

MDO-723-0044/

B-MT(SRME)-52

Page 115

\begin{tabular}{|c|c|c|c|c|c|c|c|c|c|}
\hline \multicolumn{10}{|c|}{\begin{tabular}{|ll} 
& Table IV.A-2: JOYO-1 Rig B Gas Mixture Hardware List \\
DRAWING NO & PART
\end{tabular}} \\
\hline DRAWING NO & PART & $80-20$ & $\frac{\text { AREA }}{\mathrm{in}^{2}}$ & $30-70$ & $\frac{\text { AREA }}{\text { in }^{2}}$ & $100 \mathrm{He}$ & $\frac{\text { AREA }}{\mathrm{in}^{2}}$ & TOTAL & $\frac{\text { I. AREA }}{i^{2}}$ \\
\hline SK-AMT-2221-H01 & CAPSULE & 13 & 74.43 & 12 & 68.70 & 5 & 28.63 & 30 & 171.75 \\
\hline SK-AMT-2222-H01 & HEAD & 13 & 10.66 & 12 & 9.84 & 5 & 4.10 & 30 & 24.60 \\
\hline SK-AMT-2239-H01 & WAVE SPRING & 13 & 6.24 & 12 & 5.76 & 5 & 2.40 & 30 & 14.40 \\
\hline SK-AMT-2223-H01 & LOWER SPACER & 13 & 7.28 & 12 & 6.72 & 5 & 2.80 & 30 & 16.80 \\
\hline SK-AMT-22224-H05 & LOWER INSULATOR - SHORT & 8 & 4.72 & 8 & 4.72 & 3 & 1.77 & 19 & 11.21 \\
\hline SK-AMT-2224-H06 & LOWER INSULATOR - MEDIUM & 1 & 0.59 & 2 & 1.18 & 1 & 0.59 & 4 & 2.36 \\
\hline SK-AMT-2224-H07 & LOWER INSULATOR - LONG & 4 & 2.36 & 2 & 1.18 & 1 & 0.59 & 7 & 4.13 \\
\hline SK-AMT-2223-H02 & UPPER SPACER & 13 & 7.28 & 12 & 6.72 & 5 & 2.80 & 30 & 16.80 \\
\hline SK-AMT-2224-H01 & UPPER INSULATOR - SHORT & 4 & 2.36 & 2 & 1.18 & 2 & 1.18 & 8 & 4.72 \\
\hline SK-AMT-2224-HO2 & UPPER INSULATOR - MEDIUM & 1 & 0.59 & 3 & 1.77 & 0 & 0.00 & 4 & 2.36 \\
\hline SK-AMT-2224-HO3 & UPPER INSULATOR - LONG & 3 & 1.77 & 4 & 2.36 & 2 & 1.18 & 9 & 5.31 \\
\hline SK-AMT-2224-HO4 & UPPER INSULATOR - XLONG & 5 & 2.95 & 3 & 1.77 & 1 & 0.59 & 9 & 5.31 \\
\hline SK-AMT-2220-H01 & THERMAL SHEILD - SHORT & 0 & 0.00 & 0 & 0.00 & 0 & 0.00 & 0 & 0.00 \\
\hline SK-AMT-2220-H02 & THERMAL SHIELD - LONG & 0 & 0.00 & 1 & 3.76 & 1 & 3.76 & 2 & 7.52 \\
\hline SK-RRM1011-G01 & FLUX WIRE ASSEMBLY & 16 & 0.72 & 18 & 0.81 & 5 & 0.23 & 39 & 1.76 \\
\hline SK216C9452 & TEMPERATURE MONITOR ( 7$)$ & 1 & 0.01 & 1 & 0.01 & 0 & 0.00 & 2 & 0.02 \\
\hline SK216C9452 & TEMPERATURE MONITOR (14) & 16 & 0.35 & 18 & 0.40 & 5 & 0.11 & 39 & 0.86 \\
\hline SK216C9452 & TEMPERATURE MONITOR (28) & 22 & 0.97 & 16 & 0.70 & 7 & 0.31 & 45 & 1.98 \\
\hline SK216C9452 & TEMPERATURE MONITOR (33) & 0 & 0.00 & 2 & 0.10 & 2 & 0.10 & 4 & 0.21 \\
\hline SK-AMT-2222-H01 & DENSITY DISK HOLDER - SHORT & 8 & 4.72 & 8 & 4.72 & 2 & 1.18 & 18 & 10.62 \\
\hline SK-AMT-2228-H02 & DENSITY DISK HOLDER - MEDIUM & 4 & 2.36 & 2 & 1.18 & 2 & 1.18 & 8 & 4.72 \\
\hline SK-AMT-2228-HO3 & DENSITY DISK HOLDER - LONG & 1 & 0.59 & 1 & 0.59 & 0 & 0.00 & 2 & 1.18 \\
\hline SK-AMT-2225-H01 & BIAXIAL CREEP OUTER HOLDER & 22 & 25.83 & 16 & 18.78 & 7 & 8.22 & 45 & 52.83 \\
\hline SK-AMT-2225-HO2 & BIAXIAL CREEP INNER HOLDER & 22 & 13.20 & 16 & 9.60 & 7 & 4.20 & 45 & 27.00 \\
\hline SK-AMT-2225-HO3 & BIAXIAL CREEP WASHER & 22 & 1.58 & 16 & 1.15 & 7 & 0.50 & 45 & 3.24 \\
\hline SK-AMT-2227-G01 & FRACTURE TOUGHNESS HOLDER & 6 & 13.65 & 8 & 18.20 & 2 & 4.55 & 16 & 36.40 \\
\hline SK-AMT-2226-G01 & TENSILE HOLDER & 10 & 22.75 & 10 & 22.75 & 3 & 6.83 & 23 & 52.33 \\
\hline SK-AMT-2235-HO1 & CT HOLDER & 5 & 2.95 & 3 & 1.77 & 2 & 1.18 & 10 & 5.90 \\
\hline SK-AMT-2228-H04 & DISK HOLDER - BEO & 4 & 236 & 4 & 2.36 & 0 & 0.00 & 8 & 4.72 \\
\hline SK-AMT-2241-H01 & COMPRESSION HOLDER - BEO & 1 & 0.59 & 1 & 0.59 & 0 & 0.00 & 2 & 1.18 \\
\hline KK-RRM1024 & BEO SPACER & 4 & 0.98 & 4 & 0.98 & 0 & 0.00 & 8 & 1.97 \\
\hline SK-AMT-2240-H02 & DISK GRAPHITE HOLDER & 0 & 0.00 & 1 & 0.41 & 1 & 0.41 & 2 & 0.81 \\
\hline SK-AMT-2240-HO3 & CERAMIC STRIP GRAPHITE HOLDER & 0 & 0.00 & 2 & 2.73 & 2 & 273 & 4 & 5.46 \\
\hline SK-AMT-2240-H01 & TENSILE GRAPHITE HOLDER & 0 & 0.00 & 0 & 0.00 & 0 & 0.00 & 0 & 0.00 \\
\hline 5D16357 & DENSITY DISK SPECIMEN & 61 & 14.70 & 47 & 11.33 & 20 & 4.82 & 128 & 30.85 \\
\hline $5 D 15996$ & BIAXIAL CREEP SPECIMEN & 22 & 6.88 & 16 & 5.00 & 7 & 2,19 & 45 & 14.06 \\
\hline 5D16338 & TENSILE SPECIMEN & 100 & 19.50 & 100 & 19.50 & 30 & 5.85 & 230 & 44.85 \\
\hline $5 D 16339$ & FRACTURE TOUGHNESS SPECIMEN & 54 & 6.37 & 72 & 8.50 & 18 & 2.12 & 144 & 16.99 \\
\hline SK216C9453 & BEO DISK SPECIMEN & 8 & 1.94 & 8 & 1.94 & 0 & 0.00 & 16 & 3.87 \\
\hline $5 D 16356$ & CT SPECIMEN & 10 & 3.77 & 6 & 2.26 & 4 & 1.51 & 20 & 7.54 \\
\hline SK216C9454 & BEO COMPRESSION SPECIMEN & 4 & 0.20 & 4 & 0.20 & 0 & 0.00 & 8 & 0.40 \\
\hline $216 \mathrm{C} 6883$ & MONOLITHIC DISK SPECIMEN & 0 & 0.00 & 3 & 0.77 & 3 & 0.77 & 6 & 1.53 \\
\hline $216 C 6883$ & COMPOSITE DISK SPECIMEN & 0 & 0.00 & 3 & 0.77 & 3 & 0.77 & 6 & 1.53 \\
\hline SK110E3981 & CERAMIC STRIP SPECIMEN & 0 & 0.00 & 8 & 0.50 & 8 & 0.50 & 16 & 0.99 \\
\hline SK239B9368 & CERAMIC TENSILE SPECIMEN & 0 & 0.00 & 0 & 0.00 & 0 & 0.00 & 0 & 0.00 \\
\hline & Total & 514 & 268.20 & 499 & 254.25 & 183 & 100.62 & 1196 & 623.07 \\
\hline
\end{tabular}


Enclosure (7) to

MDO-723-0044/

B-MT(SRME)-52

Page 116

\section{Capsule Preassembly/Assembly}

The initial step of the capsule assembly was to be the installation of the test specimen into the associated holder. This subassembly was to be built as quickly as possible to prevent the parts from being exposed to the air to preclude build up of any oxides. Preprocess planning is a key in order to assure the assembly proceeds well and stoppages are eliminated and all tools are available and ready for use.

After the subassemblies are complete and the appropriate parts have been gathered for a particular set of capsules to be assembled, all parts, tools and subassemblies can be entered into the welding glovebox. The following list includes the necessary items that were to be used in the glovebox:

- Assembled test holders (Subassemblies)

- Capsule Hardware

- Trays and racks to organize test holders and hardware

- Assembly tools

- JOYO head clamps

- Welding Fixture

A complete listing of the capsule hardware and assembled test holders needed for each gas mixture can be found below in Table IV.A-3.

\begin{tabular}{|c|c|c|c|c|c|c|c|c|}
\hline & RIG A & & & & RIG B & & & \\
\hline Subassembly & $80-20$ & $30-70$ & $100 \mathrm{He}$ & TOTAL & $82-20$ & $30-70$ & $100 \mathrm{He}$ & $\frac{\text { TOTAL }}{30}$ \\
\hline $\begin{array}{l}\text { CAPSULE } \\
\text { HEAD }\end{array}$ & 13 & 12 & 5 & 30 & & 12 & & 30 \\
\hline HEAD & 13 & 12 & 5 & 30 & 13 & 12 & 5 & 30 \\
\hline INSULATOR & 26 & 24 & 10 & 60 & 26 & 24 & 10 & 60 \\
\hline SPACER & 26 & 24 & 10 & 60 & 26 & 24 & 10 & 60 \\
\hline WAVE SPRING & 13 & 12 & 5 & 30 & 13 & 12 & 5 & 30 \\
\hline DENSITY DISK HOLDER & 13 & 11 & 3 & 27 & 13 & 11 & 4 & 28 \\
\hline BLAXIAL CREEP HOLDER & 26 & 18 & 6 & 50 & 22 & 16 & 7 & 45 \\
\hline FRACTURE TOUGHNESS HOLDER & 4 & 5 & 1 & 10 & 6 & 8 & 2 & 16 \\
\hline TENSILE HOLDER & 7 & 9 & 2 & 18 & 10 & 10 & 3 & 23 \\
\hline COMPACT TENSILE (CT) HOLDER & 5 & 4 & 1 & 10 & 5 & 3 & 2 & 10 \\
\hline BEO DISK HOLDER & 4 & 4 & 0 & 8 & 4 & 4 & 0 & 8 \\
\hline BEO COMPRESSION HOLDER & 1 & 1 & 0 & 2 & 1 & 1 & 0 & 2 \\
\hline DISK GRAPHITE HOLDER & 0 & 2 & 4 & 6 & 0 & 1 & 1 & 2 \\
\hline CERAMIC STRIP GRAPHITE HOLDER & 0 & 0 & 0 & 0 & 0 & 2 & 2 & 4 \\
\hline TENSILE GRAPHITE HOLDER & 0 & 2 & 4 & 6 & 0 & 0 & 0 & 0 \\
\hline THERMAL SHIELD & 0 & 1 & 2 & 3 & 0 & 1 & 1 & 2 \\
\hline
\end{tabular}

\section{Capsule Assembly Tools and Fixtures:}

Special tooling had been designed to aid in the assembly of the JOYO capsules. All of the tooling is to be manufactured from ASTM A276, Type 304 bar.

- Disk holder handling tool

- After the specimens are placed in the disk holder, the tool's prongs are slid down over the holder so that the three tabs located on the part's exterior surface slide 
into the three corresponding J-channels on the tool to fully capture the disk holder. The disk holder can be laced into the capsule.

- Plunger for disk holder tool removal

- The plunger is used to disengage the disk holder handling tool from within the capsule after installation of the loaded disk holder.

- Guide tool to ensure that the lower spacer and insulator are properly seated

\section{Welding}

The JOYO-1 welding specification (Attahcment $G$ to this Enclosure), issued May 9, 2005, contains both the closure weld qualification details and production welding requirements. Subsequent to the issuance of this document, the capsule design was modified from a $22 \mathrm{~mm}$ diameter $/ 1 \mathrm{~mm}$ wall thickness to $23 \mathrm{~mm}$ diameter $/ 1.5 \mathrm{~mm}$ wall thickness. The final weld procedure qualification testing and production welding was to be conducted in an inert atmospheric glove box, with stringent controls applied to inert gas purity, oxygen content and moisture control. These gas purity controls were established to prevent test specimen contamination during testing and not required by the welding process. In addition, the three capsule inert gasses establish thermodynamic heat transfer properties to control test specimen temperatures to predetermined temperatures. The glove box provided a means of ensuring an inert gas back fill for the closure weld which could not be otherwise generated. Three different gas mixtures were assigned to the differing test capsules in order to support the required internal capsule test temperatures. They were identified as $100 \%$ Helium, $80 \%$ Argon $20 \%$ Helium and $30 \%$ Argon $70 \%$ Helium; thereby necessitating a Welding Procedure Specification to be qualified for each of these three gas mixtures.

The welding power supply, weld head and associated fitting components were selected and a purchase order placed by April of 2005, with the majority of the components arriving in June and July of 2005. The orbital weld equipment is manufactured by the Swagelok Company. A key factor in selecting the Swagelok model was the fact of its superior heat rejection rates from the weld head. The high rate of heat rejection was critical to completing the capsule closure weld in a single pass while precluding a weld blow out due to the build up of internal pressure.

\section{Qualification}

The weld qualification program was to be conducted in three parts consisting of (a) development, (b) welding procedure qualification and (c) production proof verification, as briefly described below.

\section{a. Development}

Prototypical weld mockups duplicating the production weld joint, including internal gas volume restrictions, were to be welded and sectioned for metallographic examination; ensuring developed parameters met penetration, fusion and quality requirements.

\section{b. Welding Procedure Qualification}

Three full size weld procedure qualification test coupons duplicating the production weld joint were to be welded and subjected to the production Non Destructive Examination (NDE) inspections and code required mechanical tests. Two coupons were intended for the required tension tests; full section mockups, vice machining individual tension test specimens, were allotted to satisfy this requirement. The third coupon was to be 
Enclosure (7) to

MDO-723-0044I

B-MT(SRME)-52

Page 118

sectioned into the two face and two root bend specimens, along with preparation of a supplementary metallographic section.

c. Production Proof Qualification

At least one prototypical mockup was to be welded using the developed and authenticated parameters. This mockup was to be subject to the same visual, dimensional and pressure testing of the production units; certifying that the process met the functional end-item requirements.

Figure IV.C.1-1 depicts the basic weld joint that was to be qualified on a $23 \mathrm{~mm}$ diametrically shaped test coupons and mockups.

HEAD

CAPSULE

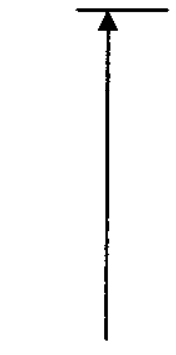

23 MM $\varnothing$

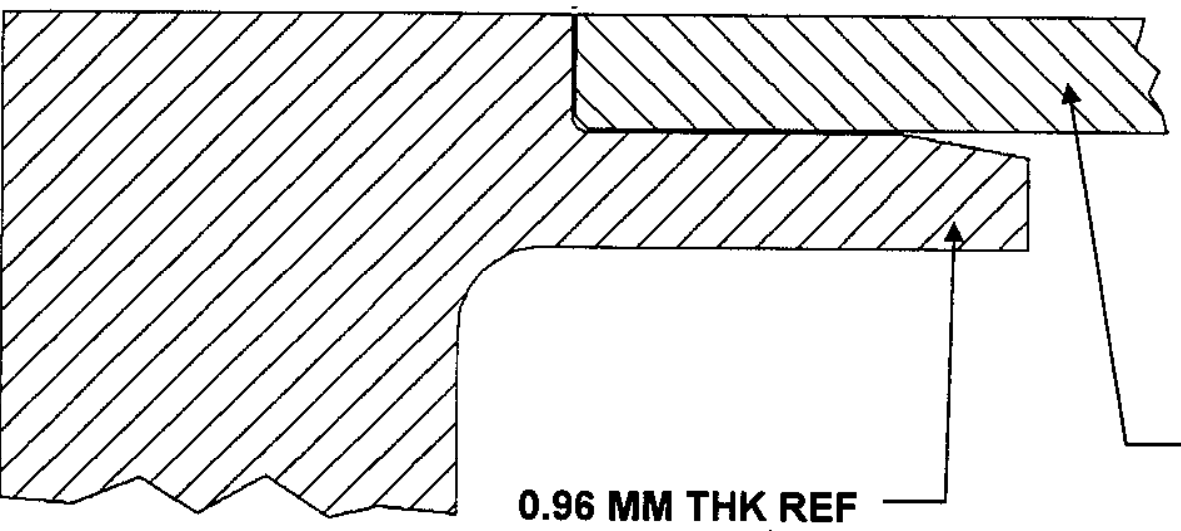

\subsection{THK REF}

Figure IV.C.1-1: Depiction of JOYO capsule closure weld joint

\section{Qualification Hardware}

\section{a. Phase I-Development}

Figure IV.C.2-1, below, depicts the mockup design intended to validate weld developmental testing. Integral to the mockup arrangement was the annular space that duplicates the free space remaining after the capsule(s) are assembled with their respective material test components. As the weld progresses circumferentially, the inert atmosphere contained within the free space is heated due to the heat imparted by the welding process resulting in some value increase of internal pressure. Therefore under these prototypical conditions, it was essential to validate that the weldment could be completed in a single continuous pass without the molten weld pool being ejected at or near the terminus of the weld due to any resultant increase of the internal gas pressure. Beyond external visual examination, metallographic sectioning was to be conducted to ensure acceptable weld penetration and fusion attributes in all four quadrants, including the start / stop location. 


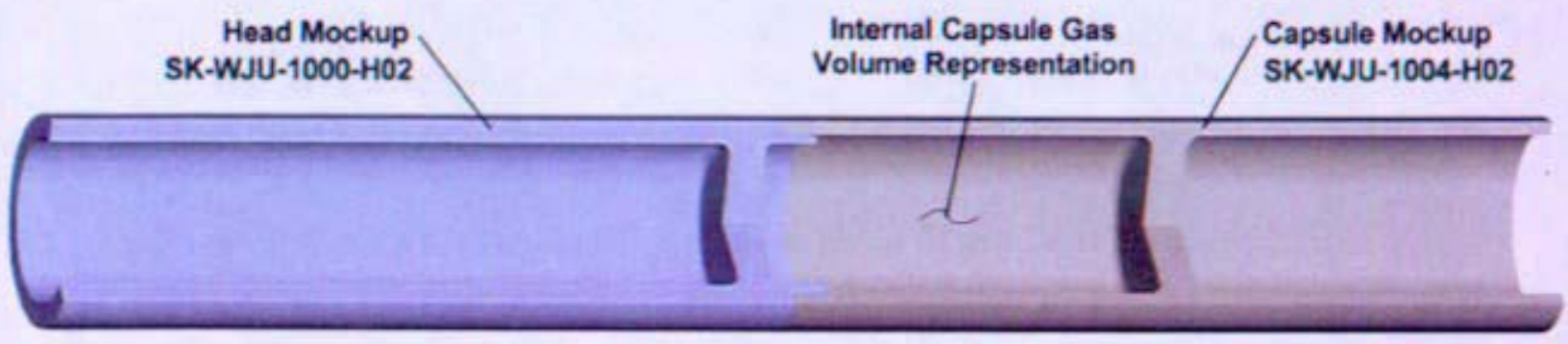

Quantity : 10 per gas

Figure IV.C.2-1 - Developmental Weld Mockups

Figure IV.C.2-2, depicts a representation of the mockups to be used to determine any instantaneous post weld increase in internal gas pressure and any residual final increase in pressure once the capsule has cooled to ambient temperature.

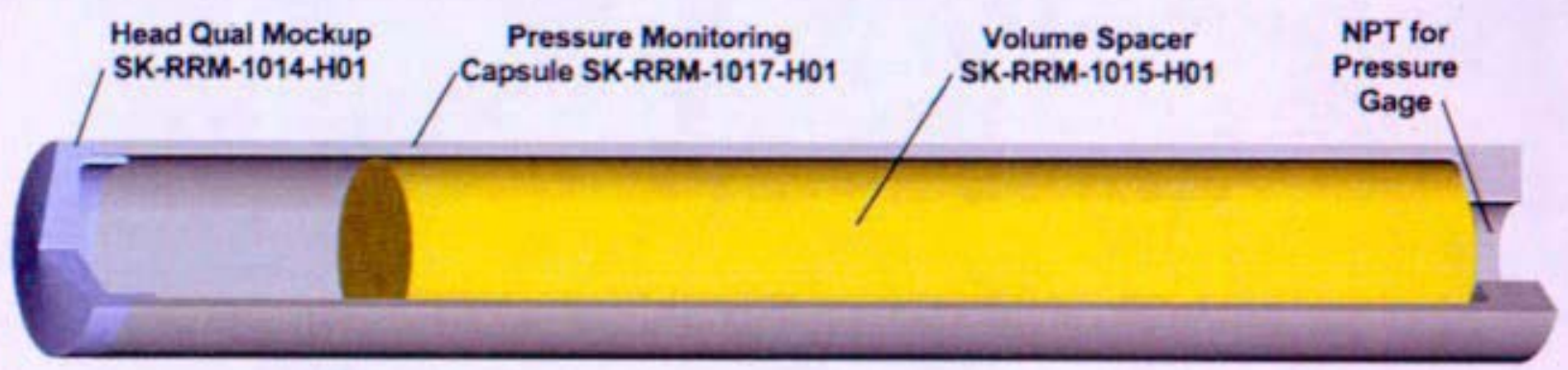

Quantity : 2

Figure IV.C.2-2 -Pressure Assessment Mockup

b. Phase II-Qualification

Figure IV.C.2-3, depicts the ASME Section IX Weld Procedure and Welder Certification Qualification test coupons. The visual, mechanical, non-destructive and destructive tests as outlined in reference (a) were to be completed in order to validate both the weld procedure qualification and welder certification for each of the three inert gas compositions. See Figures IV.C.5-1 through - 13 for photographs depicting the hardware and weld sequencing. 


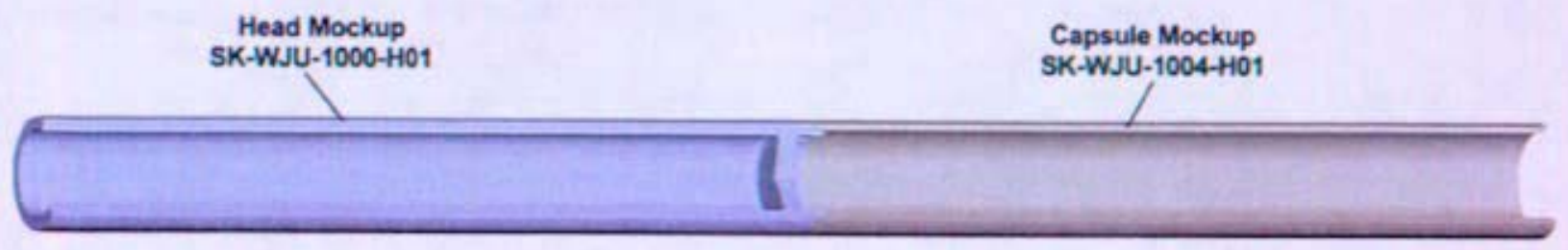

Quantity : 3 per gas and 3 per welder

Figure IV.C.2-3 -ASME Weld Qualification Mockup

\section{c. Phase III - Production Proof Qualification}

Figure IV.C.2-4, depicts the prototypical mockups to be completed for each of the three gas mixtures. These mockups, subjected to the same visual, dimensional and pressure testing of the production units, would authenticate that the developed parameters met the functional, quality and end-item requirements.

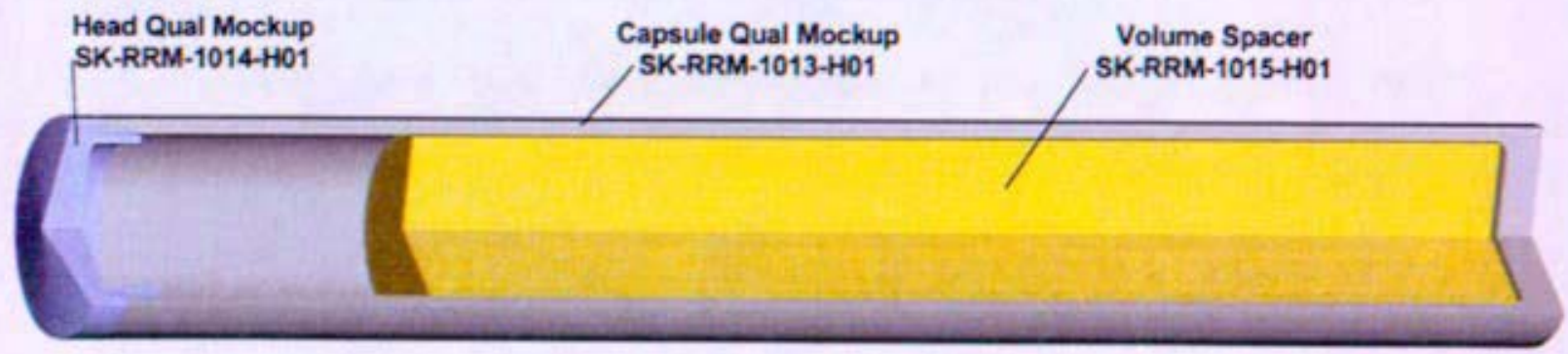

Quantity : 1 per gas and 1 per welder

Figure IV.C.2-4 -Prototypic Production Mockup

\section{Qualification Progress}

Phase I weld development testing was partially competed for the $100 \%$ Helium and $30 \% \mathrm{Ar}$ / $70 \% \mathrm{He}$ gas mixtures. The inert glove-box modifications necessary to accommodate the weld head electrical pass throughs were not completed by the expected start date; therefore out-of-glove box developmental testing was initiated in parallel to preserve the Weld Qualification completion target date. The results of this developmental work are described below.

\section{a. $100 \%$ Helium Gas}

Ten test welds were completed outside of a glove box environment using $100 \%$ Helium as the shielding gas. The purpose was to develop weld parameters to such a degree that by the time the glove box modifications were completed only minor refinements, if any, would have to be made prior to conducting the welding procedure qualification tests, under actual production conditions, inside the glove box.

The initial four test welds were simple bead-on-tube tests conducted in order to get an initial bearing on starting parameters. Figure IV.C.3-1 shows a representative cross 
section of a bead on tube test weld. The weld parameters for this test weld are

Page 121 documented in Section IV.C.7, data sheet \#1.

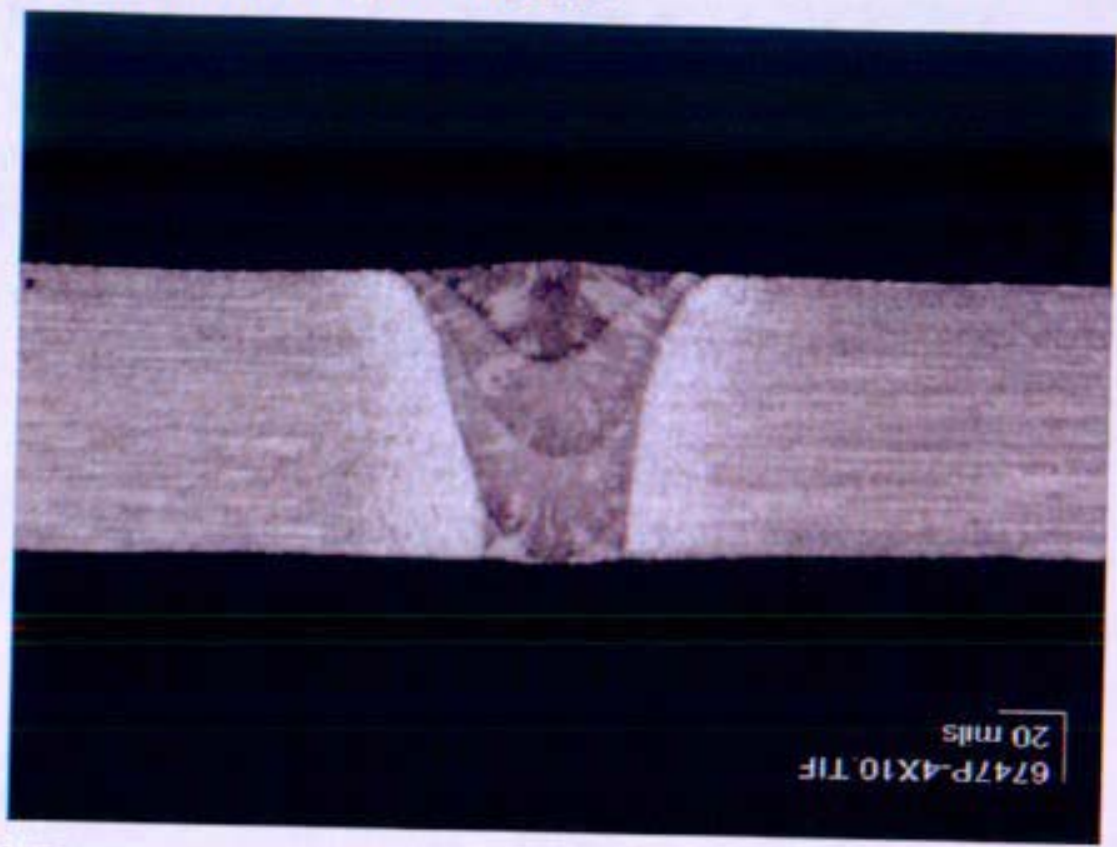

Figure IV.C.3-1: Bead on Tube Test Weld- 100\% Helium (Weld Parameter Data Sheet \#1)

The next four test welds were conducted on capsule and head mockups, using the prototypical weld joint per Figure IV.C.2-1. During this phase it was initially noted the weid centerline favored the tube side. This was thought to be attributed to the greater heat sink capacity (or material mass) of the head component as compared to the capsule side of the joint. Figure IV.C.3-2 cross section depicts this condition; refer to data sheet \#2 of SectionIV.C.7 for the weld parameters used.

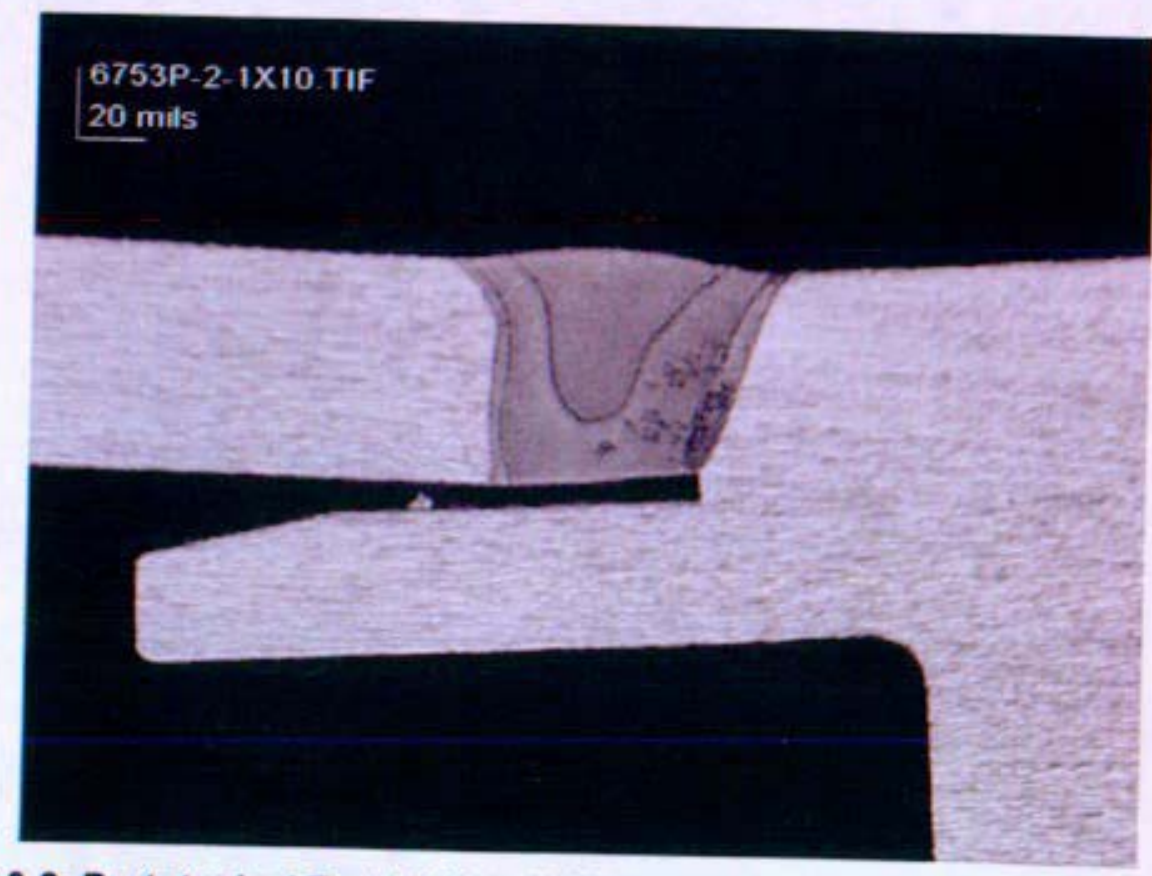

Figure IV.C.3-2: Prototypical Test Weld - 100\% Helium (Weld Parameter Data Sheet \#2) 
Enclosure (7) to

MDO-723-0044/

B-MT(SRME)-52

Page 122

The weld schedule was modified in order to rectify the weld centering and bead shape issue. Notably, the Impulse current (or high current portion of the weld pulse cycle) was maintained consistent through all four quadrants (or levels), instead of ramping down each quadrant, and the pulse rate (the cycle rate per second of high pulse current to low pulse current) was changed from 3 to 2 ; having the overall effect of maintaining a slightly higher and uniform heat input throughout all portions of the weld. Figure IV.C.3-3, shows how the above changes affected the overall weld shape and effected the weld bead bias equally between the capsule and head element. The weld parameters for this test weld documented in Section IV.C.7, data sheet \#3, were the starting basis for the glove-box weld procedure qualification testing using the $100 \%$ helium gas.

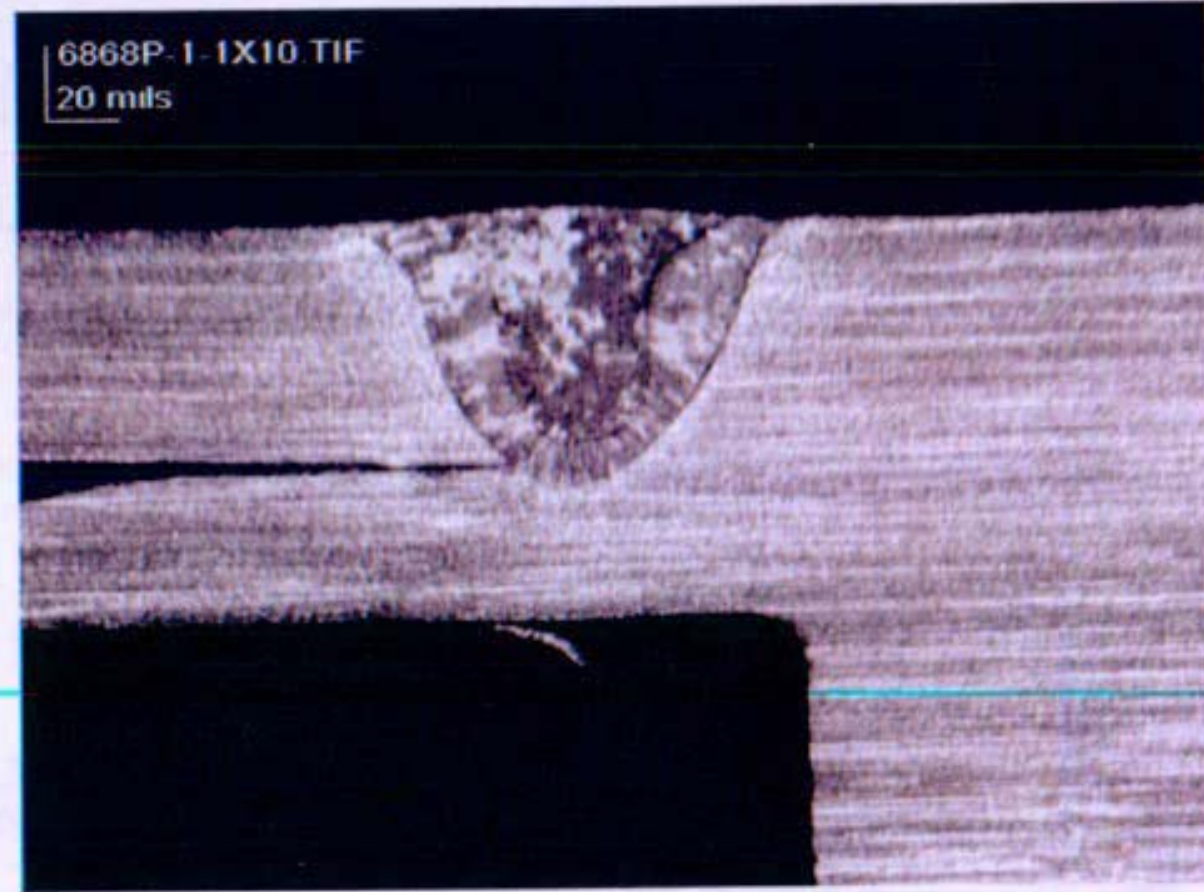

Figure IV.C.3-3: Prototypical Test Weld - 100\% Helium (Weld Parameter Data Sheet \#3)

In order to preclude joint centering issues in the weld fixture block (tungsten-to-weld joint alignment) and to confirm the relationship between weld centering, heat sink mass, technique and heat input, the last two $100 \%$ Helium gas test welds were conducted on simple tube-to-tube square-butt joints (equal material mass each side of weld joint), as typically used in industry for fluid or gas service. One test was completed using a heat input slightly lower than what would typically be required, and the second at a higher, more nominal heat input. The lower heat input tube-to-tube square-butt weld (Figure IV.C.3-4) produced some lack of penetration in two of the quadrants. The higher heat input tube-to-tube square butt weld (Figure IV.C.3-5) produced a wider weld shape with acceptable penetration for the entire joint. In both cases, however, the weld joint centerline was equally disposed between the two faying members, thus providing authentication of the earlier held relationship between the mass and shape of the head and weld heat input. Thus ruling out having to address weld joint alignment to tungsten biasing issues. The weld produced in Figure IV.C. 3 could potentially be used for future capsule applications, as apposed to the backing weld joint design for this application. If 
used, considerations should be provided for 1) Page 123 weld bead and 2) spring clearance of the underside components, 2) ensuring internal capsule heat build up would not damage internal test components, e.g. pressurized material test components.

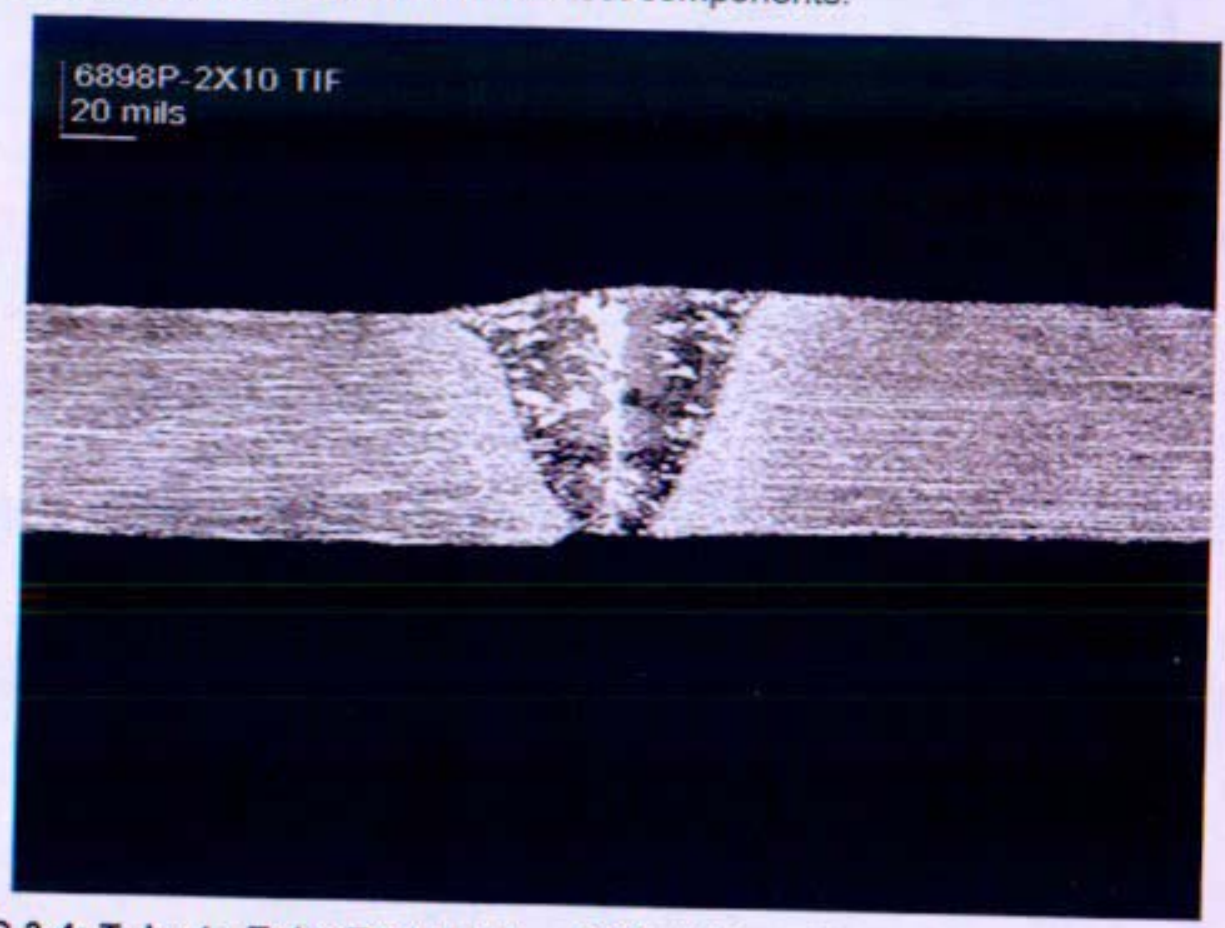

Figure IV.C.3-4: Tube-to-Tube Test Weld - 100\% Helium (Weld Parameter Data Sheet \#4)

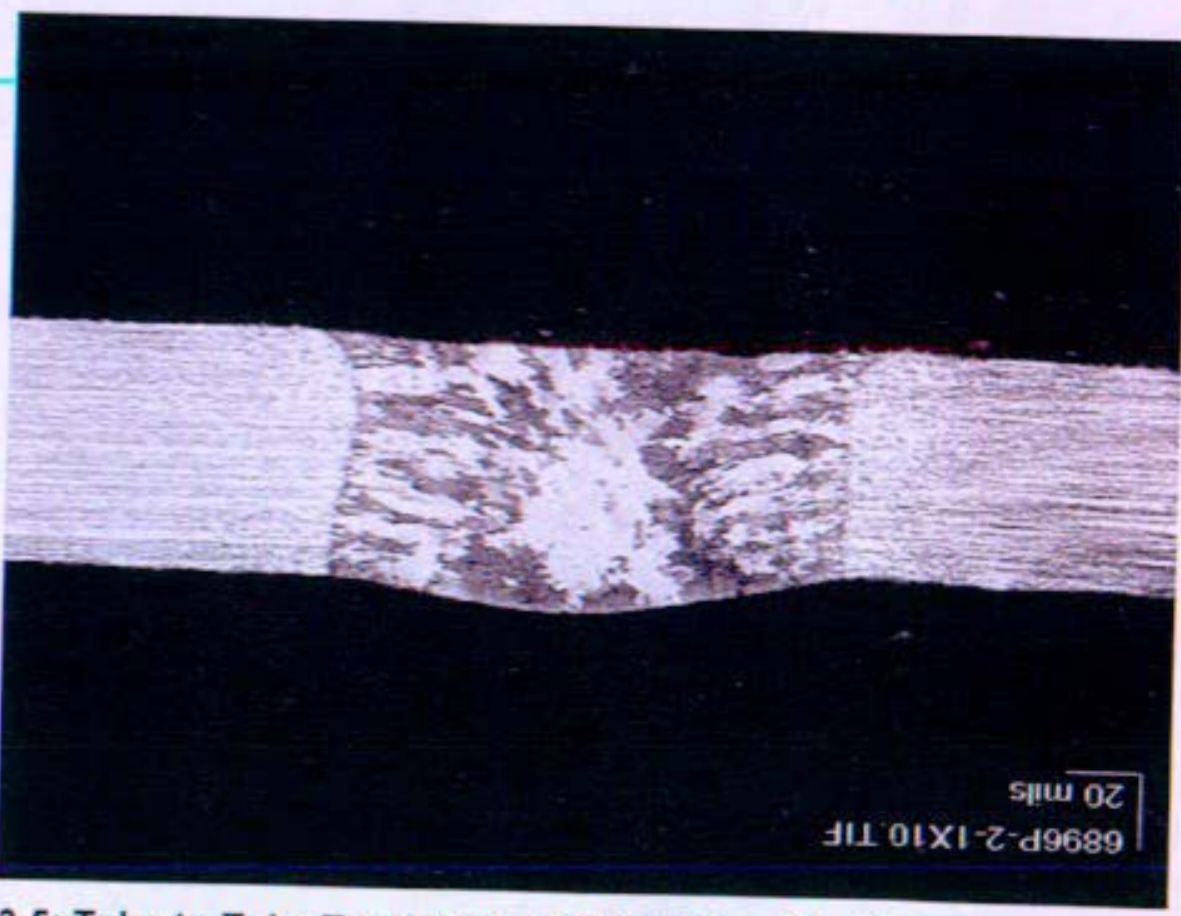

Figure IV.C.3-5: Tube-to-Tube Test Weld - 100\% Helium (Weld Parameter Data Sheet \#5) 


\section{b. $30 \%$ Argon $/ 70 \%$ Helium Gas}

One test weld was completed outside of a glove box environment using $30 \%$ Argon / $70 \%$ Helium as the shielding gas. Figure IV.C. $3-6$ depicts a representative cross section of this test weld with the parameters documented in Section IV.C.7, data sheet \#6. Not all quadrant exhibited complete penetration. The next logical path would be to increase the primary impulse current by 5 to 8 amps and to reduce the pulse rate from 3 to 2 pulses per second.

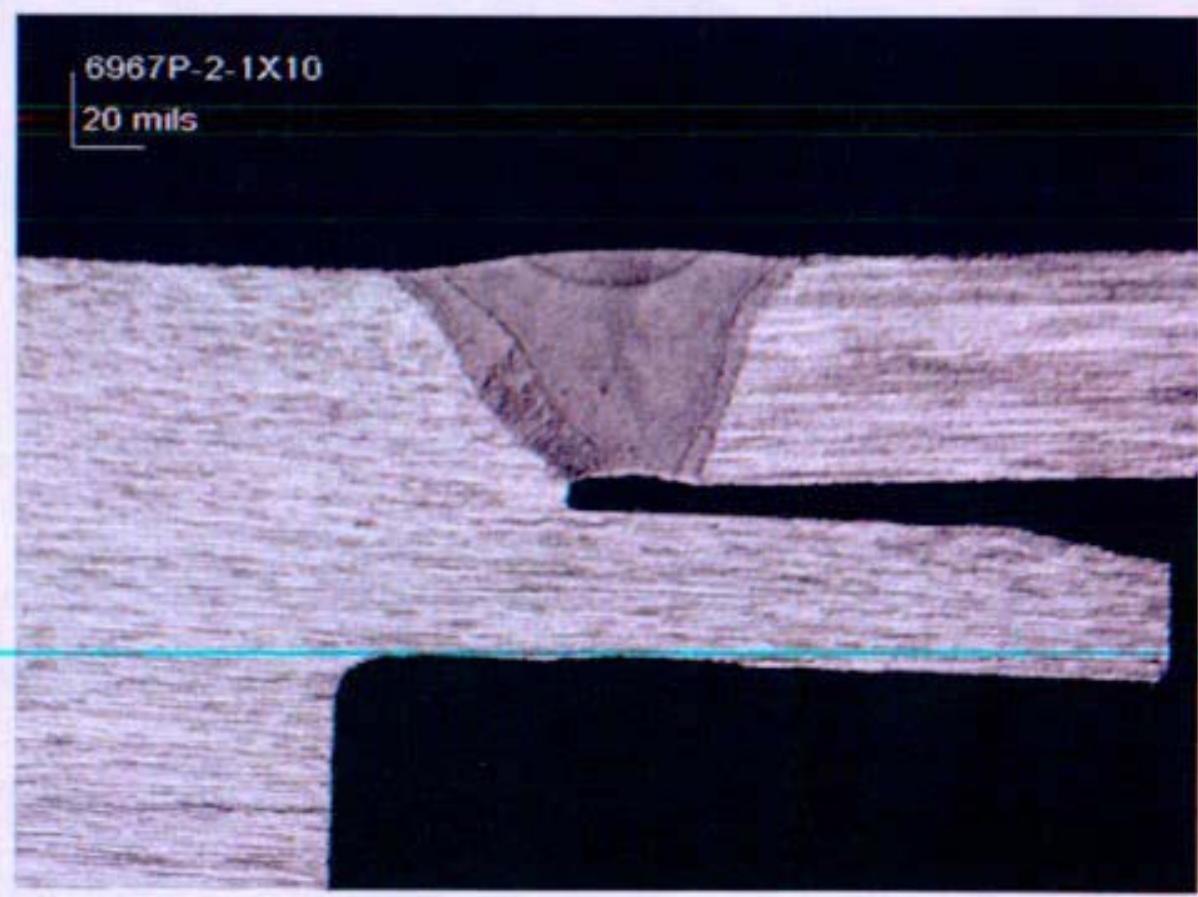

Figure IV.C.3-6: Prototypical Test Weld - 30\% Argon / 70\% Helium (Weld Parameter Data Sheet \#6)

\section{Observations}

For equivalent arc lengths at all current levels, the arc voltage obtained with helium is appreciably higher than the arc voltage with argon. Because of its high ionization potential, the use of helium shield gas produces more available heat than argon. It should also be noted that at lower currents, the volt-ampere curves pass through a minimum voltage, at current levels of approximately 90 Amperes, after which the voltage increases as the current decreases. Above approximately 90 Amperes, the voltage increases linearly as the current increases. The transition voltage for argon shield gas is approximately 50 Amperes.

Table IV.C.4-1, below, compares the effects of shielding gas on currents and voltages as a function of arc power for the test welds per Figure IV.C.3-3 (100\% Helium), Figure IV.C.3-6 ( $30 \%$ Argon $/ 70 \%$ Helium) and a typical tube-to-tube weld of like material (type $316 \mathrm{SS}$ ), equivalent square butt joint, and same approximate thickness (1/16") using $100 \%$ Argon 
shield gas. Arc power is simply the product of current and voltage. Accounting for the slightly thicker base material for the joint welded using $100 \%$ argon, the Arc Power values for the three different shield gas combinations are very nearly identical to each other. Therefore, it is apparent that to obtain equal arc power, as the percentage of argon to helium goes up, appreciably higher currents must be used.

\begin{tabular}{|c|c|c|c|c|c|c|}
\hline \multicolumn{7}{|c|}{ Table IV.C.4-1: Arc Energy as Affected by Shield Gas Composition } \\
\hline WELD & THICKNESS & SHIELD GAS & ARC GAP & $\begin{array}{c}\text { CURRENT } \\
\text { (AVERAGE') }\end{array}$ & $\begin{array}{c}\text { VOLTAGE } \\
\text { (AVERAGE) }\end{array}$ & ARC POWER \\
\hline $\begin{array}{c}\text { Figure } \\
\text { IV.C.3-3 } \\
\text { (Sq butt) }\end{array}$ & $0.059^{\prime \prime}$ nom. & $100 \% \mathrm{He}$ & $0.040^{\prime \prime}$ & $21.4 \mathrm{~A}$ & $13.2 \mathrm{~V}$ & 283 Joules \\
\hline $\begin{array}{c}\text { Figure } \\
\text { IV.C.3-6 } \\
\text { (Sq butt) }\end{array}$ & $0.059^{\prime \prime}$ nom. & $30 \% \mathrm{Ar} / 70 \% \mathrm{He}$ & $0.040^{\prime \prime}$ & $26.6 \mathrm{~A}$ & $10.75 \mathrm{~V}$ & 285 Joules \\
\hline $\begin{array}{c}\text { Tube-Tube } \\
\text { (Sq butt) }\end{array}$ & $0.065^{\prime \prime}$ nom & $100 \% \mathrm{Ar}$ & $0.045^{\prime \prime}$ & $45 \mathrm{~A}$ & $7.6 \mathrm{~V}$ & 342 Joules \\
\hline
\end{tabular}

1 Average of high (impulse) and low (maintenance) current.

2 Function of Arc gap, power supply current vs. voltage curve, shield gas and pulse rate.

During the course of the weld development work a consistent problem was discovered concerning the tungsten electrode. It was found that after completion of a weld, the tungsten electrode appeared to have some type of residual build up covering the electrode tip. If attempting to make another weld with this same electrode, the arc would not initiate. Therefore, in order to make another weld, either the electrode would have to be replaced with a new one, or the old electrode removed, and the residual metalic coating removed with an emery cloth. This being the case, once a successful arc was initiated (with a new or refurbished electrode) no negative impacts were noted with the operational behavior of the arc. The visual appearance of all test welds was good, with no spatter observed on the weld and or adjacent parent material. The only observation of note was the usual black soot found on the edges of the weld when conducting fusion operations in a helium environment; easily removed by wire brushing.

The cause of the above occurrence appeared to be associated with the use of both $100 \%$ helium and $30 \%$ argon $/ 70 \%$ helium weld atmospheres. This same electrode degradation, using the same equipment, did not occur with the use of $100 \%$ argon. The electrodes used (which came with the equipment) were of the $2 \%$ Cerium type. Time did not permit the thorough experimentation of alternative electrodes alloyed with Thorium, Lanthanum, or other proprietary binary or tertiary alloy mixes. An investigation into this phenomenon by actual analysis of either the electrode build-up constituents or the mode of transference was not able to be conducted, again due to lack of time. Therefore, the decision was made, in order to meet the near term qualification and production schedule, to replace the $2 \%$ Ceriated tungsten electrode with a new electrode after each production weld. The search for a long term solution to this problem was to be investigated after the initial production run was completed in either January or February 2006. 


\section{Sequence Overview}

Figure IV.C.5-1 illustrates the typical capsule "shell-side" weld qualification test coupon, fitted together with the item shown in Figure IV.C.5-2 duplicates the production weld joint. The length chosen was to accommodate the mechanical (Tensile \& Bend) test specimens.

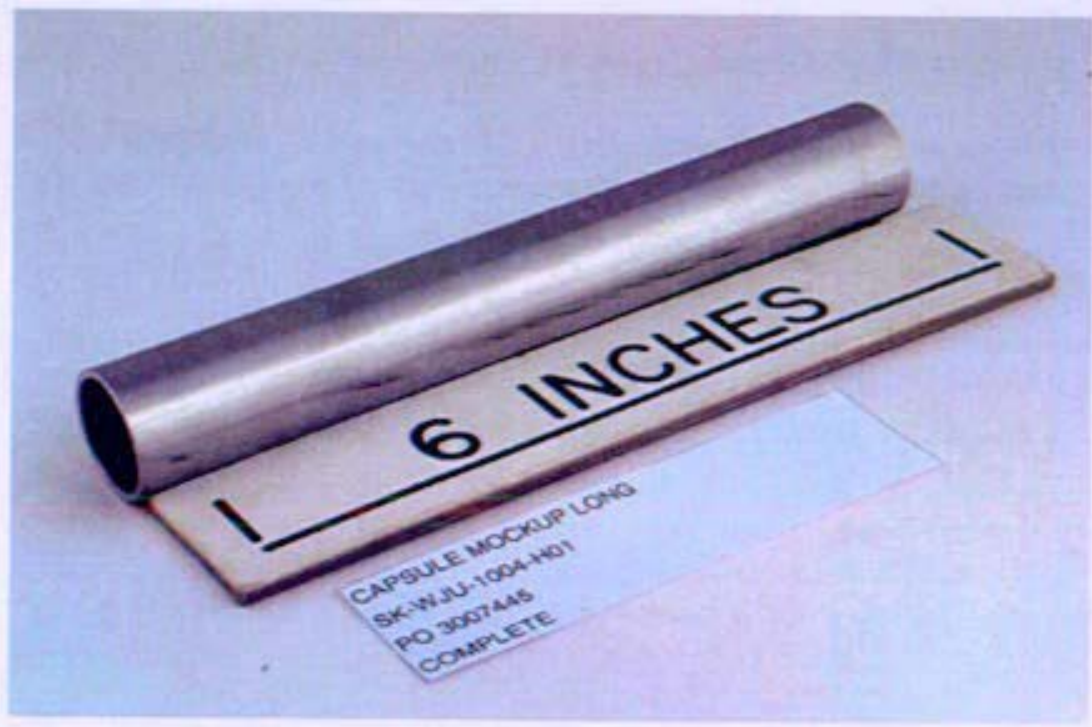

Figure IV.C.5-1: Weld Qualification Test Coupon - Capsule Shell Side

Figure IV.C.5-2 illustrates the typical capsule "head-side" weld qualification test coupon, fitted together with the item shown in Figure IV.C.5-1duplicates the production weld joint. The length chosen was to accommodate the mechanical (Tensile \& Bend) test specimens.

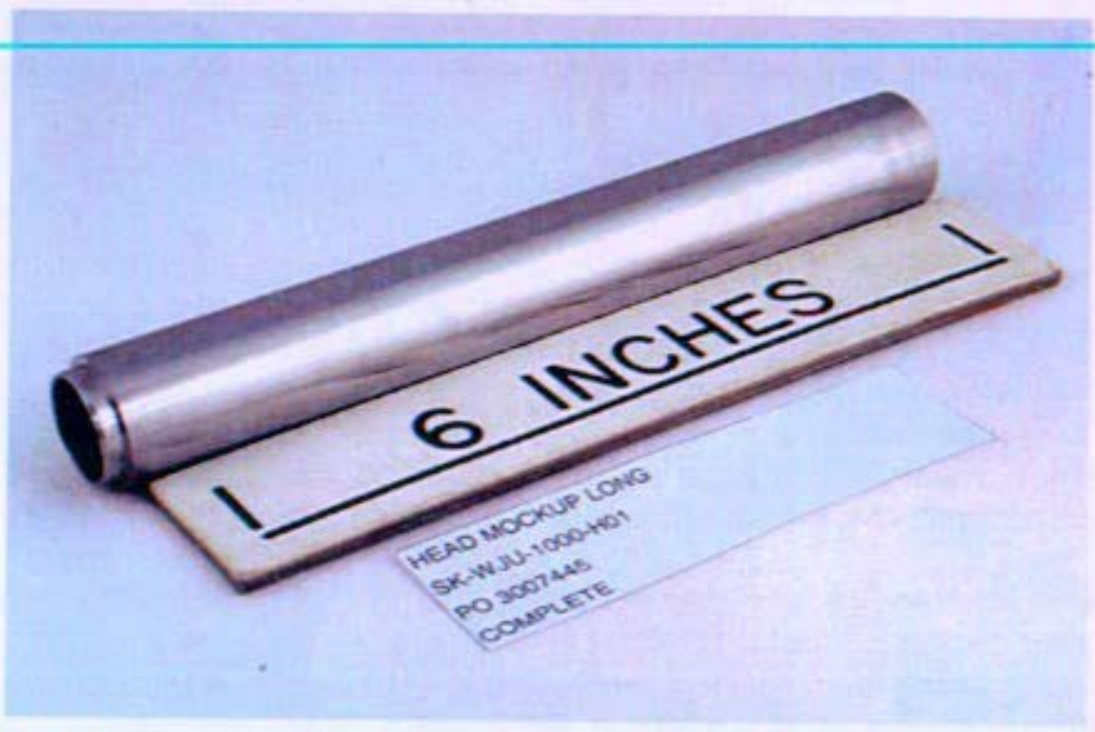

Figure IV.C.5-2: Weld Qualification Test Coupon - Capsule Head Side

Figure IV.C.5-3 illustrates the assembled weld qualification test coupon (Figure IV.C.5-1 \& IV.C.5-2) set within the weld fixture stand. The weld fixture block is in the background with the weld power supply shown on the right side. The copper tube (heat dissipation tube) on 
the right side of the test coupon was developed to dissipate heat in order to prevent a weld blow out upon completion of the final closure weld segment of a production capsule.

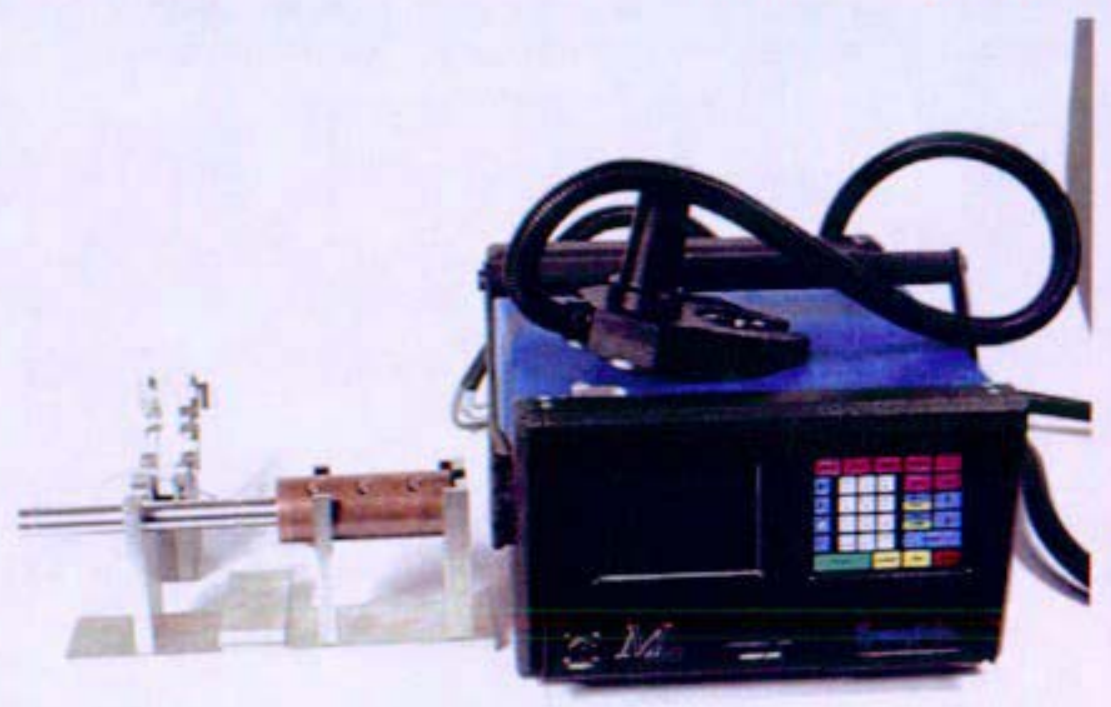

Figure IV.C.5-3: Weld Qualification Coupon Set in Weld Fixture Stand

Figure IV.C.5-4 illustrates the assembled weld qualification test coupon (items in Figures IV.C.5-1 \& IV.C.5-2) set within the weld fixture block with the orbital weld head installed. No heat dissipation tube is shown in this view. Preliminary out of glove box testing demonstrated that 1) a relatively low amount of heat is retained in the part and 2) the weld qualification coupon is opened ended and therefore not subject to internal pressure build up due to heat

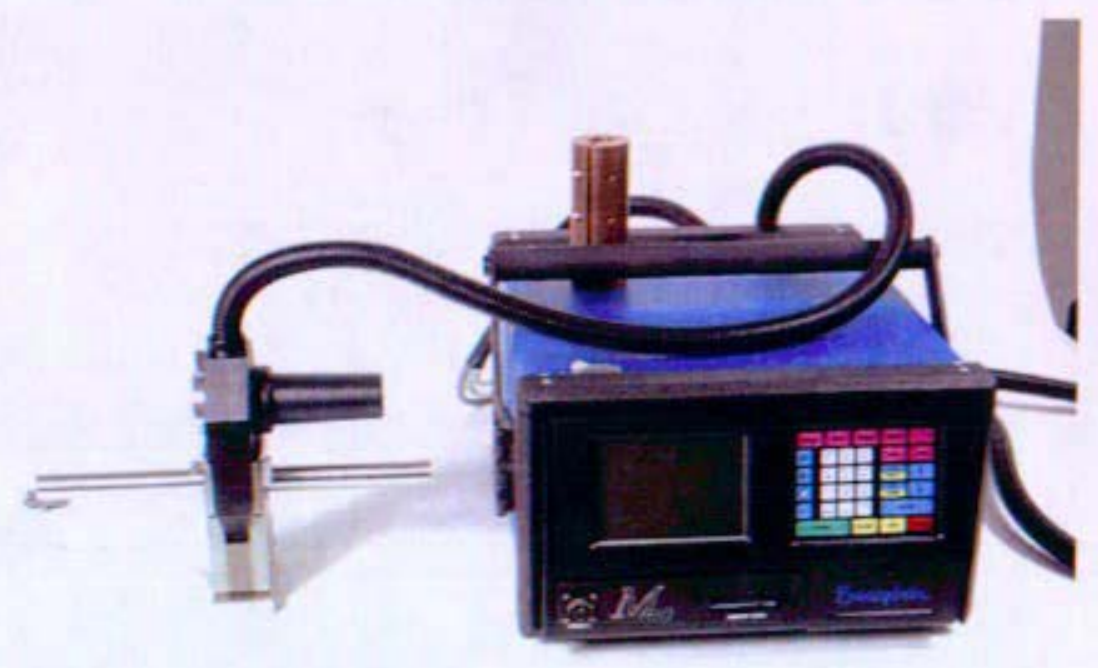

Figure IV.C.5-4: Weld Qualification Coupon in Fixture Block with Weld Head installed 


\section{Enclosure (7) to \\ MDO-723-0044/ \\ B-MT(SRME)-52 \\ Page 128}

Figure IV.C.5-5 shows a pressure gauge attached to capsule mockups. One phase of the weld qualification process was to assemble and fabricate a production mockup with an internal volume restrictor (mimics space taken up by the internal test components), thereby duplicating the calculated free space volume. The pressure gauge was to indicate the built up final pressure (due to the heat of welding).

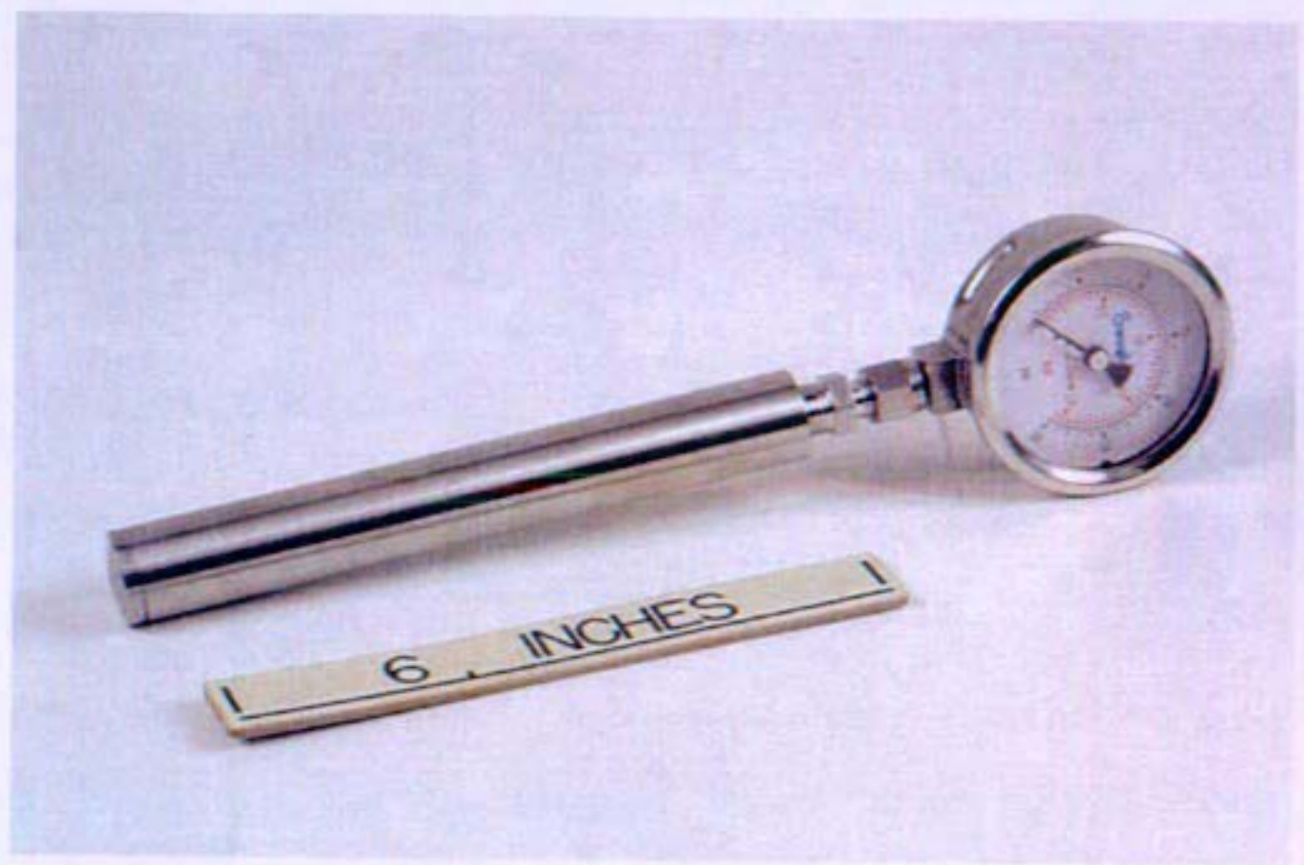

Figure IV.C.5-5: Production Proof Mockup with Pressure Gauge Installed

Figure IV.C. 5-6 illustrates a cut away of a typical test capsule showing the internal test items and how they are assembled within a capsule.

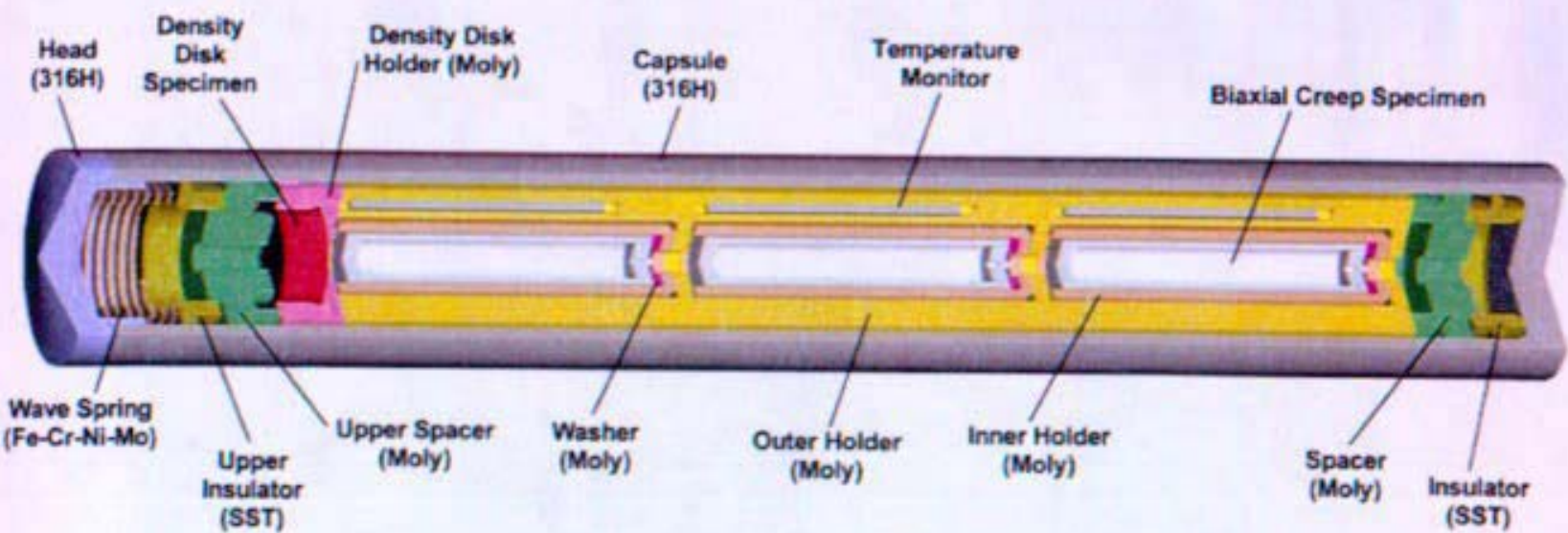

Figure IV.C.5-6: Representation of Assembled Capsule (BCD - Capsule Assembly Shown) 
Figure IV.C.5-7 illustrates a typical test mockup and the internal components with volume spacer. It was important to conduct prototypical weld testing in a glove box, duplicating the free space volume, ensuring that the closure weld could be completed in a single continuous pass without a weld blow out due to internal heat and resultant pressure build up.
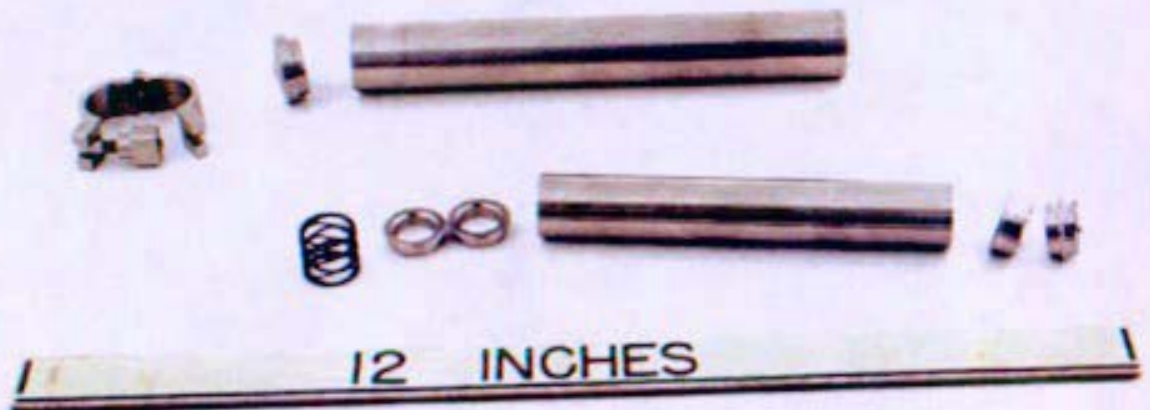

Figure IV.C.5-7: Typical Test Mockup assembly with volume spacer shown

Figure IV.C.5-8 illustrates the assembly Head-to-Shell clamp. The individual capsule assembly was to take place while the shell portion is in the vertical position. The last component to be inserted prior to the head was a "wave spring". The head required a vertical force to compress the spring, whereupon the clamp ring was then installed which temporarily holds the head secured and compressed to the shell body (Figure IV.C.5-9). The purpose was to preclude small spacers, underneath the wave spring, from becoming displaced while moving the assembly to a horizontal position to the welding fixture (Figure IV. G.5-11). Once placed in the welding fixture, an adapter would hold the head secure to the shell thereby allowing the clamp to be removed (Figure IV.C.5-12).

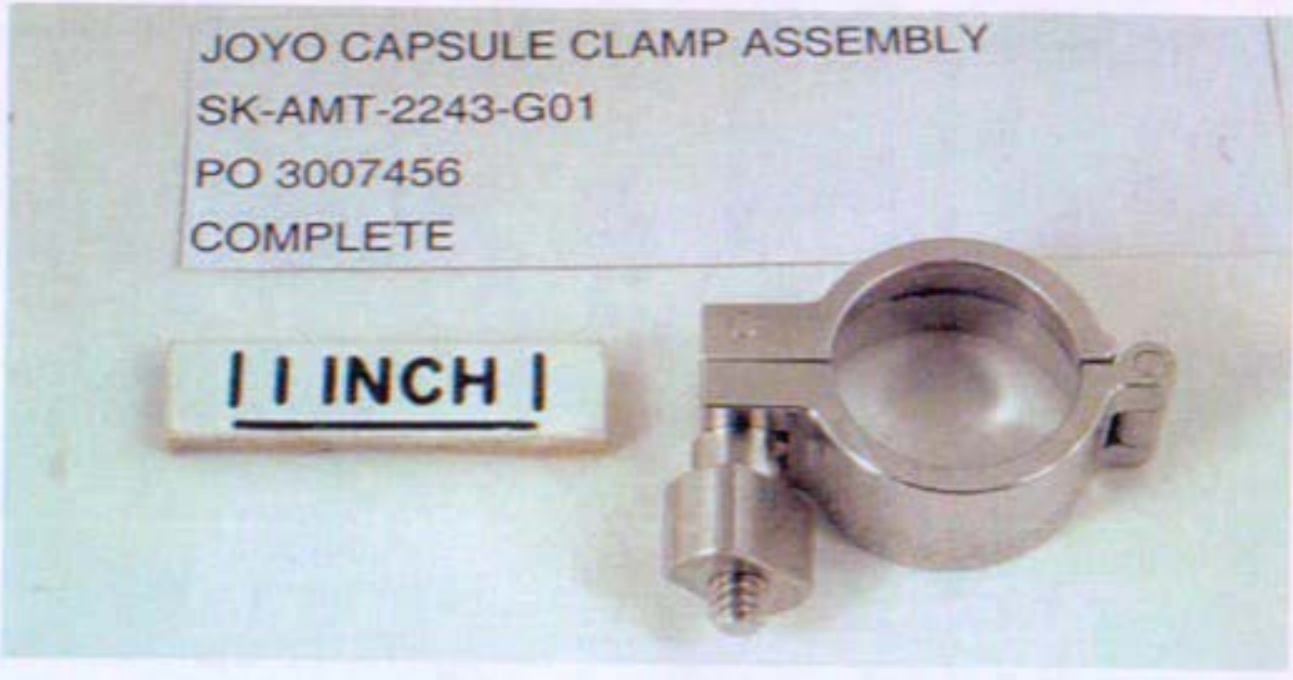

Figure IV.C.5-8: Head-to-Shell Clamp 
Enclosure (7) to

MDO-723-0044/

B-MT(SRME)-52

Page 130

Figure IV.C.5-9 shows an example of an assembled JOYO capsule with the temporary Head-to-Shell clamp mounted.
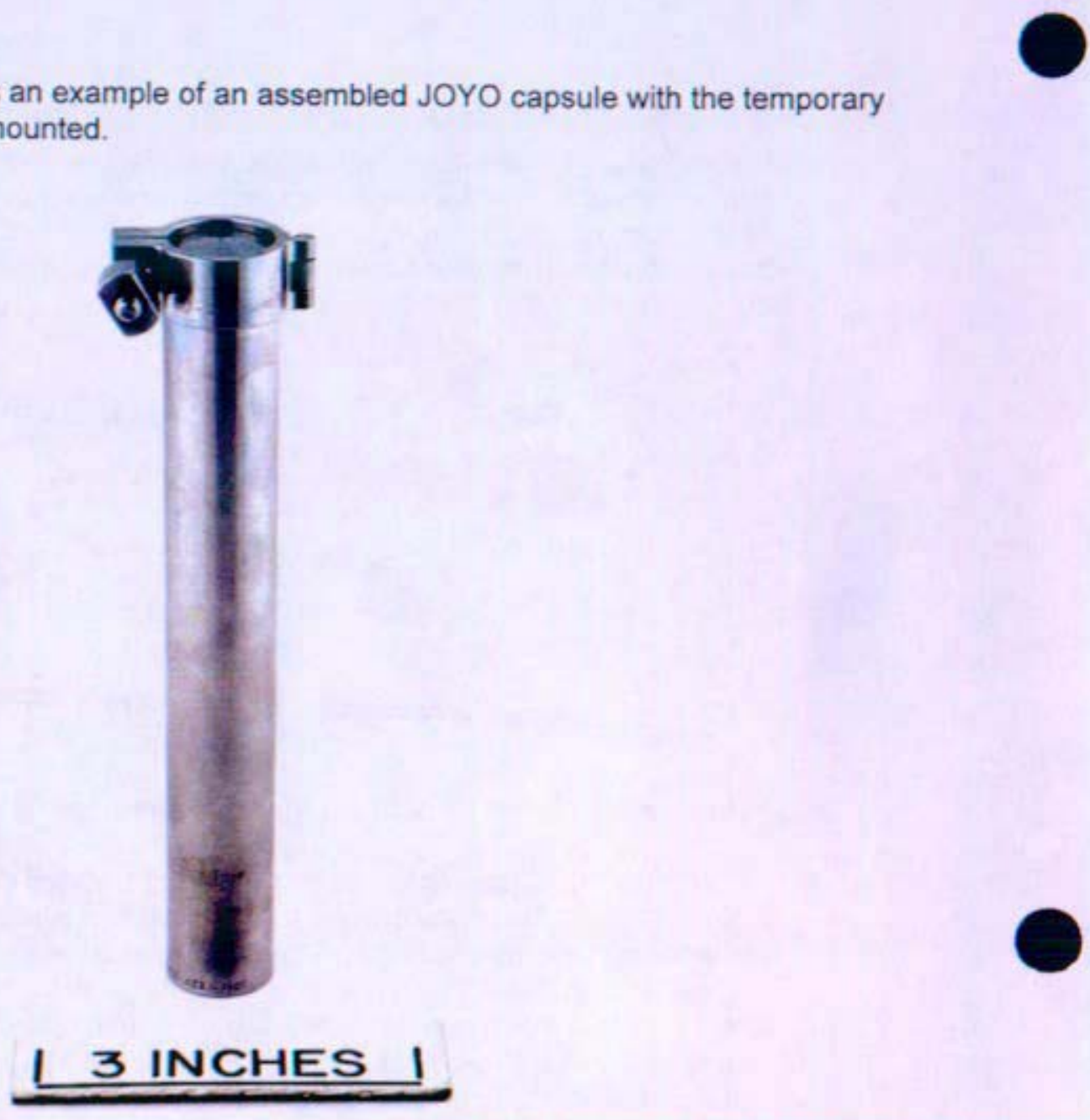

Figure IV.C.5-9: Typical Assembled JOYO Capsule (prior to welding) with Assembly Clamp Installed

Figure IV.C.5-10 illustrates an example of an assembled JOYO capsule with clamp and welding heat dissipation tube installed around the capsule.

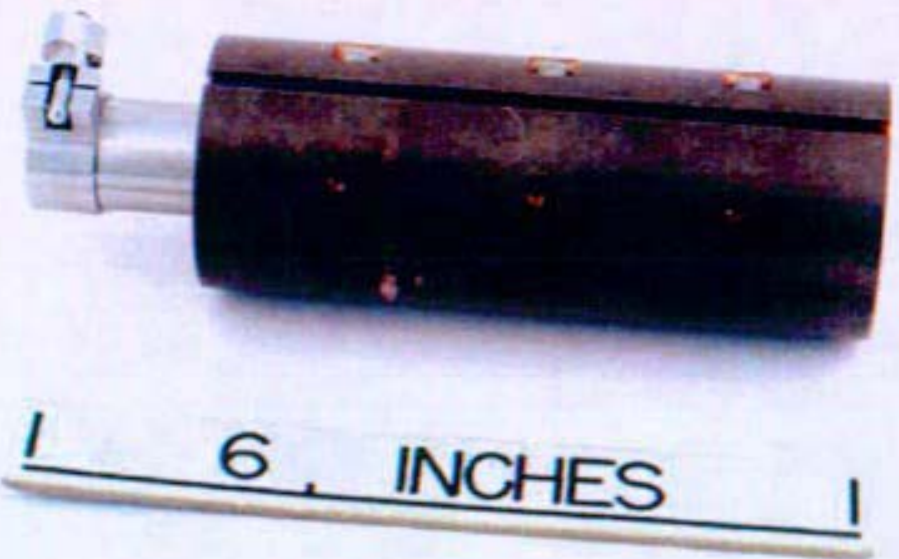

Figure IV.C.5-10: Clamped Capsule and Heat Dissipation Tube Installed 
Figure IV.C. 5-11 illustrates the JOYO assembly with clamp and heat dissipation tube placed in the open welding fixture. The smaller tiered copper cylinder on the left is a solid $\varnothing 20 \mathrm{~mm}$ adapter piece. This adapter piece has two functions, 1) allows a horizontal force (by turning the knurled adjusting knob) against the capsule head piece, thereby compressing the wave spring and ensuring a tight weld joint fit-up and 2) the larger diameter, or shoulder, of this piece comes to rest against the left outer side of the weld head fixture clamp, and thereby acts to center the weld joint between the two halves of the weld head fixture clamp, which in turn automatically ensures the weld head tungsten electrode is centered with the weld joint.

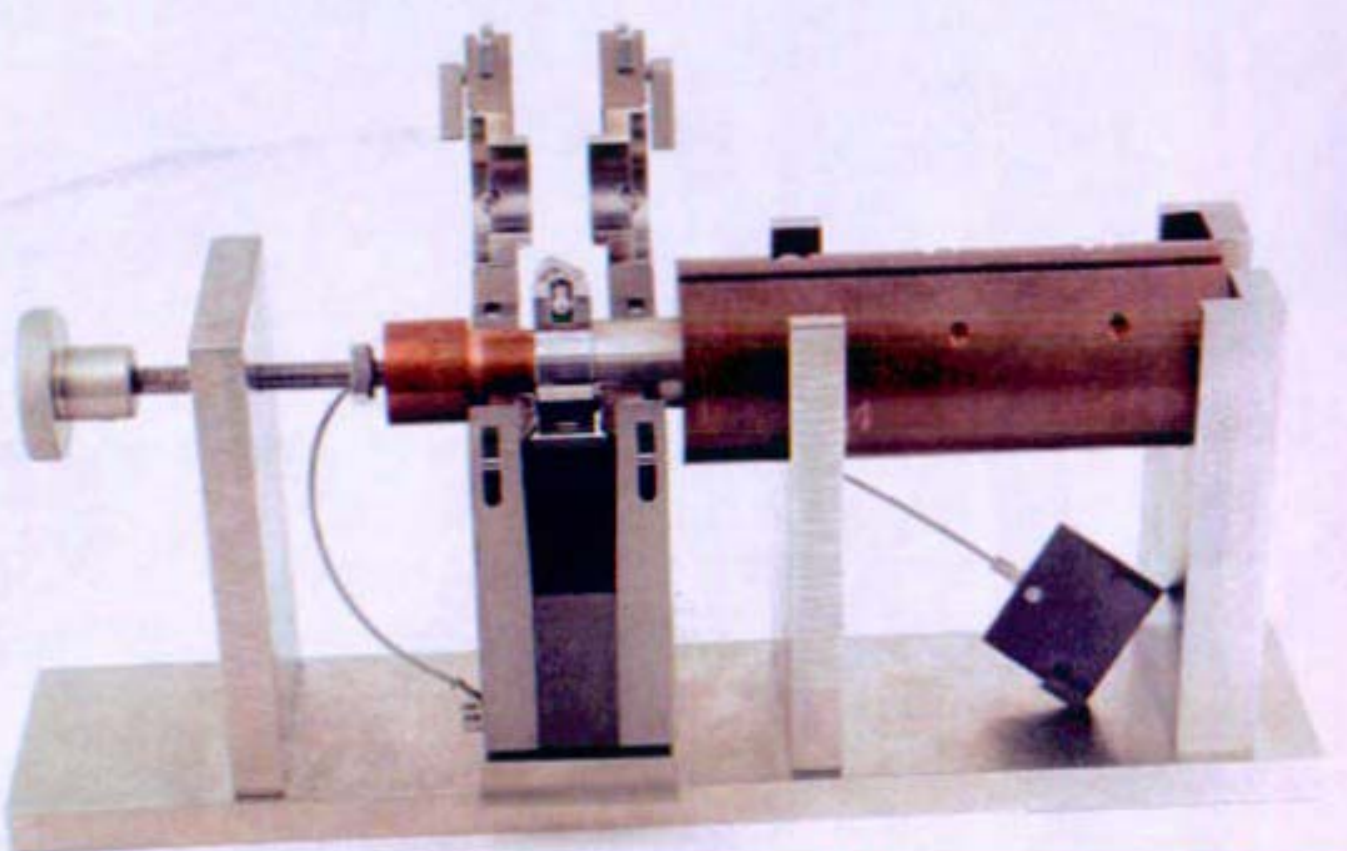

\section{Figure IV.C.5-11: Typical Capsule Assembly in Weld Fixture and Open Fixture Block}

Figure IV.C.5-12 illustrates the JOYO assembly placed in the welding fixture with the weld head fixture clamp in the closed position and the adapter tube in the tightened and seated position and the clamp ring removed. The weld head clamp fixture is in a "ready-state" to receive the orbital weld head. 
Enclosure (7) to

MDO-723-0044/

B-MT(SRME)-52

Page 132

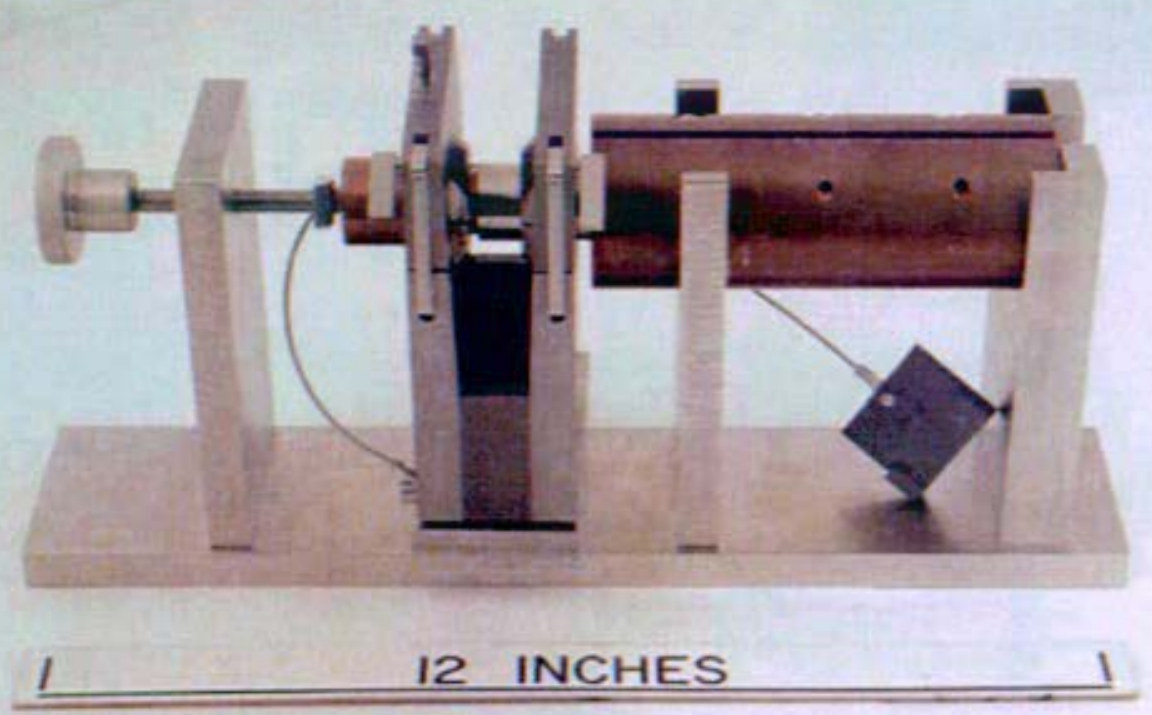

Figure IV.C.5-12: Typical Capsule Assembly in Weld Fixture and Closed Fixture Block

Figure IV.C.5-13 shows the orbital weld head installed within the two halves of the weld head fixture clamp assembly and as supported by the JOYO weld fixture. The figure depicts an assembly that is now ready to be welded.

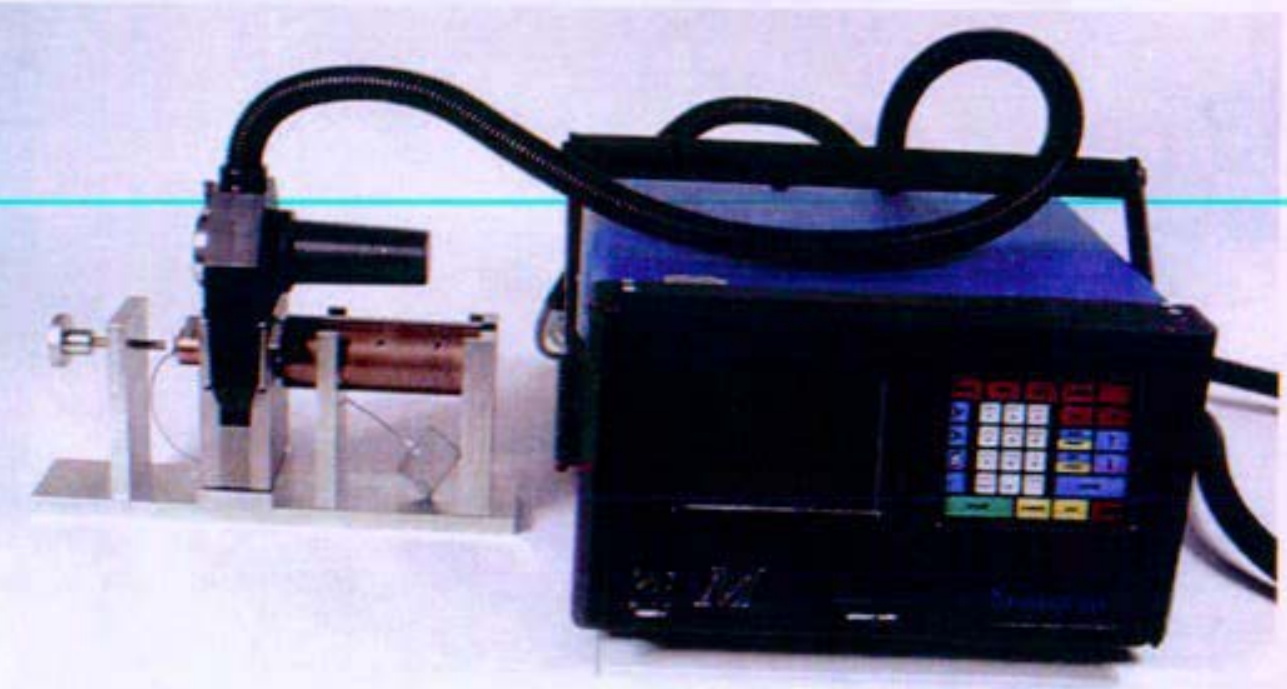

Figure IV.C.5-13: Typical Capsule Assembly in Weld Fixture and Orbital Weld Head Installed 
Enclosure (7) to

MDO-723-0044/

B-MT(SRME)-52

Page 133

\section{Weld Procedure Development Data Sheets}

\section{WELD PARAMETER DATA SHEET \#1 DATE RECORDED: 8-4-05 RECORDED BY: R. Clapp}

IDENT: Capsule SIN: n/a LAB TEST NUMBER : JDT-003 TEST DESCRIPTON: Bead on tube test

WPS: JOYO-T4 PROGRAMMER: CLAPP REF DRAWING(S):

PROCESS: AGTAW POWER SUPPLY: SWS M100-1, VER: 2.03 WELD HEAD: SWS-10H-B

TUNGSTEN: $0.062^{\prime \prime}(1.57 \mathrm{MM})$ dia/.855" (21,72 MM)lq. 2\% CERIUM PURGE ATMOSPHERE: $100 \%$ He, 0.D.: $60 \mathrm{chh}$ I.D.: flood

ARC GAP: 1.02 MM ARC GAP GAGE SETTING: 37.84 MM WELO OPERATOR: CLAPP MIN VOLTS: 9.0

CURRENT TOL: $2.5 \%$ SPEED TOL: $2.5 \%$ CUR LIMIT: $100 \%$ PURGE LIMIT: $100 \%$ GLOVEBOX $\square$ NON-GLOVEBOX $\triangle$

JOINT TYPE: BEAD -ON-TUBE MATERIAL TYPE: 316 WELD DIAMETER: 23MM WALL THICKNESS: 1.5 MM

- INPUTS -

\begin{tabular}{|c|c|c|c|c|c|}
\hline VARIABLE & VALUE & & & & \\
\hline START POWER & NORM & & & & \\
\hline $\begin{array}{c}\text { START } \\
\text { CURRENT }\end{array}$ & 16.0 AMPS & & & & \\
\hline ROTOR DELAY & $1.6 \mathrm{SEC}$ & & & & \\
\hline DOWWSLOPE & 8.3 SEC & & & & \\
\hline PREPURGE & $20 \mathrm{SEC}$ & & & & \\
\hline POSTPURGE & 20 SEC & & & & \\
\hline TACKS & DEGREES & AMPS & \multicolumn{2}{|c|}{ SECONDS } & \\
\hline \multicolumn{5}{|l|}{$\mathrm{n} / \mathrm{a}$} & \\
\hline \multicolumn{6}{|l|}{$n / a$} \\
\hline \multicolumn{6}{|l|}{$n / a$} \\
\hline \multirow{2}{*}{ LEVEL } & \multirow{2}{*}{ IMPULSE } & \multirow{2}{*}{ MAINT } & \multicolumn{2}{|c|}{-IMPULSE- } & \multirow{2}{*}{$\begin{array}{l}\text { AVG. } \\
\text { CURRENT }\end{array}$} \\
\hline & & & RATE & WDTH & \\
\hline 1 & 29.0 AMPS & 8.5 AMPS & 2 & 33 & 15.2 AMPS \\
\hline 2 & 27.5 AMPS & 8.5 AMPS & 2 & 33 & 14.7 AMPS \\
\hline 3 & 26.0 AMPS & 8.5 AMPS & 2 & 33 & 14.2 AMPS \\
\hline 4 & 24.6.3 AMPS & 8.5 AMPS & 2 & 33 & 13.8 AMPS \\
\hline LEVEL & TIME & RAMP & \multicolumn{2}{|c|}{ RMP } & $\begin{array}{l}\text { TRAVEL } \\
\text { SPEED }\end{array}$ \\
\hline 1 & 10.3 & 0.0 & \multicolumn{2}{|c|}{1.51} & $4.3 \mathrm{in} / \mathrm{min}$ \\
\hline 2 & 10.3 & 0.0 & \multicolumn{2}{|c|}{1.51} & $4.3 \mathrm{in} / \mathrm{min}$ \\
\hline 3 & 10.3 & 0.0 & \multicolumn{2}{|c|}{$1.5 t$} & $4.3 \mathrm{in} / \mathrm{min}$ \\
\hline 4 & 10.3 & 0.0 & \multicolumn{2}{|c|}{1.51} & $4.3 \mathrm{ir} / \mathrm{min}$ \\
\hline
\end{tabular}

COMMENTS

-OUTPUTS-

-AVERAGE-

LEVEL

\begin{tabular}{|c|c|c|}
\hline AMPS & VOLTS & RPM \\
\hline 15.3 & 13.5 & 1.53 \\
\hline 14.7 & 13.8 & 1.52 \\
\hline 14.2 & 13.9 & 1.52 \\
\hline 13.8 & 13.9 & 1.53 \\
\hline 7.7 & 16.6 & 1.53 \\
\hline
\end{tabular}

\begin{tabular}{|c|c|c|l|}
\hline $\begin{array}{c}\text { TRAVEL } \\
\text { SPEED }\end{array}$ & TIME & $\mathrm{KJ} / \mathrm{in}$ & \\
\hline $4.3 \mathrm{in} / \mathrm{min}$ & 10.3 & 2.8 & \\
\hline $4.3 \mathrm{in} / \mathrm{min}$ & 10.3 & 2.8 & \\
\hline $4.3 \mathrm{in} / \mathrm{min}$ & 10.3 & 2.8 & \\
\hline $4.3 \mathrm{in} / \mathrm{min}$ & 10.3 & 2.7 & \\
\hline $4.3 \mathrm{in} / \mathrm{min}$ & 8.3 & 1.8 & \\
\hline
\end{tabular}

COMMENTS: Appearance good, pen adequate for bead on tube. Acceptable test. 
Enclosure (7) to

MDO-723-0044/

B-MT(SRME)-52

Page 134

WELD PARAMETER DATA SHEET \#2

DATE RECORDED: 8-8-05 RECORDED BY: $\underline{\text { R. Clapp }}$

IDENT: Capsule SNN: n/a LAB TEST NUMBER : JDT-006 TEST DESCRIPTION: Tube-10-Tube

WPS: JOYO-7 PROGRAMMER: CLAPP REF DRAWNG(S): SK-WJU-1004 (SHT 2) \& SK-WJU-1000 (SHT 2)

PROCESS: AGTAW POWER SUPPLY: SWS M100-1, VER: 2.03 WELD HEAD: SWS-10H-B

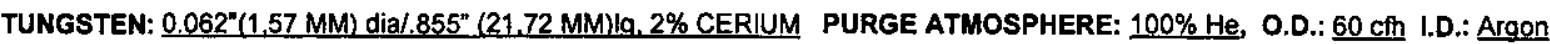

ARC GAP: 1.02 MM ARC GAP GAGE SETTING: 37.84 MM WELD OPERATOR: CLAPP MIN VOLTS: $\underline{9.0}$

CURRENT TOL: $2.5 \%$ SPEED TOL: $2.5 \%$ CUR LIMIT: 100\% PURGE LIMIT: $100 \%$ GLOVEBOX $\square$ NON-GLOVEBOX $\square$

JOINT TYPE: TB-TB BUtI MATERIAL TYPE: 316 WELD DIAMETER: 23 MM WALL THICKNESS: $1.5 \mathrm{MM}$

- INPUTS .

\begin{tabular}{|c|c|c|c|c|c|}
\hline VARIABLE & VALUE & & & & \\
\hline START POWER & NORM & & & & \\
\hline $\begin{array}{c}\text { START } \\
\text { CURRENT }\end{array}$ & 22.4 .0 AMPS & & & & \\
\hline ROTOR DELAY & 1.6 SEC & & & & \\
\hline DOWNSLOPE & 8.3 SEC & & & & \\
\hline PREPURGE & 20 SEC & & & & \\
\hline POSTPURGE & $20 \mathrm{SEC}$ & & & & \\
\hline TACKS & DEGREES & AMPS & \multicolumn{2}{|c|}{ SECONDS } & \\
\hline \multicolumn{5}{|l|}{$n / a$} & \\
\hline \multicolumn{6}{|l|}{$n / a$} \\
\hline \multicolumn{6}{|l|}{$\mathrm{n} / \mathrm{a}$} \\
\hline \multirow{2}{*}{ LEVEL } & \multirow{2}{*}{ IMPULSE } & \multirow{2}{*}{ MAINT } & \multicolumn{2}{|c|}{-IMPULSE- } & \multirow{2}{*}{$\begin{array}{l}\text { AVG. } \\
\text { CURRENT }\end{array}$} \\
\hline & & & RATE & WIDTH & \\
\hline 1 & 40.6 AMPS & 11.9 AMPS & 3 & 33 & 21.3 AMPS \\
\hline 2 & 38.5 AMPS & 11.9 AMPS & 3 & 33 & 20.6 AMPS \\
\hline 3 & 36.4 AMPS & 11.9 AMPS & 3 & 33 & 19.9 AMPS \\
\hline 4 & 34.4 AMPS & 11.9 AMPS & 3 & 33 & 19.3 AMPS \\
\hline LEVEL & TIME & RAMP & \multicolumn{2}{|c|}{ RMP } & $\begin{array}{l}\text { TRAVEL } \\
\text { SPEED }\end{array}$ \\
\hline 1 & 10.9 & 0.0 & \multicolumn{2}{|c|}{1.43} & $4.0 \mathrm{in} / \mathrm{min}$ \\
\hline 2 & 10.9 & 0.0 & \multicolumn{2}{|c|}{1.43} & $4.0 \mathrm{in} / \mathrm{min}$ \\
\hline 3 & 10.9 & 0.0 & \multicolumn{2}{|c|}{1.43} & $4.0 \mathrm{ir} / \mathrm{min}$ \\
\hline 4 & 10.9 & 0.0 & \multicolumn{2}{|c|}{1.43} & $4.0 \mathrm{in} / \mathrm{min}$ \\
\hline
\end{tabular}

COMMENTS

\begin{tabular}{|c|c|c|c|c|c|c|c|}
\cline { 2 - 8 } -AVERAGE- & AMPS & VOLTS & RPM & $\begin{array}{c}\text { TRAVEL } \\
\text { SPEED }\end{array}$ & TIME & KJ / in & \\
\hline LEVEL & 21.4 & 12.8 & 1.45 & $4.0 \mathrm{in} / \mathrm{min}$ & 10.9 & 4.1 & \\
\hline 1 & 20.7 & 12.6 & 1.45 & $4.0 \mathrm{in} / \mathrm{min}$ & 10.9 & 3.9 & \\
\hline 3 & 20.0 & 13.0 & 1.45 & $4.0 \mathrm{in} / \mathrm{min}$ & 10.9 & 3.9 & \\
\hline 4 & 19.4 & 13.7 & 1.45 & $4.0 \mathrm{i} / \mathrm{min}$ & 10.9 & 3.9 & \\
\hline DIS & 10.1 & 15.6 & 1.46 & $4.0 \mathrm{in} / \mathrm{min}$ & 8.4 & 2.4 & \\
\hline
\end{tabular}

COMMENTS: Outer appearance acceptable, Full penetration through tube wall side / no underbead reinforcement, weld centerline favors tube side, approx 20-25 mils, (suspect heat sink differential with cap side). 
- INPUTS -

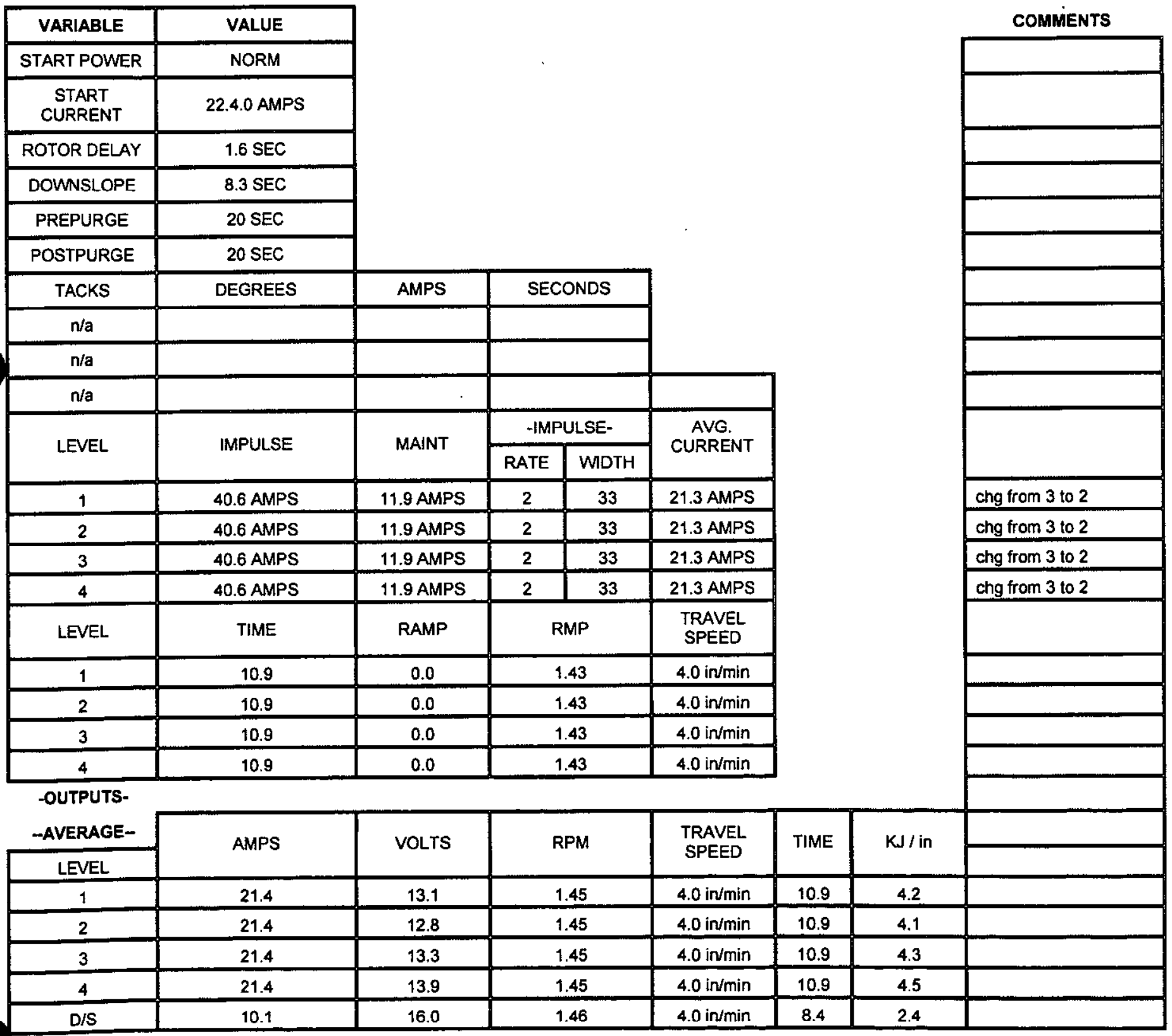

COMMENTS: Note: mounted tube side 1st piece in left block and center qauged from right side. Outer appearance acceptable, Full penetration achieved in all quadrants, joint appears centerd. 
Enclosure (7) to

MDO-723-0044I

B-MT(SRME)-52

Page 136

\section{WELD PROCEDURE DATA SHEET \#4}

DATE RECORDED: 8-12-05 RECORDED BY: R. Clapp

IDENT: Capsule S/N: n/a LAB TEST NUMBER: JDT-009 TEST DESCRIPTION: Tube-to-Tube

WPS: JOYO-5 PROGRAMMER: CLAPP REF DRAWING(S): N/A

PROCESS: AGTAW POWER SUPPLY: SWS M100-1, VER: 2.03 WELD HEAD: SWS-10H-B

TUNGSTEN: $0.062^{\prime \prime}(1.57 \mathrm{MM})$ dia/.855" (21.72 MM)la. 2\% CERIUM PURGE ATMOSPHERE: $100 \%$ He, O.D.: $60 \mathrm{ch}$ 1.D.: Argon

ARC GAP: 1.02 MM ARC GAP GAGE SETTING: 37.84 MM WELD OPERATOR: CLAPP MIN VOLTS: 9.0

CURRENT TOL: $2.5 \%$ SPEED TOL: $2.5 \%$ CUR LIMIT: $100 \%$ PURGE LIMIT: $100 \%$ GLOVEBOX $\square$ NON-GLOVEBOX $\square$

JOINT TYPE: IB-TB BUtt MATERIAL TYPE: 316 WELD DIAMETER: 23 MM WALL THICKNESS: $1.5 \mathrm{MM}$

- INPUTS .

\begin{tabular}{|c|c|c|c|c|c|}
\hline VARIABLE & VALUE & & & & \\
\hline START POWER & NORM & & & & \\
\hline $\begin{array}{c}\text { START } \\
\text { CURRENT }\end{array}$ & 16.0 AMPS & & & & \\
\hline ROTOR DELAY & 1.6 SEC & & & & \\
\hline DOWNSLOPE & 8.4 SEC & & & & \\
\hline PREPURGE & 20 SEC & & & & \\
\hline POSTPURGE & 20 SEC & & & & \\
\hline TACKS & DEGREES & AMPS & \multicolumn{2}{|c|}{ SECONDS } & \\
\hline \multicolumn{5}{|l|}{$n / a$} & \\
\hline \multicolumn{6}{|l|}{$n / a$} \\
\hline \multicolumn{6}{|l|}{$n / a$} \\
\hline \multirow{2}{*}{ LEVEL } & \multirow{2}{*}{ IMPULSE } & \multirow{2}{*}{ MAINT } & \multicolumn{2}{|c|}{-IMPULSE- } & \multirow{2}{*}{$\begin{array}{c}\text { AVG. } \\
\text { CURRENT }\end{array}$} \\
\hline & & & RATE & WIDTH & \\
\hline 1 & 29 AMPS & 8.5 AMPS & 3 & 33 & 15.2 AMPS \\
\hline 2 & 27.5 AMPS & 8.5 AMPS & 3 & 33 & 14.7 AMPS \\
\hline 3 & 26.0 AMPS & 8.5 AMPS & 3 & 33 & 14.2 AMPS \\
\hline 4 & 24.6 AMPS & 8.5 AMPS & 3 & 33 & 13.8 AMPS \\
\hline LEVEL & TIME & RAMP & \multicolumn{2}{|c|}{ RMP } & $\begin{array}{l}\text { TRAVEL } \\
\text { SPEED }\end{array}$ \\
\hline 1 & 10.9 & 0.0 & \multicolumn{2}{|c|}{1.43} & $4.0 \mathrm{in} / \mathrm{min}$ \\
\hline 2 & 10.9 & 0.0 & \multicolumn{2}{|c|}{1.43} & $4.0 \mathrm{in} / \mathrm{min}$ \\
\hline 3 & 10.9 & 0.0 & \multicolumn{2}{|c|}{1.43} & $4.0 \mathrm{in} / \mathrm{min}$ \\
\hline 4 & 10.9 & 0.0 & \multicolumn{2}{|c|}{1.43} & $4.0 \mathrm{in} / \mathrm{min}$ \\
\hline
\end{tabular}

-OUTPUTS-

\begin{tabular}{|c|c|c|c|c|c|c|c|}
\hline -AVERAGE- & AMPS & VOLTS & RPM & $\begin{array}{c}\text { TRAVEL } \\
\text { SPEED }\end{array}$ & TIME & KJ/in & \\
\hline LEVEL & 15.3 & 13.2 & 1.45 & $4.0 \mathrm{in} / \mathrm{min}$ & 10.9 & 3.0 & \\
\hline 1 & 14.8 & 12.9 & 1.45 & $4.0 \mathrm{in} / \mathrm{min}$ & 10.9 & 2.9 & \\
\hline 2 & 14.3 & 13.0 & 1.45 & $4.0 \mathrm{in} / \mathrm{min}$ & 10.9 & 2.8 & \\
\hline 3 & 13.9 & 13.5 & 1.45 & $4.0 \mathrm{in} / \mathrm{min}$ & 10.9 & 2.8 & \\
\hline 4 & 7.6 & 16.3 & 1.46 & $4.0 \mathrm{in} / \mathrm{min}$ & 8.4 & 1.8 & \\
\hline D/S & & & & & \\
\hline
\end{tabular}

COMMENTS: Note: Typical tube - tube joint, non-part-prototypical, Verify Tungsten ioint centering. Outer appearance acceptable. LOF $4^{\text {th }}$ Quadrant. 
IDENT: Capsule S/N: nia LAB TEST NUMBER : JDT-010 TEST DESCRIPTION: Tube-to-Tube

WPS: JOYO-8 PROGRAMMER: CLAPP REF DRAWING(S): N/A)

PROCESS: AGTAW POWER SUPPLY: SWS M100-1, VER: 2.03 WELD HEAD: SWS-10H-B

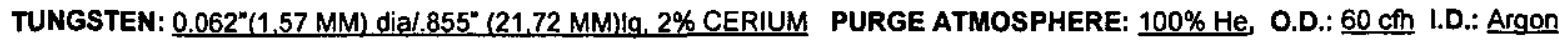

ARC GAP: 1.02 MM ARC GAP GAGE SETTING: 37.84 MM WELD OPERATOR: CLAPP MIN VOLTS: 9.0

CURRENT TOL: $2.5 \%$ SPEED TOL: 2.5\% CUR LIMIT: 100\% PURGE LIMIT: $100 \%$ GLOVEBOX $\square$ NON-GLOVEBOX $\triangle$

JOINT TYPE: IB-TB Buth MATERIAL TYPE: 316 WELD DIAMETER: 23 MM WALL THICKNESS: 1,5 MM

- INPUTS -

\begin{tabular}{|c|c|c|c|c|c|}
\hline VARIABLE & VALUE & & & & \\
\hline START POWER & NORM & & & & \\
\hline $\begin{array}{c}\text { START } \\
\text { CURRENT }\end{array}$ & 22.4.0 AMPS & & & & \\
\hline ROTOR DELAY & 1.6 SEC & & & & \\
\hline DOWNSLOPE & 8.4 SEC & & & & \\
\hline PREPURGE & $20 \mathrm{SEC}$ & & & & \\
\hline POSTPURGE & $20 \mathrm{SEC}$ & & & & \\
\hline TACKS & DEGREES & AMPS & \multicolumn{2}{|c|}{ SECONDS } & \\
\hline \multicolumn{5}{|l|}{ n/a } & \\
\hline \multicolumn{6}{|l|}{ n/a } \\
\hline \multicolumn{6}{|l|}{$\mathrm{Na}$} \\
\hline \multirow{2}{*}{ LEVEL } & \multirow{2}{*}{ IMPULSE } & \multirow{2}{*}{ MAINT } & \multicolumn{2}{|c|}{-IMPULSE- } & \multirow{2}{*}{$\begin{array}{c}\text { AVG. } \\
\text { CURRENT }\end{array}$} \\
\hline & & & RATE & MDTH & \\
\hline 1 & 40.6 AMPS & 11.9 AMPS & 3 & 33 & 21.3 AMPS \\
\hline 2 & 40.6 AMPS & 11.9 AMPS & 3 & 33 & 21.3 AMPS \\
\hline 3 & 40.6 AMPS & 11.9 AMPS & 3 & 33 & 21.3 AMPS \\
\hline 4 & 40.6 AMPS & 11.9 AMPS & 3 & 33 & 21.3 AMPS \\
\hline LEVEL & TIME & RAMP & \multicolumn{2}{|c|}{ RMP } & $\begin{array}{l}\text { TRAVEL } \\
\text { SPEED }\end{array}$ \\
\hline 1 & 10.9 & 0.0 & \multicolumn{2}{|c|}{1.43} & $4.0 \mathrm{in} / \mathrm{min}$ \\
\hline 2 & 10.9 & 0.0 & \multicolumn{2}{|c|}{1,43} & $4.0 \mathrm{in} / \mathrm{min}$ \\
\hline 3 & 10.9 & 0.0 & \multicolumn{2}{|c|}{1.43} & $4.0 \mathrm{in} / \mathrm{min}$ \\
\hline 4 & 10.9 & 0.0 & \multicolumn{2}{|c|}{1.43} & $4.0 \mathrm{in} / \mathrm{min}$ \\
\hline
\end{tabular}

COMMENTS

-OUTPUTS-

-AVERAGE-

LEVEL

1

\begin{tabular}{l|l}
2 & 214 \\
\hline
\end{tabular}

\begin{tabular}{l|l}
3 & 21.4 \\
\hline
\end{tabular}

D/S

\begin{tabular}{|c|c|c|c|}
\hline AMPS & VOLTS & RPM & $\begin{array}{c}\text { TRAVEL } \\
\text { SPEED }\end{array}$ \\
\hline 21.4 & 12.9 & 1.45 & $4.0 \mathrm{in} / \mathrm{min}$ \\
\hline 21.4 & 13.4 & 1.45 & $4.0 \mathrm{in} / \mathrm{min}$ \\
\hline 21.4 & 13.6 & 1.45 & $4.0 \mathrm{in} / \mathrm{min}$ \\
\hline 21.4 & 13.9 & 1.45 & $4.0 \mathrm{in} / \mathrm{min}$ \\
\hline 11.1 & 16.0 & 1.46 & $4.0 \mathrm{in} / \mathrm{min}$ \\
\hline
\end{tabular}

\begin{tabular}{|c|c|c|}
\hline TIME & KJ / in & \\
\hline & & \\
\hline 10.9 & 4.1 & \\
\hline 10.9 & 4.3 & \\
\hline 10.9 & 4.4 & \\
\hline 10.9 & 4.5 & \\
\hline 8.4 & 2.6 & \\
\hline
\end{tabular}

COMMENTS: Note: Typical tube - tube ioint, non-part-prototypical. Verify Tungsten joint centering. Outer appearance acceptable, penetration acceptable. 
Enclosure (7) to

MDO-723-0044/

B-MT(SRME)-52

Page 138

\section{WELD PROCEDURE DATA SHEET \#6}

DATE RECORDED: 9-14-05 RECORDED BY: R. Clapp

IDENT: Capsule SIN: nla LAB TEST NUMBER : JDT-011 TEST DESCRIPTION: Tube-to-Tube

WPS: JOYO-10 PROGRAMMER: CLAPP REF DRAWING(S): N/A)

PROCESS: AGTAW POWER SUPPLY: SWS M100-1, VER: 2.03 WELD HEAD: SWS-10H-B

TUNGSTEN: 0.062"(1.57 MM) dial.855" (21.72 MMilg. 2\% CERIUM PURGE ATMOSPHERE: 30Ar-70He, O.D.: 40 cfh 1.D.: Argon

ARC GAP: 1.02 MM ARC GAP GAGE SETTING: 37.84 MM WELD OPERATOR: CLAPP MIN VOLTS: 9.0

CURRENT TOL: $2.5 \%$ SPEED TOL: $2.5 \%$ CUR LIMIT: 100\% PURGE LIMIT: $100 \%$ GLOVEBOX $\square$ NON-GLOVEBOX $\square$

JOINT TYPE: TB-TB BUt1 MATERIAL TYPE: 316 WELD DIAMETER: 23 MM WALL THICKNESS: 1.5 MM

- INPUTS -

\begin{tabular}{|c|c|c|c|c|c|}
\hline VARIABLE & VALUE & & & & \\
\hline START POWER & NORM & & & & \\
\hline $\begin{array}{c}\text { START } \\
\text { CURRENT }\end{array}$ & 22.4.0 AMPS & & & & \\
\hline ROTOR DELAY & $1.6 \mathrm{SEC}$ & & & & \\
\hline DOWNSLOPE & 8.4 SEC & & & & \\
\hline PREPURGE & 20 SEC & & & & \\
\hline POSTPURGE & 20 SEC & & & & \\
\hline TACKS & DEGREES & AMPS & \multicolumn{2}{|c|}{ SECONDS } & \\
\hline \multicolumn{5}{|l|}{$\pi / a$} & \\
\hline \multicolumn{6}{|l|}{$\mathrm{n} / \mathrm{a}$} \\
\hline \multicolumn{6}{|l|}{ n/a } \\
\hline \multirow{2}{*}{ LEVEL } & \multirow{2}{*}{ IMPULSE } & \multirow{2}{*}{ MAINT } & \multicolumn{2}{|c|}{-IMPULSE- } & \multirow{2}{*}{$\begin{array}{c}\text { AVG. } \\
\text { CURRENT }\end{array}$} \\
\hline & & & RATE & WDTH & \\
\hline 1 & 50 AMPS & 15 AMPS & 3 & 33 & 26.5 AMPS \\
\hline 2 & 50 AMPS & 15 AMPS & 3 & 33 & 26.5 AMPS \\
\hline 3 & 50 AMPS & 15 AMPS & 3 & 33 & 26.5 AMPS \\
\hline 4 & 50 AMPS & 15 AMPS & 3 & 33 & 26.5 AMPS \\
\hline LEVEL & TIME & RAMP & \multicolumn{2}{|c|}{ RMP } & $\begin{array}{l}\text { TRAVEL } \\
\text { SPEED }\end{array}$ \\
\hline 1 & 10.9 & 0.0 & \multicolumn{2}{|c|}{1.43} & $4.0 \mathrm{in} / \mathrm{min}$ \\
\hline 2 & 10.9 & 0.0 & \multicolumn{2}{|c|}{1.43} & $4.0 \mathrm{in} / \mathrm{min}$ \\
\hline 3 & 10.9 & 0.0 & \multicolumn{2}{|c|}{1.43} & $4.0 \mathrm{in} / \mathrm{min}$ \\
\hline 4 & 10.9 & 0.0 & \multicolumn{2}{|c|}{1.43} & $4.0 \mathrm{in} / \mathrm{min}$ \\
\hline
\end{tabular}

-OUTPUTS-

\begin{tabular}{|c|c|c|c|c|c|c|c|}
\cline { 2 - 7 } -AVERAGE- & AMPS & VOLTS & RPM & $\begin{array}{c}\text { TRAVEL } \\
\text { SPEED }\end{array}$ & TIME & KJ/in & \\
\hline 1 & 26.6 & 10.3 & 1.45 & $4.0 \mathrm{in} / \mathrm{min}$ & 10.9 & 4.1 & \\
\hline 2 & 26.6 & 10.6 & 1.45 & $4.0 \mathrm{in} / \mathrm{min}$ & 40.9 & 4.2 & \\
\hline 3 & 26.6 & 11.0 & 1.45 & $4.0 \mathrm{in} / \mathrm{min}$ & 10.9 & 4.4 & \\
\hline 4 & 26.6 & 11.1 & 1.45 & $4.0 \mathrm{in} / \mathrm{min}$ & 10.9 & 4.4 & \\
\hline D/S & 13.5 & 12.8 & 1.46 & $4.0 \mathrm{in} / \mathrm{min}$ & 8.4 & 2.6 & \\
\hline
\end{tabular}

COMMENTS: Note: Outer appearance acceptable, penetration $95 \%$ acceptable; upping current 5-6 amps should provide acceptable penetration. 


\section{Conclusion}

The developmental results and planning completed thus far provides sufficient information to conclude that the identified Autogenous Automatic Gas Tungsten Arc Welding process could successfully be developed, qualified, and implemented in sufficient order to support all facets of the JOYO-1 material test capsule closure welds production runs.

\section{Post Weld Inspections}

Post weld inspections are performed on each capsule to ensure a quality weld and to conform with ASME B\&PVC and JNC requirements. These inspections include dimensional, visual, liquid penetrant, pressure and helium leak test, and radiography on pre-selected capsules. The assembly sketches, assembly route cards, and local inspection procedures will define the specific inspection requirements and acceptance criteria.

\section{Dimensional}

The capsule weld area will be dimensionally inspected for undercuts and excess reinforcement. Should weld undercuts exceed allowable values, the area will be re-welded. Alternately, if reinforcement (excess weld beyond the capsule) is exceeded, the weld will be filed to ensure the maximum diameter is not exceeded. The as built capsule length, diameter and weight will be measured and recorded on the assembly route card.

\section{Visual}

Visual examinations at $1 X$ magnification will be performed to look unacceptable conditions such as cracks, linear indications, weld spatter, or arc strikes.

\section{Liquid Penetrant}

Liquid penetrant examination will be performed be ASME B\&PVC Section III, Class 3 using local procedures to detect discontinuities which are open to the surface. Acceptance criteria is defined on the assembly sketches.

\section{Pressure and Leak Test}

An external pressure will be applied to the welded capsule using a gas mixture of Argon and a minimum of $3 \%$ Helium. An internal pressure test is not feasible since the weld seals the pressure boundary and no penetrations exist through the pressure boundary. This pneumatic test is conducted at minimum of 1.2 times the design pressure. Immediately following the pressure test, the capsule is removed from the pressure vessel, sprayed with nitrogen and inserted into a bell jar for helium leak detection using local procedures.

Pressure and leak test requirements and acceptance criteria are defined on the sketches.

\section{Radiography}

Pre-selected capsules will undergo radiography for information to evaluate the welded area for weld penetration and defects using a local procedure. Because of the wave spring behind the weld and the small size of the capsule, accurate radiographic inspection is difficult. Figure IV.D-5-1 shows radiographs with and without a wave spring in a similar 
designed capsule. Since radiography is not feasible, the analysis uses Code joint efficiency factors corresponding to neither fully or spot radiography.
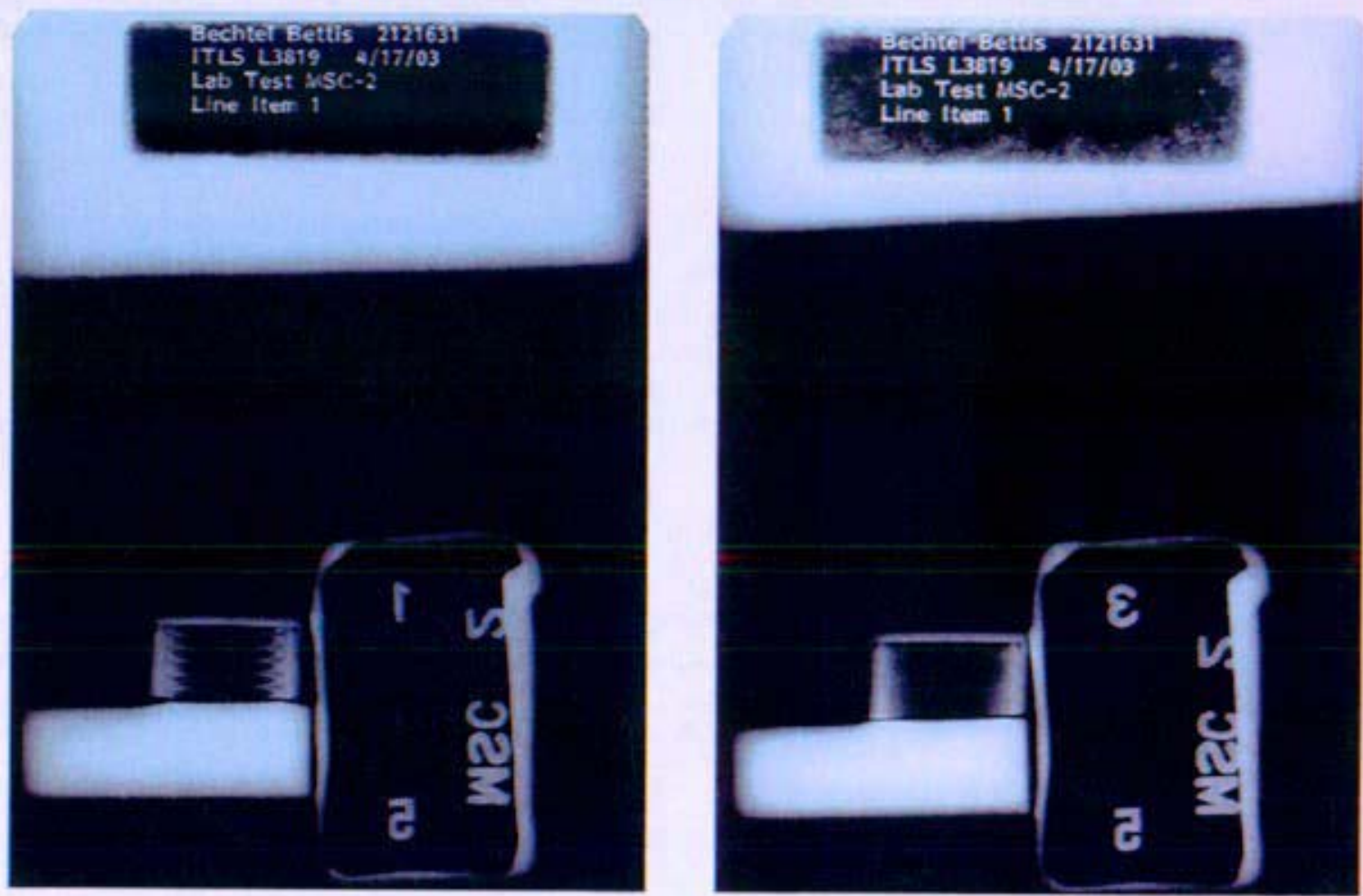

Figure IV.D.5-1 Radiographs With and Without Wave Spring

\section{E. Inspection Plan}

\section{NRPCT Quality Assurance Plan}

The Nineteen Points of Laboratory Quality (Bettis' quality plan) establishes the quality requirements for all technical work conducted by the Laboratory. The Nineteen Points of Laboratory Quality, in conjunction with 11 functional area Level II Manuals, and various Level III documents, provide the requirements and define the overall structure of the Laboratory Quality Program. The Laboratory Quality Program was developed by representatives from the technical community and provides a structured system for implementing the quality requirements of the Prime Contractor Quality Specification (PCQS). The PCQS is the NR Program quality standard for Prime Contractor organizations. The requirements of PCQS are aligned with those contained in the Code of Federal Regulations, Title 10, Part 50, Appendix B, for facilities licensed by the Nuclear Regulatory Commission.

\section{Capsule Fabrication Quality Requirements}

Quality control inspections were to be invoked during the procurement phase and post weld examinations to ensure that the raw materials met the material specifications requirements and the post weld examinations met the acceptance criteria. These inspections were to be performed by independent personnel not performing the work. In addition, an Engineering 
review was to be performed to ensure the hardware fabrication and the assembly processes were performed in accordance with the procurement specifications and assembly procedures. Table IV.E-1 list the various inspections and engineering oversight for the JOYO-1 capsules:

\begin{tabular}{|c|c|c|}
\hline \multicolumn{3}{|c|}{ Table IV.E-1 Specific JOYO Inspections and Quality Controls } \\
\hline Inspection & Quality & Performed by \\
\hline \multicolumn{3}{|c|}{$\begin{array}{l}\text { A. Fabrication of Capsule Components (includes capsule, head, insulators, spacers, and } \\
\text { holders) }\end{array}$} \\
\hline \multirow[t]{2}{*}{ 1. Material } & $\begin{array}{l}\text { Material chemical composition and mechanical } \\
\text { properties will be confirmed to meet the material } \\
\text { specifications called out on the drawings or } \\
\text { sketches. }\end{array}$ & $\begin{array}{l}\text { NRPCT Inspecto } \\
\text { or } \\
\text { NRPCT Enginee }\end{array}$ \\
\hline & $\begin{array}{l}\text { Traceability from the part serial number to the } \\
\text { material certification heat number will be verified. }\end{array}$ & $\begin{array}{l}\text { NRPCT Inspecto } \\
\text { or } \\
\text { NRPCT Enginee }\end{array}$ \\
\hline 2. Component dimensional & $\begin{array}{l}\text { Critical dimensions to the assembly of parts will be } \\
\text { confirmed with Fabricator's data sheet. }\end{array}$ & $\begin{array}{l}\text { NRPCT } \\
\text { Inspector. }\end{array}$ \\
\hline \multicolumn{3}{|l|}{ B. Welding Qualification } \\
\hline 1. Procedures & $\begin{array}{l}\text { Approved Route Cards (also called procedures or } \\
\text { travelers) for each capsule will be followed to } \\
\text { qualify the weld in accordance with Section IX of } \\
\text { the Code. Traceability to part serial numbers. } \\
\text { Route Cards will be reviewed and approved by: } \\
\text { Cognizant Manager for Facility where work will be } \\
\text { performed, Manager SMI, Cognizant Engineer, } \\
\text { Quality Control, Welding Engineer, and Welding } \\
\text { Manager. } \\
\text { Route Cards will be signed by individuals } \\
\text { performing the work. Double verification of serial } \\
\text { numbers will be performed by Technician and } \\
\text { Assembly Engineer. NRPCT Inspectors sign for } \\
\text { examinations. } \\
\end{array}$ & $\begin{array}{l}\text { NRPCT } \\
\text { Technicians, } \\
\text { Welding } \\
\text { Engineer, and } \\
\text { Managers. } \\
\text { NRPCT } \\
\text { Inspectors will } \\
\text { perform NDE } \\
\text { examinations. }\end{array}$ \\
\hline 2. Qualification & $\begin{array}{l}\text { The weld qualification plan is identified in B- } \\
\text { MT(EDT)S-029, NRPCT JOYO Pressure } \\
\text { Boundary Weld Qualification Process. }\end{array}$ & \\
\hline 3. Certification & $\begin{array}{l}\text { Independent Testing Laboratory Welder and Weld } \\
\text { Procedure Certification. }\end{array}$ & $\begin{array}{l}\text { Independent } \\
\text { Laboratory }\end{array}$ \\
\hline \multicolumn{3}{|l|}{ C. Capsule Assembly } \\
\hline 1. Procedures & $\begin{array}{l}\text { Each capsule will have its own route card with } \\
\text { Traceability to part serial numbers. } \\
\\
\text { Route Cards will be reviewed and approved by: } \\
\text { Specimen Test Sponsors, Cognizant Manager for } \\
\text { Facility where work will be performed, Manager } \\
\text { SMI, Cognizant Engineer, Quality Assurance, } \\
\text { Welding Manager (if welding is involved) }\end{array}$ & $\begin{array}{l}\text { NRPCT } \\
\text { Engineers and } \\
\text { Managers }\end{array}$ \\
\hline
\end{tabular}


Enclosure (7) to

MDO-723-0044/

B-MT(SRME)-52

Page 142

\begin{tabular}{|c|c|c|}
\hline \multicolumn{3}{|c|}{ Table IV.E-1 Specific JOYO Inspections and Quality Controls } \\
\hline Inspection & Quality & Performed by \\
\hline 2. Assembly Process & $\begin{array}{l}\text { Assembly includes cleaning, bake-out, installation } \\
\text { of specimens in holders, and capsule assembly in } \\
\text { the glove box. } \\
\text { Serial numbers of specimens will be double } \\
\text { verified at the time of insertion into the holders } \\
\text { (two independent NRPCT personnel). }\end{array}$ & $\begin{array}{l}\text { NRPCT } \\
\text { Technicians and } \\
\text { Engineers }\end{array}$ \\
\hline \multicolumn{3}{|l|}{ D. Capsule Welding } \\
\hline 1. Welding & $\begin{array}{l}\text { Welding is called out from the Assembly Route } \\
\text { Card. } \\
\text { The following information will be recorded and } \\
\text { compared to limits prior to and during welding: } \\
\text { Oxygen, Moisture, Welding Voltage and Current. }\end{array}$ & $\begin{array}{l}\text { NRPCT Welding } \\
\text { Technicians }\end{array}$ \\
\hline \multicolumn{3}{|l|}{ E. Post Examinations } \\
\hline 1. Visual Examination & $\begin{array}{l}\text { Visual Exams are performed invoked by the } \\
\text { Assembly Route Card and performed in } \\
\text { accordance with Assembly Sketch } \\
\text { Requirements. } \\
\text { See Capsule Assembly Sketches for visual } \\
\text { inspections and acceptance criteria. }\end{array}$ & NRPCT Inspes \\
\hline 2. Dimensional & $\begin{array}{l}\text { Dimensional inspections are invoked by the } \\
\text { Assembly Route Card and performed in } \\
\text { accordance with Assembly Sketch Requirements } \\
\text { using calibrated measuring equipment. } \\
\text { See Capsule Assembly Sketches for dimensional } \\
\text { inspections and acceptance criteria. }\end{array}$ & NRPCT Inspec \\
\hline 3.Liquid Penetrant Inspection & $\begin{array}{l}\text { Liquid Penetrant inspections are called out from } \\
\text { the Assembly Route Card and performed in } \\
\text { accordance with Assembly Sketch Requirements. } \\
\text { A Liquid Penetrant Inspection procedure will be } \\
\text { followed per DIP-661 with acceptance criteria as } \\
\text { specified on the Assembly Sketch and per ASME } \\
\text { BPVC Sect. III, ND-5000. }\end{array}$ & NRPCT Inspecto \\
\hline 4. Weights & $\begin{array}{l}\text { Capsule weighing is called out from the Assembly } \\
\text { Route Cards using calibrated scales. Weight will } \\
\text { be recorded in the Route Card. }\end{array}$ & $\begin{array}{l}\text { NRPCT Inspecto } \\
\text { or NRPCT } \\
\text { Certified } \\
\text { Technician }\end{array}$ \\
\hline 5. Radiography. & $\begin{array}{l}\text { Radiography will be performed on selected } \\
\text { capsules using the Assembly Route Cards and } \\
\text { Radiography procedure. } \\
\text { Radiography is performed in accordance with } \\
\text { Assembly Sketch Requirements and DIP-662. }\end{array}$ & NRPCT Inspecto \\
\hline
\end{tabular}




\begin{tabular}{|c|c|c|}
\hline \multicolumn{3}{|c|}{ Table IV.E-1 Specific JOYO Inspections and Quality Controls } \\
\hline Inspection & Quality & Performed by \\
\hline $\begin{array}{l}\text { 6. Pressure Test and Leak } \\
\text { test. }\end{array}$ & $\begin{array}{l}\text { Pressure testing and Leak testing is called out } \\
\text { from the Assembly Route Cards with pressure } \\
\text { test/leak test being performed per procedure } \\
\text { DMP-LD-703 with the acceptance criteria } \\
\text { specified on the Assembly Sketch. Leak rate will } \\
\text { be recorded in the Assembly Route Card. }\end{array}$ & $\begin{array}{l}\text { NRPCT Certified } \\
\text { Technician }\end{array}$ \\
\hline \multicolumn{3}{|l|}{ F. Certifications } \\
\hline $\begin{array}{l}\text { 1. Component procurement } \\
\text { Certification }\end{array}$ & $\begin{array}{l}\text { The component fabrication will be reviewed to } \\
\text { ensure the component was fabricated in } \\
\text { accordance with the Purchase Order and sketch. } \\
\text { Material certifications will be reviewed o ensure } \\
\text { the materials meet the material or Purchase } \\
\text { Order specification. }\end{array}$ & $\begin{array}{l}\text { Assembly } \\
\text { Engineer and SM } \\
\text { Manager }\end{array}$ \\
\hline 2. Assembly Certification & $\begin{array}{l}\text { Assembly Route cards will be reviewed to ensure } \\
\text { the capsule was assembled per the Route Card } \\
\text { instructions and all the examinations are found } \\
\text { acceptable. }\end{array}$ & $\begin{array}{l}\text { QA Engineer, } \\
\text { Assembly } \\
\text { Engineer, SMI } \\
\text { Manager. }\end{array}$ \\
\hline \multicolumn{3}{|l|}{ Definitions } \\
\hline NRPCT Certified Technician & \multicolumn{2}{|c|}{$\begin{array}{l}\text { Personnel who are to perform operations in this procedure shall be trained until } \\
\text { familiar with the use of equipment and operations employed in the performance of } \\
\text { this procedure by working with qualified operators or under the guidance of the area } \\
\text { manager. Personnel qualification shall be determined by the issuing DMP } \\
\text { engineering activity and/or the individual's immediate manager based on successful } \\
\text { mock-up or dry-run operational performance. Personnel to be qualified shall be } \\
\text { identified by the issuance of an Engineering Notice (Form 73307) documenting the } \\
\text { qualification, with a copy placed in training records accordingly. Requalification is } \\
\text { not required unless an individual is disqualified, in which case an Engineering Notice } \\
\text { shall be issued to document requalification. }\end{array}$} \\
\hline NRPCT Inspector: & \multicolumn{2}{|c|}{$\begin{array}{l}\text { Personnel who are to perform operations in this procedure shall be trained and } \\
\text { qualified per SNT-TC-1A, Personnel Qualification and Certification in } \\
\text { Nondestructive. Personnel are to be issued Certifications }\end{array}$} \\
\hline NRPCT Welding Technicians & \multicolumn{2}{|c|}{$\begin{array}{l}\text { Personnel who are to perform operations shall perform the welding qualifications per } \\
\text { B-MT(EDT)S-010, NRPCT JOYO Pressure Boundary Weld Qualification Process. A } \\
\text { Welder Qualification will be issued by an independent Testing Laboratory. }\end{array}$} \\
\hline NRPCT Technicians & \multicolumn{2}{|c|}{$\begin{array}{l}\text { Personnel who are to perform operations in this procedure shall demonstrate by } \\
\text { performing operations on mockups to show that the individual is competent to do so } \\
\text { in accordance with the Route Cards. No training Certificate is issued. }\end{array}$} \\
\hline NRPCT Engineers & \multicolumn{2}{|c|}{$\begin{array}{l}\text { Personnel who have a University Degree in Engineering and competent in their fieid. } \\
\text { The Engineer will review material certifications to ensure compliance with Material } \\
\text { Certifications. ensure the assembly procedures are followed with specimen serial } \\
\text { numbers in the properly assigned location, and certify that the component hardware } \\
\text { or specimens were manufactured according to drawing and purchase order } \\
\text { requirements. No training Certificate is issued. }\end{array}$} \\
\hline
\end{tabular}

\section{Quality Requirements}

The NRPCT requires certifications on all manufactured components received from vendors and test capsules assembled by the NRPCT. These certifications ensure that all test hardware identifications are unique and that the vendors have met all requirements for the manufacturing of the design. The test sponsors will be responsible to have their test specimens certified to NRPCT specifications. 
Enclosure (7) to

MDO-723-0044I

B-MT(SRME)-52

Page 144

The NRPCT engineer will gather all records about the fabrication, inspection and preirradiation testing of the test hardware. The NRPCT engineer will review the material to make sure the vendor has met all requirements and has reported any findings to the NRPCT.

Once the review has been completed, the NRPCT engineer will have the certification package signed by a Technical Reviewer as a peer review to assure all certifications are appropriate.

Management reviews the certification package, and once management signs, the certification package is complete.

All assembled capsules at the NRPCT go through the same certification process as the parts received from the vendors. All completed certification packages were planned to be sent to PNNL and JNC for completeness to provide them assurance that the capsules were fabricated to high quality standards.

At a meeting in June 2005 in Japan at JNC, the NRPCT presented the Quality Assurance plan for the capsule assembly, inspection, and certification. The plan utilizes standard QA oversight practices used by the NRPCT in the assembly of irradiation hardware for testing. JNC agreed that the QA plan was acceptable. Therfore, contrary to an original request for JNC to observe capsule assembly operations, JNC stated that they did not need to have personnel witness the capsule assembly and inspection process as an additional oversight.

\section{F. Shipping Plan}

\section{Regulations}

Title 49 Code of Federal Regulations (CFR) was to be followed for the unirradiated capsule shipment from NRPCT through PNNL to JNC. U.S. domestic shipping requirements will meet international requirements as stated in Title 49 Subchapter $C$ part 171.1.

The BCD capsules contain the pressurized helium biaxial creep specimens which have the following restrictions:

a. Nonflammable gases are regulated by the USDOT as Hazardous Material if the material exerts a pressure of $280 \mathrm{kPa}(40.6 \mathrm{psia})$ at $20^{\circ} \mathrm{C}\left(68^{\circ} \mathrm{F}\right)$ on the container. [part 173.115 paragraph (b) subpart (1)]

b. No label required (except when offered for transportation by air). [part 173.306 paragraph (a)]

c. Package weight is below $30 \mathrm{~kg}$. [part 173.306 paragraph (a)]

d. Containers of not more than 4 fluid ounces capacity (7.22 cubic inches). [part 173.306 paragraph (a) subpart 1]

\section{Export Controls}

PNNL has researched the following with regard to export controls:

a. DOE Sensitive Subjects List which identifies Nuclear Reactor Systems, Subsystems, and associated technologies (for space reactors) as an area that may contain sensitive information and need export control and refers to the following docs for more detailed information 
1.) Department of Commerce Export Administration Regulations (15 CFR Parts 730774)

2.) Nuclear Regulatory Commission Regulations (10 CFR Part 110)

3.) Department of Energy Regulations (10 CFR Part 810), and

4.) Department of State International Traffic in Arms Regulations (22 CFR Parts 120130)

b. Dept. of Commerce (DOC) Export Administration Regulations (EAR) defers to the Dept. of State (DOS) Directorate of Defense Trade Controls (DDTC) with regard to reactor technology

c. DOS International Traffic in Arms Regulations (ITAR) has nothing specific relating to space reactor power systems

d. Nuclear Regulatory Commission Regulations (10 CFR Part 110) pertains to commercial reactor technology and duel-use applications not specific to space reactor technology

e. Any review against the Department of Energy Regulations (10 CFR Part 810) would take place in conjunction with a Dept. of Commerce Export Control determination. Therefore, if there are not DOC issues then there should not be a DOE issue.

f. Dept. of Defense (DoD) Militarily Critical Technologies List (MCTL). Section 13 identifies the following as key technologies for developing, building, and operating nuclear fission reactor systems:

1.) Fuel fabrication techniques,

2.) Critical instrumentation and control technologies.

g. Department of Commerce controls were reviewed for Nickel and Beryllium alloys

Export Control Screening Conclusion

a. PNNL has reviewed Nickel and Beryllium against the Country List and found that none of the JOYO-1 test specimen materials are controlled when shipping to Japan.

b. Export license is not required to ship the proposed JOYO-1 structural materials to Japan. However, Japan may require JNC to obtain an import license.

\section{Required Technical Data}

The following technical data must be prepared for the import inspection under the Japanese high-pressurized gas safety law.

a. Certificate of imported gas such as chemical analysis data, purity, total volume, maximum filling pressure, etc.

b. Name and address of gas filler.

c. Name and address of manufacturer of gas container, i.e. in-pile creep specimen.

d. Certificate of gas filling such as, specimen ID, design test temp, design hoop stress, fill gas, internal pressure, fill gas volume, for each specimen.

e. Table (list) of imported specimen and irradiation capsules.

f. Technical drawing of in-pile creep specimen which shows structure and gas filling volume.

g. Technical data of in-pile creep specimen, such as material data, metallurgical data for welded region (plug-cladding and gas filling hole) to assure the safety. 
Enclosure (7) to

MDO-723-0044I

B-MT(SRME)-52

Page 146

The NBD and NBU capsules contain the BeO (Beryllia) ceramic specimens. There are no U.S. Department of Transportation hazardous material regulations which apply to the packaging and labeling of this product.

\section{Shipping Package}

A purchase order was placed with Melmat Inc. to make ABS plastic shipping cases. The shipping case inside dimensions were 18.0 inches in length, 13.0 inches in width, 8.0 inches in base depth, and 3.0 inches in lid depth. Each case was to have two metal latches. There was to be one spring loaded handle located at the center of the lid and oriented parallel to the long side of the case. The location of the handle was determined such that the capsules would be oriented vertically at all times while in transit. Each case was to have at least two hinges; the number and location of hinges was to be determined by Melmat Inc. The polyurethane cushioning material shown in Figure IV.F-1 was to be die-cut by Melmat Inc. according to sketch SK-JPH1111 Rev Orig. The size of the cutouts was 0.1"-0.2" smaller than the actual part being inserted into the foam to allow some compression on the part in order to combat rattling. The foam was also sized so that $0.5^{\prime \prime}$ of foam would compress in the vertical direction when the shipping case was shut. Each cutout was positioned approximately 2" from the next nearest cutout so that there would be no interference with neighboring components.

A wooden crate was made to store the plastic shipping cases. The crate would be stored in the warehouse until the cases were needed for transporting capsules during the assembly process or for shipping.

Four shock indicators from Impact-o-graph were to be located in each shipping container as shown in Figure IV.F-1. One shock indicator would measure an impact of 20G, one of 30G, and two of 50G (Impact-o-graph item numbers 930020,930030, and 930050, respectively). A shock harness was to be made so that two shock indicators could be mounted to one capsule in each shipping container. The shock harness would be made of Nylon $6 / 6$ as shown in sketch SK-JAM-1001 and would be mounted to the capsule as shown in SK-JAM1003. The shock indicators mounted to the capsule were to be from AGM Container Controls, Inc. and would measure $30 \mathrm{G}$ and $50 \mathrm{G}$ impacts (AGM item numbers TA280-018030 and TA280-018-050, respectively). NRPCT would evaluate a course of action to be taken by JNC if the highest G-load indicators were tripped. NRPCT would provide JNC with guidance on capsule radiography parameters to assist with inspection setup based on NRPCT experience in radiographing capsules at Bettis.

Each shipping case would hold 6 capsules. The shipping case was to be surrounded with two inches of foam plank packing material and enclosed in a wooden box. There would be a total of 10 wooden boxes shipped to JNC with an approximate size of $25 \times 19 \times 17$ inches.

\section{Shipping}

Shipping of the wooden boxes was to be subcontracted to Nippon Express. Although the details were not complete, Nippon Express would have picked up the packages at the Bettis facility or the packages would have been shipped to Nippon Express Seattle office utilizing FedEx Custom Critical Shipping. Custom Critical Shipping involves two drivers in a specially equipped truck enabling delivery in a secure and timely manner. Special handling was considered prudent due to the delicate nature of the capsule specimens and the cost for this method is low compared to the cost of the 60 capsules. Nippon Express would have 
shipped five packages in one plane and five packages in another plane to JNC located at 4002, Narita-cho, O-arai-machi, Ibarake, Japan.

The NRPCT desired to have a trial shipment of mockup capsules to ensure the method of shipment and receipt would be successful. These capsules would be welded at Bettis and could be used by JNC to inspect the quality of the capsule fabrication process and perform trial fits of the capsules into the compartments. JNC agreed that a trial shipment would be worthwhile and added that as a line item to the Phase $1 \mathrm{C}$ contract to facilitate this shipment.

ABS Plastic Shipping Case

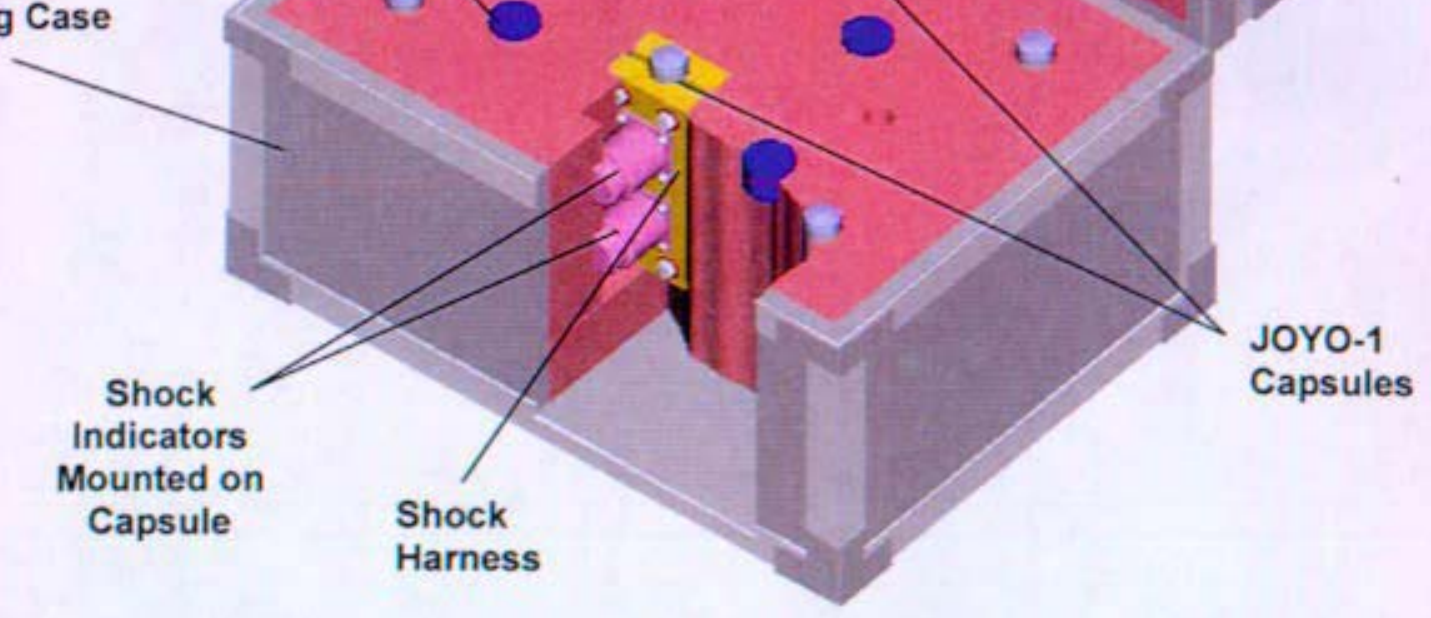

Figure IV.F-1 The shipping case showing cushioning material and capsules. 
Enclosure (7) to MDO-723-0044I

B-MT(SRME)-52

Page 148 


\section{A. Interfaces with JNC}

Communications with JNC were challenging because of the language barrier, time difference (13 hour time difference between Pittsburgh and Tokyo), and the restricted technology exchange due to not having an international agreement (between DOE and JNC in the field of JIMO space reactor materials irradiation testing) or a contract in place. In particular, the lack of a working agreement severely limited the amount of technical reactor information that JNC would provide to the NRPCT to support the design of the test capsules.

Following the initial meeting with the Japanese in November 2004, telephone conferences took place between the NRPCT, PNNL, and JNC to discuss open items relating to the Specific Memorandum Agreement, contracts, capsule design, and miscellaneous items. A teleconference agenda was sent ahead of time so that each party would be prepared. Although both sides were prepared, the language barrier and low audio problems hindered design progress.

Emails between the parties were also used and proved to be good a communication practice. Again, because of the lack of an agreement and contract, technology exchange was limited. This information included sodium temperatures, gamma heating rates, reactor conditions to establish the ASME B\&PVC service levels, material specifications, quality assurance requirements, and transportation issues. This information was crucial for the capsule designers and analysts to proceed.

The best communication and informational exchange was the face-to-face communications during February, June, and August 2005 meetings at JNC. These meetings resolved design questions by overcoming the language barrier with presentations containing pictures and sketches as well as having the opportunity with follow-up questions to ensure complete understanding of the technical issues and responses. The face-to-face meetings were established with an agenda that covered at least three days for each meeting so sufficient time was allocated to address all subject matter. Should future interfaces be required with JNC, an employee on-site at JNC that greatly enhance the ability to answer the volley of questions from the design team and test sponsors and assure they are properly interpreted and complete answers returned. Further, when budgeting for this type of effort, consideration should be given to the cost and time required for regular monthly or bi-monthly face-to-face meetings at JNC to support design progress in the US. If this regular meeting format cannot be accommodated or deemed impractical, then additional effort must be applied to establish a good working agreement with JNC (e.g. an SMA) that will allow JNC to provide the US technical information more freely.

\section{B. Design Considerations}

\section{Nuclear, Thermal and Structural Considerations}

As with any capsule design slated for insertion into a reactor, design and analysis iterations are required between the nuclear gamma heating rates and internal capsule components designs to incrementally achieve the best temperature predictions for the specimens. In the case of the JOYO design work, the nuclear analysis was performed by JNC and/or PNNL and the thermal and structural analysis was performed by the 
Enclosure (7) to

MDO-723-0044/

B-MT(SRME)-52

Page 150

NRPCT. The fact that the work was performed at different locations and with the communication complications described previously, future efforts should consider a consolidation of this analytical work were practical to help minimize transfer and downtime between the agencies. The following summarizes the analysis effort for the design of the capsules and iterative process required.

The first step in the capsule design process is for Nuclear analysts to estimate the gamma and neutron material heat rates based on neutronic evaluations of the core configuration using estimated material make-ups of the irradiation tests. Estimated material make-ups in the neutronic models are generally assumed to the based on the majority material of construction for the hardware. As for the JOYO case, the majority of the hardware material was stainless steel, molybdenum, and graphite.

For the JOYO-1 testing program, these initial gamma heat rates came from JNC because the NRPCT nuclear designers did not have knowledge of the JOYO core design including dimensions, power or core loadings. JNC used an analytical model that represented an early Cycle 1 of the JOYO reactor core loading. Note that the JOYO- 1 test program was to be tested in Cycle 5 and 6 of the JOYO reactor operation. These cycles would entail new or different core loadings from previous cycles. The results of the initial analysis supported the initial gamma heat estimates (Revision 0) for the JOYO-1 test program. Oversight of this initial analysis was provided by PNNL which has personnel experienced in the nuclear modeling of the JOYO reactor.

Capsule designers and thermal analysts took the Revision 0 gamma heating rates and established hardware and gap sizing to provide the best specimen temperature distribution to meet the specimen functional requirements. This sizing established quantity and configuration of the specimens inside the capsule as well as establishing the capsule pressure boundary thicknesses based on estimated worst case service pressures and temperatures. This initial design effort required a thermal model of each capsule type which required a significant amount of engineering time (roughly four weeks). The highest and lowest gamma heating rates based on the capsule core location were then applied to each capsule type model. By changing the gas and internal gaps, the design was enveloped between the highest and lowest gamma heat rates.

For JOYO, ten capsule designs were used in order to include all the various test specimen materials and shapes. Constant communication was required with the material scientist (test sponsor) and capsule designers to ensure that the specimen configuration within the test matrix was acceptable. The specimen test matrix identified specimen material, fluence and temperature requirements. Eventually a preliminary test configuration or test matrix was established.

Once the preliminary design (Revision 0 ) was established, the material specimen and capsule design information was compiled and provided back to PNNL and JNC for a second iteration (Revision 1) of the gamma heat rate analysis. The Revision 0 capsule design information established additional detailed input to the nuclear model to better predict the affects of the reactor on the capsules. At this point, PNNL took the lead in performing the nuclear analysis and used an updated Cycle $5 / 6$ model that represented the reactor core loading to be used for the JOYO-1 testing. The Revision 1 nuclear analysis results were provided to the NRPCT for the Revision 1 thermal and structural 
analysis to support capsule design adjustments to better established the predicted material specimen temperatures.

One last iteration of the analysis process was completed whereby the Revision 1 capsule design and material information was provided back to PNNL for another refinement to the nuclear model (Revision 2). These Revision 2 results were again provided to the NRPCT for the final thermal and structural analysis for JOYO-1 testing. At the time of the termination of the NRPCT involvement in the Space Reactor Program, PNNL was in the process of finalizing the Revision 2 nuclear analysis. For completeness, PNNL completed the Revision 2 analysis and provided it to the NRPCT. The NRPCT performed the Revision 2 thermal and structural analysis as much as practical as part of the preparation of this final JOYO testing report.

The goal of the entire analysis effort was to release the capsule design for fabrication after the Revision 1 thermal and structural analysis in order to support the demanding schedule to meet the reactor insertion window to support a test start in June 2006. The primary objective of the Revision 2 series of analysis was to provide the test sponsors the final temperature predictions for the materials in the JOYO-1 program.

\section{Capsule Assembly}

\section{Beryllium Handling}

Beryllium is considered a sensitive material in the NRPCT program and the NRPCT Laboratories objective is to minimize the use of toxic substances where technically feasible. Because Beryllium was a candidate material for use in the Space Reactor design, the material was recommended for testing in the JOYO-1 test matrix.

Initial planning for the assembly of the JOYO test capsules considered all assembly work to be done at the Bettis lab because all the necessary equipment and personnel experience existed and the cost to due so would be less expensive than outside contracts. Therefore, early in the JOYO test program, arrangements were made to schedule the appropriate time and manpower to prepare capsules to support delivery by March 2006. The JOYO-1 testing was scheduled to start in June 2006.

Because the Beryllium needed to be handled at Bettis for the capsule assembly, extensive effort was applied to provide adequate means to make sure that no personnel contact with the beryllium would be feasible during he assembly process. Bettis met with the Beryllium vendor to establish inspection and verification processes to assure that no loose Beryllium would be shipped to Bettis. Review of the capsule assembly process was conducted with the Bettis Environmental and Safety personnel to make sure the process was capable of precluding personnel contact. Further Bettis considered moving portions of the capsule assembly process to the Knolls Atomic Power Laboratory (KAPL) or to a subcontracted vendor to preclude any handling of the substance within the Bettis Lab. KAPL has handled Beryllium previously with certified personnel.

A drawback to establishing all Beryllium handling work outside of Bettis was the additional time required to establish outside vendor contracts and stage the appropriate equipment to complete the assembly at the vendors or at KAPL. Since the initial plan was to perform all work at Bettis and the schedule to meet the JOYO June 2006 
Enclosure (7) to

MDO-723-0044/

B-MT(SRME)-52

Page 152

availability date was tight, that added time required to deviate form the Bettis assembly plan would have put the delivery of the Beryllium laden capsule in jeopardy.

At the time of the termination of the NRPCT Space Program effort, the details of handling the Beryllium at Bettis were being finalized and agreed to, but were never put into action. No Beryllium was received at Bettis and all Beryllium contracts were canceled with the Beryllium supplier. Future work in this type of testing should provide for an up front review of all potential sensitive materials and plan for them accordingly if at all possible. In the case of the JOYO-1 planning, the window was extremely tight for additional effort to be incorporated into the schedule to circumvent Bettis handling of the Beryllium.

\section{Manpower}

The JOYO-1 materials test program provided a unique challenge to the NRPCT in that the test required 70 (60 test and 10 spare) test capsules to be prepared in a short amount of time. The NRPCT had previously not made more than 12 capsules at any one time. In order to support this large quantity of capsules, trained personnel for all aspect of the assembly from assembly and welding to inspection would be needed. The time allotted for the assembly process was from mid-November 2005 to late January 2006.

Strategic planning and communication was required to organize the various aspects of capsule assembly since many different organizations were involved. These organizations provided technicians and engineering support for procurement, quality control, cleaning, assembly, welding, and testing. In addition to on-site manpower needs, five different local machine shops were used to fabricate the numerous capsule components in order to have the parts delivered to meet the assembly schedule. Since the schedule was so unyielding, at least two specialized technicians were planned for each process. Having backups maintained continuous operations when a technician may be unavailable due to unforeseen situations. Component mockups were to be made available in advance to provide familiarity with the components as well to refine assembly procedures.

\section{Oversight by JNC}

As part of the inspection process for $\mathrm{JNC}$ to be assured that the capsules to be placed within the JOYO reactor were adequate, JNC requested on-site overview of the capsule assembly process. The NRPCT is made up of facilities under clearance with the Department of Energy and therefore limit access to the sites by uncleared personnel, foreign nationals, and, in general, personnel without a need-to-know. Access for JNC was denied. The NRPCT offered to provide JNC photographs of the assembly process which they considered acceptable at the June 2005 capsule design review meeting. The fact that the entire NRPCT quality assurance plan and inspection processes were reviewed with JNC in June 2005 provided support that the NRPCT could provide a viable capsule design for use in the JOYO reactor. Future work in this regard should consider all requests for $Q A$ oversite and plan for them accordingly. 


\section{Open Items}

The following is summary list of items that remained to be completed as part of the process to provide assembled capsule for installation into the JOYO reactor by June 2006:

- Report all final analysis results to JNC for their review (Final design review)

- Acquire all material specimens

- Complete machining of all capsule parts

- Complete capsule assemblies

- Develop the appropriate means of shipping the capsules to JNC and conduct a trial run of the process to shake out any issues inclusive of:

- Customs

- Licensing

- Containers and packaging

- Agencies involved

- Use of actual capsule and representative contents. Limiting case was expected to be the pressurized biaxial creep specimens with regard to Customs and or government regulations for pressure vessels.

- Establish the post-irradiation exam program and identify shipping methods for waste and/or specimens for disposal or the US for further examination. The NRPCT established a goal to not start the JOY-1 test without establishing the post test program.

- Review the need for long term on-site NRPCT representation at JNC to support capsule receipt, inspection, installation and test follow. 
Enclosure (7) to

MDO-723-0044I

B-MT(SRME)-52

Page 154

THIS PAGE IS INTENTIONALLY BLANK 
Enclosure (7) to

MDO-723-0044/

B-MT(SRME)-52

Page 155

VI. Drawings and Sketches

A. Capsule and Hardware

\begin{tabular}{|c|l|c|}
\hline \multicolumn{2}{|c|}{ Table - Sketches } \\
\hline Sketch Number & \multicolumn{1}{|c|}{ Title } & $\begin{array}{c}\text { Revision - } \\
\text { date }\end{array}$ \\
\hline SK-AMT-2214 & NRPCT/JOYO BCD Capsule Assembly & $12 / 8 / 2005$ \\
\hline SK-AMT-2215 & NRPCT/JOYO TFT Capsule Assembly & $12 / 8 / 2005$ \\
\hline SK-AMT-2219 & NRPCT/JOYO SCA Capsule Assembly & $12 / 8 / 2005$ \\
\hline SK-AMT-2221 & NRPCT/JOYO Capsule & Original \\
\hline SK-AMT-2222 & NRPCT/JOYO Head & A \\
\hline SK-AMT-2223 & NRPCT/JOYO Spacers & Original \\
\hline SK-AMT-2224 & NRPCT/JOYO Insulators & Original \\
\hline SK-AMT-2225 & NRPCT/JOYO Biaxial Creep Holders & A \\
\hline SK-AMT-2226 & NRPCT/JOYO Tensile Holder & Original \\
\hline SK-AMT-2227 & NRPCTJOYO Fracture Toughness Holder & Original \\
\hline SK-AMT-2228 & Assembly & Original \\
\hline SK-AMT-2230 & NRPCT/JOYO Capsule weld requirements & $9 / 26 / 2005$ \\
\hline SK-AMT-2231 & NRPCT/JOYO DBU/DBD Capsule Assembly & $12 / 8 / 2005$ \\
\hline SK-AMT-2233 & NRPCT/JOYO A5U/A5D Capsule Assembly & $12 / 8 / 2005$ \\
\hline SK-AMT-2235 & NRPCT/JOYO CT Holder & Original \\
\hline SK-AMT-2236 & NRPCT/JOYO NBU/NBD Capsule Assembly & $12 / 8 / 2005$ \\
\hline SK-AMT-2238 & NRPCT/JOYO BSR Capsule Assembly & $12 / 8 / 2005$ \\
\hline SK-AMT-2239 & NRPCT/JOYO Wave Spring & Original \\
\hline SK-AMT-2241 & NRPCT/JOYO BeO Compression Holder & Original \\
\hline SK-RRM1011 & NRPCT/JOYO Flux Wire Assembly & A \\
\hline SK-RRM1023 & NRPCT/JOYO Tungsten Holder & A \\
\hline SK-RRM1024 & NRPCT/JOYO BeO Spacer & Original \\
\hline SK-RRM1025 & NRPCT/JOYO Grafoil Liners & $12 / 12 / 05$ \\
\hline SK216C9452 & Indicator, Passive Temperature & Original \\
\hline & & \\
\hline
\end{tabular}


Enclosure (7) to

MDO-723-0044/

B-MT(SRME)-52

Page 156

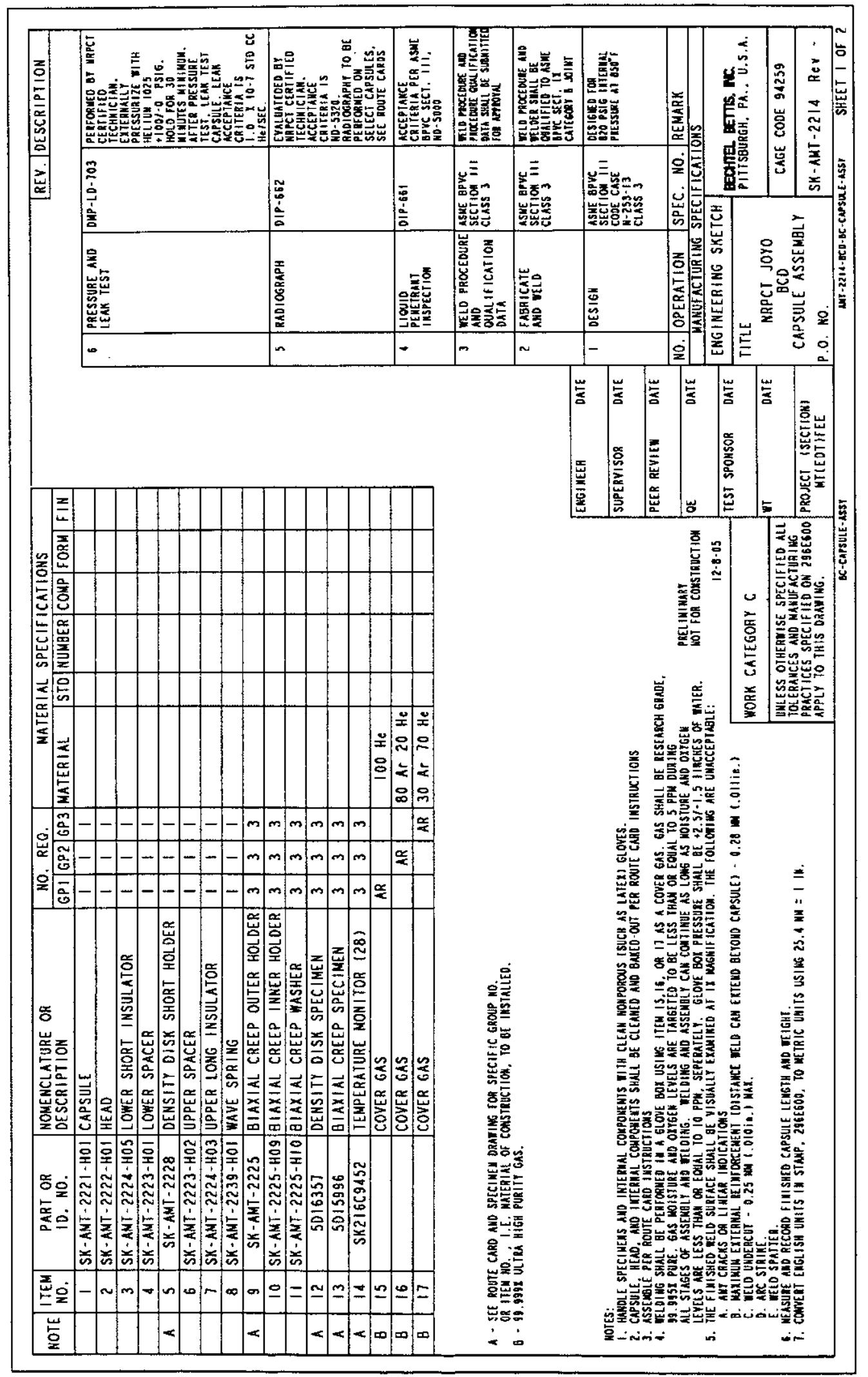


Enclosure (7) to

MDO-723-0044/

B-MT(SRME)-52

Page 157

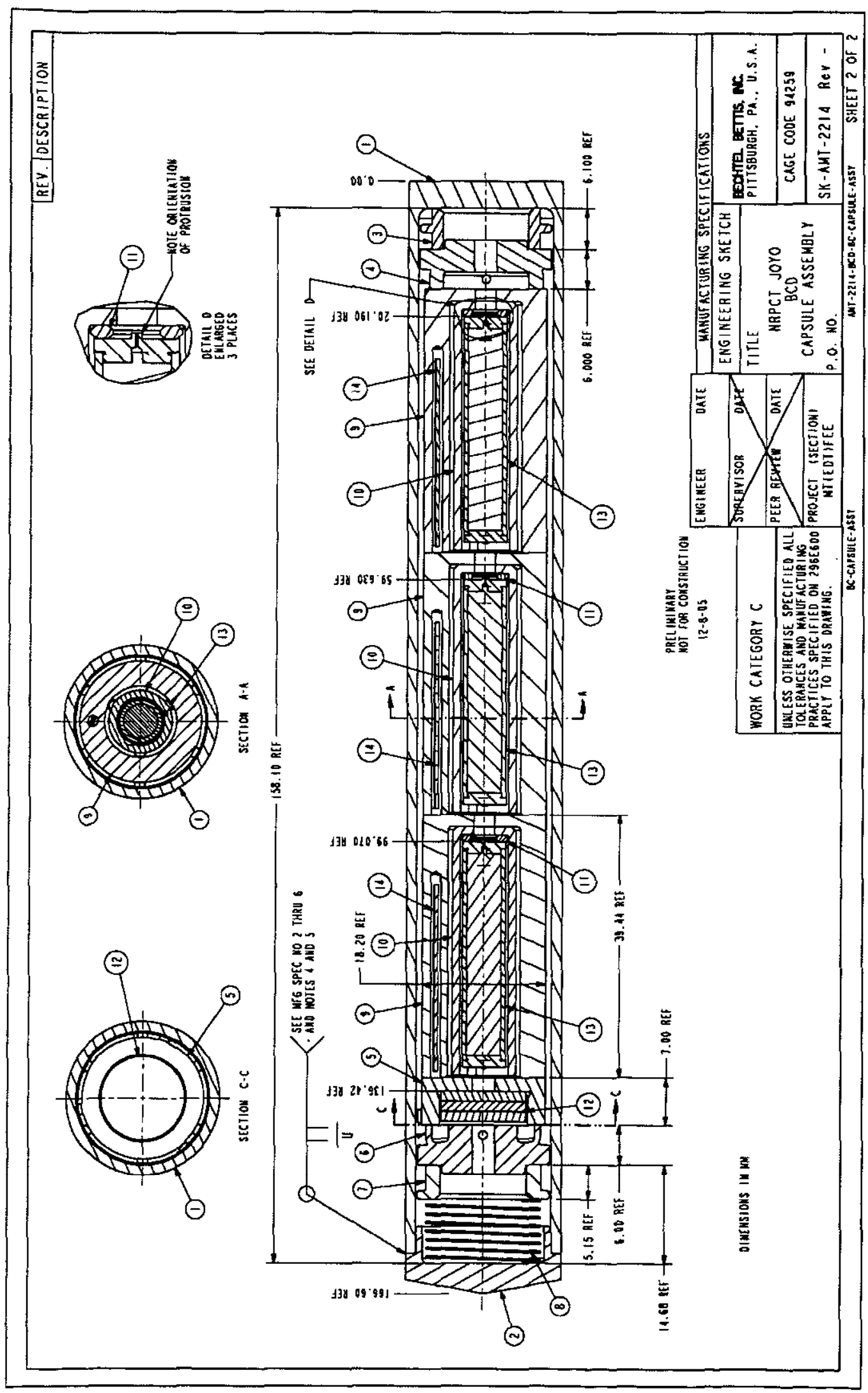


Enclosure (7) to

MDO-723-0044I

B-MT(SRME)-52

Page 158

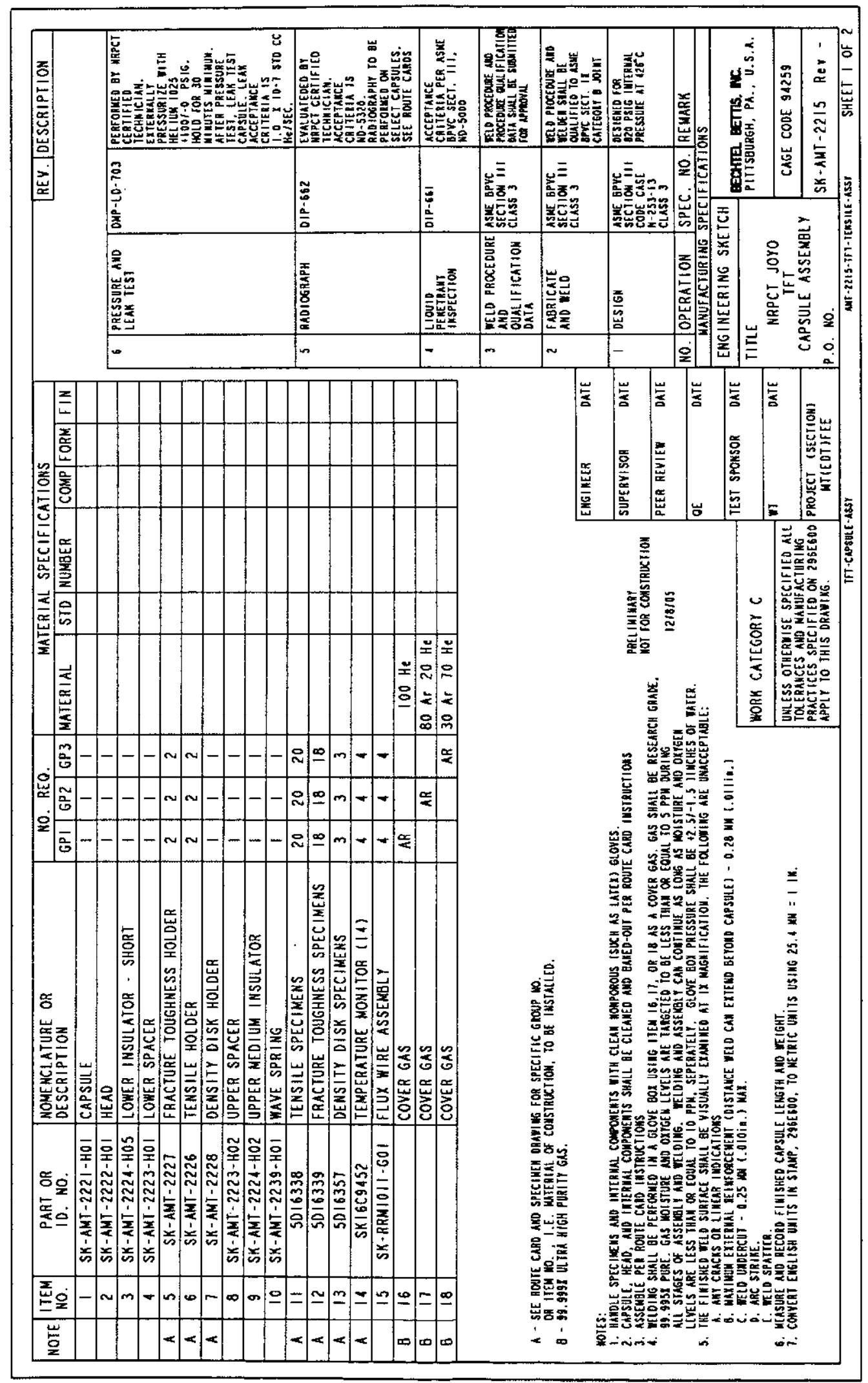


Enclosure (7) to

MDO-723-0044/

B-MT(SRME)-52

Page 159

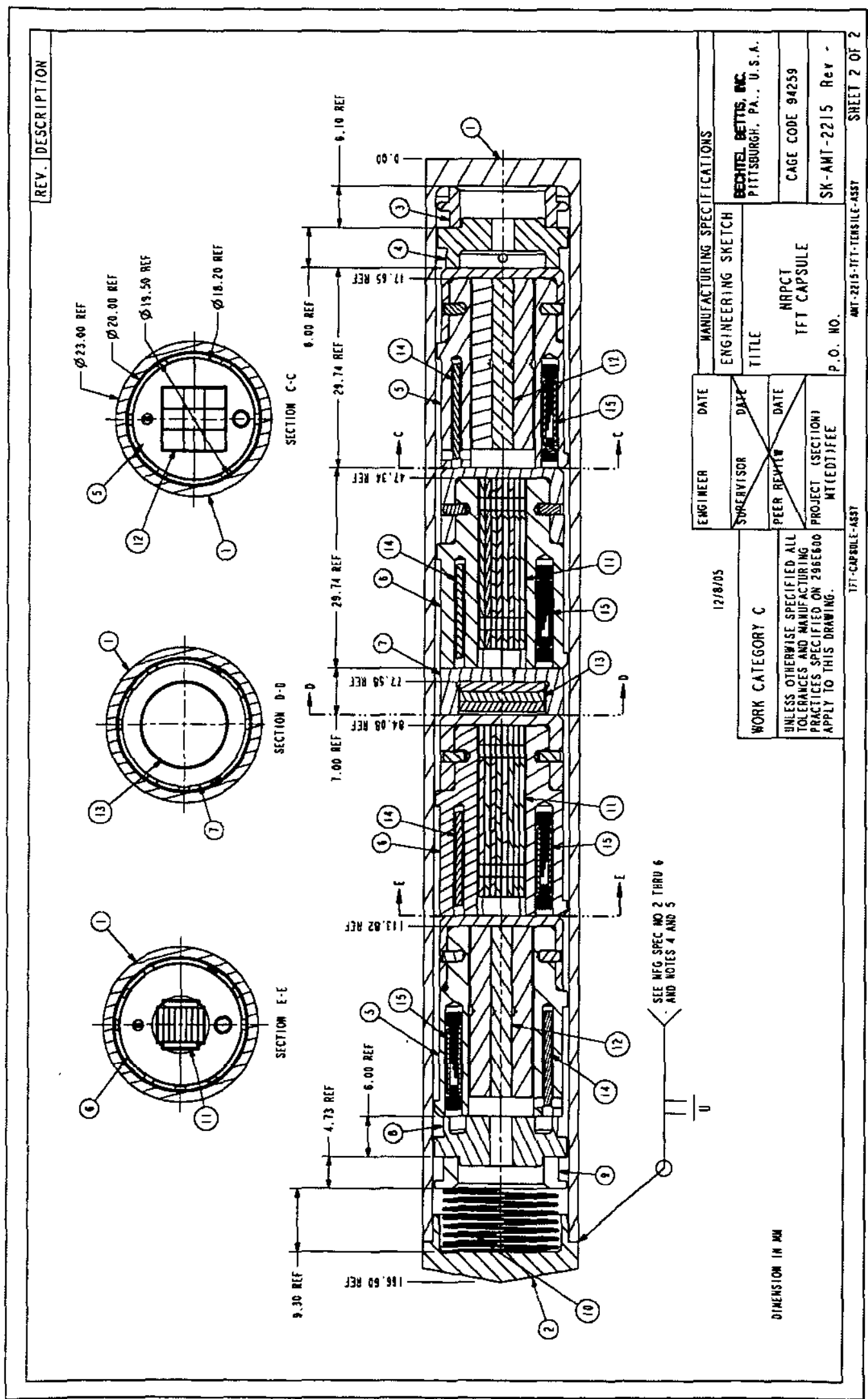




\section{Enclosure (7) to \\ MDO-723-0044/ \\ B-MT(SRME)-52 \\ Page 160}

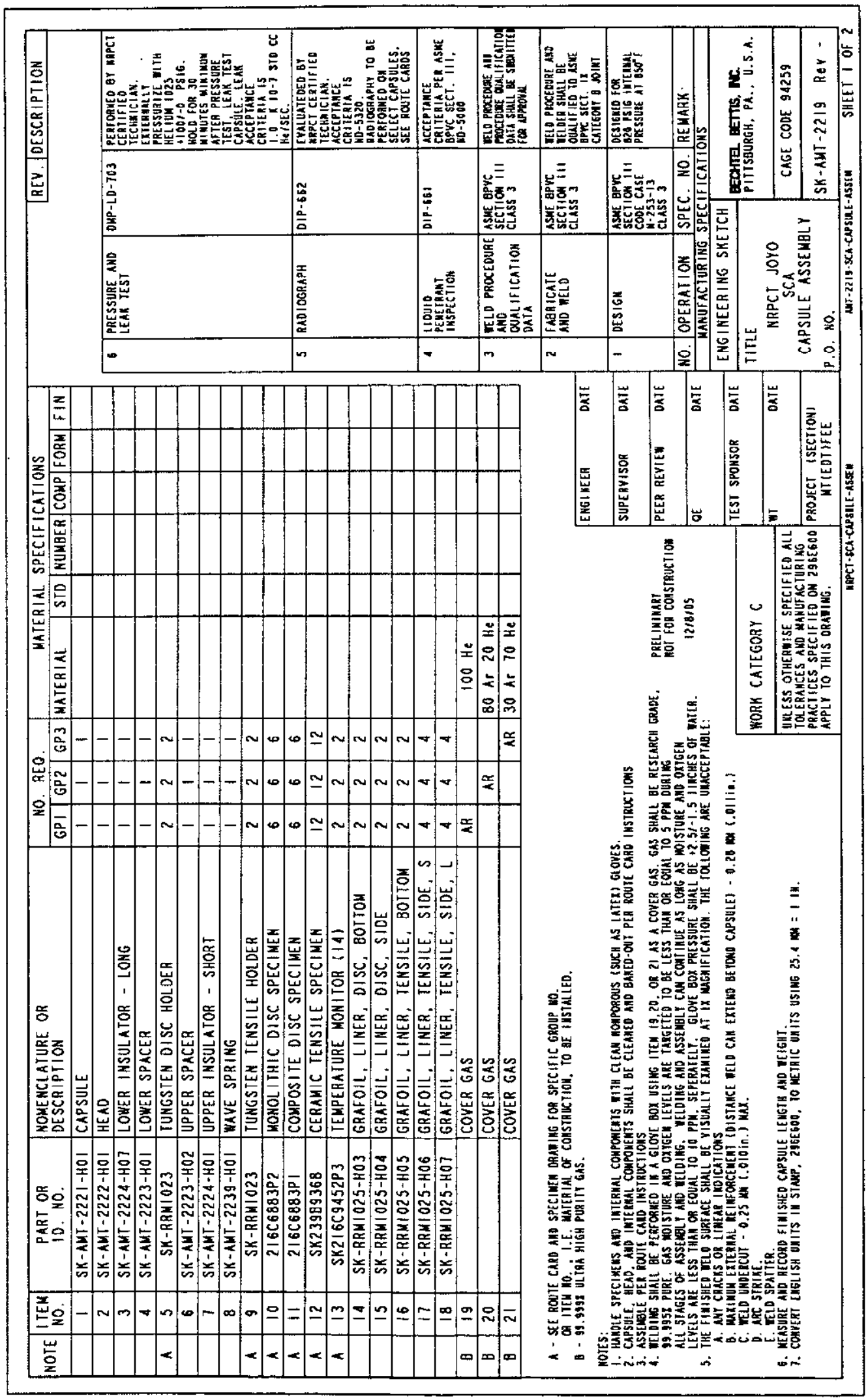


Enclosure (7) to

MDO-723-0044/

B-MT(SRME)-52

Page 161

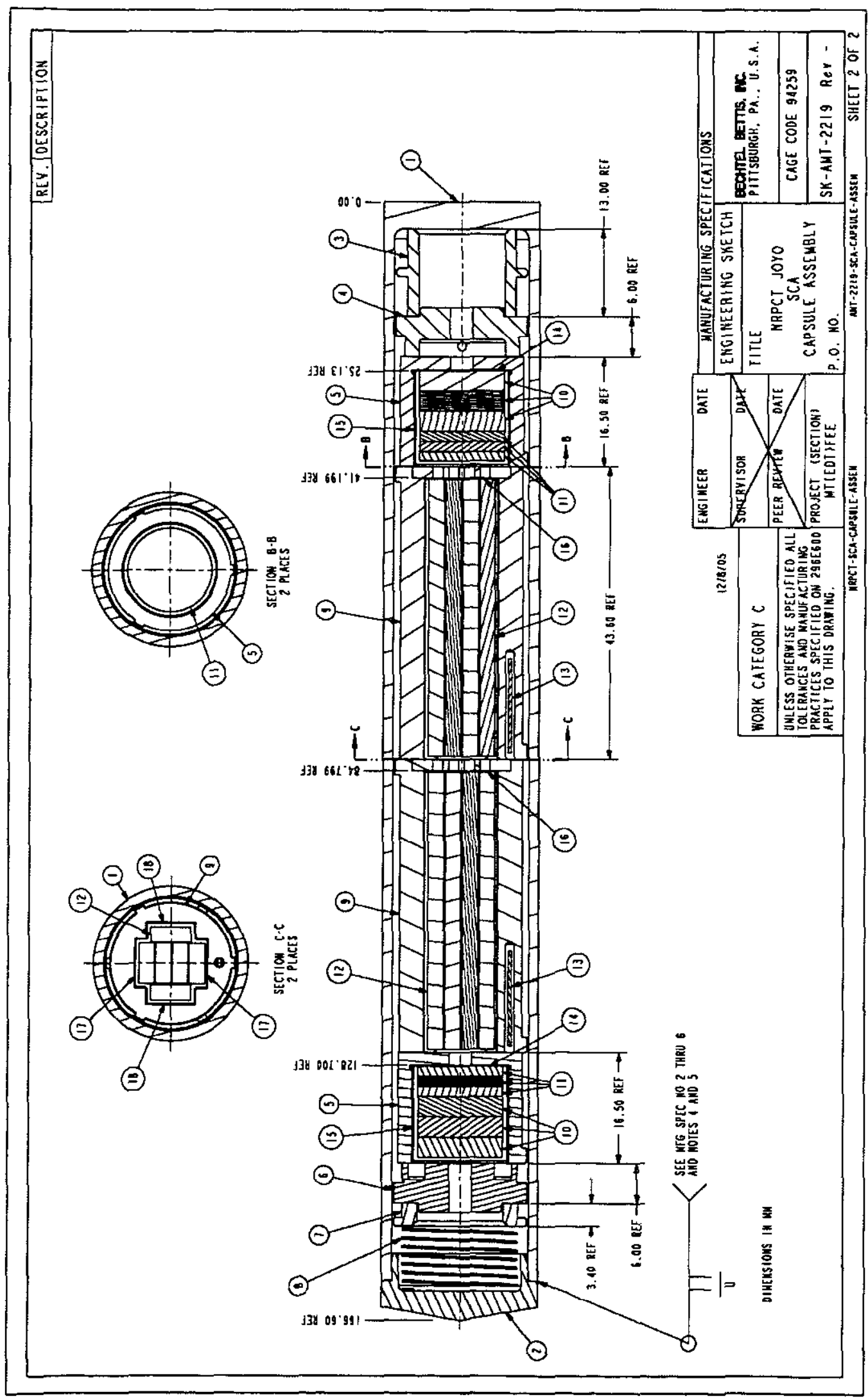


Enclosure (7) to

MDO-723-0044/

B-MT(SRME)-52

Page 162

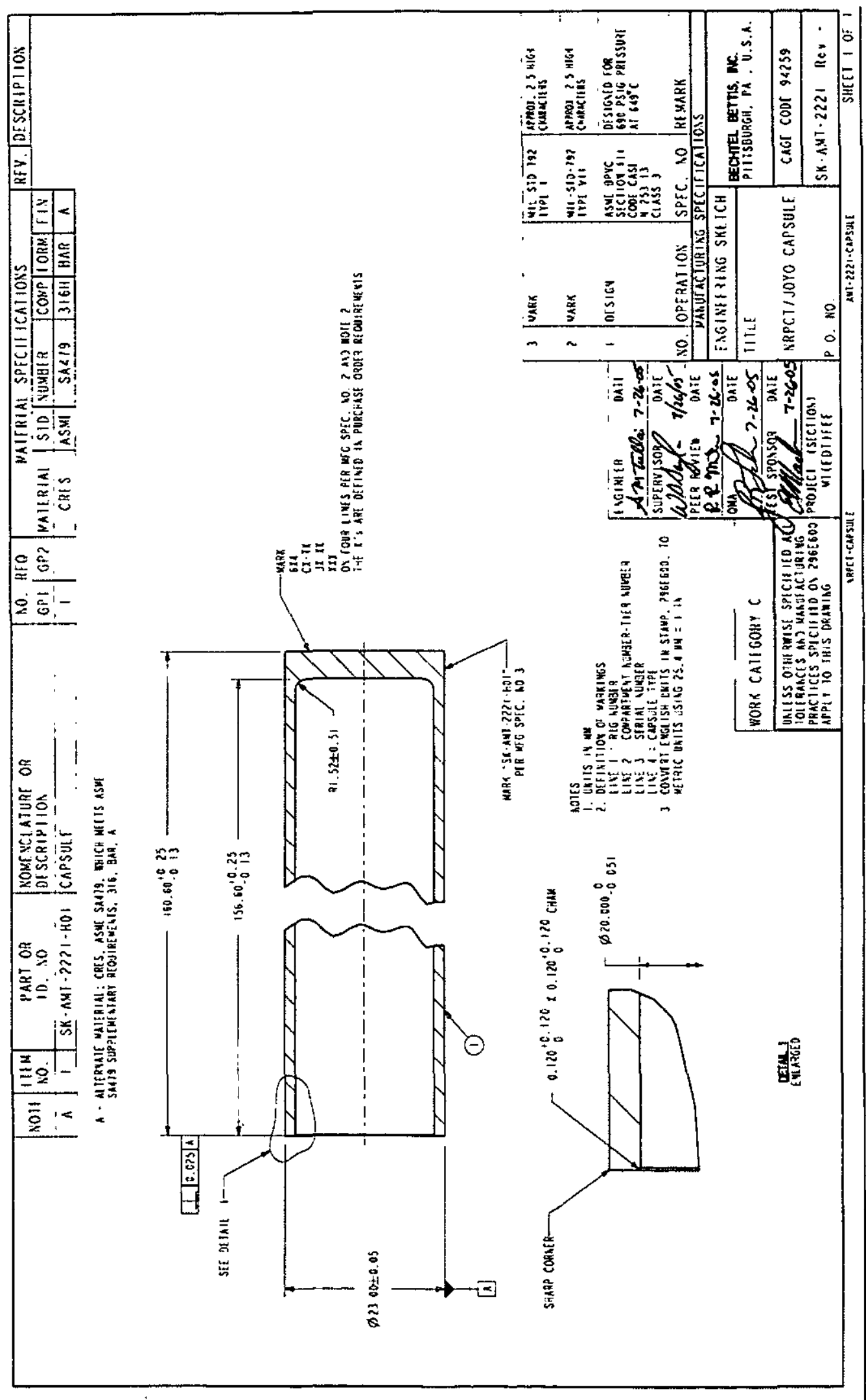


Enclosure (7) to

MDO-723-0044/

B-MT(SRME)-52

Page 163

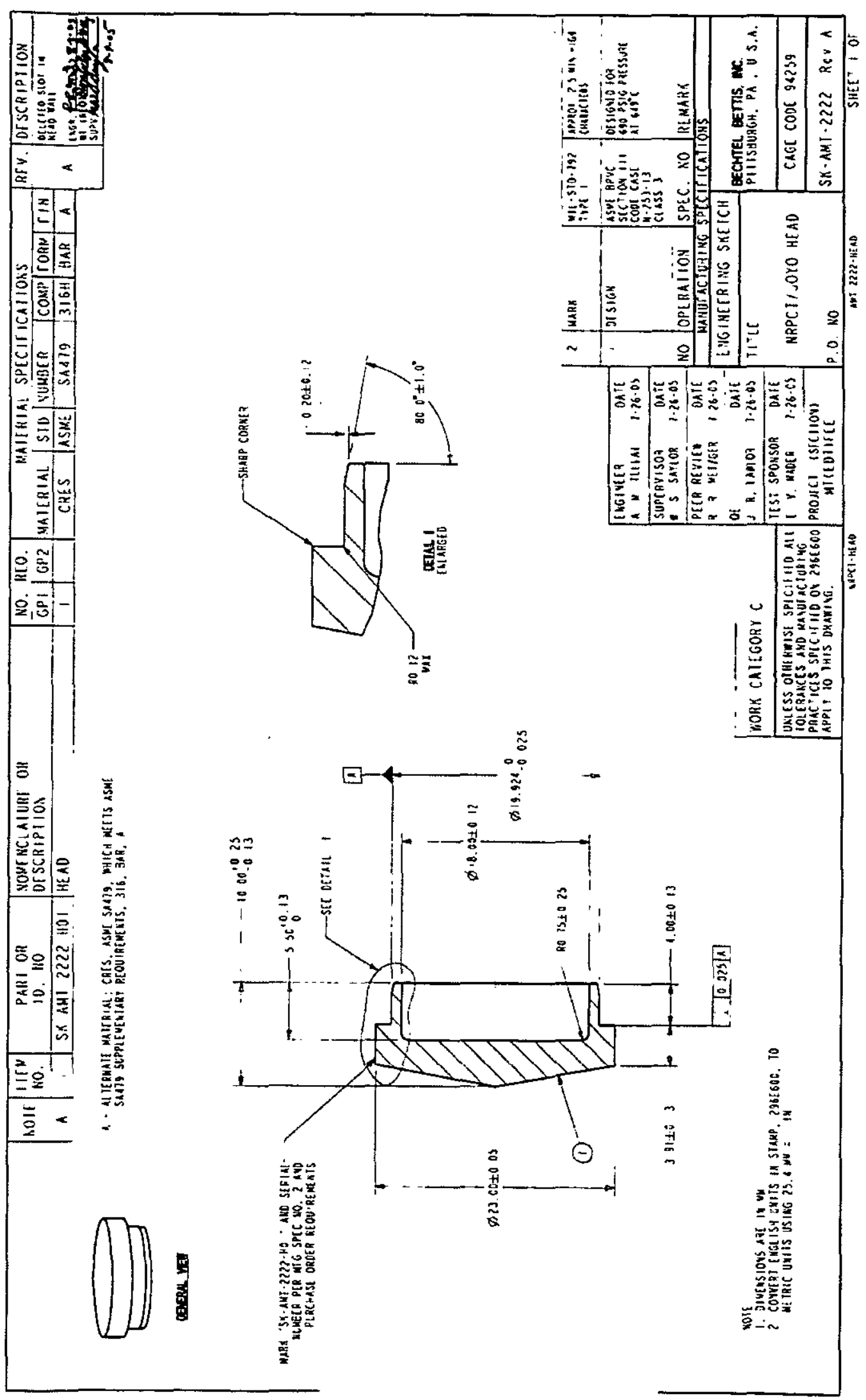


Enclosure (7) to

MDO-723-0044/

B-MT(SRME)-52

Page 164

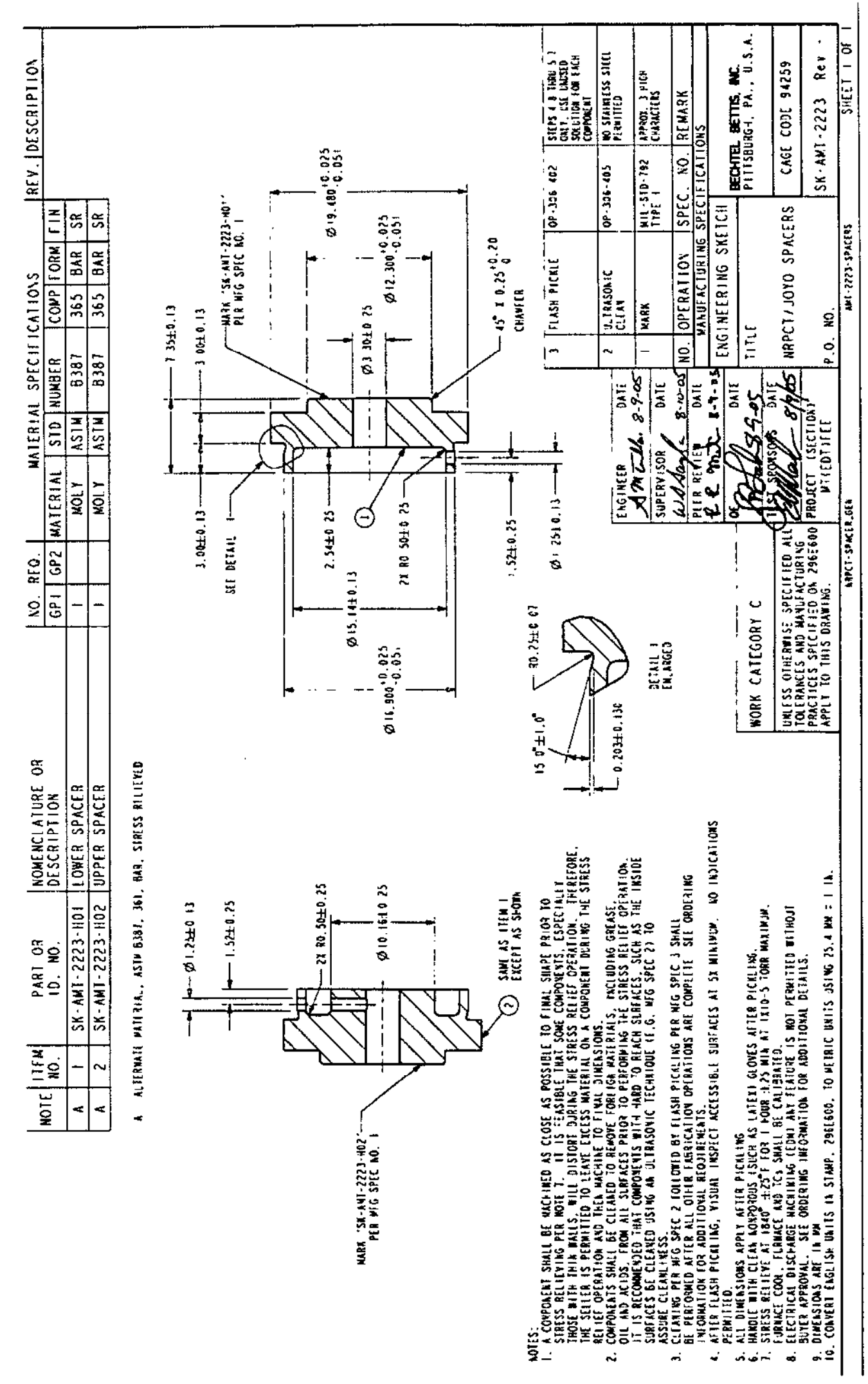


Enclosure (7) to

MDO-723-0044!

B-MT(SRME)-52

Page 165

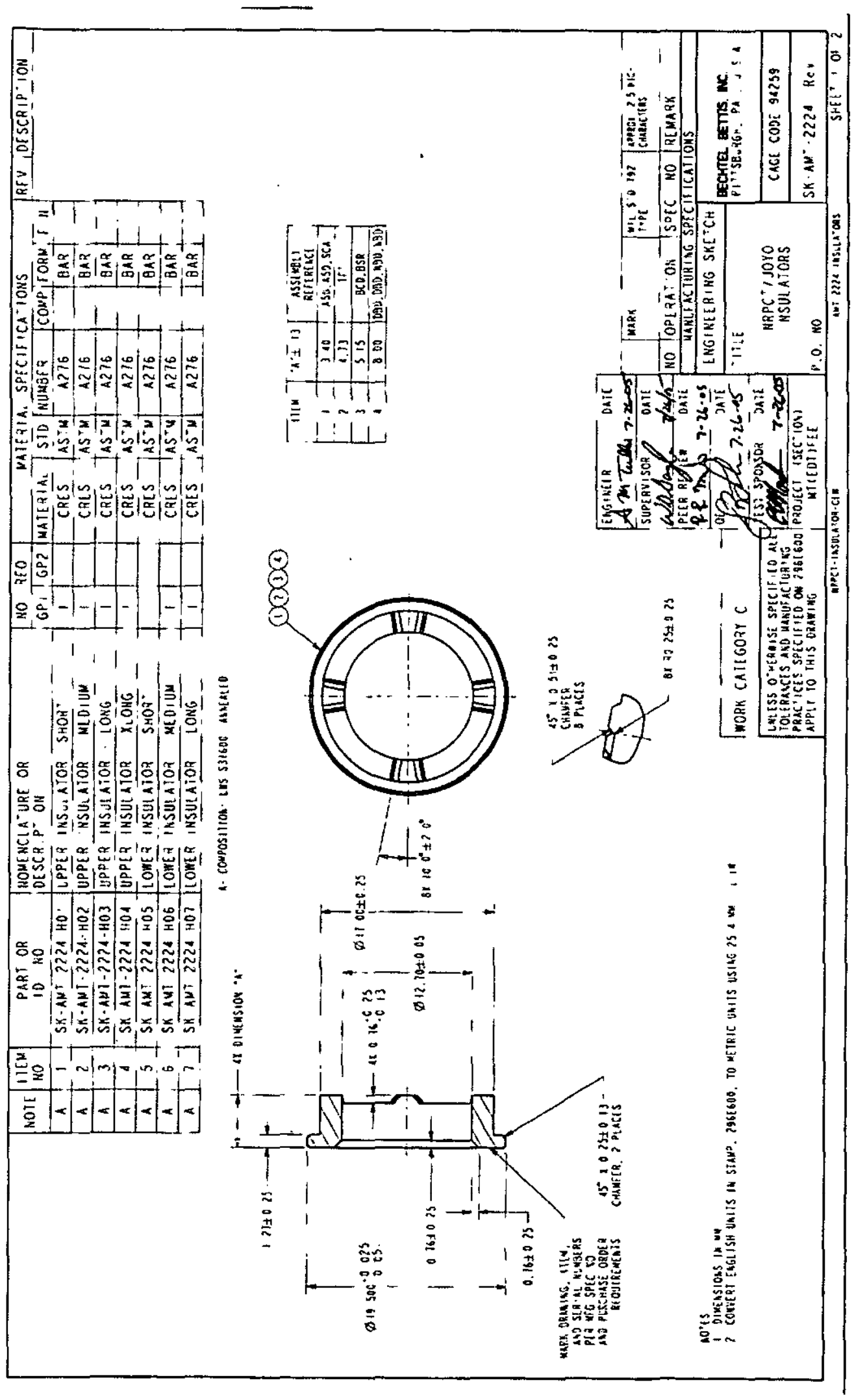


Enclosure (7) to

MD0-723-0044/

B-MT(SRME)-52

Page 166

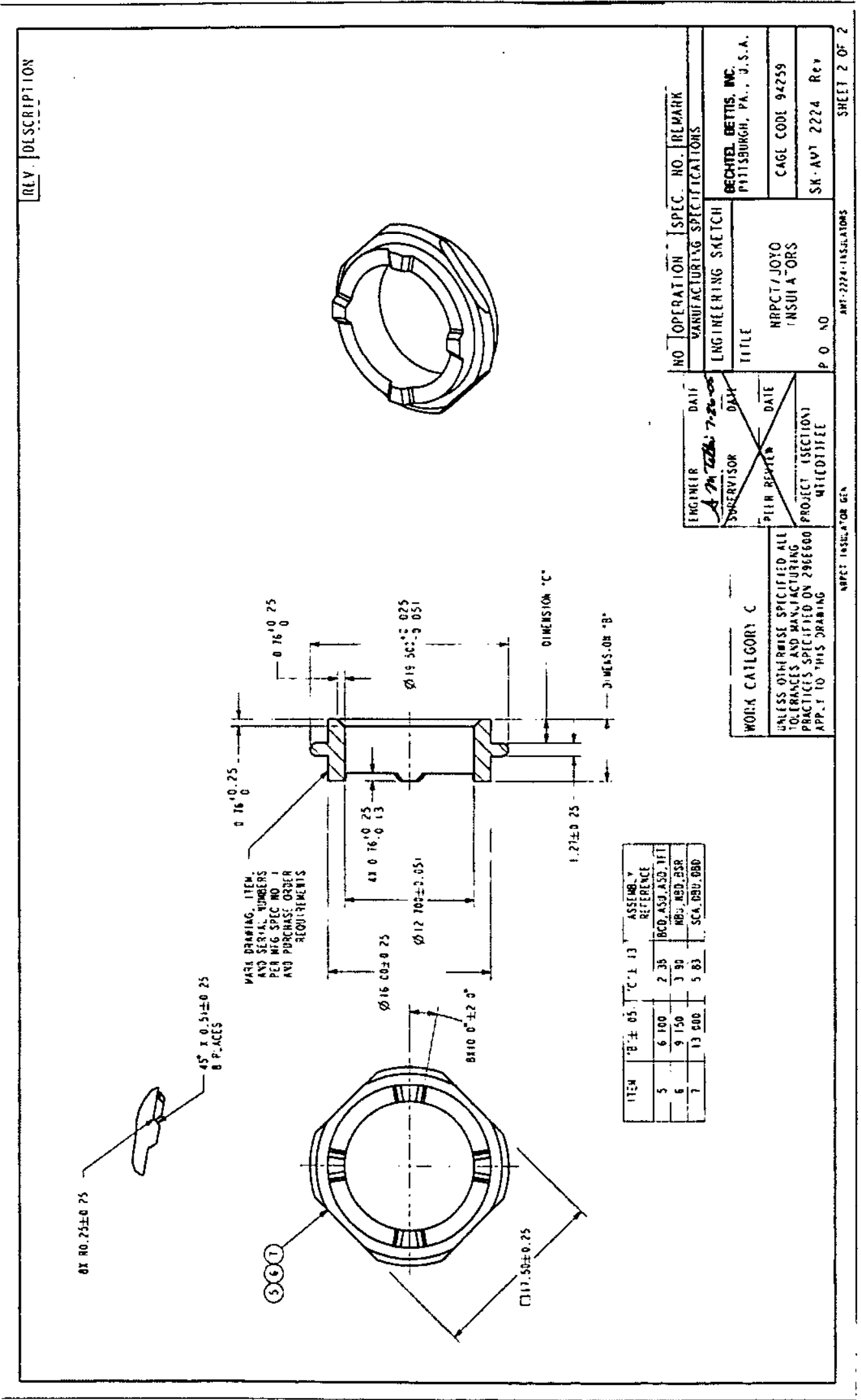


Enclosure (7) to

MDO-723-0044/

B-MT(SRME)-52

Page 167

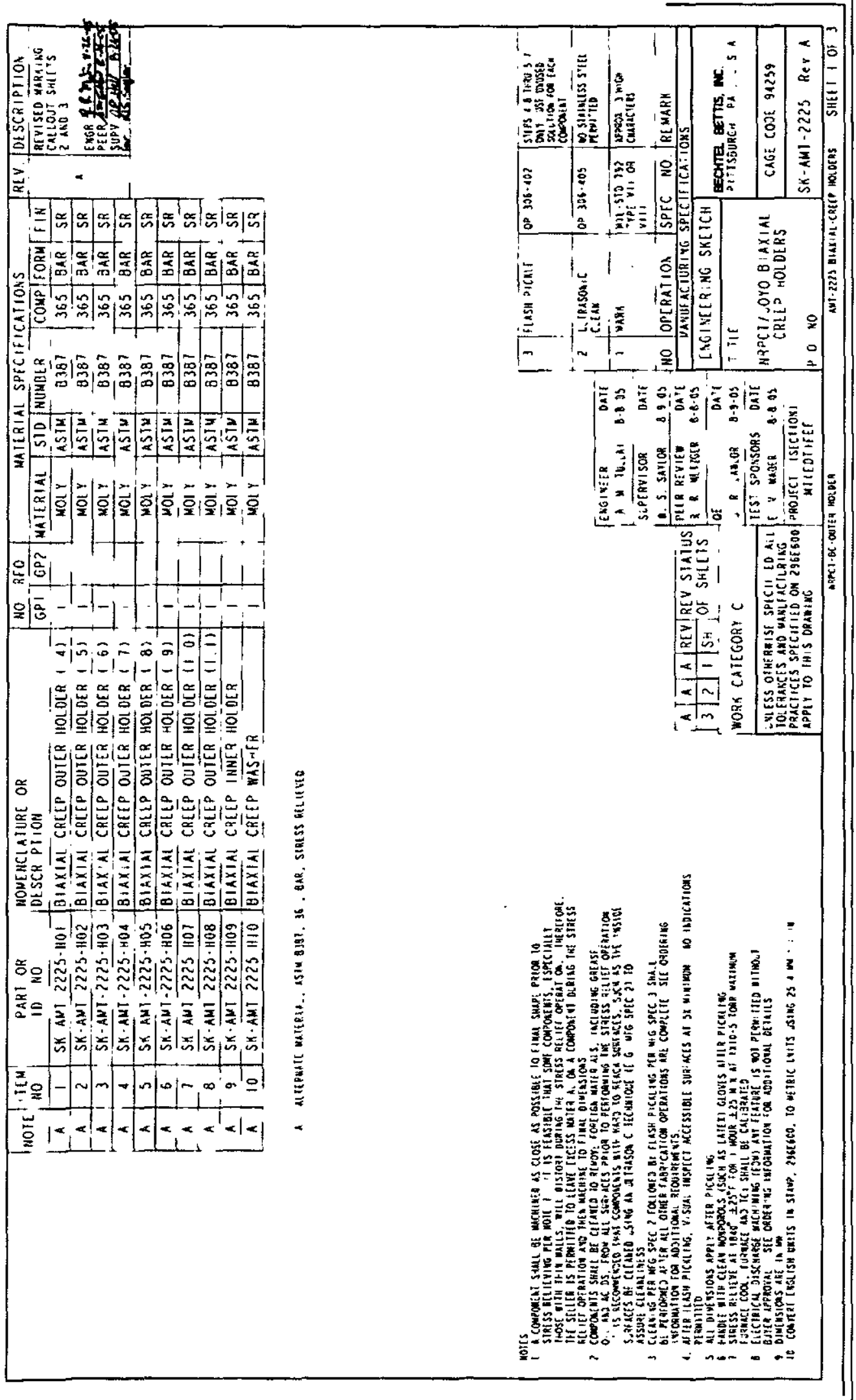




\section{Enclosure (7) to \\ MDO-723-0044/ \\ B-MT(SRME)-52 \\ Page 168}

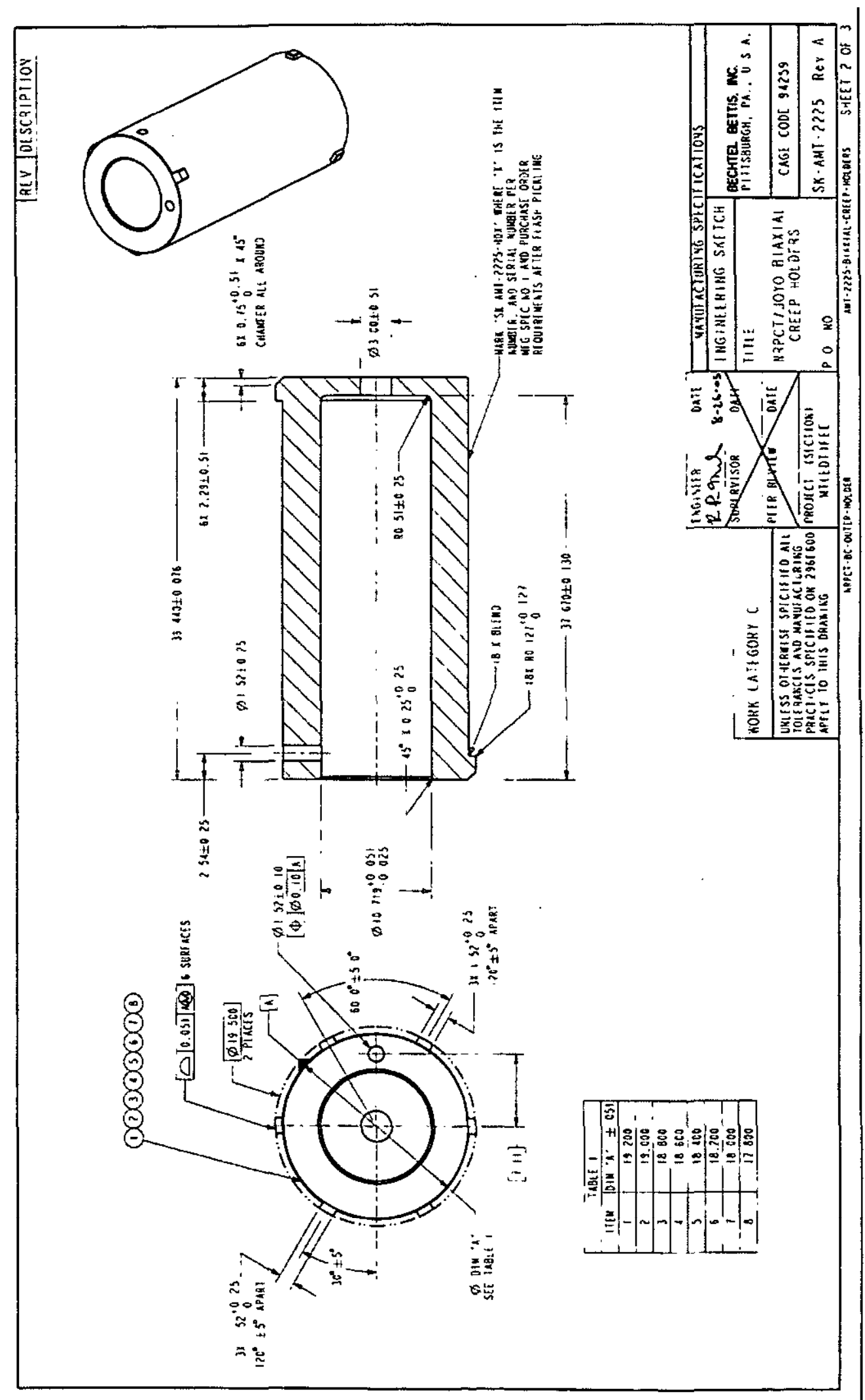


Enclosure (7) to MDO-723-0044/

B-MT(SRME)-52

Page 169

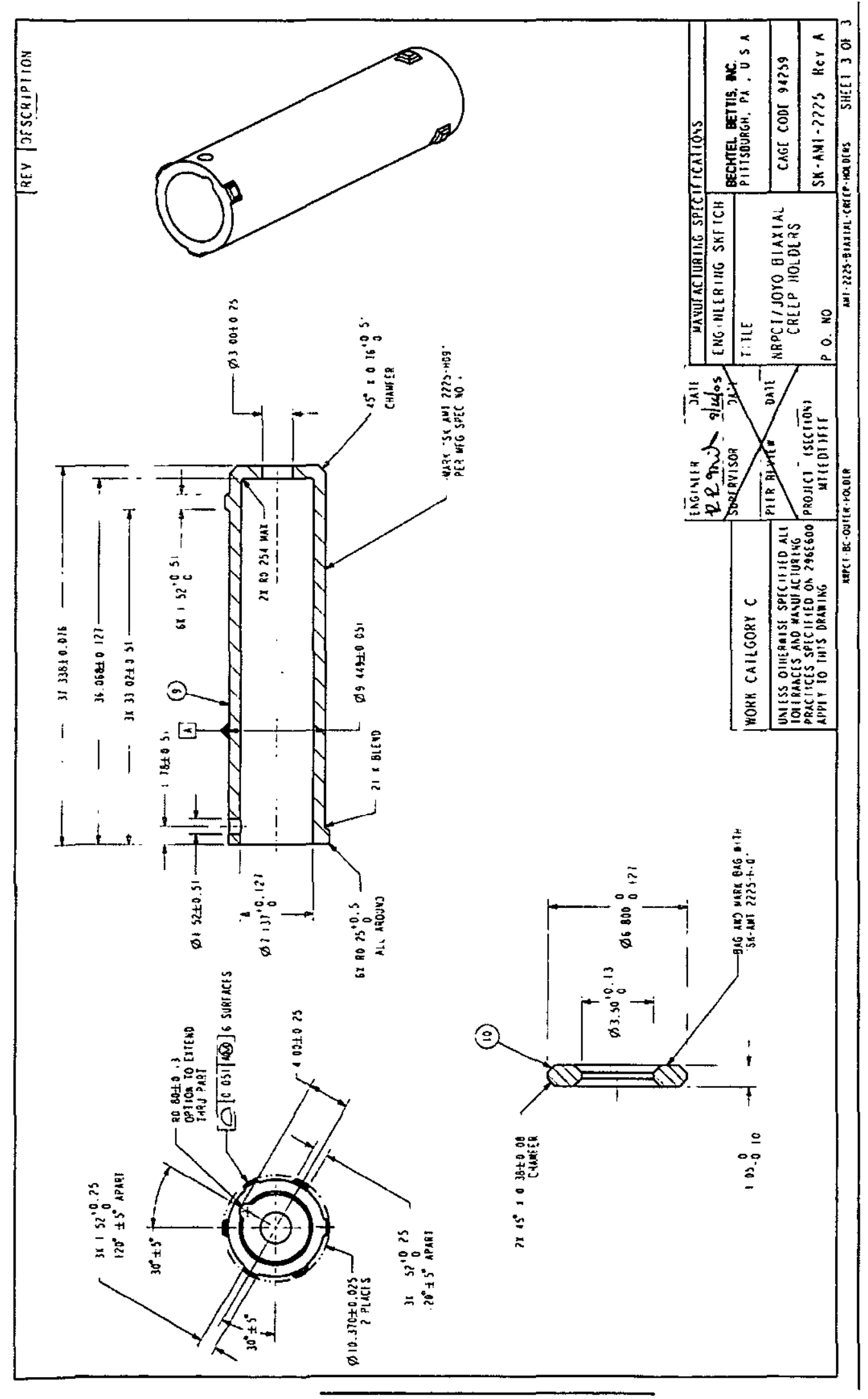


Enclosure (7) to

MDO-723-0044/

B-MT(SRME)-52

Page 170

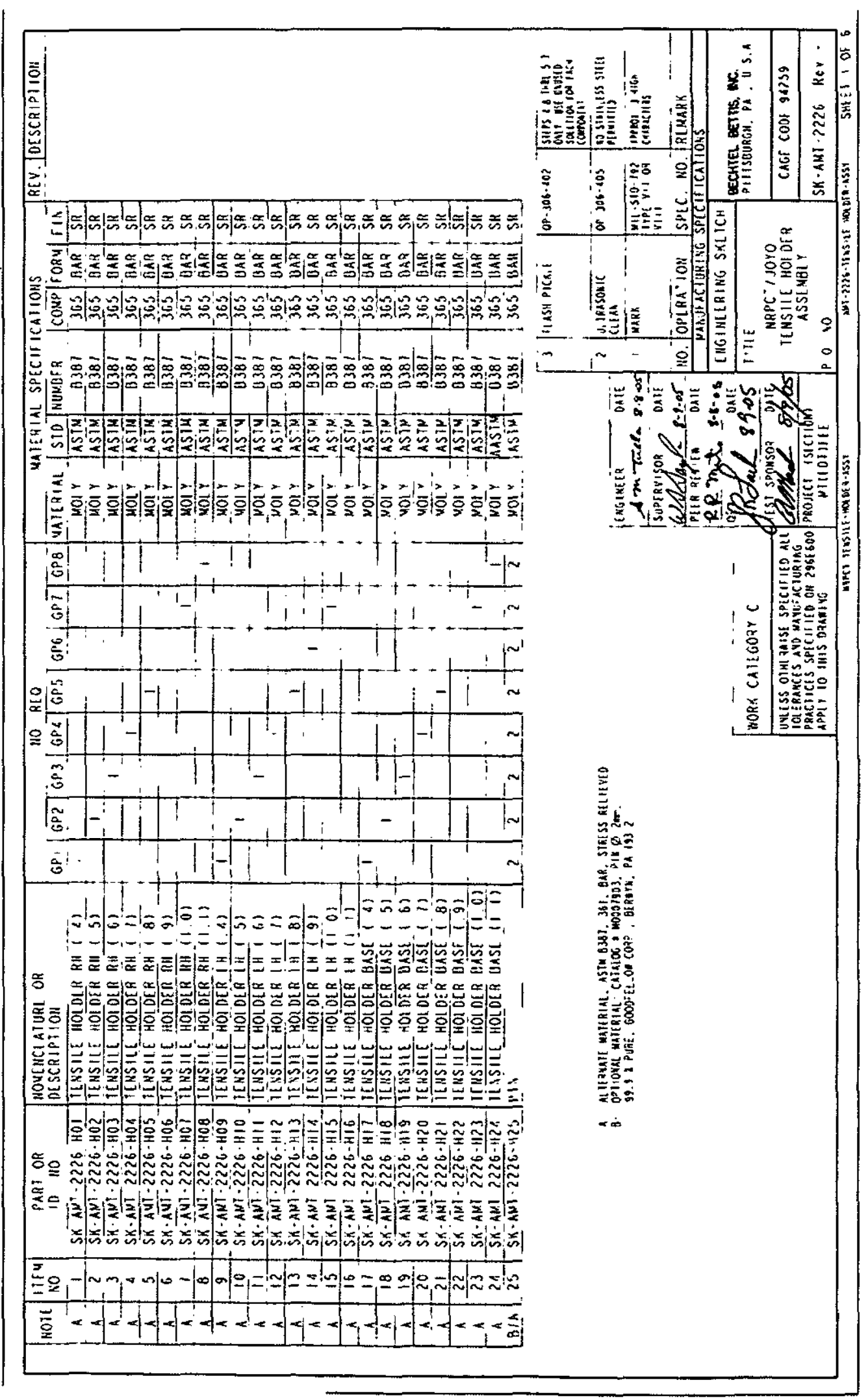


Enclosure (7) to MDO-723-0044/

B-MT(SRME)-52

Page 171

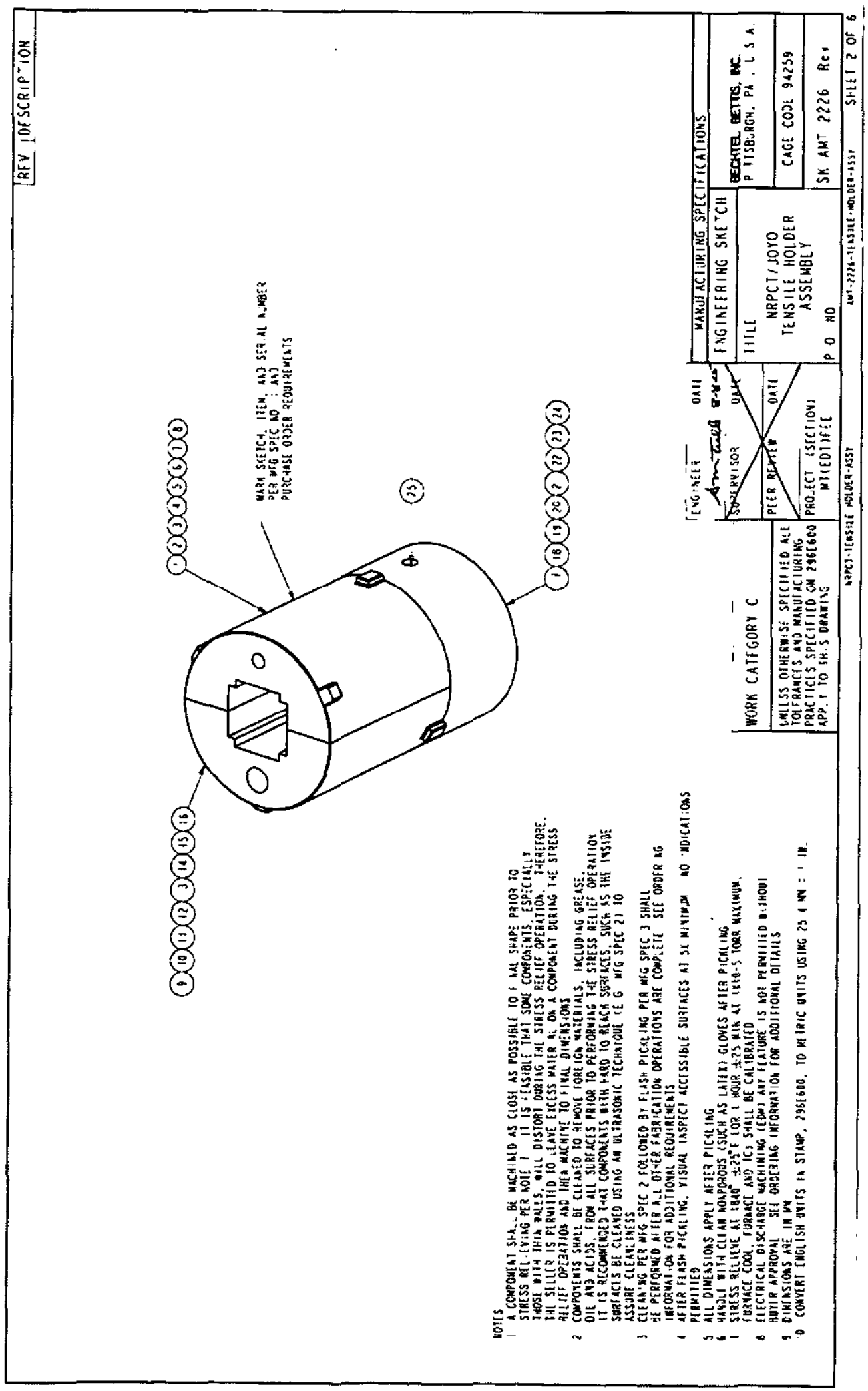


Enclosure (7) to

MDO-723-0044/

B-MT(SRME)-52

Page 172

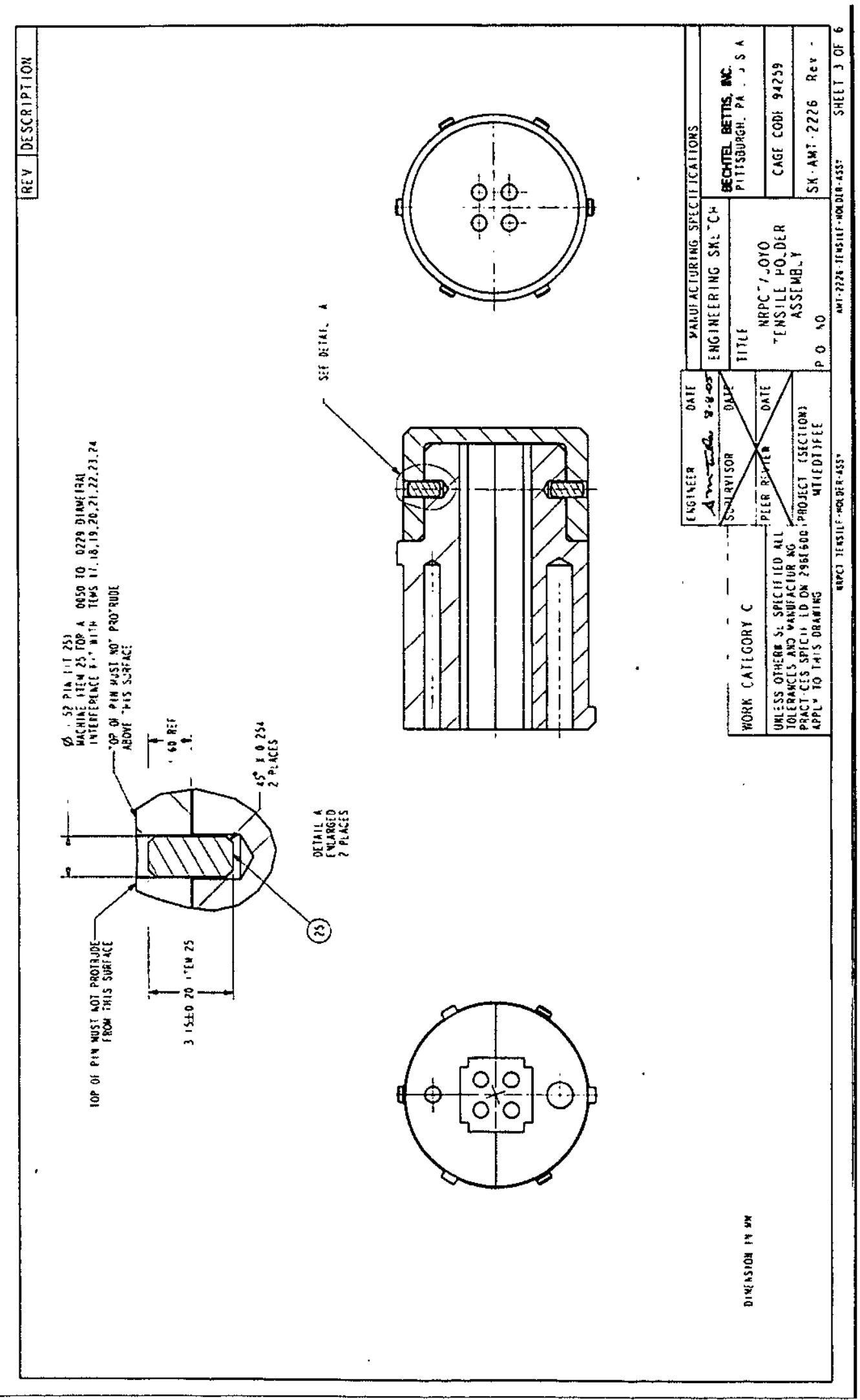


Enclosure (7) to

MDO-723-0044/

B-MT(SRME)-52

Page 173

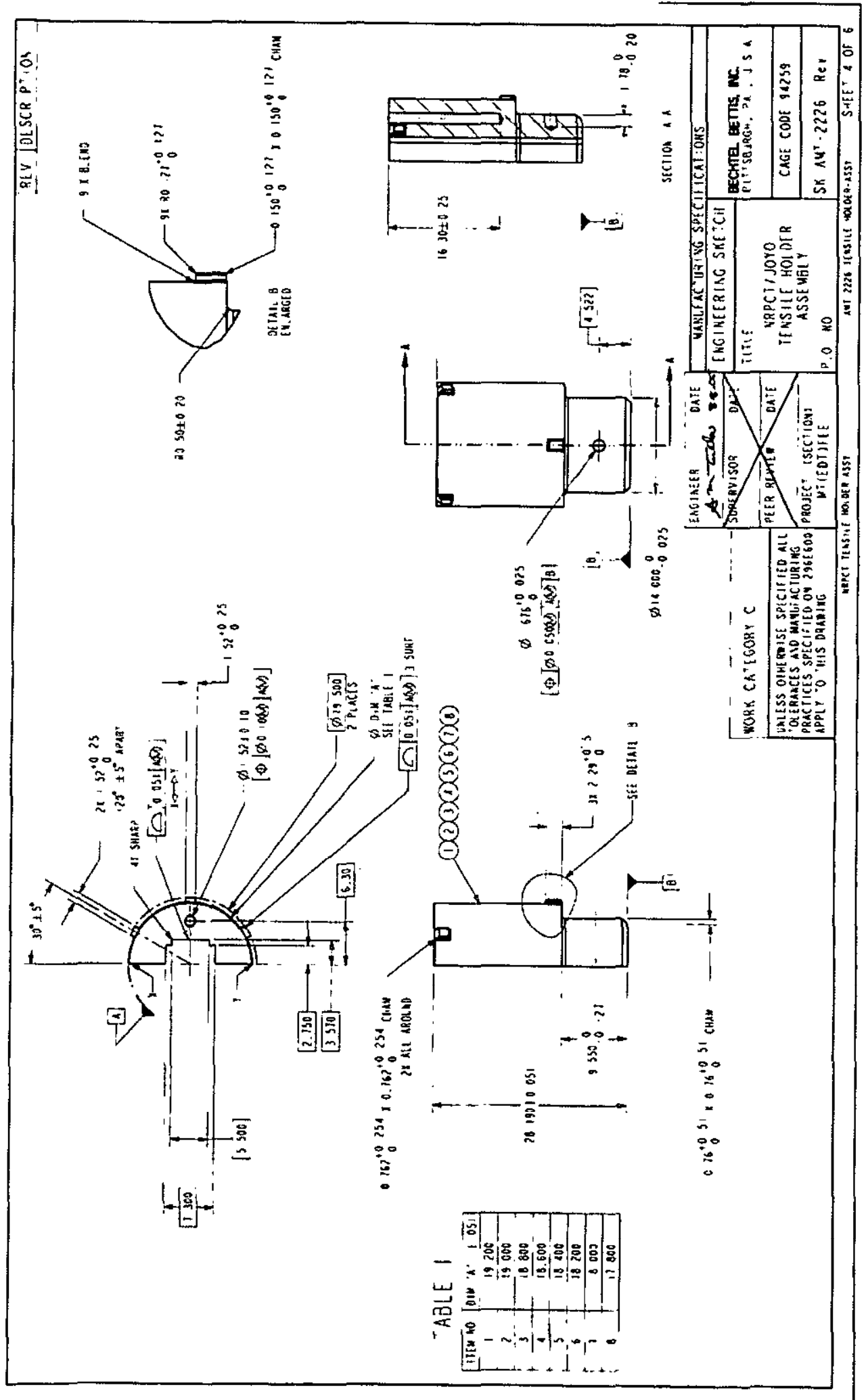




\section{Enclosure (7) to \\ MDO-723-0044/ \\ B-MT(SRME)-52 \\ Page 174}

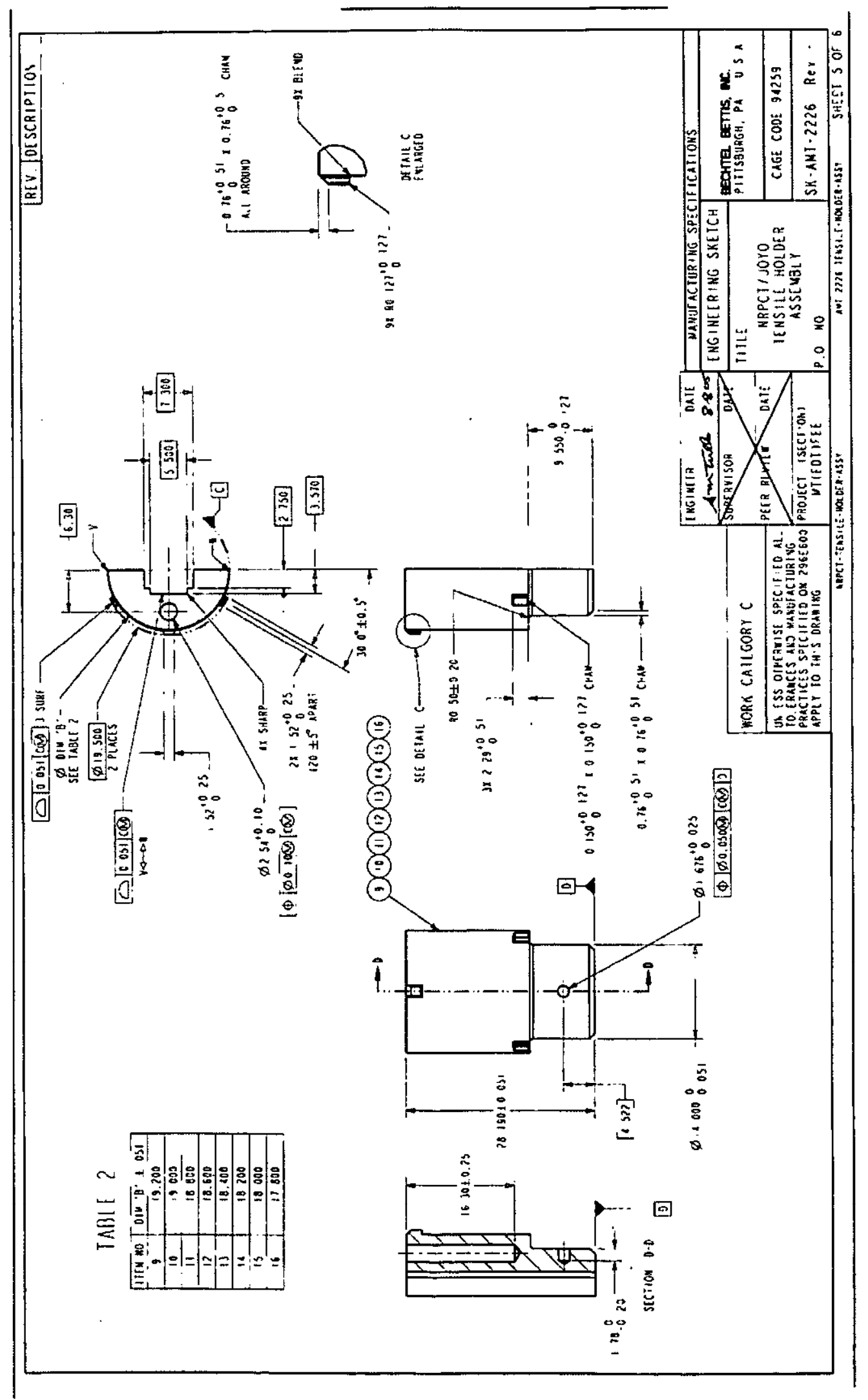


Enclosure (7) to MDO-723-0044/ B-MT(SRME)-52

Page 175

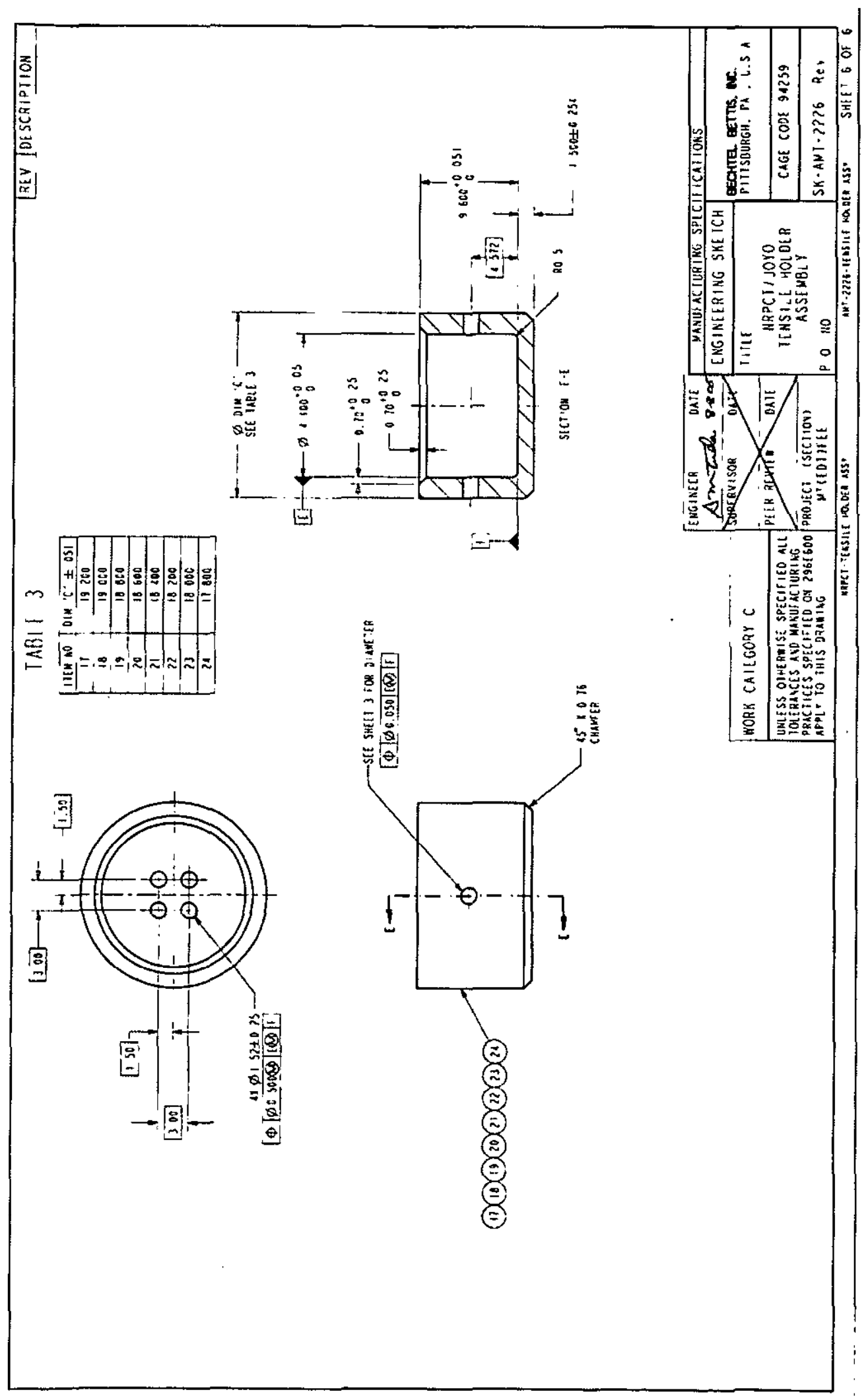




\section{Enclosure (7) to \\ MDO-723-0044/ \\ B-MT(SRME)-52 \\ Page 176}

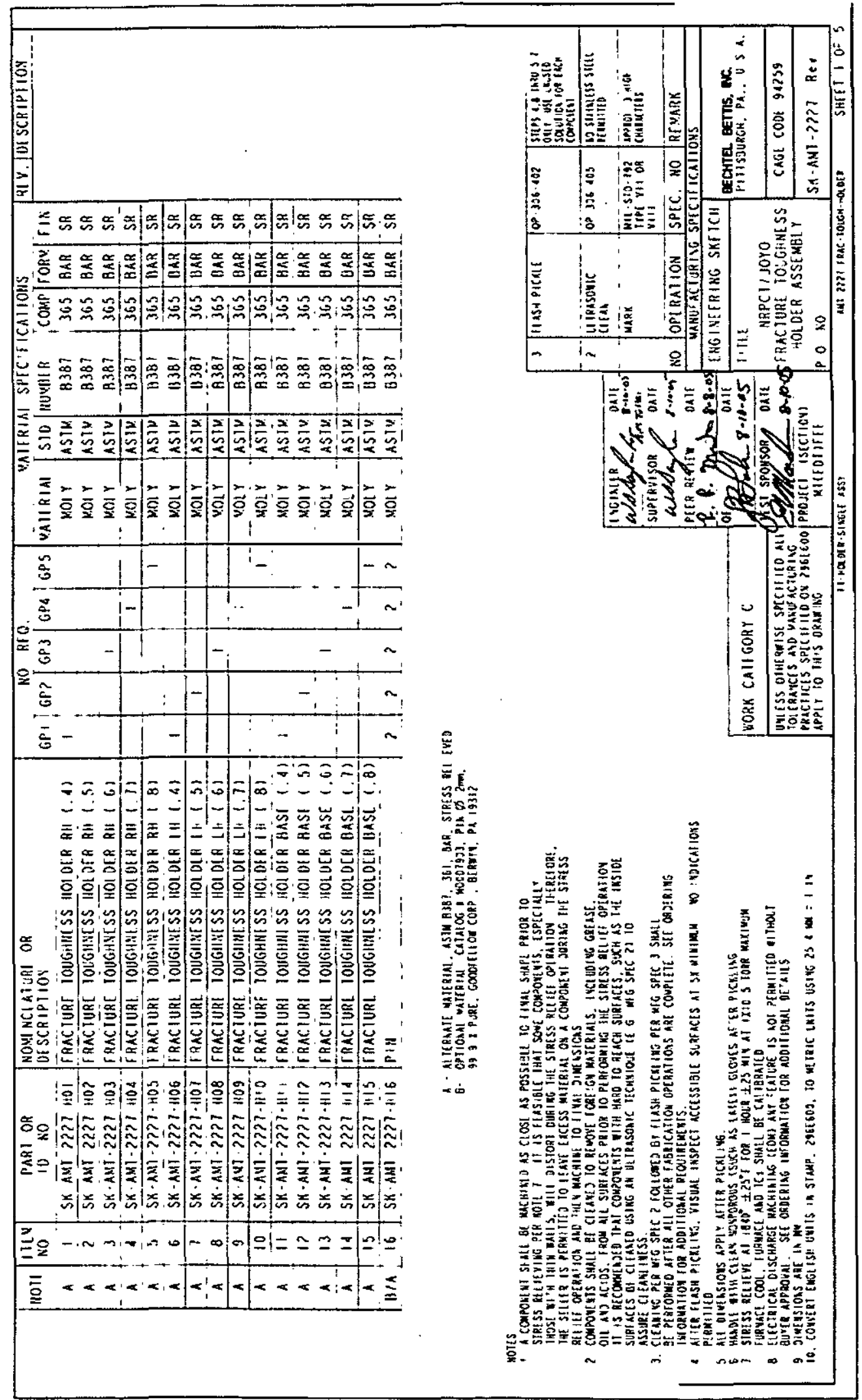


Enclosure (7) to MD0-723-0044/

B-MT(SRME)-52

Page 177

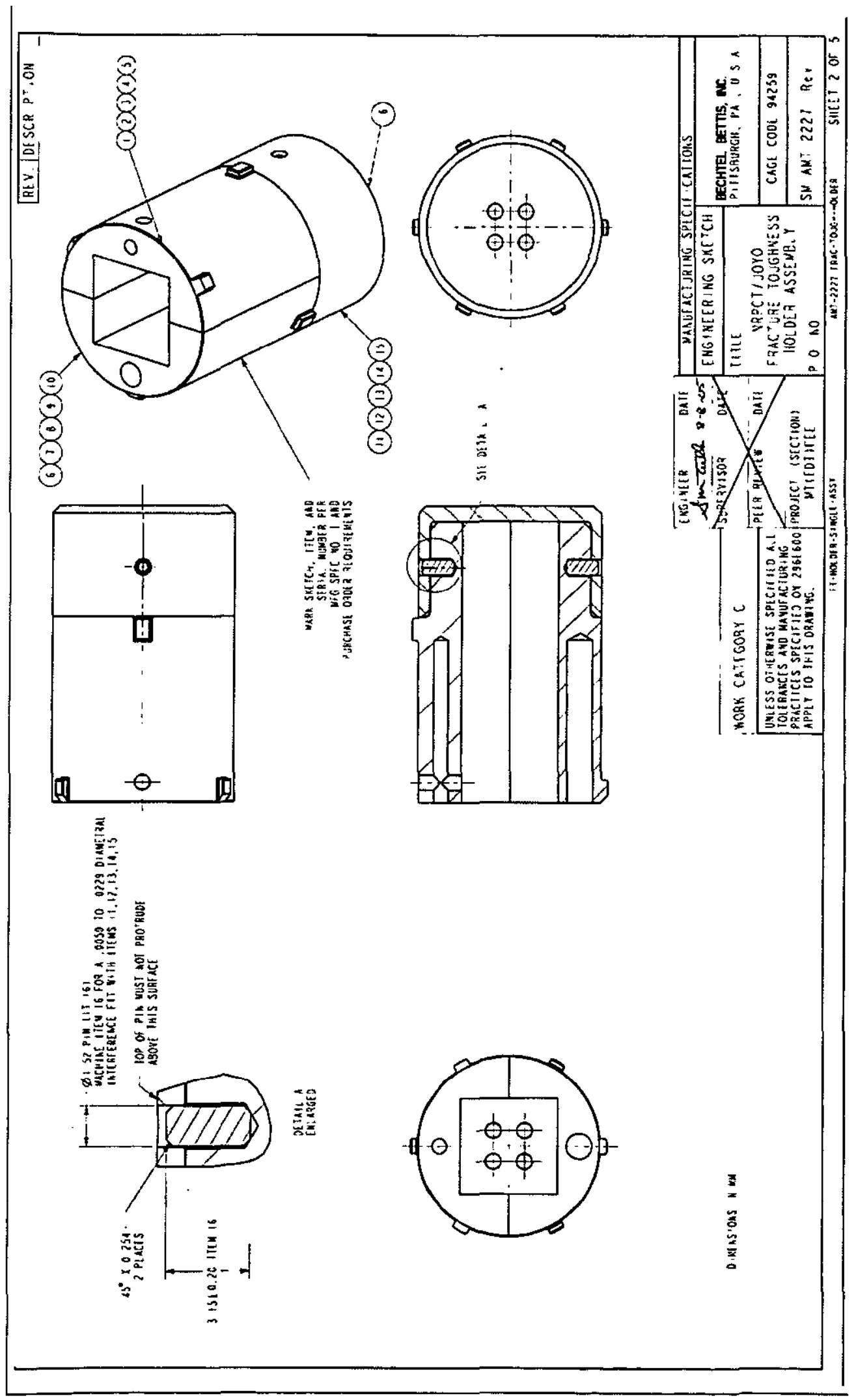


Enclosure (7) to

MDO-723-0044/

B-MT(SRME)-52

Page 178

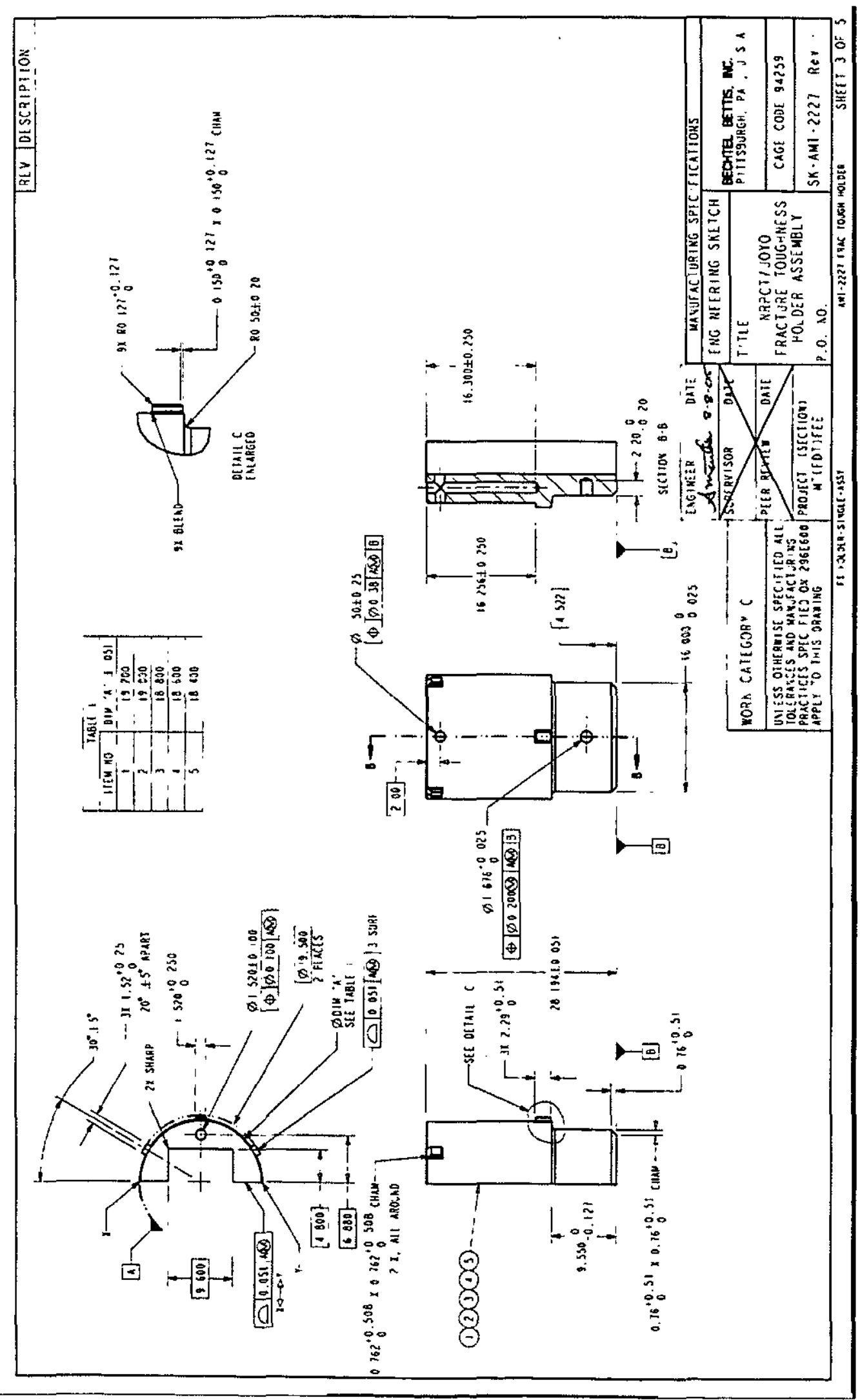


Enclosure (7) to MDO-723-0044I

B-MT(SRME)-52

Page 179

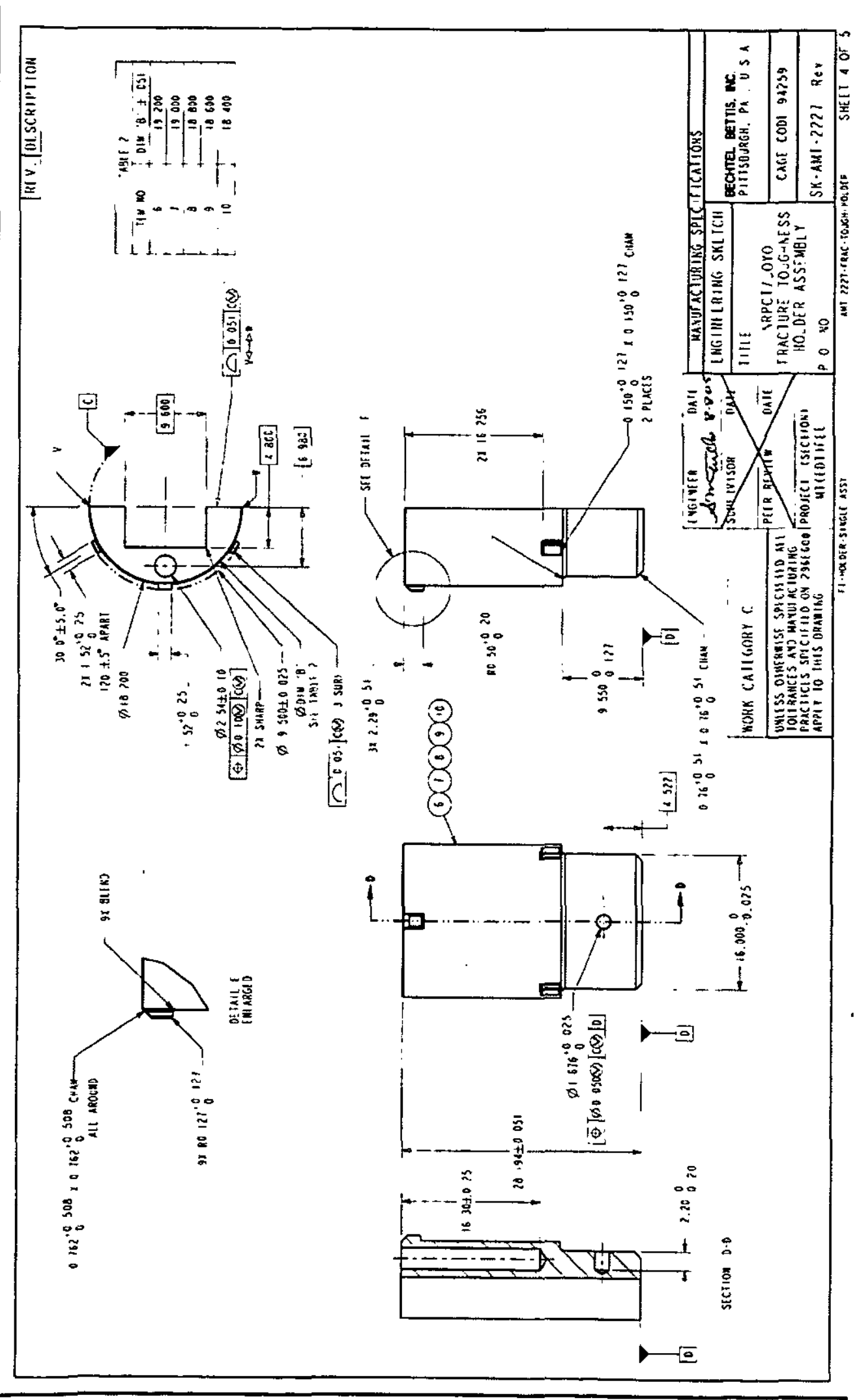


Enclosure (7) to

MDO-723-0044/

B-MT(SRME)-52

Page 180

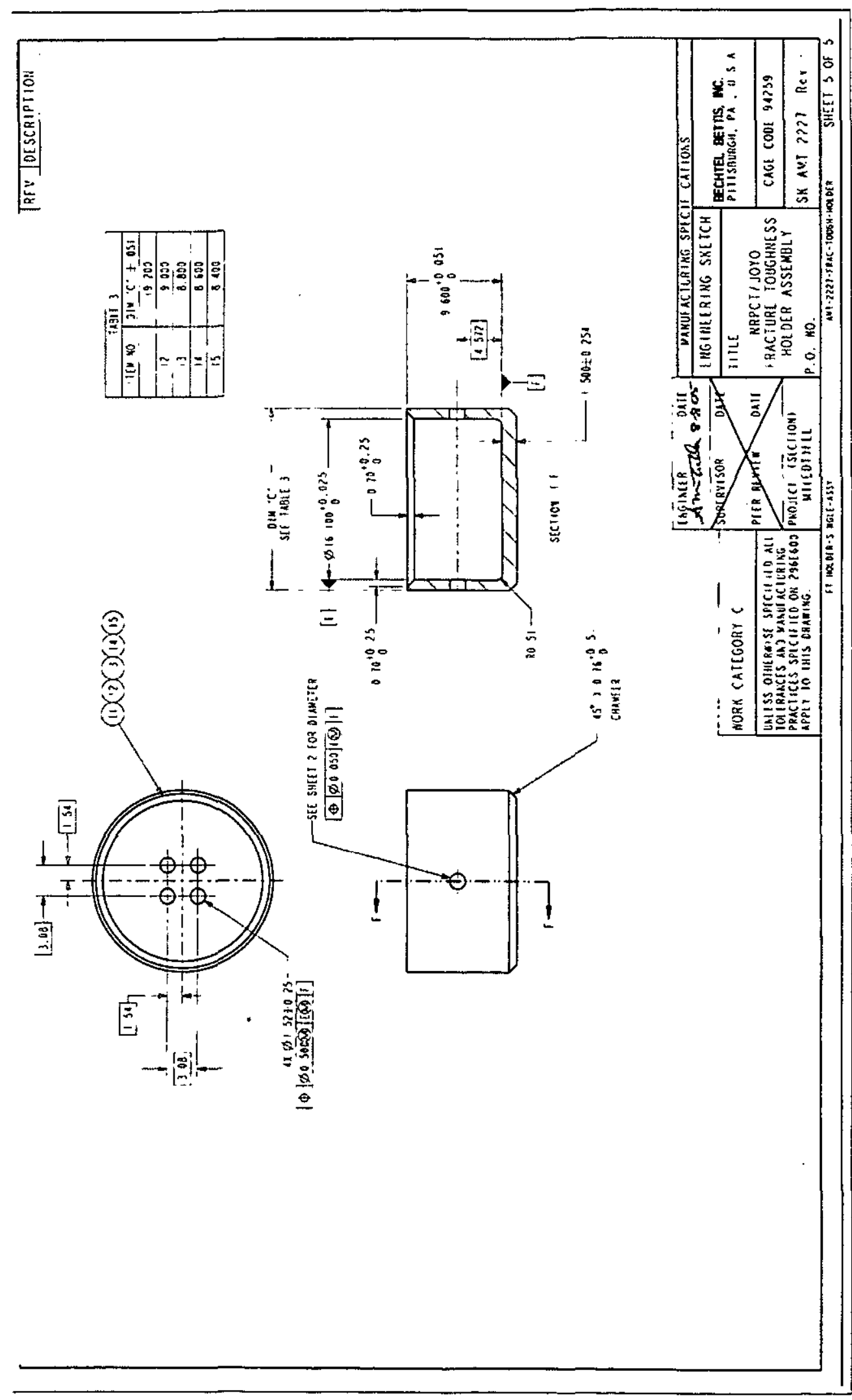


Enclosure (7) to

MDO-723-0044/

B-MT(SRME)-52

Page 181

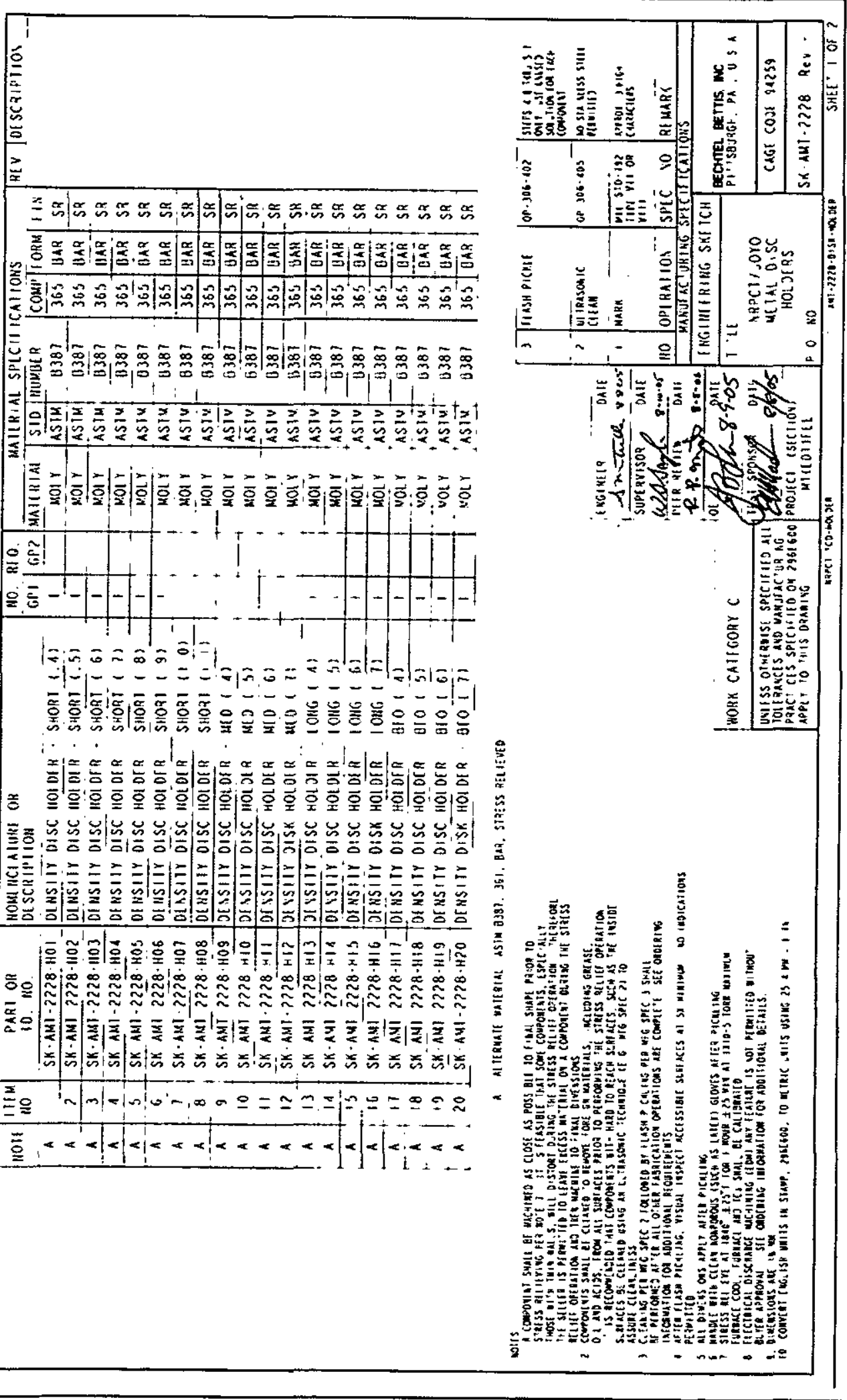


Enclosure (7) to

MDO-723-0044/

B-MT(SRME)-52

Page 182

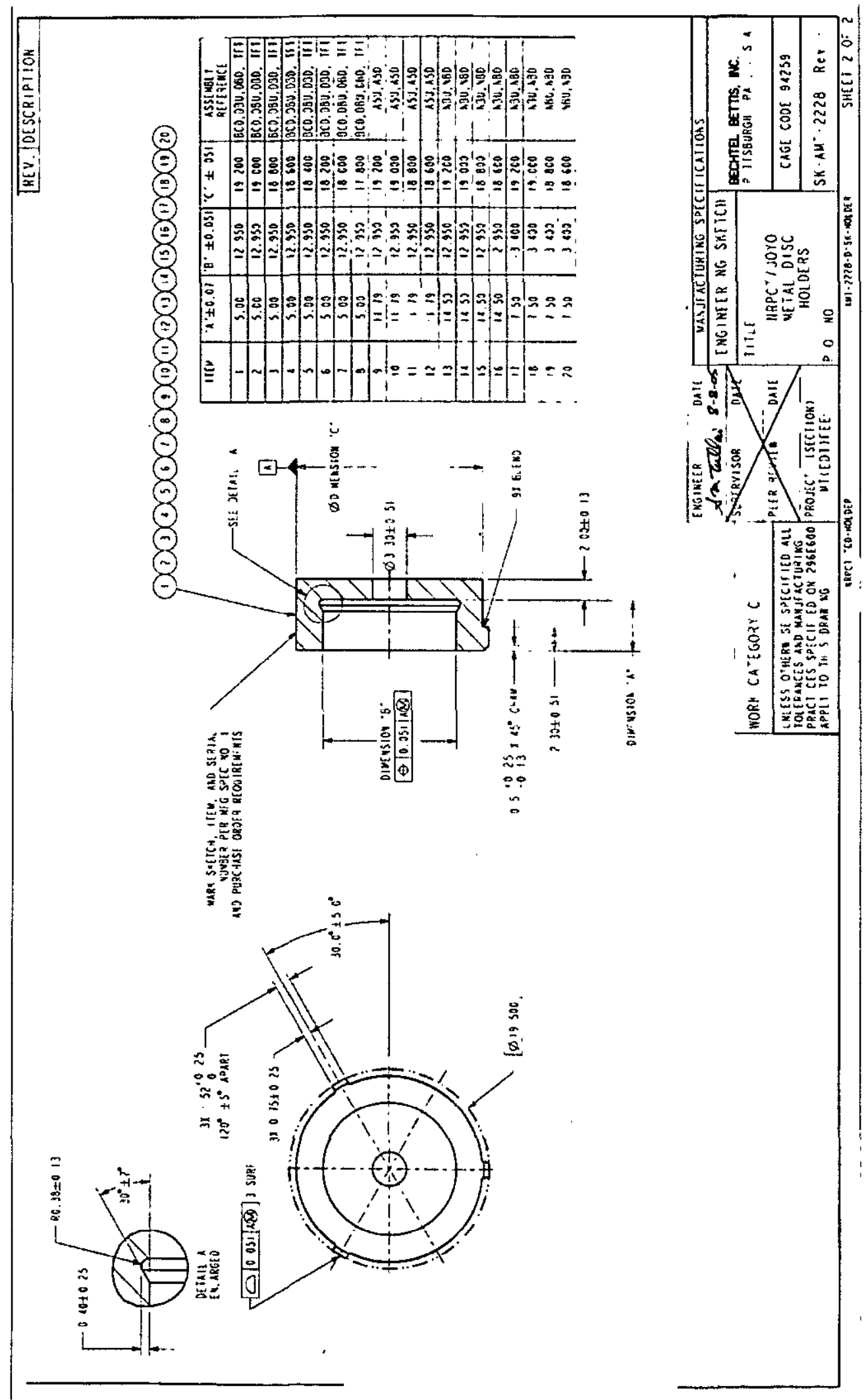


Enclosure (7) to

MDO-723-0044/

B-MT(SRME)-52

Page $\uparrow 83$

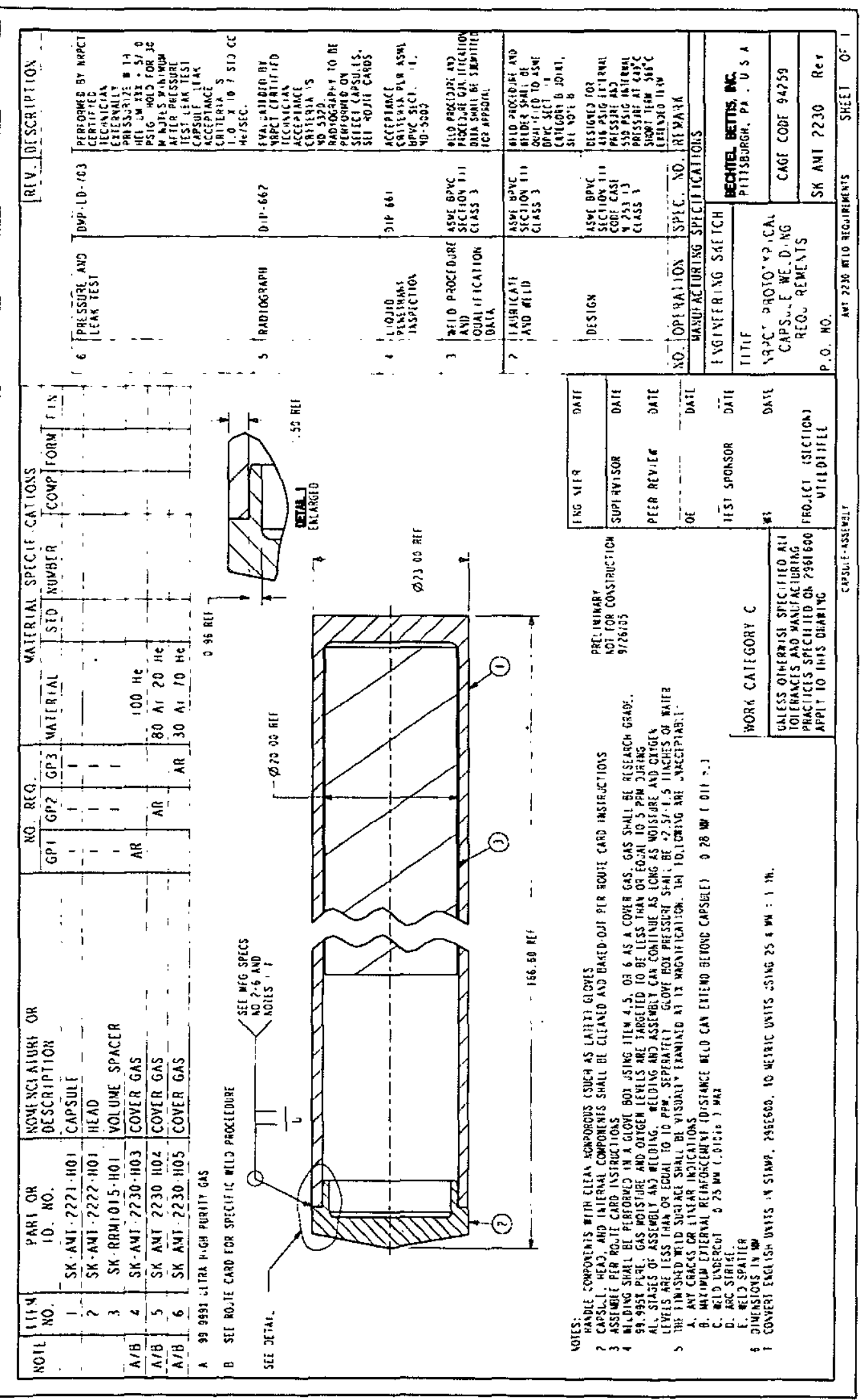


Enclosure (7) to

MDO-723-0044/

B-MT(SRME)-52

Page 184

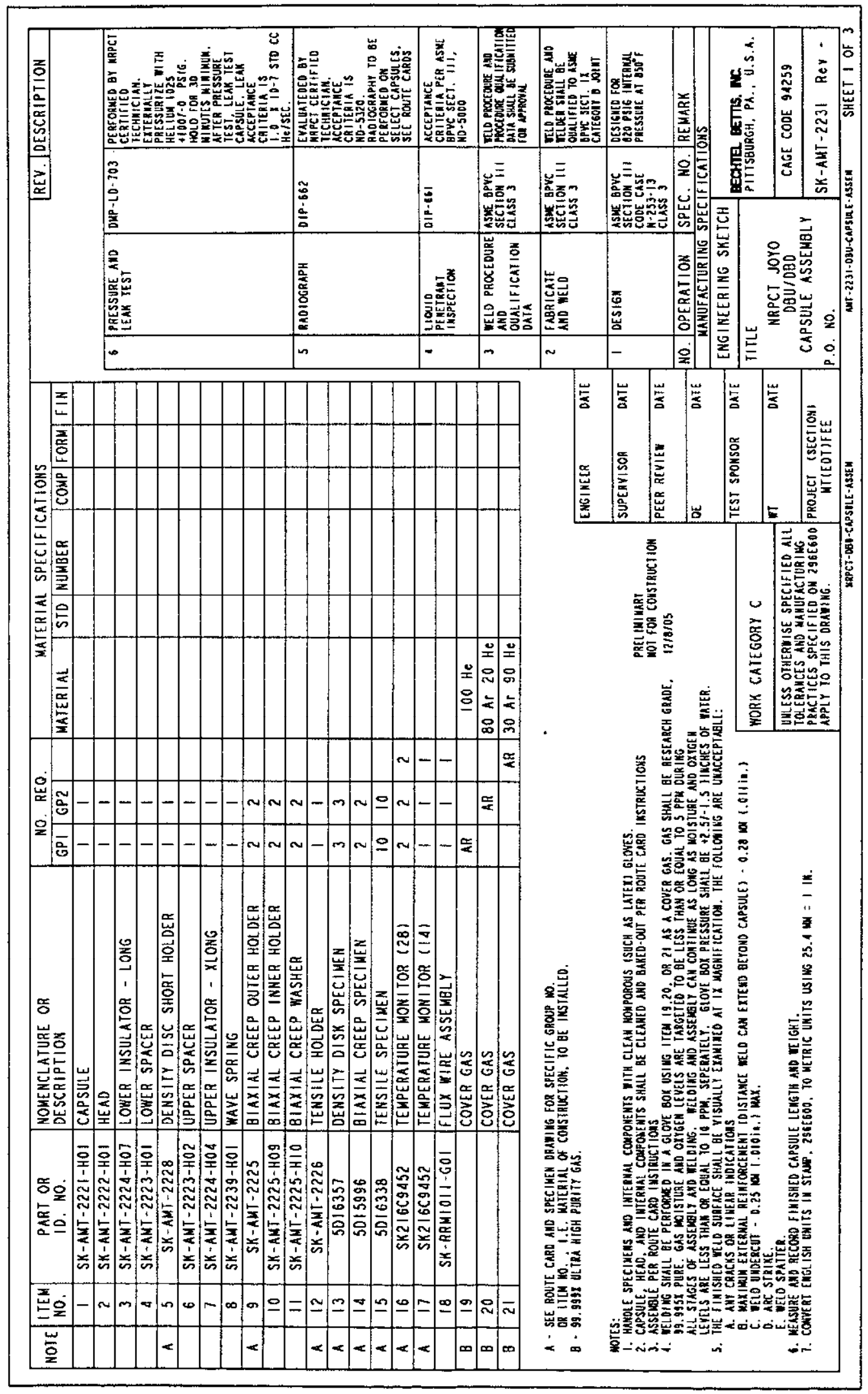


Enclosure (7) to

MDO-723-0044/

B-MT(SRME)-52

Page 185

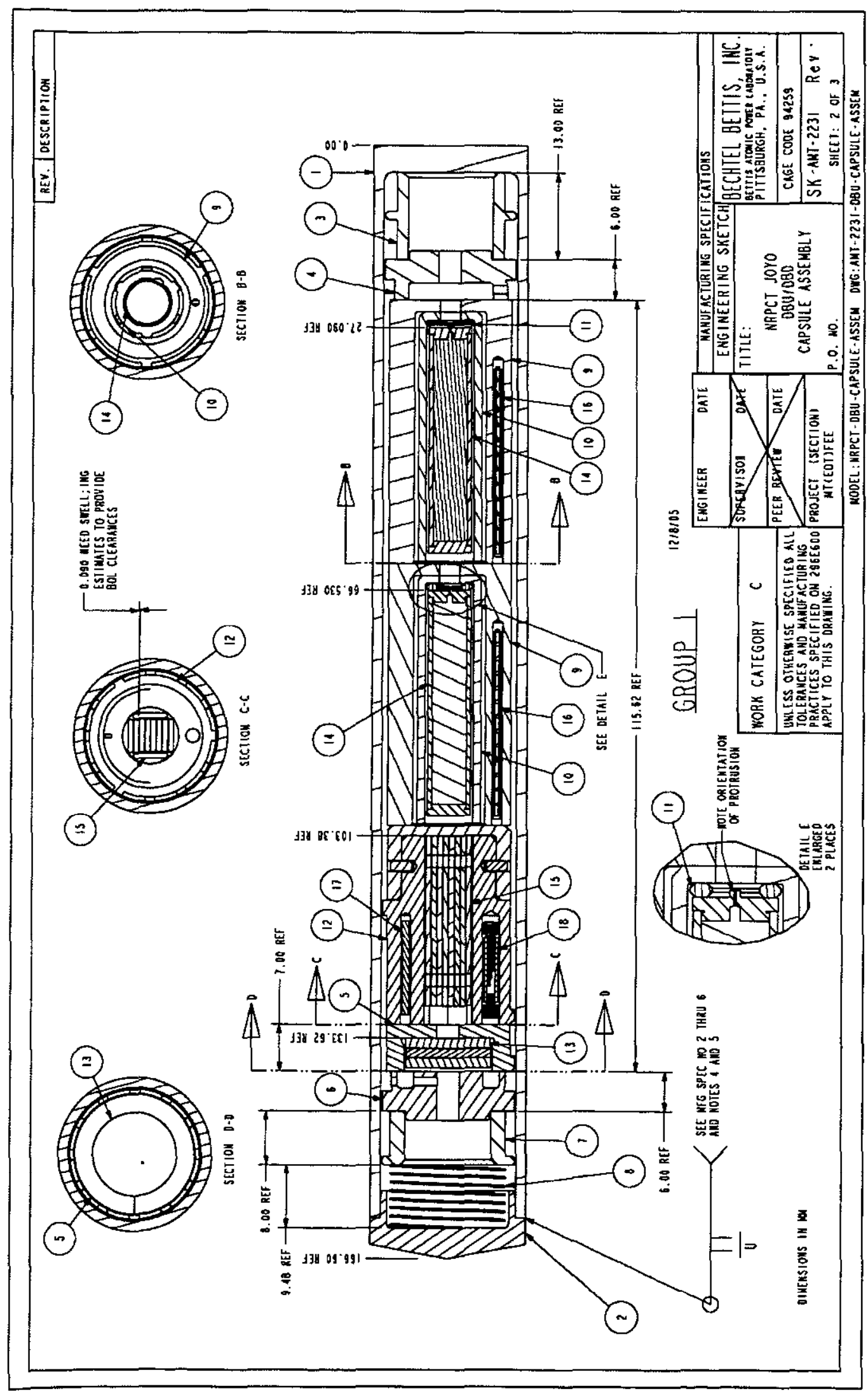


Enclosure (7) to

MDO-723-0044/

B-MT(SRME)-52

Page 186

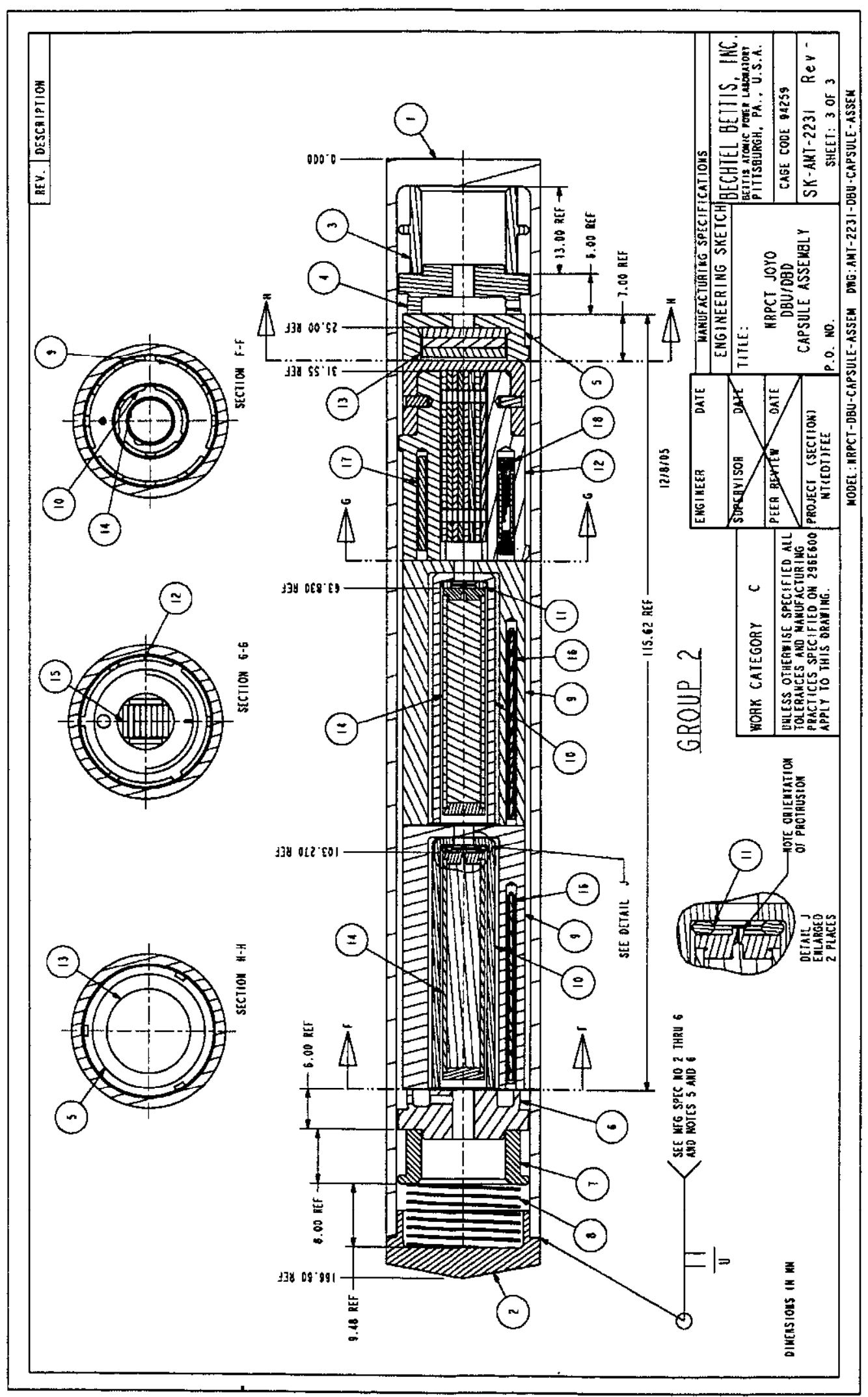


Enclosure (7) to

MDO-723-0044l

B-MT(SRME)-52

Page 187

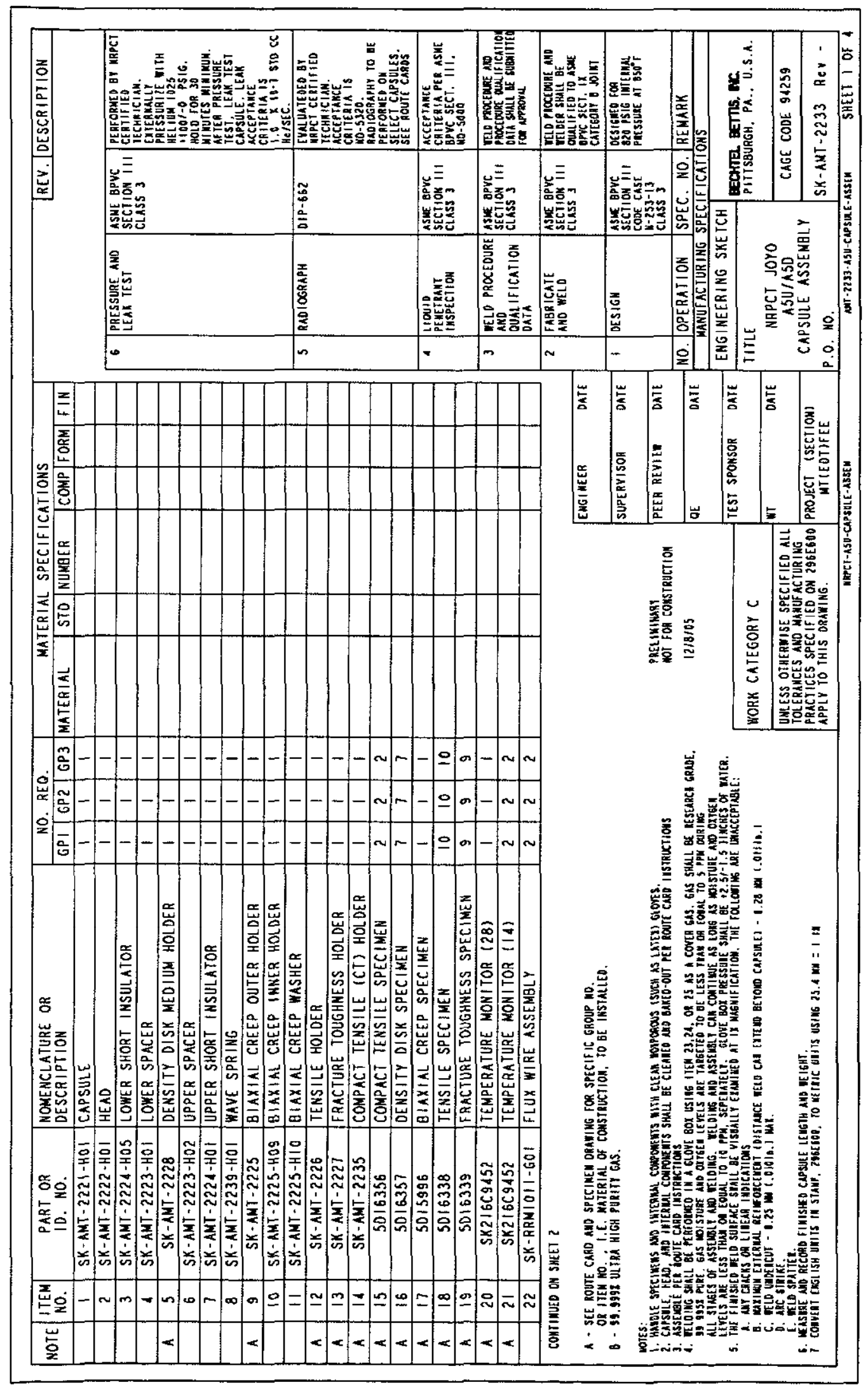


Enclosure (7) to

MDO-723-0044/

B-MT(SRME)-52

Page 188

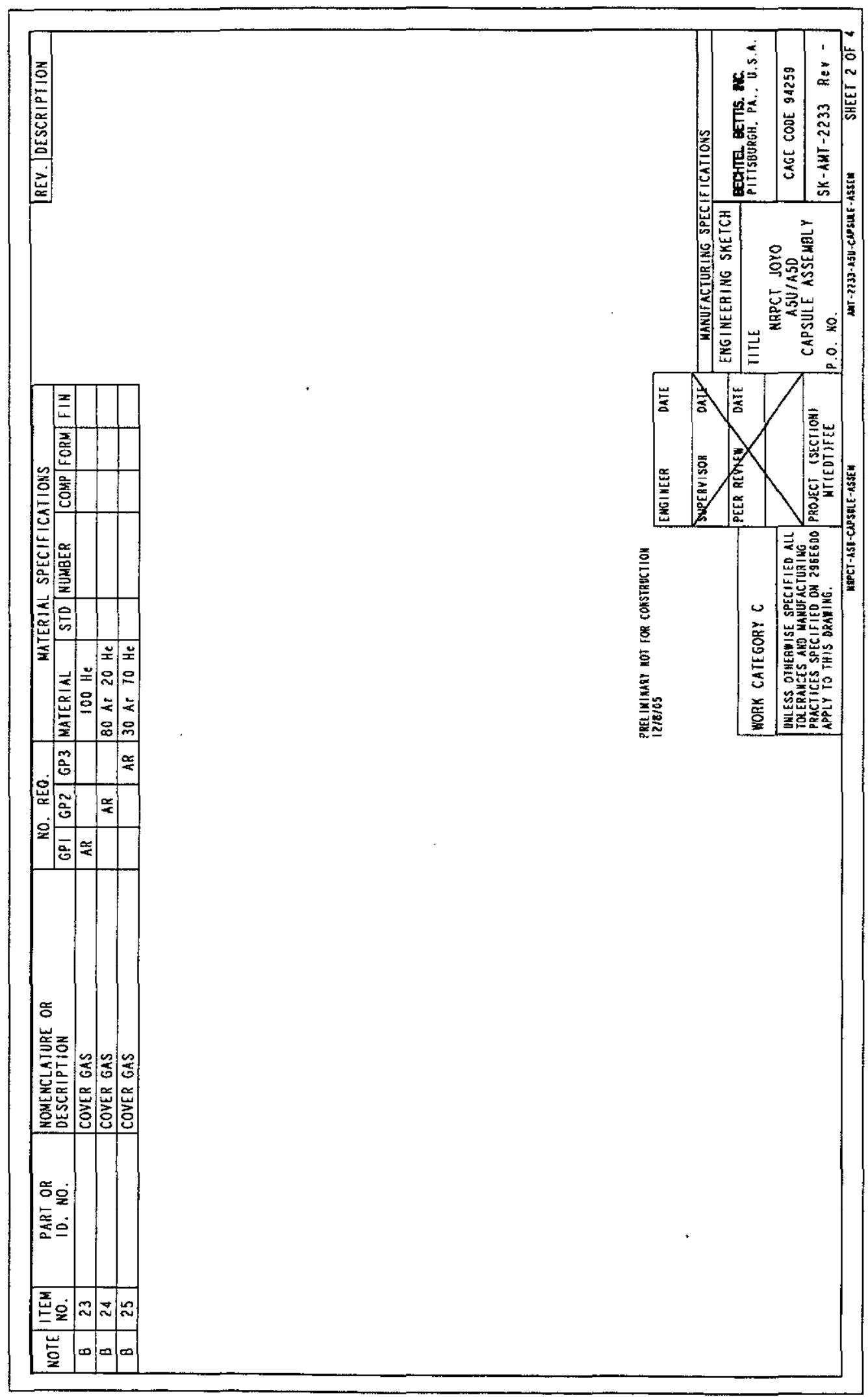


Enclosure (7) to

MDO-723-0044/

B-MT(SRME)-52

Page 189

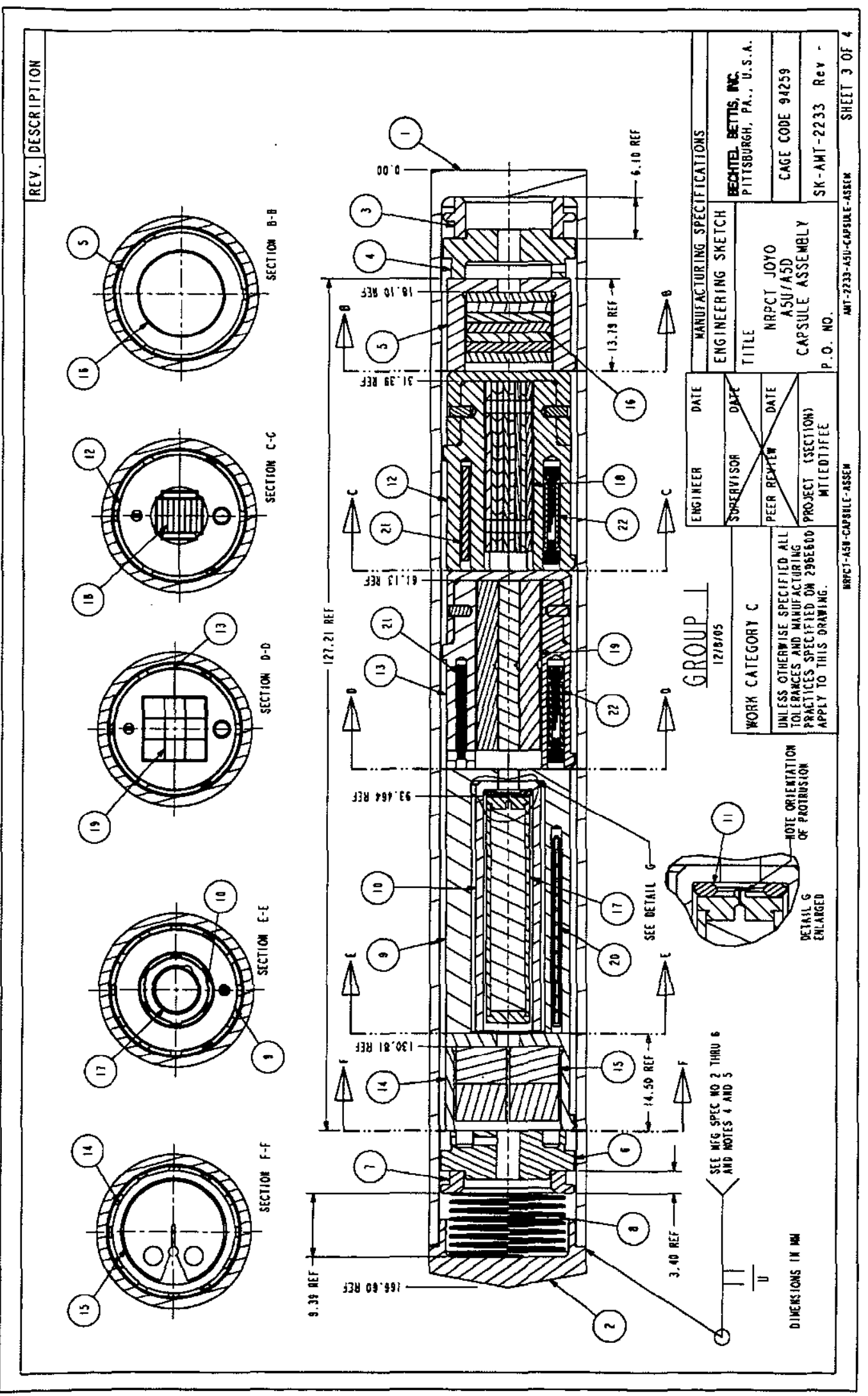


Enclosure (7) to

MDO-723-0044/

B-MT(SRME)-52

Page 190

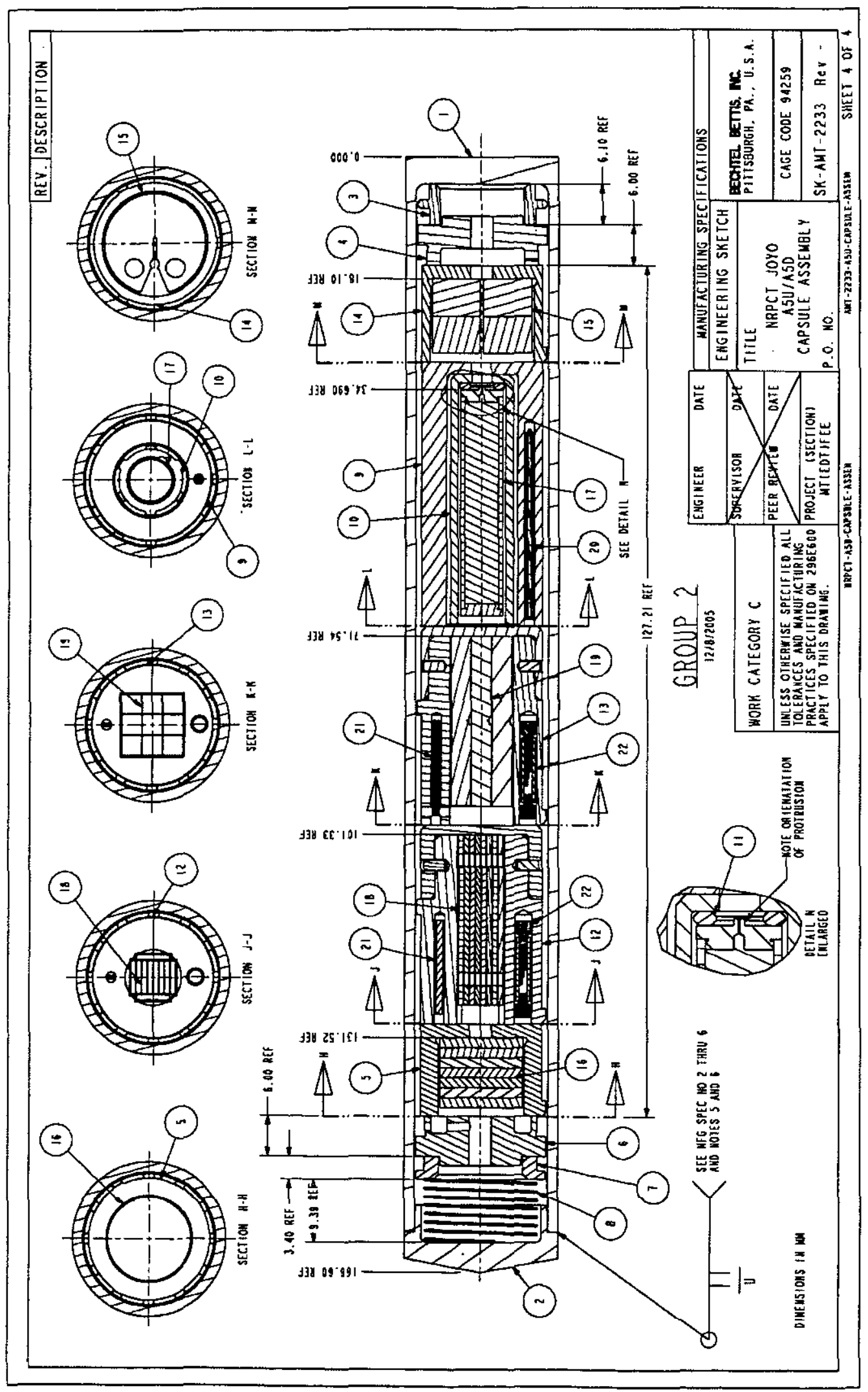


Enclosure (7) to

MDO-723-0044/

B-MT(SRME)-52

Page 191

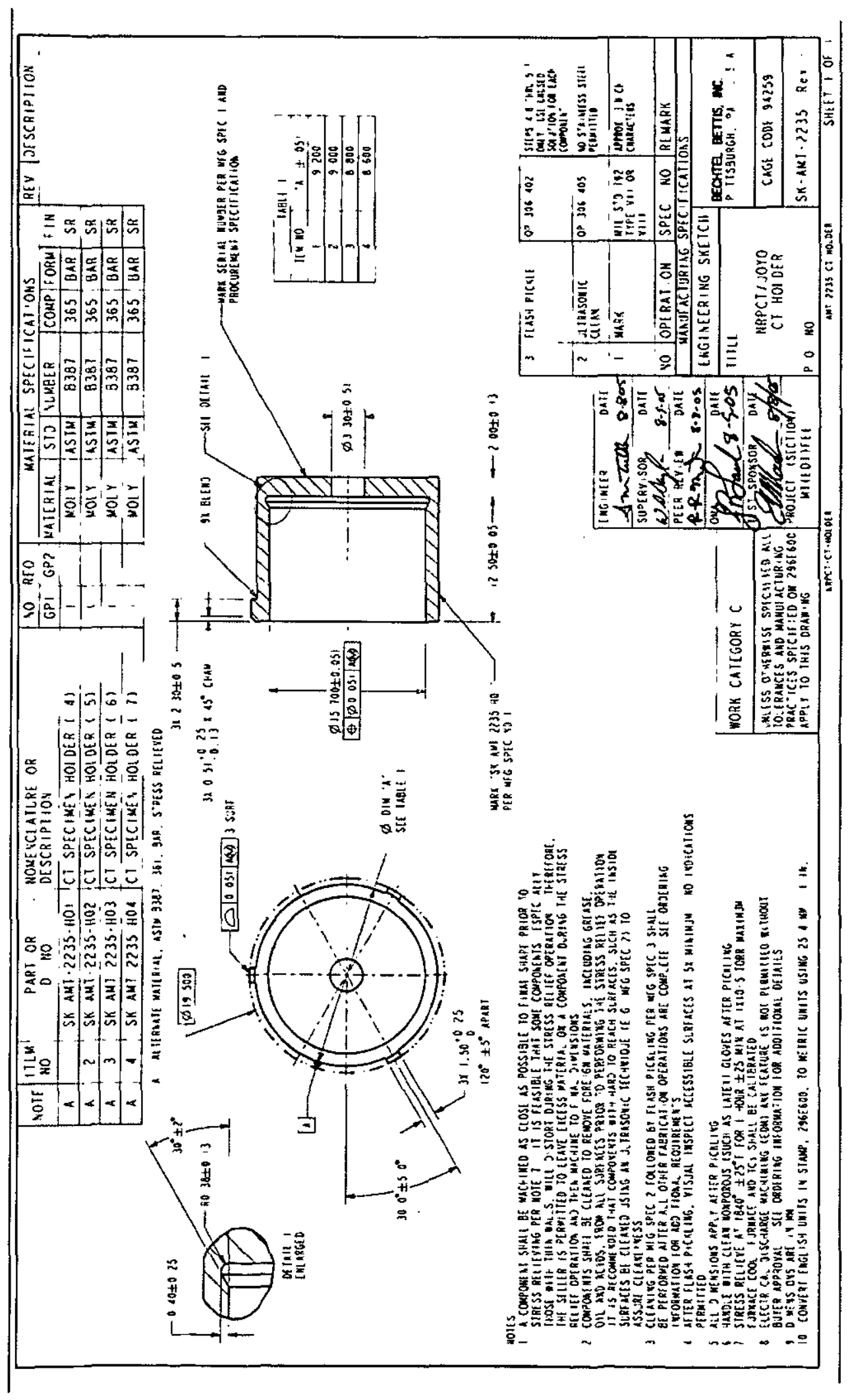




\section{Enclosure (7) to \\ MDO-723-0044I \\ B-MT(SRME)-52 \\ Page 192}

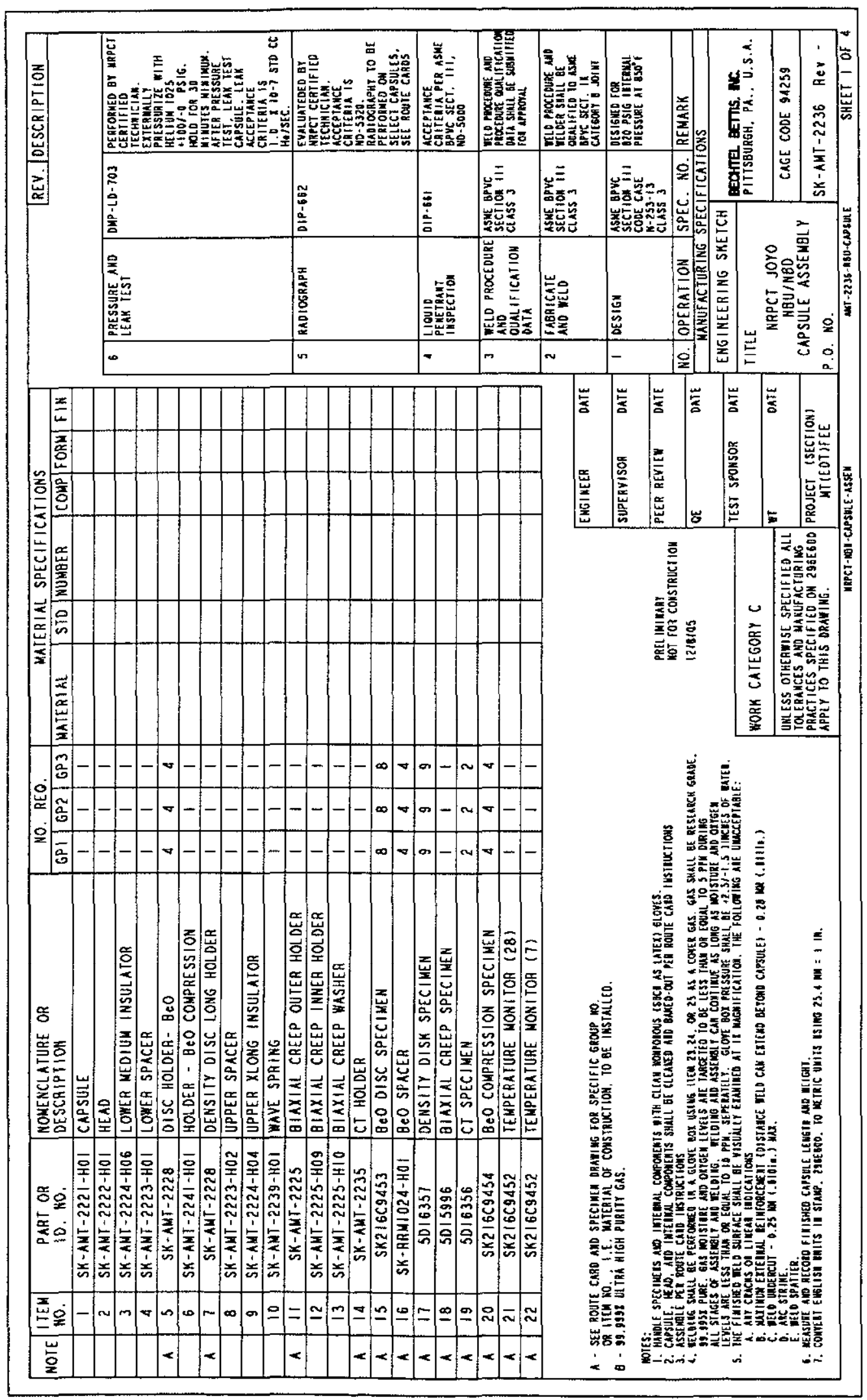




\section{Enclosure (7) to MDO-723-0044/ \\ B-MT(SRME)-52 \\ Page 193}

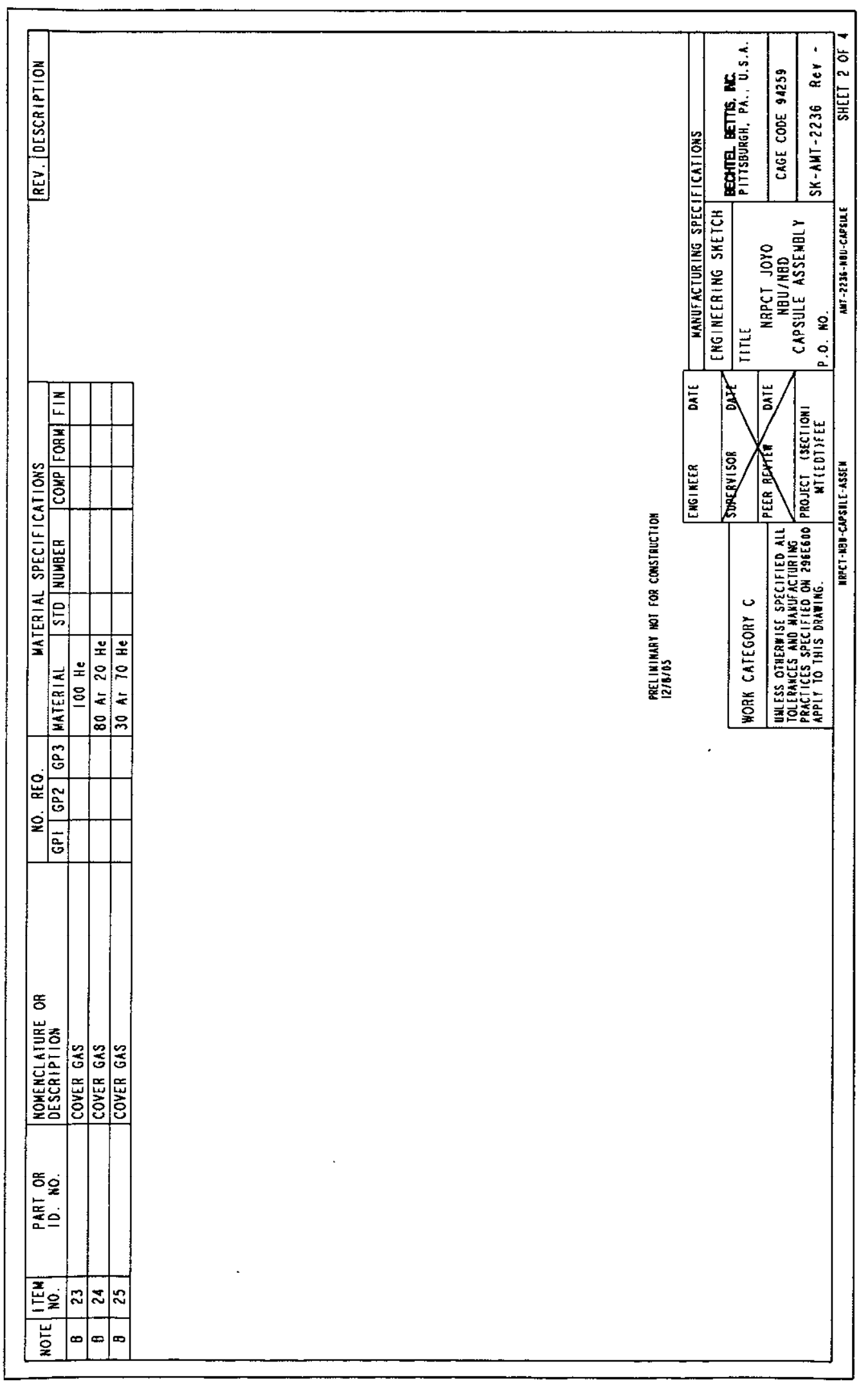


Enclosure (7) to

MDO-723-0044/

B-MT(SRME)-52

Page 194

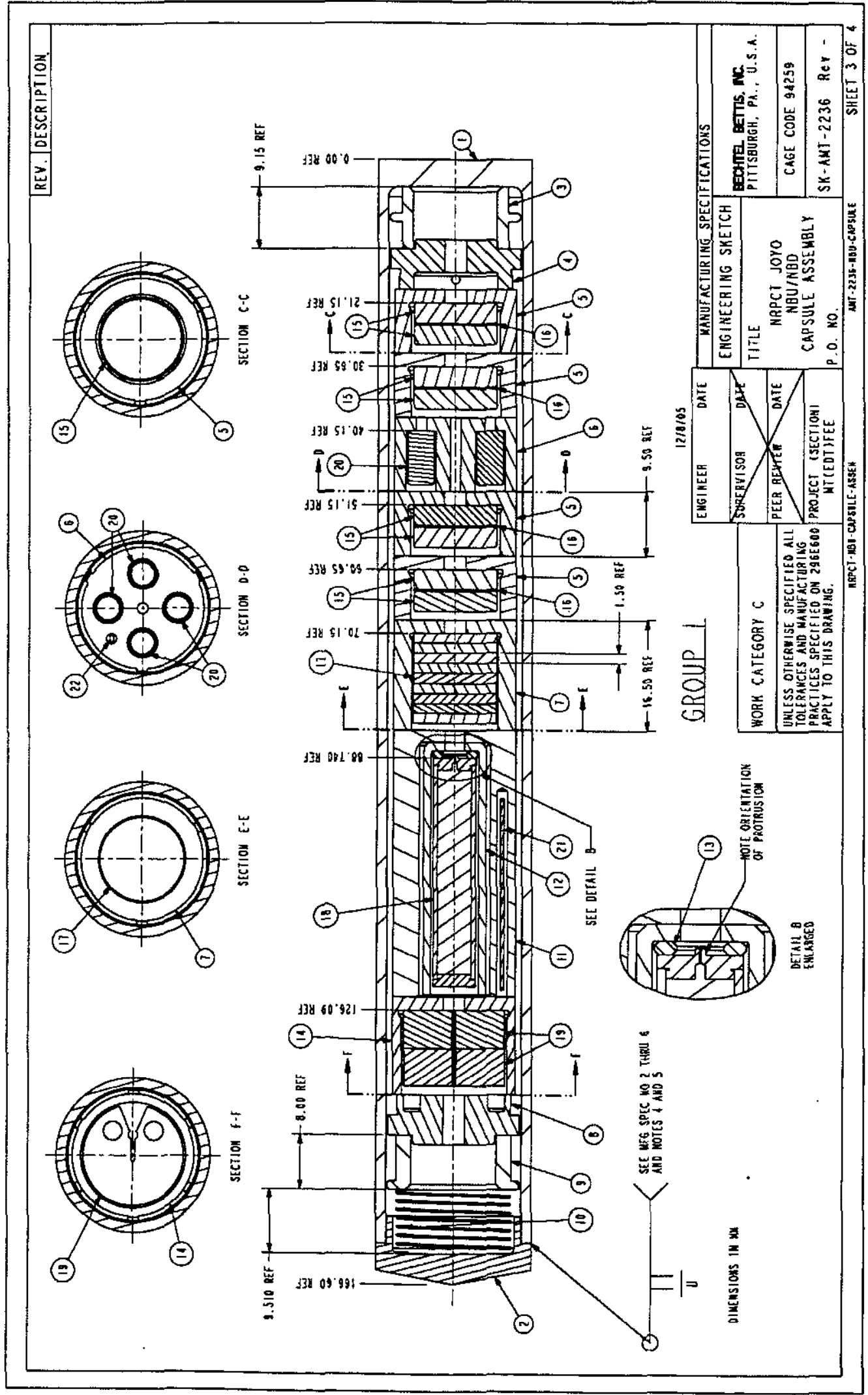



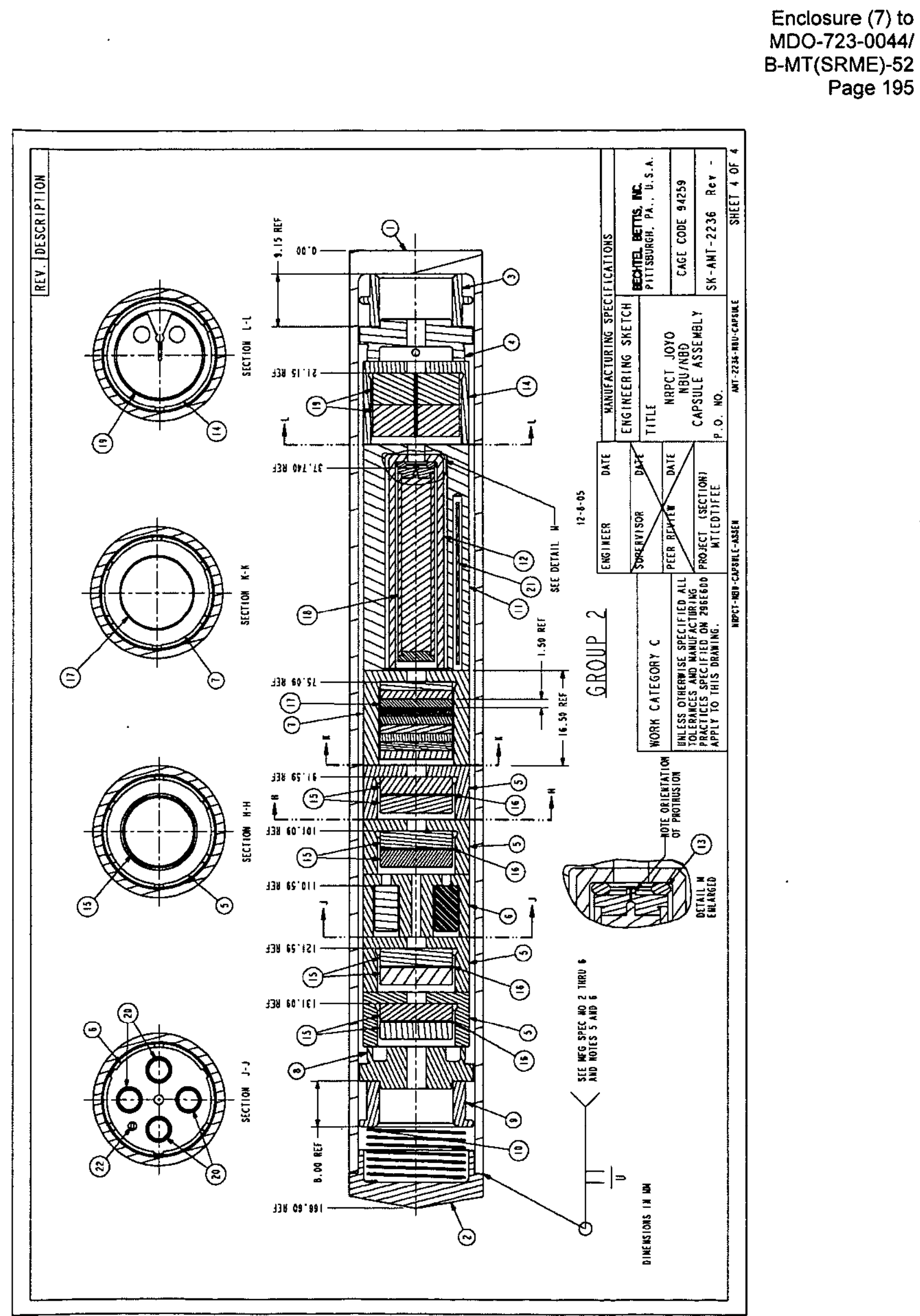


\section{Enclosure (7) to \\ MDO-723-0044/ \\ B-MT(SRME)-52 \\ Page 196}

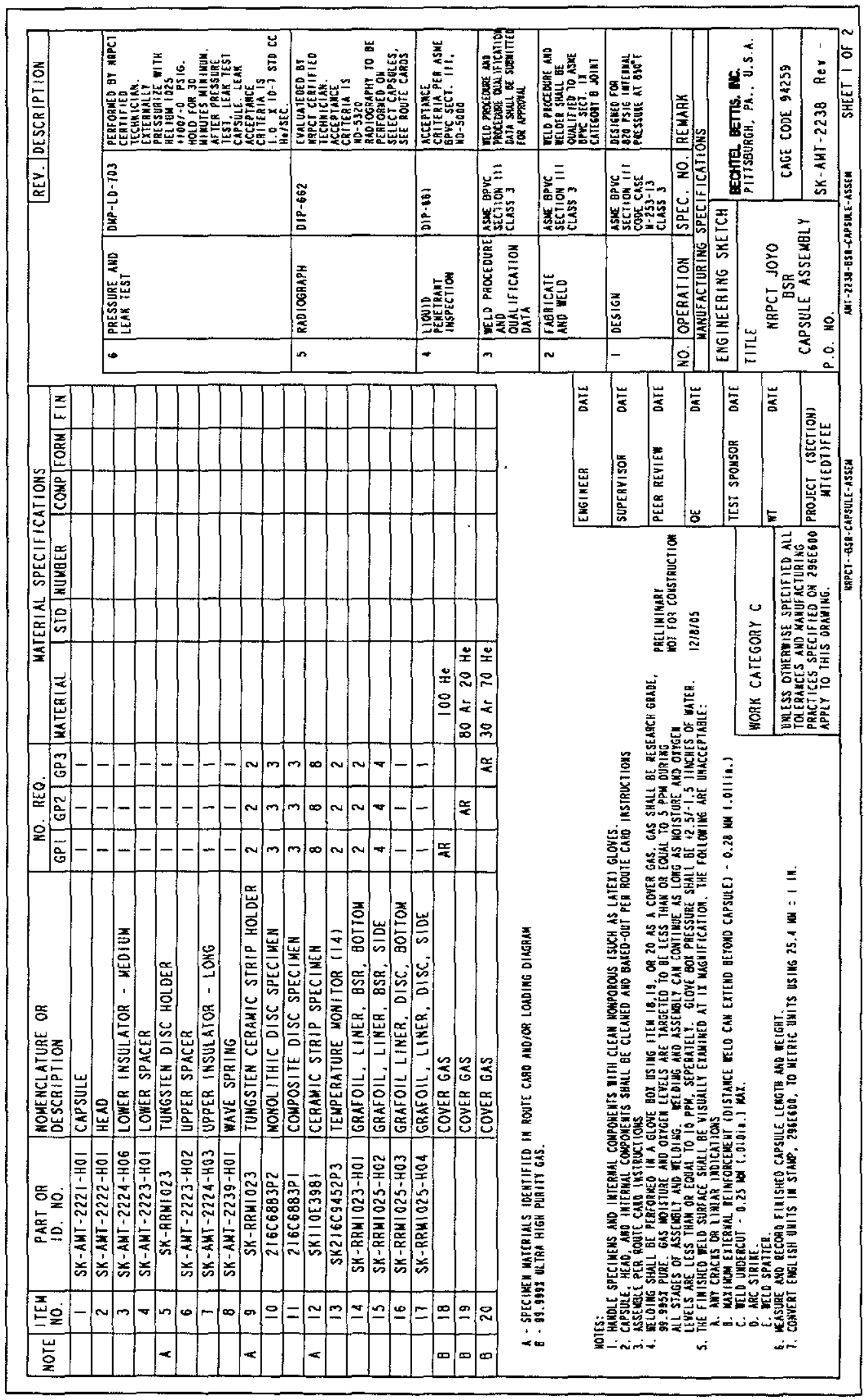


Enclosure (7) to

MDO-723-0044/

B-MT(SRME)-52

Page 197

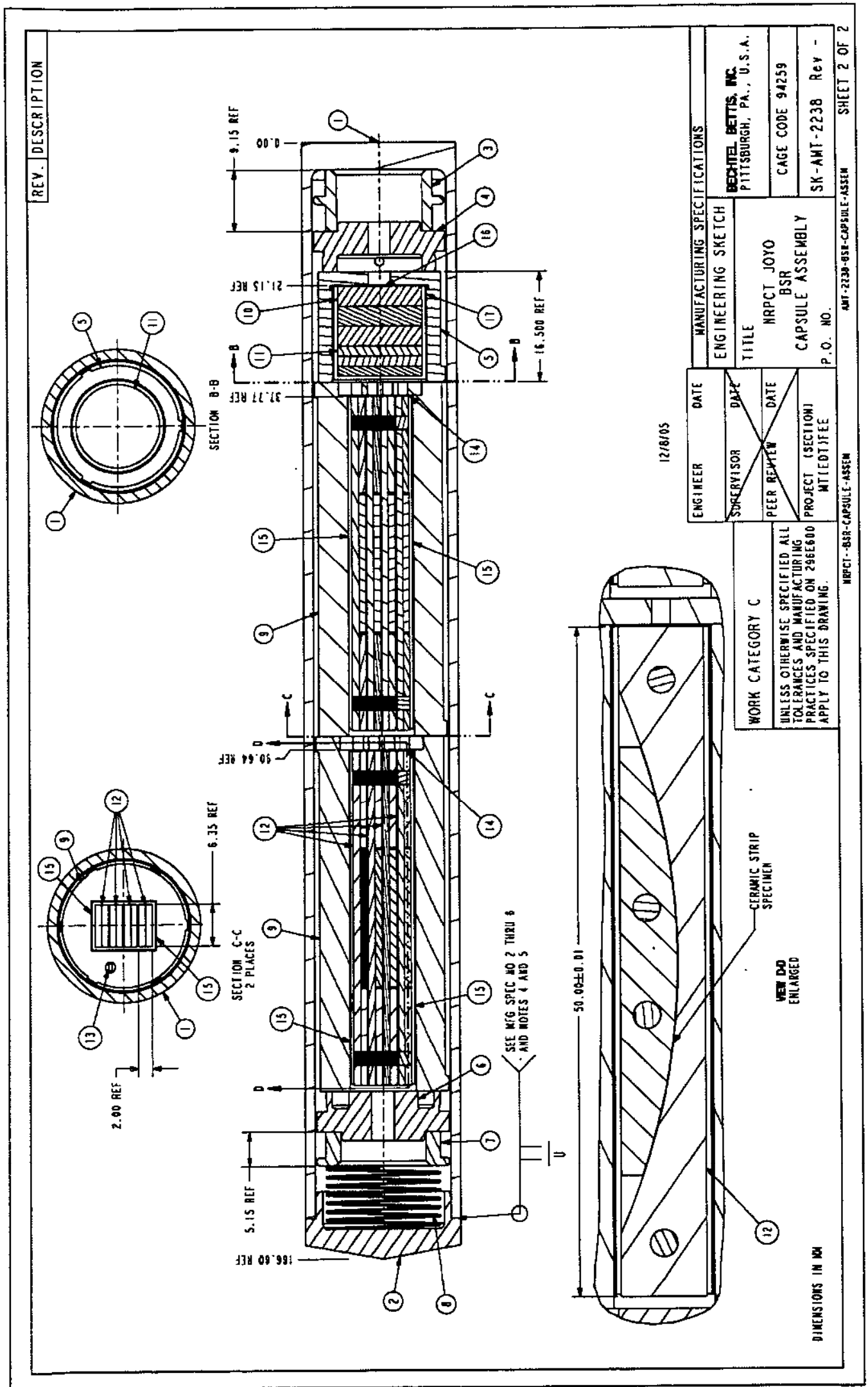


Enclosure (7) to

MDO-723-0044/

B-MT(SRME)-52

Page 198

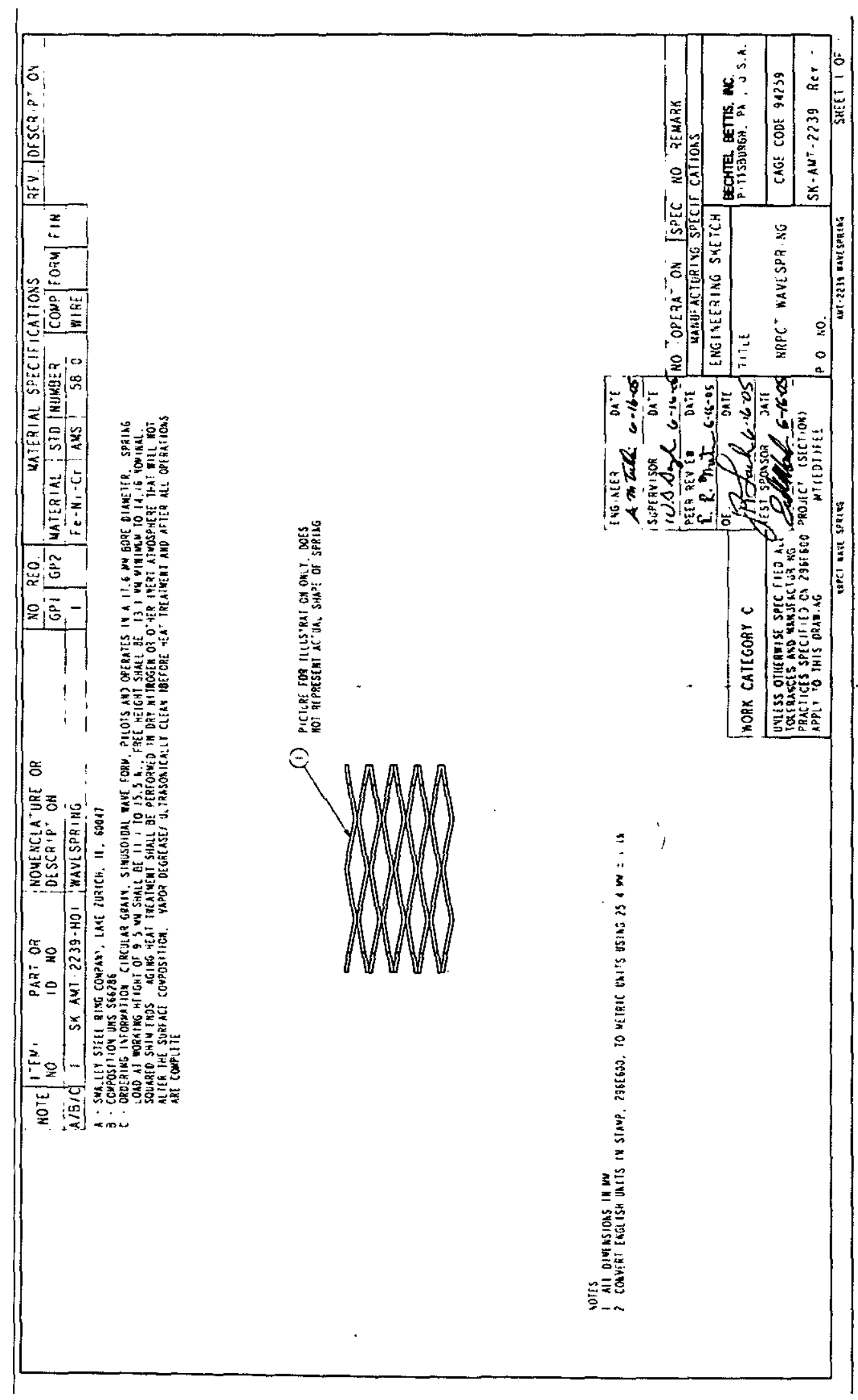


Enclosure (7) to

MDO-723-0044I

B-MT(SRME)-52

Page 199

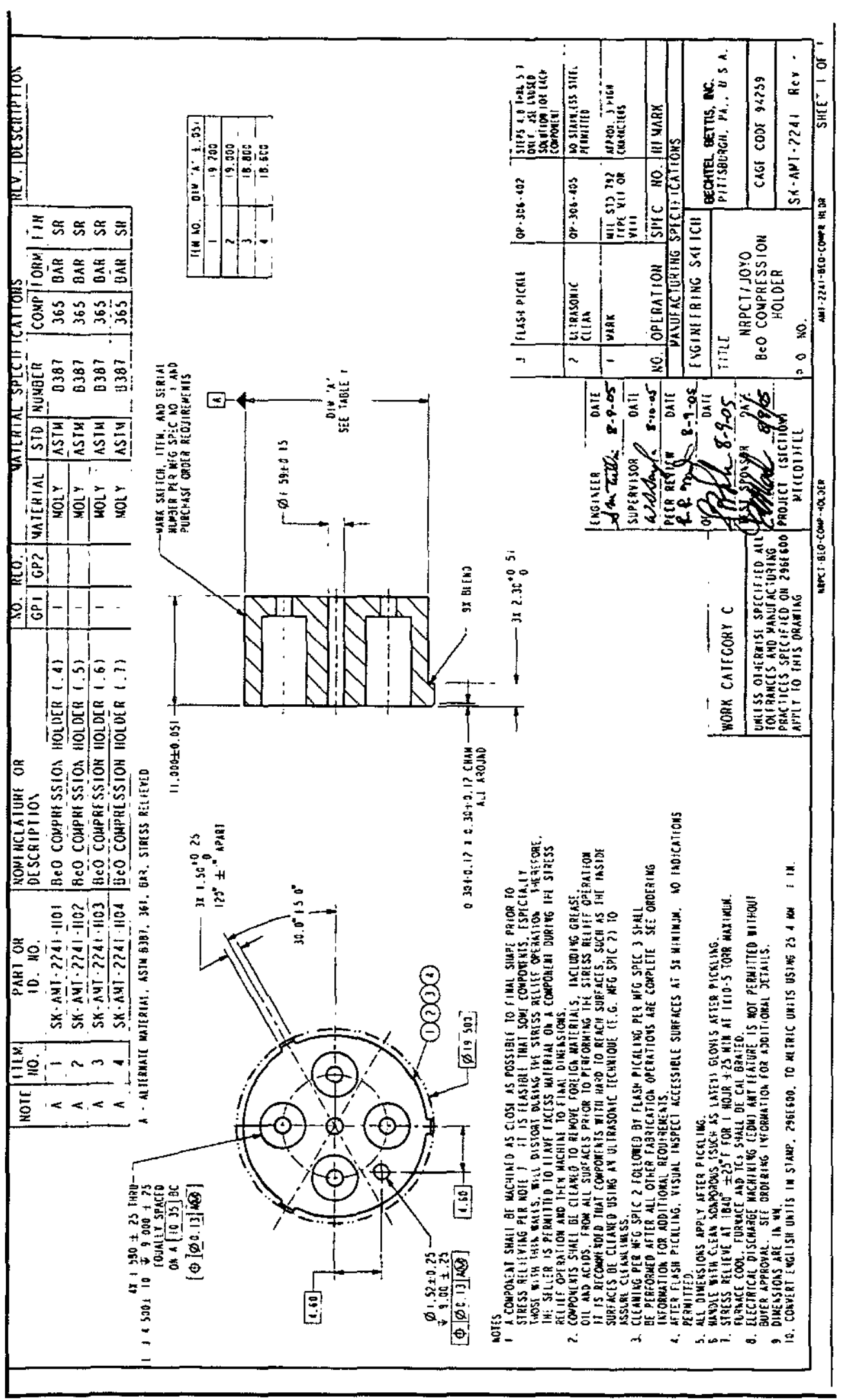


Enclosure (7) to

MDO-723-0044/

B-MT(SRME)-52

Page 200

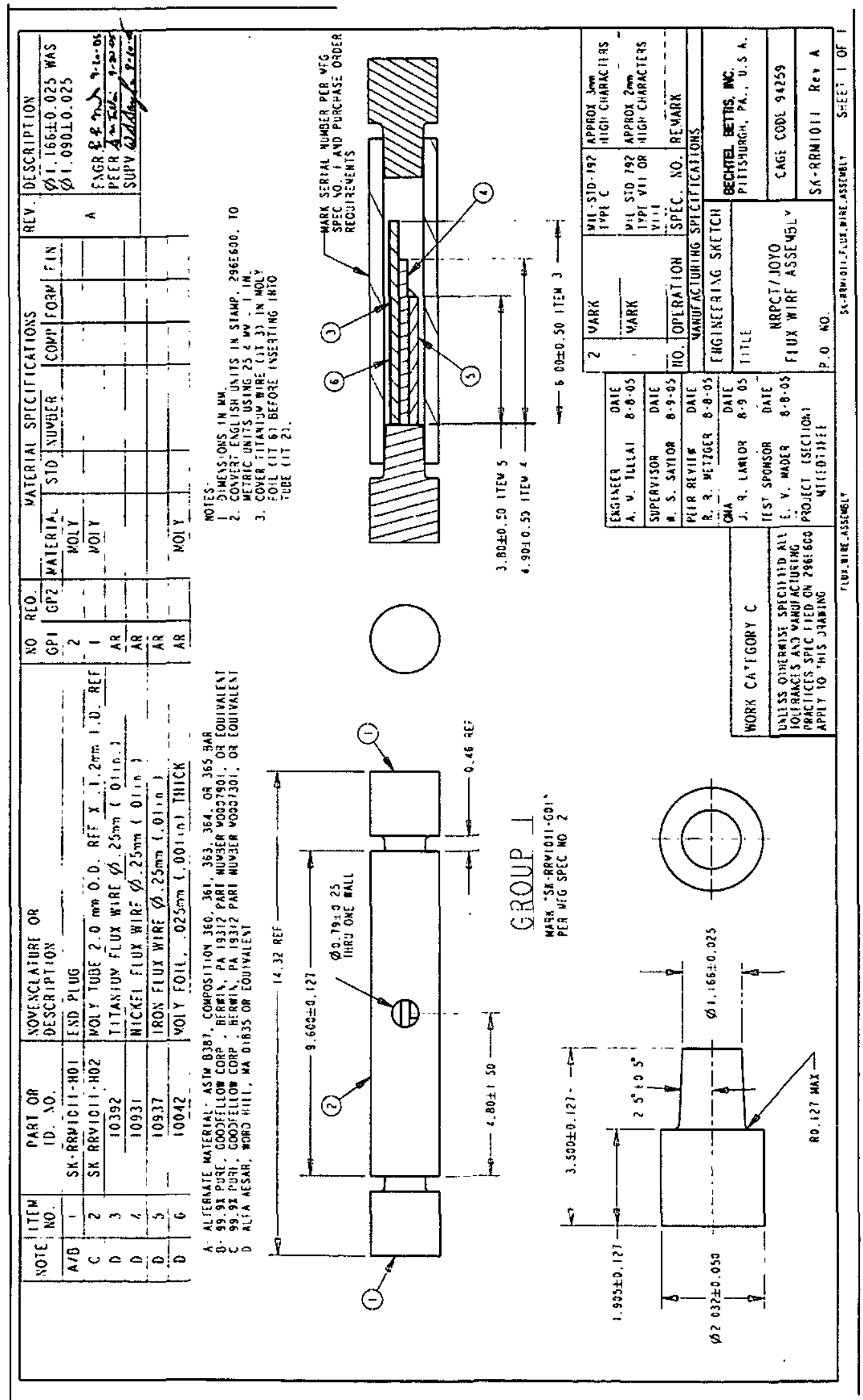


Enclosure (7) to

MDO-723-0044/

B-MT(SRME)-52

Page 201

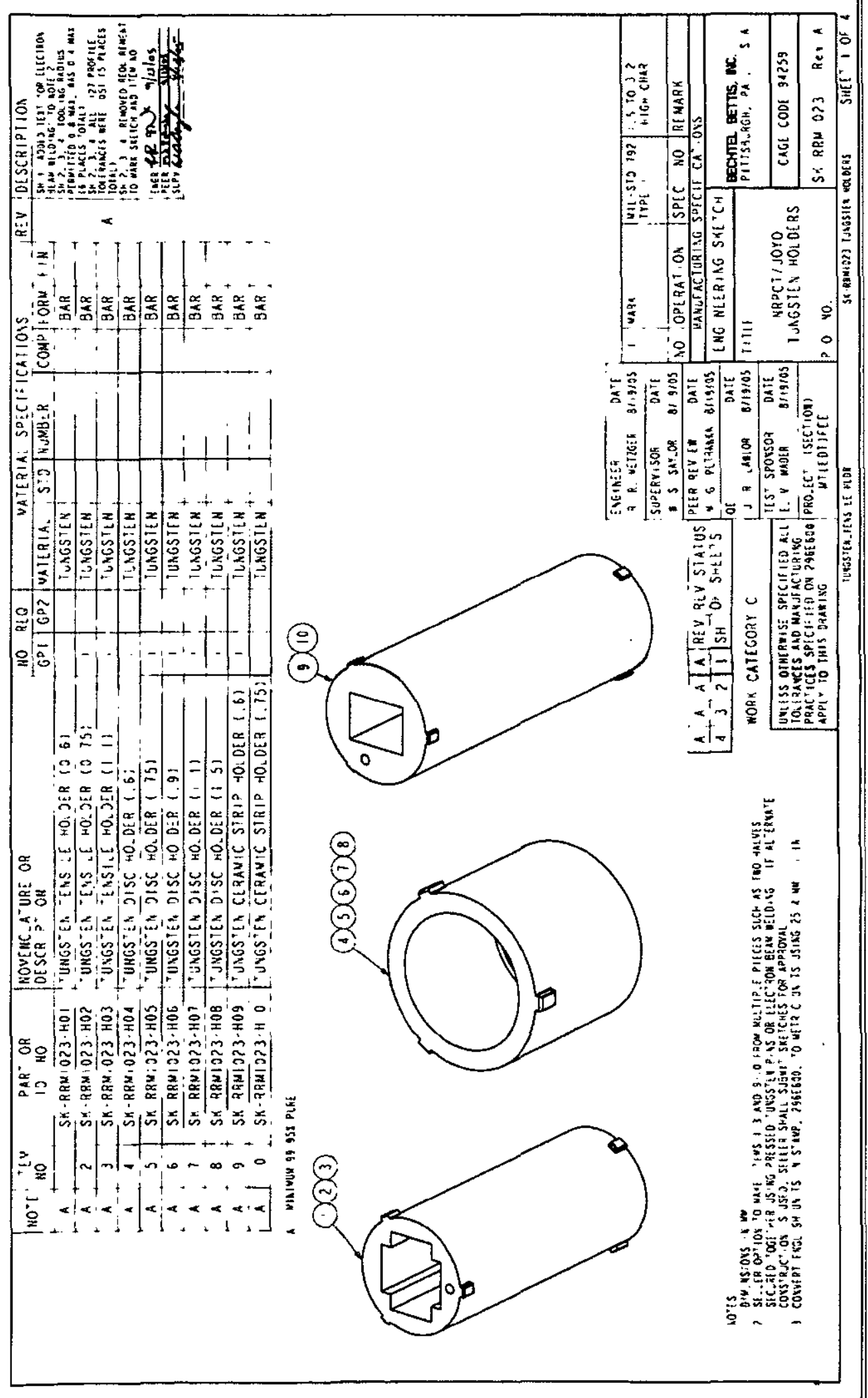


Enclosure (7) to

MDO-723-0044I

B-MT(SRME)-52

Page 202

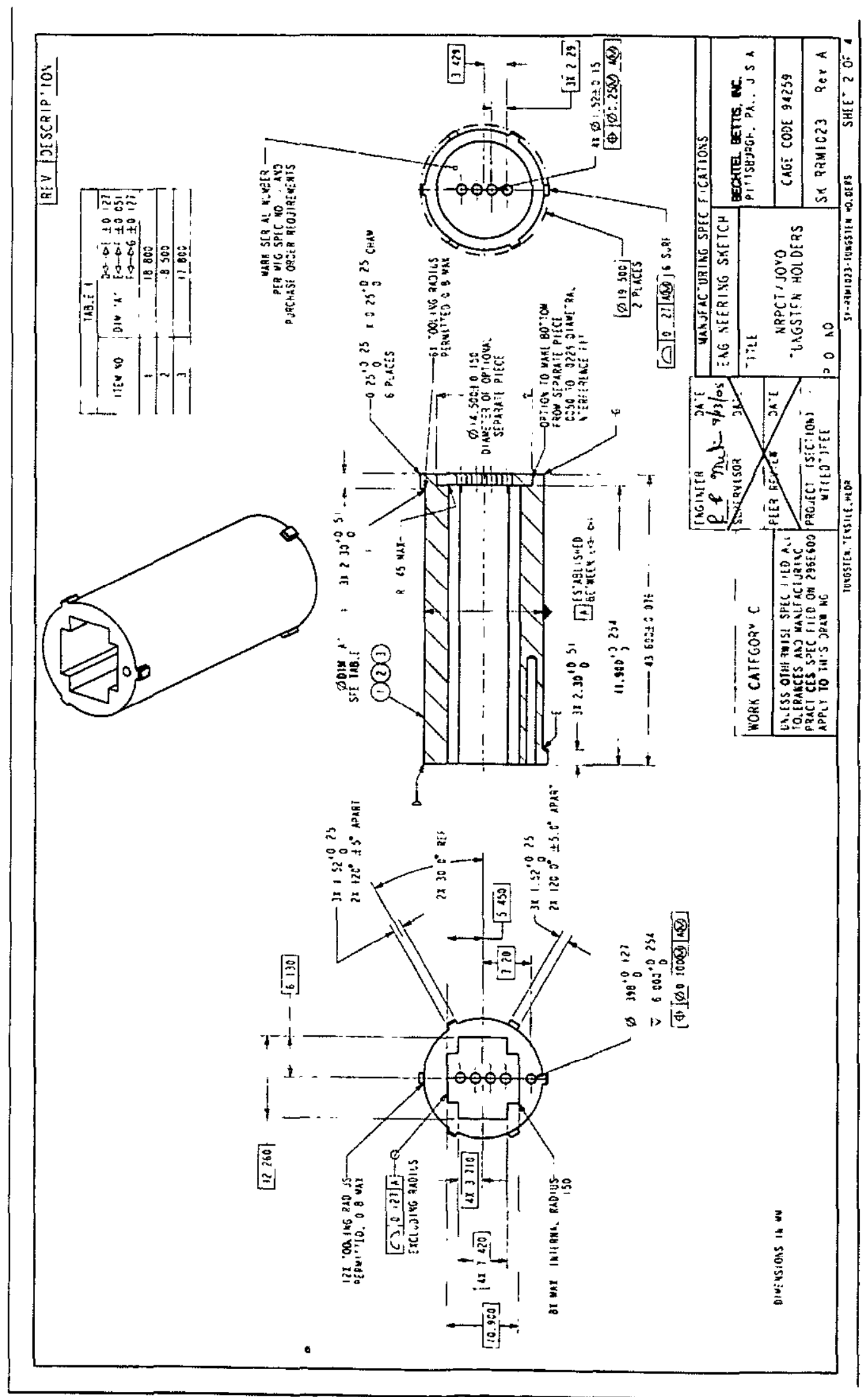


Enclosure (7) to MDO-723-0044/

B-MT(SRME)-52

Page 203

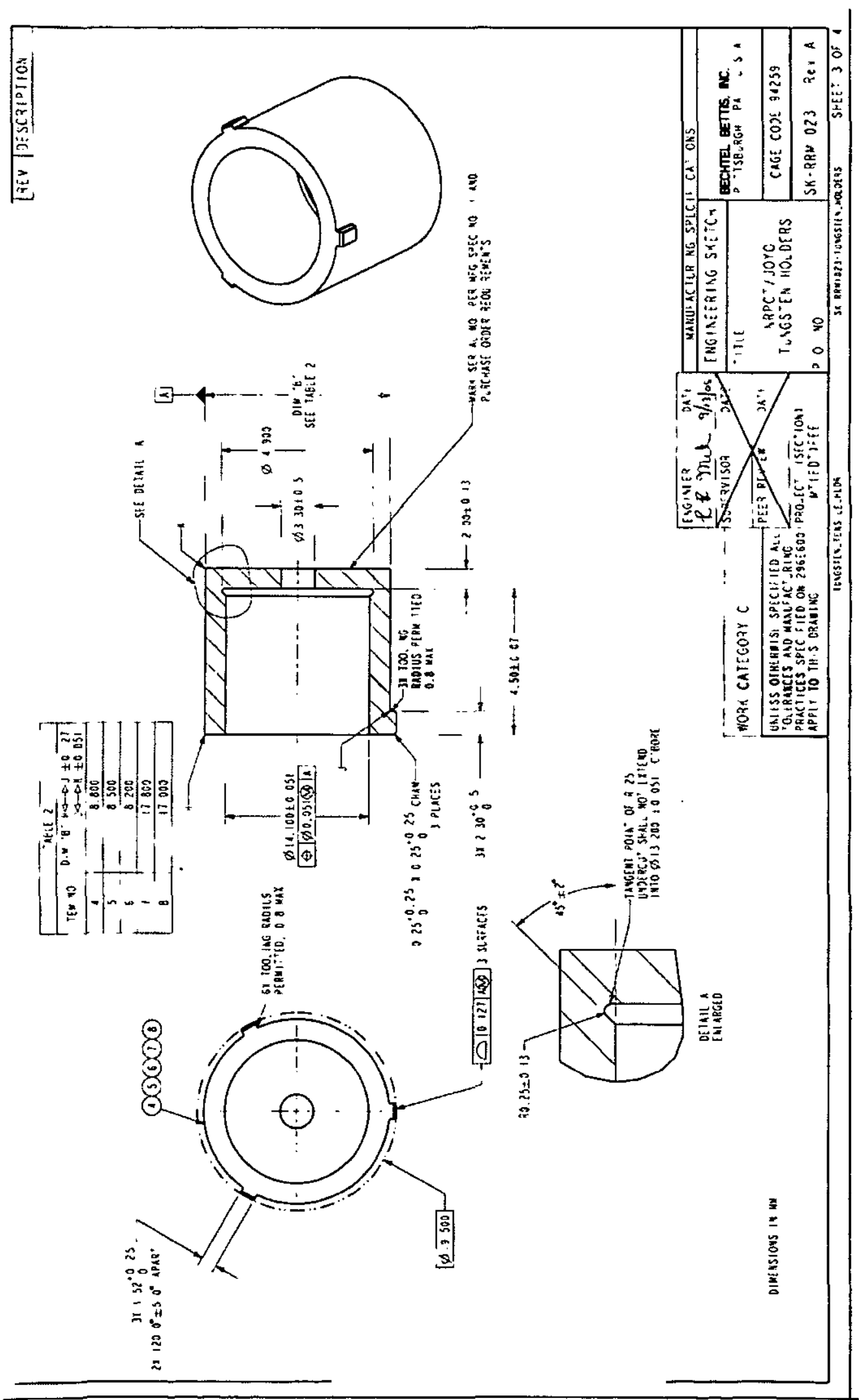


Enclosure (7) to

MDO-723-0044I

B-MT(SRME)-52

Page 204

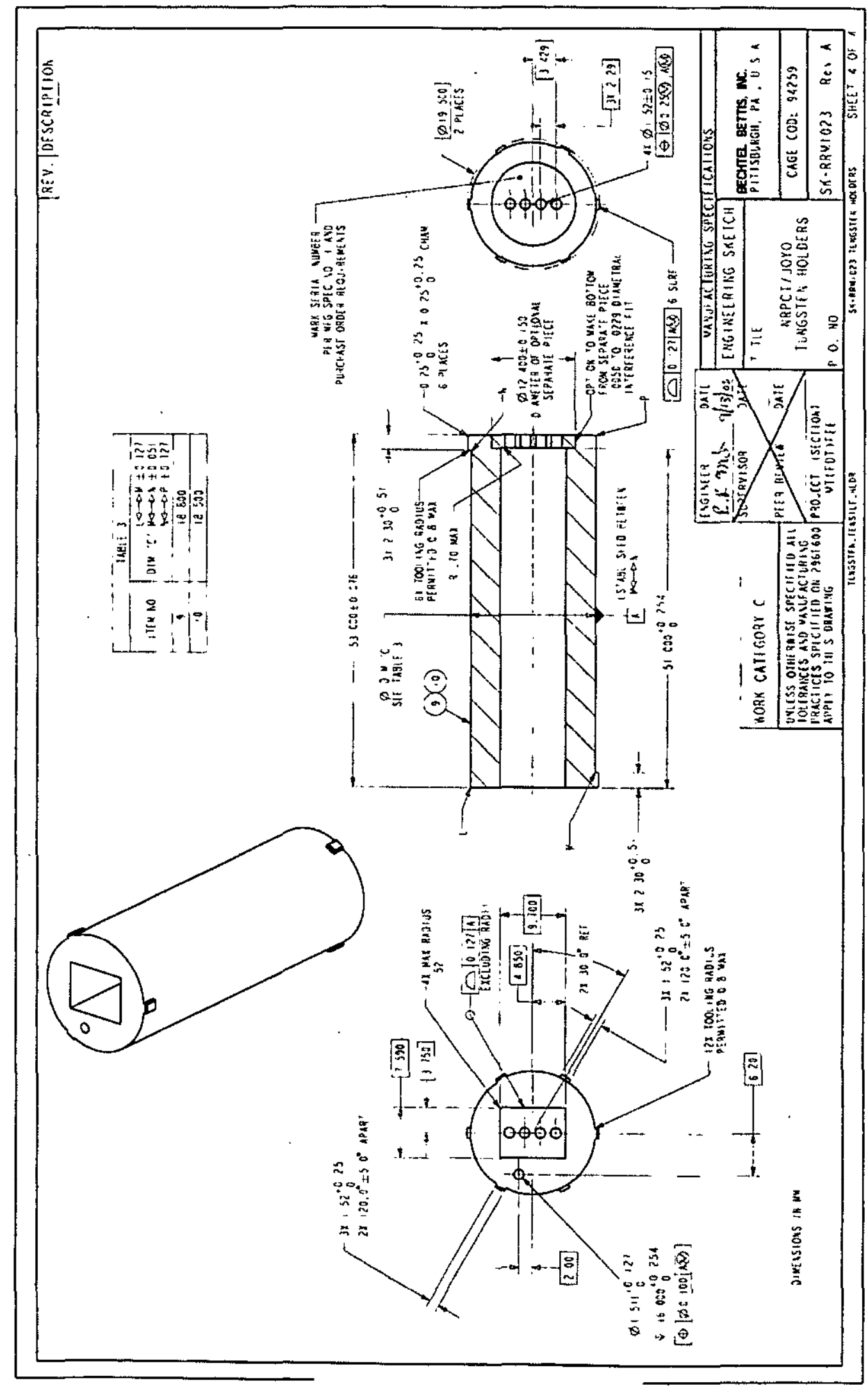




\section{Enclosure (7) to \\ MDO-723-0044/ \\ B-MT(SRME)-52 \\ Page 205}

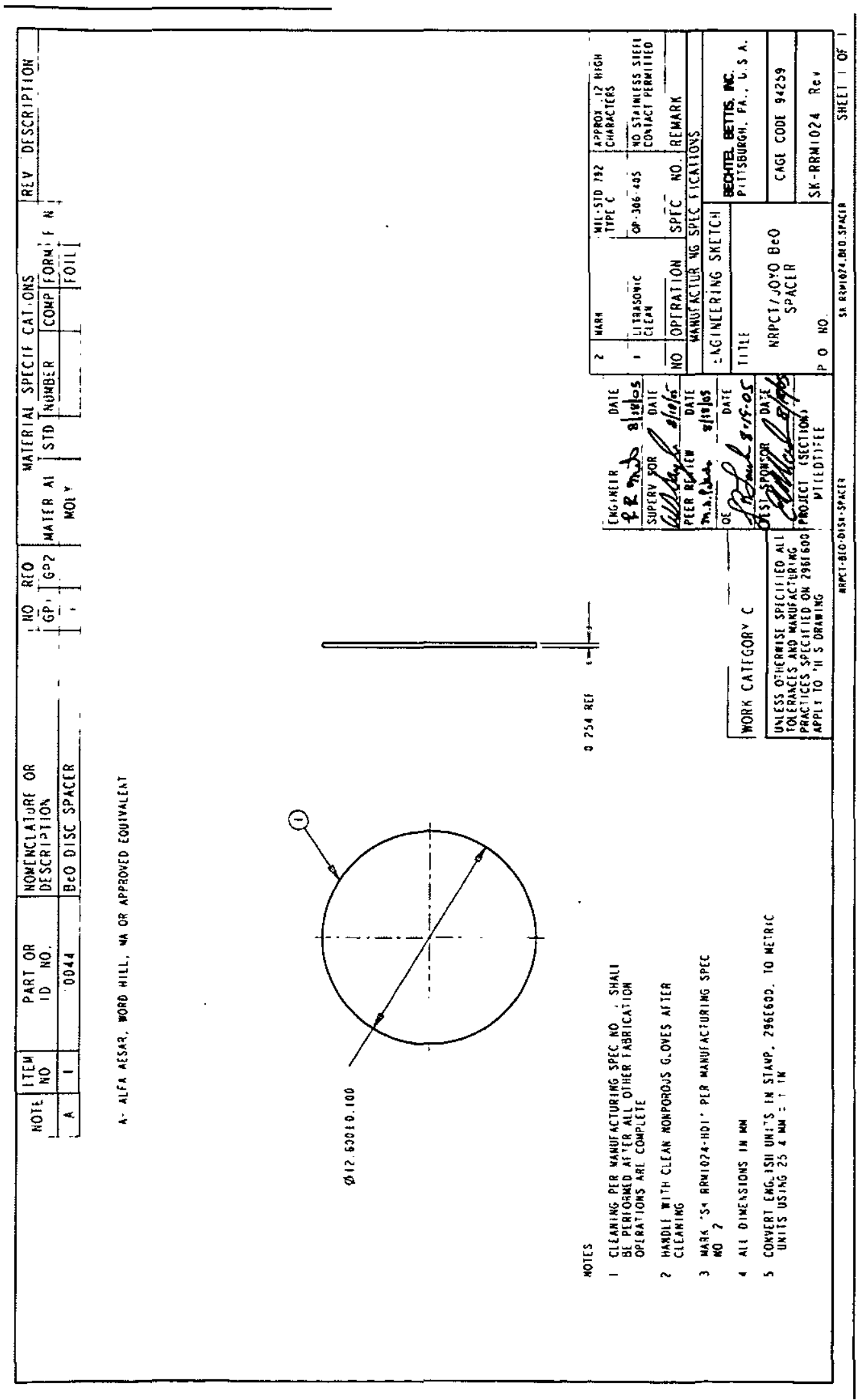




\section{Enclosure (7) to \\ MDO-723-0044I \\ B-MT(SRME)-52 \\ Page 206}

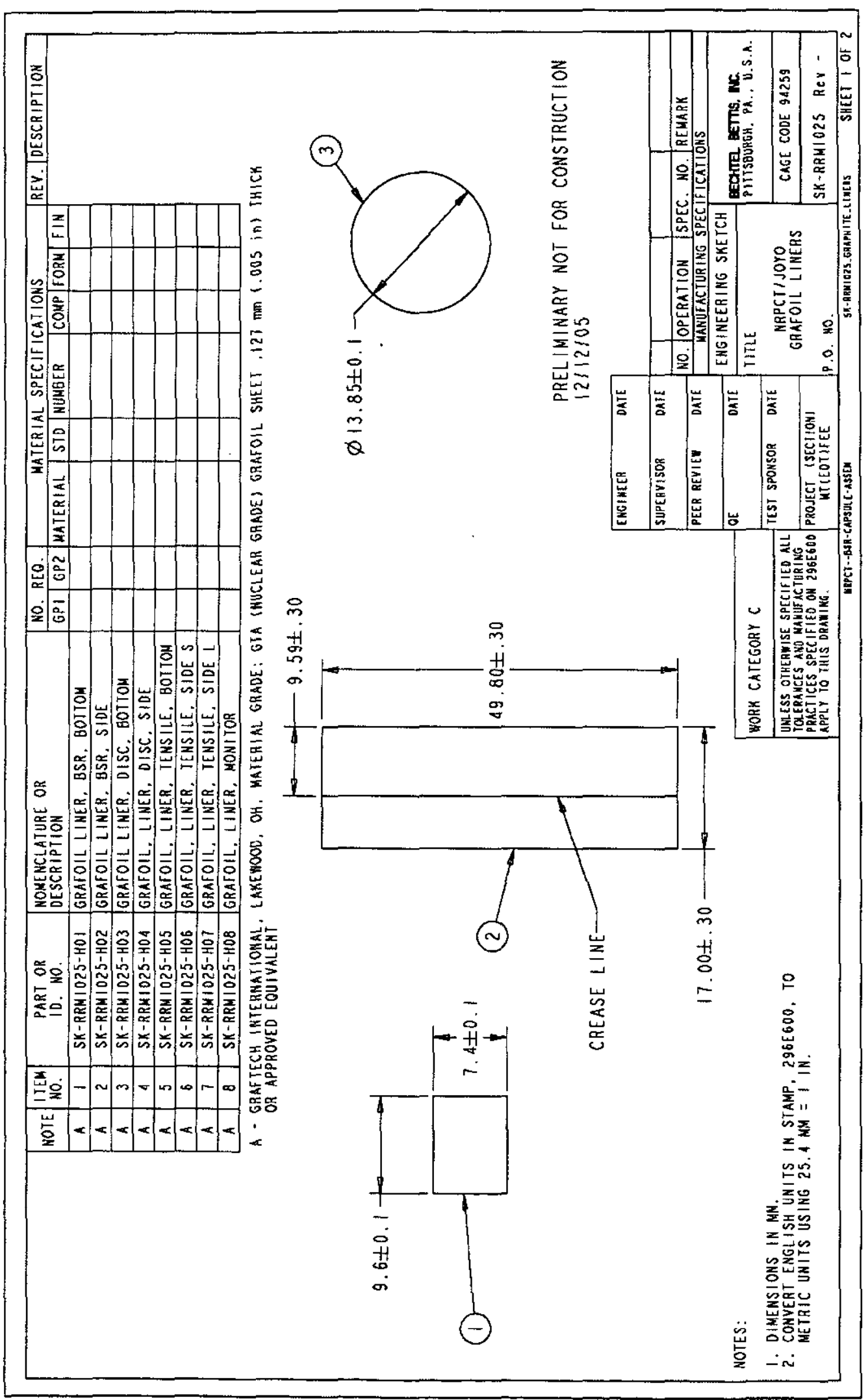


Enclosure (7) to

MDO-723-0044I

B-MT(SRME)-52

Page 207

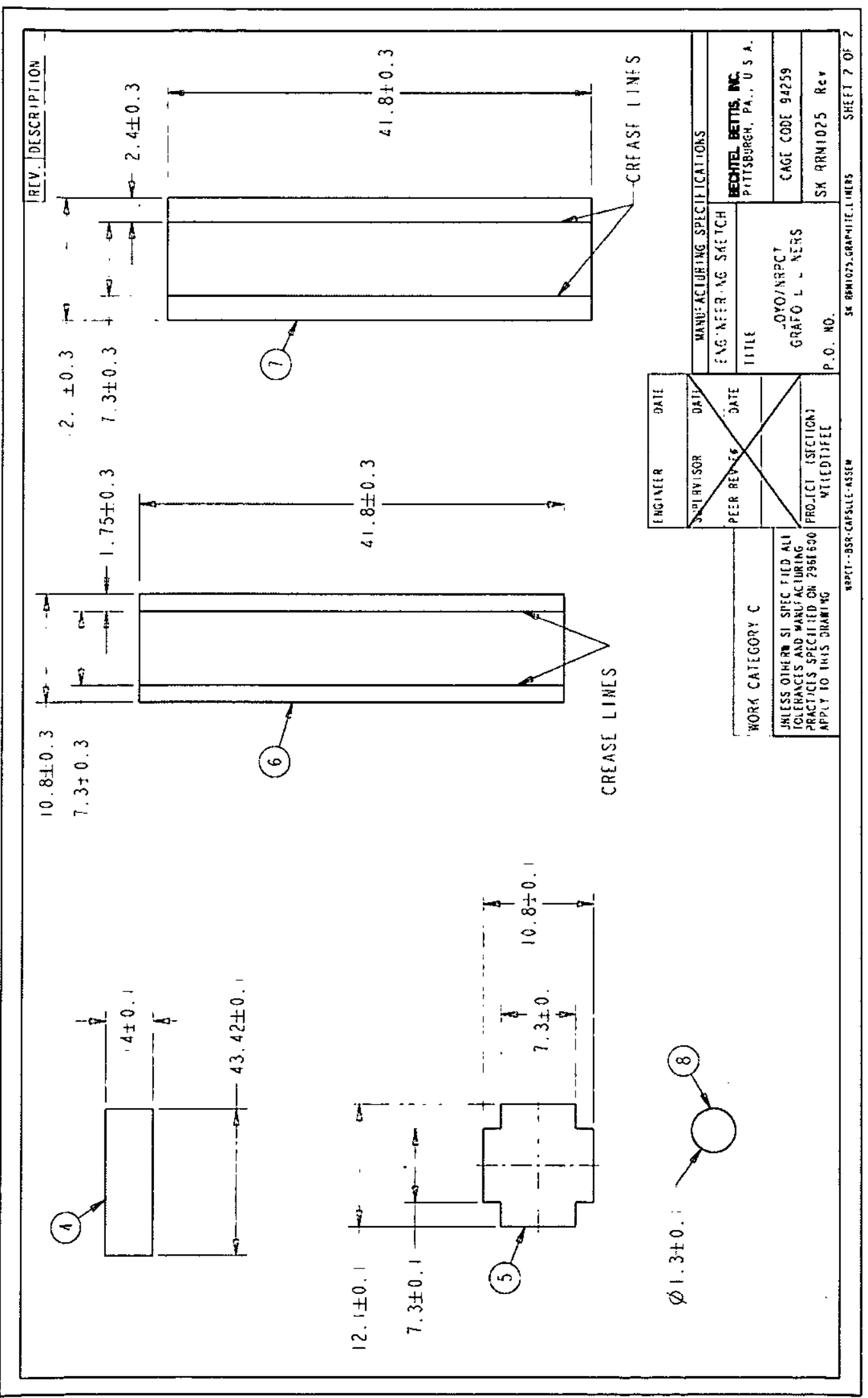


Enclosure (7) to

MDO-723-0044/

B-MT(SRME)-52

Page 208

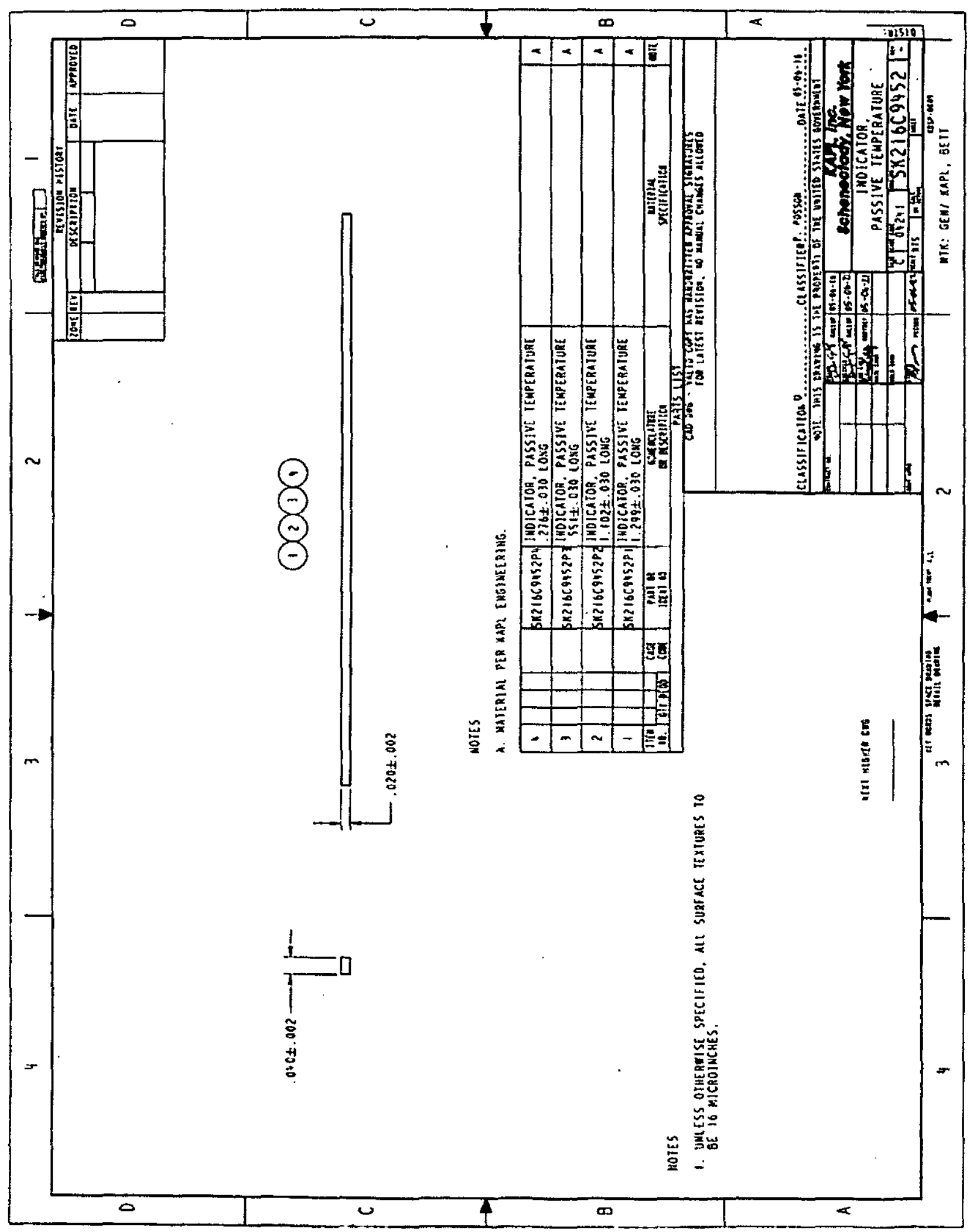


B. Specimens, Fixtures, Tools, and Packaging

1. Metallic Specimens:

\begin{tabular}{|l|c|c|}
\hline \multicolumn{1}{|c|}{ Specimen Type } & Drawing or Sketch & $\begin{array}{c}\text { Drawing or } \\
\text { Sketch Status }\end{array}$ \\
\hline Biaxial creep & Original \\
\hline & & \\
\hline Tensile & & \\
\hline & $5 D 16996$ & A \\
\hline \begin{tabular}{l} 
Fracture toughness \\
\hline
\end{tabular} & 5D16339 & Original \\
\hline $\begin{array}{l}\text { Thermal diffusivity } \\
\text { that will be included not } \\
\text { shown in figure) }\end{array}$ & 6288E55 (assembly) & $10 / 19 / 05$ \\
\hline
\end{tabular}


Enclosure (7) to

MDO-723-0044I

B-MT(SRME)-52

Page 210

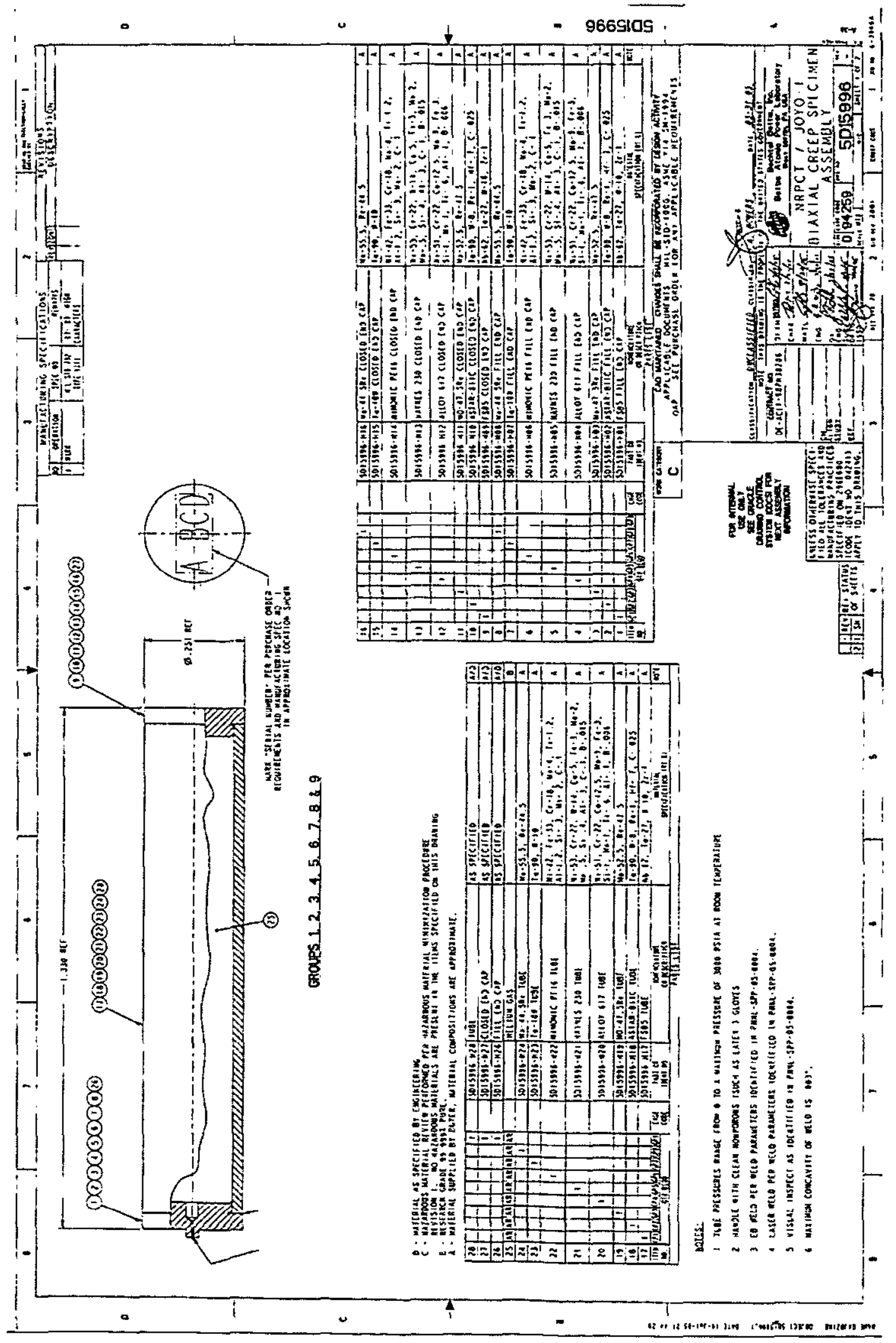




\section{Enclosure (7) to MDO-723-0044I \\ B-MT(EDT)S-029 \\ Page 211}

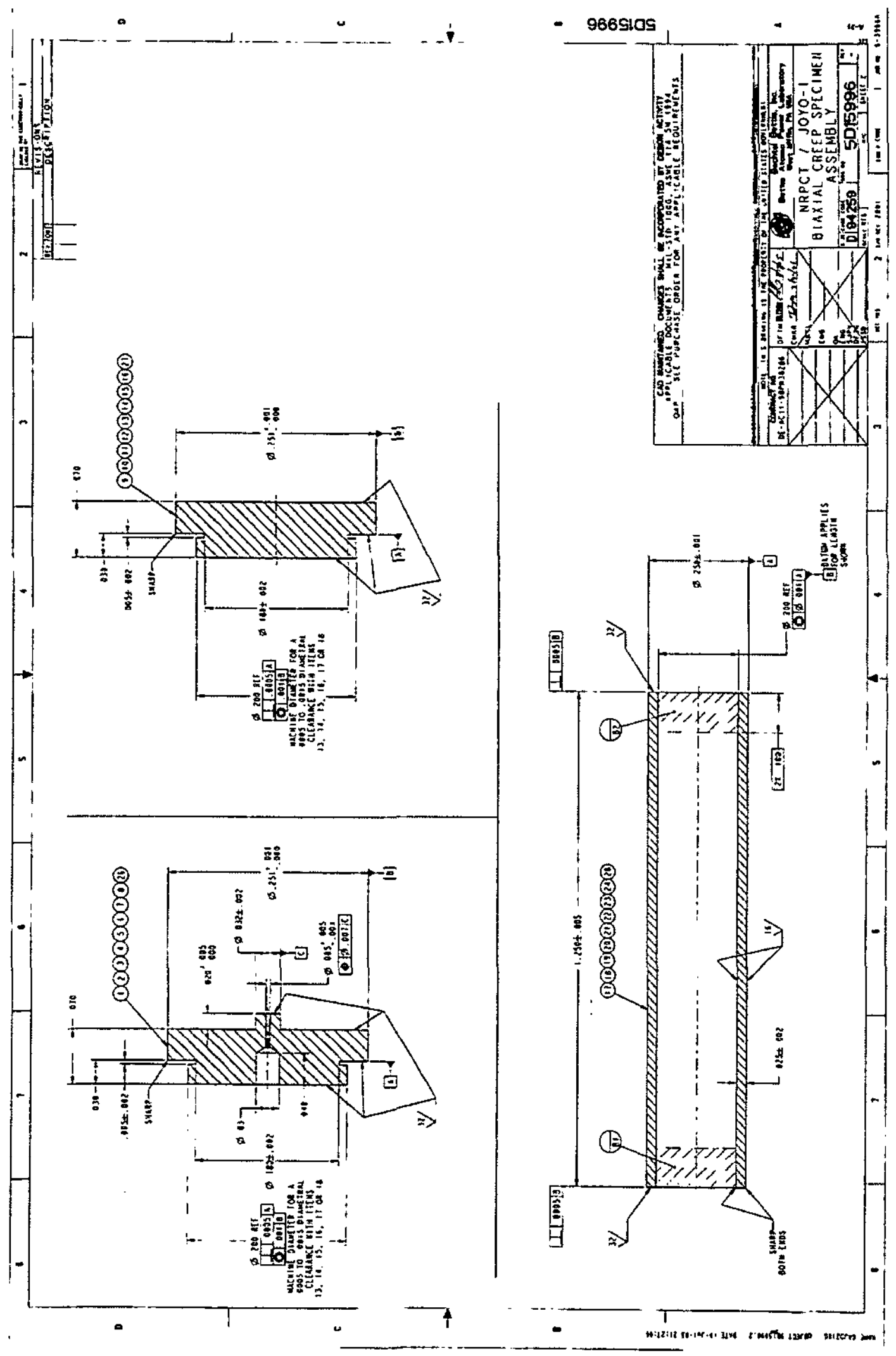


Enclosure (7) to

MDO-723-0044/

B-MT(SRME)-52

Page 212

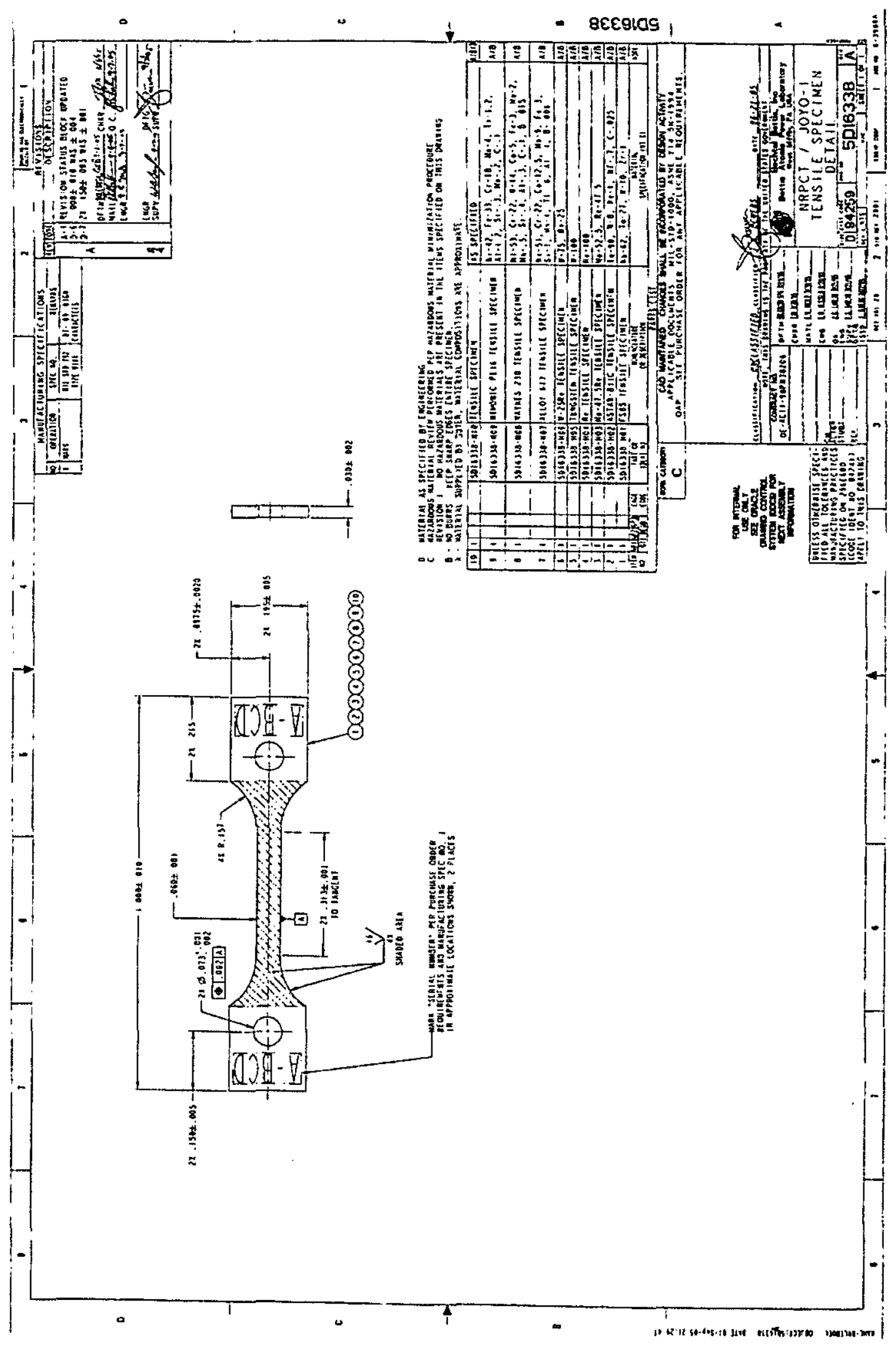


Enclosure (7) to

MDO-723-0044/

B-MT(EDT)S-029

Page 213

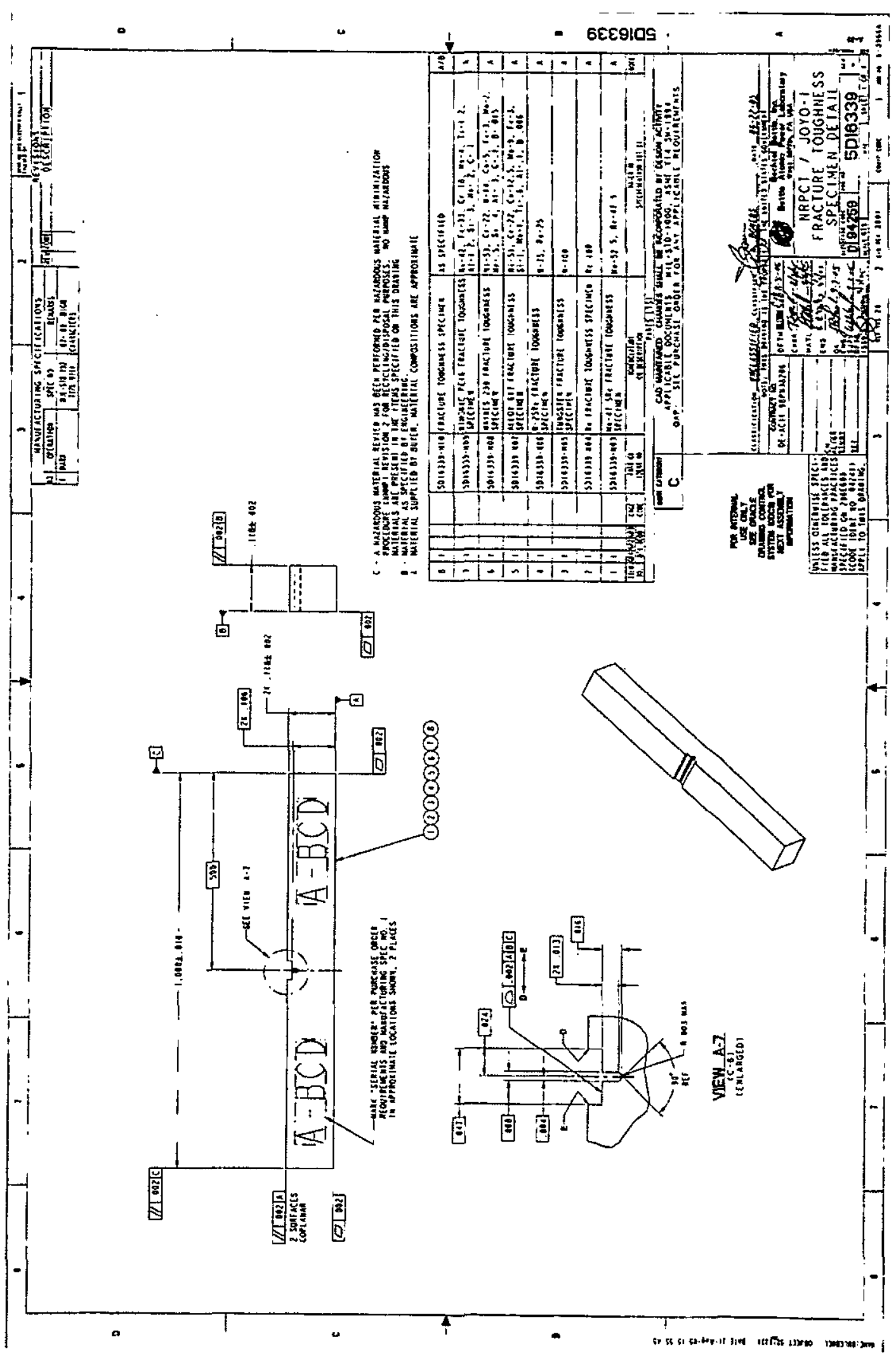


Enclosure (7) to

MDO-723-0044/

B-MT(SRME)-52

Page 214

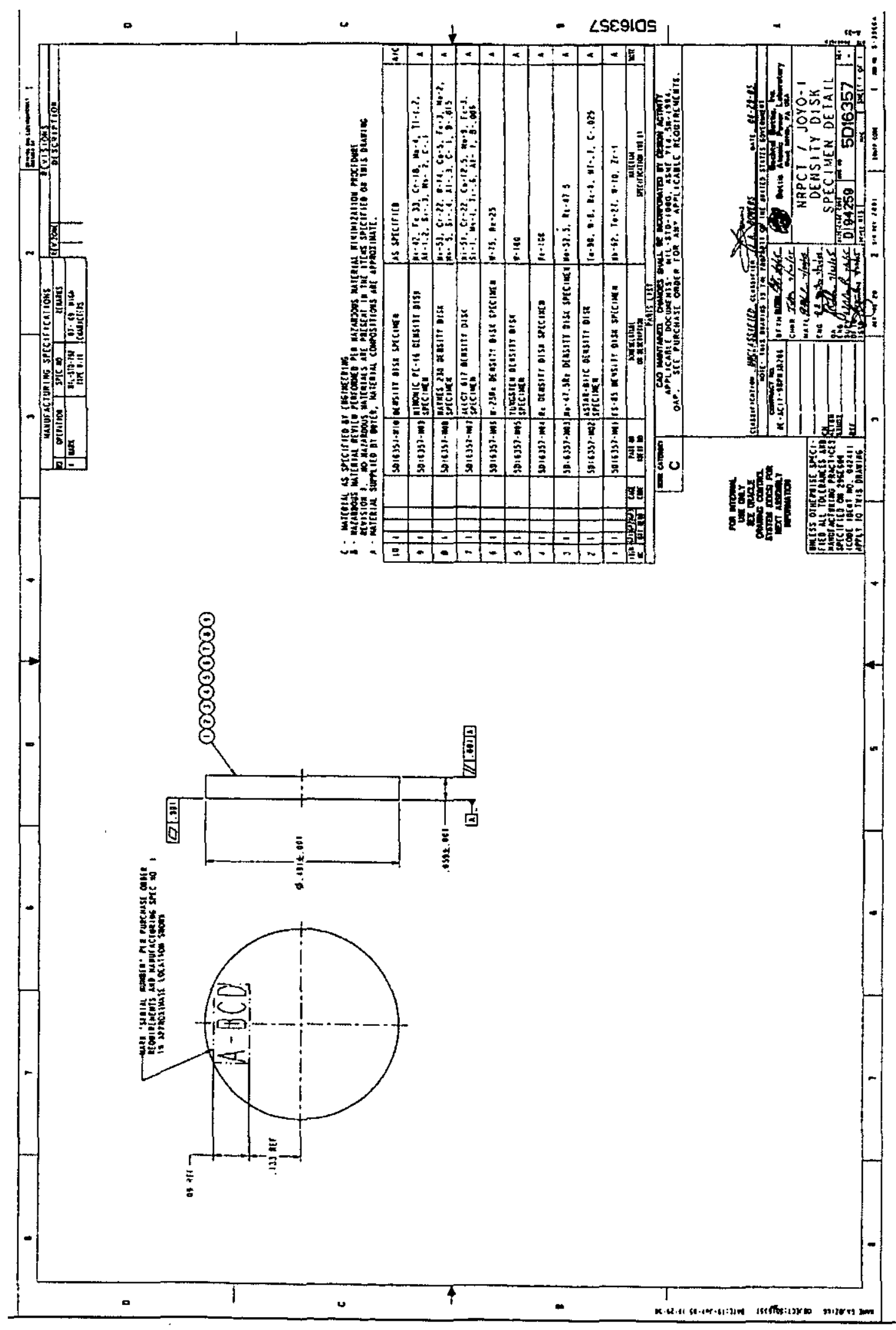


Enclosure (7) to MDO-723-0044/

B-MT(EDT)S-029

Page 215
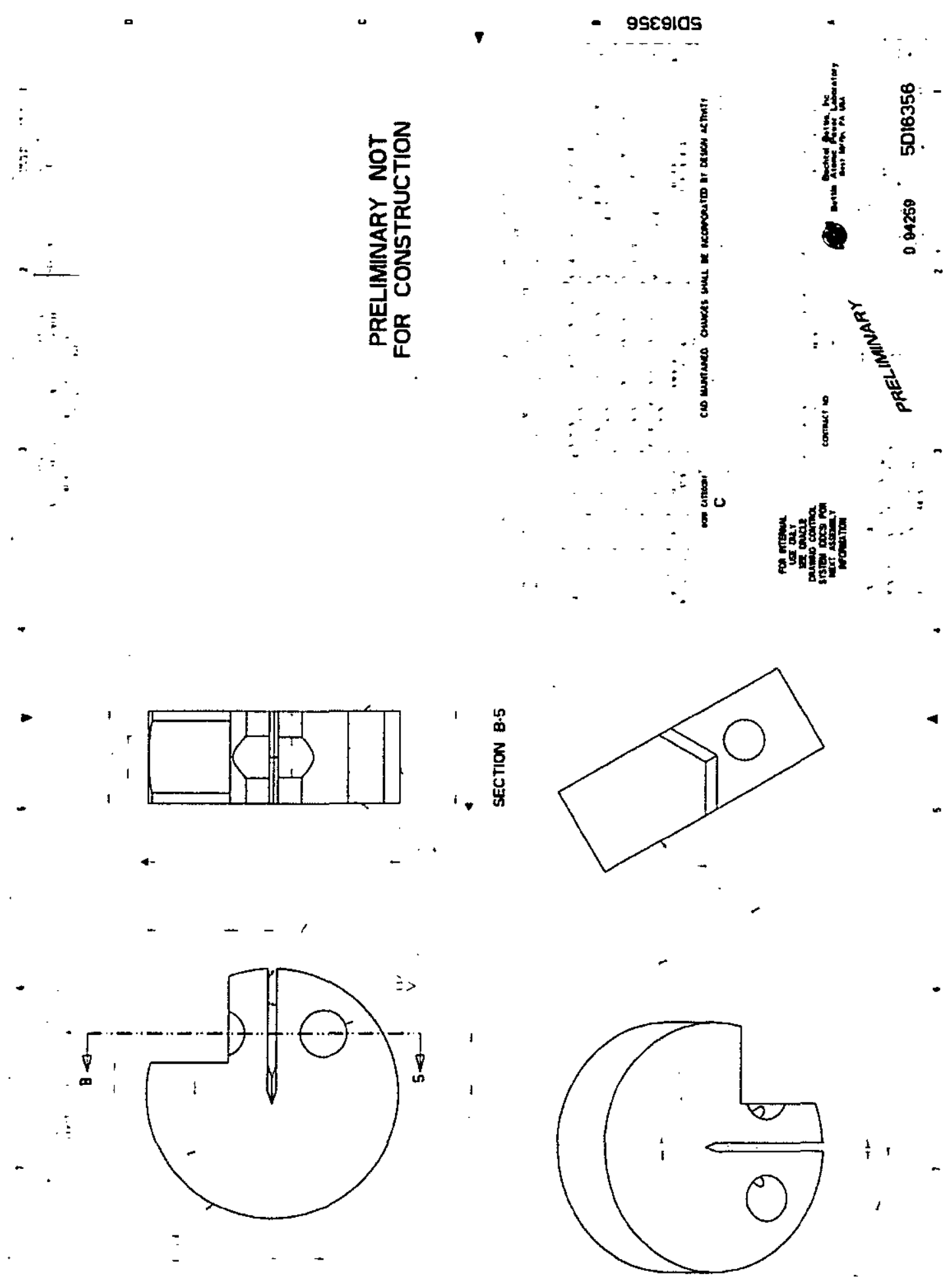
Enclosure (7) to

MDO-723-0044/

B-MT(SRME)-52

Page 216

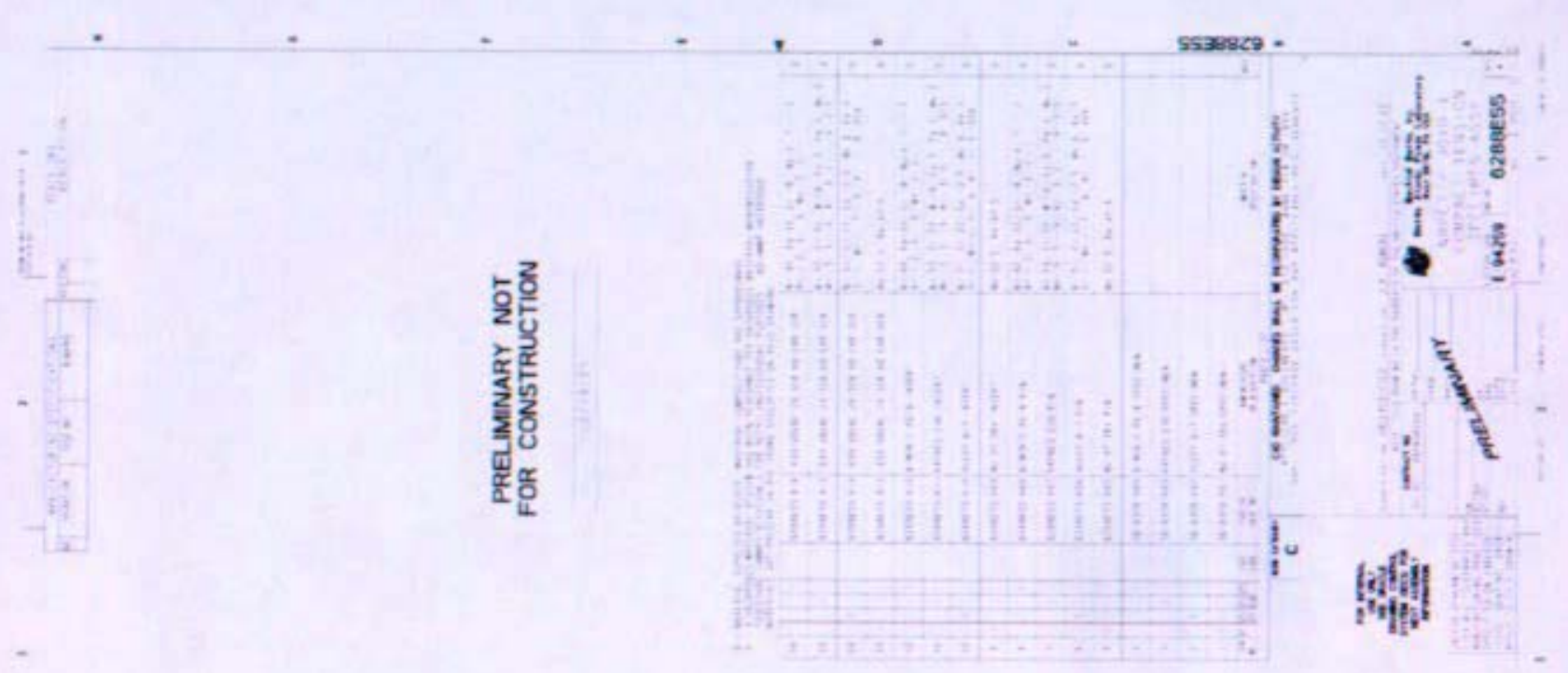

-
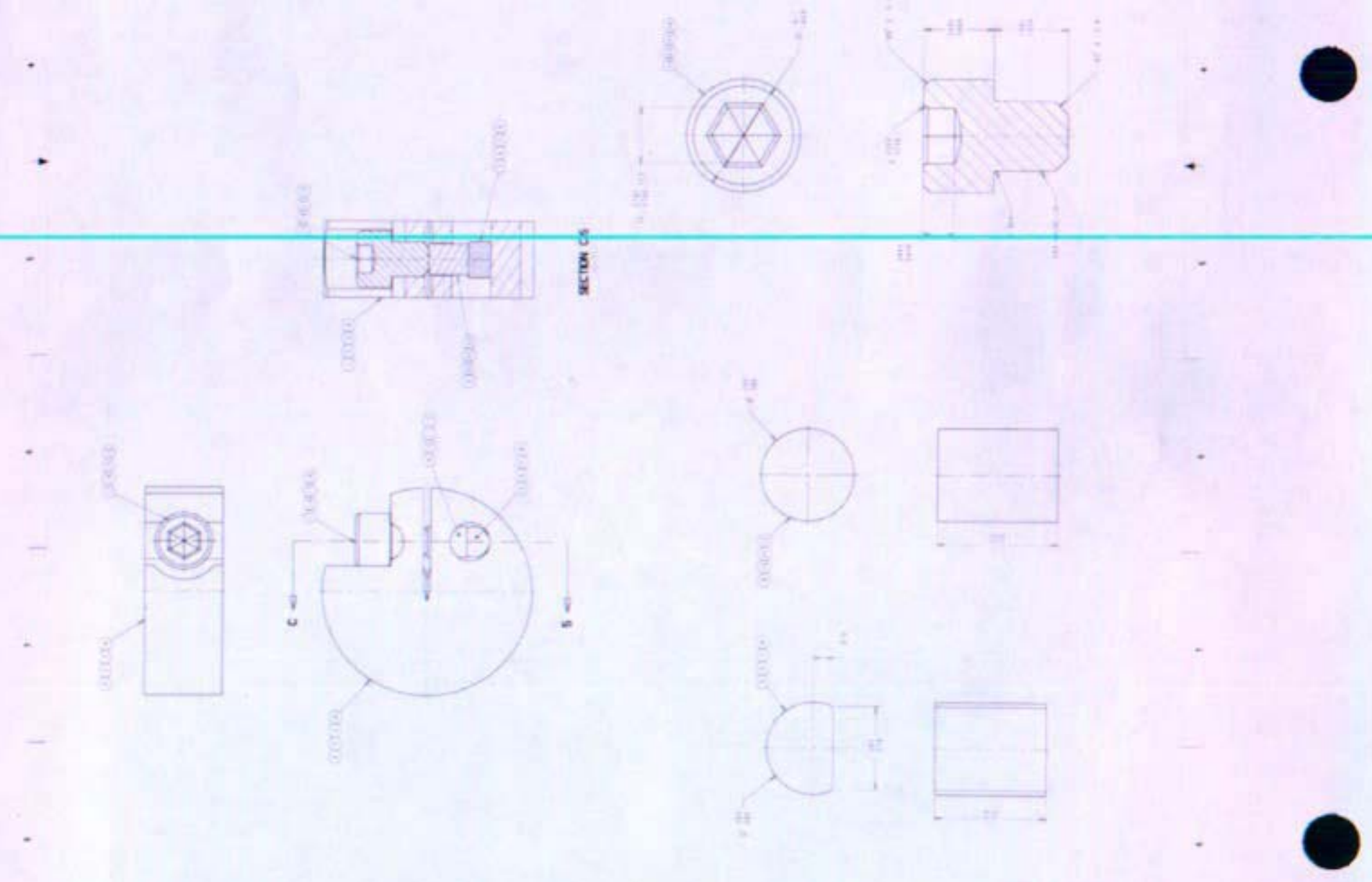
Enclosure (7) to MDO-723-0044/

B-MT(EDT)S-029

Page 217

2. Ceramic Specimens:

\begin{tabular}{|l|c|c|}
\hline \multicolumn{1}{|c|}{ Specimen Type } & Drawing or Sketch & $\begin{array}{c}\text { Drawing or } \\
\text { Sketch Status }\end{array}$ \\
\hline \begin{tabular}{l} 
Tensile \\
\hline $\begin{array}{l}\text { SK239B9368 } \\
\text { Conposite Thermal }\end{array}$
\end{tabular} & $\begin{array}{c}\text { Original } \\
\text { diffusivity, Item \#1 }\end{array}$ & C \\
\hline $\begin{array}{l}\text { MonolithicThermal } \\
\text { Conductivity }\end{array}$ & $\begin{array}{c}\text { 216C6883-SiC-thermal- } \\
\text { diffusivity, Item \#2 }\end{array}$ & C \\
\hline BeO Density Disk & SK216C9453 & A \\
\hline
\end{tabular}


Enclosure (7) to

MDO-723-0044/

B-MT(SRME)-52

Page 218

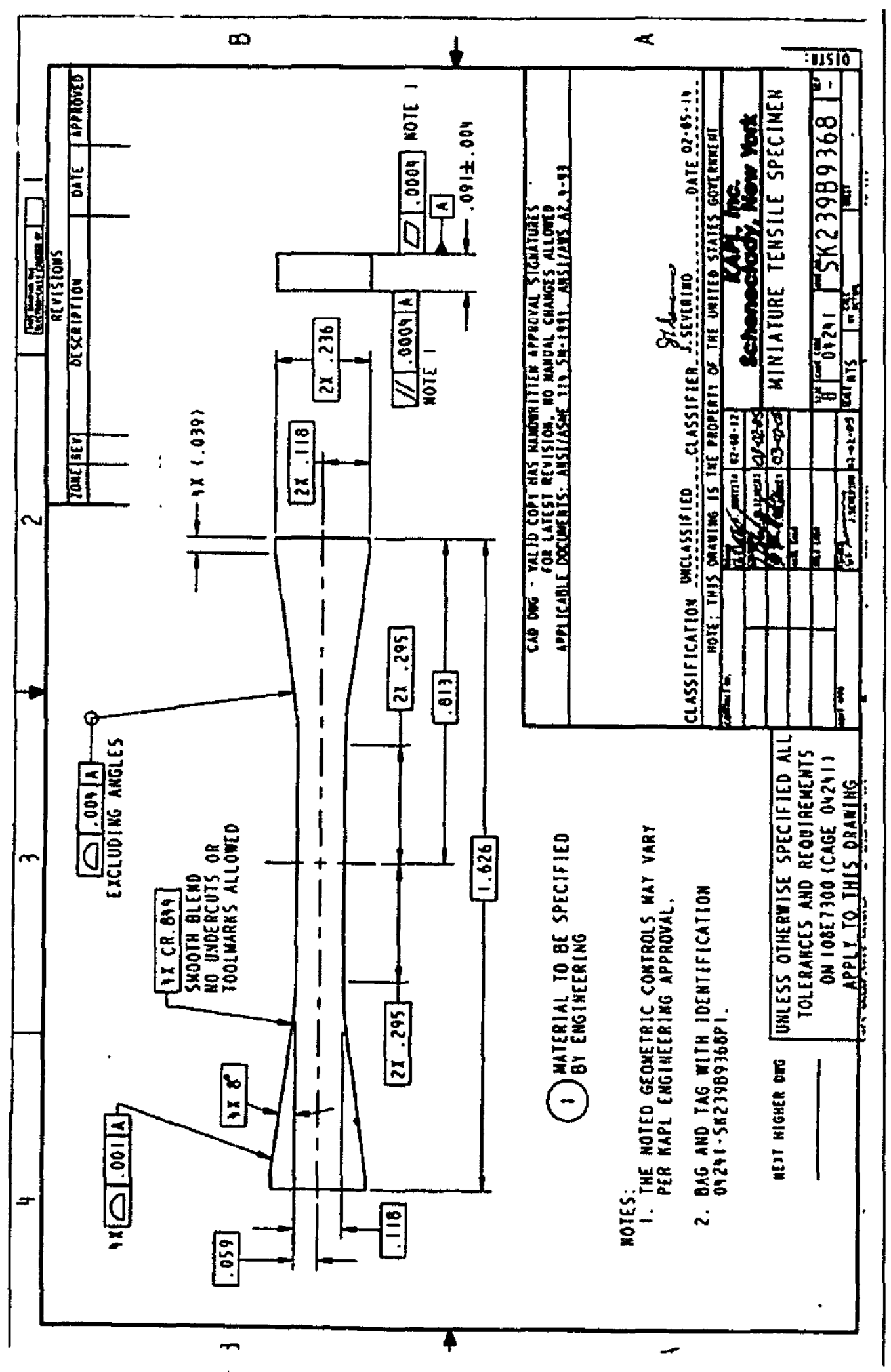



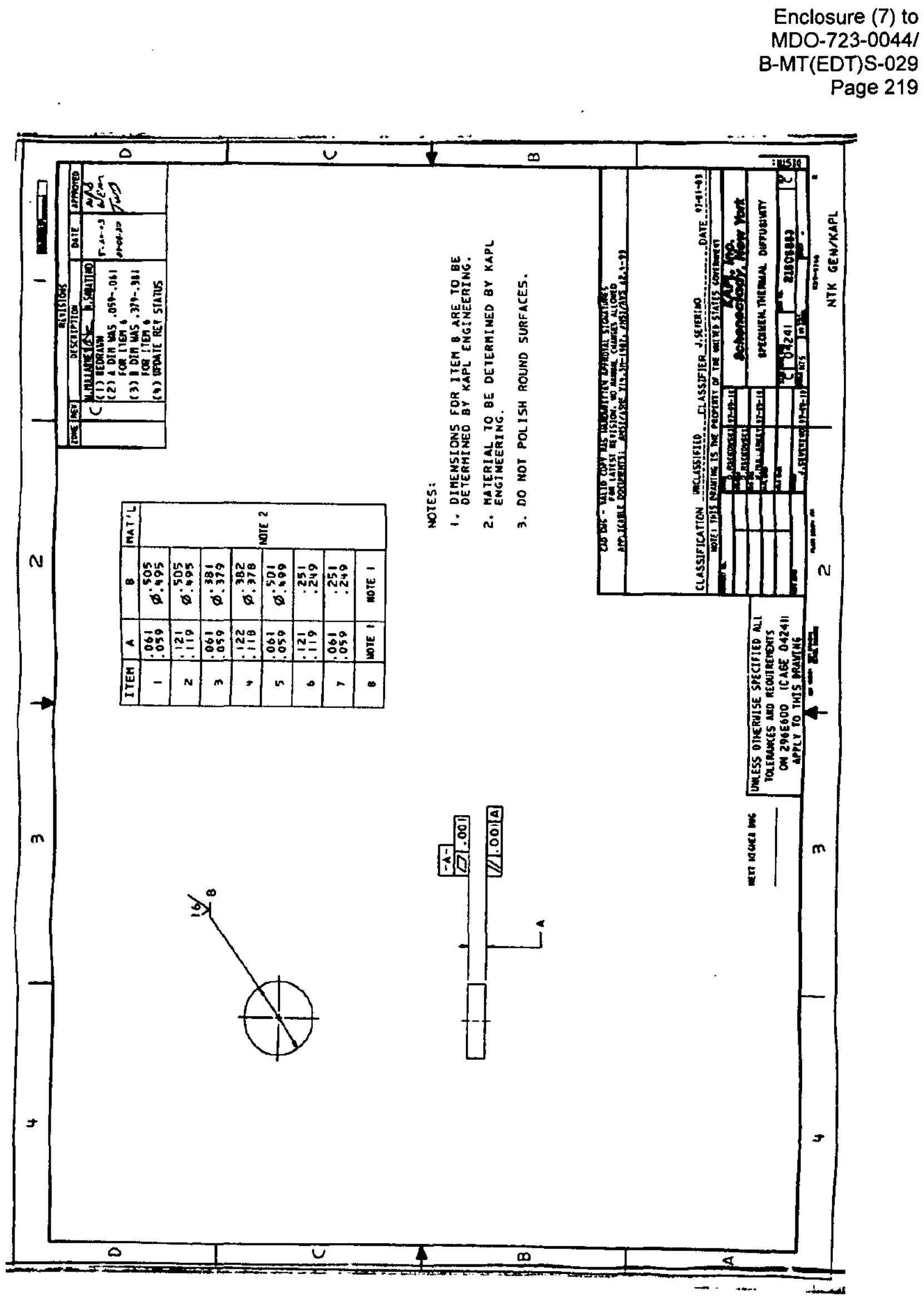
Enclosure (7) to

MDO-723-0044I

B-MT(SRME)-52

Page 220

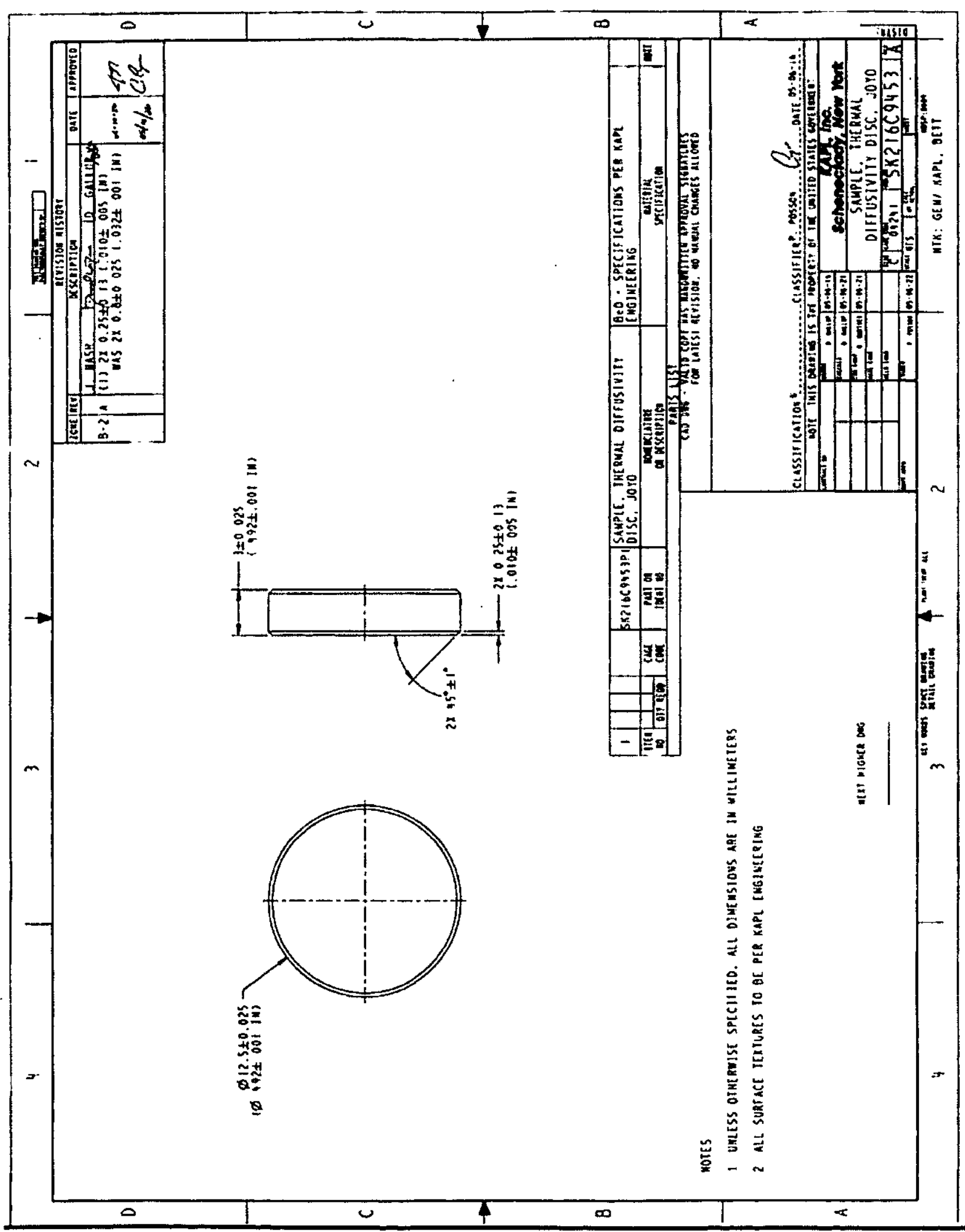


Enclosure (7) to

MDO-723-0044/

B-MT(EDT)S-029

Page 221

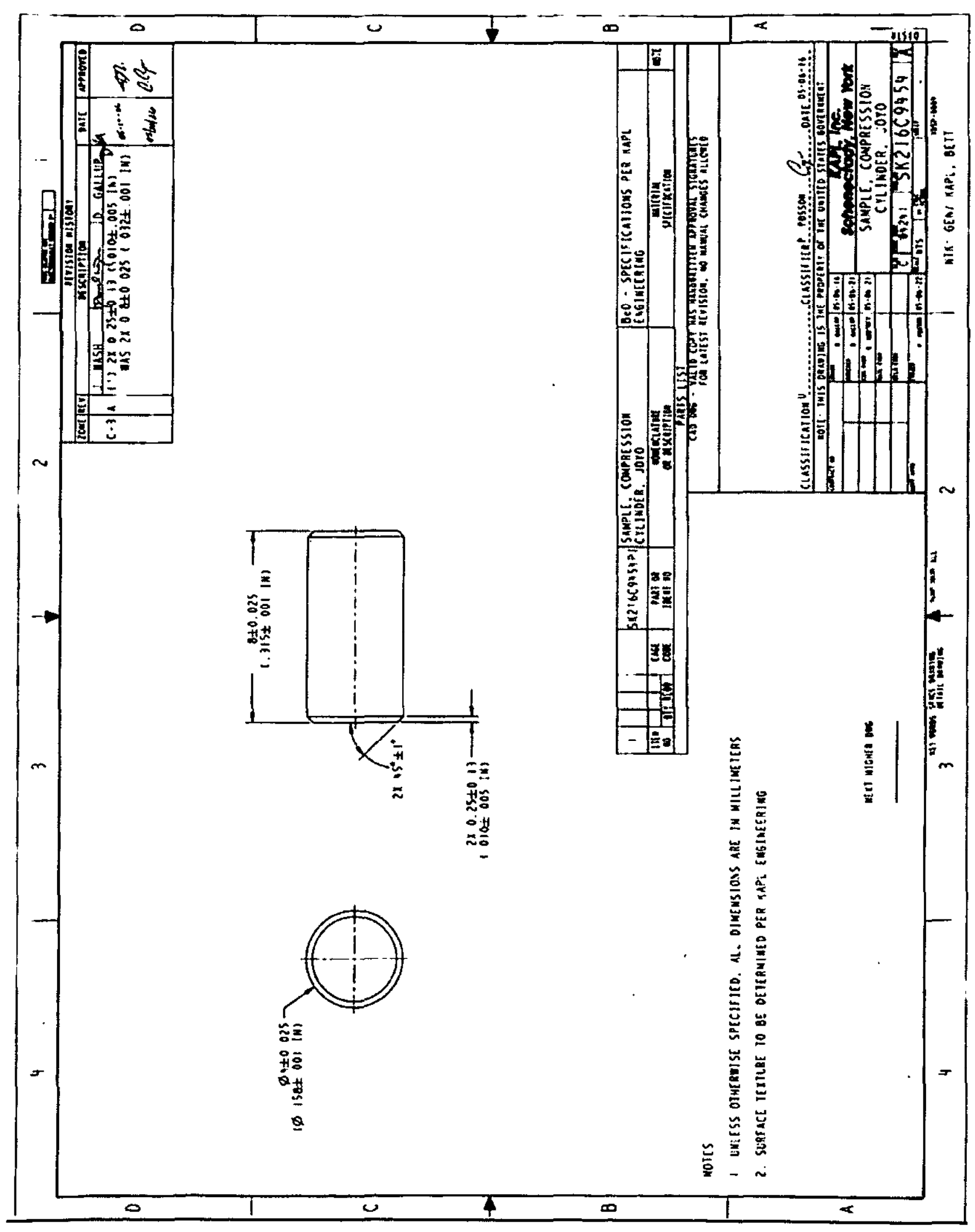


Enclosure (7) to

MDO-723-0044/

B-MT(SRME)-52

Page 222

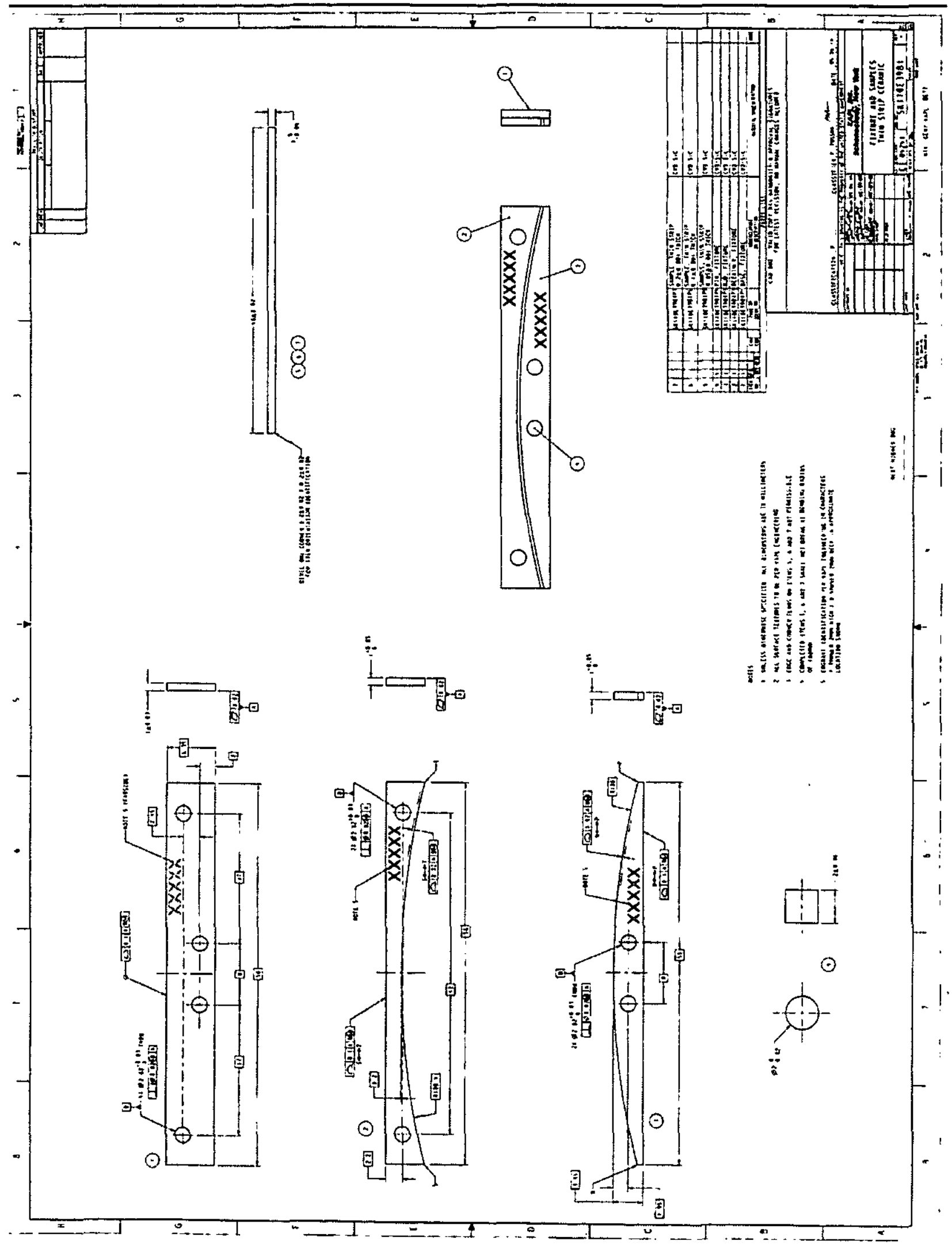


3. Fixtures, Tools, and Packaging

\begin{tabular}{|c|l|c|}
\hline \multicolumn{2}{|c|}{ Table - Fixtures, Tools, Packaging } \\
\hline Sketch Number & \multicolumn{1}{|c|}{$\begin{array}{c}\text { Revision - } \\
\text { date }\end{array}$} \\
\hline SK-AMT-2243 & JOYO Capsule Clamp Assembly & Original \\
\hline SK-JAM-1001 & JOYO Capsule Shipping Harness Detail & Original \\
\hline SK-JAM-1003 & JOYO Capsule Shock Harness & Original \\
\hline SK-JPH1110 & $\begin{array}{l}\text { JOYO Capsule Adapter Holder (replacement } \\
\text { for SK-WJU-1007) }\end{array}$ & Original \\
\hline SK-JPH1111 & JOYO Shipping Foam & Original \\
\hline SK-WJU-1000 & NRPCT/JOYO Head Weld Qualification & B \\
\hline SK-WJU-1004 & NRPCT/JOYO Weld Qualification & A \\
\hline SK-WJU-1006 & Back Stop & Original \\
\hline SK-WJU-1007 & Adapter Holder & Original \\
\hline SK-WJU-1008 & Large Bracket Holder & Original \\
\hline SK-WJU-1009 & JOYO Weld Fixture Base Plate with Dowels & Original \\
\hline SK-WJU-1010 & Mockup Support & Original \\
\hline SK-WJU-1011 & Tension Hold & Original \\
\hline SK-WJU-1015 & JOYO Welding and Assembly Fixture & Original \\
\hline SK-JLD-1000 & Disk Holder Handling Tool & $10 / 05 / 05$ \\
\hline SK-JLD-1001 & LG. Disk Holder Handling Tool & $10 / 05 / 05$ \\
\hline SK-JLD-1002 & Plunger & $10 / 05 / 05$ \\
\hline SK-JLD-1003 & Spacer Installation Tool & $10 / 05 / 05$ \\
\hline & & \\
\hline & & \\
\hline & & \\
\hline & & \\
\hline
\end{tabular}


Enclosure (7) to

MDO-723-0044/

B-MT(SRME)-52

Page 224

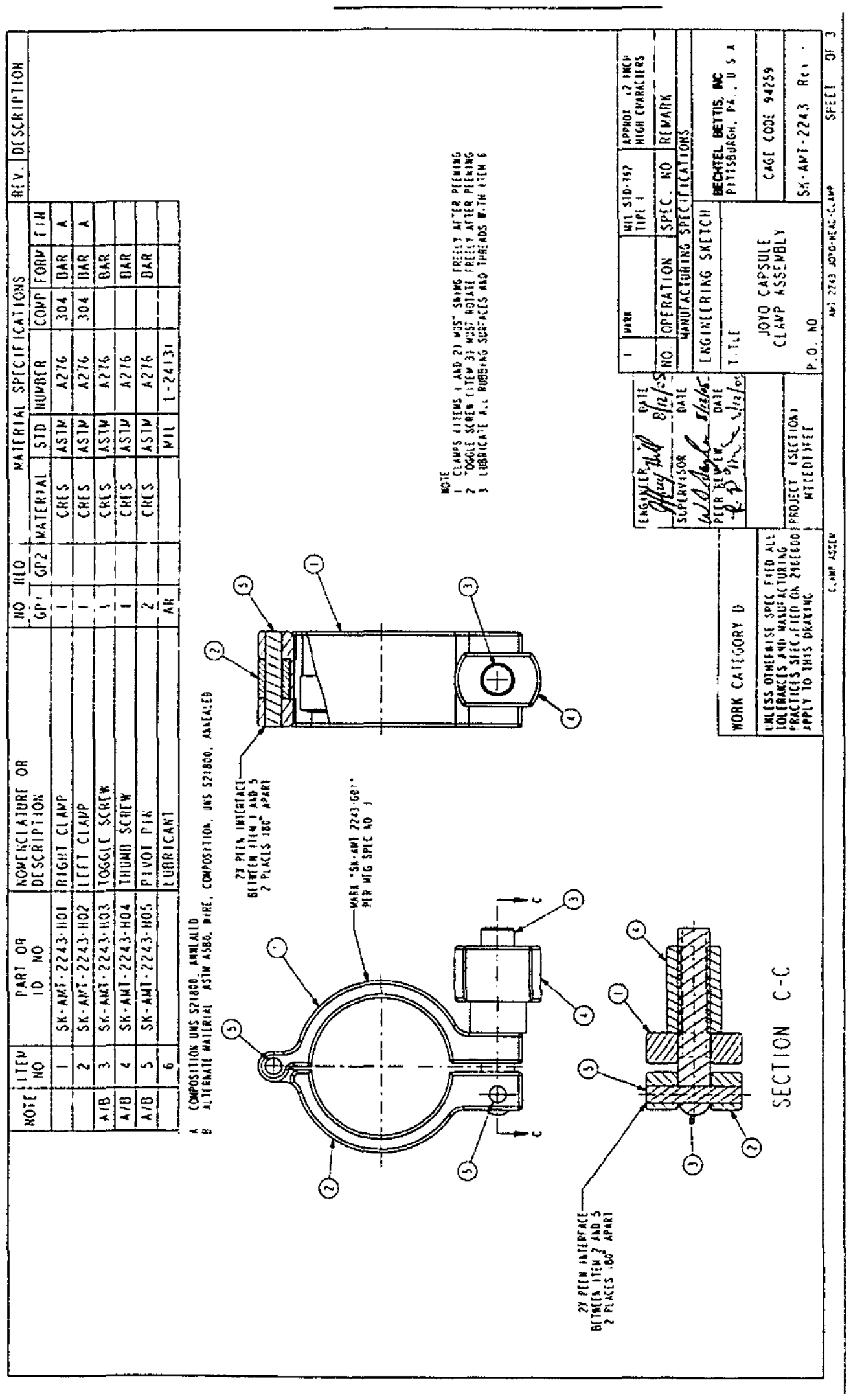




\section{Enclosure (7) to \\ MDO-723-0044/ \\ B-MT(EDT)S-029 \\ Page 225}

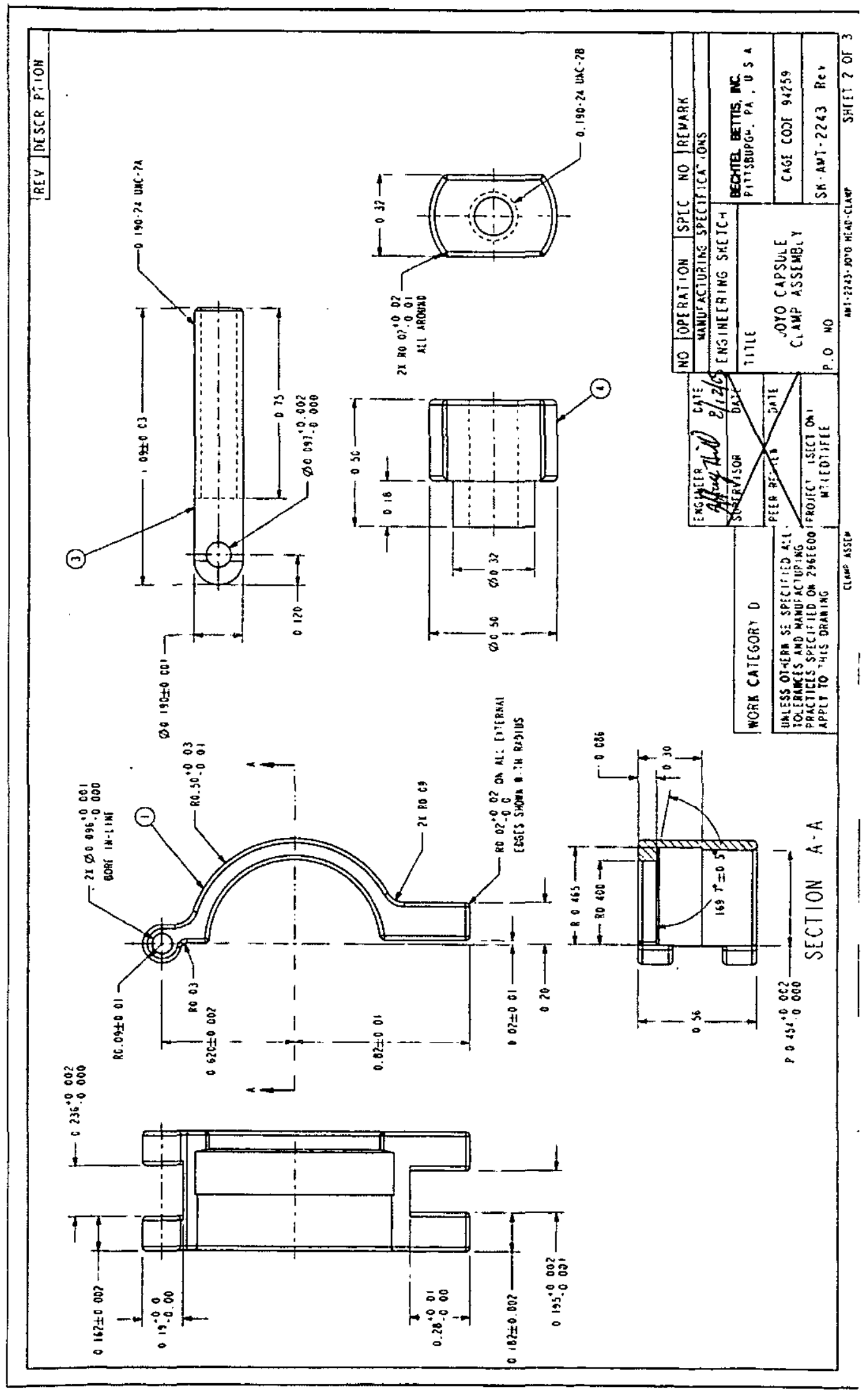


Enclosure (7) to

MDO-723-0044/

B-MT(SRME)-52

Page 226
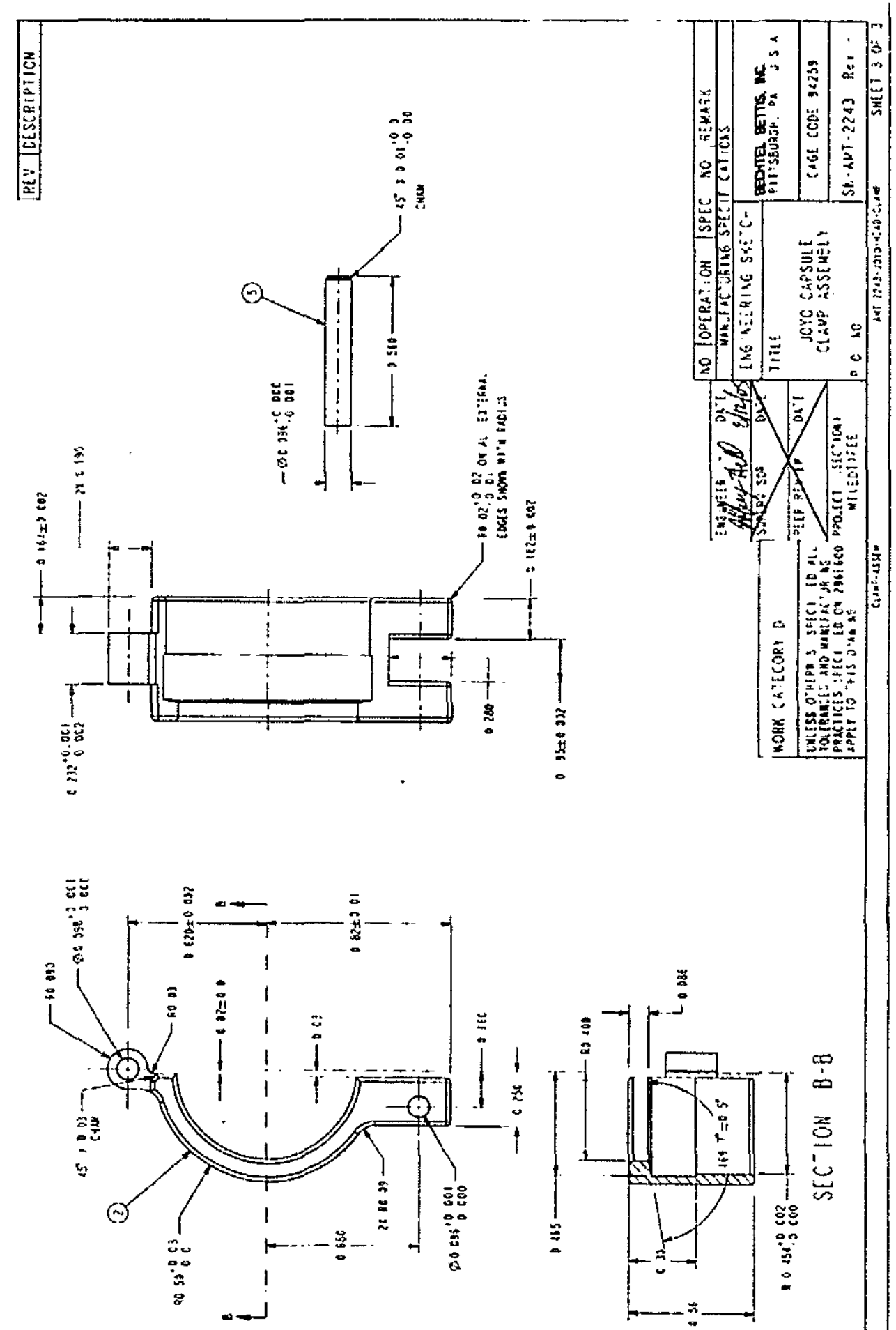


\section{Enclosure (7) to \\ MDO-723-0044/ \\ B-MT(EDT)S-029 \\ Page 227}

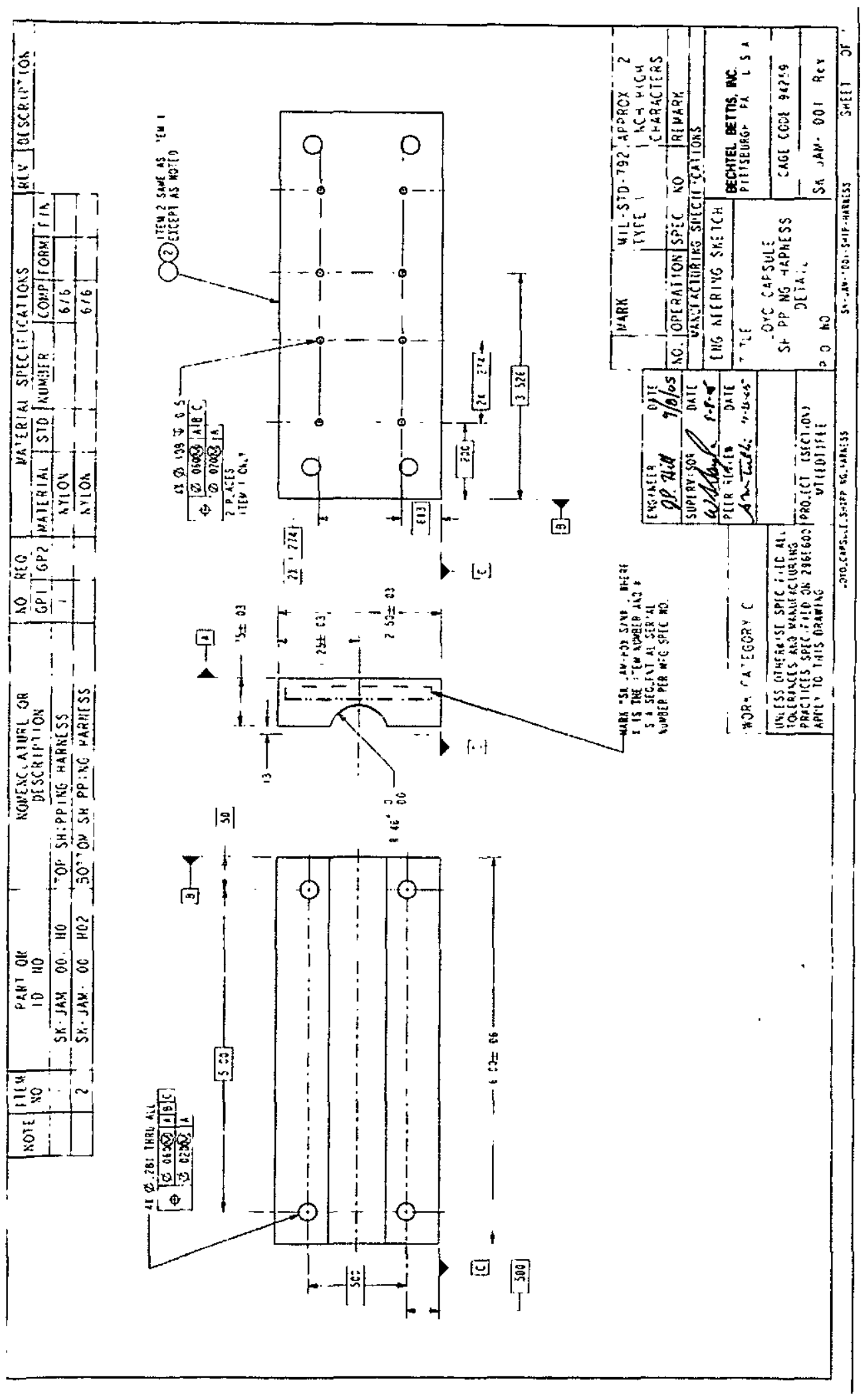




\section{Enclosure (7) to \\ MDO-723-0044/ \\ B-MT(SRME)-52 \\ Page 228}

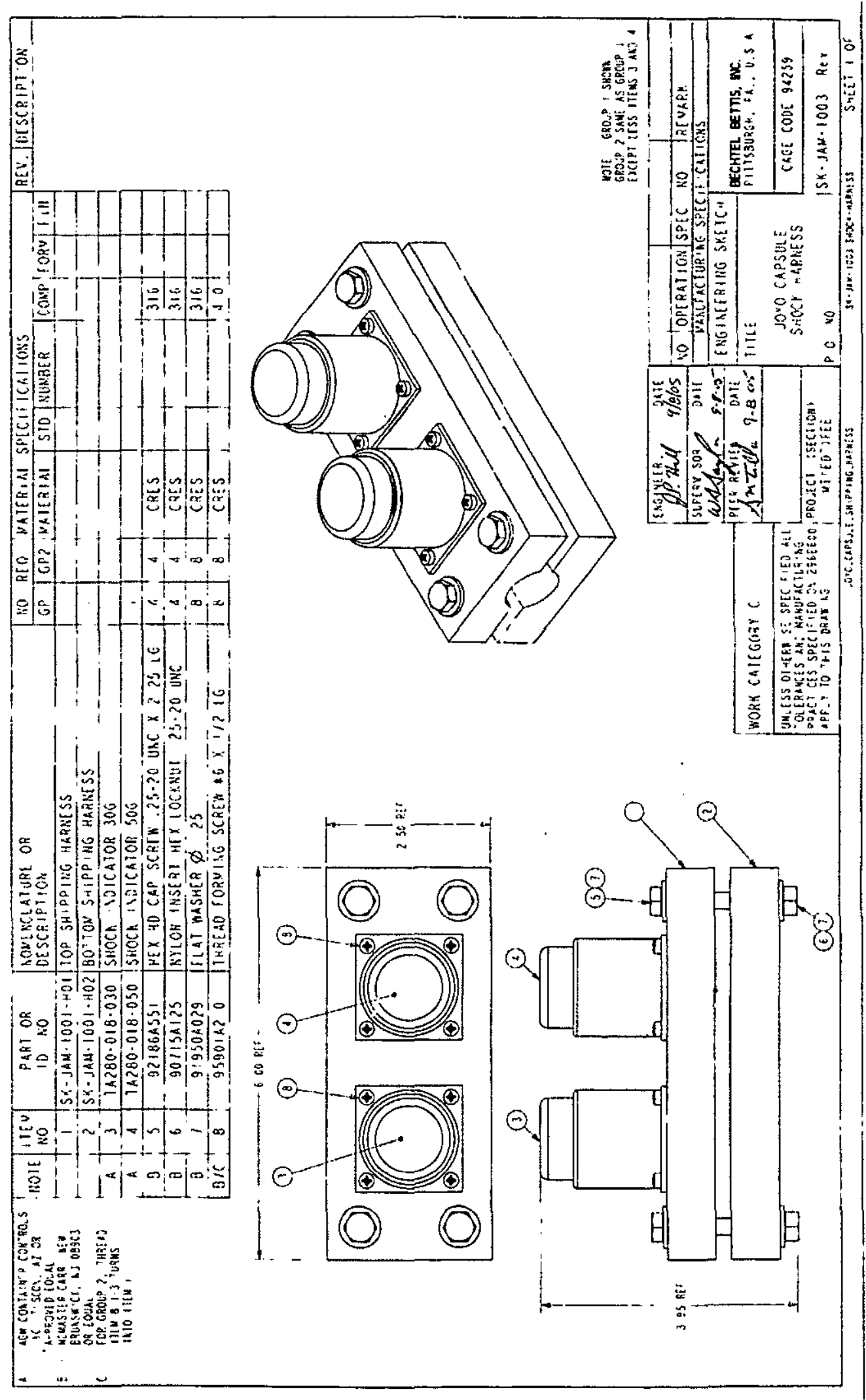


Enclosure (7) to

MDO-723-0044/

B-MT(EDT)S-029

Page 229

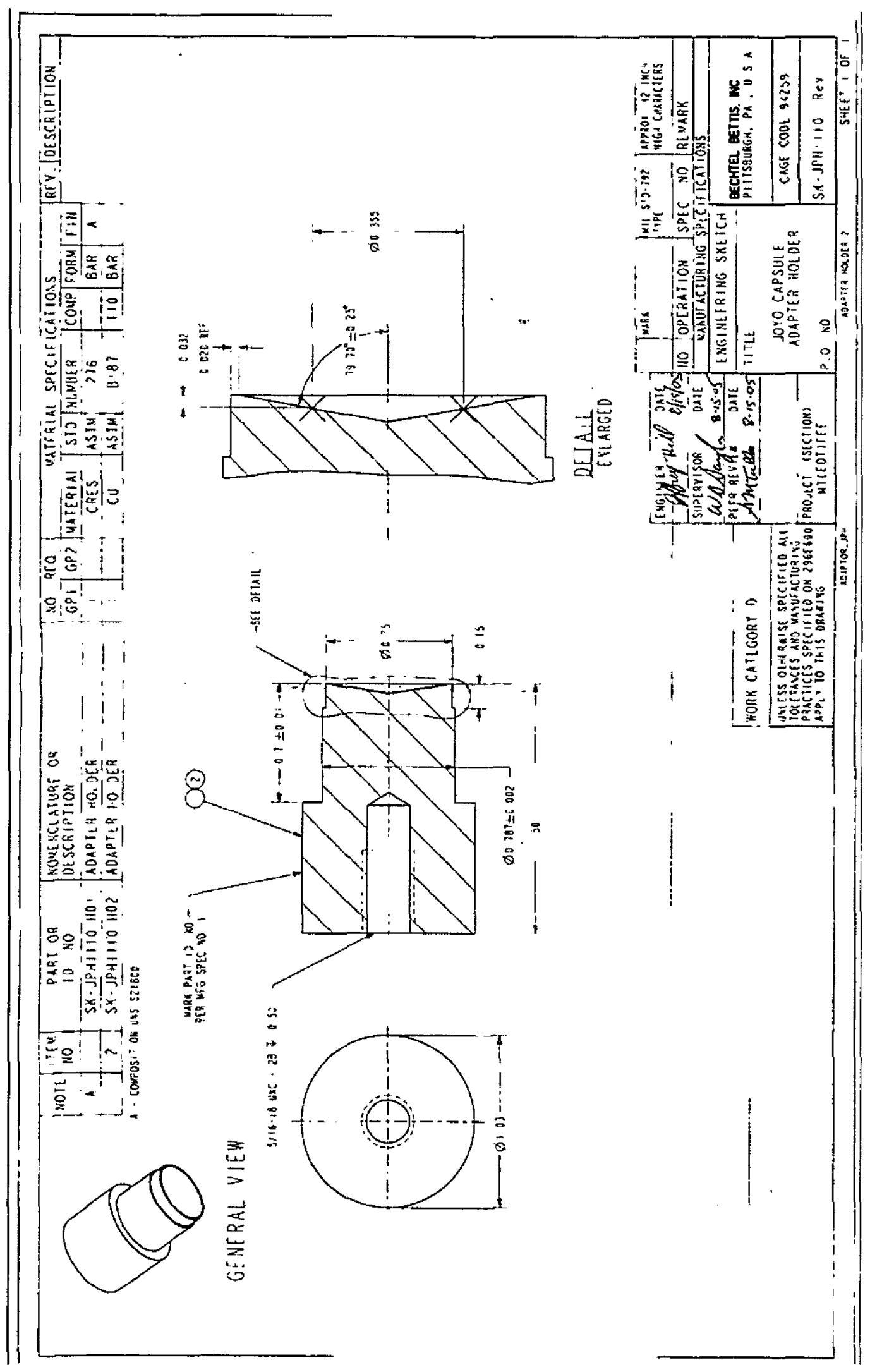




\section{Enclosure (7) to MDO-723-0044/ \\ B-MT(SRME)-52 \\ Page 230}

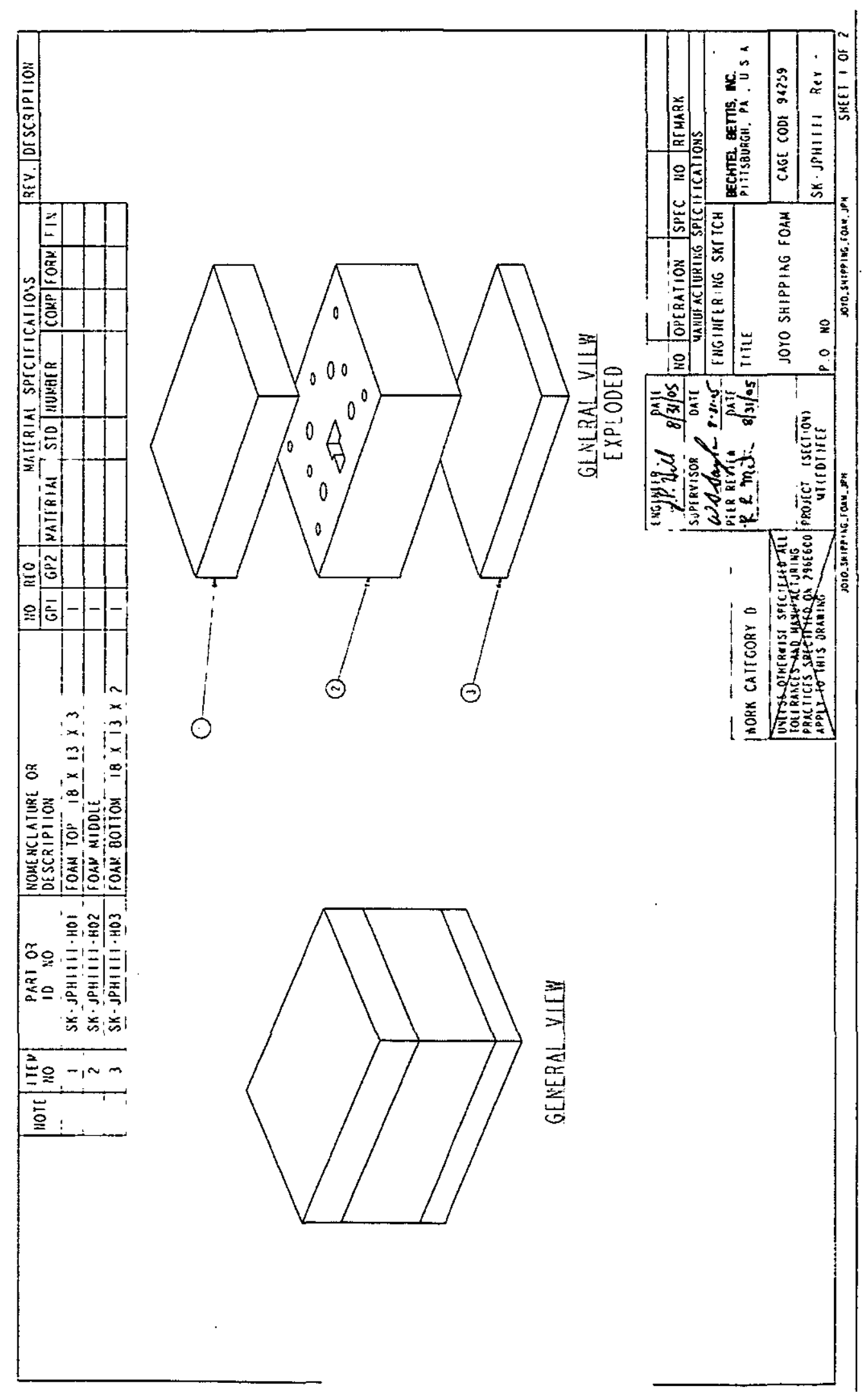




\section{Enclosure (7) to MDO-723-0044/ B-MT(EDT)S-029 Page 231}

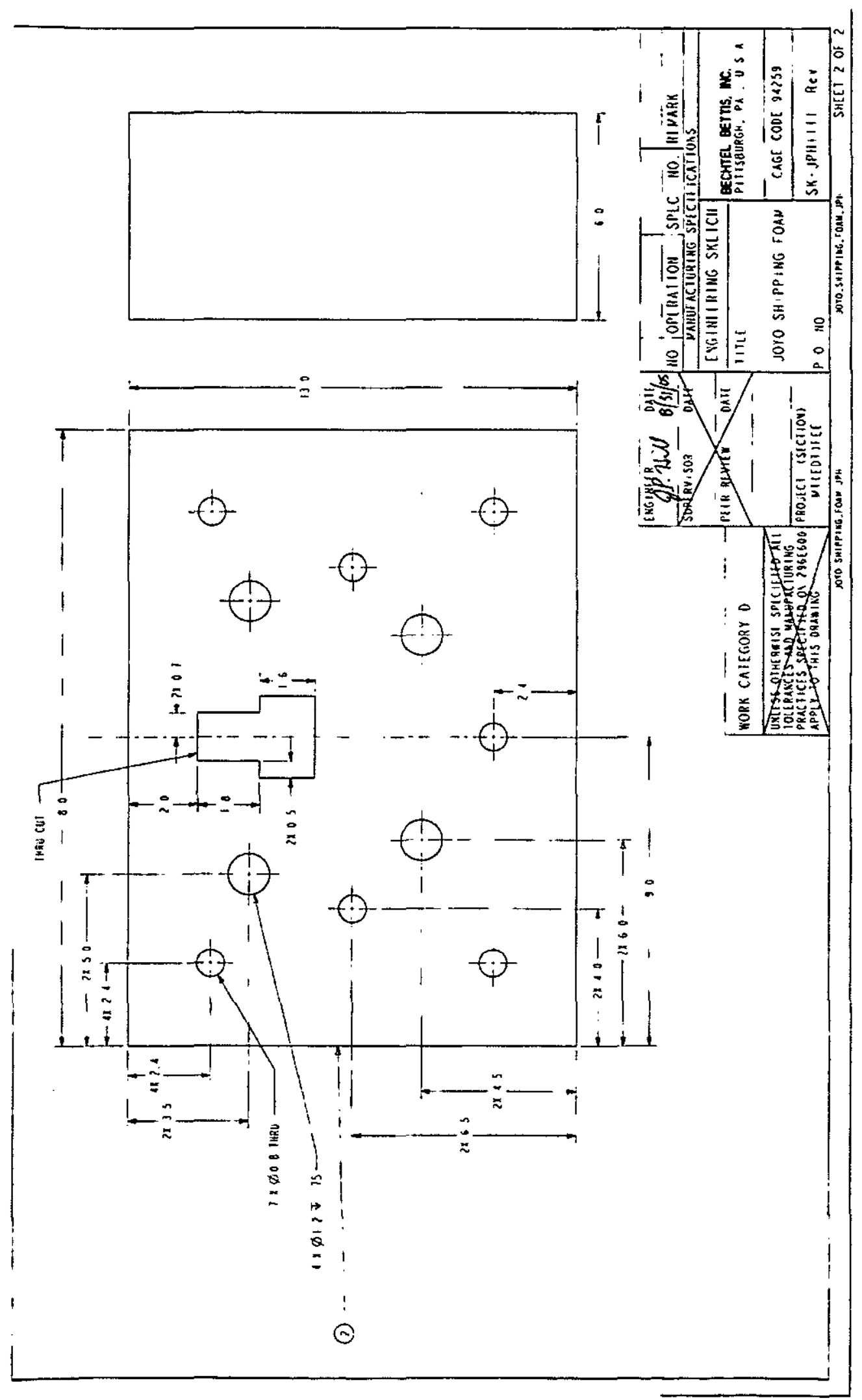


Enclosure (7) to

MDO-723-0044/

B-MT(SRME)-52

Page 232

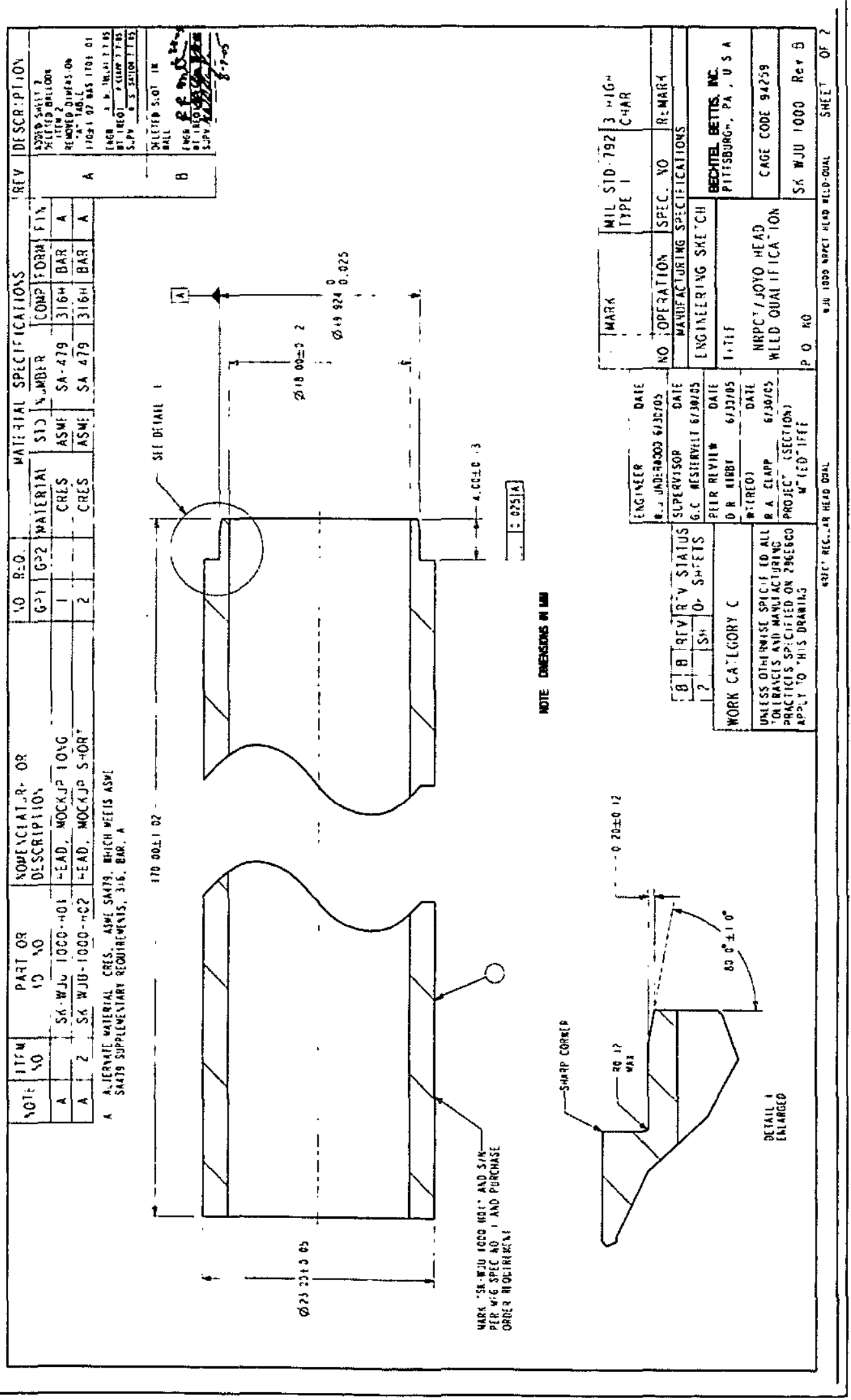




\section{Enclosure (7) to MDO-723-0044/ B-MT(EDT)S-029 Page 233}

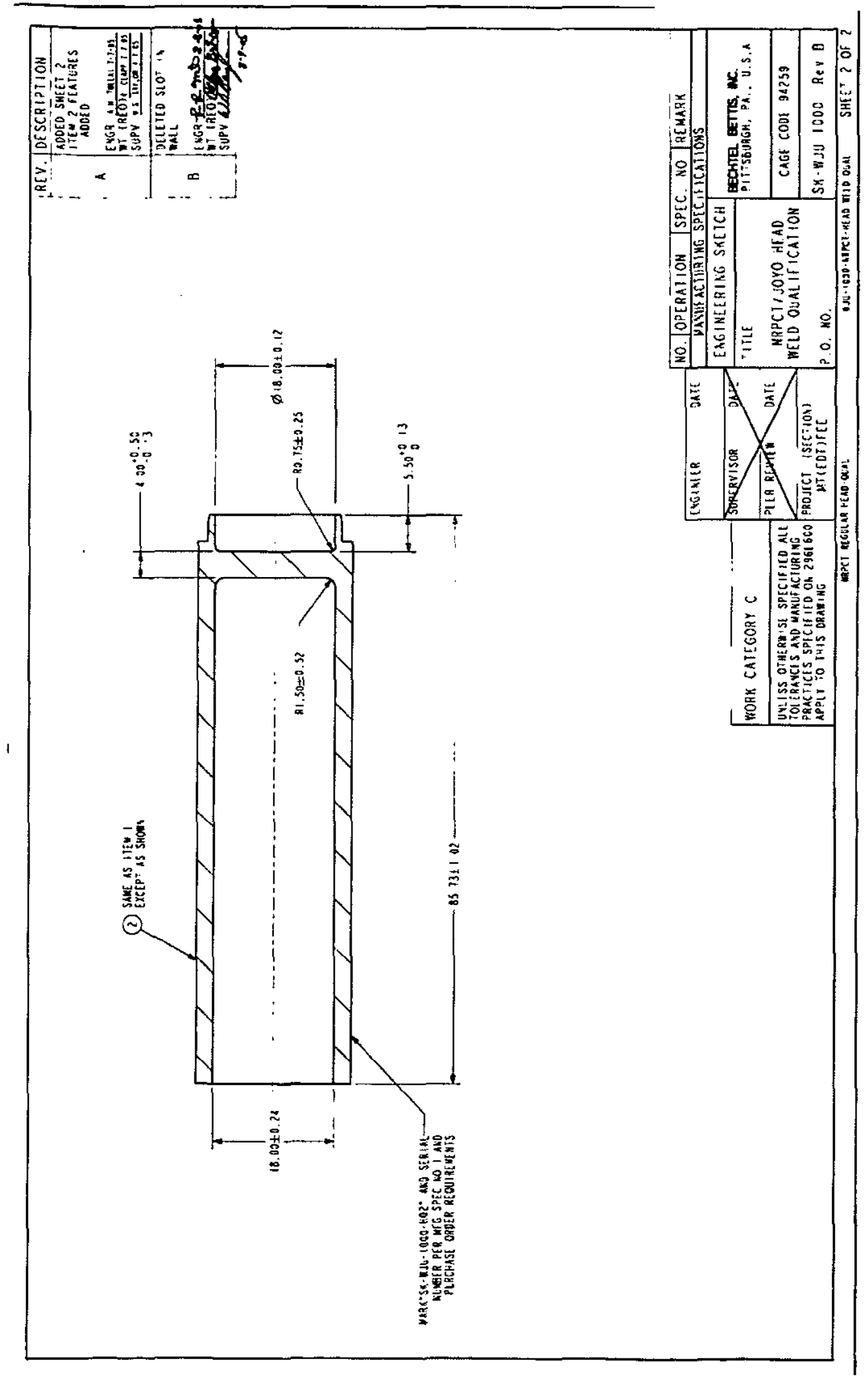


Enclosure (7) to

MDO-723-0044/

B-MT(SRME)-52

Page 234

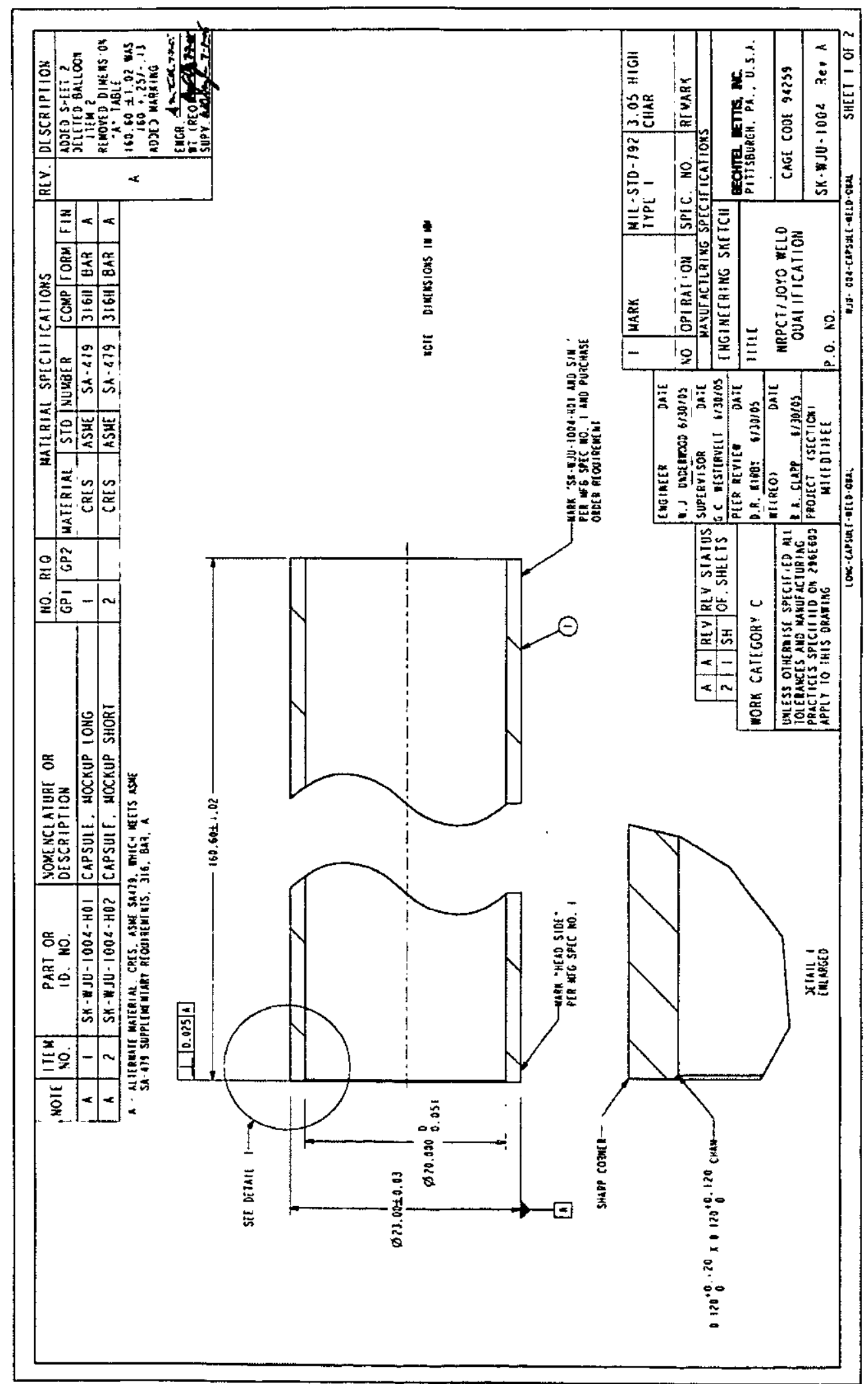


Enclosure (7) to

MDO-723-0044/

B-MT(EDT)S-029

Page 235

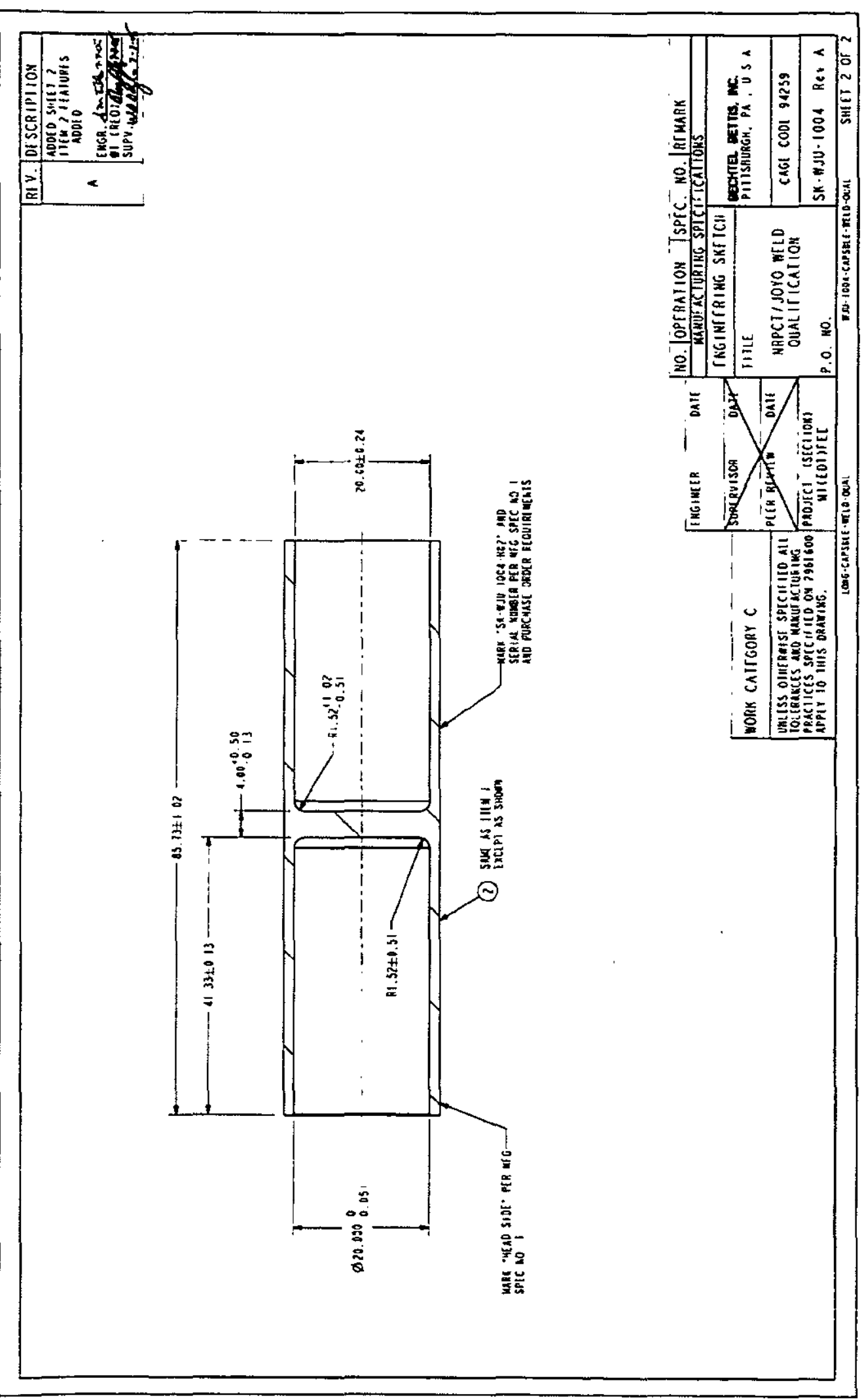


Enclosure (7) to

MDO-723-0044/

B-MT(SRME)-52

Page 236

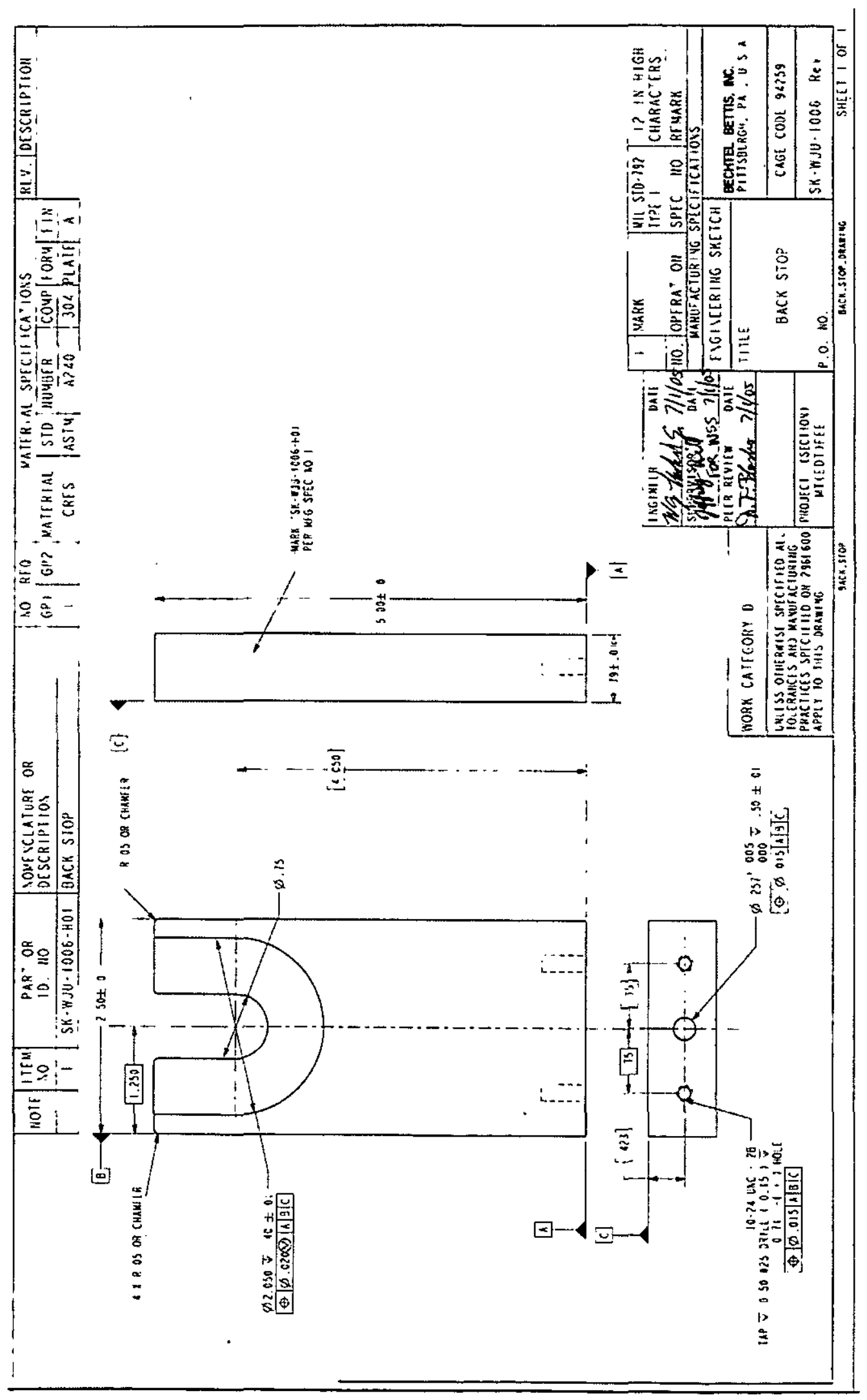




\section{Enclosure (7) to \\ MDO-723-0044l \\ B-MT(EDT)S-029 \\ Page 237}

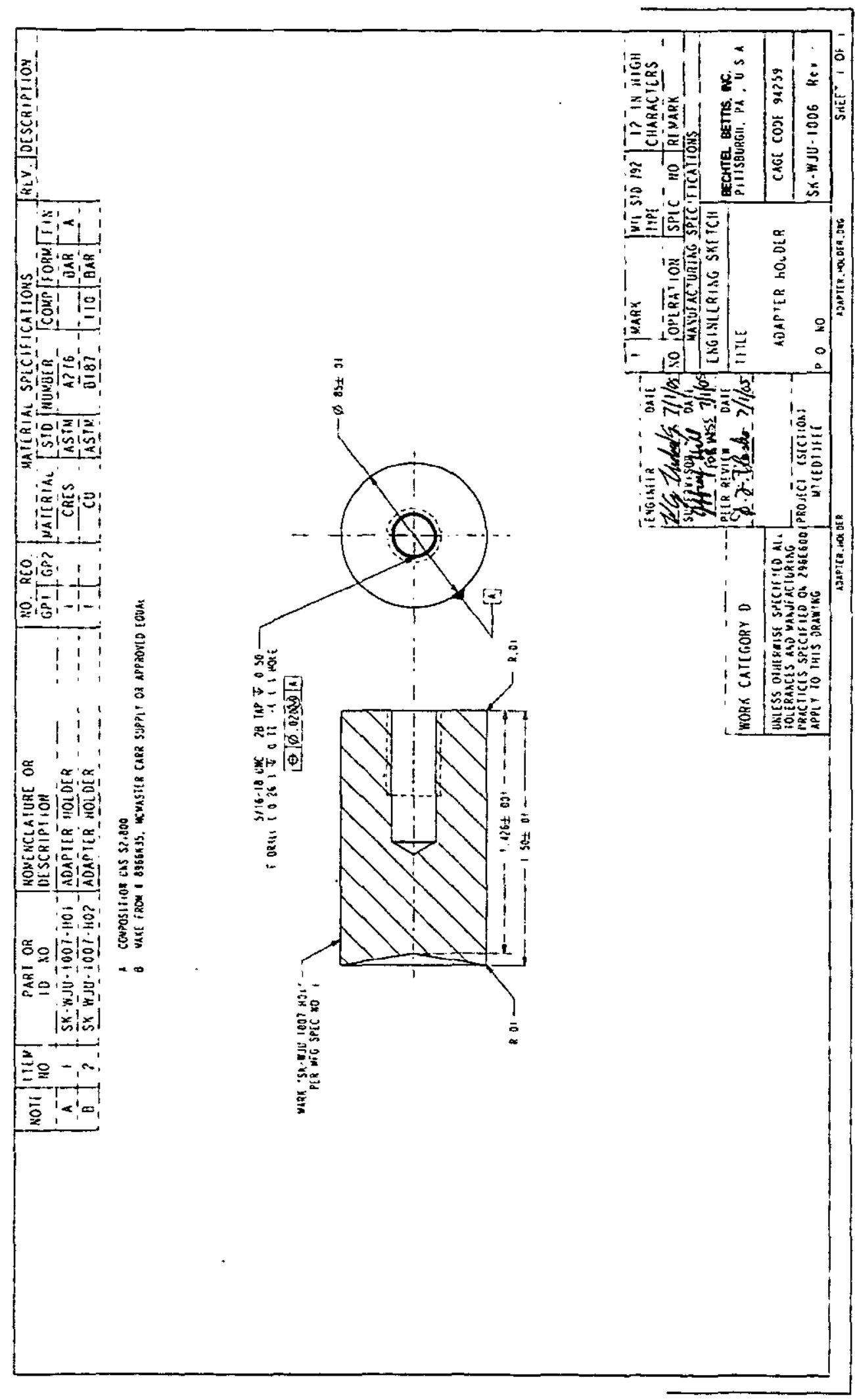


Enclosure (7) to

MDO-723-0044/

B-MT(SRME)-52

Page 238

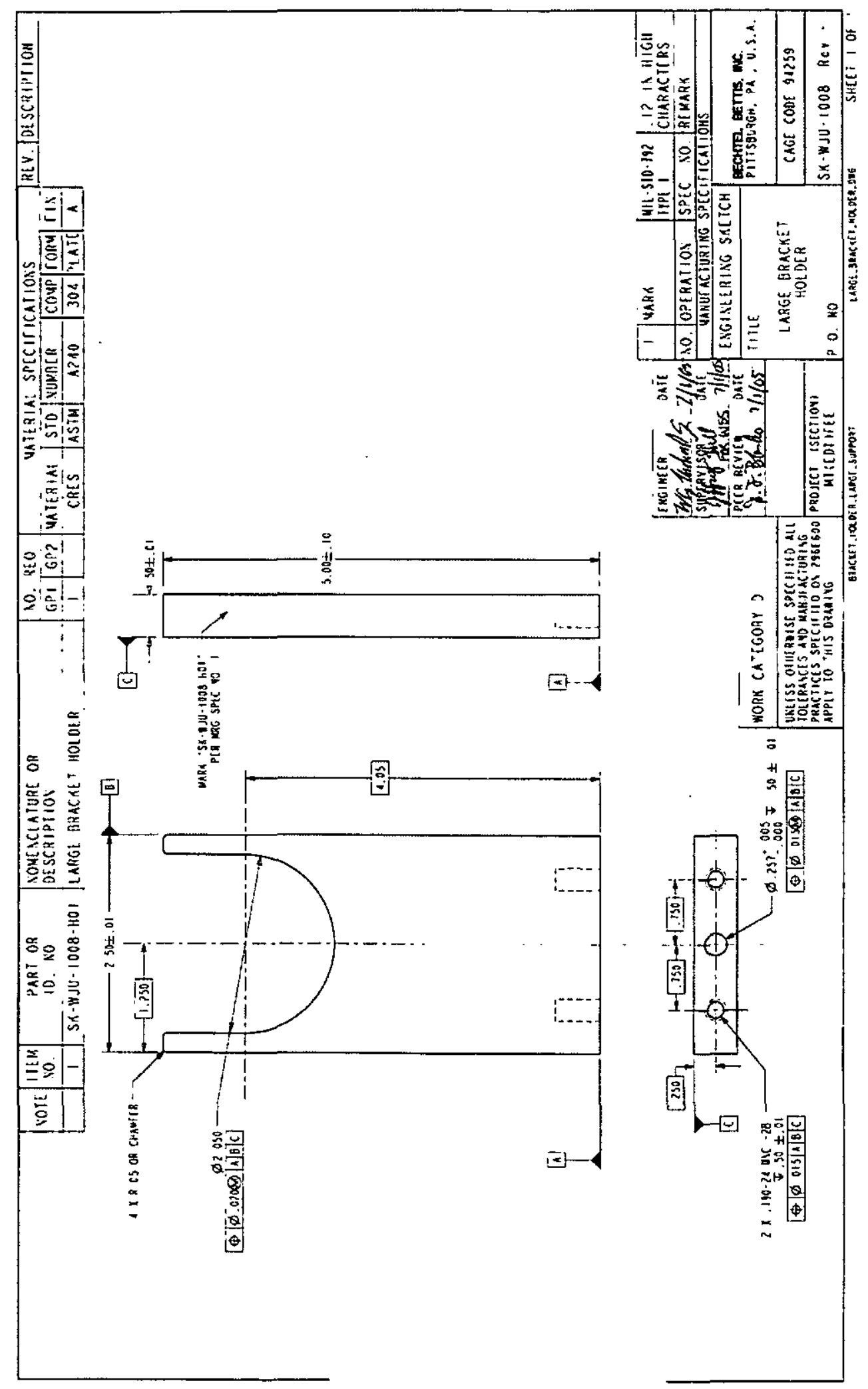



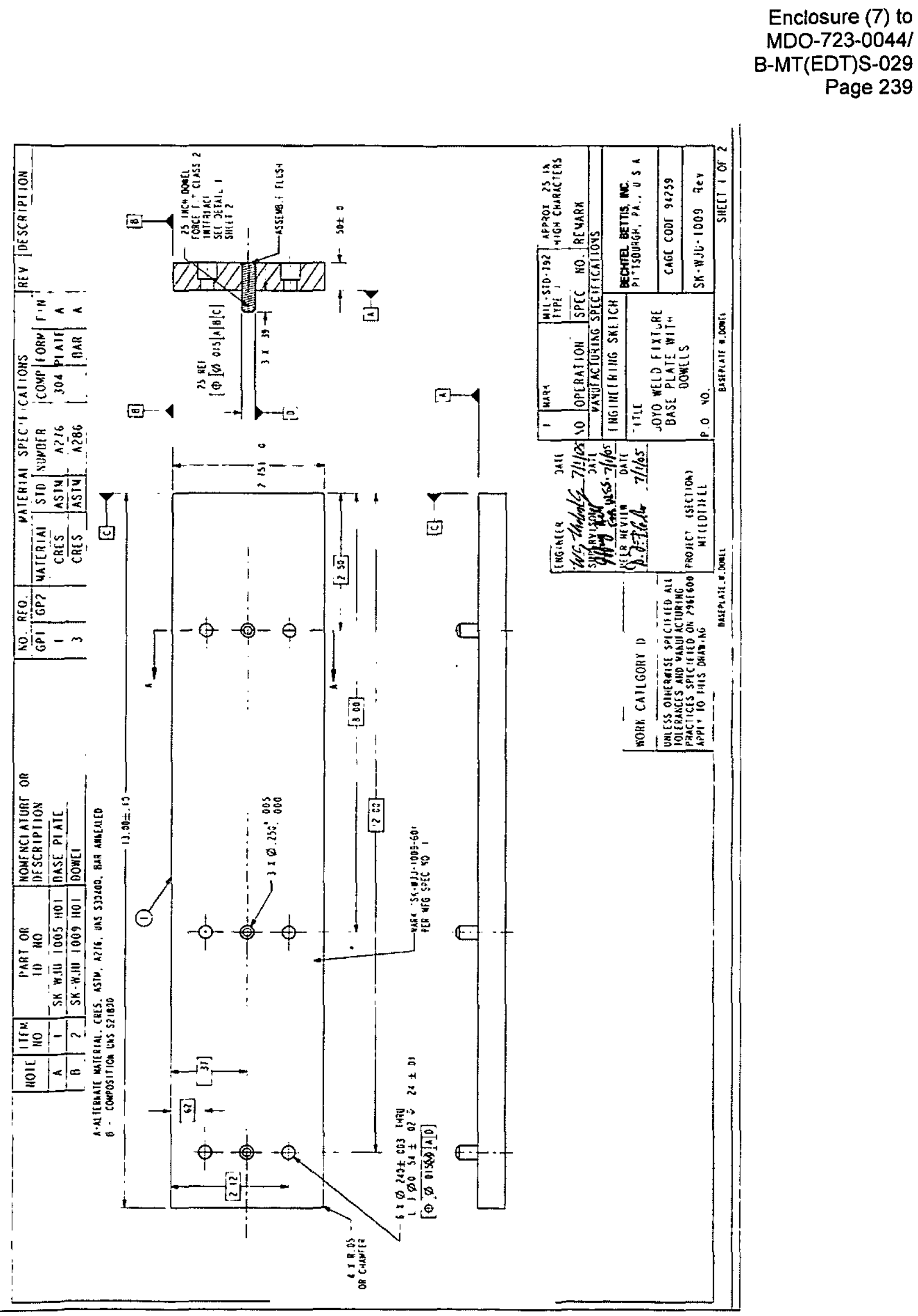
Enclosure (7) to

MDO-723-0044/

B-MT(SRME)-52

Page 240

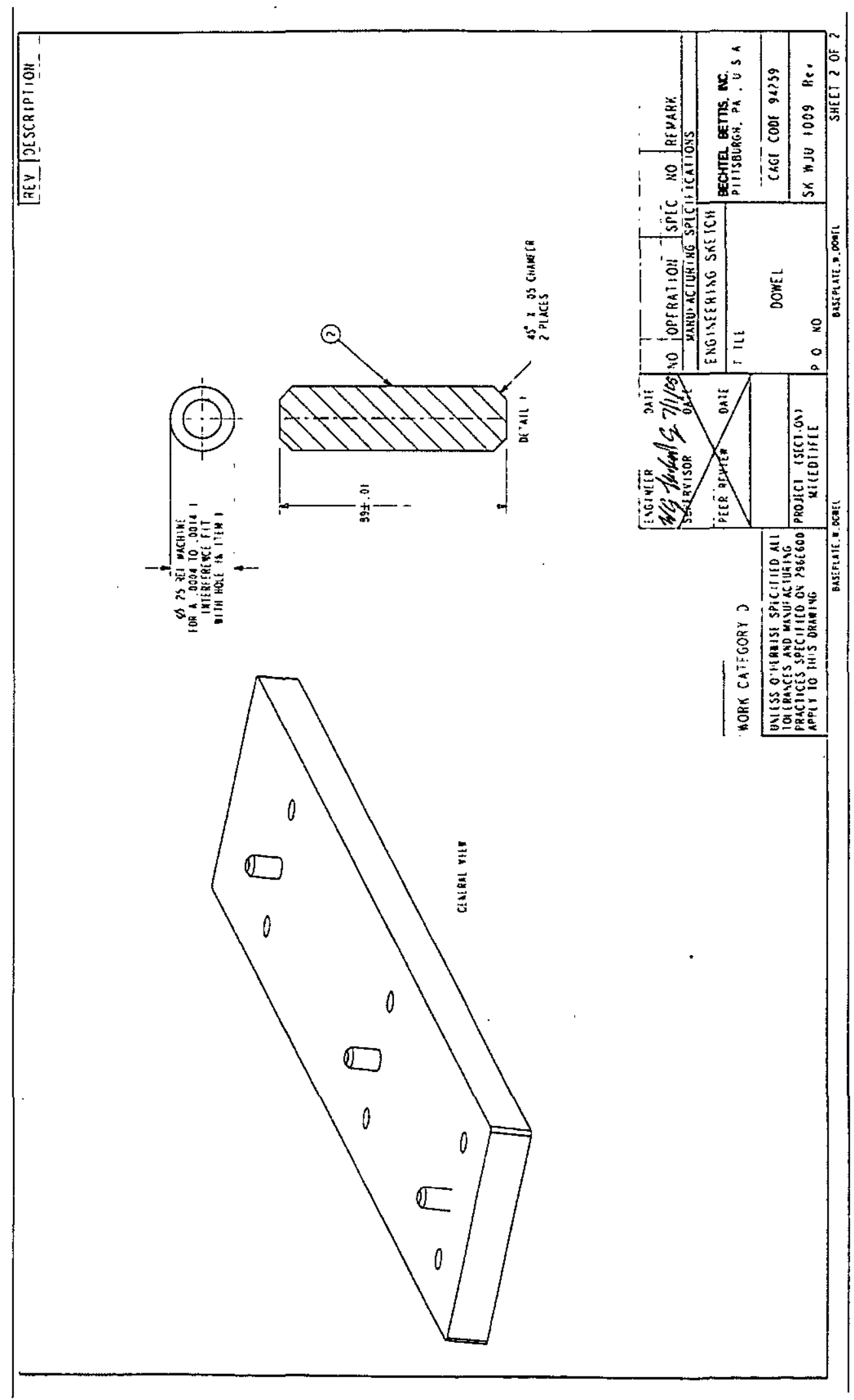




\section{Enclosure (7) to MDO-723-0044/ B-MT(EDT)S-029 \\ Page 241}

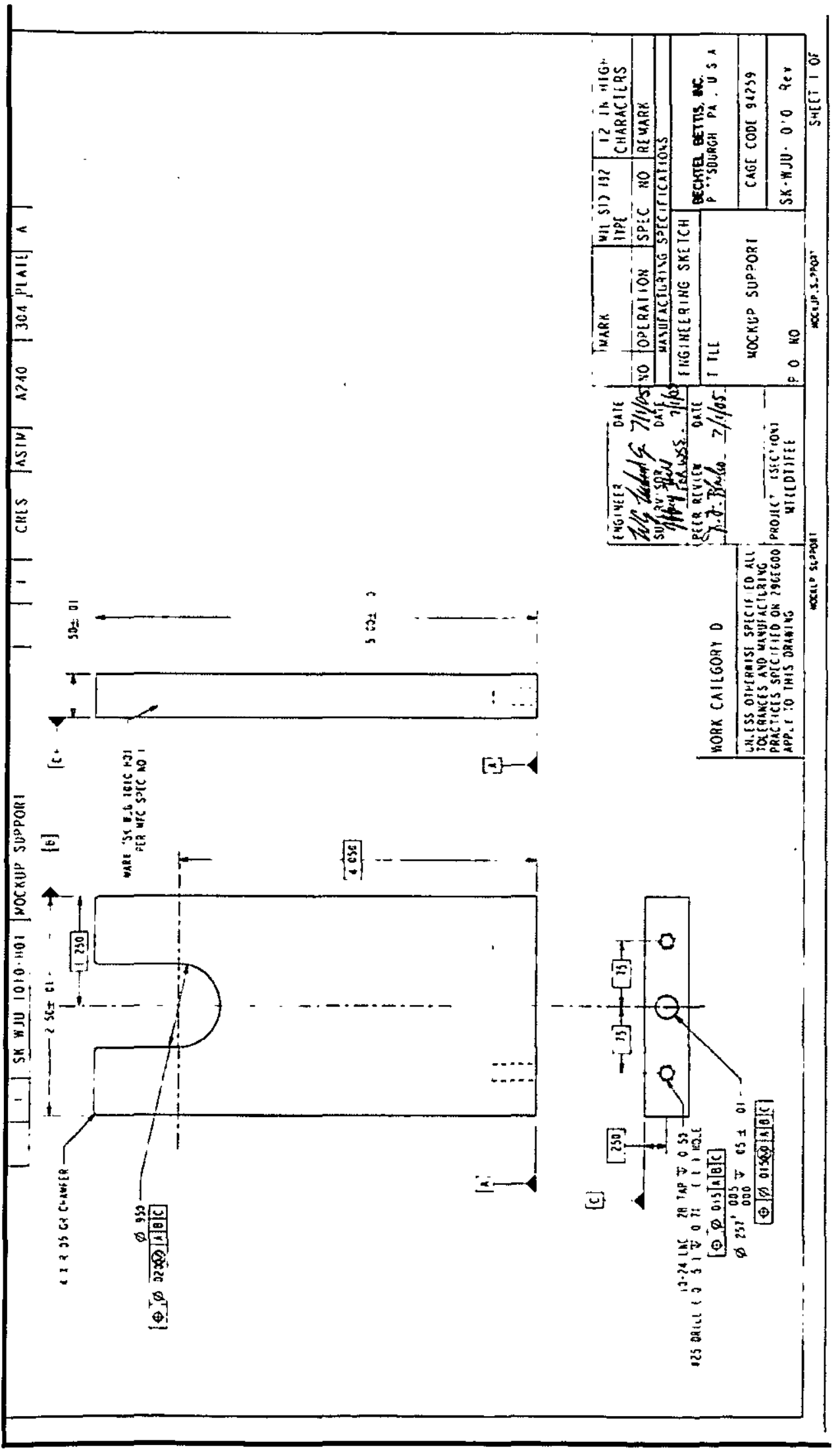




\section{Enclosure (7) to \\ MDO-723-0044/ \\ B-MT(SRME)-52 \\ Page 242}

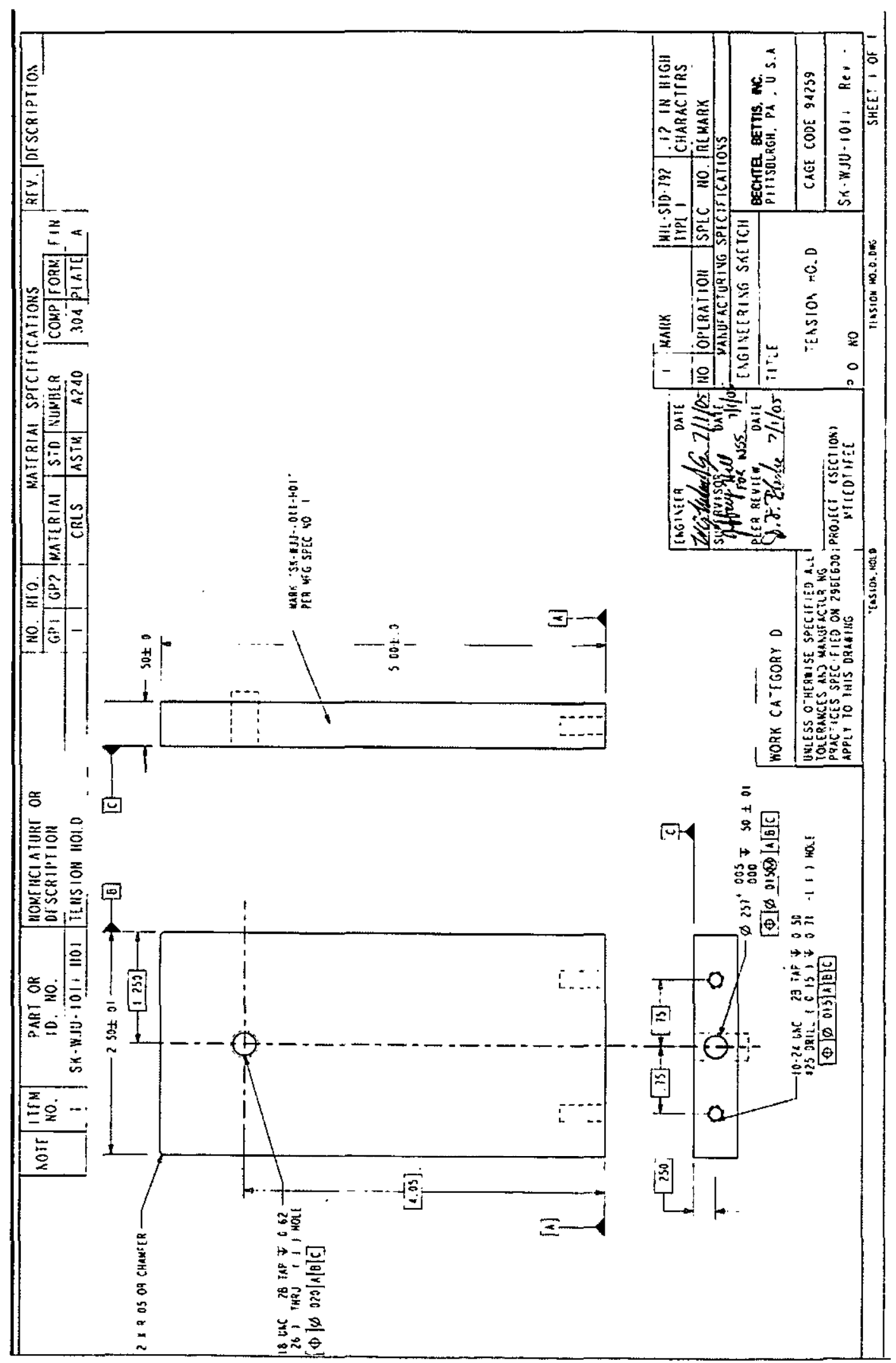


Enclosure (7) to

MDO-723-0044/

B-MT(EDT)S-029

Page 243

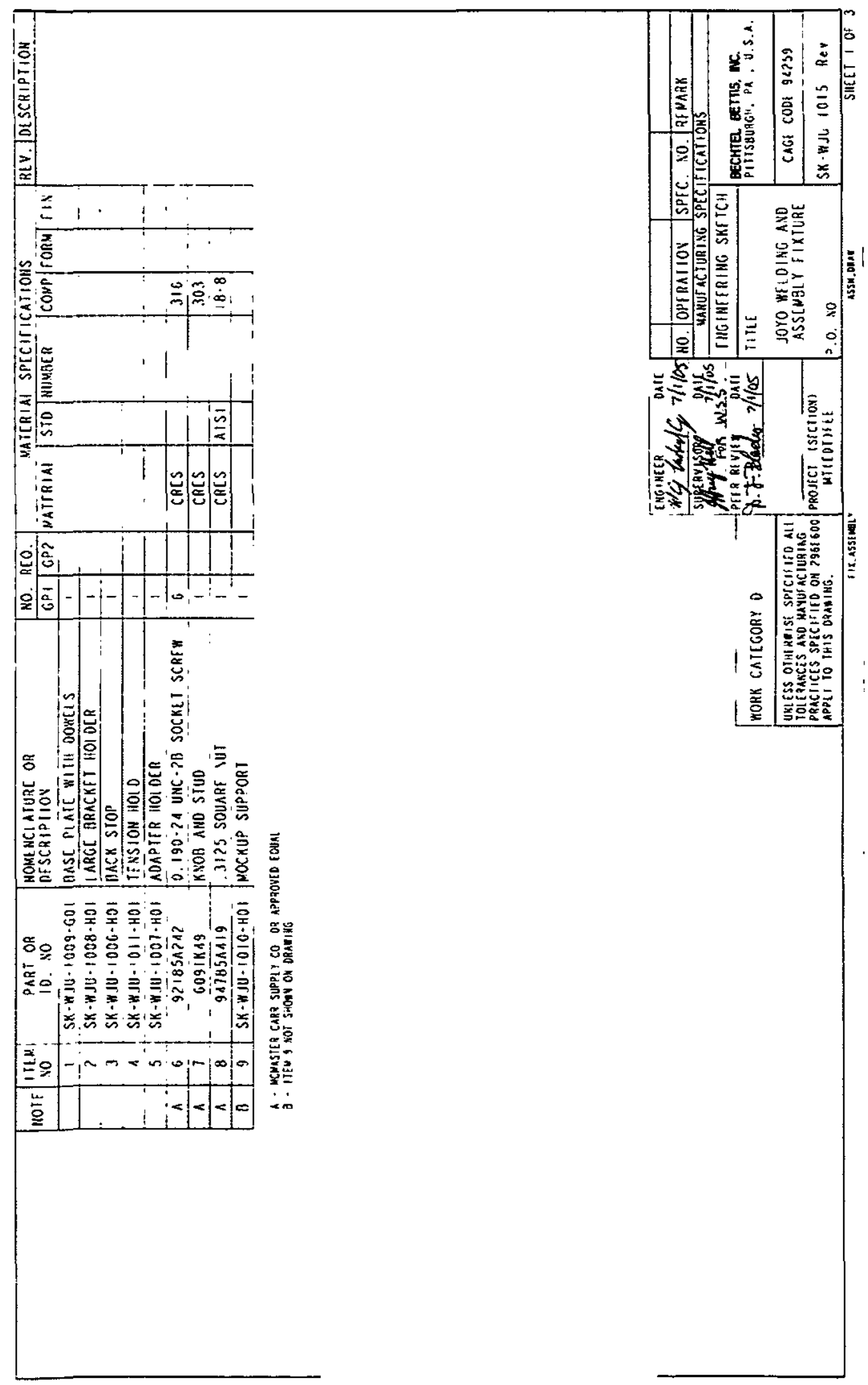


Enclosure (7) to

MDO-723-0044/

B-MT(SRME)-52

Page 244

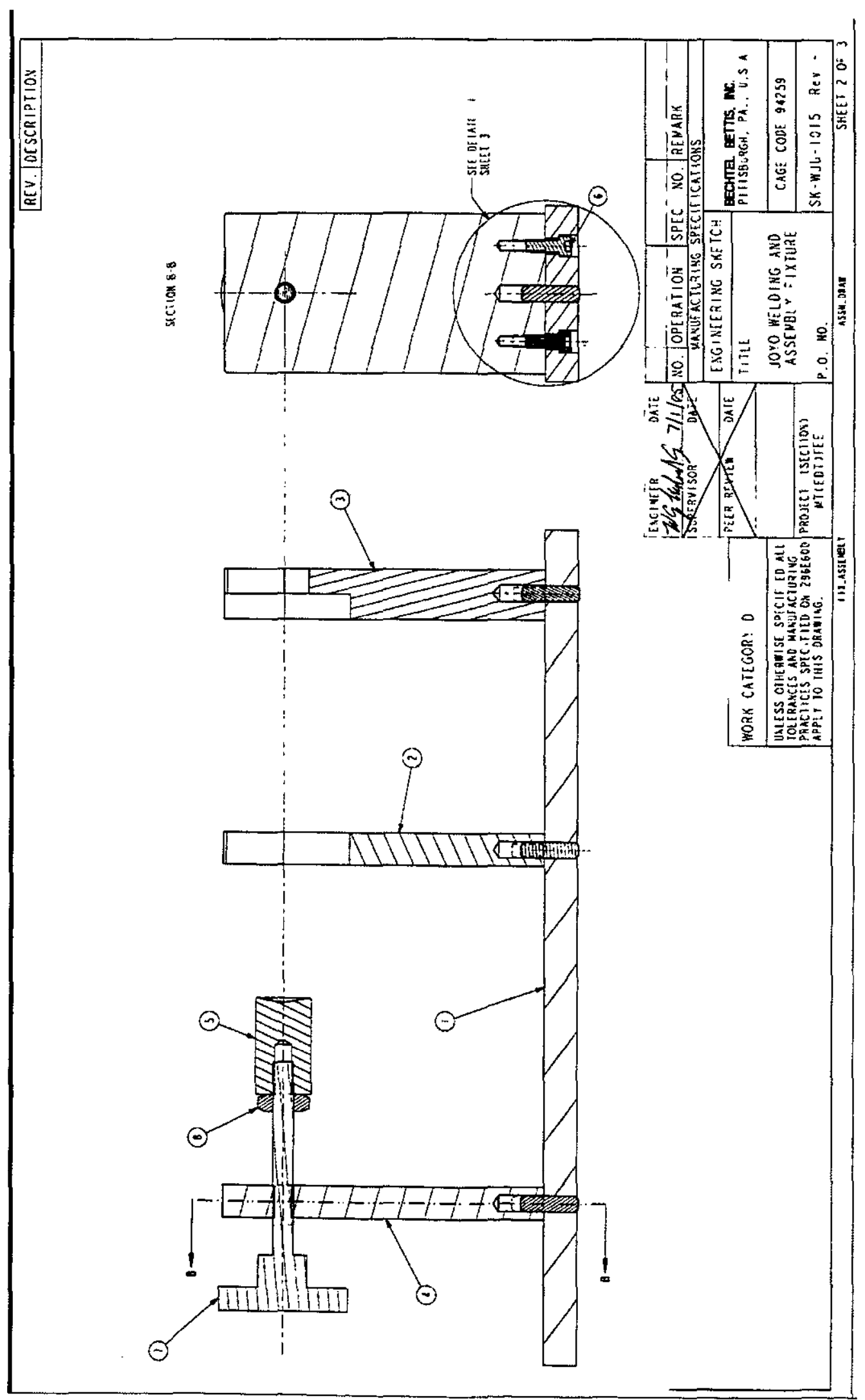



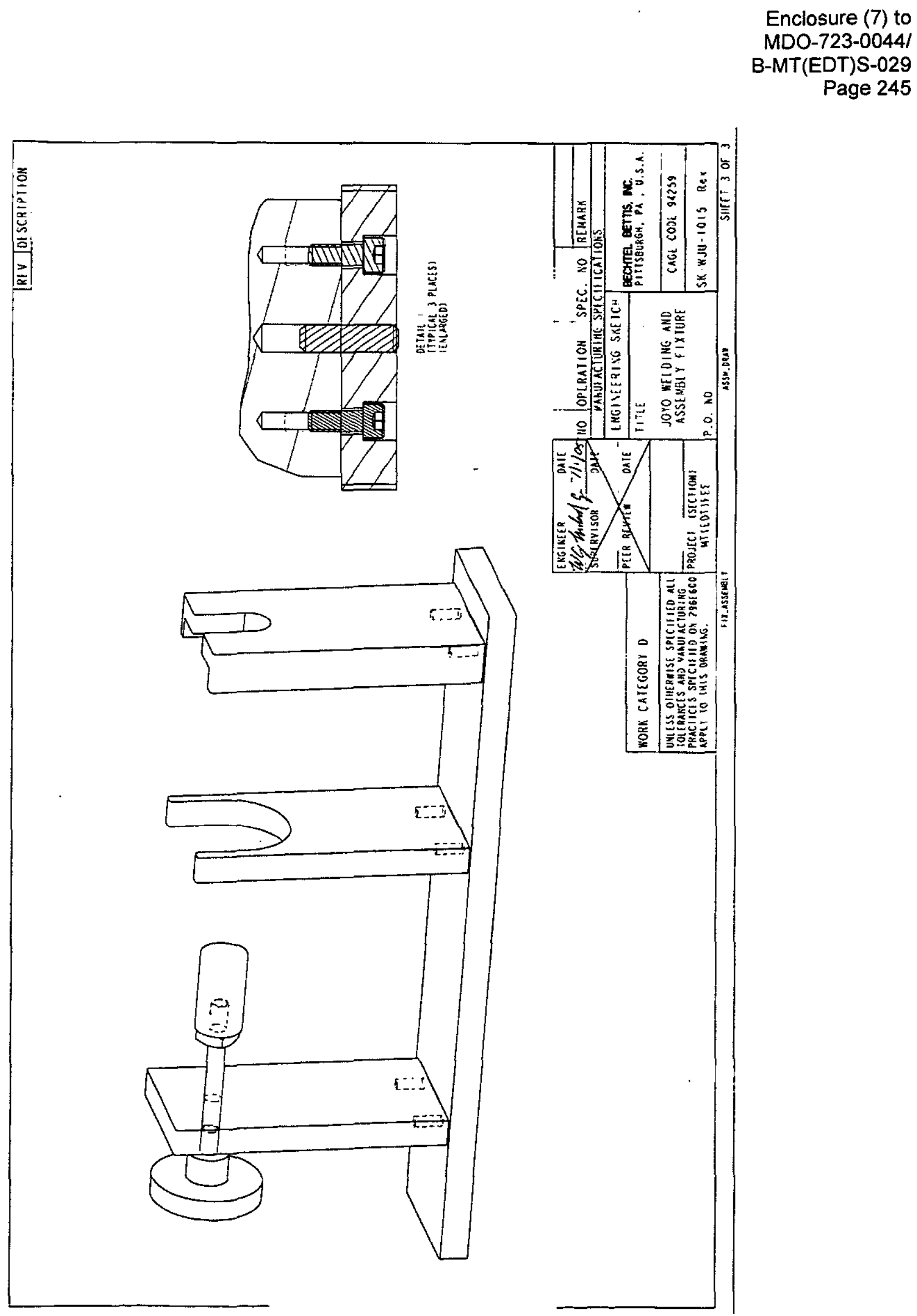
Enclosure (7) to

MDO-723-0044/

B-MT(SRME)-52

Page 246

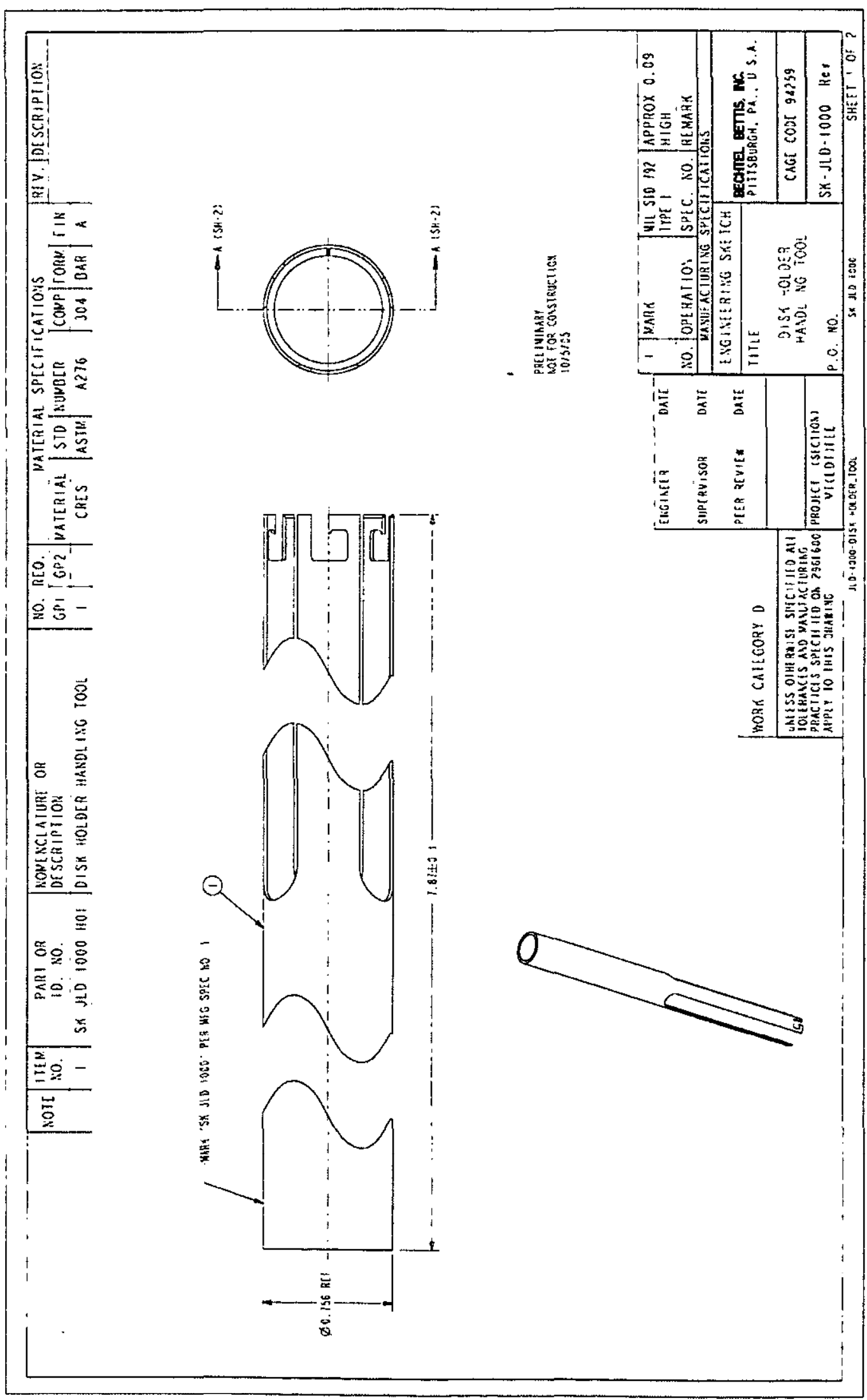



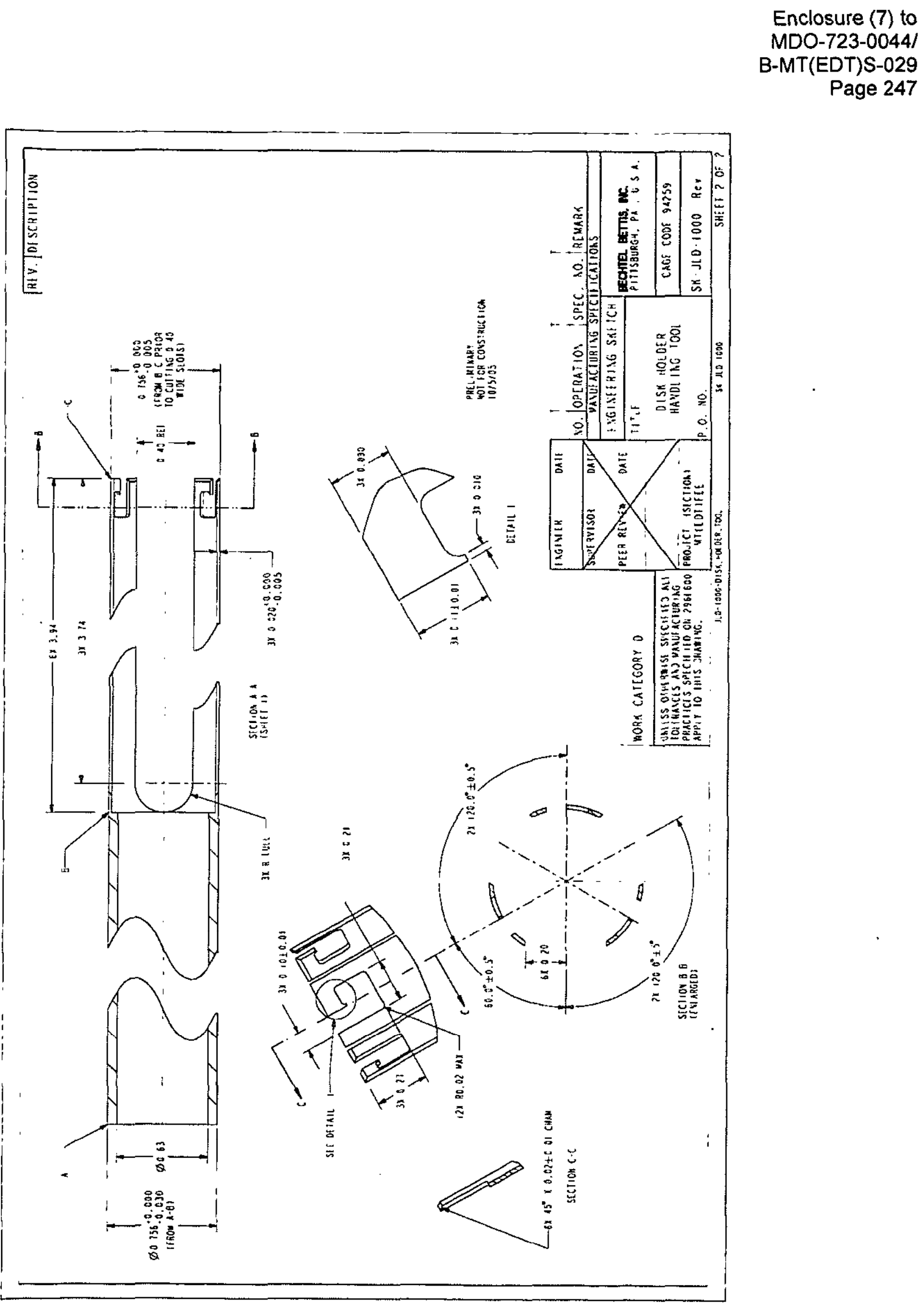
Enclosure (7) to

MDO-723-0044I

B-MT(SRME)-52

Page 248

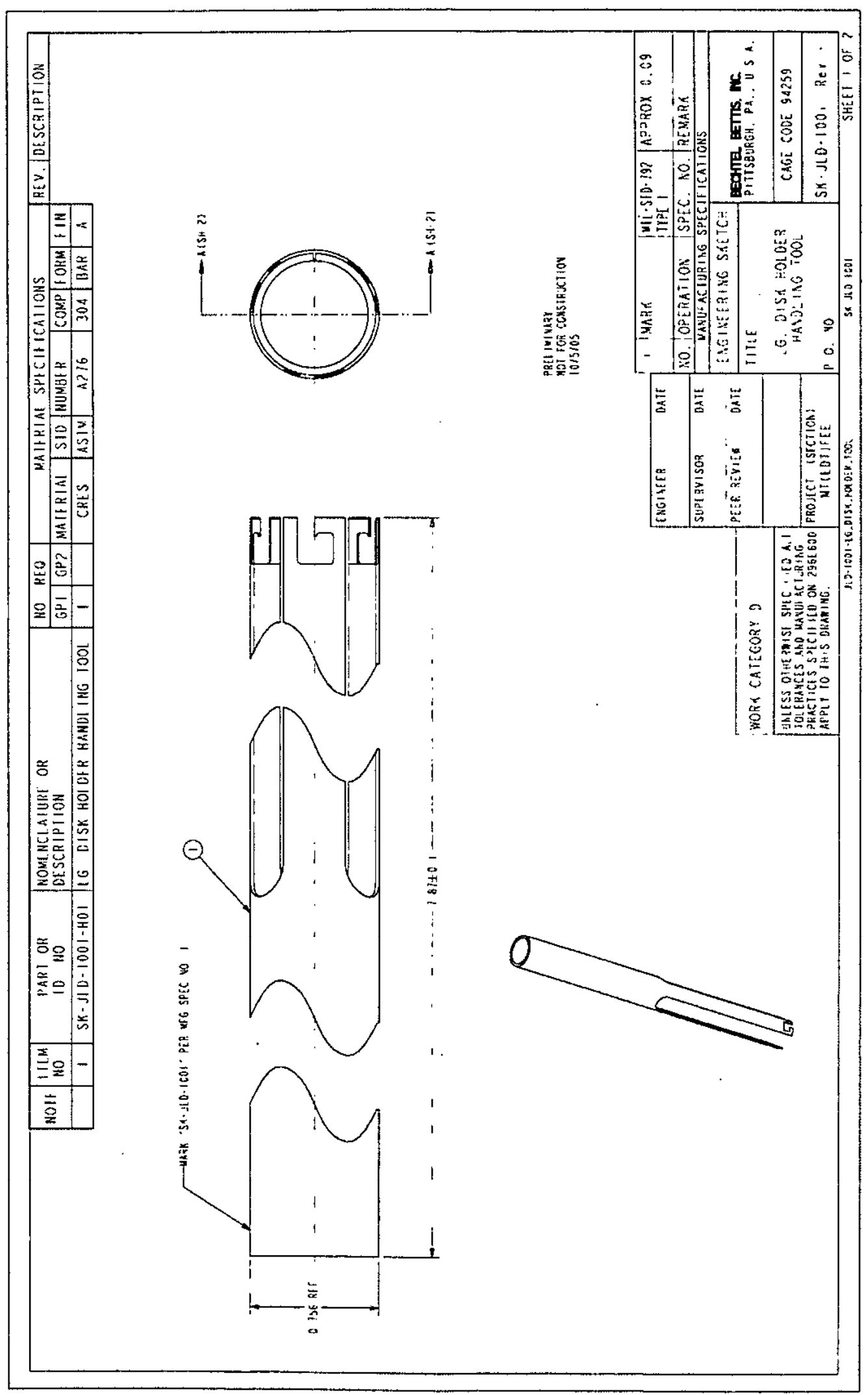




\section{Enclosure (7) to MDO-723-0044/ B-MT(EDT)S-029 Page 249}

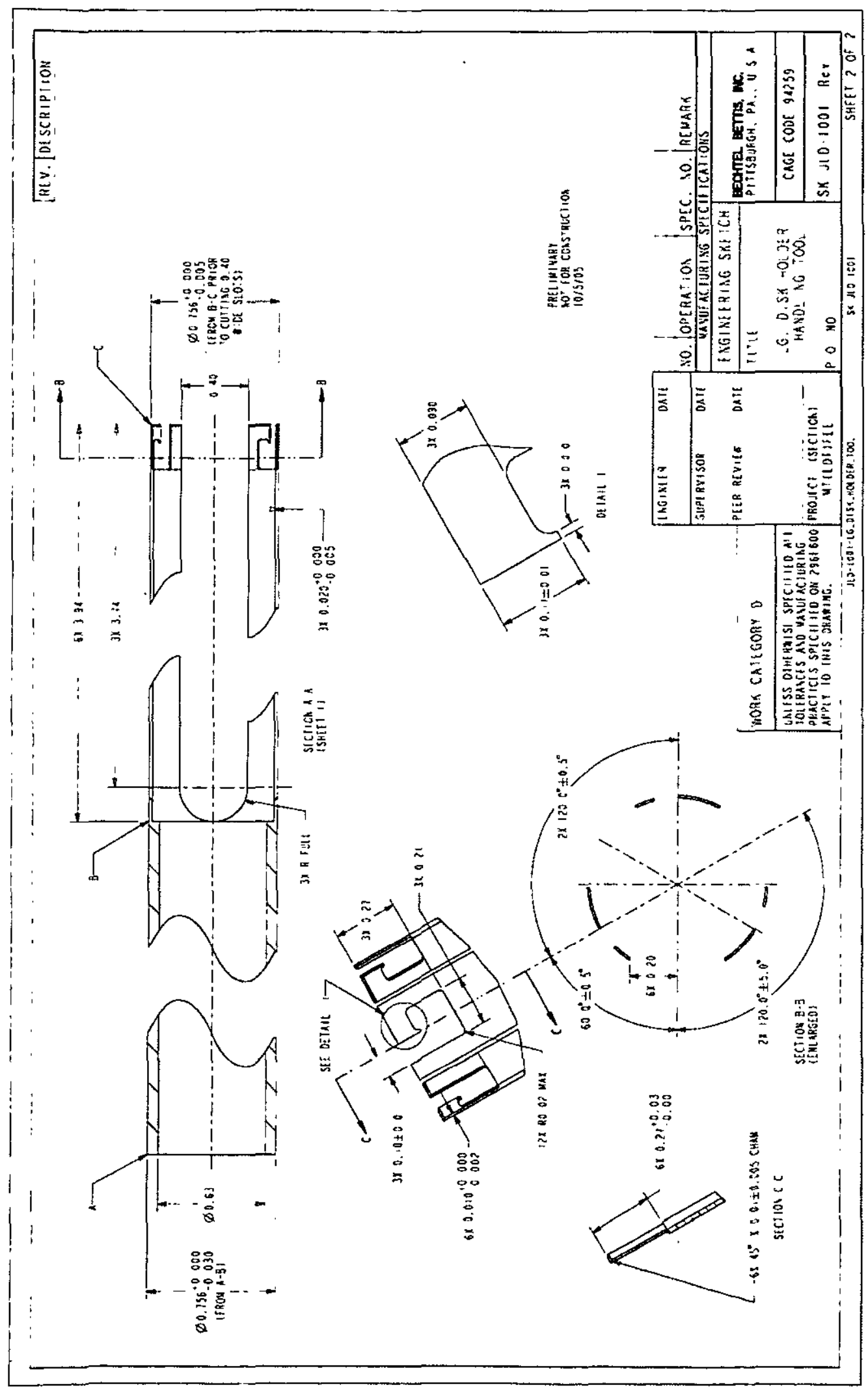


Enclosure (7) to

MDO-723-0044/

B-MT(SRME)-52

Page 250

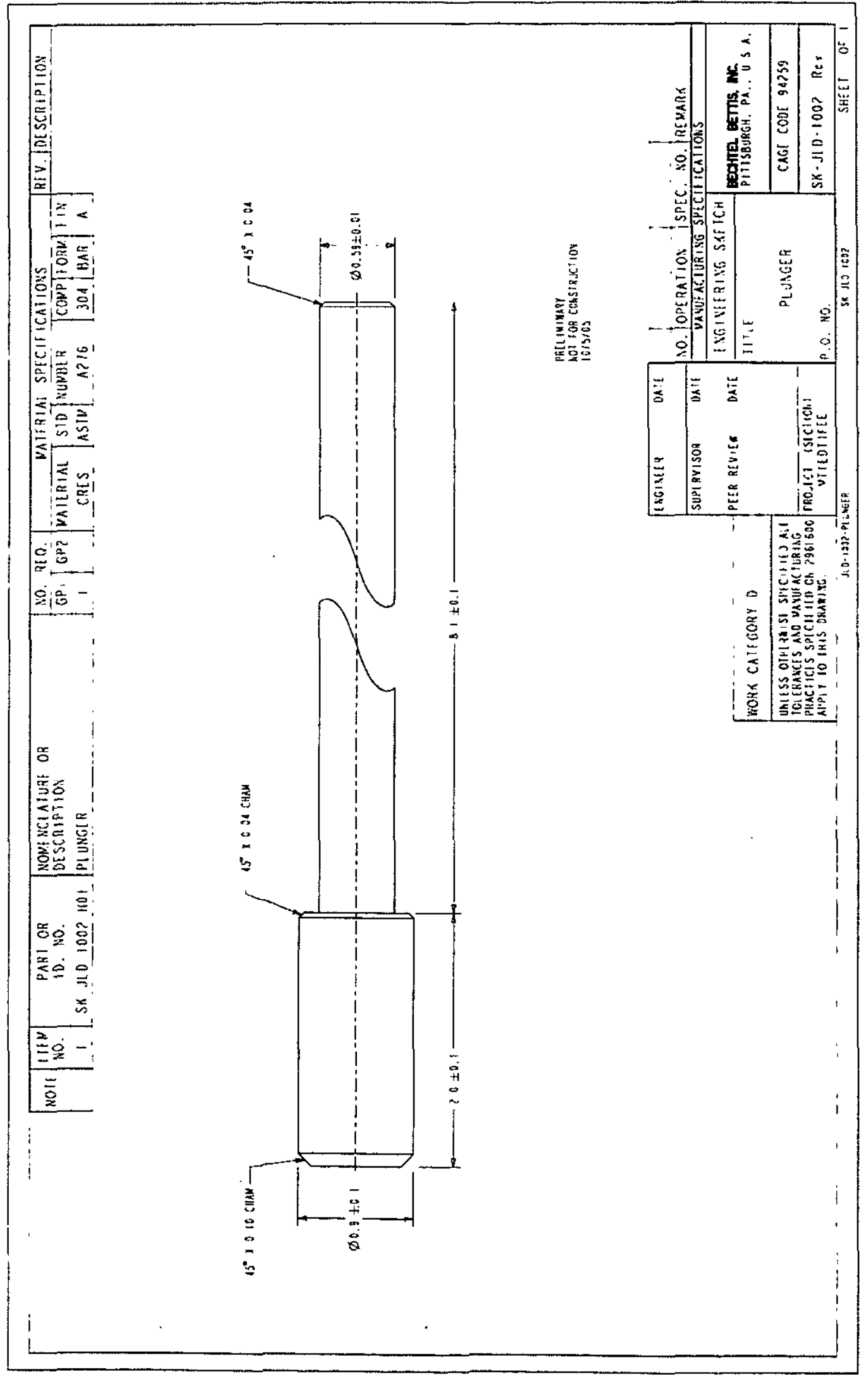


Enclosure (7) to

MDO-723-0044/

B-MT(EDT)S-029

Page 251

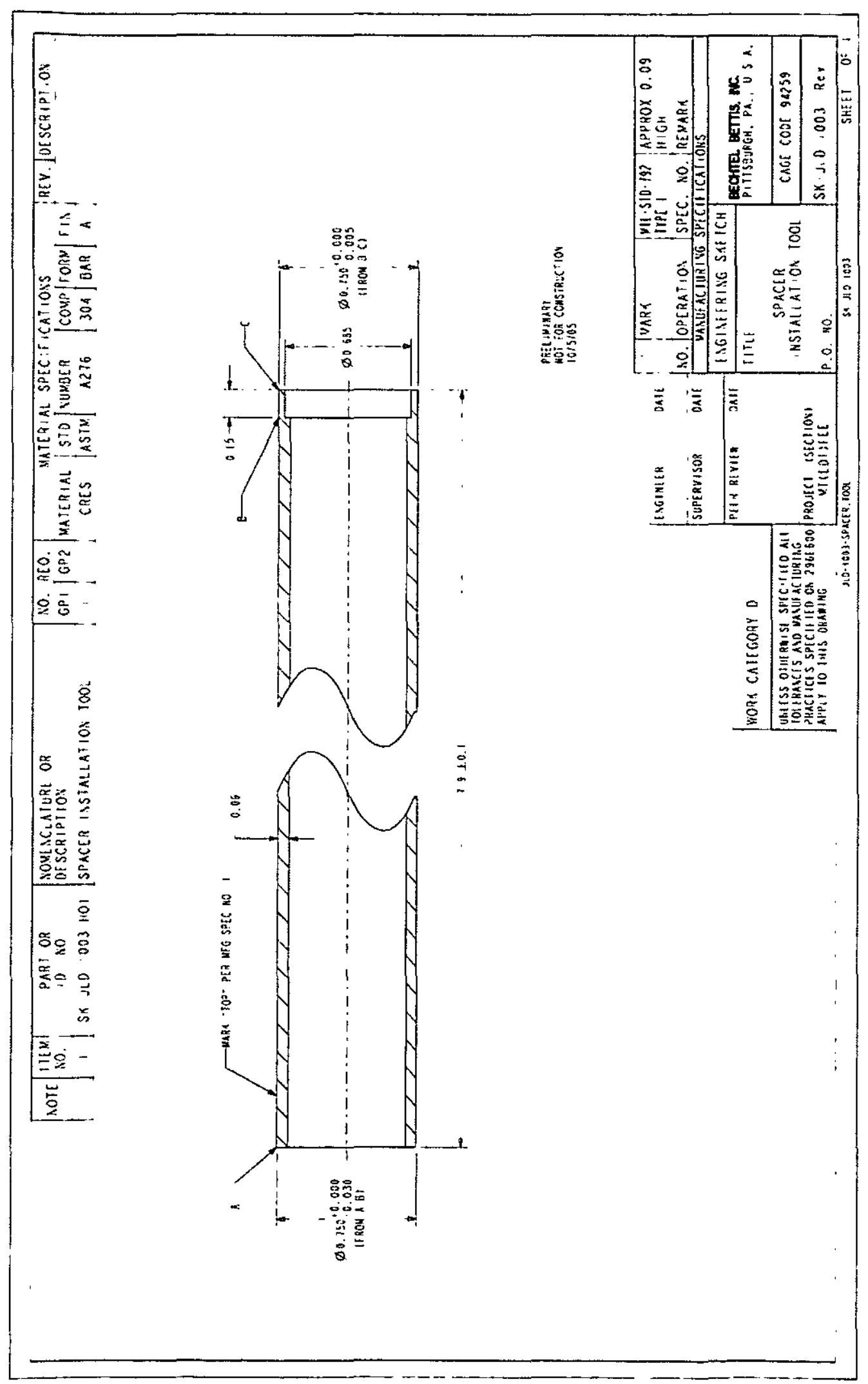


Enclosure (7) to

MDO-723-0044I

B-MT(SRME)-52

Page 252

THIS PAGE IS INTENTIONALLY BLANK 


\section{References}

I-1 B-MT(EDT)S-018 JOYO-1 Design for Permission report presentation to the Japan Nuclear Cycle Development Institute (JNC) for testing of Space structural materials in the JOYO reactor

I-2 Contract: PNNL-SPP-05-0009, Project \#48552, SRM-LRPT-005, PNNL-15295, July 2005, Adjacent Fuel Effects for JOYO-1 Irradiation Tests

III.B-1 B-MT(SMRE)-32, Mader, Borges, Northey, Nash "Material Properties and Specimen Dimensions for Capsule Design to Support the JOYO-1 Irradiation Test, Revision 0"

III.B-2 FED-Vol. 208, Turbulent Flows, ASME 1995, Vertical Natural Convection Heat Transfer Data for an Enclosed Fluid. 
Enclosure (7) to

MDO-723-0044/

B-MT(SRME)-52

Page 254

THIS PAGE INTENTIONALLY BLANK 
Attachment $A$ of

Enclosure (7) to

MDO-723-0044/

B-MT(SRME)-52

Page 1

Attachment A of

\section{Enclosure 7 to MDO-723-0044/B-MT(SRME)-52}

Adjacent Fuel Affects for JOYO-1 Irradiation Test 
Attachment $A$ of

Enclosure (7) to

MDO-723-0044/

B-MT(SRME)-52

Page 2

THIS PAGE INTENTIONALLY BLANK 


\title{
Adjacent Fuel Effects for JOYO-1 Irradiation Tests
}

\author{
Author: David Wootan
}

\begin{abstract}
The impact of the JOYO-1 irradiation experiment on the JOYO reactor power distribution was evaluated using the same MCNP full-core model that was used in calculating the heating rates in the test capsules. The pin powers in the outer row of fuel pins in fuel subassemblies adjacent to the tests were found to be slightly lower compared to pin powers adjacent to a standard reflector subassembly.

\section{Objective}

An important consideration for reactor safety and performance is the effect on adjacent driver fuel subassemblies of the JOYO Structural Materials Irradiation Rigs (SMIR) containing the sixty NRPCT structural irradiation capsules that are to be located in rig positions $6 \mathrm{~A} 4$ and $6 \mathrm{D} 4$ in JOYO Cycle 5 . Most of the test capsules contain only molybdenum, steel, and other metals. These capsules are expected to provide less reflection and more absorption than the high nickel steel used in the radial reflector subassemblies, and thus have a negative impact on adjacent fuel power. However, the 'SCA' and 'BSR' type capsules contain silicon carbide and graphite structural material samples and sample holders along with reduced amounts of molybdenum. Additionally, the 'NBU' and 'NBD' type capsules contain small samples of beryllium oxide. Concerns were raised because these types of materials have moderating properties and any low energy neutrons returning to the core might result in a localized increase in fuel pin powers since the ${ }^{235} \mathrm{Pu}$ and ${ }^{235} \mathrm{U}$ fission cross sections are higher at low energies. The nominal impact of the SMIR tests on the core is needed, along with an assessment of the localized effect of the moderating materials in the capsules. This is described as Task 1.2 in the Statement of Work (Reference 1).
\end{abstract}

\section{MCNP Cycle 5 Core Model}

The preliminary whole core model for the JOYO beginning of cycle 5 core configuration was provided by $\mathrm{JNC}$. This model was generated directly from the $\mathrm{JNC}$ core management compositions and included thermal expansion effects on dimensions and densities. This original core model represented the two SMIR irradiation tests as typical steel reflector assemblies. This model was subsequently modified to include the additional ex-core regions, beginning of cycle control rod elevations, and the test subassembly geometry and compositions.

The impact of the SMIR irradiation on the Joyo reactor power distribution was evaluated using the same MCNP full-core model used to calculate the test heating rates (Reference 2). Three variations of the model were used. The reference case for evaluating the test impact had the original standard homogenized radial reflector subassemblies in the 6A4 and 6D4 core locations designated for the SMIR tests. The nominal case model had the detailed heterogeneous test descriptions in those locations. This was the same model used in calculating heating rates in the 
test capsules. For a bounding case model, all of the test capsule compositions in both SMIRs were replaced with the type 'SCA' capsule composition, which contained a low amount of molybdenum and high amount of low-Z material compared to the other capsule types.

\section{MCNP Test Subassembly Model}

Table 1 lists the three models used for evaluating the effects of the SMIR tests on adjacent fuel pin powers.

Table 1. MCNP Cases for Evaluating Adjacent Fuel Effects

\begin{tabular}{|l|l|}
\hline Case & \multicolumn{1}{|c|}{ Description } \\
\hline 5 sf 2 & Reference case with homogenized steel reflectors \\
\hline $5 \mathrm{cf9}$ & $\begin{array}{l}\text { Nominal case with two heterogeneous SMIRs with specified } \\
\text { capsule compositions }\end{array}$ \\
\hline 5 bf9 & $\begin{array}{l}\text { Bounding case with two heterogeneous SMIRs with all SCA-type } \\
\text { capsules containing silicon carbide and graphite }\end{array}$ \\
\hline
\end{tabular}

The original cycle 5 core model used for the reference case $(5 \mathrm{sf} 2)$ contained homogeneous reflector subassemblies in the test locations. The reflector subassemblies were represented by the hexagonal unit cell filled with the homogenized steel reflector pins, wrapper tube, and sodium.

For the nominal case ( $5 \mathrm{cf} 9$ ), these reflectors were replaced with a heterogeneous model of the pin compartments in each of the tests. The heterogeneous compartment model extended 41.675 $\mathrm{cm}$ above and below core midplane and included the steel wrapper tube and seven compartments surrounded by sodium. Six compartments include steel cladding, a gas gap, and the 5 tiers of homogenized capsule material. The Elemental Makeup Revision IA total capsule elemental masses were converted to MCNP input compositions for each capsule. Each capsule was modeled as $16.67 \mathrm{~cm}$ long and $2.2 \mathrm{~cm}$ in diameter. Five capsules were stacked in each compartment, centered at core midplane. Each of the six compartments was modeled as a steel cladding tube with $2.6 \mathrm{~cm}$ outside diameter and $2.47 \mathrm{~cm}$ inside diameter. The pitch between compartments was $2.7 \mathrm{~cm}$. The center compartment was modeled as steel cladding tube with 2.5 $\mathrm{cm}$ outside diameter and $2.38 \mathrm{~cm}$ inside diameter, containing a solid steel rod of $2.0 \mathrm{~cm}$ diameter. The subassembly steel wrapper tube was the same as other subassemblies, with a wall thickness of $0.38 \mathrm{~cm}$. Figure 1 shows a cross section of the test subassembly model for core location $6 \mathrm{~A} 4$.

For the bounding case ( 5 bf9) all 60 of the test capsule compositions in both SMIRs were replaced with the type ' $\mathrm{SCA}$ ' capsule composition, which contained a low amount of molybdenum and high amount of low- $Z$ material. The model geometry was not changed. This composition was judged to bound the 'NBU' and 'NBD' type capsules with beryllium oxide, since the 3.6 grams of beryllium in those capsules is a factor of ten less than the $\sim 30$ grams of 
carbon in the 'SCA' capsules, and the 'NBU' and 'NBD' type capsules also contain approximately double the amount of molybdenum, which acts as a neutron absorber.

The sum over the fuel column of the pin fission powers in the fuel pins in the fuel subassemblies in core locations $5 \mathrm{~A} 3$ and $5 \mathrm{~A} 4$ adjacent to standard reflector subassemblies were compared to the nominal case of the two SMIR tests and to the bounding case with the graphite and silicon carbide capsules. The nominal case provides the "best estimate" of the impact of the test subassembly on adjacent fuel, while the bounding case addresses localized power peaking effects.

\section{Computer Calculations}

All computer calculations were made with the MCNP5 computer code (Reference 3 ) and associated cross section library that is maintained and controlled at PNNL in accordance with Reference 4. The most recent MCNP cross sections were used where available (mostly based on ENDF/B-VI.8). All MCNP calculations were run on a dual processor Dell Dimension 670 workstation computer ID\#WD47989.

\section{Assumptions and Limitations}

Details of the SMIR irradiation rig are not available yet, so the model was based on dimensions from similar irradiation rigs. The final SMIR design is expected to be very close to the assumed model, but the content of the center compartment is not known at this time.

MCNP calculations of adjacent fuel effects utilized primarily ENDF/B-VI cross sections. Comparisons of core-wide power distributions between the MCNP and the JNC core management codes are recommended to identify any differences in methods.

\section{Comparison of Fission Powers in Adjacent Fuel Pins for Nominal Case}

Table 2 summarizes the effects on the core of the nominal test compositions compared to the reference case with steel reflectors.

Table 2. Nominal Case with Tests Compared to Reference Case with Reflectors

\begin{tabular}{|l|c|}
\hline \multicolumn{1}{|c|}{ Parameter } & Ratio test/reflector \\
\hline Adjacent Fuel Subassembly Power & 0.98 \\
\hline Adjacent 1st row fuel pin power & $0.92-0.97$ \\
\hline Core reactivity & $-0.0007 \Delta \mathrm{k} / \mathrm{k}$ \\
\hline
\end{tabular}

Figures 2 and 3 show the ratio of the fission power in adjacent fuel pins in 5A3 and 5A4 core locations for the nominal test compared to the reference steel reflector. Figures 4 and 5 show the corresponding radial pin fission power distributions adjacent to the SMIR test. Figures 6 and 7 show the radial pin fission power distributions adjacent to the reflector. The highest pin powers 
in the row 5 fuel subassemblies are in the fuel pins closest to core center. All of the pin powers in the adjacent fuel subassemblies were lower for the SMIR case compared to the reflector case.

The uncertainty values are the MCNP statistical uncertainties at the one-sigma confidence level.

\section{Comparison of Fission Powers in Adjacent Fuel Pins for Bounding Case}

Table 3 summarizes the effects on the core of the bounding test compositions compared to the reference case with steel reflectors.

Table 3. Nominal Case with Tests Compared to Reference Case with Reflectors

\begin{tabular}{|l|c|}
\hline \multicolumn{1}{|c|}{ Parameter } & Ratio test/reflector \\
\hline Adjacent Fuel Subassembly Power & 1.00 \\
\hline Adjacent 1st row fuel pin power & $0.99-1.01$ \\
\hline Core reactivity & $0.0002 \Delta \mathrm{k} / \mathrm{k}$ \\
\hline
\end{tabular}

Figures 8 and 9 show the ratio of the fission power in adjacent fuel pins for the bounding test compared to the reference steel reflector. Figures 10 and 11 show the corresponding radial pin fission power distributions adjacent to the bounding SMIR tests. The pin powers in the adjacent fuel subassemblies for the bounding case were equivalent within uncertainties to the reflector case.

\section{Conclusions}

These evaluations demonstrate that the main effect on the JOYO core of the SMIR structural material irradiation tests, including the small amounts of low atomic weight materials, will be a small decrease in adjacent fuel pin powers compared to the reference radial reflector subassemblies. This small negative effect should satisfy concerns over potential JNC licensing issues related to the impact on the core for these tests. Further evaluation by $\mathrm{NC}$ core management will determine if the small negative reactivity effect of the tests will need to be compensated by adjustment of the Cycle 5-6 core loadings.

\section{References}

1. B-MT (EDT) S-013, Revision 0, Nuclear Calculations and Thermal Properties for Prometheus I Structural Material Irradiation Capsule in the JOYO Reactor.

2. Wootan, D., PNNL-15252, Heating Rates and Neutron Flux for JOYO-1 Irradiation Tests Revision 1, Pacific Northwest National Laboratory, Richland, Washington, 2005.

3. X-5 Monte Carlo Team, LA-UR-03-1987, MCNP - A General Monte Carlo N-Particle Transport Code, Version 5, Los Alamos National Laboratory, Los Alamos, New Mexico, 2004. 
4. Doherty, A., PNL-MA-875, Computer Code Maintenance Software Quality Assurance Manual, Revision 0, Pacific Northwest National Laboratory, Richland, Washington, 2001. 
Pacific Northwest

National Laboratory

Operated by Battelle for the

U.s. Dejurtment of Energy
Project $\$ 48552$

SRM-LRPT-005

PNNL-15295

July 2005

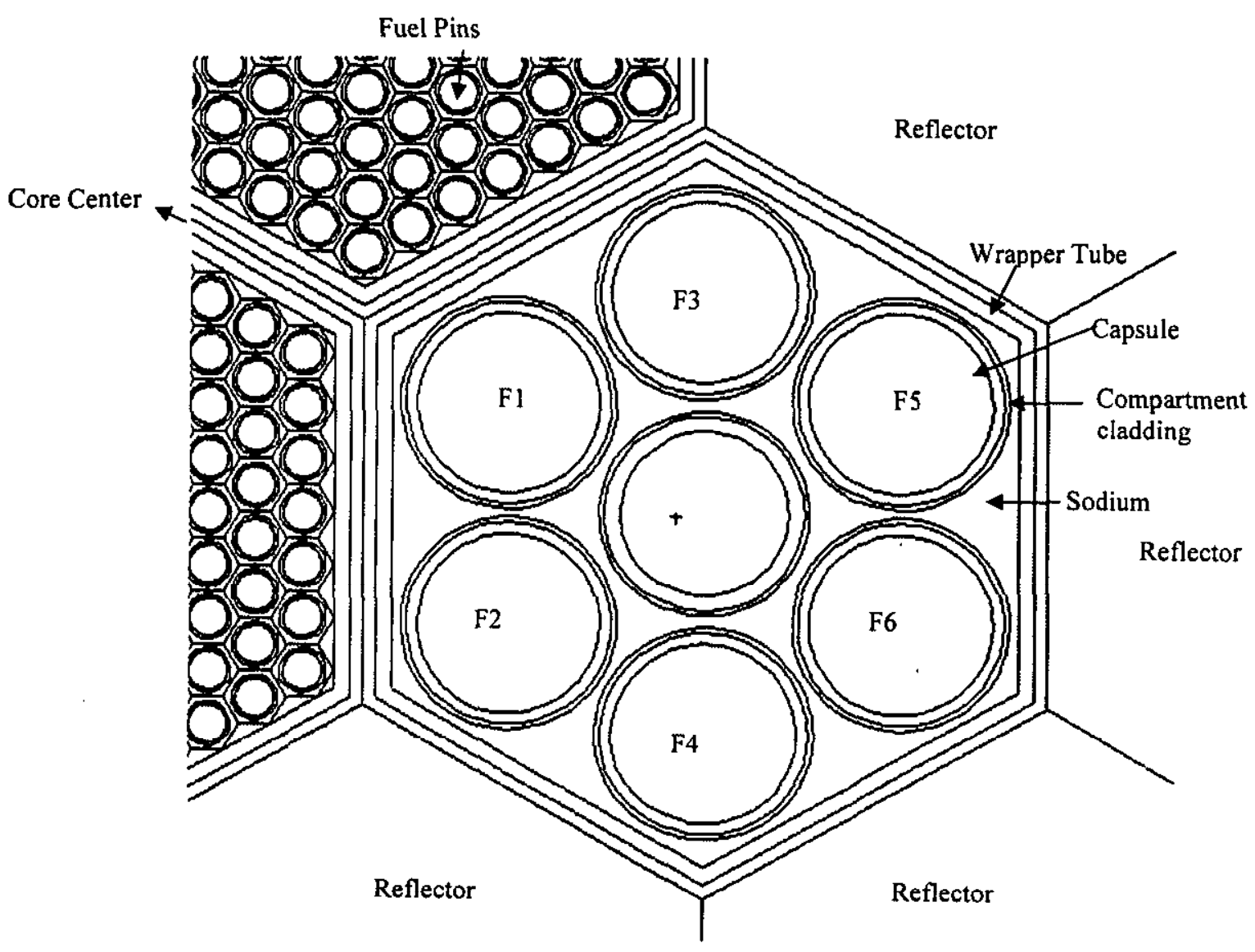

Figure 1. Cross Section View of MCNP Model of Test in JOYO Core Location 6A4 


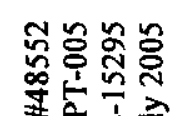

步起点
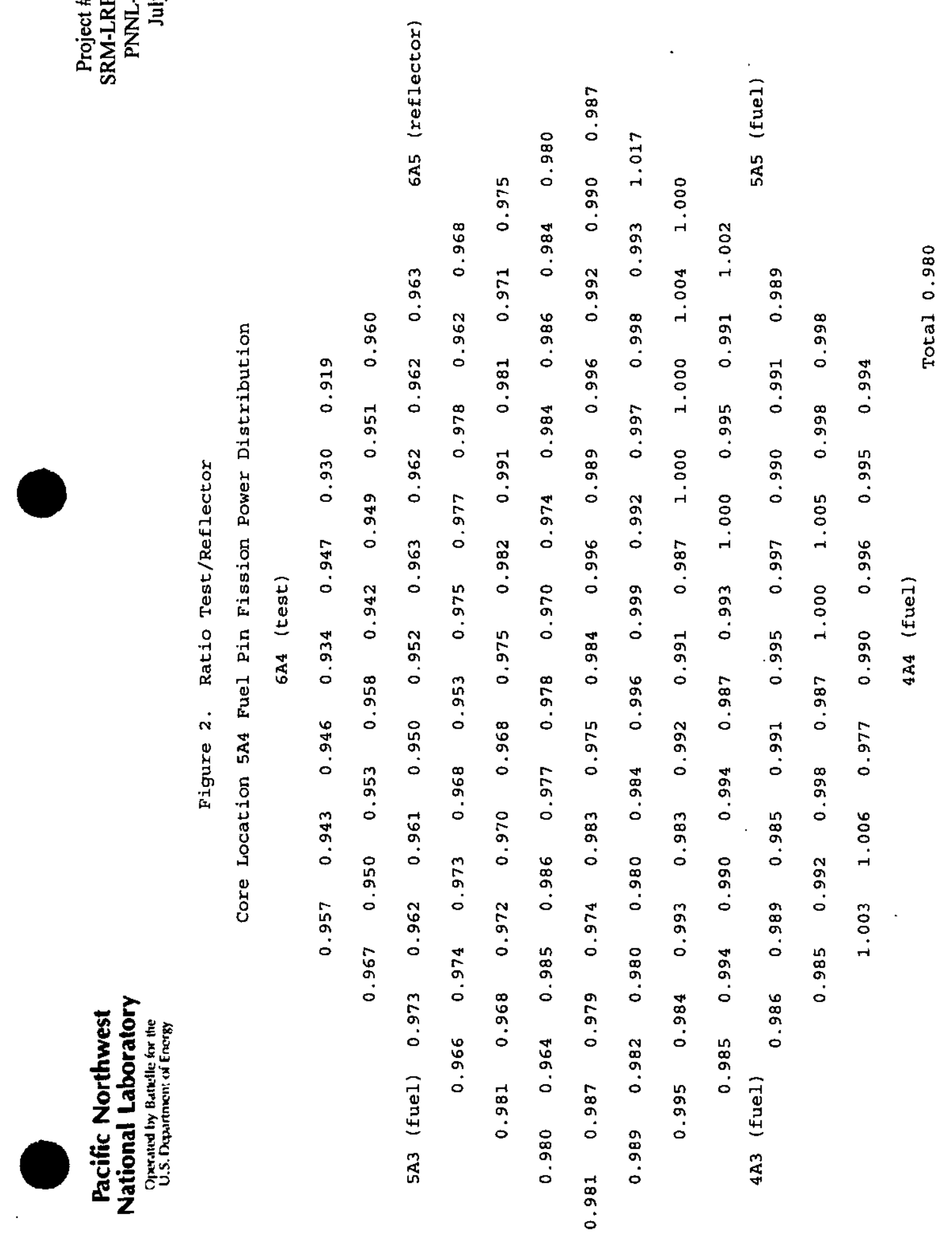

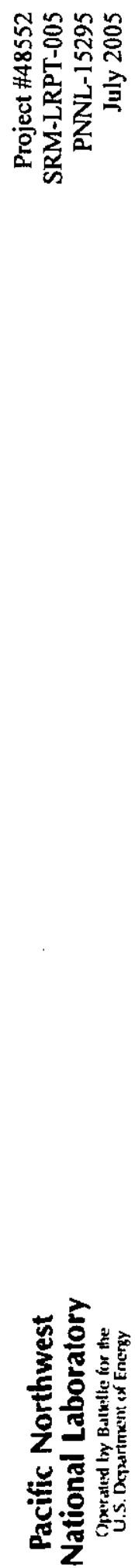

$$
\text { Wh }
$$


둥ํㅇ

के 궁

表

s.

造

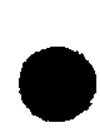

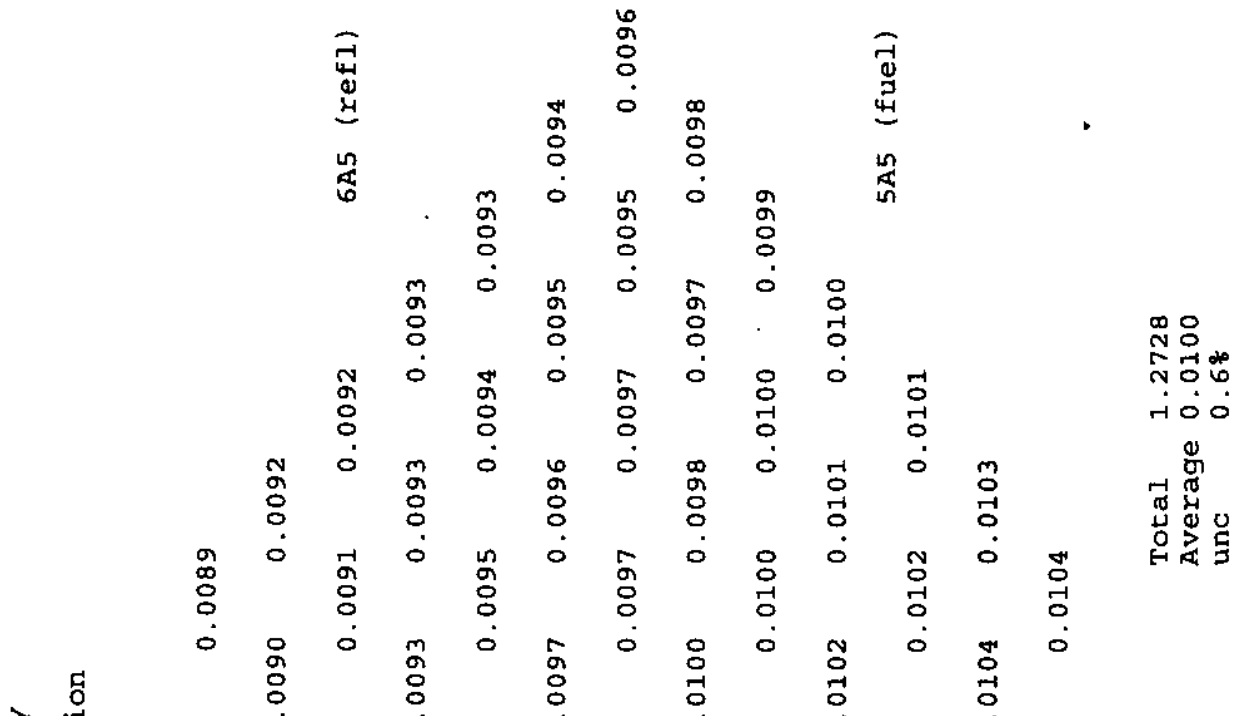

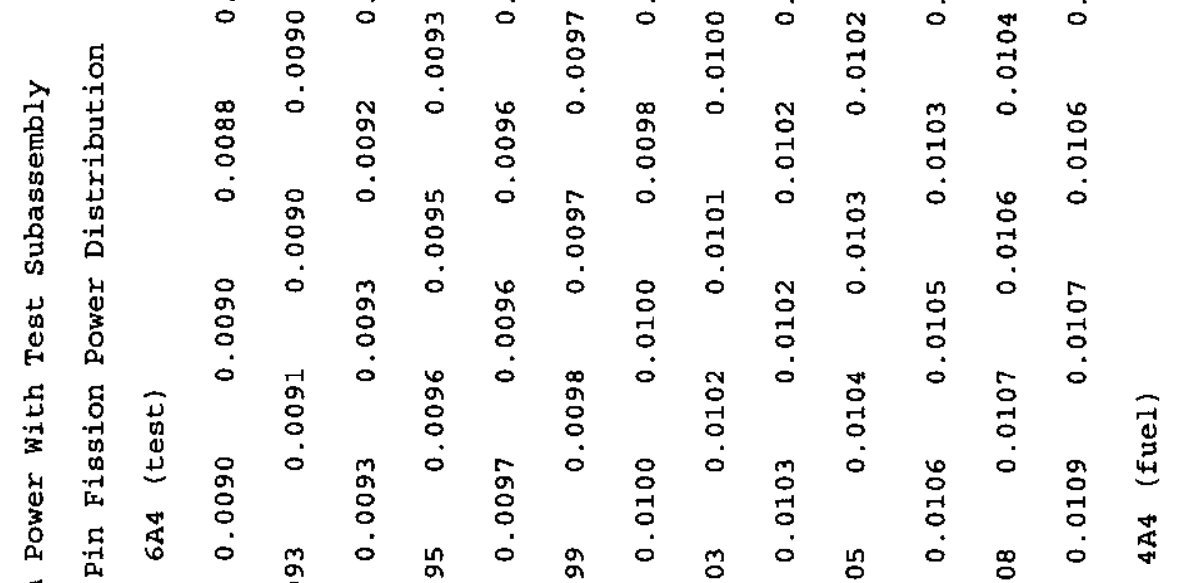

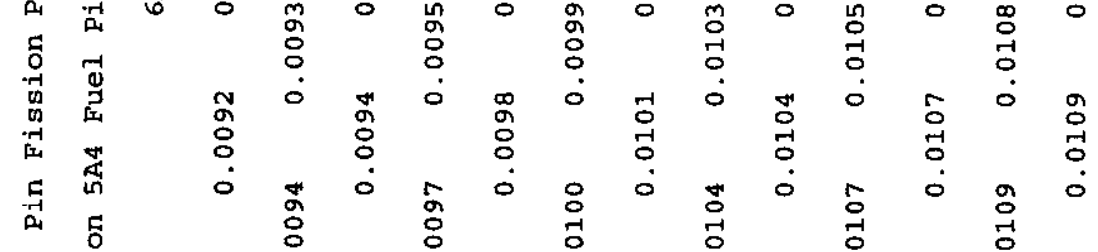

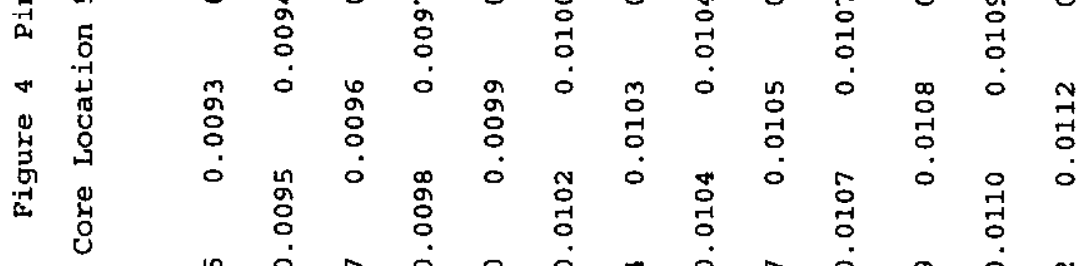

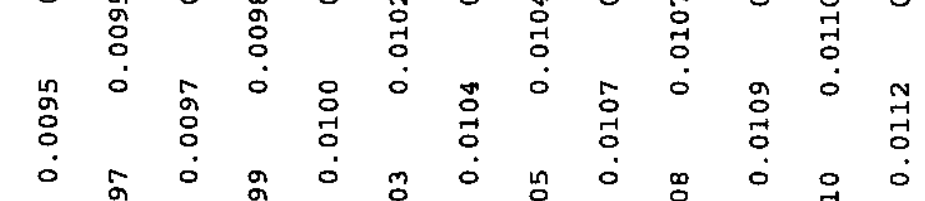

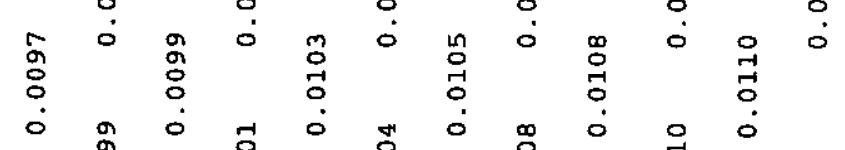

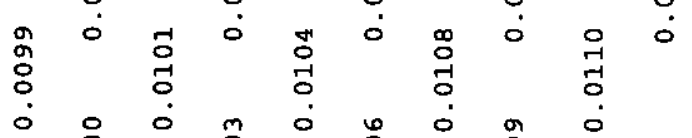

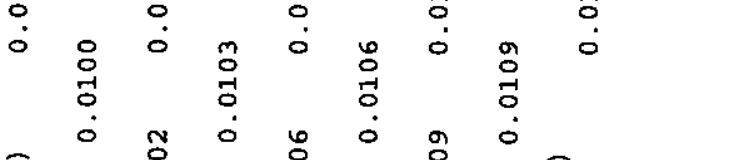

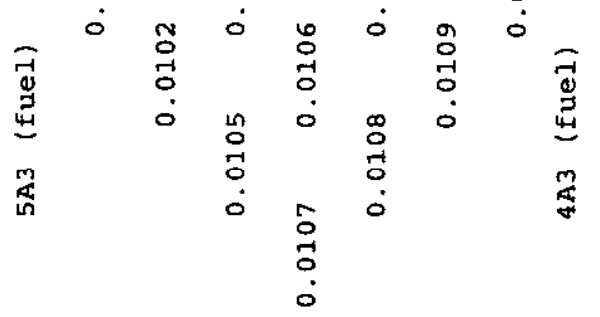

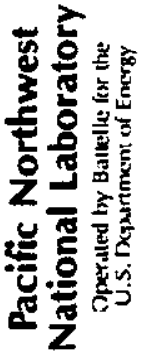

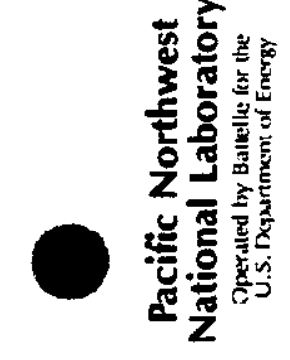




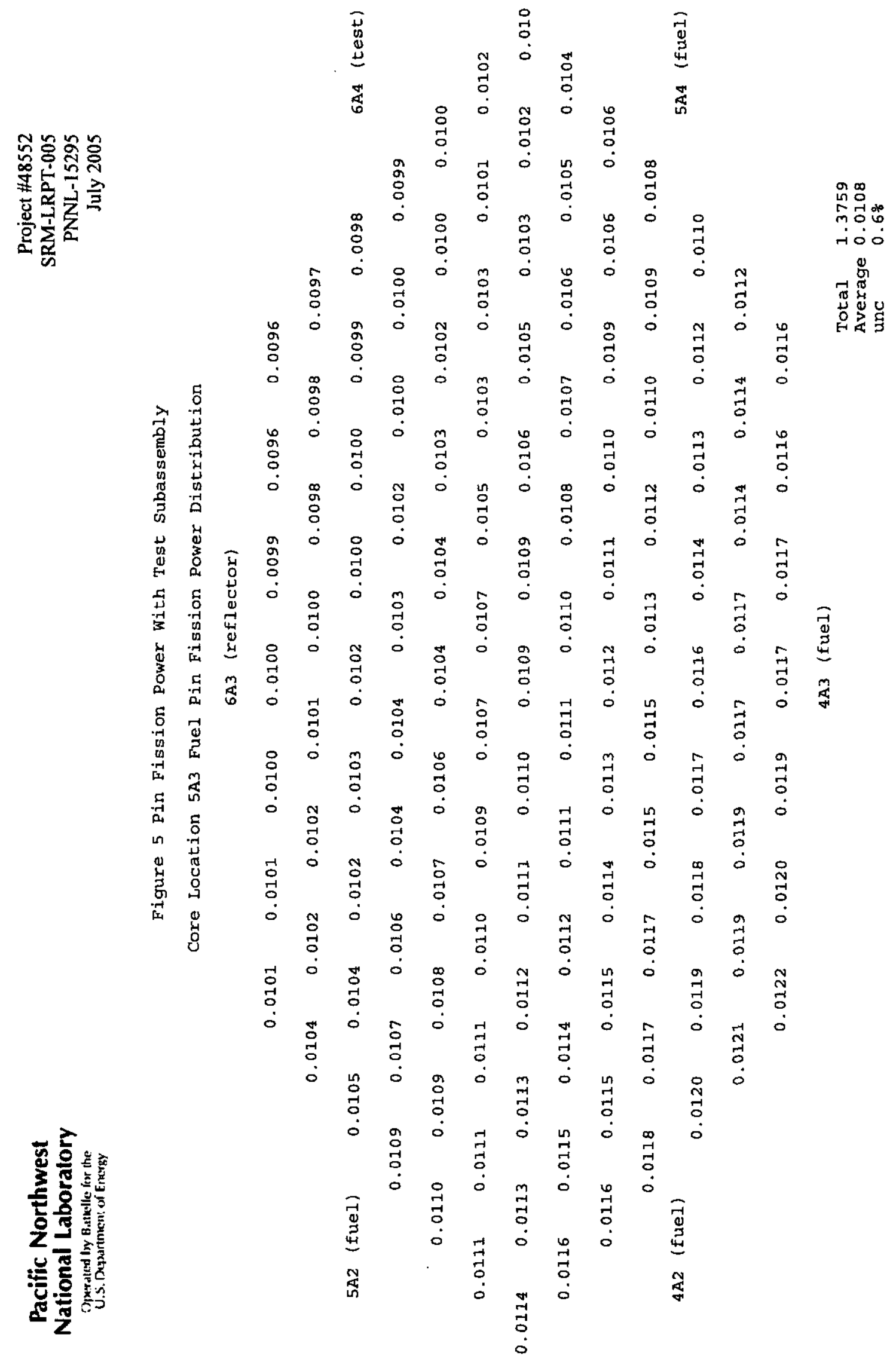



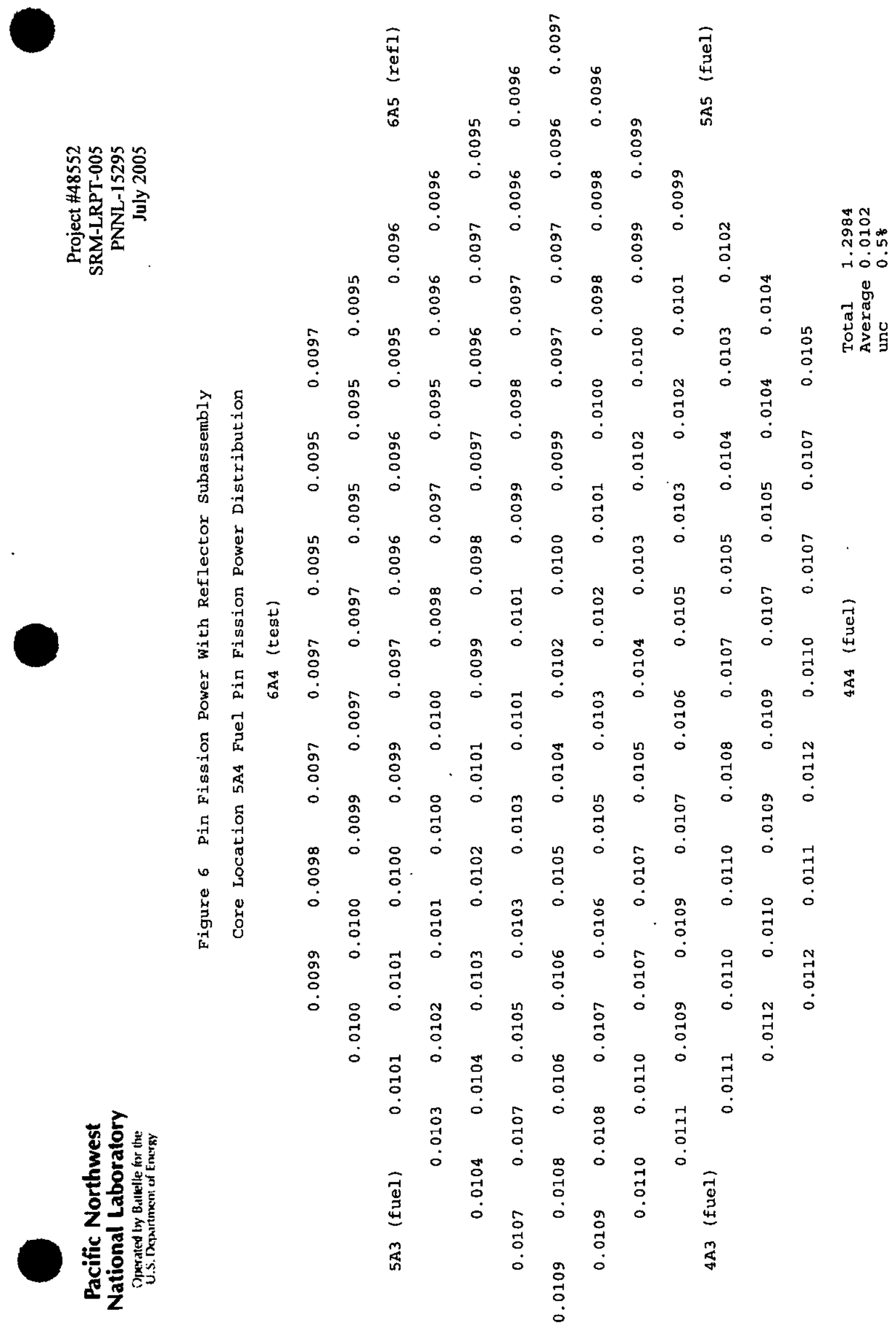


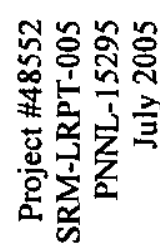

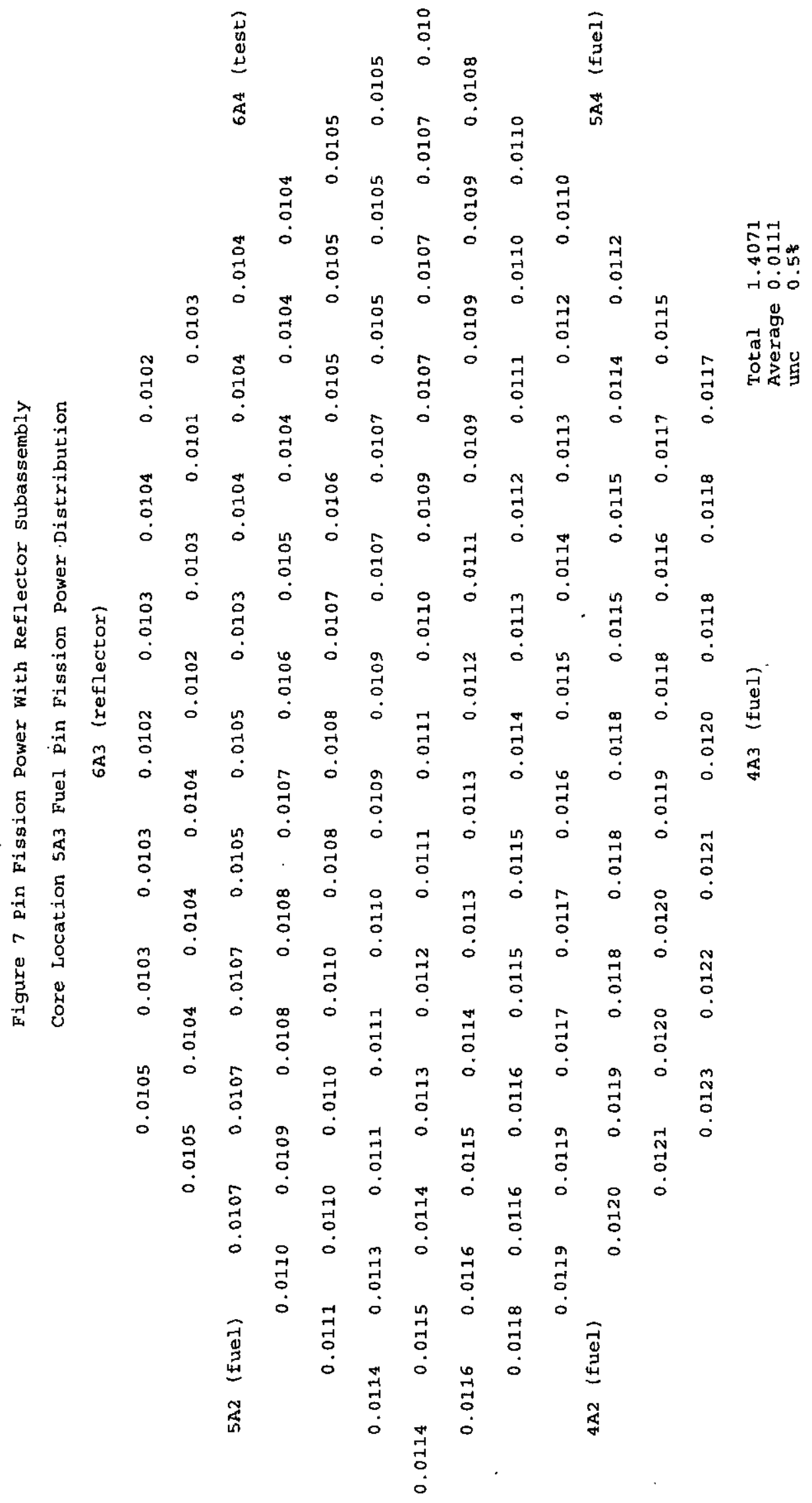

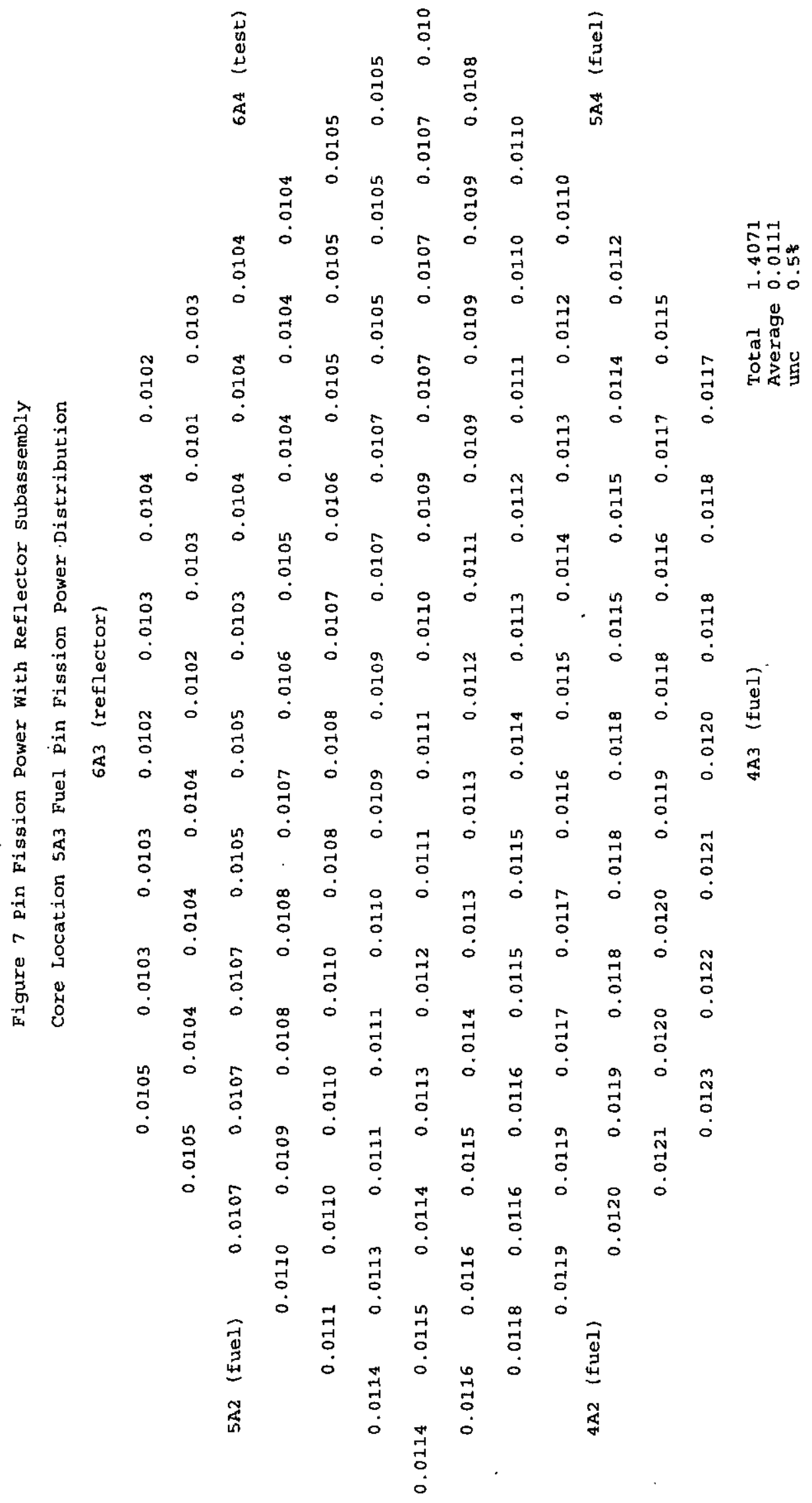

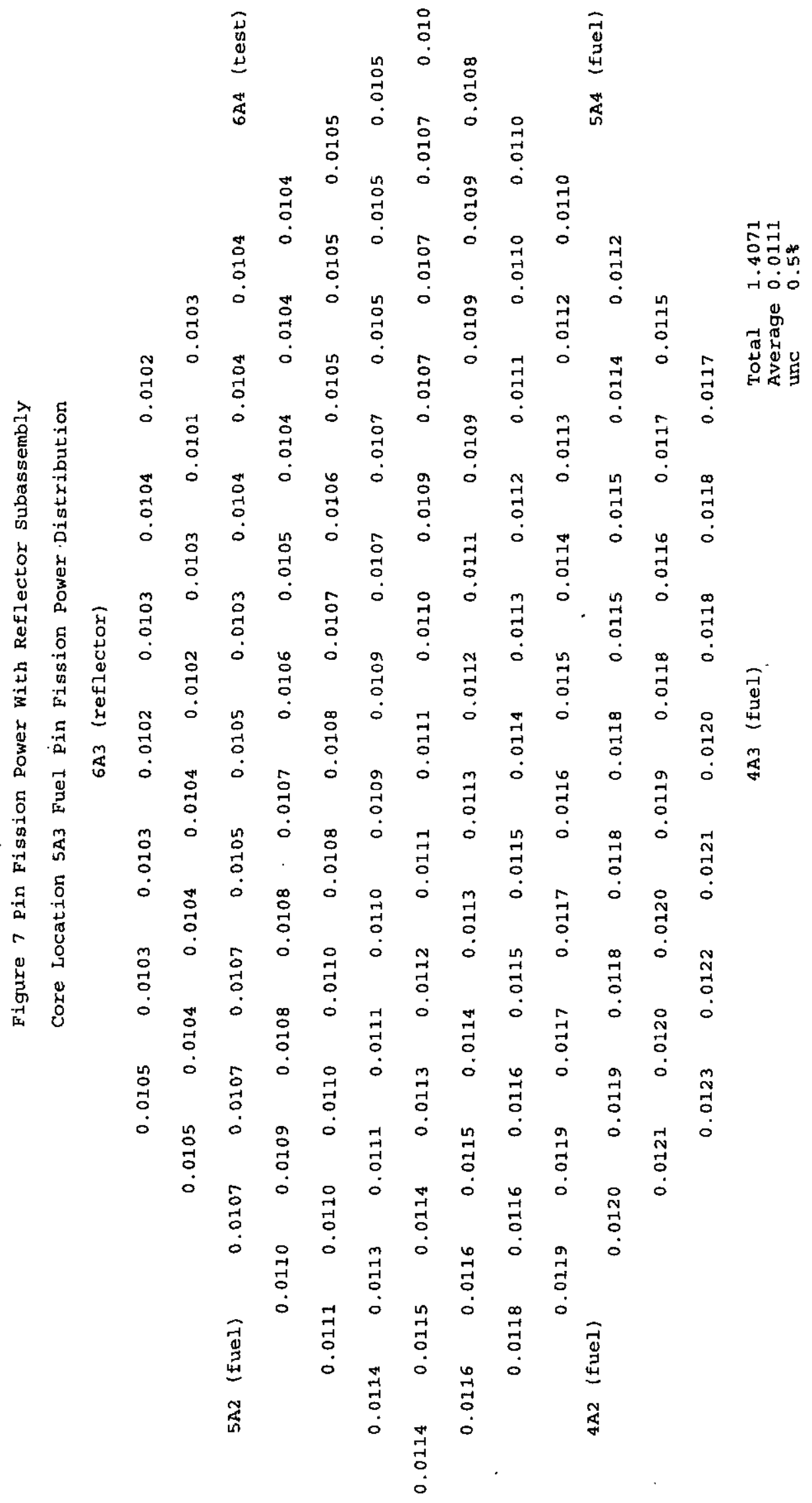

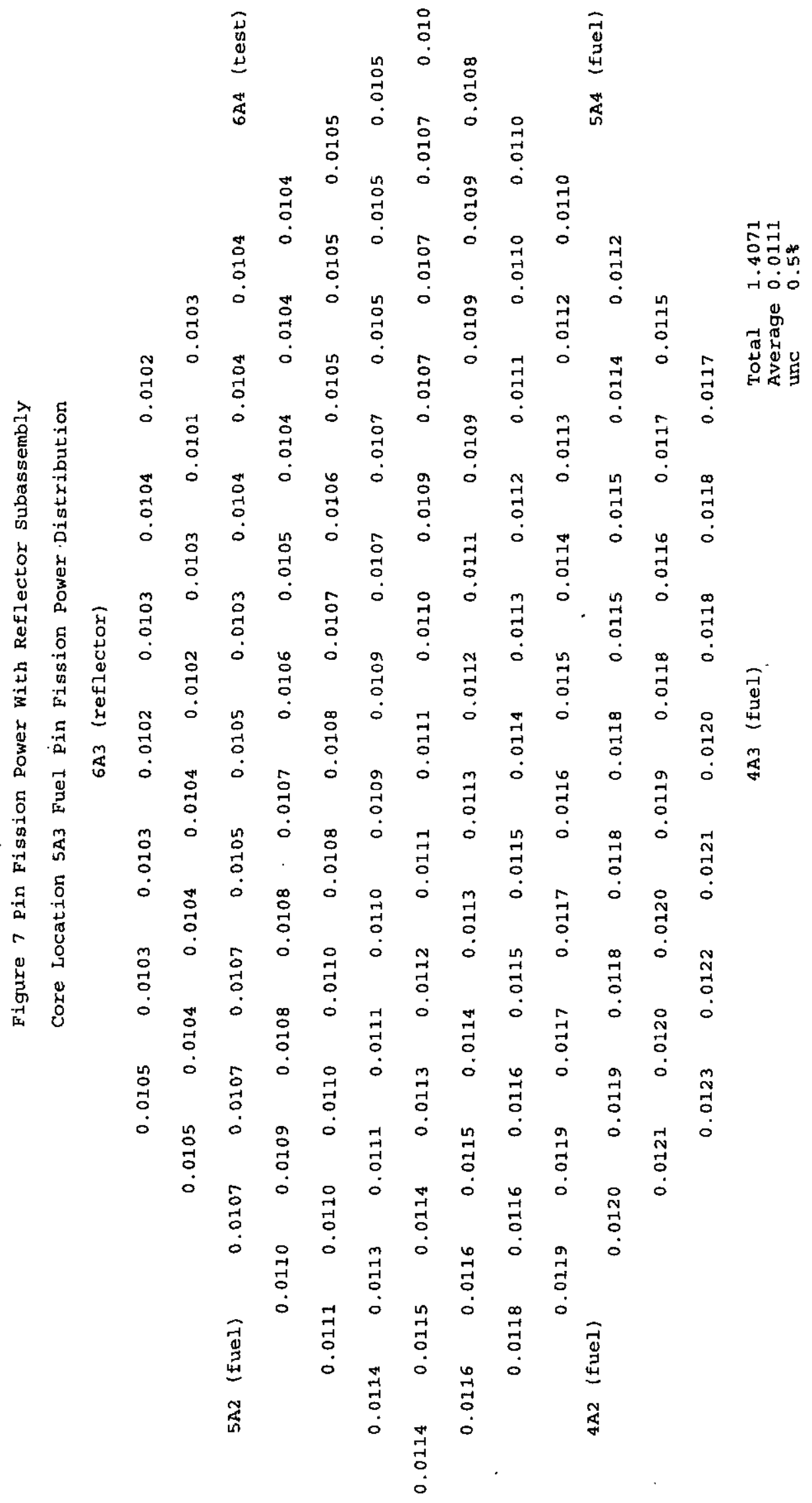

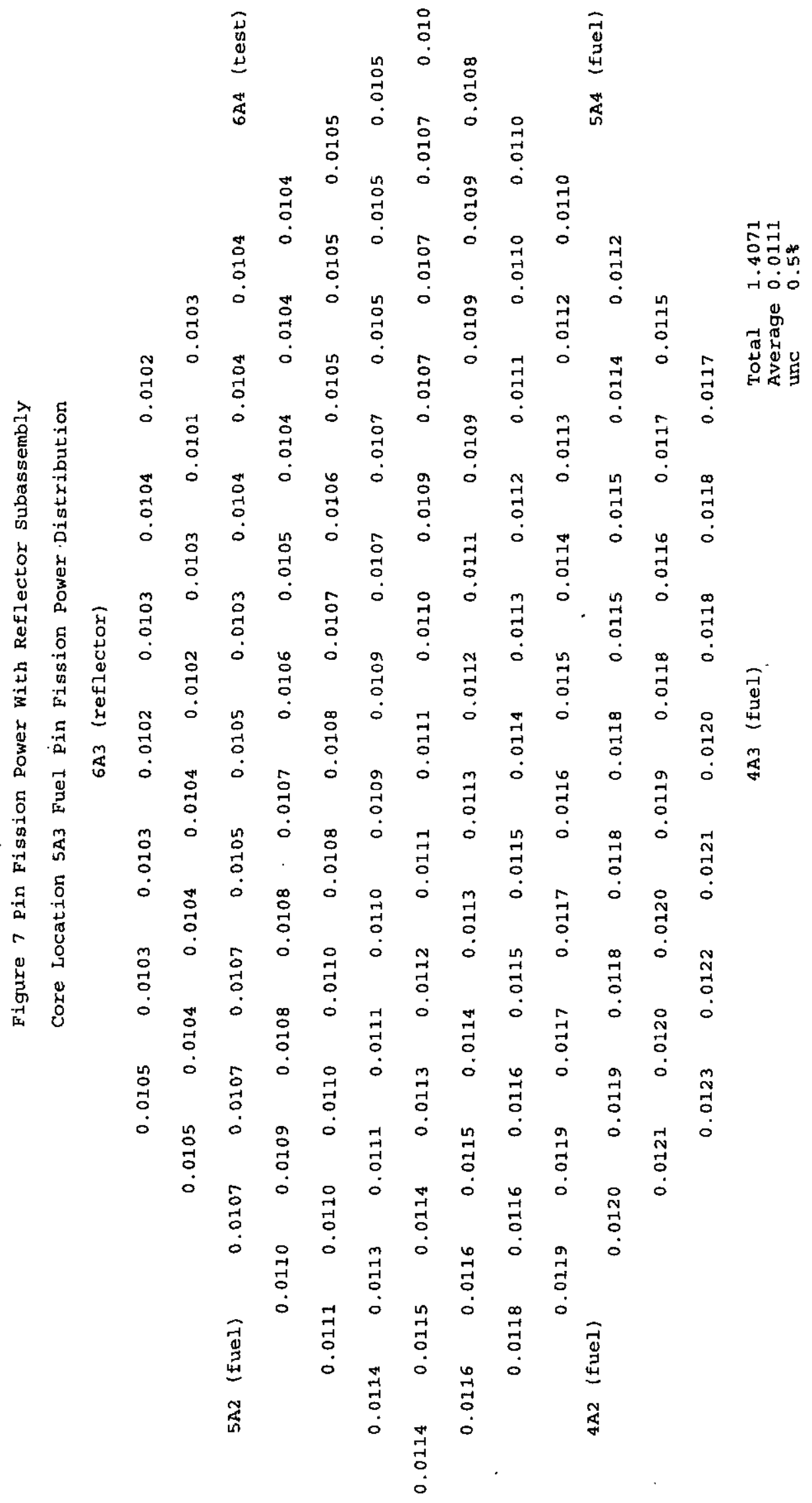

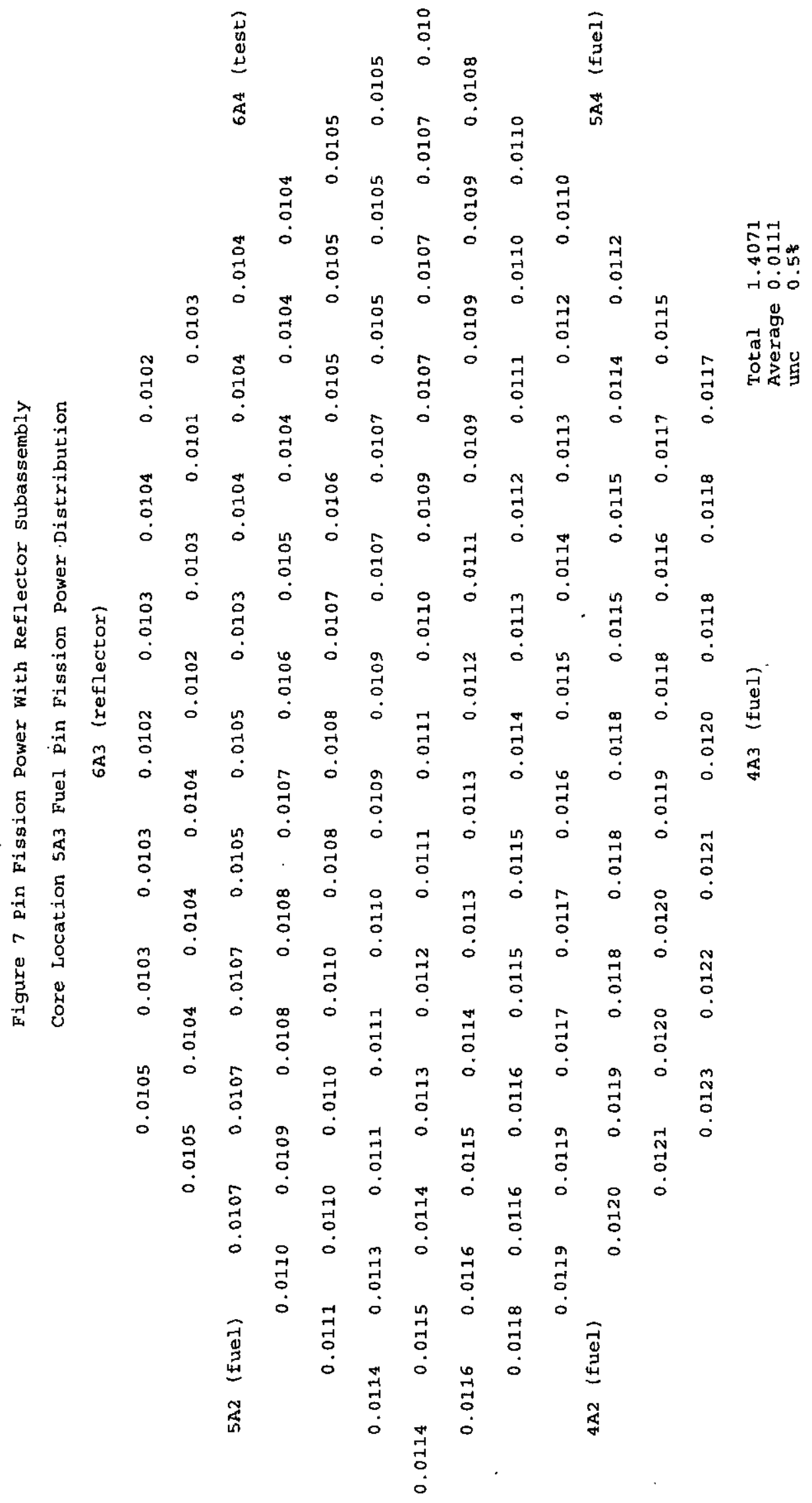

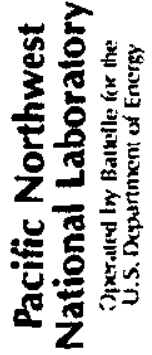




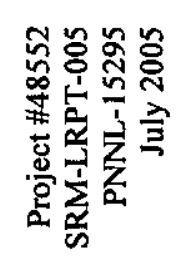

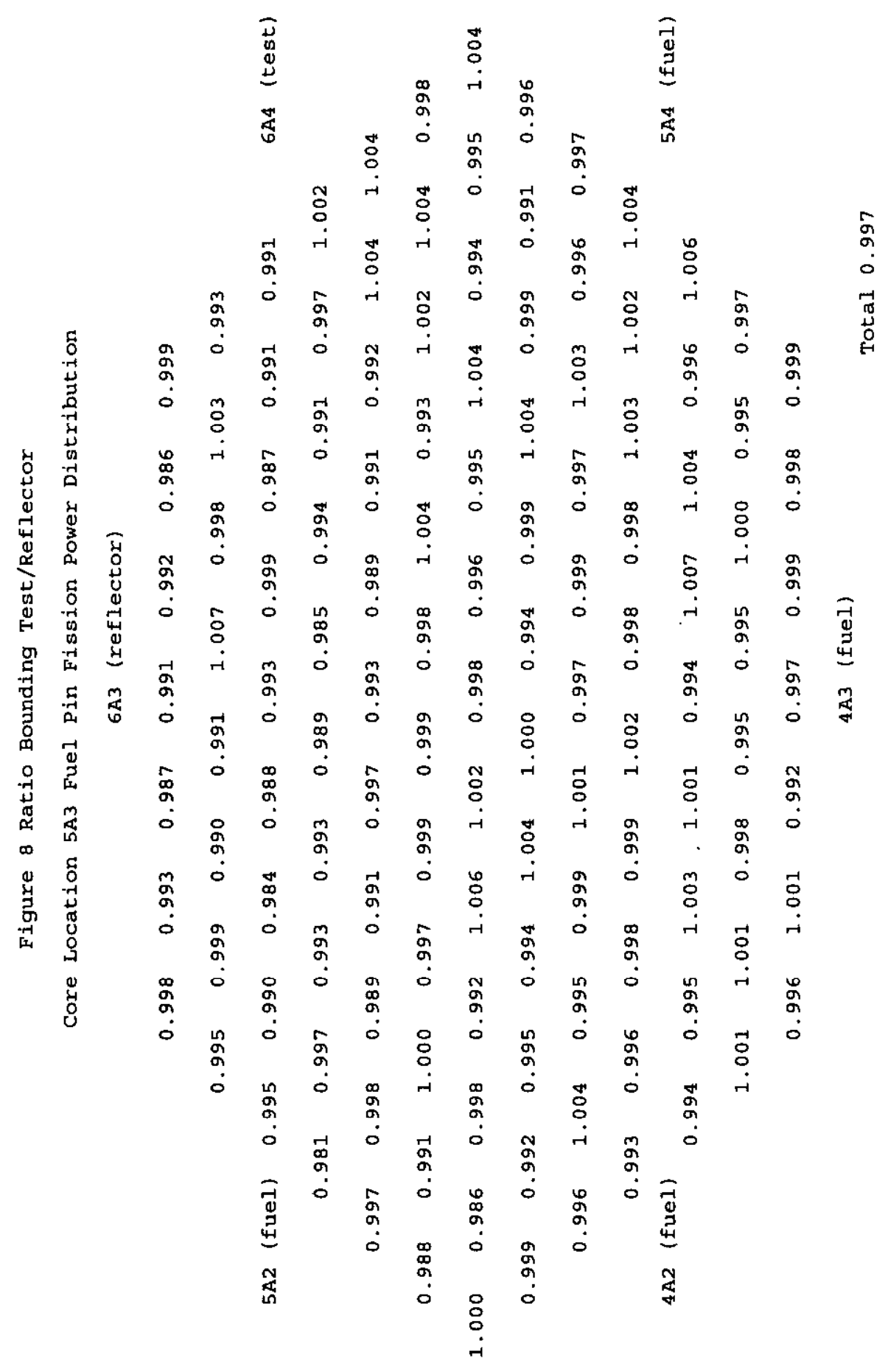



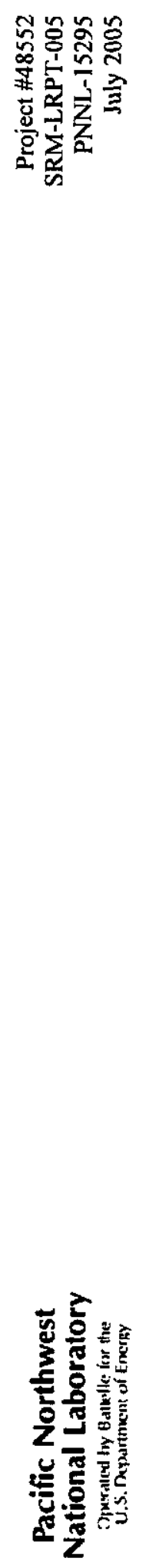

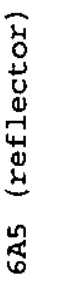

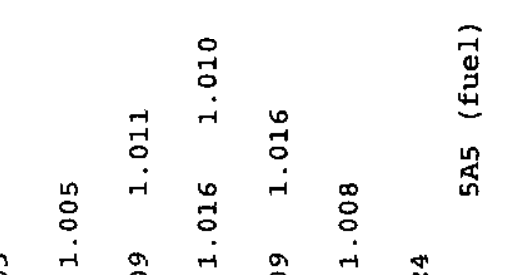

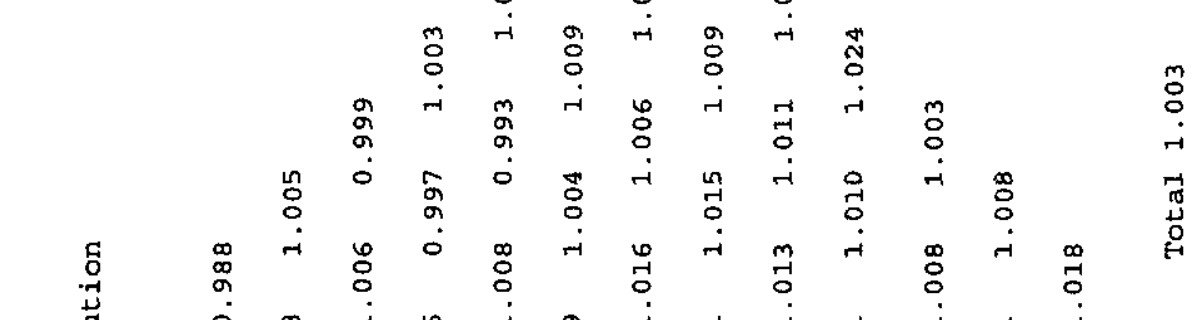

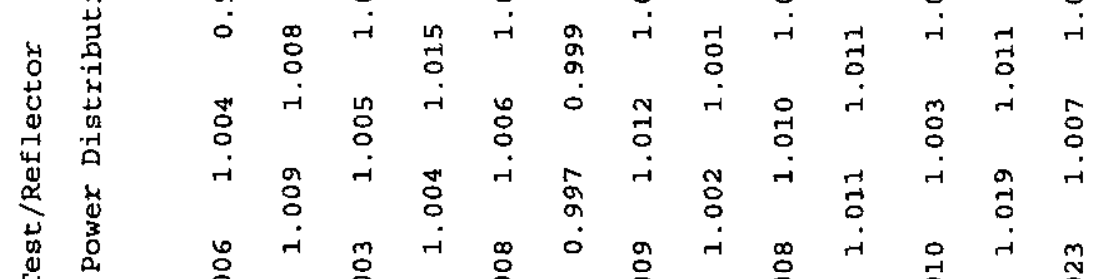

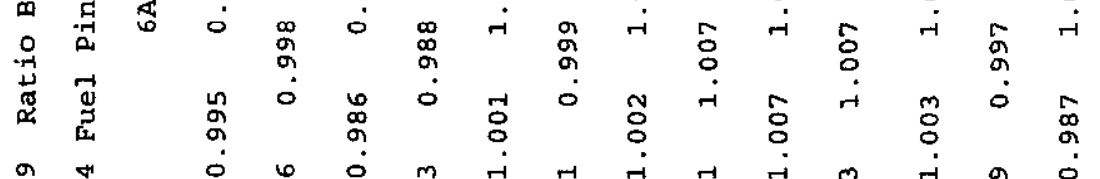

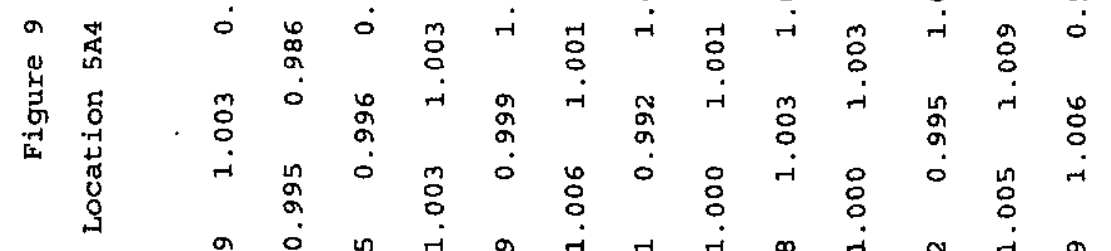

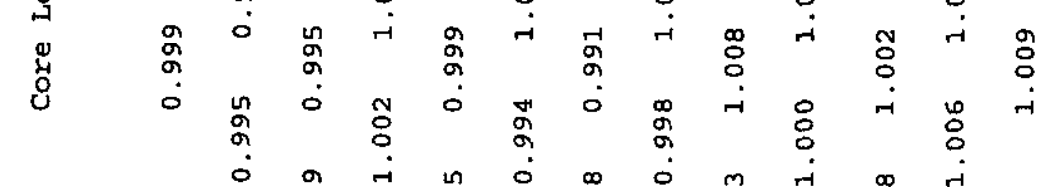

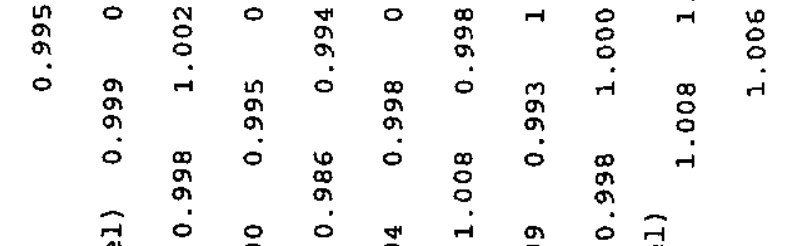

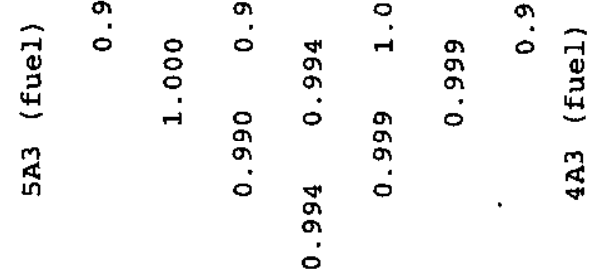




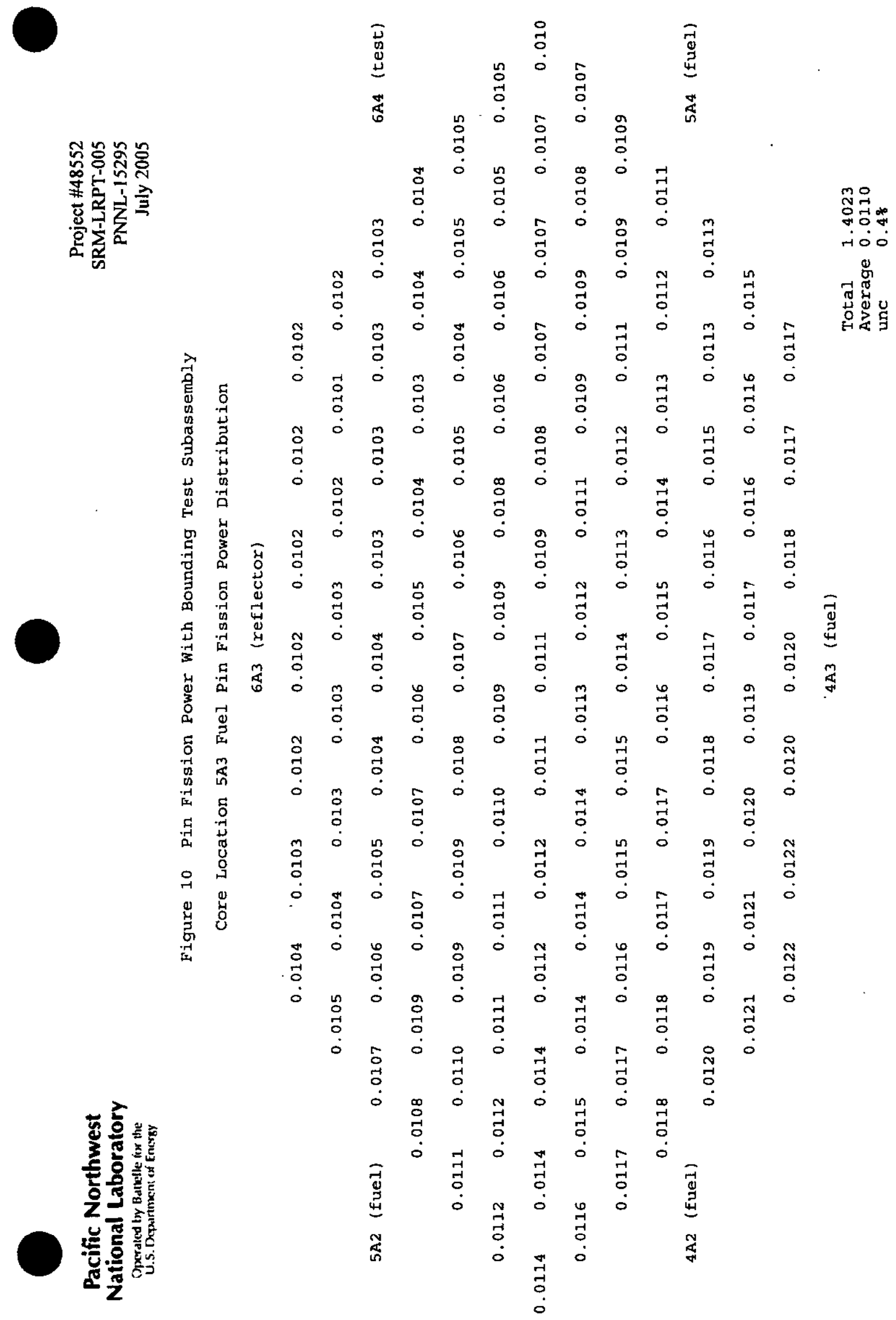



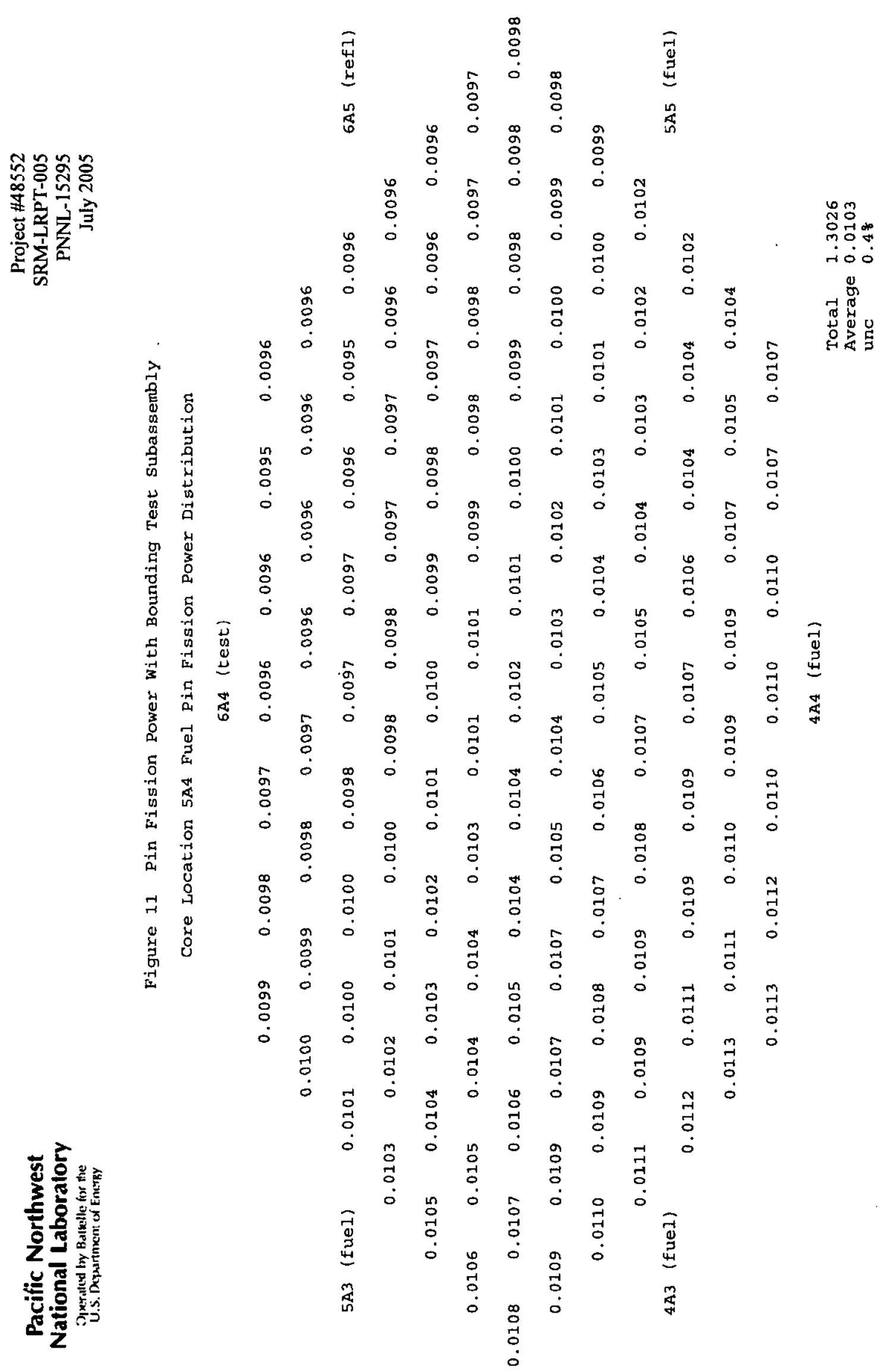
Attachment B of

Enclosure (7) to

MDO-723-0044!

B-MT(SRME)-52

Page 1

Attachment B of

Enclosure 7 to MDO-723-0044/B-MT(SRME)-52

Review of NRPCT Interpretation of JNC Supplied

Gamma Heating Rates 
Attachment B of Enclosure (7) to MDO-723-0044I

B-MT(SRME)-52

Page 2 


\title{
Review of NRPCT Interpretation of JNC Supplied Gamma Heating Rates
}

\author{
Author: Dr. David Wootan
}

\begin{abstract}
In March, 2005, JNC supplied calculated gamma heating rates for a preliminary MK-III cycle 5 core loading using their standard core analysis methods. A review of the NRPCT interpretation of the $\mathrm{JNC}$ supplied heating rates did not discover any errors in the interpretation. This review was limited to the NRPCT interpretation of gamma heating rates that were supplied by $\mathrm{NNC}$ in a spreadsheet. Actual heating in materials must consider the combination of both gamma and neutron heating.

\section{Objective}

Confirm the NRPCT interpretation of JNC supplied gamma heating rates as described in Attachment A of the SOW (reference). If the interpretation is incorrect, identify the correct interpretation of gamma heating of each compartment within the SMIR.
\end{abstract}

\section{JNC Calculations}

In March, 2005, JNC calculated gamma heating rates for a preliminary MK-III cycle 5 core loading using their standard core analysis methods. The gamma heating rates were calculated using the DORT transport code and $2 \mathrm{D} \mathrm{X}-\mathrm{Y}$ and $\mathrm{R}-\mathrm{Z}$ whole core models with Japanese evaluated cross sections (based on JENDL3.2). JNC provided to NRPCT an Excel spreadsheet (heat_rate.xls) summarizing the results of the calculations, including $\mathrm{X}$-Y mesh heating values (watts/cc) in steel, sodium, molybdenum, and carbon at core midplane and four row 6 axial distributions for heating.

The 2D X-Y whole core model used a rectangular mesh, with each hexagonal subassembly represented by a homogenized rectangular zone containing $4 \times 4$ mesh points. Each rectangular subassembly in the $X-Y$ model was $8.15 \times 7.058 \mathrm{~cm}$, compared to the actual hexagonal subassembly which is $8.15 \mathrm{~cm}$ flat-to-flat. The Row 6 compositions were homogenized steel pin-type reflector compositions. The $\mathrm{X}-\mathrm{Y}$ model extended in both $\mathrm{X}$ and $\mathrm{Y}$ dimensions from -107 to $+107 \mathrm{~cm}$ from core center.

The 2D R-Z whole core model used radial and axial mesh, with annular zones of varying thickness representing the different rows of fuel, reflectors, shields, etc. The annular zone representing the Row 6 reflectors was subdivided into 4 annular regions, with Row 6-1 adjacent to the Row 5 fuel and Row 6-4 adjacent to Row 7 reflectors. The R-Z model extended axially from -155.5 to $+570.6 \mathrm{~cm}$ from core midplane. 


\section{NRPCT Interpretation}

The NRPCT interpretation of the JNC supplied gamma heating values is described in Attachment A of the SOW. The preferred locations for the two Row 6 SMIR tests are in the mid-flat positions (JOYO core locations 6A4 and 6D4). In these locations, the SMIR hex key is used to maintain the compartment orientation relative to the core such that compartment \#1 is closest to the core center, compartments \#2 and \#6 are equidistant, compartments \#3 and \#5 are equidistant, and compartment \#4 is furthest from core center. The $16 \mathrm{X}-\mathrm{Y}$ mesh values corresponding to each location were identified and a correspondence was established between the $16 \mathrm{X}-\mathrm{Y}$ mesh points and the 6 compartments in the hexagonal subassembly. The heating rates at core midplane in each of the six compartments were obtained by combinations of the $X-Y$ mesh values. The four axial heating distributions were also mapped to the six compartments, with the Row 6-1 distribution used for compartment \#1, Row 6-2 used for \#2 and \#6, Row 6-3 used for \#3 and $\# 5$, and Row 6-4 used for \# 4. Axial heating distributions in each compartment were obtained by normalizing the $R-Z$ axial distribution values at $Z=-1.32 \mathrm{~cm}$ (approximate core midplane) values and then multiplying by the $X-Y$ model derived core midplane heating values for each compartment.

\section{Assumptions and Limitations}

This analysis was limited to confirming the NRPCT interpretation of gamma heating rates supplied by $\mathrm{NC}$ in a specific spreadsheet. No new computer or hand calculations were required for this analysis. Engineering judgement was used that was based on previous experience with JNC DORT calculations and conversations with JNC staff.

The NRPCT interpretation in Appendix A of the SOW only describes the X-Y mesh mapping for the 6D4 core location. The NRPCT mesh mapping for the $6 \mathrm{~A} 4$ core location was not provided. It is assumed that a consistent mesh mapping was also used for the $6 \mathrm{~A} 4$ values.

$\mathrm{JNC}$ has not provided details of the radial mesh and zoning used in the $2 \mathrm{D} \mathrm{R}-\mathrm{Z}$ model to allow confirmation of the details of the Row 6 segmentation into the four radial zones (Row 6-1 through 6-4). However, the 2D R-Z model appears consistent with the 2D X-Y model.

The JNC supplied heating rates are for homogenized reflector subassemblies in Row 6 core locations. Actual SMIR test subassembly compositions include materials such as molybdenum with neutron and gamma ray absorption cross sections higher than steel that may affect the gamma heating rates.

\section{Discussion of NRPCT Interpretation}

A review of the NRPCT description of their interpretation of the NNC supplied heating rates has not discovered any errors in the interpretation. General comments are as follows: 
1. The $\mathrm{X}-\mathrm{Y}$ mesh and their orientation that were identified for the $6 \mathrm{D} 4$ core location by NRPCT are correct.

2. The $\mathrm{X}-\mathrm{Y}$ heating values in the spreadsheet provided by $\mathrm{N} \mathrm{NC}$ were confirmed to represent core midplane values.

3. The mapping of core midplane heating rates between the $16 \mathrm{X}-\mathrm{Y}$ mesh values in the calculation model and the 6 compartments in the subassembly appears to be adequate to reflect the radial gradients across the subassembly. The mapping has the proper orientation relative to the core center, and approximates the location of the six compartments. A variety of mapping schemes could be envisioned with varying degrees of complexity, such as fitting the data points to a quadratic surface and then evaluating the fitting equation for the points corresponding to the centers of the compartments. However, the inherent approximation in representing hexagonal subassemblies by fairly coarse rectangular mesh makes it difficult to justify more elaborate mapping techniques.

4. The mapping of the four axial profiles to the 6 compartments adequately captures the small flattening effect in the axial peak to average heating as the distance from the core center is increased across the subassembly. The average of the Row 6-1 through 6-4 axial heating values at core midplane from the $\mathrm{R}-\mathrm{Z}$ model corresponds to the average of the row 6 heating values from the $X-Y$ model.

\section{Caution}

Actual heating in materials will be a combination of gamma and neutron heating. The gamma heating values supplied by $\mathrm{JNC}$ appear to be only gamma heating. For heavy materials like tungsten, the neutron heating is negligible. For steel, the neutron heating may add up to $10 \%$. However, for light elements like carbon, oxygen, beryllium, etc., the total neutron plus gamma heating may be substantially higher than the gamma heating alone.

Table 1 shows preliminary neutron and gamma heating rates in JOYO MK-III core for materials in a row 6 mid-flat reflector location averaged across the homogenized hexagonal subassembly in a $5 \mathrm{~cm}$ axial slice at core midplane. These preliminary values are provided to illustrate the general characteristics of the heat deposition in this type of location in a fast reactor such as JOYO. They are based on a Cycle 1 core loading instead of Cycle 5, and were generated to test the MCNP heating tallies and to provide a rough check on the $\mathrm{NNC}$ gamma heating values. Note that the gamma heating is mainly from electron interactions and thus increases smoothly with the atomic weight of the material. The neutron heating is mainly from nuclear interactions and can vary depending on particle reactions such as (n,p) (n,alpha) but generally decreases with increasing atomic weight. Note that the axial distribution of neutron heating may be different from the axial distribution of gamma heating. 
Table 1. Preliminary Neutron and Gamma Heating Rates in Row 6 Using MCNP

\begin{tabular}{|l|r|r|r|}
\hline & \multicolumn{3}{|c|}{ Row 6 core midplane heating } \\
& \multicolumn{3}{|c|}{ w/g } \\
\hline material & neutron & gamma & total \\
\hline $\mathrm{B}$ (nat) & 64.1 & 1.3 & 65.3 \\
\hline $\mathrm{Be}$ & 7.0 & 1.2 & 8.2 \\
\hline $\mathrm{C}$ & 3.6 & 1.4 & 5.0 \\
\hline $\mathrm{O}$ & 2.4 & 1.4 & 3.8 \\
\hline $\mathrm{Al}$ & 0.9 & 1.4 & 2.2 \\
\hline $\mathrm{Si}$ & 0.7 & 1.4 & 2.1 \\
\hline $\mathrm{Ti}$ & 0.3 & 1.4 & 1.7 \\
\hline $\mathrm{V}$ & 0.3 & 1.4 & 1.7 \\
\hline $\mathrm{Cr}$ & 0.2 & 1.5 & 1.7 \\
\hline $\mathrm{Mn}$ & 0.2 & 1.5 & 1.7 \\
\hline $\mathrm{Fe}$ & 0.2 & 1.5 & 1.7 \\
\hline $\mathrm{Co}$ & 0.3 & 1.5 & 1.9 \\
\hline $\mathrm{Ni}$ & 0.3 & 1.6 & 1.9 \\
\hline $\mathrm{Zr}$ & 0.2 & 2.0 & 2.2 \\
\hline $\mathrm{Nb}$ & 0.2 & 2.0 & 2.3 \\
\hline $\mathrm{Mo}$ & 0.1 & 2.1 & 2.2 \\
\hline $\mathrm{Hf}$ & 0.04 & 4.1 & 4.1 \\
\hline $\mathrm{Ta}$ & 0.04 & 4.2 & 4.2 \\
\hline $\mathrm{W}$ & 0.04 & 4.3 & 4.3 \\
\hline $\mathrm{Re}$ & 0.04 & 4.3 & 4.3 \\
\hline $\mathrm{steel}$ & 0.2 & 1.6 & 1.7 \\
\hline & & & \\
\hline
\end{tabular}

Table 2 compares the preliminary MCNP gamma-only heating rates with the JNC provided gamma heating rates at core midplane for core location 6A4. Good agreement was found. The minor differences between the MCNP and JNC heating values are likely due to the different core loadings and other model differences.

Table 2. Comparison of JNC Supplied Gamma Heating with MCNP

\begin{tabular}{|c|c|c|}
\hline & \multicolumn{2}{|c|}{ gamma heating rate, $\mathrm{w} / \mathrm{g}$} \\
\hline material & MCNP & JNC \\
\hline $\mathrm{C}$ & 1.4 & 1.5 \\
\hline steel & 1.6 & 1.7 \\
\hline Mo & 2.1 & 2.3 \\
\hline
\end{tabular}


Pacific Northwest

Project $\# 48552$

National Laboratory

SRM-LRPT-001

Operated by Batelle for the

May 2005

\section{Reference}

B-MT(EDT)S-012, Revision 1, Nuclear Neutron Flux and Gamma Heating Rates and Independent Thermal Analysis Review for a Prometheus I Structural Material Capsule in the JOYO Reactor Scope of Work. 
Attachment $\mathrm{C}$ of

Enclosure (7) to

MDO-723-0044/

B-MT(SRME)-52

Page 1

\section{Attachment C of}

\section{Enclosure 7 to MDO-723-0044/B-MT(SRME)-52}

Heating Rates and Neutron Flux for JOYO-1 Irradiation Tests Revision 2 
Attachment $C$ of

Enclosure (7) to

MDO-723-0044/

B-MT(SRME)-52

Page 2

THIS PAGE INTENTIONALLY BLANK 


\title{
Heating Rates and Neutron Flux for JOYO-1 Irradiation Tests - Revision 2
}

\author{
Author: David Wootan
}

\begin{abstract}
Neutron and gamma heating rates and fast flux values were calculated at $2 \mathrm{~cm}$ axial increments and in three radial regions in the sixty Naval Reactor's Prime Contactor Team (NRPCT) structural irradiation capsules in proposed JOYO-1 Structural Materials Irradiation Rigs (SMIRs) in the JOYO MK-III test reactor core locations 6A4 and 6D4 during Cycle 5 and Cycle 6 . The calculations were made with the MCNP code using a whole core model that was based on a preliminary Cycle 5 core loading. Subassembly test capsule compositions were based on Elemental Makeup Revision 2.
\end{abstract}

\section{Objective}

The most important consideration for safety and performance is the neutron and gamma heating of the NRPCT capsule and specimen holders. Since the gamma heating rates are dependent upon the materials located within the JOYO reactor, several nuclear iterations are required.

JNC initially provided preliminary core midplane fast flux values and gamma heating rates for selected materials along with axial and radial distributions for determining heating in the sixty NRPCT structural irradiation capsules installed in the JOYO SMIRs that are to be located in core positions 6A4 and 6D4 in JOYO Cycle 5. An evaluation of the interpretation of these heating rates was previously provided by PNNL (Reference 1).

Based on the smeared capsule elemental composition data in Elemental Makeup Revision 1 A provided by NRPCT, an initial neutronic evaluation of the irradiation tests was performed to provide axial-dependent gamma and neutron heating rate coefficients (watts/gram) for each of the 60 capsules for each material (Reference 2).

Elemental Makeup Revision 2, containing revised compositions and elemental masses for each capsule and separate specimen masses for each capsule, was subsequently provided by NRPCT. Notable changes from Revision 1A included changing graphite specimen holders to tungsten for capsules containing $\mathrm{SiC}$ specimens. Revision 2 included separate elemental masses for the total capsule, the capsule steel, and the material specimens. Based on the heterogeneous elemental composition data in Elemental Makeup Revision 2, neutronic evaluation of the irradiation tests was performed to provide the gamma and neutron heating rate coefficients (watts/gram) for each of the 60 capsules. The heating rates were generated with $2 \mathrm{~cm}$ axial resolution specific to each of three regions (capsule steel, holder, and specimens) per capsule for each element $(\operatorname{Re}, \mathrm{W}, \mathrm{Ta}$, Hf, Mo, Nb, Zr, Co, Ni, Fe, Mn, Cr, V, Ti, Si, Al, O, C, B, Be, He). This is described as Task 1.1 in the Statement of Work (Reference 3). 
The NRPCT will use the Revision 2 heating rates to reanalyze the capsule designs to meet specimen target temperatures. It is possible that minor changes in specimen or holder dimensions may occur due to modification of gas gap thicknesses to achieve the target temperatures.

\section{MCNP Cycle 5 Core Model}

A preliminary whole core model for JOYO beginning of Cycle 5 core configuration was provided by JNC. This model was generated directly from the JNC core management compositions and includes thermal expansion effects on dimensions and densities. This core model represented the two SMIR irradiation tests as typical steel reflector assemblies. This model was subsequently modified to include the additional ex-core regions, average cycle control rod elevations, and the heterogeneous SMIR test subassembly geometry and compositions. This same core model was previously used for the Revision $1 \mathrm{~A}$ analysis.

\section{MCNP Test Subassembly Model}

The original Cycle 5 core model contained homogeneous reflector subassemblies in the test locations. These were replaced with a heterogeneous model of the pin compartments in each of the tests. The heterogeneous compartment model extends $41.675 \mathrm{~cm}$ above and below core midplane and includes the steel wrapper tube and seven compartments surrounded by sodium. The subassembly model contains six compartments with steel cladding, a gas gap, and 5 tiers of capsules, with the $7^{\text {th }}$ center compartment containing a solid steel rod. Each of the six compartments was modeled as a steel cladding tube with $2.6 \mathrm{~cm}$ outside diameter and $2.47 \mathrm{~cm}$ inside diameter. The pitch between compartments was $2.7 \mathrm{~cm}$. The center compartment was modeled as steel cladding tube with $2.5 \mathrm{~cm}$ outside diameter and $2.38 \mathrm{~cm}$ inside diameter, containing a solid steel rod of $2.0 \mathrm{~cm}$ diameter. The subassembly steel wrapper tube was the same as other subassemblies, with a wall thickness of $0.38 \mathrm{~cm}$.

Five capsules were stacked in each compartment, centered at core midplane. Each capsule was modeled as $16.67 \mathrm{~cm}$ long and $2.2 \mathrm{~cm}$ in diameter, divided into 8 equal axial segments (approximately $2 \mathrm{~cm}$ ) and 3 annular regions. The outer annular region included the capsule steel and was $2.0 \mathrm{~cm}$ outside diameter and $1.82 \mathrm{~cm}$ inside diameter. The middle annular region included the specimen holder material and was $1.82 \mathrm{~cm}$ outer diameter and $0.5 \mathrm{~cm}$ inside diameter. The center cylindrical region included the specimen material and was $0.5 \mathrm{~cm}$ in diameter. The Elemental Makeup Revision 2 capsule elemental masses were converted to MCNP input compositions for the three regions (capsule steel, holder, and specimens) for each capsule. Figure 1 shows a cross section of the test subassembly model for core location 6 A4. Figure 2 shows an axial slice through a capsule and compartment in this subassembly. 
Specific gamma and neutron heating rate coefficients (watts/gram) were calculated for each of the sixty capsules, with eight axial segments (approximately $2 \mathrm{~cm}$ axial resolution) per capsule and three radial regions, specific to each of the six compartments in each of the two SMIRs. Total neutron and gamma heating rates were calculated for each element (Re, W, Ta, Hf, Mo, Nb, Zr, Co, Ni, Fe, Mn, Cr, V, Ti, Si, Al, O, C, B, Be, $\mathrm{He}$ ), whether or not it was present in each region of each capsule.

\section{Computer Calculations}

All computer calculations were made with the MCNP5 computer code (Reference 4) and associated cross section library that is maintained and controlled at PNNL in accordance with Reference 5 . The most recent MCNP cross sections were used where available (mostly based on ENDF/B-VI.8). All MCNP calculations were run on a dual processor Dell Dimension 670 workstation computer, ID\#WD47989.

\section{Assumptions and Limitations}

Details of the SMIR irradiation rig are not available yet, so the model was based on dimensions from similar irradiation rigs. The final SMIR design is expected to be very close to the assumed model, but the content of the center compartment is not known at this time.

The preliminary Cycle 5 core loading could change as a result of normal JOYO cycle core management activities. Changes that would significantly affect the heating rates are not likely.

MCNP calculations of heating rates utilized primarily ENDF/B-VI cross sections. Comparisons of core-wide power distributions between the MCNP and the JNC core management codes are recommended to identify any differences in methods, and also identify potential variations through the two cycles of irradiation.

The MCNP code does not include gamma heating contributions from delayed gamma and beta rays from fission. This was estimated to be approximately $6 \%$ of the total core energy production. However previous studies indicated that nearly all of this energy would be deposited locally in the fuel. A correction for the delayed gamma and beta energy contribution was made for the total reactor power normalization.

\section{Heating Rates}

Table 1 ('total heating' worksheet in the attached spreadsheet) provides the total neutron plus gamma heating rates (watts/gram) for the 21 elements, 3 radial regions and 8 axial segments per capsule, six capsules per tier, five tiers per subassembly, and two subassemblies. The values are ordered by subassembly, tier, material, compartment, axial segment, and radial region. The compartments are ordered by the NRPCT labeling of F1-F6, with F1 being closest to the core center. Average heating rates over the length of each capsule are in the column labeled "whole". Averages of the six compartments on 
each tier are also included for comparison. The uncertainty values are the MCNP statistical uncertainties at the one-sigma confidence level. Tables 2 and 3 provide separate neutron and gamma heating rates in worksheets labeled "neutron heating" and "gamma heating".

\section{Fast and Total Flux}

Table 4 ('flux' worksheet in the attached spreadsheet) provides the fast $(>0.1 \mathrm{MeV}$ ) and total flux values with the same detail and ordering as the heating rates.

\section{Heterogeneous to Homogeneous Capsule Model Effects on Heating Rates}

Previous heating rate calculations utilized homogeneous capsule compositions, with each capsule as a single region. In reality, each capsule is a heterogeneous arrangement of capsule steel, specimen holders, and various shapes of specimens. The specimen shapes include a variety of pressurized cylinders, solid disks, and solid flat pieces. Holder materials of molybdenum or tungsten generally surround the specimens and include gas gaps to maintain the specimen temperatures. Several layers of different size specimens and holders are typically in each capsule. The specimens and holders are sealed inside steel capsules, with end-caps, insulators, and springs on each end. The grouping of materials, with steel on the outside, holders in the middle, and specimens in the center could potentially have an impact on specific heating rates due to the radial gradients and gamma self shielding effects. To model each of the sixty capsules in full "as-built" geometry would be a daunting task. In order to assess the heterogeneous effect on heating rates, a three-region model for each capsule was generated. All of the specimen material was placed in the central region, the holder material was placed in the middle region, and the capsule steel was placed in the outer region. The outer capsule steel annular region was $2.0 \mathrm{~cm}$ outside diameter and $1.82 \mathrm{~cm}$ inside diameter. The middle specimen holder annular region was $1.82 \mathrm{~cm}$ outside diameter and $0.5 \mathrm{~cm}$ inside diameter. The center specimen cylindrical region was $0.5 \mathrm{~cm}$ in diameter. The Elemental Makeup Revision 2 capsule elemental masses were converted to MCNP input compositions for the three regions (capsule steel, holder, and specimens) for each capsule. Heating rates were calculated in each separate region and compared to the heating rates using the homogeneous capsule model. Detailed results can be found in Appendix A. The main observations from this comparison include:

- Heterogeneous iron heating rates in the outer steel capsule region were generally lower than the homogeneous values for tiers 1 and 5 .

- Ratios of heterogeneous to homogeneous.heating rates for the center specimen region ranged from 0.80 to 1.39 , depending on the material, location, and capsule type.

- Ratios of heterogeneous to homogeneous heating rates for the middle holder region ranged from 0.97 to 1.12 , depending on the material, location, and capsule type. 
These differences appeared significant enough to justify applying the three-region heterogeneous model to the test heating calculations.

\section{Capsule Front-to-Back Heating Gradients}

The radial gradient in the heating rates across a capsule was investigated by comparing the heating rates on the side facing the core to the side away from the core. The three region capsule model was used, with separate compositions for the capsule steel, holder region, and specimen region. Each of these regions was divided in half by a plane perpendicular to the direction of core center. The ratio of front-half to back-half heating rates was calculated for capsules in the $F 1, F 2$, and $F 3$ positions, in tiers 3 and 5 , in Assembly A. Detailed results can be found in Appendix B. Table 3 summarizes the results. The largest gradients are in the capsule steel regions, but these are still less than $20 \%$. These heating gradients would likely have little impact on material temperatures since the capsule materials generally have circumferential metal-to-metal contacts. NRPCT agreed that additional front-to-back heating studies were not required.

Table 3. Ratio of Front (towards core) to Back (away from core) Heating Rates in Selected Capsules

\begin{tabular}{|l|c|c|}
\hline & Tier 3 & Tier 5 \\
\hline Outer steel & $1.12-1.19$ & $1.03-1.09$ \\
\hline Middle holder & $1.09-1.12$ & $1.00-1.05$ \\
\hline Inner specimens & $1.02-1.07$ & $0.98-1.03$ \\
\hline
\end{tabular}

\section{Control Rod Effects}

The control rods in JOYO contain $\mathrm{B}_{4} \mathrm{C}$ pellets in pins and are inserted from the top of the core. Each control rod pin contains a $65 \mathrm{~cm}$ long poison region. When fully inserted, the poison region is centered at core midplane, with the bottom of the poison region at 32.5 $\mathrm{cm}$ below core midplane. The control rod elevation is measured in $\mathrm{cm}$ withdrawn from this fully inserted position. The control rods are withdrawn to a critical position for the beginning of cycle, and are further withdrawn during the irradiation cycle to compensate for decreased reactivity due to fuel burnup. The critical control rod elevation at the beginning of a cycle is typically around $40-45 \mathrm{~cm}$ withdrawn. By the end of a cycle, the control rods are typically around the top of the fuel column $(57.5 \mathrm{~cm}$ withdrawn), but could be up to fully withdrawn ( $65 \mathrm{~cm}$ withdrawn). Changes in control rod elevation will change the axial heating rate profile. This results in increasing heating rates in upper tier capsules and decreasing heating rates in lower tier capsules from the beginning of cycle to end of cycle. The impact of changes in control rod elevation during the irradiation cycle on the axial distribution of heating rates was evaluated by comparing heating rates for beginning of cycle control rod elevations with heating rates for average mid-cycle control rod elevations. The base-case MCNP calculations used an assumed average midcycle control rod elevation of $50 \mathrm{~cm}$ that provided a $\mathrm{k}_{\mathrm{eff}}$ of 1.019 with the beginning of cycle fuel compositions. The BOC calculation used a control rod elevation of $40 \mathrm{~cm}$ that 
provided a $k_{\text {eff }}$ of 0.998 . This is conservatively lower than the preliminary NNC predicted BOC5 control elevation of $44.8 \mathrm{~cm}$. The EOC calculation used a fully withdrawn control rod elevation of $65 \mathrm{~cm}$ that provided a $\mathrm{k}_{\mathrm{eff}}$ of 1.035 . Table 4 shows the average ratio of heating rates by tier for the $\mathrm{BOC}$ and $\mathrm{EOC}$ cases to the mid-cycle base-case. Tier 1 is the bottom tier and tier 5 is the top tier of capsules. These results indicate that tier 3 is relatively insensitive to the control rod position, but heating rates in other tiers may differ from the mid-cycle values by typically up to $4 \%$ over a cycle. Differences of up to $10 \%$ from the mid-cycle values could occur for tier 5 capsules if the control rods are fully withdrawn. The calculated differences were meant to provide bounds for the expected differences, since the exact positions at beginning, mid, and end of cycle are not known. Differences for the BOC condition will likely be smaller if the BOC control rod position is near the preliminary predicted value. Differences for EOC conditions may be less if the EOC control rod position is near the top of the fuel column rather than fully withdrawn.

Table 4. Ratio of Heating Rates at BOC and EOC to Mid-Cycle Control Rod Positions

\begin{tabular}{|c|c|c|}
\hline Tier & BOC/MID & EOC/MID \\
\hline 1 & 1.04 & 0.96 \\
\hline 2 & 1.03 & 0.97 \\
\hline 3 & 1.00 & 0.99 \\
\hline 4 & 0.97 & 1.04 \\
\hline 5 & 0.97 & 1.10 \\
\hline
\end{tabular}

\section{References}

1. Wootan, D., PNNL-15204, Review of NRPCT Interpretation of JNC Supplied Heating Rates, Pacific Northwest National Laboratory, Richland, Washington, 2005 .

2. Wootan, D., PNNL-15252, Heating Rates and Neutron Flux for JOYO-1 Irradiation Tests - Revision 1, Pacific Northwest National Laboratory, Richland, Washington, 2005.

3. B-MT(EDT)S-013, Revision 0, Nuclear Calculations and Thermal Properties for Prometheus 1 Structural Material Irradiation Capsule in the JOYO Reactor.

4. X-5 Monte Carlo Team, LA-UR-03-1987, MCNP - A General Monte Carlo NParticle Transport Code, Version 5, Los Alamos National Laboratory, Los Alamos, New Mexico, 2004.

5. Doherty, A., PNL-MA-875, Computer Code Maintenance Software Quality Assurance Manual, Revision 0, Pacific Northwest National Laboratory, Richland, Washington, 2001. 


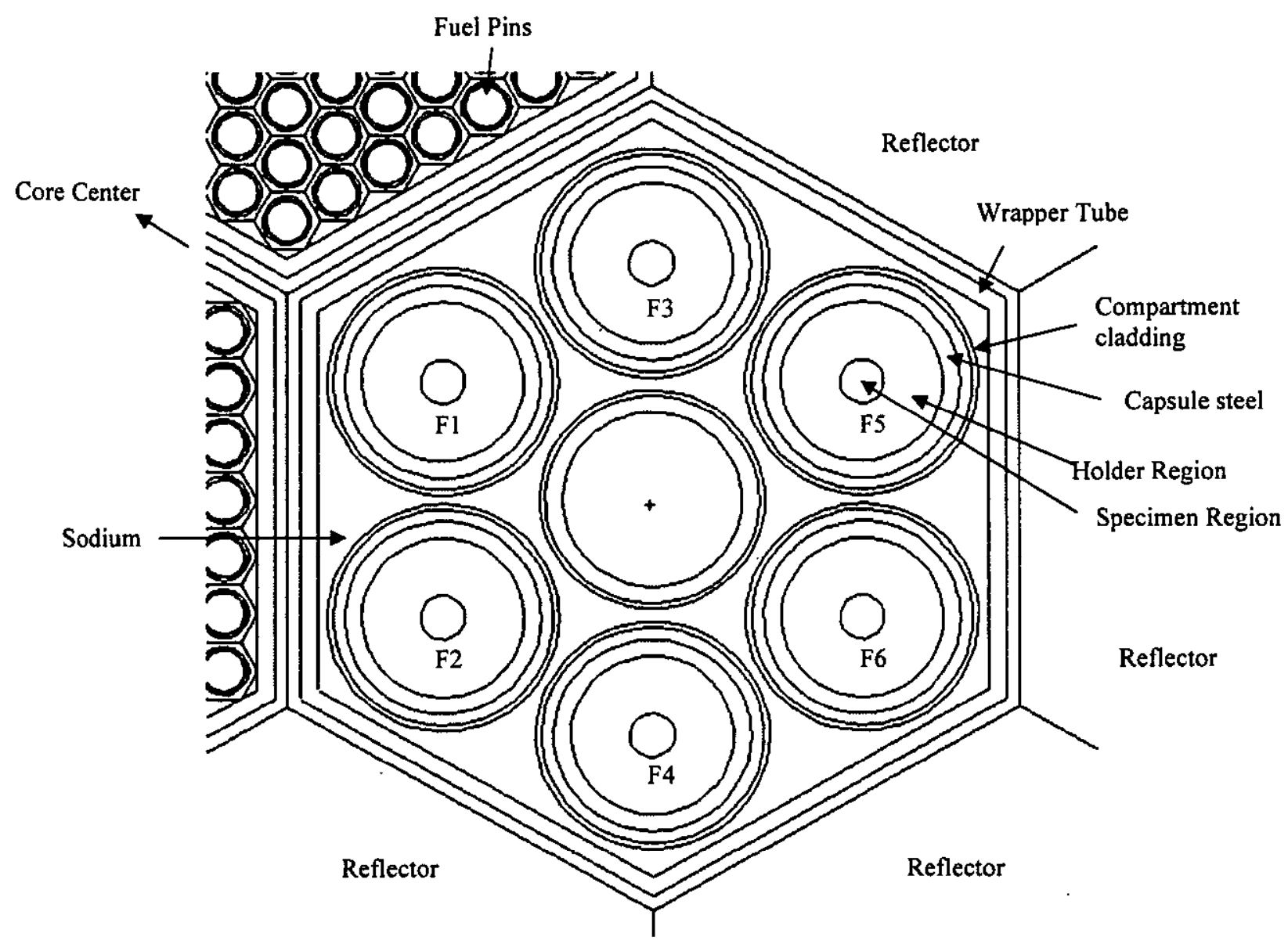

Figure 1. Cross Section View of MCNP Model of Test in JOYO Core Location 6A4 
Pacific Northwest

National Laboratory

Operalied by Batrelle for the
U.5. Department of Energy
Project \#48552

PNNL-15457

September 2005
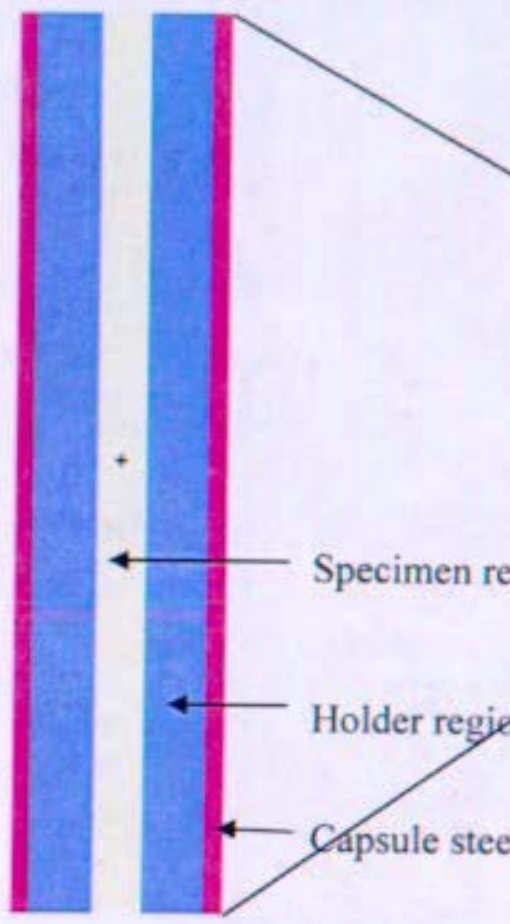

Capsule

Compartment

Tier 5

Tier 4

Tier 3

Tier 2

Tier 1 
Table A1. Comparison of Heating Rates for Heterogeneous 3-Region Model vs. Homogeneous Capsule

(Note: statistical uncertainties are $\sim 1 \%$ )

\begin{tabular}{|c|c|c|c|c|c|c|c|c|c|c|c|}
\hline \multicolumn{2}{|c|}{ A heating } & \multicolumn{10}{|c|}{ Ratio of heterogeneous/homogeneous } \\
\hline & $\mathrm{He}$ & tier & 1 & tier & 2 & tier & 3 & tier & 4 & tier & 5 \\
\hline F1 & outer capsule steel & 1.02 & A5U & 1.03 & TFT & 1.01 & SCA & 1.01 & SCA & 0.98 & A5D \\
\hline F2 & outer capsule steel & 1.01 & NBU & 1.01 & A5U & 1.01 & A5U & 1.02 & $\mathrm{~A} 5 \mathrm{U}$ & 0.99 & NBD \\
\hline$\overline{F 3}$ & outer capsule steel & 1.03 & A5U & 1.02 & $\mathrm{BCD}$ & 1.02 & $\mathrm{BCD}$ & 1.02 & $\mathrm{BCD}$ & 0.99 & A5D \\
\hline F4 & outer capsule steel & 1.02 & SCA & 1.01 & $\overline{\mathrm{DBU}}$ & 1.01 & $\mathrm{BCD}$ & 1.01 & $\mathrm{BCD}$ & 1.02 & A5D \\
\hline F5 & outer capsule steel & 1.02 & $\overline{\mathrm{DBD}}$ & 1.01 & $\mathrm{BCD}$ & 1.01 & DBU & 1.02 & $\overline{\mathrm{DBU}}$ & 1.00 & DBU \\
\hline F6 & outer capsule steel & 1.02 & DBD & 1.00 & DBU & 1.02 & $\mathrm{BCD}$ & 1.00 & $\overline{\mathrm{BCD}}$ & 1,01 & DBU \\
\hline $\overrightarrow{F l}$ & middle holder & 1.03 & ASU & 1.03 & TFT & 1.00 & SCA & 1.00 & SCA & 0.99 & A5D \\
\hline F2 & middle holder & 1.02 & NBU & 1.00 & A5U & 1.00 & ASU & 1.02 & A5U & 0.99 & NBD \\
\hline F3 & middle holder & 1.03 & ASU & 1.01 & $\mathrm{BCD}$ & 1.01 & $\mathrm{BCD}$ & 1.02 & $\mathrm{BCD}$ & 0.99 & $\overline{\mathrm{ASD}}$ \\
\hline F4 & middle holder & 1.02 & SCA & 1.01 & $\overline{\text { DBU }}$ & 1.01 & $\overline{B C D}$ & 1.01 & $\mathrm{BCD}$ & 1.02 & A5D \\
\hline F5 & middle holder & 1.03 & DBD & 1.01 & $\mathrm{BCD}$ & 1.01 & $\overline{D B U}$ & 1.02 & $\overline{\mathrm{DBU}}$ & 1.00 & DBU \\
\hline F6 & middle holder & 1.03 & DBD & 1.00 & $\overline{\text { DBU }}$ & 1.02 & $\mathrm{BCD}$ & 1.00 & $\mathrm{BCD}$ & 1.01 & DBU \\
\hline$\overline{F 1}$ & center specimens & 1.02 & ASU & 1.02 & TFT & 0.99 & SCA & 0.99 & $\mathrm{SCA}$ & 0.97 & A5D \\
\hline F2 & center spec & 1.00 & NBU & 0.97 & A5U & 0.97 & $\mathrm{~A} 5 \mathrm{U}$ & 0.99 & A5U & 0.97 & NBD \\
\hline F3 & center spe & 0.99 & ASU & 1.00 & $\mathrm{BCD}$ & 1.01 & $\mathrm{BCD}$ & 1.00 & $\mathbf{B C D}$ & 0.97 & A5D \\
\hline F4 & center spe & 1.00 & SCA & 1.02 & DBU & 1.01 & $\mathrm{BCD}$ & 1.02 & $\mathrm{BCD}$ & 1.00 & A5D \\
\hline F5 & center & 1.03 & DBD & 1.01 & $\overline{B C D}$ & 1.01 & DBU & 1.02 & $\overline{\mathrm{DBU}}$ & 1.00 & $\overline{D B U}$ \\
\hline F6 & cente & 1.07 & DBD & 1.00 & $\mathrm{DBU}$ & 1.02 & $\mathrm{BCD}$ & 1.02 & BCD & 1.03 & $\overline{D B U}$ \\
\hline & $\mathrm{B}$ (nat) & tier & 1 & tier & 2 & tier & 3 & tier & 4 & tier & 5 \\
\hline F1 & outer c & 1.15 & $\mathrm{ASU}$ & 1.03 & TFT & 1.09 & $\mathrm{SCA}$ & 1.07 & $\mathrm{SCA}$ & 1.06 & $\mathrm{A5D}$ \\
\hline F2 & outer & 1.14 & NBU & 1.06 & ASU & 1.07 & $\mathrm{ASU}$ & 1.10 & A5U & 1.06 & NBD \\
\hline F3 & outer capsule steel & 1.21 & A5U & 1.04 & BCD & 1.08 & $\mathrm{BCD}$ & 1.08 & $\mathbf{B C D}$ & 1.09 & A5D \\
\hline $\mathrm{F} 4$ & outer capsule steel & 1.16 & SCA & 1.05 & $\overline{\mathrm{DBU}}$ & 1.07 & BCD & 1.09 & $\overline{B C D}$ & 1.18 & ASD \\
\hline$\overline{F 5}$ & outer capsule steel & 1.15 & DBD & 1.06 & $\mathrm{BCD}$ & 1.06 & $\overline{\mathrm{DBU}}$ & 1.07 & $\overline{\mathrm{DBU}}$ & 1.16 & $\overline{\mathrm{DBU}}$ \\
\hline F6 & outer capsule steel & 1.11 & DBD & 1.06 & $\overline{\mathrm{DBU}}$ & 1.07 & $\mathrm{BCD}$ & 1.09 & $\mathrm{BCD}$ & 1.09 & DBU \\
\hline $\mathrm{F} 1$ & middle holder & 1.12 & A5U & 1.03 & TFT & 1.05 & SCA & 1.02 & SCA & 1.01 & A5D \\
\hline F2 & middle holder & 1.13 & NBU & 1.05 & $\mathrm{~A} 5 \mathrm{U}$ & 1.05 & A5U & 1.08 & $\mathrm{~A} 5 \mathrm{U}$ & 1.03 & NBD \\
\hline F3 & middle holder & 1.14 & A5U & 1.03 & $\mathrm{BCD}$ & 1.07 & $\mathrm{BCD}$ & 1.05 & $\mathrm{BCD}$ & 1.07 & A5D \\
\hline F4 & midd & 1.06 & SCA & 1.02 & DBU & 1.04 & $\mathrm{BCD}$ & 1.06 & $\mathrm{BCD}$ & 1.12 & A5D \\
\hline F5 & midd & 1.13 & DBD & 1.03 & $\mathrm{BCD}$ & 1.06 & DBU & 1.05 & $\mathrm{DBU}$ & 1.12 & DBU \\
\hline F6 & midd & 1.09 & DBD & 1.02 & $\mathrm{DBU}$ & 1.06 & $\mathrm{BCD}$ & 1.07 & $\mathrm{BCD}$ & 1.04 & DBU \\
\hline F1 & cente & 1.07 & A5U & 1.01 & TFT & 1.03 & $\mathrm{SCA}$ & 1.02 & $\mathrm{SCA}$ & 1.01 & A5D \\
\hline $\mathrm{F} 2$ & cente & 1.11 & NBU & 0.91 & A5U & 0.97 & $\mathrm{~A} 5 \mathrm{U}$ & 0.98 & A5U & 0.97 & NBD \\
\hline F3 & center $\mathrm{sp}$ & 0.91 & A5U & 0.96 & $\mathrm{BCD}$ & 1.03 & $\mathrm{BCD}$ & 0.98 & $\mathrm{BCD}$ & 1.03 & A5D \\
\hline $\mathrm{F} 4$ & center specimens & 1.09 & SCA & 0.96 & DBU & 0.98 & $\mathrm{BCD}$ & 1.06 & $\mathrm{BCD}$ & 0.93 & A5D \\
\hline F5 & center specimens & 1.06 & DBD & 0.98 & $\mathrm{BCD}$ & 1.00 & DBU & 1.03 & DBU & 1.08 & DBU \\
\hline F6 & center specimens & 0.95 & DBD & 0.94 & DBU & 0.96 & $\overline{B C D}$ & 0.98 & $\mathrm{BCD}$ & 0.95 & DBU \\
\hline & $\mathrm{Be}$ & tier & 1 & tier & 2 & tier & 3 & tier & 4 & tier & $\underline{5}$ \\
\hline Fl & outer capsule steel & 1.00 & ASU & 1.02 & TFT & 1.01 & SCA & 1.01 & SCA & 0.98 & A5D \\
\hline F2 & outer capsule steel & 1.00 & $\mathrm{NBU}$ & 1.01 & A5U & 1.00 & $\mathrm{~A} 5 \mathrm{U}$ & 1.01 & $\mathrm{~A} 5 \mathrm{U}$ & 0.98 & $\overline{\mathrm{NBD}}$ \\
\hline $\mathrm{F3}$ & outer capsule steel & 1.01 & $\mathrm{~A} 5 \mathrm{U}$ & 1.02 & $\mathrm{BCD}$ & 1.01 & $\mathrm{BCD}$ & 1.01 & $\mathrm{BCD}$ & 0.98 & A5D \\
\hline
\end{tabular}




\begin{tabular}{|c|c|c|c|c|c|c|c|c|c|c|c|}
\hline $\mathrm{F} 4$ & outer capsule steel & $0 . \overline{99}$ & $\mathrm{SCA}$ & 0.99 & DBU & 1.00 & $B C D$ & $1 . \overline{0}$ & $\overrightarrow{\mathrm{BCD}}$ & 1.00 & A5D \\
\hline F5 & outer capsule steel & 0.99 & DBD & $1 . \overline{0}$ & $\mathrm{BCD}$ & 0.99 & $\mathrm{DBU}$ & 1.00 & $\overline{\mathrm{DBU}}$ & 0.98 & $\overline{D B U}$ \\
\hline $\mathrm{F} 6$ & outer capsule steel & 0.98 & $\overline{\mathrm{DBD}}$ & 0.99 & $\overline{\mathrm{DBU}}$ & 1.00 & $\mathrm{BCD}$ & 0.99 & $\overline{B C D}$ & 0.97 & $\overline{D B U}$ \\
\hline $\mathbf{F} 1$ & middle holder & 1.02 & $\mathrm{~A} 5 \mathrm{U}$ & 1.03 & TFT & 1.00 & $\widehat{\text { SCA }}$ & 1,00 & $\widehat{S C A}$ & 1.00 & A5D \\
\hline $\bar{F} 2$ & middle holder & 102 & $\mathrm{NBU}$ & 1.01 & ASU & 1.01 & $\mathrm{~A} 5 \mathrm{U}$ & 1.02 & A5U & 1.00 & NBD \\
\hline F3 & middle holder & 1.03 & $\overline{A S U}$ & 1.02 & $\overline{\mathrm{BCD}}$ & 1.01 & $\mathrm{BCD}$ & 1.02 & $\mathrm{BCD}$ & 1.00 & A5D \\
\hline F4 & middle holder & 1.01 & $\overline{S C A}$ & 1.01 & $\overline{\mathrm{DBU}}$ & 1.01 & $\mathrm{BCD}$ & 1.02 & $\overline{B C D}$ & 1.02 & $\mathrm{~A} 5 \mathrm{D}$ \\
\hline F5 & middle holder & 1.02 & DBD & 1.01 & $\mathrm{BCD}$ & 1.01 & DBU & 1.02 & DBU & 1.00 & $\mathrm{DBU}$ \\
\hline F6 & middle holder & 1.02 & $\mathrm{DBD}$ & 1.01 & DBU & 1.02 & $\mathrm{BCD}$ & 1.01 & $B C D$ & 1.01 & $\overline{\mathrm{DBU}}$ \\
\hline Fl & center specimens & 1.02 & A5U & 1.01 & TFT & 0.99 & SCA & 1.00 & SCA & 0.98 & A5D \\
\hline $\mathrm{F} 2$ & center specimens & 1.01 & NBU & 0.98 & $\mathrm{~A} 5 \mathrm{U}$ & 0.97 & $\mathrm{~A} 5 \mathrm{U}$ & 0.99 & $\mathrm{~A} 5 \mathrm{U}$ & 0.99 & $\sqrt{B D}$ \\
\hline $\mathrm{F} 3$ & center specimens & 1.01 & A5U & 1.01 & $\mathrm{BCD}$ & 1.00 & $\mathrm{BCD}$ & 1.00 & $\mathrm{BCD}$ & 0.99 & A5D \\
\hline F4 & center specimens & 0.99 & SCA & 1.03 & DBU & 1.02 & BCD & 1.03 & $B C D$ & 1.01 & $\mathrm{~A} 5 \mathrm{D}$ \\
\hline F5 & center specimens & 1.06 & DBD & 1.02 & $\mathrm{BCD}$ & 1.03 & DBU & 1.04 & DBU & 1.02 & $\mathrm{DBU}$ \\
\hline F6 & center specimens & 1.11 & $\mathrm{DBD}$ & 1.04 & $\mathrm{DBU}$ & 1.04 & $B C D$ & 1.04 & $\mathrm{BCD}$ & 1.06 & $\overline{\mathrm{DBU}}$ \\
\hline & C & tier & 1 & tier & 2 & tier & 3 & tier & 4 & tier & 5 \\
\hline $\mathrm{F} 1$ & outer & 0.98 & A5U & 1.02 & TFT & 1.02 & $\mathrm{SCA}$ & 1.02 & $\mathrm{SCA}$ & 0.96 & A5D \\
\hline $\mathrm{F} 2$ & outer & 0.98 & NBU & 1.00 & $\mathrm{~A} 5 \mathrm{U}$ & 1.00 & $\mathrm{ASU}$ & 1.01 & A5U & 0.97 & NBD \\
\hline F3 & steel & 0.99 & ASU & 1.01 & $\mathrm{BCD}$ & 1.00 & $B C D$ & 1.00 & BCD & 0.96 & A5D \\
\hline $\mathrm{F} 4$ & outer & 0.97 & SCA & 0.98 & DBU & 0.99 & $\mathrm{BCD}$ & 0.99 & $\mathrm{BCD}$ & 0.98 & $\mathrm{~A} 5 \overline{\mathrm{D}}$ \\
\hline F5 & outer & 0.97 & DBD & 0.98 & $\mathrm{BCD}$ & 0.98 & $\mathrm{DBU}$ & 0.99 & DBU & 0.96 & DBU \\
\hline F6 & steel & 0.95 & DBD & 0.97 & $\mathrm{DBU}$ & 0.98 & BCD & 0.97 & $\mathrm{BCD}$ & 0.95 & DBU \\
\hline $\mathrm{F} 1$ & $\mathrm{mid}$ & 1.02 & $\mathrm{~A} 5 \mathrm{U}$ & 1.03 & TFT & 1.00 & SCA & 1.01 & SCA & 1.00 & A5D \\
\hline F2 & mido & 1.03 & $\mathrm{NBU}$ & 1.02 & $\mathrm{~A} 5 \mathrm{U}$ & 1.01 & A5U & 1.03 & $\mathrm{A5U}$ & 1.01 & NBD \\
\hline F3 & midd & 1.04 & ASU & 1.02 & $\mathrm{BCD}$ & 1.02 & $\mathrm{BCD}$ & 1.02 & $\mathrm{BCD}$ & 1.00 & $5 \mathrm{D}$ \\
\hline $\mathrm{F} 4$ & midd & 1.01 & $\mathrm{SCA}$ & 1.02 & $\mathrm{DBU}$ & 1.01 & $\mathrm{BCD}$ & 1.03 & $B C D$ & 1.03 & A5D \\
\hline F5 & mido & 1.03 & DBD & 1.02 & $\mathrm{BCD}$ & 1.01 & DBU & 1.02 & DBU & 1.01 & DBU \\
\hline F6 & midd & 1.03 & DBD & 1.01 & DBU & 1.02 & $\mathrm{BCD}$ & 1.01 & $\mathrm{BCD}$ & 1.01 & DBU \\
\hline Fl & cente & 1.02 & $\mathrm{~A} 5 \mathrm{U}$ & 1.01 & TFT & 0.99 & SCA & 0.99 & SCA & 0.98 & A5D \\
\hline F2 & cente & 1.02 & NBU & 0.97 & A5U & 0.97 & $A 5 U$ & 0.98 & A5U & 1.00 & NBD \\
\hline F3 & cente & 1.01 & A5U & 1.01 & $\mathrm{BCD}$ & 1.01 & $\mathrm{BCD}$ & 1.00 & $B C D$ & 1.00 & A5D \\
\hline $\mathrm{F} 4$ & cente & 0.98 & $\mathrm{SCA}$ & 1.04 & DBU & 1.03 & BCD & 1.05 & $\mathrm{BCD}$ & 1.02 & A5D \\
\hline F5 & cent & 1.08 & $\mathrm{DBD}$ & 1.04 & $\mathrm{BCD}$ & 1.05 & DBU & 1.06 & DBU & 1.05 & DBU \\
\hline F6 & cent & 1.17 & DBD & 1.07 & $\overline{\mathrm{DBU}}$ & 1.06 & $B C D$ & 1.07 & BCD & 1.11 & DBU \\
\hline & 0 & tier & 1 & tier & 2 & tier & 3 & tier & 4 & tier & 5 \\
\hline $\mathrm{FI}$ & outer & 0.97 & $\mathrm{~A} 5 \mathrm{U}$ & 1.01 & TFT & 1.02 & SCA & 1.02 & SCA & 0.96 & A5D \\
\hline $\mathrm{F} 2$ & oute & 0.97 & $\mathrm{NBU}$ & 1.00 & $\mathrm{~A} 5 \mathrm{U}$ & 1.00 & ASU & 1.01 & $\mathrm{~A} 5 \mathrm{U}$ & 0.97 & NBD \\
\hline F3 & oute & 0.99 & A5U & 1.00 & BCD & 1.00 & BCD & 1.00 & $\mathrm{BCD}$ & 0.95 & A5D \\
\hline F4 & outer & 0.97 & SCA & 0.98 & DBU & 0.98 & $B C D$ & 0.98 & $B C D$ & 0.97 & A5D \\
\hline F5 & outer & 0.97 & DBD & 0.97 & $\mathrm{BCD}$ & 0.97 & $\mathrm{DBU}$ & 0.98 & DBU & 0.94 & $\mathrm{DBU}$ \\
\hline F6 & outer & 0.94 & DBD & 0.95 & DBU & 0.97 & $\mathrm{BCD}$ & 0.97 & $\mathrm{BCD}$ & 0.93 & DBU \\
\hline $\mathrm{F} 1$ & midd & 1.03 & $\mathrm{~A} 5 \mathrm{U}$ & 1.03 & TFT & 1.00 & $\mathrm{SCA}$ & 1.01 & SCA & 1.01 & $\mathrm{~A} 5 \mathrm{D}$ \\
\hline $\mathbf{F} 2$ & midd & 1.03 & NBU & 1.02 & $\mathrm{ASU}$ & 1.02 & $\mathrm{~A} 5 \mathrm{U}$ & 1.03 & $\mathrm{~A} 5 \mathrm{U}$ & 1.02 & NBD \\
\hline F3 & midd & 1.04 & A5U & 1.02 & $\mathrm{BCD}$ & 1.02 & $\mathrm{BCD}$ & 1.02 & $\mathrm{BCD}$ & 1.01 & A5D \\
\hline $\mathbf{F} 4$ & midd & 1.01 & SCA & 1.02 & DBU & 1.02 & $\mathrm{BCD}$ & 1.03 & $\mathrm{BCD}$ & 1.03 & A5D \\
\hline F5 & middle h & 1.04 & DBD & 1.02 & $\overline{\mathrm{BCD}}$ & 1.01 & $\overline{\mathrm{DBU}}$ & 1.03 & DBU & 1.01 & DBU \\
\hline F6 & middle holder & 1.03 & DBD & 1.01 & DBU & 1.02 & $\mathrm{BCD}$ & 1.02 & $\mathrm{BCD}$ & 1.01 & $\mathrm{DBU}$ \\
\hline F1 & center specimens & 1.03 & A.5U & 1.01 & TFT & 0.98 & SCA & 0.99 & $\mathrm{SCA}$ & 0.98 & A5D \\
\hline F2 & center specimens & 1.03 & $\overline{\mathrm{NBU}}$ & 0.96 & $\mathrm{~A} 5 \mathrm{U}$ & 0.97 & $\mathrm{ASU}$ & 0.98 & $\mathrm{~A} 5 \mathrm{U}$ & 1.00 & $\mathrm{NBD}$ \\
\hline F3 & center specimens & 1.01 & AsU & 1.01 & $\mathrm{BCD}$ & 1.01 & $B C D$ & 0.99 & $\mathrm{BCD}$ & 1.00 & A5D \\
\hline F4 & center specimens & 0.98 & $S C A$ & 1.05 & DBU & 1.04 & $\mathrm{BCD}$ & 1.06 & $\mathrm{BCD}$ & 1.02 & A5D \\
\hline
\end{tabular}




\begin{tabular}{|c|c|c|c|c|c|c|c|c|c|c|c|}
\hline F5 & center specimens & 1.10 & DBD & 1.04 & $\mathrm{BCD}$ & 1.06 & DBU & 1.08 & DBU & 1.06 & $\overline{\mathrm{DBU}}$ \\
\hline F6 & center specimens & 1.20 & DBD & 1.08 & $\overline{\text { DBU }}$ & 1.07 & $\mathrm{BCD}$ & 1.09 & $\mathrm{BCD}$ & 1.14 & $\overline{\mathrm{DBU}}$ \\
\hline & $\mathrm{Al}$ & tier & 1 & tier & $\overline{2}$ & tier & $\overline{3}$ & tier & 4 & tier & 5 \\
\hline F1 & outer capsule steel & 0.95 & $\overline{\mathrm{A} 5 \mathrm{U}}$ & 1.00 & TFT & 1.02 & $\mathrm{SCA}$ & 1.03 & SCA & 0.93 & A5D \\
\hline$\overline{\mathrm{F} 2}$ & outer capsule steel & 0.94 & NBU & 1.00 & $\mathrm{~A} 5 \mathrm{U}$ & 1.00 & $\mathrm{~A} 5 \mathrm{U}$ & 1.00 & $\mathrm{~A} 5 \mathrm{U}$ & 0.95 & $\overline{N B D}$ \\
\hline F3 & outer capsule steel & 0.96 & $\mathrm{A5U}$ & 0.99 & $\mathrm{BCD}$ & 0.99 & $\mathrm{BCD}$ & 0.98 & $\mathrm{BCD}$ & 0.93 & A5D \\
\hline F4 & outer capsule steel & 0.94 & SCA & 0.96 & DBU & 0.96 & BCD & 0.97 & $\mathrm{BCD}$ & 0.94 & A5D \\
\hline F5 & outer capsule steel & 0.94 & $\overline{\mathrm{DBD}}$ & 0.95 & $\overline{B C D}$ & 0.95 & DBU & 0.96 & $\mathrm{DBU}$ & 0.92 & DBU \\
\hline F6 & outer capsule steel & 0.90 & DBD & 0.93 & DBU & 0.95 & $\mathrm{BCD}$ & 0.94 & $\mathrm{BCD}$ & 0.89 & DBU \\
\hline $\mathbf{F 1}$ & middle ho & 1.02 & $\mathrm{~A} 5 \mathrm{U}$ & 1.04 & TFT & 1.00 & $\overline{\mathrm{SCA}}$ & 1.01 & $\mathrm{SCA}$ & 1.01 & A5D \\
\hline $\mathrm{F} 2$ & mide & 1.04 & NBU & 1.03 & ASU & 1.03 & ASU & 1.04 & $\overline{\mathrm{A} 5 \mathrm{U}}$ & 1.03 & NBD \\
\hline F3 & $\mathrm{mid}$ & 1.04 & $\mathrm{~A} 5 \mathrm{U}$ & 1.03 & $\mathrm{BCD}$ & 1.03 & $\mathrm{BCD}$ & 1.02 & BCD & 1.01 & ASD \\
\hline $\mathbf{F 4}$ & & 1.01 & SCA & 1.03 & $\overline{D B U}$ & 1.02 & $\mathrm{BCD}$ & 1.04 & BCD & 1.04 & A5D \\
\hline F5 & & 1.04 & $\overline{\mathrm{DBD}}$ & 1.02 & $\mathrm{BCD}$ & 1.02 & DBU & 1.03 & DBU & 1.01 & DBU \\
\hline F6 & mid & 1.04 & DBD & 1.01 & $\overline{\mathrm{DBU}}$ & 1.03 & $\mathrm{BCD}$ & 1.03 & $\mathrm{BCD}$ & 1.01 & $\mathrm{DBU}$ \\
\hline F1 & cent & 1.03 & A5U & 1.01 & TFT & 0.98 & $\mathrm{SCA}$ & 0.99 & SCA & 0.98 & A5D \\
\hline$\overline{F 2}$ & center & 1.05 & NBU & 0.95 & A5U & 0.95 & A5U & 0.97 & A5U & 1.02 & NBD \\
\hline F3 & center spe & 1.01 & A5U & 1.01 & $\mathrm{BCD}$ & 1.01 & BCD & 0.99 & $\mathrm{BCD}$ & 1.01 & A5D \\
\hline $\mathrm{F} 4$ & center $\mathrm{sp}$ & 0.97 & SCA & 1.08 & DBU & 1.06 & $\mathrm{BCD}$ & 1.08 & $\mathrm{BCD}$ & 1.02 & A5D \\
\hline F5 & cent & 1.13 & DBD & 1.06 & $\mathrm{BCD}$ & 1.09 & DBU & 1.12 & DBU & 1.09 & $\mathrm{DBU}$ \\
\hline F6 & cen & 1.27 & DBD & 1.13 & $\mathrm{DBU}$ & 1.11 & $\mathrm{BCD}$ & 1.13 & $\mathrm{BCD}$ & 1.19 & DBU \\
\hline & $\mathbf{S i}$ & tier & 1 & tier & 2 & tier & 3 & tier & 4 & tier & 5 \\
\hline$F 1$ & oute & 0.95 & A5U & 1.01 & TFT & 1.03 & SCA & 1.03 & SCA & 0.93 & A5D \\
\hline $\mathrm{F} 2$ & & 0.94 & $\mathrm{NBU}$ & 1.00 & $\mathrm{ASU}$ & 1.00 & A5U & 1.00 & $\mathrm{~A} 5 \mathrm{U}$ & 0.95 & NBD \\
\hline F3 & ou & 0.96 & A5U & 0.99 & $\mathrm{BCD}$ & 0.99 & $\mathrm{BCD}$ & 0.99 & $\mathrm{BCD}$ & 0.93 & A5D \\
\hline F4 & ou & 0.94 & $\mathrm{~A}$ & 0.96 & DBU & 0.96 & $\mathrm{BCD}$ & 0.97 & $\mathrm{BCD}$ & 0.94 & A5D \\
\hline F5 & out & 0.94 & $\mathrm{DBD}$ & 0.95 & $\mathrm{BCD}$ & 0.95 & DBU & 0.96 & DBU & 0.92 & $\mathrm{DBU}$ \\
\hline $\mathrm{F} 6$ & out & 0.90 & $\mathrm{DBD}$ & 0.93 & $\mathrm{DBU}$ & 0.95 & $\mathrm{BCD}$ & 0.94 & $\mathrm{BCD}$ & 0.89 & DBU \\
\hline$\overline{F 1}$ & $\mathrm{mid}$ & 1.02 & A5U & 1.04 & TFT & 1.00 & SCA & 1.01 & SCA & 1.01 & A5D \\
\hline F2 & $\mathrm{mid}$ & 1.04 & $\mathrm{NBU}$ & 1.04 & $\overline{A S U}$ & 1.03 & A5U & 1.04 & A5U & 1.03 & NBD \\
\hline$\overline{F 3}$ & $\mathrm{mi}$ & 1. & A5U & 1.03 & $\mathrm{BCD}$ & 1.03 & $\mathrm{BCD}$ & 1.03 & BCD & 1.02 & A5D \\
\hline $\mathbf{F} 4$ & $\mathrm{mi}$ & 1.0 & SCA & 1.03 & $\mathrm{DBU}$ & 1.03 & BCD & 1.05 & $\mathrm{BCD}$ & 1.03 & A5D \\
\hline F5 & & 1.04 & DBD & 1.02 & $\mathrm{BCD}$ & 1.02 & $\mathrm{DBU}$ & 1.04 & $\mathrm{DBU}$ & 1.02 & DBU \\
\hline F6 & middle ho & 1.04 & DBD & 1.01 & DBU & 1.03 & $B C D$ & 1.03 & $\mathrm{BCD}$ & 1.01 & DBU \\
\hline F1 & cer & 1.03 & $\mathrm{~A} 5 \mathrm{U}$ & 1.01 & TFT & 0.97 & SCA & 0.99 & SCA & 0.99 & A5D \\
\hline $\mathbf{F} 2$ & & 1.05 & $\mathrm{NBU}$ & 0.95 & ASU & 0.95 & $15 \mathrm{U}$ & 0.97 & $\mathrm{~A} 5 \mathrm{U}$ & 1.02 & NBD \\
\hline $\mathbf{F 3}$ & & 1.01 & $\mathrm{U}$ & 1.01 & CD & 1.00 & $\mathrm{BCD}$ & 0.99 & $\mathrm{BCD}$ & 1.01 & A5D \\
\hline $\mathbf{F 4}$ & -8 & 0.96 & $A$ & 1.08 & $\mathrm{DBU}$ & 1.06 & $B C D$ & 1.09 & $\mathrm{BCD}$ & 1.02 & ASD \\
\hline F5 & -1 & 1.13 & DBD & 1.07 & $\mathrm{BCD}$ & 1.09 & $\overline{\mathrm{DBU}}$ & 1.13 & DBU & 1.10 & DBU \\
\hline $\mathrm{F} 6$ & cen & 1.28 & DBD & 1.13 & $\overline{\mathrm{DBU}}$ & 1.11 & $\mathrm{BCD}$ & 1.14 & $\mathrm{BCD}$ & 1.20 & $\overline{\mathrm{DBU}}$ \\
\hline & $\mathrm{Ti}$ & tier & 1 & tier & 2 & tier & 3 & tier & 4 & tier & 5 \\
\hline $\mathrm{F} 1$ & outer & 0.93 & A5U & 1.00 & TFT & 1.03 & SCA & 1.03 & SCA & 0.92 & A5D \\
\hline F2 & outer caps & 0.93 & NBU & 1.00 & A5U & 1.00 & A5U & 1.00 & A5U & 0.94 & NBD \\
\hline F3 & outer capsu & 0.95 & A5U & 0.98 & $\mathrm{BCD}$ & 0.98 & $\mathrm{BCD}$ & 0.98 & $\mathrm{BCD}$ & 0.92 & A5D \\
\hline $\mathbf{F 4}$ & outer capsule steel & 0.93 & SCA & 0.95 & DBU & 0.95 & $\mathrm{BCD}$ & 0.96 & $\mathrm{BCD}$ & 0.93 & ASD \\
\hline F5 & outer capsule steel & 0.93 & DBD & 0.94 & $\mathrm{BCD}$ & 0.93 & DBU & 0.95 & DBU & 0.90 & DBU \\
\hline F6 & outer capsule steel & 0.89 & DBD & 0.91 & DBU & 0.93 & $\mathrm{BCD}$ & 0.93 & $\mathrm{BCD}$ & 0.87 & DBU \\
\hline F1 & mid & 1.02 & A5U & 1.04 & TFT & 1.00 & SCA & 1.01 & SCA & 1.02 & A5D \\
\hline $\mathbf{F} 2$ & midd & 1.04 & NBU & 1.05 & $\mathrm{~A} 5 \mathrm{U}$ & 1.05 & $\mathrm{~A} 5 \mathrm{U}$ & 1.05 & $\mathrm{~A} 5 \mathrm{U}$ & 1.04 & NBD \\
\hline F3 & middle $h$ & 1.04 & A5U & 1.04 & BCD & 1.04 & $\mathrm{BCD}$ & 1.03 & $\mathrm{BCD}$ & 1.02 & A5D \\
\hline$\pi$ & middle holder & 1.00 & $\mathrm{SCA}$ & 1.04 & $\mathrm{DBU}$ & 1.03 & BCD & 1.06 & $\mathrm{BCD}$ & 1.04 & 5D \\
\hline
\end{tabular}




\begin{tabular}{|c|c|c|c|c|c|c|c|c|c|c|c|}
\hline$\overline{F 5}$ & middle holder & 1.04 & DBD & 1.03 & $\overline{B C D}$ & 1.02 & DBU & 1.04 & $\overline{\mathrm{DBU}}$ & 1.02 & DBU \\
\hline F6 & middle holder & 1.04 & DBD & 1.01 & DBU & 1.03 & $\mathrm{BCD}$ & 1.04 & $\mathrm{BCD}$ & 1.01 & DBU \\
\hline F1 & center specimens & 1.03 & A5U & 1.01 & TFT & 0.96 & SCA & 0.98 & $\mathrm{SCA}$ & 0.98 & A5D \\
\hline F2 & center specimens & 1.06 & NBU & 0.93 & ASU & 0.94 & A5U & 0.95 & A5U & 1.03 & NBD \\
\hline$\overline{F 3}$ & center specimens & 1.00 & $\mathrm{~A} 5 \mathrm{U}$ & 1.01 & $\mathrm{BCD}$ & 1.01 & $\overline{B C D}$ & 0.98 & $\overline{B C D}$ & 1.02 & A5D \\
\hline F4 & center specimens & 0.96 & SCA & 1.10 & $\mathrm{DBU}$ & 1.07 & $\mathrm{BCD}$ & 1.11 & $\overline{\mathrm{BCD}}$ & 1.02 & A5D \\
\hline $\mathrm{F5}$ & center specimens & 1.15 & DBD & 1.08 & $\mathrm{BCD}$ & 1.12 & DBU & 1.16 & DBU & 1.11 & $\overline{\mathrm{DBU}}$ \\
\hline $\overrightarrow{F 6}$ & center specimens & 1.32 & DBD & 1.17 & DBU & 1.14 & $\mathrm{BCD}$ & 1.18 & $\mathrm{BCD}$ & 1.24 & DBU \\
\hline & $\mathrm{V}$ & tier & 1 & tier & 2 & tier. & 3 & tier & 4 & tier & 5 \\
\hline F1 & outer capsule steel & 0.94 & $45 \mathrm{U}$ & 1.00 & TFT & 1.03 & SCA & 1.03 & $\mathrm{SCA}$ & 0.93 & A5D \\
\hline $\mathrm{F} 2$ & outer capsule steel & 0.94 & NBU & 1.00 & $\mathrm{ASU}$ & 1.00 & $\mathrm{ASU}$ & 1.00 & A5U & 0.94 & NBD \\
\hline $\mathrm{F} 3$ & outer capsule steel & 0.96 & A5U & 0.98 & $\mathrm{BCD}$ & 0.98 & $\mathrm{BCD}$ & 0.98 & $\mathrm{BCD}$ & 0.92 & A5D \\
\hline $\mathrm{F} 4$ & outer capsule steel & 0.94 & SCA & 0.95 & DBU & 0.95 & $\mathrm{BCD}$ & 0.96 & $\mathrm{BCD}$ & 0.93 & A5D \\
\hline F5 & outer capsule steel & 0.93 & DBD & 0.94 & $\mathrm{BCD}$ & 0.94 & DBU & 0.95 & DBU & 0.91 & DBU \\
\hline F6 & outer capsule steel & 0.89 & DBD & 0.91 & DBU & 0.94 & $\mathrm{BCD}$ & 0.93 & $\mathrm{BCD}$ & 0.88 & DBU \\
\hline F1 & middle holder & 1.02 & A5U & 1.04 & TFT & 1.00 & SCA & 1.01 & $\overline{\mathrm{SCA}}$ & 1.02 & A5D \\
\hline F2 & mide & 1.04 & NBU & 1.05 & A5U & 1.05 & A5U & 1.05 & A5U & 1.04 & NBD \\
\hline $\mathrm{F} 3$ & mid & 1.04 & $\mathrm{ASU}$ & 1.04 & $\mathrm{BCD}$ & 1.04 & $\mathrm{BCD}$ & 1.03 & $\mathrm{BCD}$ & 1.02 & A5D \\
\hline F4 & mid & 1.00 & SCA & 1.04 & DBU & 1.03 & $\mathrm{BCD}$ & 1.06 & $\mathrm{BCD}$ & 1.04 & A5D \\
\hline F5 & midd & 1.04 & DBD & 1.03 & $\mathrm{BCD}$ & 1.02 & DBU & 1.04 & DBU & 1.02 & DBU \\
\hline F6 & mid & 1.04 & DBD & 1.01 & DBU & 1.03 & $\mathrm{BCD}$ & 1.04 & $\mathrm{BCD}$ & 1.01 & DBU \\
\hline F1 & nens & 1.03 & $\mathrm{~A} 5 \mathrm{U}$ & 1.01 & TFT & 0.96 & SCA & 0.98 & $\mathrm{SCA}$ & 0.98 & A5D \\
\hline $\mathrm{F} 2$ & cen & 1.06 & $\mathrm{NBU}$ & 0.93 & $\mathrm{~A} 5 \mathrm{U}$ & 0.94 & $\mathrm{~A} 5 \mathrm{U}$ & 0.95 & ASU & 1.03 & NBD \\
\hline F3 & cent & 1.00 & A5U & 1.01 & $\mathrm{BCD}$ & 1.01 & $\mathrm{BCD}$ & 0.98 & $\mathrm{BCD}$ & 1.02 & A5D \\
\hline F4 & cent & 0.96 & $\mathrm{SCA}$ & 1.09 & DBU & 1.07 & $\mathrm{BCD}$ & 1.10 & $B C D$ & 1.01 & A5D \\
\hline F5 & cent & 1.15 & DBD & 1.08 & $\mathrm{BCD}$ & 1.12 & $\mathrm{DBU}$ & 1.16 & $\mathrm{DBU}$ & 1.11 & DBU \\
\hline F6 & nens & 1.32 & DBD & 1.17 & DBU & 1.14 & $\mathrm{BCD}$ & 1.17 & $B C D$ & 1.23 & DBU \\
\hline & $\mathrm{Cr}$ & tier & 7 & tier & 2 & tier & 3 & tier & 4 & tier & 5 \\
\hline Fl & outer capsule steel & 0.93 & A5U & 0.99 & TFT & 1.03 & SCA & 1.03 & SCA & 0.92 & $\overline{A 5 D}$ \\
\hline $\bar{F} 2$ & outer capsu & 0.93 & NBU & 1.00 & $\overline{A S U}$ & 1.00 & A5U & 1.00 & A5U & 0.94 & NBD \\
\hline F3 & outer capsule steel & 0.95 & A5U & 0.98 & $\mathrm{BCD}$ & 0.98 & BCD & 0.97 & $\mathrm{BCD}$ & 0.92 & A5D \\
\hline $\mathrm{F} 4$ & outer cap & 0.93 & $\mathrm{SCA}$ & 0.95 & DBU & 0.95 & $\mathrm{BCD}$ & 0.96 & $B C D$ & 0.93 & $\overline{\mathrm{A} 5 \mathrm{D}}$ \\
\hline $\mathrm{F} 5$ & esteel & 0.93 & DBD & 0.94 & $\mathrm{BCD}$ & 0.93 & DBU & 0.95 & DBU & 0.90 & DBU \\
\hline F6 & le steel & 0.89 & DBD & 0.91 & DBU & 0.93 & $\mathrm{BCD}$ & 0.93 & $\mathrm{BCD}$ & 0.87 & DBU \\
\hline $\mathrm{Fl}$ & mid & 1.03 & A5U & 1.05 & TFT & 1.00 & SCA & 1.01 & $\mathrm{SCA}$ & 1.02 & A5D \\
\hline $\mathrm{F} 2$ & $\operatorname{mid}$ & 1.04 & NBU & 1.05 & $A 5 \mathrm{U}$ & 1.05 & $\mathrm{~A} 5 \mathrm{U}$ & 1.06 & A5U & 1.04 & NBD \\
\hline F3 & & 1.05 & A5U & 1.04 & $\mathrm{BCD}$ & 1.04 & $\mathrm{BCD}$ & 1.03 & $\mathrm{BCD}$ & 1.02 & $\widehat{A 5 D}$ \\
\hline $\mathrm{F} 4$ & $m$ & 1.00 & $\mathrm{SCA}$ & 1.04 & $\mathrm{DBU}$ & 1.04 & $\overline{B C D}$ & 1.06 & $\overline{\mathrm{BCD}}$ & 1.04 & A5D \\
\hline F5 & mid & 1.05 & DBD & 1.03 & $\mathrm{BCD}$ & 1.02 & DBU & 1.05 & $\overline{\mathrm{DBU}}$ & 1.02 & DBU \\
\hline F6 & middl & 1.04 & DBD & 1.01 & DBU & 1.03 & $\mathrm{BCD}$ & 1.04 & $\mathrm{BCD}$ & 1.01 & DBU \\
\hline Fl & cent & 1.03 & $\mathrm{~A} 5 \mathrm{U}$ & 1.01 & TFT & 0.96 & $\mathrm{SCA}$ & 0.98 & $\mathrm{SCA}$ & 0.98 & A5D \\
\hline F2 & cent & 1.06 & $\mathrm{NBU}$ & 0.93 & $\mathrm{~A} 5 \mathrm{U}$ & 0.94 & $\mathrm{~A} 5 \mathrm{U}$ & 0.95 & $\mathrm{~A} 5 \mathrm{U}$ & 1.03 & NBD \\
\hline $\mathrm{F3}$ & cent & 1.00 & ASU & 1.01 & $\mathrm{BCD}$ & 1.01 & $\mathrm{BCD}$ & 0.98 & $\mathrm{BCD}$ & 1.02 & ASD \\
\hline F4 & cente & 0.96 & $\mathrm{SCA}$ & 1.10 & $\mathrm{DBU}$ & 1.08 & $\mathrm{BCD}$ & 1.11 & $\mathrm{BCD}$ & 1.02 & A5D \\
\hline F5 & center ss & 1.15 & DBD & 1.08 & $\mathrm{BCD}$ & 1.13 & DBU & 1.17 & $\mathrm{DBU}$ & 1.12 & DBU \\
\hline F6 & center specimens & 1.34 & DBD & 1.17 & DBU & 1.15 & $\mathrm{BCD}$ & 1,18 & $\mathrm{BCD}$ & 1.25 & $\mathrm{DBU}$ \\
\hline & $\mathrm{Mn}$ & tier & 1 & tier & 2 & tier & 3 & tier & 4 & tier & 5 \\
\hline F1 & outer cap & 0.94 & ASU & 1.00 & TFT & 1.03 & SCA & 1.03 & SCA & 0.92 & A5D \\
\hline F2 & outer capsule steel & 0.93 & $\mathrm{NBU}$ & 1.00 & $\mathrm{~A} 5 \mathrm{U}$ & 1.00 & $\mathrm{A5U}$ & 1.01 & $\overline{\mathrm{A}} 5 \mathrm{U}$ & 0.94 & NBD \\
\hline F3 & outer capsule stee] & 0.95 & $\mathrm{~A} 5 \mathrm{U}$ & 0.98 & $\mathrm{BCD}$ & 0.98 & $\mathrm{BCD}$ & 0.98 & $\mathrm{BCD}$ & 0.92 & ASD \\
\hline F4 & outer capsule steel & 0.94 & $\mathrm{SCA}$ & 0.95 & $\mathrm{DBU}$ & 0.95 & $\mathrm{BCD}$ & 0.96 & $\overline{B C D}$ & 0.93 & ASD \\
\hline
\end{tabular}




\begin{tabular}{|c|c|c|c|c|c|c|c|c|c|c|c|}
\hline F5 & outer capsule steel & 0.93 & DBD & 0.94 & $\mathrm{BCD}$ & 0.93 & $\overline{\text { DBU }}$ & 0.95 & DBU & 0.90 & DBU \\
\hline F6 & outer capsule steel & 0.89 & DBD & 0.91 & DBU & 0.93 & $\mathrm{BCD}$ & 0.93 & $\mathrm{BCD}$ & 0.87 & DBU \\
\hline FI & middle holder & 1.03 & $\mathrm{~A} 5 \mathrm{U}$ & 1.05 & TFT & 1.00 & $\overline{S C A}$ & 1.01 & SCA & 1.02 & $\overline{A 5 D}$ \\
\hline F2 & middle holder & 1.04 & NBU & 1.05 & $\mathrm{ASU}$ & 1.05 & $5 \mathrm{U}$ & 1.06 & $\mathrm{~A} 5 \mathrm{U}$ & 1.04 & $\overline{B D}$ \\
\hline F3 & middle holder & 1.05 & $\mathrm{ASU}$ & 1.04 & $\mathrm{BCD}$ & 1.04 & $\mathrm{BCD}$ & 1.03 & $\mathrm{BCD}$ & 1.02 & $5 \mathrm{D}$ \\
\hline F4 & middle holder & 1.00 & SCA & 1.04 & $\mathrm{DBU}$ & 1.04 & $\overline{\mathrm{BCD}}$ & 1.06 & $\mathrm{BCD}$ & 1.05 & $5 \mathrm{D}$ \\
\hline F5 & middle holder & 1.05 & DBD & 1.03 & $\mathrm{BCD}$ & 1.02 & $\overline{\mathrm{DBU}}$ & 1.04 & $\overline{\text { DBU }}$ & 1.02 & $\mathrm{BU}$ \\
\hline F6 & middle holder & 1.04 & DBD & 1.01 & $\mathrm{DBU}$ & 1.03 & $\mathrm{BCD}$ & 1.04 & $\mathrm{BCD}$ & 1.01 & DBU \\
\hline $\mathbf{F l}$ & center specimens & 1.03 & $\mathrm{~A} 5 \mathrm{U}$ & 1.01 & TFT & 0.96 & SCA & 0.98 & SCA & 0.98 & A5D \\
\hline F2 & center specimens & 1.06 & $\mathrm{NBU}$ & 0.93 & $\mathrm{~A} 5 \mathrm{U}$ & 0.94 & $\mathrm{~A} 5 \mathrm{U}$ & 0.95 & A5U & 1.03 & NBD \\
\hline F3 & center specimens & 1.00 & $\mathrm{~A} 5 \mathrm{U}$ & 1.01 & $\mathrm{BCD}$ & 1.01 & $\mathrm{BCD}$ & 0.98 & $\mathrm{BCD}$ & 1.02 & $\mathrm{ASD}$ \\
\hline F4 & center specimens & 0.96 & SCA & 1.10 & DBU & 1.08 & $\mathrm{BCD}$ & 1.11 & $\mathrm{BCD}$ & 1.01 & A5D \\
\hline F5 & center specimens & 1.15 & DBD & 1.08 & $\mathrm{BCD}$ & 1.12 & DBU & 1.17 & DBU & 1.12 & DBU \\
\hline F6 & center & 1.33 & DBD & 1.18 & $\mathrm{DBU}$ & 1.15 & $\mathrm{BCD}$ & 1.18 & $\mathrm{BCD}$ & 1.24 & $\mathrm{DBU}$ \\
\hline & $\mathrm{Fe}$ & tier & 1 & tier & 2 & tier & 3 & tier & 4 & tier & 5 \\
\hline F1 & outer & 0.93 & $\mathrm{~A} 5 \mathrm{U}$ & 1.00 & TFT & 1.03 & SCA & 1.03 & SCA & 0.92 & A5D \\
\hline F2 & outer & 0.93 & $\mathrm{NBU}$ & 1.00 & A5U & 1.00 & A5U & 1.00 & $\mathrm{~A} 5 \mathrm{U}$ & 0.94 & NBD \\
\hline F3 & outer caps & 0.95 & $\mathrm{~A} 5 \mathrm{U}$ & 0.98 & $\mathrm{BCD}$ & 0.98 & $\mathrm{BCD}$ & 0.98 & $\mathrm{BCD}$ & 0.92 & A5D \\
\hline F4 & outer & 0.93 & SCA & 0.95 & DBU & 0.95 & $\mathrm{BCD}$ & 0.96 & $\mathrm{BCD}$ & 0.93 & $5 \mathrm{D}$ \\
\hline F5 & outer & 0.93 & $\mathrm{DBD}$ & 0.94 & $\mathrm{BCD}$ & 0.93 & DBU & 0.95 & DBU & 0.90 & $\mathrm{BU}$ \\
\hline $\mathrm{F} 6$ & outer & 0.89 & DBD & 0.91 & DBU & 0.93 & $\mathrm{BCD}$ & 0.93 & $\mathrm{BCD}$ & 0.87 & $3 \mathrm{U}$ \\
\hline F1 & middl & 1.03 & $\mathrm{~A} 5 \mathrm{U}$ & 1.05 & TFT & 1.00 & SCA & 1.01 & $\mathrm{SCA}$ & 1.02 & A5D \\
\hline F2 & midd & 1.05 & $\mathrm{NBU}$ & 1.05 & $\mathrm{~A} 5 \mathrm{U}$ & 1.05 & $\mathrm{~A} 5 \mathrm{U}$ & 1.06 & $\mathrm{~A} 5 \mathrm{U}$ & 1.04 & NBD \\
\hline F3 & midd & 1.05 & $\mathrm{~A} 5 \mathrm{U}$ & 1.04 & $\mathrm{BCD}$ & 1.04 & $\mathrm{BCD}$ & 1.04 & $\mathrm{BCD}$ & 1.02 & A5D \\
\hline F4 & midd & 1.00 & $\mathrm{SCA}$ & 1.04 & DBU & 1.04 & $B C D$ & 1.06 & $\mathrm{BCD}$ & 1.05 & $\mathrm{ASD}$ \\
\hline F5 & midd & 1.05 & DBD & 1.03 & $\mathrm{BCD}$ & 1.02 & $\mathrm{DBU}$ & 1.05 & DBU & 1.02 & $\mathrm{DBU}$ \\
\hline F6 & midd & 1.04 & DBD & 1.01 & DBU & 1.04 & $\mathrm{BCD}$ & 1.04 & $\mathrm{BCD}$ & 1.01 & DBU \\
\hline $\mathrm{Fl}$ & cente & 1.03 & $\mathrm{~A} 5 \mathrm{U}$ & 1.01 & TFT & 0.96 & $\mathrm{SCA}$ & 0.98 & SCA & 0.98 & A5D \\
\hline F2 & cente & 1.07 & $\mathrm{NBU}$ & 0.93 & $\mathrm{ASU}$ & 0.94 & ASU & 0.95 & ASU & 1.03 & NBD \\
\hline $\mathrm{F} 3$ & pecimens & 1.00 & $\mathrm{ASU}$ & 1.01 & $\mathrm{BCD}$ & 1.01 & $\mathrm{BCD}$ & 0.98 & $\mathrm{BCD}$ & 1.02 & A5D \\
\hline F4 & center & 0.96 & $\mathrm{SCA}$ & 1.10 & DBU & 1.08 & $B C D$ & 1.11 & $\mathrm{BCD}$ & 1.01 & A5D \\
\hline F5 & center specimens & 1.16 & DBD & 1.08 & $\mathrm{BCD}$ & 1.13 & $\overline{\mathrm{DBU}}$ & 1.17 & $\mathrm{DBU}$ & 1.12 & $\mathrm{DBU}$ \\
\hline $\mathrm{F} 6$ & center specimens & 1.34 & DBD & 1.18 & DBU & 1.16 & $\mathrm{BCD}$ & 1.19 & BCD & 1.25 & $\mathrm{DBU}$ \\
\hline & $\mathrm{Ni}$ & tier & 1 & tier & 5 & tier & 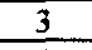 & tier & 4 & tier & 5 \\
\hline $\mathrm{F} 1$ & outer caps & 0.94 & $\mathrm{~A} 5 \mathrm{U}$ & 1.00 & TFT & 1.03 & $\mathrm{SCA}$ & 1.04 & SCA & $0 . \overline{93}$ & $\mathrm{ASD}$ \\
\hline $\mathrm{F} 2$ & outer capsule steel & 0.94 & $\mathrm{NBU}$ & 1.01 & A5U & 1.00 & $\mathrm{~A} 5 \mathrm{U}$ & 1.01 & $\mathrm{~A} 5 \mathrm{U}$ & 0.94 & NBD \\
\hline F3 & outer capsule steel & 0.96 & $\mathrm{~A} 5 \mathrm{U}$ & 0.99 & $\mathrm{BCD}$ & 0.98 & $\mathrm{BCD}$ & 0.98 & $\mathrm{BCD}$ & 0.92 & $\mathrm{~A} 5 \mathrm{D}$ \\
\hline F4 & outer capsule steel & 0.94 & $\mathrm{SCA}$ & 0.95 & $\mathrm{DBU}$ & 0.96 & $\mathrm{BCD}$ & 0.96 & $\mathrm{BCD}$ & 0.94 & ASD \\
\hline F5 & outer cap & 0.93 & DBD & 0.94 & $\mathrm{BCD}$ & 0.94 & DBU & 0.96 & DBU & 0.91 & $\mathrm{DBU}$ \\
\hline F6 & esteel & 0.89 & DBD & 0.91 & $\mathrm{DBU}$ & 0.94 & $\mathrm{BCD}$ & 0.93 & $\mathrm{BCD}$ & 0.87 & $\mathrm{DBU}$ \\
\hline $\mathrm{F} 1$ & midd! & 1.03 & A5U & 1.05 & TFT & 1.00 & $\mathrm{SCA}$ & 1.01 & SCA & 1.02 & A5D \\
\hline $\mathrm{F} 2$ & midd! & 1.05 & $\mathrm{NBU}$ & 1.06 & $\mathrm{~A} 5 \mathrm{U}$ & 1.05 & A5U & 1.06 & A5U & 1.04 & NBD \\
\hline F3 & midd & 1.05 & $\mathrm{~A} 5 \mathrm{U}$ & 1.04 & BCD & 1.04 & $\mathrm{BCD}$ & 1.04 & $\mathrm{BCD}$ & 1.03 & A5D \\
\hline F4 & midd & 1.00 & SCA & 1.04 & $\overline{\mathrm{DBU}}$ & 1.04 & $\mathrm{BCD}$ & 1.06 & $\mathrm{BCD}$ & 1.05 & A5D \\
\hline F5 & middl & 1.05 & DBD & 1.03 & $\mathrm{BCD}$ & 1.02 & $\overline{\mathrm{DBU}}$ & 1.05 & DBU & 1.02 & $\mathrm{DBU}$ \\
\hline F6 & middle holder & 1.04 & DBD & 1.01 & DBU & 1.04 & $\mathrm{BCD}$ & 1.04 & $\mathrm{BCD}$ & 1.01 & DBU \\
\hline $\mathbf{F l}$ & center specimens & 1.03 & A5U & 1.01 & TFT & 0.96 & SCA & 0.98 & $\mathrm{SCA}$ & 0.98 & A5D \\
\hline F2 & center specimens & 1.06 & NBU & 0.93 & $\mathrm{~A} 5 \mathrm{U}$ & 0.93 & $\mathrm{ASU}$ & 0.95 & A5U & 1.03 & NBD \\
\hline F3 & center specimens & 1.00 & $\mathrm{~A} 5 \mathrm{U}$ & 1.01 & $\mathrm{BCD}$ & 1.01 & $\mathrm{BCD}$ & 0.98 & $\mathrm{BCD}$ & 1.02 & A5D \\
\hline $\mathrm{F} 4$ & center specimens & 0.95 & SCA & 1.10 & $\mathrm{DBU}$ & 1.08 & $B C D$ & 1.11 & $\mathrm{BCD}$ & 1.01 & A5D \\
\hline F5 & center specimens & 1.16 & DBD & 1.09 & $B C D$ & 1.13 & DBU & 1.17 & $\mathrm{DBU}$ & 1.12 & $\overline{\mathrm{DBU}}$ \\
\hline
\end{tabular}




\begin{tabular}{|c|c|c|c|c|c|c|c|c|c|c|c|}
\hline F6 & enter specimens & 1.34 & DBD & 1.18 & $\overline{\text { DBU }}$ & 1.16 & $\overline{B C D}$ & 1.19 & $\mathrm{BCD}$ & 1.25 & $\overline{\mathrm{DBU}}$ \\
\hline & $\mathrm{Co}$ & tier & 1 & tier & 2 & tier & 3 & tier & 4 & tier & 5 \\
\hline F1 & outer capsule steel & 0.94 & A5U & 1.00 & TFT & 1.03 & $\mathrm{SCA}$ & 1.03 & SCA & 0.93 & A5D \\
\hline $\mathrm{F} 2$ & outer capsule steel & 0.94 & NBU & 1.00 & A5U & 1.00 & $\mathrm{ASU}$ & 1.01 & $\mathrm{~A} 5 \mathrm{U}$ & 0.94 & NBD \\
\hline F3 & outer capsule steel & 0.96 & A5U & 0.98 & $\mathrm{BCD}$ & 0.98 & $\mathrm{BCD}$ & 0.98 & $\mathrm{BCD}$ & 0.92 & A5D \\
\hline F4 & outer capsule steel & 0.94 & $\mathrm{SCA}$ & 0.95 & DBU & 0.95 & $\mathrm{BCD}$ & 0.96 & $\mathrm{BCD}$ & 0.94 & A5D \\
\hline F5 & outer capsule steel & 0.93 & DBD & 0.94 & BCD & 0.94 & DBU & 0.95 & DBU & 0.90 & $\mathrm{DBU}$ \\
\hline F6 & outer capsule steel & 0.89 & DBD & 0.91 & DBU & 0.94 & BCD & 0.93 & $\mathrm{BCD}$ & 0.88 & DBU \\
\hline F1 & middle holder & 1.03 & ASU & 1.05 & TFT & 1.00 & SCA & 1.01 & $\mathrm{SCA}$ & 1.02 & ASD \\
\hline $\bar{F} 2$ & middle holder & 1.05 & NBU & 1.05 & $\overline{\mathrm{A} 5 \mathrm{U}}$ & 1.05 & A5U & 1.06 & ASU & 1.04 & NBD \\
\hline F3 & middle holder & 1.05 & A5U & 1.04 & $\mathrm{BCD}$ & 1.04 & $\mathrm{BCD}$ & 1.04 & $\mathrm{BCD}$ & 1.02 & $\overline{A 5 D}$ \\
\hline$\overline{\mathrm{F} 4}$ & middle holder & 1.00 & SCA & 1.04 & DBU & 1.04 & $\mathrm{BCD}$ & 1.06 & $\overline{B C D}$ & 1.05 & A5D \\
\hline F5 & middle holder & 1.05 & DBD & 1.03 & $\mathrm{BCD}$ & 1.02 & DBU & 1.05 & DBU & 1.02 & $\overline{\mathrm{DBU}}$ \\
\hline F6 & middle holder & 1.04 & DBD & 1.01 & DBU & 1.04 & BCD & 1.04 & $\mathrm{BCD}$ & 1.01 & DBU \\
\hline F1 & center specimens & 1.03 & $\mathrm{~A} 5 \mathrm{U}$ & 1.01 & TFT & 0.96 & $\mathrm{SCA}$ & 0.98 & SCA & 0.98 & A5D \\
\hline F2 & center specimens & 1.06 & NBU & 0.93 & A5U & 0.94 & ASU & 0.95 & A5U & 1.03 & NBD \\
\hline F3 & cente & 1.00 & $\mathrm{~A} 5 \mathrm{U}$ & 1.01 & $\mathrm{BCD}$ & 1.01 & BCD & 0.98 & $\mathrm{BCD}$ & 1.02 & ASD \\
\hline F4 & cente & 0.96 & SCA & 1.10 & $\overline{\mathrm{DBU}}$ & 1.08 & $\mathrm{BCD}$ & 1.11 & $\mathrm{BCD}$ & 1.01 & ASD \\
\hline F5 & center $\mathrm{sp}$ & 1.15 & DBD & 1.08 & $\mathrm{BCD}$ & 1.13 & $\overline{\mathrm{DBU}}$ & 1.17 & DBU & 1.12 & $\overline{\mathrm{DBU}}$ \\
\hline F6 & cente & 1.34 & DBD & 1.18 & DBU & 1.15 & BCD & 1.18 & $\mathrm{BCD}$ & 1.25 & $\mathrm{DBU}$ \\
\hline & $\mathrm{Zr}$ & tier & 1 & tier & 2 & tier & 3 & tier & $\overline{4}$ & tier & 5 \\
\hline F1 & outer capsule steel & 0.97 & ASU & 1.02 & TFT & 1.05 & SCA & 1.06 & $\mathrm{SCA}$ & 0.95 & A5D \\
\hline $\mathrm{F} 2$ & outer capsule steel & 0.96 & NBU & 1.05 & $\mathrm{~A} 5 \mathrm{U}$ & 1.05 & A5U & 1.05 & A5U & 0.97 & NBD \\
\hline F3 & outer capsule steel & 1.00 & A5U & 1.02 & $\mathrm{BCD}$ & 1.02 & $\mathrm{BCD}$ & 1.01 & $\mathrm{BCD}$ & 0.95 & A5D \\
\hline $\mathrm{F} 4$ & outer capsule steel & 0.97 & SCA & 0.99 & DBU & 0.99 & $\mathrm{BCD}$ & 1.00 & $\mathrm{BCD}$ & 0.98 & ASD \\
\hline F5 & outer capsule steel & 0.96 & DBD & 0.98 & $\mathrm{BCD}$ & 0.98 & DBU & 0.99 & DBU & 0.93 & $\overline{\mathrm{DBU}}$ \\
\hline F6 & e steel & 0.91 & DBD & 0.95 & $\overline{\mathrm{DBU}}$ & 0.97 & $\mathrm{BCD}$ & 0.97 & $\mathrm{BCD}$ & 0.90 & DBU \\
\hline F1 & midd & 1.01 & $\mathrm{~A} 5 \mathrm{U}$ & 1.04 & TFT & 0.99 & SCA & 1.01 & SCA & 1.01 & A5D \\
\hline $\mathrm{F} 2$ & middl & 1.03 & NBU & 1.05 & ASU & 1.06 & $\mathrm{~A} 5 \mathrm{U}$ & 1.06 & A5U & 1.03 & $\overline{\mathrm{NBD}}$ \\
\hline F3 & mids & 1.05 & ASU & 1.03 & $\mathrm{BCD}$ & 1.03 & $\mathrm{BCD}$ & 1.03 & $\mathrm{BCD}$ & 1.01 & A5D \\
\hline F4 & mid & 1.00 & SCA & 1.03 & $\mathrm{DBU}$ & 1.03 & $\mathrm{BCD}$ & 1.05 & $\mathrm{BCD}$ & 1.04 & A5D \\
\hline F5 & mid & 1.04 & DBD & 1.02 & $\mathrm{BCD}$ & 1.01 & DBU & 1.03 & $\overline{\mathrm{DBU}}$ & 1.01 & $\overline{\mathrm{DBU}}$ \\
\hline F6 & midd & 1.02 & DBD & 1.00 & DBU & 1.02 & $\mathrm{BCD}$ & 1.03 & $\mathrm{BCD}$ & 1.00 & $\overline{\mathrm{DBU}}$ \\
\hline F1 & cents & 1.04 & $\mathrm{~A} 5 \mathrm{U}$ & 1.03 & TFT & 0.96 & SCA & 0.98 & SCA & 0.99 & A5D \\
\hline $\mathrm{F} 2$ & cent & 1.08 & NBU & 0.91 & $\mathrm{~A} 5 \mathrm{U}$ & 0.92 & $\mathrm{~A} 5 \mathrm{U}$ & 0.93 & A5U & 1.05 & NBD \\
\hline F3 & center sp & 0.97 & $\mathrm{~A} 5 \mathrm{U}$ & 1.00 & $\mathrm{BCD}$ & 1.00 & $\mathrm{BCD}$ & 0.97 & $B C D$ & 1.03 & $\overline{A 5 D}$ \\
\hline $\mathbf{F} 4$ & center sp & 0.95 & $\mathrm{SCA}$ & 1.10 & DBU & 1.08 & $\mathrm{BCD}$ & 1.11 & $\mathrm{BCD}$ & 0.99 & A5D \\
\hline F5 & center specimens & 1.16 & DBD & 1.08 & $\mathrm{BCD}$ & 1.15 & DBU & 1.19 & DBU & 1.12 & $\mathrm{DBU}$ \\
\hline F6 & center sp & 1.38 & DBD & 1.20 & DBU & 1.18 & $\mathrm{BCD}$ & 1.21 & $\mathrm{BCD}$ & 1.29 & DBU \\
\hline & $\mathrm{Nb}$ & tier & 1 & tier & 2 & tier & 3 & tier & 4 & tier & 5 \\
\hline F1 & outer capsule steel & 0.97 & $\mathrm{~A} 5 \mathrm{U}$ & 1.02 & TFT & 1.06 & SCA & 1.06 & $\mathrm{SCA}$ & 0.96 & A5D \\
\hline F2 & outer capsule steel & 0.97 & $\mathrm{NBU}$ & 1.06 & $\mathrm{ASU}$ & 1.05 & ASU & 1.06 & ASU & 0.97 & NBD \\
\hline F3 & outer capsule steel & 1.00 & A5U & 1.02 & $\mathrm{BCD}$ & 1.02 & $\mathrm{BCD}$ & 1.01 & $\mathrm{BCD}$ & 0.95 & A5D \\
\hline F4 & outer capsule steel & 0.97 & $\mathrm{SCA}$ & 0.99 & DBU & 1.00 & BCD & 1.01 & $\mathrm{BCD}$ & 0.98 & A5D \\
\hline F5 & outer capsule steel & 0.96 & DBD & 0.98 & $\mathrm{BCD}$ & 0.98 & DBU & 0.99 & DBU & 0.94 & $\overline{\mathrm{DBU}}$ \\
\hline F6 & outer capsule steel & 0.92 & DBD & 0.95 & DBU & 0.98 & $\mathrm{BCD}$ & 0.97 & $\mathrm{BCD}$ & 0.90 & $\overline{\mathrm{DBU}}$ \\
\hline F1 & middle holder & 1.01 & $\mathrm{~A} 5 \mathrm{U}$ & 1.04 & TFT & 0.99 & $\mathrm{SCA}$ & 1.01 & $\mathrm{SCA}$ & 1.01 & A5D \\
\hline $\mathrm{F} 2$ & middle holder & 1.03 & $\mathrm{NBU}$ & 1.05 & $\mathrm{~A} 5 \mathrm{U}$ & 1.06 & A5U & 1.06 & $\mathrm{~A} 5 \mathrm{U}$ & 1.03 & NBD \\
\hline F3 & middle holder & 1.05 & A5U & 1.03 & $\mathrm{BCD}$ & 1.03 & $\mathrm{BCD}$ & 1.03 & $\mathrm{BCD}$ & 1.01 & A5D \\
\hline F4 & middle holder & 1.00 & SCA & 1.03 & DBU & 1.03 & $\mathrm{BCD}$ & 1.05 & $\mathrm{BCD}$ & 1.04 & ASD \\
\hline F5 & middle holder & 1.04 & DBD & 1.02 & $\mathrm{BCD}$ & 1.01 & DBU & 1.03 & $\overline{\mathrm{DBU}}$ & 1.01 & $\overline{\mathrm{DBU}}$ \\
\hline
\end{tabular}




\begin{tabular}{|c|c|c|c|c|c|c|c|c|c|c|c|}
\hline F6 & iddle holder & 1.02 & DBD & 1.00 & $\overline{\mathrm{DBU}}$ & 1.02 & $\overline{\mathrm{BCD}}$ & 1.03 & $\mathrm{BCD}$ & 1.00 & DBU \\
\hline F1 & center specimens & 1.04 & $\mathrm{~A} 5 \mathrm{U}$ & 1.03 & TFT & 0.96 & $\overline{S C A}$ & 0.98 & $\mathrm{SCA}$ & 0.99 & $45 D^{-1}$ \\
\hline F2 & center specimens & 1.09 & NBU & 0.90 & $\overline{\mathrm{A} 5 \mathrm{U}}$ & $\overline{0.91}$ & $5 \mathrm{U}$ & & & 55 & BD \\
\hline F3 & center specimens & 0.97 & $\overline{\mathrm{A} 5 \mathrm{U}}$ & 0.99 & $\mathrm{BCD}$ & 1.00 & 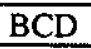 & 0.97 & & 03 & SDD \\
\hline F4 & center specimens & 0.95 & SCA & 1.10 & DBU & 1.08 & $\mathrm{BC}$ & 111 & $\mathrm{CD}$ & 0.99 & $5 \mathrm{D}$ \\
\hline F5 & center specimens & 1.16 & DBD & 1.08 & $\mathrm{BCD}$ & 1.15 & DBU & 1.19 & $\mathrm{DBU}$ & 1.12 & $\mathrm{DBU}$ \\
\hline F6 & center specimens & 1.38 & $\overline{\mathrm{DBD}}$ & 1.20 & DBU & 1.18 & $\mathrm{BCD}$ & 1.21 & $\mathrm{BCD}$ & 1.29 & $\mathrm{DBU}$ \\
\hline & Mo & tier & 1 & tier & 2 & tier & 3 & tier & 4 & tier & 5 \\
\hline $\mathrm{Fl}$ & outer & 0.97 & $\mathrm{A5U}$ & 1.03 & TFT & 1.06 & SCA & 1.07 & $\mathrm{CA}$ & 0.96 & A5D \\
\hline F2 & outer & 0.97 & $\mathrm{NBU}$ & 1.06 & A.5U & 1.06 & $\overline{\mathrm{ASU}}$ & 1.06 & $\overline{\mathrm{A} 5 \mathrm{U}}$ & 0.98 & NBD \\
\hline F3 & outer & 1.01 & $\mathrm{~A} 5 \mathrm{U}$ & 1.03 & $\mathrm{BCD}$ & 1.03 & BCD & 1.02 & $\overline{B C D}$ & 0.95 & ASD \\
\hline F4 & outer & 0.97 & $\mathrm{SCA}$ & 1.00 & DBU & 1.00 & $\mathrm{BCD}$ & 1.01 & $\mathrm{BCD}$ & 0.99 & ASD \\
\hline F5 & outer & 0.97 & DBD & 0.99 & $\mathrm{BCD}$ & 0.98 & $\mathrm{DF}$ & 1. & $\mathrm{U}$ & 0.94 & DBU \\
\hline F6 & outer & 0.92 & DBD & 0.95 & $\mathrm{DBU}$ & 0.98 & $\mathrm{BCD}$ & 0.98 & D & 0.91 & DBU \\
\hline F1 & midd & 1.01 & $\mathrm{ASU}$ & 1.03 & TFT & 0.99 & SCA & 1.01 & SCA & 1.01 & A5D \\
\hline $\mathrm{F} 2$ & midd & 1.03 & $\mathrm{NBU}$ & 1.05 & $\bar{A}$ & 06 & A5U & 1.06 & & 1.02 & NBD \\
\hline F3 & midd & 1.04 & A5U & 1.03 & $\mathrm{CD}$ & 1.03 & D & 1.03 & & 1.01 & $\mathrm{~A} 5 \mathrm{D}$ \\
\hline F4 & midd & 1.00 & SCA & 1.03 & $\mathrm{BU}$ & 1.03 & D & 1.05 & & 1.04 & A5D \\
\hline 15 & midd & 1.03 & DBD & 1.02 & $\mathrm{BCD}$ & 1.01 & $8 B U$ & 1.03 & 3U & 1.00 & $\mathrm{DBU}$ \\
\hline F6 & midd & 1.02 & DBD & 1.00 & DBU & 1.02 & $\mathrm{CD}$ & 1.03 & $\mathrm{BCD}$ & 0.99 & $\mathrm{DBU}$ \\
\hline F1 & cente & 1.04 & A5U & 1.03 & TFT & 0.96 & SCA & 0.98 & SCA & 1.00 & A5D \\
\hline $\mathrm{F} 2$ & cente & 1.09 & $\mathrm{NBU}$ & 0.90 & $\mathrm{~A} 5 \mathrm{U}$ & 0.91 & 150 & 0.92 & A5U & 1.05 & NBD \\
\hline F3 & cente & 0.97 & A5U & 0.99 & $\mathrm{BCD}$ & 1.00 & BCD & 0.9 & BCD & 1.04 & A5D \\
\hline $\mathrm{F} 4$ & cente & 0.95 & $\mathrm{SCA}$ & 1.10 & $\mathrm{DBU}$ & 1.08 & $\mathrm{BC}$ & 1.12 & $\mathrm{BCD}$ & 0.99 & A5D \\
\hline F5 & cente & 1.16 & DBD & 1.09 & BCD & 1.15 & & 1.20 & DBU & 1.12 & $\mathrm{DBU}$ \\
\hline F6 & ce & 1.39 & DBD & 1.21 & $\overline{\mathrm{DBU}}$ & 1,19 & $B C D$ & 1.22 & $\mathrm{BCD}$ & 1.30 & $\mathrm{DBU}$ \\
\hline & $\mathrm{Hf}$ & tier & 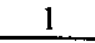 & tier & 2 & $r$ & 3 & $r$ & 4 & tier & 5 \\
\hline F1 & outer & 1.01 & $\mathrm{~A} 5 \mathrm{U}$ & 1.05 & TFT & 1.23 & A & 1.24 & $S C A$ & .00 & $45 \mathrm{D}$ \\
\hline $\mathrm{F} 2$ & Outer & 1.01 & $\mathrm{NBU}$ & 1.17 & $\underline{\mathrm{ASS}}$ & 1.16 & $\bar{A}$ & 1.16 & $\mathrm{~A} 5 \mathrm{U}$ & 1.02 & NBD \\
\hline$F 3$ & outer & 1.11 & $\mathrm{~A} 5 \mathrm{U}$ & 1.08 & $\overline{B C D}$ & 1.07 & $\mathrm{BCD}$ & 1.07 & $\overline{\mathrm{BCD}}$ & 1.00 & A5D \\
\hline $\mathrm{F4}$ & outer & 1.13 & SCA & 1.05 & $\mathrm{DBU}$ & 1.06 & $\mathrm{BCD}$ & 1.07 & $\mathrm{BCD}$ & 1.09 & A5D \\
\hline F5 & outer & 1.02 & $\mathrm{DBD}$ & 1.05 & $B C D$ & 1.06 & DBU & 1.07 & $\overline{\mathrm{DBU}}$ & 0.99 & $\overline{\mathrm{DBU}}$ \\
\hline $\mathrm{F} 6$ & outer & 0.97 & $\mathrm{DBD}$ & 1.03 & $\overline{\mathrm{DBU}}$ & 1.06 & BCD & 1.05 & $\mathrm{BCD}$ & 0.97 & $\mathrm{DBU}$ \\
\hline $\mathrm{Fl}$ & mid & 1.01 & $\mathrm{~A} 5 \mathrm{U}$ & 1.03 & TFT & 0.97 & $S C$ & 0.99 & SCA & 1.00 & A5D \\
\hline $\mathrm{F} 2$ & $\mathrm{mi}$ & 1.02 & $\underline{\mathrm{BU}}$ & 1.11 & A5U & 1.11 & $\overline{A 5 U}$ & 1.1 & A5U & 1.02 & NBD \\
\hline F3 & & 1.09 & $\mathrm{~A} 5 \mathrm{U}$ & 1.04 & $\mathrm{BCD}$ & 1.0 & $\mathrm{BCD}$ & 1.0 & $\mathrm{BCD}$ & 1.00 & A5D \\
\hline $\mathrm{F} 4$ & & 0.98 & SCA & .03 & $\overline{\mathrm{DBU}}$ & 1.03 & $\mathrm{BCD}$ & 1.05 & $\mathrm{BCD}$ & 1.09 & A5D \\
\hline F5 & & 1.03 & D & 1.03 & $\mathrm{BCD}$ & 1.03 & DBU & 1.05 & DBU & 1.00 & DBU \\
\hline $\mathrm{F} 6$ & midd & 1.03 & $\mathrm{D}$ & 1.02 & DBU & 1.04 & $\mathrm{BCD}$ & 1.04 & $\mathrm{BCD}$ & 1.00 & DBU \\
\hline $\mathrm{Fl}$ & cente & 1.02 & $\mathrm{~A} 5 \mathrm{U}$ & 1.02 & TFT & 0.97 & SCA & 0.99 & SCA & 1.00 & A5D \\
\hline $\mathrm{F} 2$ & cente & 1.04 & NBU & 0.80 & $\mathrm{ASU}$ & 0.80 & $\mathrm{~A} 5 \mathrm{U}$ & 0.82 & $\mathrm{~A} 5 \mathrm{U}$ & 1.01 & NBD \\
\hline F3 & center & 0.87 & $\mathrm{~A} 5 \mathrm{U}$ & 0.89 & $\mathrm{BCD}$ & 0.90 & $\mathrm{BCD}$ & 0.88 & $\mathrm{BCD}$ & 1.01 & A5D \\
\hline F4 & cente & 0.96 & $\mathrm{SCA}$ & 0.99 & $\mathrm{DBU}$ & 0.98 & $\mathrm{BCD}$ & 1.00 & $\mathrm{BCD}$ & 0.88 & A5D \\
\hline $\mathrm{F5}$ & cente & 1.07 & DBD & 0.97 & BCD & 0.99 & $\overline{\mathrm{DBU}}$ & 1.02 & $\mathrm{DBU}$ & 1.03 & DBU \\
\hline F6 & cente & 1.23 & DBD & 1.04 & $\overline{\mathrm{DBU}}$ & 1.02 & $\mathrm{BCD}$ & 1.05 & $\mathrm{BCD}$ & $1 . \overline{14}$ & $\mathrm{DBU}$ \\
\hline & $\mathrm{Ta}$ & tier & 1 & tier & 2 & tier & 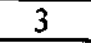 & tie & 4 & tier & 5 \\
\hline$F 1$ & outer cap & 1.01 & $5 \mathrm{U}$ & 1.05 & TFT & 1.24 & $\mathrm{SCA}$ & 1.25 & SCA & 1.00 & A5D \\
\hline F2 & outer capsule steel & 1.01 & $\mathrm{NBU}$ & 1.17 & ASU & 1.16 & $\mathrm{~A} 5 \mathrm{U}$ & 1.16 & $\mathrm{~A} 5 \mathrm{U}$ & 1.02 & NBD \\
\hline F3 & outer capsule steel & 1.11 & ASU & 1.08 & $\mathrm{BCD}$ & 1.07 & $\mathrm{BCD}$ & 1.07 & $\mathrm{BCD}$ & 1.00 & A5D \\
\hline F4 & outer capsule steel & 1.14 & SCA & 1.05 & DBU & 1.06 & $\mathrm{BCD}$ & 1.07 & $\mathrm{BCD}$ & 1.09 & $\mathrm{~A} 5 \mathrm{D}$ \\
\hline & outer capsule steel & .02 & DBD & 1.05 & $\mathrm{BCD}$ & 1.06 & DBU & 1.08 & DBU & 0.99 & $\mathrm{DBU}$ \\
\hline
\end{tabular}




\begin{tabular}{|c|c|c|c|c|c|c|c|c|c|c|c|}
\hline F6 & uter capsule steel & 0.97 & DBD & 1.03 & DBU & 1.06 & $\mathrm{BCD}$ & 1.05 & $\mathrm{BCD}$ & 0.97 & DBU \\
\hline F1 & ijddle holder & 1.01 & $\mathrm{ASU}$ & 1.03 & TFT & 0.97 & SCA & 0.99 & SCA & 1.00 & A5D \\
\hline F2 & iiddle holder & 1.02 & NBU & 1.11 & A5U & 1.11 & A5U & 1.12 & ASU & 1.02 & NBD \\
\hline F3 & jddle holder & 1.09 & ASU & 1.04 & $\mathrm{BCD}$ & 1.04 & $\mathrm{BCD}$ & 1.05 & $\mathrm{BCD}$ & 1.00 & A5D \\
\hline F4 & ijddle holder & 0.98 & SCA & 1.03 & DBU & 1.03 & $\mathrm{BCD}$ & 1.05 & $\mathrm{BCD}$ & 1.09 & A5D \\
\hline F5 & iddle holder & 1.03 & DBD & 1.03 & $\mathrm{BCD}$ & 1.04 & $\overline{\mathrm{DBU}}$ & 1.06 & $\overline{\mathrm{DBU}}$ & 1.00 & $\overline{\mathrm{DBU}}$ \\
\hline F6 & middle holder & 1.03 & DBD & 1.02 & $\overline{D B U}$ & 1.05 & $\mathrm{BCD}$ & 1.04 & $\mathrm{BCD}$ & 1.00 & $\overline{\mathrm{DBU}}$ \\
\hline F1 & center specimens & 1.02 & ASU & 1.01 & TFT & 0.97 & SCA & 0.99 & $\mathrm{SCA}$ & 1.00 & A5D \\
\hline F2 & center specimens & 1.04 & $\mathrm{NBU}$ & 0.80 & A5U & 0.80 & ASU & 0.82 & $\mathrm{ASU}$ & 1.01 & NBD \\
\hline F3 & center specimens & 0.86 & A5U & 0.89 & $\mathrm{BCD}$ & 0.90 & $\mathrm{BCD}$ & 0.88 & $\mathrm{BCD}$ & 1.01 & A5D \\
\hline F4 & cente & 0.96 & SCA & 0.99 & DBU & 0.97 & $\mathrm{BCD}$ & 1.00 & $\mathrm{BCD}$ & 0.88 & A5D \\
\hline F5 & cente & 1.06 & DBD & 0.97 & $\mathrm{BCD}$ & 0.98 & $\overline{D B U}$ & 1.02 & $\overline{\mathrm{DBU}}$ & 1.03 & $\overline{D B U}$ \\
\hline F6 & imens & 1.22 & DBD & 1.04 & $\mathrm{DBU}$ & 1.01 & $\mathrm{BCD}$ & 1.04 & $\mathrm{BCD}$ & 1.14 & DBU \\
\hline & W & tier & 1 & tier & 2 & tier & 3 & tier & 4 & tier & 5 \\
\hline $\mathrm{Fl}$ & outel & 1.01 & A5U & 1.05 & TFT & 1.24 & SCA & 1.25 & SCA & 1.00 & A5D \\
\hline F2 & oute: & 1.01 & NBU & 1.17 & $\mathrm{~A} 5 \mathrm{U}$ & 1.16 & $\mathrm{~A} 5 \mathrm{U}$ & 1.17 & $\overline{\mathrm{A} 5 \mathrm{U}}$ & 1.02 & NBD \\
\hline$F 3$ & o & 1.11 & A.5U & 1.08 & $\mathrm{BCD}$ & 1.07 & $\mathrm{BCD}$ & 1.07 & $\mathrm{BCD}$ & 1.00 & A5D \\
\hline F4 & oute & 1.14 & $\mathrm{SCA}$ & 1.05 & DBU & 1.06 & $\mathrm{BCD}$ & 1.07 & $\mathrm{BCD}$ & \begin{tabular}{|l|}
1.09 \\
\end{tabular} & A5D \\
\hline F5 & oute & 1.02 & DBD & 1.05 & BCD & 1.06 & DBU & 1.08 & $\overline{\mathrm{DBU}}$ & 0.99 & DBU \\
\hline$\overline{F 6}$ & out & 0.97 & DBD & 1.03 & $\overline{\mathrm{DBU}}$ & 1.06 & $\mathrm{BCD}$ & 1.05 & $\mathrm{BCD}$ & 0.97 & $\mathrm{DBU}$ \\
\hline F1 & $\mathrm{mid}$ & 1.01 & A5U & 1.04 & TFT & 0.97 & SCA & 0.99 & SCA & 1.00 & ASD \\
\hline $\mathrm{F} 2$ & $\mathrm{mid}$ & 1.02 & NBU & 1.12 & A5U & 1.11 & $\mathrm{~A} 5 \mathrm{U}$ & 1.12 & $\overline{A 5 U}$ & 1.02 & NBD \\
\hline F3 & mid & 1.09 & A5U & 1.04 & $\mathrm{BCD}$ & 1.04 & $\mathrm{BCD}$ & 1.05 & BCD & 1.00 & A5D \\
\hline F4 & & 0.98 & SCA & 1.03 & DBU & 1.03 & $\overline{\mathrm{BCD}}$ & 1.05 & $\mathrm{BCD}$ & 1.09 & A5D \\
\hline F5 & & 1.03 & DBD & 1.03 & $\mathrm{BCD}$ & 1.04 & DBU & 1.06 & $\overline{\mathrm{DBU}}$ & 1.00 & DBU \\
\hline F6 & & 1.03 & DBD & 1.02 & DBU & 1.05 & $\mathrm{BCD}$ & 1.04 & $\mathrm{BCD}$ & 1.00 & $\overline{\mathrm{DBU}}$ \\
\hline F1 & ce & 1.02 & $\mathrm{ASU}$ & 1.01 & TFT & 0.97 & SCA & 0.99 & SCA & 1.00 & $\mathrm{~A} 5 \mathrm{D}$ \\
\hline F2 & 7 & 1.04 & NBU & 0.79 & $\mathrm{~A} 5 \mathrm{U}$ & 0.80 & ASU & 0.82 & $\mathrm{~A} 5 \mathrm{U}$ & 1.01 & NBD \\
\hline F3 & cente & 0.86 & ASU & 0.89 & BCD & 0.90 & $\mathrm{BCD}$ & 0.87 & $\mathrm{BCD}$ & 1.01 & A5D \\
\hline F4 & ens & 0.96 & SCA & 0.99 & DBU & 0.97 & $\mathrm{BCD}$ & 1.00 & $\mathrm{BCD}$ & 0.88 & A5D \\
\hline F5 & nt. & 1.06 & DBD & 0.97 & BCD & 0.98 & DBU & 1.02 & DBU & 1.02 & DBU \\
\hline F6 & cent & 1.22 & DBD & 1.04 & DBU & 1.01 & $\mathrm{BCD}$ & 1.04 & $\mathrm{BCD}$ & 1.14 & DBU \\
\hline & $\operatorname{Re}$ & tier & $\overline{1}$ & tier & 2 & tier & 3 & tier & 4 & tier & 5 \\
\hline $\bar{F} 1$ & outer & 1.01 & A5U & 1.05 & TFT & 1.24 & SCA & 1.25 & $\mathrm{SCA}$ & 1.00 & ASD \\
\hline F2 & outer capsule steel & 1.01 & NBU & 1.17 & A5U & 1.16 & A5U & 1.17 & $\mathrm{~A} 5 \overline{\mathrm{U}}$ & 1.02 & NBD \\
\hline$\overline{F 3}$ & outer capsule steel & 1.11 & A5U & 1.08 & $\mathrm{BCD}$ & 1.07 & $\mathrm{BCD}$ & 1.07 & $\mathrm{BCD}$ & 1.00 & ASD \\
\hline F4 & outer capsule steel & 1.14 & $\mathrm{SCA}$ & 1.05 & $\overline{\text { DBU }}$ & 1.06 & $\mathrm{BCD}$ & 1.07 & $\mathrm{BCD}$ & 1.09 & ASD \\
\hline F5 & le steel & 1.02 & DBD & 1.05 & $\mathrm{BCD}$ & 1.06 & $\overline{\mathrm{DBU}}$ & 1.07 & $\overline{\mathrm{DBU}}$ & 0.99 & DBU \\
\hline F6 & out & 0.97 & DBD & 1.03 & $\overline{\mathrm{DBU}}$ & 1.06 & $\mathrm{BCD}$ & 1.05 & $\mathrm{BCD}$ & 0.97 & DBU \\
\hline F1 & & 1.01 & $\mathrm{~A} 5 \mathrm{U}$ & 1.04 & TFT & 0.97 & SCA & 0.99 & $\mathrm{SCA}$ & 1.00 & A5D \\
\hline $\bar{F} 2$ & & 1.02 & $\overline{\mathrm{NBU}}$ & 1.12 & $\mathrm{ASU}$ & 1.11 & A5U & 1.12 & $\mathrm{~A} 5 \mathrm{U}$ & 1.02 & NBD \\
\hline. $\mathrm{F3}$ & & 1.09 & ASU & 1.04 & $\mathrm{BCD}$ & 1.04 & $\mathrm{BCD}$ & 1.05 & $\mathrm{BCD}$ & 1.00 & ASD \\
\hline F4 & $\operatorname{mid}$ & 0.98 & SCA & 1.03 & DBU & 1.03 & $\mathrm{BCD}$ & 1.05 & $B C D$ & 1.09 & A5D \\
\hline F5 & mid & 1.03 & DBD & 1.03 & $\mathrm{BCD}$ & 1.04 & DBU & 1.06 & $\overline{\mathrm{DBU}}$ & 1.00 & $\mathrm{DBU}$ \\
\hline F6 & mid & 1.03 & DBD & 1.02 & DBU & 1.05 & $\mathrm{BCD}$ & 1.04 & $\mathrm{BCD}$ & 1.00 & DBU \\
\hline $\mathrm{Fl}$ & mens & 1.02 & A5U & 1.01 & TFT & 0.97 & SCA & 0.99 & SCA & 1.00 & ASD \\
\hline $\mathrm{F} 2$ & mens & 1.04 & NBU & 0.79 & A5U & 0.80 & $\mathrm{~A} 5 \mathrm{U}$ & 0.81 & $\mathrm{~A} 5 \mathrm{U}$ & 1.01 & NBD \\
\hline F3 & imens & 0.86 & $\mathrm{ASU}$ & 0.89 & $\mathrm{BCD}$ & 0.90 & $\mathrm{BCD}$ & 0.87 & $\mathrm{BCD}$ & 1.01 & $\overline{\mathrm{ASD}}$ \\
\hline F4 & center $\mathrm{s}$ & 0.96 & $\mathrm{SCA}$ & 0.99 & $\overline{\mathrm{DBU}}$ & $0 . \overline{97}$ & $\overline{\mathrm{BCD}}$ & 1.00 & $\overline{B C D}$ & 0.88 & A5D \\
\hline F5 & center spe & 1.06 & DBD & 0.97 & $\mathrm{BCD}$ & 0.98 & $\overline{\mathrm{DBU}}$ & 1.02 & $\overline{\mathrm{DBU}}$ & 1.02 & DBU \\
\hline F6 & center specimens & 1.22 & DBD & 1.04 & $\overline{\text { DBU }}$ & 1.01 & $\mathrm{BCD}$ & 1.04 & $\overline{\mathrm{BCD}}$ & 1.14 & DBU \\
\hline
\end{tabular}


Appendix B

Front-to-Back Heating Gradients in Capsules

Table B.1. Ratio of Front (towards core) to Back (away from core) Heating Rates in Selected Capsules

(Note: statistical uncertainties $\sim 0.5 \%$ )

\begin{tabular}{|c|c|c|c|c|c|c|}
\hline & \multicolumn{3}{|c|}{ A Tier 3} & \multicolumn{3}{|c|}{ A Tier 5} \\
\hline Compartment & F3 & F2 & Fl & F3 & F2 & $\mathrm{F} 1$ \\
\hline Capsule Type & $\mathrm{BCD}$ & $\mathrm{A} 5 \mathrm{U}$ & SCA & A5D & NBD & A5D \\
\hline \multicolumn{7}{|l|}{$\mathrm{He}$} \\
\hline outer capsule steel & 1.20 & 1.20 & 1.17 & 1.12 & 1.12 & 1.11 \\
\hline middle holder & 1.13 & 1.13 & 1.10 & 1.09 & 1.09 & 1.09 \\
\hline center specimens & 1.05 & 1.05 & 1.03 & 1.02 & 1.02 & 1.02 \\
\hline \multicolumn{7}{|l|}{$\mathrm{B}$ (nat) } \\
\hline outer capsule steel & 0.98 & 0.98 & 1.00 & 1.03 & 1.03 & 0.98 \\
\hline middle holder & 0.99 & 0.99 & 1.00 & 1.00 & 1.00 & 1,01 \\
\hline center specimens & 0.98 & 0.98 & 1.00 & 1.01 & 1.01 & 1.01 \\
\hline \multicolumn{7}{|l|}{$\mathrm{Be}$} \\
\hline outer capsule steel & 1.18 & 1.18 & 1.15 & 1.09 & 1.09 & 1.07 \\
\hline middle holder & 1.12 & 1.12 & 1.09 & 1.05 & 1.05 & 1.06 \\
\hline center specimens & 1.05 & 1.05 & 1.03 & 1.01 & 1.01 & 1.03 \\
\hline \multicolumn{7}{|l|}{$\mathrm{C}$} \\
\hline outer capsule steel & 1.18 & 1.18 & 1.16 & 1.07 & 1.07 & 1.05 \\
\hline middle holder & 1.12 & 1.12 & 1.10 & 1.05 & 1.05 & 1.04 \\
\hline center specimens & 1.05 & 1.05 & 1.03 & 1.01 & 1.01 & 1.01 \\
\hline \multicolumn{7}{|l|}{0} \\
\hline outer capsule steel & 1.17 & 1.17 & 1.16 & 1.06 & 1.06 & 1.03 \\
\hline middle holder & 1.12 & 1.12 & 1.10 & 1.04 & 1.04 & 1.02 \\
\hline center specimens & 1.05 & 1.05 & 1.03 & 1.00 & 1.00 & 1.01 \\
\hline \multicolumn{7}{|l|}{$\mathrm{Al}$} \\
\hline outer capsule steel & 1.18 & 1.18 & 1.17 & 1.05 & 1.05 & 1.00 \\
\hline middle holder & 1.12 & 1.12 & 1.10 & 1.03 & 1.03 & 1.00 \\
\hline center specimens & 1.06 & 1.06 & 1.02 & 1.00 & 1.00 & 0.99 \\
\hline \multicolumn{7}{|l|}{$\overline{\mathrm{Si}}$} \\
\hline outer capsule steel & 1.19 & 1.19 & 1.18 & 1.04 & 1.04 & 1.00 \\
\hline middle holder & 1.12 & 1.12 & 1.11 & 1.03 & 1.03 & 1.00 \\
\hline center specimens & 1.06 & 1.06 & 1.03 & 1.00 & 1.00 & 0.99 \\
\hline \multicolumn{7}{|l|}{$\mathrm{Ti}$} \\
\hline outer capsule steel & 1.18 & 1.18 & 1.18 & 1.04 & 1.04 & 0.98 \\
\hline middle holder & 1.12 & 1.12 & 1.11 & 1.03 & 1.03 & 0.99 \\
\hline center specimens & 1.06 & 1.06 & 1.02 & 1.00 & 1.00 & 0.98 \\
\hline \multicolumn{7}{|l|}{$\overline{\mathrm{V}}$} \\
\hline outer capsule steel & 1.18 & 1.18 & 1.17 & 1.04 & 1.04 & 0.98 \\
\hline middle holder & 1.12 & 1.12 & 1.10 & 1.03 & 1.03 & 0.99 \\
\hline center specimens & 1.06 & 1.06 & 1.02 & 0.99 & 0.99 & 0.98 \\
\hline
\end{tabular}




\begin{tabular}{|c|c|c|c|c|c|c|}
\hline \multicolumn{7}{|l|}{$\mathrm{Cr}$} \\
\hline outer capsule steel & 1.18 & 1.18 & 1.17 & 1.03 & 1.03 & 0.98 \\
\hline middle holder & 1.12 & 1.12 & 1.10 & 1.03 & 1.03 & 0.99 \\
\hline center specimens & 1.06 & 1.06 & 1.02 & 0.99 & 0.99 & 0.98 \\
\hline \multicolumn{7}{|l|}{$\mathrm{Mn}$} \\
\hline outer capsule steel & 1.17 & 1.17 & 1.17 & 1.03 & 1.03 & 0.98 \\
\hline middle holder & 1.12 & 1.12 & 1.10 & 1.03 & 1.03 & 0.99 \\
\hline center specimens & 1.06 & 1.06 & 1.02 & 0.99 & 0.99 & 0.98 \\
\hline \multicolumn{7}{|l|}{$\mathrm{Fe}$} \\
\hline outer capsule steel & 1.18 & 1.18 & 1.18 & 1.03 & 1.03 & 0.98 \\
\hline middle holder & 1.12 & 1.12 & 1.11 & 1.03 & 1.03 & 0.99 \\
\hline center specimens & 1.06 & 1.06 & 1.02 & 0.99 & 0.99 & 0.98 \\
\hline \multicolumn{7}{|l|}{$\mathrm{Ni}$} \\
\hline outer capsule steel & 1.18 & 1.18 & 1.18 & 1.04 & 1.04 & 0.98 \\
\hline middle holder & 1.12 & 1.12 & 1.11 & 1.03 & 1.03 & 0.99 \\
\hline center specimens & 1.07 & 1.07 & 1.02 & 0.99 & 0.99 & 0.99 \\
\hline \multicolumn{7}{|l|}{$\mathrm{Co}$} \\
\hline outer capsule steel & 1.17 & 1.17 & 1.17 & 1.03 & 1.03 & 0.98 \\
\hline middle holder & 1.12 & 1.12 & 1.10 & 1.03 & 1.03 & 0.99 \\
\hline center specimens & 1.06 & 1.06 & 1.02 & 0.99 & 0.99 & 0.98 \\
\hline \multicolumn{7}{|l|}{$\overline{\mathrm{Zr}}$} \\
\hline outer capsule steel & 1.15 & 1.15 & 1.16 & 1.04 & 1.04 & 0.98 \\
\hline middle holder & 1.11 & 1.11 & 1.10 & 1.03 & 1.03 & 0.99 \\
\hline center specimens & 1.06 & 1.06 & 1.02 & 0.98 & 0.98 & 0.98 \\
\hline \multicolumn{7}{|l|}{$\mathrm{Nb}$} \\
\hline outer capsule steel & 1.15 & 1.15 & 1.16 & 1.04 & 1.04 & 0.99 \\
\hline middle holder & 1.11 & 1.11 & 1.10 & 1.03 & 1.03 & 0.99 \\
\hline center specimens & 1.06 & 1.06 & 1.02 & 0.98 & 0.98 & 0.98 \\
\hline \multicolumn{7}{|l|}{ Mo } \\
\hline outer capsule steel & 1.15 & 1.15 & 1.16 & 1.04 & 1.04 & 0.98 \\
\hline middle holder & 1.11 & 1.11 & 1.10 & 1.03 & 1.03 & 0.98 \\
\hline center specimens & 1.06 & 1.06 & 1.02 & 0.98 & 0.98 & 0.98 \\
\hline \multicolumn{7}{|l|}{$\mathrm{Hf}$} \\
\hline outer capsule steel & 1.12 & 1.12 & 1.15 & 1.05 & 1,05 & 1.00 \\
\hline middle holder & 1.10 & 1.10 & 1.10 & 1.04 & 1.04 & 0.99 \\
\hline center specimens & 1.07 & 1.07 & 1.02 & 0.99 & 0.99 & 0.99 \\
\hline \multicolumn{7}{|l|}{$\mathrm{Ta}$} \\
\hline outer capsule steel & 1.12 & 1.12 & 1.15 & 1.05 & 1.05 & 1.00 \\
\hline middle holder & 1.10 & 1.10 & 1.10 & 1.04 & 1.04 & 0.99 \\
\hline center specimens & 1.07 & 1.07 & 1.02 & 0.99 & 0.99 & 0.99 \\
\hline \multicolumn{7}{|l|}{ W } \\
\hline outer capsule steel & 1.12 & 1.12 & 1.15 & 1.05 & 1.05 & 1.01 \\
\hline middle holder & 1.10 & 1.10 & 1.10 & 1.04 & 1.04 & 0.99 \\
\hline center specimens & 1.07 & 1.07 & 1.02 & 0.99 & 0.99 & 0.99 \\
\hline \multicolumn{7}{|l|}{$\operatorname{Re}$} \\
\hline Outer capsule steel & 1.12 & 1.12 & 1.15 & 1.05 & 1.05 & 1.01 \\
\hline middle bolder & 1.10 & 1.10 & 1.10 & 1.05 & 1.05 & 1.00 \\
\hline center specimens & 1.07 & 1.07 & 1.02 & 0.99 & 0.99 & 0.99 \\
\hline
\end{tabular}


Attachment $D$ of

Enclosure (7) to

MDO-723-0044I

B-MT(SRME)-52

Page 1

\section{Attachment D of}

\section{Enclosure 7 to MDO-723-0044/B-MT(SRME)-52}

Initial JOYO Initial Design Conditions 
Attachment $D$ of

Enclosure (7) to

MDO-723-0044/

B-MT(SRME)-52

Page 2 

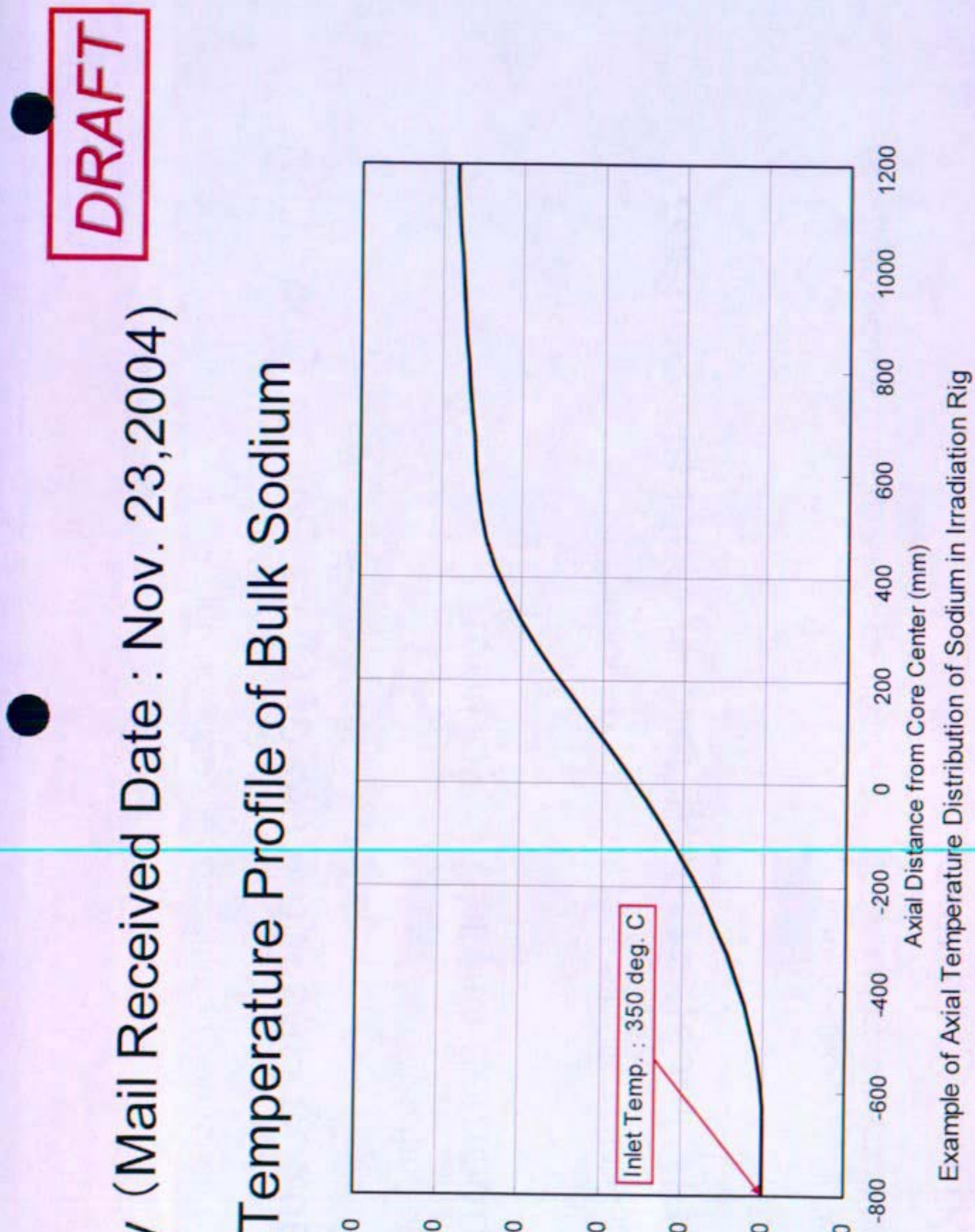

希

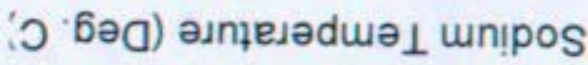



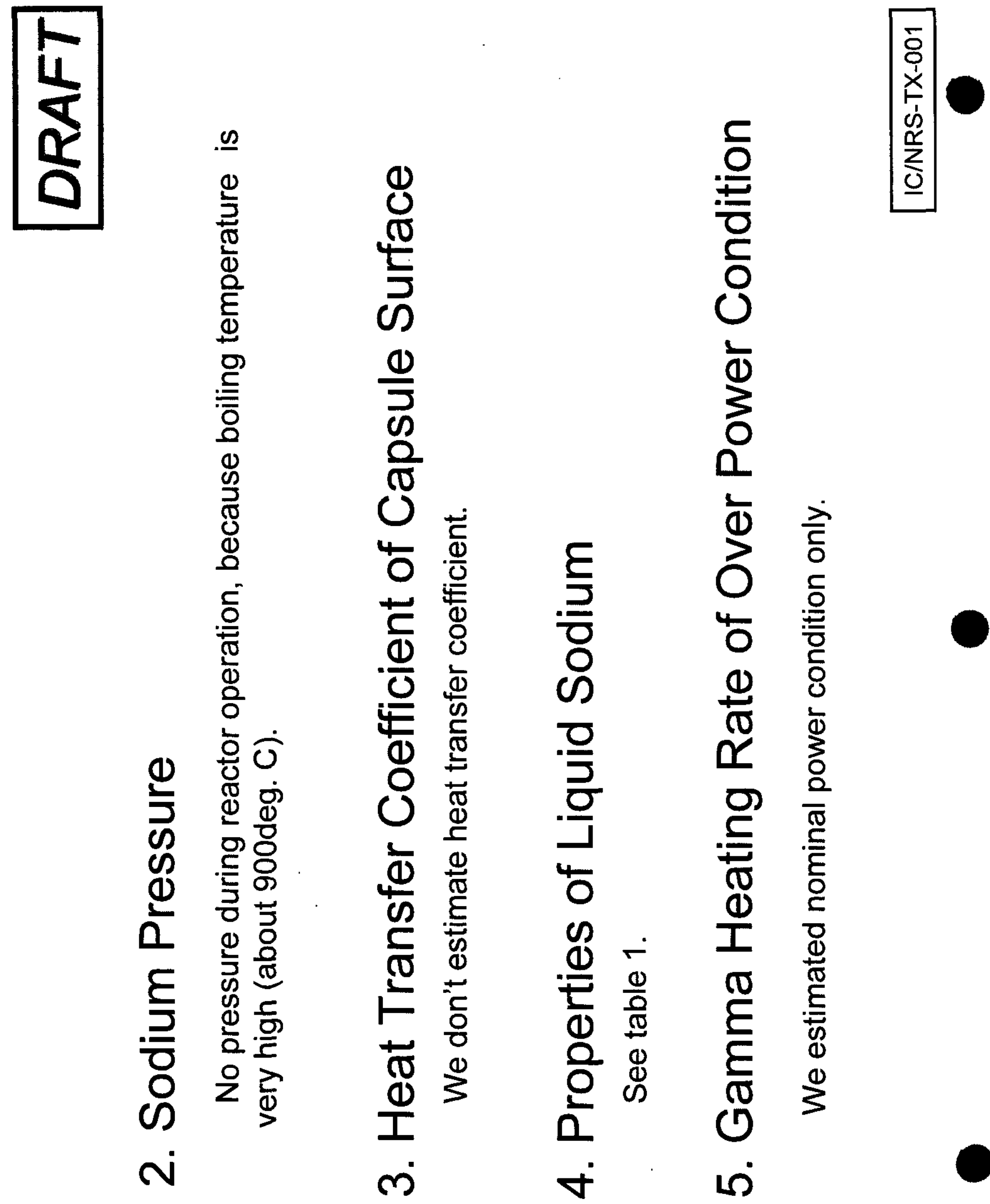

口

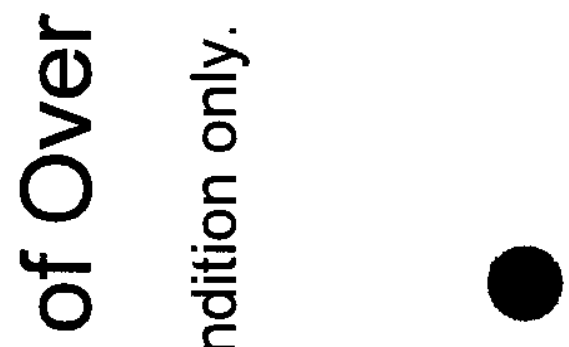

(1)

வ

을

の. I

(1) I

$=0$

(1)

(1)

응

응

음

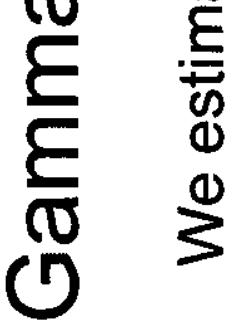

$\dot{8}$

م 


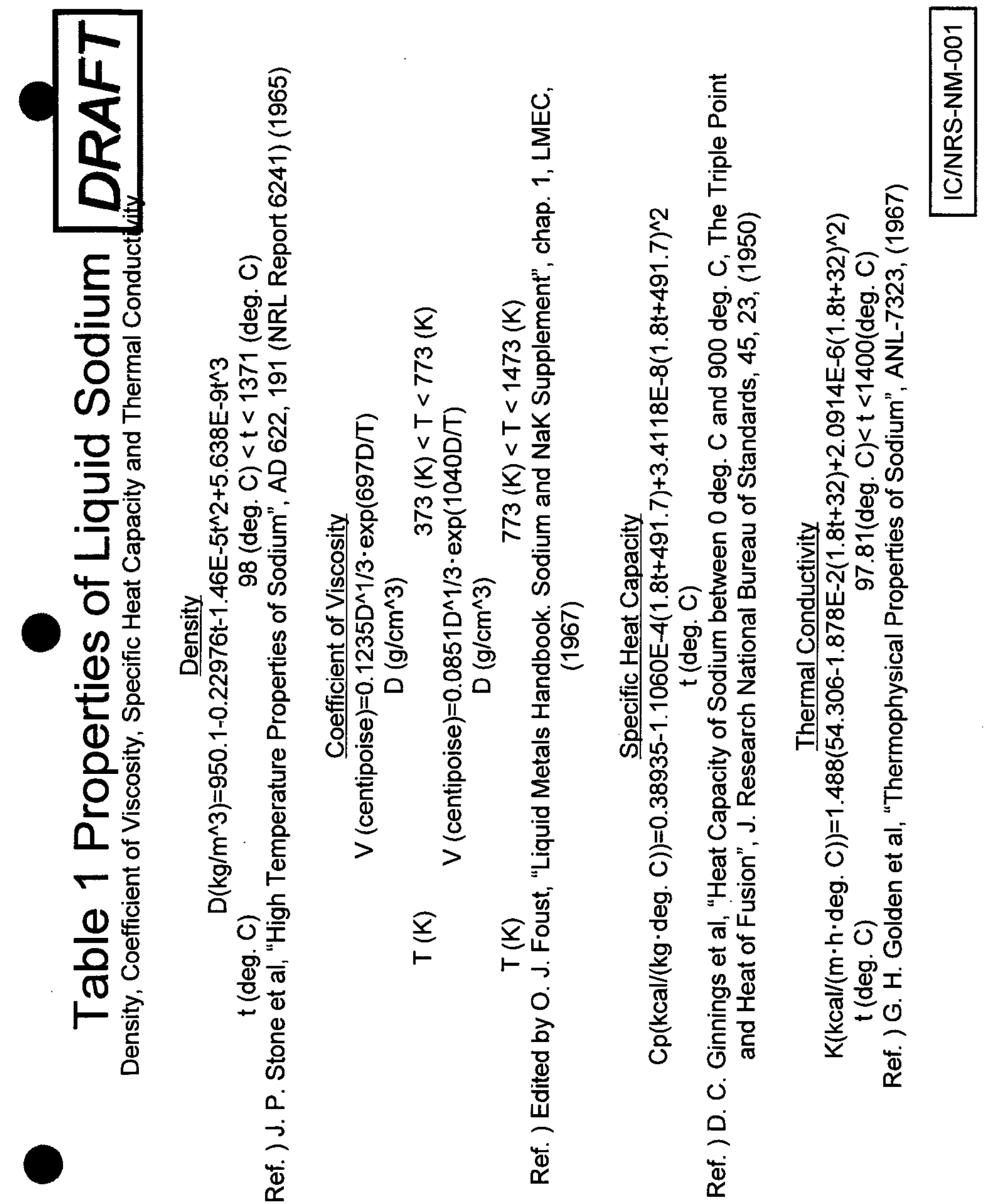


Attachment $E$ of

Enclosure (7) to

MDO-723-0044/

B-MT(SRME)-52

Page 1

Attachment E of

Enclosure 7 to MDO-723-0044/B-MT(SRME)-52

JOYO-1 Hardware Cleaning Procedure 
Attachment $E$ of

Enclosure (7) to

MDO-723-0044/

B-MT(SRME)-52

Page 2

THIS PAGE INTENTIONALLY BLANK 
FORM 76128

DMP-LD-702, Rev. 0.

Page 1 of 7

DETAILED MANUFACTURING PROCEDURE FOR

CLEANING OF JOYO IRRADIATION TEST COMPONENTS

Prepared by

W.J. Underwood
Date

$6 / 6 / 05$

REVIEWED AND APPROVED BY

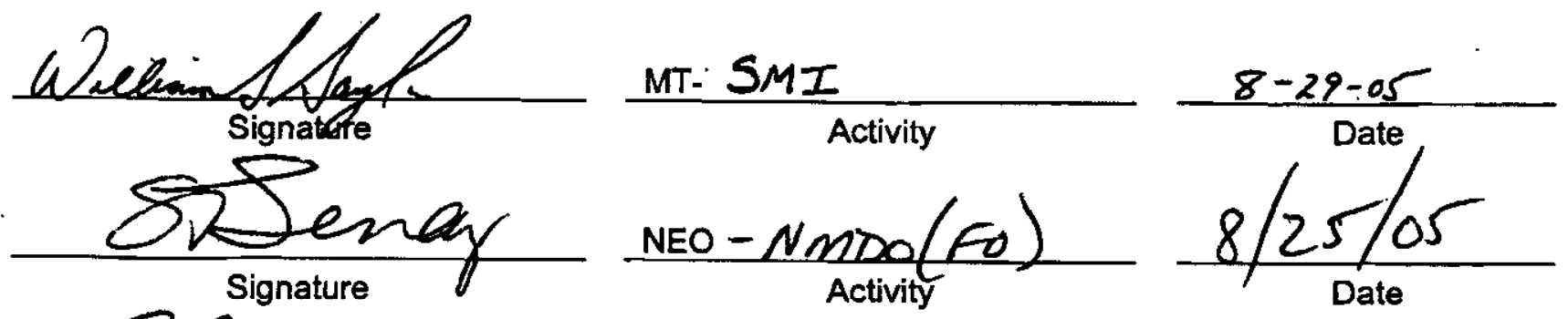

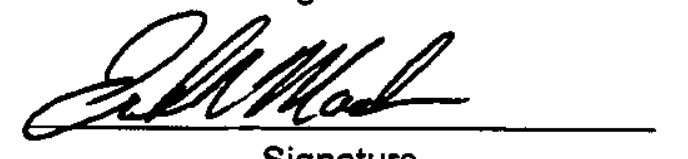

Signature

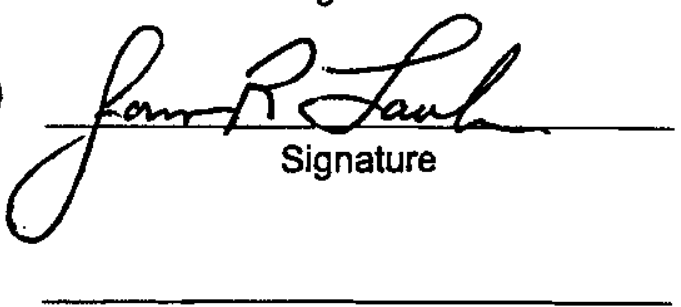

Signature

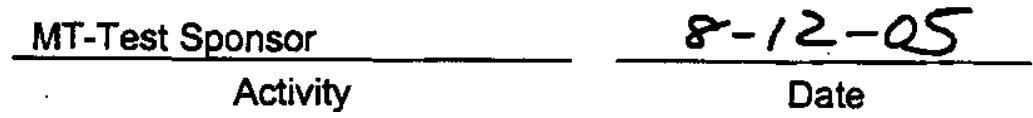

QUA
$8-12-05$

Date

Activity

Date 
ISSUE INFORMATION

\begin{tabular}{lll}
\hline Revision Date Brief Note on Revision & B
\end{tabular}

Original $6 / 06 / 05$

!. 


\section{$\underline{\text { SCOPE }}$}

This procedure describes the cleaning of JOYO Irradiation testing components. This DMP includes calibration, personnel qualification, and cleanliness requirements in compliance with appropriate sections of Bettis quality documents.

II. SAFETY PRECAUTIONS

A. Observe all applicable radiological controls and health, environmental, safety, prohibited material, and criticality control procedures in force in the work area during the performance of the work outlined by the work document and this procedure.

B. Cleaning shall be done in a hood equipped with an exhaust fan.

\section{REFERENCED DOCUMENTS}

None.

\section{EQUIPMENT, MATERIALS, STANDARDS AND FORMS}

\section{A. Equipment}

1. 2 stainless steel cleaning PANS. An exhaust hood equipped with an exhaust fan shall be located over the cleaning PANS.

3. Nitrogen gas cylinders or house supply nitrogen gas line. Only dry and oil-free nitrogen shall be used.

4. Nylon or other lint free, talc free gloves.

5. A desiccator made of stainless steel and capable of holding the components to be cleaned.

6. Nylon scrubbing brushes.

7. Stainless steel pipe cleaners with Nylon Bristles from McMaster Carr \#4754A23 or equivalent.

8. Lint free, talc free cloths.

B. Materials

1. Reagent Grade Acetone.

2. Tungsten carbide scrapers.

3. Clear polyethylene sleeving.

4. Reagent Grade 2-Propanol . 


\section{QUALIFICATION REQUIREMENTS}

Personnel who are to perform operations in this procedure shall be trained until familiar with the use of equipment and operations employed in the performance of this procedure by working with qualified operators or under the guidance of the area manager. Personnel qualification shall be determined by the issuing DMP engineering activity and/or the individual immediate manager based on successful mock up. Qualified personnel shall be identified by the issuance of an Engineering Notice (Form 73307) documenting the qualification, with a copy placed in training records accordingly. Re-qualification is not required unless an individual is disqualified, in which case an Engineering Notice shall be issued to document requalification.

\section{CALIBRATION REQUIREMENTS}

All equipment and instruments used to carry out these DMP instructions shall be in calibration, where a calibration is applicable, per the requirements of Appendix $C$ of the Bettis Quality Assurance and Administrative Manual. When portable equipment is used that is not directly associated with nor an integral part of the cleaning equipment, the WB number of that equipment shall be recorded on the work document.

VII. PROCEDURE

A. The operator shall ensure that all the components are available as required by the work document provided by the requisitioning engineer.

B. Starting bath of each cleaning agent shall be new. The same bath may be used to clean different materials without replacing the cleaning agent provided the following requirements are met:

1. Parts made of the same material may be placed simultaneously into the same bath if the following cleaning sequence is followed a) Molybdenum b) Nickel or Stainless Steel c) Graphite.

2. Dissimilar material parts are not allowed to be placed simultaneously into the same bath. For example, Molybdenum components cannot be placed simultaneously into a bath with graphite components.

3. A cleaning agent must be replaced after graphite components are cleaned in the bath.

C. Cleaning Steps for Parts Fabricated out of Molybdenum

1. All components should be handled with the proper nylon or other lint free talc free gloves at all times.

2. Turn on the fan for the exhaust hood over the cleaning tanks.

3. Fill PAN 1 with acetone bath. Fill PAN 2 with a 2-propanol bath.

4. Immerse each part in the PAN 1 (acetone) bath for 3-5 minutes. Parts made of the same material may be placed into the same bath all at once or in several loads. 
5. Using Nylon brush lightly scrub each part in the acetone bath. A new unique brush shall be used for each type of material. For small diameter hole and crevices use the Stainless Steel pipe cleaner. Ensure that the pipe cleaner reaches the bottom of any blind holes.

6. Remove the parts from bath and drip dry for a minimum of 30 seconds on a lint free talc free cloth (Observe material contact restrictions in Table 1).

7. Immerse each part in PAN 2 (2-propanol) for 3-5 minutes. Parts made of the same material may be placed into the same bath all at once or in several loads.

8. Remove the parts from bath and drip dry for 30 seconds and rinse each part with fresh 2-propanol (Observe the material contact restrictions in Table 1).

9. Allow to air dry on lint free taic free cloth for at least 15 minutes or until parts appear to be dry. Turn the parts over during the drying process. (Observe material contact restrictions in Table 1). The parts can also be dried per paragraph $\mathbf{E}$.

D. Cleaning Steps for Parts Fabricated out of Non-Molybdenum Materials

1. All components should be handied with the proper nylon or lint free talc free gloves at all times.

2. Turn on the fan for the exhaust hood over the cleaning PANs.

3. Fill PAN 1 with acetone. Fill PAN 2 with 2-propanol. Procedure can be done with one PAN by draining the PAN of acetone and allowing it to completely dry after step 4.

4. Immerse each part in the PAN 1 (acetone) bath for 3-5 minutes. Parts made of the same material may be placed into the same bath all at once or in several loads.

5. Using Nylon brush, lightly scrub each part in the acetone bath. A new unique brush shall be used for each type of material. For small diameter hole and crevices use the Stainless Steel pipe cleaner. Ensure that the pipe cleaner reaches the bottom of any blind holes.

6. Remove the parts from bath and drip dry for a minimum of 30 seconds on a lint free talc free cloth (Observe material contact restrictions in Table 1).

7. Immerse each part in PAN 2 (2-propanol) bath for 3-5 minutes. Parts made of the same material may be placed into the same bath all at once or in several loads.

8. Remove the parts from bath and drip dry for a minimum of 30 seconds and rinse each part with fresh 2-propanol (Observe material contact restrictions in Table 1).

9. Immerse each part in ultrasonic bath of deionized water (approximately $165^{\circ} \mathrm{F}$ ) for 3-5 minutes.

10. Rinse thoroughly with fresh deionized water for approximately 30 seconds. 
11. Allow to air dry on lint free talc free cloth for at least 15 minutes or until parts appear to be dry. Turn the parts over during the drying process. (Observe material contact restrictions in Table 1). The parts can also be dried per paragraph $E$.

E. Dry the part using one of the following options:

1. Blow dry with Nitrogen.

2. Place the components into a desiccator with desiccant or a vacuum chamber.

F. Visual Examination

1. Visually examine all of the cleaned components for surface contamination. All evidence of lint, dust, or other contamination shall be removed. To remove all loose contaminants, blow components with nitrogen.

a. Components having an adhering contaminant may be recycled through the cleaning (steps VII.A-E). Any components bearing a consistently adherent contaminant shall be set aside and the requisitioning engineer shall be notified.

b. Components can be hand scraped (with tungsten carbide scrapers only) to remove burrs or foreign material after which they shall be wiped clean with a lint free cloth saturated with 2-propanol and recycled through the scrub and wash cycle (Steps VII.A-E).

c. When components are of acceptable cleanliness and completely dry, package each part separately in a new clear poly bag and seal closed. Mark each package with a:

- Route Card Number

- Purchase Order Number

- Serial Number

- Part Name

- Date of Completion 
Table 1. Approved Contact Materials For NRPCT Test Specimens and Capsule Internal Components

\begin{tabular}{|l|}
\hline Silicon Carbide(SiC), Graphite, Stainless Steel, Inconel, or Zirconia \\
\hline Lint free, talc free cloth \\
\hline Diamond \\
\hline Graphite \\
\hline Hot cell acceptable rubber \\
\hline Nylon (except in cloth form) \\
\hline Polyethelene (HDPE or LDPE) \\
\hline Quart \\
\hline SiC \\
\hline Molybdenum \\
\hline Lint free, talc free cloth \\
\hline Hot cell acceptable rubber \\
\hline Granite \\
\hline Molybdenum \\
\hline Niobium \\
\hline Nylon \\
\hline Polyethelene (HDPE or LDPE) \\
\hline Pyrex Glass \\
\hline Quart \\
\hline Tungsten \\
\hline
\end{tabular}


Attachment $F$ of Enclosure (7) to MDO-723-0044I

B-MT(SRME)-52

Page 1

\section{Attachment F of}

\section{Enclosure 7 to MDO-723-0044/B-MT(SRME)-52 \\ JOYO-1 Hardware Bake-Out Procedure}


Attachment $F$ of

Enclosure (7) to

MDO-723-0044/

B-MT(SRME)-52

Page 2

THIS PAGE INTENTIONALLY BLANK 


\section{DETAILED MANUFACTURING PROCEDURE FOR}

BAKE-OUT PROCEDURE OF IRRADIATION TEST COMPONENTS

\begin{tabular}{ll} 
Prepared by & Date \\
W.J. Underwood & $07 / 26 / 2005$ \\
\hline
\end{tabular}

REVIEWED AND APPROVED BY

Signature

Signature

Signature

Signature
Activity

Activity

Activity

Activity

Activity
Date

Date

Date

Date 
ISSUE INFORMATION

Issue Date Brief Note on Revision




\section{SCOPE}

This procedure describes the Bake-out process of the Irradiation testing components. This DMP includes calibration, personnel qualification, and cleanliness requirements in compliance with appropriate sections of Bettis quality documents.

\section{SAFETY PRECAUTIONS}

Observe all applicable radiological controls and health, environmental, safety, prohibited material, and control procedures in force in the work area during the performance of the work outlined by the work document and this DMP.

\section{REFERENCED DOCUMENTS}

None.

IV. EQUIPMENT, MATERIALS, STANDARDS AND FORMS

A. Equipment

1. 10 inch Furnace in the G-building

2. Molybdenum tray holder

3. Molybdenum gettering top

4. Small Desiccators filled with desiccant

B. Materials

1. Lint free talc free gloves

\section{QUALIFICATION REQUIREMENTS}

Personnel who are to perform operations in this procedure shall be trained until familiar with the use of equipment and operations employed in the performance of this procedure by working with qualified operators or under the guidance of the area manager. Personnel qualification shall be determined by the issuing DMP engineering activity and/or the individual immediate manager based on successful mock up. Qualified personnel shall be identified by the issuance of an Engineering Notice (Form 73307) documenting the qualification, with a copy placed in training records accordingly. Re-qualification is not required unless an individual is disqualified, in which case an Engineering Notice shall be issued to document requalification. 


\section{CALIBRATION REQUIREMENTS}

All equipment and instruments used to carry out these DMP instructions shall be in calibration, where a calibration is applicable, per the requirements of Appendix $C$ of the Bettis Quality Assurance and Administrative Manual. The S/N or W/B number of all calibrated equipment and instruments used to carry out these DMP instructions shall be recorded in the work document or accompanying calibration forms/data sheets.

\section{PROCEDURE}

A. The operator shall assure that all the components are available as required by the work document provided by the requisitioning engineer.

B. The operator shall handle the parts with lint free talc free gloves at all times.

C. Pre-Bake out of furnace

1. Establish a vacuum of $5 \times 10^{\wedge} 5$ torr or better

2. Ramp at $18^{\circ} \mathrm{F} /$ minute to $1000^{\circ} \mathrm{F}(\sim 56$ minutes)

3. Hold at $1000^{\circ} \mathrm{F} \pm 30^{\circ} \mathrm{F}$ for one hour in a dynamic vacuum

4. Cool at $18^{\circ} \mathrm{F} /$ mintue or slower in a vacuum ( -60 minutes or greater)

5. Record all data during the run and provide the data to the requisitioning engineer.

6. The pre-bake out of the furnace is only required:

a. Before the bake out of the first test components

b. After any other material is placed into the furnace other then the test components

D. Bake-Out Procedure

1. The operator shall assure that all the components are available as required by the work document provided by the requisitioning engineer

2. All test component parts will be handled with lint-free talc free gloves

3. Bake-out like parts together i.e. do not bake out the molybdenum parts with the stainless steel or Inconel parts.

4. Place the similar material test components onto the molybdenum tray ensure that the parts are not stack on each other and each part has $\sim 1 / 8^{\prime \prime}$ separation between each making sure they do not touch.

5. Do not place parts on the first or last five inches of the Molybdenum tray 
6. Place Molybdenum Top over the Molybdenum tray holding the parts

7. Insert tray and parts into the furnace

8. Establish a vacuum of $5 \times 10^{\wedge} 5$ or better

9. Ramp at $18^{\circ} \mathrm{F} /$ minute to $752^{\circ} \mathrm{F}(\sim 38$ minutes $)$

10. Hold at $752^{\circ} \mathrm{F} \pm 18^{\circ} \mathrm{F}$ for 2 hours $+1 /-0$ in a dynamic vacuum

11. Cool at $18^{\circ} \mathrm{F} /$ minute or slower in vacuum ( -60 minutes)

12. Once the furnace has cooled all of the parts should be removed from the furnace

13. When components are completely cooled off, package the parts separately in a new clear poly and seal closed. Mark each package with a:
a. Route Card Number
b. Purchase Order Number
c. Serial Number
d. Part Name
e. Date of Completion

14. Store the components in desiccators

15. All data temperature and pressure data recorded during the bake out process shall be provide to the requisitioning engineer with the work document upon its completion 
Attachment $\mathrm{G}$ of

Enclosure (7) to

MDO-723-0044I

B-MT(SRME)-52

Page 1

Attachment G of

Enclosure 7 to MDO-723-0044/B-MT(SRME)-52

JOYO-1 Pressure and Helium Leak Test Procedure 
Attachment $\mathrm{G}$ of

Enclosure (7) to

MDO-723-0044/

B-MT(SRME)-52

Page 2

THIS PAGE INTENTIONALLY BLANK 


\section{DETAILED MANUFACTURING PROCEDURE FOR}

PRESSURE AND HELIUM LEAK TEST OF IRRADIATION TEST CAPSULES

$$
\begin{aligned}
& \text { Prepared by } \\
& \text { W.J. Underwood }
\end{aligned}
$$

Date

$7 / 11 / 2005$

REVIEWED AND APPROVED BY

Signature

Signature

Signature

Signature
Activity

Activity

Activity

Activity

Activity
Date

\section{Date}

Date

Date 


\section{SCOPE}

This DMP provides instructions required for pressure testing and helium leak test inspection of irradiation test capsules. This DMP includes calibration, personnel qualification, and cleanliness requirements in compliance with appropriate Bettis quality documents. Equipment qualification to fulfill specific product design requirements must be addressed by the test sponsor.

\section{SAFETY PRECAUTIONS}

Observe all applicable radiological controls and health, environmental, safety, prohibited material, and control procedures in force in the work area during the performance of the work outlined by the work document and this DMP.

III. REFERENCED DOCUMENTS

A. Operating procedure for the Varian Helium Leak, Model 979 or equivalent

B. Operating procedure or system description for the pressure and vacuum systems

IV. EQUIPMENT, MATERIALS, STANDARDS AND FORMS
A. Equipment

Varian Helium Leak Model 979 or equivalent

Helium Leak Test pressure and vacuum systems

B. Material

Helium: Minimum purity of 99.95 percent as per Federal Specifications BB-H-1168B

Nitrogen: Minimum purity of 99.5 percent as per Federal Specifications BB-N-411B

Alcohol: 2-propanol

Gloves: Lint-free or white nylon

Wiping cloth: White lint-free cotton or equivalent

C. Standards

Calibrated leak: Varian Model SC 7D or equivalent

D. Forms

Helium Leak Test Data Form (See Enclosure) 


\section{QUALIFICATION REQUIREMENTS}

Personnel who are to perform operations in this procedure shall be trained until familiar with the use of equipment and operations employed in the performance of this procedure by working with qualified operators or under the guidance of the area manager. Personnel qualification shall be determined by the issuing DMP engineering activity and/or the individual's immediate manager based on successful mock-up or dry-run operational performance. Personnel to be qualified shall be identified by the issuance of an Engineering Notice (Form 73307) documenting the qualification, with a copy placed in training records accordingly. Requalification is not required unless an individual is disqualified, in which case an Engineering Notice shall be issued to document requalification.

VI. CALIBRATION REQUIREMENTS

All equipment and instruments used to carry out these DMP instructions shall be in calibration, where a calibration is applicable, per the requirements of Appendix $C$ of the Bettis Quality Assurance and Administrative Manual. The S/N or W/B number of all calibrated equipment and instruments used to carry out these DMP instructions shall be recorded in the work document or accompanying calibration forms/data sheets.

The following items shall be calibrated according to the following schedule:

Pressure Gage - Semiannually

Calibrated Leak Rate Standard - Annually

Vacuum Gage - Semiannually

Data Acquisition System - Semiannually

VII. PROCEDURE

A. Engineering Instructions

The helium leak test system is to be calibrated prior to the initial leak test of the shift. Additional calibration may be performed during the shift if deemed necessary by the operating technician. Calibration is performed as listed in section VII.B. If the power to the leak detector is turned off during the day a recheck of the calibration must be done. Measure helium background level in the vacuum system and calibrate for helium sensitivity prior to the initial use of the day.

Visually examine each component for defects. Do not test component(s) that have cracks or any other external void that may be evidence of a leak path that can be distinguished from surface scratches and pitting. Clean white gloves must be worn when handling hardware and components.

Move component(s) smoothly and carefully. Avoid bumping, scratching or up ending components. Transfer of component(s) from pressurization chamber to vacuum chamber must be accomplished within 15 minutes of opening pressure chamber but not sooner than 10 minutes.

Reject component(s) if leak rate exceeds $9.0 \times 10^{-7}$ std. cc He/Sec. if an instantaneous off scale helium response is detected. Isolate leak detector from vacuum chamber. A Material Review 
Tag (MRT) must be issued for a rejected component(s). If possible indicate on MRT the location of the leak(s).

B. Calibration

1. Follow the manufacturer's instructions in turning on the leak detector.

2. Tune the spectrometer tube and calibrate the leak detector with a helium leak rate standard.

3. Put leak detector into test mode. Once leak detector is stabilized, adjust zero $\left(0 \times 10^{-8}\right)$ by adjusting the coarse and fine zero buttons, if applicable.

4. Evacuate vacuum chamber to $<2 \times 10^{-3}$ torr. (Note time taken to achieve $<2 \times 10^{-3}$ torr). Record time to evacuate chamber on Helium Leak Test Data Calibration Form.

5. Isolate vacuum chamber from vacuum pumps.

6. Open leak detector to vacuum chamber.

7. Measure background leak by observing leak detector reading for three minutes after leak detector is open to vacuum chamber. Verify that the helium background level is < $3.0 \times 10^{-9}$ standard cc helium/second. Record helium background level measurement on Helium Leak Test Data Calibration Form.

8. Isolate leak detector from vacuum chamber. 9. Backfill vacuum chamber with nitrogen to atmospheric pressure. Open vacuum
chamber door.

10. Place helium leak rate standard into vacuum chamber at the farthest point from the leak detector. Ensure the valve on the standard is open and the outlet end is facing the vacuum chamber door. Close vacuum chamber door. 11. Evacuate vacuum chamber to $<2 \times 10^{-3}$ torr. (Note time taken to achieve $<2 \times 10^{-3}$ torr)
Record on Helium Leak Test Data Form.

12. Isolate vacuum chamber from vacuum pumps.

13. Open leak detector to vacuum chamber and measure the helium leak rate from calibration standard after three minutes. Record measurement on Helium Leak Test

14. Isolate leak detector from vacuum chamber.

15. Backfill vacuum chamber with nitrogen to atmospheric pressure. Open vacuum chamber door. Remove standard.

16. Evacuate vacuum chamber until needed. 
C. Detailed Procedure

1. Place component(s) to be tested into pressurization chamber.

2. Pressurize component(s) to the specified pressure in the work document for a minimum of thirty minutes but not for more than one hour. Record pressure and test duration on component route card.

3. Vent pressurization chamber of helium and remove component(s).

4. Carefully wipe the component(s) with 2 propanol to remove any residual helium. Pay special attention to holes, stampings and other area that could trap helium. Cotton swabs may also be used.

5. Dry the component(s) with nitrogen. Transfer of component(s) from pressurization chamber to vacuum chamber must be accomplished within fifteen minutes of opening pressure chamber but not sooner than ten minutes.

6. Move the component(s) to the vacuum chamber. Move component(s) smoothly and carefully. Avoid bumping, scratching or up ending components.

7. Evacuate vacuum chamber to $<2 \times 10^{-3}$ torr.

8. Once evacuation is achieved, isolate vacuum chamber from vacuum pumps.

9. Open leak detector to vacuum chamber. Measure helium leak rate for three minutes minimum. Reject component(s) if leak rate exceeds $9.0 \times 10^{-7} \mathrm{std}$. cc He/Sec. or if an instantaneous off-scale helium response is detected. Isolate leak detector from vacuum chamber. Record leak rate on component route card.

A Material Review Tag (MRT) must be issued for a rejected component(s). The rejected component(s) may be submersed in a small quantity of 2-propanol. Visually inspect component for evidence of helium bubbling. If possible indicate on MRT the location of the leak(s). Record on component route card.

10. Backfill vacuum chamber with nitrogen to atmospheric pressure. Open vacuum chamber door. Remove component(s).

11. Evacuate vacuum chamber. Chamber may be under a dynamic vacuum or isolate vacuum chamber from vacuum pumps and turn off vacuum pumps. 


\section{Enclosure to \\ DMP-LD-703, DRAFT \\ HELIUM LEAK TEST DATA CALIBRATION FORM \\ Page 1 of 2}

\begin{tabular}{l} 
WORK DOCUMENT : \\
\hline OPERATION NUMBER : \\
\hline TEST SERIAL NUMBER : \\
TEST TYPE: $\square$ RETEST \\
$\square$ INITIAL $\square$ RPERATOR : \\
DATE : \\
\hline
\end{tabular}

\begin{tabular}{|l|}
\hline VACUUM GAGE S/N: \\
LEAK DETECTOR S/N: \\
EVACUATION TIME: \\
BACKGROUND HE LEAK : \\
Std CC He/SEC \\
EVACUATION TIME :
\end{tabular}

COMMENTS: NOTE: The knowing and willful recording of false, fictitious, or fraudulent statements on this document may be punishable 2
as a felony under Federal Statutes. 
Enclosure to

DMP-LD-703, Rev. DRAFT

Page 2 of 2

THIS PAGE IS INTENTIONALLY BLANK 
Attachment $\mathrm{H}$ of

Enclosure (7) to

MDO-723-0044I

B-MT(SRME)-52

Page 1

\section{Attachment $\mathrm{H}$ of}

\section{Enclosure 7 to MDO-723-0044/B-MT(SRME)-52}

NRPCT JOYO Pressure Boundary Weld Qualification Process 
Attachment $\mathrm{H}$ of

Enclosure (7) to

MDO-723-0044/

B-MT(SRME)-52

Page 2

THIS PAGE INTENTIONALLY BLANK 
B-MT(EDT)S-010

Page 1

B-MT(EDT)S-010

NRPCT JOYO Pressure Boundary Weld Qualification Process

Revision: Original

May 9, 2005

Authored by:

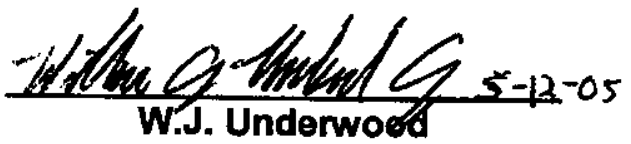

$\frac{A m \text { zedeat } 5-12-05}{\text { A.M. Tullal }}$

$\operatorname{RGClg}_{\text {R. A. Slap }} 5-12.05$

Approved by:
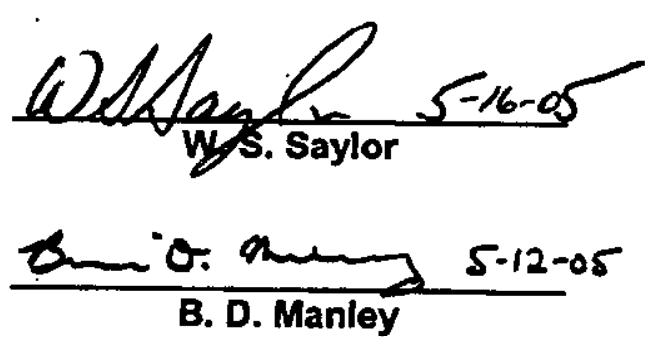


\section{NRPCT JOYO Pressure Boundary Weld Qualification Process}

\section{Introduction}

This document describes the process in which NRPCT will qualify the Type 316, Stainless pressure boundary weld between the ASME SA 479, Composition UNS S31651 capsule (SK-AMT-2221) and head (SK-AMT-2222). The NRPCT is planning to use an Orbital Automatic Gas-Tungsten Arc Weld (GTAW) with a square butt autogenous (without filler metal) joint design. This will be used for the process as described in Appendix A. The pressure boundary houses Prometheus 1 structural material specimens that will be tested in the JOYO reactor. The design and fabrication are in accordance with ASME Boiler and Pressure Vessel Code (BPVC) Section III, Subsection ND, Class 3. The Welding Procedure shall be qualified in accordance with the requirements of ASME BPVC Section IX, and as supplemented herein.

\section{Discussion}

\section{Process Outline}

The purpose of the Welding Procedure Specification (WPS) and Procedure Qualification Record $(P Q R)$ is to determine that the weldment proposed for fabrication is capable of having the required properties for its intended application. It is presupposed that the welding operator performing the welding procedure qualification test is a skilled workman. The procedure qualification test(s) are to establish the properties of the weldment and not the skill of the personnel performing the welding. Performance Qualification is described in Appendix D.

The WPS is a guidance document describing the variables required to make a successful joint, as supported and proven by the mechanical and quality conformance tests as documented by the supporting PQR. This process is summarized in Figure 1 below.

1. Procedure Qualification Record (PQR) - Record of welding data used to weld mockups of same geometry and material. This $P Q R$ is a record of the actual variables recorded during the welding qualification process and includes the test results

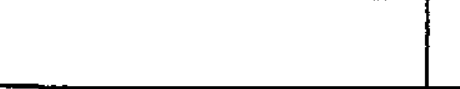

2. Weld Procedure Specification (WPS) - Procedure that will be used to weld the NRPCT Capsule and head. This document includes:

- Essential and nonessential weld variables

- References the supporting PQR.
}

Figure 1 Weld Procedure Specification Process 


\section{Procedure Qualification Record}

The process to qualify the NRPCT Capsule weld shall be conducted in three parts, as further described below; (I) development, (II) welding procedure qualification and (III) production proof verification. The information from parts II and III shall be documented on the Procedure Qualification Record (PQR). The type, number and testing requirements are illustrated in Table 1, while the intended $P Q R$ contents will be presented in similar format as shown in Appendix A.

I) Development

Prototypical Development mock up drawings (same as production capsules): SK-AMT-2230

Prototypical weld mockups duplicating the production weld joint, including internal gas volume restrictions, shall be welded and sectioned for metallographic examination; ensuring developed parameters meet penetration, fusion and quality requirements.

II) Welding Procedure Qualification

Weld Procedure Qualification mockup drawings (extended cap and body open-ended); SKWJU-1002

Three full size mockups duplicating the production weld joint shall be welded and subjected to the inspections and mechanical tests as shown in Table 1 below.

III) Production Proof Mockup

Prototypical Development mock up drawings (same as production capsules): SK-AMT-2230

At least one prototypical mockup shall be welded using the developed and authenticated parameters. This mockup shall be subject to the same visual, dimensional and pressure testing of the production units; certifying the process has met the functional end-item requirements. The results are to be documented on the PQR, as shown in Table 1 below.

\begin{tabular}{|c|c|c|c|c|c|c|}
\hline \multirow[b]{2}{*}{$\begin{array}{l}\text { WELD TEST } \\
\text { COUPONS }\end{array}$} & \multirow[b]{2}{*}{ ASSY DWG } & \multirow{2}{*}{$\begin{array}{c}\text { INSPECTION } \\
\text { VT } \\
\text { (Visual) } \\
\text { RT } \\
\text { (Radiography) } \\
\text { DIM } \\
\text { (Dimensional) }\end{array}$} & \multirow[b]{2}{*}{$\begin{array}{l}\text { DESTRUCTIVE } \\
\text { EXAMINATION }\end{array}$} & \multicolumn{3}{|c|}{$\begin{array}{c}\text { TYPE and NUMBER OF TEST } \\
\text { REQUIRED (Per ASME BPVC Section IX) }\end{array}$} \\
\hline & & & & $\begin{array}{l}\text { Tension } \\
(Q w-150)\end{array}$ & $\begin{array}{l}\text { Face Bend } \\
(\mathrm{QW}-160)\end{array}$ & $\begin{array}{l}\text { Root Bend } \\
(Q W-160)\end{array}$ \\
\hline 1 & SK-WJUJ-1002 & $V T \& R T$ & - & $\begin{array}{l}\text { Full Section }{ }^{2} \\
\text { (QW 462.1(e) }\end{array}$ & $\cdots$ & - \\
\hline 2 & SK-WJU-1002 & $V T \& R T^{1}$ & - & $\begin{array}{l}\text { Full Section }{ }^{2} \\
\text { (QW 462.1(e) }\end{array}$ & --- & - \\
\hline 3 & SK-WJU-1002 & VT \& RT' & $\begin{array}{l}\text { METALLOGRAPHIC } \\
\text { SECTION }\end{array}$ & & 2 & 2 \\
\hline 4 & SK-AMT-2230 & $\begin{array}{c}\text { VT, DIM \& } \\
\text { PRESSURE }\end{array}$ & - & -- & -- & - \\
\hline
\end{tabular}

Table 1. Type, number, and tests to be documented on the PQR.

Notes:

1. Radiographic examination is supplementary to ASME BPVC IX.

2. Alternative Reduced Section Tension testing per WQ-462.1(c).

3. Prototypical inspections and tests are in supplement to ASME BPVC IX.

4. Metallographic sectioning is supplementary to ASME BPVC IX. 
All welding and capsule assembly will take place in an inert atmosphere glove box. The inert gas is expected to be $100 \% \mathrm{Ar}, 80 \% \mathrm{Ar} 20 \% \mathrm{He}$, and $30 \% \mathrm{Ar} 70 \% \mathrm{He}$. The inert atmosphere within the capsule is governed by thermal analysis in order to obtain specimen temperatures goals.

Specifications for the inert gas include $99.995 \%$ purity with moisture and Oxygen levels maintained below 5 ppm separately, as a goal but limited to $10 \mathrm{ppm}$ moisture and Oxygen, considered separately.

Subsequent to successful completion of the phase I development, the formal qualification process shall ensue with the four weld test coupons as described in phase II and III.

\section{PHASE II, Mechanical Testing}

The test coupons designated 1,2 and 3 will undergo the inspections, examinations and physical testing as prescribed in table 1 above. Metallographic examination shall be described in Appendix " $\mathrm{C}$ ". Tension and Bend testing are conducted as follows:

\section{Tension Test}

Specimen: With the capsule outside diameter less then 3 inches $(76 \mathrm{~mm})$, a full section tension test may be used.

Procedure: The Tension test specimen shall be ruptured under tensile load. The tensile strength shall be computed by dividing the ultimate total load by the least cross-sectional area of the pipe as calculated from the actual measurements made before the load is applied

Acceptance Criteria:

In order to pass the tension test, the specimen shall have a tensile strength that is not less than:

a) the minimum specified tensile strength of the base metal or

b) if the specimen breaks in the base metal outside of the weld or fusion tine, the test shall be accepted as meeting the requirements, provided the strength is not more than $5 \%$ below the minimum specified tensile strength of the base metal

\section{Transverse Face Bend and Root Bend tests}

Specimen: The ductility of a welded joint, as evidenced by its ability to resist tearing and the presence of discontinuities on the tension surface, is determined in a guided bend test. Guided test specimens are prepared by cutting the mockup (Figure 2) to form specimens of approximately rectangular crosssection. The cut surfaces are designated as the sides of the specimen. The other two surfaces are called the face and root surfaces; the face surface representing the top of the weld, conversely the bottom or underside of the weld representing the root surface. See Figure 3 for nomenclature.

For the NRPCT capsule weld, the weld is perpendicular or transverse to the longitudinal axis, therefore, a transverse Guided-Bend tests is conducted. The surfaces of interest for this test are the face and root surfaces. The bend tests are conducted with the face and root surfaces making it the convex side of the specimen after bending.

The bend test jig dimensions are specified to subject the specimen to a calculated outer fiber elongation equal to at least that of the base material with the lower minimum elongation as specified in the base or weldment material specification. 


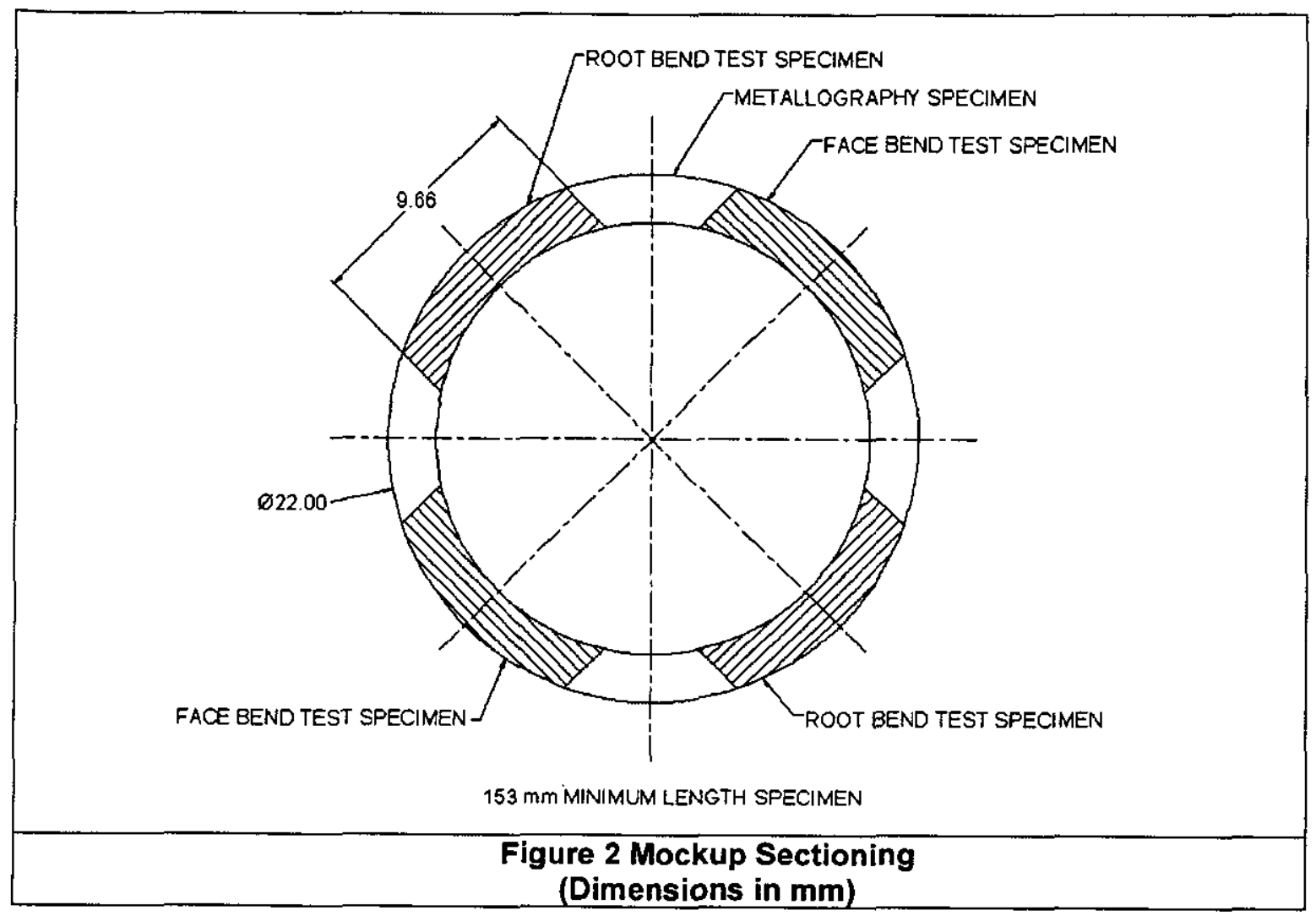

\section{Side View}

\section{Transverse/Perpendicular} Weld

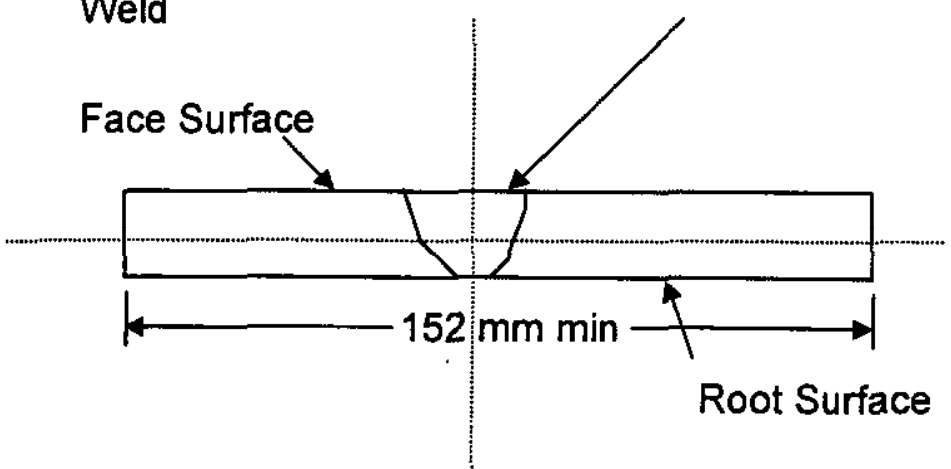

\section{Front View}

Figure 3 Cut Bend Specimen nomenclature.

Acceptance Criteria:

The guided-bend specimens shall have no open defects in the weld or heat affected zone exceeding $3 \mathrm{~mm}$, measured in any direction on the convex surface of the specimen after bending. Open defects occurring on the corners of the specimen during testing shall not be considered unless there is definite evidence that they result from lack of fusion, slag inclusions, or other internal defects. 


\section{B-MT(EDT)S-010}

Page 6

PHASE III, Production Proof Mockup

The test coupon designated number 4 will undergo the weld and dimensional inspections and testing examinations as prescribed in table 1 above and detailed in table 2 below.

\begin{tabular}{|c|c|l|}
\hline \multicolumn{2}{|c|}{ Table 2. Production Dimensional, NDE \& Pressure/Leak Testing Requirements } \\
\hline Test & \multicolumn{1}{|c|}{ Specification } & \multicolumn{1}{c|}{ Acceptance Criteria } \\
\hline $\begin{array}{c}\text { Weld and Dimensional } \\
\text { Inspection }\end{array}$ & Performed per Appendix B & $\begin{array}{l}\text { Max external reinforcement } \\
\text { (distance weld can extend } \\
\text { beyond capsule) }-.38 \mathrm{~mm} \\
\text { Weld undercut }-0.25 \mathrm{~mm} \text { max } \\
\text { \& shall not encroach on required } \\
\text { section thickness. }\end{array}$ \\
\hline Pressure Test & Performed per Appendix B & $\begin{array}{l}\text { No visual permanent } \\
\text { deformation and confirmed with } \\
\text { leak test results. }\end{array}$ \\
\hline Helium Leak Test & Performed per Appendix B & $\begin{array}{l}\text { Leak rate acceptance criteria is } \\
1.0 \times 10^{-7} \text { std. cc He/sec. }\end{array}$ \\
\hline
\end{tabular}

Table 3. Drawings for Welding Mockups (Preliminary Drawings not for Construction)

\begin{tabular}{|c|c|}
\hline Title & Number \\
\hline NRPCT/JOYO Capsule Mockup & SK-WJU-1004-H01 \\
\hline NRPCT/JOYO Head Mockup & SK-WJU-1000-H01 \\
\hline NRPCT/JOYO Capsule Weld Qualification & SK-WJU-1002-H01 \\
\hline NRPCT Capsule & SK-AMT-2221-H01 \\
\hline NRPCT Head & SK-AMT-2222-H01 \\
\hline
\end{tabular}




\section{Appendix A \\ Weld Procedure Qualification Record (PQR)}

Joint Design: Square butt, fit up gap $.05 \mathrm{~mm}$ max with integral backing. Cylinder axis horizontal, Weld Procedure Development mock drawings: SK-WJU-1002 and SK-AMT-2230'

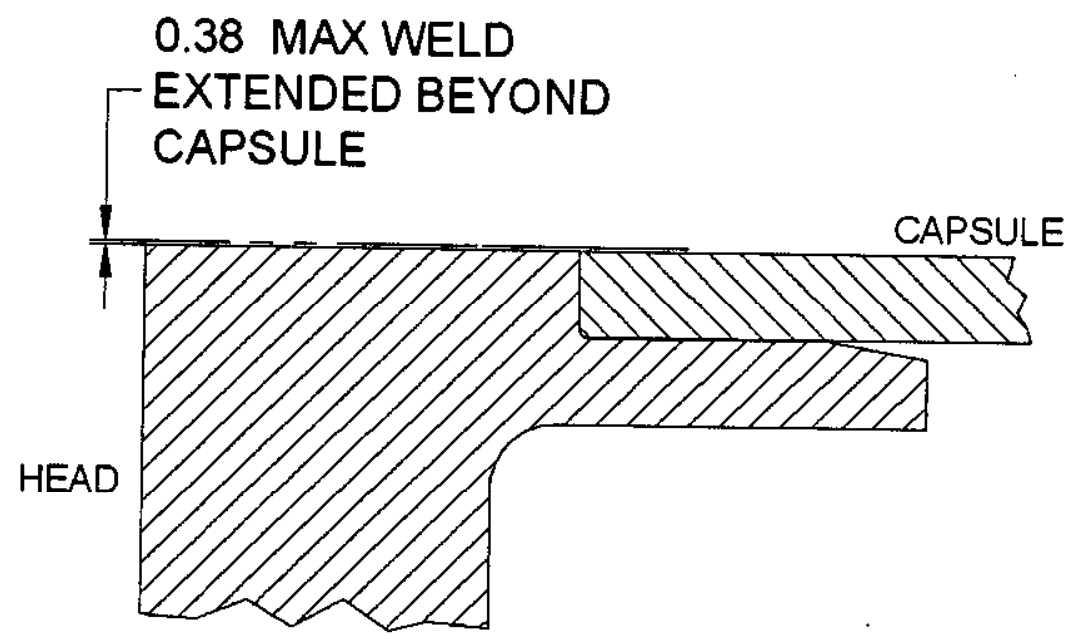

Figure 1 Joint Design

Dimensions in $\mathbf{m m}$

Process Automatic Orbital GTAW-Pulsed

Base Metals: $\quad$ Capsule and Body consist of ASME SA479, composition UNS S31651, Bar

ASME P-No.: P-8

Pipe Dia: $\quad 22 \mathrm{~mm}$

Groove: $\quad 1 \mathrm{~mm}$

Filler Metals: None

Pre-Heat: $\quad 60^{\circ} \mathrm{F}$ minimum

Interpass Temp: N/A

PWHT: none

Position of Joint: $5 \mathrm{G}$

Environment: Inert Atmosphere, $99.995 \%$ pure $\mathrm{Ar}, 20 / 80 \mathrm{He} / \mathrm{Ar}$, or $70 / 30 \mathrm{He} / \mathrm{Ar}$ Target less than $5 \mathrm{ppm}$ of $\mathrm{O}_{2}$, less than $5 \mathrm{ppm} \mathrm{H}_{2} \mathrm{O}$. Must not exceed $10 \mathrm{ppm}$ separately. Glove

Torch Flow Rate: TBD box pressure maintained below 3 psig.

Electrical Characteristics:

Current:

Voltage (range):

Current (range):

Tungsten Electrode:

Electrode Size:

Technique:

Part Rotation:

DC - SP Pulsed

To Be Determined (TBD)

TBD

EWTH-2

TBD

Automatic

$\begin{array}{ll}\text { String or Weave: } & \text { String } \\ \text { Upslope: } & \text { TBD } \\ \text { Weld: } & \text { TBD } \\ \text { Downslope: } & \text { TBD } \\ \text { Multiple or Single Pass: } & \text { Single }\end{array}$


Appendix A to

B-MT(EDT)S-010

Page 2

Tensile Test Results

\begin{tabular}{|c|l|l|l|l|l|l|}
\hline $\begin{array}{c}\text { Specimen } \\
\text { Number }\end{array}$ & Width & Thickness & Area & $\begin{array}{c}\text { Ultimate } \\
\text { Total Load }\end{array}$ & $\begin{array}{c}\text { Ultimate } \\
\text { Unit Stress }\end{array}$ & $\begin{array}{c}\text { Type of } \\
\text { Failure \& } \\
\text { Location }\end{array}$ \\
\hline 1 & & & & & & \\
\hline 2 & & & & & & \\
\hline
\end{tabular}

Guided-Bend Test Results

\begin{tabular}{|c|c|}
\hline Type and Figure Number & Results \\
\hline & \\
\hline & \\
\hline & \\
\hline
\end{tabular}

Other Test Results

Dimensional test: Performed by Bechtel Bettis in accordance with assembly Route Card Number , results recorded as: $\square$ Acceptable $\square$ Unacceptable

Pressure and Helium Leak tests: Performed by Bechtel Bettis in accordance with assembly Route Card Number , results recorded as: $\square$ Acceptable $\square$ Unacceptable

Metallography:

Welder's Name Badge Number

Test Conducted by Laboratory Test Number

We Certify that the statements in this record and that the test welds were prepared, and tested in accordance with the requirements of Section IX of the ASME Code.

Date By 


\section{Appendix B \\ Nondestructive Testing}

\section{Weld Inspection}

The finished weld surface shall be visually examined at $1 \mathrm{X}$ magnification. The surface of the weld shall be sufficiently free from course ripples, grooves, overlaps, and abrupt ridges and valleys. Imperfections producing the following indications are unacceptable:

(1) any cracks or linear indications;

(2) maximum external reinforcement (distance weld can extend beyond capsule) $-0.38 \mathrm{~mm}$;

(3) weld undercut $-0.25 \mathrm{~mm}$ max \& shall not encroach on required section thickness;

(4) Rounded indications opened to the surface shall be evaluated as follows:

\begin{tabular}{ccc}
\hline Maximum Size of Acceptable Rounded Indication & $\begin{array}{c}\text { Maximum Size of } \\
\text { Nonrelevant Indication }\end{array}$ \\
\hline Random & Isolated & $0.10 \mathrm{~mm}$ \\
\hline $0.25 \mathrm{~mm}$ & $0.33 \mathrm{~mm}$ & \\
\hline
\end{tabular}

The maximum permissible size of any indication shall be that shown for Random indications, except that an isolated indication separated from an adjacent indication by $25 \mathrm{~mm}$ or more may be that shown for Isolated indications. Aligned rounded indications are acceptable when the summation of the diameters of the indications is less than $1 \mathrm{~mm}$ in a length of $12 \mathrm{~mm}$.

\section{Dimensional Inspection}

Measure to the nearest $0.025 \mathrm{~mm}$ the outside diameter (OD) of the Capsule base metal (at least $25 \mathrm{~mm}$ away from the weld) at a minimum of three locations. Calculate the average of these measurements.

Record the average base metal OD:

With the capsule in a V-block or rotary fixture, zero a dial indicator on the capsule OD in the region where the OD measurements where taken. Move the capsule so that the indicator contacts the weld. Rotate the capsule $360^{\circ}$ and record the maximum indicator deflection in both the positive $(+)$ and negative (-) directions.

+ deflection: $(+0.38 \mathrm{~mm}$ max)

- deflection: $(-0.25 \mathrm{~mm}$ max)

If maximum positive deflection is exceeded, file or grind the weld OD to be within tolerance, taking care not to reduce the base metal OD.

If maximum negative deflection is exceeded, build up the undersized weld $O D$ by welding in accordance with the referenced weld procedure.

If any repair was performed, record the new maximum deflection below:

+ deflection: $(+0.38 \mathrm{~mm}$ max $)$

- deflection: $(-0.25 \mathrm{~mm}$ max $)$ 
Appendix $B$ to

B-MT(EDT)S-010

Page 2

\section{Pressure and Helium Leak Test}

Subject the assembly to a $950+50 /-0$ psig (preliminary value) external pressure for 30 minutes minimum. Most efficient ramp time to pressure is acceptable. Use a minimum of $3 \%$ helium with argon or Nitrogen balance.

After removal from the pressure chamber, blow dry assembly with nitrogen (or most readily gas) that does not contain helium.

As soon as possible after pressurizing, use the helium leak test pressurization and vacuum system or bell jar apparatus setup to leak test the assemblies. No data sheets required.

Record background Helium. std. cc He/sec

Record calibration leak rate. std. $\mathrm{cc} \mathrm{He} / \mathrm{sec}$

Record leak rate here std. cc He/sec

Record leak detector serial number

Record calibration standard serial number

Leak rate acceptance criteria is $1.0 \times 10^{-7} \mathrm{std}$. cc He/sec. 


\section{Appendix C}

Metallographic Examination

\section{Sample Preparation}

One cross section shall be taken for metallographic examination, as specified per Table 1 and as depicted in Figure 2. The specimen shall be prepared, mounted, polished and etched in accordance with ASTM E3 with a suitable etchant (see QW-470 of ASME B\&PV Section IX) to give a clear definition of the weld metal and heat affected zone.

\section{Acceptance Criteria}

The surface shall be examined at $\times 10$ to $\times 15$ magnification and a photograph prepared at the same magnification.

In order to be considered acceptable:

(a) visual examination of the cross section of the weld metal and heat-affected zone shall show complete fusion and be free of cracks, incomplete fusion and;

(b) porosity in excess of that shown as acceptable in appendix VI of section III, Division 1 Appendixes of ASME B\&PV code. 
Appendix D

Page 1

\section{Welder Performance Qualification}

Weld Operator Pefformance Qualification shall be conducted in accordance with the requirements of the ASME B\&PV code section IX, and as supplemented herein. The type and number of examinations and test specimens required for each weld operator is specified in Table 1 below.

The qualification test coupon shall include any manually applied GTAW tack welds as required per the production item.

\begin{tabular}{|c|c|c|c|c|c|}
\hline $\begin{array}{c}\text { Method of } \\
\text { Evaluation }\end{array}$ & $\begin{array}{c}\text { Weld Test } \\
\text { Coupon(s) } \\
\text { Required }\end{array}$ & $\begin{array}{c}\text { Visual } \\
\text { Examination } \\
\text { per QW-302.4 } \\
\text { [note 2] }\end{array}$ & $\begin{array}{c}\text { Face Bend } \\
\text { QW-462.3(a) }\end{array}$ & $\begin{array}{c}\text { Bend } \\
\text { QW-462.3(a) }\end{array}$ & $\begin{array}{c}\text { Radiographic } \\
\text { Examination } \\
\text { QW-302.2 } \\
\text { [note 3] }\end{array}$ \\
\hline $\begin{array}{c}\text { Option 1 } \\
\text { By Mechanical } \\
\text { Testing }\end{array}$ & 1 required & $\mathrm{X}$ & 2 required & 2 required & -- \\
\hline $\begin{array}{c}\text { Option 2 } \\
\text { By NDE }\end{array}$ & $\begin{array}{c}3 \\
\text { consecutive }\end{array}$ & $\mathrm{X}$ & - & -- & $\times$ \\
\hline
\end{tabular}

Table 1, Type and Number of Examinations and Test Specimens Required [note 1] Notes:

1. All paragraph references correspond to ASME B\&PV code section IX.

2. In addition to the referenced ASME visual inspection requirements, the following applies:

The finished weld surface shall be visually examined at $1 X$ magnification. The surface of the weld shall be sufficiently free from course ripples, grooves, overlaps, and abrupt ridges and valleys. Imperfections producing the following indications are unacceptable:

(a) any cracks or linear indications;

(b) maximum external reinforcement (distance weld can extend beyond capsule) $-0.38 \mathrm{~mm}$;

(c) weld undercut $-0.25 \mathrm{~mm}$ max $\&$ shall not encroach on required section thickness;

(d) Rounded indications opened to the surface shall be evaluated as follows:

\begin{tabular}{ccc}
\hline Maximum Size of Acceptable Rounded Indication & $\begin{array}{c}\text { Maximum Size of } \\
\text { Nonrelevant Indication }\end{array}$ \\
\hline Random & Isolated & $0.10 \mathrm{~mm}$ \\
\hline $0.25 \mathrm{~mm}$ & $0.33 \mathrm{~mm}$ & \\
\hline
\end{tabular}

The maximum permissible size of any indication shall be that shown for Random indications, except that an isolated indication separated from an adjacent indication by $25 \mathrm{~mm}$ or more may be that shown for Isolated indications. Aligned rounded indications are acceptable when the summation of the diameters of the indications is less than $1 \mathrm{~mm}$ in a length of $12 \mathrm{~mm}$.

3. Radiographic acceptance criteria shall be in accordance with paragraph ND-5300 of ASME B\&PV code section III, Division 1, class 3. 


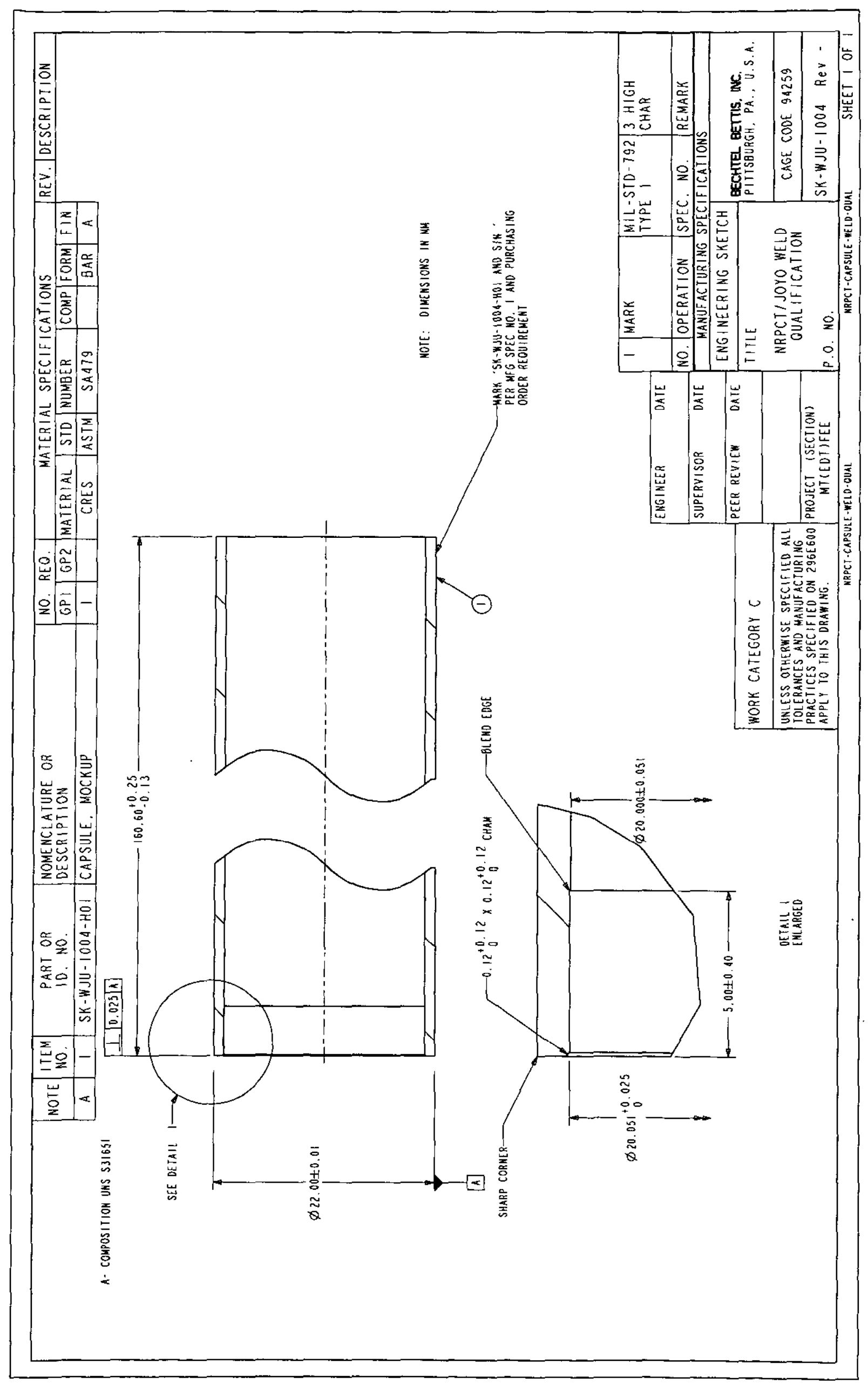




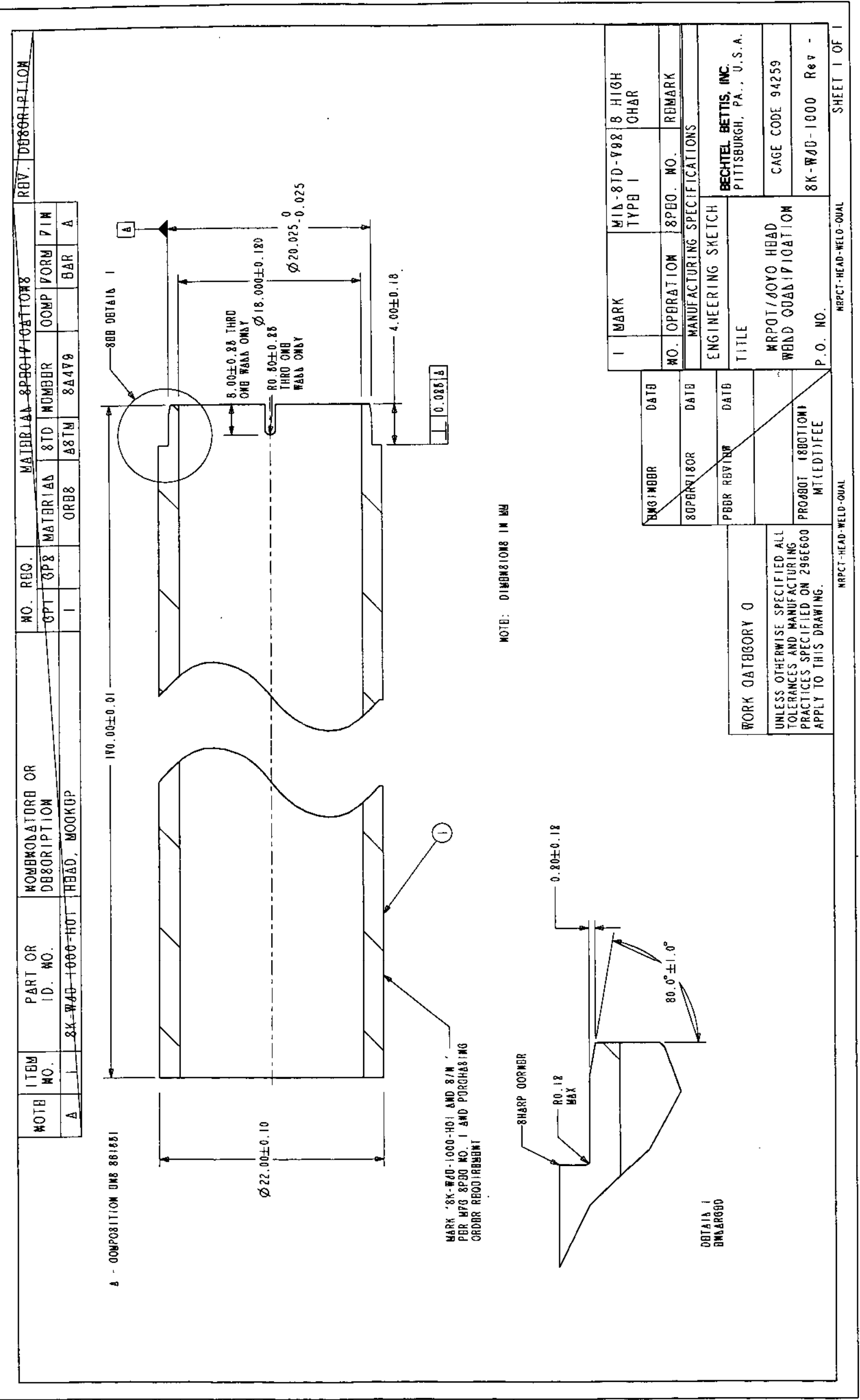




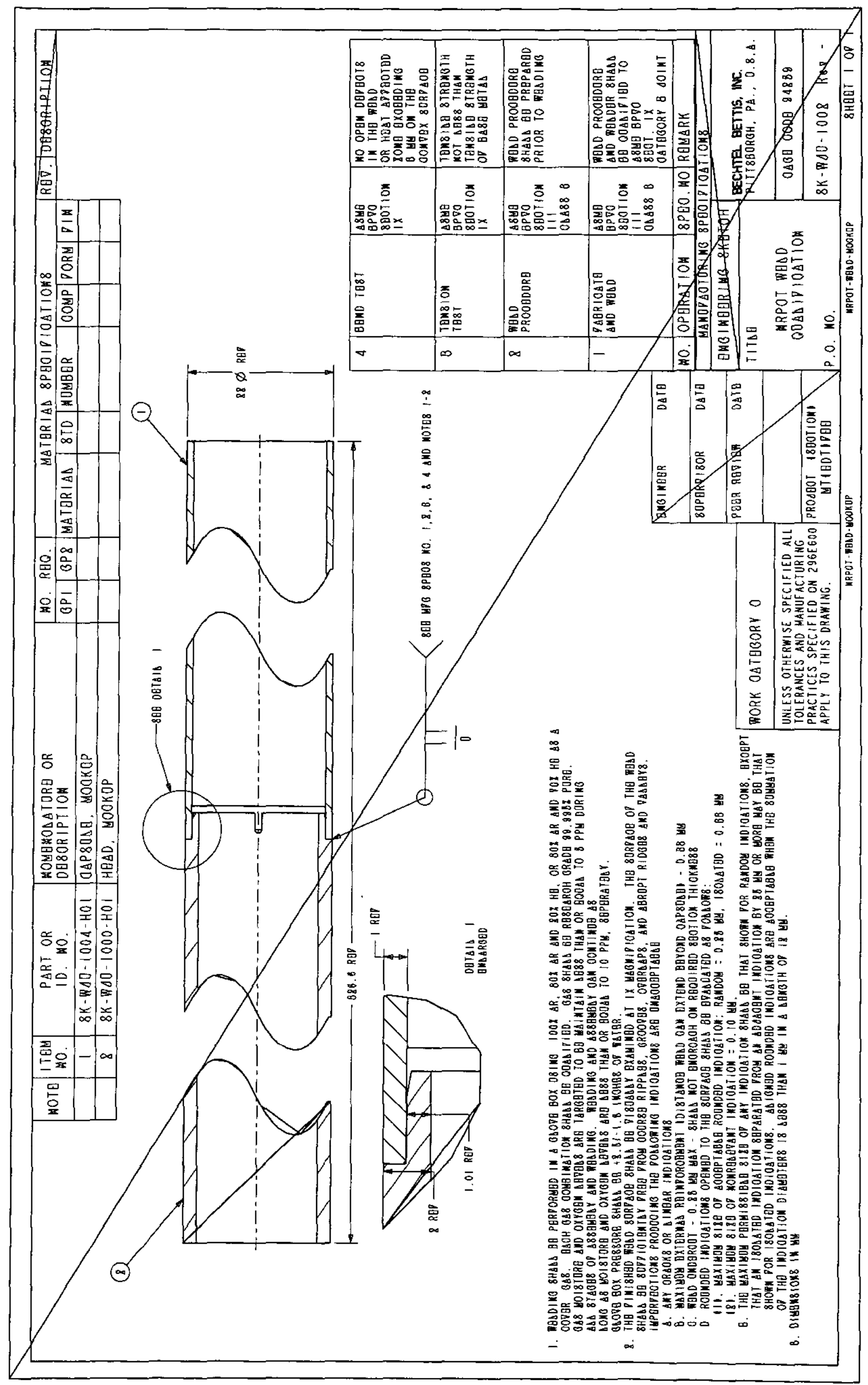




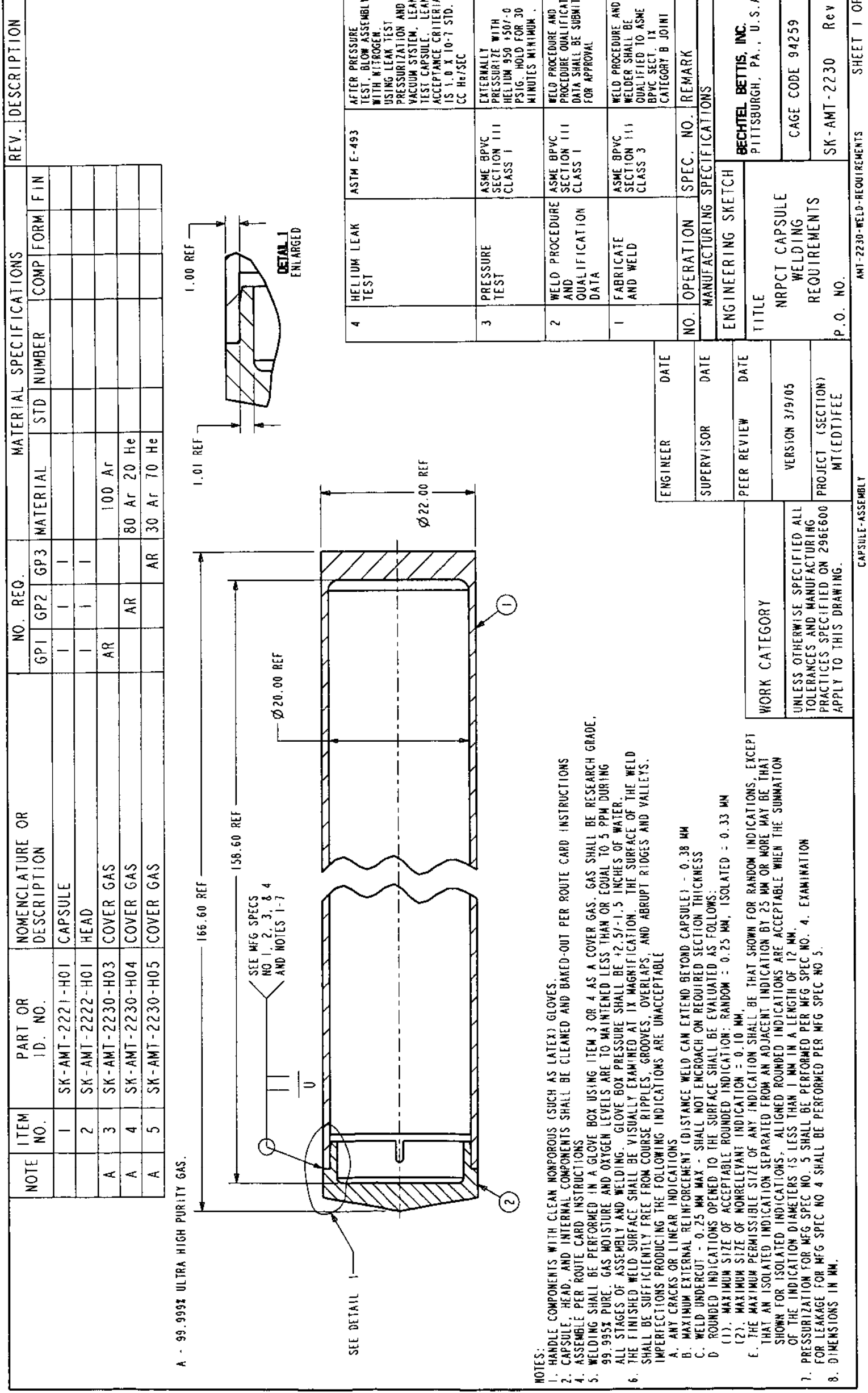




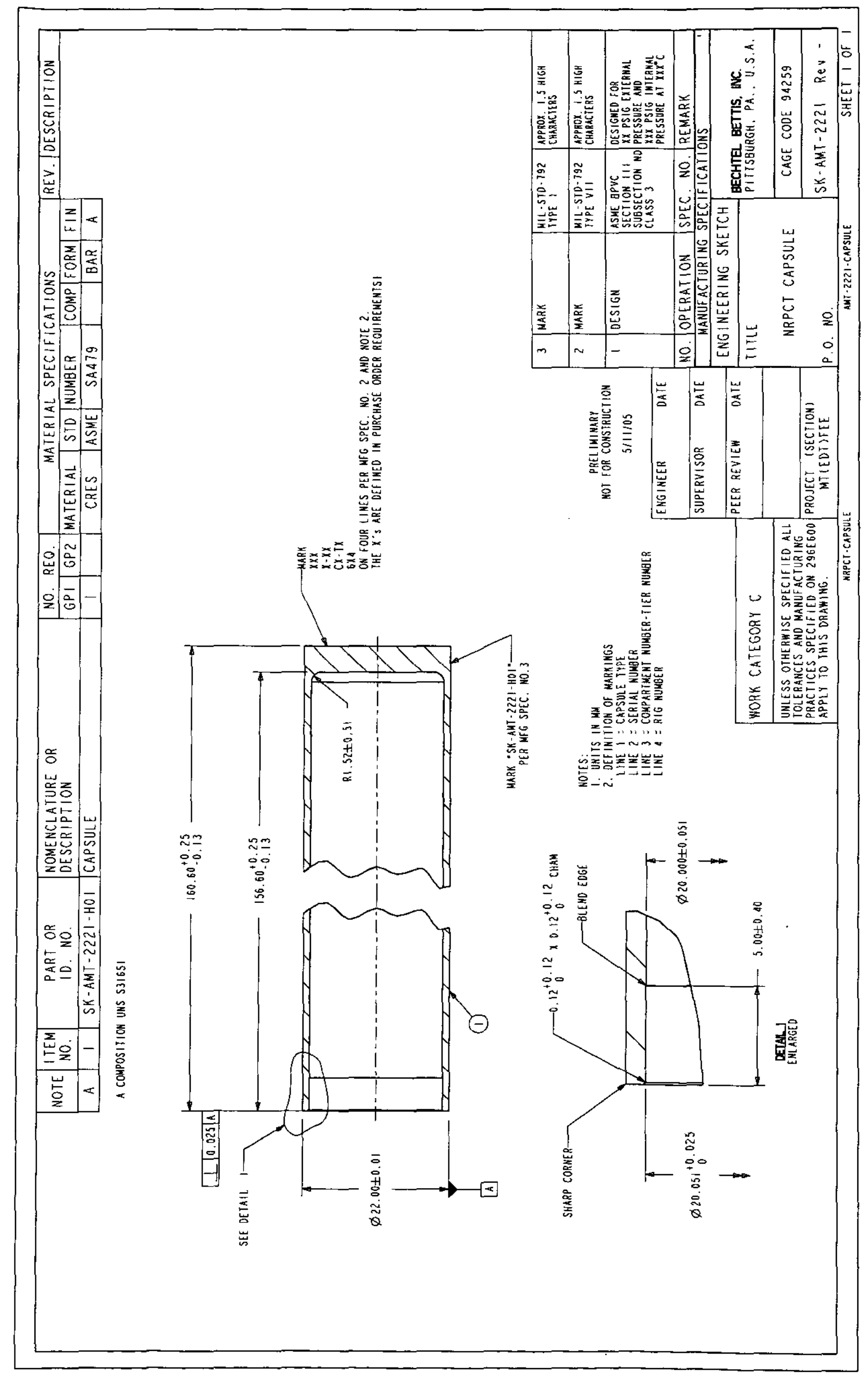




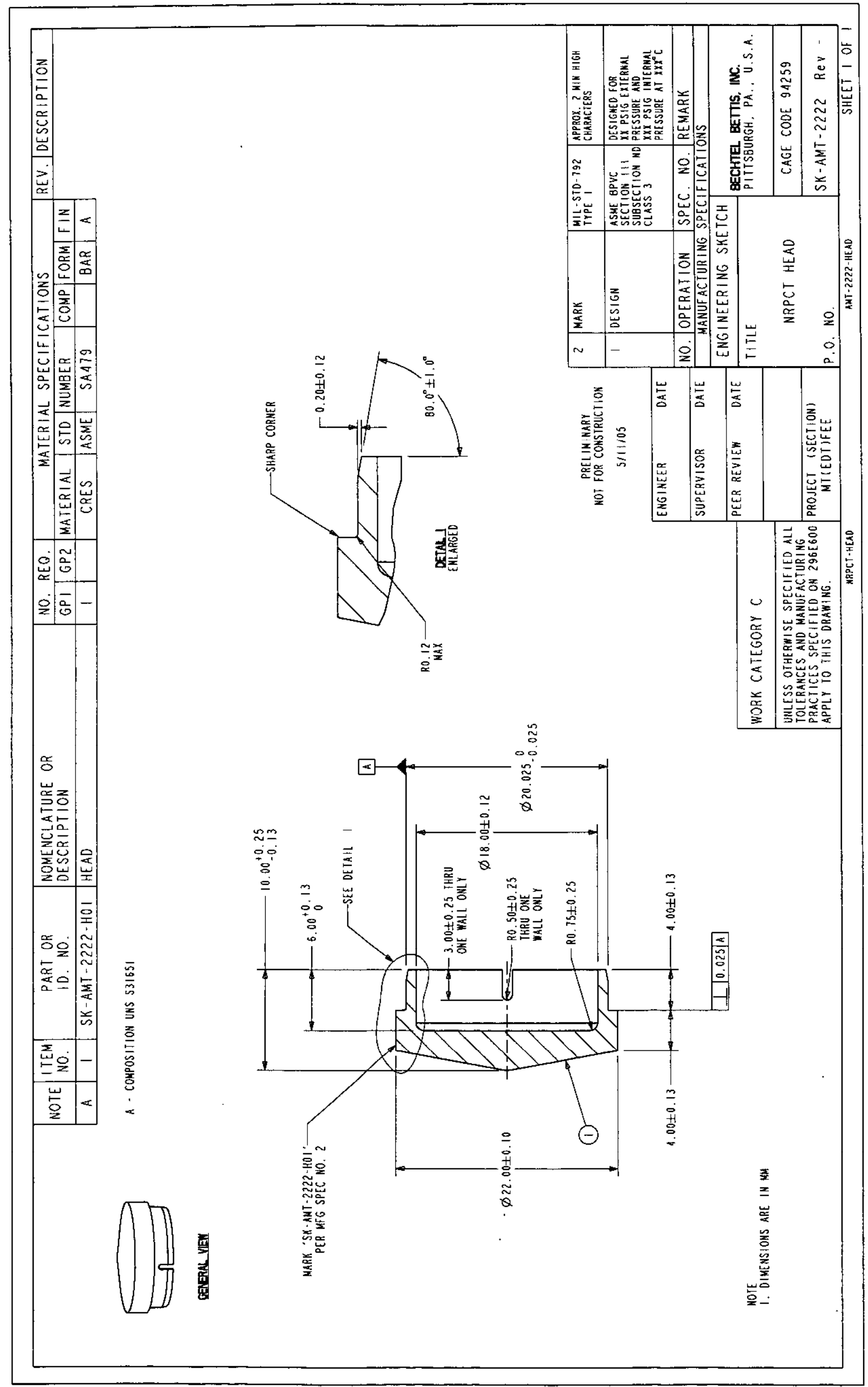


Enclosure 8 to MDO-723-0044 /

B-MT(SRME)-52

Page 1

\begin{abstract}
Enclosure 8
to

MDO-723-0044 / B-MT(SRME)-52

Alternative Disposition of JOYO-1 Space Structural Materials Irradiation Rigs (SMIR's)
\end{abstract}

George A. Newsome

William S. Saylor

January 2006 


\section{Summary}

This enclosure describes the Naval Reactor Prime Contractor Team (NRPCT) efforts to identify potential United States users for the two JOYO-1 Structural Materials Irradiation Rigs (SMIR's) in lieu of scrapping or allowing Japanese Atomic Energy Agency (JAEA) unrestricted use of the rigs. After the announcement that the Naval Reactors (NR) program was ending its collaboration with National Aeronautic and Space Agency (NASA) to deliver a space reactor for the Prometheus Project, an assessment was made of all work and contracts in progress at that time. Based on the SMIR fabrication status review, which included a cost assessment to complete fabrication versus terminate the contract, the NRPCT concluded that the most beneficial option was to complete fabrication of the SMIR's. As such, parts for these rigs will be available after January 2006 following completion of the SMIR fabrication contract. If a U.S. user is not identified, JAEA plans to find a Japanese user for the SMIR's. The most likely U.S. users of these rigs would be the U.S. DOE Advanced Fuel Cycle Initiative (AFCl) and Generation IV Programs (Gen IV). After further investigation (including a face to face meeting with JAEA), neither the AFCI nor the Gen IV Programs are interested in using the JOYO-1 SMIR's. Therefore, as instructed by the NRPCT, Pacific Northwest National Laboratory (PNNL) has issued a letter to JAEA releasing all rights to the SMIR's.

\section{Background}

A key part of the irradiation test plan developed to support NR Program efforts to deliver a Prometheus space reactor was materials testing in the JOYO Experimental Reactor in O-arai, Japan. The NRPCT material irradiation testing plan assumed that a series of tests in the JOYO reactor would occur. JOYO-1 represented the initial test series for early screening of candidate materials and was to be initiated in June 2006. The test start date was defined by the next availability of the JOYO reactor for insertion, timing to design and procure test fixturing, and the design and analysis required to meet the testing window. This resulted in a highly expedited schedule for the NRPCT to define materials, design test components, complete analysis to assure adequate test compatibility, and complete fabrication of all parts prior to support the May 2006 reactor insertion date. In addition, JAEA fabrication of the irradiation test rigs (i.e. SMIR's) to house the test capsules was also required to be done in parallel with other preparation activities. The SMIR's provide the housing for and proper placement of the test specimen capsules within the JOYO reactor. JAEA was contracted to design and fabricate these rigs because of their prior experience in building SMIR's, Japanese regulatory requirements, and their knowledge of JOYO reactor interfaces. SMIR fabrication was completed in January 2006. Conversely, the NRPCT considered having JAEA design the specimen capsules, but due to the multitude of specimen types and capsule pressure boundary conditions, the NRPCT chose to keep cognizance of the specimens and capsule designs. This would allow for maximum flexibility and design control to accommodate changes and assure NR standards are met for the designs. Also, JAEA did not have previous experience designing capsules that would contain the range of test specimen material and range of irradiation temperatures. This decision was especially important for the NRPCT when considering the communication barriers and the differences between Japanese and American design standards to assure the capsules were designed adequately.

Early negotiations and fact-finding with JAEA regarding the potential for testing in the JOYO reactor identified various locations within the reactor and the associated structures (rigs) for insertion into the locations. The NRPCT and JAEA settled on two diametrically opposite 
locations in the first ring of the reflector region of the reactor. In this region, the standard rig design used by JAEA was the SMIR. Two SMIR's would be required to house the capsules in the selected reactor locations. Figure 1 provides a schematic diagram of a SMIR and identifies the key parts including the wrapper tube and compartments. Figure 2 presents photographs of some actual SMIR hardware, similar to hardware fabricated for JOYO. Each SMIR would have seven compartments that could house five capsules each. The seven compartments were arranged such that six outer compartments surrounded a center seventh compartment to form the hexagonal cross-section of the SMIR. The center compartment was to be left empty for use by JAEA for reactor monitoring or for structural considerations, if needed at a later date. The JOYO-1 test program was established to completely utilize all of the six outer compartments in each of the SMIR's, for a total of sixty irradiation test capsules. Enclosure 7 provides much more detail on the SMIR configuration and design.

\section{Identification of Potential Users}

When the Prometheus Project was restructured, NR requested that the NRPCT investigate other US entities that may be able to utilize the SMIR's rather than have JAEA find alternative (Japanese) users or dispose of them. As an outcome of the NRPCT participation in the Space Reactor effort, the NRPCT has interacted with all of the groups that may be interested in conducting an irradiation test in the JOYO reactor.

In considering any potential future user of this equipment, the first consideration involves a restriction for conducting tests in JOYO (or any other Japanese reactor). The work must not be associated with military programs, based on the "Peaceful Uses" principle that Japan must abide by according to their Constitution. The scientific nature of the Prometheus Program and the fact that the test data from the NRPCT JOYO testing was to support a peaceful use objective in space were key reasons why the NRPCT was allowed access to the JOYO reactor.

The only U.S. programs actively conducting irradiation tests in foreign test reactors are the AFCI and Gen IV programs. These two programs are jointly coordinating their material development efforts (including irradiation test programs). Currently they are sponsoring two tests in the Phenix reactor in France. One is a fuel irradiation test and the other is a materials irradiation test (MATRIX-MI).

The AFCl/Gen IV programs have expressed interest in conducting irradiation tests in Japan. To facilitate this, they have recently put in place an INERI agreement between the United States and Japan entitled, "Implementing Arrangement Between the Department of Energy of the United States of America and the Ministry of Education, Culture, Sports, Science and Technology of Japan Concerning Cooperation in the Field of Research and Development of Innovative Nuclear Energy Technologies". Their first priority is to conduct a fuel irradiation test in JOYO tentatively planned for the 2010 time frame. The JOYO-1 SMIR's are not appropriate for this fuel test because they intend to have instrumented capsules that require a different configuration than the SMIR, plus they intend to irradiate their test in the core region rather than the reflector region.

However, after the NRPCT approached the AFCI/Gen IV programs regarding the availability of the JOYO-1 SMIR's, they replied that they could consider using them for a materials irradiation test that would complement their Phenix MATRIX-MI test. The test program that they could put together would target a 2008 insertion and would consist of only three to ten capsules. This is a 
Enclosure 8 to MDO-723-0044 I

B-MT(SRME)-52

Page 4

much smaller scope than the JOYO-1 test and would only use a small fraction of the total available space.

The NRPCT recommended to the AFCl program that they need to make contact with JAEA as soon as possible to initiate discussions regarding a potential materials irradiation test in JOYO using these SMIR's. JAEA will be searching for other users, most likely from Japan, so the U.S. programs need to inform JAEA that they are seriously interested.

The DOE AFCI program contacted JAEA and included a discussion of the potential use of the JOYO-1 SMIR's in a face-to-face meeting held in Japan in December 2005. Based on the needs and resources of the AFCl program, they indicated that they are not interested due to their higher priority of a fuel test and the lower flux locations where the JOYO- 1 SMIR's can be used in JOYO.

After conducting this research and associated discussions, it was concluded that no near-term U.S. users exist and NRPCT instructed PNNL to issue a letter to JAEA releasing all rights to the SMIR's. 
Enclosure 8 to MDO-723-0044 /

B-MT(SRME)-52

Page 5

Spacer Pad

Handing Head

LockNut

Spring

Cap

Compartment

Capsule

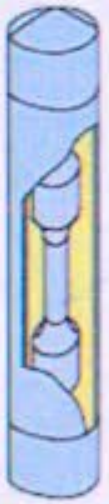

Specamen

Spacer Pad

Wrapper Twe

View of Capsule

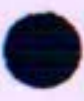

Compartment

View of Compartment

Compartmert

Entrance Nozzle

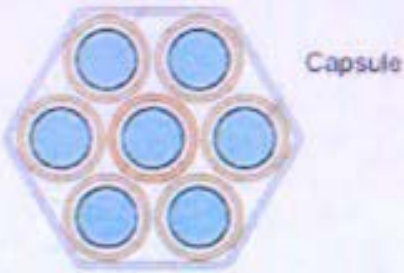

Cross Section of Materials Irrad ation Rog

Figure 1 Schematic View of Materials Irradiation Rig 
Enclosure 8 to

MDO-723-0044 /

B-MT(SRME)-52

Page 6

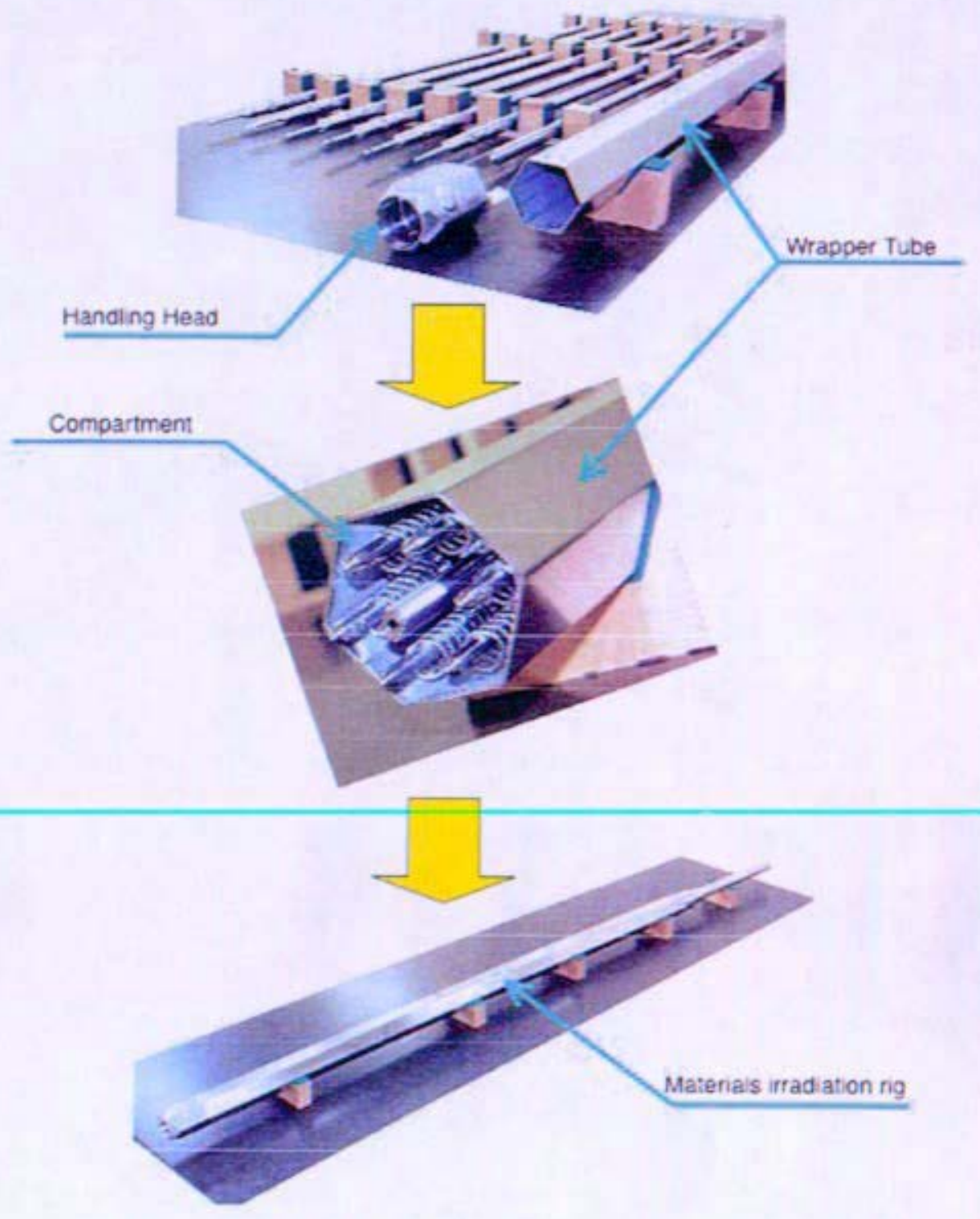

Figure 2 Components of materials irradiation rig 
Enclosure 9 to MDO-723-0044 /

B-MT(SRME)-52

Page 1

\title{
Enclosure 9 \\ to \\ MDO-723-0044 / B-MT(SRME)-52 \\ JOYO-1 Post-irradiation Examination
}

\author{
R. S. Northey \\ E. V. Mader
}

January 2006 


\section{Summary}

PIE plans for JOYO-1 were being developed at the time of project restructuring. Several facilities were being evaluated for the JOYO-1 post irradiation examinations (PIE) including Oak Ridge National Laboratory (ORNL), Pacific Northwest National Laboratory (PNNL), Japan Atomic Energy Agency (JAEA), or any combination of the three. The final PIE location(s) would have been chosen based on several factors, including costs (i.e. current facility capabilities and required upgrades), potential shipping issues and waste acceptance/burial requirements at the forefront of that decision. PIE testing was delineated into two sections by material type, ceramic or metallic. The large number of subsized specimens, multiple types of specimens and materials, and strict testing conditions (e.g., high temperature and high vacuum) necessitated that careful assessments be made of the candidate facilities.

The Naval Reactors Prime Contactor Team (NRPCT) presented the PIE technical requirements for JOYO-1 irradiation test specimens to Japan Atomic Energy Agency (JAEA) in order to discuss and clarify the testing needs (Reference 2). Further meetings with JAEA defined key testing issues, provided an understanding of current JAEA capabilities, and identified upgrades required to perform the examinations. A detailed cost analysis of performing PIE at the candidate locations had not been completed. NRPCT considered the most cost effective location to be either ORNL or PNNL; however, performing PIE at JAEA had advantages from a timing standpoint, if facility capabilities, shipping and waste stream issues could be overcome.

\section{Introduction}

This enclosure discusses the post-irradiation examination (PIE) of specimens that were to be irradiated in the JOYO-1 test campaign in the JOYO Experimental Fast Reactor, located in O-arai, Japan. JOYO is operated by the Japan Atomic Energy Agency (JAEA). JAEA was formed in an October, 2005 merger between the Japanese Nuclear Cycle Research Institute (JNC) and Japan Atomic Energy Research Institute (JAERI). Tables 1 and 2 provide details on materials that were to be tested and the respective specimen counts categorized by specimen type. Table 3 summarizes the desired test data for each specimen type. The PIE campaign was expected to be conducted in three phases:

Phase 1: $\quad$ Examination of a small subset of samples irradiated at intermediate fluences, to ensure all testing equipment meets desired testing requirements, and to guide testing parameters for higher fluence specimens.

Phase 2: $\quad$ Examination of the majority of samples irradiated at high and intermediate fluences, with some testing of low fluence specimens.

Phase 3: Examination of the remaining samples at lower fluences in addition to replicate samples. This phase would also have included optical and analytical electron microscopy examination of specimens from Phases 1 and 2.

At project restructuring, PIE plans were still under development, and no decisions had been made regarding the PIE facility(s) to be used. Options included performing all PIE in Japan, all PIE in the U.S., or a combination of both. Difficulties were encountered for each of these options and were
under evaluation the Prometheus Project was restructured. 
Enclosure 9 to

MDO-723-0044 I

B-MT(SRME)-52

Page 3

\section{PIE Options}

Performing all of the PIE in Japan was investigated as a way to generate the data in sooner. NRPCT members visited JAEA in February, March and August 2005 to discuss detailed PIE requirements and assess capabilities of JAEA and other Japanese facilities (Reference 2). During the August trip, it became clear that only a portion of the PIE testing could be performed at JAEA (as described later in this enclosure). This may have eliminated the option of performing all of the PIE at JAEA alone or with JAEA in addition to other Japanese facilities considered.

The second option, performing all of the PIE in the US, was actively under investigation. This option required the successful resolution to cask shipping issues outlined in Reference 1 . At the time of the restructuring of the Prometheus Project, the NRPCT was on the verge of formally requesting bids from two national laboratories that could likely perform the PIE operations. Attachments 1 and 2 to this enclosure are draft versions of INFORMATION TO VENDOR ("IV's") to Oak Ridge National Laboratory (ORNL) and Pacific Northwest National Laboratory (PNNL), respectively, which detailed the individual PIE tasks by specimen type. These two IVs were never issued and therefore, preliminary responses were not obtained. Attachment 3 to this enclosure is a PNNL white paper on a recommended PIE strategy for the JOYO-1 matrix. This white paper concluded that ORNL and PNNL were each suited to perform some of the PIE tasks, but not all of them. This conclusion was not acted on by the NRPCT, and no formal recommendation had been formulated by the NRPCT.

Splitting the PIE work between Japan and the US was the final option considered. However, a capsule waste disposal path was a concern, and had not been identified by the NRPCT.

Table 1: Materials included in the JOYO-1 Test Matrix

\begin{tabular}{|c|c|}
\hline & Metals \\
\hline & FS-85 (62Nb-27Ta-10W-1Zr) \\
\hline $\begin{array}{l}\text { Structural } \\
\text { Refractory Metals }\end{array}$ & ASTAR-811C (90Ta-8W-1Re-0.7Hf-0.025C-0.0014O, $\mathrm{N}-0.0003 \mathrm{H})$ \\
\hline & Mo-47Re $(52.5 \mathrm{Mo}-47.5 \operatorname{Re}-0.01 \mathrm{C}-<0.002 \mathrm{O})$ \\
\hline & $\operatorname{Re}$ \\
\hline Linet retals & $\bar{W}$ \\
\hline & W-25Re (75W-25Re) \\
\hline Structural Nickel & \begin{tabular}{|l} 
Nimonic PE-16 (42Ni-33Fe-18Cr-4Mo-1.2Ti-1.2Al-0.3Si-0.2Mn-0.1C) \\
Alloy 617 (51Ni-22Cr-12.5Co-9Mo-3Fe-1Si-1Mn-0.6Ti-0.1Al-0.006B)
\end{tabular} \\
\hline Based Superalloys & $\begin{array}{l}\text { Haynes } 230(53 \mathrm{Ni}-22 \mathrm{Cr}-5 \mathrm{Co}-14 \mathrm{~W}-3 \mathrm{Fe}-2 \mathrm{Mo}-0.4 \mathrm{Si}-0.3 \mathrm{Al}-0.5 \mathrm{Mn}-0.1 \mathrm{C}- \\
0.015 \mathrm{~B})\end{array}$ \\
\hline & Ceramics \\
\hline & \begin{tabular}{|l} 
Monolithic Silicon Carbide \\
Composite SiC/SiC with $\mathrm{Hi}-\mathrm{Ni}$ \\
\end{tabular} \\
\hline & \begin{tabular}{|l}
$\mathrm{BeO}$ \\
\end{tabular} \\
\hline
\end{tabular}


Enclosure 9 to

MDO-723-0044 /

B-MT(SRME)-52

Page 4

Table 2: PIE Specimens and Respective Testing

\begin{tabular}{|c|c|c|c|c|c|c|c|}
\hline \multicolumn{8}{|c|}{ JOYO-1 Revised Specimen Count Summary } \\
\hline Material & $\begin{array}{l}\text { Biaxial } \\
\text { Creep }\end{array}$ & $\begin{array}{c}\text { Fracture } \\
\text { Toughness } \\
\text { Bars }\end{array}$ & $\begin{array}{c}\text { Compact } \\
\text { Tension } \\
\text { Discs }\end{array}$ & Tenșile & $\begin{array}{c}\text { Density and } \\
\text { Conductivity } \\
\text { Discs }\end{array}$ & $\begin{array}{c}\text { Compression } \\
\text { Cylinders }\end{array}$ & $\begin{array}{c}\text { Bend Stress- } \\
\text { Relaxation }\end{array}$ \\
\hline FS85 (Nb alloy) & 24 & & & 51 & 18 & & \\
\hline ASTAR-811C (Ta alloy) & 22 & & & 50 & 17 & & \\
\hline Mo-47.5Re & 22 & 48 & 16 & 63 & 29 & & \\
\hline $\mathrm{Re}$ & & 8 & & 22 & 25 & & \\
\hline$W$ & & 8 & & 22 & 24 & & \\
\hline W-25Re & & 8 & & 22 & 22 & & \\
\hline Alloy 617 (Ni alloy) & 9 & 54 & 7 & 62 & 38 & & \\
\hline Haynes 230 (Ni alloy) & 10 & 36 & 9 & 39 & 45 & & \\
\hline Nimonic PE16 (Ni alloy) & 11 & 54 & 8 & 59 & 31 & & \\
\hline SiC monolith & & & & 12 & 30 & & 8 \\
\hline SiC composite & & & & 24 & 30 & & 8 \\
\hline $\mathrm{BeO}$ & & & & & 32 & 16 & \\
\hline Specimen Totals & 98 & 216 & 401 & 426 & 341 & 16 & $1 \overline{16}$ \\
\hline
\end{tabular}

Table 3: PIE Specimen Type and Respective Testing

\begin{tabular}{|l|l|}
\hline \multicolumn{2}{|l|}{ Metallic Specimens } \\
\hline Biaxial Creep & Irradiation Creep \\
\hline Disc & Swelling and Thermal Diffusivity \\
\hline \multirow{2}{*}{ Tensile } & Irradiated Tensile Properties \\
\cline { 2 - 3 } & Ductile Brittle Transition Temperature (DBTT) \\
\hline Fracture Toughness & Toughness and DBTT \\
\hline Compact Tension & Irradiation Creep Crack Growth \\
\hline & Ceramic Specimens \\
\hline Tensile & Irradiated Tensile Properties \\
\hline Disc & Swelling and Thermal Diffusivity \\
\hline $\begin{array}{l}\text { Bend Stress } \\
\text { Relaxation }\end{array}$ & Irradiation Induced Creep Effects \\
\hline $\begin{array}{l}\text { Compression } \\
\text { Cylinders }\end{array}$ & Irradiated Compressive Strength \\
\hline
\end{tabular}


Enclosure 9 to

MDO-723-0044 /

B-MT(SRME)-52

Page 5

\section{PIE Test Requirements}

\section{Metallic Specimens}

Five metallic specimen types were included in the JOYO-1 test matrix. Table 4 summarizes the PIE operations and test conditions required for each specimen type, as well as the material.

\section{Biaxial Creep Characterization}

Post-irradiation testing of biaxial creep specimens includes an assessment of the degree of expected end-of-life irradiation creep. Irradiation creep is detected by a series of five precise post-irradiation dimensional measurements via laser profilometry as compared to similar pre-irradiation measurements. Reference 3 discusses the expected dimensional changes in cylinder diameter, primarily as a function of irradiation temperature and internal pressure from four sources: elastic strain, swelling, irradiation creep, and thermal creep. Plans were to measure the diameter at five axial locations at every degree for 180 or 360 degrees. The laser profilometry measurement process was expected to be performed at room temperature in air with careful attention to employing identical procedures, preferably using the machine. Although if the same machine could not be used for both exams, the same type of machine with an appropriate use of standard control specimens would be required for the both pre-irradiation and post-irradiation measurements.

\section{Density Measurement}

PIE of the disc specimens was to include two examinations: (1) a density measurement to assess irradiation swelling and (2) a laser flash measurement to assess thermal diffusivity and, by inference, thermal conductivity. Density measurements were to be performed using the Archimedes method preferably with an immersion liquid denser than water. Density measurements could be performed in air at room temperature, but the actual test temperature should be precisely known and stable to ensure sufficient measurement accuracy. This degree of accuracy $(<0.1 \%)$ was achieved for miniature tensile specimens machined from some of the same JOYO-1 materials in the High Flux Isotope Reactor (HFIR) Pathfinder Irradiation Test Program (Reference 4).

\section{Thermal Diffusivity Measurement}

Measurement of thermal diffusivity by the laser flash method (ASTM E1461-92) was to be employed on a small subset of the total number of disc specimens. Measurement would be performed at irradiation temperature in a vacuum or inert gas. Thermal conductivity is related to thermal diffusivity through density and specific heat. Hence, thermal conductivity could be inferred from the laser flash data. Alternatively, inference of thermal conductivity from an electrical resistivity measurement (ASTM B193-02) could have been employed on tensile specimens prior to tensile testing, as was performed in the HFIR Pathfinder Program (Reference 4). Discs would not be appropriate for an electrical resistivity measurement due to variable cross sectional widths.

\section{Tensile Testing}

Tensile testing of refractory metal alloy specimens (SS-3 type, discussed in Enclosure 3) requires high vacuum and high temperature capability. Testing of the nickel-based superalloys requires either vacuum or inert gas environments at elevated temperatures (discussed in Enclosure 2). Stress/strain curves (ASTM E8-04) were to be generated to assess changes in strength and ductility properties due 
to irradiation. Irradiated specimens would be compared to similar measurements on unirradiated and thermally aged specimens, again as was performed in the HFIR Pathfinder Program (Reference 4).

\section{Fracture Toughness Testing}

Fracture toughness measurements (ASTM E1820-01) were to be performed at a high vacuum or inert gas over a series of temperatures up to the irradiation temperature. Due the subsized dimensions of the fracture toughness bars, difficulties were anticipated with extensometry necessitating acquisition of experience and proficiency with the technique. This had not been obtained at the time of the Prometheus Project restructuring. The small size of the fracture toughness specimens for JOYO-1 raised concern about the validity of the PIE toughness measurements. A goal of JOYO-1 was to screen materials; therefore the validity issue was not of large concern to the NRPCT because fracture toughness comparisons between materials would still meaningful. Future irradiation test programs would have to consider larger sized ${ }^{1}$ specimens to obtain validated data suitable for a design bases.

\section{Compact Tension Testing}

The bolt-loaded compact tension (CT) discs were added to the JOYO-1 matrix late in its formulation process due to concerns regarding crack growth enhancement due to irradiation creep. The specimens would be pre-cracked and stressed by the load from the bolt. Again, because of the subsized dimension of the specimens (approximately $0.2 \mathrm{~T}$ or $20 \%$ of a full sized CT specimen), detailed PIE procedures were still uncertain. Ideally, clip gages would be affixed to measure crack mouth opening displacement, as the specimen would be tested near the irradiation temperature. The final load imposed by the bolt following irradiation would be measured by moving the crosshead and documenting the first load recorded by the load cell. A concern with this testing method is that at irradiation completion it is possible that the bolt-loads may be removed due to relaxation of stresses through thermal or irradiation creep. This concern was potentially mitigated by the incorporation of ceramic spacers located between the bolt and CT specimen face accepting the load. This procedure would have either been performed over a one to two month period to measure creep crack growth in the post-irradiated state or over several hours to measure fracture toughness. These options were being investigated by the NRPCT but the research did not advance sufficiently to determine their efficacy. At a minimum, heat tinting of the crack surface that grew in reactor due to irradiation and thermal creep was to be measured by optical microscopy or high resolution photography. This process was envisaged to be accomplished by heat tinting in low temperature air $\left(\sim 200^{\circ} \mathrm{C}\right.$ to $\left.300^{\circ} \mathrm{C}\right)$ followed by pulling the specimen apart (with or without extensometry) to visually examine both fracture surfaces to assess the extent of crack growth during irradiation exposure. Apart from the difficulty of obtaining extensometry to measure the crack mouth opening, specimen loading in the fixture may have required bolt removal. This removal would have precluded measurement of the final bolt loads at the end of irradiation. The NRPCT was in the process of exploring novel bolt loading schemes through modifications of the specimen design to allow final bolt load measurements, however no resolution had been obtained prior to the Prometheus Project restructuring.

\footnotetext{
'Current SMIR design limits specimen size to less than approximately $16 \mathrm{~mm}$. Alternative SMIR designs could be explored to increase this limit (e.g., by combining compartments) provided the design could be licensed by
Japanese authorities.
} PRE-DECISIONAL - For Planning and Discussion Purposes Only 
Enclosure 9 to

MDO-723-0044 /

B-MT(SRME)-52

Page 7

Table 4: PIE Requirements for Metallic Specimens

\begin{tabular}{|c|c|c|c|}
\hline Specimen Type & PIE Operation & Test Conditions & Material \\
\hline \multirow{5}{*}{ Biaxial Creep } & \multirow{5}{*}{$\begin{array}{c}\text { Laser } \\
\text { Profilometry } \\
\text { (accuracy } 5+/-3 \mu \mathrm{m} \text { ) } \\
\text { A minimum of } 180 \text { diameter } \\
\text { measurements (every } 1^{\circ} \text { ) at } \\
\text { each of } 5 \text { axial locations }\end{array}$} & \multirow{5}{*}{$\begin{array}{c}\text { Room Temp } \\
\text { In Air } \\
\text { (STP) }\end{array}$} & $\begin{array}{c}\text { FS-85 } \\
\text { ASTAR-811C }\end{array}$ \\
\hline & & & Mo-47.5Re \\
\hline & & & Alloy 617 \\
\hline & & & PE-16 \\
\hline & & & Haynes 230 \\
\hline \multirow{8}{*}{ Density Disc } & \multirow{8}{*}{$\begin{array}{c}\text { Density } \\
\text { Measurement } \\
\text { (Archimedes } \\
\text { Type, }+1-0.1 \% \text { accuracy) }\end{array}$} & \multirow{8}{*}{$\begin{array}{l}\text { Room Temp } \\
\text { In Air } \\
\text { (STP) }\end{array}$} & FS-85 \\
\hline & & & ASTAR-811C \\
\hline & & & Rhenium \\
\hline & & & Tungsten \\
\hline & & & W-25Re \\
\hline & & & Alloy 617 \\
\hline & & & Haynes 230 \\
\hline & & & PE-16 \\
\hline \multirow{9}{*}{$\begin{array}{c}\text { Thermal } \\
\text { Diffusivity } \\
\text { Disc }\end{array}$} & \multirow{9}{*}{$\begin{array}{l}\text { Thermal } \\
\text { Diffusivity } \\
\text { Measurement } \\
\text { (ASTM E1461-92) }\end{array}$} & \multirow{6}{*}{$\begin{array}{l}\text { Room Temp } \\
\text { To } 1350 \mathrm{~K}\end{array}$} & FS-85 \\
\hline & & & ASTAR-811C \\
\hline & & & Mo-47.5Re \\
\hline & & & Rhenium \\
\hline & & & Tungsten \\
\hline & & & W-25Re \\
\hline & & \multirow{3}{*}{$\begin{array}{l}\text { Room Temp } \\
\text { To } 1050 \mathrm{~K}\end{array}$} & Alloy 617 \\
\hline & & & Haynes 230 \\
\hline & & & PE-16 \\
\hline \multirow{8}{*}{ Tensile } & \multirow{8}{*}{$\begin{array}{c}\text { Tensile Testing } \\
\text { (ASTM E8-04) } \\
\& \\
\text { Electrical Resistivity } \\
\text { (ASTM B193-02) }\end{array}$} & \multirow{5}{*}{$\begin{array}{l}\text { Room Temp } \\
\text { To } 1450 \mathrm{~K} \\
\text { UHP Inert Gas or } \\
\text { HV }\left(\sim 10^{-6} \text { torr }\right)\end{array}$} & FS-85 \\
\hline & & & ASTAR-811C \\
\hline & & & $\frac{\text { Mo-47.5Re }}{\text { Rhenium }}$ \\
\hline & & & Tungsten \\
\hline & & & W-25Re \\
\hline & & \multirow{3}{*}{$\begin{array}{c}\text { Room Temp } \\
\text { To } 1050 \mathrm{~K} \\
\text { UHP Inert Gas or } \\
\text { HV }\left(\sim 10^{-6} \text { torr }\right)\end{array}$} & Alloy 617 \\
\hline & & & Haynes 230 \\
\hline & & & PE-16 \\
\hline \multirow{7}{*}{$\begin{array}{l}\text { Fracture } \\
\text { Toughness }\end{array}$} & \multirow{7}{*}{$\begin{array}{c}\text { Three point } \\
\text { slow bend test } \\
\text { (ASTM E1820-01) }\end{array}$} & \multirow{4}{*}{$\begin{array}{l}\text { Room Temp } \\
\text { to } 1450 \mathrm{~K}\end{array}$} & Mo-47.5Re \\
\hline & & & Rhenium \\
\hline & & & Tungsten \\
\hline & & & W-25Re \\
\hline & & \multirow{3}{*}{$\begin{array}{l}\text { Room Temp } \\
\text { To } 1100 \mathrm{~K}\end{array}$} & Alloy 617 \\
\hline & & & Haynes 230 \\
\hline & & & PE-16 \\
\hline
\end{tabular}


Enclosure 9 to

MDO-723-0044/

B-MT(SRME)-52

Page 8

\begin{tabular}{|c|c|c|c|}
\hline Specimen Type & PIE Operation & Test Conditions & Material \\
\hline \multirow{4}{*}{$\begin{array}{l}\text { Compact } \\
\text { Tension }\end{array}$} & \multirow{4}{*}{$\begin{array}{c}\text { Measure final bolt load at } T_{\text {irr }} \\
+ \text { final crack depth due to } \\
\text { irradiation exposure at JOYO } \\
\text { from heat tint optical analysis } \\
+ \\
\text { Option A: post-irradiation } \\
\text { creep crack growth rate at } T_{\text {irr }} \\
\text { (EPD measurement of crack } \\
\text { depth with long term testing) } \\
\text { Or } \\
\text { Option B: post-irradiation } \\
\text { fracture toughness (clip gage } \\
\text { to determine displacement) }\end{array}$} & $\begin{array}{c}\text { Room Temp to } \\
1350 \mathrm{~K}\end{array}$ & Mo-47.5Re \\
\hline & & \multirow{3}{*}{$\begin{array}{l}\text { Room Temp } \\
\text { To } 1100 \mathrm{~K}\end{array}$} & Alloy 617 \\
\hline & & & Haynes 230 \\
\hline & & & PE-16 \\
\hline
\end{tabular}

\section{Ceramic Specimens}

Three ceramic materials were included in the JOYO-1 irradiation test: beta-phase silicon carbide derived from chemical vapor deposition (CVD), composite SiC consisting of Hi-Nicalon Type S fibers in a chemical vapor infiltration (CVI)-derived $\mathrm{SiC}$ matrix and beryllium oxide (BeO). Planned ceramic PIE included tensile testing, thermal diffusivity, density, bend stress relaxation measurements, and compression testing. Test conditions and ASTM standards for these examinations are listed in Table 5. These post irradiation $\mathrm{SiC}$ tests require intricate handling; therefore, if activity levels were low enough, depending on the PIE site, it would have been advantageous to manipulate specimens out of cell in a radiologically controlled area. Unlike $\mathrm{SiC}$ specimens, BeO specimens are hazardous and should be handled in a hot cell. Inhaling particulate beryllium may cause a serious lung disease called chronic beryllium disease (CBD), while skin contact may cause skin irritations. Due to this health risk, extensive measures must be taken to control this hazard including protective equipment and engineered ventilation controls at source points and throughout the facility (Additional information on $\mathrm{BeO}$ can be found in Enclosure 5).

\section{Tensile Testing}

JOYO-1 testing was to include 12 monolithic and 24 composite tensile specimens at two irradiation temperatures. PIE tensile testing was to be conducted at or near the irradiation temperature to provide insight to failure modes at working conditions. Elevated temperature testing requires the use of high temperature fixtures, extensometers and an inert gas environment. It is possible that the postirradiation tensile specimens may be at a low enough activation energy to handle out of cell (in a glove box), therefore easing handling requirements.

This tensile test included a miniature dog-bone shaped design, shown in Figure 1. Additional test parameters include a cross-head speed of $0.5 \mathrm{~mm} / \mathrm{min}$, allowable bending below $5 \%$, and edge loaded passive grip interfaces. 


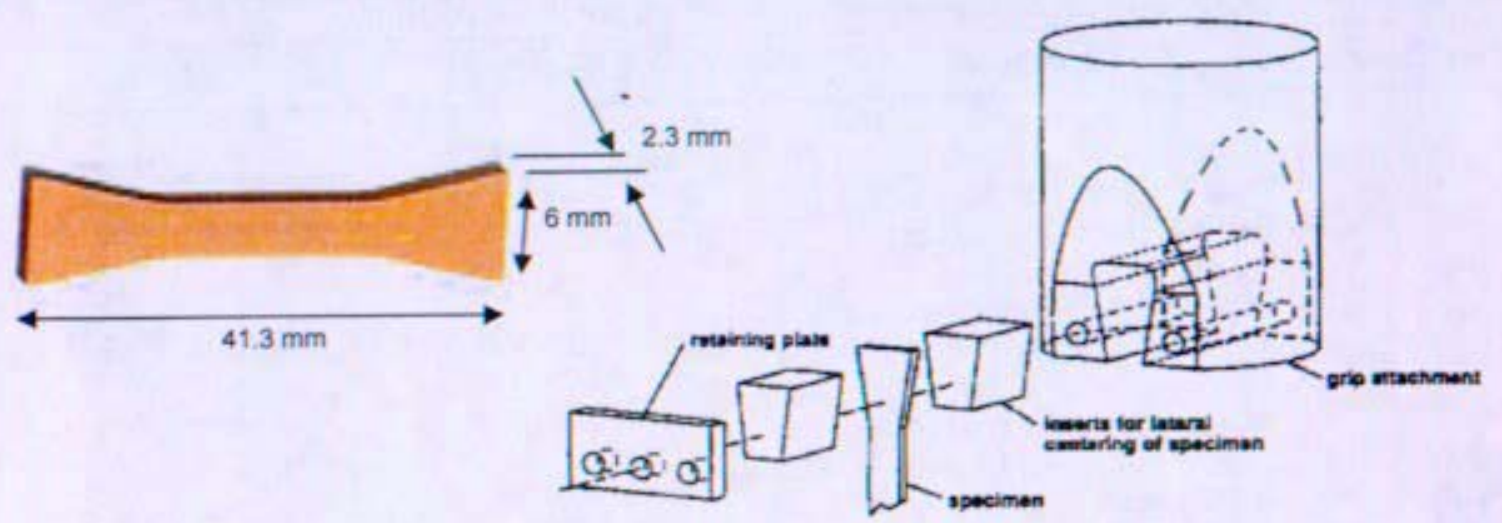

Figure 1: The dog-bone shaped design used for specimens in JOYO-1 and an example of an edgeloaded, passive grip interface for ceramic tensile PIE.

\section{Thermal Diffusivity Measurements}

JOYO-1 irradiation test plans included 24 monolithic SiC, 24 composite SiC and $8 \mathrm{BeO}$ thermal diffusivity specimens. Thermal diffusivity testing requires a laser flash unit capable of temperatures up to $1473 \mathrm{~K}$ in an inert environment, preferably argon.

Prior to conducting post irradiation measurements, the monolithic discs may require surface preparation. High purity monolithic Morton CVD SiC with its columnar grain structures may be translucent and possibly transparent, if highly orientated, under intense visible light near the infrared spectrum (Reference 5). This intrinsic material property of the as-machined monolithic discs prevents an accurate reading from the laser flash unit, due to photon penetration from the laser beam. In order to eliminate this effect, a carbon colloidal system should be applied to the flat surface directly exposed to the laser beam. Although this coating will allow testing of the monolithic specimens, it may reduce thermal diffusivity readings by $3-8 \%$ and should be removed following the test via an ultrasonic bath and isopropyl alcohol.

\section{Density Measurements}

Post irradiation density measurements were to be performed on 24 monolithic SiC, 24 composite SiC and $24 \mathrm{BeO}$ specimens. For the $\mathrm{SiC}$ specimens, the gradient column method should be applied using a series of halogenated hydrocarbons to measure material density. This method requires a strict temperature control of $23 \pm 0.1^{\circ} \mathrm{C}$. For $\mathrm{BeO}$, a room temperature Archimedes test should be conducted.

\section{Bend Stress Relaxation (BSR)}

The bend stress relaxation technique, designed by Oak Ridge National Laboratories (ORNL), is used to gain understanding of irradiation induced creep of monolithic SiC. JOYO-1 irradiation test plans included 16 holders containing up to 5 thin strip specimens per holder (Figure 2). The thin strip specimens had thicknesses of $0.200,0.100$ and $0.050 \mathrm{~mm}$ corresponding to initial stresses of 400 , 200 and $100 \mathrm{MPa}$ respectively. This test requires intricate post-irradiation disassembly and curvature readings. To determine the extent of bend stress relaxation, PIE techniques require the use of an optical microscope and recording equipment to capture an image of the final radius of curvature. The 
Enclosure 9 to

MDO-723-0044 /

B-MT(SRME)-52

Page 10

initial and final radiuses of curvature are combined to determine the BSR ratio, a measurement of irradiation induced relaxation. Enclosure 4 provides additional detail on the BSR specimens and holder.

$6.35 \mathrm{~mm}$

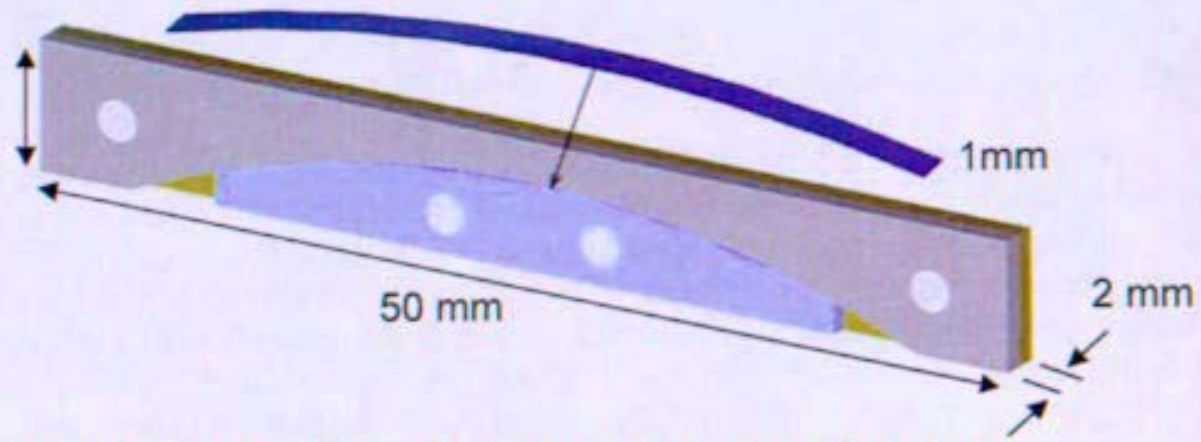

Figure 2: This picture depicts the BSR fixture and thin strip specimen.

\section{Compression Testing}

Planned compression testing included PIE of $16 \mathrm{BeO}$ samples at $1050 \mathrm{~K}$ and $850 \mathrm{~K}$. This testing requires an inert gas atmosphere in addition to high temperature fixtures and diametral strain gauges. Test conditions for this PIE were not determined at project restructuring. To clarify the post irradiation test specifics, initial compression testing on unirradiated $\mathrm{BeO}$ would have been required.

Table 5: PIE Requirements for Ceramic Specimens

\begin{tabular}{|c|c|c|c|}
\hline Specimen Type & PIE Operation & Test Conditions & Material \\
\hline Tensile & SiC (ASTM C1366-04) & $1050 \mathrm{~K}$ and $1350 \mathrm{~K}$ in & $\mathrm{SiC} / \mathrm{SiC}$ \\
\hline & SiC/SiC (ASTM C1359-96) & 1 atm inert gas & \\
\hline \multirow{3}{*}{ Density Disc } & $\mathrm{SiC}$ and $\mathrm{SiC} / \mathrm{SiC}$ & \multirow{3}{*}{ Room Temperature } & $\mathrm{SiC}$ \\
\hline & (ASTM D1505-03) & & $\mathrm{SiC} / \mathrm{SiC}$ \\
\hline & $\begin{array}{c}\mathrm{BeO} \\
\text { (ASTM C373-88) } \\
\end{array}$ & & $\mathrm{BeO}$ \\
\hline \multirow{2}{*}{$\begin{array}{l}\text { Thermal } \\
\text { Diffusivity Disc }\end{array}$} & \multirow{2}{*}{$\begin{array}{l}\text { Thermal } \\
\text { Diffusivity Measurement } \\
\text { (ASTM E1461-01) }\end{array}$} & $\begin{array}{c}\text { Room Temperature to } \\
1350 \mathrm{~K} \text { in } 1 \mathrm{~atm} \text { inert gas }\end{array}$ & $\frac{\mathrm{SiC}}{\mathrm{SiC} / \mathrm{SiC}}$ \\
\hline & & $\begin{array}{c}\text { Room Temperature to } 1250 \mathrm{~K} \text { in } \\
1 \mathrm{~atm} \text { inert gas }\end{array}$ & $\mathrm{BeO}$ \\
\hline $\begin{array}{c}\text { Compression } \\
\text { Cylinders }\end{array}$ & $\begin{array}{l}\text { Compressive Strength Testing } \\
\text { (ASTM C773-88) }\end{array}$ & $\begin{array}{c}850 \mathrm{~K} \text { and } \\
1050 \mathrm{~K}\end{array}$ & $\mathrm{BeO}$ \\
\hline BSR Samples & Optical Microscopy & Room Temperature & $\begin{array}{c}\mathrm{SiC} \\
(2.379 \mathrm{~g})\end{array}$ \\
\hline
\end{tabular}

\section{Temperature Monitors and Neutron Dosimetry}

As described in Enclosure 7 to this letter, each capsule was to have several passive monitors to assess irradiation conditions. Temperature monitors were to be examined to assess the accuracy of each capsule's specimen irradiation temperature, and flux wires were to be examined to assess the accuracy of neutron spectrum and fluence calculations by JAEA. Not all temperature monitors or flux 
Enclosure 9 to

MDO-723-0044 /

B-MT(SRME)-52

Page 11

wires were expected to be tested - only a subset of the monitors would have been required to validate temperature and fluence calculations. The number of monitors tested following irradiation would have been iteratively decided and inversely proportional to the quality of the predictions with respect to the initial measurements.

\section{Temperature Monitors}

For JOYO-1 irradiation testing, silicon carbide $(\mathrm{SiC})$ and zirconium carbide $(\mathrm{ZrC})$ passive temperature monitors were procured to determine irradiation temperatures. $\mathrm{ZrC}$ was to be used for the higher temperature capsules similar to the strategy employed in the HFIR Pathfinder Program (Reference 4). $\mathrm{PIE}$ procedures include recording electrical resistivity changes and/or dimensional changes following sequential isochronal anneals (References 6 and 7). The series of anneals should start at temperatures well below the estimated irradiation temperature. Between each sequential anneal, temperature monitor lengths and/or electrical resistivity should be measured and recorded. These two techniques may be used alone or together for additional measurement certainty. Examples of these PIE techniques are illustrated in Figure 3.

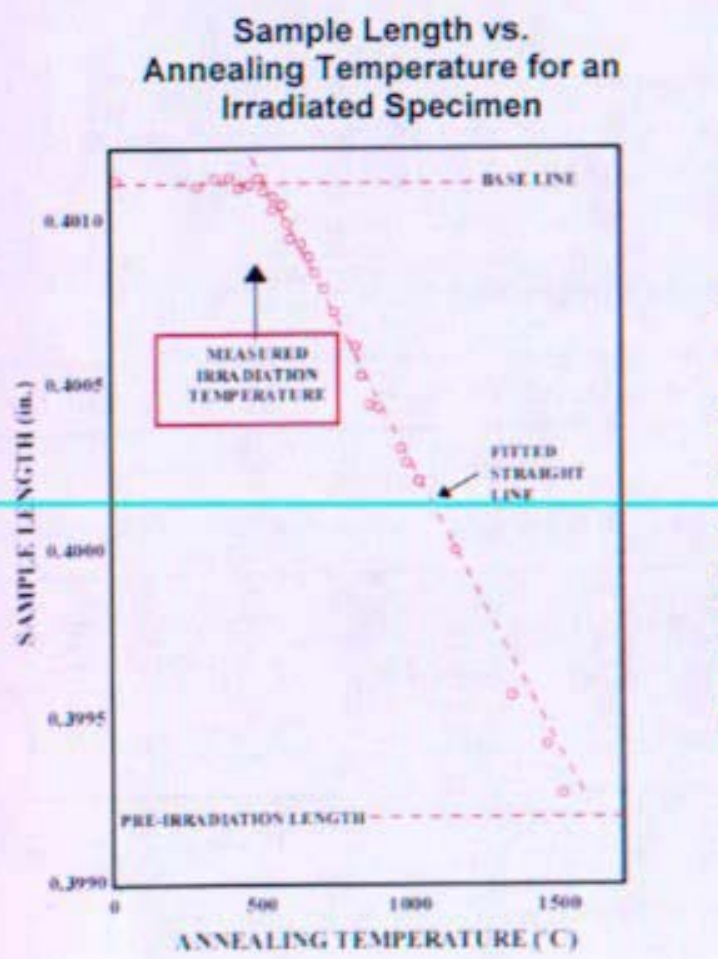

a.
Electrical Resistivity vs. Isochronal Anneal Temperature

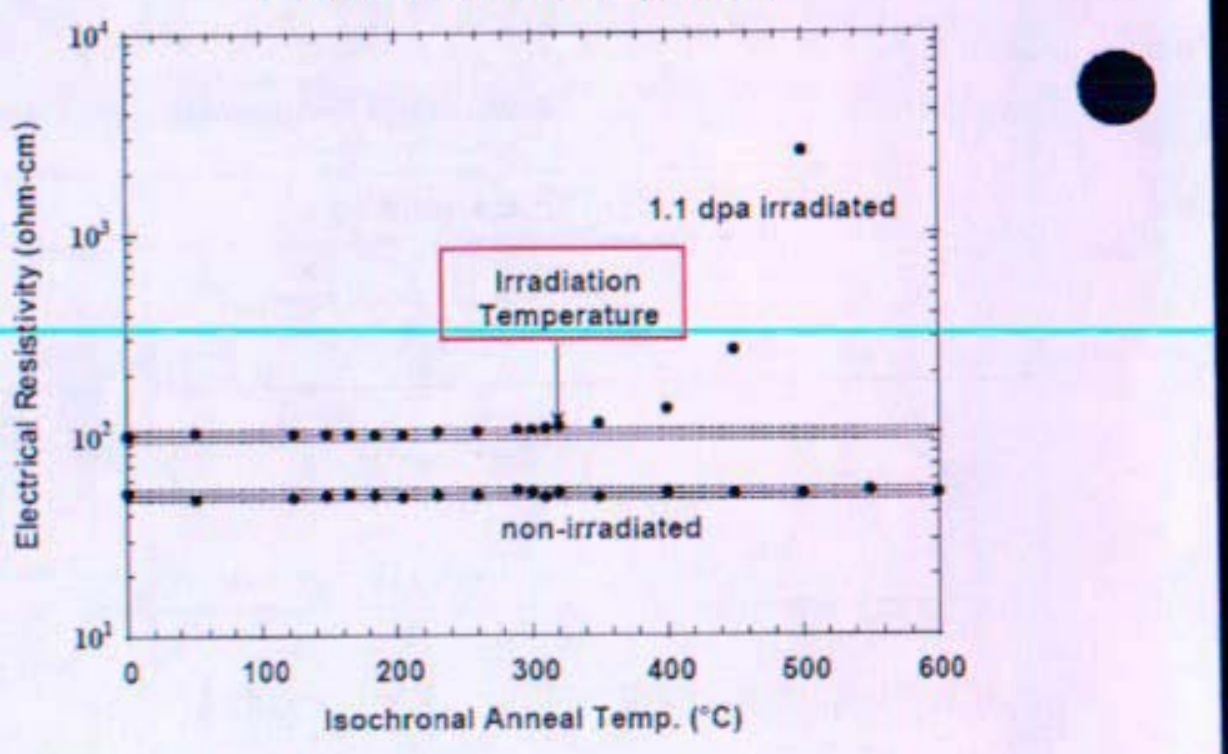

b.

Figure 3: a. Annealing temperature effect on sample length. Through the application of intersecting isochronal annealing lines the irradiation temperature can be determined. $b$. Annealing temperature effect on electrical resistivity for irradiated monolithic SiC (Reference 2).

\section{Flux Monitors}

Neutron flux dosimetry sets for the JOYO-1 irradiation test campaign consisted of Iron, Nickel, and Titanium wires, which were judged to provide sufficient neutron spectral sensitivity to confirm (or adjust) calculated neutron fluence values. Flux dosimetry analysis typically begins with an acid dissolution step with pre-weighed, irradiated wires. Dilutions may be on the order of a million to one 
to reduce sample activity to acceptable levels for removal from the hot cell and counting of the radioactive isotopes. Count data is converted into fluence values using standard calculation techniques. Depending on the dilution, equipment accuracy/calibration, and the duration from reactor shutdown to the actual counting time, measured flux wire fluence precision can be within $5 \%$ to $7 \%$. Uncertainties can be reduced to approximately $3 \%$ to $5 \%$ by calibration at the energies of interest and adjusting counting times to minimize errors due to statistical fiuctuation.

\section{PIE Facilities Assessment}

A preliminary assessment of facilities capable of performing PIE for the JOYO-1 specimens was under development at the time of the Prometheus Project restructuring. Individual facilities were assessed to different degrees either by NRPCT staff visits or by reviews of published documentation.

\section{Oak Ridge National Laboratory (ORNL)}

Due to the HFIR Pathfinder Program (Reference 4), ORNL has many of the required PIE capabilities in place. The Pathfinder Program focused on SS-3 tensile specimens (identical to the JOYO-1 metallic tensile specimens) of refractory metal alloys from which tensile, density, microhardness, and electrical resistivity measurements were performed. Furthermore, transmission electron microscopy (TEM) and scanning electron microscopy (SEM) were performed on unirradiated materials. ORNL has also successfully performed laser profilometry on biaxial creep specimens similar to the JOYO-1 specimens. ORNL had demonstrated the requisite expertise to handle some of the PIE work planned for JOYO- 1 in the related Pathfinder Program (References 8, 9 and 10). Attachment 4 to this enclosure summarizes capabilities of some ORNL facilities by individual hot cells or workstations that were part of the HFIR Pathfinder Program (Reference 4).

\section{Pacific Northwest National Laboratory (PNNL)}

Due to the involvement of PNNL in the manufacturing of the JOYO-1 biaxial creep specimens (see Enclosure 1); a limited assessment of PNNL's capability was achieved. Attachment 3 to this enclosure outlines PNNL's suggested PIE strategy for JOYO-1, which recommended splitting the PIE according to the individual operations between PNNL and ORNL. PNNL was identified as a good candidate for receiving the cask from Japan and fully capable of specimen sorting and repackaging for shipment to other US facilities. Splitting the PIE among different U.S. facilities would have allowed the NRPCT to obtain PIE data more effectively, provided shipment within the U.S. could have been performed efficiently. A visit by cognizant NRPCT staff to PNNL for a more detailed capability assessment was planned but not performed.

PNNL was identified as having the necessary expertise and capability to perform many of the PIE operations in the JOYO-1 test matrix. Flux wire and passive temperature monitor analyses are routinely performed at PNNL. Laser profilometry of biaxial creep specimens was also identified as a likely candidate for PIE at PNNL due to the involvement of PNNL in the manufacturing of the JOYO-1 biaxial creep specimens (see Enclosure 1). They performed welding and pre-irradiated measurements on the biaxial creep specimens therefore they are the best and most logical place to conduct PIE on these specimens. Thermal diffusivity measurements of both metallic and ceramic specimens are also routinely performed at PNNL. Due to the lower activation of the ceramic specimens in JOYO-1, all PIE operations on these specimens were possible at PNNL because they could be handled out-of-cell. 
Enclosure 9 to

MDO-723-0044/

B-MT(SRME)-52

Page 13

Mechanical property testing of refractory metal alloy and nickel-based superalloy specimens was identified as a capability that PNNL currently lacks. PNNL identified that ORNL was a good candidate to perform PIE operations on the tensile, fracture toughness, and compact tension specimens.

\section{Japan Atomic Energy Authority (JAEA)}

The NRPCT presented the PIE technical requirements for JOYO-1 irradiation test specimens to JAEA to discuss and clarify technical and mechanical testing needs (Reference 2). PIE testing was delineated into two sections by material type, ceramic or metallic. Tables 6 and 7 show the feasibility for PIE at JAEA for the metal and ceramic specimens, respectively.

Table 6: PIE requirements and JAEA capabilities for metallic specimens of the JOYO-1 irradiation test campaign.

\begin{tabular}{|c|c|c|c|c|}
\hline $\begin{array}{c}\text { Specimen } \\
\text { Type }\end{array}$ & Type of Exam & Currently Capable & $\begin{array}{l}\text { Likely with } \\
\text { Upgrades }\end{array}$ & Not Likely \\
\hline Biaxial Creep & $\begin{array}{c}\text { Diameter } \\
\text { measurement } \\
\text { using laser } \\
\text { profilometry } \\
\end{array}$ & $X$ (Cell \#4) & & \\
\hline Density disc & $\begin{array}{c}\text { Archimedes-type } \\
\text { density }\end{array}$ & $X$ (Cell \#4) & & \\
\hline $\begin{array}{l}\text { Thermal } \\
\text { diffusivity disc }\end{array}$ & Laser flash & & & $\begin{array}{l}X \text { (Instrument } \\
\text { is out of cell) }\end{array}$ \\
\hline Tensile & Tensile test & & $X($ Cell \#3) & \\
\hline Tensile & $\begin{array}{l}\text { Electrical } \\
\text { resistivity }\end{array}$ & & $\begin{array}{l}\mathbf{X} \text { (No equipment } \\
\text { or expertise but } \\
\text { relatively easy) }\end{array}$ & \\
\hline Broken tensile & Fractography & $\begin{array}{l}X \text { (Metallographic } \\
\text { and Microstructural } \\
\text { cells) }\end{array}$ & & \\
\hline $\begin{array}{l}\text { Fracture } \\
\text { Toughness }\end{array}$ & 3-Point bend & & & $\begin{array}{c}\mathbf{X} \text { (Fixture } \\
\text { exchange and } \\
\text { clip gage } \\
\text { attachment } \\
\text { difficulties) }\end{array}$ \\
\hline $\begin{array}{l}\text { Compact } \\
\text { Tension }\end{array}$ & Tensile test & & & $\begin{array}{c}\bar{X} \text { (Fixture } \\
\text { exchange and } \\
\text { clip gage or } \\
\text { EPD contact } \\
\text { attachment } \\
\text { very difficult) }\end{array}$ \\
\hline
\end{tabular}




\section{Metallic specimens}

JAEA has the existing capability to measure diametral strain for biaxial creep specimens. Quoted accuracies of $\pm 3 \mu \mathrm{m}$ is sufficient for characterization of expected strain levels. However, specimen throughput was an issue due to high activation levels of the tantalum alloys. It was originally thought that a minimum time frame of 6 to 9 months would be needed after the end of irradiation before testing could commence. However, lower fluence levels of the NRPCT tantalum bearing specimens (due to placement in the lower flux compartments in the rigs), predict lower specimen activation levels. This is more in line with JAEA's hot cell isotope-specific activity limits, somewhat alleviating specimen throughput concerns. More detailed calculations of specimen activation were ongoing at JAEA and specimen throughput discussions were delayed pending the activation results.

Density measurements of metallic discs are also within JAEA's existing capability, with the exception of an unresolved issue regarding use of an immersion liquid other than water. JAEA only uses water and NRPCT preferred to use a higher density liquid to improve the accuracy of the Archimedes measurement. JAEA requested NRPCT to provide standards of pure materials so that they could provide an accuracy estimate for their measurement equipment that was to be used for NRPCT materials. These standards were not provided once the Prometheus Project was restructured.

PIE of NRPCT metallic tensile specimens would require installation of a new machine in Hot Cell \#3. Guidance was requested by JAEA to help set up a new tensile machine with high vacuum and high temperature capability. Expertise to conduct tensile testing and data analysis currently exists at JAEA and if used, was not expected to be problematical.

An electrical resistivity measurement on intact tensile specimens is not an existing JAEA capability, although both JAEA and NRPCT considered this to be a realistic upgrade to JAEA's facility. Fractography of broken tensile specimens is an existing capability but could be enhanced with improved optical equipment.

Fracture toughness and compact tension testing of NRPCT metallic specimens does not appear to be realistic for PIE operations at JAEA. High temperature extensometry required on very small specimens requires considerable expertise, training and practice. This would likely take too long given NRPCT's schedular requirements. Additionally, installing the required equipment upgrades to the proposed in-cell tensile machine would be difficult.

\section{Ceramic Specimens}

Tensile testing of ceramic specimens was discussed with the JAEA staff. Although they had extensive knowledge of metallic tensile tests, they did not have experience working with $\mathrm{SiC}$. Unlike historical metallic tensile tests performed at JAEA, ceramics require an inert, high-temperature atmosphere using an edge-loaded fixture. In addition, this test requires use of high temperature extensometers to ensure proper sample alignment. This test should be completed-out-of cell and could have been done by JAEA with proper upgrades, including training, new instrumentation and fixtures. Following tensile tests, examination of broken specimens would require use of an optical microscope. At the time of project restructuring, JAEA had low magnification capabilities (2x to 4x). Upgrades, such as a SEM, would have been required to obtain the higher magnification $(\sim 500 \mathrm{x})$
needed for the analysis.

$\mathrm{BeO}$ compression testing necessitates different fixtures, but requires the same instrumentation as the ceramic tensile test. JAEA is familiar with the equipment, although they do not have required fixtures, 
equipment, or experience with this particular test. In addition, further investigation was necessary to resolve BeO handling capabilities at JAEA.

Table 7: PIE requirements and JAEA capabilities for ceramic specimens of the JOYO-1 irradlation test campaign

\begin{tabular}{|c|c|c|c|c|}
\hline $\begin{array}{l}\text { Specimen } \\
\text { Type }\end{array}$ & Type of Exam & $\begin{array}{c}\text { Currently } \\
\text { Capable }\end{array}$ & $\begin{array}{l}\text { Likely with } \\
\text { Upgrades }\end{array}$ & Not Likely \\
\hline Tensile & Tensile Machine & & $X$ (out of cell) & $\mathbf{X}$ (in cell) \\
\hline Broken Tensile & $\begin{array}{l}\text { Fractography } \\
\text { (optical) }\end{array}$ & $\begin{array}{l}X \text { (low } \\
\text { magnification } \times 2 \text { - } \\
4, \text { Cell \#4) }\end{array}$ & $\begin{array}{l}X \text { (high } \\
\text { magnification } \sim \times 500 \\
\text { SEM or another } \\
\text { apparatus) }\end{array}$ & \\
\hline $\begin{array}{l}\text { Bend Stress } \\
\text { Relaxation } \\
\text { (BSR) }\end{array}$ & $\begin{array}{l}\text { Optical } \\
\text { Microscopy } \\
\text { (Digital Camera) }\end{array}$ & & $\begin{array}{l}X \text { (No expertise, } \\
\text { although easier to } \\
\text { maneuver in a glove } \\
\text { box) }\end{array}$ & \\
\hline $\begin{array}{l}\text { Density Disc } \\
(\mathrm{BeO})\end{array}$ & $\begin{array}{l}\text { Archimedes } \\
\text { Density }\end{array}$ & $X($ Cell \#4) & & \\
\hline $\begin{array}{l}\text { Density Disc } \\
\text { (SiC) }\end{array}$ & $\begin{array}{l}\text { Gradient Column } \\
\text { Method }\end{array}$ & & $\mathbf{x}$ & \\
\hline $\begin{array}{l}\text { Thermal } \\
\text { Diffusivity Disc }\end{array}$ & $\begin{array}{l}\text { Laser Flash } \\
\text { Method }\end{array}$ & & $\begin{array}{l}X \text { (Instrument is out } \\
\text { of cell but in a } \\
\text { controlled area) }\end{array}$ & $\begin{array}{l}\mathbf{X} \text { (Helium } \\
\text { Atmosphere } \\
\text { Control) } \\
\end{array}$ \\
\hline $\begin{array}{l}\text { Compression } \\
\text { Testing (BeO) }\end{array}$ & Tensile Machine & & $\mathbf{X}$ & \\
\hline $\begin{array}{l}\text { Temperature } \\
\text { Monitors }\end{array}$ & $\begin{array}{l}\text { Electrical } \\
\text { Resistivity }\end{array}$ & & $\bar{x}$ & $\begin{array}{l}\mathbf{X} \text { (May have } \\
\text { issues using } \\
\text { Hydrofluoric } \\
\text { acid) }\end{array}$ \\
\hline
\end{tabular}

The bend stress relaxation (BSR) specimens and holder assemblies consist of eight intricate pieces. Due to the nature of the specimen, it was determined that disassembly must be completed in a glove box. These delicate specimens require optical microscopy to establish the post-irradiation angle of curvature. JAEA had no expertise in this test. A digital camera could have been easily purchased to upgrade the facility, but it may have been difficult to provide the training required for BSR disassembly.

The density of BeO is measured using the Archimedes method. JAEA had the capabilities for this test in their Mechanical Cell (identified as cell \#4). Although they had the capability, BeO handling requirements needed further investigation. The gradient column method should be used to determine the density of SiC. JAEA did not have this capability or training; however these upgrades were possible for a glove box application.

JAEA had the equipment and expertise to determine thermal diffusivity of ceramic materials using the laser flash method. Their machine could pull a vacuum, although it did not have environmental controls. The NRPCT anticipated conducting this particular measurement in helium, a standard NRPCT procedure. The JAEA PIE team indicated that this upgrade was not possible, therefore indicating that this test could not be conducted at JAEA.

For PIE of the temperature monitors, training and technical capability was easily attainable, but the test required use of hydrofluoric acid (HF), which JAEA did not want to use. Further investigation 
indicated that there was no viable substitute for HF; therefore, it was uncertain that this test could be performed at JAEA.

\section{Nippon Nuclear Fuel Development Company (NFD)}

NFD is involved in the PIE of fuel material systems. Although the NRPCT was not actively pursuing PIE of JOYO-1 specimens at NFD, while the NRPCT was in Japan their hot cells were evaluated.

The following is a list of applicable PIE instrumentation available at NFD.

- Metallography

- Advanced characterization instrumentation, including a field emission SEM (scanning electron microscope), a field emission TEM (transmission electron microscope) with a focused ion beam (FIB) system for sample preparation, an electron microprobe, and an $\mathrm{x}$-ray diffractometer, a quadrupole mass spectrometer.

- Mechanical testing including tensile, fatigue, and microhardness

- Tensile test system with testing capability to $1300^{\circ} \mathrm{C}$ in air. There are plans to add the capability for testing in argon or other inert gas environment. The heating rate is $50^{\circ} \mathrm{C} / \mathrm{min}$ and the tensile specimen size is $60 \mathrm{~mm}$ long $\dot{\times} 15 \mathrm{~mm}$ wide $\times 3 \mathrm{~mm}$ thick. The same mechanical test equipment is used for CT samples with a maximum furnace temperature of $400^{\circ} \mathrm{C}$.

- DSC (differential scanning calorimetry) - Maximum temperature is $1400^{\circ} \mathrm{C}$. This instrument is usually used for heat capacity measurements.

- Laser flash thermal diffusivity instrument. Samples are measured in vacuum ( $1 \times 10^{-5}$ Torr) at a maximum temperature of $1750^{\circ} \mathrm{C}$.

\section{Conclusions}

Generation of test data from the JOYO-1 specimens would have been complex. JOYO-1 was intended to screen candidate materials and to pave the way for subsequent irradiation tests that would have been needed to provide validated property data to the reactor design bases. Shipping, waste, facility logistical and testing capabilities were significant issues that had not been resolved at the time of project restructuring. Post irradiation examination requirements were also significant and it was not clear which facilities would be utilized. The large number of subsized specimens, multiple types of specimen and materials, and strict testing conditions (e.g., high temperature and high vacuum) necessitated that careful assessments be made of the candidate facilities. The PIE campaign was envisioned to be conducted in at least three phases such that high priority test data would have been obtained in the most rapid and cost-effective manner to provide input to reactor design studies. The leading candidate facilities were ORNL, PNNL, and JAEA. JAEA did not have the expertise or existing capability to perform all PIE operations, but PIE at JAEA would have avoided shipping issues prior to PIE. PNNL had substantial capability and expertise except for mechanical testing of the metallic materials. ORNL also had substantial capability and expertise and likely could have tested all specimens and materials. NRPCT's need to rapidly obtain data may have necessitated use of multiple testing facilities, but because cost information had not been obtained, it was not possible to lay out a detailed plan for PIE. 
Enclosure 9 to

MDO-723-0044 /

B-MT(SRME)-52

Page 17

\section{References}

1. MDO-723-0036 / B-MT(SRME)-37, "Space Nuclear Propulsion Program (SNPP) - Overview of Information Related to and Progress in Addressing Issues Associated With Domestic and International Shipping of Irradiated Structural Materials and Handling Associated Waste, for NR Information (U)," Issued January, 2006.

2. SM-7234-0010, "Trip Report to JNC," Issued November 22, 2005.

3. B-MT(SRME)-39 / MDO-723-0058, "Recommended Stress and Strain Levels for Biaxial Creep Specimens in the JOYO-1 Test Matrix," Issued November 18, 2005.

4. MDO-723-0011 / B-MT(SRME)-53, "Refractory Metal Irradiation Testing at ORNL," To Be Issued.

5. Y. Kim, A. Zangvil, J.S. Goela and R.L. Taylor, "Microstructure Comparison of Transparent and Opaque CVD SiC", J. Am. Ceram. Soc., Volume 78, 1995, pp 1571.

6. R. J. Price, "Annealing Behavior of Neutron-Irradiated Silicon Carbide Temperature Monitors," Journal of Nuclear Technology Volume 16, Dec. 1972.

7. L.L. Snead, A.M. Williams, and A.L. Qualls, "Revisiting the Use of SiC as a Post Irradiation Temperature Monitor," The Effects of Radiation on Material: $21^{\text {st }}$ Int. Symposium, ASTM STP 1447, M.L. Grossbeck, T. R. Allen, R.G. Lott, and A.S. Kumar, Eds., ASTM International, West Conshoshocken, PA, 2004.

8. B-MT(SRME)-19, “Trip to Oak Ridge National Laboratory to discuss the possibility of fabrication, mechanical testing, and irradiation of specimens for the Space Reactor initiative", Issued March 3, 2005.

9. SM-7234-008, Trip Report, Issued October 17, 2005.

10. B-MT(SRME)-42, "Trip to Oak Ridge National Laboratory (ORNL) to Evaluate Post Irradiation Examination (PIE) Equipment and to Discuss initial PIE Data from HFIR-Irradiated Materials", Issued October 19, 2005. 
Enclosure 9 to MDO-723-0044 I

B-MT(SRME)-52

Page 18

\section{Attachments}

1. Draft IV to ORNL for PIE

2. Draft IV to PNNL for PIE

3. PNNL White Paper on PIE Strategy

4. ORNL Hot Cell Capabilities

Enclosure 9 to MDO-723-0044 / B-MT(SRME)-52 
Attachment 1 to

Enclosure 9 to MDO-723-0044I

B-MT(SRME)-52

Page 1

Attachment 1

Draft IV to ORNL for PIE Bid

Enclosure 9 to MDO-723-0044/B-MT(SRME)-52 


\section{DRAFT}

Attachment 1 to

Enclosure 9 to

MDO-723-0044/

B-MT(SRME)-52

Page 2

KAPL, Inc.

PO Box 1072

Schenectady, NY 12301

Telephone: 518-395-4000 Facsimile: 518-395-7804

INFORMATION TO VENDOR

(Space Power Program)

Number ORNL-SPP-05-00XX

Issue

Date Page 1 of 10

To: Oak Ridge National Laboratory

Attention: Bruce B. Bevard

\begin{tabular}{|l|l|}
\hline $\begin{array}{l}\text { Purchase Order No. } \\
\text { EA0100007a }\end{array}$ & $\begin{array}{l}\text { Seller Reference Document } \\
\text { No. }\end{array}$ \\
\hline Charge Number: N/A \\
\hline $\begin{array}{l}\text { Subject: } \\
\text { Post-Irradiation Examination of Materials Irradiated } \\
\text { in JOYO-1 Campaign }\end{array}$ \\
\hline
\end{tabular}

Topics covered in the following pages:

A. Post-Irradiation Examination of Materials Irradiated in JOYO-1 Campaign.

page 2

\begin{tabular}{ll|ll}
\hline Cognizant Engineer & Date & $\begin{array}{l}\text { Cognizant Contract Administrator } \\
\text { Sign Name }\end{array}$ & Print Name Robert M. Dalton \\
$\begin{array}{l}\text { Sign Name } \\
\text { Bldg. A2 Room. 209 }\end{array}$ & Print Name Steven Z. Hayden & & \\
\hline
\end{tabular}

\section{Notice - Disclaimer}

KAPL does not have the authority to modify the prime contract between the Seller and the Government, therefore, if the action contained herein is considered by the Seller to require a change in any aspect or provision (Price, Contract Term, Clauses, et al.) of the Seller's prime contract, the Seller shall not proceed with the action contained herein but should promptly, and in any event within 20 days of receipt of this document, notify the Seller's Cognizant Government Contracting Officer of the facts and the reasons for considering that a contract change is required, and concurrently notify KAPL.

NOTICE TO VENDOR: This information is considered by KAPL to be within the order requirements and is to be incorporated into the work without modification to any provision of the order. If you consider this information does modify any provision of the order, take no action to incorporate this information but advise KAPL of your position within twenty (20) days after receipt of this IV. In the absence of such advice within said time it is agreed that the information will be incorporated into the work without modification to any provision of the order. Sign one copy below as evidence of receipt and acceptance of this information.

No acknowledgment required.

Acknowledged by:

Date: 


\section{DRAFT}

Attachment 1 to

Enclosure 9 to

MD0-723-0044I

B-MT(SRME)-52

Page 3

Work is not vet authorized. KAPL requests costs and schedule by September 23,2005 for the following work.

\section{Post-Irradiation Examination of Materials Irradiated in JOYO-1 Campaign}

This work involves evaluating the post-irradiation examination (PIE) of specimens irradiated in the JOYO-1 test campaign in the Experimental Fast Reactor JOYO located in O-arai, Japan [operated by the Japanese Nuclear Cycle Research institute (JNC)]. Tables 1 and 2 provide specimen counts and weights per material, fluence range experienced, and desired PIE operation and testing conditions. The specimen counts are broken into three phases for PIE (See Tables 1 and 2), which are:

Phase 1: $\quad$ A small subset of samples irradiated at intermediate fluences to ensure all testing equipment meets desired testing requirements and to guide testing parameters of higher fluence specimens.

Phase 2: $\quad$ The majority of samples irradiated at high and intermediate fluences, with some testing of low fluence specimens.

Phase 3: $\quad$ The remaining samples at lower fluences and replicate samples. This phase will also include optical and analytical electron microscopy examinations of specimens from Phases 1 and 2.

Tables 3 and 4 provide supplemental information on the specimen dimensions and chemical compositions.

For this IV, ORNL shall:

1. Provide a cafeteria-style cost estimate for PIE based upon: specimen type, material composition and testing conditions. ORNL can submit an estimate on performing all PIE of the JOYO specimens or certain portions. ORNL shall determine the most cost effective method to perform the PIE phases. Estimates shall also provide costs for disposal of waste generated from any PIE performed. It should be noted that the specimens may be shipped in the stainless steel (SS) capsules in which they were irradiated. ORNL shall include the opening of capsules in both the cost estimate and waste disposal.

2. Provide current testing capabilities (or possible capabilities with upgrades), handling capacity, and throughput. Also, a list of cask receiving capabilities shall be provided.

3. Supply a schedule for PIE assuming PIE to begin the end of March 2007.

4. Provide biographies on ORNL personnel having relevant experience working with the materials listed in Tables 1 and 2.

Deliverables shall include:

An estimate (due by September 23,2005 ) that contains, at a minimum, the following:

1. Costs broken down by specimen type, material composition, and testing conditions. ORNL shall determine the most cost effective method to perform the PIE Phases.

2. Cost associated with opening SS capsules.

3. Cost to measure electrical resistivity on a per specimen basis for a to-be-determined (TBD) subset of tensile specimens.

4. Cost on a per specimen type basis to perform dimensional inspection on a TBD subset of specimens (i.e. tensile, fracture toughness, etc.).

5. Cost on a per specimen basis for performing optical and analytic electron microscopy testing techniques (i.e. SEM, TEM (including punching discs from grips of tensile specimens and foil prep), and Auger) on a TBD subset of fracture toughness and tensile specimens. ORNL should also provide estimates of the time needed for cooling of each metal alloy prior to performing TEM analysis.

6. Feasibility of installing a focused ion beam (FIB) in a hot cell. 


\section{DRAFT}

Attachment 1 to

Enclosure 9 to MDO-723-0044/

B-MT(SRME)-52

Page 4

7. Cost on a per specimen basis to perform examination on a subset of $\mathrm{Ni}, \mathrm{Ti}$, and Fe flux wires (Figure 1) per ASTM E263-05, E264-02, and E526-97.

8. Cost on a per specimen basis to perform examination on a subset of $\mathrm{SiC}$ and $\mathrm{ZrC}$ temperature monitors (Figure 2) using electrical resistivity method.

9. Waste disposal costs associated with materials tested (including SS capsules and Mo and W/grafoil specimen holders).

10. Current hot cell and PIE capabilities (or possible capabilities with upgrades), handling capacity, throughput and cask receiving capabilities.

11. Schedules for testing assuming PIE to start end of March 2007.

12. Quality Assurance requirements that will be associated with this work effort.

13. Radiological controls and environmental, health and safety controls to mitigate associated risk.

14. Brief biographies on laboratory personnel having relevant experience working with the materials listed in Tables 1 and 2.

The ORNL technical contact for this task is Steve Zinkle (865-576-7220). The Naval Reactors Prime Contractor Team (NRPCT) technical contacts for this task are Jessica Bump (412-476-3912) at Bettis and Ryan Nelson (518-395-6652) at KAPL. 
Table 1. Metallic specimens in JOYO-1 test matrix.

\begin{tabular}{|c|c|c|c|c|c|c|c|}
\hline $\begin{array}{c}\text { Specimen } \\
\text { Type }\end{array}$ & $\begin{array}{c}\text { PIE } \\
\text { Operation }\end{array}$ & $\begin{array}{c}\text { Test } \\
\text { Conditions }\end{array}$ & $\begin{array}{c}\text { Material } \\
\text { (Mass) }\end{array}$ & $\begin{array}{c}\text { Fast } \\
\text { Fluence } \\
\text { Range }\end{array}$ & & $\begin{array}{c}\text { Nominal \# } \\
\text { of } \\
\text { Specimens }\end{array}$ & \\
\hline & & & & $\begin{array}{l}(1 \mathrm{E} 20 \\
\left.\mathrm{n} / \mathrm{cm}^{2}\right) \\
\end{array}$ & Phase 1 & Phase 2 & Phase 3 \\
\hline \multirow{7}{*}{ Biaxial Creep } & \multirow{7}{*}{$\begin{array}{c}\text { Laser } \\
\text { Profilometry } \\
\text { (accuracy } \leq+/-3 \\
\mu \mathrm{m} \text { ) } \\
\text { A minimum of } 180 \\
\text { diameter } \\
\text { measurements } \\
\text { (every } 1^{\circ} \text { ) at each } \\
\text { of } 5 \text { axial locations }\end{array}$} & \multirow{7}{*}{ Room Temp } & $\begin{array}{l}\text { FS-85 } \\
(4.3 \mathrm{~g}) \\
\end{array}$ & $47-150$ & 7 & 17 & 0 \\
\hline & & & $\begin{array}{c}\text { ASTAR-811C } \\
(7.649 \mathrm{~g})\end{array}$ & $30-150$ & 8 & 14 & 0 \\
\hline & & & $\begin{array}{c}\mathrm{Mo}-47.5 \mathrm{Re} \\
(6.275 \mathrm{~g})\end{array}$ & $47-170$ & 7 & 15 & 0 \\
\hline & & & $\begin{array}{c}A 617 \\
(3.829 \mathrm{~g})\end{array}$ & $15-72$ & 2 & 7 & 0 \\
\hline & & & $\begin{array}{c}\text { PE16 } \\
(3.664 \mathrm{~g})\end{array}$ & $18-67$ & 2 & 7 & 0 \\
\hline & & & $\begin{array}{c}\mathrm{H} 230 \\
(4.108 \mathrm{~g})\end{array}$ & $20-65$ & 2 & 7 & 0 \\
\hline & & & Sum & & 28 & 67 & 0 \\
\hline \multirow{10}{*}{ Density Disc } & \multirow{10}{*}{$\begin{array}{c}\text { Density } \\
\text { Measurement } \\
\text { (Archimedes } \\
\text { Type, }+/-0.1 \% \\
\text { accuracy) }\end{array}$} & \multirow{10}{*}{ Room Temp } & $\begin{array}{c}\text { FS-85 } \\
(1.950 \mathrm{~g})\end{array}$ & 28-144 & 4 & 9 & 0 \\
\hline & & & $\begin{array}{c}\text { ASTAR-811C } \\
(3.073 \mathrm{~g})\end{array}$ & $21-144$ & 5 & 6 & 0 \\
\hline & & & $\begin{array}{c}\text { Mo-47.5Re } \\
(2.521 \mathrm{~g})\end{array}$ & $28-168$ & 5 & 18 & 0 \\
\hline & & & $\begin{array}{c}\operatorname{Re} \\
(3.871 \mathrm{~g})\end{array}$ & $31-169$ & 6 & 12 & 0 \\
\hline & & & $\begin{array}{c}\mathrm{W} \\
(3.560 \mathrm{~g})\end{array}$ & $27-170$ & 6 & 10 & 0 \\
\hline & & & $\begin{array}{l}\text { W-25Re } \\
(3.625 \mathrm{~g})\end{array}$ & $28-150$ & 6 & 11 & 0 \\
\hline & & & $\begin{array}{c}A 617 \\
(1.583 \mathrm{~g}) \\
\end{array}$ & $13-151$ & 9 & 11 & 7 \\
\hline & & & $\begin{array}{c}\mathrm{H} 230 \\
(1.650 \mathrm{~g})\end{array}$ & $10-150$ & 6 & 14 & 8 \\
\hline & & & $\begin{array}{c}\text { PE16 } \\
(1.472 \mathrm{~g})\end{array}$ & $11-149$ & 6 & 15 & 8 \\
\hline & & & Sum & & 53 & 106 & 23 \\
\hline \multirow{10}{*}{$\begin{array}{l}\text { Thermal } \\
\text { Diffusivity }\end{array}$} & \multirow{10}{*}{ (ASTM E1461-92) } & \multirow{6}{*}{$\begin{array}{c}\text { Room Temp } \\
\text { to } \\
1350 \mathrm{~K}\end{array}$} & $\begin{array}{c}\text { FS-85 } \\
(1.950 \mathrm{~g})\end{array}$ & $28-134$ & 2 & 3 & 0 \\
\hline & & & $\begin{array}{c}\text { ASTAR-811C } \\
(3.073 \mathrm{~g})\end{array}$ & $21-63$ & 3 & 3 & 0 \\
\hline & & & $\begin{array}{c}\text { Mo-47.5Re } \\
(2.521 \mathrm{~g}) \\
\end{array}$ & 28-166 & 3 & 6 & 0 \\
\hline & & & $\begin{array}{c}\mathrm{Re} \\
(3.871 \mathrm{~g})\end{array}$ & $21-72$ & 2 & 2 & 0 \\
\hline & & & $\begin{array}{c}\mathrm{W} \\
(3.560 \mathrm{~g})\end{array}$ & $27-165$ & 2 & 3 & 0 \\
\hline & & & $\begin{array}{l}\text { W-25Re } \\
(3.625 \mathrm{~g})\end{array}$ & $27-83$ & 1 & 3 & 0 \\
\hline & & \multirow{4}{*}{$\begin{array}{c}\text { Room Temp } \\
\text { to } \\
1050 \mathrm{~K}\end{array}$} & $\begin{array}{c}\mathrm{A} 617 \\
(1.538 \mathrm{~g})\end{array}$ & $12-87$ & 5 & 8 & 0 \\
\hline & & & $\begin{array}{c}\mathrm{H} 230 \\
(1.650 \mathrm{~g})\end{array}$ & 13-53 & 5 & 8 & 0 \\
\hline & & & $\begin{array}{c}\text { PE16 } \\
(1.472 \mathrm{~g})\end{array}$ & $12-45$ & 5 & 7 & 0 \\
\hline & & & Sum & & 28 & 43 & 0 \\
\hline
\end{tabular}


DRAFT

Attachment 1 to

Enclosure 9 to

MDO-723-0044/

Table 1 cont'd. Metallic specimens JOYO-1 test matrix.

\begin{tabular}{|c|c|c|c|c|c|c|c|}
\hline \multirow[t]{2}{*}{$\begin{array}{c}\text { Specimen } \\
\text { Type }\end{array}$} & \multirow[t]{2}{*}{$\begin{array}{c}\text { PIE } \\
\text { Operation }\end{array}$} & \multirow[t]{2}{*}{$\begin{array}{c}\text { Test } \\
\text { Conditions }\end{array}$} & \multirow[t]{2}{*}{$\begin{array}{c}\text { Material } \\
\text { (Mass) }\end{array}$} & \multirow{2}{*}{$\begin{array}{c}\begin{array}{c}\text { Fast } \\
\text { Fluence } \\
\text { Range }\end{array} \\
(1 \mathrm{E20} \\
\left.\mathrm{n} / \mathrm{cm}^{2}\right) \\
\end{array}$} & & \multirow{2}{*}{$\begin{array}{c}\text { Nominal \# } \\
\text { of } \\
\text { Specimens } \\
\text { Phase } 2\end{array}$} & \multirow{2}{*}{\begin{tabular}{|r|} 
Page \\
Phase 3 \\
\end{tabular}} \\
\hline & & & & & Phase 1 & & \\
\hline \multirow{10}{*}{ Tensile } & \multirow{10}{*}{$\begin{array}{c}\text { Tensile } \\
\text { Testing } \\
\text { (ASTM E8-04) } \\
\& \\
\text { Electrical } \\
\text { Resistivity } \\
\text { (ASTM B 193-02) }\end{array}$} & \multirow{6}{*}{$\begin{array}{c}\text { Room Temp } \\
\text { to } \\
1450 \mathrm{~K} \\
\& \mathrm{HV} \\
\left(<10^{-6} \text { torr }\right)\end{array}$} & $\begin{array}{c}F S-85 \\
(0.619 g)\end{array}$ & $31-128$ & 12 & 29 & 12 \\
\hline & & & $\begin{array}{c}\text { ASTAR-81 1C } \\
(0.975 \mathrm{~g})\end{array}$ & 24-147 & 16 & 35 & 16 \\
\hline & & & $\begin{array}{c}\mathrm{Mo-47.5 \textrm {Re }} \\
(0.800 \mathrm{~g})\end{array}$ & $32-172$ & 0 & 28 & 11 \\
\hline & & & $\begin{array}{c}\mathbf{R e} \\
(1.229 \mathrm{~g}) \\
\end{array}$ & 24-172 & 8 & 16 & 0 \\
\hline & & & $\begin{array}{c}W \\
(1.130 \mathrm{~g})\end{array}$ & $29-172$ & 8 & 15 & 0 \\
\hline & & & $\begin{array}{l}\text { W-25Re } \\
(1.150 \mathrm{~g})\end{array}$ & $32-172$ & 8 & 16 & 0 \\
\hline & & \multirow{4}{*}{$\begin{array}{l}\text { Room Temp to } \\
1050 \mathrm{~K} \\
\text { \& UHP gas or } \\
\text { HV (<10 tor) }\end{array}$} & $\begin{array}{c}\mathrm{A} 617 \\
(0.488 \mathrm{~g})\end{array}$ & $12-158$ & 16 & 33 & 16 \\
\hline & & & $\begin{array}{c}\mathrm{H} 230 \\
(0.524 \mathrm{~g})\end{array}$ & $12-158$ & 15 & 37 & 2 \\
\hline & & & $\begin{array}{c}\text { PE16 } \\
(0.467 \mathrm{~g}) \\
\end{array}$ & $12-158$ & 15 & 37 & 9 \\
\hline & & & Sum & & 98 & 246 & 66 \\
\hline \multirow{8}{*}{$\begin{array}{l}\text { Fracture } \\
\text { Toughness }\end{array}$} & \multirow{8}{*}{ (ASTM E1820-01) } & \multirow{4}{*}{$\begin{array}{c}\text { Room Temp } \\
\text { to } \\
1450 \mathrm{~K}\end{array}$} & $\begin{array}{c}\text { Mo-47.5Re } \\
(3.091 \mathrm{~g})\end{array}$ & $39-173$ & 32 & 16 & 0 \\
\hline & & & $\begin{array}{c}\mathrm{Re} \\
(4.747 \mathrm{~g})\end{array}$ & $39-173$ & 2 & 6 & 0 \\
\hline & & & $\begin{array}{c}W \\
(4.365 \mathrm{~g}) \\
\end{array}$ & 39.173 & 2 & 6 & 0 \\
\hline & & & $\begin{array}{l}\text { W-25Re } \\
(4.444 \mathrm{~g})\end{array}$ & $39-173$ & 2 & 6 & 0 \\
\hline & & \multirow{4}{*}{$\begin{array}{c}\text { Room Temp } \\
\text { to }\end{array}$} & $\begin{array}{c}A 617 \\
(1.886 \mathrm{~g}) \\
\end{array}$ & $15-172$ & 8 & 36 & 10 \\
\hline & & & $\begin{array}{c}\mathrm{H} 230 \\
(2.024 \mathrm{~g})\end{array}$ & $15-172$ & 7 & 29 & 18 \\
\hline & & & $\begin{array}{c}\text { PE16 } \\
(1.805 \mathrm{~g})\end{array}$ & $15-172$ & 6 & 31 & 17 \\
\hline & & & Sum & & 59 & 130 & 45 \\
\hline \multirow{5}{*}{ Compact } & \multirow{5}{*}{$\begin{array}{l}\text { Measure final bolt } \\
\text { load at Tirt } \\
\text { + final crack depth } \\
\text { due to irradiation } \\
\text { exposure at JOYO } \\
\text { from heat tint } \\
\text { optical analysis + } \\
\text { Option A* post- } \\
\text { irradiation creep } \\
\text { crack growh rate at } \\
\text { Tirr (EPD } \\
\text { measurement of } \\
\text { crack depth with } \\
\text { long term testing) } \\
\text { Option B: post- } \\
\text { irradiation fracture } \\
\text { toughriess (clip } \\
\text { gage to determine } \\
\text { displacement) }\end{array}$} & $\begin{array}{l}\text { Room Temp to } \\
1350 \mathrm{~K}\end{array}$ & $\begin{array}{c}\text { Mo-47.5Re } \\
(13.558 \mathrm{~g})\end{array}$ & $41-166$ & 0 & 0 & 16 \\
\hline & & \multirow{4}{*}{ Room Temp } & $\begin{array}{c}\text { A617 } \\
(8.273 \mathrm{~g})\end{array}$ & 23-87 & 0 & 0 & 8 \\
\hline & & & $\begin{array}{c}\mathrm{H} 230 \\
(8.877 \mathrm{~g})\end{array}$ & $34-77$ & 0 & 0 & 8 \\
\hline & & & $\begin{array}{c}\text { Pel6 } \\
(7.917 \mathrm{~g})\end{array}$ & $34-90$ & 0 & 0 & 8 \\
\hline & & & Sum & & 0 & 0 & 24 \\
\hline
\end{tabular}


Table 2. Ceramic specimens in JOYO-1 test matrix.

\begin{tabular}{|c|c|c|c|c|c|c|c|}
\hline \multirow[t]{2}{*}{$\begin{array}{c}\text { Specimen } \\
\text { Type }\end{array}$} & \multirow[t]{2}{*}{$\begin{array}{c}\text { PIE } \\
\text { Operation }\end{array}$} & \multirow[t]{2}{*}{$\begin{array}{c}\text { Test } \\
\text { Conditions }\end{array}$} & \multirow[t]{2}{*}{ Material } & \multirow{2}{*}{$\begin{array}{c}\text { Fast } \\
\text { Fluence } \\
\text { Range } \\
(1 \mathrm{E20} \\
\left.\mathbf{n} / \mathrm{cm}^{2}\right) \\
\end{array}$} & \multirow[b]{2}{*}{ Phase 1} & \multicolumn{2}{|l|}{$\begin{array}{c}\text { Nominal \# } \\
\text { of } \\
\text { Specimens }\end{array}$} \\
\hline & & & & & & Phase 2 & Phase 3 \\
\hline \multirow{3}{*}{ Tensile } & \multirow{3}{*}{$\begin{array}{c}\text { Tensile } \\
\text { Testing } \\
\text { Option A: } \\
\text { SiC (ASTM } \\
\text { C1366-04 \& } \\
\text { SiC/SiC (ASTM } \\
\text { C1359-96) } \\
\text { Option B: } \\
\text { SiC (ASTM } \\
\text { C1273-95a \& } \\
\text { SiC/SiC (ASTM } \\
\text { C1275-00) }\end{array}$} & \multirow{3}{*}{$\begin{array}{c}\text { Option A } \\
1050 \mathrm{~K} \text { and } 1350 \\
\mathrm{~K} \text { in } 1 \text { atm inert } \\
\text { gas } \\
\text { Option B } \\
\text { Room } \\
\text { Temperature }\end{array}$} & $\begin{array}{c}\mathrm{SiC} \\
(1.9 \mathrm{~g}) \\
\end{array}$ & $127-194$ & 0 & 8 & 4 \\
\hline & & & $\begin{array}{c}\mathrm{SiC} / \mathrm{SiC} \\
(1.9 \mathrm{~g})\end{array}$ & $42-196$ & 2 & 14 & 8 \\
\hline & & & Sum & & 2 & 22 & 12 \\
\hline \multirow{4}{*}{ Density Disc } & \multirow{4}{*}{$\begin{array}{c}\text { Density } \\
\text { Measurement } \\
\text { SiC \& SiC/SiC } \\
\text { (ASTM D1505- } \\
\text { 03) } \\
\text { BeO (ASTM } \\
\text { C373-88) }\end{array}$} & \multirow{4}{*}{ Room Temp } & $\begin{array}{c}\mathrm{SiC} \\
(1.178 \mathrm{~g}) \\
\end{array}$ & $34-193$ & 24 & 0 & 0 \\
\hline & & & $\begin{array}{l}\mathrm{SiC} / \mathrm{SiC} \\
(0.589 \mathrm{~g})\end{array}$ & $35-194$ & 24 & 0 & 0 \\
\hline & & & $\begin{array}{c}\mathrm{BeO} \\
(0.554 \mathrm{~g})\end{array}$ & $16-50$ & $\begin{array}{c}32 \text { dises } \\
16 \mathrm{cyl} .\end{array}$ & 0 & 0 \\
\hline & & & Sum & & 96 & 0 & 0 \\
\hline \multirow{4}{*}{ Diffusivity Disc } & \multirow{4}{*}{$\begin{array}{c}\text { Thermal } \\
\text { Diffusivity } \\
\text { Measurement } \\
\text { (ASTM E1461- } \\
01 \text { ) }\end{array}$} & \multirow{2}{*}{$\begin{array}{l}\text { Room Temp to } \\
1350 \mathrm{~K} \text { in } 1 \mathrm{~atm} \\
\text { inert gas }\end{array}$} & $\begin{array}{c}\mathrm{SiC} \\
(1.178 \mathrm{~g})\end{array}$ & $34-192$ & 0 & 24 & 0 \\
\hline & & & $\begin{array}{r}\mathrm{SiC} / \mathrm{SiC} \\
(0.589 \mathrm{~g}) \\
\end{array}$ & $35-193$ & 0 & 24 & 0 \\
\hline & & \multirow{2}{*}{$\begin{array}{l}\text { Room Temp to } \\
1250 \mathrm{~K} \text { in } 1 \text { atm } \\
\text { inert gas }\end{array}$} & $\begin{array}{c}\mathrm{BeO} \\
(0.554 \mathrm{~g})\end{array}$ & $16-47$ & 0 & 32 & 0 \\
\hline & & & Sum & & 0 & 80 & 0 \\
\hline $\begin{array}{c}\text { Compression } \\
\text { Cylinders }\end{array}$ & $\begin{array}{c}\text { Compressive } \\
\text { Strength Testing } \\
\text { (ASTM C773-88) }\end{array}$ & $\begin{array}{c}850 \mathrm{~K} \text { and } \\
1050 \mathrm{~K}\end{array}$ & $\begin{array}{c}\mathrm{BeO} \\
(0.303 \mathrm{~g})\end{array}$ & $18-44$ & 4 & 8 & 4 \\
\hline BSR Samples & $\begin{array}{c}\text { Optical } \\
\text { Microscopy }\end{array}$ & Room Temp & $\begin{array}{c}\mathrm{SiC} \\
(2.379 \mathrm{~g})\end{array}$ & $53-75$ & 4 & 8 & 4 \\
\hline
\end{tabular}




\section{DRAFT}

Attachment 1 to

Enclosure 9 to MDO-723-0044/

B-MT(SRME)-52

Page 8

Table 3. Description of metallic specimens in JOYO-1.

Holders for metallic specimens will be molybdenum.

Metallic Specimens:

\begin{tabular}{|c|c|c|c|c|}
\hline Specimen Type & Shape & Size $(\mathrm{mm})$ & $\begin{array}{l}\text { Volume } \\
\left(\mathrm{mm}^{3)}\right.\end{array}$ & $\begin{array}{l}\text { Total Number } \\
\text { of Specimens }\end{array}$ \\
\hline Biaxial creep & $\begin{array}{l}\text { Circular } \\
\text { cylinder, } \\
\text { pressurized } \\
\text { tube }\end{array}$ & $\begin{array}{c}6.25 \text { diameter } \\
\times 33 \text { long }\end{array}$ & 458 & 95 \\
\hline Tensile & $\begin{array}{l}\text { Rectangular, } \\
\text { dog bone } \\
\text { shaped, flat }\end{array}$ & $\begin{array}{c}5 \text { wide } \times 25.4 \\
\text { long } \times 0.75 \\
\text { thick }\end{array}$ & 59 & 410 \\
\hline Fracture toughness & $\begin{array}{l}\text { Single-edge } \\
\text { notched bars }\end{array}$ & $\begin{array}{l}3 \text { wide } \times 25.4 \\
\text { long } \times 3 \text { thick }\end{array}$ & 226 & 234 \\
\hline Thermal diffusivity & $\begin{array}{l}\text { Circular } \\
\text { cylinder disks }\end{array}$ & $\begin{array}{l}12.5 \text { diameter } \\
\times 1.5 \text { thick }\end{array}$ & 184 & 253 \\
\hline $\begin{array}{l}\text { Compact Tension (bolt } \\
\text { that will be included not } \\
\text { shown in figure) }\end{array}$ & $\begin{array}{c}\text { Notched } \\
\text { Circular Disk }\end{array}$ & $\begin{array}{l}15 \text { diameter } \mathrm{x} \\
5.6 \text { thick }\end{array}$ & 840 & 40 \\
\hline & & & & \\
\hline
\end{tabular}

Chemical compositions :

Metallic Specimens: ( $w t \%)$

FS-85

$(62 \mathrm{Nb}-27 \mathrm{Ta}-10 \mathrm{~W}-1 \mathrm{Zr})$

ASTAR-811C (90Ta-8W-1Re-0.7Hf-0.025C)

$\mathrm{Mo}-47 \mathrm{Re} \quad(52.5 \mathrm{Mo}-47.5 \mathrm{Re})$

$\mathrm{Re} \quad(100 \mathrm{Re})$

W (100W)

W-25Re (75W-25Re)

Nimonic PE-16 (42Ni-33Fe-18Cr-4Mo-1.2Ti-1.2Al-0.3Si-0.2Mn-0.1C)

Alloy $617 \quad(51 \mathrm{Ni}-22 \mathrm{Cr}-12.5 \mathrm{Co}-9 \mathrm{Mo}-3 \mathrm{Fe}-1 \mathrm{Si}-1 \mathrm{Mn}-0.6 \mathrm{Ti}-0.1 \mathrm{Al}-0.006 \mathrm{~B})$

Haynes $230 \quad(53 \mathrm{Ni}-22 \mathrm{Cr}-5 \mathrm{Co}-14 \mathrm{~W}-3 \mathrm{Fe}-2 \mathrm{Mo}-0.4 \mathrm{Si}-0.3 \mathrm{Al}-0.5 \mathrm{Mn}-0.1 \mathrm{C}-0.015 \mathrm{~B})$

(all nominal compositions in weight percent) 
Table 4. Description of ceramic specimens in JOYO-1

Holders for $\mathrm{SiC}$ capsules will be tungsten and grafoil; $\mathrm{BeO}$ will use molybdenum

Ceramic Specimens:

\begin{tabular}{|c|c|c|c|c|}
\hline Specimen Type & Shape & Size $(\mathrm{mm})$ & $\begin{array}{l}\text { Volume } \\
\left(\mathrm{mm}^{3}\right)\end{array}$ & $\begin{array}{c}\text { Total } \\
\text { Number of } \\
\text { Specimens }\end{array}$ \\
\hline Tensile & $\begin{array}{l}\text { Rectangular, } \\
\text { dog bone } \\
\text { shaped, flat }\end{array}$ & $\begin{array}{l}6 \text { wide } \times 41.3 \\
\text { long } \times 2.3 \text { thick }\end{array}$ & 372 & 36 \\
\hline $\begin{array}{l}\text { Composite Thermal } \\
\text { Conductivity }\end{array}$ & $\begin{array}{c}\text { Circular } \\
\text { cylinder disks }\end{array}$ & $\begin{array}{l}12.5 \text { diameter } \\
\times 1.5 \text { thick }\end{array}$ & 184 & 24 \\
\hline Thermal Conductivity & $\begin{array}{c}\text { Circular } \\
\text { cylinder disks }\end{array}$ & $\begin{array}{l}12.5 \text { diameter } \\
\times 3 \text { thick }\end{array}$ & 368 & 24 \\
\hline BeO Density Disk & $\begin{array}{c}\text { Circular } \\
\text { cylinder disks }\end{array}$ & $\begin{array}{c}12.5 \text { diameter } \\
\times 3 \text { thick }\end{array}$ & 368 & 32 \\
\hline BeO Compression & $\begin{array}{c}\text { Circular } \\
\text { cylinder disks }\end{array}$ & $\begin{array}{l}4 \text { diameter } x \\
8 \text { tall }\end{array}$ & 100 & 16 \\
\hline Ceramic Strip & $\begin{array}{l}\text { Thin } \\
\text { Rectangular } \\
\text { Strip }\end{array}$ & $\begin{array}{c}1 \text { height } x \\
40 \text { long } \times 0.05 \text {, } \\
0.1 \text {, or } 0.2 \\
\text { width }\end{array}$ & $\begin{array}{c}1.3 \\
\text { (specimen) } \\
625 \\
\text { (fixture) }\end{array}$ & 16 \\
\hline
\end{tabular}

Chemical compositions :

Ceramic Specimens:

$\mathrm{SiC}$ - chemical vapor deposited silicon carbide (monolithic)

$\mathrm{SiC} / \mathrm{SiC}$ - Woven Hi-Nicalon Type S (SiC) fiber in a chemical vapor infiltration (CVI) SiC matrix

BeO - Beryllia 


\section{DRAFT}

Attachment 1 to

Enclosure 9 to

MDO-723-0044/

B-MT(SRME)-52

Page 10

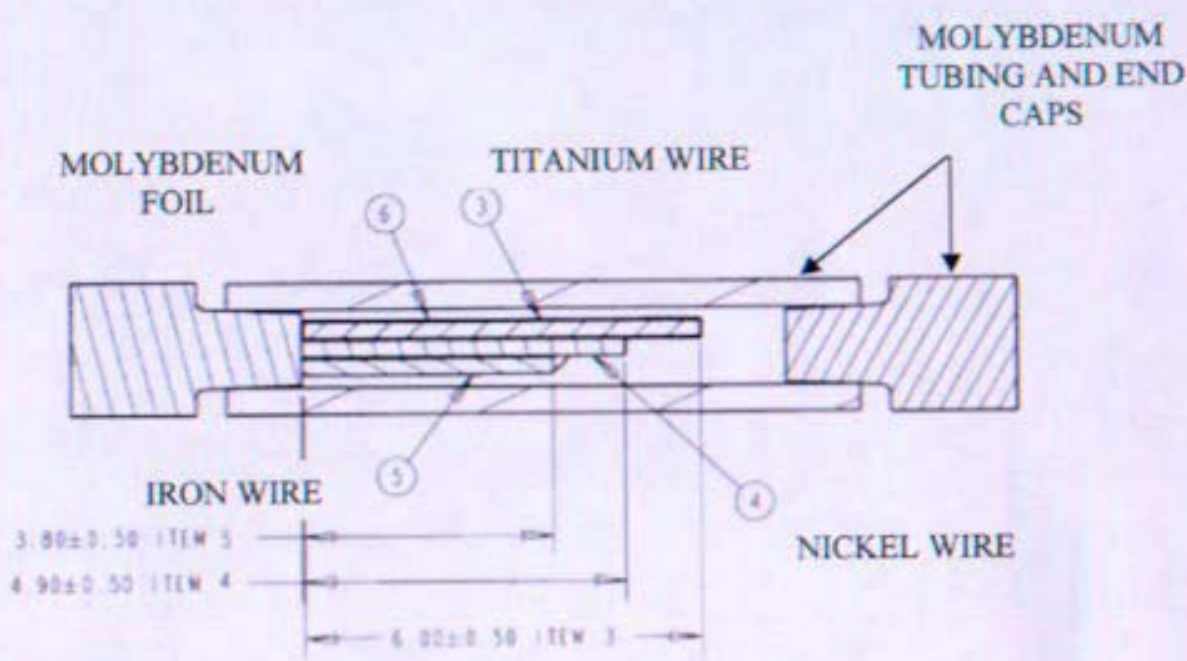

Figure 1. Nickel, titanium, and iron flux wires used in JOYO-1 campaign. Dimensions in mm. Flux wire diameters are $0.25 \mathrm{~mm}$. Total assembly is $2.03 \mathrm{~mm}$ O.D. $x 14.32 \mathrm{~mm}$ long.

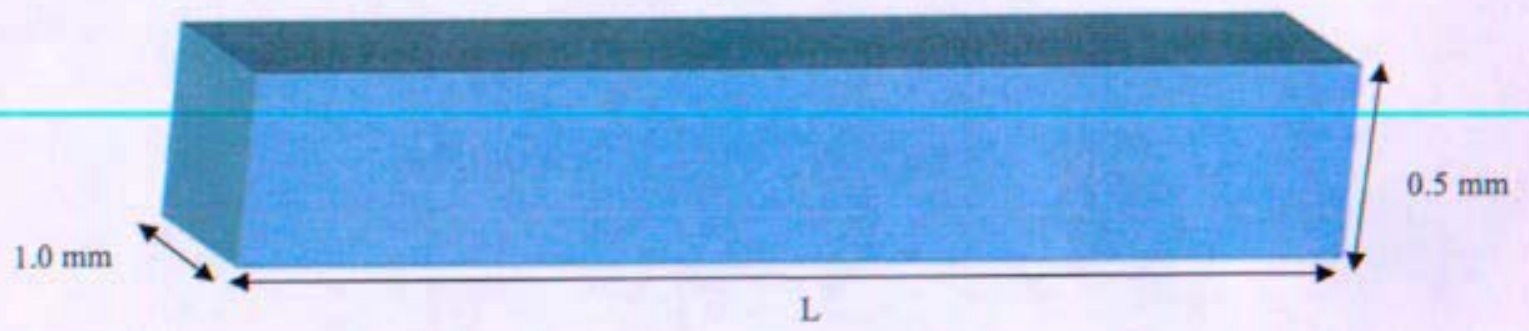

Figure 2. $\mathrm{SiC}$ and $\mathrm{ZrC}$ temperature monitors used in JOYO-1 campaign. $\mathrm{L}=7.0,14.0,28.0$, and $33.0 \mathrm{~mm}$ the $\mathrm{SiC}$ monitors needed for various capsule designs. $\mathrm{L}=14.0,28.0$, and $33.0 \mathrm{~mm}$ for $\mathrm{ZrC}$ monitors. 
Attachment 2

Draft IV to PNNL for PIE Bid

Enclosure 9 to MDO-723-0044/B-MT(SRME)-52 


\section{DRAFT}

Attachment 1 to

Enclosure 9 to MDO-723-0044/

B-MT(SRME)-52

Page 2

KAPL, Inc.

PO Box 1072

Schenectady, NY 12301

Telephone: 518-395-4000 Facsimile: 518-395-6068

INFORMATION TO VENDOR (Space Power Program) Number PNNL-SPP-05-00XX

Issue

Date

Page 1 of 10

To: Battelle-Pacific Northwest National Laboratory

(PNNL)

Attention: Kenneth A. Buxton

\begin{tabular}{|l|l|}
\hline $\begin{array}{l}\text { Purchase Order No. } \\
\text { EA0100007d }\end{array}$ & $\begin{array}{l}\text { Seller Reference Document } \\
\text { No. }\end{array}$ \\
\hline Charge Number: N/A \\
\hline $\begin{array}{l}\text { Subject: } \\
\text { Post-Irradiation Examination of Materials Irradiated } \\
\text { in JOYO-1 Campaign }\end{array}$ \\
\hline
\end{tabular}

Topics covered in the following pages:

A. Post-Irradiation Examination of Materials Irradiated in JOYO-1 Campaign.

page 2

Cognizant Engineer

Sign Name

Bldg. A2 Room. 209
Date

Print Name Steven Z. Hayden
Cognizant Contract Administrator

Sign Name
Date

Print Name Robert M. Dalton

\section{Notice - Disclaimer}

KAPL does not have the authority to modify the prime contract between the Seller and the Government, therefore, if the action contained herein is considered by the Seller to require a change in any aspect or provision (Price, Contract Term, Clauses, et al.) of the Seller's prime contract, the Seller shall not proceed with the action contained herein but should promptly, and in any event within 20 days of receipt of this document, notify the Seller's Cognizant Government Contracting Officer of the facts and the reasons for considering that a contract change is required, and concurrently notify KAPL.

NOTICE TO VENDOR: This information is considered by KAPL to be within the order requirements and is to be incorporated into the work without modification to any provision of the order. If you consider this information does modify any provision of the order, take no action to incorporate this information but advise KAPL of your position within twenty $(20)$ days after receipt of this IV. In the absence of such advice within said time it is agreed that the information will be incorporated into the work without modification to any provision of the order. Sign one copy below as evidence of receipt and acceptance of this information.

$\square$ No acknowledgment required.

Acknowledged by:

Date: 
Work is not yet authorized. KAPL requests costs and schedule by September 23,2005 for the following work.

\section{Post-Irradiation Examination of Materials Irradiated in JOYO-1 Campaign}

This work involves evaluating the post-irradiation examination (PIE) of specimens irradiated in the JOYO-1 test campaign in the Experimental Fast Reactor JOYO located in O-arai, Japan [operated by the Japanese Nuclear Cycle Research Institute (JNC)]. Tables 1 and 2 provide specimen counts and weights per material, fluence range experienced, and desired PIE operation and testing conditions. The specimen counts are broken into three phases for PIE (See Tables 1 and 2), which are:

Phase 1: $\quad$ A small subset of samples irradiated at intermediate fluences to ensure all testing equipment meets desired testing requirements and to guide testing parameters of higher fluence specimens.

Phase 2: $\quad$ The majority of samples irradiated at high and intermediate fluences, with some testing of low fluence specimens.

Phase 3: $\quad$ The remaining samples at lower fluences and replicate samples. This phase will also include optical and analytical electron microscopy examinations of specimens from Phases 1 and 2.

Tables 3 and 4 provide supplemental information on the specimen dimensions and chemical compositions.

For this IV, PNNL shall:

1. Provide a cafeteria-style cost estimate for PIE based upon: specimen type, material composition and testing conditions. PNNL can submit an estimate on performing all PIE of the JOYO specimens or certain portions. PNNL shall determine the most cost effective method to perform the PIE phases. Estimates shall also provide costs for disposal of waste generated from any PIE performed. It should be noted that the specimens may be shipped in the stainless steel (SS) capsules in which they were irradiated. PNNL shall include the opening of capsules in both the cost estimate and waste disposal.

2. Provide current testing capabilities (or possible capabilities with upgrades), handling capacity, and throughput. Also, a list of cask receiving capabilities shall be provided.

3. Supply a schedule for PIE assuming PIE to begin the end of March 2007.

4. Provide biographies on PNNL personnel having relevant experience working with the materials listed in Tables 1 and 2.

\section{Deliverables shall include:}

An estimate (due by September 23,2005 ) that contains, at a minimum, the following:

1. Costs broken down by specimen type, material composition, and testing conditions. PNNL shall determine the most cost effective method to perform the PIE Phases.

2. Cost associated with opening SS capsules.

3. Cost to measure electrical resistivity on a per specimen basis for a to-be-determined (TBD) subset of tensile specimens.

4. Cost on a per specimen type basis to perform dimensional inspection on a TBD subset of specimens (i.e. tensile, fracture toughness, etc.).

5. Cost on a per specimen basis for performing optical and analytic electron microscopy testing techniques (i.e. SEM, TEM (including punching discs from grips of tensile specimens and foil prep), and Auger) on a TBD subset of fracture toughness and tensile specimens. PNNL should also provide estimates of the time needed for cooling of each metal alloy prior to performing TEM analysis. 


\section{DRAFT}

6. Feasibility of installing a focused ion beam $(F \mid B)$ in a hot cell.

7. Cost on a per specimen basis to perform examination on a subset of $\mathrm{Ni}, \mathrm{Ti}$, and $\mathrm{Fe}$ flux wires (Figure 1) per ASTM E263-05, E264-02, and E526-97.

8. Cost on a per specimen basis to perform examination on a subset of $\mathrm{SiC}$ and $\mathrm{ZrC}$ temperature monitors (Figure 2) using electrical resistivity method.

9. Waste disposal costs associated with materials tested (including SS capsules and Mo and W/grafoil specimen holders).

10. Current hot cell and PIE capabilities (or possible capabilities with upgrades), handling capacity, throughput and cask receiving capabilities.

11. Schedules for testing assuming PIE to start end of March 2007.

12. Quality Assurance requirements that will be associated with this work effort.

13. Radiological controls and environmental, health and safety controls to mitigate associated risk.

14. Biographies on laboratory personnel having relevant experience working with the materials listed in Tables 1 and 2.

The PNNL technical contact for this task is Dave Senor (509-376-5610). The Naval Reactors Prime Contractor Team (NRPCT) technical contacts for this task are Jessica Bump (412-476-3912) at Bettis and Ryan Nelson 
DRAFT

Table 1. Metallic specimens in JOYO-1 test matrix.

\begin{tabular}{|c|c|c|c|c|c|c|c|}
\hline \multirow[t]{2}{*}{$\begin{array}{c}\text { Specimen } \\
\text { Type }\end{array}$} & \multirow[t]{2}{*}{$\begin{array}{c}\text { PIE } \\
\text { Operation }\end{array}$} & \multirow[t]{2}{*}{$\begin{array}{c}\text { Test } \\
\text { Conditions }\end{array}$} & \multirow[t]{2}{*}{$\begin{array}{c}\text { Material } \\
\text { (Mass) }\end{array}$} & \multirow{2}{*}{$\begin{array}{c}\begin{array}{c}\text { Fast } \\
\text { Fluence } \\
\text { Range }\end{array} \\
(1 \mathrm{E20} \\
\text { n/cm } \\
\end{array}$} & \multirow[b]{2}{*}{ Phase 1} & \multicolumn{2}{|c|}{$\begin{array}{c}\text { Nominal \# } \\
\text { of } \\
\text { Specimens }\end{array}$} \\
\hline & & & & & & Phase 2 & Phase 3 \\
\hline \multirow{7}{*}{ Biaxial Creep } & \multirow{7}{*}{$\begin{array}{c}\text { Laser } \\
\\
\text { Profilometry } \\
(\text { accuracy } \leq+/-3 \\
\mu \mathrm{m}) \\
\text { A minimum of } 180 \\
\text { diameter } \\
\text { measurements } \\
\left(\text { every } 1^{\circ} \text { ) at each }\right. \\
\text { of } 5 \text { axial locations }\end{array}$} & \multirow{7}{*}{ Room Temp } & $\begin{array}{l}\text { FS-85 } \\
(4.3 \mathrm{~g}) \\
\end{array}$ & $47-150$ & 7 & 17 & 0 \\
\hline & & & $\begin{array}{c}\text { ASTAR-811C } \\
(7.649 \mathrm{~g})\end{array}$ & $30-150$ & 8 & 14 & 0 \\
\hline & & & $\begin{array}{c}\mathrm{Mo}-47.5 \mathrm{Re} \\
(6.275 \mathrm{~g}) \\
\end{array}$ & $47-170$ & 7 & 15 & 0 \\
\hline & & & $\begin{array}{c}A 617 \\
(3.829 \mathrm{~g}) \\
\end{array}$ & $15-72$ & 2 & 7 & 0 \\
\hline & & & $\begin{array}{c}\text { PE16 } \\
(3.664 \mathrm{~g}) \\
\end{array}$ & $18-67$ & 2 & 7 & 0 \\
\hline & & & $\begin{array}{c}\mathrm{H} 230 \\
(4.108 \mathrm{~g})\end{array}$ & $20-65$ & 2 & 7 & 0 \\
\hline & & & Sum & & 28 & 67 & 0 \\
\hline \multirow{10}{*}{ Density Dise } & \multirow{10}{*}{$\begin{array}{c}\text { Density } \\
\text { Measurement } \\
\text { (Archimedes } \\
\text { Type, }+/-0.1 \% \\
\text { accuracy) }\end{array}$} & \multirow{10}{*}{ Room Temp } & $\begin{array}{c}\begin{array}{c}\text { FS-85 } \\
(1.950 \mathrm{~g})\end{array} \\
\end{array}$ & $28-144$ & 4 & 9 & 0 \\
\hline & & & $\begin{array}{c}\text { ASTAR-811C } \\
(3.073 \mathrm{~g}) \\
\end{array}$ & $21-144$ & 5 & 6 & 0 \\
\hline & & & $\begin{array}{c}\text { Mo-47.5Re } \\
(2.521 \mathrm{~g})\end{array}$ & $28-168$ & 5 & 18 & 0 \\
\hline & & & $\begin{array}{c}\mathrm{Re} \\
(3.871 \mathrm{~g})\end{array}$ & $31-169$ & 6 & 12 & $\underline{0}$ \\
\hline & & & $\begin{array}{c}\mathrm{W} \\
(3.560 \mathrm{~g})\end{array}$ & $27-170$ & 6 & 10 & 0 \\
\hline & & & $\begin{array}{r}\text { W-25Re } \\
(3.625 \mathrm{~g}) \\
\end{array}$ & $28-150$ & 6 & 11 & 0 \\
\hline & & & $\begin{array}{c}\mathrm{A617} \\
(1.583 \mathrm{~g})\end{array}$ & 13-151 & 9 & 11 & 7 \\
\hline & & & $\begin{array}{c}\mathrm{H} 230 \\
(1.650 \mathrm{~g})\end{array}$ & $10-150$ & 6 & 14 & 8 \\
\hline & & & $\begin{array}{c}\text { PEl6 } \\
(1.472 \mathrm{~g})\end{array}$ & 11-149. & 6 & 15 & 8 \\
\hline & & & Sum & & 53 & 106 & 23 \\
\hline \multirow{10}{*}{$\begin{array}{l}\text { Thermal } \\
\text { Diffusivity }\end{array}$} & \multirow{10}{*}{ (ASTM E1461-92) } & \multirow{6}{*}{$\begin{array}{c}\text { Room Temp } \\
\text { to } \\
1350 \mathrm{~K}\end{array}$} & $\begin{array}{c}\begin{array}{c}\text { FS-85 } \\
(1.950 \mathrm{~g})\end{array} \\
\end{array}$ & $28-134$ & 2 & 3 & 0 \\
\hline & & & $\begin{array}{c}\text { ASTAR-811C } \\
(3.073 \mathrm{~g})\end{array}$ & $21-63$ & 3 & 3 & 0 \\
\hline & & & $\begin{array}{c}\text { Mo-47.5Re } \\
(2.521 \mathrm{~g})\end{array}$ & $28-166$ & 3 & 6 & 0 \\
\hline & & & $\begin{array}{c}\operatorname{Re} \\
(3.871 \mathrm{~g}) \\
\end{array}$ & $21-72$ & 2 & 2 & 0 \\
\hline & & & $\begin{array}{c}W \\
(3.560 \mathrm{~g})\end{array}$ & 27.165 & 2 & 3 & 0 \\
\hline & & & $\begin{array}{l}\text { W-25Re } \\
(3.625 \mathrm{~g})\end{array}$ & $27-83$ & 1 & 3 & 0 \\
\hline & & \multirow{4}{*}{$\begin{array}{c}\text { Room Temp } \\
\text { to } \\
1050 \mathrm{~K}\end{array}$} & $\begin{array}{c}A 617 \\
(1.538 \mathrm{~g}) \\
\end{array}$ & $12-87$ & 5 & 8 & 0 \\
\hline & & & $\begin{array}{c}\mathrm{H} 230 \\
(1.650 \mathrm{~g})\end{array}$ & 13.53 & 5 & 8 & 0 \\
\hline & & & $\begin{array}{c}\text { PE16 } \\
(1.472 g) \\
\end{array}$ & $12-45$ & 5 & 7 & $\underline{0}$ \\
\hline & & & Sum & & 28 & 43 & 0 \\
\hline
\end{tabular}


DRAFT

Table 1 cont"d. Metallic specimens JOYO-1 test matrix.

Attachment 1 to

Enclosure 9 to

MDO-723-0044

B-MT(SRME)-52

\begin{tabular}{|c|c|c|c|c|c|c|c|}
\hline \multirow[t]{2}{*}{$\begin{array}{c}\text { Specimen } \\
\text { Type }\end{array}$} & \multirow[t]{2}{*}{$\begin{array}{c}\text { PIE } \\
\text { Operation }\end{array}$} & \multirow[t]{2}{*}{$\begin{array}{c}\text { Test } \\
\text { Conditions }\end{array}$} & \multirow[t]{2}{*}{$\begin{array}{c}\text { Material } \\
\text { (Mass) }\end{array}$} & \multirow{2}{*}{$\begin{array}{c}\text { Fast } \\
\text { Fluence } \\
\text { Range } \\
(1 \mathrm{E} 20 \\
\left.\mathrm{a} / \mathrm{cm}^{2}\right)\end{array}$} & & \multirow{2}{*}{$\begin{array}{c}\text { Nominal \# } \\
\text { of } \\
\text { Specimens } \\
\text { Phase } 2\end{array}$} & \multirow{2}{*}{$\begin{array}{r}\text { Pag } \\
\text { Pbase } 3\end{array}$} \\
\hline & & & & & Phase 1 & & \\
\hline \multirow{10}{*}{ Tensile } & \multirow{10}{*}{$\begin{array}{c}\text { Tensile } \\
\text { Testing } \\
\text { (ASTM E8-04) } \\
\& \\
\text { Electrical } \\
\text { Resistivity } \\
\text { (ASTM B 193-02) }\end{array}$} & \multirow{6}{*}{$\begin{array}{c}\text { Room Temp } \\
\text { to } \\
1450 \mathrm{~K} \\
\& \mathrm{HV} \\
\left(<10^{-6} \text { torr }\right)\end{array}$} & $\begin{array}{c}\text { FS-85 } \\
(0.619 \mathrm{~g}) \\
\end{array}$ & $31-128$ & 12 & 29 & 12 \\
\hline & & & $\begin{array}{c}\text { ASTAR-811C } \\
(0.975 \mathrm{~g}) \\
\end{array}$ & $24-147$ & 16 & 35 & 16 \\
\hline & & & $\begin{array}{c}\mathrm{Mo}-47.5 \mathrm{Re} \\
(0.800 \mathrm{~g}) \\
\end{array}$ & $32-172$ & 0 & 28 & 11 \\
\hline & & & $\begin{array}{c}\mathrm{Re} \\
(1.229 \mathrm{~g}) \\
\end{array}$ & $24-172$ & 8 & 16 & 0 \\
\hline & & & $\begin{array}{c}W \\
(1.130 \mathrm{~g})\end{array}$ & $29-172$ & 8 & 15 & 0 \\
\hline & & & $\begin{array}{l}\text { W-25Re } \\
(1.150 \mathrm{~g})\end{array}$ & $32-172$ & 8 & 16 & 0 \\
\hline & & \multirow{4}{*}{$\begin{array}{l}\text { Room Temp to } \\
1050 \mathrm{~K} \\
\& \mathrm{UHP} \text { gas or } \\
\text { HV }\left(<10^{-6} \text { torr }\right)\end{array}$} & $\begin{array}{c}\mathrm{A} 617 \\
(0.488 \mathrm{~g})\end{array}$ & $12-158$ & 16 & 33 & 16 \\
\hline & & & $\begin{array}{c}\mathrm{H} 230 \\
(0.524 \mathrm{~g}) \\
\end{array}$ & $12-158$ & 15 & 37 & 2 \\
\hline & & & $\begin{array}{c}\text { PE16 } \\
(0.467 \mathrm{~g})\end{array}$ & $12-158$ & 15 & 37 & 9 \\
\hline & & & Sum & & 98 & 246 & 66 \\
\hline \multirow{8}{*}{$\begin{array}{l}\text { Fracture } \\
\text { Toughness }\end{array}$} & \multirow[b]{4}{*}{ Three point } & \multirow{4}{*}{$\begin{array}{c}\text { Room Temp } \\
\text { to } \\
1450 \mathrm{~K}\end{array}$} & $\begin{array}{c}\text { Mo-47.5Re } \\
(3.091 \mathrm{~g}) \\
\end{array}$ & $39-173$ & 32 & 16 & 0 \\
\hline & & & $\begin{array}{c}\mathrm{Re} \\
(4.747 \mathrm{~g}) \\
\end{array}$ & $39-173$ & 2 & 6 & 0 \\
\hline & & & $\begin{array}{c}W \\
(4.365 \mathrm{~g}) \\
\end{array}$ & $39-173$ & 2 & 6 & 0 \\
\hline & & & $\begin{array}{l}\text { W-25Re } \\
(4.444 \mathrm{~g})\end{array}$ & $39-173$ & 2 & 6 & 0 \\
\hline & \multirow{4}{*}{$\begin{array}{l}\text { slow bend test } \\
\text { (ASTM E1820-01) }\end{array}$} & \multirow{4}{*}{$\begin{array}{c}\text { Room Temp } \\
\text { to }\end{array}$} & $\begin{array}{c}A 617 \\
(1.886 \mathrm{~g})\end{array}$ & $15-172$ & 8 & 36 & 10 \\
\hline & & & $\begin{array}{c}\mathrm{H} 230 \\
(2.024 \mathrm{~g})\end{array}$ & $15-172$ & 7 & 29 & 18 \\
\hline & & & $\begin{array}{c}\text { PE16 } \\
(1.805 \mathrm{~g})\end{array}$ & $15-172$ & 6 & 31 & 17 \\
\hline & & & Sum & & 59 & 130 & 45 \\
\hline \multirow{5}{*}{ Compact } & \multirow{5}{*}{$\begin{array}{l}\text { Measure final bolt } \\
\text { load at Tirr } \\
\text { + final crack depth } \\
\text { due to irradiation } \\
\text { exposure at JOYO } \\
\text { from heat tint } \\
\text { optical analysis + } \\
\text { Option A: post- } \\
\text { irradiation creep } \\
\text { crack growth rate at } \\
\text { Tirr (EPD } \\
\text { measurement of } \\
\text { crack depth with } \\
\text { long term testing) } \\
\text { Option B: post- } \\
\text { irradiation fracture } \\
\text { toughness (clip } \\
\text { gage to determine } \\
\text { displacement) }\end{array}$} & $\begin{array}{l}\text { Room Temp to } \\
1350 \mathrm{~K}\end{array}$ & $\begin{array}{c}\mathrm{Mo-47.5 \textrm {Re }} \\
(13.558 \mathrm{~g})\end{array}$ & $41-166$ & 0 & 0 & 16 \\
\hline & & \multirow{4}{*}{ Room Temp } & $\begin{array}{c}A 617 \\
(8.273 \mathrm{~g})\end{array}$ & $23-87$ & 0 & 0 & 8 \\
\hline & & & $\begin{array}{c}\mathrm{H230} \\
(8.877 \mathrm{~g})\end{array}$ & $34-77$ & 0 & 0 & 8 \\
\hline & & & $\begin{array}{c}\text { Pel6 } \\
(7.917 \mathrm{~g})\end{array}$ & $34-90$ & 0 & 0 & 8 \\
\hline & & & Sum & & 0 & 0 & 24 \\
\hline
\end{tabular}


DRAFT

Attachment 1 to

Enclosure 9 to

MDO-723-0044/

B-MT(SRME)-52

Page 7

Table 2. Ceramic specimens in JOYO-1 test matrix.

\begin{tabular}{|c|c|c|c|c|c|c|c|}
\hline \multirow[t]{2}{*}{$\begin{array}{c}\text { Specimen } \\
\text { Type }\end{array}$} & \multirow[t]{2}{*}{$\begin{array}{c}\text { PIE } \\
\text { Operation }\end{array}$} & \multirow[t]{2}{*}{$\begin{array}{c}\text { Test } \\
\text { Conditions }\end{array}$} & \multirow[t]{2}{*}{ Material } & \multirow{2}{*}{$\begin{array}{c}\text { Fast } \\
\text { Fluence } \\
\text { Range } \\
(\mathbf{1 E 2 0} \\
\left.\mathbf{n} / \mathbf{c m}^{2}\right)\end{array}$} & \multirow[b]{2}{*}{ Phase 1} & \multicolumn{2}{|l|}{$\begin{array}{c}\text { Nominal \# } \\
\text { of } \\
\text { Specimens }\end{array}$} \\
\hline & & & & & & Phase 2 & Phase 3 \\
\hline \multirow{3}{*}{ Tensile } & \multirow{3}{*}{$\begin{array}{c}\text { Tensile } \\
\text { Testing } \\
\text { Option A: } \\
\text { SiC (ASTM } \\
\text { C1366-04 \& } \\
\text { SiC/SiC (ASTM } \\
\text { C1359-96) } \\
\text { Option B: } \\
\text { SiC (ASTM } \\
\text { C1273-95a \& } \\
\text { SiC/SiC (ASTM } \\
\text { C1275-00) }\end{array}$} & \multirow{3}{*}{$\begin{array}{c}\text { Option A } \\
1050 \mathrm{~K} \text { and } 1350 \\
\mathrm{~K} \text { in } 1 \text { atm inert } \\
\text { gas } \\
\text { Option B } \\
\text { Room } \\
\text { Temperature }\end{array}$} & $\begin{array}{c}\mathrm{SiC} \\
(1.9 \mathrm{~g}) \\
\end{array}$ & $127-194$ & 0 & 8 & 4 \\
\hline & & & $\begin{array}{c}\mathrm{SiC} / \mathrm{SiC} \\
(1.9 \mathrm{~g})\end{array}$ & $42-196$ & 2 & 14 & 8 \\
\hline & & & Sum & & 2 & 22 & 12 \\
\hline \multirow{4}{*}{ Density Disc } & \multirow{4}{*}{$\begin{array}{c}\text { Density } \\
\text { Measurement } \\
\text { SiC \& SiC/SiC } \\
\text { (ASTM D1505- } \\
\text { 03) } \\
\text { BeO (ASTM } \\
\text { C373-88) }\end{array}$} & \multirow{4}{*}{ Room Temp } & $\begin{array}{c}\mathrm{SiC} \\
(1.178 \mathrm{~g})\end{array}$ & $34-193$ & 24 & 0 & 0 \\
\hline & & & $\begin{array}{c}\mathrm{SiC} / \mathrm{SiC} \\
(0.589 \mathrm{~g})\end{array}$ & $35-194$ & 24 & 0 & 0 \\
\hline & & & $\begin{array}{c}\mathrm{BeO} \\
(0.554 \mathrm{~g})\end{array}$ & $16-50$ & $\begin{array}{c}32 \text { discs } \\
16 \mathrm{cyl} .\end{array}$ & 0 & 0 \\
\hline & & & Sum & & 96 & 0 & 0 \\
\hline \multirow{4}{*}{ Diffusivity Disc } & \multirow{4}{*}{$\begin{array}{c}\text { Thermal } \\
\text { Diffusivity } \\
\text { Measurement } \\
\text { (ASTM E1461- } \\
01 \text { ) }\end{array}$} & \multirow{2}{*}{$\begin{array}{l}\text { Room Temp to } \\
1350 \mathrm{~K} \text { in } 1 \mathrm{~atm} \\
\text { inert gas }\end{array}$} & $\begin{array}{c}\mathrm{SiC} \\
(1.178 \mathrm{~g}) \\
\end{array}$ & $34-192$ & 0 & 24 & 0 \\
\hline & & & $\begin{array}{r}\mathrm{SiC} / \mathrm{SiC} \\
(0.589 \mathrm{~g}) \\
\end{array}$ & $35-193$ & 0 & 24 & 0 \\
\hline & & \multirow{2}{*}{$\begin{array}{l}\text { Room Temp to } \\
1250 \mathrm{~K} \text { in } 1 \text { atm } \\
\text { inert gas }\end{array}$} & $\begin{array}{c}\mathrm{BeO} \\
(0.554 \mathrm{~g})\end{array}$ & $16-47$ & 0 & 32 & 0 \\
\hline & & & Sum & & 0 & 80 & 0 \\
\hline $\begin{array}{l}\text { Compression } \\
\text { Cylinders }\end{array}$ & $\begin{array}{c}\text { Compressive } \\
\text { Strength Testing } \\
\text { (ASTM C773-88) }\end{array}$ & $\begin{array}{c}850 \mathrm{~K} \text { and } \\
1050 \mathrm{~K}\end{array}$ & $\begin{array}{c}\mathrm{BeO} \\
(0.303 \mathrm{~g})\end{array}$ & $18-44$ & 4 & 8 & 4 \\
\hline BSR Samples & $\begin{array}{c}\text { Optical } \\
\text { Microscopy }\end{array}$ & Room Temp & $\begin{array}{c}\mathrm{SiC} \\
(2.379 \mathrm{~g}) \\
\end{array}$ & $53-75$ & 4 & 8 & 4 \\
\hline
\end{tabular}




\section{DRAFT}

Attachment 1 to

Enclosure 9 to MDO-723-0044/

B-MT(SRME)-52

Page 8

Table 3. Description of metallic specimens in JOYO-1.

Holders for metallic specimens will be molybdenum.

Metallic Specimens:

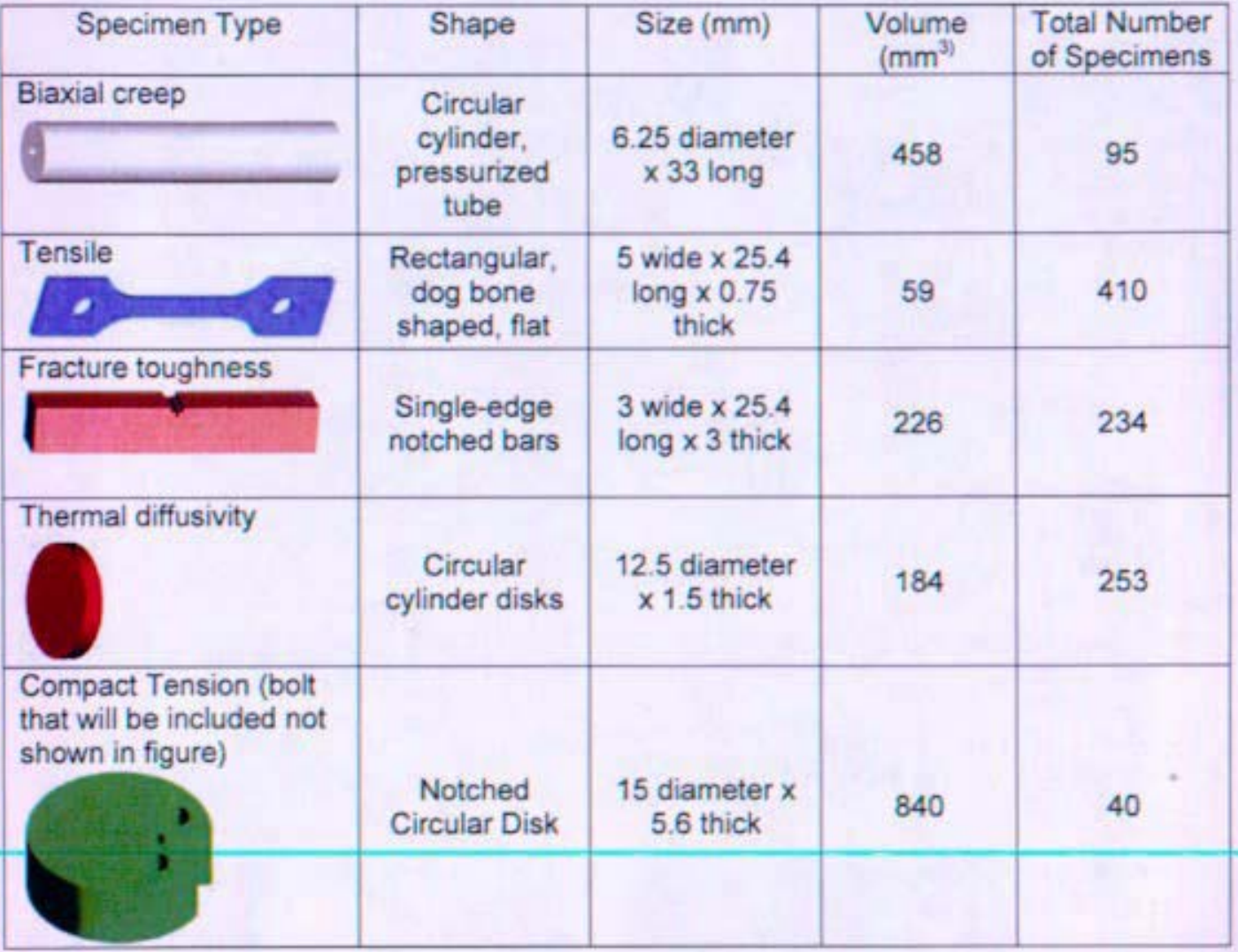

Chemical compositions :

Metallic Specimens: (wt\%)

FS-85

(62Nb-27Ta-10W-1Zr)

ASTAR-811C (90Ta-8W-1Re-0.7Hf-0.025C)

Mo-47Re (52.5Mo-47.5 Re)

$\operatorname{Re}$

(100Re)

W (100W)

W-25Re (75W-25Re)

Nimonic PE-16 (42Ni-33Fe-18Cr-4Mo-1.2Ti-1.2Al-0.3Si-0.2Mn-0.1C)

Alloy $617 \quad(51 \mathrm{Ni}-22 \mathrm{Cr}-12.5 \mathrm{Co}-9 \mathrm{Mo}-3 \mathrm{Fe}-1 \mathrm{Si}-1 \mathrm{Mn}-0.6 \mathrm{Ti}-0.1 \mathrm{Al}-0.006 \mathrm{~B})$

Haynes $230 \quad(53 \mathrm{Ni}-22 \mathrm{Cr}-5 \mathrm{Co}-14 \mathrm{~W}-3 \mathrm{Fe}-2 \mathrm{Mo}-0.4 \mathrm{Si}-0.3 \mathrm{Al}-0.5 \mathrm{Mn}-0.1 \mathrm{C}-0.015 \mathrm{~B})$

(all nominal compositions in weight percent) 
Table 4. Description of ceramic specimens in JOYO-1

Holders for SiC capsules will be tungsten and grafoil; BeO will use molybdenum

Ceramic Specimens:

\begin{tabular}{|c|c|c|c|c|}
\hline Specimen Type & Shape & Size $(\mathrm{mm})$ & $\begin{array}{l}\text { Volume } \\
\left(\mathrm{mm}^{3}\right)\end{array}$ & $\begin{array}{c}\text { Total } \\
\text { Number of } \\
\text { Specimens }\end{array}$ \\
\hline Tensile & $\begin{array}{l}\text { Rectangular, } \\
\text { dog bone } \\
\text { shaped, flat }\end{array}$ & $\begin{array}{l}6 \text { wide } \times 41.3 \\
\text { long } \times 2.3 \text { thick }\end{array}$ & 372 & 36 \\
\hline $\begin{array}{l}\text { Composite Thermal } \\
\text { Conductivity }\end{array}$ & $\begin{array}{c}\text { Circular } \\
\text { cylinder disks }\end{array}$ & $\begin{array}{c}12.5 \text { diameter } \\
\times 1.5 \text { thick }\end{array}$ & 184 & 24 \\
\hline Thermal Conductivity & $\begin{array}{c}\text { Circular } \\
\text { cylinder disks }\end{array}$ & $\begin{array}{c}12.5 \text { diameter } \\
\times 3 \text { thick }\end{array}$ & 368 & 24 \\
\hline BeO Density Disk & $\begin{array}{c}\text { Circular } \\
\text { cylinder disks }\end{array}$ & $\begin{array}{c}12.5 \text { diameter } \\
\times 3 \text { thick }\end{array}$ & 368 & 32 \\
\hline BeO Compression & $\begin{array}{c}\text { Circular } \\
\text { cylinder disks }\end{array}$ & $\begin{array}{c}4 \text { diameter } x \\
8 \text { tall }\end{array}$ & 100 & 16 \\
\hline Ceramic Strip & $\begin{array}{l}\text { Thin } \\
\text { Rectangular } \\
\text { Strip }\end{array}$ & $\begin{array}{c}1 \text { height } x \\
40 \text { long } x 0.05 \text {, } \\
0.1 \text {, or } 0.2 \\
\text { width }\end{array}$ & $\begin{array}{c}1.3 \\
\text { (specimen) } \\
625 \\
\text { (fixture) }\end{array}$ & 16 \\
\hline
\end{tabular}

Chemical compositions

Ceramic Specimens:

$\mathrm{SiC}$ - chemical vapor deposited silicon carbide (monolithic)

$\mathrm{SiC} / \mathrm{SiC}$ - Woven Hi-Nicalon Type S (SiC) fiber in a chemical vapor infiltration (CVI) SiC matrix

$\mathrm{BeO}$ - Beryllia 


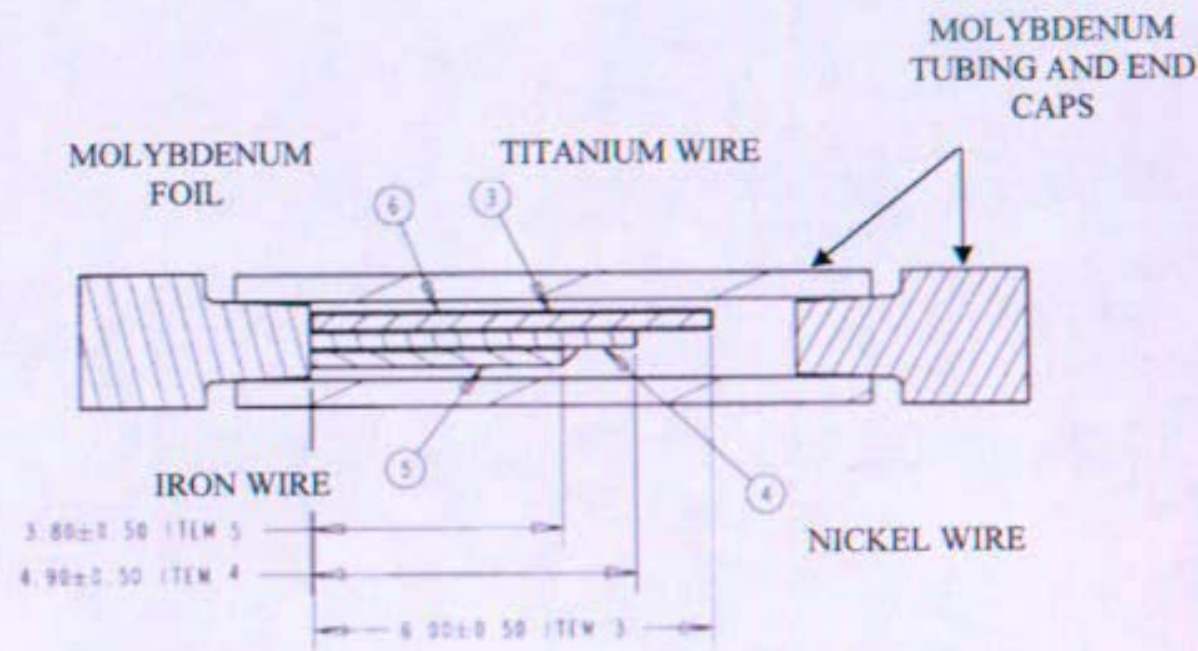

Figure 1. Nickel, titanium, and iron flux wires used in JOYO-1 campaign. Dimensions in mm. Flux wire diameters are $0.25 \mathrm{~mm}$. Total assembly is $2.03 \mathrm{~mm}$ O.D. x $14.32 \mathrm{~mm}$ long.

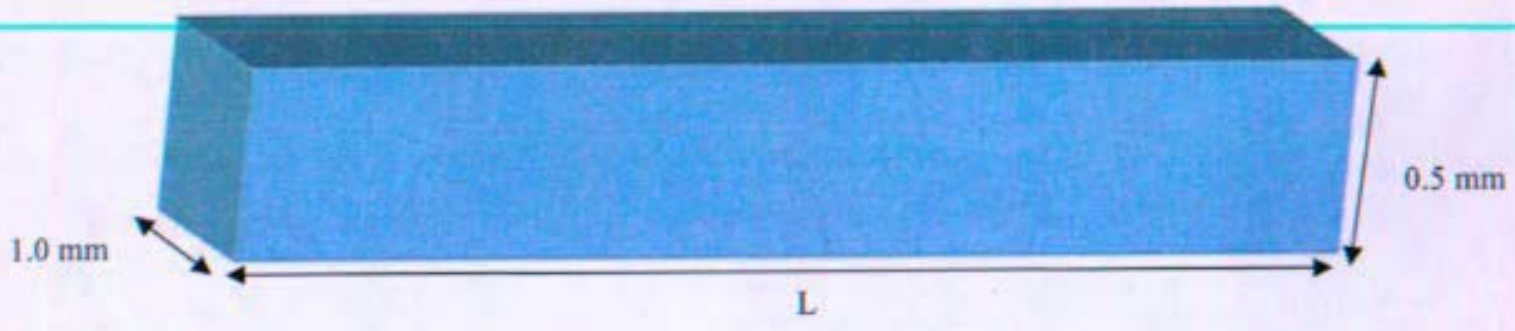

Figure 2. $\mathrm{SiC}$ and $\mathrm{ZrC}$ temperature monitors used in JOYO-1 campaign. $\mathrm{L}=7.0,14.0,28.0$, and $33.0 \mathrm{~mm}$ the $\mathrm{SiC}$ monitors needed for various capsule designs. $\mathrm{L}=14.0,28.0$, and $33.0 \mathrm{~mm}$ for $\mathrm{ZrC}$ monitors. 
Attachment 3 to

Enclosure 9 to

MDO-723-0044/

B-MT(SRME)-52

Page 1

Attachment 3

PNNL White Paper on PIE Strategy

Enclosure 9 to MDO-723-0044/B-MT(SRME)-52 


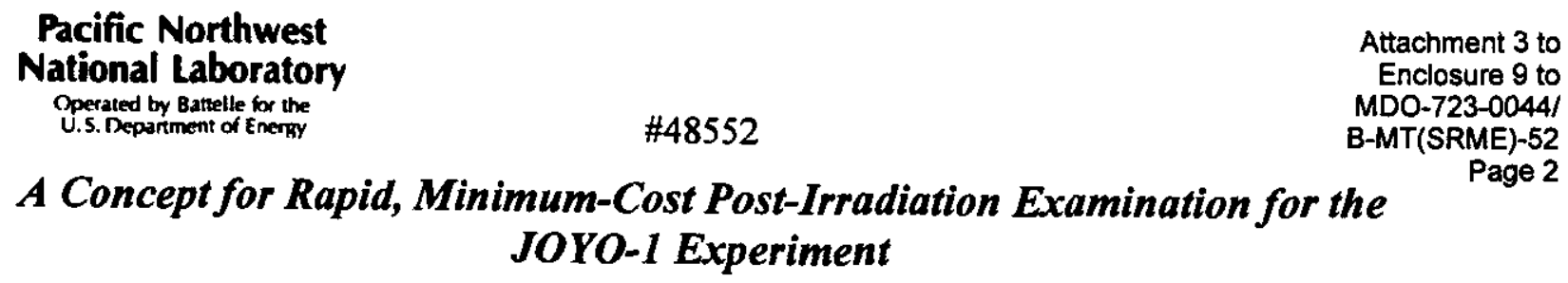

\author{
White Paper \\ David Senor and Chad Painter \\ Space Reactor Materials Irradiation Project \\ July 8, 2005
}

Information provided to date on the status of negotiations between the US Department of Energy (DOE) and the Japan Nuclear Cycle Development Institute (JNC) on the Specific Memorandum of Agreement for performing irradiation tests for Project Prometheus under the existing DOEJNC Nuclear Technology Agreement indicates there is a reluctance on the part of the Japan government to accept US materials and secondary waste generated during post-irradiation examination (PIE) for radioactive material disposal in Japan. Added to these challenges are the potential issues associated with the importation of radioactive waste into the US. These circumstances may make it difficult, expensive, and time-consuming to perform destructive postirradiation examination (PIE) in Japan and return capsule pieces, capsule internals, test specimens, and secondary waste generated during PIE for disposal in the US (Crane 2005). Moreover, investment by NRPCT in PIE capability at JNC would need to be initiated now (July 2005 ) in order for mechanical properties testing of the refractory alloys to be ready in FY2007. Therefore, if all US-supplied materials (capsules, capsule internals, and test specimens) must ultimately be returned to the US for disposition after irradiation, including secondary waste, it is reasonable to consider performing most of the PIE activities in the US where the capabilities already exist and waste issues could be handled within the domain of U.S. Government entities.

The concept described in this white paper provides a path forward for conducting PIE on the JOYO-1 irradiation experiment under two basic assumptions. First, the concept is designed to maximize throughput by splitting the PIE work among multiple facilities. The quantity of test specimens in the irradiation test matrix is very large (approximately 1200). Add to this number a significant quantity of dosimetry wires and passive temperature monitors that will require analysis. Performing PIE on even a fraction of these specimens would be a huge effort for a single laboratory. Therefore, it is reasonable to distribute specimens to multiple laboratories where particular capabilities provide the best possibility to perform the PIE as quickly as possible. Second, the concept is designed to minimize installation of new capability. Testing is proposed only at facilities that currently have the necessary capability and expertise. This approach will minimize preparation costs and time and help ensure that the data can be available in FY2008. Finally, the proposed path forward would leverage contracting vehicles put in place and relationships established between representatives of the Japanese and US governments to date.

To expedite the initial post-irradiation analysis of the capsules, some non-destructive PIE could be performed in Japan soon after removing the 60 capsules from the two irradiation rigs (SMIRs). For example, visual inspection, weighing, profilometry, gamma scanning, and x-ray tomography on the intact capsules could provide valuable information regarding the state of the capsules and test specimens. The capsules would be cleaned of sodium and extracted from the SMIRs in the JNC Fuels and Materials Facility (FMF). Each of the proposed non-destructive examinations are routinely done in the FMF on irradiated fuel pins (Itoh and Koyama 2005). Gamma spectroscopy will provide a preliminary indication of the irradiation dose received by the capsules, while weighing and profilometry will provide an indication of the condition of the capsule internals (for 
example, excessive weight change or deformation of the capsule could indicate failure of biaxial creep specimens). The $x$-ray tomography capability in the FMF is very unique and will allow non-destructive inspection of the internal configuration of the capsules (Katsuyama et al. 2003). Aside from specific fixturing to accommodate the capsule dimensions, little modification will be required to these existing non-destructive $\mathrm{N}$ C capabilities. After all of the non-destructive examinations are completed in the FMF, the capsules would be packaged and inserted in a shipping cask in FMF (JNC 2000). Thus, all the proposed JNC activities can be done in one facility, avoiding potentially time-consuming transfers of materials from one facility to another.

A transportation task would need to be established to manage and coordinate the shipment of capsules back to the US. A contract would need to be established with NNC to obtain Japanese government permission to ship irradiated capsules in their TN6-4 cask AND obtain a US Department of Transportation certificate to enter the US. Discussions with JNC and the US NRC estimate the time to obtain these permissions will be about $1 \frac{1 / 2}{2}$ years. (NOTE: The US Department of Transportation is the certifying authority for packaging containing radioactive waste; however, the US NRC provides technical reviews for DOT). It is very likely that an inner cask package would need to be designed and constructed by $\mathrm{JNC}$ to protect the capsules from damage during shipment. The design of the inner package would need to be reviewed by the organization that would ultimately receive the cask in the US to ensure it could be efficiently handled. Shipment of the cask would be made by sea and could enter the US in the port of Tacoma (the shortest sea route from Japan). From there the cask would be moved by truck to PNNL, if the laboratory was selected to disassemble the capsules. A recent estimate of the resultant activation of isotopes in terms of curie content and heat load has been completed and this information has been provided to JNC to estimate how many shipments would be needed to move all 60 capsules back to the US (Prichard 2005). At 90 days after irradiation the curie content of Ta-182 is estimated to be 10,000 curies, which will likely determine the number of shipments.

Given the existing contractual relationship between PNNL and NNC, it is reasonable to arrange for shipping the cask or casks containing the capsules to PNNL for disassembly and specimen distribution. The proposed TN6-4 cask would be received and the capsules disassembled using existing facilities in the Radiochemical Processing Laboratory (325 Building). This work is very similar to past and current (the next shipment is due to arrive July 15, 2005) efforts on the DOENNSA Tritium Technology Program in which irradiated tritium-producing burnable absorber rods (TPBARs) are received in commercial casks and disassembled for PIE. The planning and preparation time to receive a new cask is typically 1 year.

Using PNNL as the central point for receiving, disassembly, and distribution also would expedite the dosimetry analysis, a particular area of expertise for the laboratory. To supplement the dosimetry of the flux wires included in the experiment, it would be valuable to perform retrospective dosimetry on slices taken from representative capsules and possibly specimen holders. This is a technique that has been used effectively by PNNL in previous irradiation experiments (Senor et al. 2000a). These data will provide much greater resolution on fluence and irradiation dose (displacements per atom, dpa), which will be important given the location of the two SMIRs in the first row of the JOYO reflector (Greenwood and Smither, 1985). Nuclear analyses performed to date indicate a factor of up to 1.5 variation in radial fast flux and a factor of 4.5 variation in axial fast flux across the SMIRs in that location (Wootan 2005).

PNNL has significant expertise in analysis of passive temperature monitors (e.g. SiC) using highresolution metrology, dilatometry and thermal diffusivity (Youngblood et al. 2004, Senor et al. 


\section{Pacific Northwest National Laboratory \\ Operated by Battelle iox the \\ U.S. Depantment of Erergy}

Attachment 3 to

Enclosure 9 to

MDO-723-0044/

B-MT(SRME)-52

Page 4

2003). Thus, the temperature monitors could be analyzed at PNNL in parallel with the dosimetry analysis to yield rapid feedback on the temperatures experienced by the capsules and test specimens in-reactor.

PNNL is assembling, pressurizing, and inspecting the biaxial creep specimens before irradiation. The pre-irradiation inspections include laser profilometry of the pressurized specimens. Therefore, it is reasonable to perform the post-irradiation laser profilometry using the same equipment at PNNL to eliminate as much bias as possible. This work would be accommodated at PNNL in the same hot cell that would be used for capsule disassembly (A-Cell in the $\mathbf{3 2 5}$ Building). Preliminary dose rate calculations indicate A-Cell would accommodate all of the irradiated biaxial creep specimens simultaneously (Fountain 2005).

PNNL has significant expertise with thermal diffusivity testing of irradiated materials (Youngblood et al. 2004, Senor et al. 2003, Youngblood et al. 2003, Senor et al. 2000a, Senor et al. 2000b, Senor et al. 1996a) and the laboratory recently installed a new Netzsch thermal diffusivity testing apparatus in a lead-shielded glovebox that is suitable for testing the irradiated metallic thermal diffusivity specimens. The lower-activity ceramic thermal diffusivity specimens would be accommodated in existing thermal diffusivity testing systems located outside the hot cell facility in the Material Sciences Laboratory (326 Building).

Given the existing capabilities and experience of PNNL in post-irradiation mechanical property testing and characterization of $\mathrm{SiC}, \mathrm{SiC}_{\mathrm{f}} / \mathrm{SiC}$ and other ceramic materials (Senor et al. 2000a, Senor et al. 1996b, Henager et al. 1992, Henager et al. 1991), the PIE on the ceramic specimens would be accommodated at PNNL using existing facilities in the 326 Building. This would include dimensional measurements on the bend-stress-relaxation (BSR) specimens, tensile tests, four-point bend tests, compression tests, metrology, and other necessary characterization techniques including optical microscopy, scanning electron and transmission electron microscopy, electron and $\mathrm{x}$-ray diffraction, and high-resolution He measurements.

The remainder of the test specimens (approximately $58 \%$ of the total, not including flux wires and temperature monitors) are metallic mechanical property specimens including tensile, fracture toughness, and compact tension specimen types. There currently is no existing in-cell capability at PNNL for performing mechanical property tests on high-activity specimens. Therefore, this portion of the work would be better performed at another facility that has this capability. For example, Oak Ridge National Laboratory has existing in-cell mechanical testing capability that is suitable for the JOYO-1 specimens, as well as significant expertise in the area of refractory and Ni-base alloys. Regardless of which laboratory was selected to perform this portion of the work, the relevant specimens could be packaged in the 325 Building at PNNL and shipped using a variety of casks that are currently licensed and available for shipment within the US. Presumably, the metallography and other characterization of the metallic mechanical property specimens, along with waste disposal, would be the responsibility of the laboratory selected to perform the work.

With regards to waste disposal and facility availability many discussions with PNNL management have occurred in recent weeks. The number of waste disposal packages is highly dependent on the Cobalt- 60 content of the stainless steel used to build the capsule end caps and walls-PNNL will need to know this value before any good estimate of waste disposal cost can be provided. However, disposal paths exist for all of the materials included in the JOYO-1 irradiation experiment and there do not appear to be any barriers to disposal of the material at Hanford for waste generated at PNNL. 
At Hanford, efforts are underway as part of the DOE Office of River Protection's mission to close facilities in the 300 area. Discussion with PNNL management and the local DOE field office confirm that the PIE work proposed in this white paper will not be affected by the effort to close 300 area facilities. PNNL's capability to perform radiological work will be transitioned and fully operable in new facilities before the legacy facilities are closed, thereby eliminating any disruption of ongoing activities for not only the Space Reactor program, but several other critical national security missions.

\section{References}

Crane, PJ. 2005. "Space Reactor Materials Irradiation Testing Project: Evaluation of Impact of Washington State Initiative 297 and Stipulation Order," Space Reactor Material Irradiation Testing Project (48552) White Paper. Richland, WA: Pacific Northwest National Laboratory.

Fountain, MS. 2005. "HLRF Dose Rates, Rev. 2," PNNL Memo from MS Fountain to RE Thomhill Dated 14 March 2005. Richland, WA: Pacific Northwest National Laboratory.

Greenwood, LR and RK Smither. 1985. "SPECTER: Neutron Damage Calculations for Materials Irradiations," $A N L / F P P-197$. Richland, WA: Pacific Northwest National Laboratory.

Henager, CH, GW Hollenberg, ML Hamilton, SA Simonson and GA Newsome. 1991. "KAPL Irradiation Screening Test (KIST1)," PNL-7864. Richland, WA: Pacific Northwest Laboratory.

Henager, $\mathrm{CH}$, GW Hollenberg, GE Youngblood and ML Hamilton. 1992. "KAPL Irradiation Screening Test (KIST2)," PNL-8112. Richland, WA: Pacific Northwest Laboratory.

Itoh, M and S Koyama. 2005. "Present Status of Post-Irradiation Examination Facilities for Fuels and Materials Development of Fast Reactor." Oarai, Ibaraki, Japan: Japan Nuclear Cycle Development Institute.

NC. 2000. "Fuels and Materials Division." Oarai, Ibaraki, Japan: Japan Nuclear Cycle Development Institute.

Katsuyama, K, T Nagamine, S Matsumoto and M Itoh. 2003. "Application of X-Ray Computer Tomography for Observing the Deflection and Displacement of Fuel Pins in an Assembly Irradiated in FBR," Journal of Nuclear Science and Technology, 40(4):220-226.

Prichard, AW. 2005. "Post Irradiation Radioactivity and Heating Rates for JOYO-1 Irradiation Tests," PNNL-15258. Richland, WA: Pacific Northwest National Laboratory.

Senor, DJ, GE Youngblood, CE Moore, DJ Trimble, GA Newsome and JJ Woods. 1996a. "Effects of Neutron Irradiation on Thermal Conductivity of SiC-Based Composites and Monolithic Ceramics," Fusion Technology, 30(3):943-955.

Senor, DJ, CH Henager, Jr., GE Youngblood, JL Brimhall, and MA McKinnon. 1996b. "The KARE Irradiation Experiment: Post-Irradiation Examination and Material Property Testing," PNL-10861. Richland, WA: Pacific Northwest Laboratory.

Senor, DJ, GE Youngblood, CL Painter, LR Greenwood, CR Eiholzer, DV Archer, GK Whiting and DL Alexander. 2000a. "KAPL Advanced Materials Examination and Testing Project: Final Report," PNNL12204. Richland, WA: Pacific Northwest National Laboratory. 
Senor, DJ, KC Cartwright, DL Alexander, DV Archer, SD Knowles and SV Forbes. 2000b. "KAPL Thermal Properties Screening Project: Final Report," PNNL-13300. Richland, WA: Pacific Northwest National Laboratory.

Senor, DJ, GE Youngblood, LR Greenwood, DV Archer, DL Alexander, MC Chen and GA Newsome. 2003. "Defect Structure and Evolution in Silicon Carbide Irradiated to 1 dpa-SiC at $1100^{\circ} \mathrm{C}$," Journal of Nuclear Materials, 317(2-3):145-159.

Wootan, DW 2005. "Heating Rates and Neutron Flux for JOYO-1 Irradiation Tests - Revision 1," PNNL15250. Richland, WA: Pacific Northwest National Laboratory.

Youngblood, GE, DJ Senor and RH Jones. 2003. "Thermal Diffusivity/Conductivity of Irradiated HiNicalon 2D-SiC $/ \mathrm{SiC}$ Composite," in Fusion Materials Semiannual Progress Report for Period Ending 30 June 2003, DOE/ER-0313/34, Washington, DC: US Department of Energy, pp. 41-47.

Youngblood, GE, DJ Senor and RH Jones. 2004. "Effects of Irradiation and Post-Irradiation Annealing on the Thermal Conductivity/Diffusivity of Monolithic and $\mathrm{SiC}_{\mathrm{f}} / \mathrm{SiC}$ Composites," Journal of Nuclear Materials, 329-333:507. 
Attachment 1 to

Enclosure 9 to

MDO-723-0044/

B-MT(SRME)-52

Page 1

Attachment 4

ORNL Hot Cell Capabilities

Enclosure 9 to MDO-723-0044/B-MT(SRME)-52

PRE-DECISIONAL - For Planning and Dicussion Purposes Only 


\begin{tabular}{|c|c|c|c|}
\hline \multicolumn{3}{|c|}{ LAMDA (Low-activation) Hot cells } & \\
\hline \multirow{3}{*}{$\begin{array}{l}\text { Tensile } \\
\text { Testing }\end{array}$} & \multicolumn{2}{|c|}{ Mo grips, pins, pull rods, tungsten heating elements, } & \multirow[b]{3}{*}{ B-MT(SRME)-42 } \\
\hline & \multicolumn{2}{|c|}{ Currently - 500tb load cell, also has 1,000 lb load cell } & \\
\hline & \multicolumn{2}{|l|}{ atmospheric controls $=10^{\wedge}-6$ torr } & \\
\hline \multirow{2}{*}{$\begin{array}{c}\text { Density } \\
\text { Measurements }\end{array}$} & \multicolumn{2}{|l|}{ Archimedes Method } & \multirow[t]{2}{*}{ B-MT(SRME)-4i } \\
\hline & \multicolumn{2}{|l|}{ Fluorinert FC-43 ( $2 X$ density of water) } & \\
\hline \multicolumn{4}{|l|}{$\begin{array}{l}\text { Microhardness } \\
\text { Measurements }\end{array}$} \\
\hline \multirow[t]{2}{*}{ Thermometry } & \multicolumn{2}{|c|}{$\begin{array}{l}\text { SiC temperature monitors - isochronal anneals/resistivity } \\
\text { technique }\end{array}$} & \multirow[t]{2}{*}{$\overline{B-M T(S R M E)-42}$} \\
\hline & \multicolumn{2}{|l|}{ 4-point probe- copper contacts } & \\
\hline \multicolumn{4}{|c|}{ IMET (High activation/JOYO-1 Specimens) } \\
\hline \multirow{2}{*}{\multicolumn{4}{|c|}{$\begin{array}{l}\text { The Irradiationed Materials Examination and Testing BIdg. } 3025 E \\
6 \text { interconnected steel lined hot cells containing } 320 \text { square feet of of } \\
\text { Class III nuclear Facility }\end{array}$}} \\
\hline & & & \\
\hline \multicolumn{4}{|c|}{ Cell \#1 (No fuel/No alpha) Heavily used for specimen sorting and identification } \\
\hline \multirow{2}{*}{$\begin{array}{c}\text { Laser } \\
\text { Profilometer }\end{array}$} & BETA Laser Mike Model 162 & \multicolumn{2}{|c|}{ ORNL Webpage } \\
\hline & $\begin{array}{l}\text { Laser Mic on translation stage -- diameter of } \\
\text { pressurized tubes }\end{array}$ & \multicolumn{2}{|c|}{ Attachment 3 to $7248-1156-R J D$} \\
\hline Densitometer & $\begin{array}{l}\text { Radiation Induced Swelling }+1-0.2 \% \\
\text { Accuracy (for TEM specimens } \sim 15 \mathrm{mg} \text { mass) }\end{array}$ & \multicolumn{2}{|c|}{ Attachment 3 to $7248-1156-R J D$} \\
\hline \multirow{2}{*}{$\begin{array}{c}\text { Video } \\
\text { Equipment }\end{array}$} & Digital recording capabilities & \multirow{2}{*}{\multicolumn{2}{|c|}{$\begin{array}{l}\text { Attachment } 3 \text { to 7248-1156-RJD and } \\
\text { (ORNL Webpage) }\end{array}$}} \\
\hline & Fracture Surface Observation & & \\
\hline \multicolumn{4}{|l|}{ Cell \#2 } \\
\hline \multirow[t]{10}{*}{ Tensile Testing } & Instron tensile machine & \multirow{5}{*}{\multicolumn{2}{|c|}{ B-MT(SRME)-42 }} \\
\hline & $\begin{array}{l}\text { Mo grips, pull rods, tungsten heating } \\
\text { elements, soon to have Mo-47.5Re pins }\end{array}$ & & \\
\hline & $500 \mathrm{lb}$ load cell/1000lb cell & & \\
\hline & $10^{\wedge}-7$ torr vacuum & & \\
\hline & may be able to preform bend bar testing & & \\
\hline & 20kip load frame & \multirow{4}{*}{\multicolumn{2}{|c|}{ Attachment 3 to 7248-1156-RJD }} \\
\hline & Merlin Data Acquisition System upgrade & & \\
\hline & High vac with turbo pump & & \\
\hline & Temp capability $(1350 \mathrm{C})$ & & \\
\hline & Screw Driven Machine & & Webpage \\
\hline $\begin{array}{c}\text { Automated Ball } \\
\text { Indentation (ABI) }\end{array}$ & $\begin{array}{l}\text { Tension/compression testing at room } \\
\text { temperature }\end{array}$ & Attachment 3 & 48-1156-RJD \\
\hline Flow Properties & Variety of ball indenters are available for & ORNL Webpa & \\
\hline Testing & $\begin{array}{l}\text { determination of the yield and plastic flow } \\
\text { properties }\end{array}$ & Attachment 3 & 48-1156-RJD \\
\hline & Screw Driven Machine & Attachm & 7248-1156-RJD \\
\hline & Capable of very low strain rates & & \\
\hline & $\begin{array}{l}\text { up-gradable to reduced or elevated } \\
\text { temperatures }\end{array}$ & & \\
\hline Hardness Tester & $\begin{array}{l}\text { Mitutoyo automated microindintation } \\
\text { hardness tester }\end{array}$ & & Webpage \\
\hline & Loads 10-1000grams & & \\
\hline & Analyzed using $0.3 \mathrm{~s}$ reading speed & & \\
\hline
\end{tabular}




\begin{tabular}{|c|c|c|}
\hline Cell \#3 & & $\begin{array}{l}\text { Attar } \\
\text { Enc } \\
\text { MDO }\end{array}$ \\
\hline \multirow{5}{*}{$\begin{array}{l}\text { Charpy Impact } \\
\text { Testing }\end{array}$} & Tinius-olsen $\otimes$ charpy impact machine & \multirow{5}{*}{$\begin{array}{c}\text { Attachment } 3 \text { to } 7248-1156-R J D \text { and B- } \\
\text { MT(SRME)-42 }\end{array}$} \\
\hline & High temps (-196 to $400 \mathrm{C})$ & \\
\hline & $265 \mathrm{ft}-\mathrm{lb}$ capacity & \\
\hline & $\begin{array}{l}\text { Full size, } 1 / 2 \text { size, } 1 / 3 \text { size Charpy V-Notch } \\
\text { specimens }\end{array}$ & \\
\hline & Semi-automatic loading system & \\
\hline \multirow[t]{6}{*}{$\begin{array}{c}\text { Compact } \\
\text { Tension Testing }\end{array}$} & $\begin{array}{l}\text { MTS® Universal Testing Machine } \\
\text { (Servohydraulic) }\end{array}$ & \multirow[t]{4}{*}{ Attachment 3 to $7248-1156-R J D$} \\
\hline & 20 kip load frame & \\
\hline & Room temperature to $400 \mathrm{C}$ test in air & \\
\hline & $\begin{array}{l}\text { Fracture Toughness, 3-point bend, large } \\
\text { tensile crack-arrest capabilities }\end{array}$ & \\
\hline & High temps & \multirow[t]{2}{*}{ B-MT(SRME)-42 } \\
\hline & High vacuum & \\
\hline Cell \#4 & (Confict between Jessica's paper and the we & site and previous documents) \\
\hline \multirow[t]{7}{*}{ Tensile Testing } & $\begin{array}{l}\text { High Temperature (Room Temperature to } \\
700 \mathrm{C} \text { ) }\end{array}$ & B-MT(SRME)-42/ORNL Webpage \\
\hline & No Vacuum & B-MT(SRME)-42 \\
\hline & $\begin{array}{l}\text { MTS } \otimes \text { Universal Testing Machine } \\
\text { (Servohydraulic) }\end{array}$ & \multirow[t]{5}{*}{ Attachment 3 to $7248-1156-R J D$} \\
\hline & 20KIP Load Frame & \\
\hline & Vacuum Chamber $1.5 \times 10^{\wedge}-8$ torr & \\
\hline & strain and load control extensometry & \\
\hline & configured for hourglass fatigue testing & \\
\hline $\begin{array}{l}\text { Annealing } \\
\text { Furnace }\end{array}$ & Air Atmosphere $150-1000 \mathrm{C}$ & Attachment 3 to $7248-1156-R \mathrm{JD}$ \\
\hline $\begin{array}{l}\text { Resistlvity } \\
\text { Measurement } \\
\text { Equipment }\end{array}$ & 4-point probe- copper contacts & Attachment 3 to 7248-1156-RJD \\
\hline \multicolumn{3}{|l|}{ Cell \#5 } \\
\hline \multirow[t]{6}{*}{ SEM } & $\begin{array}{l}\text { FEI@ (Phillips) } \otimes \text { XL30 Scanning Electron } \\
\text { Microscope }\end{array}$ & B-MT(SRME)-42 \\
\hline & $\mathrm{LaB}_{6}$ Gun & \multirow[t]{5}{*}{ Attachment 3 to $7248-1156-\mathrm{RJD}$} \\
\hline & Mouse Driven, PC/ Windows Operation & \\
\hline & $\begin{array}{l}\text { Oxford/Link X-ray EDS Micro Compositional } \\
\text { Analysis }\end{array}$ & \\
\hline & Semi-remote web-based operation & \\
\hline & Web Interfaced Data Transfer & \\
\hline \multicolumn{2}{|c|}{ Cell \#6 SEM Sample Preparation Capabilities } & B-MT(SRME)-42 \\
\hline \multirow{4}{*}{$\begin{array}{c}\text { EMCOQ TM02 } \\
\text { Computer } \\
\text { Numeric Control } \\
\text { (CNC, } \\
\text { CAD/CAM) } \\
\text { Milling Machine }\end{array}$} & Automated, Programmable & \multirow[t]{4}{*}{ Attachment 3 to 7248-1156-RJD } \\
\hline & 10 tool stations for limming Drilling, etc. & \\
\hline & $\begin{array}{l}\text { Programmable for fabrication of charpy } \\
\text { impact, compact tension, and tensile } \\
\text { specimens }\end{array}$ & \\
\hline & Minor capsule Disassembly & \\
\hline $\begin{array}{c}\text { Wachs } \\
\text { Guillotine Saw }\end{array}$ & 4 inch max. dia. & Attachment 3 to $7248-1156-R J D$ \\
\hline $\begin{array}{l}\text { Slow speed } \\
\text { diamond saw }\end{array}$ & 1 in. dia. & Attachment 3 to $72 \overline{48-1156-R J D}$ \\
\hline
\end{tabular}

High temps (-196 to 400C) specimens

Semi-automatic loading system

(Servohydraulic)

20kip load frame

Room temperature to $400 \mathrm{C}$ test in ai

tensile crack-arrest capabilities

High temps

High vacuum

(Conflict between Jessica's paper and the website and previous documents)

$700 \mathrm{C}$

No Vacuum

(Servohydraulic)

20KIP Load Frame

acuum Chamber $1.5 \times 10^{\wedge}-8$ torr

train and load control extensometry

A fured for hourglass fatigue testing

PRE-DECISIONAL - For Planning and Dicussion Purposes Only 
Small bench type micro lathe

Specimen sorting/ identification station

Cell is equlpped with a 1-ton bridge crane for positioning of heavy equipment

\begin{tabular}{|l|l|l|}
\hline \multicolumn{2}{|l|}{} & \\
\hline Specimen Preparation Laboratory & \\
\hline 3 Glove boxes & $\begin{array}{l}\text { Struners Tenupol 3 Electropolishing Unit, } \\
\text { Twin Jet thinning, <0 C temp. control }\end{array}$ & \\
& Attachment 3 to 7248-1156-RJD \\
\cline { 2 - 2 } & Buehler slow-speed diamond saw & \\
\cline { 2 - 2 } & FIM specimen Preparation Capability & \\
\hline 3 ft. Fume Hood & & \\
\hline Metrology & Low activity level specimens & \\
\hline
\end{tabular}

Attachment 4 to

Enclosure 9 to MDO-723-0044/ B-MT(SRME)-52 Page 4 
The Manager, SNR

The Manager, PNR
Distribution to

MDO-723-0044

B-MT(SRME)-52

CONCURRENCE/DESIGN CHECK FORM FOR DOCUMENT NO. MDO-723-0044/B+MT(SRME)-52 Date: 1/31/06 DOCUMENT TITLE: JOYO-1 Irradiation Test Campaign Technical Close-out, for NR Information - (U)

REFERENCES MDO-723-0021 / B-MT(SRME)-21;

B-MT(SMAT)-005 / MDO-723-0019;

B-MT(SRME)-32 / MDO-723-0041;

MDO-723-0046 / B-MT(SPME)-23;

SM-7231-0009 / B-MT(SPME)-3;

MDO-723-0036 / B-MT(SRME)-37

B-MT(SRME)-29T; MDO-723-0043;

MDO 720-1137; $\quad$ B-MT(ME)-293;

B-MT(SRME)-51; $\quad$ B-MT(SRME)-50;

B-MT(SRME)-49; MDO-723-0057;

B-MT(EDT)S-018; $\quad$ B-MT(EDT)-014;

SM-7234-0010

1. ADSARS: PERMANENT RECORD: Yes $X$ No
ATTACHMENTS::

(1) JOYO-1 Structural Refractory Metal Materials Specimen

Fabrication Efforts

(2) JOYO-1 Superalloy Specimen Fabrication Efforts

(3) JOYO-1 Refractory Metal (Liner Materials) Specimen

Fabrication Efforts

(4) JOYO-1 Silicon Carbide Specimen Fabrication Efforts

(5) JOYO-1 Beryllium Oxide Specimen Fabrication Efforts

(6) JOYO-1 Temperature Monitors and Dosimetry Fabrication

Efforts

(7) JOYO-1 Capsule Design Close-out Report

(8) Alternative Disposition of JOYO-1 Structural Materials

Irradiation Rigs

(9) JOYO-1 Post-Irradiation Examination
FLIB Corporate KAPL NR PROGRAM S

Repository MFLIB Corporate KAPL NR PROGRAM S

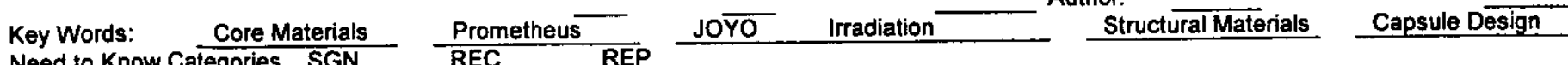

$\begin{array}{llll}\text { Need to Know Categories SGN } & \text { REC } \\ \text { Available Sites: } & \text { PRNR } & \text { REP }\end{array}$

Design File Location(s)

2. DESIGN CHECK

Type of Check

\begin{tabular}{|c|}
\hline A. No check considefed necessay \\
\hline B. Check vs. previous results/issues \\
\hline C. Checked calculations made \\
\hline D. Checked computer input and/or output \\
\hline Computer Programs approved/qualified \\
\hline f. Performed independent audit \\
\hline G. Spot checked significant points \\
\hline H. Reviewed methods used \\
\hline I. Reviewed results for reasonableness \\
\hline J. Comparison with test data \\
\hline K. Reviewed vs. drawings \\
\hline L. Verified procedures \\
\hline M. Technical content reviewed \\
\hline $\begin{array}{l}\text { N. Management verification of adequate } \\
\text { review by others }\end{array}$ \\
\hline O. Performed Lessons Learned Search \\
\hline P. Used Measurement Uncertainty Methods \\
\hline Q. Other Checks (Describe) \\
\hline
\end{tabular}

3. CONCURRENCE REQUIREMENTS: ARP MANAGER

\begin{tabular}{l} 
NUCLEAR ENGINEERING \\
REACTOR THMECH DES \\
REACTOR EQUIPMENT \\
POWER PLANT MECHANICA \\
POWER PLANT ELECTRICAL \\
PINANCE \\
\hline \\
PEW SHIP PROGRAMS \\
\hline
\end{tabular}

\begin{tabular}{l} 
NUCLEAR ENGINEERING \\
REACTOR THMMECH DESIGN \\
REACTOR EQUIPMENT \\
POWER PLANT MECHANICAL \\
POWER PLANT ELECTRICAL \\
\hline PINANCE \\
\hline PEW SHIP PROGRAMS \\
\hline
\end{tabular}

\begin{tabular}{l} 
NUCLEAR ENGINEERING \\
REACTOR THMECH DES \\
REACTOR EQUIPMENT \\
POWER PLANT MECHAN \\
POWER PLANT ELECTRICA \\
PINANCE \\
\hline
\end{tabular}

\begin{tabular}{l} 
NUCLEAR ENGINEERING \\
REACTOR THMMECH DESIGN \\
\hline \\
REACTOR EQUIPMENT \\
POWER PLANT MECHANICAL \\
POWER PLANT ELECTRICAL \\
FINANCE \\
PEW SHIP PROGRAMS \\
\hline
\end{tabular}

\begin{tabular}{l} 
NUCLEAR ENGINEERING \\
REACTOR THMECH DESIGN \\
REACTOR EQUIPMENT \\
POWER PLANT MECHANICAL \\
POWER PLANT ELECTRICAL \\
PINANCE \\
\hline \\
PEW SHIP PROGRAMS \\
\hline
\end{tabular}

\begin{tabular}{l} 
NUCLEAR ENGINEERING \\
REACTOR THMECH DES \\
REACTOR EQUIPMENT \\
POWER PLANT MECHAN \\
POWER PLANT ELECTRICA \\
PINANCE \\
\hline
\end{tabular}

\begin{tabular}{l} 
NUCLEAR ENGINEERING \\
REACTOR THMECH DES \\
REACTOR EQUIPMENT \\
POWER PLANT MECHAN \\
POWER PLANT ELECTRICA \\
PINANCE \\
\hline
\end{tabular}

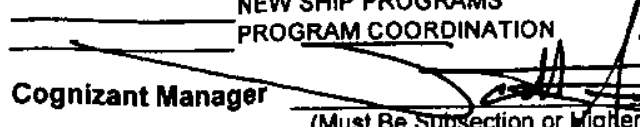

(Must Be Subsection or Mighter TorExtemartetters)

\section{AUTHORIZED CLASSIFIER:}

RELATED SUBJECTS:

UTRS Implication (Y/N)

Safety Council Review (Y/N)
Roviewed By:

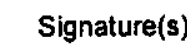

Signature(s)

Indicate signatures required by $X$ :

NCSG

ADVANCED CONCEPTS

NOISE \& ELEC. TECH.

SHIELDING

REACTOR SAFETY

To

RSO

FSO

SMDO
Comments: (Including Reference to Check Document If Appropriate)
FLUID DYNAM

STRUC. ENGRG DRAFTING

QA OTHER

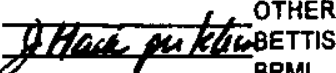

BPMI

ADMIN REVIEW
Commitment Made (Y/N)

Design Basis Info. (Y/N)

Design Review (Y/N)

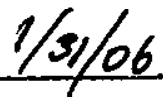

CLASSIFICATION: Unclassified

Commitment Complete $(\mathrm{Y} / \mathrm{N}) \quad \mathrm{Y}$ UTRS Doc. \# 
The Manager, SNR

The Manager, PNR
Distribution to

MDO-723-0044

B-MT(SRME)-52

Page 2 of 2

\section{DISTRIBUTION:}

\section{(*electronic copy only)}

\section{NR/SNR/PNR}

DI Curtis (5) (for information) -08S

JD Yoxtheimer 08S

C Oosterman

$\mathrm{J}$ Mosquera 08C

S. T. Bell - 08I

$H$ Miller/D Potts / $G$ Millis (SNR)

HA Cardinali (cover letter only)/JF Koury/JP Bannon/J. Andes(PNR)

\begin{tabular}{|c|c|c|c|}
\hline BETIIS & $\begin{array}{l}\text { MJ Zika* } \\
\text { CD Eshelman* } \\
\text { DP Hagerty* } \\
\text { WL Ohlinger* } \\
\text { SD Harkness* } \\
\text { JE Hack* } \\
\text { EV Mader } \\
\text { WS Saylor } \\
\text { R Baranwal } \\
\text { JL Bump } \\
\text { RF Luther* } \\
\text { ME Petrichek* } \\
\text { AM Tullai } \\
\text { DC Stambolis* } \\
\text { GD Carpenter* } \\
\text { CM Rodenbush* } \\
\text { DC Noe* } \\
\text { RA Reitz* } \\
\text { J Molitoris* } \\
\text { V Perone* } \\
\text { ADSARS/LIB }\end{array}$ & KAPL & $\begin{array}{l}\text { MJ Wollman* } \\
\text { DF McCoy* } \\
\text { C Dempsey* } \\
\text { J Ashcroft* } \\
\text { H Schwartzman* } \\
\text { SZ Hayden* } \\
\text { J Prybylowski* } \\
\text { KC Loomis* } \\
\text { C Jordan* } \\
\text { RA Mulford* } \\
\text { CM Regan* } \\
\text { S Simonson } \\
\text { BC Campbell* } \\
\text { L Kolaya* } \\
\text { T Angeliu* } \\
\text { T Schumaker* } \\
\text { Y Ballout } \\
\text { GA Newsome } \\
\text { MH Lane } \\
\text { JM Nash* } \\
\text { RS Northey }\end{array}$ \\
\hline
\end{tabular}

